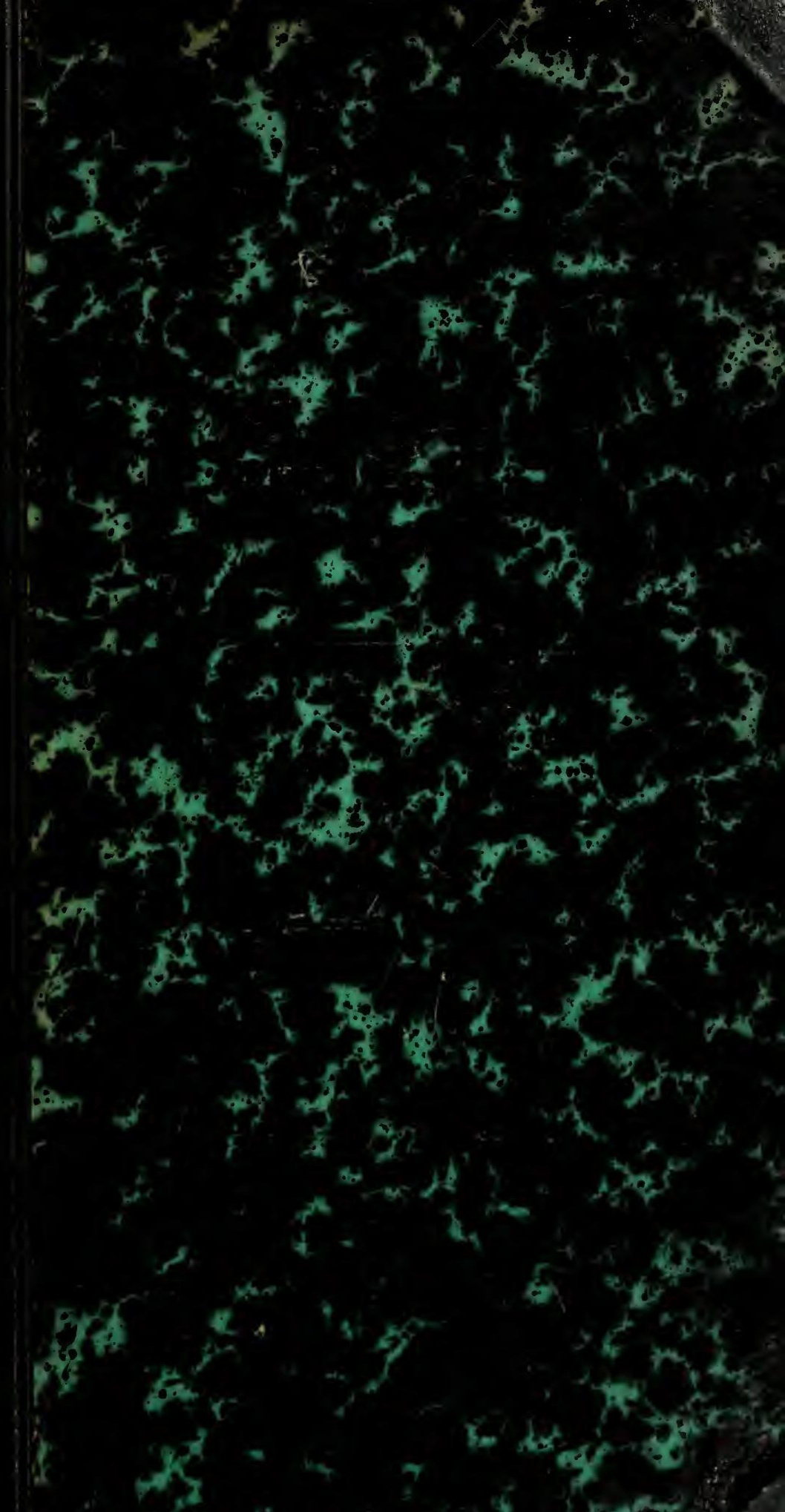




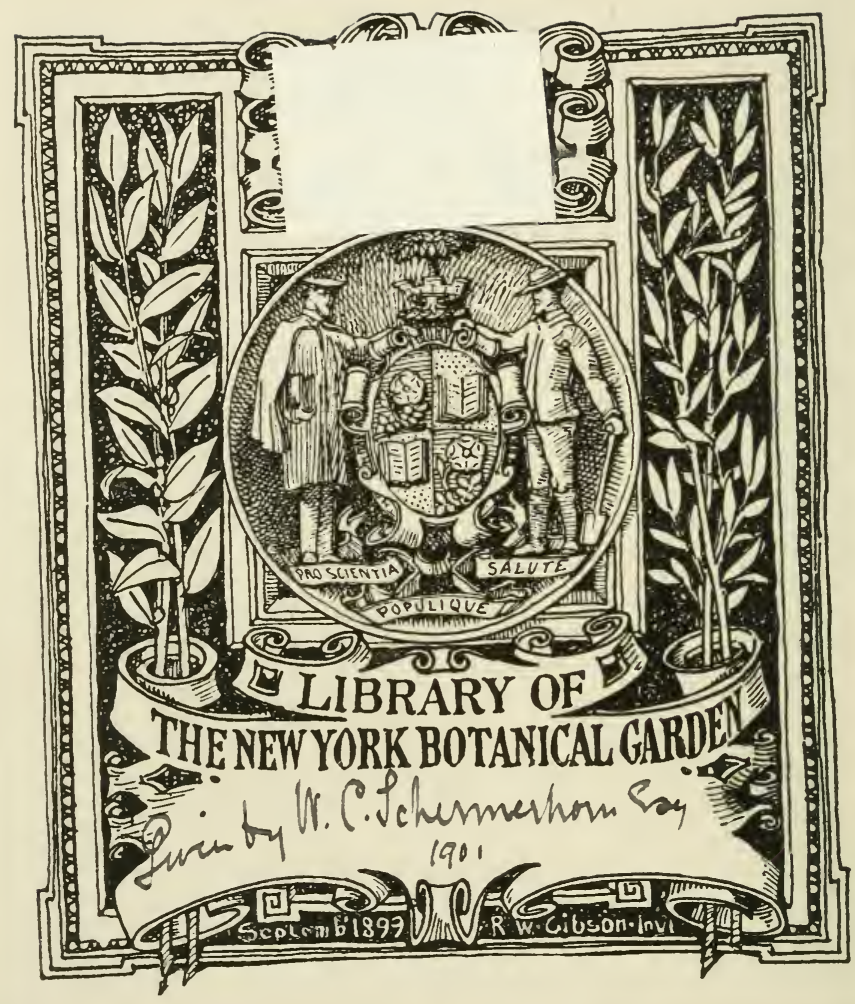







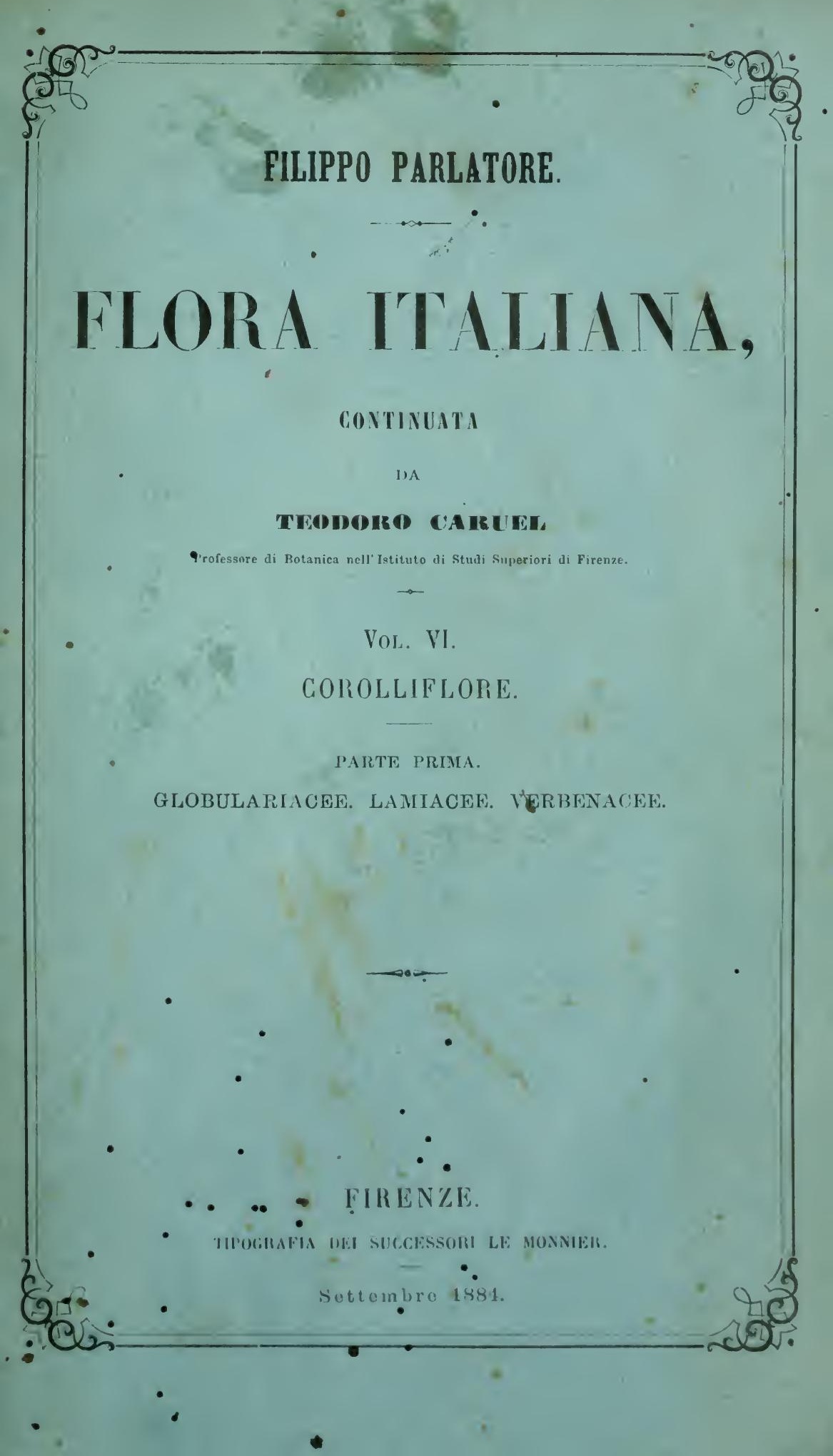




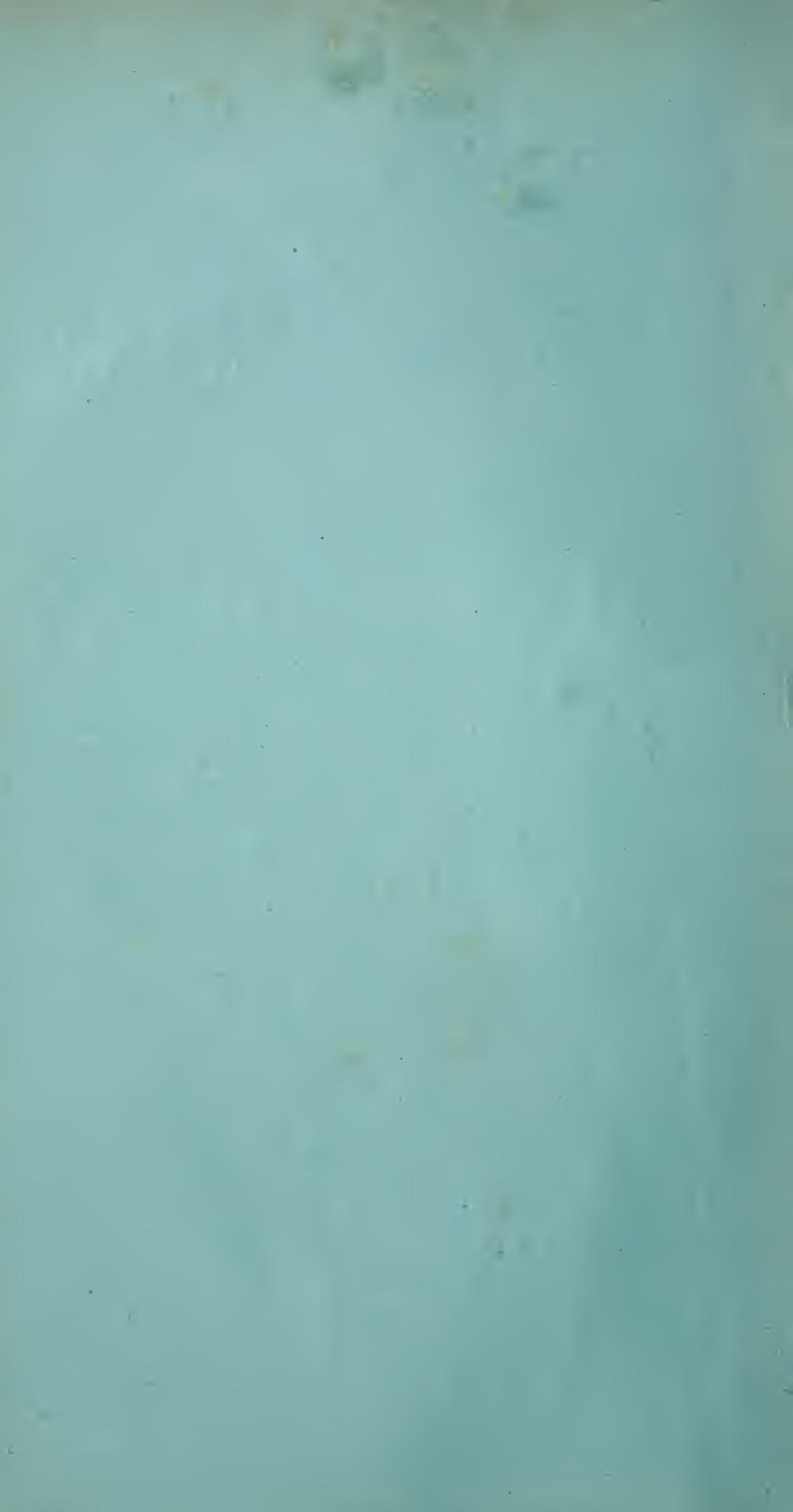




\section{FLORA ITALIANA.}





\section{FILIPPO PARLATORE.}

\section{FLORA ITALIANA,}

\section{CONTINUATA}

DA

\section{TEODORE CAIRUNA}

Professore di Botanica nell' Istituto di Studi Superiori di Firenze.

VoL. VI.

COROLLIFLORE.

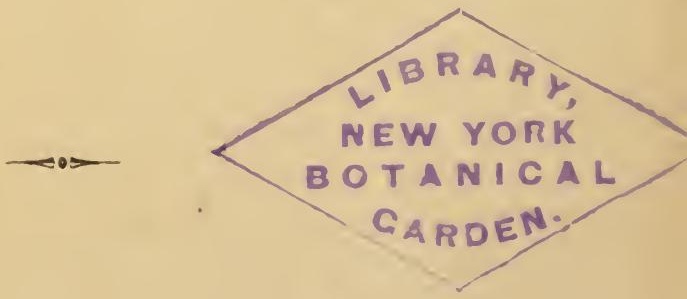

FIRENZE.

TIPOGMAFIA DEI SUCCESSOII LE MONNIER. 



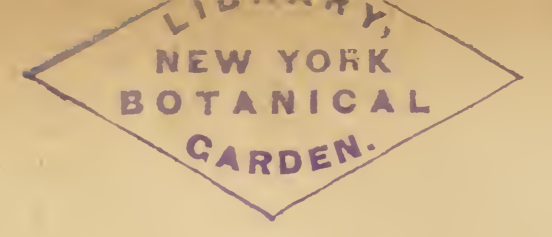

\section{AVVERTENZA.}

Il primo fascicolo della Flora Italiana comparve nel 1848, e nel 1875 l' ultimo sinora pubblicato, che completa il volume V, e porta l'opera sino alla specie posta sotto il numero 1381. Poichè le specie fanerogamiche d'Italia sommano a pressochè 5000 , la parte pubblicata dell' opera ne costituisce adunque meno del terzo. Della rimanente parte il lodatissimo Autore lasciò, morendo, il manoscritto, peró in istato incompleto, più o meno secondo le famiglie, e da non potersi stampare.

Dolente di vedere cosi rimasto in tronco un tanto libro, e stimando doveroso per me di continuarlo, e, potendo, ultimarlo, io mi sono determinato all'impresa, per quanto immane sia; speranzoso d'altronde che mi sarà alleviata dall' aiuto di colleghi botanici, i quali vorranno dividere con me il lavoro: fra i quali sono lieto di potere giả fare assegnamento sui signori prof. Arcargeli, L. Caldesi, prof. Gibelli, S. Sommier.

Nel dare mano alla pubblicazione dell'opera, Parlatore si era ripromesso condurla a fine con un numero non grande di volumi e di anni. La cosa è proceduta altrimenti, per più ragioni, fra le quali non ultima il modo sempre piủ largo col quale sono stati successivamente trattati i vari gruppi di piante, in maniera che la Flora Italiana aveva preso in fine piuttosto l'aspetto di una serie di elaborate monorrafie. In questa conti. uuazione, pure conservando la forma usata nei rolumi precedenti, io ho ricercalo una maggiore concisione, che diminuisca la mole dell'opera, senza togliere i suoi pregi migliori, e permetta di 
ultimarla in un numero minore di anni. A tale scopo ho creduto bene fare le seguenti modificazioni:

1. ${ }^{\circ}$ Per gli ordini e i sottordini, io ho ristretta la sinonimia, e sostituita una diagnosi alla descrizione latina. Piuttosto mi sono diffuso nelle osservazioni intorno a un argomento nuovo, i criteri cioè di distinzione delle famiglie nell'ordine o nel sottordine; e ho aggiunto un articolo nuovo, sulla distribuzione geografica dell'ordine.

2. Per le famiglie, io ho grandemente ristretta la loro sinonimia, limitandola in generale alla citazione del primo fondatore e di chi ne ha proposto il nome in quella forma che ho prescelta. Alla descrizione latina, anche qui ho sostituita una succinta diagnosi della famiglia, paragonata alle altre del medesimo ordine o sott' ordine. L'articolo detto portamento è stato chiamato col nome più esatto di descrizione, essendo infatti una descrizione della famiglia, fatta sulle sole specie italiane.

3. Anche per $\mathrm{i}$ generi ho limitato quasi esclusivamente la sinonimia alla citazione del fondatore, dei Genera plantarum di Bentham e Hooker, ultima opera comparsa sui generi delle piante, e del Compendio della flora italiana di Cesati, Passerini e Gibelli, ch'è anzitutto una illustrazione dei generi italiani. La lunga descrizione è stata surrogata al solito da una breve diagnosi differenziale.

4.0 La diagnosi delle specie è stata foggiata sulle sole specie italiane. Per i sinonimi, ho creduto bene valermi della Flora italica di Bertoloni, fatta molto bene in questa parte, omettendo, per tutte quelle specie dove mi trovo d'accordo con lui, che sono le più, la citazione degli autori giả da lui citati; cosi facendo, risparmio molto spazio, senz' alcun inconveniente, giacchè lo studioso è avvertito che per gli autori anteriori a Bertoloni bisogna ricorrere al suo libro, di cui non puó fare a meno chi si dà a studi estesi sulla flora d'Italia. Ho giudicato altresi poter tralasciare, salvo che in casi eccezionali, le opere sulla flora dei paesi contermini all' Italia. Ho poi soppressa del tutto la rubrica del nome italiano delle specie, perchè, ad eccezione di certi nomi notissimi e veramente italiani, come olivo o vite, i cosi detti nomi italiani delle piante sono mere finzioni, 
ignote nell' uso comune. Avrei voluto surrogarli coi nomi vernacoli usati nelle varie provincie, ma questi sono d'ordinario raccolti con troppo poca cura dagli autori perchè sia possibile prestarci molta fede. Infine, ritornando in questa come in altre cose al concetto spiegato nella prefazione alla Flora Italiana e praticato nel primo volume, la descrizione non è stata messa, fuorchè per piante non comuni e studiate sul vivo. Piuttosto ho serbato molto posto all' abitazione italiana delle specie, ritenen. dola parte importantissima dell'opera, e fra le più originali, perchè quella che in altri libri non si può trovare ${ }^{1}$ ed è anche (occorre soggiungere) la più difficile forse ad eseguire bene, per l'impossibilità di conoscere con tutta precisione ogni luogo in un páese cosi esteso come l' Italia, e per la frequente incertezza in cui la disuguale esplorazione botanica delle varie parti d'Italia pone lo studioso riguardo al grado della frequenza delle piante. Per contro ho indicata la distribuzione geografica in termini assai generali.

Le suddette modificazioni non sono state fatte che nella por. zione dell' opera scritta da me. Quanto ha lasciato scritto Parlatore è stampato tutto senza alterazione, e in modo da distinguersi dal testo non suo.

La classazione seguita è quella che resi di pubblica ragione nel 1881. ${ }^{2}$ Per procedere ordinatamente, ho dovuto ritornare indietro, e fare una succinta revisione dei gruppi maggiori delle Monocotiledoni, già trattate nei tre primi volumi della Flora, innanzi di cominciare l'illustrazione di quelle Dicotiledoni che stanno prime nel mio sistema. Le famiglie dicotiledoni già trattate nella Flora saranno ricordate in appresso al loro posto.

Firenze, 29 seltemtre 1883.

\section{T. Caruel.}

- Rammenterò in proposito che il punto esclamativo dopo una località vuol dire che io stesso vi ho veduta la pianta, dopo un nome di persona che ho veduta la pianta da essa racculta nell indicato luogo, e che il nome di un autore non seguito da punto esclamativo vuol dire che la localiti è data in un suo scritto

${ }^{2}$ Porsieri sulla lassinomia bolanica (Atti dell' Accademia dei Lincei). 



\section{DIVISIONE I.}

\section{FANEROGAME.}

Plante FlORIfERE veter.

Phenostemones Gleditsch.

Phenoganle Willd. 1804.

Vasculares phanerugane Bartl.

Phanerogame Brongn.

Anthophyta A. Braun.

Plantæ trimorphæ, alternantes, forma altera neutralis, duas sexuales producens. Forma neutralis indefinita, ex oosplææra in proembryone conversa ut embryo longitrorsus orta. Forma mascula definita: pollen cum favilla. Forma fœminea definita: gemmula dein semen, oosphæras in oogonio clauso continens.

\section{CLASSE PRIMA.}

\section{ANGIOSPERIME.}

Angiosperme Brongn.

Pollen subunicellulare. Gyneceum completum. Gemmula in gemmulario contenta, amygdalum post foecundationem gignens in oogonio apicali. 


\section{SOTTOCLASSE PRIMA.}

MOINOCOTILEDONI.

Monocotyledones Juss. gen. Parl. fl. ital. 1. p. 27.

Embryo cum embryophyllo unico raginante, rare nullo.

\section{COORTE PRIMA.}

HIRIANTE.

LiRianthe Car. pens. tass. bot. p. 35.

Flores e verticillis 5 alternantibus, quorum 2 pro perianthio, 2 pro androceo, 1 pro gyneceo, completis, vel sæpe varia imminutione incompletis aut uno alterove deficiente usque ad 1 reductis, rarissime multiplicatis.

\section{ORIDINE PRIMO.}

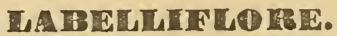

Labelliflore Car. pens. tass. bot. p. 39.

Inflorescentia nec spadix nec spicula. Flores irregulares, præsertim in androceo, cum perianthio completo, rarissime ob petalum 1 deficiens incompleto, cum androceo ad stamina 5-1 reducto, rare completo regulari, cum gyneceo nunc incompleto nunc completo regulari.

Osservazioni. - Quest'ordine si scinde agevolmente in 3 sottordini, Ginandre, Scitamini ed Efemeri. In ognuno le famiglie formano una serie, che da quelle di fiore irregolare in sommo grado tende ad altre di fiore poco irregolare, od anche regolare affatto. Mancando in Europa il sottordine degli Efemeri, contraddistinto dal gemmulario supero (eccettochè in un genere di Tecofileacee), e mancando agli altri due sottordini le famiglie meno irregolari, ne risulta 


\section{LABELLIFLORE.}

una diagnosi molto più esclusiva per le Labelliflore europee, che formano perciò un gruppo ben distaccato da tutte l' altre Nonocotiledoni.

Le affinità prossime delle Labelliflore sono con le Liliiflore, di cui riproducono il tipo fatto irregolare, e con cui si connettono inti. mamente per le Commelinacee regolari e massime per le Pontederiacee; e tali affinità sono specialmente con quelle famiglie, come le Bromeliacee, che hanno distinzione di calice e di corolla, e con quelle, come le Iridacee, le Burmanniacee ecc., che hanno l'androceo ridotto.

Distribuzione geografica. - Essendo ordine più abbondante nella zona torrida, ne mancano in Italia parecchie famiglie, e quelle ivi esistenti sono rappresentate da un numero ristretto di generi e di specie.

\section{SOTTORDINE PRIMO.}

\section{GINANDRE.}

Gynandre Lindl. nix. plant. Parl. fl. ital. 3. p. 331.

Gemmularium inferum. Semen examygdalosum. Embryo aphyllus.

Osservazioni. - Le modificazioni dell'androceo, e secondariamente il gemmulario 1-loculare o 3-loculare, danno il modo di spartire il sottordine in 4 famiglie: Orchidacee, Cipripediacee, Apostasiacee A. Rich. e Corsiacee Becc. Malesia p. 238, di cui le ultime due esotiche.

\section{FAMIGLIA PRIMA.}

ORCHIDACEE.

OrChides Lindl. nix. plant. Parl. fl. ilal. 3. p. 333 (excl. Cypripedeae).

\section{FAMIGLIA SECONDA.}

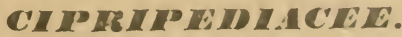

Cipripedie Lindl. nix. plant.

Crirriplede trib. Orchid. Parl. fl. ital. 3. p. 564. 


\section{SOTTORDINE SECONDO.}

SCITAIIINI.

Scitamina Linn. phil. bot.

Scitaminea Linn. Parl. fl. ital. 3. p. 320.

Gemmularium inferum. Semen amygdalosum. Embryo monophyllus.

\section{FAMGLA TERZA.}

C.INNACEL.

Cannez $R$. Br.prodr. fl. Nov. Holl.

Cannace e Agardh aphorism. Parl. fl. ital. 3. p. 321.

\section{ORIDINE SECONDO.}

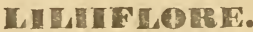

Liliflore Car. pens. tass, bot. p. 39 (non Agardh nec alior.)

Inflorescentia nec spadix nec spicula. Flores regulares vel subregulares, cum perianthio completo, rarissime incompleto aut etiam deficiente, cum androceo completo, rare ob verticillum unum alterumve deficiens incompleto, rare aucto, cum gyneceo completo, rarissime incompleto.

Osservazioni. - Sono già state accennate le affinità delle Liliiflore con l'ordine precedente. Dall'altra parte si connettono mediante la loro famiglia delle Fenicacee intimamente con le Spadiciflore, e mediante le Restionacee, le Eriocaulonacee ecc., si accostano in qualche modo alle Glumiflore. Hanno anche relazioni con la famiglia delle Juncaginacee fra le Alismiflore.

La lunga serie delle farniglie che ho riunito in quest'ordine si spartisce in due sottordini di assai ineguale estensione, le Orchioidi, ristrette alle Burmanniacee e Tismiacee, e le Coronarie, che abbracciano tutto il resto. 
Distribuzione geografica. - Le Liliflore sono largamente sparse tanto per i paesi temperati che per i caldi; per cui sono anche largamente rappresentate in Italia. Se vi manca è vero metà delle famiglie, sono quasi tutte delle minori, o di quelle localizzate, come le Bromeliacee americane, le Restionacee dell' emisfero australe ecc. Dei due sottordini, il secondo solamente, delle Coro. narie, trovasi in Europa.

\section{SOTTORDINE.}

\section{CORONARIE.}

Phenicordee et Lilioidee Parl. fl. ital. 2. p. $271,280$.

Coronarie (non Linn. nec alior.).

Semen amygdalosum. Embryo monophyllus.

Osservazioni. - Ad eccezione delle Fenicacee o Palme, che formano tale una famiglia da non confondersi con altre, le rimanenti Coronarie hanno fra di loro legami si stretti, da renderne difficile assai la distribuzione fra famiglie bene distinte; talchè, se si tolgono le Iridacee, già costituite solidamente sin dai tempi di B. de Jussieu c di Adanson, di tutte l'altre famiglie di Coronarie si puo dire che non hanno ancora limiti certi, ma vanno soggette a vederli spo. stati di continuo. I recenti lavori di Bentham e soprattutto di Baker, pubblicati nei vol. XI-XVII del Journ. of the Linn. soc., hanno schiarito molte questioni relative all'argomento, ma senza dare un risultato più soddisfacente che per l'innanzi in quanto alla' costituzione stessa delle famiglie; ciò senza dubbio perchè la estesissima cognizione del gruppo nci sullodati autori non è stata sorretta dal principio della equivalenza necessaria fra i gruppi minori da stabilirsi in esso, vale a dire per le famiglie; onde, come del resto universalmente si usa, i medesimi caratteri qua ritenuti validi a contrassegnare certe distinzioni, là no, essendo costretti a cedere il passo ad altri caratteri, i quali altrove perdono ogni valore. I caratteri devonsi non soltanto pesare e misurare, ma il peso e la misura devono essere uguali in tutta l'estensione di un gruppo quando si tratta di suddividerlo.

Impertanto dacgli studi fatti sembra essere risultato un accordo in quanto al poco o niun valore familiare di certi caratteri, ai quali altra volta si dava importanza, come sarebbero le modificazioni del 
corpo vegetativo, l'infiorescenza, il gemmulario uniloculare o triloculare, la natura del guscio seminale. Degli altri caratteri, più 0 meno adoperati, conviene indagare l'importanza.

Dietro esame attento, sembrami che due siano gli ottimi fra tutti, perchẻ congiungono i pregi di una gran generalità, e della massima determinatezza. Il primo si riferisce alla gemmula, secondochẻ sia diritta, ortotropa, o rovescia in qualche maniera, anatropa, semi-anatropa, campilotropa ecc. (essendovi tutti gli intermedi fra queste modificazioni); carattere questo che ne implica altro per l'em. brione, il quale è antitropo nel caso della gemmula ortotropa, omo. tropo (o vago) negli altri casi. Il secondo carattere è pure dell'embrione, secondochè sia estrario, situato esternamente alla mandorla, 0 intrario, circondato da questa.

Altro buonissimo carattere è somministrato dall' androceo, che tipicamente diciclico, talora si riduce monociclico per la soppressione, ora del verticillo esterno, ora del verticillo interno. Il fatto stesso della soppressione non ha in sè alcun valore, perchè si palesa quanto mai incostante in molti gruppi riconosciuti, perfino nei generi e nelle specie; ma devesi accordare per contro molto valore, per la sua assoluta costanza, alla natura del verticillo soppresso: è questo per esempio il principalissimo carattere che distingue le Iridacee dalle famiglie affini.

Il perianzio somministra altro carattere generale al pari del precedente, ma di molto minore determinatezza e perciò inferiore, giusta le sue modificazioni di consistenza e di colorito, per cui ora è distinto in calice e in corolla, ora è un perigonio, che può variare dall' essere corollino, o pure calicino quando è scarioso.

Tra i caratteri da considerarsi ancora come generali sonovi le modificazioni del frutto. Non vi si accorda d'ordinario tutta quell' im. portanza che a senso mio dovrebbero avere, forse per la inesattezza con la quale bene spesso sono descritte, conseguente allo stato poco soddisfacente in cui versano tuttora gli studi carpologici. Eppure studiato più accuratamente, il frutto delle Coronarie offre nelle sue principali modificazioni tale una determinatezza, nonostante certi passaggi, da fornire un carattere distintivo che va apprezzato; secondochè esso frutto si presenti foggiato a bacca, o ad achenio, 0 a carcerulo (come l'avrebbero chiamato Mirbel e Desvaux), o a setticido, o a cassula vera loculicida (con la quale si potrebbe confondere i pochi casi quando è settifraga), o.a legume.

Fra i caratteri piủ particolari il primo posto va dato senza con- 
trasto al gemmulario, infero, o supero; con esempi è vero non rari di passaggi dall'uno all'altro, che diminuendo la determinatezza del carattere ne diminuiscono ancora il valore.

Ora vengono sotto esame le modificazioni dell'antera, molto adoperate per la classazione del gruppo, ma intese non poco confusamente. Sono relative alla inserzione, ch'è dorsale, o ventrale, o basilare; oppure ai modi della deiscenza, che si fa per fendicure longitudinali, nella faccia dorsale, o nella ventrale, o nei lati dell'antera; talora accadendo che la fenditura ha i suoi labbri discosti ad una estremità, da simulare ivi un poro, che come tale è stato descritto, così è certissimamente nei generi Galanthus, Leucoium, Calectasia, probabilmente in altri come Conanthera, Cumingia, Dianella ecc. in cui le figure mostrano un solco longitudinale in proseguimento del foro, e forse in tutti i casi di creduta deiscenza apicale. Ad ogni modo, i passaggi fra la inserzione dorsale e la ventrale mediante la basilare, e fra la deiscenza introrsa e la estrorsa mediánte la latrorsa, diminuiscono valore a siffatti caratteri.

In ultimo il carattere dello stilo, tratto dalla sua interezza 0 dalla divisione. Ma sono tanti i casi intermedi, fra lo stilo il piủ intero, e quello diviso fino alla base, trovandosi essi anche esemplificati quasi fino agli estremi in una medesima famiglia bene assodata qual'è quella delle Iridacee, che dessi non lasciano altro che un minimo di valore da accordare al carattere, e non superiore a quello che generalmente si accorda nel gruppo alla unisessualità 0 bisessualità dei fiori.

Cosicchè, dalle precedenti riflessioni io sarei condotto a graduare i caratteri familiari delle Coronarie come segue: in prima linea quelli della gemmula, e dell' embrione, - in seconda quelli dell'androceo, - in terza, del perianzio e del frutto, - in quarta, del gemmulario e dell'antera, - in ultima, dello stilo e della sessualità.

Procedendo su queste basi alla costituzione delle famiglie delle Coronarie, io trovo da riconoscere le seguenti:

$1^{\circ}$ Le Vellosiace e (Vellosiece Brongn.). Martius e Zuccarini (Nova gen. et sp. plant. Bras. 1. p. 13) e Seubert (in Fl.bras. fasc. 9. p. 66) ne descrivono l'embrione come intrario, ma senz'addurre figure in appoggio); per contro Le Nlaout e Decaisne (Tr. gén. p. 564, 565 ) ricisamente lo descrivono e figurano estrario, e la cosa sembra confermata da qualche espressione di Baker (in Journ. Linn. soc. 17. p. 94); e se realmente è cosi, la famiglia riesce distintissima dalle Amarillidacee, con le quali altrimenti si confonderebbe. 
2. Le Bromeliaces vere, con gemmulario infero, e con una bacca per frutto.

3. Le Tillandsiace e, da togliersi dalle Bromeliacee degli autori perchẻ hanno il gemmulario supero o semisupero, e un setticido per frutto: sia un vero setticido, sia un frutto che vi si avvicina, e non una cassula come dicono gli autori, cosi ho verificato in parte sul vivo, in parte su saggi secchi, o in parte su figure.

$4 .^{\circ}$ Forse le Kingiacese, arendo aspetto di famiglia buona questo gruppo proposto da Endlicher (Gen. plant. p. 132) in calce alle Iuncacee. L' embrione della Kingia è descritto come "semi-immerso: " intendasi estrario; le gemmule, basilari erette, sono descritte come anatrope; ma nel Dasypogon bromeliaefolius le ho vedute orto. trope, con doppio integumento, e cinte alla base da un terzo invoglio obliquo; il frutto è un achenio; il perigonio è scarioso. Bentham e Mueller ( $\mathrm{Fl}$. austral. 6) inseriscono in questo gruppo anche la $\mathrm{Ca}$ lectasia $\mathrm{R}$. Br., le di cui gemmule sono certamente anatrope, e la Baxteria R. Br. (in Lond. journ. of bot. 2. p. 492) che ha per frutto un settifrago; dell'una e dell'altra va studiato l'embrione.

$50^{\circ}$ Le Rapateace Schomb.

6. Le Mayacace. Walp.

7. Le Xyridacee Lindl.

8. ${ }^{\circ}$ Le Eriogaulonace (Eriocaulece auct.).

$90^{\circ}$ Le Restionacese (Restiacece auct.).

10. Le Flagellariacese (Flagellariece Brongn. e Gris in Bull. soc. bot. de Fr. 8. p. 268), famiglia distinta dalle precedenti affini per il frutto ch'è una bacca. Con essa si chiude la serie delle Coronarie coll' embrione estrario. Passando all' altra serie coll' embrione intra. rio, vien dato naturalmente un posto estremo alle

11. Smilacace (Smilacece Lindl.), unica famiglia della serie che abbia gemmule ortotrope. Quindi

12. Le Stemonacee (Roxburghiacece Lindl.), distintissime per lo spermoforo centrale e per il frutto ch'è un legume.

13..$^{\circ}$ Le Iridace e Lindl.

$14 .^{\circ}$ Le Taccacere Dum. Il frutto, descritto dagli autori come una bacca, "par che sia invece un frutto secco indeiscente 0 non facilmente deiscente, con molti semi, insomma un carcerulo.

- 15. Le Hypoxidace e Lindl. Tengo separata questa famiglia, non per i semi che non hanno (secondo si assevera) particolari che bastino a ciò, come ho dimostrato altrove (in Nuovo giorn. bot. ital. 10. p. 94), ma per la natura del frutto, il quale, descritto 
ora come cassulare, ora come baccato, ho ragione di ritenere, stando principalmente alle figure di Wight (Ic. plant. Ind. or. 6. t. 2042-2046), che sia sempre quale l'ho veduto in più specie di Hypoxis, membranoso indeiscente, un carcerulo.

16. Le Dioscoreacese (Dioscoridece Dum.), ristrette a quelle di frutto cassulare, o almeno secco, per non escludere il gen. Rajania.

17. Le Tamnacee (Tamnece Dum.), costituite con le Diosco. riacee aventi una bacca per frutto.

$18 .^{\circ}$ Le Haemanthaces, ossia Amarillidacee baccate; vicinissime invero alle precedenti, che non se ne distinguono se non per le antere ventrifisse estrorse, e per i caratteri infimi che sono la unisessualità dei fiori e la divisione dello stilo.

19. Le Amaryludacee, con l'aggiunta delle Emodoracee dal gemmulario infero, e coll'esclusione dei generi haccati.

20. Le Liriopaceæ (Liliacece Liriopece Bak.), singolari per il frutto che si rompe irregolarmente prima della maturazione dei semi.

21. Le Asparagacee, nel senso in cui Baker le considera quale suddivisione delle Liliacee (in Journ. Linn. soc. 14), e con l'aggiunta di altri generi baccati come Lapageria, Philesia, Astelia.

22. Le LiLIACE E, ristrette assolutamente a quelle dotate di una vera cassula loculicida; e con l'aggiunta delle Emodoracee dal gemmulario supero, del genere Narthecium, del genere Aphyllanthes ed altri affini, che all'infuori del portamento nulla hanno per differenziarli dalle Liliacee tipiche, dei generi Conanthera e Cumingia che, come il Neclaroscordum, hanno il gemmulario alquanto infero, e perciò pendono verso le Amarillidacee.

23. Le Uvulariaces Walp., esattamente intermedie alle Liliacee, di cui hanno il frutto, e alle Colchicacee, di cui hanno le antere e lo stilo.

24. Le Colchicaces, dal frutto setticidale e non altrimenti.

25. ${ }^{\circ}$ Le Juncace Koch, alle quali si connettono senza diffi. coltà certi generi come Xerotes, Yanthorrhaea, che ne hanno tutti i caratteri più essenziali.

20. Infine le Piroenicaces Lem., o Palme. 


\section{FAMIGLIA PRIMA.}

SIITLACACER.

Surace Lindl. intr. nat. syst. A. de Cand. mon. phan. 1. Parl. fl. ital. 3. p. 5.3 (trib. Asparag.).

\section{FAMIGLIA SECONDA.}

\section{IRTACEE.}

Iridea Vent. tabl. règne vég. 2. Parl. fl. ital. 3. p. 209.

- Iridaces Lindl. nat. syst. bot.

\section{FAMIGLIA TERZA.}

TAMINACEE.

Tamnes Dum. anal. fam.

Dioscoreace.e trib. Tames Parl. fl. ital. 3. p. 62.

\section{FAMIGLIA QUARTA.}

AITARITIDACEE.

Amaryllidee R. Br. et auct. (excl. gen.). Parl. fl. ital. 3. p. 66 .

\section{FAMIGLIA QUINTA.}

ASPARA GACEE.

Asparaginee Parl. $f l$ ital. 3. p. 5 (excl. Smilac.).

Asparagaceas ser. Liliac. Bak. in journ. linn. soc. 14. 


\section{FAMIGLIA SESTA.}

COLCHICACEE.

Colchigace a Cand. fl. fr. 3.

Melanthace $R$. Br. et auct. (pro parte). Parl. fl. ilal. 3. p. 166.

\section{FAMIGLIA SETTIMA.}

\section{HILICEE.}

Narthecię trib. Juncac., Aphyllanthace e et Liliace $e$ Parl. $f$. . ital. 2. p. 360, 362, 369 .

LILIACE E VERE Bak. in journ. linn. soc.

\section{FAMIGLIA OTTAVA.}

IUNCACER.

JunCACE K Koch syn. fl. germ. Parl. fl. ital. 2. p. 282 (excl. Narthec.).

\section{FAMIGLIA NONA.}

FEVICACEE.

Palme Linn. phil. bot. Parl. fl. ital. 2. p. 272.

Phoenicacee Lem.

\section{ORDINE TERZO.}

SIPADICHFT,DIEL.

Spadiciflora: Endl. gen. pl p. 232. Car. pens. tass, bot. p. 39. Aroides et pars Naj.d. Parl. /l. ilal. 2. p. 221, 3. p. 619. 
Inflorescentia spadix. Flores cum perianthio completo regulari, vel saepius incompleto aut deficiente, cum androceo completo v. in. completo, cum gyneceo saepius incompleto.

Distribuzione geografica. - L'ordine essendo più specialmente della zona calda, trovasi peraltro rappresentato in Italia da quasi tutte le sue famiglie autentiche, ma la principalissima, delle Aracee, con un numero ben scarso di generi e di specie.

\section{FAMIGLIA PRIMA.}

\section{ARACEE.}

AROIDE Juss. gen. pl. (excl. gen.).

Araces Schott melet. Parl. fl. ital. 2. p. 222.

\section{FAMIGLIA SECONDA.}

TEMINACEE.

Lemnacee Duby bot. gall. Parl. fl. ital. 3. p. 666.

\section{FAMIGLIA TERZA.}

TIIACEE.

TYPHe Juss. gen. pl.

Typhacese Cand. fl. fr. Parl. fl. ital. 2. p. 259.

\section{ORDINE QUARTO.}

\section{GLUMLELIRE.}

Glumaces Parl. fl. ital. 1. p. 33.

Glumiflores Car. pens, tass. bot. p. 39.

Inflorescentia spicula. Flores cum perianthio atrophico, sæpius incompleto aut deficiente, rare completo aut aucto, cum androceo 
completo aut incompleto, rarissime aucto, cum gyneceo completo aut saepius incompleto, rarissime aucto.

Distribuzione geografica. - Se la famiglia piccolissima delle Centrolepidacee è assente dalla nostra flora, le due altre vastissime delle Poacee e delle Ciperacee ne formano gran parte, a segno che le Glumiflore formano una metà delle Monocotiledoni italiane, e stanno al quarto posto per numero di generi e di specie fra gli ordini fanerogamici.

\section{FAMIGLIA PRIMA.}

POACEE.

Gramina Hall. en. stirp. Helv.

Gramine $Æ$ Juss. gen. $p l$.

Graminace L Lindl. veg. kingd. Parl. fl. ital. 1. p. 34.

\section{FAMIGLIA SECONDA.}

\section{CIPERACEE.}

Cyperoideæ Juss. gen. pl.

Cyperace e Cand. n. fr. Parl. fl. ital. 2. p. 5.

\section{COORTE SECONDA.}

IDIRNTE.

Hydranthe Car. pens, tass, bot. p. 35.

Flores $e$ verticillis $1-\infty$ alternantibus, varie inter perianthium, androceum et gyneceum distributis, rare incompletis, rare perianthium deficiens. 


\section{ORDINE PRIMO.}

\section{AEISIIITLIDERE.}

Helobie Parl. fl. ital. 3. p. 568 (non alior.). Alismiflora Car. pens. tass. bot. p. 39.

Flores cum perianthio, rarissime nudi. Embryo micropodus.

Distribuzione geografica. - Piccolo ordine, che anche in Italia figura scarsamente nell' insieme della flora.

\section{SOTTORDINE PRIMO.}

INFERE.

INFere Car. pens, tass. bot. p. 40.

Gemmularium inferum.

\section{FAMIGLIA PRIMA.}

\section{IDAROCARITACEE.}

Hydrocharides Cand. $f . f r$. Parl. f. ital. 3. p. 570. Hydrocharitacee Asch. fl. prov. Brandenb.

\section{SOTTORDINE SECONDO.}

SUPERE.

Superæ Car. pens. tass. bot. p. 40.

Gemmularium superum. 


\section{FAMIGLIA SECONDA.}

\section{IBUTOIIA CWE.}

Butoneæ L. C. Rich. in mém. mus.

Butomace Gray arr. brit. pl. Parl. fl. ital. 3. p. 586.

\section{FAMIGLIA TERTA.}

ALISUICEE.

Alisuacee L. C. Rich. in mém. mus. Parl. fl. ital. 3. p. 590.

\section{FAMIGLA QUATTA.}

DENCATINACEE.

Jungagineae $L$. C. Rich. in mem. mus. Parl. A. ital. 3. p. 607.

\section{ORDINE SECONDO.}

\section{FEUVIITLOTE.}

NAJAdes pro parte Parl. fl. ilal. 3. p. 619.

Fluvilflore Car. pens. tass. bot. p. 40.

Flores nudi. Embryo macropodus.

Distribuzione geografica. - L' unica famiglia di quest' orline annovera in Italia $1 / \mathrm{s}$ delle sue specie.

\section{FAMIGLIA UNICA.}

\section{POTARIOERTONACEE.}

РОtamogetonea Reich. consp. regni veg. p. 43.

Nasadace.e part. Parl. 凡. ital. 3. p. 620. 


\section{COORTE TERZA.}

Centrante.

Centranthe Car. pens. tass. bot. p. 35.

Flores e stamine centrali aut e pistillo centrali efformati, cum perigonio vaginante duplici aut simplici aut nullo.

\section{ORDINE UNICO.}

CEN'TIEITARE.

Centriflore Car. pens. tass. bot. p. 40 (non Fries).

Distribuzione geografica. - Questo rninimo ordine, di unica famiglia, unico genere e una $10^{\text {a }}$ di specie, ne conta 2 in Italia.

\section{FAMIGLIA UNIGA.}

\section{NAIAIDACEE.}

Najadace e trib. Najade e Parl. fl. ital. 3. p. 661. NAJAdEe Gren. in Gren. et Godr. fl. de Fr. 3. p. 321. NAJADACE Magnus in nuovo giorn. bot. ital. 2. p. 186.

\section{SOTTOCLASSE SECONDA.}

\section{DICOTILEDONI.}

Dicotyledones Juss. (part.). Parl. fl. ital. 4. p. 5 (part.).

Embryo cum embryophyllis duobus oppositis, rare nullis. 


\section{COORTE PRIMA.}

\section{DICLAMIDANTE.}

Dichlamydanthe Car. pens. tass. bot. p. 60.

Flores regulares vel sæpissime irregulares, bisexuales monomorphi, rarius et tantum atrophia aut abortu unisexuales, rarissime dimorphi, plerumque 5 -meri. Perianthium e verticillis 2 isomeris alternantibus, quorum 1 pro calyce, 1 pro corolla, rare (in floribus regularibus) anisomeris vel non rite alternantibus, nunc uno alterove (rarissime utroque) abortu deficiente. Androceum plerumque e verticillo unico cum perianthialibus (in floribus regularibus) isomero, aut e verticillis 2 isomeris alternantibus, rarius meiomerum, vel pleiomerum et tunc etiam helicatum.

\section{SOTTUCOORTE PRIMIA.}

SPiANatio.

Explanate Car. pens. tass. bot. p. 60.

Thalamus (hypogynus vel epigynus) convexus, planus vel leviter incavatus.

\section{ORDINE PRIMT.}

COHOHETHARE.

Monopétales hypogynes Adr. de Juss. cours élém. d'hist. nat., bot. Bicarrellate (series Gamopet.) Benth. et Hook. gen. pl. 2. p. IV (excl. fam.).

Conolliflohe Car. pens. tass. bot. p. 72 (non Cand.).

Flores regulares, vel ex toto zygomorphice et fere semper recte irregulares. Calyx manifestus, hypogynus, rare epigynus. Corolla cum calyce isomera alternans, rarissime pleiomera, hypogyna, rare 
epigyna, gamopetala. Androceum cum corolla isomerum alternans, in floribus irregularibus tantum meiomerum, epicorollinum. Gyneceum rare cum androceo isomerum alternans, plerumque reductum et fere semper ad pistilla 2 , recte aut oblique antero-posteriora. Gemmularium superum, rare semi-inferum vel inferum.

Osservazioni. - Come l' ho circoscritto, quest' ordine è dei piủ naturali; tanto è vero che i reciproci rapporti delle sue famiglie sono ricordati da tutti $\mathrm{i}$ tassinomisti. Corrisponde al gruppo delle Bicarpellatee di Bentham e Hooker, esclusione fatta delle Oleacee, Salvadoracee, Columelliacee e Plantaginacee, che riferisco ad altri ordini, e con dubbia ammissione delle Loganiacee: almeno questa famiglia, fattasi, cosi eterogenea fra le mani di Endlicher, ed ancora rimasta tale abbenchè appurata da Bureau, accanto a tipi che sono certamente delle Corolliflore ne racchiude certuni, come il genere Usteria, che non combinano più coi caratteri dell'ordine.

Adr. de Jussieu o. c. aveva già indicata la più ovvia spartizione del gruppo in altri due, fondati sulla regolarità del fiore con isomeria dell'androceo, e sulla sua irregolarità con meiomeria dell'androceo. Io li ho accettati quali sottordini coi nomi di Meiostemones e d'Isostemones, per la considerazione che il carattere addotto è assai generale, essendo esteso a tutto quanto il fiore, e ch' è sufficientemente determinato, nonostante che l'irregolarità sia ridotta a minimi termini in certi generi di Verbenacee, $o$ in quei generi intermedi alle Solanacee e alle Scrofulariacee ove l'androceo non è incompleto, ma neppure tutti gli stami sono ugualmente bene sviluppati. Non credo che sia da tenere conto in proposito dei generi $L a$ chnostachys ed affini, che ascritti dapprima alle Amarantacee, ne sono stati tolti (Bentham, Fl. austral., Hooker e Bentham, Gen. plant.) per essere posti, nonostante la completa regolarità del fiore, nelle Verbenacee, ma che non vi possono stare, per essere la loro pretesa corolla un androforo come quello delle Amarantacee, nelle vicinanze delle quali vanno ricondotte.

Distribuzione geografica. - Il vastissimo ordine occupa tutto il globo. Una metà delle famiglie è più riccamente rappresentata nella zona calda, l'altra metà nelle zone temperate; e quest' ultima è quella che spinge i suoi rappresentanti nelle zone fredde. A que. sto riguardo quel che vale per l'ordine in complesso, vale per ognuno dei suoi sottordini.

Dalle 26 famiglie che vi ammetto, 12, le Frimacee, Selaginacee, Mioporacee, Stilbacee, Pedaliacee, Gesneracee, Cirtandracee, 
Bignoniacee, Cestracee, Loganiacee, Idrofillacee, e Nolanacee, sono assolutamente estranee alla flora italiana. Le rimanenti 14 esistono in Italia, alcune, tali soprattutto le Lamiacee, le Scrofulariacee, le Borraginacee ecc., in buon numero di generi c di specie, altre in un numero proporzionale ristretto, essendo quest' ultime tutte, ad ec. cezione delle Polemoniacee, famiglie più proprie della zona calda che s'inoltrano alcun poco in questa nostra temperata.

\section{SOTTORDINE PRIMO.}

\section{MEIOSTEMONI.}

Labiatiflonæ (excl. Plantaginaceis) Eichl. Blïthendiagr. 1. p. 206. Personales (excl. Columelliaceis) et Lamiales Benth. et Hook. gen. pl. 2.

Meiostenones Car. pens. tass. bot. p. 73.

Flores irregulares, androceo prae corolla meiomero.

Osservazioni. - Le famiglie di questo gruppo sono strette fra loro da tali vincoli di affinità, che bene spesso ne risultano incerti e variabili i limiti. I caratteri differenziali sono presi principalmente da quanto si attiene alle gemmule e ai semi; e dalla loro indagine critica si può, precisandoli meglio, arrivare a circoscrivere più uniformemente le famiglie.

Un primo carattere è dato dagli spermofori. V' ha un solo spermoforo centrale nelle Utriculariacee, che per ciò restano ricisamente staccate da tutte l'altre famiglie, dove gli spermofori sono o parietali o septali (assili). Circa a questi duc ultimi modi d'essere $v^{\prime}$ ha qualche caso dubbio, nelle Scrofulariacee per esempio; ma per lo più la distinzione dall' uno all' altro modo è manifesta, e dà un buon carattere. Lo darelsbe ancora più, se ci potessinı valcre in un lavoro generale delle ricerche genetiche fatte su alcune poche specie, le quali hanno dimostrato che talora (Scrofulariacee, Bignoniacee) il setto divide fin dalla sua origine il gemmulario, e poi si trasforma in spermoforo, e talora (Lamiacec, Verbenacee) risulta ulteriormente dalla saldatura di spermofori parictali clie s' incontrano nel centro del gemmulario. Nel caso poi degli spermofori parietali, si lia che d'ordinario essi corrispondono al margine dei pistilli, ossia, essendo questi nella regola due, l'uno anteriore e l'altro poste- 
riore, gli spermofori sono laterali; però nelle Globulariacee, e molto probabilmente nelle Frimacee, corrispondono al mezzo dei pistilli.

Variano grandemente il numero delle gemmule sugli spermofori, la loro disposizione, e la direzione. Numerosissime, sono seriate, le serie essendo ora poche e regolari, ora molte e allora facili a perdere la regolarità. In questo caso la direzione delle gemmule sembra essere poco costante, fra l'orizzontale, la pendente e l'ascen. dente. Di numero ridotto, possono ancora éssere seriate, cioè so. vrapposte; ma in generale sono allora collaterali, ed hanno costantissima l'inserzione e la direzione, che doventano perciò caratteri buoni. Non è a tacere peraltro che si danno casi di transizione che diminuiscono il valore al carattere desunto da siffatte particolarità delle gernmule; cosi nel genere Veronica certe specie hanno gem. mule più numerose seriate, altre le hanno ridotte a due collaterali: onde la necessità di lasciare nelle Scrofulariacee i generi Melampyrum o Tozzia con gemmule appaiate, come si lascia nelle Acantacee il genere Thunbergia che trovasi nel medesimo caso.

In quanto a struttura, le gemmule sono sempre ricurve, variando facilmente fra l' anatropo, l'emianatropo e il campilotropo; fuorchè nel solo genere Phryma, dove dicesi che sieno ortotrope; e sono quasi ortotrope in specie di Aiuga e di Teucrium. Da siffatte modificazioni di struttura, e dalla direzione delle gemmule, deriva il carattere dell' embrione nel seme, ch' è ora con radicetta supera, ora con infera, salvo qualche caso come quello degli Acanti (Plan-

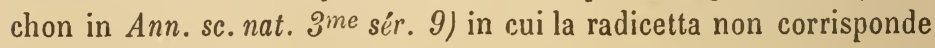
piủ al micropilo.

Il seme propriamente detto somministra un carattere buono, secondochè è fornito di mandorla o ne manca; dico.buono, nonostante la varia proporzione dell'embrione con la mandorla quando v'è, per cui anche questo carattere non è affatto determinato.

Il gemmulario somministra un carattere da non tralasciarsi, per essere talora più o meno infero, e ciò nelle vere Gesneracee. Meno attendibili sono la tendenza all'aborto di uno dei due pistilli, e la tendenza alla formazione di setti secondari, le quali costanti in alcune famiglie, sono ambedue incostanti nelle Verbe. nacee.

Il gemmulario convertito in frutto dà esso pure un carattere da non tralasciarsi, qualora si attenda, passando sopra a molte sue variazioni, soprattutto al frutto ch'è deiscente nella regola quando ha molti semi, dirompente o indeiscente quando ne ha pochi. 
L'antera, ordinariamente 2-loculare, è 1-loculare nelle sole Selaginacee.

All' infuori dei precedenti caratteri, non vedo che altre parti del fiore 0 il frutto ne diano che si possano considerare valevoli a segnare famiglie, ma solamente tribủ o generi.

Sulla scorta di quelli prescelti come migliori si puỏ distinguere le famiglic delle Corolliflore Mleiostemoni come segue:

1.0 Le Phrymaceæ Schauer, dal gemmulario 1-loculare, dalla gemmula solitaria, probabilmente mediopistillare, eretta, ortotropa, dal frutto ch' è un achenio, dal seme senza mandorla, dall' em. brione con radicetta supera.

2. Le Globulariace£ Link, dal gemmulario 1-loculare, dalla gemmula solitaria, mediopistillare, pendente, anatropa, dal frutto ch'è un achenio, dal seme con mandorla, dall' embrione con radicetta supera.

5. ${ }^{\circ}$ Le Selaginaceæ Chois., distintissime per l' antera 1-loculare. Hanno il gemmulario 2-loculare, gli spermofori septali (ma non sappiamo se tali dall' origine o se da prima parietali), le gemmule solitarie, pendenti, il frutto dirompente, il seme con mandorla, l'embrione con radicetta supera.

4. Le Myoporaces Lindl., che hanno il gemmulario 2-locu. lare, spesso tramezzato secondariamente, gemmule pendenti, colla. terali o seriate, un frutto drupaceo o dirompente, pressochẻ punta mandorla, la radicetta dell' embrione supera.

$5 .^{\circ}$ Le Stilbacee Lindl., differiscono dalle Verbenacee per avere semi con mandorla, e ordinariamente un achenio per frutto.

6. Le Lamiacee Lindl., che differiscono appena dalle Verbe. nacee per il gemmulario costantemente 4-loculare, e profondamente 4-lobato, e per il frutto ch' è sempre tetracocco.

7. Le Verbenaces Juss., con gemmulario reso 2-loculare da saldatura di spermofori parietali, e facilmente 4-loculare da forma. zione di setti secondari, gemmule collaterali, ascendenti, un frutto dirompente, o indeiscente (drupa o bacea), seni pressochè senza mandorla, embrione con radicetta infera.

8. Le Pedaliaces, gruppo rimasto sempre eterogeneo, nonostante i lavori di cui è stato oggetto. La sua tribù delle Pretrece, quale proposta da Bentham e Ilooker, è vicinissima alle Verbenacee, anzi è difficile trovare in che cosa ne differisca, fuorchè per l'abito diverso. La tribù delle Pedalieae è altrettanto affine alle Mioporacee. Le Martyniea sono vere Cirtandracee. Con esse le Sesamea si allon- 
tanano dalle tribù precedenti per il frutto deiscente. In mancanza di migliori studi, parni che per ora la famiglia si possa ristringere alle Pedaliee e le Sesamee.

9. Le Acanthacee Juss., con gemmulario 2-loculare, spermoforo septale (parietale in origine), gemmule parecchie, seriate, ascendenti (fuorchè nelle Thunbergiece dove sono 2 collaterali), una cassula per frutto, mandorla punta o quasi punta, radicetta infera.

10. Le Gesneracese, ristrette a quelle di gemmulario infero o semi-infero. Con esse principia la serie delle famiglie con molte gemmule pressochè orizzontali. Qui le gemmule sono sopra spermo. fori parietali. I semi sono con mandorla.

11. Le Orobanchace E Lindl,, differiscono dalle Gesneracee unicamente per il gemmulario affatto supero.

12. Le Cyrtandraces Jack, differiscono da ambedue le famiglie precedenti per la mancanza quasi assoluta di mandorla.

15. Le Utriculariace£, distintissime per lo spermoforo cen. trale.

14. Le Bignoniacel R. Br., con spermofori septali, e semi senza mandorla.

15. Le Scrofulariacee Lindl., con spermofori septali, e semi con mandorla.

\section{FAMIGLA PRIMA.}

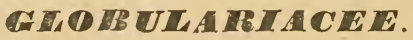

Globularie Cana. fl. fr. 3. p. 427 (1805).

Globulariace e Link handb. der gew. 1. p. 675 (1829).

Gemmularium 1-loculare, gemmula solitaria, ex apice parietis posterioris pendula, anatropa, cum raphe anteriori. Fructus siccus indehiscens. Semen amygdalosum. Embryo axilis, radicula supera.

Osservazioni. - Come notò benissimo Candolle quando costitui la famiglia restringendola al solo genere Globularia, essa è distintissima; e malamente hanno fatto coloro che l' hanno dipoi congiunta alle Selaginacee, le quali ne differiscono per l'antere 1-loculari, per il gemmulario 2-loculare con spermoforo septale, e per il frutto ch'è dirompente. Lo spermoforo che, giusta le osservazioni di Payer (Traité d'org. comp. de la fl. p. 584), è impiantato nel mezzo 
di un pistillo, anzichè essere o sui margini dei pistilli o sopra un tramezzo di origine stipitale, è tale carattere che distacca ricisamente le Globulariacee da tutte l'altre Corolliflore, ad eccezione forse delle sole Frimacee, le quali hanno perỏ la gemmula eretta, ortotropa, e mancano di mandorla.

Descrizione. - Fra le diverse specie delle Globulariacee nostrali havvi una simiglianza nell'aspetto, che deriva dalla infiorescenza a capolino e dal colore turchino dei fiori; ma nel resto differiscono. Una (Globularia Alypum) è un frutice eretto ramoso, altre due (G. incanescens e G. cordifolia) sono suffrutici sdraiati cespugliuti, altre due ancora (G. vulgaris e $G$. nudicaulis) sono erbe perenni scapigere e con le foglie a rosetta.

Le foglie hanno sempre apparenza di essere durature sulla pianta. Sono sparse, le inferiori sovente riunite a rosette, sono senza stipole, consistenti, glabre, pressochè intere, di forma che varia fra la lanciolata, l' ovale e l' arrotondita, nel quale ultimo caso sono picciuolate. Le prime sui polloni sono facilmente ridotte allo stato di scaglia; e facilmente pure le ultime in vicinanza all'infiorescenza passano allo stato di brattee.

L'infiorescenza è costantemente un capolino, quasi sempre solitario terminale, di molti fiori sopra un ricettacolo fatto a cupola o a cono. Ogni fiore è all' ascella di una paglietta o brattea scaglio. sa; delle quali pagliette poche esteriori sono talora vuote all' ascella, soltanto nella Globularia Alypum sono molte e costituiscono un vero involucro.

I fiori sono irregolari, in tutta la loro estensione, con sviluppo maggiore anteriormente in tutti i verticilli.

Il calice è conico a rovescio, gamosepalo, diviso piủ o meno profondamente in 5 lobi, pochissimo disuguali, dei quali 1 posteriore, 2 laterali, 2 anteriori, a quanto pare di preflorazione aperta. Li persistente.

La corolla è sempre di color turchino. É spiccatamente irregolare, essendo divisa in due labbri, dei quali l' inferiore 3-lobo, il superiore 2-lolo, piủ piccolo dell' altro e talora quasi nullo. La pre. Horazione n' è aperta. Ė marcescente, infine caduca.

Gli stami sono 4, 2 anteriori e 2 laterali, mancando totalmente il posteriore. Essendo inseriti in cima al tubo corollino, sono lungamente sporgenti, specialmente gli anteriori che hanno il filamento alquanto più lungo. L'antera, piccola, reniforme, è inserita nel mezzo del dorso, ed è biloculare; le sue caselle si aprono inentro 
per lungo, confondendosi in una; secondo Chatin (in Bull. soc. bot. de Fr. 20. p. 42) sono gli stami laterali ultimi ad aprire le loro antere; infine l' antera cade. Il polline è bianchiccio, ovale, pieghettato per lungo.

Il gineceo, ora è accompagnato da un piccolo disco, ora non ne ha. È 2-mero, risultando da due pistilli antero-posteriori. Il gemmulario è 1-loculare; racchiude una sola gemmula pendente dall' apice della casella, anatropa, con integumento scempio, con rafe anteriore. Lo stilo, filiforme, lungo presso a poco quanto gli stami, ha due lobi appuntati, piccolissimi, soprattutto il posteriore, che spesso anche manca.

Il frutto è piccolo, rinchiuso nel calice, terminato in una punta costituita dalla base persistente dello stilo. $\dot{\mathrm{E}}$ un achenio, con pericarpio membranoso strettamente applicato sul seme. Il quale ha mandorla carnosa, e un embrione collocato nell' asse di questa, poco più corto di essa, con embriofilli bislunghi, piano-convessi, quasi uguali al fusticino, la cui radicetta è supera.

Considerazioni geografiche. - Sono circa 12 le specie della famiglia, abitatrici dell' Europa, particolarmente della meridionale, e dell' Oriente, nonchè dell' Africa settentrionale. Di esse la metà, e precisamente 5 , sono in Italia: 1 largamente sparsa, 1 ristretta al littorale mediterraneo, e 3 proprie dei monti. Fra quest'ultima spicca la Globularia incanescens, affatto speciale delle nostre Alpi Apuane.

\section{chomularea.}

Globularia Tourn. inst. p. 466. Benth. et Hook.gen.pl. 2. p. 1130. Ces.

Pass. Gib. comp. fl. it. p. 290. t. 43.

Carradoria et Globularia A. de Cand. in Cand. prodr. 12. p. 610,

611. Willk. rech. sur l’org. et la class. des Globul.

Calyx quinquefidus, æqualis vel subbilabiatus. Gorollæ hypogynæ tubus cylindricus, limbus bilabiatus, labio superiore minore profunde bifido, ${ }^{1}$ inferiore trifido. Stamina 4 , summo tubo corollæ inserta, limbi laciniis alterna, quintum inter lacinias superiores deficiens. Filamenta filiformia, recta. Antheræ reniformes, in alabastro biloculares, loculis sub anthesi confluentibus univalves, longitudinaliter dehiscentes. Pollen ellipticum, utrinque obtusum, laeve.

1 In G. canescenle plerumque integro. 
Ovarium oblonguin, uniloculare, uniovulatum. Ovulum ex apice loculi pendulım, anatropum. Stylus terminalis, filiformis, exsertus. Stigma brevissime emarginato-bifidum. (Parl. ms.).

Portamento. - Vedasi la descrizione della famiglia.

Osservazioni. - Il genere Carradoria è stato costituito per la Globularia incanescens a motivo del labbro superiore della corolla intero e non diviso, e dell' assenza di un disco ipogino. Ora questo nelle Globularie è dimezzato, minimo, o ridotto a una glandola anteriore, e ben poco ci corre alla sua totale mancanza; e dalla descrizione qui appresso della $G$. incanescens fatta da Parlatore, nonchè dalle osservazioni di Cesati ricordate nel fascicolo 30 delle sue Stirpes italicre, risulta che il labbro superiore della corolla, nella pianta in discorso, $s$ ' $\dot{e}$ per lo più intero, può àche essere più o meno profondamente diviso.

\section{Globulnaia nudicanlis.}

G. herbacea, foliis rosulatis oblongo-spathulatis, scapo subnudo, corollae labio superiore brevissimo subnullo.

Globularia nudicaulis Linn. sp. pl. ed. 1. p. 97. Com. fl. com. 1. p. 170. Bert. fl. ital. 2. p. 11. De Not. rep. fl. lig. p. 338. Rota prosp. fl. Berg. p. 73. Hausm. fl. Tir. p. 724. Car. prodr. 11. losc. p. 530. Suppl. p. 4.4. Ard. A. Alpes-mar. p. 314. De Vis. Sacc. cat. piant. Ven. p. 142. Archb. A. Alto-Serch. p. 61. Ces. Pass. Gib. comp. fl. ital. p. 291. Zersi prosp. piant. Brescia p. 175. Arc. comp. fl. ital. p. 569. Gib. Pir. fl. Mod. p. 135. t. $19 \%$.

Figure. - Willk. rech. Glob. t. 4. Reich. ic. R. germ. 20.

Stazione, Abitazione e Fioritura. - Nei lnoghi pietrosi dei pascoli e dei boschi nelle Alpi: alpi di Tenda e di Clans (Ard.), monti Valdensi, fin versi) Piossasco (All. /l. ped.), monte Braulio in Valtellina (Com.), alpi Comasche al monte Barbisino (Daenen!), nel monte Barro fra 8-900 m. (Parl.!), nei monti Codeno, San Primo, Arengo, Pizzo di Gino, Resegone, nell'alpe di Zana, e quasi alla riva del lagno di Como alle Crosgralle (Com.), alpi Beryamasche al Caremo (Rota!), sopra Zngno (Bert.), alpi Bresciane alla Corna Blacea, al monte Ario e al Dos alto fra 1700-1800 m., al monte Gerle a Ifion m. (Parl!), al Dragone, al fronden (\%ersi), alpi Veronesi nel Baldo, a Fondone e Campogrosso, al monte Magrgiure, monte Spinale in Tirolo, val di Bregnzzo nelle Gundicarie (Hansm.), alpi Treviciane al monte Cirappa (Kellner!), al Frontale de Cre- 
spano, al Bocoar (Montini!). Trovasi ancora nell'Appennino toscano dove la scopri Pietro Savi, al Cimone di Caldaia e a Mandromini (Beccari). Fiorisce da giugno ad agosto.

Distribuzione geografica. - In tutta la catena delle Alpi, dalle Alpi Marittime alla Croazia; nell'indicato luogo dell' Appennino; nei Pirenei, e nella Spagna settentrionale e centrale.

\section{Globularia vulgaria.}

G. herbacea, foliis rosulatis obovato-spathulatis, scapis vestitis a foliis parvis lanceolatis, corollæ labio superiore dimidium inferioris aequante 2-secto.

Globularia vulgaris Linn. sp. pl. ed. 1. p. 96. Com. fl. com. p. 169. Bert. fl. ital. 2. p. 5 P'ucc.! syn. pl. luc. p. 77. Trev. prosp. f. eug. p. 25. De Not. rep. fl. lig. p. 338. Zan. prosp. fl. ven. p. 26. Simi! fl. alp. vers. p. 28. Hausm. fl. Tir. p. 724. Rota prosp. fl. Berg. p. 72. Car. prodr. fl. tosc. p. 529. Ard. cat. pl. Ment. p. 30. Tassi fl. sen. p. 51. Bot. Siena p. 90. G. Bert. not. piante Porr. p. 50. Veg. monti Porr. p. 91. Ard. fl. Alpes-mar. p. 314. De Vis. Sacc. cat. piant. Ven. p. 142. Zersi prosp. piant. Bresc. p. 175. Ces. Pass. Gib. comp. fl. ital. p. 291. Ing. cat. sp. Mond. p. 39. Groves contr. fl. Otr. p. 66. Cald. fl. fuv. tent. p. 184. Arc. comp. fl. ital. p. 563. Gib. Pir. R. Mod. p. 135.

Globularia nudicaulis Ten. prodr. fl. nap. p. 11 (e non " multicaulis " come per errore tipografico sta scritto nella syll. $\mu$. neap. p. 58 del medesimo autore).

G!obularia Wilkommii Nym. syll. fl. eur. p. 140. Ces. el. piant. Maiella p. 24.

Figure, - Bot. mag. t. 2256. Willk. rech. Glob. t. 1. Reich. ic. fl. germ. 20. t. 196.

Stazione, Abitazione e Fioritura. - Comune nei luoghi aridi, erbosi o selvatici, specialmente nei montuosi, di tutta l'Italia superiore e media, spingendosi nella meridionale fino ai monti di Gaeta, e d'Itri (Ten. syll., Terracciano!), a Monte Casino e al Matese (Ten.), ed è stata trovata anche in Terra d'Otranto (Groves). Non è molta l'altezza alla quale sale nei monti: cosi Parlatore l'ha trovata a $4-500 \mathrm{~m}$. sulle pendici del monte Barro di faccia a Lecco, e in Toscana il suo limite superiore è poco sopra i $1000 \mathrm{~m}$. Manca affatto nelle isole. Fiorisce da marzo a giugno secondo i luoghi.

Distribuzione geografica. - Occupa tutta l'Europa occidenta- 
le, avendo il limite nord nella Francia, nell'()landa e a Danzig sul Baltico, e il limite sud nella Spagna centrale, nelle suindicate parti dell' Italia, e nel monte Emo dei Balcani. Trovasi anche in Crimea e al Caucaso, ma non altrove in Oriente come si credeva (Boiss. R. or.).

Descrizione. - Pianta perenne, alta da uno a due centimetri, verde, glabra. La radice è grossotta, dura, ramosa, flessuosa, rossiccioscura e quasi nericcia, glabra. Il fusto è ramoso alla base, con i rami cortissimi, duri e quasi legnosi, i quali mandano molte foglie in cespu. glietto e da mezzo ad esse i rami horiferi: questi sono ascendenti o eretti, erbacei, cilindrici, fortemente striati, il doppio o poco più lunghi delle foglie inferiori. Tali foglie sono in rosetta, consistenti, quasi coriacee, eretto-palenti, obovate, smarginate e come tridentate, con i denti scarsi, l'intermedio molto più corto e più piccolo dei due la. terali, assottigliate lungamente in basso quasi in un picciólo alato, il quale è scanalato-solcato di sopra, angolato-rarenato di sotto; sono verdi e un po'lucenti di sopra ed ivi con pochi e leggieri solchi che corrispondono ai nervi laterali della pagina inferiore, di un verde più chiaro ed opaco di sotto ed ivi con un nervo longitudinale e con due nervi laterali per parte, i quali sono rilevati, si partono dalla base della foglia, si dirigono un po'curvi verso il margine e l'apice e si diramano poco. Le foglie del fusto sono molte, rivestono il fusto sino all'apice, sono meno consistenti delle inferiori, assai piccole, sparse, eretto-patenti, sessili, lanceolate, acute, con un nervo longitudinale e due nervetti iaterali, per cui sono quasi trinervi. I fiori sono molti, fitti, sessili, piccoli, in un capolino dapprima quasi emisferico, poi quasi tondo all'apice del fusto; ciascun fiore è accompagnato da una brattea, la quale è avvicinata a' fiori, poco più lunga del calice, ovalelanceolata o lanceolata, acuta, verdognolo-violetta e pelosctta verso l'apice e con ciglia lunghette: le brattee esterne sono glabre. Il calice è tubuloso, con il tubo quasi turbinato, hianchiccio, peloso, con i peli lunghi e bianclii, e con peli lunghetti, anche bianchi di dentro verso la gola: è diviso sotto la metà in cincuue lacinie, quasi eguali, due inferiori un po' piu grandi delle tre superiori, eretto-patule, lanceolato-acuminate, verdognole, con ciglial lunghette e hianche. La corolla è quasi il doppio puì lunga del calice, di color violetto-chia. ro, tubulosa, con il tuho stretto. lungo quanto it calice, con il lembo diviso in due labbra, l'inleriore piii grande, liviso profondamente in tre lacinie lueari, ottuse, o il smperiore quasi la meta piu corto dell' inferiore, diviso profondamente in due lacinie stretta. 
mente lineari ed ottusette. Gli stami sono quattro, alterni con le lacinie della corolla, mancando quello che dovrebbe corrispondere in mezzo alle due lacinie del labbro superiore, inseriti nella parte superiore del tubo, un po' disuguali, quasi tanto lunghi quanto le lacinie del labbro inferiore. I filamenti sono filiformi, eretti, di color violetto chiaro, glabri. Le antere sono quasi bislunghe, leggermente bilobe, aprentisi per una fessura longitudinale, uniloculari, inserite nel mezzo del dorso, di color violetto scuro, glabre. Il polline è bianco. Il pistillo è quasi la metà più corto delle lacinie del labbro inferiore della corolla. L' ovario è piccolo, bislungo, glabro, bianchiccio, uniloculare e contiene un solo ovolo. Lo stilo si continua quasi con l'apice dell'ovario, è lungo, diritto, filiforme, di color violetto verso alto, glabro. Lo stimma è piccolo, bifido, con le lacinie filiformi, quasi uguali, erette 0 appena appena divergenti, del colore dello stilo. (Parl. ms., descr. della pianta forentina).

Osservazione. - Pare che Linneo confondesse con questa specie un'altra assai rara, delle isole Oeland e Gottland del Baltíco, e che trovasi ancora in Spagna, la Globularia spinosa di Lamarck. Per questo motivo il Nyman ha proposto di chiamare la nostra pianta G. Willkommii, serbando il nome di G. vulgaris alla pianta di Svezia. Ma non si troverà giustificata la proposta, qualora si rifletta che Linneo nelle sue Species, opera generale e non flora locale, intese anzitutto per G. vulgaris la pianta volgarissima in Europa, come risulta dal cumulo delle citazioni da lui addotte; fra le quali, quelle errate della Flora suecica e dell'Iter oelandicum, dove egli ebbe in inira l'altra specie, non possono valere a distruggere l'effelto dell'altre citazioni, e a cambiare nome alla pianta in discorso.

\section{Globularia inconescens.}

G. suffrutescens, foliis sparsis, infimis late obovato-spathulatis, ramis floriferis vestitis a foliis minoribus ovalibus lanceolatisre, corollæ labio superiore inferiorem fere æquante integro vel nonnumquam diviso.

Globularia incanescens Viv. fl. ital. fragm. fasc. 1.p. 2. Bert.! fl. ital. 2. p. 7. Ces. stirp. ital. fasc. 3. Pucc.! syn. pl. luc. p. 76. Simi! fl. vers. p. 29. Car. prodr. fl. tosc. p. 529. Sec. suppl. p. 23. Archb. Al. Alto-Serch. p. 61. Ces. Pass. Gib. comp. fl. ital. p. 291. Arc.! comp. fl. ital. p. 563. Gib. Pir. fl. Mod. p. 135. 
Carradoria incanescens A. de Cand. in Cand. prodr. 12. p. 610. Willk. rech. Glob. p. 16.

Figure.-Zan, et Mont. rar. stirp. hist. t. 84. Viv. o. c. t.3. Reich. ic. bot. cent. 9. f. 1097, 1098. Pucc. o. c. Cies. o. c. Willk. o.c. t. 1. Reich. ic. fl. germ. 20. t. 195.

Stazione, Abitazione e Fioritura. - Sulle rupi marmoree o altrimenti calcaree delle Alpi Apuane, dov' è comune assai, dalle loro radici sino alle cime piủ alte, nonchè dell'Appennino piủ prossimo, cosi in Garfagnana alle Grotte e alla Pania di Corfino!, in vari luo. ghi dell'alpe di Barga (Parl.!), a Pratofiorito (Archb.), nel Rondinaio! e sue vicinanze, e giù in Val-di-Lima a Casoli (Pucc.!), presso Boscolungo (Parl.!), al Corno alle scale (Gennari!). Fiorisce da maggio ad agosto secondo i luoghi.

Distribuzione geografica. - Specie propria dei suddetti monti. Il Savi (Bot.etr.2.p .80) l' indica ancora del monte Argentaro nella maremma Toscana, ma nessuno ve l'ha più trovata; e soltanto per confusione con la G. punctata di Lapeyrouse (ch'è un sinonimo della $G$. cordifolia) è stata indicata nei Pirenei.

Descrizione. - La pianta forma dei graziosi cespuglietli che sporgono dalle fessure delle rupi. La radice è dura, scura, ramosa e manda molte fibre capillari. Il fusto è ramoso alla base, e tanto esso che i rami sono lunghi da uno a due pollici, sono curvati in fuori e in alto e leggermente flessuosi, delicati, quasi quadrangolari, verdi e privi di peli. Le foglie sono alterne, di un verde pallido, più pallido anche di sotto, le inferiori sono obovate, rotondate all'apice, e la loro lamina è prolungata alquanto alla base nel picciòlo ch' è lunghetto e scanalato di sopra; le superiori sono gradatamente piu piccole e di forma ovata, la loro base si prolunga purc in un picciòlo ch'è gradatamente più corto. Il peduncolo è solitario all'apice del fusto e dei rami, quasi quadrangolare e diritto, e porta i fiori distribuiti in un capolino emisferico, piano di sotto e convesso di sopra. Alla base del capolino che corrisponde all' apice del fusto ri è spesso una fogliolina come quella della parte superiore del fusto ma piu piccola. I fiori sono di color violetto, ciascuno di essi è accompagnato da una brattea lanceolato-acuminata e quasi arestata, lunga quasi quanto il calice, quasi scagliosa, di colore scuro, fornita di alcuni peli bianchi sulla pagina inferiore e di corte ciglia scabre nel margine: le brattee dei fiori inferiori, che sono maggiori di quelle dei finri superiori, formano quasi un involıcro al capolino. Il calice è tubuloso inferiormente ed ivi di culor verde chiaro e coperto di lun- 
ghi e densi peli biarıchi che lo rendono irsuto; le lacinie sono cinque, lanceolato-acuminate e quasi arestate, quasi uguali tra loro, fornite di lunghe ciglia bianche inferiormente nei margini e scabre in essi verso l'apice; la gola poi del calice è coperta di lunghi peli bianchi che sporgono in parte fuori tra la base delle lacinie. Il tubo della corolla è cilindrico, lungo quasi quanto le lacinie del calice, è piủ corto del lembo della corolla stessa e di colore più chiaro di questo. Il lembo è diviso profondamente in due labbri, uno superiore più piccolo e più corto diviso sino alla base in due lacinie lineari, strettissime ed ottuse, ed uno inferiore diviso fin verso il quarto inferiore in tre lacinie, il doppio circa piủ lunghe di quelle del labbro superiore, lineari, un poco però più larghe alla base ed ottuse all'apice. Gli stami sono quattro, talvolta se ne trovano cinque in qualche fiore, inseriti nella parte superiore del tubo presso alle fauci, sono diritti, lunghi quanto le due lacinie del labbro superiore e quasi uguali al pistillo: i filamenti sono filiformi e dello stesso colore del lembo della corolla. Le antere sono di color violetto, quasi in forma di rene, fissate per il mezzo del dorso, dapprima biloculari ma poi per la unione delle logge uniloculari, si aprono per una fessura longitudinale e si rovesciano in modo che diventano non solo piane ma con. vesse superiormente. Il polline è giallo, ellittico, otluso ad ambedue le estremità. L'ovario è piccolo, allungato, verde; lo stilo è diritto, filiforme, di color violetto; lo stimma è quasi bifido con le lacinie cortissime e alquanto acute. (Parl. ms., descr. della pianta di presso Boscolungo).

\section{Globularia cordifolia.}

G. suffruticosa, foliis confertis obovato vel oblongo-spathulatis aut cuneatis, ramis floriferis subnudis, corollæ labio superiore dimidium inferioris æquante 2-secto.

Globularia cordifolia Linn. sp. pl.ed.1. p. 96. Avé-Lallem. pl. Ital. bor. p. 7. Com. fl. com. 1. p. 170. Bert. fl. ital. 2. p. 8. Pucc.l syn. pl. luc. p. 76. De Not. rep. fl. lig. p. 338. Koch syn. fl. germ. ed. 2. p. 681. Simi! fl. vers. p. 28. Hausm. fl. Tir. p. 724. Rota! prosp. fl. Berg. p. 73. Car. prodr. fl. tosc. p. 529. Ard. cat. pl. Ment. p. 30. Fl. Alpes-mar. p. 314. De Vis. Sacc. cat. piant. Ven. p. 142. Zersi prosp. piant. Bresc. p. 175. Ces. el. piant. Maiella p. 24. Terr. sec. rel. Terra di Lav. p. 90. Archb. fl. Allo-Serch. p. 61. Ces. Pass. Gib. comp. fl. ital. p. 291. Terr. quarta rel. Terra 
di Lav. p. 108. Paól. el. piant. Sib. p. 12. Arc. comp. fl. ital. p. 563. Gib. Pir. fl. Mod. p. 135.

Globularia bellidifolia Ten.! viagg. in Abruzzo. Ces. o. c. p. 24.

Figure. - Lam. ill t. 56. f. 2. Ten. fl. nap. t. 109 (G. bellidifolia). Willk, rech. Glob. t. 4. f. 1. Reich. ic. fl. germ. 20. t. 195. f. 1,2 .

$\beta$ nana, foliis angustissimis, ramis floriferis abbreviatis.

Globularia cordifolia $\beta$ nana Camb, mon. Glob. p. 27. De Vis. Sacc. l. c.

Figure. - Cand. ic. pl. Gall. t. 3. Willk. rech. Glob. t. 4. f. 2. Reich. ic. A. germ. 20. t. 195. f. 3.

Stazione, Abitazione e Fioritura. - Comune assai nelle rupi e nei luoghi sassosi dei monti, dai pascoli alpini fin giủ presso alla loro base: alpi di Tenda (Bourgeau!), da dove scende sino a $800 \mathrm{~m}$. nei monti sopra Nizza e Mentone (Ard.), vallate Valdensi (Rostan!), Cenisio a $2000 \mathrm{~m}$. (Arc.! ecc.), e giủ presso Susa (Parl.! ecc.), Cogne in val d'Aosta (Carestia!), monti Novaresi (Bir. fl. acon.), monte Generoso e monte Lucomagno nel Ticino (Franzoni!), monti del lago di Como, e di Como stesso, delle valli Intelvi, Sassina, e Cavragna, Iella Valtellina (Com.) fino allo Stelvio a 2000-2500 m. (Parl.!), alpi Bergamasche (Rota! ecc.) e Bresciane a 1800-2000 m. (Parl.!), da dove scende coi fiumi, cosi trovasi nei greti dell'Oglio (Zersi), monte Baldo (Bert.), monte Pestello(Nanganotti!), il Tirolo nel Trentino (Perini!), a Bolzano, in Pusteria (Hausm. ecc.), monti di Bassano (Montini! ecc.), di Belluno (Kiellner!), di Udine (Pirona! eec.), Trieste (Koch) al Carso (Bert.), Appennino ligure a Triora (Panizzi!), da Nava a Carlino (Ricca!), sopra Ceriale (Bert.), tutte l'Alpi Apuane, principalmente nelle cime!, ma anche in basso come ai Fanti-scritti (Bert.) e verso Stazzema (Simi), Appennino toscano al Corno (Vitm. ist. erb.), monte S. Vicino a' $1000 \mathrm{~m}$. (Bucci!) e nonte Birro (Bert.) nelle Ilarche, Appennino Piceno al Vettore!, alla Sibilla (Marzialetti!) ecc., Abruzzi al monte de'Fiori!, al Corno (Orsini!), al Velino (Cherici!), al Sirente (Groves!), al Morrone (Ces.), alla Maiella a $2.400-2500$ m., e giủ presso Caramanico (Levier! ecc.), monti di Segni (Rolli!), monti della Terra di Lavoro a monte Meta (liert.), a Pescosolido, a Picinisco (T'err. 1) e al Taburno (Guss.!), monti di Castellamare e isola di Capri (Pasquale! ecc.), monte !'ollino in Basilicata ('Ten.). La var. è piuttosto rara: Mpi di Tenda a 1900 m. (Parl.!), di Viosenne (liicca!) ecc. Fiorisce da maggio ad agosto secondo i luoghi. 
Distribuzione geografica. - Dai Pirenei e dalle parti finitime della Spagna va a traverso la Francia meridionale alle Alpi, che occupa in tutta la loro estensione, spingendosi al Giura e ai Vosgi, occupa poi tutto l'Appennino, si stende lungo. l'Adriatico insino alla Grecia, trovasi in tutta la regione Danubiana, e infine in Crimea.

Descrizione. - Pianta suffruticosa, procumbente, glabra, verde, fornita di un rizoma legnoso, molto esteso e ramosn, che si abbarbica con fibre radicali delicate e scure, e manda all' apice dei rami delle foglie in cespuglietto e dei rami fioriferi. Le foglie sono patenti o eretto-patenti, carnosette, verdi, obovato-cuneiformi, assottigliate lungamente alla base, smarginate quasi in cuore all' apice ovvero ivi ottusissime, intere o tridentate, quasi piegate di sopra, con un nerro sporgente a guisa di carena di sotto. Il peduncolo è eretto, e un po'flessuoso, cilindrico, striato, glabro, verdognolo sfumato di rossiccio, per lo più due o tre volte più lungo delle foglie, talvolta poco più lungo di queste, nudo o con una o due piccole brattee membranacee, quasi in forma di scaglie, erette o patenti, allungatolanceolate, acute, con corte ciglia nei margini e pelosette di fuori. I fiori sono distribuiti in un capolino emisferico, fitti e sessili sopra un ricettacolo e accompagnati da brattee che formano quasi un involucro al capolino. Tali brattee sono ovali-lanceolate, acuminate, scure e pelosette nel dorso, con lunghe ciglia bianche nel margine, sono avvicinate ai fiori, dei quali sono molto più corte, uguagliando solo in lunghezza il calice. Questo è tubuloso, verdognolo, peloso con peli lunghi e bianchi di funri e con un ciuffo di peli anche bianchi e lunghi di dentro vicino alla gola, diviso per più della metà in cinque lacinie eretto-patenti, lanceolato-acuminate, fornite di ciglia nel margine. La corolla è quasi due volte più lunga del calice, di color violetto chiaro, bilabiata, con il labbro superiore diviso in due lacinie lineari ed ottuse, e il labbro inferiore in tre lacinie anch'esse lineari ed ottuse ma un po'più larghe e un po' piủ lunghe delle superiori : queste sono anche più patenti e rivolte un po' in giù, di un colore un po' più carico e leggermente scanalate di sopra. Gli stami sono quattro, appena più lunghi della corolla, eretto-patenti, inseriti in alto del tubo. I filamenti sono filiformi, di color violetto chiaro, glabri. Le antere sono giallicce, aprentisi in una loggia. Il pistillo è piủ corto degli stami e un po'più corto anche della corolla. L'ovario è piccolo, allungato-cilindrico, glabro, bianchiccio, uniloculare. Lo stilo è molto più lungo dell' ovario, filiforme, eretto, di color vio. 
letto chiaro. Lo stimma è bifido, papilloso, ottuso, gialliccio. (Parl. ms., descr. della pianta di Dos Alto nelle Alpi Bresciune).

Osservazioni. - Come le specie vicine, questa varia molto per la grandezza e il taglio delle foglie e per la lunghezza dei rami fioriferi; la var. nana è una forma estrema: Buissier ( $F l$. orient. 4. $p$. 530) la ritiene per specie buona, ma non dice per quale motivo. La Globularia bellidifolia di Tenore corrisponde a una pianta media, come si presenta piü abitualmente, la sua G. cordifolia a una forma di sviluppo massimo.

\section{Globuannia Alyumm.}

G. fruticosa, foliis sparsis lanceolatis, ramis floriferis vestitis, corollæ labio superiore brevissimo suhnullo.

Globularia Alypum Linn. sp, pl. ed. 1. p. 95. Bert. fl. ital. 2. p. 4. Guss. fl. sic. syn. 1. p. 168. De Not. rep. fl. lig. p. 3:97. Mor.! fl. sard. 3. p. 345. Car. prodr. fl. tosc. p. 530. Ard. cat. pl. Ment. p. 30. Tass. 1. prov. sen. p. 51. Ard. fl. Alpes-mar. p. 314. Mars. cat. pl. Cors. p. 119. Ces. Pass. Gib. comp. R. ital. p. 291. Arc. comp. fl. ital. p. 563.

Figure. - Willk. rech. Glob. t. 4. f. 5. Reich. ic. A. germ. 20. t. 197. f. 1, 2. Moggr. contr. M. Ment. t. 34.

Stazione, Abitazione e Fioritura. - - Nei luoghi aridi e in specie nei colli calcarei del littorale Mediterraneo : nella Liguria occidentale dov'è comune, così a Nizza (Parl.! ecc.), Villafranca (Durando!), Mentone (Moggr.), S. Remo, Taggia (Panizzi!), Porto Maurizio (Ricca!), Oneglia (All. $l$. ped.); quindi nella Mla. remma toscana a Castagneto (Amidei!), Piombino, Talamone (Savi ecc.), nel monte Argentaro!; nell' Elba (P. Savi! ecc.); in Corsica a Pastia (Pucci! ecc.), a Brando, a S. Finrenzo (Nar's.), ad Aiaccio (Bert.) ed altrove; in Sardegna a S. Elia presso Cagliari (Mor.!), e nelle isole S. Pietro e S. Antioco (Mor.); in Sicilia a Cefalù (Guss.), Trapani (Parl.!), Marsala, Mazzara, Terranova, e nelle isole di Maretimo e di Leranzo (Guss. ecc.); nell'isola di Lam. pedusa (Aiuti! ecc.). l'iorisce dall'autumno alla primavera.

Distribuzione geografica. - Del Mediterranen, trovasi dalla Tunisia al Maroceco, in Spagna, in Francia, in Lalia, in Grecia e nell' Arcipelagn, in Creta, in Anatolia, nel Libano.

Descrizione. - Caulis rubidus, vel inferne cinereus, ramuli rubili vel vires(entes, striatn-angulati. Folia glanca, carnosulocoriacea, erecta, erecto-patula aut arcuato-subreflexa, saepe vix con- 
torta, basi angustato-petiolata, supra saepe concaviuscula, subtus nervo longitudinali vix prominente notata, utrinque sub vitro punctis albis prominentibus notata, apice sæpe tridentata; folia superiora sensim angustiora. Capitula semiglobosa, bracteis fuscis, lanceolatis, acuminatis submucronatis, quoque flore adpressis, margine dense et longiuscule albo-ciliatis, inferioribus dorso apicem versus pilosis, calyce longioribus. Calyx albo-villosus, tubo brevi, recto, albo, laciniis" limbi subulatis, rectis, subpatulis, 3 inf. paullo longioribus, 2 sup. paulo brevioribus, omnibus corollæ tubo longioribus et labio inferiore ejus brevioribus, viridibus, apicem versus albidis, undique albo-villosis. Corolla pallide violacea; tubus superne sensim vix dilatatus, subcompressus a dorso antice, albido-violaceus, glaber, intus etiam glaber; limbi dentes 2 superiores brevissimi, subtriangulares, obtusi, laciniæ 3 inferiores in labium oblongum, apicem versus sensim vix dilatatum, patulo-subreflexurn, apice tridentatum, dentibus obtusis, subæqualibus, lateralibus vix brevioribus et vix angustioribus, lineis tribus paulo saturatioribus ab apice ad basim notatum. Stamina 4, prope apicem tubi corollæ inserta, subæqualia, labium corollae longit. subæquantia, glabra. Filamenta erecta, vix curvata, subulata, violacea; antheræ violaceæ, medio affixæ, oblongæ, obtusæ, biloculares? an 4-loculares? Pistillum exsertum, staminibus subæquale. Ovarium oblongum, subcompressum, viride, glabrum. Stylus ovario 3-4plo longior, cum ovario continuus, rectus, subcompressus, violaceus; stigma bifidum, laciniis vix divergentibus, acutiusculis, apice albidis. (Parl. ms., descr. della pianta di Nizza).

\section{FAMIGLIA SECONDA.}

\section{EATIRACIEE.}

Labiate Adans. fam. des pl. 2. p. 180.

LAMIAGE K Lindl. nat. syst. ed. 2. p. 275.

Gemmularium 4-loculare, 4-lobatum, gemmulis solitariis, erectis anatropis, vel adscendentibus hemianatropis aut amphitropis, cum raphe interiori. Fructus 4-coccus. Semen examygdalosum vel parce amygdalosum. Embryo radicula infera.

Osservazioni. - Da mezzo secolo a questa parte Bentham è la grande autorità per le Lamiacee, seguito e fedelmente copiato dai più dopo la comparsa della sua classica monografia: Labialarum ge- 
nera et species (1832-1836). Non è quindi meraviglia se, confortato da si generale appoggio, egli abbia nelle seguenti opere ripetuto se stesso, con poche variazioni e senza i miglioramenti che sarebbero stati richiesti dai progressi della scienza: a mo'd'esempio si noti che nel XII volume del Prodromus pubblicato nel 1848 la ubicazione delle specie in Italia è tuttora data sulle scarse indicazioni dell'Allioni, del Savi o del Tenore, senza che sia tratto partito da quelle copiosissime del Bertoloni già rese di pubblica ragione fra il 1844 e il 1847. Il fatto sta che al presente le Lamiacee sono fra le famiglie che più abbisognano di un monografo, il quale, ammaestrato nei moderni metodi d'investigazione morfologica, studi profondamente la struttura di queste piante, e ne tragga oculatamente i caratteri migliori per la spartizione della famiglia in gruppi subordinati. Se quelli ora vigenti, voglio dire le tribù e $i$ generi, sono cosi spesso d'incerta e confusa limitazione, gran parte di colpa ne va data altresi ad un principio tassinomico erratissimo, e non pertanto adottato da molti autori, e dallo stesso Bentham espressamente proclamato a pp. $46-48$ della prefazione alla sua monografia, secondo il quale i medesimi caratteri non dovrebbero avere lo stesso valore in tutta l'estensione di un gruppo: il che nella pratica significa sostituire a criteri accertati dalla discussione, e ad una valutazione sempre uguale dei caratteri, a tutti intelligibile, una salutazione in costante ed arbitraria, in obbedienza al sentimento personale che il classatore può avere concepito intorno alla naturalezza o alla convenienza del tale o tal altro gruppo. L'appresso lavoro sulle Lamiacee italiane è informato al principio affatto contrario, della equivalenza dei gruppi di medesimo grado, da fondarsi sopra caratteri paralleli. Nella scelta dei caratteri io mi sono attenuto ai resultati delle osservazioni piủ note, che hanno dimostrato l' incostanza dı alcuni caratteri, la maggiore costanza di altri; pur sempre desideroso che qualcuno, avendo tempo a ció, possa fare della famiglia tıtta un lavoro più completo e più profondo, e stabilirue la classazıone sopra base piủ sicura. I particolari di quella da me provisoriamente proposta vengono in appresso; iutanto ne do qui il prospetto riassuntivo:

\section{STACHYMTEN.}

1. LaVANulez.

1. Lavandula Linn. . . . . . . . . 5

๖. Sideritis Linn. . . . . . . . . . . 5

3. Marrubium Tourn ......... 4 
2. STAchydee.

1. Tendanea.

4. Tendana Reich. fil. . . . . . . . . .

2. Mentheж.

5. Lycopus Tourn.............. . 2

6. Mentha Tourn............ 8

7. Maiorana Tourn. ........... 1

8. Hyssopus Tourn. ........... 1

3. LAMIEA.

$\S$ Satureia.

9. Thymus............... 7

10. Satureia. . . . . . . . . . 26

11. Cuspidocarpus Spenn.......... 1

12. Ziziforum Linn. . . . . . . . . . 1

13. Melissa Moench.......... 1

14. Horminum Linn. . . . . . . . . . 1

15. Melittis Linn. . . . . . . . . . 1

16. Brunella Tourn. . . . . . . . . . 3

17. Stachys Benth........... 17

18. Ballota Benth. . . . . . . . . . 4

19. Leonurus Linn. . . . . . . . . . . 2

20. Moluccella Linn. . . . . . . . . . . 1

21. Lamium Tourn. . . . . . . . . . 10

22. Phlomis Tourn. ........... 4

25. Prasium Linn. . . . . . . . . . . . . 1

24. Galeopsis Tourn. ............ 2

25. Salvia Linn. . . . . . . . . . . . 16

$\S \S$ Nepete Benth.

26. Nepeta Benth. . . . . . . . . . 9 9

27. Dracocephalum Linn. . . . . . . . 2

II. Teucrinea.

28. Rosmarinus Tourn........... 1

29 Teucrium Linn. . . . . . . . . . 13

30. Aiuga Linn. . . . . . . . . . . . . . . 7

III. Scutellarinee De Vis.

31. Scutellaria Linn.

Species ....... $16 \frac{7}{166}$ 
Descrizione. - Le 166 specie nostrali di Lamiacee sono erbe annue o perenni, suffrutici e frutici, alti da pochi centimetri a un metro e due, di portamento assai svariato, e che nonpertanto si la. sciano facilmente riconoscere quali membri della famiglia pei molti caratteri comuni.

Sono sovente pelose, in vario grado e in varia maniera. Fra i peli, meritano speciale menzione i glanduliferi, frequentissimi, ora piủ lunghi e sporgenti sulla superficie dell'epidermide, ora cortissimi ed infossati nell' epidermide stessa, tanto da sembrare glandole inter. ne, come ben ha rilevato il Martinet (Sur les organes glanduleux des Labices in Bull. soc. bot. de Fr. 18. p. 239, e Organes de sécrétion des végétaux in Ann. sc. nat. $5^{a}$ ser. 14). Sono dessi, che segregando oli essenziali canforati, fanno delle Lamiacee piante aromatiche, e al gusto pizzicanti, quando non sono piuttosto amare per la prevalenza di principi di tal natura.

Il fusto è tetragono, con gli spigoli spesso ben marcati, talora oscuri. Porta sulle faccie le fogtie, che sono costantemente opposte, senza stipole, semplici, non articolate; variabili del resto per grandezza, da qualche millimetro a mezzo metro, per forma, dalla strettissima alla rotondata, per interezza o divisione poco sentita, cioè dentatura, rari essendo i casi di maggiore divisione.

Sulla struttura interna del fusto e delle foglie si può consultare: Mirbel, Sur l'anatomie el la physiologie des plantes de la famille des Labies (in Amn. mus. 15), Steinheil, Observations sur la tige du La. mium album (in Ann. sc. nat. $2^{a}$ ser. 1), Kiirchoff, De Labiatarum organis vegetativis commentarium, 1861, Guillard, Des caractères dislinctifs des familles à puiser dans l'organisme interne (in Adansonia, 8 ), Petersen, Om Barkens bygning ecc. (in Bot. Tidsstrift, $3^{a}$ ser. 1).

La infiorescenza è sommamente uniforme in sostanza, diversis. sima per l'aspetto. Sono sempre dicasi ascellari, ed opposti : talora palesemente dicasi (delle Nepela, delle Satureia), con gli stipiti liorali sufficientemente sviluppati, per quanto in generale con poea regolarità; ma molto più spesso i dicasi sono contratti, e convertiti in glo. meruli, di molti fiori e allora irregolarissimi, o li pochi fiori, spesso di tre, o anche ridotti a un fiore solo, nei quali casi si hanno due tiori opposti, o sei o un maggior numero in verticillo apparente: $\mathrm{i}$ cosi detti verlıcillastri. lira mezzo ai liori sonovi le bratteole, poco appariscenti in generale per essere ora lunghe a hastanza ma sottili, ora corte, e non di rado sono soppresse del tutto; di rado sono vistose, come nei Phlomis o nella Salureia Clinopodium, o nella Mlohucella dore 
sono convertiti in spine; mentrechè nel Rosmarinus e altrove sono sostituite da vere foglie. In quanto alle foglie fiorali alla cui ascella sono i dicasi, possono essere nolı diverse dalle altre, stando a distanza da palco a palce di fiori (Melissa, dei Teucrium, delle Scutellaria); possono anche andare diminuendo in grandezza, nel qual caso facilmente i fiori eziandio si ravvicinano sempre più fra di loro, da costituire nel loro insieme una infiorescenza foggiata a pannocchia, a grappolo, a spiga o a capolino: quando spesso altresi le foglie fiorali passano a brattee, piccole, o grandi, talora colorate (Thymus Origanum, delle Salvia). In certe Lavandula, e nella Salvia Horminum, le brattee terminali, grandi, colorate, sterili alla loro ascella, formano un ciuffo all'apice dell'infiorescenza. Su questo argomento della infiorescenza si può riscontrare le osservazioni di Wydler in Flora, 1851.

I singoli pedicelli fiorali sono sempre corti o cortissimi.

Il perianzio è 5 -mero, rarissimamente 4 -mero (Lycopus).

Quando è 5 -mero il calice, ha il sepalo dispari posteriore. Ė gamosepalo. Può essere regolare, diviso in 5 , o rarissimamente 4,0 10 (dei Marrubium) lobi uguali fra di loro; ma piủ spesso è irregolare, i lobi essendo più o meno disuguali, e tendenti a spartirsi fra due labbri, dei quali d' ordinario il posteriore è costituito da 3 lobi, e l'anteriore da 2, ma in certi Sideritis o Tencrium il labbro posteriore è di un lobo solo e l'anteriore di 4 ; la prevalenza per grandezza è ordinariamente del labbro superiore. L'unione dei sepali nei labbri può essere totale, cosi nelle Maiorana e nelle Scutellaria; nel quale ultimo genere si ha la singolarità del calice chiuso strettamente innanzi e dopo la fioritura. La gola del tubo calicino è sovente guarnita di peli erelti, che convergendo la chiudono. Degna poi di speciale studio è la nervazione del calice. In primo luogo esistono costantemente 5 nervature mediane ai sepali; oltracciò ne esistono altre 5 alternanti con le prime, cioè 10 in tutto; o pure rnancando queste i sepali ne hanno 3 , e sono 15 in tutto le nervature; e se le due modificazioni si combinano nelle varie parti di un medesimo calice, il numero delle nervature risulta intermedio, e per lo più è 15. Le nervature bene spesso sporgono, da essere vere costole; altre volte non sono così salienti; raramente sono invece affossate (Moluccella). La preflorazione del calice è aperta, eccettochè in pochi casi in cui è valvåre. $\grave{E}$ persistente, anzi un tantino accrescente.

- La corolla, gamopetala, ipogina, caduca eccettochè nelle Aiuga 
dov'è marcescente, è sempre irregolare, ma in vario grado, dalla irregolarità appena accennata di una Lavandula o di una Mentha, alla estrema di un Teucrium. Ad eccezione appunto dei Teucrium, in cui la corolla è fessa in dietro e si butta tutta in avanti formando un solo labbro, tutte l'altre Lamiacee l'hanno bilabiata, il labbro superiore risultando da 2 petali uguali fra loro, l'inferiore da $\bar{\jmath}$ disuguali, il mediano essendo diverso dai laterali e piủ grande. La diversità di grandezza fra i due labbri ora è a favore del posteriore, ora dell'anteriore; $\dot{e}$ al massimo nelle Ainga e in certi Sideritis, che hanno piccolissimo il labbro posteriore. Del resto molte sono le modifica. zioni secondarie nella forma della corolla, sia dei labbri, che del tubo; il quale talora è nudo nell'interno, talora provvisto di un anello di peli che lo chiude in vicinanza alla sua base sopra il gemmulario. Nella preflorazione il lobo mediano del labbro inferiore è coperto dai lobi laterali, e il tutto è coperto dal labbro superiore.

Mancando costantemente to stame posteriore, l'androceo non ne ha che $\dot{4}$, oppure (di rado) 2 quando mancarıo altresi gli stami la. terali. Sono inseriti nel tubo della corolla a varia altezza, sia asso. lutamente, sia per rispetto gli uni agli altri; più spesso sono sporgenti, o no (Lavandulece). Quando sono 4 non hanno mai la medesima precisa grandezza, ma la differenza può essere poco (Mentha) o molto pronunziata, ed allora sono maggiori gli stami anteriori, ad eccezione delle Nepetece in cui la cosa sta al contrario. Il filamento n' $\dot{\mathrm{e}}$ sottile, senz'appendici, o pure ne presenta una in forma di gohba, Ji dentino o di sprone, sia all' apice, sia verso basso (Phlomis, Ros. marinus). L'antera è continua col filamento, mediante un connettivo ordinariamente poco sviluppato, da lasciare che le due caselle dell'antera sieno contigue, di rado più sviluppato da far si che le caselle stiano discoste (Satureia, Thymus); il solo genere Salvia presenta la singolarità di un conteltivo straordinarianente sviluppato per lungo, e articolato sul filameuto, in guisa da assumere aspetto fi un bilancino, Irizzato e portante in alto una casella ad un estremo, mentre l'altro estremo pende in giù e non ha che un rudimento di casella. Anche nel genere S'sutellaria rrli stami superiori sono dimezzaci, cioc con una sola casella; e il Rosmarimus ha pure l'antere 1-loculari. Le sole Ǵaleopsis hanno trasversile la deiscenza delle antere; in tutte l'altre lamiacee è longitudinale; e per la contiguità delle caselle, e per la frequente mossa che famno aprendosi di sco. starsi in basso mettendosi in linea, facile ne risulta la confluenza in 
una sola; la qual cosa puó anche avvenire innanzi la deiscenza (Aiuga, Lavandula). Il polline è ovale, pieghettato.

Il gineceo riposa sopra un grosso disco, più o meno evidentemente 4-lobato, e cio in gerierale ugualmente e in alternanza coi 4 lobi nei quali trovasi diviso il gemmulario. La quale divisione è profondissima nella maggior parte della famiglia, e fa sì che i lobi stessi poggino con la loro estrema base sul disco. Ciò nella sottofamiglia delle Stachidinee. In quella delle Teucrinee invece la divisione è molto meno profonda, e non arriva al più che a mezzo il gemmulario; per cui l' inserzione dei lobi va dalla loro base sin oltre la metà del loro lato interno. In quella delle Scutellarinee poi il disco ha una forma specialissima, e induce modificazioni corrispondenti nel gemmulario; nella parte centrale è allungato, conico, e porta in cima il gemmulario profondamente diviso, posto obliquamente in avanti, cosicchè i lobi se ne trovano in due piani, e la loro inserzione è tutta laterale e non basilare. Lo stilo occupa sempre il centro del gineceo, vale a dire si trova sempre impiantato in fondo all' incavo apicale del gemmulario. Ė sporgente, o incluso unitamente agli stami (Lavandulece); è filiforme, e termina in due corti lobi antero-posteriori, talora sensibilmente uguali fra di loro, più spesso disuguali, essendo mag. giore il lobo anteriore, d'ordinario sono lesiniformi e divergenti, nelle Lavandulece peraltro e nelle Tendanece sono appianati e accostati; sono stimmatici nella punta o nei margini. Lo stilo è caduco. I 4 lobi del gemmulario sono la esterna indicazione delle 4 caselle in cui desso trovasi completamente diviso. In ogni casella v'ha una gemmula, attaccata nell' angolo interno, rovescia, ascendente, con integumento scempio, e che in corrispondenza alla inserzione dei lobi gemmulariani presenta le seguenti tre modificazioni: dove l'inserzione è basilare, la gemmula è attaccata dal fondo a metà altezza della casella, ed è più o meno anatropa; dove l'inserzione dei lobi si estende dalla loro base cospicuamente sul lato, la gemmula è attaccata dal mezzo in su della casella, ed è emianatropa e spesso quasi atropa; dove l'inserzione dei lobi è laterale mediana, la gem: mula ha pari attacco, ed è anfitropa.

Gli studi genetici hanno pienamente dimostrato il gineceo delle Lamiacee essere costituito da due pistilli. Vedansi su di ciò Clos, Observations sur le fruit des Labiées (in Bull. soc. bot. de Fr. 2. p. 169), Schacht, Das Mikroskop, $3^{a}$ ed. p. 234; e in genere sulla genesi fiorale della fainiglia Barnéoud in Ann. sc. nat. $3^{\mathrm{a}}$ ser. 6. p. 286, e Payer, Trailé d'organog. comp. de la fl. p. 553. t. 114. 
Frequenti assai, e già da gran tempo avvertite, sono in questa famiglia le variazioni di grandezza nei fiori della medesima specie, collegate ad una distinzione sessuale: essendo i fiori più grandi con androceo e gineceo ugualmente bene sviluppati, i più piccoli col solo gineceo perfetto, o talora (secondo Delpino) col solo androceo. Le relative notizie sono compendiate da Darwin, Des différentes formes de Reurs, pp. 305.31\%, e più estesamente esposte da Müller, Die Befruchtung der Blumen durch Insekten, pp. 306-332, insieme ai pro. cessi dell'impollinazione, già diffusamente studiati da Delpino (Ulteriori osservazioni sulla dicogamia). Non mancano neppure i fiori chiusi cleistogami, e sono ben noti quelli del Lamium amplexicaule.

Il frutto riproduce le fattezze del gineceo, poco cambiato e non molto aumentato in grandezzza. E circondato e nascosto dal calice, eccettochè nel Prasium dore questi si fa patulo. Nel genere Scutella. ria il calice è completamente chiuso per il combaciamento dei suoi labbri, talché soltanto alla maturità resta scoperto il frutto, quando il labbro inferiore del calice si disarticola e cade. Il colore del frutto varia dal cenerino al nero. Maturo, si dirompe in 4. cocchi, staccandosi i suoi lobi dalla loro base d'inserzione. Non di rado il numero dei cocchi è ridotto, fino ad 1. Il pericarpio n'è asciutto, crostoso; nel solo Prasium i cocchi sono drupacei, con mesocarpio carnoso ed endocarpio crostoso. In ogni cocco v' ha naturalınente un solo seme, con guscio sottile membranoso, con poca o punta mandorla. L'embrione è diritto, con embriofilli larghi, piani, con fusticino cortis. simo, infero; nel solo genere Scutellaria il fusticino è più lungo, ricurvato lungo il dorso di un embriofillo.

Sul germogliamento e sui processi vegetativi delle Lamiacee enropee si deve vedere Irmisch, Beitrïge zur vergleichenden Morpholo. gie der Pllanzell.

Considerazioni geografiche. - Sulla totalità delle Lamiacee no. strali una $50^{2}$ si trovano per tutta Italia, e sono in buona parte di quelle piante comunissime clie si presentano in gran copia l'individui dove hauno confacente la stazione. Un numero alquanto may. giore, una 60", sono per una metil abitatrici eselusive dell'Italia neridionale, e per l'altra meta specie che, avendo pur ivi la sede, s'inoltrano chi più chi meno nell'Italia centrale; a questa cateroria appartengnno per intero i generi Maiorana, Molucella, Phlomis e Prasium. Minore assai, di una ()$^{2}$ di specie, i la cateroria contraria, di piante esclusice dell'Italia setlentrionale, o che ha questa si avanzano nella centrale; $i$ generi esclusivi de questa categoria sono: 
Cuspidocarpus, Horminum, Leonurus e Dracocephalum. L'Italia centrale non ha tipi propri molto diffusi, essendochè le non molte specie che vi si trovano esclusivamente sono di quelle localizzate, delle cosi dette rare. Nondimeno, verificandosi in questa parte media d'Italia l'incontro di molti tipi e meridionali e settentrionali, essa può vantare una ricchezza in Lamiacee uguale a quella della Bassa Italia, ossia $2 / 3$ buoni del novero totale, mentre l'Alta Italia ne ha appena più della metà.

Se si pongono in riscontro il lato occidentale e l'orientale delI'Italia, non v'è più quel divario nella flora, dovuto alla differenza di clima; ma tuttavia alcuni caratteri speciali vi sono. Il lato orientale possiede in proprio 8 specie, e il lato occidentale ne possiede il quadruplo, proporzione maggiore dovuta sopratutto alle piante proprie della Corsica e Sardegna da una parte, della Sicilia e Calabria dall'altra parte.

Fra le specie piủ localizzate sono da notarsi: i due Dracocephalum delle Alpi; nella Liguria occidentale: Sideritis hirsuta, Tendana Piperella, Salureia thymoides, Brunella hyssopifolia, Ballota spinosa; in Corsica: Satureia filiformis, S. corsica, Nepeta agrestis; in Sardegna: Satureia Thymbra, S. cordata, Nepeta foliosa; in Corsica e Sardegna: Mentha Requieni, Thymus Herba-barona, Satureia glandulosa, Stachys corsica, S. glutinosa, Teucrium massiliense; in Sicilia: Maiorana Onites, Thymus nitidus, Salvia canariensis, Nepeta tuberosa, N. Apuleii, Teucrium creticum (di Lampedusa), Scutellaria Linnaeana; in Calabria: Phlomis lanata, Salvia ceratophylloides; in Sicilia e Calabria: Lavandula multifida, Moluccella spinosa, Aiuga orientalis; nell'Italia occidentale : Stachys marrubiifolia, Teucrium Marum; nella Penisola orientale settentrionale: Marrubium peregrinum, $S a$ tureia Hostii, Cuspidocarpus rupestris; nella orientale meridionale: Satureia cuneifolia, S. cristata, S. graveolens; nella orientale in genere: Satureia marifolia; ecc.

Considerata la distribuzione secondo l'altezza dei luoghi, risalta un primo fatto, ed è l'enorme preponderanza delle Lamiacee di luoghi bassi sopra quelle esclusive di luoghi alti, essendo le prime al quadruplo delle seconde. Però, siccome buonissima parte, $\mathrm{i}^{2} / 5$, di quelle di luoghi bassi s'inoltrano su pei monti fino ad altezze notevoli, ne viene minorata la sproporzione, e la flora lamiacea montanina sta all'altra $:: 2: 3$. Come specie più propriamente rnontanine si possono annoverare le seguenti: Sideritis sicula; $S$. hyssopifolia, Tendana Piperella, Mentha Requieni, Hyssopus officinalis, Thymus 
Herba-barona, Satureia cordata, S. fliformis, S. glandulosa, S. grandiflora, S. corsica, S. alpina, S. graveolens, Horminum pyrenaicum, Brunella grandifora, Slachys Alopecuros, S. densiflora, S. alpina, S. corsica, Lamium garganicun, Salvia Aethiopis, S. argenlea, Nepeta Nepetella, N. agrestis, N.:foliosa, Dracocephalum Ruyschiana, D. austriacum, Teucrium lucidum, T. montanum, Aiuga pyramidalis, A. acaulis, Scutellaria alpina, S. commulata. Notisi che in questo elenco stanno 9 sulle 12 specie proprie (quanto all' Italia) della flora Corso-sarda.

Le specie che piủ s'innalzano sui monti, dai $1500 \mathrm{~m}$. in sı, sono principalmente le seguenti: Thymus Herba-barona, T. Serpillum (sino a $2500 \mathrm{~m}$.), Salureia grandiflora, S. corsica (sino a $2200 \mathrm{~m}$.), S. alpina, Horminum pyrenaicum. Melittis Melissophyllum, Brunella grandiflora, B. vulgaris, Stachys Alopecuros, S. densiflora, S. Belonica, S. alpina, S. recta, Lamium garganicum (sino a $2500 \mathrm{~m}$.), L. album, L. maculalum, Caleopsis Telrahit, Salvia glutinosa, S. pratensis, Nepeta Nepetella, N. agrestis, N. nuda, Dracocephahum Ruyschiuna. D. austriacum, Teucrium Chamcedrys, T. montanum, Aiuga reptans, A. generensis, A. pyramidalis, A. acaulis, Scutellaria alpina. L'ailezza che raggiungono non è maggiore negii Appennini a latitudine più bassa che nelle Alpi più settentrionali; il che non sorprenderà coloro clie conoscono le condizioni inospitali delle eccelse vette appennine, in paragone alla protezione data nelle Alpi da molti luoghi riparati posti alla medesima altezza di quelle.

Esistono in Italia $\%$ delle Lamiacee europec. Essa possiede in proprio 14 specie, delle quali 1 assai diffusa, la Satureia temuifolia, 5 dell' Italia meridionale: Satureia consentina, S. fasciculata e Aiuga acaulis, 1 ristretta all'isola Marettimo dell'Egadi, il Thymus nitidus, 1 alta Liguria occidentale, la Satureia thymoides, e 8 proprie del gruppo Corso-sardo: Mentha Requieni (che la anche a Montecristo), Thymus Ilerba-barona, Satureia cordata, S. glandulosa, S. corsica, Stachys corsica, S. glutinosa, Nepeta foliosa.

Due specie, la Salvia hispanica dell'America tropicale, e la S. canariensis delle Canaric, sono di recente accertata naturaliti.

Le rimanenti 150 Lamiace italiane appartengono in m.ıggiore numero (\$j) alla categoria delle piante dette mediterrance, e si possono spartire come segue, secondochẻ sono presenti :

In tulto il bacino del Mediterraneo ........ 91

Nel solo Mediterraneo ocridentale . . . . . 14 
Nel solo Mediterraneo orientale . . . . . . . . 10

In tutta l'Europa meridionale ......... 11

Nella sola Europa meridionale occidentale . . . . 17

Nella sola Europa meridionale orientale . . . . 10

Di questa categoria mediterranea sono tutte le specie caratteristiche delle parti basse più calde d'Italia, abbenchè non poche altre s'inalzino nei monti, e d'assai come sarebbe il Lamium garganicum.

Un numero minore (66) è di piante nordiche per rispetto all'Italia, ossia di specie, per una metà dell'Europa media, per l'altra metà dell'Europa settentrionale e della media, che da quelle più alte latitudini si estendono in basso sul Mediterraneo. Alla quale categoria spettano a preferenza le piante esclusive dell'Alta Italia, o che da questa s'inoltrano verso la Bassa Italia, prescegliendo più spesso la via dei monti; e vi spettano poi quasi tutte quelle più largamente diffuse, le specie che si dicono comuni.

Una sola specie, l'Horminum pyrenaicum, è della ristrettissima categoria propria dei Pirenei e delle Alpi.

In conclusione, la flora lamiacea d'Italia è costituita da elementi nordici e da elementi mediterranei, con prevalenza di questi ultimi (nella proporzione di $3: 2$ ), e in essi degli occidentali sugli orientali (nella proporzione di $2: 1$ ).

L'area di abitazione delle nostre Lamiacee, prese in massa, $\dot{e}$ limitata a ponente dall'Atlantico, che pochissime specie varcano per ritrovarsi nell'America settentrionale; Asa Gray (Syn. fl. of North Amer. 2), fra molte specie europee naturalizzate, non ne menziona che 3 veramente indigene: Scutelluria galericulata, Brunella vulgaris e Stachys palustris, alle quali bisogna forse aggiungere il Lamium hybridum trovato in Terranova (Cand. prodr.). Il limite a sud si può mettere verso il $30^{\circ}$ grado di latitudine, giacchè molto al di là non trovansi notati che la Mentha Pulegium, il Thymus Serpillum, il Teucrium Scordium e la Scutellaria Linnaeana in Abissinia, senza far menzione della quasi cosmopolita Brunella vulgaris che fa sino in Australia. Il limite a nord può essere segnato dal circolo polare, al di là del quale non si spingono che pochissime specie fino al $70-71^{\circ}$ : il Thymus Serpillum, la Nepeta Glechoma, la Galeopsis Tetrahit, la Stachys silvatica, il Lamium purpureum, il L. maculatum (Lecoq géogr. bot. de l'Eur. 8). In quanto ai limiti orientali, in basso si possono assegnare come tali quelli dell'Asia occidentale, fino ai paesi del Caspio, ma in alto restano affatto indeterminati, le varie specie 
spingendosi chi piủ chi meno avanti a traverso la Russia, la Siberia occidentale e la Siberia orientale fino all'estremo continente asiatico, dove giungono il Lycopus europæus, il Thymus Origanum, il T. Serpillum, la Nepeta Glechoma, la Satureia Clinopodium, il Lamium maculatum, il L. album, la Galeopsis Tetrahil, la Stachys palustris, la Scutellaria galericulata, la Brunella vulgaris, ecc.

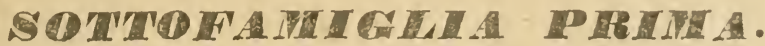

\section{STCAHHTIEE.}

STACHYDINEe.

Gemmularium profunde fere ad basim 4-partitum. Gemmulæ a basi ad medium loculorum insertæ, anatropæ vel hemianatropæ. Fructis cocci areola insertionis parra, basilari, sæe obliqua. Embryo cauliculo brevi, recto.

\section{TRIBU் PRIMA.}

\section{La va un dillece.}

LAVAXDULEE (non alior.).

Stamina tubo corollæ inclusa. Stylus inclusus, lobis (saltem in. feriore) complanatis.

\section{MA YANTUUMA.}

Lavandula el Stoechas Tourn. inst. p. 198, 201. 1. 93, 95.

Lavandula Linn. gen. pl. ed. 1. p. MGS. Mirb. in ann. mus. 15. 1. 17.

1. 1-6. Ging. hist. nat. des Lav. 18:6. Gen. 11. 1. germ 6.t.

2. Benlh. IIool. gen. pl. 2. p. I179. Ces. Pass. Gib. comp. R. it. p. 295. 1. 43. . . .

Cialyx tubulosus, valide 15-15-nervatus. Corolla vix bilabiata, labio superiore patulo, planiusculo. Stamina 4, filamentis edentulis, 
antheris 2-locularibus, loculis longitudinaliter dehiscentibus etiam ante dehiscentiam confluentibus, conniventia, inferiora longiora. Stylus lobis aqualibus. Cocci apice convexi leves.

Portamento. - Frutici o suffrutici bassi, cenerognoli, con fitte foglie di mezzana grandezza, intere o divise, e fittissimi fiori piccoli, violetti, in infiorescenze terminali, ciliniriche, compatte o diradate, bratteate, le brattee terminali sono talora colorate e formano una chioma.

Osservazione. - Il disco in questo genere è spartito in quattro squammette, contrapposte ai lobi del gernmulario; quando questi, passati allo stato di cocchi del frutto, si distaccano, portan via seco la relativa squammetta, bianca, la quale da alcuni osservatori (Gin. gins, Bentham) è stata shagliata per la cicatrice d'inserzione del cocco, descritta come estrorsa, mentrechè la vera cicatrice è basilare.

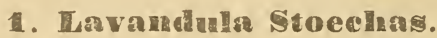

L. fruticosa, foliis integerrimis, bracteis latis rotundatis, supre. mis sterilibus magnis coloratis comam efformantibus, calycis dente superiore dilatato coteris maiore.

Lavandula Stoechas Linn. sp.pl.ed. 1. p. 5\% 3. Bert. fl. ital. 6. p. 79. Pucc. syn. pl. luc. p. 300. De Not. rep. fl. lig. p. 314. Gren. Godr. fl. de Fr. 2. p. 647. Simi! R. alp. vers. p. 125. Guss. en. pl. In.p. 252. Mor. fl. sard. 3. p. 26\% Car. prodr. fl. tosc. p. 500. Ard. cat. pl. Ment. p. 28. Tass. A. prov. sen. p. 50. Car. fl. Montecr. p. 28. Tass. cenno bot. Sien. p. 85. Ard. Al. Alpes-mar. p. 294. Mars. cat. pl. Corse p. 111. Genn. fl. Capr. p. 112. Archb. fl. AltoSerch. p. 59. Cies. Pass. Gib. comp. A. ital. p. 269. Arc.! comp. fl. ital. p. 535 .

Figure. - Savi mat. med. veg. tosc. t. 55. Ging. hist. nat. Lav. t. 3. Reich. ic. fl. germ. 18. t. 26. f. 3.

Stazione, Abitazione e Fioritura. - Nei luoghi aridi selvatici sul Mediterraneo, e s'interna anclıe sui poggi a qualche distanza dal mare: comunissima in tutto il littorale Ligure, da Nizza alla Spezia (Bert. ecc. ecc.), e nel littorale Toscano, dalle parti basse delle Alpi Apuane (Simi!) al monte Argentario!, innalzandosi a 500 metri all'incirca, e risalendo il Val-di-Serchio fino al Ponte a Moriano soprá Lincea (Calandrini! ecc.), e il Val-d'Arno fino al monte Albano e ai colli Fiorentini che ne dipendono!, e si ritrova a Siena (Cam. pani! ecc.); nelle isole tutte dell'Arcipelago toscano, ad eccezione 
di Giannutri e di Pianosa (Car. ecc.); in Terra di Lavoro a Sperlonga (Terracciano!), a Gaeta (Bert.), nclle isole d'Ischia (Bolle ! ecc), e di Capri (Rert.), al Capo Palinuro (Ten. syll.); in Calabria a Nonteleone (Ricca!), a S. Venere (Arc.!); in Corsica a Bastia (Debeaux! ecc.), a Corte (Requien!), a Calvi (Soleirol!), ad Aiaccio (Kralik!); in Sardegna, comunissima (Mor.); in Sicilia nella costa settentrionale da Trapani a Cefalù (Guss. syn., ecc.); nell'isola di Pantellaria (Calcara!). Fiorisce da marzo ed aprile a maggio e gingno.

Distribuzione geografica. - In tutto il bacino del Mediterraneo, nelle isole Canarie, in Madera.

Descrizione. - Sufrutice in cespuglio, di un verde bianchiccio, tomentoso per peli corti stellati, odoroso. Il fusto è legnoso in basso, ramosissimo con i rami eretti, quadrangolari, con gli angoli ottusi e sporgenti, verdognoli-bianchicci. Le foglie sono opposte, eretto-patenti, le inferiori e quelle dei ranetti non fioriferi arvicinate in modo da parere in fascetti, strette, lineari, o lineari-bislunghe, ottuse o ottusette, saldate tra loro all'infima base, intere e con i margini rovesciati, di un verde biancliccio e tomentose in ambedue le pagine, con un solco longitudinale nella pagina superiore e con un nervo grosso longitudinale più chiaro nella inferiore, dal quale partono dei nervetti laterali poco obliqui, visibili quando si guarda le foglie contro la luce. I fiori sono molti, piccoli, folti, distribuiti in 4 fila longitudinali in una spiga ovale o bislunga, fitta, portata all' apice di un peduncolo il quale è corto, quadrangolare, di colore violetto, la spiga ha in alto dei fiori sterili. Le brattee dei fiori fertili sono larghe, ovato-romboidali, quasi trilobe, con una punta corta all' apice, di un violetto rossiccio con nervi ramosi piủ scuri, tomentose di fuori, concave e glabre di deutro, con lunghe e folte ciglia bianche nel margine; quelle dei fiori sterili che sono in alto della spiga, sono molto grandi, e formano quasi una chioma a questa: esse sono erette o eretto-patenti, larghe, obovate, piegate e come increspate, di un violetto chiaro con nervi ramosi più carichi, stellato-tomentose di fuori, con pochi peli stellati di dentro, sono ristrette in basso quasi in una unghia. Il calice è tuboloso, un po gonfio, verdognolo-violetto, con nervi longitudinali verdognoli, peloso, lıa cinçue denti corti, quasi uguali, larghetti, ottusi, il superiore dei quali lıa una appendice la quale è larghelta, tonda-reniforme, con una parte più strelta che l'unisce al dente, violelta, pelosetta. La corolla is jiỉ del doppio piủ lunga del calice. Il lubo è quasi il doppio piủ lungo di questo, curvato, piu largo in alto verso 
la gola, violetto-chiaro e quasi bianchiccio, poco tomentoso, di dentro è irșuto per peli bianchi e lunghi. Il lembo ha due labbri, uno superiore bilobo, uno inferiore trilobo, tutti e due con i lobi quasi uguali e quasi tondi, e di colore porporino scuro, quelli del lobo superiore diretti in alto, quelli dell' inferiore diretti in fuori ed in giủ. Gli stami fertili sono 4 , didinami, due poco più lunghi e due poco più corti del tubo della corolla, i primi avvicinati al labbro inferiore. I filamenti sono quasi filiformi, corti, glabri. Le antere quasi ovali, di un violetto scuro, pelose, uniloculari, aprentisi in semicerchio. Il polline è giallo. Il pistillo è più corto degli stami più corti. Lo stilo è eretto, cilindrico, bianchiccio, glabro. Lo stimma è in capolino, quasi bilobo, di color violetto scuro. (Parl. ms., descr. della pianta di Sestri nella Riviera di Ponente).

Osservazione. - Gingins (Hist. nat. Lav. p. 130), Bentham (Lab. p. 147), Gussone (Fl. sic. syn. 2. p. 65), Moris distinguono una varietà macrostachya, che non merita davvero di essere separata dal tipo specifico.

\section{Lavandenta dentata.}

L. fruticosa, foliis inciso-crenatis, bracteis latis rotundatis, supremis sterilibus magnis coloratis comam efformantibus, calycis dente superiore dilatato coeteris maiore.

Lavandula dentata Linn. sp. pl. ed. 1. p. 572. Zer. fl. mel. thes. p. 27. Ten. syll. fl. neap. p. 280. Ces. Pass. Gib. comp. fl. it. p. 296. Arc. comp. fl. it. p. 535.

Figura. - Ging. hist. nat. Lav. t. 5.

Abitazione e Fioritura. - Nell' isola di Malta secondo Zerapha; nel Gargano secondo Baselice (Ten.); in Sicilia secondo Bentham (Lab. p. 149), ma non figura nella Flora di Gussone; e in genere è molto dubbia la qualità d'indigena per questa pianta, che coltivata di frequente ne' giardini, può talvolta e temporaneamente essersi mostrata fuori di quelli. Fiorisce in primavera.

Distribuzione geografica. - In Spagna, in Madera, in Algeria.

\section{Tirvandalm of}

L. suffruticosa, foliis integerrimis, bracteis latis ovatis, supremis liaud comantibus, calycis dente superiori dilatato coeteris maiore.

Lavandula Spica Linn. sp. plant. ed. 1. p. 572 (excl. var. ß). 
Zer.? fl. mel. thes. p. 26. Trev. prosp. fl. eug: p. 25. Bert. fl. ital. 6. p. 75. Mor. fl. sard. 3. p. 263. Bicchi! agg. fl. lucch. p. 15. Ard. cat. pl. Ment. p. 28. Zersi prosp. piante Brescia p. 163. Ing.? cat. sp. Mond. p. 46.

Lavandula officinalis Chaix in Vill. hist.pl. Dauph. i. p. 355 (1786). Car. prodr. 凡. tosc. p. 500. Tass. 月. prov. sen. p. 50. Genno bot. Sien. p. 85. Ard. R. Alpes-mar. p. 29't. Ces. Pass. Gib. comp. R. ital. p. 296. Arc. comp. fl. itnl. p. 535.

Lavandula angustifolia Moench meth. p. 389 (1799).

Lavandula vera Cand. fl. fr. 5. p. 398 (18/5). De Not.! rep. n. lig. p. 313 (var. ligustica). Koçh syn. 1. germ. ed. 2. p. 631. Hausm. R. Tir. p. 669. De Vis. Sacc. cat. piant. Ven. p. 131.

Figure. - Ging. hist. nat. Lav. t. 6. Reich. ic. fl. germ. 18. t. $26 . f .1$.

Stazione, Abitazione e Fioritura. - Sui poggi aridi sassosi, per tutta l'Italia, ma sparpagliata: in Piemonte fra Nus e Villa. franca in val d'Aosta (All. fl.), nel tratto da Susa a Oulx (Parl.! Aiuti! ecc.), abbonda poi nei subalpini Valdensi, di Vinadio, di Valdieri (All.), cosi in val S. Mlartin Perosa (Rostan!), tra Borgo S. Dalmazzo e Valdieri, tra Vernante e Limone (Parl.!), e sul versante inediterraneo a Tenda (Bourgeau!), nella Riviera di Ponente a Nizza (Durando! ecc.), Mentone (Ard.), S. Remo (Panizzi!), Pietrabruna (Bert.), Dolcedo (Berti!), Diano (Ricca!), e in parecchi altri luoghi in prossimità di quelli, quindi sopra Genova a Bavari (De Not.!) e a Rati (Carrega!); in Tirolo a Trento, a Bolzano, a Merano, ma, dicesi, non spontaneo (Koch, Hausm.); sul Lago di Crarda, a Verona verso Aresa e S. Martino, nei colli Berici (Poll. f., Piert.) ed Euganei (Trev.); a Trieste (Bert.); a Bologna nel monte Paderno (Bert.); in Toscana nel monte Pisano (Sav.), presso Firenze nel monte Rinaldi, nel monte Ceceri e nei colli di Pontassieve (Bert.), e a Porto S. Stefano in Maremma (Aiuti!); nel Gargano (T'en.); in Terra di Lavoro a Sperlonga ('Terracciano!); nel Pollıwo alla Scala di Morano (Ten. syll.); in Corsica a Calvi (Solei. rol!); in Sardegna nei monti Orgosolo, verso Dorgali (Mor.); in Sicilia a Noara (Munafó!), presso Messina a Mili (Seguenza!), presso Catania (Parl.!), fra Noto ed Avola (Gisss, syn.); in Malta? (Zer... l'iorisce nell' estate e l'autunno nelle parti settentrionali, nella priinavera e l'estate nelle parti meridionali.

Distribuzione geografica. - Nel bacino occidentale del Mledi. terraueo. 
Descrizione. - Pianta di un verde bianchiccio per corti e piccoli peli stellati, frutescente, alta sino a 6 decimetri, formante graziosi cespugli, di odore grato. Fusti legnosi in basso, molto ramosi alla base con i rami ascendenti o eretti, in parte sterili, in parte fioriferi. Pami fioriferi eretti, delicati, quadrangolari, con ggli angoli ottusi, e con un solco nelle faccie, verdognoli-bianchicci con peli corti e stellati. Foglie opposte, spesso con fascetti di giovani foglie che sono corte, strette, lineari e di un bianco sericeo : eretto-patenti, lineari o lineari-lanceolate, ottusette, un po'ristrette alla base, di un verde chiaro di sopra ed ivi con un solco longitudinale, di un verde-bianchiccio di sotto, col nervo longitudinale concolore, sporgente, coi margini rovesciati, interi. Fiori in pochi giri (ciascuno di circa sei fiori) alquanto vicini in alto, più lontani in basso e formanti così una spiga delicata, bislunga, lineare, interrotta e portata sopra un peduncolo molto lungo, quadrangolare, con un solco nelle facce. I pedicelli sono molto corti, grossotti, bianchicci. Le brattee sono larghette, scagliose, ovato-romboidali, prolungate all' apice in una punta lunghetta, scure, con i soliti peli stellati. Le bratteole sono setacee, scagliose e scure come le brattee, talvolta mancano. Il calice è tubuloso, con dieci costole ottuse, con cinque denti corti, oftusissimi, conniventi, il dente superiore ha una piccola appendice quasi orbicolare; è pubescente-stellato per peli lunghetti di colore violetto, più chiaro alla base. La corolla è di un bel colore violetto, quasi il doppio più lunga del calice. Il suo tubo è più lungo di questo, striato, quasi irsuto per corti peli diretti in giù, più largo in alto, verso la gola di dentro è pubescente ed ha quasi un anello di peli lunghi. Il labbro superiore è diretto in alto ed in dietro, è quasi ovale, smarginato-bilobo, l'inferiore è appena più corto del superiore, diretto in avanti ed un po' in giủ, trilobo con i lobi uguali ed ovali, ottusi: ambedue i labbri sono pubescentiglandolosi. Stami 4 , inclusi, due poco più alti degli altri due, inseriti in alto del tubo. Filamenti lesiniformi, bianchicci, glabri. Antere bilobe in basso, con i lobi tondi, gialliccie, poi scure, pelose di fuori e un po'verso la incavatura dei lobi, uniloculari, aprentisi in un disco tondo. Polline quasi dorato. Pistillo più corto degli stami. Ovario quadrilobo, con i lobi quasi tondi, verdognoli e glabri, sopra un disco più grosso di lui e verdognolo. Stilo erelto, quasi cilindrico, bianchiccio, pubescente. (Parl. ms., descr. della pianta delle alpi di Valdieri.)

Osservazioni. - Questa specie varia per la larghezza delle fo. 
glie, per la maggiore o minore radezza delle infiorescenze, e per la grandezza delle brattee, che peró non arrivann mai nella pianta italiana a quell' ampiezza che mostrano nclla pianta dei Pirenei.

Uso. - Gli steli fioriti si legano in inazzetti, e si mettono negli armadi di biancheria e di panni per fugare le tignole. La pianta essendo coltivata a questo scopo, tende a insalvatichire, e poi a sparire, per cui risultano dubbie varie sue localitả: in Toscana per esempio non l' ho mai incontrata.

\section{Eavandula latifolia.}

L. suffruticosa, foliis integerrimis, bracteis angustissimis fere filiformibus, supremis haud comantibus, calycis dente superiore dilatato coeteris maiore.

Lavandula Spica $\beta$ Linn. sp. plant. ed. 1. p. 572.

Lavandula latifolia Vill. hist.pl. Dauph. 2. p. 363. Bert. fl. ital. 6. p. 77. Car. prodr. 月. tosc. p. 50\%. Ard. rat. pl. Ment. p. 28. Fl. Alpes-mar. p. 294. Ces. Pass. Gib. comp. fl. ital. p. 296. Arc. comp. A. ital. p. 59j.

Lavandula Spica De Not. rep. fl. lig. p. 3/3. Zan. prosp. $l$. ven. p. 26.

Figure. - Ging. hist. nat. Lav. t. 7. Reich. ic. fl. germ. 18. t. 26. p. 2 .

Stazione, Abitazione e Fioritura. - Dove la specie precedente, per i poggi aridi sassosi, ma molto piủ ristretta nella sua distribuzio. ne: in Piemonte (Colla herb.); nei colli Euganei ('Zannichelli); nella Riviera di Ponente a Nizza (Parl.!), Mentone (Ard.), Porto Maurizio (I)e Not.), Capo delle Nele (Berti!), Finale (Caldesi!), Capo di Noli (Piccone!); intorno a Firenze nei colli di Fiesole!, di Pontassieve (Parl.!), dell' Incontro (Ricasoli!), dell' Inpruneta (Bucci!), di ('ireve (Reboul!); presso R'erugia nel monte della Guardia (Bert.); nel Napoletano e in Sicilia (Ces. Pass. (iib.). Fiorisce nell' estate e l'antumno.

Distribuzione geografica. - Nel bacino occidentale del Mediterranen.

Descrizione. - Pianta ndorosa, hianchiecin-verdognola, tomentosa, tomento piccolo, stellato. Fusto leguoso, ramosissimo alla base, rami ascendenti, cespitosi, - in basso legnosi e seuri, rami dell'anno ascendenti o crelli, yua irangolari, con gli angoli ottusi e le facce yuasi scanalate. Fuglie lineari-lanceolate, piủ strette in basso, 
ottuse, piane, un po'convesse di sopra e un po' concave di sotto per i margini leggermente rovesciati, con un nervo longitudinale più sporgente e piủ bianco di sotto che di sopra, tomentoso-bianchicce in ambedue le pagine, erette, le inferiori avvicinate quasi in fascetti, quelle del fusto lontane, più strette delle inferiori, erette anch'es. se, gradatamente più piccole. Bratteole strette lineari, ottuse, più corte del calice 0 quasi uguali a questo, erette ed avvicinate al calice stesso, tomentose. Calice tuboloso, quasi cilindrico, con costole grosse longitudinali, con cinque denti cortissimi e ottusi, il superiore dei quali ha all' apice una appendice quasi orbicolare e avvicinata al tubo della corolla, tomentoso, verdognolo-turchiniccio. Corolla meno del doppio più lunga del calice, turchina, di fuori tomentoso-stellata. (Parl. ms., descr. della pianta Fiorentina.)

Uso. - Il medesimo che per la specie precedente.

\section{Kayandula muluifita.}

L. suffruticosa, foliis bipinnatisectis, bracteis latis rotundatis, supremis haud comantibus, calycis dente superiori cæteris vix latiore.

Lavandula multifida Linn. sp. pl. ed. 1. p. 572. Guss. pl. rar. p. 231. Ten. fl. nap. 5. p. 8. Syll. p. 280. Guss. fl. sic. syn. 2. p. 66. Bert. fl. ital. 6. p. 78.

Figura. - Ging. hist. nat. Lav. t. 11.

Stazione, Abitazione e Fioritura. - Nelle colline aride marittime dell' estrema Calabria al Capo dell'Armi (Guss., Pasquale! ecc.), e della vicina Sicilia fra Messirra e Catania a Capo Scaletta (Guss.) e alla locanda di Latien (Tineo!). Fiorisce in marzo, aprile e maggio.

Distribuzione geografica. - Sul Mediterraneo in Portogallo, Spagna, Italia, Egitto, Tunisia e Algeria.

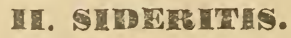

Marrubiastri sp. et Sideritis Tourn. inst. p. 190, 191. t. 89, 90. Sideritis Linn. gen. pl.ed. 1. p. 171. Mirb. in ann. mus. 15. t. 17. f. 7-12. Benth. Hook. gen. pl. 2. p. 1205. Ces. Pass. Gib. comp. fl. ital. p. 313. t. 46.f. 4 .

Sideritis, Burgsdorfia, Hesiodia Gen. pl. fl. germ. 6. t. 35-37.

Calyx tubuloso-campanulatus vel infundibuliformis, valide aut leviter 10-nervatus. Corolla bilabiata, labio superiore erecto vel pa- 
tentiusculo, planiusculo. Stamina 4, filamentis edentulis, antheris 2-locularibus, loculis longitudinaliter dehiscentibus, confluentibus, conniventia, inferiora longiora sæpe cum antheris abortivis. Stylus lobis inæqualibus, inferiore superiorem amplectente. Cocci apice convexi leves.

Portamento. - Le poche specie italiane del genere si dividono per il portamento in due gruppi. Nel primo stanno piante suffruticose, con falsi verticilli di liori accompagnati da grandi brattee; nel secondo sono piante annue, con foglie fiorali ai verticilli. Del resto questi sono numerosi, ravicinati fra loro o distanti, i fiori, piutto. sto piccoli, sono gialli o bianchi, le foglie sono ovali allungate, in. tere o dentate, variamente pelose.

Osservazione. - E difficile indovinare cosa sia la Sideritis incana, pianta di Spagna, indicata da Allioni (Fl. ped. 1. p. 26) nel Nizzardo tra l'Escarena e Roccatalliada.

\section{Gideritis sieula.}

S. suffruticosa, lanata, foliis oblongis, denticulatis, bracteis late ovatis, acuminatis, integris, dentibus calycinis sulæqualibus.

Sideritis sicula Ucria pl. ad Liın. op. add. Bert. fl. ital. 6. p. 81. Ces. Pass. Gib. comp. fl. ital. p. 314. Arc. comp. fl. ital. p. 549.

Sideritis brutia Pasq. Lic. viagg. Garg. p. 23.

Figure. - Barr. ic. f. 1187. Ten. R. nap. t. 51.

Stazione, Abitazione e Fioritura. -- Nei luoghi sassosi asciutti dei monti della Penisola nella sua metà inferiore, e della Sicilia : nell'Appennino di Foligno da Colfiorito a Muccia!, sopra Ascoli ad Arquata (Bert.), in Abruzzo a Cerqueto (Orsini!), presso A quila (Groves!), a Massa d'Albe sotto il Velino, nel Morrone all'Avrellanito sopra Solmona a 1000 metri, tra Solmona e Canzano (Levier!), a Castel di Sangro (Hıet!), a Salviano (Ten. syll.), nel Lazio sopra Arsoli (Rolli!) e a Riofreddo (Bert.), in Molise a Pettorano (Ten.), e sui monti di Campobasso (Pasquale!, , nel Gargano sopra Manfredonia (T'en.), e a Monte S. Angelo (Porta e Rigono!), in Basilı'ata nel monte di Viggiano (Pasquale!), nell'dlpe di Latronico, a Ruggia (Ten.), in Calabria al Pollino (Thomas!), a Tempone, Cocuzzo (Ten.), Campotenese (Bert.), in Sicilia nelle Madonie (I'arl.! ece.) a 1500-1800 metri (lluet!). Fïrisce in giugno e luglio.

Distribuziono geografica. - Dicesi nascere inoltre in Spagna. 
Descrizione. - Suffrutice fittamente cespugliuto, mollemente cotonoso e biancheggiante, aromatico, co'rami eretti o ascendenti, alti fino a $1 / 2$ metro, semplici. Foglie bislunghe-lanciolate, le inferiori ristrette in basso, acute. Glomeruli di 5 fiori, numerosi, distanti fra loro in modo da costituire infiorescenze allungate. Brattee slargate, arrotondite, acute, abbraccianti, gialle. Fiori di un giallo pallido, sessili, senza bratteole. Calice fittamente cotonoso, tubuloso, ristretto in basso, 5-fido, coi lobi eretti, lanciolati, acuti, pressochè uguali fra loro, i 2 inferiori essendo appena più piccoli degli altri. Corolla dal tubo chiuso nel mezzo da un anello di peli, cilindrico di sotto, di sopra slargato e campanulato; dal labbro superiore eretto, 2-fido, coi lobi acuti; dal labbro inferiore più corto, J-partito, coi lobi uguali, ricurvati. Stami superiori inseriti alla metà del tubo, gli inferiori sotto alla sua gola. Disco più grande in avanti. Stilo corto, coi lobi di diversa forma, l'inferiore slargato e fatto a doccia che ab. braccia il superiore più lungo, cilindrico, troncato. (v..v. nell'Appennino di Foligno).

Osservazioni. - Questa pianta appartiene a un gruppo di specie Orientali, sommamente affini fra di loro. Delle quali un'altra, la Sideritis perfoliata, è stata indicata sull'Etna dall'Ucria (Hort. pan. p. 242), ma nessun altro botanico (ch'io sappia) ve l'ha trovata, e non so perchè sia stata ammessa nella flora italiana dagli autori dei Compendi.

\section{Sideritis hirsuts.}

S. suffruticosa, hirsuta, foliis cuneato-oblongis ovalibusve, inciso-dentatis, bracteis late ovatis, dentato-spinosis, dentibus calycinis subæqualibus.

Sideritis hirsuta Linn. sp. $\mu l$. ed. 1. p. 575. Bert. fl. ital. 6 . p. 86. Ard.cal. pl. Ment.p. 29. Fl. Alpes-mar. p. 302. Ces. Pass. Gib. comp. fl. ital. p. 314. Arc. comp. fl. ital. p. 549.

Sideritis scordioides De Not. rep. fl. lig. p. 328 (non Lirn.).

Figure.-Barr. ic. f. 1160. Cav. ic. f. 302. Reich. ic. fl. germ. 18. t. 24. f. 1.

Stazione, Abitazione e Fioritura. - In luoghi aridi sassosi della Piviera di ponente, rara: Nizza (All., Bert., De Not.) a Vinaigrier (Sarato!), Villafranca (Ard.), Turbia (Bert.), Roccabruna, Levenzo, Utelle, col di Braus (Ard.), valle di Tenda (Bourgeau!), fiu- 
mara di Cervo (Ricca!), Albenganese (De Not.). Fiorisce da maggio e giugno a luglio e agosto.

Distribuzione geografica. - Nell'Europa meridionale, dal Por. togallo all'Italia.

\section{Sideritis Inysapifolia.}

S. suffruticosa, parce pilosa, foliis cuneato-oblongis linearibusve, subdentatis, bracteis late oratis, dentato-spinosis, dentibus calycinis subæqualibus.

Sideritis hyssopifolia Linn. sp. pl. ed. 1. p. 575. Ed.2. p. 803. Vitm.? ist. erb. p. 32, 45.

Sideritis scordioides Linn. sp. pl. ed. 2. p. 803. Com. fl. com. 4. p. 299. Rota prosp. fl. Berg. p.70. Ces, Pass. Gib. comp. fl. ital. p. 314. Arc. comp. fl. ital. p. 529.

Sideritis hirsuta Mass.? prodr. fl. valt. p. 176 (ex loco).

Figure.-Reich. ic. fl. germ. t. 24. f. 2-4.

Stazione, Abitazione e Fioritura. - Benchè indicata da Linneo in Toscana, ed ivi da Vitman nell'Appennino pistoiese e nelle Alpi apuane, da Ucria e da Cupani in Sicilia, questa pianta non vi nasce. Più di recente Bentham l'ha data del Piemonte, e più specificatamente delle Alpi piemontesi ( $L a b$. gen. et $s p$.), Mlassari della Valtellina sulla fede dell' erbario Ferrari, se tant'è che la sua Sideritis hirsuta sia questa specie, Comolli del Comasco al di sopra di Aquate alle falde del Resegone, e del Bergamasco nel letto del torrente Bione, trovata in quest'ultimo luogo dal Rota, che a sua volta scrive che fa presso Somasca: dai quali luoghi tutti non ho reduto alcun saggio. Fiorisce in luglio e agosto.

Distribuzione geografica. - Nell'Europa ineridionale, dalla Spagna alla Svizzera e all' Italia.

Osservazione. - Molto probabilmente questa specie dorrà unirsi alla precedente.

\section{Sideritis montuma.}

S. annua, hirsuta, foliis lanceolatis, subintegris, floralibus subconformilus, dentilus calycinis inequalibus, tribus superioribus paullo maioribus. 
Sideritis montana Linn. sp. pl.ed. 1. p. 575. Trev. prosp. 1 . eug. p. 26. Bert. fl. ital. 6. p. 83. Com. fl. com. 4. p. 301. Car. prodr. fl. tosc. p. 515. De Vis. Sacc. cat. piant. Ven. p. 138. Ces. Pass. Gib. comp. fl. ital. p. 314. Cald.! fl. fuv. tent. p. 182. Arc. comp. fl. it. p. 550 .

Figure. - Col. ecphr. p. 196. Fl. graec. t. 551. Reich. ic. fl. germ. 18.t. 25. f. 3.

Stazione, Abitazione e Fioritura. - In luoghi asciutti, selvatici o campestri, della Penisola settentrionale e centrale, non comune: in Piemonte presso Dagonney (All. auct.), e sopra Vogogna e Premosello (Bir. $f l$. acon.), sul S. Salvatore presso Lugano, nel Comasco sopra Villa Albese ed Erba, e fra Bonzanigo, Viano e Tremezzo (Com.), negli Euganei verso Arquà (Rigo! ecc.), a Ravenna (Pasolini!, Beccari!), intorno a Faenza in parecchi siti (Cald.), a Castrocaro (Cald.!), a Firenze verso l'Incontro (Ricasoli!), nel monte Pisano (Savi $f l$. pis.), nelle Marche presso Albacina e nei monti della Serra S. Quirico a 500 metri (Bucci!), nel Piceno sul Vettore sopra S. Gemma!, a Rieti (Rolli!), in Abruzzo nei monti di Cesa e di Castelvecchio (Bert.), a Salviano e nel monte di Mezzo (Ten. syll.), presso Castelrecchio Subequo alle falde del Sirente (Groves!), e presso Massa d'Albe alle radici del Velino (Levier!). L'Ucria l'aveva indicata nelle Madonie in Sicilia; ma i botanici moderni non ve l'hanno trovata. Fiorisce da maggio a luglio.

Distribuzione geografica. - Nell'Europa meridionale, dalla Spagna alla Russia, nell'Asia occidentale fino all' India, nell'Affrica settentrionale.

\section{Sideritis romana.}

S. annua, hirsuta, foliis ovali-oblongis, dentatis, floralibus conformibus, dentibus calycinis valde inæqualibus, supremo maximo.

Sideritis romana Linn. sp. pl. ed. 1. p. 575. Bert. fl. ital. 6. p. 84; et auct. omn.

Figure.-Cav. ic. t. 187. Fl. grcec. t. 552. Reich. ic. fl. germ. 18.t.25.f.1.

Stazione, Abitazione e Fioritura. - Per quasi tutta Italia nei luoghi incolti, nei campi asciutti, ecc. Nell'Alta Italia è rara, non essendovi stata trovata che a Venzago nel Bresciano (Zersi prosp.), nei colli del Mantovano (Barbieri!), in vari siti intorno a Verona 
(Bracht!, Manganotti! ccc.), nel lido di Venezia (Kellner! ecc.), in Istria a Parenzo, Rovigno, Pola (Bert.), a Ravenna (Caldesi!), a Faenza nel monte Mauro (Cald. lent.). Quindi nella Penisola orientale tro. vasi a Civitanova, a Serravalle nell'Appennino, a Grottamare (Bert.), a Montefortino dove abboṇla (Marzialetti!), ad Ascoli (Parl.!), a Pescara (Kuntze!), a Caramanico (Ces. el. Maiell.), nel Gargano a S. Nicandro (Porta e Rigo!), sul Vulture dove abbonda (Terr. $l$. Vult.), a Otranto (Groves!). Dall' altro lato della Penisola è comunissima in tutta la Liguria, come pure in tutta la Toscana nelle pianure e le vallate e per le falde inferiori dei monti, e vi nasce nelle isole di Gorgona (P. Savi!) e di Capraia (Ior. De Not. fl. Capr.); più giu trorasi a Viterbo e Canino (Bert.), a Roma, comunissima (Seb. Maur. prodr.), a Napoli, pure (Ten. syll.), in Ischia (Guss, enum.) e Capri (Pasị. 月. ves.), infine in Calabria da Pizzo a Staiti

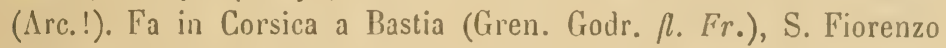
(Soleirol!), Calvi (Bert.), Corte, Aiaccio (Gren. Godr.), Bonifacio (erb. Webb! ecc.); in Sardegna, dov'è comune (Mor. $l$. sard.), a Caprera, La Maddalena (Gren. $l$. Capr.), Cagliari (Ascherson!), S. Antioco (Bert.); ovunque in Sicilia, e in tutte le isolette circostanti, fino a Lampedusa (Aiuti!); infine in Nlalta (Duthie! ecc.). Fiorisce secondo $i$ lnoghi in aprile e maggio, o maggio e giugno, o giugno e luglio.

Distribuzione geografica. - In tutto il bacino del Mediterraneo.

Osservazione. - La Sideritis approximata Gasparrini! (in Guss. suppl.), fatta con individui evidentemente stentati della forma comune, non è neanco una varielà.

\section{MIARRUIBUMI.}

Marruljium Tourn. inst. p. 192. 1. 91. Mirb. in ann. mus. 15. t. 18. f. 16-19. Gen. pl. 1l. germ.6.1. 38. Benth. Ilook. gen. pl. ?. p. 1206. Ces. Pass. Gib. comp. R ital. p. 31.1. 1. 46. 1. 万.

Calyx tubuloso-campanulatus vel infundibuliformis, valide 10 nervatus. Corolla bilabiata, lahio superiore erecto, planiusculo. Sta. mina 4, filamentis edentulis, antheris 2-locularibus, loculis longitudinaliter dehiscentibus, confluentibus, comniventia, inferiora longiura. Stylus lobis inequalibus, inferiore superiorem amplectente. Cocci apice planiusculi leves, 
Portamento. - Erbe perenni, che grandemente si rassomigliano fra di. Inro, alte $1 / 2$ metro, semplici o ramose a corimbo, più 0 meno biancheggianti per abbondante lanugine, con foglie picciuolate, ovali o arrotondite, deritate, rugose, e con grossi glomeruli, distanti fra di loro, fogliati, di fiori piccolini, bianchi.

Osservazione. - Genere appena distinguibile dal precedente.

\section{Prompubiuman Aysson.}

M. foliis cuneato-rotundatis, inciso-crenatis, bracteolis nullis, dentibus calycinis 5 , subæqualibus, lanceolato-subulatis, rectis.

Mlarrubium Alysson Linn. sp. pl. ed. 1. p. 582. Bert. h. ital. 6. p. 176. De Not. rep. fl. lig. p. 329. Mor.! fl. sard. 3. p. 318. Ces. Pass. Gib. comp. fl. ital. p. 315. Arc. comp. fl. ital. p. 550.

Figura. - Riv. pl. fl. irreg. monnp. t. 55. fig. dextr.

Stazione, Abitazione e Fioritura. - Nei luoghi incolti o campestri solatii dell' Italia meridionale, rara: in Puglia a Foggia, Tressanti, Casaltrinità (Bert.), Barletta (Calcara!), e nel Gargano al lago di Lesina e a Mlanfredonia (Porta e Rigo!); quindi in Sardegna a Cagliari (Gennari!). E stata indicata anche la Calabria come luogo nativo, ma io ritengo che ciò sia per un equivoco riferibile a Berto. loni, che (o.c. p. 177) scrisse "Calabria o dove avrebbe dovuto scrivere "Apulia "; ed altra località sospetta è quella di Genova, dove dicesi che Viviani avesse trovata la pianta (De Not. l. c.). Fiorisce da marzo ed aprile a giugno.

Distribuzione geografica. - Pianta Mediterranea, nasce pure in Spagna, in Soria e Palestina, e nell'Affrica settentrionale.

\section{VIfarrebinu peregrinum.}

M. foliis ovali-lanceolatis, crebre dentatis, bracteolis paucis, calycibus multo brevioribus, dentibus calycinis 5 , subæqualibus, sı. bulatis, rectis.

Marrubium peregrinum Linn. sp. pl. ed. 1. p. 582. Poll. fl. . ver. 2. p. 287. Pir. fl. for. syll. p. 113. De Vis. Sacc. cat. piant. Ven. p. 139. Ces. Pass. Gib. comp. fl. ital. p. 315. Arc. comp. 1 . ital. p. 550 .

Marrubium creticum Poll. l. c.

Figure.-Reich. ic. bot.t. 288. Ic. fl. germ. 18.t. 23. f. 3, 4. 
Stazione, Abitazione e Fioritura. - In luoghi incolti e simili dell'Istria a Pola e verso Parenzo secondo Zannichelli (Poll. fl. ver.), del Friuli a Duino secondo Host, e sul lago di Garda (Poll.). Fiorisce in luglio e agosto.

Distribuzione geografica. - Nell'Europa media e meridionale dall'Austria verso l'est, nell'Asia Minore.

\section{Marombium camatiolissinnum.}

II. foliis ellipticis, crebre dentatis, bracteolis numerusis, calyces fere æquantibus, dentibus calycinis 5 , subæqualibus, subulatis, rectis.

Marrubium candidissimum Linn. sp.pl. ed. 1. p. 583. Bert. 1. ital. 6. p. 177. Koch syn. fl. germ. ed. 2. p. 657. Zan. prosp. fl. ven. p. 26. Car. prodr. fl. 10sc. p. 515. Pir. pl. for. sijll. p. 113. Tass. fl. prov. sen. p. 5\%. De Vis. Sacc. cat. piant. Ven. p. 139. Ces. el. Maiell. p. 24. Ces. Pass. Gib. comp. fl. ilal. p. 31j. Arc.! comp. f. ilal. p. 550 .

Marrubium peregrinum Ces. l. c. (non Linn.).

Figure. - Reich. ic. bot. t. 312. Ic. 1. germ. 18. t. 23. f. 2.

Stazione, Abitazione e Fioritura. - In luoghi aridi sassosi della Penisola. Nasce in Istria a Fiume (A. M. Smilh!), a Trieste (Barbieri!), a Iluino (Koch) o llonfalcone (Bert.), nel Veneto a Cividale di Friuli (Pir.) e al Lido di Venezia (Barbieri !); quindi ricomparisce a Fano (Bert.) nelle Marche, dove si trova poi nei monti Catria (Parl.! erc.), e S. Vicino a 800 metri (Bucci!), a Rocca S. Angelo (Paolucci!), presso Perosara e Serra S. Quiriro (Bucci!), a Civitanuova, presso Macerata (Nurducci! ecc.), Tolentino (Ricci!), Colfiorito!, nel monte Birro (Bert.), a Montefortino (Marzialetli!), verso Acrquasanta!; e più griù nell'Aluruzzo al Ciorno (Ten. syll.), nei monti di Roccamnrice e d'Aquila (Bert.), presso Massa d' Albe, sopra Solmona, e Caramanico (Levier! ece.), a Castel di Sangro (Iluet!); infine al (iargano (Pasquale!) a Monte S. Augelo (Ten.). Nell'altro - lato occidentale della Penisola comparisee nella Toscana mrientalemeridionale, cioè a Mnntalone in val Tiberina (Amidri!), Rapolano nel Senese (Sommier!), Pienza (Sant. viagg.), an B.tgni di Vignone!, a liadicofani (Campani!), S. Casciano ai lasni (1re. !), . Ireidosso nel monte Amiata, Busceto nel moute Lahhru!. Castellazzara, Pitigliano, Roselle presso Girosseto (Santi), presso Purto S. Stefino nel monte 
Argentario (Levier!); quindi seguita in giủ per la Somma nell'Umbria, Canino nel Lazio (Bert), per i monti di Tivoli e di Subiaco (Rolli !), cosi nel monte Catillo (WVebb.!), a Santo Polo, sui Cimini (Seb. Maur. prodr.); e termina nella Campania ad Itri (Terracciano!), nel Matese (Ten.), e a Circemaggiore (Avellino!). Fiorisce da giugno ad agosto.

Distribuzione geografica, - Questa pianta occupa inoltre la parte superiore della penisola Balcanica.

Descrizione. - Tota planta canescens. Caulis basi lignosus, fibris radicalibus præditus, ramosus, ramis adscendentibus, tetragonis, faciebus planis, lana alba tectis. Folia canescentia, mollia, supra tomentoso-sericea, subtus opaca, patenti-recurva, supra sulcis nervis in pagina inferiore prominentibus respondentibus notata, ovato-oblonga, obtusa, crenata. Petiolus brevis, supra leviter canaliculatus, subtus convexiusculus. Bracteolæ lineari-setaceæ, dorso convexiusculæ, calycis tubum subæquantes, canescentes. Calycis tubulosi tubus costis 10 longit. crassis notatus, sulcis intermediis divisis; dentes patentes recurvi, inæquales, 2 inferiores paullo majores, omnes subulati, acuti et mucronati, fauce calycis barbata pilis albis. Corolla alba calyce vix duplo longior; tubus rectiusculus, inferne glaber, superne, ut limbus, pubescens et latior, calycis tubo sublongior; limbus bilabiatus, labium superius planum, erecto-recurvum, bilobum laciniis oblongis, obtusis, inferius brevius, patenti-reflexum, planum, trilobum lobis obtusitisculis, intermedio paulo majore. Stamina 4, didynama, inclusa, supra medium tubi corollæ inserta. Filamenta alba, basi fere orbiculari dilatata et barbata, reliqua parte subulata et glabra. Antheræ fuscæ, glabræ, biloculares, loculis superpositis rima longitudinali communi dehiscentibus. Pollen viridulum. Pistillum staminibus brevius. Ovarium 4-lobum lobis oblongis, obtusis, viridulis, glabris, apice glandulis minutis albis conspersis. Stylus rectiusculus, filiformis, albus, glaber. Stigma bifidum laciniis inæqualibus, divergentibus, albis, glabris. Nuculæ oblongæ, obtuse triquetræ, dorso planæ, viridulæ, glabræ, apice glandulis albis minutis - notatæ. (Parl. ms., descr. della pianta dei monti Catria e S. Vicino).

\section{VIrrobium vulgare.}

II. foliis rotundatis, crenatis, bracteolis numerosis, calyces fere æquantibus, dentibus calycinis 10 , valde inæqualibus, subulatis, uncinatis. 
Marrubium vulgare Linn. sp. pl. ed. 1. p. 583: Bert. fl. ital. 6. p. 179; et auct. omn.

Figure.-- Savi mat. med. veg. tosc. 1. 33. Ten. fl. nap. t. 154. Reich. ic. 1. germ. 18. t. 23. f. 1.

Stazione, Abitazione e Fioritura. - Nei luoghi aridi incolti, lungo le strade, nei calcinacci ccc. Nell'Alta Italia nasce qua e là: in Piemonte sembra abbastanza comune, vi nasce a Susa (Parl.!) e Bardonecchia, verso Moncalieri, verso Fussano e Cherasco, ad Alba, nell'Astigiano, nel Monferrato, sopra Ivrea, nel Novarese (Colla herb. ped. ecc.); in Lombardia fa nel Pavese verso Casteggio e altrove (Nocc. Balb. f. tic.), nel Comasco, ma raro, sopra Bonzanico in Tremezzina (Com. $l$. com.), in Valtellina comunissimo da Sondrio a Bormio (Mass. prodr.), nel qual ultimo luogo trovasi a un' altezza di 1530 metri (Parl.!), wel Bergamasco (Rota prosp.), al Lago di Carda, presso Pozzolengo (Zersi prosp.), a Volta (Bert.), a Valeg. gio (Rigo!); in Tirolo fa in Fiemme, qua e là intorno a Bolzano, tra Wals e Tisens, a Mals (Hausm. fl. Tir.); nel Veneto nasce a Verona (Bracht!), negli Euganei (Trev. prosp.), a Bassano (Bert.), a Venezia (Zan. prosp.), a Chioggia (Bert.), a Gradisca e Monfalcone (Pir. ll. for.); in Istria a 'Trieste (Bert.); nell' Emilia al castello di Tabiano uel Parmense (Passerini!), presso Querzola nel Modenese (Gib. Pir. fl. Mod.), nel Bolognese ad Ancognano, Rio Verde, Ca. stello di Casio, Montespechio (Cocc. l. bol.); in Romagna a Faenza (Cald. tent.). In Liguria è raro anzichè no, essendo stato finora trovato soltanto a Nizza (Bert.), Nentone (Ard. cat.), Bordighera (Ricca!), e Finale (Bert.). In Toscana si rende molto piủ frequente, trovandosi a Corsanico presso Viareggio (Cialandrini!), a l'artigliano nel Lucchese (Pucc. syn.), nel monte Pisano (Savi $/$. pis.), abbondante nel Fiorentino!, in Mugello (Bert.), nel Casentino a Romena!, a Volterra (Amidei!), comune nel Senese (Campani! ecc.), in Maremma a S. Vincenzo, al monte Argentaro, nel monte Amiata e tutt'attorno (Parl.! ecc.), come pure nelle isole dell'Elba (P. Savi! ecc.), e del Ligglio (Bert.); quindi seguita per Viterbo (Bert.), Roma dov'è comme (Sel. Maur. prodr.), Vicalvi (Terracciano!) e. Frasso (Ten syll) in Terra di Lavoro, Napoli, dov'e frequente (Ten.), Melfi (Terr. fl. Vult.) e Castelyrande in Basilieata (Bert.), Mereato del sabato e Nadunna della stella nel Principato citeriore 'T'en ), fino in Calabria a Pizzo, P'alizzi ecc (Are. !), Grotteria ('asquale! ece.). Dall'altro lato della Penisola trovasi nelle Marche presso Tolentino (Ricci!) e sul monte Vettore (Gemmi!), in Abruzzo a Caramanico 
(Ces. el. Maiell.), in Puglia al Tavoliere, a Manfredonia, al Gargano (Ten.), infine presso Taranto (Aiuti!) e ad Otranto (Groves! ecc.). Pare che sia frequente tanto in Corsica (Mars. cat.) quanto in Sardegna (Hor. $f:$ sard.). In Sicilia è dichiaraco trovarsi ovunque (Guss. syn.), come pure nelle isolette circostanti fino a Linosa e Lampedusa (Aiuti!), e in Malta (Grech $f l$. mel.). Fiorisce da aprile a luglio nei luoghi più caldi, e da maggio o giugno alla fine dell'estate nei meno caldi.

Distribuzione geografica. - In tutta l'Europa, meno la piú nordica, nell'Asia occidentale fino al Cascemir, nell'Affrica settentrionale, nelle due Americhe.

\section{TRIBU் SECONDA.}

Gtachidee.

STACHydee (non alior.).

Stamina e tubo corollæ exserta. Stylus exsertus.

\section{SOTTOTRIBÙ PRIMA.}

Trematranee.

Tendanea.

Stamina inter se distantia, omnia erecta. Stylus lobis complanatis.

\section{TENDANA.}

Tendana Reich. fil. ic. fl. germ. 18. p. 39.

Calyx tubulosus, valide $15-1 \bar{z}$-nervatus. Corolla manifeste bilabiata, labio superiore suberecto, planiusculo. Stamina 4, filamentis edentulis, antheris 2-locularibus, loculis longitudinaliter dehiscentibus, a connectivo seiunctis. Stylus lobis æuqualibus. Cocci apice mucronati leves.

Osservazione. - Genere distintissimo per lo stilo bilamellato, 
e per la forma dei cacchi, e che per gli stami divergenti non ha che vedere con i generi Satureia ell affini che sono di altro gruppo.

Portamento. - Sulfrutice glabro, alto 1-2) decim., cespugliuto, da' rami sottili, dalle foglie piccole, tondeggianti, intere, da' fiori porporini, non grandi ma. lunghi fino a $1 \frac{1}{2} / 2$ centim., iu dicasi pauciflori all' ascella delle foglie superiori.

\section{Tendan Tipenella.}

Thymus Piperella All. l. ped. 1. p. 21 (non Linn.).

Nicromeria Piperella Benth. lab. p. 379. De Not. rep. fl. lig. p. 320. Ard. R. Alpes-mar.p. 295.

Satureia Piperella Bert. fl. ital. 6. p. 50. Ces. Pass. Gith.comp. fl. ilal. p. 302. Arc. comp. fl. ital. p. 540.

Tendana Piperella Reich. fil. ic. fl. germ. 18. p. 39.

Tendia (sic) Piperella Ard. cal. pl. Ment. p. 28.

Figure, - Reich. o c. t. 70. f. 1. Moggr. contr. /l. Ment. t. 32.

Stazione, Abitazione e Fioritura. - Sulle rupi delle Aipi marittime, verso l'altezza di $1000 \mathrm{~m}$., essendovi frequente, per eseinpio a Tenda (Reuter! ecc.), a Briga (All.), sopra Mentone al Bress e all'Agel (Ard.), nei monti d' Ormea (Bert.), a Garessio (All.), sopra Oneglia (Ardissone!), ad Albenga (De Not.), ecc. Fiorisce in luglio, agosto e settembre.

Distribuzione geografica. - Nasce oltraccio in Croazia, Dalmazia, Montenegro, Erzegovina e Serbia, ma in forma di una varietá pelosa (Thymus croalicus Pers.).

\section{SOTTOTRIBÜ SECONDA.}

\section{Mrestee.}

MEATHex (non alior.).

Stamina inter se distantia, omnia erecta. Stylus lobis subulatis:

\section{HYC'THU:}

Lycopus Tourn. inst. p. 190. 1. 89. Mirb. in ann. mus. 15.

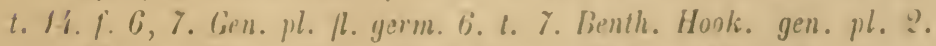
p. 1183. C'es. Pass, Gib. comp. /1. ilal. p. 2998. 1. 43. f. 6. 
Calyx campanulatus, validiuscule $8-10$-nervatus. Corolla vix ac ne vix bilabiata, labio superiore patentiusculo, subplano. Stamina, 2 , filamentis edentulis, antheris 2-locularibus, loculis longitudinaliter dehiscentibus, distinctis. Stylus lobis æqualibus. Cocci crasse unarginati, apice complanati obtusissimi leves.

Portamento. - Sono lilue piante perenni, robuste, alte da $1 / 2 \mathrm{~m}$. a $1 \mathrm{~m}$. e più, ambedue stolonifere, fittamente rivestite di foglie grandi, ovate o lanciolate, più o meno divise. I fiori sono in fitti glomeruli ascellari, sono piccolini, screziati di bianco e di rosso.

\section{Lycopus enropreus.}

L. folis grosse dentatis, basim versus pinnatifidis etiam partitis, lobis integris.

Lycopus europælis Linn. sp. plant.ed.1.p. 21. Mass. prodr. fl. valt. p. 108. Bert. fl. ital. 1. p. 199. Com. fl. com. 1. p. 2\%. Parl.! 1. pan. p. 16. Pucc. syn. pl. luc. p. 19. Trev. prosp. fl. eng. p. 25. Guss. fl. sic. syn. 1. p. 20. De Not. rep. 1l. lig. p. 315. Zan. prosp. n. ven. p. 26. Rota prosp. piant. Pav. p. 27\%. Hausm. R. Tir. p. 672. Rola prosp. fl. Berg. p. 69. Pir. fl. for. syll. p. 109. Mor.! /. sard. 3. p. 272. Ard. cat. pl. Ment.p. 28. Car. prodr. R. tosc. p. 503 (ex parte). Tassi 月. prov. sen. p. 50. Cenno bot. prov. Siena p. 86 . G. Bert. not. piant. Porr. p. 47. Veg. Porr. p. 86. Ard. fl. Alpesmar. p. 293. De Vis. Sacc. cat. piant. ven. p. 132. Zersi prosp. piant.Bresc. p. 163. Terr. fl. Vult. syn. p. 136. Mars. cat. pl. Corse p. 111. Archib. 1. Alto-Serch. p. 59. Ces. Pass. Gib. comp. fl. ital. p. 298. Ing. cat. sp. Mond. p. 49. Groves contr. /l. Terra d'Otr. p. 65. Cald.! fl. fav. tent. p. 178. Arc. comp. fl. ital. p. 537. Gib. Pir. fl. Mod. p. 127.

Lycopus menthifolius Mab.! rech. pl. cors.

Figure.-Fl. dan. t. 1081. Reich. ic. A. germ. 18. t. 20. f. 1.

Stazione, Abitazione e Fioritura. - Nei luoghi paludosi, o altrimenti umidi o inondati, comune per tutta Italia, fuorchè nelle piccole isole: in Piemonte (All. ecc.), in Lombardia (Parl.! ecc.), in Valtellina (Nlass.), nel Veneto (Nontini! ecc.), in vari luoghi del Tirolo (Ambrosi! ecc.) fino in val di Taufer a $1000 \mathrm{~m}$. (Ausserdarfer!), in Emilia nel Parmense, e sino a Borgotaro (Parl.!), nel Modenese, e sino a Ronconuovo sul Ventasso (Gib. Pir.), a Bologna (Bert.), e nei monti Porrettani (G. Bert.), in Romagna 
(Cald.!), in i.iguria da Nizza (Durando!) a Genova (Carrega!), in Toscana nel littorale, su per Val-di-Seichio sino a Tereglio (Giannini!), nel Val-d'Arno!, nell'Appennino Casentinese (Parl.!), nel Senese!, nel monte Amiata (Santi) ecc., nel Romano (Seb. Maur. ecc.), nel Piceno (Parl.!), a Napoli (Parl.! ecc.), nel Principato citeriore (Ten.), a Melfi (Terr.), a Otranto (Groves), in Corsica (Mab.! ecc.), in Sardegna (Nor.!), in Sicilia a Palermo (Parl.!), ad Arola (Bianca!) ecc. Fiorisce da giıgno e luglio all'antunno.

Distribuzione geografica. - In tutta Europa, eccettuata l'artica, nell'Affrica settentrionale, nell'Asia occidentale, settentrionale e centrale.

Descrizione. - Pianta perenne, alta da 5 decimetri a un metro o poco piú, di un verde chiaro, scabrosetta, fornita di un rizoma che manda molte fibre radicali, flessuose, e dei rami lunghi radicanti a guisa di stoloni ma privi di foglie. Il fusto è erelto, durotto, quadrangolare, con gli angoli un po' rilevati, bianchicci e scabrosetti, con un solco largo e alquanto profondo in ciascuna faccia, con qualchic pelo curvo, lunghetto e bianco; è per lo piu ramoso, con $\mathrm{i}$ rami opposti e distribuiti in quattro linee, patenti e un po' curvi in su, più corti del fusto. Le foglie sono opposte, alquanto lontane, patenti o patentissime, erbacee, le inferiori ovali-lanceolate, acute, pennatifile alla base, inciso-dentate nel resto, con i denti ottusetti, fornite di un picciòlo corto, scanalato di sopra, convesso di sotto, le foglie di mezzo sono più stretle e piü piccole, ovali-lanceolate o quasi lanceolate, quasi sessili o sessili, inciso-dentate e le supreme ancora più strette e piủ piccole, sessili, soltanto dentate: tutte di un verde chiaro di sopra ed ivi quasi glabre o glabre e con solchi che corrispondono ai nervi di sotto, di un verde ancora piủ chiaro di sotto ed ivi con un nervo longitudinale grosso e bianchiccio, da cui partono dei nervi laterali rilevati, i quali si dirigono obliquamente verso il margine dove terminano all' apice dei denti dopo aver mandato qualche rametto laterale un po'rilevato: hanno dei peli curvi, ruviletti e bianchi nel nervo principale e meno nei nervi laterali e nelle loro divisioni, e delle fossette tonde, assai piccole, visibili con una forte lente, nel parenchima di sotto. I liori sono molto piccoli, sessili, circa 10 e fitti in glomeruli pirceoli, all' ascella di tutte le foglie tei rami e delle foglie del fusto che slanuo sopra degli ul. timi rami: cosi per ciascuna coppia di fogglie vi sono cirea venti fiori; tali glomeruli sonn piecoli, molte volte piu corti delle foglie. Le brattee sono piccole, erette, lunghe quanto il calice, lineari o li. 
neari-lanceolate, acute, verdognole, puberule. Il calice è campanulato, verdognolo, puberulo di fuori, glabro di dentro, con 5 denti quasi uguali tra loro, più lunghi del tubo, eretto-patenti, ovalilanceolati, acuminati e quasi spinescenti all' apice con uno o tre leggieri nervi. La corolla è appena più lunga del calice, bianca con qualche macchiolina di color violetto chiaro alla base dei lobi laterali e in maggior numero nel lobo inferiore. Il tuho è lungo quasi quanto il calice, larghetto, peloso di dentro, massime verso la gola con i peli lunghi e bianchi. Il lembo è cortissimo, diviso in quattro lobi, poco disuguali e patuli-recurvi, l'inferiore essendo un po' più grande di tutti, come troncato all' apice e diretto in giù, il superiore appena smarginato, e i due laterali un po' piủ piccoli di questo e quasi tondeggianti. Gli stami sono due, opposti ai due lobi laterali della corolla, e inseriti nella parte superiore del tubo di questa, inclusi. I filamenti sono cortissimi, bianchi. Le antere quasi tonde, introrse, bianche, glabre. Il pistillo è più lıngo della corolla. L'ovario è diviso in quattro lobi, troncati all' apice, verdognoli, glabri. Lo stilo è lungo, avvicinato al lobo superiore della corolla, cilindrico, bianco, glabro. Lo stimma è bifido, con le lacinie disuguali, acute, divergenti, bianche.

In altre piante ho veduto gli stami piủ lunghi dei lobi laterali della corolla, eserti, lunghi quasi quanto il pistillo, le antere profondamente bifide, quasi a ferro di cavallo, di color violetto chiaro, e gli stimmi di questo medesimo colore. (Parl. ms., descr. della pianta di Grumone in Lombardia).

Osservazione. - Questa specie varia alquanto per la forma delle foglie, e più per il grado della pelurie, ora più o meno abbondante, ora scarsa e quasi nulla.

\section{HECOMUS exnItaten.}

L. foliis pinnatifidis partitisque, lobis subdentatis.

Lycopus exaltatus Linn. fil. suppl. p. 87. Seb Maur. fl. rom. prodr. p. 8. Bert. f. ilal. 1. p. 130. Com. fl. com. 1. p. 28. Pucc.! syn. pl. luc. p. 20. Trev. prosp: fl. eug. p. 25. Koch syn.fl. germ. ed. 2. p 636. Zan. prosp. fl. ven. p. 26. Rota prosp. piant. Pav. p. 271. Prosp. fl. Berg. p. 69. Pir. fl. for. syll. p. 109. De Vis. Sacc. cat. piant. Ven. p. 132. Zersi prosp. piant. Bresc. p. 163. Ces. Pass. Gib. comp. fl. ital. p. 298. Arc. comp. A. ital. p. 537. Gib. Pir. A. Mod. p. 127. 
Lycopus europæus (ex parte) Car. prodr. fl. tosc. p. 503.

Figure. - Fl. grcec. t. 12. Reich. ic. /. germ. 18. t. 90. f. 2.

Stazione, Abitazione e Fioritura. - Dore la sperie precedente, ma rreno comune: Torino a S. Ilauro, Alba (Bert.), Pavia (Rota), Canzo nel Comasco (Com.), Valle Caleppio nel Bergamaseo (Rota), Mantova (Barbieri!), Lago di Garda, Verona, Vicenza (Poll. $f$.), colli Enganei (Kellner!), S. Michele di Angarano (Montini!) e altri luoghi de! Veneto, Villaraspa e Ronchi nel Friuli (Pir.), isola Veglia (Kioch), Ozzano nel Parmense (Parl.!), Reggiolo, Revere, Salto di Montese e Ronconuovo sul Ventasso nel Modenese (Gib. Pir.), Bologna a Gandazollo, Bagnacavallo, Ravenna (Bert.), Lucca a Colle di Conpito (Pucc.!), Tolentino (Ricci!), Roma alla Caffarella (Seb. Mlaur.), paludi Pontine (Bert.), Napoli al Fusaro (Ten. syll.). Manca più in basso, e manca nelle isole. Fiorisce da luglio a settembre.

Distribuzione geografica. - Nell' Europa inedia orientale, l'Italia segnandovi il confine occidentale e meridionale, e neli'Asia media.

Descrizione. - Il fusto manda degli stoloni, è diritto o ascendente, ramoso alla base e talvolta con rami verso alto, quadrango. lare, con un solco alquanto profondo in ciascuma faccia, glabro o quasi glabro, di color verdognolo o un po'rossiccio inferiormente. Le foglie sono opposte, sessili, patenti, di color verde e un po piu pallido di sotto, le inferiori hanno una circonferenza largamente ovata, le superiori lanceolate, tutte profondamente pinnatifide, con le lacinie che giungono a poca distanza dal nervo mediano, lanceolato-lineari, con qualche dente o intere. I fiori sono molti e assai piccoli, uniti in un glomerulo posto all'ascella della foglia, della quale sono assai piủ corti: i glomeruli dei due lati sono vicini tra loro in modo che ne resulta apparentemente un verticillo; ciascun fiure è sessile, accompagnato da una piccola brattea lincare-lanceolata o lineare, acuminata, verde, con i margini hianchicci e fornita di piccolissine ciglia : le brattee dei fiori inferiori sono poco piủ lunghe dei fiori stessi. II calice $i$ quasi in forma di campana, verde, pelosetto, liviso in cinque denti profondi, più lunghi del tubo, ovato-acuminati e quasi trinervi alla base : di dentro è verde. La corolla è piccola, appena più lunga del calice, a gnisa di una campaniua, divisa in quattro lacinie o lobi, quasi uruali, quasi tondi, patenti, ì bianca con punti o lineette di colore amarante distribuite quasi in due line nelle lacinie: è pelosetta di dentro nella gola e verso l'origine delle lacinie. Cili stami sono due, inseriti presso la base del tubo della corolla, piu lunghi 
di questa. I filamenti sono filiformi, diritti, un poco curvati in dentro verso l'apice in modo che ivi convergono tra loro, bianchi e quasi glabri. Le antere sono profondamente bifide, inserite nel mezzo della divisione, biloculari, aprentisi longitudinalmente, di color rossiccio. Il polline è ninuto, sferico, liscio, gialliccio. L'ovario è quasi tondo, con quattro lobi schiacciati e manifesti solo in alto, verde e glabro. Lo stilo è filiforne, glabro, bianco, poco piủ lungo degli stami, avvicinato a uno dei lobi della corolla, bifido all' apice con le lacinie disuguali. Gli stimni sono piccoli. (Parl. ms., descr. della pianta di $0 z \approx a n 0)$.

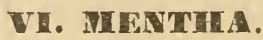

Mentha Tourn. inst. p. 188. t. 89. Mirb. in ann. mus. 15. t. 16. f.14-17. Benth. Hook. gen. pl. 2. p. 1182. Ces. Pass. Gib. comp. fl. ital. p. 296. t. 43. f. 5 (excl. Preslia).

Mentha et Pulegium Gen. pl. fl. germ. 6.t. 5, 6.

Calyx campanulatus, valide aut leviter 10-nervatus. Corolla vix bilabiata, labio superiore suberecto, planiusculo. Stamina 4, filamentis edentulis, antheris 2-locularibus, loculis longitudinaliter dehiscentibus, distinctis. Stylus lobis subæqualibus. Cocci apice convexi leves.

Portamento. - Le Mente sono erbe perenni, stolonifere, di odore aromatico fortissimo e tutto speciale, robuste, alte da 2 decimetri a $\%$ di metro, generalmente più o meno pelose, con molte foglie sessili o brevemente picciuolate, dentate, e fiori piccolini, di color lilla chiaro, in glomeruli folti, i quali ora sono all' ascella di coppie di foglie distanti le une dalle altre, ora sono ravvicinati in infiorescenze ordinariamente lunghe, cilindriche e riunite in pannocchia nell'estremità dei fusti, nel qual caso le foglie sono sostituite da brattee piccoline. Una sola specie, la Mentha Requieni, si discosta dalle congeneri per essere piccolina, gracile, sdraiata, con fiori solitari o pochi insieme.

Osservazione. - Le piante della prima sezione di questo genere sono polimorfe in sommo grado, per la pelurie, per la conformazione delle foglie, e per quella dei fiori, che hanno variabile il calice e la corolla, e glistami ora rinchiusi ora sporgenti in relazione a pseudounisessualità dioica. Aggiungasi la frequenza degli ibridi, che si suol 
riconoscere dalla loro sterilità; e nessuno si maraviglierà dell’ immenso numero di nomi assegnati a specie, a varietà, a ibridi, che ingombra questo genere, il di cui studio è diventato una specialità per alcuni botanici, e che possiede in proprio tutta una letteratura. Ho pensato meglio non entrare nel ginepraio, e raccogliere le forme ita. liane in 6 specie, di cui due a dir vero sono discutibili, le indiscusse essendo 4; Mentha rotundifolia, M. silvestris, M. aquatica e M. arvensis.

Usi. - Sono piante medicinali. Se ne distilla un' acqua, e se ne estrae un olio essenziale, che serve per le pasticche dette di Menta. A questo scopo si coltiva negli orti la Mentha piperita, con la quale si confonde spesso (anzi sempre secondo Bertoloni) la var. glabra della $M$. silvestris detta $M$. viridis.

\section{Mentha rotundifolia.}

M. foliis subsessilibus, late ovalibus, crenato-serratis, supra pubescentibus, subtus plus minusve lanatis, glomerulis in inflorescentias cylindrico-conicas plerumque paniculatas approximatis, calyce fauce nuda.

Nlentha rotundifolia Linn. sp. pl. ed. 2. p. 805. Mass. prodr. l. valt. p. 113. Trev. prosp. 1l. elig. p. 25. Pucc. syn. pl. luc. p. 301. De Not. rep. n. lig. p. 314. P. Savi /l. gorg. p. 27\%. Com. fl. com. 4. p. 303. Rota prosp. piant. I'av. p. 271. Prosp. /l. Berg. p. 68. Pir. fl. foroj. syll. p. 109. Mor. fl. sard. 3. p. 265. Ard. cal. pl. Ment. p. 28. Car. prodr. fl. tosc. p. 501. Tassi fl. prov. sen. p. 50. Ard. fl. Alpes-mar. p. 292. De Vis. Sace. cat. piant. Ven. p. 131. Zersi prosp. piant. Bresc. p. 162. Archb. 1. Alto-Serch. p. 59. Ces. Pass. Gib. comp. 11. ital. p. 297. Ing. cat. sp. Mond. p. 50. Groves contr. fl. Terra d'Otr. p. 65. Call.? Il. fav. tent. p. 176. Cocc. quarto contr. 1. Bol. p. 15, 16. Arc. comp. A. ital. p. 535. Pir. Ciib. A. Mod. p. 126.

Nentha macrostachya Ten.! ก. nap. prods. p. 33. Bert. A. ital. 6. p. 91. Zan. prosp. /l. ven. p. 2G. Guss. enum. pl. Ina im. p. 253. G. Bert. not. piant. Porr. p. 47. Veg. Porr. p. SG. Pasq. R. ves. p. 78. Terr. R. Vull. syn. p. 13\%. Sec. rel. Terra di Lau. $p$ 94. Quart. rel. id. p. 112. Cald. 1t. fav. tent. p. Iiti.

Mentha insularis Req! ! lett. in giorn. bot. ital. ann. ? par. 3. p. 111, 116. Cren. Godr. 11. de Fr. 2. p. 6.19. Bert. /. ital. 10. p. 
519. Genn. fl. Capr. p. 112. Mars. cat. pl. Corse p. 111. Ces. Pass. Gib. l. c. Arc. l. c.

Figure. - Ten. fl. nap. t. 56, 15\%. f. 1. Reich. ic. 月. germ 18. $t .81$.

Stazione, Abitazione e Fioritura. - Per tutta Italia, nel continente e nelle isole, comunissima lungo i fossi, e generalmente in tutti i luoghi umidi. Non pare che salga molto nei monti: in Toscana poco oltre i 1000 metri. Fiorisce da giugno e luglio a settembre.

Distribuzione geografica. - In Eur.spa dalle Isole Britanniche e dall' Olanda in giù, e da Nadera alla Siberia, nell'Affrica settentrionale, anche nella Nord-America.

Descrizione. - Pianta odorosa, perenne, alta da 3 a 6 decimetri, di un verde bianchiccio per un tomento formato di peli bianchi, lunghetti, un po'ruvidetti, parte semplici, parte ramosi, fornita di un rizoma che manda fibre radicali e in alto $\mathrm{i}$ fusti e degli stoloni piuttosto corti, grossetti, radicanti e con piccole foglie. I fusti sono eretti, quadrangolari, cou gli angoli rilevati e ottusi, peloso-tomen. tosi, con i peli più abbondanti e più lunghi negli angoli che nelle facce le quali sono piane, ramosi, con i rami piuttosto corti, patenti o eretto-patenti. Le foglie sono un po'consistenti, opposte, sessili, patentissime, ovali-orbiculari, quasi tondeggianti o un po' acute all'apice, ed ivi con un corto mucrone, crenate o crenato-seghettate nei margini, di un verde bianchiccio di sopra ed ivi pelose con corti peli e con una rete di solchi a maglia stretta, che le rende un po' ri. levate in mezzo ai solchi, bianchicce-verdognole di sotto per un tomento piuttosto fitto ed ivi con un nervo longitudinale e con molti nervi laterali i quali si uniscono tra loro ad arco verso il margine e con i loro rami formano una rete rilevata in tutta la pagina inferiore della foglia. Le foglie superiori e quelle dei rami fioriferi sono gradatamente più piccole. I fiori sono distribuiti in glomeruli formanti delle spighe cilindriche acute, con i verticilli alquanto vicini tra loro. Le brattee sono patentissime, quasi uguali ai fiori, ovali-lanceolate, acuminate, verdi, nervose, pelose; le bratteole sono più strette, lineari o lineari-setacee, più corte dei fiori ma più lunghe dei pedicelli. Questi sono piủ corti o quasi uguali al calice, cilindrici, verdognoli, pelosi, con peli curvati in giù. Il calice è quasi campanulato, verdognolo, in parte violetto, peloso, con i peli corti e bianchicci, con i nervi poco o punto rilevati, diviso in cinque denti, disuguali, i superiori più corti, triangolari, acuti, gl'inferiori triangolari-lan- 
ceolati, acuminati, tutti più corti del tubo: sono eretto-patenti, poi conniventi quando il calice si gonfia un poco e diventa ventricoso alla maturità. La corolla è da due a quattro volte più lunga del calice, di color bianco o bianco-violetto, con quattro lobi eretto-patenti, ottusi e uno dei superiori un po' più largo e smarginato, pelosetta nel dorso dei lobi, glabra nel resto anche di dentro. Gli stami sono quattro, il doppio più lunghi della corolla, un po'disuguali tra loro, cretto-patenti, inseriti in alto del tubo. I filanienti sono filiformi, bianchicci, glabri. Le antere piccole, quasi tonde, ottuse, smarginate alla base, inserite nel mezzo del dorso, introrse, biloculari, aprentisi longitudinalmente, di color violetto. Il pistillo è più corto o quasi uguale agli stami. L'ovario è quadrilobo, liscio, verdognolo, inserito sopra un disco rerdognolo e glabro. Lo stilo è lungo, filiforme, di. ritto o currato e quasi inginocchiato in alto, bianchiccio, glabro. Lo stimma è bifido, con le lacinie un po'disuguali, erette, quasi acute, e bianchicce. (Parl. ms., descr. della pianla di Olmenteia in Lom. bardia).

Osservazione. - Questa specie varia per avere le foglie talvolta segheltate con i denti acuti, e la corolla ora più grande e ora più piccola, bianca o di color violetto chiaro. (Parl. ms.). - La varietà qui indicata è una delle forme intermedie per le foglie fra la Mentha rotundifolia e la $\boldsymbol{H}$. silvestris, che oggigiorno si considerano quali ibridi delle due specie, e si dicono $M$. rolundifolia $\backslash$ silvestris o $M$. silvestris $\times$ rotundifolia. Nell'Erbario centrale se ne trovano saggi raccolti da Parlatore a Fiumedinisi in Sicilia, e a Olmeneta e Grumone in Lombardia.

Non trovo nella Mentha insularis di Corsica alcuno di quei carattcri che si ritengono specifici in questo genere.

\section{Menthr silvestriss.}

M. folis sulsessilibus, ovalibus vel lanceolatis, argute serratis, supra plus minusve pilosis, subtus tomentosis, glomerulis in inflorescentias cylindrico-conicas plerumque paniculatas approximatis, calỵ ce fauce nuda.

Mentha silvestris Linn. sp. pl. ed. 2. p. S0i Mass. prodr. fl. valt. p. 113. Trev. Mosp. /l. eug. p. 25. I'ucc. syn. ll. luc. p. 301. Bert.! /l. ilıl 6. p SS. De Nol. rep. /l. lig. p. 80t. Com. Al. com.' 4. p. 30i. Sim fl. alp. vers. p. 197. Ruta prosp. piant. Pau. p. ?il. 
Hausm. f1. Tir. p. 669. Rola prosp. fl. Berg. p. 68. Pir. 1. for. syll. p. 109. (excl. var. r). Bicchi! agg. fl. lucch. p. 16. Ard. cat. pl. Ment. p. 28. Car. prodr. fl. tosc. p. 501. Tass. fl. prov. sen. p. 50. Ard. fl. Alpes-mar. p. 292. De Vis. Sacc. cat. piant. Ven. p. $13 \%$ Zersi prosp. piant. Bresc. p. 162. Terr. fl. Vult. syn. p. 134. Ces. el. piant. Maiella p. 23. Terr. sec. rel. Terra di Lav. p. 94. Ces. Pass. Gib. comp. fl. ital. p. 297. Ing. cat. sp. Mond. p. 50. Cocc. terzo contr. fl. Bol. p. 27. Arc. comp. fl. ital. p. 536. Gib. Pir. A. Mod. p. 126 .

Mentha serotina Ten.! fl. med. univ. Guss. enum. pl. Inarim. p. 254. Terr. nota piant, Volt. p. 3. Ces, el. piant. Maiella p. 23. Cald. fl. fav. tent. p. 177.

Mentha Rosani Ten.! f. nap. prodr. app. quinta p. 18.

Mentha Lamarckii Ten. fl. nap. prodr. app. quinta p. 19. Cald. o. c. p. 176 .

Mentha ambigua Guss. o. c. p. 253. Cald. o. c. p. 176.

Mentha candicans Ard. fl. Alpes-mar. p. 292. Ces. l. c.

Mentha niliaca Cald. o. c. p. 176.

Mentha balsamea Cald. o. c. p. $17 \%$.

Figure. - Ten. f.nap. t. 156. Reich. ic. bot. t. 982-984. Guss. o. c. t. 11.f. 1 .

$\beta$ glabra, foliis utrinque glabris.

Mentha viridis Linn. sp. pl. ed.2.p. 804. Zer. fl. mel. thes. p. 53. Trev. prosp. fl. eug. p. 25. Bert. fl. ital.6. p. 93. Com. fl. com. 4. p. 306. Guss. enum. plant. Inarim. p. 254. Mor. fl. sard. 3. p. 267. Car. prodr. fl. tose. p. 502. De Vis. Sacc. cat. piant. Ven. p. 131. Zersi prosp. piant. Bresc. p. 162. Ces. Pass. Gib. comp. Al. ital. p. 297. Arc. comp. fl. ital. p. 536 .

Mentha silvestris var. glabra Koch syn. fl. germ. Gib. Pir. fl. Mod. p. 126.

Figure. - Reich. ic. bot. t. 981. Guss. o. c. t. 10. f. 2.

Stazione, Abitazione e Fioritura. - Lungo i fossi e i corsi d'acqua, nei prati umidi, come pure in looghi incolti e asciutti, comunissima da un capo all'altro della Penisola. Sale nelle Alpi fin sopra 1500 metri. Trovasi pure in Sicilia, e nell' isola d'Ischia (Guss.); pare che marıchi nelle altre isole. La var. $\beta$ è meno comune, trovasi qua e là in luoghi freschi: in Piemonte, per esempio nel Monferrato (All. fl.), nel Novarese (Bir. $f l$.); in Lombardia a Como (Com.), a Pontoglio (Zersi), in Valtellina a Montagna (Com.); nel Friuli (De Vis. Sacc.); nel Veneto a Bassano (Bert.), a Treviso (erb. Reboul!), 
negli Euganei (Trev.); nell'Emilia a Fiumalbo (Gib. Pir.), Berceto (Passerini!), Borgotaro (Bert.); in Liguria presso Pegli, a Genova, a S. Stefano d'Aveto (Bert.); a Firenze!; a Roma (Seh. Maur. prodr.); nell'Isola d'lschia (Guss Basilicata (Pasquale!); nella Sardegna centrale (Mor.); in Malta (Zer.). Fiorisce da giugno e luglio a settembre.

Distribuzione geografica. - Nell'Europa tutta quanta, dalla Scozia e dalla Srezia meridionale in giù, nell' Asia occidentale e settentrionale, nell'Affrica dalle isole Canarie all'Abissinia, noncliè al Capo di Buona Speranza, e anche nelle due Americhe, credesi ivi inselvatichita.

Descrizione. - Pianta perenue, un po' odorosa, alta da 4 a 12 decimetri, di un verde bianchiccio, tomentosa, fornita di un rizoma duro, bianchiccio, che manda molte fibre radicali delicate e lunghette e degli stoloni orizzontali, grossetti, radicanti. Il fusto è eretto, quadrangolare, tomentoso, con pochi rami verso l'apice, i quali sono corti, opposti, eretto-patenti. Le foglie sono opposte, patenti e un po curvate ad arco in giù, vicine alquanto tra loro, sessili, ovalilanceolate, acute, leggermente scavate in cuore o tondeggianti alla base, dentato-seghettate $\mathrm{c}$ un po' ondeggianti nei margini, con i denti poco sporgenti e aventi all'apice un piccolo callo ottuso; sono di un verde chiarn di sopra ed ivi pelosette e con solchi in rete che corri. spondono ai nervi di sotto con il parenchima un po'in bolle, di un verde bianchiccio di sotto ed ivi tomentose e con un nerro longitudinale grossetto e di colore piủ chiaro, dal quale partono molti nervi laterali che si dirigono un po' curvi verso il margine dove si uniscono ad arco tra loro e con i loro rami, formando una rete rilevata in tutta la pagina inferiore della foglia. 1 fiori sono piccoli, distribuiti in glomeruli, che formano delle spighe cilindriche, un po'acute, fitte, talvolta interrotte alla base, all apice del fusto e dei rami di questo. Le brattee sono lineari-lesiniformi, quasi uguali o poco, più corte dei fiori, verdognolo-rossicce, pelose. I pedicelli sono filiformi, più corti del calice, rossicci, pelosi. Il calice è tubuloso-campanulato, con molti nervi, verilognolo o in parte rossiccio, peloso, con peli corti, bianchi, rivolti in su, con quattro denti ovato-lanceolati, acuminati, piì corti del tubo, eretti. La corolla ie infundibuliforme, il doppio più lunga del ralice, di un rosso-violetto, pelosetta di fuori; il suo tubo ì quasi cilindrico, lungo quanto il calice; il lembo ì diviso in cinque lobi, quasi uguali, eretto-patenti, ovali, ottusi anzi un po'tondeg. gianti all'apiee. Gli stami sono quattro, alterni con i lobi della co-

Floma ITALIANA - Vol VI. 
rolla, circa la metà più corti dei lobi di questa, eretti, inseriti in alto del tubo. I filamenti sono filiformi, bianchicci, glabri. Le antere piccole e gialle. Il pistillo è lungo quanto o appena più lungo della corolla. Lo stilo è lungo, eretto, filiforme, roseo, bifido, con le lacinie un po' curvate in fuori ed ottusette. (Parl. ms., descr. della pianta di Boscolungo nell'Appennino toscano).

Osservazioni. - Pianta variabilissima per le foglie, in quanto: a grandezza, da $1 / 3$ di decimetro a 1 decimetro; a forma, dipendente dalla maggiore 0 minore lunghezza e dalla base cuoriforme 0 attenuata; a pelurie, più 0 meno abbondante 0 anche mancante, per cui ora sono verdi, ora biancheggianti di sotto 0 in ambedue le pagine; a superficie, piana o crespa; a consistenza. Varia anche per la gros. sezza delle infiorescenze, per gli stami sporgenti o no, ecc. Da ciò innumerevoli e intralciate distinzioni specifiche. Di quelle istituite da Tenore non lıo citato che alcune appoggiate a saggi autentici di lui: avvertendo peraltro che, come altri già l'hanno osservato, gli stessi saggi non corrispondono sempre alle sue descrizioni.

\section{Fentha piperita.}

II. foliis petiolatis oblongo-lanceolatis, argute serratis, utrinque glabris vel subtus pilosis, glomerulis in inflorescentias cylindrico-conicas sæpe paniculatas approximatis, calyce fauce nuda.

Mentha piperita Linn.? sp. plant.ed. 1.p. 576. Wirtg. ! exsicc. n. 1. Com. fl. com. 4. p. 308. Rota prosp. fl. Berg. p. 69. Mor. A. sard. 3. p. 268. Tass. fl. prov. sen. p. 50. Cenno bot. Sien. p. 86 De Vis. Sace. cat. piant. Ven. p. 13\% Ces. Pass. Gib. comp. fl. ital. p. 297. Arc. comp. fl. ital. p. 536.

Stazione, Abitazione e Fioritura. - Pianta coltivata negli orti. Li indicata nel.Napoletano dal Compendio di Cesati, Passerini e Gihelli, ma col sospetto che sia meramente inselvatichita. Vi riferisco un saggio raccolto dal Marzialetti in giugno 1838 a Montefortino all'Acquaviva, che però si allontana dal tipo, per avere le foglie cotonose di sotto.

Distribuzione geografica. - In quasi tuitta l'Europa, in Egitto, nell'Asia media, nell'India, nelle due Americhe, difficile a sapere dove sia veramente spontanea.

Osservazione. - C'è chi ritiene questa pianta un ibrido fra la Mentha viridis e la $M$. aquatica. 


\section{A. Nilentha pyramidalis.}

M. foliis petiolatis, ovatis vel ovato-lanceolatis, serratis, utrinque pubescenti-hirtis raro glabris, glomerulis in inflorescentias cylindricn-conicas sæpe paniculatas approximatis, calyce fauce nuda.

Mentha pubescens Willd.? enum. hort. berol. p. 608 (1809).

Mentha pyramidalis Ten.! fl.nap. 1. prodr.p. 34 (18/1). Benth. lab. p. 175. Guss. fl. sic. syn. 2. p. 69.

Mentha urticæfolia Ten.! fl. nap. 4. syll. p. 82 (1826).

Mentha suavis Guss. pl. rar. p. 387. Ces. Pass. Gib. comp. R. ital. p. 297. Cocc. contr. f. Bol. p. 14. Arc. comp. fl. ital. p. 536. Gib. Pir. fl. Mod. p. 127.

Mentha hirsuta var. $\delta, \varepsilon, \varphi, \gamma$. Bert. fl. ital. 6 p. 96.

Mentha Avellini Tod.l in Bert. l. c. (1844).

Mentha nepetoides Rota? prosp. fl. Berg. p. 69.

Mentha inarimensis Guss. en. pl. In. p. 255. Terr.! fl. Vult. syn. p. 134.

Mentha aquatica var. Car. prodr. fl. tosc. p. 502. Ces. Pass. Gib. o. c. p. 298. Arc. l. c. Gib. Pir. o. c. p. 126.

Mentha viridis $\beta$ Ces. Pass. Gib. l. c. Arc. l. c.

Mentha hirta Cald.? fl. fav. tent. p. 178.

Mentha Lloydii Cald.? l. c.

Figure.-Ten. fl. nap. t. 55, 2.12. f. 3. Guss. pl. rar. t. 66 . Reich. ic. bot. t. 978.

Stazione, Abitazione e Fioritura. - Nei luoghi umidi di tutta l'Italia, ma non comune: nel Tirolo a Fiemme (Bert.), presso Bergamo a Caprino (?) (Rota), nelle provincie di Milano e di Pavia (Moretti!), a Parma (Passerini!), nell'Appennino Parmense (Bert.), nell'Appennino Modenese tra il Salto e Montese (Gilb. Pir.), a Bologna lungo il Canale Naviglio (Coce.), a Faenza (?) (Cald.), presso Lueca a San Lazzaro (Beccari!) e Montecarlo (Chiostri!), a Firenze lungo l'Arno, a Siena, a lioma (Bert.), nell'Abruzzo a Caramanico a S. Croce (Guss.), in Terra di Lavoro a Sura verso il Fibreno (Terr. !) e a Caserta (Bert.), a Napoli al Pascone e ai Bagnuoli (Ten, Bert.), nell'isola d'Ischia alle (Yuianole del Testaccio (Guss., Levier!), in Basilicata alla Ferrara presso Melfi (Terr.!), a Castelgrande (Guss.) e a Potenza ('Ten., Bert.), in Sicilia a Palermo al Fiume Oreto (Tod.!), a Boccadifalco, a Polizzi (Guss.), a Lercara (Sorrentino!). Fiorisce da giugno e luglio a ottobre. 
Distribuzione geografica. - Nella Francia meridionale, e in Italia.

Osservazione. - Le forme che ho riunito sotto la Mentha pyramidalis rassomigliano molto a quelle che sono state distribuite da Wirtgen come ibridi fra la $M$. aquatica, e la $M$. rotundifolia o la $M$. silvestris; e siccome, al pari di questi, le ho trovate sterili in tutti i saggi che ho potuto esaminare, ho un forte dubbio che tale difatti sia la loro natura, trattarsi cioè di prodotti d'iłıridazione.

\section{Mentha aquatica.}

M. foliis petiolatis, ovatis, serratis, utrinque plus minus pubescenti-hirtis vel etiam glabris, glomerulis in inflorescentias globosas vel ovales sæpe corymbosas aut paniculatas approximatis, vel insuper nonnullis axillaribus, calyce fauce nuda.

Mentha aquatica Linn. sp.pl.ed. 1.p.576. Zer. fl. mel. thes. p. 53. Mass. prodr. fl. valt. p. 113. Trev. prosp. fl. eug. p. 25. Pucc. syn. pl. luc. p. 302. De Not. rep. fl. lig. p. 314. Com. fl. com. 4. p. 309. Rota prosp. piont. Pav. p. 271. Hausm. fl. Tir. p. 670. Rota prosp. A. Berg. p. 69. Grech Del. fl. mel. p. 26. Pir. fl. foroj. syll. p. 109. Mor. fl. sard. 3. p. 268. Bicch. agg. fl. lucch. p. 15. Ard. cat. pl. Ment. p. 28. Car. prodr. 凡. tosc. p. 502 (excl. var.). Tass. fl. prov. sen. p. 50. Ard. fl. Alpes-mar. p. 292. De Vis. Sacc. cat. piant. Ven. p. 132. Zersi prosp. piant. Bresc. p. 162. Mars. cat. pl. Corse p. 111. Archb. fl. Alto-Serch. p. 59. Ces. Pass. Gib. comp. fl. ital. p. 297. Ing. cat. sp. Mond. p. 50. Cocc. contr. fl. Bol. p. 13. Terzo contr. fl. Bol. p. 27. Cald. fl. fav. tent. p. 178. Arc. comp. fl. ital. p. 536 Gib. Pir. fl. Mod. p. 126.

Mentha hirsuta Bert. fl. ital. 6. p. 95 (excl. var. $\delta-\gamma)$. Zan. prosp. fl. ven. p. 26. G. Bert. not. piant. porr. p. 47. Veg. Porr. p. 86. Cald. l. c.

Figura. - Reich. ic. bot. t. 977.

Stazione, Abitazione e Fioritura. - Comunissima in tutta Italia nei fossi e neiluoghi paludosi. S'innalza nei monti non poco: in Toscana fin sopra i 1000 metri. Fiorisce da luglio a settembre e ottobre.

Distribuzione geografica. - In tutta l'Europa eccettuata l'artica, nella Siberia occidentale, nell'Affrica settentrionale, al Capo di Buona Speranza, nell'America settentrionale. 
Descrizione. - Pianta odorosa, perenne, alta da 5 a 6 decimetri, verde, pelosetta o pelosa, fornita di un rizoma il quale manda fibre radicali bianchicce $\mathrm{e}$ in alto $\mathrm{i}$ fusti $\mathrm{e}$ alla base di questi alcuni stoloni lunghi o lunghissimi, delicati, radicanti, che portano piccole foglie. Il fusto è eretto, quadrangolare, con gli angoli rilevati ed ot. tusi, verdi, pelosi negli angoli e in parte nelle facce, le quali sono piane, per peli piuttosto corti, curvati in giủ, bianchi; è ramoso con i rami patenti. Le foglie sono molli, opposte, patenti o patentissime, ovate o ovali-lanceolate per essere talvolta ristrette un po'alla base nel picciòlo invece di essere ivi quasi tondeggianti, ottusette o acute, seghettate, con i denti ottusetti e disuguali; sono verdi e per lo più glabre di sopra ed ivi con solchi corrispondenti ai nervi di sotto, di un verde pallido di sotto ed ivi con poche e piccole glandole a guisa di vescichette trasparenti, con un nervo longitudinale e con molti nervetti laterali, rilevati, i quali si uniscono ad arco tra loro e formano con i loro rami una rete un po'rilevata e a larghe mạglie vicino al margine: in questi nervi sono peloselte con peli curvi e bianchi; il picciòlo è più corto delle foglie, profondamente solcato di sopra, convesso di sotto, peloso ivi e sui margini. I fiori sono distribuiti in glomeruli, dei quali i superiori sono in un capolino grande, quasi tondo, ovale, ottuso e gl'inferiori un po'lontani sono peduncolati all'ascella di due grandi foglie fiorali. Il peduncolo di questi è quasi tanto lungo quanto il picciòlo delle foglie corrispondenti; tutti i fiori sono portati sopra pedicelli i quali sono delicati, eretti, poco più corti o uguali al calice, di color violetto-rossiccio, irsuti, con peli corti, bianchi e curvati in giù e accompagnati oltre alle brattee da bratteole strette, lineari o setacee, piu corte o quasi uguali ai pedicelli stessi, patenti, verdi e fornite di corte ciglia nei margini. Il calice è tubuloso, verdognolo-rossiccio, con molti nervi longitudinali pelosetti, con cinque denti, la metà circa più corti del tubo, triangolari-lanceolati, acuminati, eretti. La corolla è poco meno del doppio più lunga del calice, di color violetto-bian. chiccio, pelosetta di fuori, con peli diritti, e pelosa di dentro nel tubo, divisa in quattro lobi quasi uguali, i quali sono eretto-patenti, ottusi e uno dei superiori smarginato all'apice e un po'piu largo de. gli altri. Gli stami sono quattro, quasi uguali, quasi il doppio piu lunghi della corolla, corrispondono in mezzo ai lohi di questa e sono eretto-patenti, inseriti in alto del tubo. I filamenti sono filiformi, diritti, bianchicci, glabri. Lo antere sono quasi ovali, ottuse, smarginate alla base, inserite nel mezzo del dorso, introrse, hiloculari, 
aprentisi longitudinalmente, di colore violetto, glabre. Il pistillo è appena più lungo o quasi uguale agli stami. L'ovario è diviso in - quattro lobi, ottusi, lisci, inserito sopra un disco circolare, verde, glabro e più alto dell' ovario. Lo stilo è filiforme, lungo, bianchiccio, glabro. Lo stimma è bifido, con le lacinie un po'disuguali, un po'divergenti, quasi acute, bianchicce e glabre. (Parl. ms., descr. della pianta di Olmeneta in Lombardia).

Osservazione. - Esiste una forma affine, rappresentata dalla tav. 976 di Reich. ic. bot., che alcuni ritengono una varietà verticillata della Mentha aquatica, altri un ibrido fra essa e la $M$. arvensis. In Italia è stata trovata dal Marzialetti nel 1838 nel Piceno a Montefortino all'Acqua-viva.

\section{Mentha arvensis.}

M. foliis petiolatis, ovatis vel ovali-lanceolatis, serratis, utrin. que plus minus pubescenti-hirtis vel etiam glabris, glomerulis axillaribus remotis, calyce fauce nuda.

Mentha gentilis et M. arvensis Linn. sp. pl.ed. 1.p. 577. Com. fl. com. 4. p. 312, 313. Zan. prosp. fl. ven. p. 26. Rota prosp. piant. Pav. p. 271. Hausm. fl. Tir. p. 671. Rota prosp. fl. Berg. p. 69 (incl. M. rubra et M. pratensis). Pir. fl. for. syll. p. 109.

Mentha sativa Linn. sp. pl. plant. ed. 2. p. 805. Com. o. c. p. 311. Hausm. 0. c. p. 670. Rota ll. cc. Pir. l. c. De Vis Sacc. cat. piant. Ven. p. 132. Zersi prosp. piant. Bresc. p. 162.

Mentha arrensis Benth. lab. p. 178. Trev. prosp. fl. eug. p. 25. Pucc. syn. pl. iuc. p. 302. Bert. fl. ilal. 6. p. 99. Car. prodr. fl. tosc. p.502. Tassi fl. prov. sen. p. 50. Ard. fl. Alpes-mar. p. 292. De Vis. Sacc. l. c. Zersi l. c. Ces. Pass. Gib. comp. fl. ital. p. 298. Ing. cat. sp. Mond. p. 50. Cocc. contr. fl. Bol. p. 13. Arc. comp. fl. ital. p. 536. Gib. Pir. fl. Mod. p. 126.

Mentha pratensis Zersi? l. c. Gib. Pir.? l. c

Figure. - Fl. dan. t. 512. Reich. ic. bot. t. 968-975.

Stazione, Abitazione e Fioritura. - Nei campi umidi, nei luo. ghi incolti inondati, nelle paludi ecc. Abbonda nell'Alta Italia: in tutto il Piemonte (All. ecc.), cosi a Mondovi (Ing.), e varcando il confine a S. Martino Lantosca nelle Alpi marittime (Ard.), nelle vallate Valdesi (Rostan!), a Torino, nel Canavese (All.), a Riva di Sesia (Carestia!), a Novara (Bír. fl.), in Val Intrasca (De Not.!), in 
Val Vegezzo (Franzoni!); in Lombardia a Pavia, a Milano (Rampoldi!), in Brianza (Parl.!), nel Comasco, sino al fondo del lago (Com.), a Grumone nel Cremonese (Parl.!), nel Bergamasco (Rota), nel Bresciano a Brescia (Zersi), a Iseo, sopra Collio a 900 metri (Parl.!), a Pontedilegno (Ricca! ecc.), in Tirolo nel Trentino (Perini! ecc.), a Bolzano, a Merano (Hausm.), e altrove; nel Veronese (Poll. $\boldsymbol{n}_{\text {.) }}$, nel Vicentino, nel Trivigiano, nel Bellunese (De Vis. Sacc. ecc.), nel Friuli (Pir. ecc.), a Venezia (Kellner!), a Chioggia (Bert.), nel l'adovano (Trev. ecc.), nel Nantovano (Barbieri! ece.), nell'Emilia a Guastalla, a Parma (Passerini!), a Borgotaro (Bert.), nei monti di Querzola (Re), a Bologna (Coce.). Doventa molto rara nella Media Italia, dov'è indicata soltanto presso Lucca alla Badia di Pozzeveri (Pucc.), a Siena fuori Porta Fontebranda e altrove (Bart. cat.), e giù nell'Abruzzo a Pescocostanzo (Bert.). Il Compendio di Cesati, Passerini e Gibelli l'indica ancora in Sicilia. Fiorisce in luglio, agosto e setternbre.

Distribuzione geografica. - In tutta Europa; meno l'artica, ma piủ scarsa nella sua parte mediterranea, e nell'Asia settentrionale e media.

Descrizione. - Pianta perenne, odorosa, alta da 4 a 5 decimetri, ispicla per peli lunghetti, rigidi, curvati in gii e bianchi, e aventi nelle parti superiori delle glandolette verdognole quasi tonde, fornita di un rizoma ramoso, il quale manda molte fibre radicali ramose e bianclicce e in alto degli stoloni radicanti, lunghetti, rossicci e aventi piccole foglie e i fusti. Questi sono quadrangolari, con gli angoli rilevati ed ottusi, ivi ispidi per i peli di sopra descritti, leg. germente scanalati nelle facce, le quali nella parte superiore del fusto hanno delle glandolette tonde e verdognole, ramosi fin dalla base, con i rami opposti, piuttosto corti e patenti, simili al fusto e come questo in parte rossicci. Le foglie sono un po'consistenti, opposte, poco lontane tra loro, patentissime e rivolte un po'in gii, ovate, ottusette o quasi acute, dentate, con $\mathrm{i}$ denti poco rilevati, ot. tusi e diretti verso l'apice della foglia, quasi intere alla base, verdi di sopra ed ivi con profondi solchi corrispondenti ai nervi della pagina inferiore, di un verde un po'pallido di sotto ed ivi con glandole rade, quasi tonde e trasparenti, e con un nervo lomgitudinale rilevato da cui partono molti nervi laterali, egualmente rilevati, obliqui c. curvi, diretli versn l'apice e il inargine, in vicinanza del quale si uniseono ad areo tra loro; sono pelosette e ruvidette nella pagina smeriore e nei nervi della inferiore, fornite di un picciólo corto, sol- 
cato di sopra, convesso di sotto, di un colore più chiaro e peloso in ogni parte. I fiori sono assai piccoli, distribuiti in glomeruli all'apice di un corto peduncolo che è all' ascella delle foglie superiori, e sono da circa 15 a 20 in ciascun glomerulo in modo che in ciascun verticillo vi sono da 30 a 40 fiori; ciascun verticillo è quasi tondo e poco più lungo o quasi uguale al picciòlo della foglia fiorale corrispondente; i verticilli superiori sono meno numerosi o quasi mancano, per cui i rami terminano con piccole foglie. Il peduncolo è peloso, angolato, verdognolo-rossiccio. Le brattee sono due, patenti, piủ lunghe dei pedicelli in modo da uguagliare quasi il calice, lanceolate, acuminate, uninervi, verdi, fornite di ciglia. I pedicelli sono delicati, patenti, lunghi quasi quanto il calice, cilindrici, rossicci, ispidetti, glandolosi. Il calice è tubuloso, un po' più largo gradatamente verso alto, verdognolo-rossiccio, con circa dieci nervi grossi a guisa di costole e ispidetti, con molte glandole quasi distribuite in linee in mezzo ai nervi, diviso in cinque denti $\mathrm{i}$ quali sono da tre a quattro volte più corti del tubo del calice, eretto-patuli, trian. golari-lanceolati, acuti. La corolla è poco piủ lunga del calice, di color violetto-roseo assai chiaro; il suo tubo è quasi cilindrico, un po' più largo verso alto, glabro di fuori in basso, pelosetto in alto, e peloso di dentro con peli lunghetti e orizzontali; il lembo è diviso in 4 lacinie, poco più corte del tubo, patule, quasi uguali in lunghezza, però la lacinia superiore è un po' più larga, ovale, e smarginata all'apice, le altre sono ovate, ottusissime e quasi tondeggianti all' apice: tutte pelosette di fuori e glabre di dentro. Gli stami sono pelosi, giungono fino alla gola, e sono inseriti nella parte superiore del tubo. I filamenti sono filiformi, bianchi, corti e glabri. Le antere ovali, ottuse, smarginate alla base, inserite nel dorso, introrse, biloculari, bianchicee, glabre. Il pistillo è lungo quanto gli stami o appena più lungo di questi e perciò molto piủ corto della corolla. L'ovario è piccolo, con quattro lobi, ovali, glabri, verdognoli, in. serito sopra un disco verde quasi tanto alto quanto l'ovario. Lo stilo è filiforme, bianchiccio, glabro. Lo stimma è bifido, con le lacinie quasi uguali, un po'divergenti, filiformi, ottusette, glabre. (Parl. ms., descr. della pianta di Grumone in Lombardia).

Osservazione. - Specie variabile al solito per il grado della pe. lurie; varia anche per il calice, ora più corto più decisamente campanulato; ora più lungo alquanto cilindrico, ciò che principalmente induce alcuni botanici a distinguerla in parecchie specie; ma sono variazioni che si ritrovano nelle congeneri. 


\section{ข. Venthu Puleginm.}

M. foliis petiolatis, ovalibus, denticulatis, utrinque plus minus pubescenti-hirtis vel glabris, glomerulis axillaribus remotis, calyce fauce villis clansa.

Mentha Pulegium Linn. sp. pl. ed. 1. p. 577. Zer. fl. mel. thes. p. 29. Mass. prodr. fl. valt. p. 113. J. Bert. it. apenn. bon. p. 24. Trev. prosp. fl. eug. p. 25. Pucc. syn. pl. luc. p. 302. Bert. f. ital. 6. p. 102. De Not. rep. fl. lig. p. 314. Com. R. com. 4. p. 315 Req. lett. in giorn. bot. p. 8. Zan. prosp. fl.ven. p. 26. Rota prosp. piant. Pav. p. 27\% Prosp. fl. Berg. p. 69. Genn. fl. Capr. p. 112. Mor. R. sard. 3. p. 2i0. Art. cat. pl. Ment. p. 28. Car. prodr. A. tosc. p 503. Fl. Montecr. p. 28. Tassi A. prov. sen. p. 50. Ard. 1l. Alpes-mar. p. 292. Zersi prosp. piant. Bresc. p. 162. Pasq. 1. ves. p. 78. Terr. fl. Vult. syn. p. 134. Mars. cat. pl. Corse p. 111. Arclib. fl. Alto-Serch. p. 59. Ces. Pass. Gib. comp. R. ital. p. 298. Ing. cat. sp. Mond. p. 50. Groves contr. A. Terra d'Otr. p. G5. Terr. quarta rel Terra di Lav. p. 112. Lojac. is. Eol. p. 122. Cald. 1l. fav. tent. p. 178. Arc. comp. A. ital. p. 537. Gib. Pir. /l. Mod. p. 126.

Pulegium vulgare Mill. dict. Hausm. R. Tir. p.672. Grech Del. 1l. mel. p. 26. Pir. Rl. foroj. syll. p. 109. De Vis. Sacc. cal. piant. Ven. p. 132.

Figura.-Reich.ic. /l. germ. 18. 1. 89. f. 2.

Stazione, Abitazione e Fioritura. - Sui margini dei fossi, lıogn le strade, nei campi e nei prati umidi, e anche in lıoghi asciutti, per tutta l'Italia, piủ o meno comune. Nell' Alta Italia s'interna nei monti fino a Locarno nel Ticino (Franzoni!), in Valtellina (Mass.), in Tirolo a Roveredo (Hausm.), in Valsugana (Ambrosi!), in Campo Trentino (Perini!) e verso Bolzano (Hausm.); nella Media Italia sale a piccole altezze, cosi non arriva a 1000 metri. Fiorisce nelle parti meridionali in maggio, giugno e luglio, nelle settentrio. nali in luglio, agosto e settembre.

Distribuzione geografica. - In tutta Europa dall' Inghilterra e dalla Svezia in giï, nell'Africa settentrionale, in Oriente, e anche nelle due Americhe.

Descrizione. - Pianta odorosa, perenne, di un verde bianchic. cio, pubsescente tomentosa, fornita di un rizoma quasi orizzontale, 
scuro, che manda in giù fibre radicali nericce e in alto i fusti e dalla base di questi degli stoloni radicanti. I fusti sono ascendenti o giacenti alla base, poi eretti, spesso un po'flessuosi, quadrangolari, con gli angoli ottusi, non rilevati, pubescenti-tomentosi per peli disuguali, semplici, curvati in basso e bianchi; sono ramosi con i rami patenti ed opposti. Le foglie sono piccole, molte, opposte, patentissime e un po'curvate in giù, ovali, ottuse, con piccoli e rari denti nel margine, ristrette alla base in un picciỏlo corto, quasi piano di sopra, convesso di sotto, verdi e pelosette di sopra ed ivi con un solco longitudinale e pochi solchi laterali poco profondi che corrispondono ai nervi di sotto, di un verde più pallido di sotto ed ivi con molte glandole a guisa di vescichette trasparenti e con un nervo longitudinale rilevato da cui partono pochi nervetti laterali, anche essi rilevati, i quali si uniscono tra loro ad arco verso il margine. I fiori sono distribuiti in glomeruli e formano una spiga con verticilli quasi tondi e un po' lontani. Le brattee sono simili alle foglie ma gradatamente più piccole, più lunghe dei fiori e rivolte in giù. I pedicelli sono più lunghi o quasi uguali al calice, diritti, cilindrici, verdognoli o rossicci, pelosi, con peli corti e diritti. Il calice è tubuloso, con dieci nervi rilevati, cinque dei quali giungono all'apice dei denti e cinque ai seni intermedi, è verdognolo, e in parte violetto-rossiccio, peloso nei nervi con una fila di glandole trasparenti giallicce nei solchi che sono tra i nervi, quasi bilabiato, con il labbro superiore diviso in tre denti triangolari-lanceolati, e l'inferiore in due denti lanceolato-acuminati, tutti patenti e pettinatocigliati nei margini. La corolla è quasi il doppio piủ lunga dal calice, di color violetto-chiaro. Il suo tubo è stretto, cilindrico, pelosetto, ad un tratto ingrossato alla gola la quale è ventricosa ed ha in avanti una sporgenza un po'schiacciata a guisa di carena; le lacinie sono quattro, quasi uguali, allungate, ottuse, eretto-patule, pelose di fuori con peli lunghetti, un po' concave dalla parte interna, glabre di dentro. Gli stami sono quattro, poco più lunghi della corolla, eretto-patenti, inseriti nella gola. I filamenti sono filiformi, glabri, del colore della corolla. Le antere ovali, ottuse, smarginate alla base, inserite nel dorso, biloculari, aprentisi longitudinalmente, di colore violetto. Il polline è bianco. Il pistillo è quasi uguale agli stami. Lo stilo è filiforme, diritto, bianchiccio, glabro. Lo stimma è bifido, con le lacinie un po'disuguali, acute, divergenti. Il calice nel frutto ha i suoi denti patenti e la gola chiusa da un anello di peli lunghi e bianchi. (Parl. ms., desir. della pianta di Grumone in Lombardia). 


\section{Mentha requieni.}

M. foliis petiolatis, rotundatis, integris, pilosulis vel glabris, floribus solitariis vel paucis axillaribus, fauce villis clausa.

Mentha Requieni Benth. lab. p. 182. Bert. fl. ital. 6. p. 104. Gren. Godr. fl. de Fr. 2. p. 653. Mor. A. sard. 3. p. 27\%. Car. prodr. fl. tosc. p. 502. Fl. Monterr. p. 28. Mars. cat. pl. Corse p. 111. Genn. A. Capr. p. 112. Ces. Pass. Gib. comp. /l. ital. p. 298. Arc. comp. A. ital. p. 537.

Figura. - Mor. o. c. t. 106.

Stazione, Abitazione e Fioritura. - Nei luoghi umidi dei monti di Corsica: ai laghi di Cireno e di Nıno, a Campotille (Requien!), nell'alto Tavignano presso il lago d'Inn, al monte Cagno (Gren. Godr.), tra i colli San Rocco e Sevi, tra Vico e Aitone, nella foresta d'Aitone, ad Aullene, all'Ospedale, nella valle Restonica a circa 1000 metri (Mars.). In Sardegna nell' isola di Caprera presso il Teialone, rara (Genn.), nci monti di Arizzo (Genn.!), sopra Santa Barbara (Ascherson!) e altrove. In Montecristo nel borro del Colle del Leccio (Watson-Taylor!). Fiorisce in giugno e luglio.

Distribuzione geografica. - Specie propria delle qui indicate isole.

Descrizione. - Pianta perenne, piccola, alta da 6 o 8 centimetri a tre o quattro decimetri, odorosa, fornita di fusti molto ramosi e come i raıni giacenti per terra, che mandano fibre radicali delicate e lunghette nei nodi inferiori, quasi filiformi, ottusamente Iuadrangolari, verdognoli, puberuli per rari e corti peli orizzontali e bianchi. Le foglie sono piccolissime, opposte, patenti, orbicolari, talvolta leggermente quasi in cuore alla base, quasi piane 0 un po'concave di sopra ed ivi spesso pelosette o pelose e di un color verde chiaro, di un verde più chiaro di sotto ed ivi con un nervo longitudinale rilevato da cui partono lateralmente presso la base uno o due nervi curvi che si dirigono verso l'apice e il margine della loglia: sono di sotto meno pelose o quasi glabre, tutte fornite di un picciòlo lunghetto, piano di sopra, convesso alquanto di sotto, verdognolo, puberulo-pelosetto e quasi cigliato. I tiori sono piccoli, in gtomeruli un po'laschi, da due a sei in quelli laterali, da sei a dodici nell'ultimo glomerulo verso l'apice dei rami: sono forniti di un pedicello filiforme, poco più corto del calice, verdognolo, puberulo-scabro, 
e accompagnati dalle foglie fiorali che sono simili a quelle dei rami. Il calice è turbinato-campanulato, verdognolo, puberulo-peloso, con dieci nervi longitudinali e con cinque denti un po'disuguali, ovatolanceolati, acuti e alcuni acuminati, eretti, poi un po' patuli; la gola del tubo è larga, peloso-barbata. La corolla è piccola, una metà circa piủ lunga del calice, rosea, il tubo è stretto, un po'curvo, di un roseo più chiaro, poco più corto del lembo: questo è diviso in qualtro lobi, ovali-orbicolari, quasi uguali, interi o taluno ora il superiore ora uno dei laterali smarginato, dei quali tre rivolti in su e uno un po' in giù, in modo che la corolla pare abbia due labbri. Gli stami sono 4, più corti della corolla, ma più lunghi del suo tubo. I filamenti sono filiformi, glabri, rosei. Le antere piccole, quasi tonde, inserite nel dorso sopra della base, biloculari, introrse, di colore di viola chiaro. Il polline è bianco. Il pistillo è poco più corto degli stami. L'ovario è piccolo, diviso in quattro lobi, quasi tondi, verdognoli, glabri, con un disco a guisa di anello dello stesso colore. Lo stilo è lungo, filiforme, glabro, bianchiccio. Lo stimma è bifido, con le lacinie quasi uguali, grossette, ottuse, divergenti-ricurve, bianchicce o rosee. (Parl. ms., descr. di pianta coltivata).

\section{MA IOERANA.}

Majorana Tourn. inst.p. 199. t. 91. Moench meth. pl.p. 406. Benth. lab. p. 338. Gen. pl. fl. germ. 6.t. 15.

Origani sp. Benth. Hook.gen. pl. 2. p. 1186. Ces. Pass. Gib. comp. fl. ital. p. 299. t. 44.f. 1. a, c, d.

Calyx antice fissus ideoque explanatus, obsolete nervatus. Corolla vix bilabiata, labio superiore subpatulo, planiusculo. Stamina 4, filamentis edentulis, antheris 2-locularibus, longitudinaliter dehiscentibus, distinctis. Stylus lobis æqualibus. Cocci apice convexi leves.

Portamento. - L'unica specie nostrale tlel genere ha interamente l'aspetto del Thymus Origanum, però con le brattee più serrate, e coi fiori bianchi.

Osservazione. - Questo genere è stato sempre, o ravvicinato al genere Origanum Tourn., o riunito ad esso, a motivo dell'infiorescenza; però i caratteri fiorali sono cosi diversi, da dovere distaccare maggiormente i due generi, come ho fatto. 


\section{Manonna Dnitew.}

Origanum Onites Linn. sp. pl. ed. 1. p. 590. Bert. fl. ital. 6. p. 198. Ces. Pass. Gib. comp. fl. ital. p. 299. Arc. comp. fl. ital. p. 538.

Majorana Onites Benth. lab. p. 339.

Figura. - Fl. greec. $t .572$ (mala quoad analyses).

Stazione, Abitazione e Fioritura. - A Siracusa, in luoghi aridi sassosi (Bianca! ecc.), e a Sponti (Bert.). Fiorisce in maggio e giugno.

Distribuzione geografica. - Nell'indicato punto di Sicilia, in Dalmazia, in Grecia, nell'Asia Minore, in Soria.

\section{IIYSSGPUS.}

Hyssopus Tourn. inst. p. 200. t. 95. Mirb. in ann. mus. 15. t. 16. f. 6, 8. Gen. pl. fl. germ. 6. t. 19. Benth. Hook. gen. pl. 2. p. 1187. Ces. Pass. Gib. comp. fl. ital. p. 304. t: 44. f. 4.

Calyx tubuloso-campanulatus, valide 15-nervatus. Corolla manifeste bilabiata, labio superiore erecto, planiusculo. Stamina 4, filainentis edentulis, antheris 2-locularibus, loculis longitudinaliter dehiscentibus, confluentibus. Stylus lobis subæqualibus. Cocci apice couvexiusculi leves.

Portamento. - Suffrutice ramoso, coll rami drizzati in su, alto 3-4 decimetri. Foglie fitte, bislunghe-lineari, intere. Fiori turchini, talora rosei o bianchi, in glomeruli piủ o meno ravvicinati fra loro da formare lunghe intiorescenze terminali, interfoliate, unilaterali.

\section{Iyssopus officinalis.}

Hyssopus officinalis Linn. sp. pl. ed. 1. p. 569. Bert. ^. ital. 6. p. 63. De Not. rep. 月. lig. 1. 319. Koch syn. 月. germ. ed. 9. p. 645. Zan. prosp. R. ven. p. 26. Mansm. 1. Tir. p. 652. Pir. A. for. syll. p. 111. Ard. cat. pl. Ment. p. QS. Fl. Alpes-mar. p. 29. De Vis. Sace. cat. piant. Ven. p. 135. Zersi prosp. piant. Bresc. p. 165. Ces. Pass. Gib. comp. 17. ital. p. 305. Ing. cal. sp. Mond p. 43. Arc. comp. R. ital. p. 5 il. 
Figure. - Savi mat. med. veg. tosc. $t$. 15. Reich. ic. fl. germ. 18. $t .58$.

Stazione, Abitazione e Fioritura. - Qua e là in luoghi sassosi solatii dei monti dell'Italia settentrionale, e talora anche nel piano: in Piemonte e Liguria nella valle d'Aosta presso Giambava (Del Ponte!), nella valle d'Oulx, fra Garessio ed Ormea, nel Monferrato lungo il fiume Orba, lungo la Scrivia nel Tortonese (Colla herb. ped.), nel Novese (De Not.), a Tenda, lungo il Vesubio presso Levenzo, nella sommità dell'Agel sopra Turbia a 1120 metri (Ard.), nei monti del Ceriale (Bert.), nel Piacentino e Parmense lungo la Nura, a Bardi (Bert.), nel letto del Taro presso la via Emilia (Passerini!), in Val. tellina a Sondrio (Rampoldi!), forse nel Bresciano sulla pendice settentrionale del Guglielmo (Zersi), verso Roveredo (Hausm.), alle radici del Baldo non lungi da Pazzone (Bert.), nel monte Pastello di Verona sopra la Chiusa (Manganotti! ecc.), nel monte Sumano di Vicenza (Barbieri!), nel monte Cavallo di Belluno (Zannichelli), in Friuli ed Istria presso S. Daniele, Suzano, Gemona, Tolmezzo (Poll. fl.), Gorizia al monte Salvatino (Mattioli), Monfalcone (Pir.), Trieste a Contovello (Bert.), Pirano, Fiume (Poll.), infine a Chioggia presso il porto di Fossone (Bert.). Molto più raro nell' Italia più meridio. nale, non vi è stato trovato che a Valcimarra presso Camerino (Ricci!), nel monte Luco presso Spoleto (Arc.), in Abruzzo a Costa di Fascia e Pietra Cantavallo, nel Velino alla grotta di S. Benedetto (Levier!), e nella Maiella a Gravara alla bocca della valle presso Lama, nel Gargano, in Campania nel Matese (Ten. syll.) e alle radici del Taburno sopra Airola (Bert.). Fiorisce da luglio a ottobre.

Distribuzione geografica. - Nella Spagna, la Francia e la Sviz. zera meridionali, l'Italia, la Dalmazia, il Montenegro e l'Erzegovina, la Crimea, il Caucaso, la Siberia e la Persia, fino all'Imalaia, l'Atlante (Ball.).

\section{SOTTOTRIBÙ THRZA.}

\section{Henniee.}

LAMieE (non alior.).

Stamina simul contra labium superius corollæ applicita, inferiora adscendentia. Stylus lobis subulatis. 


\section{SEZIONE PRIMA.}

Satureiee.

Satureies (non alior.).

Stamina inferiora longiora.

\section{EX. 'HYMUS.}

Thymus, Serpyllum et Origanum Tourn. inst. p. 196, 198. $t$. 93, 94.

Thymus (part.) et Origanum (part.) Liun. gen. pl.ed. 1.p. 168, 169. Thymus et Origanum (part.) auct. rec. Mirb. in ann. mus. 15. t.20. 1. 1, 2, 7. Benth. Hool. gen. pl. 2. p. 118j, 1/86. Ces. Pass. Gib. comp. fl. ital. p. 299. t. 44.f. 1, 2.

Thymus et Origanum Gen. pl. fl. germ. 6. t. 14, 16.

Calyx tubuloso-campanulatus, valide 10-13-nervatus. Corolla manifeste bilabiata, labio superiore erecto, planiusculo. Stamina 4 , superne divergentia, filamentis edentulis, antheris 2-locularibus, longitudinaliter dehiscentibus, a connectivo seiunctis. Stylus lobis subrequalibus. Cocci apice convexi eleves.

Portamento. - I veri Timi sono frutici bassi, ramosissini, eretti, o sdraiati e allora facilmente radicanti, con foglie piccoline, sessili o brevemente picciolate, intere, con fiori piccoli, rosei, in glomeruli piủ o meno ravicinati fra loro e accompagnatí da foglie fiorali simili alle altre, o modificate e volgenti in brattee. Le loro parti erbacee sono ricchissine di glandole, che danno a siffatle piante un odore forte, v'lia pure in generale pelurie, e specialmente sul fusto. Il solo Riegamo è una pianta perenne, con fusti robusti, quasi legnosi, alti fino a $" / \frac{3}{3}$ di metro, rivestiti di foglie di mezzana grandezza, sessili o hrevernente picciuolate, ovate, debolmente dentate; i fiori, piccolini, rosei o bianchi, sono in spighe riunite in corimbi e poi in pannocchia, e sono quasi nascosti all'ascella di hrattee embri. ciate, e verdi o colnrate.

Osservazioni. - Da grau tempo erano riconosciute le strette affinità fra i generi Thymus e Oraganum, tanto che gli autori erano 
imbarazzati a distinguerli. "Genus a Thymo potius inflorescentia quam characteribus floralibus distinctum, " scrivono dell'Origanum Bentham e Hooker, senza ricordarsi che in certi Timi ( $T$. capitatus, $T$. striatus, $T$. longiflorus, $T$. cephalotus ecc.) l'infiorescenza viene ad essere, per motivo dello sviluppo grande delle brattee, sostanzialmente la stessa che negli Origani. Essendo adunque verissimo che fra i due generi non esistono caratteri differenziali di momento, io non posso esitare a riunirli, molto più dopo di avere distaccato dai veri Origani il genere Maiorana, e posto in altra sotto-tribù, come più sopra si può vedere.

\section{Thymus vulgaris.}

T. suberectus, foliis oblongis, margine revolutis, subtus canescentibus, floralibus subconformibus, glomerulis laxe vel densiuscule approximatis, tubo calycino digitaliformi.

Thymus vulgaris Linn. sp. pl.ed. 1. p. 591. Bert. fl. ital. 6. p. 210 De Not. rep. fl. lig. p. 317. Rota prosp. piant. Pav. p. 271. Koch syn.fl. germ. ed. 2. p. 640. Gren. Godr. fl. Fr. 2. p. 657. Ard. cat. pl. Ment. p. 28. Car. prodr. fl. tosc. p. 504. Ard. fl. Alpes-mar. p. 295. Ces. Pass. Gib. comp. fl. ital. p. 300. Ing. cat. sp. Mond. p. 70. Groves contr. fl. Terra d'0tr. p. 65. Terr. quarta rel. prov. Terra di Lav. p. 112. Arc. comp. fl. it. p. 539.

Figura. - Reich. ic. fl. germ. 18. t. 63. f. 1.

Stazione, Abitazione e Fioritura. - Nelle colline aride e nei monti Ligustici, dove è comune, così nel Nizzardo (Parl.!, ecc.), nell'alpi di Tenda (Reuter!), a Dolcedo (Berti!), a Genova (Bert.), a Chiavari (Delpino!), al golfo della Spezia!; trovasi ancora nel vicino Piemonte ad Arquata (Bert.), tra Garessio ed Ormea (Ing.), lungo la Bormida nell'Acquense, tra Saint Vincent e Aosta, e Giambava (All. $f l_{0}$ ), e nel Pavese nelle arene lungo il Po a Bastida de Dossi (Nocca Balb. fl.). Ritrovasi poi nella costa occidentale della Pe. nisola a Terracina (Bert.), e a Gaeta a Montespaccato (Terr.), e nella costa adriatica ad Otranto verso gli Alimini (Groves), e in Istria (Koch.). E indicato in Corsica da Grenier e Godron, ma non da altri. Fiorisce da marzo ed aprile a giugno e luglio.

Distribuzione geografica. - In Portogallo e Spagna, nella Francia meridionale, in Italia. 


\section{Thymus Merba-barona.}

T. erectus vel adscendens, foliis ovatis lanceolatisve, planis, pilosulis, floralibus subconformibus, glomerulis densiuscule approximatis, tubo calycino digitaliformi.

Thymus Herba-barona Lois. 凡. gall. ed. 1. p. 360. Bert. fl. ital. 6. p. 208. Gren. Godr. fl. Fr. 2. p. 657. Hars. cat. pl. Corse p. 112. Ces. Pass. Gib. comp. 凡. ital. p. 300. Arc. comp. fl. ital. p. 538 .

Figura. - Lois. o. c. t. 9.

Stazione, Abitazione e Fioritura. - Nei monti aridi scoperti di Corsica tra 1000 e 2000 metri (Mars.): il Capo (Nars.), Bastia nella sommità del Pigno (Mabille!), S. Fiorenzo (Gren. Godr.), Vizzavone, monte Eliseo, monte Rutondo (Mars.), Guagno, Campolitte, Tretore (Gren. Godr.), monte d'Oro (Soleirol!), monte Cagnone (Gren. Godr.), monte Renoso (Requien), Aullena (Nars.), Restonica, Mello (Gren. Godr.), monte Coscione (Kralik!), monte Cagna (erb. Webb!). In Sardegna, frequentissimo (Mor.): monti di Arizzo, di Laconi (Gennari! ecc.). Fiorisce in giugno e luglio.

Distribuzione geografica. - Specie propria di quelle due isole. Webb (It. hisp. p. 21), e sulla sua fede Willkomm e Lange (Prodr. fl. hisp. 2. p. 405), l'indicano di Spagna: ma il saggio autentico della località spagnuola giacente nell' erbario Webb è di un Thymus vulgaris a foglie strettissime.

\section{Tĥ̀mus nitidus.}

T. erectus vel adscendens, foliis ovatis, planis, glabris, floralibus subconformibus, glomerulis densiuscule approximatis, tuho calycino turbinato.

Thymus nitidus Guss. n. sic. syn. 2. p. 97. Bert. П. ital. 6. p. 205. Ces. Pass. Gih. comp. R. ital. p. 300. Arc. comp. 月. ital. p. 538 .

Stazione, Abitazione e Fioritura. - Nei luoghi pictrosi dell'isola Marettimo di Sicilia (Tineo! ecc.). Fiorisce in maggio e giugno.

Distribuzione geografica. - Pianta speciale di quell'isoletta.

Osservazione. - Le foglie sono grossotte, i calici sono colorati in scuro. 


\section{Thymens Serpillum.}

T. procumbens, foliis ovalibus, oblongis linearibusve, planis, plus minus pilosis, aut sæpius glabris sed etiam tunc frequenter basi longe ciliatis, floralibus subconformibus, glomerulis laxe vel densiuscule approximatis, tubo calycino turbinato.

Thymus Serpillum Linn. sp. pl.ed. 1. p. 590. Bert. fl. ilal. 6. p. 201. Koch syn. fl. germ. ed. 2. p. 640; et auct. omn.

Thymus pannonicus Mass. prodr. l. valt. p. 185. Trev. prosp. fl. eug. p. 26. De Not. rep. fl. lig. p. 318. Bert.! o. c. p. 209. Com. fl. com. 4. p. 363. Rota prosp. piant. Pav. p. 271. Hausm. fl. Tir. p. 677. Car. prodr. fl. tosc. p. 505. Ces. Pass. Gib. comp. fl. ital. p. 300. Arc. comp. 1. ital. p. 538.

Thymus Chamædrys Mass. o. c. p. 186. Bicch. agg. fl. lucch. p. 16. Cald. fl. fav. tent. p. 178.

Thymus citriodorus Muss. o. c. p. 186.

Thymus angustifolius Ces. Pass. Gib. l. c. Cald. o. c. p. 179.

Figure. - Waldst. Kit. pl. rar. Hung. t. 71. Reich. ic. fl. germ. 18. t. 63. f. 2, t. 64. f. 4, t. 65, 66, 67.

Stazione, Abitazione e Fioritura. - La più comune delle nostre Lamiacee, trovasi ovunque nei luoghi aridi selvatici, nei boschi asciutti ecc., specialmente di collina e di montagna, in tutta la Penisola, e in Sicilia. S'innalza nelle Alpi fin quasi al termine delle Fanerogame, cioè fin verso i 2500 metri secondo Wahlenberg: per esempio nel Cenisio (Parl.!); nell'Appennino raggiunge le maggiori cime. Manca interamente in Corsica, in Sardegna, nell'Arcipelago toscano, nelle isole del golfo di Napoli, nelle isole Eolie. Fiorisce da aprile a giugno nei luoghi più hassi, e in luglio e agosto nei monti altissimi.

Distribuzione geografica. - In tutta Europa, nell' Asia occidentale, settentrionale e centrale, in Abissinia, nell'America più settentrionale-orientale.

Osservazioni. - Pianta polimorfa. Essa varia: per la pelurie, che qualche volta è molta (Thymus pannonicus All.), qualche volta poca, anche nulla nelle foglie, e nei fusti spesso ristrettá a due soli lati in ogni internodio; per la grandezza delle foglie, quando più grandi ( $T$. Chamoedrys Fries), quando più piccole, e per la loro forma, piủ larga, o più stretta, talora strettissima bisiunga-lineare 
(T. angustifolius Pers.); per le infiorescenze, raccorciate globose, o allungate cilindriche. Al solito, tali variazioni si combinano fra loro, in modo da produrre quelle molte forme clie hanno data occasione a tante distinzioni specifiche. Oltracciò vi ha la consueta modificazione sessuale del genere, vale a dire che certi individui presentano fiori pseudo-femminei, la metà più piccoli degli altri, e con gli stami rinchiusi.

\section{Thymus striatus.}

T. procumbens, foliis oblongo-linearibus, planis, subglabris ciliolatis, floralibus basi dilatatis ovato-lanceolatis, glomerulis laxe vel densiuscule approximatis, tubo calycino turbinato.

Thymus striatus Vahl symb. bot pars 3. p. 78.

Thymus acicularis Sang. cent. prodr. fl. rom. add. p. 85 (non Waldst. hit.).

Thymus Zygis Bert. f. ital. 6. p. 206 (non Linn.). Terr.! fl. Vult. syn. p. 137. Ces. Pass. Gib. comp. fl. ital. p. 300. Arc. comp. fl. ital. p. 539.

Thymus angustifolius Car. suppl. prodr. A. tosc. p. 43 (non Pers.).

Figura. - Fl.grcec. $t .574$.

Stazione, Abitazione e Fioritura. - Nei luoghi aridi montuosi dell'Italia meridionale, e della centrale. In Sicilia pare che sia comune, cosi trovasi verso Trapani, a Scopello, ad Amorosa (Guss. syn.), a Busambra (Todaro!), sopra Polizzi (Parl.!), a Petralia (Mlinà!), a Sperlingo (Guss.), a Nicosia (Minà!), a Bronte (Guss.), a Caltanisetta e in molti luoghi tutt'attorno (Guss., Parl.!), a Butera (Guss.), ecc.; nel continente comparisce a Otranto, a Lecce, a Taranto, a Grottaglie (Ten. syll.), quindi ritrovasi a Melfi (Terr.!) e sul Gargano (Porta e Rigol), nell'Abruzzo alla Maiclla (Ten.), e al monte de Fiori!, nelle Marche e nell' Umbria al Vettore!, alla Sibilla, a Montefortino (Marzialctti!), al monte Birro, al Furlo (liert.), al monte Catria fra 900 e 1000 metri (l'arl!), e al Sasso di Simone (Cherici!), e dall'altro lato occidentale della Penisola fa a Tivoli (Nauri, Rert.), sul monte Calvi nella maremma Toscana (Marcucci!), e infine sul monte Ferrato vicino a Firenze (Gemmi ). Fiorisce in maggin e gillynn.

Distribuzione geografica. - Oltre l'ltalia, abita ancora la Ilalmazia, la Grecia e l'Asia Minore. 
Osservazioni. - Le foglie fiorali sono talora semicuoiose, lavate di porporino, e in tal caso sono caduche, e nell' infruttescenza non si trovano più. Talora invece sono verdi, erbacee, persistenti : è la forma corrispondente al Thymus spinulosus Ten.!

La specie in discorso non può essere il Thymus Zygis di Linneo, pianta di Spagna, che dalle cose dette nelle Sp. plant. ed. 1. p. 591 e dalla figura citata di Barrelier Icon. 777, corrisponde assai bene al T. tenuifolius Mill. figurato da Boissier Voy. bot. Esp. t. 137; non osta la pianta conservata nell'erbario di Linneo solto il nome di $T$. Zygis, e non corrispondente al $T$. Zygis $s p . p l$., ma secondo Bentham (in Cand. prodr. 12. p. 202) al T. angustifolius, e secondo Gussone (Fl. sic. syn. 2. p. 95) al T. striatus: poichè quando un testo parla chiaro, io son di parere che debba avere maggior valore di saggi d'erbario, soggetti a tante cause di confusione. La nostra specie poi è certamente il T. striatus di Vahl, abbenchè la descrizione da lui data non sia soddisfacente in ogni punto; ma egli ha descritta una pianta del Regno Napoletano, e a nessun' altra del genere ivi esistente si può adattare la descrizione, e secondo Gussone (o. c. p. 96) e Boissier (Flor. orient. 4. p. 557) il saggio del suo erbario è del nostro $T$. striatus.

\section{Thymus capitatus.}

T. suberectus, foliis lineari-lanceolatis, concavo-carinatis, subglabris ciliolatis, floralibus latioribus oblongo vel ovali-lanceolatis crebre ciliatis, glomerulis densissime approximatis, tubo calycino turbinato-ovali applanato.

Thymus capitatus Hoffm. et Link f. port. 1. p. 123. Zer. fl. mel. thes. p. 74. Grech Del.! fl. mel. p. 26. Mor.l fl. sard. 3. p. 279. Ces. Pass. Gib. comp. f. ital. p. 300. Groves! contr. fl. Terra d'0tr. p. 65. Arc.! comp. fl. it. p. 539.

Satureia capitata Bert. fl. ital. 6. p. 61.

Figure. - Fl. græe. t. 544. Reich. ic. fl.germ. 18. t. 70.f. 2.

Stazione, Abitazione e Fioritura. - Nei colli aridi sassosi dell'Italia meridionale, principalmente sul mare: in Sicilia è comunissimo (Guss. syn.), cosi intorno a Palermo (Parl.! ecc.), in Favignana, Levanzo, Maretimo (Guss.), a Licata (Bert.), a Terranova (Sommier!), a Vittoria (Aiuti!), ad Avola (Bianca!), nell'interno a Leonforte (Heldreich!), ecc.; nasce in Pantellaria (Calcara!), in Malta 
(Grech Del.!), in Linosa e in Lampedusa (Aiuti!); in Sardegna abbonda intorno a Cagliari (Mor.! ecc.), e si ritrova a Sassari e Sorso!; fa in Calabria al Capo dell'Armi (Bert.), al Capo Spartivento e Palizzi (Arc.!), e nel lato orientale della Penisola a Otranto (Groves!), a Lecce, a Ostuni, a Mlartina (Ten. syll.), a Barletta (Bruni!), terminando nel Gargano (Porta e Rigo! ecc.). Fiorisce da maggio ad agosto.

Distribuzione geografica. - Pianta del Mediterraneo, si trova in Portogallo e Spagna, in Italia, in Dalmazia, in Grecia e nel resto dell'Oriente, compreso l'Egitto, in Barberia.

\section{ซ. Whymus Driganum.}

T. erectus, foliis ovatis, subdenticulatis, pilosulis, bracteis quadrifariam imbricatis, membranaceis, coloratis, ovatis lanceolatisve, floribus solitariis, tubo calycino turbinato.

Origanunı vulgare Bert. fl. ital. 6. p. 196; et aucl. ital. omn.

Origanum hirtum Koch syn. fl. germ. ed. 2. p. 640. Mor. 凡. sard. 3. p. 275. Ces. Pass. Gib. comp. 1. ital. p. 299. Arc. comp. fl. ital. p. 538 .

Origanum virens Guss. enum: plant. Inarim. p. 258. Terr. fl. Vult. syn. p. 136. Ces. el. piant. Maiella p. 24. Ces. Pass. Gib. l. c. Cocc. terzo contr. 凡. prov. Bol. p. 27. Cald. fl. fav. tent. p. Iis. Goir. app. bot. p. 24. Arc. o. c. p. $53 \%$.

Origanum macrostachyum Guss. l. c.

Figure. - Lam. ill. t.511. f. 1. Reich. ic. f. germ. 18. 1.61, 62. f. 1, 2. Moggr. contr. A. Ment. t. 62.

Stazione, Abitazione e Fioritura. - Comune assai per tutta l'Italia nei luoghi selvalici, principalmente di collina e di montagna. Nell' Alta Italia s'inoltra nella Valtellina (Nass. prodr.), sale in Valca. monica sino a 1320 metri fra i larici (Parl.!), e nel Tirolo alto si spinge fino in P'usteria (Hausm. $l$. .); in T'oscana non superano di molto i 1000 metri le maggiori altezze a cui si trovi; nella liassa Italia $\dot{e}$ stato trovato sulla Maiella (Pasquale!), ma non si sa a quale altezza. Pare che si faccia piủ raro in alcuni luoghi, cosi in Sardegna Moris non l'indica che di Oliena, in Corsica Marsilly l'indica soltanto fra Bastia e S. Fioreuzo, e manca affatto nelle isole dell'Arcipelago toscano. Le forme molto pelose o con brattee piu piccole sono piủ spe- 
ciali al mezzogiorno d'Italia. Fiorisce da giugno ad agosto, o da luglio a settembre, secondo i luoghi.

Distribuzione geografica. - In tutta Europa meno l'artica, nell'Asia occidentale, settentrionale e centrale.

Descrizione. - Pianta perenne, alta 3 decimetri e più, verde e in parte rossiccia, pelosetta, fornita di un rizoma obliquo o orizzontale, che manda molte fibre radicali, delicate, scurette, e dei rami sotterranei corti, carnosetti, carnei con squamette opposte, e dei rami aerei parte sterili e parte fioriferi. Questi sono eretti, spesso un po'flessuosi, semplici o ramosi, con i rami eretti, e come questi quadrangolari, di un rosso-scuro, o verdognoli in alto, pelosi, con peli lunghetti, bianchi, quasi orizzontali o appena curvati in giù. Le foglie sono opposte, poco lontane, patenti o patentissime, ovate, otluse, tondeggianti o appena scavate in cuore alla base, con pochi e piccoli denti ottusetti nel margine, verdi di sopra ed ivi con leggieri solchi che corrispondono ai nervi di sotto, di un verde chiaro e quasi bianchiccio di sotto ed ivi con un nervo longitudinale da cui partono dei nervi laterali obliqui e curvi, tutti rilevati, che si dirigono verso il margine e l'apice e si uniscono tra loro ad arco, dopo avere mandati dei rametti che fanno una rete appena rilevata sul parenchima della foglia; sono pelosette in ambedue le pagine e mas. sime nella inferiore e fornite in ambedue di molte piccole fossarelle in ciascuna delle quali è una piccolissima glandula bianchiccia; hanno un picciỏlo il quale è da due a tre volte più corto della foglia, con un leggiero solco di sopra, convesso di sotto, peloso, con peli_simili a quelli del fusto. I fiori sono piuttosto piccoli, distribuiti in spighe ovoidee e avvicinate all' apice dei rami, formando insieme una pannocchia stretta tricotoma. I peduncoli sono eretti o eretto-patenti, pelosi, con i peli pinttosto corti, curvati ad arco in giù; i pedicelli sono cortissimi. Le brattee e le bratteole sono larghette, ovali, un po'strette alla base, acute, appena concave, con una leggiera carena nel dorso, di color violetto-rossiccio, o verdognole: le bratteole sono appena piủ lunghe del calice. Questo è quasi tubuloso, con dieci leggieri nervi longitudinali, con poche glandole tonde e bianchiccie poste in fila longitudinali tra i nervi, verdognolo e glabro in basso, di color violetto rossiccio in alto ed ivi puberulo, ha cinque denti lun. ghi poco più del terzo del tubo, eretti, quasi uguali tra loro, larghetti, triangolari, acuti, e di dentro ha nella gola un cerchio di peli bianchi che sono quasi uguali ai denti. La corolla è più del doppio più lunga del calice, di color violetto-roseo, puberula di fuori. II tubo 
è piủ lungo del calice, diritto, quasi insensibilmente un poco più largo in alto. Il lembo è poco più corto del tubo, diviso in due labbri, uno superiore, un poco più corto, quasi orbicolare, smarginatobilobo, diretto in su, l'altro inferiore patente, trilobo, con i lobi poco disuguali, l' intermedio avendo dei piccoli denti ottusi all' apice. Gli stami sono quattro, diritti, un po' divergenti in alto, appena didinami, poco piủ lunghi o quasi uguali al labbro snperiore al quale sono avvicinati, inseriti nell'apice del tubo della corolla. I filamenti sono quasi filiformi, del colore di questa, glabri. Le antere sono piccole, biloculari, con le logge un po' divergenti, con ił connettivo larghetto, di color rossiccio-scure, glabre. Il pistillo è poco piủ lungo o quasi uguale agli stami. L'ovario è piccolo, diviso in quattro lobi, ovali-tondi, verdognoli, glabri, lisci, lucenti e sostenuto da un disco piủ lungo di esso, gialliccio. Lo stilo è lungo, filiforme, del co. lore della corolla. Lo stimma è bifido, con le lacinie quasi uguali, divergenti, quasi acute, del color dello stilo. (Parl.ms., descr. della pianla di Grumone in Lombardia).

Osservazioni. - Specie variabilissima: 10 per il numero dei fiori nelle spighe, che percio essendo lunghe d'ordinario 1 centimetro all'incirca, talora raggiungono 3 centimetri di lunghezza (var. prismaticum Gaud.); $2^{\circ}$ per la grandezza della corolla, essendovi anche qui una forma micrantha, e per il suo colore, roseo o raramente bianco; $5^{\circ}$ soprattutto per le brattee, che sono generalmente il doppio o una volta e mezzo piủ lunghe del calice, ma talora lo su. perano appena (Origanum hirtum Link?, Benth.); che porporine nei lagghi solatii, o lavate di verde e di porporino, sono anche totalmente verdi in luoghi aduggiati (quando la pianta è stata scambiata coll' 0 . vi rens Hoffm. Link, che sembra specie distinta); che in quanto a forma, essendo ovali, ora sono piủ larghe e passano all'obovato, ura sono piủ strette e passano al lanciolato, onde le spighe più grosse o piủ sottili; che in quanto a indumento, spesso non ne hanno, talora sono sparse di poche glandole, più di sovente lıanno peli, o pochi o molti. Le suddette modificazioni si combinano fra di loro in piu maniere, da rendere difficile la distinzione di varietà ben separate. Ne sono nate quelle creazioni specifiche dette da Linneo 0. crelicum, (). heracleolicum, 0. smyrnaum, e confuse e moltiplicate da'suoi successori.

Usi. - In Toscana e altrove condiscono le acciughe con le braltee policrizzate. 


\section{SATUREIA.}

Calamintha (part.), Clinopodium (part.), Satureia Tourn. inst.p. 193, 194, 197. $t .92$.

Satureia, Clinopodium, Acinos, Calamintha Monch meth.pl.p. 385, $405,407,408$.

Satureia, Clinopodium, Thymus (part.), Melissa (part.) Mirb. in ann. mus. 15. t. 16. f. 1-4, t. 17. f. 13, t. 20. f. 4, 6, 8, 11, 12.

Satureia, Micromeria et Melissa (part.) Benth. lab.p. 351, 369, 383. Acinos, Clinopodium, Calamintha, Satureia, Micromeria Gen. pl. fl. germ. 6.t. 11 bis, 12, 13, 17, 20.

Satureia et Clinopodium Scheele in flora 1843. 2. p. 568, 569.

Satureia, Micromeria, Calarnintha Benth. Hook. gen. pl. 2. p. 1187, $1188,1190$.

Satureia et Calamintha Ces. Pass. Gib. comp. fl. ital. p. $301,302$. t. 44.f. 3, 5 .

Calyx campanulatus vel tubulosus, valide 10-15-nervatus. Corolla manifeste bilabiata, labio superiore erecto, planiusculo. Sta. mina 4, conniventia, filamentis edentulis, antheris 2-locularibus, longitudinaliter dehiscentibus, a connectivo seiunctis. Stylus lobis inæqualibus. Cocci apice convexi leves.

Portamento. - E svariato. Sonovi nel genere erbe annue, erbe perenni, suffrutici, e frutici, sdraiati, ascendenti, o eretti, alti da pochi centimetri fin quasi a 1 metro, per lo piủ ramosi, variamente pelosi. Le foglie sono piccole o mezzane, di forma che varia fra l'arrotondita e la lineare strettissima, sono intere o dentate. I fiori, d'ordinario più o meno piccoli, raramente grandetti, sono di color carnicino o roseo, piủ di rado o porporino o bianco; sono disposti in dicasi, oppure in glomeruli, ora multiflori densi, ora di pochi fiori e radi, ora perfino ridotti a un fiore solo.

Osservazioni. - Da molto tempo e ripetutamente i migliori osservatori, quali Bentham, Koch, Spenner, De Visiani, invano cercando caratteri differenziali di qualche momento fra i moderni generi Satureia, Micromeria e Calamintha, avevano resa evidente la necessità che alfine si imporrebbe di riunirli in un genere solo; come già del resto aveva fatto Scheele sin dal 1843 (con la poco accorta sepa- 
razione perỏ del genere Clinopodium); nè si capisce perchè Bentham, biasimandolo di tale rimnione, l'abbia dichiarata "invita natura " (in Cand. prodr. 12. p. 209).

\section{Satureia hortensis.}

S. annua, pubescens, caule erecto, ramosissimo, ramis brachia. tis, foliis subsessilibus, patentissimis, lineari-lanceolatis, obtusiusculis, integerrimis, utrinque sparse foveolato-glandulosis, verticillastris 1-6-floris, folio brevioribus, bracteis linearibus, acutis, ciliatis, calyce brevioribus, calycibus campanulatis, glandulosis, quinquefidis, laciniis tubo calycis longioribus, lineari-acuminatis, longiuscule ciliatis, corolla calyce paulo longiore, labio superiore rotundato, inferiore trifido, lacinia intermedia longiore subobovata, lateralibus rotundatis. (Parl. ms.).

Satureia hortensis Linn. sp. pl. ed. 1. p. 568. Trev. prosp. . eug. p. 26. De Not. rep. 凡. lig. p. 319. Bert. fl. ilal.6. p. 53. Koch syn. fl. germ. ed. 2. p. 642. Com. fl. com. 4. p. 287. Zan. prosp. fl. ven. p. 26. Rota prosp. piant. Pav. p. 271. Hausm. fl. Tir. p. $67 \%$. Bicch. agg. fl. lucch. p. 16. Pir. fl. foroj. syll. p. 110. Car. prodr. fl. tose. p. 505. Tassi cenno bot. prov. Sien. p. 86. G. Bert. veg. mont. porr. p. 87. De Vis. Sacc. cat. piant. Ven. p. 134. Zersi prosp. piant. Brescia p. 16\%. Ces. Pass. Gib. comp. 月. ital. p. $30 \%$. Cald.! fl. fav. tent. p. 179. Arc. comp. fl. ital. p. 539.

Figura. - Reich. ic. fl. germ. 18. t. 71. f. 2.

Stazione, Abitazione e Fioritura. - Nelle ghiaie, nei luoghi sassosi e simili, nei campi, dell'Italia settentrionale e centrale, dove a di piu coltivata negli orti per uso di cucina, vi si rende facilmente spontanea: a Torino verso Battuelli (Colla herb. ped.), ad Alba (Bertero!), a Novi (De Not.), a Novara (Bir. /l. ac.), a Godiasco sopra Voghera (Nocca e Balb. R.), a Como (Com.), a Brescia (Zersi), nel Veronese verso Avesa, nelle valli di Caprino, Policella, di Roncả (Poll. fl.), Pantena (Bracht!), a Torri e Garda (Rigo!) e altrove, nel Tirolo in Valsugana al Col-rotondo sopra Primolano (Ambrosi!) e alle Tezze, e nel monte Surist alla Fratta (Hausm.), nel Mantovano (Barbieri!), nel monte Civillina di Vicenza (Poll.), nei colli Euganei ('Trev.), a Chioggia (Bert), a Venezia (Zan. eec.), nel Trivigiano (I) Vis. Sacc.), nel l'riuli in vari torrenti (Pir.), a Facnza (Cald.!), alla Porretta (G. Bert.), nel Nizzardo (All. ^.), a Dolcedo 
presso Porto Maurizio (Berti!), a Genova (De Not.), a Lucca a Monte San Quirico e altrove (Mezzetti!, Bicch.), in Garfagnana a S. Donnino (Ad. Targioni!), nel monte Pisano (Sav.), intorno Firenze all' Impruneta (Bucci!), a S. Casciano (Ricci!), sul monte Morello (Bert.), sul monte Rinaldi!, a Pontassieve (Parl.!), a Paterno! ecc., in Chianti a Brolio (Ricasoli!) e a San Giovanni!, ad Arezzo (Genmi!). Fiorisce da luglio a ottobre.

Distribuzione geografica. - In Spagna, nella Francia meridio. nale, in Italia, in Croazia, in Dalmazia, in Crimea, nell'Asia Minore; trovasi poi nell'Indie, al Capo di Buona Speranza e nell'America settentrionale, si suppone introdotta.

Descrizione. - La radice è fusiforme, ramosa, fibrillosa, flessuosa, di un bianco sudicio, glabra. Il fusto è alto da cinque a sei pollici a poco meno di un piede, eretto, ramosissimo con i rami op-. posti, brachiati, ramosi, ottusamente quadrangolari, di colore rossiccio scuro, con peli corti, fitti, curvi in basso e bianchi che li fanno biancheggiare. Le foglie sono opposte, quasi sessili, patentis. sime, lineari-lanceolate, ottusette all'apice, ristrette alla base, intere nei margini, con un nervo o vena longitudinale, poco rilevato, con un solco longitudinale di sopra, di color verde scuro, coperte di peli bianchi simili a quelli del fusto e dei rami, e con tante fosserelle tanto di sopra quanto di sotto in ciascuna delle quali si vede una gocciolina trasparente che viene dalla glandola sottoposta. I fiori sono per lo piủ 3 o 4, di raro meno o più sino a 6 , distribuiti in piccoli verticillastri, più corti delle foglie all' ascella di queste; i verticillastri inferiori sono distanti tra loro, i superiori sono più vicini e quasi facenti una spiga o racemo all' apice dei rami. Ciascun fiore ha un corto pedicello ed è accompagnato da una bratteola quasi lineare, più corta del calice verde, fornita di lunghi peli bianchi a guisa di ciglia e di qualche pelo più corto nella pagina inferiore. Il calice è campanulato, quinquefido, con le lacinie strette lineari-acuminate, quasi uguali tra loro, più lunghe del tubo del calice, poco più corte della corolla e fornite di lunghe ciglia bianche in tutto il loro margine; è rossiccio ed ha dieci nervi, cinque dei quali corrispondono alle lacinie e cinque meno manifesti sono intermedi, e diverse glandole donde escono delle gocciole trasparenti e giallicce : i nervi sono forniti di peli lunghetti e bianchi. La corolla è piccola, di color roseo-violetto, assai chiaro.' Il tubo è̀corto. Il labbro superiore è piủ piccolo dell'inferiore, diretto in avanti e in alto, quasi tondo, ottusissimo, leggermente crenulato nel margine, pubescente di fuori e 
un poco anche di dentro verso l'apice, il lobo inferiore è diretto in a vanti, trifido con le divisioni piane, le due laterali quasi tonde, l'in. tèrmedia più lunga e quasi obovata, tondeggiante e come troncata all' apice e smarginata, pubescente di fuori. La gola è pelosa, con i peli bianchicci, ivi presso la base di ciascun lobo del labbro infe. riore è una glandola gialla. Gli stami sono didinami, posti sotto il labbro superiore di cui i due più alti sono appena più corti. I filamenti sono quasi filiformi, bianchicci, glabri. Le antere di color vio. letto, biloculari, con le logge allungate, ottuse, divergenti e glabre. Il polline è bianchiccio. Il pistillo è poco più corto dei due stami più alti. L'ovario è circondato da un disco grosso, circolare e quasi quadrilobo, e giallo. I lohi dell' ovario sono tondo-ovali, verdognoli, papillosi verso alto. Lo stilo è glabro, filiforme. Gli stimmi sono divergenti, acuti, bianchicci. Le nucule sono ovali, nere, scabrosette in fondo del calice persistente. (Parl. ms., descr. della pianta.di Firenze).

\section{Satureia montana.}

S. suffruticosa, inferne glabra et superne puberula, caule erecto vel ascendente, ramoso, ramis erectis, foliis subsessilibus, patentibus, obverse vel subobovato-lanceolatis, acutis, submucronatis, integris, utrinque crebre foveolato-glandulusis, verticillastris 2-5-floris, folio brevioribus subæqualibusve, bracteis lineari-lanceolatis, acutis, margine basin versus breviter ciliatis, calyce brevioribus, calycibus subcampanulatis, quinquedentatis, dentibus tuho calycis brevioribus, lanceolato-linearibus, acutissimis, inargine basin versus et inter eos longe ciliato-pilosis, corolla calyce plus quam duplo longiore, labio superiore rotundato. (Parl. ms.).

Satureia montana Linn. sp. pl. ed. 1. p. 568. Trev. prosp. fl. eug. p. 26. De Not. rep. R. lig. p. 318. Bert. fl. ital. 6. p. 57. Rota prosp. piant. Pav. p. 27\%. Koch syn. fl. gerın. ed. 2. p 642. Com. fl. com. 4. p. 289. Zan. prosp. R. ven. p. 26. Pucc. syn. pl. luc. p. 300. Sim. R. alp, vers. p. 125. Hausm. 11. Tir. p. 678. Rola prosp. fl. prov. Berg. 1. 69. Ard. cat. pl. Ment. p. 2S. Pir. 1. for. syll. p. 110. Car. prodr. 1l. tose. p. 50i. Tassi 1. prov. sen. p. 50. Ard. /l. Alpes-mar. p 295 lle Vis. Sacc. cat piant. Ven. p. 139. Zersi prosp. piaut. prov. Biesc. p. 16\%. Ces. el. piant. Maiella p. 24. Terr.l sec. rel. prov Terra di Lav. p. 94. Archb. A. Alto-Serch. 
p. 60. Ces. Pass. Gib. comp. fl. ital. p. 301. Terr. ! quarla rel. prov. Terra di Lav. p. 112. Arc. comp. fl. ital. p. 539.

Satureia variegata Host fl. austr. Koch. l. c. Pir. l. c.

Satureia pygmæa Sieb.

Satureia subspicata De Vis.! stirp. dalm. spec. p. 11.

Figure. - Savi mat. med. tosc. t. 6. De Vis. o. c. t. 4. Fl. grac. t. 543. Reich. ic. fl. germ. 18. p. 72. f. 1, 2, 3.

Stazione, Abitazione e Fioritura. - Nei luoghi sassosi montuosi di tutta la Penisola, però distribuita disugualmente: sul Resegone sopra Lecco, sul Gordora in valle Intelvi (Com.), al lago d'Idro fra Anfo e Lavenone (Zersi); indi si fa comune dal lago di Garda in là, sui colli e sui monti bassi, cosi a Verona (Bracht! ecc.), per il monte Baldo (Barbieri! ecc.), e nel vicino Tirolo a Riva (Porta!), Ro. veredo, Stenico (Hausm.), Borgo di Valsugana (Ambrosi!) ecc., negli Euganei (Trev.), nel Vicentino (Poll. fl.), nel Bassanese (Bert. ecc.), a Feltre (Ambrosi !), a Venezia (Zan.), nel Friuli a Clausetto, Gemona, Venzone, Cividale ecc. (Pir.), a Duino (Poll.), a Gorizia (Pir.), presso Trieste (Moretti! ecc.), a Fiume (Smith!); comunissima in tutta la Liguria (De Not. ecc. ecc.), s' innalza nell'alpi di Tenda (Rostan!), varcandole anche fino a Valdieri (Parl.!), e più oltre varca l'Appennino per affacciarsi ad Alba (Bertero!) e nel Pavese (Rota); comunissima in tutte le Alpi Apuane, fin quasi alle cime più alte (Bert. ecc.) e nel monte Pisano!, risale val di Magra da Sarzana (Bert.) fin sopra Pontremoli!, e varcando l'Appennino si ritrova presso Berceto nel Parmense (Passerini!), e risale val di Serchio fino ai Bagni di Lucca (Parl.!) e a Tereglio (Giannini!); nel resto della Toscana trovasi a Volterra (Bert.), nella Montagnola senese, in val di Rosia, nel Vescovado, a Rapolano, a Montepulciano, a Pienza (Santi viagg.), a Campiglia (Bert.), a Roselle nel Grossetano (Santi), nel monte Argentaro (Aiuti!), a Pitigliano (Santi), nel monte Amiata in vari punti (Campani! ecc.); nelle Marche trovasi al Furlo (Bert.), al monte Catria (Parl.!), nell'Appennino di Fabriano (Narducci!), nel monte Gemmo, presso Brondoleto e Camerino (Ricci!), a Montefortino ecc., in Umbria nei monti di Norcia, nell'Appennino della Somma (Bert.), verso Rieti (Cherici!), e nei monti di Subiaco nel Lazio (Bert.), e nell'Abruzzo alle radici del Velino (Levier!), intorno Solmona (Groves!), nei monti di Caramanico (Ces.); si prolunga in Terra di Lavoro a Casalvieri, S. Biagio Picinisco (Terr.!), Cairo presso Montecassino, Piedimonte (Terr.), Cusano (Avellino!), Caserta, nel Napoletano a Castellammare (Ten.), Vietri (Pasquale!), Amalfi 
(Parl.!), Capri (Bert.); termina al Capo Palinuro (Ten.), e in Cala. bria ulteriore (Pasquale!). Manca nelle isole grandi e piccole, fuor. chè in quella ora rammentata di Capri. Fiorisce da luglio a ottobre.

Distribuzione geografica. - Si estende dal Portogallo e dalla Spagna per la Francia meridionale, l'Italia, la Dalmazia, la Turchia e la regione Danubiana alla Russia meridionale, e ritrovasi nell'Africa settentrionale.

Descrizione. - La radice è legnosa, ramosa, tortuosa, fibril. losa, scura. Il fusto è diritto o ascendente, alto circa un piede, ramoso fin dalla base con i rami eretti, ottusamente quadrangolari, bian. chicci o rossicci, e con peli corti, bianchi e curvati in giù che li rendono puberuli. Le foglie sono opposte, sessili, patenti, lanceolate a rovescio o quasi obovato-lanceolate, ristrette alla base, acute e quasi mucronate all'apice, intere nei margini ed ivi con qualche pic. colo ciglio, di color verde scuro con tanti piccoli punti bianchi quando si guardano con una lente e con molte fossarelle dal fondo delle quali esce una gocciolina gialliccia, in ambedue le faccie: la pagina inferiore ha un nervo longitudinale in cui come nella pa. gina stessa si vede qualche pelo. I verticillastri si compongono di due 0 tre a cinque fiori, all' ascella delle foglie superiori del fusto e dei rami delle quali sono più corti o quasi uguali: i fiori poi sono accompagnati da brattee verdi, lineari-lanceolate, più Junghe dei pedicelli i quali sono delicati e pubescenti. Il calice è quasi campanu. lato, verde, con dieci nervi, cinque dei quali più lunghi corrispondono e si prolungano nel mezzo dei cinque denti che sono lanceolatolineari, acutissimi, e forniti verso la base e in mezzo ad essi di lunghe ciglia bianche: nei nervi il calice è pubescente e in mezzo ad essi ha poche fossarelle glandolose come quelle delle foglie. La corolla i più del doppio piủ lunga del calice, di colore bianco-roseo, o violetto assai chiaro con alcune righe più cariche nel lahbro superiore e nella gola; è di fuori puberula ed ha le solite glandole, più abbon. danti nel labbro superiore. Il tubo è un poco curvo, poco più lungo del calice. Il labbro superiore è eretto, concavo, con il margine tondeggiante e quasi crenulato esmarginato all' apice. Il labbro inferiore è poco più lungo, diretto in avanti, trilido, con le lacinie quasi piane, tondeggianti.all'apice, la intermedia appena naggiore delle laterali dalle quali è distante: verso la base loro e nella gola si vedono alcuni lunghi peli del colore della corolla. Gli stami sono didinami, sotto il labbro superiore: $i$ due più lunghi sono uguali a questo, i due piủ corti un poco più corti di esso. I filannenti sono filiformi, bian- 
chicci, glabri, un poco curvi. Le antere sono violette, biloculari e per la divergenza delle due logge quasi in forma di freccia, glabre. Il polline è bianco. I lobi dell'ovario sono quasi tondi, verdognoli, lisci, papillosi all apice e stanno sopra un disco grosso, annulare, diviso quasi in quattro lobi, verdognolo e poco più alto dei lobi dell'ovario. Lo stilo è filiforme, bianco, diviso all'apice in due lacinie lesiniformi e divaricate: è più corto degli stami più corti. (Parl. ms., descr. della pianta del monte Pisano).

Osservazioni. - Questa specie varia molto per la grandezza e il colore dei fiori, per l'infiorescenza rada o fitta, per la statura, per la pelurie, per le foglie più larghe o più strette. Come già vien generalmente riconosciuto, e come io stesso ho potuto vedere sulla pianta viva, la Salureia variegata Host non differisce per nulla dalle forme più comuni della $S$. montana. Anche la pianta di Carniolia, Istria e Dalmazia (S. illyrica Host, S. subspicata De Vis., S. pygmcea Sieb.) varia allo stesso modo, e non può essere staccata a nessun titolo dalla specie.

\section{Satureia cuneifolia.}

S. suffruticosa, suberecta, foliis anguste lanceolatis subspathulatis, conduplicatis, integris, glomerulis paucifloris, breviter pedunculatis, folia floralia subæquantibus, haud secundis, bracteolis caly. cibus brevioribus, calycis tubo 10-nervato, basi æquali, fauce nuda, dentibus porrectis, inferioribus sublongioribus, corolla calyce 2 plo3 plo maiore.

Satureia cuneifolia Ten.! fl. nap. 1. prodr. p. 33. Bert. fl. ital. 6. p. 57. Pasq. Lic. viagg. bot. al Garg. p. 22. Ces. Pass. Gib. comp. fl. ital. p. 301. Arc. comp. fl. ital. p. 539.

Figure. - Ten. o. c. t. 155. f. 2. De Vis. fl.dalm. t. 18. f. 2. Reich. ic. fl. germ. 18. t. 71.f. 1.

Stazione, Abitazione e Fioritura. - Nelle colline aride marittime dell'Italia meridionale orientale, rara: nel Gargano presso Vico ai Cappuccini (Pasq. Lic.) e a Manfredonia (Ten.!) ne'sepolcreti di Siponto, a Monopoli, a Lecce (Ten.), al Capo di Leuca (Bert.), a Taranto (Ten.). Fiorisce da giugno ad agosto.

Distribuzione geografica. - Nella Spagna meridionale, nei qui indicati luoghi dell' Italia, nella parte occidentale della penisola Balcanica, in Asia Minore. 
Osservazione. - Questa specie somiglia molto alla Satureia montana, ne differisce per il minor numero dei fiori, non voltati da una stessa parte, più distanti fra loro di maniera che costituiscono una infiorescenza a foggia di spiga anzichè di grappolo o di pannocchia.

\section{Satureia Thymbra.}

S. suffruticosa, suberecta, foliis oblongo-lanceolatis subspathulatis, conduplicatis, integris, glomerulis densissirnis, sessilibus, folia floralia superantibus, bracteolis calyces subæquantibus, calycis tubo 10-nervato, basi æquali, fauce nuda, dentibus porrectis, subæqualibus, corolla calyce duplo maiore.

Satureia Thymbra Linn. sp. pl. ed. 1.p. 567. Bert. fl. ital. 6. p. 59. Mor.! fl. sard. 3. p. 281. Ces. Pass. Gib. comp. fl. ital. p. 30 \%. Arc. comp. fl. ital. p. 539.

Figura. - Fl. grcec. $t .541$.

Stazione, Abitazione e Fioritura. - In Sardegna, nei luoghi solatii aridi sul mare intorno a Cagliari, dove abbonda (Mor.), per esempio al castello di S. Michele (Gennari!). Fiorisce da aprile ad agosto. L'Allioni l'a veva indicata $(F l$.ped. 1. p. 24) a Nizza, ma nessun botanico moderno ce l'ha veduta.

Distribuzione geografica. - In Grecia e nell'Arcipelago, e nell'Asia occidentale.

\section{Saturein inliana.}

S. suffruticosa, suberecta, foliis infinis ovatis, cxteris lanceolato-linearibus, margine revolutis, integris, glomerulis densis, breviter pedunculatis, folia floralia subæquantibus, haud secundis, bracteolis calyces æquantibus vel eis paullo brevioribus, calycis tubo 13-nervato, basi aquali, fauce pilosa, dentibus porrectis vel comırventibus, subequalibus, corolla calyce paullo longiore.

Satureia juliana Linn. sp. pl. ed. 1. p. 567. Bert. R. ital. 6. p. 4.9. Pucc. syn. pl. luc. p. 300. Ces. Pass. Gib. comp. A. ilal. p. 801 . Arc. comp. R. ital. p. 5.40.

Micromeria juliana Benlh. Lab. p. 373. Zan. prosp. R. ren. 
p. 26. Car. prodr. fl. tosc. p. 506. Tassi fl. prov. sen. p. 50. De Vis. Sacc. cat. piant. Ven. p. 134. Archb. fl. Alto-Serch. p. 60. Pasq. f. ves. p. 80 .

Mlicromeria approximata Terr.! sec. rel. Terra di Lav. p. 94 (non Reich.).

Figure. - Fl. grac. t. 540. Reich. ic. fl. germ. 18. t. 79.f. 1.

$\beta$ hirsuta Benth. in Cand. prodr. 12. p. 213.

Satureia juliana b Guss. fl. sic. syn. 2. p. 87.

Stazione, Abitazione e Fioritura. - Nei luoghi aridi sassosi dei colli e delle parti basse dei monti, e sui vecchi muri. Rarissima nell'Alta Italia, non si trova che sui muri a Venezia (Kellner! ecc.), e (dicesi) nel Vicentino (Poll., De Vis. Sacc.) e nel Padovano (De Vis. Sacc.); è indicata a Nizza dall'Allioni, ma non da altri; nell'Italia centrale comparisce di sicuro in Toscana, dov'è abbastanza comune: cosi a Montignoso presso Massa (Viv., Bert.), nel monte di Quiesa (Bert.), nel monte Pisano dove abbonda, in val di Serchio tra Ponte a Moriano e Diecimo!, ai Bagni (Archb.) e a Tereglio (Giannini!), in val di Nievole a Monsummano!, nel Fiorentino (Reboul!), nei monti di Livorno, a Montecastelli di val di Cecina (Bert.), nel monte Calvi di Maremma, a Siena!, e nel Senese a Rapolano (Sommier!), la Montagnola (Santi viagg.), Rosia!, Castelnuovo (Santi), i Bagni di Vignone (Bert.), in val di Chiana a Sarteano (Santi), nel monte Argentaro!; trovasi poi ad Ascoli nelle Marche (Parl.!), a Terracina (Bert.), nel Napoletano a Vicalvi presso Sora (Terr.!), Arpino, Picinisco (Terr.), sul monte di Diecimari (Ten.), sul Vesuvio (Pasq.) e nell'isola di Capri (Ten.!); fa infine in Sicilia a Termini (Pasq.!), a Prizzi, al monte delle Rose, al monte di Cammarata (Guss.). La varietà è propria dell' Italia meridionale, dove fa in Calabria (Ten.!), e in Sicilia, essendovi anzi più comune del tipo: cosi a Palermo al Caputo, a monte Cuccio, a Santa Maria a Gesù, al Parco (Guss.), a Pizzuta, a Sagana (Parl.!), a S. Giuseppe, a Cofani, nel monte di Trapani, nei monti di Noto a Mililli, a Messina, a S. Fratello, nelle Madonie ecc. (Guss.). Fiorisce in giugno e luglio, e sin da maggio nei luoghi più caldi.

- Distribuzione geografica. - In Portogallo, nella Francia meridionale, in Italia, nella Penisola Balcanica, nell'Arcipelago, nell'Asia occidentale, nell'Affrica settentrionale.

Descrizione. - Pianta odorosa. Fusti legnosi alla base, ramosi, con i rami eretti o ascendenti, delicati, quadrangolari, rossicci in basso e con piccoli peli corti, bianchi e diretti in giù. Foglie 
opposte, patenti, piccole, lineari, ottuse, convesse e puberule di sopra, con i margini rovesciati, di un verde più chiaro di sotto. Fiori piccoli assai, in piccoli glomeruli fitti ed eretti, accompagnati di brattee simili alle foglie superiori, e di bratteole linearisubulate poco piủ corte del calice. Pedunculo comune molto corto. Calice tubuloso, stretto, quasi diritto o un po'curvo, verde, con nervi longitudinali, grossetti, puberulo, con cinque denti eretti, avvicinati al tubo della corolla, lesiniformi, puberuli e cigliati. Corolla poco più lunga del calice, di color roseo. Tubo stretto, appena curvo, bianchiccio in basso, pubescente in alto e ivi roseo. Lembo puberulo di fuori, diviso in due labbri corti, uno superiore diritto, smarginato, ed uno inferiore un poco piủ lungo ma più largo diretto in avanti e un po' in giù, trilobo, con i lobi quasi tondi, l'intermedio un po' più grande dei due laterali. Stami 4, didinami, due piủ lunghi poco più corti del labbro superiore e due più corti che giun. gono quasi alla gola. Filamenti filiformi, glabri, bianco-rosei. Antere piccole, di un violetto-scuro, glabre. Pistillo quasi uguale agli stami più lunghi. Lo stilo è filiforme, roseo, glabro, bifido all' apice con le lacinie quasi uguali, assottigliate. (Parl. ms., descr. della pianta di monte Calvi in Toscana).

Osservazioni. - Come bene osservò Gussone, la gola del calice è provvista di peli in questa specie come nelle affini. Il suo migliorc carattere distintivo è nella lunghezza delle brattee. I fiori sono piccoli, cssendo il calice lungo 3 millimetri.

\section{b. Snturein cristata.}

S. suffruticosa, suberecta, foliis infimis ovatis, cxteris lanceolato-linearibus, margine revolutis, integris, glomerulis densiusculis, breviter pedunculatis, folia floralia subæquantibus, haud secundis, bractcolis calycilous 2-3-plo brevioribus, calycis tubo 15 -nervato, basi æequali, fauce pilosa, dentibus porrectis vel connirentibus, subaqualibus, corolla calyce paullo longiore.

Thymus cristatus Ilampe.

Micromeria cristata Gris. spic. 1l. rum. ‥p. 122. Benth. in Cand. prodr. 12. p. 218.

Abitazione e Fioritura. - Trovata da Parlatore nel luglio 1856 vicino ad Acquasanta nel Piceno. 
Distribuzione geografica. - Nell'Asia Minore, in Macedonia, in Serbia, nell'indicato luogo d'Italia.

Osservazione. - Ha tutto l'aspetto della Salureia iuliana, dalla quale differisce principalmente per le brattee.

\section{y. Satureia tenuifolia.}

S. suffruticosa, suberecta, foliis infimis ovato-lanceolatis, cæteris lanceolato-linearibus, margine revolutis, integris, glomerulis densiusculis vel densis, breviter pedunculatis, a foliis floralibus plus minusve superatis, haud secundis, bracteolis calycibus plus minusve brevioribus, calycis tubo 13-nervato, basi æquali, fauce pilosa, dentibus porrectis vel parum divergentibus, subæqualibus, corolla calyce paullo longiore.

Satureia tenuifolia Ten.! fl. nap. 1. prodr. p. 33. Sang. cent. prodr. fl. rom. add. p. 85. Ten. syll. fl. neap. p. 278. Guss. fl. sic. syn. 2. p. 88 .

Satureia græca Bert. fl. ilat. 6.p. 45 (ex parte). Ces. Pass. Gib. comp. fl. ital. p. 302 (var. $\gamma$ ). Arc. comp. 月. ital. p. 540 (var. $\gamma)$.

Micromeria graeca De Not. rep. fl. lig. p. 219 (ex parte). Grech Del. f. mel. p. 26 (ex parte). Mor. fl. sard.3. p. 284 (var. ex parte). Car. prodr. fl. tosc. p. 506 (var.). Tassi fl. prov. sen. p. 50 (ex parte). Archb. fl. Alto-Serch. p. 60?

Micromeria tenuifolia Benth. in Cand. prodr. 12. p. 215 (non Reich. fl. germ. exc., quee Satureia parviflora De Vis.). Bicch. agg. fl. lucch. p. 16. Pasq. fl. ves. p. 80. Ces. el. piant. Maiella p. 24. Terr.! sec. rel. prov. Terra di Lav. p. 94.

Figura. - Ten. o. c. t. 151.f. 4.

Stazione, Abitazione e Fioritura. - Nei luoghi asciutti di tutta l'Italia: nei monti Vicentini (Barbieri!); nella Riviera di ponente ai Piani (Ricca!), al Capo di Noli (Canepa!), a Pegli (Carrega!); in Toscana a Lucca (Bicch.), a Pisa (Parl.!), a Montecatini di val di Nievole, intorno a Firenze dov'è comunissima!, a Figline (Ricasoli!), presso Anghiari (Cherici!), a Rapolano (Sommier!), in val di Cecina alle Saline (Amidei!), nell' Elba a Bagnaia! e presso Longone (Ad. Targioni!); in Umbria verso Perugia (Parl.!); nelle Marche al porto di Recanati, e di S. Elpidio (Narducci!), ad Amandola (Marzialetti!), ad Ascoli (Orsini!); in Abruzzo a Caramanico (Ces.), e sulla Maiella in luoghi subalpini della valle dell' Orfenta (Levier!); intorno a Roma 
a Tivoli (Webb!), a Frascati (Parl.!), ecc.; a Terracina (Fiorini!); in Terra di Lavoro a Roccasecca (Terr.!), a Cairo (Terr.); presso Napoli a Pompei (Ten.!), Pozzuoli, Baia, Niseno e altrove (Ten.), e nelle parti finitime al Taburno (Guss.!), a Cusano, a Sant'Angelo ecc. (Pasquale!); in Basilicata a Muro (Terr.!); in Sardegna(Mor.); in Sicilia a Termini, a Cefalu, a Castelbuono (Parl.!), a Finale, a Mistretta, a S. Fratello, a Bronte, fra Tusa e Caronia, nei monti di Canmmarata, e delle Prose (Guss.); in llalta (Grech). Probabilmente in molti altri luoghi dell' Italia centrale e meridionale, essendo stata confusa ora con la $S$. iuliana ora con la $S$. grceca. Fiorisce in giugno e luglio.

Distribuzione geografica. - Pianta italiana.

Descrizione. - Suffrutice minutamente peloso, ramosissimo, dai rami eretti o ascendenti, alti $1 / 5-1 / 2$ metro, semplici o ramosi, fioriferi per lungo tratto, oscuramente quadrangolari, coi peli rivolti in giủ, legnosi e nudi in basso. Foglie brevissimamente picciuolate, erctte, le inferiori ovate-lanciolate, l'altre lineari-lanciolate, acutette, interissime, rivoltate nel margine, solcate di sopra, con una nerva. tura sporgente di sotto, lunghe circa 1 centimetro, coi peli rivolti in su. Dicasi (non rivoltati da un medesimo lato) più corti delle foglic (piủ o meno), con 1-10 fiori, col pedunculo della lunghezza dei calici, o piủ lungo, o molte volte più corto. Fiori addensati, eretti, brevissimamente pedicellati, con numerose brattee subulate, erette, della lunghezza del calice 0 anche la metà più corte di esso. Calice tubuloso, appena curvato, con 13 nervature, coi peli rivolti in su, lungo 5 millimetri, coi denti uguali, eretti, non conniventi, subulati, la metà piủ corti del tubo, con la gola chiusa da peli. Corolla rosea, appena curvata, pubescente, all'infuori coi peli per in su, in dentro per in giủ, col tubo presso a poco uguale al calice, col lab. bro superiore corto ( $1 \frac{1}{2}-2$ millimetri $)$, sporgente, pressochè piano, smarginato-bilobo, coll' inferiore un po' più lungo, patulo, trifido, dai lobi piani, tondeggianti, presso a poco uguali, interi. Stami inseriti presso alla gola della corolla, gl'inferiori piủ lunghi, inseriti appena più in alto dei superiori (che sono rinchiusi nel tubo), c un poco più corti del labbro superiore, tutti ascendenti, ravicinati al. l'apice in coppie, glabri; filamenti filiformi, bianchi; antere poste orizzontalmente sul lato, articolate col filamento, a caselle bislunghe, porporine, divergentissime, libere, congiunte soltanto per l'apice al connettivo, e perció separate da tutta la sua larghezza, deiscenti longitudinalmente sul lato esterno a corolla chiusa, quando si fa l'im. 
pollinazione; polline bianco, pressochè globoso, largo 0,04 millimetri. Disco grosso, dell' altezza del gemmulario, uguale, non sporgente. Lobi del gemmulario equidistanti; gemmula ascendente, anatropa, con integumento scempio; stilo che raggiunge le antere degli stami più corti, glabro, filiforme, un po' ingrossato verso basso, strozzato nella base, coi lobi conniventi, lineari, ottusetti, compressi, presso a poco uguali. - Pianta fruttifera. Calice immutato. Cocchi bislunghi, ottusetti, carenati ottusamente nella faccia interna, lunghi 1 millimetro, falbi, lisci, con la cicatrice laterale; pericarpio igroscopico, sottile, crostoso. Embrione senza mandorla, con fusticino corto, con radicetta di faccia all'ilo, con embriofilli paralleli all'asse del fiore (v. v., descr. della pianta di Firenze, 1855).

Osservazione. - In questa sezione di genere composta di specie sommamente affini fra di loro, questa qui mi sembra distinta a sufficienza dalla seguente.

\section{Satureia graeca.}

S. suffruticosa, suberecta, foliis infimis ovatis, inferioribus ovalisuperioribus lineari-lanceolatis, margine subrevolutis, integris, glomerulis laxiusculis vel laxis, breviter vel longiuscule pedunculatis, folia floralia plerumque subæquantibus, secundis, bracteolis calycibus plus minusve brevioribus, calycis tubo 13-nervato, basi æquali, fauce pilosa, dentibus porrectis vel parum divergentibus, subæqualibus, corolla calyce paullo longiore.

Satureia græca Seb. Maur. fl. rom. prodr. p. 190. Ten.! syll. fl. neap. p. 278. Guss. fl. sic. syn. 2. p. 89 (excl. var.?). Bert. fl. ital. 6. p. 45 (ex parte). Ces. Pass. Gib. comp. fl. ital. p. 302 (excl. var.). Arc. comp. fl. ital. p. 540 (excl. var.).

Micromeria græca Benth. lab. p. 373 (excl. var.). De Not. rep. 1. lig. p. 319 (ex parte). Koch syn. fl. germ. ed. 2. p. 643. Gren. Godr. fl. Fr. 2. p. 661. Grech Del. fl. mel. p. 26 (ex parte). Guss. enum. pl. Inarim. p. 258. Mor. fl. sard. 3. p. 283 (excl. var.). Car. prodr. f. tosc. p. 506 (excl. var.). Fl. Hontecr. p. 28? Tassi f. prov. sen. p. 50 (ex parte). Pasq. fl. ves. p. 79. Terr. fl. Vult. syn.p. 136. Mars. cat. pl. Corse p. 112. Genn. fl. Capr. p. 112. Ces. el. piant. Maiell. p. 24. Loj. is. col. p. 122.

Figure. - Fl. gr(ec. t. 542. Ten. fl. nap. t. 151.f. 2. Reich. ic. fl. germ. 18. t. 79. f. 2. 
$\beta$ hirsuto, pilis patulis præsertim in caule et in glomerulis plus minus obducta.

Satureia canescens Guss. pl. rar. p. 228. Ten. syll. R. neap. p. 279. Guss. 1. sic. syn. 2. p. 91. Cies. Pass. Gib. comp. R. ilal. p. 302. Groves! contr. fl. Terra d' Otr. p. 65. Arc. comp. fl. ilal. p. 540.

Micromeria canescens Benth. lab. p. 376.

Figura. - Guss. pl. rar. t. 42.

Stazione, Abitazione e Fioritura. - Nei poggi aridi sassosi, specialmente marittimi, sulle rupi, sui muri ecc., di tutta Italia, ma rarissima nella settentrionale, e sempre più frequente andando dalla centrale alla meridionale: a Gandria nel Cantone Ticino (Koch); in Liguria a Porto Maurizio, Portofino (Piccone!); nella Maremma toscana nel monte Calvi (Aiuti!), sotto Campiglia, presso Follonica (P. Savi!), a Porto S. Stefano (Parl.!), ai Passionisti e alle Cannelle (Aiuti!), sul monte Argentario, e nelle isole toscane all'Elba (P. Savi!), e Montecristo (?) (Taylor); nelle Marche ad Ancona (Paolucci! ece.), Macerata (Piccinini!), Ascoli!; in Abruzzo a Chieti, Caramanico (Ces.); a Roma, dove pare che sia comune (Rolli!, Seb. Mauri ecc.); a Terracina (Fiorini!) ; a Napoli, comune (Ten.!, Pasq.), $\mathrm{e}$ in Ischia (Bolle!); nel Vulture (Terr.); nel Gargano (Ten.); presso Taranto (Aiuti!); in Calabria a Terranuova, Cosenza (Aiuti!), Pizzo, Palmi (Biondil); in Corsica a Bastia (Requien! ecc.), S. Fiorenzo, Portovecchio (Mars.), Bonifacio (Requien! ecc.); in Sardegna a Ca. gliari, comune (Ascherson!), in Naddalena e S. Stefano (Genn.); in Sicilia qua e là, principalmente nella parte meridionale (Guss.): a Palermo (Parl.!), Castelbuono (Minà!), Messina (Seguenza!), Avola (Bianca!), Noto (Inzenga!), Girgenti (Aiuti!), isole Eolie (Loj.), di Ustica (Calcara!), Lgadi (Guss.), di I'antellaria (Calcara!); iı Malta (Grech). La var. in Terra d'Otranto a Taranto, Punta di Cutaro, Gallipoli, Torricella (Guss.), Otranto (Groves!), in Calabria ulteriore (Pasq.!), e nella Sicilia merilionale a Siracusa (Cassia!), Avola (Bianca!), Scicli, Modica, Ragusa (Guss.), Comiso, Vittoria (Aiuti!), Terranova (Guss.). Fiorisce da aprile e maggio per tutta l'estate.

Distribuzione geografica. - Specie Mediterranea, trovasi in Portogallo e Spigna, nella Francia meridionale, in Italia, in Turchia e Grecia, nell'Arcipelago, ncll'Asia occidentale, in Egitto.

Osservazione. - In Sicilia questa pianta va soggetta nei fiori a una deformazione, opera senza duhbio di qualche insetto, per cui il calice si manifesta molto ingrandito, e coperto di folta pelurie. 


\section{Satureia thymoides.}

S. suffruticosa, suberecta, foliis infimis ovalibus, cæteris lanceolato-linearibus, margine revolutis, integris, glomerulis laxiusculis, breviter pedunculatis, folia floralia subæquantibus, subsecundis, bracteolis calycibus multo brevioribus, calycis tubo 13 -nervato, basi æquali, fauce vix pilosa, dentibus 2 sublongioribus strictis, 3 paullo divergentibus, corolla calyce vix longiore.

Micromeria thymoides De Not. rep. fl. lig. p. 319. Benth. in Cand. prodr. 12. p. 219.

Satureia thymoides Nym. syll. fl. eur. p. 102. Ces. Pass. Gib. comp. fl. ital. p. 302. Arc. comp. fl. ital. p. 540.

Stazione, Abitazione e Fioritura. - Nella Riviera di ponente nelle ghiaie de'torrenti a Oneglia (Berti! ecc.) e a Piani (Gentili!). Fiorisce nell' estate.

Distribuzione geografica.- Pianta propria dell'indicato luogo.

Descrizione. - Specie ben distinta, anche per l'aspetto. Forma fitti cespugli, alti $1-2$ decimetri. I fiori son' piccolini, il calice non avendo che 3 millimetri. Sono d'ordinario numerosi nei glomeruli; i quali sono talora tutti distanti gli uni dagli altri, quando la pianta rammenta una Satureia grceca in miniatura; ma più spesso i superiori sono ravvicinati fra di loro, da formare una fitta infiorescenza cilindrica (v. s.).

\section{Satureia consentinn.}

S. suffruticosa, diffusa adscendens, foliis infimis ovatis, inferioribus ovali- superioribus lineari-lanceolatis, margine revolutis, integris, glomerulis laxis, breviter vel longiuscule pedunculatis, folia floralia subæquantibus, vel ab illis sæpe longe superatis, secundis, bracteolis calycibus plus minusve brevioribus, calycis tubo 13-nervato, basi æquali, fauce pilosa, dentibus 2 sublongioribus porrectis, 5 paullo divergentibus, corolla calyce paullo vel etiam duplo longiore.

Satureia consentina Ten.! ad fl. neap. prodr. app. quinta $p .17$. - Fl. nap. 3. syll. p. 81. Guss. 月. sic. prodr. suppl. Ten. 月. nap. 5. p. 4. Guiss. fl. sic. syn. 2. p. 88 .

Satureia sicula Guss.? fl. sic. syn. 2. p. 89 . 
Satureia græca Bert. n. ital. 6. p. 45 (ex parte). Ces. Pass. Gib. comp. fl. ital. p. 302 (var. $\beta, \delta$ ). Arc. comp. f. ital. p. 540 (var. $\beta, \delta)$.

Micromeria græca Mor. 凡. sard. 3. p. 284 (var. ex parte).

Figura. - Ten. 月. nap. t. 151.f. 1.

$\beta$ hirsutu, pilis patulis obducta.

Satureia sicula b Guss.? fl. sic. syn. 2. p. 89.

Stazione, Abitazione e Fioritura. - Nelle colline aride sassose dell'Italia piủ meridionale: a Muro in Basilicata (Terracciano!); in Calabria dove pare che si faccia comune: a Porta Piana e Donnici presso Cosenza (Ten.), Mezzapraia (Ricca!) e Santa Venere presso Pizzo, al Telegrafo presso Palmi, a Bagnara (Arc.!), Reggio!, Roccella (Huter Porta Rigo!), in Aspromonte (Avellino!), ecc.; in Sicilia, pure comune a bastanza lungo la costa orientale e settentrio. nale: a Catania!, Acireale (Guss.), Giarre, sull'Etna (Parl.!), a Taormina, Capo S. Alessio, Scaletta (Guss.), Messina!, Milazzo (Borzi !), Patti (Profeta! ecc.), Castelbuono (Parl.!), intorno a Palermo in molti luoghi (Parl.!, Todaro! ece.), nelle isole Eolie (Guss.); in Sardegna (Mor.). La var. a Palermo a San Martino (Parl.!) e altrove, a Sagana (Parl.!), ecc. Fiorisce da aprile e maggio a attobre.

Distribuzione geografica. - Specie italiana.

Descrizione. - Pianta perenne, formante graziosi cespuglietti, verde 0 di un verde bianchiccio, pelosetta o pelosa, alta da uno a tre o quattro decimetri. II fusto è delicato, decumbente, ascendente o quasi diritto, oscuramente quadrangolare o quasi cilindrico, di colore rossiccio scuro in hasso, verdognolo in alto, pubescente o peloso con i peli corti, curvati ad arco in giii, ramoso, con i rami ascendenti 0 eretti. Le foglie sono patentissime, opposte, le inferiori e quelle dei rami sterili corti sono piccole, ovate, acute, intere e scabrosette nei margini, verdi di sopra ed ivi con un solco longitudinale superficiale, di un verde più pallido e spesso rossiccie di sotto ed ivi con un nervo delicato longitudinale da cui partono dei nervetti laterali, anche dclicati e curvi che si dirigono verso il margine e l'apice, pelosette nei nervi, con i peletti corti e curvati verso l'apice, con alcune glatidolette tonde e trasparenti in questa pagina inferiore; fornite di un picciölo corto e delicato, solcato di sopra, convesso di sotto e pelosetto o peloso come i nervi. Le foglie superiori soun lanceolato-lineari o lineari, larghe da 5 millimetri a un millimetro e mezzo, ottusette o ottuse, con un solco longitudinale di sopra e un nervo longitudinale di sotto da eni partono pochi nerretti laterali molto obliqui che si dirigono verso l'apice, con il margine $u$ po' ro- 
vesciato di sotto, del resto simili alle foglie inferiori. I fiori sono da 3 a 6 e talvolta sino a 9 distribuiti in piccoli corimbi laterali, posti all' ascella delle foglie, delle quali sono quasi uguali o poco piu lunghi. Il peduncolo è corto, delicato, quasi cilindrico, patente, pubescente o peloso con i soliti peli; i pedicelli sono più corti del calice, quasi capillari, diretti o patenti, simili del resto al peduncolo. Le bratteole sono opposte, lineari-setacee, eretto-patenti, più corte del calice o poco più lunghe dei pedicelli. Il calice è stretto, tubuloso, un po' curvo, verdognolo o in parte rossiccio, con molti nervi rilevati, $\mathrm{i}$ quali sono ispidetti per peli corti, diritti o curvati in su: le sue lacinie sono un po'disuguali, più corte del tubo e la inferiore un po' più lınga delle altre ma sempre più corta o quasi uguale al tubo, tutte eretto-patenti, lesiniformi, cigliate, con la gola pelosetta o pelosa per peli diritti e bianchi come quelli delle ciglia delle lacinie. La corolla è piủ del doppio più lunga del calice, di color lilla, pubescente o pelosetta di fuori per peli corti e diritti. Il tubo è lungo quasi il doppio del calice, un po'curvo, gradatamente un po' piủ lungo verso alto; il lembo è diviso in due labbri : il labbro superiore è eretto con l'apice rivolto un po' in dietro, quasi ovale, tondeggiante all'apice e ivi smarginato, un po' concavo davanti, un po' convesso di dietro, il labbro inferiore è poco più lungo e più largo, patente, trilobo, con il lobo medio un po'più lungo e più largo dei laterali, quasi obovato, smarginato all'apice con i lobetti tondeggianti, e i lobi laterali quasi quadrati, con gli angoli attusi. La gola è puberula dalla parte del labbro inferiore. Gli stami sono quattro, didinami, due quasi uguali al labbro superiore e due poco più alti della gola. I filamenti sono filiformi, quasi diritti, bianchicci, glabri. Le antere sono piccole, cor le logge le quali sono distanti per un connettivo lungo, e grossetto, bianco, e inserito quasi nel mezzo sul filamento, stando in posizione verticale a questo; sono di colore violetto-rossiccio scuro, glabre e si aprono per una fessura longitudinale. Il polline è gialliccio. Il pistillo è poco piủ lungo degli stami superiori. L' ovario è molto piccolo, con i quattro lobi quasti distinti e ovali, ottusi, bianchicci e glabri, inseriti sopra un disco grosso, carnoso, verdognolo. Lo stilo è lungo, diritto, filiforme, bianchiccio e glabro. Lo stimma è bifido, con le lacinie lesiniformi, un po' disuguali, l'inferiore un po' più lunga e curvata un po'in giù e la superiore diritta e diretta in su, ambedue di color lilla. (Parl. ms., descr. di pianta coltivata alquanto diversa dalla spontanea).

Osservazioni. - In mancanza di saggi autentici della Satureia 
sicula Guss., non posso dire che cosa sia precisamente; perỏ lo stesso Gussone mostra di avere esitato a ritenerla distinta dalla $S$. consentina.

Per quanto questa specie sia molto vicina ad alcune forme della S. greeca, in generale però se ne distingue bene.

\section{Satureia fasciculata.}

S. fruticosa, cæspitoso-decumbens, viridescens, foliis lanceolato-linearibus, margine revolutis, integris, floribus subsolitariis, breviter vel longiuscule pedicellatis, folia floralia superantibus, haud secundis, bracteolis calyces vix attingentibus, calycis tubo 15-nervato, basi equali, fauce pilosa, dentibus 2 sublongioribus porrectis, 3 paullo divergentibus, corolla calyce paullo longiore.

Satureia fasciculata Raf. préc. des déc. Bert. n. ital. 6. p. 48. Ces. Pass. Gib. comp. fl. ital. p. 302. Groves contr. fl. Terra d'Otr. p. 65. Arc. comp. fl. ital. p. 540.

Satureia approximata Biv. stirp. in Sic. man. 4. p. 13.

Micromeria approximata Reich.? fl. germ. exc. p. 859. Benth. lab. p. 375.

Figura. - Salureia saxatilis compactis foliis Bocc. mus. di piant. rare $t .119$.

Stazione, Abitazione e Fioritura. - Nei colli aridi sassosi dell'Italia più meridionale: in Sicilia intorno a Palermo in più luoghi (Parl.! ecc.), nell' isola Alicuri, a Trapani, nelle isole Egadi, a Siculiana, Palma, Licata, in Pantellaria e Lampedusa (Guss. $\mu$. sic. syn.); in Terra d'Otranto a Grottaglie (Ten. syll.) e Otranto (Groves); nel Napoletano ad Amalfi (Parl.!), Sorrento e in Capri (Pasquale!). Fiorisce in autunno, da settembre in poi, e a quanto pare per tutto l'inverno sino a marzo.

Distribuxione geografica. - Si troverebbe a di piủ in Albania secondo Reichenbach, ma dalle sue poche parole di descrizione risul. terebbe che la sua é pianta diversa dalla nostra.

Descrizione. - Radice ramosa, legrnosa, torta, scuretta. Fusto legnoso alla base, ramoso in questa, con i rami decumbenti ramulosi formanti dei cespuglietti, con la scorza bianchiccia e coperta di peli bianchi, corti, quasi orizzontali, la quuale in basso si stacca per cui ivi i rami sono denudati, glabri e di color di legno secco. Le foglie sono avvicinate tra loro per il poco srolgimento dei rametti, per 
cui pare che sieno molte in fascetti, un po' convesse e assai vicine tra loro: sono piccole, parte erette, parte patenti, strette, lineari-lanceolate, ottusette, di un verde chiaro, e quasi biancheggianti di sopra, ivi irsute per peli corti, bianchi e patenti, di un verde più chiaro di sotto con i margini roresciati in giù e ivi con un nervo longitudinale sporgente, meno irsute che di sopra. I fiori sono per lo più solitarii all' apice di un peduncolo delicato, eretto, piủ corto del calice, irsuto per i soliti peli, talvolta bifloro: vi sono pochi fiori in ciascun fascetto di foglie. I peduncoli sono accompagnati da due brattee, le quali sono alla loro base, erette, quasi uguali al peduncolo, strette, lineari, simili alle foglie. II calice è stretto, tubuloso, quasi cilindrico, verdognolo 0 in alto rossiccio, con 10 strie grosse longitudinali, un po'irsuto per i soliti peli, con 5 denti i quali suno stretti, lanceolati-acuminati, un po' patuli, forniti di ciglia. Il tubo è fornito nella gola di molti peli bianchi, lunghi. La corolla è roseovioletta, la metà circa più lunga del calice, peloso-irsuta di fuori, glabra di dentro. Il tuho è slargato in alto, spesso piủ lungo del calice. Il labbro superiore è eretto, smarginato, l'inferiore è più lungo e piủ largo, diretto in avanti, piano, quasi obovato. Gli stami sono 4 e didinami, due quasi uguali al labbro superiore, due poco più corti, tutti avvicinati all' apice. I filamenti filiformi e le antere sono del colore della corolla e glabri. Le antere sono 2-loculari con le logge divergenti. Il pistillo è lungo quasi quanto il tubo della corolla. Lo stilo cilindrico e lo stimma sono del colore della corolla. Lo stimma è bifido con le lacinie disuguali, strette, acute, divergenti. (Parl. ms., deser. della pianta di Palermo).

\section{Satureia cordinta.}

S. suffruticosa, diffusa, foliis infimis cordatis, inferioribus ora. tis, superioribus oblongo-linearibus, margine (incrassatis?) revolutis, integris, floribus subsolitariis, longiuscule pedicellatis, folia floralia subæquantibus..., bracteolis calyces hand attingentibus, calycis tul) $0 . .$. , basi æquali, fauce pilosa, dentibus 2 sublongioribus..., corolla calyce duplo (?) longiore.

Micromeria cordata Mor. diagn. stirp. Sard. nov. Fl. sard. 3. p. 285.

Satureia cordata Mor. in Bert. fl. ital. 10. p. 519.

Stazione, Abitazione e Fioritura. - Nelle rupi calcaree dei monti 
di Sardegna, cioè nei monti d'Oliena a circa 850 metri, e nel monte S. Giovanni Orgosolo (Mor.). Fiorisce in giugno e luglio.

Distribuzione geografica. - Specie propria della Sardegna.

Osservazione. - Non ho veduta la pianta. Noris la paragona alla Saturein parviflora De Vis. e alla S. microphylla Guss.: specie invero molto differenti l'una dall'altra.

\section{Satureia nervosa.}

S. suffruticosa, suberecta, foliis ovatis ovalibusve, aut superioribus oblongis, planis, integris, glomerulis laxiusculis, breviter pedunculatis, folia floralia subæquantiluus, subsecundis, bracteolis caly. cibus multo brevioribus, calycis tubo 13-nervato, basi æquali, fauce pilosa, dentibus paullo divergentibus, subæqualibus, corolla calyce paullo longiore.

Satureia nervosa Desf.? fl. atl. 2. p. 9 (non herb.!). Bert. /l. ilal. 6. p. 49. Ces. Pass. Gib. comp. n. ital. p. 302. Arc. comp. fl. ital. p. 540 .

Satureia plumosa Hampe in fl. 1842. beibl. p. 127.

Micromeria nervosa Benth. lab. p. 376.

Figura. - Desf. o. c. t. 121. f. 2.

Stazione, Abitazione e Fioritura. - Nei colli aridi, special. mente marittimi, dell'Italia più meridionale: in Terra d'Otranto a Taranto, Punta di Cutaro, Gallipoli, Ugento, Lecce (Guss. pl. rar.), Otranto (Bert.), Grottaglie, e in Terra di Bari a Polignano (Ten. syll.); in Sicilia a Castelvetrano, Sciacca, Montallegro (Guss. syn.), Girgenti (Todaro!), Cannicatti, Palmi, Alicala (Guss.), Terranova (Somnier!), Vittoria, Comiso, Scoglitti (Aiuti!), Spaccaforno, da Noto a Siracusa (Guss.). Fiorisce in aprilc e maggio.

Distribuzione geografica. - Trovasi inoltre in Grecia, nell'Arcipelago e in Soria e Palestina; l'hanno indicata ancora in Spagna e nell' Africa settentrionale, ma con dubbio.

Osservazione. - Ordinariamente $\mathrm{i}$ calici sono molto pelosi, ma talora quasi glabri.

\section{Satureia microphylla.}

S. suffruticosa, diffusa, foliis nvatis, planis, integris, glomerulis laxis, breviter pedunculatis, folia floralia subsuperantibus, sub. 
secundis, bracteolis calyces vix attingentibus, calycis tubo 13-nervato, basi æquali, fauce pilosa, dentibus paullo divergentibus, 2 sublongiorilus, corolla calyce paullo longiore.

Thymus micranthus Guss.! pl. rar. p. 243 (non Brot.). Ten. syll. fl. neap. p. 296.

Satureia microphylla Guss. fl. sic. prodr. 2. p. 120. Fl. sic. syn. 2. p. 91. Bert. fl. ital. 6. p. 51. Ces. Pass. Gib. comp. fl. ital. p. 302. Arc. comp. fl. ital. p. 540.

Thymus microphyllus Ten. fl. nap. 5. p. 27. Syll. f. neap. l.c.

Micromeria microphylla Benth. lab. p. 377. Grech Del. fl. mel. p. 26 .

Figura. - Clinopodium nigricantibus foliis siculum Bocc. mus. di piant. $t .89$.

Stazione, Abitazione e Fioritura. - Nelle colline aride della Penisola piủ meridionale ad Andria, Monopoli (Guss.), Martina (Ten.), Taranto (Guss.), Gallipoli (Guss.!), Lecce, Otranto (Guss.), di Sicilia ạd Avola, Noto, Nodica, Comiso, Vittoria (Guss.!), Terranova (Todaro!), Sciacca, Castelvetrano (Guss.), Favignana (Huet!), Maretimo (Guss.), e delle isole lontane di Malta (Calcara!) dov'è comune (Grech), di Linosa e di Lampedusa (Aiuti!). Fiorisce in aprile e maggio.

Distribuzione geografica. - Trovasi inoltre in Creta. pelurie.

Osservazione. - Essendo pelosa questa pianta, varia per la

\section{Satureia fliformis.}

S. suffruticusa, diffusa, foliis ovatis, planis, integris, floribus subsolitariis, longiuscule pedicellatis, folia floralia superantibus, secundis, bracteolis minimis, calycis tubo 13-nervato, basi æquali, fauce subpilosa, dentibus 2 sublongioribus porrectis, 3 paullo diver. gentibus, corolla calyce paullo longiore.

Thymus filiformis Ait. hort. kew. 2. p. 313.

Micromeria filiformis Benth. lab. p. 378. Gren. Godr. fl. Fr. 2. p. 662. Mars. cat. pl. Corse p. 112.

Satureia filiformis Nym. syll. fl. eur. p. 102. Ces. Pass. Gib. comp. fl. ital. p. 302. Arc. comp. fl. ital. p. 541.

Stazione, Abitazione e Fioritura. - In Corsica, sulle rupi del Golo, a Ponte di Golo (Mabille!). Fiorisce in maggio e giugno.

Distribuzione geografica. - Trovasi inoltre nelle isole Baleari. 


\section{Satureia marifolia.}

S. suffruticosa, suberecta, foliis subovatis, planis, subintegris, dichasiis breviter pedunculatis, folia floralia (sensim minora) superantibus, laxis vel densiusculis, haud secundis, bracteolis parvis, calycis tubo 15-nervato, basi æquali, fauce pilosa, dentibus (brevissimis) æqualibus, porrectis, corolla calyce subduplo longiore.

Melissa fruticosa Linn. sp. pl.ed. 1. p. 593.

Calamintha cretica Lam.

Micromeria marifolia Benth. lab. p. 382.

Figura. - Cav. ic. t. 576. Reich. ic. bot. t. 219.

Stazione, Abitazione e Fioritura. - Questa pianta rarissima fa sul Carso tra Duino e Trieste (Moretti!), ed è stata scoperta nel 1875 dai signori Porta e Rigo in Puglia sulle rupi calcaree presso Manfredonia. Fiorisce in giugno e luglio.

Distribuzione geografica. - In Spagna, nei sopraindicati luo. ghi d'Italia e in Albania, e nell' Asia occidentale: perỏ Boissier ( $F l$. or. 4. p. 574) distingue specificamente la pianta orientale.

\section{1\%. Satureia Iostii.}

Calamintha thymifolia Ilost $l$. austr. De Vis. Sacc. cat. piant. Ven. p. 134. Ces. Pass. Gib. comp. 月. ital. p. 303. Arc. comp. /l. ilal. p. 511 .

Stazione, Abitazione e Fioritura. - In luoghi rocciosi nel Veneto e presso 'Trieste, fiorita d'estate (Ces. Pass. (Gib.). gुine.

Distribuzione geografica. - Specie propria dell'indicata re-

Osservazione. - Non conosco altrimenti questa pianta.

18. Satureia glandulosa.

S. perennis, suberecta, foliis ovatis, planis, obscure crenatis, dichasiis pedunculatis, folia floralia (sension minora) superantilus, lavis, subsecundis, bracteolis parvis, calycis tubo (2-3 millim. Iongo) 15-nervato, basi aquali, fauce pilosissima pilis exsertis, denti- 
bus subæqualibus vel parum inæqualibus, suberectis, corolla calyce vix longiore.

Thymus glandulosus Req.! in ann. sc. nat. 5. p. 386. Bert. $f$. ital. 6. p. 222.

Thymus Nepeta var. Salis-Marsch, in fl. 1834. beibl. p. 17.

Calamintha glandulosa Benth. in Cand. prodr. 12. p. 227. Gren. Godr. fl. Fr. 2. p. 665. Mars. cat. pl. Corse p. 112.

Calamintha Nepeta var. Mor. fl. sard. 3. p. 288.

Calamintha parviflora var. Ces. Pass. Gib. comp. fl. ital. p. 303. Arc. comp. fl. ital. p. 542.

Stazione, Abitazione e Fioritura. - In Corsica lungo i corsi d'acqua, nel monte Niolo (Req.!), al fume di Calarima (Req.), sotto il ponte di Golo (Salis), a Calusiaca, tra Calusiaca e la valle di Stagno, a Corte lungo la Restonica (Req.!), a Calvi (Gren. Godr.); in Sardegna nei luoghi montuosi (Mor.). Fiorisce in agosto.

Distribuzione geografica. - Specie propria di quelle due isole.

Osservazione. - Per quanto questa specie si distacchi poco dalla seguente per caratteri tecnici, però se ne distingue bene per la scarsa pelurie, per le glandole abbondanti sulla pagina inferiore delle foglie e sui calici, e per i fiori più piccoli.

\section{Satureia Nepeta.}

S, perennis, suberecta, foliis ovatis, planis, crenatis, dichasiis pedunculatis, folia floralia (sensim minora) superantibus, laxis, subsecundis, bracteclis parvis, calycis tubo (3-4 millim. longo) 13 -nervato, basi æquali, fauce pilosissima pilis exsertis, dentibus parum inæqualibus, suberectis, corolla calyce vix vel etiam duplo longiore.

Melissa Nepeta Linn. sp. pl. ed. 1. p. 593. Mass. prodr. fl. valt. p. 112. Trev. prosp. fl. eug. p. 26. Zan. prosp. fl. ven. p. 26. De Not. rep. fl. lig. p. 321.

Calamintha parviflora Lam. fl.fr. 2.p. 396. Car. prodr. fl.tosc. p. 507. Fl. Montecr. p. 28. Tassi R. prov. sen. p. 50. G. Bert. not. monti porr. p. 47. Veg. mont. Porr. p. 87. Archb. fl. Alto-Serch. p. 60. Cies. Pass. Gib. comp. fl. ital. p. 303 (excl. $\beta$ ). Arc. comp. fl. ital. p. 541 (excl. $\beta)$.

Calamintha Nepeta Sav. R. pis. 2. p. 63. Com. fl. com. 4. p. 373. Pucc. syn. pl. luc. p. 312. Rola lnusp. piant. Pav. p. 27. 
Hansm. A. Tir. p. 680. Rota prosp. fl. Berg. p. 69. Grech Del. fl. mel.p 26. Guss, en. pl. Inarim. p. 259. Mor. fl. sard.3.p. 287. Ard. cat. pl. Ment.p. 28. Pir. fl. for. syll. p. 111. Ard. fl. Alpes-mar. p. 296. De Vis. Sace. cat. piant. Ven. p. 134. Zersi prosp. piant. Bresc. p. 165. Pasq. 1. ves. p. 80. Terr. fl. Vult. syn. p. 137. Mars. cat. pl. Corse p. 112. Loj. is. eol. p. 122. Cald.! fl. fav. tent. p. 179. Gib. Pir. 11. Mod. p. 128.

Melissa cretica Zer.? fl. mel. thes. p. 29 (non Linn.).

Satureia Nepeta Schcele in flora 1849. 2. p. $57 \%$.

Melissa Calamintha P. Sav.! fl. gorg. p. 274 (non Linn.).

Thymus Nepcta Bert. fl. ital. 6. p. 220. Sim. R. alp. vers. p. 131.

Calamintha nepetoides Ard. f. Alpes-mar. p. 296. Wars. l. c. Cald. l. c.

Calamintha officinalis var. Ces. Pass. Gib. l. c. Arc. l.c.

Figure. - Reich. ic. fl. germ. 18. t. 76. f. 2, t. 77. f. 2. Moggr. contr. fl. Ment. t. 62. f. C , D.

Stazione, Abitazione e Fioritura. - Comunissima sui muri, lungo le strade, e generalmente nci luoghi asciutti di tutta quanta I'Italia, in pianura e nei monti non altissimi, nessuna parte eccettuata. Nell'Alta Italia s'inoltra nel Cantone Ticino, nella Valtel. lina a Bormio a 1500 metri, in val Trompia sopra Brescia a 750 metri (Parl.!), in Tirolo a Trento e Castell Brughier in val di Non (Ilausm.); nell' Italia centrale s'inalza in montagna sino a circa IOU() metri. Fiorisce da gingno e luglio sino al autunno inoltrato.

Distribuzione geografica. - Nell' Europa media e soprattutto meridionale, nell'Asia occidentale, nell'Africa scttentrionale.

Descrizione. - Pianta perenne, alta 4 decimetri e piü, pulescente, odorosa, di un verde bianchiccio. La radice è llessuosa, fibrillosa, scura. Il fusto è giacente per terra alla base ed ivi radicante, poi eretto, llessuoso, tetragono, rossiccio in basso, rerdognolo in alto ma ovunque biancheggiante per peli fini, orizzontali, lunghetti e bianchi, ramoso, con i rami eretto-patenti, un po'curvati in su. Le foglie sono opposte, patenti, o dirette un po'in giii, pircole, quasi ovali-romboidali, ottuse, con poche e leggiere crenature nel margine, verdi di un verde hianchiccio di sopra ed ivi con solchi che corrispondono ai nervi di sotto, di un verde più chiaro e quasi hianchiccio nella pagina inferiore, ed ivi con un nerso longitudinale alquanto rilevato, dal quale partono dei nerretii, anche puco rilevati che si dirigono oblipuamente e un po'curvi verso il 
margine e l'apice dove si uniscono ad arco tra loro e mandano dei rami alle smerlature del margine, sono pelose in ambedue le facce e nel margine con i peli corti un po' curvi e quasi avvicinati ad esse, e fornite di un picciòlo corto, piano di sopra, convesso di sotto, di colore più chiaro e quasi bianchiccio, pubescente-peloso. I fiori sono piccoli, in piccole cime peduncolate all'ascella delle foglie della parte superiore del fusto e dei rami, dirette da un lato, più lunghe delle foglie fiorali corrispondenti. Il peduncolo è corto, quasi patente, duretto, i pedicelli diritti, più corti del calice, tutti pubescenti, verdognolo-bianchicci o rossicci. Le bratteole sono avvicinate ai pedicelli, quasi uguali o più corte di questi, lanceolato-lineari, acute, verdognole, pubescenti. Il calice è verdognolo, puberulo, con molti nervi o strie assai rilevate longitudinali, e con poche glandolette gialliccie nei solchi, ha il tubo cilindrico, diritto, un po' più gonfio in basso nella maturità del frutto, fornito nella gola di molii peli orizzontali, bianchi che chiudono quasi questa, e il lembo con cinque denti, tre superiori, più corti, ovato-triangolari, acuti, diretti in su, divergenti l'uno dall' altro e i due inferiori più lunghi, diretti in avanti, un po' curvi o avvicinati al tubo della corolla, lesiniformi, tutti con rade e corte ciglia. La corolla è piccola, il doppio piủ lunga del calice, puberula di fuori, di color violetto assai chiaro quasi bianco, con macchioline disuguali più cariche nel labbro inferiore verso la gola. Il tubo è quasi diritto, gradatamente piủ largo da basso in alto, il lembo è diviso in due labbri, uno superiore piủ corto, diretto in avanti e in su, quasi orbicolare, smarginato, quasi piano con una leggiera carena longitudinale, l'altro inferiore diretto in avanti ed in giù, trilobo, con i lobi laterali più piccoli quasi orbicolari e l'intermedio più lungo e poco più largo dei laterali, leggermente concavo di sopra, troncato e appena smarginato: vi sono dei peli rari quasi distribuiti in due linee alla base di questo labbro verso la gola tra il lobo medio e i lobi laterali. Gli stami sono quattro, didinami, i due superiori quasi uguali o poco più corti del labbro superiore, i due inferiori poco più lunghi del tubo, tutti avvicinati a quel labbro e inseriti sopra della metà del tubo. I filamenti sono eretti, filiformi, glabri, del colore della corolla. Le antere piccole, con il connettivo largo e quasi quadrato, con le logge distanti, quasi ovali, oblique, di colore più carico del connettivo, glabre. Il pistillo è quasi uguale al labbro superiore. L' ovario è piccolo, diviso in quattro lobi, quasi tondi, verdognoli, lisci, sopra un disco quasi tondo, poco più alto e più largo dell' ovario, di colore ranciato. Lo 
stilo è lungo, gradatamente più stretto dalla base in su, del colore della corolla, glabro. Lo stimma è bifido, con le lacinie disuguali, l'anteriore più lunga e più larga, curvata in avanti e in giủ, la posteriore quasi acuta ed eretta. (Parl. ms., descr. della pianta di Grumone in Lombardia).

Osservazioni. - Non è punto esatto che, con' è stato detto, a differenza della specie seguente questa qui abbia i cocchi del frutto bislunghi: ambedue li hanno ovali tondeggianti.

Come tante altre Lamiacee, anche questa ha i fiori dimorfi, altri completi bisessuali con la corolla più grande, altri pseudo-femminili con la corolla piủ piccola e gli stami atrofici.

\section{Satureia Calamintha.}

S. perennis, suberecta, foliis ovatis, planis, serrato-crenatis, dichasiis pedunculatis, folia floralia (sensim minora) inferiora subxquantibus, laxis, subsecundis, bracteolis parvis, calycis tubo (4-5 millim. longo) 13-nervato, basi æquali, fauce pilosula pilis inclusis, dentibus valde inæqualibus, 2 inferioribus longioribus incurvatis, 3 superioribus recurvatis, corolla calyce paullo vel etiam subduplo longiore.

Melissa Calamintha Linn.sp.pl. ed. 1. p. 593. Trev. prosp. fl. eug. p. 26. De Not. rep. fl. lig. p. 321. Zan. prosp. A. ven. p. 26.

Calamintha montana Lam. fl. fr. 2. p. 396 (excl.var. ß). Car. prodr. fl. tosc. p. 507. Tassi fl. prov. sen. p. 50. Archb. fl. Alto-Serch. p. 60 .

Calamintha officinalis Monch meth. p. 409. Com. fl. com. 4. p. 371. Pucc. syn. pl. luc. p. 312. Rota prosp. piant. Pav. p. 271. Hausm. 凡. Tir. p. 679. Rota prosp. 月. Berg. p. 69. Guss. enum. pl. Inarim. p. 259. Pir. fl. foroj. syll. p. i1f. Ard. /l. Alpes-mar. p. 296. De Vis. Sacc. cat. piant. Ven. p. 195 (excl. ß). Zersi prosp. piant. Bresc. p. 165. Pasq. /l. ves. p. 80. Terr. /l. Vull. syn. p. 136. Mars. cal. pl. Corse p. 112. Ces. Pass. Gib. comp. N. ital. p. ¿0: (excl. var.). Arc. comp. h. ital. p. 512 (excl. var.). Gib. Pir. A. Mod. p. 128.

Satureia Calamintha Scheele in fora 1813. 2. 1). 5 57.

Thymus Calamintha Berl. . ital. 6. p. 293.

Calamintha silvatica Bromf. in suppl. engl. bot. Ces. Pass. Gib. Frora Italiara. - Voz. VI. 
l. c. Gocc. nuovo contr. fl. Bol. p. 24. Cald. fl. fav. tent. p. 179. Arc.

l. c. Gib. Pir. l. c.

Figura. - Reich. ic. fl. germ. 18. t. 75. f. 2.

Stazione, Abitazione e Fioritura. - Nei luoghi boschivi di tutta l'Italia, comune anzichè no nel settentrione, meno comune nel mezzogiorno: in Piemonte a Susa (Re), a Torino (All.), nelle vallate Valdesi (Rostan!), ad Alba (Bertero!), nel Novarese (Bir.) ecc.; nel Cantone Ticino a Mendrisio, Lugano, Locarno (Com.); in Lombardia nel Comasco (Com.), a Milano (Rampoldi!), Pavia (Rota), Grumone (Parl.!), nel Bergamasco (Rota), nel Bresciano (Zersi), per esempio tra Bovegno e Collio a 850 metri (Parl.!), nel Mantovano (Barbieri!), ecc.; nel Tirolo. a Roveredo, Stenico, Tione, in val di Fiemme, e val Fassa, intorno a Bolzano sino a 1100 metri (Hausm.); nel Veneto a Verona (Poll.), nel Bassanese (Bert.), negli Euganei (Trev.), a Venezia (Zan.), nel Friuli (Pir.), ecc.; a Trieste (Bert.); nell' Emilia e la Romagna a Parma (Passerini!), sul Ventasso (Gib. Pir.), a Bologna (Bert.), Montese, Porretta (Cocc.), Faenza (Cald.); in Liguria a Sospello (Ard.), sopra Voltri (Figari!) ed altrove; in Toscana a Sàrzana (Bert.), Lucca (Bert.), ai Bagni di Lucca (Archb.), nella Selva pisana (P. Sav.), intorno Firenze!, in Casentino (Parl.!), nel Senese (Bert. ecc.); nelle Marche al Catria (Piccinini!); nel Romano ad Acquapendente, intorno Roma (Parl.!), ecc.; in Abruzzo a Pescara (Kuntze!); nel Napoletano a Cusano (Pasq.!), Napoli (Parl.!), nell' isola d'Ischia (Guss.), sul Vulture (Terr.); in Corsica al Capo, a Bocognano (Mars.), Vico (Req. !); in Sicilia a Piazza, Mineo, nelle Madonie, in Valdemone (Guss.). Fiorisce da giugno e luglio ad autunno inoltrato.

Distribuzione geografica. - In tutta l'Europa media e meri. dionale, nell' Asia Minore.

Descrizione. - Pianta perenne, di un odore soave, verde, pelosa, alta da 3 a 7 decimetri, fornita di un rizoma delicato, che manda di tratto in tratto delle fibre radicali e degli stoloni lunghi e radicanti. I fusti sono eretti, flessuosi, quadrangolari con gli angoli ottusi, verdognoli, pelosi con i peli bianchi, ramosi con i rami eretto-patenti. Le foglie sono opposte, un po'lontane, patenti, erbacee, molli, le inferiori piccole quasi orbicolari o ovato-orbicolari, quasi intere, le altre ovali o ovato-ovali, quasi acute, ristrette un po'alla base nel picciòlo, dentato-seghettate, verdi di sopra ed ivi con leggieri solchi che corrispondono ai nervi di sotto, di un verde più pallido nella pagina inferiore ed ivi con un nervo longitudinale 


\section{SATUREIA.}

rilevato ma delicato da cui partono dei nervi laterali, anch'essi rilevati e delicati, i quali si dirigono verso il margine el'apice e si uniscono ivi ad arco tra loro, mandando un nervetto a ciascun dente: sono pelose in ambedue le pagine, massime nella inferiore, e fornite di un picciòlo più corto della foglia, con un leggiero solco di sopra, convesso di sotto, peloso e verdognolo. Le brattee o foglie fiorali sono piủ piccole, ovali-orbicolari, quasi intere o con pochi denti, con un picciỏlo più corto. I fiori sono piủ grandi di quelli della $\mathrm{Ca}$ lamintha Nepeta, in piccole cime ascellari, rade e unilaterali, le inferiori delle quali sono fornite di un peduncolo lunghetto, verdognolo, peloso, con i peli bianchi, e portano tre e di raro quattro fiori accompagnati da bratteole strette, quasi lesiniformi, verdognole, pelose. Ciascun fiore ha un pedicello delicato, piủ corto o quasi uguale al calice, rossiccio, puberulo. Il calice è verde, in parte rossiccio, peloso, quasi cilindrico, con circa dodici strie longitudinali, barbato nella gola, con il lembo diviso in due labbri, dei quali il superiore ha tre denti patenti, ovato-acuminati, e l'inferiore due denti piủ lunghi e piủ stretti, avvicinati alla corolla, lanceolato-acuminati, con lunghe e forti ciglia bianche: nel frutto è un po'curvato in giủ e un po'tondeggiante in basso. La corolla è circa tre volte piủ lunga del calice, di un roseo-violetto, con macchioline piủ cariche verso la gola nella parte di mezzo del labbro inferiore: è pelosa di fuori, glabra di dentro eccetto nel tubo verso la parte qui notata della gola. Il tubo è un po' curvato ad arco in giù, insensibilmente slargato nel lembo: questo è diviso in due labbri, il superiore più piccolo, diretto in alto, quasi orbicolare, smarginato, l'inferiore diretto in avanti ed in giủ, trilobo, con i lobi laterali tondeggianti, un po' piu piccoli del lobo di mezzo il quale è quasi orbicolare, non smarginato e legs. germente piegato a onde nel margine. Gli-stami sono quattro, didinami, due poco piủ corti del labbro superiore e due poco piủ corti o quasi uguali al tubo in modo che si vedono dalla gola, inseriti in alto del tubo. I filamenti sono filiformi, lontani tra loro alla base, in ciascun paio convergenti all'apice, bianchi, glabri. Le antere sono biloculari, con le logge un po'disuguali, divergenti, ristrette alla base, quasi tonde ma schiacciate in alto ed ivi di color piu carico della corolla, aprentisi per una fessura trasversale in ciascuna loggia, glabre. Il polline è bianchiccio. Il pistillo è quasi uguale o poco più lungo degli stami superiori, e talvolta supera il labbro superiore. L' ovario é piccolissimo, diviso in quattro lobi, ovali, ottusi, verdo. gnoli, glabri, cireondati alla base da un disco grosso, hianchiccio. 
Lo stilo è lungo, filiforme, appena più grosso alla base, glabro, di un roseo-bianchiccio. Lo stimma è bifido, con le lacinie disuguali, un po'divergenti, acute, di color roseo-violetto. I frutti sono ovalitondi, scuri, finamente punteggiati quando si guardano con una forte lente. (Parl. ms., descr. della pianta di Grumone in Lombardia).

Osservazione. - Per Satureia Calamintha ho intesa più par. ticolarmente la forma detta oggigiorno Calamintha silvatica. Oltre i caratteri indicati, questa ha le foglie membranose, e più grandi rispetto alla pianta. Ho stimato meglio riferire alla $S$. Nepeta certe forme intermedie, ma che hanno le foglie più grossotte.

\section{Sntureir grandiflora.}

S. perennis, suberecta, foliis ovatis, planis, grosse dentatis, dichasiis pedunculatis, folia floralia (sensim minora) inferiora haud æquantibus, laxis, secundis, bracteolis parvis, calycis tubo (1 decim. longo) 11 -nervato, basi subæquali, fauce pilosa pilis exsertis, den. tibus inæqualibus, inferioribus porrectis, superioribus recurvatis, corolla calyce subduplo longiore.

Melissa grandiflora Linn. sp. pl.ed. 1. p. 592. Mass. prodr. fl. valt. p. 112. Trev. prosp. fl. eug. p. 26. De Not. rep. fl. lig. p. 320.

Calamintha grandiflora Moench meth. p. 408. Koch syn. fl. germ. ed. 2. p. 643: Com. fl. com. 4. p. 369. Pucc. syn. pl. luc. p. 311. Rota prosp. piant. Pav. p. 271. Hausm. fl. Tir. p. 679. Rota prosp. fl. Berg. p. 69. Pir. fl. for. syll. p. 111. Car. prodr. fl. tosc. p. 506. Tassi? fl. prov. sen. p. 50. G. Bert. not. piant. porr. p. 47. Veg. mont. Porr. p. 87. Ard. fl. Alpes-mar. p. 296. De Vis. Sacc. cat. piant. Ven. p. 135. Zersi prosp. piant. Brescia p. 165. Ces. el. piant. Maiella p. 24. Archb. fl. Alto-Serch. p. 60. Ces. Pass. Gib. comp. fl. ital. p. 303. Sacc. Bizz. agg. fl. trev. p. 29. Arc. comp. fl. ital. p. 542. Gib. Pir. fl. Mod. p. 128.

Thymus grandiflorus Mass.? o. c. p. 186. Bert. fl. ital. 6. p. 226.

Satureia grandiflora Scheele in flora 1843. 2. p. 577.

Figure, - Bot. mag.t. 208. Reich. ic. fl. germ. 18. t. 75. f. 1.

Stazione, Abitazione e Fioritura. - Nei boschi di montagna nella Penisola, non rara al settentrione e al centro, assai più verso mezzogiomo: in Piemonte nei monti di Dronero (Manuel!), Vinadio (All.), Valdieri a 1200 metri, Tenda (Parl.! ecc.), a Bardonec- 
chia (Aiuti!), a Courmayeur (Graudin); nel Canton Ticino a Lugano (Gaudin), e Locarno (Franzoni!); in Lombardia nel Pavese oltre Po (Rota), vicino a Como, sopra la Madonna del Honte di Varese, a Dumenza, nella Valsolda (Com.), a Montagna sopra Sondrio (Mass.), nel Bergamasco ne'monti Epolo e Campelli (Rota), nel Bresciano alla Corna Blacca a 1600-1700 metri (Parl.!), e fra Gargnano e Tignale (Zersi); in Tirolo a Daone (Porta!), Roveredo, Folgaria (Hausm.), in Valarsa (lierner!), in Valsugana (Ambrosi!), a Tione, Trento, Predazzo, in val di Non, a Bolzano (Hausm.); nel Baldo, in val Novese a 1600-1700 metri (Bracht!), e in basso come nelle valli Bassiana (Ilanganotti!) e Ime (Pollini), in val di Portole (Ball!), e a Recoaro (Meneghini!) nel Vicentino, negli Euganei (Kellner!), nel Bassanese (Bert.), nel Trevigiano sopra Valdobbiadene, nei monti Collalti e Grappa (Sacc. Bizz.), nel Bellunese, nel Friuli (De Vis. Sacc.), nel Littorale e in Istria (Koch ecc.); in Liguria sopra Nizza (Barla!), a Rezzo (Berti!) e altrove; in Toscana nei monti Gotro e Orsaio!, nelle alpi Apuane al Pizzo d'uccello e nell'alpe di Gramolazzo (Ad. Targioni!), nell'Appennino di Garfagnana!, e di val di Lima fino a Boscolungo a 1300 metri e al Teso (Parl. !); sul versante settentrionale dell'Appennino alla Porretta (G. Bert.), la Madonna - dell'Acero (Gennari!), Civago (Gib. Pir.); in Abruzzo al Velino (Rol. li!), al Morrone a 1600-1700 metri (Groves!); in Basilicata (Avellino!); in Calabria al Pollino, la Sila (Ten.), Aspromonte (Pasquale!). Il Gussone l'indica sulla fede di Tineo in Sicilia nei boschi di Caronia (fl. sic. syn.). Fiorisce in luglio ed agosto.

Distribuzione geografica. - Nell'Europa meridionale, dalla Spagna al Caucaso, nell'Asia Minore, nell'Affrica settentrionale.

Descrizione. - Pianta perenne, di odore piuttosto forte, alta da 2 a 5 decimetri, di un verde chiaro, pubescente, fornita di un rizoma, che manda fibre radicali delicate e degli stoloni corti e dei fusti. Questi sono ascendenti o eretti, un po'flessuosi, semplici o quasi semplici, quadrangolari, verdognoli, puberuli-irti con altri peli più lunglii e più rari. Le foglie sono erbacec, molli, opposte, un po'lontane, patenti, ovate o ovali-bislunghe, acute o un po'ottuse, un po'cuneiformi alla base, con grandi denti acuti a guisa di sega nel margine, di un verde chiaro di sopra ed ivi con un solco longitudinale e dei solchi laterali tutti superficiali, che corrispondono ai nervi di sotto, di un verde piủ chiaro di sotto ed ivi con un nervo longitudinale rilevato, da cui partono dei nervetti laterali, anche rilevati, e ramosi che terminano all' apice di ciascun dente: sono puberule in 
ambedue le facce, fornite di corte ciglia nel margine e di un picciòlo il quale è quasi uguale o più corto delle foglie, con un solco longitudinale di sopra dove è peloso, e convesso di sotto. I fiori sono molto grandi, in piccole cime di due fiori all' ascella di ciascuna foglia superiore, le quali sono patenti, rivolte da un solo lato, e sono più corte della foglia corrispondente; i peduncoli e i pedicelli sono cilindrici, puberuli-glandolosi, con le glandole piccolissime, accompagnati da brattee e bratteole patenti, piccole, lineari o lineari-lanceolate, verdi e puberule: i pedicelli sono più corti assai del calice. Questo è tubuloso, con il tubo quasi cilindrico, con molti nervi longitudinali, circa 13, che lo rendono quasi angolato, verde, puberulo-glandoloso di fuori, barbato di dentro verso la gola, con il lembo diviso in due labbri corti, il superiore con tre denti rivolti in su, ovato-acuminati con poche ciglia nel margine, e l'inferiore con due denti avvicinati alla corolla, un po'curvati in su, poco più lunghi dei denti del labbro superiore, lanceolato-acuminati, con le ciglia più lunghe e più numerose. La corolla è molto grande, da due a tre volte più lunga del calice, di un color roseo-violetto, puberula di fuori, peloso-barbata alla gola alla base dei lobi del labbro inferiore e in due linee 0 pieghe rilevate che si avanzano dalla gola nel tubo; con il tubo lungo, un po'curvato ad arco in su, stretto nella metà inferiore, quasi a un tratto slargato nella metà superiore, con il lembo diviso in due labbri, uno superiore più piccolo diretto in avanti e in su, quasi piano, tondeggiante all'apice e ivi smarginato, e l'altro inferiore patente, trilobo, con i lobi laterali tondeggianti all'apice e l'intermedio un po'più lungo dei laterali e smarginato. Gli stami sono quattro, didinami, due sono più corti del labbro superiore sotto il quale si vedono, e due poco piủ lunghi della metà del tubo della corolla, inseriti tutti e quattro a poca distanza tra loro verso la metà del tubo medesimo. I filamenti sono un po'distanti tra loro in basso, più vicini in alto, un po'curvi, quasi filiformi, di un bianco roseo, glabri. Le antere sono situate trasversalmente, inserite per la base, biloculari, con le logge strette, distanti per un largo connettivo, distinte alla base, aprentisi per una fessura longitudinale, bianchicce e glabre. Il pistillo è poco piủ lungo degli stami piủ lunghi e quasi uguale al labbro superiore, al quale è avvicinato. L'ovario è piccolo, quadrilobo, con i lobi eretti, piani di dentro, un po'convessi di fuori, ottusi, verdognoli, glabri, ed è circondato alla base da un disco grande, quasi tondo, gialliccio. Lo stilo è lunghissimo, filiforme, curvo in su, bianchiccio roseo, puberulo. Lo stimma è bifido, con le lacinie 
disuguali scanalate di dentro, un po'patenti, quasi acute, rosee. (Parl. ms., descr. della pianta di Boscolungo in Toscana).

\section{Satureia Clinopedium.}

S. perennis, erecta, foliis ovatis, planis, crenulatis, glomerulis densissimis, breviter pedunculatis, folia floralia (subconformia) nune haud æquantibus nune superantibus, bracteolis calyces fere æquantibus, calycis tubo (5-6 millim. longo) 13-nervato, basi æquali, fauce pilosa pilis exsertis, dentibus inæqualibus, inferioribus incurvatis, superioribus recurvatis, corolla calyce dimidio-duplo longiore.

Clinopodium vulgare Mass. prodr. fl. valt. p. 49. Bert. fl. ital. 6. p. 192. Com. fl. com. 4. p. 356. Pucc. syn. pl. luc. p. 309. Simi! fl. alp. vers. p. 130. Rota prosp. piant. Pav. p. 271. Hausm. fl. Tir. p. 681. Rota prosp. fl. Berg. p. 69. Guss. enum. plant. Inarim. p. 260. Pir. 1. for. syll. p. 111. De Vis. Sacc. cat. piant. Ven. p. 135. Zersi prosp. piant. Bresc. p. 165. Pasq. f. ves.p. 80. Terr. fl. Vult. syn. p. 137. Sec. rel. Terra di Lav. p. 95. Quart. rel. id. p. 112. Loj. is. eol. p. 123. Cald. fl. fav. tent. p. 180. Gib. Pir. fl. Mlod. p. 128.

Melissa vulgaris T'rev. prosp. fl. eug. p. 26.

Melissa Clinopodium Mor. De Not. fl. capr. p. 106. De Not. rep. n. lig. p. 320. Zan. prosp. fl. ven. p. 26.

Calamintha Clinopodium Mor.: en. sem. hort. taur. 181, Fl. sard. 3. p. 289. Ard. cat. pl. Ment. p. 28. Car. prodr. fl. tosc. p. 508. Tassi fl. prov. sen. p. 50. G. Bert. not. piant. porr. p. 47. Veg. Porr. p. 87. Ard. 月. Alpes-mar. p. 297. Nars, cat. pl. Corse p. 112. Archb. fl. Alto-Serch. p. 60. Ces. Pass. Gih. comp. fl. ital. p. 30.t. Arc. comp. fl. ital. p. 5.11.

Figure, - Fl. dan. t. 930. Reich. ic. fl. germ. 18. t. 73. f. 1.

Stazione, Abitazione e Fioritura. - Comunissima nelle siepi, nei luoghi boschivi ecc., per tutta Italia, in pianura ma specialmente in collina e in inontagna, dove s'inalza assai, per esempio in 'Tirolo sul monte Ritten presso Bolzano fino a 1200-1500 metri (Ilausm.), nell'Appennino toscano fino alla regione superiore dei boschi!. Delle piccole isole abita quelle dell'Elba!, di Capraia (Mor. De Not.), di Capri (Pasq.), d'Ischia, di Lipari e Saline nelle Lolie (Giuss.), di Pantellaria (Calcara!). Fiorisce da maggio, giugno o luglio secondo i luoghi, alla fine dell' estate. 
Distribuzione geografica. - In tulta Europa meno la piủ nordica, nell'Asia occidentale fino all' Imalaia, nell'Africa settentrionale, nell'America settentrionale ma introdotta.

Descrizione. - Pianta perenne, alta da 3 a 6 decimetri, di un verde chiaro e in parte bianchiccia per peli lunghetti e bianchi. Il fusto è eretto 0 ascendente, flessuoso, radicante alla base, quadrangolare, semplice o più spesso ramoso con $\mathrm{i}$ rami opposti eretto-patenti, peloso con peli piuttosto fitti, bianchi, orizzontali. Le foglie sono opposte, alquanto lontane, patenti, ovali, ottuse, con pochi e leggieri denti quasi in forma di sega, e con i margini un poco rovesciati, di un verde chiaro di sopra ed ivi con leggieri solchi che corrispondono ai nervi di sotto, di un verde-bianchiccio di sotto ed ivi con un nervo longitudinale alquanto rilevato da cui partono dei nervetti laterali, un po'rilevati, obliqui e un po'curvi che vanno verso il margine e l'apice e si uniscono ad arco tra loro mandando anche un nervetto ai denti del margine della foglia; sono pelose in ambedue le pagine e segnatamente di sotto e fornite di un picciỏlo corto, quasi piano di sopra, convesso di sotto, peloso e bianchiccio. I fiori sono molti in piccole cime formanti dei glomeruli all' ascella delle foglie supreme del fusto e dei rami, accompagnati da bratteole, lunghe quasi o poco piủ corte del calice, setacee, verdognolo-bianchicce, irsuto-cigliate con i peli lunghi. I pedicelli sono molto più corti del calice, irsuti, verdognolo-bianchicci. Il calice è irsuto, verdognolo e in parte violetto-rossiccio, con molti nervi rilevati e verdognoli, senza glandolette nei solchi; ha il tubo un po' curvo, fornito di peli lunghi orizzontali nella gola ma non ivi barbato, con il lembo diviso in due labbri, uno superiore più corto, diretto in avanti ed in su, con tre denti divergenti l'uno dall'altro, corti, largamente lanceolato-acuminati, e uno inferiore diviso in due lacinie o denti più lunghi dei superiori, stretti, quasi lesiniformi, curvati in avanti e in su. La corolla è da 2 a 3 volte più lunga del calice, di color violettochiaro e quasi roseo-porporino. Il tubo è diritto, piủ lungo del calice, stretto in basso, si slarga quasi ad un tratto verso alto; il lembo ch'è circa un terzo del tubo, è bilabiato, il labbro superiore piủ corto è diretto in avanti ed in su, piano, ovale-orbicolare, smarginato, l'inferiore è diretto in avanti ed in giủ, trilobo, con i lobi laterali più piccoli, quasi troncati, e l'intermedio piủ largo all'apice, smarginato, tutti con qualche crenatura irregolare all'apice: vi sono dei peli grossi quasi in forma di clava e bianchicci o del colore stesso della corolla distribuiti quasi in due linee alla base del labbro infe- 
riore vicino alla gola tra $\mathrm{i}$ lobi laterali e il lobo di mezzo. Gli stami sono quattro, didinami, i due superiori poco meno della metà piủ corti del labbro superiore della corolla, al quale sono tutti avvicinati, inseriti nella parte superiore del tubo di questa. I filamenti sono eretti, filiformi, glabri, di color roseo-bianchiccio. Le antere sono piuttosto piccole con le logge distanti, divise da un largo connettivo quasi quadrato, glabre, del colore quasi della corolla, con il polline bianchiccio. Il pistillo è più lungo degli stami e quasi uguale al lab. bro superiore della corolla, al quale è avvicinato. L'ovario è piccolo, diviso in quattro lobi quasi tondi, verdognoli, lisci, ed ha alla base un disco grosso, alto quasi quanto l'ovario, ma sporgente ai lati dell'ovario e di color gialliccio. Lo stilo è lungo, eretto, filiforme, con qualche corto peletto verso alto, del colore della corolla. Lo stimma è bifido con le lacinie disuguali, divergenti, lunghette e quasi peta. loidee, l'anteriore piủ grande. (Parl. ms., descr. della pianta di Grumone in Lombardia).

\section{Satureia corsica.}

S. fruticosa, procumbens, foliis rotundatis, planis, integerrimis, glomerulis sessilibus, vel floribus solitariis, folia floralia (conformia) superantibus, bractcolis minimis, calycis tubo (4-5 millim. longo) 13-nervato, inferne antice vix gibbo, fauce pilosissima pilis exsertis, dentibus subæqualibus, suberectis, corolla calyce duplo longiore.

Thymus corsicus Pers, ench. 2.p. 131. Bert. fl. ital. 6.p. 219.

Calamintha corsica Benth. in Cand. prodr. 12. p. 291. Gren. Godr. fl. Fr. 2. p. 667. Mar's. cat. pl. Corse p. 112. Ces. Pass. Gib. comp. fl. ital. p. 303. Arc. comp. fl. ital. p. 542.

Stazione, Abitazione e Fioritura. - Nei pascoli degli altissimi monti di Corsica, sul Renoso (Requien! ecc.), sul Cagnone (erb. Jordan! ecc.), e sul Coscione a 2200 metri (Soleirol!). Fiorisce in luglio e agosto.

Distribuzione geografica. - Specie ritenuta propria della Corsica. Peraltro Nyman (Consp. M. enr. p. 589) la dit ancora di Sardegna; c nell' crbario Webb havene un saggio, proveniente dall' erbario Desfontaines, che porta sul cartellino l'indicazione: A liroguard pyrénées. "

Descrizione. - Pianta sdraiata, radicante, piủ o meno pelosa. 
Le foglie sono piccoline e fitte, consistenti, col margine ingrossato. I fiori, pochi e ravvicinati nell'estremitả dei rametti, sono 1-2 al. l'ascella delle foglie fiorali; il calice n' è cilindrico, piuttosto curvato nell' insieme che veramente gobbo da un lato nel basso; la corolla n'è porporina (v. s.).

\section{Satureia alpina.}

S. perennis suffrutescens, diffusa, foliis ovali-rotundatis ellipticis lanceolatisve, planis, subserratis, glomerulis sessilibus, haud secundis, bracteolis minimis, folia floralia (conformia) subæquantibus, calycis tubo (4-5 millim. longo) 13-nervato, inferne antice gibho, fauce pilosissima pilis exsertis, dentibus inæqualibus, inferioribus porrectis, superioribus recurvis, corolla calyce subduplo longiore.

Thymus alpinus Linn. sp. pl. ed. 1. p. 591. Mass. prodr. fl. valt. p. 186. Bert. fl. ital. 6. p. 215.

Calamintha alpina Lam. fl. fr. 2. p. 394. Com. fl. com. 4.p. 368. Pucc. syn. pl. luc. p. 311. Hausm. fl. Tir. p. 679. Rota prosp. fl. Berg. p. 69. Ard. cat. pl. Ment.p. 28. Pir. 1. for. syll. p. 110. Car. prodr. fl. tosc. p. 508. Tassi fl. prov. sen. p. 50. Ard. fl. Alpes-mar. p. 296. Zersi prosp. piant. Bresc. p. 165. Ces. el. piant. Maiella p. 24. Terr.! sec. rel. Terra di Lav. p. 94. Archb. fl. Alto-Serch. p. 60. Ces. Pass. Gib. comp. fl. ital. p. 304. Terr.! quarta rel. Terra di Lav. p. 112. Cald. fl. fav. tent. p. 180. Paol. primo el. piant. sib. p. 13. Arc. comp. fl. ital. p. 543. Gib. Pir. fl. Mod. p. 128.

Melissa alpina Benth. lab. p. 390. De Not. rep. fl. lig. p. 321.

Satureia alpina Scheele in flora 1843. 2. p. 577.

Acinos alpinus De Vis. Sacc. cat. piant. Ven. p. 135.

Calamintha patavina Ces. Pass. Gib. o. c. p. 303. Cocc. nuovo contr. fl. prov. Bol. p. 24. Arc.! o. c. p. 542. Gib. Pir. l. c.

Calamintha rotundifolia Ces. Pass. Gib. o. c. p. 303. Arc. o. c. p. 543.

Figure - Bot. mag. t. 2153. Reich. ic. fl. germ. 18. t. 74. f. 1,2 .

Stazione, Abitazione e Fioritura. - Nei pascoli e nei luoghi scoperti sassosi dei monti di tutta la Penisola, e di Sicilia. E comune nelle Alpi, da parti altissime fino ai colli al loro piede, e talora scende nelle pianure col corso dei torrenti: cosi al Col di Tenda a 1900 me- 
tri (Parl.!) e nel resto delle Alpi Marittime, nel Cenisio e sui monti vicini, fin giù presso Susa (Aiuti! ecc.), nelle alpi di Ceresole (Bert.). a Riva di Valsesia (Carestia!), sopra Craveggia in valle di Vigezzo (Bir. fl. acon.), nel Cantone Ticino (Com.) fino al Gottardo (Heldreich!), in Valtellina a Bormio (Levier!), e nella valle Malenco (Mass.) ecc., a Chiavenna (Rampoldi!), nel Comasco (Com.), nel Bergamasco (Rota), nel Bresciano, per esempio nei monti di val Trompia fra 1700 e 1300 metri (Parl.!), in tutto il Tirolo (Hausm.), sul Baldo a 6000 piedi (Bracht!) e nel resto del Veronese (Poll. $f$. veron.), nel Vicentino sui monti Portole e Grappa (Bert.), e giù a Marostica (Barbieri!), nel Trevigiano, nel Bellunese (De Vis. Sacc.), nel Friuli intorno a Gemona (Bert.) ecc. Abbonda nei monti Ligustici (De Not.), così nel Nizzardo, dove scende fin presso Mentone (Ard.). E pure comune nelle parti superiori delle Alpi Apuane!. Nell'Appennino si stende senza interruzione dal monte Orsaio in Lunigiana! all'alpe della Luna in val Tiberina (Gherici!), scendendo nel versante nord-est fino ai contorni di Parma (Passerini!), di Bo. logna (Bert.) e di Faenza (Cald.!), e inoltrandosi dall'altra parte per il Pratomagno e i monti del Mugello fin presso Firenze!; per i monti delle Marche, quali il Catria (Piccinini!), e il S. Vicino (Bucci!), per i dintorni di San Severino (Aiuti!) e di Macerata (Narducci!), giunge ai monti Sibillini e Abruzzesi!, dove si trova nelle maggiori cime, e in luoghi bassi come Montefortino (Marzialetti!). Distaccata, trovasi a levante al Gargano (Bert.), e a ponente nei monti Amiata e Labbro di Toscana!, a Viterbo (Bert.), nei monti Albani (Polli!), verso Sora (Terr.!), a S. Angelo (Pasquale!), Potenza (Ten.), Cosenza (Aiuti!), Stilo, in Aspromente (Arc.!). In Sicilia fa sull’Etna (Bivona! ecc.), presso Noara (Seguenza!), nelle Madonie (Parl.!), nel monte Camerata (Guss.), a Busambra (Lojacono!), alla Pizzuta (Parl.!). Fiorisce da maggio a luglio, o da giugno ad agosto, secondo i luoghi.

Distribuzione geografica. - Per tutti i monti dell'Europa me. ridionale e media, dalla Spagna alla Transilvania.

Descrizione. - Pianta perenne, alta da 2 a 4 decimetri, fornita di molti fusti ramosi, e come i rami legnosi alla base e che man. dano ivi fibre delicate radicali, giacenti dapprima per terra, poi rilevati o ascendenti, parte lioriferi, parte sterili nell'anno, e tutti formanti graziosi cespuglietti; tali fusti e rami sono quadrangolari, con due facce più strette e aventi un solco longitudinale, verdognoli o rossicci, pubescenti, con i peli corti, curvati in giù ad arco e bian- 
chi. Le foglie sono opposte, patenti, patentissime e alcune talvolta un po' rivolte in giù, ovate o ovato-ovali, acute, verdi di sopra e ivi con un solco longitudinale e pochi solchetti leggieri laterali che corrispondono ai nervi di sotto, di un verde bianchiccio di sotto ed ivi con un nervo longitudinale rilevato da cui partono due o tre paja di nervetti laterali, lontani tra loro, alquanto rilevati, un po' curvi, che si dirigono verso l'apice e i margini della foglia: questi sono rovesciati; le foglie sono pubescenti nei nervi e con qualche pelo nella pagina superiore, o quasi glabre, fornite di un picciòlo corto, quasi piano di sopra, un po'convesso di sotto, di colore un po'più chiaro della foglia e pubescente. I fiori sono per lo più 3 o 2 all' ascella di ciascuna foglia superiore, per cui vi sono circa sei fiori in ciascun giro, forniti di un peduncolo corto, eretto, peloso. Il calice è un po' curvo, come gobbo in basso ed in avanti, stretto ad un tratto sopra al gobbo e ivi cilindrico, è verdognolo, con molti nervi di colore più carico, peloso, con i peli disuguali, quasi orizzontali e bianchi; ha il lembo diviso in due labbri, uno superiore con all'apice tre denti corti, quasi uguali, e uno inferiore diviso in due lacinie che giungono sino ad uguagliare i denti del superiore: tanto i denti quanto le lacinie sono acuminate, fornite di ciglia, avvicinate alla corolla, eretto-patenti nel frutto; il calice ha la gola chiusa allora da peli lunghi e bianchi. La corolla è quasi due volte piủ lunga del calice, di un bel colore violetto con la base del labbro inferiore di essa più chiara e quasi bianchiccia ma ivi con piccole macchioline violette: è pelosa di fuori massime nel tubo; questo è molto più lungo del calice, stretto nella parte racchiusa da questo, poi si slarga quasi ad un tratto. Il labbro superiore è piccolo, diretto in alto e un po' in dietro nell'apice, ovale, tondeggiante e smarginato all'apice: l'inferiore è quasi il doppio più grande, patente, trilobo, con i lobi laterali quasi orbicolari e il lobo di mezzo più grande, ora più ora meno smarginato. La gola è larga, con molti peli bianchi che la rendono quasi barbata da ambe le parti, essendo glabra nelle parte depressa. Gli stami sono didinami, inseriti sopra la metà del tubo, i due piủ lunghi sono più corti del labbro superiore. I filamenti sono quasi filiformi, un po'curvi, glabri, bianchi. Il pistillo è lungo quanto gli stami più lunghi. L'ovario è piccolo, diviso in quattro lobi, bislunghi, verdognoli, glabri; il disco è grosso, verdognolo. Lo stilo è lungo, un po'curvo, più assottigliato in alto, ivi di color violetto chiaro, bianchiccio in basso, glabro. Lo stimma è bifido, con le lacinie disuguali, divergenti. (Parl. ms., descr. della pianta di Prataglia nell'appennino Casentinese in Toscana). 
Osservazioni. - Come era da aspettarsi da una pianta cosi largamente sparsa, e abitatrice di climi diversi, essa varia non poco per la grandezza dell'insieme e delle parti, per la pelurie, abbondante 0 scarsa, per la forma delle foglie; e varia anche per la natura dei peli, e per la direzione e la forma dei denti calicini, coi quali caratteri hanno cercato i botanici di avvalorare certe distinzioni specifiche che non reggono all'esame. La Calamintha rotundifolia auct. è una forma rigogliosa, che non presenta altra piủ sostanziale differenza dalle forme più ordinarie della specie; la $C$. granatensis Boiss. Reut., stata trovata sull'Etna, sul Velino, è una forma stentata, con foglie assai piccole e fiori ravvicinati fra loro.

Come bene osservò Bertoloni (o. c. p. 215), il Thymus patavinus fu creato da Jacquin (Obs. bot. 4. p. 7. t. 87) sopra pianta coltivata, ed egli vi riferi un sinonimo di Boccone (Mus. di piante rare $p .60 . t .45 . f . B$ ) ugualmente di pianta coltivata nell'orto botanico di Padova: d'onde il nome. Le descrizioni dei due autori non dicono nulla per distinguere la specie dalle affini, ma dalle loro figure niente osta a che si riunisca alla Satureia alpina.

\section{Satureia Acinos.}

S. annua, foliis ovali vel oblongo-lanceolatis, subserratis, glomerulis sessilibus, folia floralia (conformia) haud vel vix æquantibus, bracteolis minimis, calycis tubo (4 millim. longo) 13-nervato, inferne antice valde gibbo, fauce pilosissima pilis exsertis, dentibus inæqualibus, inferioribus incurvatis, superioribus recurvis, corolla calyce vix vel dimidio longiore.

Calamintha arvensis Lam. fl. fr. 2.p. 394. Car. prodr. fl. tosc. p. 507. Tassi R. prov. sen. p. 50. G. Bert. not. monti porr. p. 47. Veg. mont. Porr. p. 87. Archb. 凡. Alto-Serch. p. 60. Groves contr. fl. Terra d'Otr. p. 65.

Calamintha Acinos Clairv. in Gaud. /l. helv. 4. Com. $\mu$. com. 4. p. 366. Pucc. syn. pl. luc. p. 311. Rota prosp. piant. Pav. p. 271. Hansm. n. Tir. p. 678. Rota prosp. 凡. Berg. p. 69. Mor.! l. sard. 3. p. 290. Pir.! /l. for. syll. p. 110. Ard. /. Alpes-mar. p. 296. Zersi prosp. piant. Bresc. p. 164. Terr. 月. Vult. syn. p. 137. Ces. el. piant. Maiell. p. 2.t. Ces. Pass. Gib. comp. 1. ital. p. 303. Cald. n. fav. tent. p. 179. Arc. comp. R. ital. p. $5: 12$ (excl. var.). Gib. Pir. M. Mod. p. 128. 
Thymus acinoides Ten.! fl. nap. 1.prodr. p. 35. Bert.! fl. ital. 6. p. 214.

Melissa Acinos Benth. lab. p. 389. Trev. prosp. fl. eug. p. 26. De Not. rep. fl. lig. p. 321. Zan. prosp. fl. ven. p. 26.

Satureia Acinos Scheele in flora 1843. 2. p. $57 \%$.

Thymus Acinos Mass. prodr. fl. valt. p. 186. Bert. o. c. p. 212. Sim. fl. alp. vers. p. 181. Pasq. fl. ves. p. 80.

Acinos thymoides De Vis. Sacc. cat. piant. Ven. p. 135.

Acinos vulgaris Pasq. Lic. viagg. Garg. p. 22.

Figura. - Reich. ic. fl. germ. 18. t. 73. f. 4.

Stazione, Abitazione e Fioritura. - Nei campi, nei luoghi incolti, nei luoghi asciutti erbosi o boschivi, e simili. É comune nell'Alta Italia, dalla pianura a parti assai alte dei monti: cosi in Piemonte sale sul Cenisio, e sopra i Bagni di Valdieri nel monte Stella (Parl.!), e nel Cantone Ticino sul Gottardo (Bert.), in Valtellina trovasi nella valle Malenco (Mass.), e a Bormio oltre 1200 metri, a Ponte di legno in val Camonica a 1500 metri (Parl.!), in Tirolo nell' estrema Pusteria a Welsberg (Hausm.), e nell' appennino Modenese sale fino nell'alpe di Succiso (Gib. Pir.); in Liguria preferisce i monti (De Not.), ma si trova anche sul mare, come a Nizza (Durando!); anche in Toscana è più comune nei colli e nei monti, in tutta la loro parte boschiva, ma fa anche al mare, corne nell'Elba (Bert.); nelle Marche e nell' Abruzzo fa ad Urbino (Federici!), Senigaglia (Bert.), nel monte S. Vicino (Bucci!), a Macerata, S. Elpidio (Bert.), Montefortino (Marzialetti!), Ascoli (Bert.), Caramanico (Ces.); è indicata nel Romano (Seb. Mauri); nasce al Gargano (Pasq. Lic.), a Barletta (Bruni!), Matera (Bert.), Potenza, Martina, Taranto, Lecce (Ten.), nel Vulture (Terr.), nel Vesuvio, e in Capri (Pasq.); manca in Corsica; ma trovasi in Sardegna nei monti d'Oliena, Tonneri d'Irgini, Gennargentu (Mor.), in Sicilia parimente nei monti, a Marineo, sull'Etna, a Caronia, Pizzuta, Prizzi, Palazzo Adriano e altrove, infine nell'isola di Lampedusa (Calcara!). Fiorisce in maggio e giugno, o in luglio e agosto secondo i luoghi.

Distribuzione geografica. - In tutta Europa, meno l'artica.

Osservazione. - Non ho citato la figura del Thymus acinoides di Tenore ( $F l$. nap. $t .155 . f .1)$, perchẻ dả i fiori con la corolla assai più grande del vero, giudicando da un saggio autentico dell' autore nell'Erbario Centrale, che nel resto corrisponde alla figura. Quel saggio, come medesimamente la figura, mancano della parte inferiore della pianta; e giudico che Tenore e Bertoloni, di. 
cendo di essa ch'è suffruticosa, abbiano creduto a qualche apparenza fallace.

\section{Satureia graveolens.}

S. annua, foliis late ovalibus ovatisve, subserratis, glomerulis sessilibus, folia floralia (conformia) haud vel vix æquantibus, bracteolis minimis, calycis tubo (4 millim. longo) 13-nervato, inferne antice valde gibbo, fauce pilosissima pilis exsertis, dentibus inæqualibus, inferioribus incurvatis, superioribus recurvis, corolla calyce vix longiore.

Thymus graveolens Bieb. fl. taur.-cauc. 2. p.60. Bert.! fl. ital. 6. p. 218.

Thymus pallescens Ten.! fl. nap. 5. p. 26.

Calamintha graveolens Benth. in Cand. prodr. 12. p. 231. Ces. Pass. Gib. comp. fl. ital. p. 303. Arc. comp. fl. ital. p. 542.

Figure. - Fl. grcec. t. 575. Reich. ic. fl. germ. 18. t. 74. f. 3.

Stazione, Abitazione e Fioritura. - Questa rarissima specic nasce nei campi montuosi dell'Abruzzo, dove fu trovata da Tenore, Orsini e Mauri nel 1829 presso Aquila ad Assergio, e piủ precisamente tra Assergio e Aragno (Ten.) a Camarda (Bert.), ed è stata ritrovata dal dott. Levier nel 1882 presso Solmona, tra Pettorano e la valle Vozz.

Distribuzione geografica. - Nell'Asia occidentale, la Grecia e la Turchia, la Crimea, la Moldavia e la Transilvania, l'indicato luogo d'Italia, la Spagna.

\section{CUSTIDOCA IRTUS.}

Satureise, Calaminthx vel Micromerix sp. auct.

Cuspidocarpus Spenn. gen. $p l$. $l$. germ. 6 .

Calyx tubuloso-campanulatus, valide 13-nervatus. Corolla manifeste bilabiata, labio superiore erecto, planiusculo. Stamina 4, conniventia, filamentis edentulis, antheris 9-locularibus, loculis longitudinaliter dehiscentibus, a connectivo seiunctis. Stylus lobis inæqualibus. Cocci apice mucronati atque pilosi et glandulosi.

Portamento. - È quello della Satureia marifolia, eccettochè 
ivi i fiori sono meno numerosi e più radi; e non è nemmen lontano da quello di certe forme della comune $S$. Nepeta.

Osservazione. - Genere vicinissimo al precedente, non ne differisce che per il carattere del frutto; il quale essendo pertanto ritenuto sufficiente a distinguere fra loro altri generi di Lamiacee, come Sideritis e Marrubium, deve valere anche qui per far accettare il genere Cuspidocarpus.

\section{Cuspidocarpus rupestris.}

Satureia thymifolia Scop. fl. carn. ed. 1. p. 428 (1772).

Satureia rupestris Wulf. in Jacq. coll. 2. p. 130 (1788). Bert. fl. ital. 6. p. 58. Ces. Pass. Gib. comp. fl. ital. p. 302. Arc. comp. fl. ital. p. 541 .

Calamintha thymifolia Reich. fl. germ. exc. p. 328 (1830) (non Host). Pir. fl. for. syll. p. 111.

Calamintha rupestris Host fl. austr. 2 (1831).

Cuspidocarpus rupestris Spenn. l. c.

Micromeria rupestris Benth. in Cand. prodr. 12. p. 225.

Figure. - Scop. o. c. t. 29. Spenn. o. c. t. 18. Reich. ic. fl. germ. 18. t. 78. f. 2, 3 .

Stazione, Abitazione e Fioritura. - Sulle rupi, sui vecchi muri, in luoghi sassosi dell' Istria a Fiume (Noé!), a Trieste (erb. Reboul!), e del Friuli alla bocca dell' Isonzo (Bert.), a Gorizia (Pir.), Flitsch (Huter!), Gemona, Venzone, e presso Sauris (Pir.). Fiorisce in luglio ed agosto.

Distribuzione geografica. - Da tutto il littorale est dell'Adriatico si estende per la Croazia e la Carniolia nell'Ungheria.

\section{ZIVIFORUM.}

Ziziphora Linn. hort. cliff. p. 305. Mirb. in ann. mus. 15.t. 14. f. 8. Benth. Hook. gen. pl. 2. 1198.

Ziziforum Adans. fam. pl. 2. p. 189. Zizifora Ges. Pass. Gib. comp. fl. ital. p. 308. t. 45. f. 4.

Calyx tubulosus, valide 13-nervatus. Corolla manifeste bilabiata, labio superiore erecto, demum recurvo, plano. Stamina 2 (inferiora), 
conniventia, filamentis edentulis, antheris (in specie nostra) dimidiatis 1-locularibus, longitudinaliter dehiscentibus. Stylus lobis valde inæqualibus. Cocci apice convexi leves

Portamento. - Vedasi la descrizione qui appresso della specie italiana.

Osservazioni. - Linneo nominò il genere dal nome volgare Orientale della presente specie, riferito da Morison (Hist. pl. 3. $p$. 37.) nella forma Ziziforum; malamente adunque lo tramuto in Zizi. phora, dandogli falso sembiante di etimologia greca.

\section{Ziziforum capitstum.}

Ziziphora capitata Linn. sp. pl. ed. 1.p. 21. Savi! bot. etr. 2. p. 160. Ten. fl. nap. 2. p. 379. Bert. A. ilal. 1.p. 132. Car. prodr. fl. tosc. p. 512. Ces. el. piunt. Maiella p. 24. Ces. Pass. Gib. comp: f. ital. p. 309. Cald. fl. fav. tent. p. 181. Arc. comp. fl. ital. p. 546. Ziziphora serpyllacea Ten. syll. fl. neap. p. 16 (non Bieb.).

Figure. - Pluk. phyt. t. 164.f. 4. Fl. groec. t. 13.

Stazione, Abitazione e Vita. - Questa pianta fu scoperta in Italia nel 1795 da Gaetano Savi presso la Certosa di Firenze; ai tempi di Micheli non esisteva nell' agro Fiorentino, poichè non si trova nel suo erbario, e neppure nel catalogo manoscritto da esso lasciato delle piante di quell'agro; ora vi si è resa comune per i campi, specialmente di grano, sulle colline firo a Malmantile (Scaffai!), S. Casciano (Ricci!), Incisa (Maire!), Pontassieve (Ricasoli!) e monte Se-。 nario (Parl.!). Savi pure la trovó dipoi nel letto dell'Arno presso Pisa, ed io mi rammento di averla veduta lungo l'Arno a S. Pierino sotto Empoli Bertoloni ricorda nel $185 \overline{3}$ il suo ritrovamento dal Tas. sinari, 'un suo scolaro, nell' agro Faentino, dove Caldesi la nota dei monti di Riolo. Giả nel 1820 Tenore l'avera inserita nella sua Flora, per essere stata scoperta nella Puglia. Fa altresi nel Maceratese a Corneto (Gennari!), Mungini, Cincinelli (Narducci!), nell'Ascolano a S. Giusto (Marzialetti!), in Abruzzo tra S. Valentino e S. Tom. maso (Ces.). Nasec nel marzo ofl aprile, fiorisce in giugno, la sua fioritura durando una rentina di giorni, matura $i$ frutti nel mese seguente.

Distribuzione geografica. - Mhita tutto l'Oriente, e si trova anche nella Russia meridionale, in Serbia ed Erzegovina, in Spagna, nell' Affrica settentrionale. In Italia è molto probabilmente importata, certamente in Toscana. 
Descrizione. - Erba annua, eretta o ascendente, semplice o ramosa, alta $1-3$ decim. Padice sottile, bianchiccia. Fusto tetragono, pubescente in basso, velloso in alto, coi peli rivolti in giu. Foglie hrevemente picciuolate, bislunghe-lanciolate o lineari-lanciolate, acutette, dentellate oscuramente e a distanza, pressochè glabre, cigliolate, 1-nervate, lunghe fino a 4 centim., le inferiori più corte e più lungamente picciuolate ed intere, le fiorali eziandio più corte ma larghissime, ovate-aguzzate, pubescenti di sopra, lungamente cigliate, con picciuolo cortissimo e largo, addossate ai fiori, che sorpassano o (le piu interne che sono quasi tonde) uguagliano. Fiori odorosi, addensati come in capolini terminali, quasi senza bratteole, eretti, pedicellati, col pedicello alquanto compresso, lungo $1-3$ millimetri. Calice lungo 1. centin., tubuloso, appena curvato, con 13 nervature, con pubescenza patula, glabrissimo inentro, con la gola chiusa da peli eretti, bilabiato, il labbro superiore essendo 5-dentato, l'inferiore più profondamente 2-dentato co' denti lunghi quasi 3 millim., tutti eretti, riuniti, lesiniformi, cigliati, un poco ricurvi. Corolla di un porporino chiaro, con pubescenza minuta in fuori; tubo sottile, un po'più lungo del calice, alquanto dilatato alla gola, coperto in den. tro di peli rivolti in giù e clavati; labbri glabri di sopra, il superiore piano, al fine ricurvato, lungo 2 millim., bislungo, arrotondito all'apice, intero, l'inferiore appena più lungo, patulo, 3-fido, coi lobi laterali arrotonditi, interi, col lobo medio appena più piccolo, smarginato. Stami inseriti presso alla gola, ascendenti, diritti, ap. pena appena più corti del labbro superiore, glabri, porporini chiari; filamenti filiformi; antere erette, 1-loculari (per la totale mancanza della casella esterna), per lo più agglutinate insieme, con la casella bislunga, inserita presso alla base del lato esterno, essendo presente il connettivo, deiscente per lungo in avanti; polline bianco, pressochè globoso, pieghettato, largo $0 \mathrm{~mm}, 045$. Disco grosso, "iguale. Stilo glabro, volgente al porporino, filiforme-subulato, ristretto fra mezzo ai lobi del gemmulario, alfine un po'più lungo della corolla e raddrizzato, dapprima piegato in giủ, coi lobi subulati, il superiore essendo piccolissimo; lobi del gemmulario equidistanti fra loro, bislunghi; gemmula ascendente, anatropa, con integurnento scempio, introrsa. Cocchi bislunghi, ottusi, lisci, fulvi, lunghi $1 \frac{1}{2}$ mil. limetri, minutissimamente punteggiati sotto alla lente; pericarpio crostoso. Seme dal guscio sottilissimo; embrione senza mandorla, diritto, col fusticino corto, con gli embriofilli ottusi, paralleli all'asse del frutto. (v. v. dai campi Fiorentini). 


\section{KMII. IRELISSA.}

Melissa (part.) Toum . inst. p. 193. t. 91. Linn. gen. ph. ed. I. p. 172. Hirb. in ann. mus. 15. 1. 20. .. \%.

Helissa Moench meth. pl. p. 408. Gen. pl. /l. germ. 6 t. II. Bicuth.

Hook. gen. pl. 2. p. 1191. Ces. Pass. Git. comp. fl. ital. p. jut. t. $45 .+1$.

Calyx campanulatus, valide 13 -nervâtus. Corolla manifeste bilabiata, labio superiore erecto, planiusculo. Stamina 4, conniventia, filamentis edentulis, antheris 2-locularibus, loculis longitudinaiter dehiscentibus, conlluentibus. Stylus lobis subæqualibus. Cucci apice convexi leves.

Portamento. -- Erba perenne, ransosissima, carica di foglie piuttosto grandi, e di fiori piecoli, bianchicci. Vedasi la descrizione qui appresso.

\section{Vlelissa offeintalis.}

Melissa officinalis Linn. sp. pl. ed. 1. p. 592. Bert. 11. Ilul. 66. p. 229 ; et unct. omn.

Melissa altissima Sibth. Sin. Al. gruec. prodr. 1. p. 徒. Guss. enum.pl. Inarim. p. 261. Terr.! 1l. Vult. syn. p. 198. Ciroves conlr. fl. Terra d'0tr. p. 65.

Figure, - Savi mat. med. veg. tosc. t. 80 . 1'l. gr'Ret. t. 579. Reich. ic. 11. germ. 18. t. 60.

Stazione, Abitazione e Fioritura. - Nelle siepi, nei boschi, ed altri luoghi ombrosi freschi di tutta la Penisola, e delle grand isole, comune assai, e fa anche nelle piccole isole di Malta (finlia!), di Capri (Paş.), d'Ischia Bolle!) e dell'Elba!!. In Sicilia s'inalza nei monti fin nella regione boschuva più elevata (Ciuss. syn.); nell'Italia centrale si ferma in generale a metà dei monti, ussa a poco piu di 1000 metri; nella settentrionale é stata trovata m P'ienonte presso Vinadio (Reuter!), ch'i a 912 metri, s'inoltra nel 'Tricino presso Locarno (Com. 1l. com.), pressu Gandria sul lagno de lagano, e in valle Anzasea contermine al Vallese (liant. h. helv., in Valtel. lina a Nontagna e Trevisio (Mass. prodr. H. cult.), in Tirolo verso liondu, Bolyano, Merano (llansm. M. Thr.), intine nel firifi pare 
che non oltrepassi i dintorni d'Udine (Pir. fl. for. syll.). Fiorisce in giugno e luglio.

Distribuzione geografica. - Nell' Europa meridionale, dal Portogallo al Caucaso, nell'Asia occidentale, nell'.Africa settentrionale.

Descrizione. - Læte viridis, vix odora. Folia patentia, subtus pallide viridia, supra sulcis nervis in pagina inferiore prominentibus respondentibus. Petioli suhcernui, teretes, pilosi, virides. Calyx pilosus, viridis, nervoso-angulatus, bilabiatus, labio superiore lato, ascendente, tridentato, dentibus brevibus mucroniformibus, labio inferiore corollæ adpresso, dentibus 2 lanceolatis aristatis, apice parum convergentibus; tubus extus et fauce pilosus, basi intus glaber. Corolla alba, calyce vix duplo longior; tubus curvus, superne parum dilatatus; labium superius brevius, ovali-orbiculare, concavum, extus puberulum, apice emarginato-bilobum, lobis erecto-retroflexis, labium inferius 3-lobum, lobis lateralibus ovalibus, patentibus, glabris, intermedio majore ovali-orbiculari, apice subdependente, prope basim pilosulo. Corollæ tubus intus sparse pilosus. Stamina 4, didynama, sub galea. Filamenta tubi parte superiore inserta, erecta, apice incurva, glabra, alba. Antheræ luteolæ. Pistillum staminibus brevioribus longius, longioribus brevius. Ovarium parvum, 4-lobum, lobis distinctis, ovalibus, albidis, glabris, disco crasso, ovario æquilongo, albo insidentibus. Stylus filiformis, albus. Stigna bifidum, laciniis subæqualibus, longis, recurvis. ( Parl. ms., descr. della pianta del monte Catria nelle Marche).

\section{HORMINUM.}

Horminum Linn. hort. cliff. p. 309. Gen. pl. fl. germ. 6.t. 10. Benth. Hook. gen. pl. 2. p. 1103. Ces. Pass. Gib. comp. fl. ital. p. 305. t. $48 . f .6$.

Calyx campanulatus, valide 13-nervatus. Corolla manifeste bilabiata, labio superiore erecto, planiusculo. Stamina 4, conniventia, filamentis apice denticulo auctis, antheris 2-locularibus, loculis longitudinaliter dehiscentibus, confluentibus. Stylus lobis subæqualibus. Cocci apice convexi leves.

Portamento. - Vedasi la descrizione dell'unica specie del genere. 


\section{Iоrminum pyrenaicum.}

Horminum pyrenaicum Linn. sp. pl. ed. 1. p. 596. Mass. prodr. fl. valt.p. 88. Bert. fl. ital. 6. p. 235. Koch syn. fl. germ. ed. 2. p. 615. Com. fl. com. 4. p. 378. Hausm. 1. Tir. p. 682. Rota prosp. fl. Berg. p. 69. Pir. fl. for. syll. p. 111. Car. prodr. fl. tosc. p. 509. De Vis. Sacc. cat. piant. Ven. p. 135. Zersi prosp. piant. Bresc. p. 165. Archb. fl. Allo-Serch. p. 60. Ces. Pass. Gib. comp. fl. ital. p. 305. Anzi auct. fl. nov.-com. p. 196. Arc. comp. fl. ital. p. 543.

Figure. - Lam. ill. t. 515. Reich. ic. fl. germ. $t .59$.

Stazione, Abitazione e Fioritura. - Nei luoghi erbosi asciutti della catena orientale delle Alpi, dalle Carniche alle Retiche : abbonda nel Friuli (Pir.), cosi a Tolmezzo ed Ampezzo (A. M. Smith!); fa al monte Cavallo del Bellunese (Kellner I), alle Vette di Feltre, ai monti Vallerana e Grappa, ai monti Bassanesi, nel monte Portole, a Mariana (Bert.), a Recoaro (A. M. Smith!), nel Baldo da 1500 a 2000 metri (Bracht!); è comune pure sui monti calcarei del Tirolo, cosi in Pusteria sull' Höhlenstein tra 3800 e 5500 piedi (Ausserdorfer!), presso Peitelstein, sul Kolkfus e sull'Enneberg, a Welsberg, sui monti di Brunecco, e di Bressanone (Hausm.), sull' Odai e il Daroi (Bert.), in Fassa e Fiemme, sul Bondone, sullo Spinale (Perini!), sul Tonale a 2000 metri (Parl.!), in Valsugana sul Manasso e il Civerone, nelle Giudicarie, al lago di Garda (Hausm.) ecc. ecc.; frequente pure nel Bresciano, da S. Eusebio su pel Dragone (Zersi), nei monti Gerle, Paio, Corna Blacca, Ario e Dos alto da 1400 a 1900 metri (Parl.!); net Bergamasco al lago d'Iseo (Bert.), sul monte Epolo di Schilpario (Parl.!), a Vilminora in val di Scalve (Bert.), a Valleve in val Brembana (Rampoldi!); al lago di Como, nelle valli Sassina e Cavargna, sui Corni di Canzo (Com.), sul Resegone (Aiuti!) e il Barro (Parl.!), e discende quasi alle spiaggie del lago sulle Grosgalle, a Griante e Rezzonico (Com.), ecc.; piủ rara in Valtellina, vi si trova sullo Stelvio a 2000-2400 metri (Parl.!), sul Braulio dal piede alla cima (Com.), in valle Zebrú, valle di Rezzola, monte Cristallo (Anzi); rara pure nel Tíino, vi si trova sul Camogheo (Com.) e sul Calbege (Kiveh, Bert). Recentemente è stata scoperta dal signor Burnat nelle Alpi Marittime, sul colle della Garbella tra Entraque e Pallanfre. II Bertoloni l'indica nelle Alpi Apuane, alla Tambura e ai Vetulini, ma vi dev'essere molto rara, perchè non ne ho veduto esemplari raccolti dipoi. Fiorisce da giugno ad agrosto. 
Distribuzione geografica. - Nelle Alpi, da Salisburgo e la Carniola alla Svizzera e agl'indicati luoghi d'Italia; nei Pirenei, e nella Castiglia.

Descrizione, - Pianta perenne, alta da 1 a 2 decimetri e mezzo e talvolta fino a tre decimetri, pubescente, fornita di un rizoma quasi orizzontale, un po'obliquo, grossetto, legnoso, scuro, che manda in giì delle fibre radicali fibrillose e scure e all'apice una rosetta di foglie e un fusto o ramo fiorifero. Le foglie sono quasi carnosette, patenti, ovali o quasi orbicolari, ristrette alquanto alla base, o ivi quasi in cuore, in un picciòlo più lunga della lamina, larghetto, bianco, piano di sopra, convesso di sotto con i margini acuti e cigliati, appena allargato alla base ed ivi spesso di color lilla: tali fo. glie hanno il margine crenato e cigliato, con le crenature più piccole verso la base, sono di un color verde pallido di sopra con leggier solchi che corrispondono ai nervi di sotto, di un verde ancora più pallido di sotto ed ivi con un grosso nervo longitudinale sporgente da cui partono fin dalla hase dei nervi laterali, anch'essi sporgenti e verdognoli come il mediano, obliquamente diretti verso l'apice e il margine ma che si diramano per formare una rete rilevata nella foglia e mandare rametti che terminano all'apice di ciascuna crenatura. I fusti sono ascendenti o eretti, semplici, quadrangolari, o rossiccio-scuri o verdognoli, pubescenti, nudi ovvero con uno, due o tre paja di foglie piccole, opposte, patenti o patenti-riflesse, della stessa consistenza e colore delle inferiori ma sessili, allungate, ottuse o quasi acute, crenato-incise o intere nei margini. I fiori sono un po'pendenti, per lo piủ a tre a tre all' ascella di brattee opposte, in modo che in ciascun giro vi sono per lo più sei fiori e formano cosi una spiga lunga quasi quanto la parte inferiore del fusto o poco più corta e interrotta. Le brattee sono membranacee, rivolte in giù, ovate 0 ovali, acute e fornite all' apice di una punta, gradatamente da sotto in su un poco più piccole, intere, con ciglia lunghette, bianche. I pedicelli sono cilindrici, nutanti, più corti del calice, di color violetto scuro, pubescenti, con peli all'apice glandolosi come quelli della parte superiore del fusto. Il calice è un po'pendente, poi nel frutto rivolto in giù : è verde, di color violetto scuro alla base dove s'inserisce il pedicello, con molti nervi longitudinali, puberulo-glandoloso, tubuloso-campanulato, bilabiato, con il labbro superiore ascendente, tridentato e i denti corti, lanceolati, acuminati, e con il labbro inferiore bifido e le lacinie lanceolato-acuminate. La corolla è due volte piì lınga del calice, di color violetto-carico, puberula di fuori, con 
il tubo gradatamente più largo sino alla gola, fornito di dentro di peli lunghi e bianchi distribuiti quasi in due linee! che sono in fuori dei due stami piủ lunghi, bilabiato, con le labbra corte, il superiore un po' più piccolo e più corto, diretto in avanti, un po'concavo e smarginato, con ilobetti tondeggianti, l'inferiore trilobo, con i lobi quasi orbicolari, patenti, un po'disuguali, essendo l'inferiore un po' maggiore e smarginato. Gli stami sono quattro, didinami: $i$ due più lunghi quasi uguali al labbra superiore dal quale sono coperti, i due più corti quasi uguali al tubo, inseriti verso il terzo superiore del tubo della corolla. I filamenti sono filiformi, di un bianco-violetto, curvati ad arco in alto in dentro, glabri e forniti di un dente esternamente sotto dell'antera. Le antere sono biloculari con le logge di. vergenti in direzione interamente opposta e confluenti, avvicinate a paja e aderenti tra loro quelle degli stami della stessa altezza, di cn. lor violetto, glabre. Il polline è di color giallo pallido. Il pistillo è lungo quanto gli stami più corti. L'orario è piccolo, diviso in quattro lobi profondi, quasi tondi, verdognolo-giallicei, glabri, e inserito sopra un ginobasio carnoso, più alto e più grosso dell' ovario, verdognolo con macchioline violette. Lo stilo è lungo, filiforme, di color violetto chiaro. Lo stimma è bifido con le lacinie quasi lineari, un po'divergenti e violetti. (Parl. ms., deser. della piranla di Corna Blarca).

XV. MEIITTIS

Melittis Linn. gen pl. elt. 1. p. 172. Gen. pl. fl. germ. (i. 1. 2 i (2)). Benth. Hool. gen. pl. 2. p. 1205. Ces. Pass. Gib. comp. Il. Ital. p. 31.3. 1. 46. f. 3.

Calyx late campanulatus, obsolete nervatus. Corolla manifeste bilabiata, labio superiore suberecto, planiusculo. Stamina 4, comniventia, filamentis apice denticnlo anctis, antheris 2 -locularibus, loculis longitudinaliter debiscentibus, distinctis. Stylus lohis subrynalibus. Cocci apice convexi leves.

Portamento. - Liunica specie del genere $i$ fra le piii vistosp Lamiacee nostrali. E' un' erba pereune, di ndore non grato, pelnsetta, che da un rizoma ohliquo, definito manda polloni alti linn a mezzo metro, semplici, o ramosi in hasso soltanto, guarniti di loglir 
sul fare di quelle della Melissa, verdichiare. I fiori sono da 1-3 all'ascella delle foglie, sono assai grandi, rosei, inchinati.

Osservazione. - Irmisch ha studiato particolarmente la vegeta. zione di questa pianta, principiando dal suo germogliamento, che ne ha manifestato ipogei gli embriofilli, contrariamente a quanto si osserva in generale nella famiglia. Il suo lavoro è nella Botanische Zeitung pel 1858.

\section{Melittis Melissoplnyllum.}

Melittis Melissophyllum Linn. sp. pl.ed. 1.p.597. Mass. prodr. fl. valt. p. 113. Trev. prosp. fl. eug. p. 26. Pucc. syn. pl. luc. p. 313. De Not. rep. fl. lig. p. 324: Bert. A. ital. 6. p. 237. Com. fl. com. 4. p. 380. Sim. fl. alp. vers. p. 132. Car. prodr. fl. tosc. p. 514. Rota prosp. piant. Pav. p. 272. Hausm. fl. Tir. p. 685. Rota! prosp. fl. Berg. p. 69. Pir. fl. for. syll. p. 111. Ard. cat. pl. Ment. p. 29. Tassi f. prov. sen. p. 51. Cenno bot. Sien. p. 88. G. Bert. not. porr. p. 48. Veg. Porr. p. 88. Ard. fl. Alpes-mar. p. 302. Zersi prosp. piant. Bresc. p. 166. De Vis. Sacc cat. piant. Ven. p. 136. Mars. cat. pl. Cors. p. 114. Ces. el. piant. Maiell. p. 24. Archb. fl. AltoSerch. p. 60. Ces. Pass. Gib. comp fl. ital. p. 313. Cald. fl. fav. tent. p. 182. Arc. comp. fl. ital. p. 549. Gib. Pir. fl. Mod. p. 129. Cocc. fl. Bol. p. 383.

Melissa grandiflora Terr.! fl. Vult. syn. p. 137. Sec. rel Terra di Lav. p. 95. Quarta rel. id. p. 113.

Melissa sylvestris Ing. cat. sp. Mond. p. 50.

Figure. - Riv. pl. fl. irreg. monop. t. 21. Reich. ic. bot. t. 241, 242. Ic. fl. germ. 18. $t$. 1.

Stazione, Abitazione e Fioritura. - Nei boschi, principalmente montuosi, più o meno frequente in tutta la Penisola e in Sicilia. In Piemonte trovasi a Torino (Parl.!), a Mondovi (Ing.), ai Bagni di Valdieri (Ball!), a Riva di Sesia (Carestia!), a Villarboit (Malinver. ni!), nel Novarese, dove del resto si dice comune (Bir. fl. acon.), ad Arona (Ricca!); nel Cantone Ticino abbonda, nonchè nella provincia di Como (Com.) e in Valtellina (Mass., Com.), e trovasi allo Spluga (Bert.); altrove in Lombardia fa in molti luoghi del Pavese (Nocc. Balb. fl. tic. ecc.), nel Milanese a Cassano d'Adda (Rampoldi!), nel Bergamasco dov'è comunissimo (Rota), nel Bresciano parimente (Zersi), e fa nel Dos alto e sulla Corna Blacca a 1600-1700 metri (Parl.!); in Tirolo nasce a Marlinga, a Bolzano dov'è comune, 
e fa presso Klobenstein a 1400 metri, Salorno, Zambana (Hausm.), Trento (Perini!), Pergine, Levico, Borgo, Tione (Hausm.); nel Veneto nasce a Verona in val Pantena (Bracht!), nel Mlantovano (Bar. bieri!), negli Euganei (Trev.), nei Colli Berici, nel Bassanese, nei Sette Comuni, a Asolo (Bert.), nel Friuli a Fagagna, Faedis, S. Da. niele, Tolmezzo ecc. (Pir.); in Istria nasce a Trieste (Bert.) e Fiume (Müller!); in Romagna a Faenza (Cald.); e nell'Emilia a Bologna dov'è comune (Cocc. ecc.), fino alla Porretta (G. Bert.), e a Modena, fin sul Ventasso (Gib. Pir.). Abbonda nella Liguria (De Not.), dal Nizzardo dove scende da Tenda (Webb!) fin presso Mentone (Ard.), a Genova (Ardissone! ecc.); abbonda pure in tutta la Toscana, dalle faggete dell' Appennino fino al piè de' colli presso al littorale maremmano: manca perỏ nell'arcipelago Toscano. Ugualmente comune nelle Marche, vi nasce da Sassofeltrio (Bert.) ad Ascoli (Parl.!); nell'Abruzzo è stato trovato nel monte Costa presso Pizzoli (Bert.), e nella Maiclla (Pedicino! ecc.); e fa infine in Molise a Campobasso, e nel Gargano (Ten. syll ). A Roma è comunissimo (Webb! ecc.); in Campania fa ad Arpino, Pastena, Lenola (Terr.), Itri (Terr.!), Sant'Angelo di Castellamare, Trinità della Cava, Avvocatella, Sessa, Valle, Omignano (Ten.); in Basilicata sul Vulture (Terr.!); in Calabria a Palmi e S. Cristina (Arc.!). In Sicilia abbonda nei monti (Guss. syn.), e si trova segnatamente nella costa settentrionale da Palermo (Parl ! ecc.) a Tortorici (Citarda!). - In Corsica è rarissimı, essendovi stato trovato soltanto tra Bastia e Carilo (Mars.), e ad Orezza (Bert.). Manca del tutto in Sardegna. Fiorisce in maggio, giugno o luglio secondo i lunghi.

Distribuzione geografica. - Europa media e meridionale.

XVI. IRU UNUIA.

Brunella Tourn. inst. p. 182. t. 84. Mirb. in ann. mus. 15. t. 21. f. 15, 16 (Prunella). IIum. not. mon. in Ser. bull. bot. 1. p. 153. t. 7. Gen. pl. 1. germ. 6. 1. 91. Benth. Hook. gen. pl. 2. p. 1203. Ces. Pass. Gib. comp. R. ital. p. 311. t. 46. f. I.

Calyx tubulnso-campanulatus a dorso compressus, valide 10-et ultra nervatus. Corolla nanifeste bilabiata, labio superiore galeato. Stamina 4, conniventia, filamentis apice denticulo auctis, antheris 
2-locularibus, loculis longitudinaliter dehiscentibus, confluentibus. Stylus lobis suhæqualibus. Cocci apice convexi leves.

Portamento. - Piante perenni, grandemente rassomiglianti fra di loro, ramose, piuttosto basse, con foglie mezzane, intere o divise, e fiori grandetti, violacei o bianchi, in infiorescenze terminali compatte, cilindriche, fornite di larghe brattee semimembranose.

\section{Hewnella hyss@pifolire.}

B. foliis subsessilibus, lineari-lanceolatis, integerrimis, calyce dentibus lateralibus labii superioris intermedio productioribus, corolla $1 \frac{1}{2}$ centim. longa.

Brunella hyssopifolia Linn. sp. pl.ed. 1. p. 600 (Prunella). De Not. rep. fl. lig. p. 322. Bert. fl. ital. 6. p. 256. Gren. Godr. fl. Fr. 2. p. 703. Ard. cat. pl. Vient. p. 29. Fl. Alpes-mar. p. 303. Mars. cat.pl. Cors. p. 114. Ces. Pass. Gib. comp. fl. ital. p. 311. Arc. comp. fl. ital. p. 548.

Figure. - Reich. ic. bot. t. 243. Ic. fl. germ. 18. t. 22. f. 1.

Stazione, Abitazione e Fioritura. - In luoghi erbosi, campestri 0 salvatici, della Riviera di Ponente: nel Nizzardo, dov'è piuttosto comune (Durando!, Barla!, Ard. ecc.), ai colli di Bruis e di Braus (Reichenbach! ecc.), verso S. Remo a Camporosso (Panizzi!), sopra Porto Maurizio (De Not.), a Diano Cervo (Ricca!), a Ortovero (Bert.), nei monti di Savona (De Not.). Grenier e Godron la danno anche di Bastia in Corsica. Fiorisce in giugno e luglio.

Distribuzione geografica. - Nasce inoltre nella Francia meri. dionale e in Spagna.

\section{Brunella greandiflora.}

B. foliis petiolatis, ex ovato ellipticis oblongisve, integris denticulatis aut inciso-dentatis, calyce dentibus lateralibus labii superioris intermedio productioribus, corolla 2 centim. longa.

Brunella grandiflora Jacq. fl. austr. 4 (1776) (Prunella). Moench meth. p. 414 (1794). Mass. prodr. fl. valt. p. 146. Trev. prosp. fl. eug. p. 26. De Not. prosp. fl. lig. p. 322. Bert. fl. ital. 6. p. 252. Com. fl. com. 4. p. 388. Rota prosp. piant. Pav. 272. Hausm. fl. Tir. p. 698. Rota prosp. fl. Berg. p. 70. Pir. fl for. syll. p. 114. Ard. 
17. Alpes-mar. p. 303. Zersi prosp. piant. Bresc. p. 169. De Vis. Sacc. cat. piant. Ven. p. 140. Terr.? fl. Vult. syn. p. 139. Gib. Pir. fl. Mod. $p$ 132.

Prunella vilgaris grandiflora Linn. sp. pl. ed. 1. p. 600. Ces. Pass. Gib. comp. fl. ital. p. 311. Cocc. quart. rontr. fl. Bol. p. 16. Arc. comp. fl. ital. p. 549. Coce. A. Bol. p. 382.

Figure.-Reich. ic. fl. germ. 18. t. 22. f. 4, 5.

Stazione, Abitazione e Fioritura. - Nei pascoli e nei luoghi boschivi dei colli e dei monti dell'Alta Italia, dal loro piede finn ad una grande altezza: a Torino presso la Venaria e intoruo all'Eremo (Colla herb. ped.), attorno Susa, sul Cenisio vicino al lago (Parl.!), presso Bardonecchia (Aiuti!), in val d'Aosta presso Courmayeur (Webb!), a Novara (Bir. fl. acon.), nel Cantone Ticino a Lugano (Bert.), nel Comasco in Valintelvi (Bert.), in Tremezzina (Rampoldi!), sui Corni di Canzo a 1000-1500 metri, e sui monti Barro (Parl.!) e Resegone (Aiuti!), in Valtellina e Bormio a 1220-1400 metri (Parl.!), nel Bergamasco (Rota), nel Bresciano, frequente (Zersi), per esempio in val Camonica sotto Edolo a 670 metri, e a Ponte di legno a $15 \overline{5} 0$ metri, in val Trompia a 950-1500 metri (Parl.!), e a Rocca d'Anfo (Bert.), in Tirolo a Brentonico, Roveredo (Hausm.), Trento (Perini!), Tione, in Fiemme e Fassa, a Bolzano, sul Ritten fino a 1800 metri, a Merano, Laas, Taufers, Welsberg (Hausm.), e sul Brennero (Parl.!), nel Bassanese (Bert.), sulle Vette di Feltre (Hansm.), in Friuli a Faedis, Gemona, Venzone, " nelle alpi Carniche (Pir.), nel monte Slavnick presso Trieste (Bert.), negli Euganei (Kellner!), nel Modenese intorno a Modena, a Montese e Salto di Montese (Gib. Pir., Cncc.), nel Parmense al monte Prinzera (Passerini!), nel Pavese ai colli di S. Colombano (Nocc. Balb. fl. tic.), in Liguria nei monti del Bracco, dove abbonda, presso Genova nei monti Fasce (Bert.) e Gazzo (Beccari!), a Ormea (Ricca!), a Dolcedo (Berti!), e nel Nizzardo (Ard.). Il Terracciano dà poi questa pianta di Melfi al Sierro del Priore, che come si vede sarebhe una localita molto all'infuori della sua abitazione ordinaria. Fiorisce da gingno ad agosto .

Distribuzione geografica. - In Europa, dalla Svezia meridionale e dalla Danimarca alla Spagna, ai paesi Danubiani e alla Russia meridionale.

Descrizione. - Fusto rossiccio in hasso, verdognolo in alto, quadrangolare, angoli ottusi, talvolta con qualche pelo, grossotto, curvo e rivolto in su, fucce due un po'convesse e due scanalate al. 
ternativamente. Foglie opposte, patenti, avvicinate, un po'consistenti, ovali-bislunghe, ottuse 0 ottusette, intere o quasi intere nei margini che sono appena rovesciati ed hanno dei peli curvi diretti verso l'apice della foglia, di un verde pallido di sopra e con solchetti che corrispon. dono ai nervi di sotto, più chiare di sotto e ivi con un nervo longitudinale grossetto che poco sopra della base manda due nervi laterali anche longitudinali, un po' curvi, delicati che si perdono verso la metà della foglia: sono strette alla base in un picciòlo con un solco longitudinale stretto di sopra, convesso di sotto. Due foglie superiori sessili, erette alla base e ivi quasi scanalate dalla parte interna, erettopatenti nel resto, con qualche raro dente ottuso nel margine e con le solite ciglia. Spiga quasi bislunga stretta, con due brattee larghe, ovato-orbicolari, a un tratto acuminate, verdognole rossicce con vene in rete, con ciglia lunghe e fitte, pelose nel dorso verso la carena, più corte dei fiori. I fiori sono pedicellati, eretti o eretto-patenti. I pedicelli sono corti, molto più corti del calice, quasi schiacciati da sopra in sotto, larghetti, grossetti, verdognoli, peloso-irsuti per peli corti e bianchi di sotto. Il calice è granue, tubuloso-campanulato, con molti nervi alquanto sporgenti che rendono angolato il tubo e si prolungano nei labbri del calice il quale è corto, verdognolo-bianchiccio; è diviso in due labbri, grandi, due a tre volte più lunghi del tubo, quasi uguali in lunghezza tra loro, quasi piani, verdognolo-rossicci, il superiore più largo appena curvato in su nella metà superiore, con tre denti corti, l'intermedio quasi triangolare, i due laterali quasi rappresentassero la metà longitudinale di questo, tutti e tre acuti e con una piccola punta sporgente, l'inferiore è diviso fino al terzo superiore in due denti più profondi quasi due la. cinie, acuminate, dirette appena in su verso l'apice: i nervi sono piủ sporgenti e saldi nei denti laterali del labbro superiore e nelle lacinie dell'inferiore e in questo più pelosi ancora. La corolla è quasi il doppio più lunga del calice, di color violetto-scuro. Il tubo è quasi diritto, bianchiccio alla base, lungo quasi quanto il calice. Il lembo è diviso in due labbri quasi uguali in lunghezza, uno superiore diretto in su e in avanti, a guisa di un elmo, con un angolo o inginocchiatura, smarginato all'apice, con una serie di peli curvati in avanti nel margine superiore, un po'schiacciato lateralmente, uno inferiore trilobo, patente, con i lobi disuguali, l'intermedio maggiore dentato, i due laterali interi. Gli stami sono quattro, poco disuguali, paralleli, inseriti in alto del tubo poco piủ in sopra dell' anello dei peli. I filamenti sono ascendenti, un po' più grossi in basso, di color vio. 
letto, glabri, ed hanno all'apice dietro dell'antera una piccola sporgenza a guisa di un' appendice corta ed ottusa. Le antere restano na. scoste sotto l' elmo del labbro superiore; sono biloculari, con le logge ovali-bislınghe, ottuse alle estremità, divergenti, aprentisi per una fessura propria a ciascuna loggia, bianchicce. Il polline è bianco. Il pistiilo è piủ lungo degli stami. L'ovario è piccolo, quadrilobo, con i lobi ovali-tondi, lisci, verdognoli, aventi all'apice poche e piccole papille bianche: è sostenuto e circondato da un disco quasi tondo, piủ lungo dell'ovario, quadrilobo, con i lobi ottusi, alterni con quelli dell' ovario e piủ corti di questi. Lo stilo è molto lungo, un po'curvo in alto in avanti, filiforme, glabro, bianchiccio. Lo stimma è bifido con le lacinic quasi uguali, acute o divergenti-recurve. (Parl. ms., descr. della pianta del monte Barro).

\section{Brunella vulgaris.}

B. foliis petiolatis, ex ovato ellipticis oblongisve, integris aut dentatis, calyce dentibus lateraliljus labii superioris intermedio bre. vioribus, corolla $1-1 \frac{1}{2}$ centim. longa.

Brunella vulgaris Linn. sp. pl. ed. 1. p. 600 (part.) (Prunella). Bert. fl. ital. 6. p. 250; et auct.

Figure. - Tilli cat. hort. pis. t. 17. f. 2, 3. Reich. ic. fl.germ. 18. t. 22. f. 2.

$\beta$ laciniata, foliis, præsertim superioribus, inciso-dentatis partitisque.

Brunella vulgaris laciniata Linn. l. c. (Prunella).

Brunella laciniata Bartulini! cat. piant. Sien. p. 22 (1776). Bert. o. c. p. 254; et auct. plur.

Brunella alba Pall. in Bieb. R. taur-cauc. 2. p. 67; et auct. plur.

Figure.-Reich. ic. bot. t. 23S. Ic. fl. germ. l. c. f. 3.

Stazione, Abitazione e Fioritura. - Comune assai nei prati ed in altri luoghi erbosi, e la var. di preferenza nei luoghi asciutti, dalle pianure a parti altissime dei monti, per tutta la Penisola e le grandi isole, ma delle piccole non truvasi che in quelle dell' Elba, la var. (Marcucci!), di Capraia, la var. (Mor. De Not. R. Capr.), d'Ischia (Guss. enum. pl. Inarim.), e di Capri (Pası. R. ves.). Sale nelle Alpi a 11 10 metri ai Bagni di Valdieri (Parl. 1), sul Ritten in Tirolo sino a 1700 metri, a la var. sino a 1100 metri (IIausm. $f$. 
Tir.), e nell'Appennino in Toscana fin entro l'ultima regione boschiva che termina ai 1700 metri. Fiorisce da aprile e maggio insino all'autunno nei luoghi bassi, e in luglio e agosto nei piủ alti.

Distribuzione geografica. - In tutto l'emisfero boreale, freddo, temperato e anche caldo, e nell'Australia.

Descrizione. - Perenne, alta da 1 a 3 decimetri, di un verde chiaro. Rizoma obliquo, torto, quadrangolare che manda fibre radicali lunghette e delicate. Fusto ascendente 0 decumbente, radicante nei nodi inferiori, quadrangolare con due delle facce scanalate e due quasi piane alternativamente negli internodii, con gli angoli e in parte ancora le facce fornite di peli diretti in su, applicati, rigidetti e bianchi, semplice o ramoso, con i rami opposti ascendenti, o decumbenti come il fusto. Foglie opposte, patenti, e le superiori patentissime o patentissime-reflesse, inferiormente bislunghe-ovali o ovali, ottuse, tondeggianti in basso, e quasi ad un tratto ristrette nel picciỏlo, intere o con pochi e piccoli denti più manifesti verso la base, di un verde chiaro di sopra e ivi con solchi leggieri che corrispon. dono ai nervi di sotto, di un verde ancora più chiaro di sotto e ivi con un nervo longitudinale da cui partono pochi nervi laterali che si dirigono obliquaniente e un po'curvi verso il margine e l'apice, e si uniscono tra loro e mandano dei rametti ai denti del margine: sono glabre. Il picciỏlo è lungo quasi quanto la foglia, stretto, con un solco longitudinale di sopra, convesso di solto, verde-chiaro. Le foglie superiori sono gradatamente un po' più piccole, più lunghe, con il picciỏlo piủ corto o quasi sessili. I fiori sono distribuiti in una spiga ovale-bislunga, ottusa, fitta, accompagnata nella base da due larghe brattee, le quali sono poco più lunghe del calice, largamente cordatotonde, un po'convesse di fuori ed ivi con un nervo longitudinale che le rende quasi carenate, pelose di fuori, un po'concave di dentro, verdognolo-bianchicce con vene ramoso-reticolate e verdognole, cigliate e fornite all'apice di una punta lunga, quasi lanceolata, acuta e verdognola. Le altre brattee sono simili. I fiori sono per lo più tre all' ascella di ciascuna brattea, con un corto pedicello, grossetto, schiacciato d' avanti in dietro, di color verdognolo o violetto, un po'irto per peli corti. Il calice è tubuloso-campanulato, verdognolo in basso, vio. letto in alto, con molti nervi longitudinali, ispidetto, diviso sino alla metà in due labbri, uno superiore il quale è piano di sopra, come troncato all'apice ed ivi con tre piccoli denti quasi in forma di corta cresta, lontani l'uno dall'altro e con altri dentini o smerlature appena manifeste e irregolari intermedie, l'inferiore lungo quasi quanto il supe- 
riore ma più stretto, bifido, con le lacinie lanceolato-acuminate, quasi arestate e un po'convergenti e cigliato-scabre nei margini. La corolla è la metà circa piủ lunga del calice, di color violetto, piủ chiaro e quasi bianchiccio alla base del labbro inferiore. Il tubo è lungo quasi quanto il calice, piủ largo in alto, ivi violetto chiaro con righe più cariche, bianchiccio in basso, fornito di dentro yerso la metà di un anello di peli lunghi e bianchicci. Il lembo è diviso in due labbri, uno superiore diretto in su e in avanti, convesso e quasi con una carena ottusa nel dorso e in questa ispidetto, concavo d'avauti, quasi ovale peró piủ largo in alto, con il margine irregolarmente e leggermente smerlato. Il labbro inferiore è diretto in giủ ed in avanti, quasi uguale in lunghezza al superiore, trilobo con il lobo interme. dio un po' più lungo dei laterali e frangiato dentato nel margine verso l'apice, e con i lobi laterali tondeggianti, troncati all'apice, con corte e rade ciglia. Stami 4 , didinami, inseriti in alto del tubo. Filamenti un po'curvi in avanti, filiformi, violetti, glabri, forniti sotto del. l'apice di una appendice lesiniforme clì è piủ corta nei due stami più corti e più lunga nei due più alti. Antere biloculari, con le logge distinte, divergenti, schiacciate lateralmente, aprentisi ciascuna per una fessura longitudinale, violette, glabre. Polline bianchiccio. Pistillo poco più lungo degli stami più lunghi e del labbro superiore. Ovario diviso in 4 lobi o parti bislunghe, verdognole, glabre, lisce. Stilo lungo, un po'curvato in avanti, filiforme, bianchiccio, glabro, bifido all' apice con le divisioni quasi uguali, divergenti, acute. Acheni bislunghi, piani da una parte, convesso-angolati dall'altro, glabri, lisci, giallo-scuri, racchiusi dentro il calice thiuso e scaglioso. (Parl. ms., descr. della pianla di Grumone in Lombardia).

Osservazioni. - Ai caratteri distimtivi della varietà si possono aggiungere $\mathrm{i}$ fiori bianchi gialloguoli e non violacei, e la pelurie ab. bondante rigrida e non scarsa o mancante. Ma tanto per questi carat. teri, quanto per la divisione delle foglie, vi sono parecchie combinazioni, per cui oltre i due lipi estremi qui ricordati esistono altri intermediari.

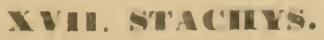

Stachys el Betonica T'ourn. inst. p. 186, 2919.1.86, 96. Linn. gen. pl. ed. 1.p. 174, 171. Mirb. in ann. mus. 15. 1. 18. 1. 6-15.

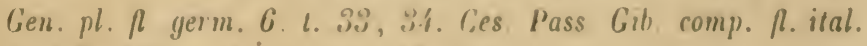
p. ¿̈/6. 315. 1 4\%. f. 1, t. 46. f. 6.

Stachys Benth. Lab. p. i25. Benth. Ilook. gen. pl. 2. p. 120 ' 
Calyx campanulatus, leviter 5-10-nervatus. Corolla manifeste 2-labiata, labio superiore erecto, concavo. Stamina 4, conniventia, anteriora post anthesin sæpius ad latera deiecta, filamentis edentulis, antheris 2-locularibus, loculis longitudinaliter dehiscentibus, confluentibus. Stylus lobis subæqualibus. Cocci apice convexi leves.

Portamento. - É vario. Una specie (Stachys glutinosa) è un frutice basso, ramosissimo; un'altrà ( $S$. corsica) è un' erbolina delicata, sdraiata; la più parte sono erbe annue o più spesso perenni, generalmente robuste, erette 0 ascendenti, di varia altezza, semplici o ramose, ora poco pelose verdi, ora moltissimo biancheggianti, con foglie di mezzana grandezza, di più forme fra l'ovata e la lanciolata, dentate, rugose. I fiori, che sono di varie tinte di rosso, di giallo o di bianco, sono piuttosto grandi, per lo più in glomeruli più 0 meno forniti, ora distanti fra loro ora ravvicinati in infiorescenze bislunghe 0 ovali, e accompagnati da foglie fiorali simili alle altre ma più piccole, o pure tramutate in vere brattee. I calici hanno costantemente i denti spinescenti all'apice.

Osservazione. - Oltre le specie registrate qui appresso, i due Compendi annoverano la Stachys spinulosa Sibth. Sn., Orientale, come italiana perchè dell'Istria. Siccome non danno altro schiarimento, io suppongo che si siano fidati per ciò all'autorità di Peichenbach nella $\mathrm{Fl}$. germ. exc.; il quale però dice semplicemente (1.p.318) che la pianta, oltrechè della Dalmazia, si "supponen di Pola in Istria. In mancanza di qualunque saggio Istriano, io non mi arrischio a mettere questa specie nelle flora italiana.

\section{Stachys Alopecuros.}

S. perennis, foliis petiolatis, cordatis, grosse crenatis, floralibus subito in bracteas lato-ovatas abeuntibus, vel nullis, floribus luteis, calyce campanulato dentibus subulato-spinescentibus, antheræ loculis parallelis.

Betonica Alopecuros Linn. sp. pl. ed. 1. p. 573. Mass. prodr. fl. valt. p. 27. Bert. fl. ital. 6. p. 139. Com. fl. com. 4. p. 336. Hausm. fl. Tir. p. 695. Rota prosp. fl. Berg. p. 70. Pir. fl. for. syll. p. 113. De Vis. Sacc. cat. piant. Ven. p. 138. Zersi prosp. piant. Bresc. p. 168. Terr.! sec. rel. Terra di Lav. p. 95. Ces. el. piant. Maiell. p. 24. Ces. Pass. Gib. comp. fl. ital. p. 316. Terr. quarta rel. p. 113. Arc. comp. fl. ital. p. 550.

Stachys Alopecuros Benth. lab. p. 531 . 
Figura. - Reich. ic. bot. t. 719.

Stazione, Abitazione e Fioritura. - Nei pascoli e nei luoghi boschivi delle Alpi e dell'Appennino, molto disugualmente distribuita. Delle Alpi occupa le catene orientali: nasce adunque nel Cantone Ticino nell'alpi di Cadro (Nuret!), nel monte Calbege (Bert.), e in valle Mesolina presso al S. Bernardino (Com.); abbonda al lago di Como (Com.), per esempio ai Corni di Canzo (Levier!), al Resegone (Aiuti!), al monte Barro a 800 metri (Parl.!), al monte Codeno (Daenen!), sul S. Primo e nelle Grosgalle presso Bellagio (Com.); si trova in valle Malenco nella Valtellina (Mass.); sembra comune pure nel Bergamasco, specialmente sul calcare e sul dolomitico (Rota), cosi a S. Salvatore (Bert.); ed è frequente nel Bresciano, cosi a Nave, S. Onofrio, nei monti Conche, Dragone (Zersi), Gerle a 1500-1600 metri, sotto il colle di Croce a 1600-1700 metri (Parl.!); nel Tirolo trovasi sui monti calcarei, donde scende qua e là nelle vallate: in Pusteria presso Welsberg e Peitelstein, e ad Ampezzo, intorno a Bolzano sui monti Mendola, Schlern e Seiser, e presso Ratzes, in Fassa e Fiemme, sull' Udai, nel Trentino sul monte Gazza, nel castagneto presso Sardagna e sull'alpe di Sardagna, sulle Vette di Feltre (Hausm.), in Valsugana (Ambrosi!), intorno a Roveredo, presso Stelle nelle Giudicarie (Ilausm.); nasce sul Baldo a 4-5000 piedi (Bracht!), agli Zocchi (Hausm.) e nelle valli Fredda, Novesa e dell'Artillon, nel monte Surriano del Vicentino (Poll. $\mu$. ver.) e nei Sette comuni, nei monti Vallerana e Grappa di Bassano (Mert.), nel monte Cavallo di Belluno, infine nel Friuli intorno a Tolmezzo, Amaro, Gemona e altrove (Poll.), e nel monte Guarda (Bert.). Passando sopra all'A ppennino settentrionale, comparisce nel centrale, da una parte nel Piceno-Abruzzo nel monte Pirro (Bert.), a Frondosa (Gernari!), nel monte della Sibilla alla Corona (Narzialetti!), nel Corno a Pietra Camela (Bert.), nel Velino (Cherici!), nel Morrone a 5000 piedi, nel Sirente a 5500-6000 piedi (Groves!), nella Naiella a Grotta Caprara (Ten. syll.), in valle Canella a 1800-2000 metri (Huet!); e dall'altra parte ma in vicinanza nei monti di Subiaco sulla sommità dell' Autore (Rolli!), e in Campania in vari luoghi intorno a Picinisco, e fra l'altri al monte Meta a 2500 metri (Terr.!), a Settefrati e a S. Biagio Saracinesco (Terr.). Fiorisce da giugno ad agosto.

Distribuzione geografica. - Nell' Europa media e meridionale dai Pirenei ai Carpazi.

Desorizione. - Pianta perenne, di un verde bianchiccio, pube. 
scente pelosa, alta da 3 a 4 decimetri, fornita di un rizoma obliquo, duro, legnosetto, nericcio, il quale manda molte fibre radicali grossette, giallicce $\mathrm{e}$ in alto $\mathrm{i}$ rami o fusti, che sono ascendenti o diritti, quadrangolari, duretti e pubescenti-pelosi con i peli bianchi, lun. ghetti e diretti verso giù. Le foglie inferiori nascono in cespuglietto dall' apice del rizoma e sono patenti, largamente ovate, ottuse, scavate in cuore alla base, con grossi denti ottusi nel margine, verdi di sopra ed ivi pubescenti e con solchi in rete che corrispondono ai nervi della pagina inferiore, di un verde bianchiccio di sotto ed ivi pelose e con nervi in rete assai rilevati e bianchicci, fornite di un picciòlo ch’è più lungo o quasi uguale alla lamina, solcato di sopra, molto convesso di sotto, verdognolo, peloso con i peli lunghetti, bianchi e rivolti verso giù. Le foglie del fusto sono per lo più due verso la base dello stesso, opposte, simili a quelle inferiori ma più piccole, talvolta mancano. Le foglie del primo verticillo fiorale ch'è Iontano dagli altri sono più piccole ancora, meno scavate in cuore alla base, sessili e rivolte in giù, del resto simili alle foglie del fusto. I fiori sono molti, distribuiti in una spiga fitta, allungata o quasi cilindrica, ottusa, formata da diversi verticilli, avvicinati tra loro eccetto l'inferiore ch' è molto lontano dagli altri. In ciascun semiverticillo sono da 7 a 8 fiori, sessili. Le brattee che accompagnano i verticilli superiori sono membranacee, patenti, ovate, ottuse o ottusette, quasi intere o poco dentate nei margini, verdi, pelose. Il calice è tuboloso, verde, peloso, ed ha cinque denti eretti, lanceolati, aventi all' apice un acume bianchiccio a guisa di una corta cresta, ha cinque nervi longitudinali più grossi che vanno ai denti e altri nervetti intermedii, longitudinali e uniti in alto tra loro. La corolla è quasi il doppio più lunga del calice, di color giallo pallido, glabra in gran parte di fuori, puherulo-glaudolosa di dentro. Il lembo è poco più corto del tubo, bilabiato con le labbra di fuori pelose; il labbro su. periore è eretto, ristretto in alto in un apice bilobo, con i lobi quasi ottusi, l'inferiore è trilobo, con il lobo intermedio quasi tanto lungo quanto il superiore, diretto in su, concavo dalla parte interna o superiore, quasi orbicolare e leggermente crenulato, e i due lobi laterali assai più piccoli, ovali e ottusetti. Gli stami sono quattro, quasi uguali o appena più corti del labbro superiore, visibili in gran parte di fuori, due esterni appena più lunghi dei due interni, inseriti nel tubo della corolla e rivolti in giù ad arco in fuori. I filamenti sono cilindrici, bianchi, pelosi, con peli orizzontali glandolosi. Le antere sono piccole, ovali, ottuse all'apice, bilobe alla base, inserite nel 
mezzo del dorso, poste quasi trasversalmente, biloculari con le logge aprentisi longitudinalmente, avendo le aperture dirette in avanti : sono gialle, glabre. Il pistillo è lungo quanto gli stami. L'ovario è piccolo, quadrilobo, verde, glabro. Lo stilo è lungo, un po' curvo in avanti verso alto, filiforme, glabro, bianchiccio. Lo stimma è bifido, con le lacinie disuguali, lineari, acute, divergenti e glabre. (Parl. ms., descr. della pianta del monte Gerle nel Bresciano).

\section{Stuchys densiflora.}

S. perennis, foliis petiolatis, ovato-oblongis, grosse crenatis, floralibus subito in bracteas ovato-lanceolatas abeuntibus, floribus rubris, calyce tubuloso dentibus subulato-spinescentibus, antheræ loculis divergentibus.

Betonica birsuta Linn. mant. p. 248. Hass. prodr. fl. valt. p. 27. P'ucc. syn. pl. luc. p. 306. Bert. fl. ital. 6. p. 138. Com. /l. com. 4. p. 334. Hausm. 17. Tir. p. 695. Rola prosp. R. Bery. p. 70. Car. prodr. A. 10se. p. 516. I'ir. fl. for. syll. p. 113. Ard. /l. Alpes-mar. p. 301. De Vis. Sacc. cat. piant. Ven. p. 138. Zersiprosp. piant. Bresc. p. 168. Archb. fl. Alto-Serch. p. 60. Ces. Pass. Gib. comp. A. ital. p. 316. Ansi aucl. fl. nov.-com. p. 196. Arc. comp. fl. ital. p. 551. Gib. Pir. fl. Hod. p. 131. Cocc. fl. Bol. p. 384.

Stachys densillora Benth. lab. p. 532. De Not. prosp. fl. lig. p. 526 .

Figure. - Reich. ic. bot. t. 7/4. Ic. fl. germ. 18. t. 15. 1. 1.

Stazione, Abitazione e Fioritura. - Nei pascoli delle Alpi, qua e là sparsa, na non comune: in Carnia al passo di monte Croce (Ball!), e nel monte P'izzul, in Friuli presso Ponteba (Pir.); sul lialdo in valle Fredda (.langanotti!), agli Zocehi, nella valle del. l'Artillon e altrove (Ilansm.), nel Tirolo in val di Ledro a 5 - 6000 piedi (Purta!), in Fassa (Bert.), in Primiero alla Neve, nelle Gindicarie sotto la cima del Frate (Hausm.) e alla Lanciada (Porta!), nel Bresciano e Bergamasco al Rondenino, a Prada, al Broflione alle Crocette (Zersi), al Tonale (Parl.!), in val Seve di val Brembana (Rampoldi!) ecc., nel Comasco nelle valli d'Agrneda sotto il Pizzo del diarolo, di Esino, nei monti S. Primo (Anzi) e Biandino (Bert.), nelle valli Carargna, Solda, Intelvi, Sissina, sul Camogheo e sul Bulia nel Cantone Ticino (Com.), nel bosen Valdone (Mass.) e a Sondrio (Bert.) in Valtellina; al Margozzo ucl Novarese (Bir. $\Re$. acon.), 
ad Oropa (Malinverni!), in val d'Aosta a S. Mar'cel e a Notre Dame de Rhemes (Carestia!), nel Cenisio al lago (Beccari!) e a Ronche (Parl.!), presso Susa alla casa d'Asti (Aiuti!), nelle vallate Valdesi (Rostan!), per esempio sopra Fenestrelle, nelle alpi di Vinadio, di Valdieri e di Viủ (All. fl. ped.); nelle Alpi Marittime alla Madonna delle Finestre (Bourgeau!), verso S. Martino Lantosca a 1200-1300 metri (Parl.!), verso Tenda (Reuter!) e altrove, scendendo a San Michele presso Eza a 2 chilom. dal mare (Ard.); infine a Mendatica sopra Porto-Maurizio (Gentili!), e a Capo di Noli secondo Viviani (De Not.). Sulle più alte cime dell'Appennino settentrionale, essendovi assai rara: nell'alpe di Mommio ai Cingi!, al Cimone di Fanano (Bert. ecc.), al Ventasso (Gib. Pir.), ai Quattro Frati nel Rondinaio (Giannini!), al Diaccion della Volpe (Parl!!), al Corno alle scale (P. Savi! ecc.), al Cimone di Caldaia (G. Bert. ecc.). Infine nell'Abruzzo a Forca-di valle nel monte Corno (Bert.). Fiorisce in luglio e agosto.

Distribuzione geografica. - Nell'Europa media-meridionale, dalla Spagna al Caucaso.

Descrizione. - Verde-chiara, pelosa, peli lunghi, bianchicci, curvati alquanto in giủ, articolati. Rizoma grossetto, scuro, fibre radicali molte, quasi porporine!, ramoso. Fusti ascendenti o eretti, quadrangolari, verdi chiari, pelosi, semplici. Foglie inferiori o radicali erette 0 eretto-patenti, bislunghe, ottuse, in cuore alla base, con larghi smerli, verdi di sopra, ivi solchi che corrispondono ai nervi di sotto, verdi pallide di sotto e ivi nervo longitudinale sporgente, bianchiccio, da cui nervetti laterali ramosi, un po'curvi, pelose, . poco di sopra e ivi molli. Picciòlo lungo quasi quanto la lamina, solco longitudinale di sopra, convesso di sotto, peloso, verde chiaro. Foglie del fusto opposte, lontane, simili alle inferiori più piccole, picciòlo più corto, supreme sessili. Fiori molti in spiga ovale, ottusa, fitta, non interrotta. Bratteole lunghe quanto il calice, erette, larghette, lanceolate, acuminato-arestate, fornite di ciglia lunglie con i peli nascenti da un tubercolo bianchiccio, sono verdi con l'aresta bianchiccia. Calice tuboloso, quasi gonfio, verdognolo, con molti nervi uniti tra loro in rete, con cinque denti, triangolari-acuminati e arestati, con l'aresta bianchiccia e lunghe ciglia bianche. Corolla grande, più del doppio più lunga del calice, di color violetto chiaro, con righe un po'più scure nel labbro inferiore e con il tubo bian. chiccio in basso, pelosetta di fuori. Tubo più lungo del calice, un po'curvo, appena più largo in alto, quasi il doppio più lungo dei 
labbri, di dentro con tanti peli corti, radi, grossetti, ottusi e bianchicci. Labbro superiore diretto in su ed in avanti, ovale-tondo, appena smarginato, concavo di davanti, convesso di fuori. Labbro inferiore lungo quanto il superiore ma piủ largo, diretto in avanti, trilobo, lobo medio quasi il doppio più largo dei laterali, con qualche dentino. Stami 4, due superiori poco più corti del labbro superiore e due un po' più corti degli altri, nella concavità del labbro superiore, inseriti quasi nella gola della corolla. Filamenti eretti, appena curvati all'apice in avanti, cilindrici, violetto-chiari, pubescenti-glandolosi per glandole piccolissime all'apice dei peli. Antere con le logge quasi ovate, schiacciate alquanto e divergenti, gialle-scure, glabre. Pistillo lungo quasi quanto gli stami piủ lunghi. Ovario diviso profondamente in 4 lobi, bislunghi, piani obliquamente di sopra e ivi pelosi. Stilo lungo, filiforme, appena curvato in avanti, bianchiccio in basso violetto-chiaro in alto, peloso sotto lo stimma. Stimma bifido, lacinie assottigliate, divergenti, quasi uguali. Calice con le lacinie patule e un po' spinescenti nel frutto. Fruttini ovali, tondeggianti all'apice, quasi piani di fuori, un po'convessi di dentro, lisci, con un margine bianchiccio, (Parl. ms., descr. della pianta delle Alpi Mariltime).

\section{Stachys Betonica.}

S. perennis, foliis petiolatis, ovato-oblongis, grosse crenatis, floralibus subito aut sensim in bracteas oblongo-lanceolatas abeunti. bus, floribus rubris, calyce campanulato dentibus subulato-spiuescentibus, antheræ loculis divergentibus.

Betonica officinalis Linn. sp. pl. ed. 1. p. 573. Dert. 1l. ital. 6. p. 135; et auct. omn.

Betonica hirsuta Ten.! syll. R. neap. p. 289 (non Linn.).

Stachys Betonica Benth. lab. p. 532. De Not. rep. /. lig. p. 326. Stachys officinalis Trev. prosp. Al. eug. p. 26.

Betonica vulgaris Rota prosp. piant. Pav. p. 272.

Figure. - Savi mat. med. veg. tosc. t. 15. Reich. ic. bot. 1 . 710-12. Ic. 1.. germ. 18. 1. 16. f. 1-3.

stazione, Abitazione e Fioritura. - Piủ o meno comune nei boschi e nei luoghi erbosi della Penisola settentrionale e media, dalle a 1520 metri a Ponte di legno in val Camonica (Parl.!), e sul litten in Tirolo fino a 1500 metri per lo meno (Hausm. $\mu$. Tir.); in Ligu- 
ria ; in Toscana, dov' è comunissima e che occupa dal littorale fino a tutta la regione boschiva dei monti; nelle Marche, dov'è stata trovata al Catria (Parl.!), ad Ancona (Paolucci!), S. Ginesio (Bert.), Ascoli (Parl.!) ecc., e nell'Appennino alla Sibilla (Bert.), e al Corno nell'Abruzzo (Orsini!), nonchè alla Maiella, e al Mattone di Pettorano ('Ten. syll.); a Roma, ancora comune (Seb. Nlaur. prodr.). Più giì si fa rara, essendo pure indicata a Cassino in Terra di Lavoro sul monte Cairo (Terr. rel.), in Basilicata ad Auletta, Sessa, Omignano al monte della Stella, e Potenza, a Ginosa e Lecce in Terra d'Otranto (Ten.), e in Calabria tra Lungro e Saracena a 2-300 metri (Huter Porta Rigo!), a Rosarno (Pasquale!) e sopra Bagnara (Bert.). Manca affatto nelle isole grandi e piccole. Fiorisce da luglio a settembre.

Distribuzione geografica. - In tutta l'Europa e nell' Asia Minore.

Descrizione. - Pianta perenne, alta da $1 \frac{1}{2}$ a 6 decimetri, verde, pelosa, fornita di un rizoma obliquo, che manda lunghe fibre radicali bianchiccie. Il fusto è eretto o ascendente, quadrangolare, verdognolo, peloso, con i peli lunghi, bianchi, diretti in giu, o glabro (Gren.), semplice. Le foglie inferiori sono eretto-patenti, ovali-bislunghe, ottuse, scavate in cuore alla base, crenate con le crenature piuttosto grandi, verdi di sopra ed ivi con solchi che corrispondono ai nervi di sotto, di un verde più chiaro di sotto ed ivi con un nervo longitudinale grosso da cui partono molti nervi laterali poco rilevati, i quali si dirigono un po'curvi verso il margine e si uniscono ivi ad arco mandaindo però dei rametti che fanno una rete a larghe maglie nel parenchima della foglia: sono fornite di un picciòlo il quale è molto più lungo e talvolta quasi uguale o più corto della lamina, ha un solco stretto di sopra, è convesso di sotto, verdognolo, pelosoo pelosetto, con i peli diretti in giù o avvicinati ad esso, un po'slargato alla base. Le foglie del fusto sono simili alle inferiori ma piủ piccole e con il picciòlo più corto della lamina, un po' più slargato alla base: sono per lo più due coppie lontane. Le foglie fiorali sono patenti, le inferiori di esse simili alle foglie superiori ma più piccole, le altre obovato-bislunghe, o bislunghe-lanceolate, ottuse o acute, intere, verdi, piủ lunghe o quasi uguali ai fiori. Questi sono per lo piủ tre, sessili all'ascella di ciascuna foglia fiorale e fanno con quelli della foglia opposta dei glomeruli, i quali sono arvicinati tra loro in una spiga terminale, bislunga, ottusa, delicata, interrotta spesso in basso. Le bratteole sono ovali-acuminate, quasi spinulose all' apice, un 
po' convesse e con un nerro longitudinale poco rilevato nel dorso, ivi verlognolo o sfumate di rossiccio, erette, lunghe quasi quanto il calice. Questo è tubuloso-canpanulato, peloso o talvolta glabro, verulognolo, con cinque nervi verdognoli che lo rendono appena angolato, e con cinque denti uguali, lunghi poco più del terzo del tubo, eretto-patenti, triangolari, lesiniforni-spinulosi all' apice: ha la gola fornita di lunghe ciglia rade e bianche, dirette in su. La corolla $\dot{e} d a$ due a tre volte piủ lunga del calice, di color violetto chiaro. Il tubo è quasi il doppio più lungo del calice, curvo in avanti, quasi cilindrico, poco più largo verso alto, pelosetto o peloso di fuori, peloso con lunghi peli di dentro ma senza anello di peli. Il lembo è diviso in due labbri, il superiore diretto in su, con l'apice un po'in dietro, poco più corto dell'iuferiore, ovale, quasi tondeggiante all'apice, intero o talvolta smarginato; l'inferiore è diretto in aranti ed un po' in giù, trilobo, con i lobi disuguali, quello di mezzo piủ largo e più lungo, obovato, crenulato, i due laterali quasi tondeggianti all’apice. Gli stami sono quattro, due poco più alti degli altri due, molto più corti del labbro superiore al quale sono arvicinati, inseriti nella parte superiore del tubo. I filamenti sono cilindrici, quasi diritti, hianchicci, pelosetti. Le antere sono biloculari, con le logge quasi parallele, bislunghe, scure, glabre, con poche glandolette quasi tonde, grossette e bianche attorno al punto d'inserzione nel filamento: si apron per una fessura comune. Il pistillo è lungo quanto gli stami. L'ovario è diviso in quattro lobi, tondo-ovali, verdognoli, glabri, ha un disco circolare poco alto. Lo stilo è lungo, filiforme, un po' curvato in a vanti verso alto, bianchiccio, glabro. Lo stimma è bifido, con le lacinie ottuse, bianchiccio. (Parl. ms., descr. di pianta della Brianza).

Osservazioni. - Specie molto variabile per la pelurie, scarsa o più o meno abbondante, e soprattutto per l'infiorescenza, ora fitta raccorciata, ora diradata allungata: per cui è stata divisa da taluni botanici in 405 specie, da altri perfino in 25 , senza che se ne possano distinguere le molte forme neanche in varietà bene stacrate.

Balla specie precedente si differenzia ottimamente anche per la grandezza del calice, ch'è lungo mezzo centimetro, mentreché nella Stachys densiflora è lungo più di 1 centimetro.

Non si puó sapere cosa sia la Belonica foliosa di Presl, da Bentham riferita come sinnonimo alla presente specie, che come si è detto non nasce affatto in Sicilia. 


\section{Stachys Teraclen.}

S. perennis, foliis petiolatis, ovato-oblongis oblongisve, crenatis, floralibus sensim in bracteas late ovatas integerrimas abeuntibus, floribus rubris, calyce tubuloso-campanulato dentibus ovatis spinescentibus, antheræ loculis divaricatis.

Stachys Heraclea All. fl. ped. 1.p. 31. Bert.! fl. ital. 6.p. 152. De Not. rep. fl. lig. p. 326. Car. prodr. 月. tosc. p. 517. Tassi fl. prov. sen. p. 51. Ard.l cat. pl. Ment.p. 29. G. Bert. not. porr. p. 48. Veg. Porr. p. 89. Ard. fl. Alpes-mar. p. 300. Terr.! fl. Vult. syn. p. 139. Mars. cat. pl. Cors. p. 114. Ces. Pass. Gib. comp. fl. ital. p. 317. Cald. fl. fav. tent. p. 183. Arc. comp. 1. ital. p. 553. Cocc. fl. Bol. p. 385 .

Figure, - All. o. c. t. 84. f. 1. Reich. ic. fl. germ. 18.t. 8. f. 1 .

Stazione, Abitazione e Fioritura. - Qua e là nei luoghi aridi sassosi, quasi sempre di collina e di montagna, dell' Italia media e meridionale. É frequente presso Bologna (Bert., Cocc.), e risale la valle del Reno sino a Granaglione (G. Bert.); e nasce presso Faenza a Quartolo (Cald.) e al monte Mauro (Bert.). Nella Riviera di ponente è comunissima da Nizza a Savona (De Not.), scendendo da Tenda fin sopra Nizza (Barla! ecc.) e Mentone a 900-1100 metri (Ard.!), e cosi sopra S. Remo (Berli!), Diano (Ricca!) ecc.; nella Riviera di levante è indicata nel monte di Portofino (De Not.). In Toscana scarseggia, vi è stata trovata a Sarzana (Cand. $f l . f r$.), in Mugello (Bert.), in vari luoghi attorno a Firenze!, in Chianti presso la Castellina (Beccari!), a Colle (Ant. Targioni!), nel Volterrano (Amidei!), a Radicondoli, e nél monte Amiata (Bert.). Quindi nasce a Canino presso Viterbo (Bert.), a Civitavecchia, a Nerola nel Romano (Rolli!), ad Ancorano nel Piceno (Orsini!), nel Gargano, nel Tavo. liere di Puglia, nel monte Vergine, nel Matese (Ten. syll.), ad Itri in Campania (Terr.!), ad Avellino, nel monte S. Angelo di Castellammare (Ten. fl. nap.), in Basilicata a Melli (Terr.!) e presso Castelgrande (Bert.), a Martina verso Taranto (Ten.), in Calabria presso i Bagni di Gerace, e verso Ciminà a 100-200 metri (Huter Porta Rigo!). Nasce in Corsica secondo Viviani (Bert.). Rara in Sicilia, vi si trova a Troina, a Nicosia al Molino delle mandre, e a Cesarò (Guss. syn.). Fiorisce in giugno e luglio. 
Distribuzione geografica. - Nell' Europa meridionale occidentale, cioè in Spagna, in Francia e in Italia.

Osservazione. - La pianta del Parmigiano rammentata nei Com. pendi spetta alla Stachys alpina, giusta un saggio autentico del Passerini stesso.

\section{Stachys germaniea.}

S. subperennis, foliis petiolatis, ovato-oblongis oblongisve, crenatis, floralibus sensim minoribus lanceolatis, floribus rubris, calyce tubuloso-campanulato dentibus ovatis aut ovato-lanceolatis acuminato-spinescentibus, antheræ loculis divaricatis.

Stachys germanica Linn. sp. pl. ed. 1. p. 581. Bert. fl. ital. 6.p.148; et auct. omn.

Stachys italica Mill. dict. Bert. o. c. p. 150. De Not. rep. fl. lig. p. 326. Koch syn. 月. germ. ed. 2. p. 652. Com. fl. com. 4. p. 342. Car. prodr. fl. tosc. p. 517. Tassi fl. prov. sen. p. 51. De Vis. Sacc. cat. piant. Ven. p. 137. Terr.! sec. rel. Terr. di Lav. p. 96. Ces. Pass. Gil. comp. 月. ital. p. 318. Groves! contr. fl. Terr. d'Otr. p. 65. Arc. comp. fl. ital. p. 553. Gib. Pir. A. Mod. p. 130. Cocc. A. Bol. p. 386.

Stachys polystachya Ten.! fl. nap.

Stachys salviaefolia Ten.! o. c. Terr. fl. Vult. syn. p. 139. Ces. el. piant. Maiell. p. 24. Terr. quart. rel. Terr. di Lav. p. 113. Stachys dasyanthes Raf. préc. Ces. Pass. Gib. l. c. Arc. l. c.

Stachys Janiana Ces. Pass. Gib. l. c. Arc. l. c. Gib. Pir. l. c. $\alpha$ viridis, foliis laxius villosis viridibus.

Figure. - Fl. dan. t. 684. Fl. nap. t. 53. Reich. ic. /. germ. 18.t. 9.f. $1,2$.

$\beta$ alba, foliis densius villoso-tomentosis incanis.

Figure. - Fl. nap. t. 54. Fl. grcec. t. 558 . Reich. l. c. f. 4.

Stazione, Abitazione e Fioritura. - Nei lnoghi aridi selvatici, nei luoghi incolti ecc., di tutta l'Italia. In Piemonte la si dice comune (All. $l$. ped.), peró non ve la trovo indicata che in provincia di Cuneo a Roascio (Ing. cat.) e a Roccavione (Parl.!), a Susa (Re), a Torino (Colla herb. ped.), e a Novara (Bir. /l. ac.); nel Cantone Ticino è stata trovata a Nendrisio (Com.); in Lombardia trovasi a Pavia (Rota!), a Milano (liampoldi!), presso Como, presso Morbegno in Valtellina (Com.), nel liergamasco (liota prosp.), nel Bresciano a Goletto e a Mlompiano (Zersi prosp.); in Tirolo nasce a Bolzano, Cal- 
daro, in val di Non a Fondo (Hausm. fl. Tir.), a Trento, ad Arco (Perini!), alla Scala di Primolano (Hausm.) e in qualche altro luogo; nel Veneto fa nel Veronese (Bert.), negli Euganei (Trev. prosp.), a Padova, a Chioggia (Bert.), a Venezia (Zan. prosp.), a Dignano in Friuli, qua e là nella Carnia (Pir. syll.); in Istria a Trieste (Bert.), a Fiume (Koch); in Romagna a Ravenna, Cervia (Bert.), Castrocaro (Cald.!), Faenza (Cald. tent.); in Emilia in parecchi luoghi del Bolognese (Gennari! ecc.), e in specie per tutta la vallata del Reno - sino a Granaglione (G. Bert. Porr., Cocc.), del Modenese, e fino all'alpe di Succiso (Gib. Pir.), del Parmense (Parl.! ecc.). In Lignria pare che sia rara, trovandosi soltanto a Nizza (Ard. fl.), al monte Baccelega presso Albenga (Bert.), nel Novese, e a Portofino (De Not.). In Toscana per contro è nolto comune, trovandosi da per tutto, dal littorale fino a tutta la regione boschiva dei monti; e nasce ancora nell'Elba (Car. stat. bot. Tosc.). Nelle Marche è stata trovata a Sasso Feltrio, a Sassoferrato (Bert.), nel Catria (Parl.! ecc.), a Serra S. Quirico (Bucci!), a S. Elpidio (Bert.), a Montefortino (Marzialetti!), ad Acquasanta!, sul Vettore al Castelluccio (Parl.!), sul monte Bove (Sang. cent.); nei monti Abruzzesi al monte dei Fiori, al Pizzo di Sivo!, al Corno, al Velino (Ten. syll.), alla Maiella, al Morrone (Levier! ecc.) ecc. In Umbria è stata trovata a Serravalle (Bert.), a Fossato (Parl.!); nel Lazio a Viterbo (Bert.), a Roma dov' è comune (Webb! ecc.); in Campania a Valvori (Terr.!), a Pastena (Terr.), a Cusano (Pasquale!), intorno a Napoli (Ten.! ecc.), a Salerno, Vallo (Ten.) ecc.; al Gargano a 100-400 metri (Porta Rigo!), in Puglia (Gussone! ecc.), ad Otranto (Groves!); in Basilicata a Melfi (Terr.!), a Potenza (Ten.), in Calabria a Pizzo (Ricca!), Giffoni (Pasquale!), Palizzi (Arc.!), ed altrove. Nasce qua e là in Corsica, a Bastia (Mlars. cat.), Corte (Kesselmeyer!), Campo di Loro, Bocognano, Vico (Mars.), Bonifacio (Jordan!); trovasi in Sardegna (Moris!); infine per la Sicilia in molti luoghi: presso Palermo alla Piana de’Greci, la Pizzuta (Parl.!), Busambra (Guss. syn.) ecc., presso Cefalù, a Castelbuono, nelle Madonie (Parl.! ecc.), a Mistretta, nei boschi di Caronia (Guss.), a Montalbano, Messina (Seguenza!), a Catania, Agosta, Lentini, Militello, Cassaro, Siracusa (Guss.), Avola (Bianca!), Ragusa, nel monte di Cammerata e altrove, nonchè in Felicuri nelle Eolie. Fiorisce in giugno e luglio, o in luglio e agosto secondo i luoghi.

Distribuzione geografica. - Nell' Europa media e meridionale, e nell'Asia Minore. 
Descrizione. - Pianta perenne, alta da 6 a 8 decimetri, tomentoso-lanata, bianchiccia, fornita di un rizorna grossotto, gialliccio, ramoso, che manda in giù fihre radicali, lunghette, giallicce e dall'apice dei rami dei fusti o rami eretli o un po'ascendenti alla base, semplici o di raro un po' ramosi, quadrangolari, con gli angoli ottusi, con le facce aventi un solco longitudinale larghetto, verdognoli-rossicci, coperti di un tomento quasi una lana bianchiccia. Le foglie sono opposte, le inferiori lontane, le superiori avvicinate alquanto tra loro, patenti, bislunghe, scavate in cuore alla base, crenate, rugose, molli, verdi di sopra ed ivi con leggieri solchi in rete, di un verde chiaro e quasi biancheggianti di sotto per un tomento piủ manifesto nei nervi, dei quali uno è longitudinale piủ grosso e gli altri che partono da questo sono obliqui molto e si dirigono verso l'apice e il margine della foglia: sono fornite di un picciỏlo il quale è gradatamente più corto nelle foglie superiori, scanalato di sopra, convesso-angolato di sotto, tomentoso-lanato e bianchiccio come il fusto. I fiori sono molti, piccoli, fitti in verticilli quasi come tanti capolini tondi, e formanti una spiga fitta, interrotta in basso. Le foglie fiorali sono quasi il doppio piủ lunghe dei verticilli inferiori, rivolte in giù, poco piủ lunghe o quasi uguali ai verticilli superiori e patenti: sono simili alle foglie del fusto ma gradatamente più piccole e ristrette alla base e ivi quasi sessili o sessili. Le bratteole sono strette, acuminate, quasi uguali o poco più corte del calice, lanatc. Il calice è verdognolo, coperto di una lana bianca, tubuloso, però un po'ristretto alla base, un po' curvo, i denti sono cinque disuguali, i due anteriori piu piccoli, i due di mezzo di mezzana grandezza e il posteriore o superiore più grande di tutti, eretti, poi un po'erettopatenti, triangolari-acuminati, forniti all' apice di un mucrone bianchiccio: la gola del calice è chiusa da una lana lunga e bianca. La corolla é piccola, di un color di rosa carico con la gola e il tubo bianchiccio: questo è lungo quanto il tubo del calice, un po'curvo, un po'gobbo di sotto poco più in su della base, glabro in questa, pelosetto nel resto, di dentro ha verso il terzo inferiore un anello di corti peli bianchi. Il labbro superiore è diretto in su, ovale-bislungo, smarginato all' apice, convesso di fuori ed ivi lanato per lunghi peli bianchi, concavo di dentro, con i margini appena rovesciati in dietro; il labbro infẹriore is lungo quasi quanto il superiore, diretto in giu, scanalato alla base, trilobo, con i lobi laterali piccoli, ottusi, e il terminale quasi orbicolare, tutti quasi interi nel margine. Gli stami sono didinami : i due piủ lunghi o esterni si curvano presto in giù 
e si seccano, $i$ due interni rimangono eretti. I filamenti sono grossetti, quasi piani, bianchi, con pochi peli a guisa di ciglia e aventi all'apice una piccolissima glandola. Le antere sono di un verde scuro, quasi nericce, glabre, con le logge opposte l'una all' estremità dell'altra aprentisi per una fessura longitudinale comune. Il polline è bianco. Il pistillo è più corto degli stami più corti. Lo stilo è filiforme, glabro, bianco; lo stimma è bilido, glabro, bianco, con le lacinie quasi uguali e divergenti. I frutti sono obovato-ovali, un po'schiacciati, glabri, lisci, verdognoli. (Parl. ms., descr. della pianta di monte Senario presso Firenze).

Osservazioni. - Ripetute investigazioni sulle piante vive, e l'esame comparativo di una vistosa serie di saggi secchi, mi hanno convinto appartenere a un medesimo tipo specifico la Stachys italica e la S. germanica, nonchẻ le altre meno generalmente separate come specie. All'infuori dell' aspetto diverso dato dalla pelurie abbondante bianca, o più scarsa e che lascia scorgere il verde della pianta, tutti gli altri caratteri differenziali addotti sono variazioni comuni alle due forme: a tal segno che gli autori hanno presa ora l'una forma, ora l'altra come $S$. italica, e viceversa come $S$. germanica. La durata della pianta è da uno a più anni, giusta le osservazioni di Irmisch (Die Keimung etc. der Labiaten, p. 77). Il fusto è alto da 3 decimetri a 1 metro, semplice o ramoso a pannocchia. Le foglie variano tra più larghe e piủ strette, appuntate o alquanto smussate, alla base arrotondite o pure cuoriformi. I glomeruli di fiori stanno fra loro piủ ravvicinati o più distanti, ed hanno le foglie fiorali più o meno larghe. I denti del calice variano anch'essi per la larghezza; ina in quanto a direzione sono sempre sostanzialmente a un modo, e cosi riguardo al loro margine ingrossato, nè questi possono essere caratteri differenziali come qualcuno ha pensato.

\section{Stachys alpina.}

S. perennis, foliis petiolatis, ovatis aut ovato-oblongis, crenatis, floralibus sensim minoribus ovato-lanceolatis, floribus rubris, calyce tubuloso-campanulato dentibus ovatis abrupte mucronato-spineseentibus, antheræ loculis divaricatis.

Stachys alpina Linn. sp. pl.ed. 1.p. 581. Mass. prodr. fl. valt. p. 182. Pucc. syn. pl. luc. p. 306. Bert. fl. ital. 6. p. 146. De Not. rep. 1l. lig. p. 327. Com. fl. com. 4. p. 339. Simi fl. alp. vers. p. 129. Rota prosp. piant. Pav. p. 272. Hausm. fl. Tir. p. 691. Rota prosp. 
fl. Berg. p. 70. Pir. fl. for. syll. p. 112. Car. prodr. fl. tosc. p. 517. Ard. fl. Alpes-mar. p. 300. De Vis. Sacc. cat. piant. Ven. p. 137. Zersi prosp. piant. Bresc. p. 167. Terr. sec. rel. Terra di Lav. p. 95. Archb. fl. Alto-Serch. p. 60. Ces. Pass. Gib. comp. fl. ital. p. 317. Ing. cat. sp. Mond. p. 69. Terr. quarta rel. Terra di Lav. p. 113. Arc. comp. fl. ital. p. 553. Gib. Pir. fl. Hod. p. 130. Cocc. fl. Bol. p. 385.

Figure. - Lap. fig. fl. Pyr. t. 8. Reich. ic. fl. germ. 18.t.8. f. 2.

$\beta$ nerpolitana, hirsuto-sericea.

Stazione, Abitazione e Fioritura. - Nei boschi, nelle siepi, nei prati di montagna, per quasi tutta la Penisola. In Piemonte nei monti di Frabosa, regione Fontane (Ing.), in Viosenne (Ricca!), nel monte Stella sopra i Bagni di Valdieri a 1200 metri (Parl.!), nella regione di Fossimagna (Re secondo Colla herb. ped.), a Riva di Sesia alla Balına (Carestia!), nel Torarese alle radici delle Alpi, comune (Bir. f. acon.); nel Cantone Ticino (Com.); in Valtellina (Mass.), nel Comasco (Com.), nel Bergamasco, sino al piano (Rota), nel Bresciano, frequente, cosi al Guglielmo, al Mufetto (Zersi), al monte Ario a $1600-$ 1700 metri, sopra Ivino a 1200 metri, al monte Gerle a $1300-1800$ metri, al Tonale (Parl.!); in Tirolo in Fiemme, in val di Rabbi, a Folgaria, sopra il Palazzo Cresseri (Hausm.), nel Baldo (Barbieri!) in val Fredda (Bert.), al Cerbiole, al Gambone e agli Zocchi (Hausm.), nel Bondone, nel Gazza (Perini!); nel Vicentino (Poll. $f$. ver.), nel Bassanese in Grappa, Colalti e Vallerana (Bert.), nel Bellunese al monte Cavallo (Kellner!), in Carnia presso Sauris, Sappadan, Forni di sopra e altrove (Pir.). Rarissima in Liguria, è indicata al col di Tenda (Ard.), e nell'appennino di Chiavari (De Not.). Nel versante settentrionale dell'Appennino trovasi in quello di Bobbio (De Not.), al Sarren, nel Monte Boglelio (Nocc. Balb. fl. tic.), sul monte Caio del Parmense e presso la Cisa (Passerini!), nel monte Orsaio!, in quello di Modena e di Bologna a Vallestra, nell'alpe di Faidello, a Fiumalbo, al Cimone, all' Acero (Gib. Pir.), presso Vidicciatico (G. Bert. !), a Rio maggiore, Lustrola, Bocca di Rio ecc. (Cocc.), nel Faentino in Valcoloreta (Bert.). In Toscana è piuttosto comune: nelle Alpi A puane sotto il monte Arni (Simi), nell' Appennino dall'alpe di Mlommio (Calandrini!) sino al Teso (P. Savi!) in parecclii luoghi, quindi a monte Senario (Ricasoli!) e a Vallombrosa!, in Casentino a Camaldoli (Parl.!), infine nell'alpe di Viamaggio in ral Tiberina (Amidei!). La var. è propria del mezzogiorno, nasce negli Abruzzi al Corno (Orsini!) a 
Cafornio a piè del Velino (Cherici!), e nel monte Amaro a Tavola rotonda, in Campania a Picinisco al monte Meta (Levier!) e al Passeggio dell'orso, e a Pietraroia sul monte Mutria (Terr.), in Basilicata a Potenza (Ten. syll.). Fiorisce in luglio e agosto.

Distribuzione geografica. - Nell' Europa media e in parte nella meridionale, dalla Spagna al Belgio e alla Penisola Balcanica, e nel Caucaso.

Descrizione. - Pianta perenne, alta da $\bar{j}$ a 8 decimetri, verde, pelosa e in alto peloso-glandolosa, odorosa, fornita di un rizoma corto, duro, scuro, ramoso che manda molte fibre radicali scurette e dall'apice dei rami delle foglie e dei fusti. Questi sono eretti o ascendenti, semplici, quadrangolari, rossiccio-scuri e irsuti in basso, verdognoli e peloso-glandolosi in alto, con i peli orizzontali e bianchi. Le foglie sono molli, le radicali erette 0 eretto-patenti, ovalibislunghe, scavate in cuore alla base, crenato-dentate, verdi di sopra ed ivi con leggieri solchi che corrispondono ai nervi di sotto, di un verde più pallido nella pagina inferiore ed ivi con un nervo lon. gitudinale rilevato da cui partono dei nervetti laterali molto obliqui clie si portano verso l'apice e il margine diramandosi e formando una rete rilevata nella stessa pagina inferiore: sono pelose e molli in am. bedue le facce e segnatarnente nella inferiore per peli bianchi, lunghetti e come articolati, hanno un picciỏlo più lungo o quasi uguale alla foglia, stretto, con un solco longitudinale di sopra, convesso di sotto, verdognolo, peloso. Le foglie del fusto sono opposte, un po' lontane, patenti o patentissime, le inferiori simili alle radicali ma un po'più grandi e con il picciỏlo un po'più corto; le altre ovali-lanceolate, acute, o quasi acuminate, con i denti quasi in forma di sega e con il picciólo molto più corto della foglia o cortissimo; le fiorali sono gradatamente più strette, più acuminate, con meno denti ma spesso più profondi nel margine, e sessili. I fiori sono cinque 0 sei all' ascella di ciascuna foglia superiore, in modo che in ogni glomerulo vi sono dieci o dodici fiori, patenti, quasi sessili o con un corto pedicello peloso, accompagnati da brattee patenti delle quali le esterne che corrispondono tra le foglie sono lanceolato-lineari, quasi uguali al calice e le interne setacee, più corte di questo. II calice è quasi campanulato, verdognolo-rossiccio, peloso-glandoloso, con dieci nervi longitudinali e con cinque denti patenti, ovato-triangolari, acuminati, quasi uguali tra loro. La corolla è poco piủ lunga del calice, di color roseo-violetto, bianchiccia verso la gola, bilabiata. Il tubo è lungo quanto il calice, quasi cilindrico, appena curvato in 
avanti, bianchiccio, glabro, pelosetto in alto, fornito di dentro di un anello di peli lunghetti, stretto e molto obliquo. Il labbro superiore è più piccolo dell' inferiore, diretto in avanti ed in alto, fatto a guisa di volta, obovato-ovale, barbato di fuori, con pochi e corti peli di sotto nella parte concava; il labbro inferiore è diretto in avanti ed in giủ, trilobo; con i lobi laterali più piccoli, quasi orbicolari, con il lobo medio quasi orbicolare e smarginato con le parti laterali dirette un po'in giù: tutti e tre i lobi e segnatamente i laterali sono barbati di sotto, quello di mezzo è pelosetto di sopra verso la base. Gli stami sono quattro, didinami, due appena più corti del labbro superiore e due un po'più corti degli altri, sotto la volta di quel labbro, inseriti verso la metà del tubo. I filamenti sono cilindrici, un po' curvati in alto in avanti, bianchicci, pelosetti. Le antere hanno il connettivo grosso, le logge divergenti e dirette verticalmente una in su una in giù, nericce, glabre. Il pistillo è lungo quanto gli stami più lunghi e talvolta anche supera la corolla. L'ovario è quadrilobo, con i lobi bislunghi, tondeggianti, verdi, glabri, circondato alla base da un disco circolare, quasi crenato, bianchiccio. Lo stilo è lungo, filiforme, glabro, bianchiccio. Lo stimma è bifido, con le lacinie quasi acute, divergenti, roseo-violette. (Parl. ms., descr. della pianta di Boscolungo nell' Appennino toscano).

\section{\%. Stachys silvatica.}

S. perennis, foliis petiolatis, ovatis, crenatis, floralibus subito in bracteas anguste lanceolatas abeuntibus, floribus rubris, calyce campanulato dentibus subulatis spinescentibus, antheræ loculis divaricatis.

Stachys silvatica Linn. sp. pl. ed. 1. p. 580. Bert.fl. ital. 6. p. 142 ; et auct. omn.

Figura. - Fl. dan. t. 1102.

Stazione, Abitazione e Fioritura. - Nei boschi, nelle siepi ecc., di tutta Italia. In Piemonte abbonda (All. fl. ped.), cosi a Mondavi (Ing. cat.), Torino, Susa (Re), Riva di Sesia (Carestia!), Novara (Bir. /l. ac.) ecc., e s'innalza nei monti, per esempio sopra i liagni di Valdieri a 1200 metri (Parl. !); in Lombardia nasce a Pavia (Nocea Balb. f. tic.), Melegnano, Levate (Rampoldi!), Como, nella ralle Intelvi (Com. M.), nella ralle d'Ambria in Valtellina (Mass. prodr.), nel Bergamasco (Rota prosp.), nel Bresciano, dov'è frequente ('Zersi 
prosp.), a Castelgoffredo (Bert.), Asola (Paglia!) ecc. ; in Tirolo sem. bra molto sparsa, trovandosi in val Venosta, a Merano, Bolzano (Hausm. fl.), Trento, sul Bondone (Perini!), in Fiemme e Fassa (Bert.), a Stenico, Roveredo, sul Baldo (Hausm.); nel Veneto ugualmente: a Bassano, Padova (Bert.), negli Euganei (Trev. prosp.), a Venezia (Kellner!), nel Friuli a Udine, Fagagna, S. Daniele, Tarcen. to ecc. (Pir. syll.); in Emilia pure: a Reggio (Bert.), Modena, Rocca di Montese, Fiumalbo (Gib. Pir. fl.), Bologna, e nei monti Bolognesi fino all'Acero, alla Porretta e a Castiglione ecc. (Bert., Cocc.); in Ro. magna nasce a Faenza (Caldesi!); quindi nelle Marche a S. Mlarino, Sasso Feltrio, Pergola (Bert.), al Catria a 1450 metri (Parl. !), al monte S. Vicino a 1000 metri (Bucci!), a Macerata (Bert.), Montefortino (Marzialetti!); e termina nell'Abruzzo al Pizzo di Sivo (Gemmi !) e alla Maiella nella valle dell' Orfenta (Ces. el.). In Liguria dicesi comune (De Not. rep.), e specialmente fa a Nizza, S. Martino Lantosca (Ard. fl.), S. Dalmazzo (Barla!), Rezzo (Berti!), Sestri ponente (Caldesi!). In Toscana è veramente comune in montagna, per tutto l'Appennino e le sue diramazioni, e fa eziandio nelle Alpi Apuane al Pizzo d' uccello e nell'alpe Gramolazzo (Ad. Targioni!), nel monte Amiata!, e secondo Santi (viagg.) nel monte Argentaro; piủ giủ nel Lazio è stata trovata a Canino (Bert.) e a Roma (Webb!), in Campania a Picinisco, nel monte Roia (Terr. rel.) e a Napoli (Bruni!, Pasquale!), nonchè nelle isole d'Ischia (Guss. enum.) e di Capri (Pasq. fl. ves.), nel Vulture (Terr. syn.), e si prolunga in Calabria a Pizzo (Arcangeli!). Manca affatto in Corsica e in Sardegna; ma si ritrova in Sicilia, nell' Etna alla contrada del Sambuco (Guss. syn.), e in parecchi luoghi della parte più settentrionale, da Messina a Palermo (Seguenza!, Todaro!, Parl.! ecc.). Fiorisce da giugno ad agosto, o da maggio a luglio secondo i luoghi.

Distribuzione geografica. - In tulta Europa, meno l'estremo nord e l'estremo sud, nell'Asia Minore, e fino alla Siberia e al Cascemir.

Descrizione. - Pianta di un odore sgradevole, perenne, alta da 3 a 5 o 6 decimetri e talvolta anche sino a un metro, di unverde un po' bianchiccio, pelosa, fornita di un rizoma che manda fibre radicali bianchicce e dei stoloni sotterranei. Il fusto è eretto, fistoloso, quadrangolare, con le facce leggermente scanalate, verdognolo, peloso, con i peli disuguali, bianchi ed in alto con altri peli glandolosi all'apice, semplice o con due rami in alto i quali sono eretto-patenti, poco più corti del fusto e portan soltanto i fiori e le foglie fiorali. 
Le foglie sono opposte, lontane, patenti, grandi, molli, cordatoovate, acuminate, verdi di sopra ed ivi con leggieri solchi corrispon. denti ai nervi di sotto, di un verde bianchiccio di sotto ed ivi con un nervo longitudinale da cui partono dei nervi laterali i quali si dirigono molto obliquamente e un po' curvi verso l'apice e il margine, diramandosi per formare una rete rilevata 1 ella pagina inferiore della foglia, tutti i nervi sono sporgenti e di un colore appena più chiaro della pagina inferiore: sono dentate nei margini con i denti grossi, un po'disuguali, ottusetti e forniti all'apice di una piccola punta quasi di un callo ottuso, hanno un picciòlo, il quale nelle foglie in. feriori è quasi uguale alla foglia, piủ corto di questa nelle superiori ed è profondamente scanalato di sopra, convesso di sotto, verdognolo, peloso. Le foglie fiorali sono sessili, le inferiori simili alle foglie superiori ma più piccole, più lunghe dei fiori, le superiori gra. datamente piu piccole, quasi uguali e poi piu corte dei fiori, ovato o ovali-lanceolate e acuminate, quasi intere o intere nei margini, tutte molto aperte 0 un po'dirette in giù massime nel frutto. I fiori sono per lo più tre, talvolta due, all' ascella di ciascunà foglia fiorale in modo che vi sono da 6 a 4 fiori in ciascun giro, sono forniti di un corto pedicello, e distribuiti in un racemo interrotto nella parte superiore del fusto e dei rami qualora questi vi sono; il racemo è piuttosto stretto e si allunga dopo la fioritura; i fiori medesimi sono patenti. I pedicelli sono molto più corti del calice, cilindrici, rossicci, pelosi. Il calice è tubuloso-campanulato, con dieci nervi, dei quali cinque meno sporgenti degli altri cinque, verdognolo-rossiccio, peloso con peli in parte glandolosi, con cinque denti lanceolati, acuminatospinosi, con una carena formata dal prolungamento del nervo corrispondente, quasi uguali tra loro, però i due inferiori curvati ad arco in giù e gli altri diritti o quasi diritti; nel frutto il calice $\dot{e}$ un po' gonfio nel tubo e ha i denti tutti aperti. La corolla è più del doppio più lunga del calice, di color porporino-violetto, con inacchioline bianche irregolari nel mezzo del lobo di mezzo e alla base dei lobi laterali del labbro inferiore. Il tubo è più lungo del calice, è cilindrico, stretto, bianchiccio e glabro quasi nel suo terzo inferiore, ivi ha uno stringimento e subito sopra questo ha un gobbo in avanti e da questo punto in poi è un po'più largo e del colore del resto della corolla, pelosetto di sopra con i peli corti, bianchi, diretti un po in giù : di dentro nello stringimento è un anello barbato con i peli bianchi; il labbro superiore è più curto dell' inferiore; liretto in su, ovale, intero, convesso e con molti peli lunghi e glandolosi nel durso, con- 
cavo e glabro davanti; il labbro inferiore è papilloso barbato nella gola, trilobo con i lobi laterali tondeggianti all'apice e più corti di quello di mezzo il quale è anche piủ largo, quasi orbicolare e appena smarginato. Gli stami sono quattro, due poco più lunghi degli al. tri due, quasi uguali al labbro superiore, eserti. I filamenti sono larghetti e quasi piani eccetto verso alto dove sono cilindrici e un po' curvati in avanti, pelosi sopra della metà, del colore della corolla. Le antere sono di un verde scuro e quasi nericce, con le logge opposte l'una all' estremità dell'altra, aprentisi per una fessura longitudinale comune. Il pistillo è lungo quanto gli stami. L'ovario è diviso in quattro lobi, ovali, verdognoli, glabri. Il disco è più corto dell'ovario, verdognolo. Lo stilo è lungo, filiforme, glabro, bianchiccio in basso, di color della corolla in alto. Lo stimma è bifido, con le lacinie quasi uguali, un po' divergenti, acute. I carpelli sono ovali, con piccoli tubercoli, neri. (Parl. ms., descr. della pianta del Casentino in Toscana).

\section{Stachys palustris.}

S. perennis, foliis fere sessilibus, oblongo-lanceolatis, crenatis, floralibus subito in bracteas lanceolatas abeuntibus, floribus rubris, calyce campanulato dentibus subulatis spinescentibus, antheræ loculis divaricatis.

Stachys palustris Linn. sp. pl. ed. 1. p.580. Bert. fl. ital. 6 . p. 144; et auct. omn.

Stachys ambigua De Not. rep. fl. lig. p. 327. Hausm. fl. Tir. p. 692. Rota! prosp. piant. Pav. p. 272. Prosp. fl. Berg. p. 70. Cald.! fl. fav. tent. p. 183.

Figure. - Fl. dan. t. 1103. Reich. ic. fl. germ. 18. t. 10. f. 1.

Stazione, Abitazione e Fioritura. - Nei luoghi paludosi, lungo i fossi ecc., della Penisola. E comunissima per tutta l'Alta Italia nei luoghi bassi, da dove si spinge nelle vallate alpine, cosi in Valtellina sino a Tirano (Nlass. prodr.), in Tirolo sino a Welsberg e a Laas (Hausm. fl.); e si prolunga nel lato orientale della Penisola per Tolentino (Ricci!) sino a S. Benedetto (Marzialetti!) e a Trisungo sopra Ascoli (Parl.!). In Liguria è indicata soltanto nel Nizzardo (Ard. fl.), ad Albenga e a Chiavari dove la si dice comune, e alla Spezia (Dc Not.). Per la Toscana è abbastanza comune, nel Lucchese (Calandrini! ecc.), nel Pisano, nel Fiorentino!, nel Volterrano (Amidei!), 
nel Senese (Bart.cat.), al lago di Chiusi (Santi viagg.); a Roma pure è comune (Rolli! ecc.); e ritrovasi in Campania a Sora, a Fondi (Terr.!), a Traetto (Terr. rel.), e sino al Sele (Ten. syll.). Fiorisce da giugno ad agosto.

Distribuzione geografica. - In Europa, eccettuata la più scttentrionale e la più meridionale, in Asia Minore, nell'Asia e l'America settentrionali.

Descrizione. - Pianta perenne, alta da 4 decimetri a circa un metro, verde, in parte ispidetta per peli duretti, rivolti in giù, fornita di un rizoma radicante e che manda degli stoloni lunghetti e delicati. Il fusto è eretto, quadrangolare, con gli angoli ottusi, rilevati, verde, spesso rossiccio alla base degl'internodii, ispidetto per peli rivolti in giù, più abbondanti negli angoli che nelle facce, ramoso, con i rami eretti. Le foglie sono molli, opposte, un po'lontane, patentissime, scavate in cuore alla base, allungato-lanceolate, acute, crenato-seghettate, verdi di sopra ed ivi puberule e con solchi corrispondenti ai nervi di sotto, di un verde pallido di sotto, ed ivi con un nervo longitudinale rilevato da cui partono dei nervi laterali molto obliqui $\mathrm{e}$ un po'curvi i quali si uniscono tra loro ad arco verso il margine e formano con i loro rami una rete rilevata in questa pagina inferiore, sono ispidette ivi, segnatamente nei nervi, fornite di un picciỏlo corto, scanalato di sopra ed ivi peloso, convesso ed ispidetto di sotto. I fiori sono per lo più tre all'ascella di ciascuna foglia fiorale, in modo che in ciascun verticillo vi sono sei fiori, talvolta ve ne $\dot{e}$ un numero maggiore sino a dodici: sono sessili, patenti e un po'divergenti tra loro e formano una spiga terminale, interrotta. Le foglie fiorali inferiori sono simili alle foglie superiori del fusto, peró quasi sessili o sessili, per cui superano più volte i fiori, le altre sono gradatamente più piccule, ovato-lanccolate, acute o acuminate, a poco per volta quasi uguali o più corte dei fiori. Il calice è tubuloso-campanulato, verdognolo-rossiccio, peloso, glandoloso di fuori, glabro di dentro, con dieci nervi un po'rilevati, cinque dei quali giungono sino all' apice dei denti e cinque ai seni lasciati da questi: con cinque denti quasi uguali tra loro, più corti del tubo, patenti, lanceolato-acuminati per un mucrone lesiniforme, corto. La corolla é più del doppio più lunga del calice, di color lilla chiaro con macchioline più cariche nel labbro inferiore. Il tubo è stretto alla base ed ivi glabro anche di dentro, allargato e quasi ventricoso nel resto ed ivi glabro in gran parte di fuori e peloso di dentro, fornito di un anello trasversale di peli lunghi nel punto intermedio. Il labbro superiore ¿̀ 
diritto, diretto in su, ovale, tondeggiante all' apice, concavo e glabro dalla parte interna, peloso-glandoloso di fuori, il labbro inferiore è piủ lungo e più largo del superiore, patente, diretto in avanti, trilobo, con il lobo mediano molto più grande dei laterali, quasi orbicolare, intero, i laterali quasi tondeggianti all'apice e diretti un po'in giù. Gli stami sono quattro, due appena più alti degli altri, appena, più corti del labbro superiore dal quale sono coperti, inseriti $i$ due esterni un po' piủ in basso dei due interni nel tubo della corolla. I filamenti sono grossetti, un po'curvi, pelosi verso la metà con peli orizzontali e papillosi, e con pochi peli glandolosi in alto distribuiti in fila: sono di color lilla scuro. Le antere sono biloculari, con le logge dapprima alquanto vicine tra loro, poi divergenti l'una alla estremità dell'altra, gialle, glabre, aprentisi per una fenditura longitudinale. Il pistillo è lungo quasi quanto gli stami. L' ovario è qua. drilobo, con i lobi ovali, di un verde pallido, glabri, inserito sopra un disco verdognolo meno alto di lui. Lo stilo è lungo, filiforme, un po'curvo, di color lilla; lo stimma è bifido, con le lacinie quasi uguali, divergenti, acute, dello stesso colore. (Parl. ms., descr. dello. pianta di Olmeneta in Lombardia).

Osservazione. - La Stachys ambigua trovata nell' Alta Italia, per esempio a Faenza (Cald.!), a Pavia (Rota!), a Milano (Rampoldi!), ad Alba (Bertero!), mi sembra appena una varietà a foglie un po' più larghe. Se la $S$. ambigua di Smith sia cosa veramente diversa, e, a quanto asseriscono, un ibrido della $S$. palustris e della $S$. silvatica, resta a indagarsi. Si noti che la $S$. ambigua d'Italia da me vista $\dot{e}$ pianta fruttifera.

\section{Stacliys consica.}

S. perennis, foliis petiolatis, ovato-rotundatis, inciso-crenatis, floralibus sensim minoribus angustatis, floribus rubicundis, calyce campanulato dentibus ovatis lanceolatisve spinescentibus, antheræ loculis divaricatis.

Stachys corsica Pers. syn. pl. 2: p. 124. Bert. fl. ital. 6. p. 154. Gren. Godr. 11. Fr. 2. p. 690. Mor.! fl. sard. 3. p. 305. Mars. cat. pl. Cors. p. 114. Ces. Pass. Gib. comp. fl. ital. p. 317. Arc. comp. fl. ital. p. 552 .

Figura. - Reich, ic. bot. t. 646.

Stazione, Abitazione e Fioritura. - In luoghi freschi dei monti 
di Corsica e di Sardegna, dove sembra abbastanza sparsa. In Corsica al capo Corso, all' isola Rossa (Gren. Godr.), al capo Revelata presso Calvi (Soleirol!), in valle di Restonica, a Corte (Mabille!), nei monti Rotondo (Soleirol!), d'Oro (Gren. Godr.), Renoso (Kralik!), a Guagno (Requien!), a Niolo (Labillardière!), presso Aiaccio (Bert.), nei monti Coscione (Jordan!), Cagna, Grosso (Bert.), in valle di Mollo, a Campolite, Tretore (Gren. Godr.), Portovecchio, al ponte dell'Oso (Mars.), a Bonifacio (Requien!), al monte della Trinita (Bert.). In Sardegna a Galtelli (Mor.), ai Sette fratelli (Gernnari!), ad Oliena, a Desulo, al Gennargentu, al monte Spada, fino a 1400-1500 metri (Mor.), sopra Aritzo (Ascherson!), alla gola di S. Giorgio sopra Osini nell'Ogliastro (Biondi!). Fiorisce da maggio a luglio, e talora rifiorisce in autunno (Mars.).

Distribuzione geografica. - Specie propria della flora corsosarda.

Descrizione. - Pianta annua, bassa, di un verde chiaro, pelosetta per peli radi, lunghi e bianchi, orizzontali o patenti. I fusti sono numerosissimi, sdraiati alla base ed ivi ai nodi con fibre radicanti, ramosi, con i nodi superiori lontani, delicati, quadrangolari, con gli angoli acuti, pelosi con peli radi, lunghi, bianchi e orizzontali. Le foglie sono molli, piccole, opposte, ovate o quasi tonde, scavate in cuore alla base, con sette o cinque crenature nel margine, di un verde chiaro di sopra ed ivi con solchi che corrispondono ai nervi della parte di sotto, di un verde più pallido di sotto ed ivi con nervi rilevati che si uniscono tra loro ad arco, sono pelose o pelo. sette in ambedue le pagine, e fornite di un picciòlo delicato, stretto, profondamente solcato di sopra, convesso di sotto, verdognolo e pelosetto, che è piủ lungo delle foglie inferiori e gradatamente quasi nguale o più corto delle foglie superiori, nelle quali è fornito di lmn. ghe e rade ciglia. I fiori sono solitarii o gemini (sec. Gr. e Godr.) all' ascella delle foglie, rivolti da una parte, piủ lunghi delle foglie fiorali, e forniti di un pedicello un po'flessuoso o contorto, appena o poco più lungo del calice, cilindrico, rerdognolo o in parte rossiccio, peloso. Il calice è tubuloso-campanulato, verde, con cinque angoli, peloso-irsuto di fuori, diviso per pii del terzo superiore in cinque denti patuli, quasi uguali, ovali, quasi acuti e terminati da una setola quasi una piccola spina. La corolla è molto più grande del calice, di un rosso-chiaro e in alcuni punti quasi bianchiccia con qualche piccola macchiolina più carica verso la hase del labbro inferiore. Il tubo è quasi il doppio piủ lungo del calice, appena curvo, Im 
po'schiacciato dalle parti, peloso di fuori, e fornito di dentro di un anello di peli quasi obliquo. Il lembo è diviso in due labbri molto disuguali; il labbro superiore è più piccolo, diretto in su, un po'concavo, quasi ovale, un po'stretto in basso, smarginato all'apice, peloso di dietro, glabro d'avanti, l'inferiore è patente, trilobo, con $\mathrm{i}$ lobi laterali piccoli e ottusi e il lobo di mezzo molto più largo e più lungo, quasi orbicolare, smarginato all' apice; è peloso di sotto, glabro di sopra eccetto verso la gola dove ha dei peli corti. Gli stami sono quattro, avvicinati al labbro superiore del quale sono molto più corti, due un poco più alti degli altri due, inseriti nella parte superiore del tubo. I filamenti sono eretti, quasi cernui all'apice dove in avanti portano l' antere ed ivi puberulo-glandolosi, roseo-bianchicci, pelosi in basso, i due piủ corti pelosi anche un po'piủ in su. Le antere hanno le logge un po'schiacciate lateralmente, poste l'una verticalmente sull'altra, rossicce scure, glabre, si aprono in avanti con una fenditura che va da una loggia all'altra. Il polline è gialliccio. Il pistillo è quasi uguale agli stami più corti. L' ovario è diviso in quattro lobi, ovali-tondi, un po'schiacciati nel dorso, di un verde chiaro, glabri, lisci e circondati alla base da un disco circolare intero, verde. Lo stilo è filiforme, quasi diritto, glabro, bianchiccio o in parte roseo. Lo stimma è bifido con le lacinie lineari, acute, divergenti, rosee. (Parl. ms., descr. di pianta coltivata nell'Orto botanico fiorentivio).

Osservazioni. - Questa pianta vien generalmente detta annua; ma Bertoloni e Moris già l'avevano riconosciuta perenne. A giudicarne. dai saggi seccati, dev'avere una vegetazione compagna alla Nepeta Glechoma; ossia i rami fioriferi eretti devono dopo la fioritura sdraiarsi per terra, e allungandosi mettere radici, e l'anno dipoi cacciare dalle lore ascelle foliari nuovi rami fioriferi.

Come tante altre Lamiacee, anche questa va soggetta a variazioni nella grandezza della corolla, che non sono peró di tanta entità da giustificare a parer mio la separazione di una varietà micrantha, come fu proposta da Bertoloni.

\section{Stachys mevensis.}

S. annua, foliis petiolatis, ovatis, crenatis, floralibus sensim minoribus angustatis, floribus rubris, calyce anguste campanulato dentibus lanceolatis spinescentibus, antheræ loculis divaricatis.

Stachys arvensis Linn. sp. pl. ed. 2. p. 814. Mass. prodr. $l$. 
valt. p. 182. Pucc. syn. pl. luc. p. 308. P. Savi fl. gorg. p. 273. Bert. f. ital.6. p. 157. De Not. rep. fl. lig. p. 327. Zan. prosp. $n$. ven.p. 26. Guss. enum. plant. Inarim. p. 263. Mor.! R.sard. 3. p. 306. Car. prodr. 1l. tosc. p. 518. Tassi fl. prov. sen. p. 51. Ard.cat. pl. Ment. p. 29. Car. fl. Montecr. p. 28. Ard. 1. Alpes-mar. p. 300. De Vis. Sacc. cat. piant. Ven. p. 138. Pasq. fl. ves. p. 81. Genn. 1l. Capr. p. 112. Mar's. cat. pl. Cors. p. 114. Archb. 1l. Alto-Serch. p. 61. Ces. Pass. Gib. comp. fl. itol. p. 317. Ing. cal.sp. Mond. p. 69. Arc. comp. fl. ital. p. 552.

Figure. - Fl. dan. t. 587. Reich. ic. Al. germ. 18. t. 11.f. I

Stazione, Abitazione e Fioritura. - Nei campi, nei luoghi erbosi di tutta Italia, ma molto disugualmente distribuita: presso San Michele-Mondovi (Ing.); lungo la Tosa in val d'Ossola, comune (Bir. fl. ac.); in Valtellina (Mass.); nel Mantovano, nel Padovano (De Vis. Sacc.), nel Lido veneto (Kellner!); a Nizza (All. l. ped.), Rocchetta, Mentone (Ard.), Oneglia (All.), Ceriale (Bert.), Pegli (Carrega!), Sestri ponente (Caldesi!), Chiavari (Bert.), e in generale comune in Liguria (De Not.); in Toscana a Calice verso Campi, comune (Bert.), a Viareggio (Ricci!), intorno a Lucca, pure abbastanza comune (Calandrini! ecc.), e ai Bagni di Lucca (Archb.), alla base del monte Pisano (Savi !), presso la Consuma in Mugello (Bert.), nel Grossetano a monte Pruno! e alla Porchereccia di Cupi (Santi viagg.), nelle isole di Gorgona (P. Savi!), di Capraia (Mor. De Not. 1.), dell'Elba a S. Rocco (Bert.), di Montecristo (Taylor) e del Giglio (Parl.!); nel Romano, rara, nei monti della Farnesina (Rolli!), e a Bravetta (Seb. Maur. prodr.); in Campania a Fondi, Terracina (Bert.), Roccamorfina ('Terracciano!), Sessa, intorno a Napoli in diversi luoghi (Ten. syll., Pasq.), nelle isole d'Ischia in molti punti (Guss.) e di Capri (Pasq.); in Corsica, ovunque (Mars.), a Bastia (Soleirol!), ad Aiaccio (Requien!); in Sardegna, pure frequente (Mor.), nell'isola di Caprera (Genn.); in Sicilia a Palermo (Parl.!), alla Piana de'greci, ad Amorosa (Guss. syn.), presso Castelbuono (Minà), a Patti, Milazzo (Guss.), Messiua (Seguenza! ecc.), liala, nelle Eolie, in Ustica, e in Pantellaria (Guss.). Fiorisce da febbraio, marzo od aprile, ad aprile, maggio o giugno secondo i luoghi.

Distribuzione geografica. - In tutta Europa, meno la più nordica e la più orientale, nell'Afirica settentrionale, c nelle due Americhe, ivi prohabilmente introdotta.

Descrizione. - Pianta aunua, alta da 1 a $\overline{3}$ decimetri, un po'irsuta, verde. La radice è delicata, ramosa, con molte fibre lunghe c 
scure. Il fusto è debole, ramoso alla base e come i rami giacente in basso per terra ed ivi talvolta mandante fibre radicali, poi ascendente o eretto, un po'flessuoso, un po'ingrossato sotto dei nodi vitali, quadrangolare, con gli angoli sporgenti e alquanto irsuti per peli piuttosto corti e alcuni più lunghi, tutti curvati ad arco in giù e bianchicci $o$ bianchi, con le facce piane, spesso rossiccie: tanto esso quanto i rami hanno dei rametti opposti, corti, eretto-patenti. Le foglie sono opposte, alquanto lontane, patenti, ovate o ovato-orbicolari, tondeggianti o appena in cuore alla base, ottuse o ottusette all'apice, smerlato-seghettate nei margini con gli smerli poco sporgenti, di un verde piuttosto chiaro di sopra ed ivi con solchi che corrispondono ai nervi di sotto, di un verde più chiaro di sotto ed ivi con un nervo longitudinale bianchiccio da cui partono dei nervi laterali i quali si dirigono un po' curvi verso il margine e l'apice per unirsi ad arco tra loro dopo avere mandato altri nervetti: tanto il nervo principale quanto i laterali e in parte i nervetti sono sporgenti; le foglie inoltre sono pelosette in ambedue le pagine e fornite di un picciòlo il quale è piủ corto della foglia, gradatamente più corto nelle superiori, piano di sopra, convesso di sotto, verdognolo o rossiccio chiaro e irsuto corne il fusto. Le foglie fiorali sono simili alle snperiori ma più piccole, e gradatamente più strette, ovato-bislunghe, o quasi ovali, il picciòlo è più corto: sono però sempre piủ lunghe dei fiori. Questi sono piccoli, per lo più da 6 a 12 all' ascella delle foglie superiori del fusto e dei rami e rametti, in modo che in ciascun verticillo vi sono da 14 a 20 e talvolta 24 fiori : questi verticilli sono piủ vicini tra loro in alto dei rami e dei rametti. I pedicelli sono delicati, più corti del calice, dapprima patenti poi un po cernui, cernui nel frutto, cilindrici, verdognoli e rosei, irsuti e accompagnati da bratteole! strette-lineari, patenti, verdognole e irsuto-cigliate, quasi uguali o più corte dei pedicelli. Il calice è tubuloso-campanulato, verdognolo o rossiccio, con dieci nervi sporgenti, irsuto, con cinque denti, tre superiori più larghi e piủ corti quasi triangolari e formanti quasi un labbro superiore, e due inferiori strettamente lineari-lanceolati, tutti acuti, ottusi all'apice e privi di spina o di mucrone, con lunghe ciglia nei margini. La corolla è piccola, la metà circa più lunga del calice, di color violetto chiaro quasi bianchiccio con due righe larghette più cariche nel labbro superiore e tre nell'inferiore. Il tulso è poco piủ lungo del calice, quasi cilindrico, poco più largo verso alto. Il labbro superiore è piccolo, rivolto in su, quasi orbicolare, smarginato, l'inferiore è poco più lungo, trilobo, con i lobi quasi 
orbicolari, quasi uguali ed interi, l' intermedio dei quali ha pochi peli grossetti, diritti verso la gola. Gli stami sono didinami: i due più lunghi sono poco più corti del labbro superiore, al quale tutti sono avvicinati. I filamenti sono diritti, un po'curvi all'apice in avanti, filiformi, bianchi, glabri: Le antere hanno le logge separate da un connettivo larghetto, sono rosee, glabre. Il pistillo ẻ lungo quanto gli stami più lunghi. L'ovario ha quattro lobi, bislunghi, ottusi, lisci, glabri, verdognoli; il disco è alto quasi quanto l'ovario, gialliccio. Lo stilo è filiforme, bianchiccio. Lo stimma è bislungo, piano d'avanti in dietro, ottusetto, curvato un po'in avanti. I carpelli sono eretti, ovali, ottusi, schiacciati un po'd'avanti in dietro, scuri, lisci, glabri. (Parl. ms., descr. di pianta coltivata).

\section{Stachys marrubiifolia.}

S. annua, foliis petiolatis, ovatis, crenatis, floralibus sensim minoribus angustatis, floribus rubris, calyce late campanulato dentibus lariccolatis spinescentibus, supremo valde maiore, antheræ loculis divaricatis.

Stachys marrubiifolia Viv. app. ad \%. cors. prodr. p. 3. Berl. $l$. ital. 6. p. 155. Guss.! enum. pl. Inarim. p. 262. Mars. cal. pl. Cors. p. 114. Ces. Pass. Gib. comp. 1. ital. p. 317. Arc. comp. R. ital. p. 552 .

Figura. - Guss. o. c. t. 11. f. 2.

Stazione, Abitazione e Fioritura. - In Corsica, alla Parata, e a Rogliano (Mars.). Nell'isola d' Ischia, sui margini erbosi arenosi solatii dei campi lungo un viottolo che intersecando le Chianole del Testaccio conduce al Vataliere (Guss.); e presso Napoli nei colli asciutti a Posilipo, rara (Ten. syll.). Fiorisce in aprile e maggio.

Distribuzione geografica. - Oltre gl'indicati luoghi d'Italia, dicesi che nasca anche in Algeria.

Osservazione. - Per quanto assai simile d'aspetto alla specic precedente, questa qui se ne distingue subito anche per i fiori molto più grandi.

\section{Stachys hirta.}

S. annua, foliis petiolatis, ovatis, crenatis, floralibus sensim minoribus angustatis et in bracteas lanceolatas integras denum abeun. 
tibus, floribus luteis, calyce late campanulato dentibus lanceolatoacuminatis longe setoso-spinescentibus, antheræ loculis divaricatis.

Stachys hirta Linn. sp. pl.ed. 2. p. 813. Bert. fl. ital. 6. p. 156. De Not.! rep. fl. lig. p. 328. Grech Del. fl. mel. p. 26. Mor. fl. sard. 3. p. 307. Car. prodr. A. tosc. p. 519. Tassi R. prov. sen. p. 51. Ard. cat. pl. Ment. p. 29. Fl. Alpes-mar. p. 301. Ces. Pass. Gib. comp. fl. ital. p. 316. Terr.! quarta rel. Terra di Lav. p. 114. Arc. comp. fl. ital. p. 551.

Figure. - Reich. ic. exot. t. 40. Ic. fl. germ. 18. t. 12. f. 1.

Stazione, Abitazione e Fioritura. - Nei luoghi asciutti, coltivati o erbosi, principalmente sul mare, dell' Italia media e meridionale. In Liguria pare che sia comune (De Not.), si trova a Nizza (Barla! ecc.), Villafranca, Monaco, Mentone (Ard.), Portofino (De Not.!). Più giù ricomparisce nella maremma Toscana, all'Alberese presso Grosseto (Parl.!), presso Talamonaccio, nel monte Argentaro (Santi viagg.), e presso Capalbio (Savi bot. etr.); quindi prosegue per il Lazio a Canino (Bert.), intorno a Roma dov'è comune (Rolli! ecc.), e per la Campania a Terracina (Bert.), Itri, Spigno (Terr.!), Salerno (Bert.). Nell'altro lato della Penisola comparisce a Pescara (Kuntze!), e si trova poi a Foggia (Bert.), e in Terra d'Otranto, e nella vicina Basilicata (Ten. syll.). Termina in Calabria dove nasce a Pizzo, Monteleone, Palmi (Arc.!), Bagnara (Ten.). Nasce in Sardegna a Sassari, e nell'altro estremo a Cabras, Cuglieri, Bosa (Mor.). In Sicilia a Palermo (Parl.! ecc.), Messina (Seguenza!), Avola (Bianca!), Girgenti (Aiutil). Infine nell' isola di Malta (Grech). Fiorisce secondo i luoghi da aprile a giugno, o da marzo a maggio.

Distribuzione geografica. - Nel bacino occidentale del Mediterraneo, e fino a Madera e alle Canarie.

\section{Strolnys annma.}

S. annua, foliis petiolatis, ovalibus lanceolatisve, crenatis, floralibus sensim minoribus angustatis, floribus albidis, calyce tubuloso-campanulato dentibus lanceolato-acuminatis vix spinescentibus, antheræ loculis divaricatis.

Stachys annua Linn. sp. pl. ed. 2. p. 813. Bert. fl. ital. 6. p. 159 ; et auct. omn.

Figura. - Reich. ic. fl. germ. 18. t. 11. f. 2.

Stazione, Abitazione e Fioritura. - Nei campi della Penisola, in generale comunissima dove si trova. In Piemonte ad Alba (Ber- 
tero!), a Mondovi, ovunque (Ing. cal.), a Torino (Delponte!), verso Susa (Re), a Novara, comune (Bir. $f l$.); in Lombardia a Pavia (Rota prosp.), presso Como, Villa-Albese, Erba, e nella Tremezzina (Com. fl.), a Bergamo (Rota prosp.), presso Brescia a Prati, frequentissima (Zersi prosp.); in Tirolo presso Welsberg, sul Ritten, in piu luoghi e in quantità, presso Pigenò, presso Castel Brughier, presso 'Tesero (Hausm. R.); nel Veneto sul lago di Garda (Rigo!), a Verona (Poll. fl.), nel Mantovano (Barbieri!), nei Sette Comuni del Vicentino, a Bassano (Bert.), negli Euganei (Trev. prosp.), nel Lido veneto (Kell. ner!), a Fagagna, Virco, S. Daniele (Pir. syll.); in Istria a Trieste (Bert.), Pola (Poll.), Fiume (A. M..Smith!); in Emilia ad Ozzano nel Parmense (Parl.!), a Mlodena, e su nei monti a Fiumalbo, sul Ventasso, sull'alpe di Succiso ecc. (Gib. Pir. fl.), a Bologna, abbondantissima (Bert.), e su nei monti alla Porretta (G. Bert. not.); in Romagna a Faenza, quasi da per tutto (Caldesi!); dall'Alta Italia scendendo poi lungo la costa orientale trovasi ad Urbino (Serpieri!), Senigallia (Ricci!), Perosara (Bucci!), Serra S. Quirico (Parl.), Sant'Elpilio (Rert.), Grottamare (Parl.!), Pescara (Kúntze!), Caramanico (Ces. el.). Nel lato occidentale della Penisola trovasi in Liguria nél Nizzardo a Drappo, Fontana, S. Martino Lantosca e al col di Braus (Ard. fl.), quindi a Diano (Ricca!), Borzi, Alassio, Genova (Bert.); in Toscana nel Lucchese a Partigliano e Casoli (Pucc. syn.), nel Pisano a Casciana (Bastianini!), nel Fiorentino, dove abbonda!, presso Radda nel Chianti (Beccari!), nel Senese, comune (Campani! ece.), ad $\Lambda$ rezzo (Pert.), a l'ratovecclio (Parl. !); in Umbria presso Fossato (Parl.!), e sotto Rieti (Rolli!); in Campania a Casalattico (Terr.!); infine nel Principato ulteriore (Bert.) e in Basilicata (Ten. syll.). Fiorisce da giugno e luglio sino nell'autunno inoltrato.

Distribuzione geografica. - Nell'Europa media, e nella meridionale eccettochẻ nella sua porzione piủ al sud, nell'Asia Minore.

Descrizione. - Pianta di 1 a 3 decimetri, verdechiara, puberula. Radice fusiforme, ramosa, flessuosa, gialliccia. Fusto eretto, quadrangolare, puberulo-peloso, con i peli corti, bianchi, curvati in gii,, verdognolo e in parte rossiccio, ramoso sin dalla base con i rami opposti, eretto-patenti. Foglie opposte, patenti e un po'curvate in giii, bislunghe ovate o quasi ovate, oltuse o ottusette, fornite di un picciỏlo lunghetto, le medie lanceolato-bislunghe anche ottusette, con il picciòlo più corto, le superiori quasi lanceolate, acute e sessili, tutte di un verde chiaro di sopra ed ivi con solchi corrispondenti ai nervi di sotto, pelosette, di un verde ancora piu chiaro di sotto ed ivi con 
un nervo longitudinale e con pochi nervi laterali molto obliqui, uniti tra loro in rete, tutti squisitamente e delicatamente rilevati, più pelose che nella pagina superiore con i peli corti, bianchi, curvati verso l'apice della foglia: sono dentate nel margine con $\mathbf{i}$ denti ottusi; il picciỏlo è stretto, scanalato di sopra, convesso di sotto, slargato alla base, ivi con lunghe ciglia, nel resto puberulo. Le foglie fiorali sono lanceolate, acute, intere, quasi il doppio più lunghe dei fiori. Que. sti sono per lo più 3 , talvolta da 1 a 4 all'ascella delle foglie fiorali, patentissimi, forniti di un pedicello cortissimo e accompagnati da una bratteola corta, filiforme, verdognola, pelosa. II calice è tubulosocampanulato, verdognolo, peloso con poche e piccole glandolette, con 10 nervi poco rilevati, con 5 denti lunghi, curvati quasi a guisa di falce, quasi uguali, lanceolato-lesiniformi, con tre nervi, una piccola spina all' apice, forniti di ciglia e pelosi sino all'apice della spina. La corolla è il doppio più lunga del calice, bianca o di un bianco şalliccio, con il labbro inferiore di un giallo chiaro, pubescente di fuori. Il tubo è ristretto in basso, si allarga poi in alto, è un po'schiacciato lateralmente, ha di dentro un anello di corti peli; il labbro superiore è curvato in avanti e poi in su, bislungo, ottuso, con il margine irregolarmente smerlato ed ondeggiante: ha talvolta davanti verso il margine una riga di color violetto chiaro interrotta a guisa di macchiolina; l'inferiore è poco piủ lung̉o del superiore, trilobo, con il lobo intermedio più grande, smarginato, con piccoli denti irregolari nel margine, i due laterali tondeggianti all'apice. Gli stami sono didinami, due poco più alti degli altri due, più corti del labbro inferiore, inseriti verso la metà del tubo. I filamenti sono filiformi, bianchi, peloso-cigliati in gran parte eccetto l'apice dove però hanno qualche peletto di dietro. Le antere sono scure, con le logge poste all'estremità l'una dell'altra, aprentisi per una fessura longitudinale comune, glabre. Il pistillo è più corto degli stami. L'ovario è piccolo, con i lobi ovali-bislunghi, quasi triangolari, glabri, bianco-rosei, con un disco circolare, piủ corto dell'ovario, quasi del colore di questo. Lo stilo è lungo, filiforme, glabro, bianco. Lo stimma è bifido con le lacinie quasi uguali, divergenti, bianche. (Parl. ms., descr. della pianta di Serra S. Quirico nelle Marche).

\section{Staelnys mareitimn.}

S. perennis, foliis petiolatis, ovalibus vel ovali-oblongis, crenatis, floralibus in bracteas lanceolatas integras abeuntibus, floribus 
luteis, calyce tubuloso-campanulato dentibus lanceolatis acutatis vix spinescentibus, antheræ loculis divaricatis.

Stachys maritima Linn. mant. p. 82. Bert. fl. ital. 6. p. 161. De Not.! rep. fl. lig. p. 328. Koch syn. fl. germ. ed. 2. p. 654. Gren. Godr. fl. Fr. 2. p. 692. Zan. prosp. fl. ven. p. 26. Pir. fl. for. syll. p. 112. Car. prodr. fl. tosc. p. 519. Ard. fl. Alpes-mar. p. 301. De Vis. Sacc. cat. piant. Ven. p. 138. Mars.cat. pl. Cors. p. 114. Ces. el. piant. Maiell. p. 24. Ces. Pass. Gib. comp. fl. ital. p. 317. Gro. ves contr. fl. Terra d'Otr. p. 65. Arc. comp. fl. ital. p. 552.

Stachys pubescens Ten.! Bert. l. c. Ces. Pass. Gib. l. c.

Figure.-Fl. nap. t. 239. f. 1. Reich. ic. bot. t. 484. Ic. /l. germ. 18. $t$. 12. f. 3.

Stazione, Abitazione e Fioritura. - Nelle arene marittime. Abbonda nella Riviera di ponente, trovandosi in moltissimi luoghi da Nizza (Ard.) a Sestri (De Not.!) ; quindi si presenta alla Spezia!, e prosegue in Toscana per la Marinella di Luni, S. Giuseppe (Bert.) e Migliarino presso bocca di Serchio!; di li con molte interruzioni passa successivamente a Civitavecchia (Seb. Maur. prodr.), a Terracina (Bert.), in Calabria (comp.), in Basilicata (Ten.!), ad Otranto (Groves) e Lecce, a Bari (comp.), a Pescara (Ten.!), e poi, facendosi più comune, a Porto d'Ascoli, Grottamare, Porto S. Elpidio (Bert.), Falconara (Paolucci!), Pesaro (Parl.!), Rimini, Ravenna (Caldesi!), Venezia (Kellner!), Grało, Monfalcone (Tommasini!), Trieste (Koch). Nasce in Corsica nelle vicinanze di Bastia, a Fiumorbo, a Bravoni(Gren. Godr., Mars.), e ad Aiaccio (erb. Webb!). Eziandio nell' Elba a Procchio (Marcucci!), e nella Pianosa (erb. centr.!). Fiorisce da maggio a luglio.

Distribuzione geografica. - Nell'Europa mediterranea.

Descrizione. - Radice semplice, robusta. Fusti numerosi, ascendenti, ripieni, vellosi. Glomeruli ravvicinati, gl'inferiori un po'distanti. Bratteole poche, subulate, piccole. Fiori quasi sessili, un po'inchinati. Calice a cono rovescio bislungo, di 8 millinetri, mollemente velloso e insieme glandoloso-peloso, con 10 nervature poco evidenti, con la gola nuda, 5-dentato fino a $1 / 3$ della sua lunghezza, coi denti eretti, lanciolati, acutissimi, pressoche uguali, il superiore essendo peró un po'minore. Corolla di un giallo pallido, infuori vellosetta, col tubo uguale al calice, diritto, compresso, gradatanente piu largo, fornito presso la hase di un anello peloso trasversale, col labbro superiore la metà più corto del tubo, eretto, ovale, concaso, dentellato in cima, col labbro inferiore un poco piu 
lungo, patulo, 3-fido, dal lobo intermedio arrotondato smarginato, e dai laterali più piccoli. Stami inseriti presso la gola, ascendenti, la metà più corti del labbro superiore, di uguale lunghezza fra loro, gl'inferiori buttati ai lati dopo la fioritura; filamenti compressi, lineari; antere degli stami inferiori gialle, dei superiori scure, glabre, dalle caselle ovali-globose confluenti, dal polline bianco. Disco uguale in giro. Stilo piủ corto degli stami, dai lobi subulati, uguali. Calice fruttifero rigonfiato campanulato. Cocchi 4 , grigio-neri, fissati per la loro base, colla cicatrice d'attacco bianca, lisci, lucenti, obovatiarrotonditi, trigoni, convessi nel dorso, ottusissimi, lunghi 2 millimetri (v. v. raccolta alla Spezia).

Osservazione. - Convengo pienamente coll'Arcangeli nel considerare la Stachys pubescens di Tenore una varietà, o piuttosto una mera forma della $S$. maritima. Il principale carattere distintivo, del tubo corollino più lungo del calice, si ritrova nelle forme ordinarie della $S$. maritima, variabile a questo riguardo per avere il tubo ora piủ corto del calice, ora uguale, ora più lungo, non mai però quanto nella figura di Tenore, ch'è un' esagerazione, e non corrisponde al saggio autentico di lui.

\section{Stachys recta.}

S. perennis, foliis fere sessilibus, ovali, oblongo aut linearilanceolatis, crenato-serratis, floralibus in bracteas ovato-lanceolates acuminatas abeuntibus, floribus luteis, calyce campanulato dentibus lanceolatis acuminato-spinescentibus, antheræ loculis divaricatis.

Stachys recta Linn. mant. p. 82. Bert. fl. ital. 6. p. 163; et auct. omn.

Stachys subcrenata De Vis.! in fl. 1829. Koch syn. fl. germ. ed. 2. p. 654. De Vis. Sacc. cat. piant. Ven. p. 138.

Stachys fragilis De Vis.! l. c.

Stachys labiosa Bert. o. c. p. 166. Rota prosp. fl. Berg. p. 70. Zersi prosp. piant. Bresc. p. 168.

Figure.-De Vis. fl. dalm. t. 16. f. 1, 2. Reich. ic. fl. germ. 18. t. 13. f. 1-4.

Stazione, Abitazione e Fioritura. - Per la Penisola, nei luoghi asciutti di ogni sorta, boschivi, erbosi, sassosi ecc., dal littorale a una grande altezza nei monti. $\dot{\mathrm{E}}$ comunissima in Piemonte (All. fl., ecc.), salendo sino al lago del Cenisio (Beccari!); nasce nel Cantone Ticino a Mendrisio (Com. fl.), e al Gottardo (Bert.); in Lom- 
bardia a Pavia (Nocca Balb. fl.), a Lainate (Bert.), sopra Como (Com.), sopra Lecco (Aiuti!), a Bormio in Valtellina (Rampoldi!), nel Bergamasco (Fota), nel Bresciano, frequentissirna (Zersi), per esempio verso il Ponte di legno a 1300 metri (Parl.!); fa in molti luoghi del Tirolo, sino in Pusteria, e salendo sul Pitten a 4000 piedi (Hausm. fl.); ovunque nel Veneto (De Vis. Sacc. ecc. ecc.); a Trieste (Bert.), a Fiume (Noë!); in Emilia al monte Prinzera nel Parmense (Parl.!), in tutto il Modenese (Gib. Pir. fl.) e il Bolognese (Cocc. ecc.), com. preso l'Appennino fino all'alpe di Succiso (Gib. Pir.) e alla Porretta (G. Bert. not.); in Romagna a Ravenna, e nel Faentino (Caldesi! ecc.). In Liguria è comune, da Nizza (Durando! ecc.) e dall'alpi di Tenda (Bourgeau!) a Sestri levante (Delpino!); comune assai in Toscana, dalle cime piủ alte dell'Appennino di Lunigiana! sino al Mugello (Savi!) e sino al monte Calvi di Maremma (Aiuti!); trovasi nelle Marche in molti luoghi, sino ad Ascoli (Parl.!), c nell'Appennino Piceno-Abruzzese al Vettore!, al Pizzo di Sivo (Orsini!), al Velino (Rolli!), alla Maiella tra $\overline{5}-5000$ piedi (Porta e Rigo!), al Morrone ecc., per terminare più lontano al Gargano (Ten. syll.); dall'altro lato della Penisola ricomparisce a Palombara presso Roma (Seb. Maur. prodr.), ritrovasi in Campania presso Gacta (Terracciano!), c Piedimonte (Terr. rel.), al monte Vergine (Ten.), a S. Angelo di Castellamare (Pasquale!), a Salerno (Ten.), nell' isola di Capri (Kiessclıneyer!), per terminare in Basilicata (Pasquale!). Fiorisce da mag. gio ad agosto nei luoghi bassi, e in lug̣lio e agosto nei piủ alti.

Distribuzione geografica. - Nell'Europa media e meridionale, dalla Spagna al Ciaucaso, ad eccezione della porzione piủ meridionale.

Descrizione. - Pianta perenne, alta da 2 a 5 decimetri, verde, irsuta per peli bianchi e lunghetti, fornita di un rizoma ramoso, da cui partono dei fusti. Questi sono ascendenti o eretti, per lo piủ semplici o ramosi, con i rami eretti, quadrangolari con gli angoli rilevati, verdi, irsuti. Le foglie sono molli, opposte, un po'lontane, patentissime e un po curvate in giù, ovali-allıngate, o quasi lanceolate, ottuse o ottusette, crenate, un po'assottigliate e intere alla base, le inferiori ivi fornite di un picciölo corto, verdi di sopra ed ivi con solchi in rete che corrispondono ai nervi di sotto, di un verde chiaro di sotto ed ivi con un nervo longitudinale rilevato e con due nervelti anche longitudinali in vicinanza dei margini, i quali peró finiscono poco piủ in sopra della metà lella foglia unendosi con i rami dei ner. vetti laterali che vengono dalla parte superiore del nervo longitudi. 
nale mediano e formando tutti una rete rilevata nella pagina inferiore della foglia: sono pelose in ambedue le facce. I fiori sono sessili o con un pedicello corto, da tre a cinque all'ascella di ciascuna foglia superiore e cosi formano dei verticilli di 6 a 10 fiori e con il loro insieme una spiga, allungata, ottusa, interrotta per la massima parte perchè i verticilli sono un po'lontani l'uno dall' altro : ciascun verticillo ha cosi due foglie fiorali, gradatamente più piccole da basso in alto in modo che le inferiori sono molto più lunghe dei fiori e le supe. riori più corte, queste sono ovato-lanceolate, acuminate, intere o quasi intere nei margini, quelle quasi uguali alle foglie. Le bratteole sono setacee, eretto-patenti, più lunghe dei pedicelli e più corte del calice, pelose. Il calice è verdognolo, peloso, campanulato, con cinque denti poco disuguali tra loro, più corti del tubo, eretto-patenti, triangolari-lanceolati, aventi all'apice un mucrone lungo e glabro: ha dieci nervi, cinque dei quali più lunghi e più rilevati giungono all'apice dei denti e cinque ai seni lasciati da questi. La corolla è il doppio più lunga del calice, di color giallo pallido, con poche macchioline di color rossiccio violetto nel labbro inferiore e talora anche inferiormente nei margini del labbro superiore, è bilabiata, con il tubo larghetto, un po'curvo in avanii, fornito in dentro di un anello di peli posto obliquamente verso il terzo inferiore dello stesso: i labbri sono quasi uguali al tubo, il labbro superiore è diretto in alto ed in avanti, ovale, quasi intero all' apice, concavo di sotto, convesso di sopra os. sia esternamente ed ivi peloso, il labbro inferiore è un po' più lungo e più largo, patente, trilobo, con i lobi laterali molto piccoli, ottusi e diretti un po'in giù e con il lobo mediano grande, diretto in avanti e smarginato. Gli stami sono quattro, didinami, piủ corti del labbro superiore. I filamenti sono quasi piani, pelosi inferiormente sino alla metà nella parte esteriore. Le antere sono quasi orbicolari, schiacciate, biloculari, con le logge un po' divergenti, giallicce, glabre. Il pistillo è lungo quanto gli stami più lunghi. L'ovario è piccolo, quadrilobo, glabro, verdognolo, inserito sopra un disco che lo circonda in basso a guisa di un cercine con il margine un po'irregolare, di colore verdognolo. Lo stilo è lungo, un po' curvato in avanti, filiforme, gradatamente un po' più grosso verso alto ed ivi con un leggero solco longitudinale in avanti ed in dietro, bianchiccio, glabro. Lo stimma è bifido, con le lacinie corte, quasi filiformi, ottusette, divergenti e glabre, di colore giallicciobianchiccio. (Parl.ms., descr. della pianta di Bovegno e di S. Colombano nella alpi Bresciane). 
Osservazioni. - Questa specie polimorfa varia per la statura, per la direzione, eretta o sdraiata ascendente, per la ramificazione, per la pelurie, abbondante o scarsa, ma soprattutto per la grandezza dei fiori e per la larghezza delle foglie. Queste vanno da una larghezza di 2 centimetri sino a non avere che 1 millimetro, e ciò a quanto pare in ragione dell'ariditả del suolo. In quanto ai fiori, essi hanno la corolla che va per grandezza dal semplice al doppio, con il labbro inferiore da mezzo centimetro a 1 centimetro, le forme grandiflore essendo più particolari ai monti alti. La Stachys labiosa Bert. è una forma grandiflora; le $S$. subcrenata e $S$. fragilis De Vis. sono forme angustifolie, variabili del resto per la grandezza dei fiori, come ho riscontrato negli stessi saggi autentici di De Visiani. Si noti che questo autore nella $\mathrm{Fl}$. dalm. 2. $p .208$ riunisce le sue due specie in una, e vi riferisce a di più la S. labiosa Bert., e accennando alla loro variabilitả mostra di dubitare egli stesso della distinzione fatta dalla S. recta.

In flore locali è possibile scindere la specie in varietà, come ho fatto io stesso nel mio Prodr. fl. tosc.; ma non mi riesce più con la caterva di saggi d'Italia che ora ho per mano, tanto variamente si combinano fra loro le variazioni dianzi ricordate.

\section{Stachys arenuria.}

S. perennis, foliis fere sessilibus, oblongo-lanceolatis, crenatoserratis, floralibus sensim in bracteas ovato-acuminatas abeuntibus, floribus rubris, calyce tubuloso-campanulato dentibus subulatis spinescentibus, anther $x$ loculis divaricatis.

Stachys arenaria Vahl symb. bot. 2. p. 64. Bert. fl. ital. 6. p. 168 (excl. syn. S. iberica et S. congesta?). Ces. Pass. Gib. comp. fl. ilal. p. 317. Arc. comp. fl. ital. p. 552.

Figure. - Desf. R. atl. t. 126. Bot. mag. t. 1959.

Stazione, Abitazione e Fioritura. - Nei campi, nelle colline, nei luoghi arenosi della Sicilia meridionale: Comiso, pianure sotto Chiaromonte, S. Michele, Caltanissetta, Pietraperzia, Piazza, Aidone (Guss. synn.), S. Maria Nicieme (Huet!). Nei campi asciutti di Calabria, e a Sessa alla fiumara de'Staffoli in Principato citeriore (Ten. syll.). A Venezia a S. Nicoló di Lido, ed al Cavallino (Moretti!). Fiorisce in aprile e maggio nell' Italia meridionale.

Distribuzione geografica. - Inoltre in Barberia, e forse in Asia Minore se a questa specic leve riferirsi la Stachys iberica Bieb.

Flora ltaliasa. - Vol. Vi. 
Osservazione. - Boissier (Fl. or. 4. p. 731) non vuole riunita a questa specie la Stachys iberica Bieb. dell' Oriente, che ha il labbro superiore della corolla intero e non diviso, e gli stami rinchiusi e non sporgenti; ma tali caratteri non sono cosi ricisi come parrebbe dall' enunciazione, e la rassomiglianza totale delle due piante è cosi grande, da far difficilmente credere che sieno specie diverse. Però la cosa va studiata.

\section{1\%. Stachyø glutinosa.}

S. fruticosa, foliis subsessilibus, lineari-lanceolatis, inferioribus serratis superioribus integris, floralibus minoribus, floribus albis, calyce oblongo-campanulato dentibus lanceolatis acuminato-spinescentibus, antheræ loculis divergentibus.

Stachys glutinosa Linn. sp. pl. ed. 1. p. 581. Bert. fl. ilal. 6. p. 167. Gren. Godr. fl. Fr. 2. p. 693. Mor.! fl. sard. 3. p. 308. Car. prodr. fl. tosc. p. 520. Genn.! fl. Capr. p. 112. Mars. cat. pl. Cors. p. 114. Ces. Pass. Gib. comp. fl. ital. p. 316. Arc. comp. fl. ital. p. 551.

Figura. - Zan. ist. bot. t. 71.

Stazione, Abitazione e Fioritura. - Nell'isola di Capraia (Mor. De Not. fl.), in Corsica e Sardegna, dov'è comune per le colline aride sassose ecc.: Bastia!, Corte (Gren. Godr.), Calvi (Soleirol!), Casaglione (Thiriaux!), Aiaccio (Requien!), Bonifacio (Jordan!), La Maddalena, Tavolara, Asinara (Mor.), S. Vito (Sommier!), Cagliari (Genn.!) ecc. Fiorisce da aprile a giugno.

Distribuzione geografica. - Specie propria delle indicate isole.

\section{BALLOTA.}

Ballote et Pseudodictamnus Tourn. inst. p. 184, 187.t.85, 89.

Ballota et Marrubii sp. Linn. gen. Mirb. in ann. mus. 15. t. 18. f. 20, 21. t. 19. f. 1, 2.

Ballote et Beringeria Neck. elem. bot. 1. p. 311, 312.

Ballota Benth. lab. p. 592. Benth. Hook. gen. pl. 2. p. 1212. Ces. Pass. Gib. comp. fl. ital. p. 321. t. 47. f. 4.

Beringeria, Ballota et Acanthoprasium Gen. plant. fl. germ. 6. t. 39, $40,41$. 
Calyx tubuloso-campanulatus subinfundibuliformis, valide 10nervatus. Corolla manifeste bilabiata, labio superiore erecto, concavo. Stamina 4, conniventia, filamentis edentulis, antheris 2-lo. cularibus, loculis longitudinaliter dehiscentibus, confluentibus. Stylus lobis subequalibus. Cocci apice convexiusculi umbonati.

Portamento. - Sono erbe perenni, facilmente suffrutescenti alla base, e una specie è addirittura un frutice spinoso. Hanno foglic ovate o tondeggianti, crenate, più o meno pelose. I fiori sono solitari nel frutice, nell'altre specie sono in glomeruli ben forniti, sempre con foglie fiorali intercalate, conformi all'altre della pianta. Le corolle sono piccole rispetto al calice, miste di bianco e di rosso.

\section{Ballota Pseudodictamnus.}

B. albo-lanata, foliis rotundatis, subcrenatis, bracteis mollibus, calycis limbo magno, patente, obtuse lobato.

Marrubium Pseudodictamnus Linn. sp. pl. ed. 1. p. 583. Zer. fl. mel. thes. p. 29. Ten. fl. nap. 5. p. 16. Syll. p. 293.

Ballota P'seudodictamnus Benth. lab. p. 594. Ces. Pass. Gib. comp. fl. ital. p. 322. Cocc. terz. contr. fl. Bol. p. 28. Arc. comp. h. ital. p. 55\%. Cocc. A. Bol. p. 388.

Figura. - Reich. ic. fl. germ. 18. t. 19. f. 2.

Abitazione e Fioritura. - In Malta (Zer.), in Terra d'Otranto a Gallipoli, in Calabria al Pollino (Ten.), a Napoli (Bruni!), in Abruzzo alla Maiella (Ten.), nei quali luoghi si puó credere che sia veramente spontanea. E stata poi trovata nel 1824 a Genova nelle basse valli verso la base di Polcevera (Figari!), nel 1842 nel Vicentino presso Lugo in vicinanza al Ponte Veglia (Kellner!), e in ultimo a Casio nel Bolognese (Cocc.): dove si puo ritenere che fosse meramente arventizia. Fiorisce da giugno ad agosto.

Distribuzione geografica. - (Itre l'Italia, in Candia e nelle isole greche. Indicata anche in Spagna, ma è molto dubbio che vi sia (Willk. Lange prodr. h. hisp.).

\section{Ballota rupestris.}

B. hirsuta, foliis late ovatis, crenatis, bracteis mollibus, calycis limbo parvo, patentiusculo, ciliato-denticulato.

Ballota saxatilis Guss. 月. sic. syn. 2. p. S2 (non Benth.). Bert. f. ilal. 6. p. 173. 
Ballota rupestris De Vis. fl. dalm. 2. p. 216 (1847). Ces. Pass. Gib. comp. fl. ital. p. 322. Arc.! comp. fl. ital. p. 556.

Ballota italica Benth. in Cand. prodr. 12. p. 519 (1848).

Figure. - Biv. stirp. rar. in Sic. man. 2. t. 1. Gen. pl. fl. germ. 6. t. 39. Reich. ic. fl. germ. 18. t. 19. f. 1.

Stazione, Abitazione e Fioritura. - In luoghi aridi sassosi dell'Italia meridionale: in Sicilia ovunque secondo Gussone, per esempio a Palermo nel monte Pellegrino e a S. Ciro (Bert.), nelle Madonie (Minà!), a Noara e Bafia (Guss.), a Bronte (Inzenga!), a Catania all’Olivetto (Parl.!), ad Avola (Bianca!), a Comiso (Aiuti!); in Calabria a Scilla e Bagnara (Ten.), presso Reggio a Valanidi (Bert.) e nel monte Catania (Huter Porta Rigo!), in Aspromonte sopra Polsi (Pasquale!), a Palizzi (Arc.!), a Gerace a Grottaglia (Huter Porta Rigo!), a Corigliano, a Cassano (Ten.); in Puglia a Manfredonia (Ten.); in Abruzzo a Popoli (Bert.); in Istria (Nym. consp. fl. eur.). L' hanno trovata anche in un luogo detto Montorio, ch'è stato supposto (di certo erratamente) del Lombardo-Veneto (Reich. ic. fl. germ. 18. p. 10), mentre ci sono luoghi di questo nome nel Napoletano e altrove dove potrebbe allignare la pianta. Fiorisce da aprile a luglio.

Distribuzione geografica. - Nasce inoltre in Dalmazia, Erzegovina e Montenegro.

Descrizione. - Perenne, suffruticosa, alta da 8 a 10 decimetri, verde pallida, pelosa per peli lunghi e molli. Rami eretti, quadran. golari, verde pallidi, pelosi, peli lunghi, bianchi, quasi orizzontali, un po' diretti in giù. Foglie opposte, patenti, alquanto vicine, cordato-ovate, acute, disugualmente smerlate, di un verde pallido di sopra e ancora più di sotto dove è un nervo longitudinale delicato dal quale partono molti nervetti laterali che si dirigono obliquamente e si diramano verso il margine e l'apice: sono mollemente pelose in ambedue le facce e con i peli lunghi e folti. Il picciòlo è circa il terzo della lunghezza della foglia, con un solco longitudinale di sopra, peloso come i rami. I fiori sono molti, sessili e fitti in verticilli all'ascella delle foglie superiori dei rami. Le bratteole sono strette, lineari-lanceolate, quasi uguali al calice, aventi all'apice una punta a guisa di una aresta corta, pelose. 11 calice è duretto, tubuloso, un po'slargato in alto quasi ad imbuto, con dieci grossi nervi verdi, diviso da dieci leggieri solchi longitudinali, peloso con peli disuguali, bianchi, alcuni dei quali aventi all' apice una glandoletta: ha dieci denti patuli, corti, cinque un po' più piccoli degli altri, quasi triangolari, termi- 
nati da una piccola aresta come quella delle bratteole, curvata in fuori. La corolla è poco meno del doppio piủ lunga del calice. Il tubo è lungo quanto il calice, quasi cilindrico, pubescente, bianchiccio con macchioline amarante. Il lembo è diviso profondamente in due labbri, uno superiore eretto, quasi lineare, con quattro denti lunghetti ed ottusi all' apice, leggermente concavo in avanti, bianchiccio e peloso per lunghi peli bianchi in ogni parte, il labbro inferiore è poco piủ corto del superiore, diretto quasi orizzontalmente in avanti e un po in giù, trilobo, con i lobi laterali piccoli, e con il lobo mediano molto più lungo e largo, tutti e tre i lobi con i margini dentati e rivolti in giù : è bianchiccio con macchie a guisa di righe longitudinali e di punti di colore amarante. Gli stami sono quattro, didinami, i due piủ lunghi poco piủ corti del labbro superiore, tutti posti avanti a questo, inseriti in alto del tubo. Filamenti grossetti, lesiniformi, eretti, amaranti, pelosi. Antere dirette in dentro, con due logge quasi ovate, divergenti e aventi di sopra in basso molte glandolette bianche e tonde come tante puntine: sono gialle e glabre. II pistillo è più corto degli stami piủ corti, però eserto. L'ovario é circondato da un disco grosso, carnoso, smerlato all' apice e bianchiccio : è quadrilobo con i lobi bislunghi, verdognoli, glabri, aventi all'apice delle glandolette tonde e bianche come quelle delle antere. Lo stilo è molto più lungo dell' ovario, quasi cretto, un po' curvo, filiforme, bianchiccio e glabro. Lo stimma è bifido con le lacinie corte, poco disuguali, divergenti, simili allo stilo per il colore. (Parl. ms., descr. della pianta delle Madonie).

\section{IBallota nigran.}

B. hirsutiuscula ant pubescens, foliis late ovatis, crenato-serratis, bracteis mollibus, calycis limbo parro, patentiusculo, grosse acute dentato.

Ballota nigra Linn. sp. pl. ed. 1. p.582. Bert. /l. ital. 6. p. 170 ; et auct.

Ballota alba Linn. sp. pl. ed. 2. p. 81\%.

Ballota foetida Lam. R. fr. 2. p. 381; et aucl. nonn.

Figure. - Reich. ic. bot. 1. 775 . Ic. /1. germ. 18. 1. 17.

Stazione, Abitazione e Fioritura. - Comune assai per tulta Italia nelle siepi, nei rottami di fahbrica ece. Cosi in Piemonte a Mondovì (Ing.cat.), Alba (Bertero!), Torino, Susa (Colla herb.), Novara 
(Bir. $f l$.$) ecc. ; in Valtellina a Capararo in valle Malenco (Mass. prodr.),$

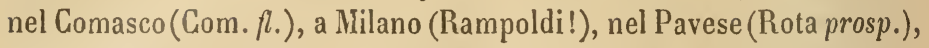
nel Cremonese (Parl.!), nel Bergamasco (Rota prosp.), nel Bresciano (Zersi prosp.), per esempio a Bovegno a 750 metri (Parl.!); in Tirolo a Roveredo (Hausm. fl.), in Valsugana (Ambrosi!), a Trento (Perini!); Tione, Cavalese, Bolzano, Laas ecc. (Hausm.); nel Veronese (Poll. fl.), a Mantova (Bert.), negli Euganei (Trev. prosp.), a Venezia (Kellner!), a Bassano (Bert.), Udine ecc. (Pir. syll.); in Istria a Lippizza (Bert.); a Faenza (Caldesi!), nel Bolognese (Cocc. fl.), alla Porretta (G. Bert. not.), nel Modenese (Gib. Pir. fl.). Cosi in Liguria nel Nizzardo (Ard. fl.), a Bordighera (Ricca!) ecc.; in tutta la Toscana, dove sale fino a mezzo Appennino ai 700 metri circa, e nell' Elba!; nelle Marche a S. Marino (Bert.), ad Albaccina (Bucci!), a Montefortino (Marzialetti!), e più giù a Pizzoli in Abruzzo (Bert.); a Roma (Seb. Maur. prodr.); in Campania a Sora, Roccasecca (Terr.!), Piedimonte (Terr. quart. rel.), Napoli (Ten. ecc.), in Capri (Pasq. fl. ves.) ed Ischia (Guss. enum.), ad Avellino (Bruni!); nel Vulture (Terr. syn.), in Calabria a Stilo (Arc.!) e altrove. In Corsica fa per esempio a Corte (Kesselmeyer!), in Sardegna per esempio nell' isola Maddalena (Genn. $f l$. Capr.), in Sicilia a Palermo (Todaro!), Castelbuono (Mlinà!), Patti (Profeta!), Messina (Seguenza!), Avola (Bianca!), Spaccaforno, Caltanisetta ecc., e nelle Eolie, in Ustica, nelle Egadi e in Pantellaria (Guss. syn.); infine nasce in Malta (Zer. $f l$., Grech $f l$.$) . Fiorisce se-$ condo i luoghi da aprile, maggio o giugno insino all' autunno.

Distribuzione geografica. - In tutta l' Europa, nell' Affrica settentrionale, nell'Asia più occidentale.

Descrizione. - Pianta perenne, alta da 3 a 5 decimetri, verde, pubescente, un po' puzzolente, fornita di un rizoma grossetto, obliquo o quasi orizzontale, che manda molte fibre radicali discendenti, piuttosto delicate e giallicce, e dall' apice dei fusti o rami. Questi sono eretti o ascendenti, un po'flessuosi, quadrangolari, pubescenti-pelosi, con i peli quasi orizzontali o un po'curvati ad arco in giù e nelle parti superiori e nei rami con i peli più molli e curvati ad arco in giù, rossicci o verdognoli, ramosi con i rami opposti, eretti o un po'ascendenti, simili al fusto ma verdognoli. Le foglie sono molli, opposte, un po'lontane, patenti, ovali, ottuse e le superiori ottusette 0 quasi acute, con grandi crenature aventi all'apice una piccola punta, la terminale essendo un po'più grande delle altre, sono verdi di sopra ed ivi con solchi un po' profondi che corrispondono ai nervi di sotto, di un verde un po'pallido di sotto ed ivi con un nervo lon- 
gitudinale rilevato di colore più chiaro da cui partono pochi nervi laterali egualmente rilevati e dello stesso colore, i quali si dirigono molto obliquamente verso l' apice e il margine per terminare alla piccola punta dell'apice delle crenature dopo aver formato con i loro rametti una rete alquanto rilevata nella pagina inferiore della foglia: sono mollemente pubescenti in ambedue le pagine e fornite di ciglia nel margine, hanno tutte un picciòlo, il quale ha circa la metả 0 il terzo della lunghezza della foglia ed è stretto, con un solco largo longitudinale di sopra, un po' convesso di sotto, di colore verdognolo chiaro, pubescente. I fiori sono 504 , in un glomerulo fornito di un corto peduncolo, e quasi patente, rivolto con quello vicino da una sola parte, all'ascella delle foglie superiori del fusto e dei rami delle quali è molto più corto. Il peduncolo è patente, molto piủ corto del calice, ramoso, o quasi cilindrico, pubescente, rossiccio o in parte verdognolo; i pedicelli sono corti o cortissimi : alla base di ciascuno di questi dalla parte esterna è una bratteola molto più corta del calice, eretto-patente, lineare-lesiniforme, verde, pubescente. Il calice è lungo, verde, pubescente di fuori con i pcli curvati in su, pulserulo di dentro con i peli corti, in parte aventi all'apice una piccolissima glandola, è diritto, tubuloso, largo in alto quasi in forma l'infundibulo, verde, pubescente, con dieci costole rilevate, cinque più lunghe che giungono sino all'apice dei denti del lembo e cinque più corte che arrivate ai seni si uniscono con un ramo laterale ai nervi più lunghi, oltre di altri nervetti trasversali più bassi; il lembo è di viso in cinque denti larghi, ovali, acuminati ma non spinosi all'apice, un po' concavi dalla parte interna, diretti in alto ed in fuori con la punta dell' apice patula: hanno poche ciglia nel margine. La corolla è poco più di una metà piủ lunga del ealice, di color roseo-violetto con righe bianche nel labbro inferiore. Il suo tubo è poco più corto del calice, diritto, appena piủ largo verso l'apice, un po' schiacciato lateralmente, bianchiccio, glabro in basso, peloso in alto, con i peli diretti alquanto in giù. Il lembo è più corto del tubo, diviso in due labbri, uno superiore poco più corto, diretto in avanti e un po'in su, ovale, sinarginato e con qualche pic colo dente ottuso nell'apice, concavo, con l'apice e il margine quasi piani, pubescente di fuori e di dentro per peli diversi; l'altro inferiore è patente, trilobo, con i lobi laterali piủ piccoli, quasi ovali, come tondeggianti all'apice, e con il lobo medio più lungo e pii largo, smarginato, con le due parti laterali rivolte alquanto in giu, tondeggianti e con piccole crenature 0 deuti irregolari. Gli stami sono quattro, due poco più alti degli altri 
due, arrivano poco sopra della metà del labbro superiore, nella cui concavità corrispondono, sono inseriti in alto della parte posteriore del tubo della corolla. I filamenti sono quasi cilindrici, eretti, appena curvi in avanti, del colore colla corolla, pelosi lateralmente con peli lunghi in basso e in avanti, con altri peli corti e grossi, sono glabri all'apice. Le antere hanno le logge bislunghe, ottuse, molto divergenti e poste quasi longitudinalmente dopo l'apertura loro, glabre, rossicce-scure, con piccole glandolette tonde a guisa di perline bianche nel punto vicino alla loro inserzione, con una fessura longitudi. nale in ciascuna loggia quasi in continuazione con quella dell' altra. Il polline è bianchiccio. Il pistillo è lungo quanto gli stami inferiori. L'ovario ha quattro lobi, bislunghi, ottusi, lisci, verdognolo-giallicci, e alla base un disco circolare, poco alto, quasi lobato, bianchiccio. Lo stilo è lungo, filiforme, curvato in alto un po'in avanti, bianchiccio, glabro. Lo stimma è bifido, con le lacinie poco disuguali, divergenti, quasi acute, del colore dello stilo. (Parl. ms., descr. della pianta di Grumone in Lombardia).

Osservazione. - La Ballota alba di Linneo, riconosciuta non diversa dalla $B$. nigra, è soltanto della seconda edizione delle Species, mentre la $B$. nigra è della prima; non v'ha dunque ragione per non mantenere quest'ultimo nome, che ha la priorità, e per volerlo cambiare in quello di $B$. foetida. La legge di priorità, applicata alle opere di autori diversi, devesi applicare anche a quelle di un medesimo autore.

\section{Ballota spinosa.}

B. hirsutiuscula, foliis ovato-rotundatis, inciso-crenatis, bracteis spinescentibus, calycis limbo parvo, patentiusculo, grosse spinescenti-dentato.

Ballota spinosa Link handb. 1. p. 475. Bert. fl. ital. 6. p. 175. De Not. rep. fl. lig. p. 329. Ard.t cat. pl. Ment. p. 29. Fl. Alpesmar. p. 302. Ces. Pass. Gib. comp. fl. ital. p. 322. Arc. comp. fl. ital. p. 556 .

Figure, - All. fl. ped.t. 2. f. 2. Reich. ic. fl. germ. 18. t. 18. Moggr. contr. fl. Ment.t. 84.

Stazione, Abitazione e Fioritura. - Nel Nizzardo, sulle rupi ombreggiate, piuttosto rara: a Nizza, Eza, Mentone al Gourg dell’Ora (Ard.), Ventimiglia a Roverino (Panizzi!), tra Castellaro e Ca. 
stiglione, tra Gorbio e S. Agnese, a Sospello (Ard.), Breglio, Giandola (Webb!), Saorgio (Ard.), Levenzo alla Vesulia (Bourgeau!), al confluente del Varo e della Tinca (Burnat!), a Villar, Duranus (Ard.), nell'alpi di Tenda (Reuter!). Fiorisce da maggio a luglio.

Distribuzione geografica. - Questa specie, propria di quella contrada, trovasi pure in qualche località vicina di Francia sulla sinistra del Varo.

Descrizione. - Fruticosa, alta circa 6-8 decimetri. Il fusto è quadrangolare ma con gli angoli ottusi, glabro inferiormente, pubescente in alto come $\mathrm{i}$ rami con peli corti, bianchi e rivolti in giù, rossiccio, ramoso. I rami sono opposti, taliolta però uno dei due rami rron si svolge, quasi cilindrici perchè gli angoli sono ottusissimi, rossicci in basso o interamente verdi, delicati. Le foglie sono opposte, piccole, ovate, ottusette o quasi acute, con 3 a 7 crenature piủ o meno profonde e talvolta quasi lobi, ottusetti, di color verde pallido e pubescenti in ambedue le pagine con cinque o tre nervi ramosi che partono dalla base e sono manifesti di sotto e assottigliati alla base in un picciòlo corto, c gradatarnerte piu corto nelle superiori, e leggermente solcato di sopra. I fiori sono quasi uguali alle foglie fiorali, per lo piủ tre all'ascella di ciascuna foglia superiore, talvolta due o un solo, portati sopra un peduncolo cortissimo, e ciascuno poi sopra un pedicello anche cortissimo, segnatamente nel fiore di mezzo: i due laterali hanno alla base del pedicello una brattea in forma di spina profondamente trifida, ciascuna spinetta è lesiniforme, rossiccia, puberula; il fiore di mezzo ne è privo. Questa brattea spinosa si vede pure all' ascella delle foglie poste al di sotto di quelle che portan fiore, quantunque in esse non si srolgan i fiori. Il calice $\dot{e}$ peloso, tubuloso-turbinato, con dieci nervi verdi e rilevati che lasciano dieci solchi larghetti di colore più chiaro, ha il lembo diviso in cinque denti, aperti, pelosi anche di dentro, ovati e forniti all'apice di una spina lesiniforme, lunghetta, verdognolo-rossiccia, pelosa. La corolla è biança, poco meno del doppio piủ lunga del calice. Il tubo è quasi incluso, cilindrico, peloso in alto di fuori, di dentro nel mezzo lia un anello di peli bianchi lunghetti e fitti. La galca è cretta, obovato-allungata, un po'concava, di fuori e nel margine irsutissima per peli lunghi, fitti c bianchi. Il labbro inferiore é piccolo, patente, trifido, con la lacinia di mezzo ovato-allun. gata e piì lunga delle laterali che sono ovate. Gli stami sono poco disuguali, poco più corti della corolla. I filamenti sono inseriti alla gola, eretti, filiformi, pelosetti in basso, glabri in alto, bianchi. 
Le antere sono rossicce scure, glabre, biloculari, con le logge un po'divergenti in basso, ovali-allungate, ottuse: diventano poi nericce. Il pistillo è poco più corto degli stami. L'ovario è quasi turbinato, glabro in basso, quadrilobo e peloso-barbato in alto, bianco. Lo stilo è filiforme, glabro, diritto, bianco. Lo stimma è bifido, con le lacinie lesiniformi ed un po'acute. (Parl. ms., descr. della pianta inviata da Panizzi).

\section{HEONURUS.}

Cardiaca et Leonurus Tourn. inst. p. 186, 187. t. 87.

Leonurus Linn. gen. pl.ed. 1.p. 176. Mirb. in ann. mus. 15. t. 19. f. 3, 4. Benth. Hook. gen. pl.2. p. 1210. Ces. Pass. Gib. comp. fl. ital. p. 319. t. 48. f. 2.

Leonurus et Chaiturus Gen. pl. fl. germ. 6. t. 30, 31.

Calyx campanulatus, valide 5-10-nervatus. Corolla manifeste bilabiata, labio superiore erecto, concavo. Stamina $\dot{4}$, conniventia, filamentis edentulis, antheris 2-locularibus, loculis longitudinaliter dehiscentibus, confluentibus. Stylus lobis subæqualibus. Cocci apice plani submarginati.

Portamento. - Le specie di questo genere si rassomigliano molto per l'aspetto. Sono erbe perenni, alte fino a 1 metro, con fusto semplice o ramoso, accannellato, foglie piuttosto grandi, membranose, picciuolate, variamente divise, e glomeruli numerosi, di. stanti, di molti fiori rossicci, piccoli.

\section{Leonurus Cardinca.}

L. foliis palmatifidis inciso-dentatis, staminibus longe exsertis, antheræ loculis parallelis.

Leonurus Cardiaca Linn. sp. pl. ed. 1.p. 584. Mass. prodr. fl. valt. p. 100. Bert. fl. ital. 6. p. 182. Trev. prosp. fl. eug. p. 26. De Not. rep. fl. lig. p. 325. Com. fl. com. 4. p. 353. Zan. prosp. fl. ven. p. 26. Rota prosp. piant. Pav. p. 272. Hausm.! fl. Tir. p. 697. Rota prosp. fl. Berg. p. 70. Pir. fl. for. syll. p. 113. Car. prodr. fl. tosc. p. 521. Tassi fl. prov. sen. p. 51. Cenno bot. Sien. p. 88. Ard. fl. Alpes-mar. p. 299. De Vis. Sacc. cat. piant. Ven. p. 139. Zersi prosp. piant. Bresc. p. 168. Ces. Pass. Gib. comp. fl. ilal. p. 319. 
Ing. cat. sp. Mond. p. 46. Cocc. contr. A. Bol. p. 14. Arc. comp. fl. ital. p. 554. Gib. Pir. M. Mod. p. 131. Cocc. 月. Bol. p. 387.

Figure.-Fl. dan. t. 727. Reich. ic. fl. germ. 18. t. 31. f.2. t. 32. f. 2.

Stazione, Abitazione e Fioritura. - Nelle maceric, nei luoghi incolti, nelle siepi, qua e là per l'Alta Italia: nelle vallate Valdensi a S. Martino (Rostan!), nella valle di Viủ (Beccari!), a Mondovi, ovunque (Ing.), nell' alto Monferrato (Delponte!), nel Novarese, comune (Bir. fl.), nel Pavese a Corteolona, Miradolo (Nocca Balb. $l$.), Mirabello, nel Milanese a Bruzzano (Rampoldi!), nel Comasco, specialmente in pianura, nel Cantone Ticino (Com.), nella Valtellina (Mass.), nel Bergamasco a Levate (Rampoldi!), nel Bresciano a Capriano (Zersi), Edolo a 700 metri, Ponte di legno a 1320 metri (Parl.!), nel Tirolo in val di Sol, presso Predazzo, e Oltrecastello (Hausm.), intorno a Bolzano (Hausm.!), nel Cremonese a Grumone (Parl.!), nel Mantovano a Castellaro (Bert.), nel Veronese (Poll. fl.), negli Euganei (Trev.), a Chioggia (Bert.), a Venezia presso S. Elisabetta (Kiellner!), nel Bassanese ad Angarano (Bert.), nel Friuli a Fagagna, e S. Daniele (Pir.), nel Triestino a Lippizza (Bert.), nel Parmense a S. Martino Sinzano (Passerini!), nel Mlodenese al Salto di Montese (Gib. Pir.), nel Bolognese al Castello degli Arienti c a Pizzocalvo (Cocc.). Trovasi ancora più in basso nella Penisola, ma reso molto raro: a Nizza, accidentalmente, a Mentone, una rolta sola (Ard.), a Chiavari (De Not.), in Toscana a Montelatrone in val d'Orcia (Santi viagg., Savi bot.), in Umbria a Pietralta sul confira abruzzese (Ten. syll., Bert.). Fiorisce da giugno a agosto.

Distribuzione geografica. - In tutta Europa, meno la più settentrionale e la più meridionale, nell'Asia Ninore, nella Siberia e I'Imalaia, e (introdotta) nell'America settentrionale.

Descrizione. - Pianta perenne, alta da 6 decimetri a un metro c più, verde. Il fusto è fistoloso, eretto, quadrangolare, con gli angoli acuti e scabrosetti per peli corti, rigidi, diretti in giủ e arvicinati al fusto, piano nelle facce, verde, ramosissimo, con i rami opposti patenti o eretto-patenti. Le foglie sono molli, patentissime. Le inferiori sono palmato-partite, per lo più con cinque lacinic acuminate, dentate, con denti pochi, grandi e disuguali, aventi all'apice un mucrone ottusissimo, scavate in cuore alla base, di un verde scuro e puberule di sopra ed ivi con leggieri solchi che corrispondono ai nervi della pagina inferiore, di un verde chiaro e juuasi bianchicce di sotto ed ivi pubescenti e con molti nervi palmati, delicati e rile- 
vati i quali si diramano per fare una rete alquanto rilevata nella pagina inferiore della foglia: sono fornite di un picciòlo piủ corto della foglia, stretto, solcato di sopra, convesso di sotto, pubescente. Le foglie superiori del fusto sono gradatamente più piccole, trilobe 0 solamente dentate, in forma di cuneo alla base, del resto simili alle foglie inferiori. I fiori sono 4 o 5 , quasi sessili all'ascella delle foglie superiori, per cui in ciascun verticillo vi sono 8 o 10 fiori, e fanno una spiga interrotta, essendo i verticilli lontani, quasi tondi e più corti del picciòlo delle foglie corrispondenti: sono accompagnati da bratteole setacee, erette, più corte del calice, verdognole, pelose di fuori con i peli curvati in su. Il calice è tuboloso-campanulato, con cinque angoli ottusi, quasi bilabiato con il labbro superiore formato da tre denti, ovato-triangolari, acuminato-spinosi, diretti in su e un po'patenti, ed un labbro inferiore un po' più lungo, bifido con le lacinie o denti lanceolati, acuminalo-spinosi e curvati un po' in giù : ha cinque nervi che terminano all'apice dei denti ${ }^{\circ}$ e che mandano un rametto laterale in su per unirsi con l'altro del nervo vicino nel seno lasciato dai denti, è verdognolo-gialliccio e glabro nel tubo in basso, verde e puberulo-glandoloso nel tubo verso alto e nei denti, diventa duro e molto spinoso nel frutto. La corolla è poco più del doppio più lunga del calice, di color roseo-bianchiccio, con macchioline rossicce-amarante nel labbro inferiore. Il tubo è più lungo del calice, ristretto poco sotto della metà e ivi di dentro con un anello di peli lunghi, glabro di fuori sotto del ristringimento, puberulo sopra di questo. Il labbro superiore è diretto in su, ovale, concavo e glabro dalla parte inferiore, convesso e pelosissimo con lunghi peli bianchi di sopra. Il labbro inferiore è quasi uguale al superiore ma un po' più largo, trilobo, con i lobi laterali molto piccoli, diretti un po'in giù, ottusi e con piccole ciglia nel margine, e con il lobo medio diretto in avanti e ovale, tondeggiante all'apice, glabro di sopra, con pochi peli lunghi di sotto verso la base. Gli stami sono quattro, didinami. I filamenti sono quasi filiformi, bianchi, pelosi, segnatamente quelli degli stami più lunghi, con peli lunghi. Le antere sono bilobe, con i lobi divergenti in basso, aprentisi longitudinalmente, giallicce, glabre. Il pistillo è lungo quanto gli stami. L'ovario è piccolo, quadrilobo, peloso in alto, con i peli diretti in su, inserito sopra un disco glabro e verdognolo. Lo stilo è filiforme, bianchiccio, glabro. Lo stimma è bifido, con i lobi corti, divergenti, oltusetti. (Parl. ms., descr. della pianta di Grumone in Lombardia). 


\section{Leonunus Manpulbiastrum.}

L. foliis crenatis, staminibus vix exsertis, antheræ loculis divaricatis.

Leonurus Marrubiastrum Linn. sp. pl.ed. 1. p. 584. Trev. prosp. 凡. eug. p. 26. Pucc.! syn.pl. luc. p. 309. Bert. 月. ital. 6. p. 183. Zan. prosp. fl. ven. p. 26. Car. prodr. fl. losc. p. 521. Zersi prosp. piant. Bresc.p. 168. Ces. Pass. Gib. comp. R. ital. p. 319. Arc. comp. fl. ital. p. 554. Gib. Pir. fl. Mod. p. 131.

Chaiturus Marrubiastrum Rota prosp. piant. Pav. p. 272. Pir. f. for. syll. p. 113. De Vis. Sacc. cat. piant. Ven. p. 139.

Figure. - Bocc. mus. di piant. t. 98. Reich. ic. fl. germ. 18. t. 32. f. 1.

Stazione, Abitazione e Fioritura. - Per le vie, lungo i fossi, nei luoghi incolti ecc. della Penisola superiore e media, raro: ad Alessandria e Novi (All. fl.), a Casalmonferrato (Negri!), presso $\mathrm{Pa}$ via a Campospinoso (Noce. Balb. fl.), Belgioioso, Alagna, a Milano (Poll. fl.), a Belgirate sul lago Maggiore una volta sola (Bir. fl.), lungo la Fiuma presso Guastalla, e a Quingentole (Bert., Gib. Pir.), nel Mantovano a Castellaro (Bert.) e in valle di Sermide (Paglia!), nel Bresciano a Venzago (Zersi!), a Lazise sul lago di Garda (Rigo!), presso Verona a Palù, nel Vicentino (Poll.), negli Euganei (Trev., Bert.), a Venezia (Zan.), presso Monfalcone a Ronchi (Pir.), presso Lucca a Porcari (Pucc.!) e a Capannori (Pucc.). Fiorisce in luglio, agosto e settembre.

Distribuzione geografica. - Nell'Europa media, $c$ in parte nella meridionale, nella Siberia Altaica, nell'America settentrionale, introdotta.

\section{MOLUCCEHAR.}

Molucea Tourn. inst. p. 187.t. 88 .

Moluccella Linn. gen. pl. ed. 1. p. 176. Mirb. in ann. mus. 15. 1. 19. f. 11. Benth. Hook. gen. pl. 2. p. 1211. Cies. Pass. Gib. comp. f. ital. p. 321. 1. AS. f. \& (err. quoad f.e ). C.hasmonia Presl fl. sic. 1. praf. p. 37.

Calyx late campanulato-infundiluuliformis, 5 -10-nervatus, nervaturis inferne excavatis, unde calyx plicatus evaditur. Corolla ma- 
nifeste bilabiata, labio superiore concavo subgaleato. Stamina 4, conniventia, filamentis edentulis, antheris 2-locularibus, loculis longitudinaliter dehiscentibus, confluentibus. Stylus lobis subæqualibus. Cocci apice plani vel planiusculi, submarginati.

Portamento. - La specie italiana del genere è una pianta annua, alta fino a un metro, glabra, con un fusto robusto, rosseg. giante, pressochè semplice, con foglie grandi, palnate, lobate, che quasi tutte accompagnano glomeruli di" grandi fiori bianchi, dotati di un ampio calice spinoso.

\section{Molucella spinosa.}

Moluccella spinosa Linn. sp. pl. ed. 1. p. 587. Bert. fl. ital. 6 . p. 190. Ces. Pass. Gib. l. c. Arc.! comp. fl. ital. p. 556.

Chasmonia incisa Presl l. c.

Figura. - Fl. græe. $t$. $56 \%$.

Stazione, Abitazione e Fioritura. - In Sicilia, nei luoghi incolti sassosi, e simili: a Palermo alle falde del monte Castellana, ai Petrazzi, S. Maria a Gesù, Carini (Guss. syn.), sotto Monreale (Todaro!), presso Termini a Buonfornello e presso Cefalủ alla Roccella (Parl.!), a Scillato sotto Polizzi, a Scopello presso Castellam. mare, a Sommatino, Spaccaforno (Guss.), Avola (Bianca!), Siracusa presso S. Cosimano, Militello di val di Nolo, Agosta (Guss.), Taormina (Arc.!), e presso Messina (Bert.). Nasce eziandio presso Palizzi in Calabria (Arc.!). Fiorisce in maggio e giugno.

Distribuzione geografica. - Nasce inoltre in Spagna, in Grecia, e in Soria e Palestina.

\section{CAMIUUI.}

Lamium Tourn. inst. p. 183. t. 85. Linn. gen. pl. ed. I. p. 175. Mirb. in ann, mus. 15. t. 12. f. 14. t. 18. f. 3-5. Benth. Hook. gen. pl. 2. p. 1210. Ces. Pass. Gib. comp. R. ital. p. 319. t. 47. f. 3.

Lamium et Galeobdolon Gen.pl. fl. germ. t. $28,29$.

Calyx campanulatus, leviter 5-10-nervatus. Corolla manifeste 2-labiata, labio superiore galeato. Stamina 4, conniventia, filamentis edentulis, antheris 2-locularibus, loculis longitudinaliter dehi- 
scentibus, confluentibus. Stylus lobis plus minus inæqualibus. Cocci apice oblique plani marginati.

Portamento. - Erbe annue o perenni, ramose, alte da 2 a 5 decimetri, molli e floscie, pelose o glabre, di odore ingrato. Le foglie sono grandi o mezzane, membranose, generalmente ovate, fortemente dentate. I fiori sono ora piccoli e poco appariscenti, ora piủ grandi, ora grandissimi e vistosi, rosei-porporini, oppure bianchi; facilmente screziati, gialli in una sola specie; sono in glomeruli abbastanza ben forniti, più o meno distaccati fra di loro, accompagnati da foglie fiorali simili alle altre, o poco dissimili.

\section{Lamium Grvala.}

L. perenne, foliis late ovatis, duplice grosse dentatis, floralibus conformibus, florilus maximis, rubris, rare albis, corolla tubo recto, intus transverse annulato, antheris glabris.

Lamium Orvala Linn. sp.pl.ed. 2. p. 808. Mass. prodr. R. valt. p. 98. Trev. prosp. fl. eug. p. 26. Bert. fl. ital. 6. p. 109. Zan. prosp. fl. ven. p. 26. Hausm. fl. Tir. p. 686. Rola prosp. fl. Bery. 1. 76. Pir.! fl. for. syll. p. 111. De Vis. Sacc. cat. piant. Ven. p. 136. Zersi prosp. piant. Bresc. p. 166. Ces. Pass. Gib. comp. Il. ital. p. 320. Arc. comp. fl. ital. p. 554.

Figure. - Bot. mag. t. 172. Reich. ic. fl. germ. 18. t. 7.

Stazione, Abitazione e Fioritura. - Sotto alle siepi, e in altri luoghi ombrosi dell'Alta Italia sulla sinistra del Po: in Istria a Castua (A. M. Smith!), Trieste (Fleischer!) e Lippizza (Bert.), nel Friuli a Udine (Pir.!), Fagagna, Dignano, Brazzano e altrove, comune (Pir.), a V'enezia (Zan., Kellner!) e Mestre, a Mogliano presso Treviso, nel Bassanese alle grotte d'Oliero, nei Berici (Bert.), negli Euganei (Trev.), nel Veronese, principalmente verso Avesa e San Martino, nonchè nel Baldo (Poll. fl.), in Tirolo presso Storo (Porta!), presso Stenico e Tione (Hausm.), a Trento (Perini!), per esempio tra Buco di Vela e Sardagna (Hausm.), in Valsugana ( $\mathrm{Am}$. brosi!), a Salorno (Hausm.), nel Bresciano a Garza, in val di Nave, a Caino, Lonato, Valsabbia (Zersi), nel Rergamasco presso l'Oglio a Sarnico, e a Paratico (Rota), linalmente in Yaltellina secondo Mas. sara. Fiorisce in aprile e maggio, piì tardi nei luoghi pii freddi.

Distribuzione geografica. - Iall' Italia, dalla Dalmazia e dalla Carinzia si stende questa pianta sino alla Transsilvania. 
Osservazione.-Nel suo viaggio al Gargano, il Micheli scopri ivi una inostruosità di questa pianta, che poi rivide coltivata negli orti di Bologna, dove ancora esiste (Bert., Cocc. $f$. Bol.). La stimò un genere nuovo, e cosi la descrisse e figurò nei Nova pl. gen. sotto il nome di Papia (p. 20.t.17), che Linneo, adottando il genere, mutò arbitrariamente in quello di Orvala (Gen. pl.), e chiamò la specie 0 . garganica (Sp. plant. ed. 1.p.578), ma già sospettando che fosse una varietà del Lamium Orvala. Siffatta mostruosità consiste in una divisione più profonda delle foglie, e in una profondissima della co. rolla, scissa in quattro lobi. I moderni erboratori non hanno ritrovata la pianta al Gargano, forse perchè vi si sono recati in stagione troppo inoltrata.

\section{Lamium garganicum.}

L. perenne, foliis late ovatis vel ex ovato elongatis, duplice grosse crenatis, floralibus sensim minoribus, floribus maximis, rubris rare albis, corollæ tubo recto, intus nudo, antheris barbatis.

a genuinum, villosum.

Lamium garganicum Linn. sp. pl. ed. 2. p. 808. Bert. fl. ital. 6. p. 110. Pasq. Lic. viagg. Garg. p. 23. Ces. Pass. Gib. comp. fl. ital. p. 390. Arc. comp. fl. ital. p. 555.

Figure. - Tilli cal. hort. pis. t. 34. f. 2. Reich. ic. fl. germ. 18. t. 6.f. 2.

$\beta$ lcevigatum, parce pilosum.

Lamium longiflorum Ten.! fl. nap. 1. prodr. p. 34. Bert. o. c. p. 111. Gren. Godr. fl. Fr. 2. p. 678. Car. prodr. fl. tosc. p. 522. Suppl. p. 43. Tassi fl. prov. sen. p. 51. Ard.! cat. pl. Ment. p. 29. Fl. Alpes-mar. p. 298. Mars. cat. pl. Cors. p. 113. Terr.! sec. rel. Terra di Lav. p. 95. Ces. el. piant. Maiell. p. 24. Terr.! quarta rel. p. 113.

Lamium grandiflorum Ten.! o. c. 1. syll. app. 4. p. 10 (an Pourr.?). Terr.! sec. rel. Terra di Lav. p. 95.

Lamium Columnæ Ten.? syll. p. 285.

Lamium pedemontanum Reich. $l$. germ. exc. 1. p. 322. De Not. rep. A. lig. p. 325.

Lamium garganicum lævigatum Ces. Pass. Gib. l. c. Arc. l. c. Lamiurn garganicum Paol. primo el. piant. mont. sib. p. 13. 
Figure.-Tilli 0. c. t. 34. f. 1. Ten. 0. c. t. 152. Reich. o. c. t. 6.f. 1 .

* Lamium corsicum Gren. Godr.? o. c. p. 679. Mars. l. c. Ces. Pass. Gib.? l. c. Arc.? l. c.

Lamium numidicum Noé! in bull. soc. bot. Fr. 2. p. 584.

Lamium longiflorum minus Mor. fl. sard. 3. p. 310. Ces. Tass. Gib. l. c. Arc. l. c.

Stazione, Abitazione e Fioritura. - La var. $\alpha$, molto rara, non è stata trovata che al Gargano a Monte S. Angelo (Tilli, Pasq. Lic., ecc.) tra 1000 e 2000 piedi (Porta e Rigo!), e in Basilicata presso Castelgrande (Bert.). La var. $\beta$ è sparsa per $i$ luoghi rocciosi dei monti dell'Italia media e meridionale. Così è frequente nelle Alpi Marittime (De Not.): al col di Tenda a 1900 metri (Parl.! ecc.), a S. Martino Lantosca (Bert.), a Giandola (Webb!), presso Levenzo (Mourgeau!), sopra Mentone fra 1200 e 900 metri (Ard.), e nell'altro versante sopra i Bagni di Valdieri tra 1450 e 1140 metri (Parl.!), sopra Viosonne (Ricca!), presso Ormea (Bertero!), ecc. Indi si ripresenta nei monti calcari della Maremma toscana al monte Calvi!, alle Cornate di Gerfalco (Amidei!), e al monte Labro!; e continua più giủ nei Cimini (Bert.), nel Lucretile della Sabina (Rolli!), nel monte Genuaro presso Poma (Bert.), nei Lepini sopra Carpineto (Rolli!), in Campania ad Itri (Terr.!), al monte Cairo presso Cassino (Terr.), nel monte Meta a 2000 metri (Levier!), a S. Biagio, Picinisco (Terr.!), S. Donato, Pietravairano, Pietraroia (Terr.), Frasso (Ten. syll.). Dall' altro lato della Penisola si presenta nel monte Catria (Piccinini!), e prosegue per il Piceno e l'Abruzzo, dove si rende comune: presso Camerino a Rio sacro (Bert.), al monte Birro (Nar. ducci!), al monte Volubrio (Narzialetti!), alla Sibilla (Paol.), al Vettore (Orsini!), al monte de'Fiori!, al Corno (Bert.), al Velino (Orsini!), al Sirente, al Morrone fra 1500 e 1600 metri (Levier!), alla Maiella in vari punti tra 1000 e 2400 metri (Porta e Rigo!, Lc. vier! ecc.), e in generale per tutto quel gruppo di monti, nonchè in Molise (Gussone!), riattaccandosi per tal modo alle località di Campania. Quindi si ritrova al Gargano, in Basilicata a Sirino presso Lagonegro (Ten.), iu Calabria a Ciavole presso il Pollino (Biondi!), c a Campotenese (Bert.). Nasce pure in Corsica alla sinistra del Fiumorbo presso il col di Sorba, a Vivario (Mlars.), nei monti Coscione, Cagnone (Gren. Ciodr.) e Cagna (Bert.), e nella somınità del moute Cinto nel Niolo? (Greu. Godr.); e in Sardegna ad Arizzo, Orgosolo (Mor.) e Oliena (Gennari!). Fiorisce da maggio e giugno a luglio c agosto. 
Distribuzione geografica. - Nella Francia meridionale occidentale, in Italia, nella Penisola Balcanica e in Asia Minore (non essendo distinguibili da questa specie il Lamium molle Boiss. Orph. e il Lamium bithynicum Boiss.! dell' Oriente), nonchè in Tunisia e Algeria.

Descrizione.- Pianta di un verde alquanto glaucescente, con i fusti e i rami rosso-scuri o glaucescenti, alti da 4 a 6 o 8 decimetri, quasi glabra. Rizoma obliquo, piuttosto delicato, bianchiccio; fibre radicali lunghette, bianchicce. Fusti ascendenti 0 eretti, ramosi, con i rami eretti o un po'curvi, e come questi fistolosi, quadrangolari, appena più grossi nei nodi vitali, rosso-scuri o verdognoli, pubescenti negli angoli per peli corti, bianchicci, curvati in giủ. Foglie patenti, verdi di sopra, ivi con solchi corrispondenti ai nervi di sotto e con sporgenze del parenchima quasi bollate, verdechiare di sotto, coi nervi, nervetti fino alle ultime diramazioni in rete sporgenti, concolori, pelosette in ambedue le pagine, massime di sotto ai nervi, coi denti ottusi. Picciòli rossicci, con solco di sopra, convessi di sotto, pelosetti come le foglie. Fiori 304 all' ascella delle foglie superiori, in modo che in ogni giro ve ne sono $6-8$, sessili. Calice tubuloso, un po'curvo, angolato, verdognolo, in parte rossiccio, glabro; cinque denti grandi, disuguali, eretto-patenti, lanceolati, acuminati, pubescenti-glandolosi nel margine per peli parte piủ lunghi e curvi e bianchi e parte cortissimi che hanno una piccolissima glandola tonda e rossiccia, rossicci nel margine stesso. Corolla grande, più del dop. pio più lunga del calice, pubescente di fuori, di color violetto più chiaro verso la gola, con righe più cariche di dentro nella parte larga del tubo e con macchioline più cariche a guisa di punti distribuiti in linee nel labbro inferiore. Tubo piủ lungo del calice, strelto, un po' schiacciato e bianchiccio nella metà inferiore, slargato quasi a un tratto e largo assai nella metà superiore. Labbro superiore eretto, largo, ovale, un pó'convesso di fuori e concavo a guisa quasi di volta di dentro, smarginato-bilobo all'apice con i due lobetti piccoli, curvati in dietro e smarginati. Labbro inferiore lungo quasi quanto il superiore, diretto in giù e in avanti, trilobo, lobo medio molto grande, smarginato-bilobo con i lobetti grandi, tondeggianti, dentati, lobi laterali del labbro piccolissimi smarginato-bilobi, ottusi, curvati in giủ e quasi fossero due piccoli denti o lobetti della parte larga del tubo della corolla. Stami 4, due più lunghi e due piủ corti, sotto la volta del labbro superiore, inseriti in alto del tubo. Filamenti quasi filiformi, un po'curvati in avanti, pubescenti e in parte puberulo-glandolosi, bianchicci. Antere bilobe, uniloculari per la con- 
fluenza delle due logge, rossicce, con lunghi peli bianchi quasi barba alla estremità e un po'lateralmente. Polline giallo. Pistillo lungo quanto gli stami piủ lunghi. Ovario piccolo, 4-lobo, lobi piani di sopra con un orlo rilevato nel margine di sopra, verdognolo, glabro. Stilo lungo, filiforme, bianchiccio, curvato in alto in avanti. Stimına bifido, lacinie poco disuguali, assottigliate in alto, del colore dello stilo. Calice slargato nel frutto, con le lacinie un po'patenti. Frutti scuri, quasi triangolari, piani di sopra e dalle facce laterali, un po'convessi di fuori. (Parl. ms., descr. della pianta dei Bagni di Valdieri).

Osservazioni. - Fra il Lamium garganicum tipico e il L. lon. giflorum Ten. non c'è proprio altra differenza che la pelurie.

Niente di piủ variabile per grandezza e per forma dei denti calicini in questa pianta. Talora (i più lunghii) non misurano che 4 milli. metri, e sono triangolari, e talora arrivano al doppio e sono lanciolatiağuzzi.

Il Lumium longiforum minus di Moris, al quale sarà forse da riferirsi il $L$. corsicum Gren. Godr. (ma non lo conosco altrimenti), è una forma a fiori più piccoli, come se ne danno solitamente nella famiglia.

Il dottor Levier ha trovata nel Sirente sopra Ovindoli una forma gracile di questa pianta, coi polloni sottili, con le foglie piccole, arrotondite, coi calici sbiancati dai denti corti, che corrisponde al L. veronicafolium Benth. d'Oriente, e che come questa ed altre affini specie (cosi dette) orientali non mi riesce disgiungere mediante caratteri sicuri dedla var. $\beta$ del $L$. garganicum.

\section{Iamium bifielum.}

L. annuum, foliis late ovatis, duplice inciso-crenatis, floralibus conformibus, floribus mediocrihus, albis, corollæ tubo recto, intus undo, antheris barbatis.

Lamium bifidum Cyr. pl. var. neap. fasc. 1. p. 29. Bert.! 1 . ital.6.p. 118. Gren. Godr. A. Fr. 2. p. 6i9. Guss, enum. pl. Inarim. p. 263. Mor. 11. sard. 3. p. 311. Car. prodr. /l. 10sc. p. 592. Tassi 1. prov. sen. p. 51. Car. A. Monterr. p. 28. Pasq. 1l. ves. p. S1. Terr.! /l. Vull. syn. p. 138. Mars. cal. pl. Cors. p. 11\%. Ces. Pass. Gil. comp. Al. ital. p. p. 555. 
Lamium incisum Car. prodr. fl. tosc. p. 523 (non Willd.). Tassi fl. prov. sen.p. 51.

Figure. - Cyr. o. c. t. 7. Ten. fl. nap. t. 153. f. 2.

Stazione, Abitazione e Fioritura. - Nei luoghi freschi, erbosi 0 boschivi, dell'Italia meridionale e media. Nasce in Sicilia a Mon. reale, alla Ficuzza (Parl.!), a Sagana, a Busambra, nelle Madonie, fra S. Gregorio e Capo di Orlando, a S. Agata, sotto Naso, a Patti, Torrenova, Messina, Giarre, e nelle isole di Ustica (Guss. syn.) e di Lipari (Mandralisca!). Sul continente trovasi in Calabria nel monte Stella presso Stilo a 7-800 metri (Huter Porta Rigo!), e a Pizzo (Ricca!), nel Principato citeriore a Sessa e altrove (Ten. syll.), in Basilicata a Melfi (Terr.!), ảl Gargano (Porta Rigo!), intorno a Napoli in parecchi punti (Ten., Heldreich! ecc.), e in Ischia (Guss.), a Caserta (Terr.!), a Terracina (Bert.), a Roma, dov'è comune (Seb. Maur. prodr., Gennari! ecc.), a Canino (Bert.), in Toscana nel monte Argentaro (Parl.!, Caldesi!), a Orbetello!, a Fercole fra Grosseto e Siena (Parl.!), nel monte Calvi (Beccari!), presso Volterra (Parl.!), nella Selva pisana (P. Savi! ecc.), ultirno limite da questo lato della Penisola; mentrechè dall'altro lato trovasi in Abruzzo al monte de'Fiori (Orsini!), in Piceno al monte Volubrio (Marzialetti!), al Subasio nell'Umbria (Gennari!), al Catria (Piccinini!), e presso la Pieve S. Stefano in val Tiberina (Amidei!), ultimo limite. Dicesi comune in Sardegna (Mor.); in Corsica nasce a Bonifacio, Portovecchio, Bocognano, Vico (Gren. Godr.), Aiaccio, Appieto, Cargese, Vizzavona (Mars.), nel monte Grosso (Soleirol!), a Bastia (Mabille!) e San Fiorenzo (Mars.); finalmente nell' arcipelago toscano a Montecristo (Wats.-Tayl.), al Giglio (Parl.!), e all'Elba nel monte Capanna (Beccari!). Fiorisce in marzo ed aprile.

Distribuzione geografica. - Oltre l'Italia, questa specie fa in Dalmazia e in Grecia.

Osservazione. - Anche questa Lamiacea va soggetta ad avere fiori minuscoli. È stata cosi trovata in Sicilia, e descritta da Gussone sotto il nome di Lamium cryptanthum, e figurata da Reichenbach (Ic. bot. t. 70\%).

\section{Iamium anaplexicaule.}

L. annuum, foliis rotundatis, crenato-fissis, floralibus conformibus, amplexicaulibus, floribus parvis, rubris rare albis, corollæ tubo recto, intus nudo, antheris barbatis. 
Lamium amplexicaule Linn. sp. pl. ed. 1. p. 579. Bert. fl. ital. 6. p. 122 (excl. L. intermedium Fries); et auct. omn.

Figure, - Fl. dan. t. 752. Reich. ic. fl. germ. 18. t. 3. f. 2.

Stazione, Abitazione e Fioritura. - Comune per i luoghi coltivati di tutta l'Italia, ma non a quanto sembra ugualmente ovunque, dalle pianure a notevoli altezze nei monti. Nell'Alta Italia a Mondovi (Ing. cat.), Torino, Susa (Golla herb. ped.), Riva di Sesia (Carestia!), nel Novarese (Bir. $\uparrow$.), a Pavia (Rota prosp.), Nlilano (Rampo!di!), nel Comasco, abbondante (Com. fl.), in Valtellina (Mass. prodr.), nel Cremonese (Parl.!), nel Bergamasco (Rota prosp.), in vari punti del Bresciano (Zersi prosp.), per esempio a Ponte di legno a 1320 metri (Parl.!), in Tirolo dal lago di Garda a Brunecco (Hausm. f.), nel Veronese (Poll. fl.), negli Euganei (Trev. prosp.), a Venezia (Kellner!), Bassano (Bert.), Monfalcone (Pir. syll.), Trieste, Pirano (Bert.), in vari punti del Modenese (Gib. Pir. $f l$.) e del liolognese (Cocc. l. $_{\text {) }}$, a Faenza (Cald. tent.), in quel di Lugo (Caldesi!), ecc. Trovasi in Liguria nel Nizzardo, a Mentone (Ard. /.), a Dolcedo (Berti!); in Toscana a Sarzana (Bert.), Lucca (Calandrini!), Pisa (Savi fl.), Firenze!, Volterra (Amidei!), Siena (Campani!), S. Vincenzo (Parl.!), Castellazzara in val di Paglia (Santi viagg.) ecc., nonchè nelle isole di Gorgona (P. Sav. fl.), di Capraia (Mor. De Not. fl.) e dell'Elba a Rio (Marcucci!); nel Piceno a Montefortino (Marzialetti!), in Abruzzo nella Maiella (Ces. el.); sul Gargano (Porta e Rigo!); a Roma, comune (Seb. Maur. prodr.); a Napoli, altresi (Ten. syll.), c in Ischia (Guss. en.) e Capri (Pasq. fl.); sul Vulture, ovunque (Terr. syn.); ad Otranto (Groves!). Ė indicata come comune in Corsica (Mars. cat.), e in Sardegna (Mor. /.), per esernpio a Sassari!. In Sicilia pare che si trovi ovunque, nonchè nelle Folie (Guss. syn. ecc.), in Ustica, Pantellaria (Calcara!), Linosa e Lampedusa (Ainti!); infine in Nalta (Grech!). Fiorisce da febbraio e marzo ad aprile e maggio, e in montagna da maggio a luglio.

Distribuzione geografica. - In tutta Europa, nell'Asia occidentale e fino all'Imalaia, nelle isole Canarie, negli Stati l'niti sull'Allantico.

Osservazione. - Questa pianta suol avere nella regola i primi fiori, invernali o primaverili che sieno, minuscoli, con la corolla (hiusa a motivo delle labbra che restano in istato di preflorazione, ed anche s'incollano fra di loro. In essa le antere sono strettamente. aderenti allo stimma, e i granelli pollinici enettono i loro fili stando in posto e traforando le pareti delle caselle. Liesistenza di tali fiori 
è conosciuta dai tempi Linneani; i nuovissimi particolari ne sono stati illustrati da Walz (in Bot. Zeit. 1864). Vedasi la figura illnstrativa della pianta in questo stato in Reich. ic. bot. $t .708$.

\section{Lamium hylbridum.}

L. annuum, foliis ovato-rotundatis, crenato-fissis, floralibus conformibus, floribus parvis, rubris, corollæ tubo recto, intus piloso-annulato aut nudo, antheris barbatis.

Lamium hybridum Vill. hist. pl. Douph. 1.p. 251 (1786). Ard. cat. pl. Ment. p. 29. Fl. Alpes-mar. p. 298. Ces. Pass. Gib. comp. fl. ital. p. 321. Ing. cat. sp. Mond. p. 45. Arc. comp. fl. ital. p. 556 .

Lamium dissectum With. arr. brit. pl. ed. 3. p. $527(1796)$.

Lamium incisum Willd. sp. plant. 3. p. 89 (1800). Rota prosp. piant. Pav. p. 272.

Figure. - Reich. ic. bot. 3. t. 223. Ic. fl. germ. 18. t. 3. f. 4.

Stazione, Abitazione e Fioritura. - Nei luoghi coltivati: a Mondovì (Ing.); nel Pavese (Rota); a Nizza, a S. Agnese sopra Mentone (Ard.). Fiorisce in aprile e maggio.

Distribuzione geografica. - Nell'Europa, principalmente media, e in Terra Nuova.

Osservazioni. - Ho riferita questa pianta come italiana sulle indicazioni degli autori, non avendone veduto alcun saggio d'Italia, quello di Toscana da me registrato nel mio Prodromo essendo di $L a$ mium bifidum senza fiori. Parecchi autori la ritengono una varietà del $L$. purpureım; i più moderni inclinano a crederla un ibrido fra il $L$. purpureum ed il $L$. amplexicaule, nel qual caso sarebbe un ibrido fertile, poichè si riproduce da seme (With. l. c.).

\section{G. Lamiun purpureum.}

L. annuum, foliis ovatis, crenatis, floralibus conformibus, floribus parvis, rubris rare albis, corollæ tubo recto vel basi vix curvato, intus piloso-annulato, antheris barbatis.

Lamium purpureum Linn. sp. pl. ed. 1. p. 579. Bert. fl. ital. 6. p. 121; et auct. omn.

Figure. - Fl. dan. $t$. 523. Reich. ic. f. germ. 18. t. 3. f. 3.

Stazione, Abitazione e Fioritura. - Nei luoghi erbosi freschi, 
nei campi ecc. È comunissimo per tutta l'Alta Italia, dove s'inoltra nelle Alpi in Valtellina (Mass. prodr.), in val Camonica a Ponte di legno a 1320 metri (Parl.!), in Tirolo sino a Welsberg in Pusteria ecc. (Hausm. fl.), e nell'Appennino a Porretta nella valle del Rieno (G. Bert. veg. porr.), e all'Acero (G. Bert. il. bon.). In Liguria nasce a Nizza (Webb!), dov' è comune, a Nentone (Ard. cal.), a Genova (De Not. prosp.); in Toscana abbonda all'infuori della regione maremmana, salendo nei monti a Tereglio nel Lucchese (Gian. nini !), a Pupiglio nel Pistoiese (Bert.), tocca appena l'anzidetta regione a Sarzana (Bert.), a Pisa (Sav. f. pis.), e manca affatto nelle isole; e parimente nell'altro lato della Penisola pare che si fermi al monte Priore del Piceno (Ilarzialetti!). Si ritrova peraltro a Rioma (Parl.!), dove dicesi comune (Seb. Maur. prodr.), a Capua (Parl.!), a Napoli (Ten. syll., Pasq. fl. ves.), Ischia (Guss. enum.) e Capri (Pasq. o. c.), sempre comune, infine nel Vulture, ancora comunissimo (Terr. syn.). Nasce pure in Corsica a Bastia (Mars. cat.), ad Aiaccio (Requien!), a Bonifacio (Bert.) e altrove. Manca in Sardegna e in Sicilia. Fiorisce da febbraio e marzo ad aprile e maggio, e nei monti in giugno e luglio; talora da capo in autunno.

Distribuzione geografica. - In tutta Europa, meno gli estremi nord e sud, nell'Asia occidentale, fino in Sibcria e nell'Altai.

Descrizione. - Pianta annua, alta da $1 \mathrm{e}^{1 / 2}$ a 2 decimetri, di un verde chiaro. La radice è fusiforme, fibrosa, bianchiceia. II fusto è erctto, un po'flessuoso, quadrangolare, con gli angoli forniti di peli rivolti in giu che lo rendono scabro, rossiccio o verdo. gnolo, ramoso sin dalla base, con i rami inferiori lunghi quanto esso ed ascendenti, i laterali eretto-patenti o patenti, pii corti. Le foglie sono erbacee, molli, opposte, lontane, le inferiori piccole, cordatoorbicolari, e le inedie cordate, patenti, le superiori cordate ma rivolte alquanto in giù, tutte ottuse, crenate, di un verde chiaro di sopra ed ivi con solchi in rete che corrispondono ai nervi di sotto, di un verde più chiaro di sotto ed ivi con nervi rilevati che si uni. scono tra loro per formare una rete: sono pelose in ambedue le pagine e fornite di un picciòlo, il quale è piủ lungo o quasi uguale alla foglia nelle foglie di mezzo, piủ corto di essa nelle foglie superiori, piano di sopra, convesso di sotto, pelosetto. I fiori sono in glomeruli di 5 a 5 , sessili all' ascella delle foglie superiori, accompagnati da bratteole lesiniformi, verdognole, pelosette, più corte del calice. Questo è tuhuloso-campanulato, con dieci costole che lo rendono angolato, verdognolo in queste, bianchiccio nei punti interme- 
dii, peloso o quasi glabro, con cinque denti, eretti, lesiniformi, forniti di ciglia disuguali, e verdognoli; diventa un po'più grande nel frutto, allora i suoi denti sono lanceolato-acuminati e divaricati. La corolla è piccola, appena il doppio più lunga del calice, di un roseo carico con macchioline più cariche nel mezzo e verso la base del labbro inferiore come pure sotto ai due denti laterali verso la gola. Il tubo è più lungo del calice, stretto, angolato in basso, slargato ad un tratto sotto della gola ed ivi puberulo di fuori, e di dentro fornito trasversalmente di un anello di peli. Il lembo è piccolo, diviso in due labbri, il superiore è diretto in alto, ovale, e intero all' apice, convesso e peloso di furi, concavo di dentro e glabro, l'inferiore ha i due lobi laterali rappresentati da due piccoli denti lesiniformi corti e diretti in avanti, e il lobo medio quasi orbicolare, smarginato-bilobo. Gli stami sono quattro, didinami, posti sotto la piccola volta formata dalla concavità del labbro superiore, due quasi uguali a questo e due poco più corti. I filamenti sono eretti, filiformi, bian. chi, pelosetti, saldati in basso con il tubo della corolla. Le antere sono biloculari, con le logge quasi tonde, scure, barbate con piccole glandole a guisa di perline bianche verso la base di fuori, aprentisi per una fessura longitudinale comune. Il polline è di color di minio. Il pistillo è quasi uguale agli stami superiori. L'ovario è diviso pro. fondamente in quattro lobi, quasi triangolari, piani di sopra, concavi di fuori, verdognoli, glabri, circondati alla base da un disco bianchiccio. Lo stilo è lungo, filiforme, bianco, glabro. Lo stimma è bifido, con le lacinie lesiniformi, quasi uguali, divergenti. I frutti sono triangolari, bislunghi, giallicci, glabri. (Parl.ms., descr. della pianta di Grumone in Lombardia).

\section{ข. Taminn album.}

L. perenne, foliis ovatis, grosse crenato-serratis, floralibus conformibus, floribus magnis, albis, corollæ tubo basi curvato, intus oblique piloso-annulato, antheris barbatis.

Lamium album Linn. sp. pl. ed. 1. p. 579 (excl. $\beta$ ). Bart. cat. piant. Sien. p. 22. Sant. viagg. 1. p. 112, 207, 292. Seb. Maur. fl. rom. prodr. p. 193. Mass. prodr. fl. valt. p. 98. Bert.! fl. ilal. 6. p. 116. Com. fl. com. 4. p. 321. Zan. prosp. fl. ven. p. 26. Rota prosp. piant. Pav. p. 272. Hausm. fl. Tir. p. 687. Rola prosp. $f$. Berg. p. 70. Pir. fl. for. syll. p. 112. Car. prodr. fl. tosc. p. 523. 
Tassi fl. prov. sen. p. 5\%. Cenn. hot. Sien. p. 89. De Vis. Sacc. cat. piant. Ven. p. 136. Zersi prosp piant. Bresc. p. 166. Ces. el. piant. Maiell. p. 23. Ces. Pass. Gib. comp. fl. ital. p. 321 . Ing. cat. sp. Mond. p. 45. Cocc. quart. contr. fl. Bol. p. 16. Arc. comp. R. ital. p. 556. Gib. Pir. R. Hod. p. 129. Cocc. fl. Bol. p. $38 \%$.

Lamium vulgatum $\beta$ Benth. lab. p. $51 \%$.

Lanium maculatum var. Trev. prosp. ^. eug. p. 26.

Figure. - Fl. dan. t. 594. Reich. ic. 1. germ. 18. t. 1. f. 1.

Stazione, Abitazione e Fioritura. - L Lungo le siepi, nei margini dei cámpi, nei lunglii incolti ecc. dell'Alta Italia : a Torino (Delponte! ecc.), nei colli della Garzegna in Mondovi (Ing.), nel Novarese (Pir. fl. ac.), nel Parese (Nocca Balb. fl. tic., liota), a Milano, a Orlate (Parl.! ecc.), nel Comasco dov'è comune (Com.), in Valtellina, per esempio presso Bormio tra 1200 e 1400 metri (Parl.!), nel Bergamasco (Rota), nel Bresciano, per esempio a Ponte di legno a 1520 metri, a Collio a 850 metri, nel monte Gerle a 1700 metri (Parl.!), in Tirolo a Borgo di Valsugana, Trento, Tione, in Fassa e Fiemme, intorno a Bolzano, a Merano, Welsberg ecc. (Hausm.), nel Mantovano (Barbieri! ecc.), a Vicenza (Caldesi!), a Bassano, ad Abano, a Chioggia (Bert.), a Venezia nel Lido (Kellner!) e a Cam. palto (Ruch. fl.), a Udine, Dignano, Resia e altrove (Pir.), nei dintorni di Mlodena, raro, cioè a S. Cataldo presso Secchia (Gib. Pir.), raro pure a Bologna, cioè a Corticella (Cocc.). Esiste pure nel resto della Penisola, ma molto scarsamente: in Toscana a Pisa (Corinaldi!), a Siena (Bart), e nel monte Amiata a Casteldelpiano, la Trinitả e Badia S. Salvatore (Sant.); in Abruzzo nei monti di Caramanico, e nel monte Coccia (Ces.); a Roma (Seb. Maur.); a Calvi in Terra di Lavoro (Terracciano!); a Pizzo in Calabria (Ricca!). Fiorisce nei luoghi bassi da marzo a maggio, e da capo in autunno, e nei lnoglii più alti da gingno ad agosto.

Distribuzione geografica. - Nell'Europa, principalmente media, nell' Asia Minore e fino in Persia, in Siberia.

Descrizione. - Rizoma che mandia fibre radicali bianchicce, e rami in alto non fiorifere ma con piccole foglie. Fusto ascendente, quadrangolare, flessuoso, rossiccio in hasso, verilognolo in alto: angoli un po'rilevati ed ottusi, facce legggermente scanalate, con la parte. di mezzo un po' convessa: pelosetto con peli radi, corti, curvati in giu. Fusto macchiato di nericcio lateralmente all'inserzione delle foglie fiorali. Foglie erlacee, patenti, di un verile chiaro di sopra ed ivi con solchi corrispondenti ai nervi di sotto i quali fanno una rete ri- 
levata nella pagina inferiore, questạ è di un verde piủ pallido di quella di sopra, pelosette in ambedue le pagine, peli sparsi nella superiore, nei nervi nella inferiore. Picciòlo gradatamente un po' piủ largo verso la base, scanalato di sopra, un po'convesso e quasi carenato di sotto, con ciglia rade, curvate in giù, più lunghe e più abbondanti verso la base. Fiori sessili, 4-5 all' ascella di ciascuna foglia superiore, più corti o quasi ugualial picciòlo. Calice quasi campanulato, largo assai in alto, senza peli di dentro, verdognolo-bianchiccio, spesso con macchie rosso-scure e quasi nericce verso la base, con dieci nervi longitudinali verdognoli, i cinque principali peloso-scabri con i peli curvati in su, tali peli sono anche nei seni, con cinque denti poco disuguali, più lunghi del tubo, eretto-patenti, lanceolato-lesiniformi, il superiore un po' più larghetto. La corolla è piủ del doppio piủ lunga del calice, bianca; il suo tubo è piủ corto dei denti del calice, curvo, più largo in alto, di dentro ha in basso un anello di peli; il lembo è diviso in due labbri, uno superiore più grande, quasi in forma di elmo, ovale, convesso-carenato di sopra, con la carena ottusa che si biforca verso l'apice, è leggermente crenulato-dentato nell'apice, concavo di sotto, pubescente di fuori, cigliato nel margine, il labbro inferiore è trilobo, con i lobi laterali molto corti e aventi all'apice un dente lineare, acuto, lunghetto, e il lobo medio grande, patente, quasi orbicolare, smarginato, con un solco longitudinale di sopra, ed ivi con macchioline verdognole. Gli stami sono didinami, quasi uguali o poco più lunghi del labbro superiore, nella cui concavità sono sempre i due più corti, inserito in alto del tubo della corolla. I filamenti sono grossetti, quasi cilindrici, appena curvi in alto, bianchi, pelosi in basso dalla parte interna, con i peli lunghi e fini. Le antere sono di un giallo scuro, quasi nericcie, fornite all'apice di cia: scuna loggia di un ciuffo di lunghi peli bianchi, si aprono per una fenditura conune. Il polline è giallo. Il pistillo è lungo quanto gli stami. L'ovario è diviso in quattro lobi, bislunghi, triangolari, con le facce interne piane, la faccia esterna un po'convessa, con l'apice troncato, appena convesso e avente di fuori un margine rilevato ed acuto: è verdognolo, glabro, liscio. Lo stilo è lungo, filiforme, curvato in alto in avanti, glabro. Lo stimma è bifido, con le lacinie lunghette, assai divergenti. (Parl. ms., descr. della pianta di Olate in Lombardia).

Osservazione. - Molte delle surriferite citazioni deronsi considerare dubbie, per essere facilmente presa per questa specie la va. rietà a fior bianco della seguente. 


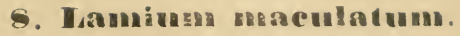

L. perenue, foliis oxatis, grosse crenato-serratis, floralibus conformibus, floribus magnis, rubris rare albis, corollx tubo basi cur. vato, intus transversim piloso-annulato, antheris barbatis.

Lamium maculatum Linn. sp. pl. ed. 2. p. 809. Bert. fl. ital. 6. p. 113 (excl. syn. L. albi Seb. Maur.?); et auct.

Lamium Columnæ Ten.! herb., anne op.? Terr.! quart. rel. Terra di Lav. p. 113.

Lamium affine Guss. Ten.! in Ten. Al. nap. 5. syll. app. 4. p. 10.

Lamium lævigatum Mass. prodr. fl. valt. p. 98.

Lamium vulgatum $\propto$ Benth. lab. p. $51 \%$.

Lamium tomentosum Ces. Pass. Gib. comp. fl. ital. p. 321 . Arc. comp. A. ital. p. 556 .

Figure, - Ten. R. nap. t. 153. f. 1. Reich. ic. bot. t. 21521\%. Ic. 月. germ. 18. t. 4. f. 2, 3.

Stazione, Abitazione e Fioritura. -- Sotto alle siepi, nei luoghi boschivi ecc., della Penisola superiore e media, dalle pianure a parti assai alte de'monti, in generale comunissimo. Così trovasi in Piemonte a Torino (Parl.!), Alba (Bert.), Mondovi (Ing. cat.), Valdieri (Bert.), Barge, Susa, Biella (Colla herb. ped.), Varallo (Carestia!), Novara (Bir. $l$. ac.) ece.; nel Ciantone Ticino, in Valtellina, in tutto il Comasco (Com. 7 . ecc.), nel Pavese, nel Bergamasco (Rota prosp.), nel Bresciano (Zersi prosp.); in Tirolo, dove sembra raro, poichè indicato nella sola località di val Aviana (Hausm. $l$.); in tutto il Veneto (De Vis. Sace. cat. ece. ecc.); in Istria a Fiume (Weiker!); nel Modenese (Gib. Pir. $l l$.) e Bolognese (Coce. fl.), e nell' annesso $\Lambda$ ppennino quasi fino alle maggiori cime (Parl.! ecc.); a l'aenza (Cald. Lent.), a Urbino (Bert.), al monte S. Vicino (Bucci!), a Macerata (Ainti!), nel monte Birro (Bert.) e nel Vettore (Orsini!), nel monte de' Fiori (Gemmi!), nel Velino (Orsini!), nel Norrone a 2000 metri (Porta e Rigo! ecc.), nella Maiella (Ten.!) e in altri monti del Piceno e dell' Abruzzo, terminando nel Gargano tra Viesti e Nonte S. Angelo (Pasq. Lic. viagg.). Dill' altro lato trovasi in Liguria, ma non comume: nel Nizzardo a Levenzo e Gorlio (Ard. fl.), in val di T'enda (Bourgeau!), a Dolcedo (Berti!), sopra Genosa (lle Not. rep.), a Chiavari (Bert.); trovasi in Toscana, dove abbonda, 
e sale in tutto l'Appennino, fin' oltre le regioni boschive!, peraltro scarseggia in Maremma, dove non è stato trovato più in basso di Fercole (Parl.!) e di Pitigliano (Bert.), e manca nelle isole, eccettochẻ in quella del Giglio secondo Bertoloni; quindi fa a Viterho (Bert.), a Roma dov'è comune (Fiorini ! ecc.), in Campania a Sora enelle vicinanze (Terr.!), a Pastena e Spigno (Terr.), nel Matese (Terr. ! ecc.), e verso Maddaloni e Avellino (Bert.), terminando in Basilicata a Muro (Terr.!) e Potenza (Ten. syll.). Fiorisce da marzo a maggio nei luoghi bassi, da giugrio ad agosto nei monti.

Distribuzione geografica. - Nell' Europa media, e in parte nella settentrionale e nella meridionale, nell'Asia Minore.

Descrizione. - Pianta perenne, alta da 3 a 6 decimetri, verde, ispidetta, fornita di un rizoma orizzontale, che manda in giù fibre radicali e in alto i rami o fusti ascendenti o eretti, flessuosi, sem. plici, fistulosi, quadrangolari, con gli angoli un po'rilevati, scabri nelle parti superiori per peli corti e rigidi e rivolti in giù, verdognoli o in parte rossicci. Le foglie sono erbacee, opposte, un po'lontane, patenti, le inferiori piccole, quasi orbicolari, cordate, ottuse, quelle di mezzo cordate, ovate, ottuse e le superiori acuminate, tutte con grosse crenature disuguali fornite all apice di una piccola punta, di un verde scurelto di sopra ed ivi con leggieri solchi in rete, di un verde chiaro di sotto ed ivi con un nervo longitudinale da cui partono dei nervi laterali che si diramano per formare una rete rilevata nella pagina inferiore e terminare all' apice dei denti, pubescenti-ru. vidette massime nella pagina superiore e con corte ciglia nel margine, hanno un picciỏlo più corto della foglia, solcato di sopra, conresso di sotto e pelosetto. I fiori sono sessili, distribuiti in gruppetti di 5 a 5 all'ascella delle foglie superiori. Il calice è quasi campanulato, verdognolo, rossiccio alla base, ispidetto, con dieci nervi, con cinque denti patenti, poco più lunghi del tubo, lanceolato-acuminati, forniti di ciglia. La corolla è tre a quattro volte più lunga del calice, di color violetto-porporino, bilabiata, con il tubo più lungo del calice, curvato sopra la base e ivi ristretto, gonfio in alto e nella gola, fornito nel margine da ciascun lato di un dente lesiniforme lunghetto diretto in avanti, con un anello peloso di dentro nella sua parte stretta. Il labbro superiore è a guisa di una piccola volta con piccoli denti nel margine, peloselto di fuori con i peli avvicinati, ed ivi con una carena nella parte posteriore che si biforca in due carene meno sporgenti e divergenti in avanti per terminare al margine del labbro verso l'apice. Il labbro inferiore è diretto in avanti, smarginato, un 
po' concavo nelle sue due metà di sopra, con tante macchioline più scure sopra un fondo violetto-chiaro. Gli stami sono poco piủ corti della corolla, protetti dal labbro superiore, didinami, inseriti verso l'alto del tubo dove questo si slarga. I filamenti sono filiformi, un po' curvi in avanti, bianchicci, pelosetti. Le antere sono verticali, con le logge divaricate, ovali, giallicee, di dietro pelose con lunghi peli bianchi. Il pistillo è lungo quanto gli stami inferiori. L'ovario è quaUrilobo, con i lobi piani di sopra, verdognolo, glabro, liscio e circondato alla base da un disco circolare, piano, dentato, di color giallo pallido. Lo stilo è lungo, filiforme, un po'curvo in avanti, bianco, glabro, bifido all'apice con le divisioni divergenti, acute, e quasi ugıali. In una varietả la pianta è più piccola, con il rizuma molto più strisciante, le foglie piccole, tutte cordato-ovate, non acuminate, la corolla con il tubo più corto e il labbro superiore più largo, gli stami più corti di quello. (Parl. ms., descr. della pianta di Bosco- lungo nell'Appennino toscano, e la var. di li presso al Valloncello).

Osservazioni. - Pianta assai variabile per il grado della pelu. ric, per le foglie macchiate di bianco o no, alquanto piủ larghe 0 piu strette, aguzze o ottuse, cuoriformi alla base 0 no, con denti ottusi o appuntati, nonchè per la grandezza e più minuti particolari del fiore; onde ne sono venute tante cattive specie, fra i fabbricatori delle quali non ultimo il nostro Tenore. La sua sinonimia rispetto al genere in complesso, come rispetto a molti altri della sua flora, $\dot{e}$ quasi inestricabile, a motivo non soltanto dei caralteri poco sicuri a' quali bene spesso si appigliava, ma soprattutto del percepimento confuso che ne aveva, per cui mutazioni continue nei suoi apprezza. menti sulle forme che ebbe a trattare, a tal segno che non di rado manca perfino la concordanza fra le sue descrizioni e $i$ saggi da lui distribuiti, i quali percio non hanno che un dubbio valore di autenticita. Per esempio, il suo Laminun Columnce, dal saggio da lui dunato all' Lirbario centrale, e dalla figura data a tav. 155 della sua flora, è lo stessissino L. maculatum tipico; nel mentre che nella Syll. p. 286 egli insiste sulla sua alfinitá col L. longiflorum.

\section{Kamminm Mexmosmun.}

L. perenne, folis ovatis, grosse duplice crenato-serratis, floralibus conformibus, floribus mediocribus vel magnis, alhis rubrisve, corollae tubo basi curvato, intus oblique piloso-annulato, antheris glahris. 
Lamium flexuosum Ten.! fl. nap. 1. prodr. p. 34 (1811). Bert. 凡. ital. 6. p. 120. Pasq. fl. ves. p. 81. Terr. fl. Vult. syn. p. 198. Ces. el. piunt. Maiell. p. 24. Ces. Pass. Gib. comp. fl. ital. p. 320. Terr.! quarla rel. Terra di Lav. p. 113. Arc. comp. fl. ital. p. 555.

Lamium rugosum Sibth. Sm. fl. grcec. 6. p. 45 (1826) (non Ait.). Ces. Pass. Gib. l. c. Arc.! l. c.

Lamium pubescens Sibth. in Benth.lab. p. 515 (1832-36). Bert. o. c. p. 117 .

Figure. - Fl. nap. t. 52. Fl. grace. t. 555. Reich, ic. bot. $t$. 706. Ic. fl. germ. 18. t. 5. f. 1.

Stazione, Abitazione e Fioritura. - Nei boschi, solto alle siepi ecc. dell'Italia meridionale: in Sicilia, dove sembra comune abbastanza, cosi tutt'intorno a Palermo (Parl.! ecc. ecc.), a Mirto (Todaro!), da Messina (Seguenza!) all'Etna (Guss. syn.), a Siracusa (erb. Webb!) ed Avola (Bianca!) ecc.; in Calabria nell'Aspromonte tra 900 e 1100 metri (Huter Porta Rigo!), intorno a Palmi, a Monteleone ecc. (Arc.!); a Melfi (Terr.); intorno a Napoli, comune (Ten.! ecc.), e nell' isola di Capri (Bert., Pasq.); a Roma (Webb!); in Abruzzo sul Morrone e in valle Orfenta (Ces.); in Piceno ad Umito (Orsini!). Fiorisce da marzo ed aprile a maggio e giugno.

Distribuzione geografica. - Nasce inoltre nella Catalogna in Spagna e nel Russiglione in Francia.

Osservazione. - Non ho creduto dovere tenere separato dal $L a$ mium flexuosum il $L$. pubescens Sibth., o L. rugosum Sibth. Sm., perchè i caratteri differenziali, cioè la maggiore o minore pelurie, il colore dei fiori, e la loro grandezza, alquanto maggiore o minore, sono di quelli che nel genere non servono a distinguere le specie.

\section{Lamium Galeobdolon.}

L. pereme, foliis ovatis, grosse duplice crenato-serratis, floralibus conformibus, floribus mediocribus, luteis, corollæ tubo basi curvato, intus oblique piloso-annulato, antheris glabris.

Lamium Galeobulolon Crantz stirp. ausir.; et auct. rec.

Galeobdolon luteum Huds. R. angl. p. 258 (1798). Bert. fl. ital. 6. p. 133 ; et auct. vet.

Figura. - Reich. ic. fl. germ. 18. t. 5. f. 3.

Stazione, Abitazione e Fioritura. - Per i boschi ombrosi, principalmente di collina e di montagna, della Penisola. E molto 
sparso per tutta l'Alta Italia: in Piemonte ovuuque secondo Allioni, cosi a Mondovi (Ing. cat.), a Torino, in quel di Susa (Colla herb.), a Riva di Sesia (Carestia!), ad Arona (Ricca!), nel Novarese (Bir. f.) ecc.; presso Bellinzona (Com. f.); a Cappararo in valle Malenco della Valtellina (Mass. prodr.); nel Ciomasco nei monti di Ponzate, di Canzo, di Bellagio e della valle Intelvi (Com., Bracht!, Parlatore! ecc.); presso Milano (Rampoldi!); nel Pavese (Rota prosp.); nel Bergamasco (id.); nel Bresciano, frequente (Zersi prosp.), cosi dal lago d'Iseo a Ponte di legno a 1520 metri, a Ciollio a 900 metri e nella Corna Blacca e il Dos alto (Parl.!); in molti luoghi del Tirolo: sul Baldo, a Roveredo (Hausm. $f l$.), nel Trentino (Perini!), a Stenico e Tione, in Fiemme e Fassa, intorno a Bolzano, presso Mlerano ecc. (Hausm.); nei monti Lessini, nel Vicentino (Poll. fl.); nel Bassanese al monte Grappa e al Buso d'Oliero (Bert.); nel monte Cavallo del Bellunese (Poll.), in Friuli a Gemona, Venzone, S. Da. niele (Pir. syll.); in Istria a Lippizza (Bert.); in Emilia nell'Appennino sopra Modena e Bologna, in molti punti (Gib. Pir. fl., Coce. f.), sino a Boscolungo a 1300 metri!, all'Acero, alla Porretta ecc. (G. Bert.). Raro in Liguria, vi è stato trovato sopra Tenda (Ard. $f$.), a Rezzo (Berti!), ad Antora nel Genovesato (Figari!), e nel monte Crusiggia presso S. Stefano d'Aveto (Bert.); in Toscana invece non è raro per tutta la catena appennina, dal monte Gotro (Parl.!) fino all'alpe della Luna (Amidei!), e nelle sue diramazioni di Vallom. brosa, di monte Senario!, di val di Serchio dove scenile fin presso Lucca (Calandrini! ecc.), nonchè nel monte Pisano! e nelle Alpi Apuane (Simi! ecc.); trovasi quindi nel monte Catria delle Marche (Piccinini!); e più giủ nel Piceno al Vettore, alla Sibilla, al Farnio (Paol. el.), ad Unito (Bert.), a Montefortino (Marzialetti!), e nell'A bruzzo (Ten. syll.); da dove salta in Calabria ad Aspromonte (Bert.), a Giffoni (Ten.!), nel bosco di Montalto, alla Sila all'Ervuso e a Basilicó (Ten.). Fiorisce da maggio e griugno a luglio e agosto.

Distribuzione geografica. - Nell' Europa media e in parte nella settentrionale e nella meridionale, nell' $\Lambda$ sia Mlinore.

Descrizione. - Pianta perenne, alta da 5 a 6 decimetri, verde, pelosetta. Il fusto è fistoloso, ascendente o eretto, spesso con fibre radicali in hasso nel primo caso, flessuoso, semplice, quadrangolare, con gli angoli ispidetti per peli corti, curvati in giii e arvicinati al fusto, e con cortissimi peli nelle facce, $i$ quali hanno una piccola glandola all'apice. Le foglie sono molli, opposte, un po' lontane, patentissime, ovato-acute o ovato-acuminate, un po'scavate 
in cuore alla base e le superne ovato-lanceolate, acuminate, doppia. mente dentate, con i denti forniti all'apice di una piccola punta ottusa, verdi di sopra ed ivi con leggieri solchi in rete che corrispondono ai nervi della pagina inferiore e pelosette per peli corti, rari e diretti verso l'apice della foglia, di un verde chiaro e quasi lucente di sotto ed ivi con un nervo longitudinale rilevato da cui partono molti nervi laterali i quali si dirigono obliquamente verso l'apice e il margine della foglia, formando con la loro unione una rete rilevata nella pagina inferiore di questa e terminando alla punta dell'apice dei denti del margine: sono pelosette anche di sotto con i peli diritti e corti; hanno un picciòlo che nelle foglie inferiori è uguale o poco più corto della foglia e va gradatamente diventando più corto, in modo che è cortissimo nelle foglie superiori, è profondamente solcato di sopra, convesso di sotto, pelosetto. I fiori sono per lo più 5 , sessili all'ascella di ciascuna foglia superiore e perciò dieci per ogni coppia di queste. Le bratteole sono patenti, poco piủ corte o quasi uguali al calice, lineari-lanceolate, acuminate, spinescenti, verdi, pelosette di fuori. Il calice è tubuloso, ristretto alla base, verde, pelosetto, con cinque denti, dei quali uno quasi superiore, due laterali e due inferiori, un po'disuguali, patenti, lanceolato-acuminati e spinescenti. La corolla è poco più del doppio piủ lunga del calice, di color giallo-pallido, un po'più carico nel labbro inferiore dove sono delle rnac. chioline a guisa di linee rossicce. Il suo tubo è corto, lungo quanto il tubo del calice, stretto, non slargato ad un tratto, con un anello peloso obliquo nella parte superiore. Il labbro superiore è a guisa di una volta ovale, bislungo, tondeggiante all'apice, peloso di fuori, con lunghe ciglia nel margine, l'inferiore è patente, dirctto un po' in giù, appena più lungo del superiore, trilobo, con i lobi leggermente rotondi nel margine, quasi lanceolati, i laterali un po' più corti e quasi acuti, l' intermedio ottusetto. Gli stami sono quattro, didinami, posti sotto la volta del labbro superiore, che i due più lunghi quasi uguagliano, inseriti in alto del tubo. I filamenti sono filiformi, un po ${ }^{\prime}$ curvi in avanti, bianchicci, puberuli e i due degli stami più corti pelosetti. Le antere sono dapprima quasi orbicolari, appena smarginate all'apice, profondamente scavate in cuore alla base, inserite sopra della base, biloculari, poi con le logge divaricate in modo da essere poste verticalmente, aprentisi con una fenditura comune alle due logge, giallicce, glabre. L'orario è quadrilobo, con i lobi bislunghi, obliquamente piani di sopra, verdognoli, glabri, circondato alla base da un disco un po' lentato. Lo stilo è lungo in modo che 
lo stimma corrisponde tra gli stami corti e lunghi, è filiforme, ma curvo in avanti, violetto-bianchiccio, glabro. Lo stimma è bifido, con le lacinie divergenti, acute, quasi uguali. (Parl. ms., descr. della pianta di Boscolungo nell'A Apennino toscano).

\section{PHLOVIS.}

Phlomis Tourn. inst. p. 177. t. 82. R. Br. prodr. fl. Nov. Holl. p. 504. Benth. Hook. gen. plant. 2. p. 1214. Ces. Pass. Gib. comp. fl. ital. p 322. t. 47. f. 5 .

Phlomidis sp. Linn. gen. pl. Mirb in ann. mus. 15. t. 19. f. 7, 8. Phlomis el Phlomitis Gen. pl. fl. germ. 6. t. 42, 43.

Calyx tubuloso - campanulatus, leviter 5-10-nervatus. Corolla manifeste bilabiata, labio superiore galeato. Stamina 4, conniventia, filarnentis posteriorum basi in appendiculum sursum curvatum pro. ductis, antheris 2-locularibus, loculis longitudinaliter dehiscentibus, confluentibus. Stylus lobis valde inæqualibus. Cocci apice attenuati planiusculi marginati.

Portamento. - Piante alte da $1 / 2$ metro a 2 metri, ramosissime, di cui tre sono frutici con fiori gialli, la quarta è un' erba perenne con fiori rossi. Sono variamente pelose, con foglie di mezzana grandezza, di forma fra l'ovata e la hislunga, intere o leggermente den. tate. I fiori sono grandi, nell' estremità dei rami in fittissimi glomeruli distanti fra loro, e accompagnati da foglie fiorali e da bratteole molto eviderti.

Osservazioni. - Oltre le specie qui annoverate, è stata descritta da Smith (Spicil bot.) una Phlomis italira, affine alla P. purpurea Linn., e indicata da Willdenow e da Persoon in Italia, dove però non si sa che esista.

La P. Lychnitis Linn. è ammessa nel Rep. f. lig. di De Notaris come pianta Nizzaria, sulla fede di un saggio dell' Erbario Torinese, e già come tale era stata segnata da Allioni ( Fl. ped. 1. p. 33). Perỏ al presente non è stata più trovata in quella contrada.

\section{Phlomia Ianuta.}

P. fruticulus densissime tomentosus, foliis ovalibus, basi attenuatis rotundatisve, bracteolis ovali-lanceolatis, dorso undique toFloak Italiaka, - Vol. Vi. 
mentosis, floribus luteis, dentibus calycinis brevissimis, obtusis vel mucronulatis.

Phlomis lanata Willd. enum. plant, hort. ber. suppl. p. 41. Bert. 1. ital. 6. p. 187. Ces. Pass. Gib. comp. fl. ital. p. 323. Arc. comp. f. ital. p. 557 .

Figure.-Alp.de plant. exot.p. 108?. daub. Spach ill.pl.orient. t. H1 (mala).

Stazione e Abitazione. - Nelle colline sassose di Calabria (Ten.!).

Distribuzione geografica. - Oltre l'Italia, in Candia.

\section{Phlomis fruticosa.}

P. frutex densissime tomentosus, foliis oblongis vel ex ovatooblongis, basi attenuatis rotundatisve, bracteolis lanceolatis, exterioribus interdum ovatis, omnibus dorso tomentosis margine setulosis, floribus luteis, dentibus calycinis brevibus, subulatis.

Phlomis fruticosa Linn sp. pl.ed. 1.p. 584. Zer. fl. mel. thes. p. 60. Bert. fl. ital. 6. p. 185. Grech Del. fl. mel. p. 26. Mor. fl. sard. 3. p. 315. Ces. Pass. Gib. comp. fl. ital. p. 323. Groves contr. fl. Terra d'Otr. p. 65. Arc.! comp. fl. ital. p. 557.

Phlomis ferruginea Groves! l. c. (non Ten.).

Figure.-Fl. grcec. t. 563. Reich. ic. fl. germ. 18. t. 21.f. 1.

Stazione, Abitazione e Fioritura. - Nei luoghi aridi sassosi dell'Italia meridionale: in Malta (Gulia! ecc.); in Sicilia nelle parti meridionale e orientale dell' isola, a Sciacca (Guss. syn.), Villafranca (Inzenga!), Siculiana (Citarda!), Girgenti (Parl.! ecc.), Avola (Bianca!), Siracusa (Parl.!), Cassaro, Militello, Caltagirone, Giarre (Guss.), Taormina, Mandanici (Segıenza! ecc.), Messina (Parl.!), Furnari (Guss.) ecc.; in Calabria a Reggio (Bert.), Vallonidi (Pasquale!), Bova, Palizzi dove ahbonda (Arc !); a Otranto, comunissima (Groves!), a Brindisi (Webb!); in Abruzzo nel monte Salviano (Orsini! ecc.); nella Sardegna meridionale a Panli Gerrei (Mor.). Trovasi inoltre nei colli Euganei, dove anticamente la raccolse lo Zannichelli, e modernamente il Barbieri; ma forse non vi è spontanea, e il Prospetto del Trevisan non l'ammette. Fiorisce da aprile a luglio.

Distribuzione geografica. - Oltre l'Italịa, dalla Dalmazia all'isola di Candia.

Osservazioni. - Le foglie variano alquanto per la larghezza; più 
le bratteole. Il colore del rivestimento peloso varia dal bianco al cenerognolo, al gialliccio, al rossigno, nel qual ultimo caso questa specie è stata talora scambiata con la seguente.

\section{Phlomis viscosn.}

P. frutex densiuscule tomentosus, insuper glanduloso-subpilosus, foliis ovatis vel ovato-oblongis, basi cordatis rotundatisve, bracteis anguste lineari-lanceolatis, dorso undique setulosis, floribus luteis, dentibus calycinis brevibus, subulatis.

Phlomis viscosa Poir. in enc. méth. 5.p. 271 (1804).

Phlomis ferruginea Ten.! f. nap. 1. prodr. p. 35 (1811). 2. p. 36. Bert. A. ital. 6. p. 187. Ces. Pass. Gib. comp. fl. ital. p. 323. Arc. comp. fl. ital. p. $55 \%$.

Phlomis virens Cand. cat. plant. hort. monsp. p. 132 (1813).

Phlomis polymorpha Clos in bull. soc. bot. de Fr. 15. p. 6.

Figura. - Ten. o. c. t. 57 (mala).

Stazione, Abitazione e Fioritura. - Nelle colline di Basilicata, e di Terra d'Otranto a Ginosa, Martina e Mlonopoli (Ten. op.); nonchè in Puglia e in Calabria (Ten.! herb.). Fiorisce da maggio a luglio.

Distribuzione. - Oltre che in Italia, nasce in Grecia ; in Candia e in Rodi, in Soria e in Palestina.

Osservazione. - Come bene ha notato Clos, il quale ha soltanto avuto il torto di dare un nome nuovo a una specie già battezzata tre volte, $\mathrm{i}$ detti tre battesimi si riferiscono a un medesimo tipo, neanco molto variabile. I peli glandolosi, abbondanti sui saggi soriani, sono scarsi ma non mancano in quelli italiani.

\section{Phlomis Merbn-venti.}

P. herba hirtula, foliis lanceolatis, bracteolis subulatis, lloribus rubris, dentibus calycinis elongatis, subulatis.

Phlomis Herba-venti Linn. sp. pl.ed. 1. p. 586. Bert. 1l. ital. 6. p. 188. Terr.! /2. Vult syn. p. 1:40. C'es. Pass, Gib. comp. Al. ital. p. 323. Arc. comp. fl. itul. p. $55 \%$.

Figura. - Fl. grece. 1. 565.

Stazione, Abitazione e Fioritura. - Nei pascoli ascintti, nei campi argillosi ecc. per i colli e i monti dell'ltalia meridınale: in Sicilia presso Palermo alla Piana de' greci, alla Pizzuta, alla Ficuzza 
(Parl.! ecc.), nei monti di Renda (Sorrentino!), nelle Madonie a Petralia, a Mannarini (Minà!), in val di Mazzara, a Bronte, a Troina (Guss. syn.); nell'Etna (Parl.!), a Castrogiovanni (Calcara!), in val di Noto (Guss.); in Basilicata a Potenza (Ten. syll.) e a Melfi (Terr.!), nel Matese (Ten.), in Molise a Pietracatella (Arellino!), a Foggia (Groves!), nel Gargano a Monte S. Angelo (Porta e Rigo!), in Abruzzo al Morrone, a Solmona (Ten.), nel Sirente presso Logliano (Groves!), ad Aquila (Orsini!), a Campo Renina presso Pizzoli(Bert.). Fiorisce da maggio a luglio.

Distribuzione geografica. - Nell' Europa meridionale, dal Por. togallo al Caucaso, nell' Asia Minore e fino in Persia, nell'Affrica settentrionale.

\section{PRASIUM.}

Prasium Linn. gen. pl. p. 345. Gen. plant. fl. germ. 6.t. 44. Benth. Hook. gen. pl. 2. p. 1217. Ces. Pass. Gib. comp. fl. ital. p. 323. t. $47 . f .6$.

Calyx late campanulatus, obsolete 10-nervatus. Corolla manifeste bilabiata, labio superiore erecto, concavo. Stamina 4, conniventia, filamentis edentulis, antheris 2-locularibus, loculis longitudinaliter dehiscentibus, conflnentibus. Stylus lobis subæqualibus. Cocci drupacei, apice convexi.

Portamento. - Vedasi la descrizione dell'unica specie del genere.

\section{Prasium maius.}

Prasium maius Linn. sp. pl. ed. 1. p. 601. Zer. fl. mel. thes. p. 64. Bert. fl. ital. 6. p. 258. P. Savi! fl. gorg. p. 274. Gren. Godr. fl. Fr. 2. p. 705. Grech Del.! fl. mel. p. 26. Guss. enum. plant. Inarim. p. 265. Mor.! fl. sard. 3. p. 326. Car. prodr. fl. tose. p. 524. Tassi fl. prov. sen. p. 52. Mars.! cat. pl. Cors. p. 115. Ces. Pass. Gib. l. c. Groves! contr. fl. Terr. d'Otr. p. 66. Terr. quart. rel. Terra di Lav. p. 113. Loj. is. eol. p. 123. Arc. comp. fl. ital. p. $55 \%$.

Figure.-Zan. ist. bot. t. 46. Fl. groec. t. 584. Reich. ic. fl. germ. 18. t. 2. f. 1. 
Stazione, Abitazione e Fioritura. - Sulle scogliere e sui colli sassosi del littorale, dell' Italia meridionale e della media sino in Toscana. In Sicilia fa nel terreno vulcanico come nel calcareo: intorno a Palermo (Parl.! ecc.), a Finale e Monticelli (Minà!), in val di Mazzara, a Milazzo, in val Demone, raro (Guss. syn.), a Panicastro (Seguenza!), a Catania (Heldreich!), in val di Noto (Guss.), ad Avola (Bianca!), e nelle Eolie (Guss., Loj., Mandralisca!), in Ustica (Calcara! ecc.), nelle Egadi (Guss.), in Pantellaria (Calcara! ecc.), Linosa e Lampelusa (Aiuti! ecc.); nonchè in Malta (Grech! ecc.). In Sardegna nasce per esempio a Cagliari (Barla!), a Capoterra (Thomas!), a Castelsardo (Bert.), nell'isolotto Figarello (Mlor.). In Corsica è stato trovato a Bonifacio (Requien!, Kralik! ecc.), e ad Aiaccio in una località dove il Marsilly lo sospetta introdotto. Fa in tutto l'a:cipelago Toscano, cioè in Gorgona (P. Sav.!), nell'Elba (P. Sav.!, Caldesi!), in Pianosa (Bert.) e nel Giglio (Parl.!). Nella Penisola occidentale trovasi in Calabria da Reggio a Tropea (Ten. syll., Arc!, Ricca! ecc.), e a Terranuova (Aiuti!); a Sorrento (Bert.), presso Napoli verso capo Miseno (Ten., Pasquale! ecc.), nonchè in Ischia (Guss., Bolle!) e in Capri (Bert.), quindi da Mondragone (Terr.!) e Gaeta (Terr.) a Terracina (Ball! ecc.); a Roma (Seb. Haur. prodr., Barbieri!); nella Maremma toscana, da Orbetello a Populonia!. Nella Penisola orientale nasce ad Otranto (Groves!), tra Monopoli e Bari (Bert.), a Trani (Bruni!), e nel Gargano (Ten.). Fiorisce da marzo ed aprile a maggio e gingno.

Distribuzione geografica. - Nell'Europa meridionale, da Mladera alla Grecia, nell'Asia più occidentale, nell'Affrica settentrionale.

Descrizione. - Suffrutice alto da 2 a 5 decimetri. Fusti ramosi, rami ascendenti o eretti, legnosi in basso, erbacei in alto, 4-angolari, verdognoli con gli angoli sporgenti, bianchicci e pelosetti, rametti corti e patenti. Foglie opposte, verdi, ovate o ovato-lanceolate, ottuse, spessu curdate alla base, crenulate, con i denti forniti all'apice di una piccola punta ottusa, verdi e un po'lucenti di sopra ed ivi con solchi che corrispondono ai nervi di sotto i quali sono sporgenti e incolori alla pagina inferiore ch'è di un verde-glaucescente: pelosette-ispidette per rari.peli nel margine e di solto nei nervi e in qualche raro punto della pagina inferiore. Picciòlo corto, solco longitudinale largo e profondo quasi canale, convesso di sotto, margini bianchicci, peloso-ispidetto. Fiori eretti solitarii, all'ascella di una brattea, formanti un racemo o spiga terminale corta od ottusa; 
pedicelli cortissimi. Brattee o foglie fiorali lunghe quanto il calice; larghe, ovato-lanceolate, intere, con ciglia rade ed ispidette, con una punta a guisa di aresta all'apice, erette, verdi, lucenti con un solco longitudinale di sopra e glabre, di un verde glauco di sotto, nervo longitudinale poco sporgente, laterali meno. Pedicello come il calice con molti peli glandolosi all'apice. Calice grande, campanulato, verde, pubescente, glanduloso, appena bilabiato, con il labbro superiore diviso in tre lacinie e l'inferiore in due, le lacinie quasi uguali, larghe, ovate, acuminate con una punta quasi are. sta all'apice: nervo longitudinale sporgente che va sino alla base del calice. Corolla più grande del calice, bianca. Tubo poco piủ corto del calice, quasi diritto, un po'schiacciato lateralmente, un po'piủ largo verso l'apice, glabro con un anello peloso obliquo di dentro verso alto, peli a guisa di squamette in basso. Lembo bilabiato, labbro superiore più piccolo ovale e bislungo, con l'apice prolungato e bidentato, denti ottusi, concavo, pubescente glandoloso di fuori, inferiormente più lungo e più largo, lungo quasi quanto il tubo della corolla medesima, patente, trilobo, lobi laterali bislunghi, ottusi, quello di mezzo più grande, quasi orbicolare, gola spesso con righe longitudinali formate da macchioline quasi amarante. Stami 4 quasi uguali di altezza, sotto la concavità del petalo superiore, inseriti in alto del tubo. Filamenti un po'curvi in avanti, più grossi in basso, ivi cigliato-barbati con i peli lunghi e bianchi, più stretti e glabri in alto, bianchicci. Antere con le logge bislunghe, ottuse, divaricate, un po'pendenti, aprentisi longitudinalmerte, glabre, violetto-scure, connettivo largo all' apice del filamento e bianchiccio. Pistillo quasi uguale agli stami. Orario piccolo, 4 lobi quasi tondi, verdognoli, lisci, lucenti, in un disco annulare grossetto verdognolo. Stilo filiforme, lungo, un po'curvo in avanti, glabro, bianco. Stimma bifido, lacinie corte, divergenti, acute, quasi uguali, bianche. Calice dilatato nel frutto, campanulato con le lacinie patenti. (Parl. ms., descr. della pianta del monte Argentaro in Toscana). Frutto generalmente dicocco, i cocchi essendo angolati-globosi, verdi, lunghi 4 millimetri, drupacei, coll'endocarpio crostoso. (v. v.).

\section{GALEPSIS.}

Galeopsis Tourn. inst. p. 185. t. 86. Mirb. in ann. mus. 15. t. 18. f. 1, 2. Gen. pl. fl. germ. 6 t. 32. Benth. Hook. gen. pl. 2. p. 1209. Ces. Pass. Gib. comp. fl. ilal. p. 318. t. 47. f. 2. 
Calyx tubuloso-campanulatus, leviter 5-10-nervatus. Corolla manifeste bilabiata, labio superiore galeato. Stamina 4, conniventia, filamentis edentulis, antheris 2-locularibus, loculis transversim dehiscentibus. Stylus lobis subæqualibus. Cocci apice convexi.

Portamento. - Le due specie di questo genere sono erbe annue, erette, semplici o ramose a pannocchia, alte da 1 decimetro fin quasi a un metro, pelose. Le foglie sonio piuttosto grandi, picciolate, per lo più dentate, variabili nella forma dalla strettissima lanciolata alla slargata ovata. I fiori, piuttosto grandi, di vario colore, sono in glomeruli ascellari, distanti fra loro e alquanto ravvicinati nell' estremità del fusto e de' rami.

\section{Galeopsis hadanum.}

G. caule æquali, foliis ovato, oblongo aut lineari-lanceolatis, villosis, calycibus villosulis dentibus lanceolatis.

Galeopsis Ladanum Linn. sp. pl. ed 1.p. 579; et auct.

a latifolia Wimm. et Grab. fl. Sil.

Galeopsis dubia Leers 凡. herb. ed. 1.

Galeopsis ochroleuca Lam. enc. méth. bot. 2. p. 600. Mor. Al. ven. Trev. prosp. fl. eug. p. 26. Zan. prosp. f. ven. p. 26. Hausm. fl. Tir. p. 689. Pir. fl. for. syll. p. 112. De Vis. Sacc. cat. piant. Ven. p. 137. Cies. Pass. Gib. comp. fl. ital. p 318. Ing. cat. sp. Mond. p. 37. Arc. comp. fl. ital. p. 553. Gib. Pir. fl. Mod p. 130.

Galeopsis intermedia Vill. hist. pl. Dauph. 2. p. 387. Car. prodr. fl. tosc. p. 521 . Archb. 1. Alto-Serch. p. 61.

Galeopsis villosa IIuds. fl. angl. ed. 2. p. 256.

Galeopsis grandiflora Balb. misc. bot. p. 28. Ruch. R. lid. ven. p. 142. Poll. fl. ver. 2. p. $27 \%$.

Galeopsis Ladanum $\beta$ Bert. fl. ital. 6. p. 126.

Figure.-Riv. pl. fl. irreg. monop. t. 24. fig. destr. Reich, ic. bot. t. 46, 47. Ic. 1. germ. 18. t. 2\%. f. 1, 3.

$\beta$ angustifolia Wimm. et Grab. o. c.

Galeopsis Ladanum Bert. o. c. p. 125.

Galeopsis angustifolia Eh, herb. Rota prosp. piant. Pav. $\mu$. 29?. Bicch. agg. fl. lucch. p. 16. Ard. cat. pl. Ment. p. 29.

Galeopsis Ladanum Car. o. c. p. 520.

Galeopsis arvatica Jord., G. glaucescens Reul., Ard M. Alpesmar. p. 299.

Galeopsis Reuteri Reich. fil. ic. 11. germ. 18. p. 17. 
Figure, - Lam. ill. t. 506.f. 2. Reich. ic. fl. germ. 18. t. 28. f. 1, 2 .

Stazione, Abitazione e Fioritura. - Nei campi, come pure nei luoghi incolti, della Penisola, e s'inalza notevolmente nei monti. Dicesi abbondare nel Piemonte (All. fl. ped. ecc.), e difatti è stata trovata in molti luoghi del tratto montuoso da Alba (Bert.) ad Arona (Ricca!), dove sale al col di Tenda a quasi 2000 metri, sul Cramont fra gli abeti (Parl.!) ecc Nasce ai laghi di Lugano, e di Corno (Com. fl. com.), in Valtellina a Montagna, in valle Malenco (Mass.) e presso Bormio a 1200-1400 metri, presso Lecco a Ballabio e sul monte Barro, in Brianza dov'è comune (Parl.!), presso Milano a Cassano d’Adda (Rampoldi!), presso Pavia, in vari siti del Bergamasco (Rota ecc.), nel Bresciano dov'è frequente (Zersi), fino a Ponte di legno a 1320 metri (Parl.!), in Tirolo al lago di Garda, a Roveredo (Hausm.), Trento (Perini!), nelle Giudicarie (Hausm.), in Fiemme (Bert.), intorno a Bolzano, e in val Venosta (Hausm.), a Mantova (Bert.), nel Veronese e nel Vicentino, dove sembra che sia comune (Poll.), negli Euganei (Trev. ecc.), a Venezia (Kellner! ecc.), nel Trivigiano a Crespano (Bert.), nel Friuli superiore e nella Carnia (Pir.), a Trieste (Bert.); e sulla riva destra del Po a Godiasco sopra Voghera (Nocc. Balb. $f l$. tic.), a Parma e nei monti Parmensi (Passerini!), cosi nel monte Prinzera (Parl.!), nei monti Modenesi a Pavullo, Salto di Montese ecc. (Gib. Pir.), nel Bolognese dov'è frequente (Cocc. fl. bol.), e nel sovrastante Appennino alla Porretta (Bert. not. Porr.) e al monte Beni (Bert.), infine a Faenza (Cald. fl. tent.). Trovasi qua e là in Liguguria (De Not. rep.), cosi a Nizza (Webb! ecc.), Mentone (Ard.), Lantosca (Ball!), Dolcedo (Berti!), Cervo (Ricca!), Genova (Bert.), Chiavari (Delpino!) ecc. E comune per la Toscana, in tutta l'estensione delle valli della Magra, del Serchio e dell'Arno, dove sale nei monti fino al limite superiore dei boschi, manca però nella regione mareminana, e precisamente fra il mare e la linea che da Pisa (Savi fl. pis.) passa a Casciana (Bastianini!), Siena e il monte Amiata!, come manca nelle isole dell'arcipelago toscano. Nelle Marche fa a S. Marino, Macerata (Bert.), Civitanuova, Camerino (Ricci!), San. t'Elpidio, Grottamare (Bert.), Montefortino, Castelluccio sul Vettore (Marzialett!!), Trisungo sopra Ascoli (Parl.!); in Abruzzo al Pizzo di Sivo, Avezzano (Ten. syll.), Caramanico (Ces. el.) e altrove; nel Lazio presso Cammarata (Rolli!); in Mulise a Capracotta (Ten., Bert.); in Campania a Casalvieri (Terracciano!), Cassino, e diversi altri siti vicini (Terr. rel.), nel Matese, a Cusano, a Gragnano (Pa- 
squale!); infine in Basilicata alla Fiumara di Pattano sotto Castellonuovo, alle sponde dell' Alento, e altrove (Ten.). Fiorisce da giugno e luglio a settembre.

Distribuzione geografica. - In tutta l'Europa, meno la piủ settentrionale e la più meridionale, nell'Asia Minore, e in Siberia.

Descrizione. - Pianta annua, alta da 1 decimetro e mezzo a piủ decimetri, di un verde chiaro, pubescente con i peli molli curvati ad arco in giu e avvicinati nel fusto e semplicemente avvicinati nelle foglie. La radice è ramosa, piuttosto delicata, flessuosa, bianchiccia. Il fusto è eretto, semplice o con qualche corto rametto in alto, piuttosto delicato, quadrangolare, non 0 appena ingrossato sotto ai nodi vitali, rossiccio, pubescente per i peli già descritti e fornito inoltre verso alto di peli radi, lunghi, orizzontali, aventi all' apice una glandoletta tonda di color violetto scuro e quasi nericcia. Le foglie sono opposte, alquanto lontane, molto aperte e le superne anche un po'dirette in giù, bislungo o ovato-lanceolate, strette alla base, di un verde piuttosto chiaro di sopra ed ivi con un solco longitudinale e con pochi solchi laterali molto obliqui, di un verde piủ chiaro di sotto ed ivi con un nervo longitudinale più sporgente, da cui partono pochi nervi laterali anche sporgenti, i quali si dirigono obliquamente verso il margine e l'apice della foglia; tutti i nervi sono di colore più chiaro della pagina inferiore di questa: le foglie inoltre hauno pochi e grossi denti a guisa di sega, ottusetti e lontani, sono pubescenti di sopra e un po' anche di sotto con i peli avvicinati alquanto ad esse e con qualche pelo glandoloso all'apice come quelli del fusto, e forniti di un picciòlo il quale è piủ corto di esse, scanalato di sn. pra, un po'convesso di fuori, verdognolo e pubescente. I fiori sono da 5 a 7 all' ascella della foglia superiore, sessili, accompagnate da bratteole disuguali, piủ corte del calice, parte crette, parte curvate in fuori, lanceolate con un apice lesiniforme-spinoso, verdognole, pubescenti, con qualche pelo glandoloso. Il calice ha il tubo verdognolo, con dieci nervi alqunanto sporgenti, è pubescente ed ha diversi peli glandolosi : ha cinque dentı poco disuguali tra loro, la metà circa più corti del tubo, triangolari, lesiniformi, spinosi, verdi, con alquanti peli glandolosi. La corolla $\dot{e}$ di color violetto-rhiaro e quasi porporino, con macchie bianchicee, larghette, lunghe, disuguali alla base del lobo medio del labbro inferiore. Ha il tubo quasi il doppio più lungo del calice, gradatamente un po' più largo verso alto, pubescente. Il labbro superiore è più corto dell'inferiore, ovato-ovale, un po'concavo e glabro in avanti, un po convesso e pubescente di die. 
tro, spesso con due piccoli denti all'apice, talvolta ottusi: il labbro inferiore è diretto in avanti, trilobo, con i lobi laterali più piccoli di quello di mezzo, quasi tondeggianti e leggermente smerlati all'apice e con il lobo di mezzo obovato-orbicolare smarginato, con un dente nella smarginatura e anch'esso leggermente smerlato. Gli stami sono didinami, i due più lunghi appena più corti del labbro superiore, tutti protetti da questo. I filamenti sono inseriti all' apice del tubo, sono eretti, un po'curvati in avanti verso alto, quasi piani, più stretli un po'in alto, bianchi, glabri. Le antere sono gialle, biloculari, con le logge opposte, quasi tonde, con una valva ovato-tonda, fornita di poche ciglia nel margine. Il pistillo è lungo quanto gli stami piủ lunghi. L'ovario è diviso in quattro lobi, ovali-tondi, un po'convessi di fuori, convesso-angolati di dentro, verdognoli, glabri. Lo stilo è lungo, filiforme, glabro, bianchiccio in basso, rosso in alto. Lo stimma è bifido, con le lacinie quasi uguali, acute, dirette l'una in su e l'altra curvata in avanti ed in giù. (Parl. ms., descr. della pianta dell' Appennino casentinese in Toscana).

Osservazioni. - Questa pianta polimorfa varia : per la larghezza delle foglie; per la presenza più o meno abbondante o assenza di peli glandolosi misti ai peli ordinari; per la-quantità di questi; per il colore della corolla, ordinariamente rossa, talora gialla; per la sua grandezza, dal doppio appena al quadruplo del calice. Tali variazioni hanno suggerito molte specie poco buone, che sono state ricondotte dipoi ad un medesimo specifico; e ben a ragione da ultimo l'Ascherson (Fl. prov. Brand. p. 527) vi ha ricondotto anche la Galeopsis $d u$ bia Leers, o G. ochroleuca Lam., dai più tenuta ancora separata.

\section{Galeopsis Tetrahit.}

G. caule sub nodis tumido, foliis ovatis, pubescentibus, calycibus plus minus hirto-pilosis dentibus subulatis.

Galeopsis Tetrahit Linn. sp. pl. ed. 1. p. 579. Bert. fl. ital. 6. p. 128; et auct.

Galeopsis cannabina Willd. sp. pl. Mass. prodr. fl. valt. p. 75.

Galeopsis pubescens Bess. prim. fl. Gal. Gaud. f. helv. 4. p. 57. Com. fl. com. 4. p. 329 Rota prosp. piant. Pav. p. 272. Prosp. $f$. Berg. p. 70. Hausm. fl. Tir. p. 690. Gib. Pir. fl. Mod. p. 130.

Galeopsis versicolor Curt. ft. lond. Bert. o. c. p. 131. De.Not. ‘ep. fl. lig. p. 325. Hausm. l. c. Rota prosp. fl. Berg. p. 70. Pir. 
f. for. syll. p. 112. Zersi prosp. piant. Bresc. p. 167. De Vis. Sacc. cat. piant. Ven. p. 13\%. Cocc. 17. Bol. p. 387.

Galeopsis bifida Bonn. in Lej. Court. comp. fl. belg. Hausm. l. c. Rota prosp. fl. Berg. p. 70. Gib. Pir. o. c. p. 129.

Galeopsis sulfurea Jord., Ard. /l. Alpes-mar. p. 299.

Figure. - Reich. ic. bot. t. 18, 49, 57, 877. Ic. 11. germ. 18. t. 29,30 .

Stazione, Abitazione e Fioritura. - Nei luoghi boschivi o campestri della Penisola, specialmente in montagna. In Piemonte trovasi ovunque (All. fl. ped.), per esempio da Garessio (Gentili!) al Cenisio, dove sale al lago e a Ronche (Parl. !), a Riva di Sesia (Care-

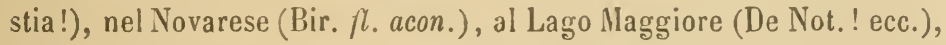
a Domodossola (Gaud.); e nel vicino Cantone Ticino a Bellinzona (Gaud.). In Lombardia nasce nel Pavese (Nocc. Balb. fl. tic..), nel Cremonese (Parl.!), in rari punti del Comasco (Com.), e della Val. tellina (Mass.), nel Bergamasco dove sembra comune (Rota), nel Bresciano ugualmente (Zersi), e risale per val Camonica fino al To. nale a 2000 metri (Parl.!), mentre scende nel piano ad Asola (Paglia!). In Tirolo è pure frequente, da Laas e Trafoi in val Venosta, e da Welsberg in Pusteria a Merano, Bolzano, val di Sole, Fassa e Fiemme, Trento, le Giudicarie, e il Baldo (Hausm. ecc.). E pure comune nel Veronese e nel Vicentino, fino ai Lessini e al monte Sumano (Poll. $f$. ver. ecc.); trovasi negli Enganei (Trev. prosp.), a Padova (Bert.), a Venezia (Zan. prosp.), nel Trevigiano (De Vis. Sacc.), Bassanese (Bert.) e Bellunese (De Vis. Sacc.), nel Friuli a S. Daniele, Gemona, Faedi (Pir.), Monfalcone (Bert.) ecc. Nell'altro lato della valle del Po nasce nell'Appennino Piacentino (Bert.), a Parma, e nell'Appennino Parmense alla Cisa (Passerini!), a Modena, e in molti luoghi dell'Appennino Modenese (Gib. Pir.) fino all'Abetone!, parimente nell'Appennino Bolognese fino all'Acero e al Cavone del Corno (Bert., Cocc.), nel Ferrarese, nell'Appennino Faentino ad Abbia e a Valcoloreta (Bert.); e poi prolungandosi nelle Marche ivi nasce nel monte Catria (Piccinini!), a Pioraco nel Camerinese (Bert), nel Piceno ad Amandola (Marzialetti!) e a Colle d'Arquata (Bert.), terminando nell'Abruzzo (Ten. syll.) e in Molise nel monte di Mezzo (Bert.). Nel lato occidentale della Penisola nccupa la Liğuria, ma scarsamente, cosi fa a Tenda (Burnat!), S. Martino Lantosca, Robione, S. Albano, Nizza (Art.), Porto Maurizio (De Not.), Dolcedo, Rezzo (Berti!), Chiavari (Delpino!); ampiamente poi la Toscana nella sua parte montuosa boschiva, fino alle radici dei 
monti, cioè le Alpi Apuane (Bert. ecc.), tutto l'Appennino e le sue diramazioni dal monte Gotro! all'Alvernia (Parl.!), e a Vallombrosa!, S. Marco in Chianti (Beccari!), Siena, monte Amiata a S. Fiora (Bert.) e alla Badia S. Salvadore (Campani!), Acquapendente (Parl.!); quindi scendendo ritrovasi presso Roma al monte Gennaro, alla caduta delle Marmore (Sang. cent.), e a Rocca di Papa (Rolli!), in Campania a Cassino (Terr. rel.), a Picinisio (Terr. !), nel Mlatese (Terr. rel.), a Cusano (Avellino!) Fiorisce da luglio a settembre.

Distribuzione geografica. - In tutta Europa, meno la piủ meridionale, nell'Asia Minore e in Siberia.

Descrizione. - Pianta annua, alta da 3 a 10 decimetri e talvolta anche più, di un verde chiaro, peloso con i peli lunghi e diretti un po'in giù nelle parti inferiori e con i peli corti, e curvati in giù e avvicinati nelle parti superiori, dove pure sono dei peli diritti aventi all' apice una glandola nericcia. La radice è fibrosa, ramosa, bianchiccia. Il fusto è eretto, quadrangolare, con gli angoli ottusi, con le facce leggermente scanalate verso alto, ingrossato sotto il nodo vitale, ivi ispido per peli lunghi, rigidetti, articolati e diretti un po'in giù, verdognolo, ramoso, con i rami opposti, eretto-patenti. Le foglie sono delicate, opposie, patenti, ovate o allungato-lanceolate, acuminate, quasi tonde e un po'in forma di cuneo alla base, dentato-seghettate nel margine, con i denti ottusi aventi all'apice una punta ottusa, di un verde un po chiaro di sopra ed ivi pelose per peli lunghetti e rari, con un solco longitudinale e molti solchi laterali obliqui che vanno dal nervo longitudinale al margine: di un verde assai più chiaro di sotlo ed ivi pubescenti, segnatamente nei nervi, con un nervo longitudinale rilevato, dal quale partono molti rami laterali obliqui anche rilevati, interi o bifidi all' apice dove si uniscono tra loro in mezzo ai denti del margine, come pure pei rametti laterali: sono fornite di un picciòlo, lungo quasi un terzo o un quarto della foglia, con un solco stretto di sopra e convesso di sotto. I fiori sono da 3 a 5 , sessili in ciascuna metà del verticillo all'ascella delle foglie superiori, in modo che in ciascun verticillo vi sono da 6 a 10 fiori, facendo una spiga corta ed interrotta e accompagnata dalle foglie fiorali più lunghe o più corte dei fiori, e ciascun fiore poi ha una bratteola setacea, più corta del calice, un po'patula, verdognolobianchiccia, ispida e con peli lunghi aventi una glandola nericcia all'apice. It calice è tuboloso, con il tubo membranaceo, bianchiccio o rossiccio con cirque nervi rilevati che vanno ai denti ed altri intermedi più delicati e pelosetti per peli corti e delicati, ha cinque denti 
piủ corti del tubo, un po' patenti, strettamente lanceolati-lesiniformi, terminati in una punta spinescente, verdognoli, pelosetti e con pochi peli lunghi e glandolosi, nei seni vi sono dei peli molto lunghi: cresce un poco nel frutto e i denti diventano patenti. La corolla è molte volte più lunga del calice. Il tubo è molto piủ lungo del calice!, cilindrico e bianchiccio per gran tratto, un po'slargato in alto ed jvi di color violetto-roseo come le labbra, e gialliccio nel punto intermedio, è puberulo di fuori e con pochi e corti peli di dentro verso la base. Il labbro superiore è diretto in su ed in avanti, ovale, eroso-dentellato verso l'apice, convesso e puberulo e di color violet. to-roseo di fuori, concavo, glabro e violetto-bianchiccio di dentro. Il labbro inferiore è piủ largo ma della stessa lunghezza del superiore, patente, trilobo, con i lobi laterali più piccoli, quasi quadrati, dentellato-laceri all'apice, di color roseo-violetto, e con il lobo di mezzo anche quasi quadrato, smarginato o intero, di colore più carico nel mezzo e assai più chiaro nel margine e nell'apice: questo lobo alla base nel punto di unione con i due lobi laterali ha due appendici a guisa di denti ottusi, un po'divergenti tra loro, diretti in alto e del colore stesso della corolla. Gli stami sono quattro, didinami, i due più lunghi sono appena più corti del labbro superiore dalla concavità del quale sono coperti. I filamenti sono un po' piani inferiormente, lesiniformi in alto, un po'curvati in avanti verso l'apice, bianchi; $i$ due più corti hanno alcuni peli corti nel margine verso basso e $\mathrm{i}$ due più lunghi soltanto ivi nel inargine interno. Le antere sono bilocu. lari, con le logge opposte e divise dal connettivo grossetto quasi cilindrico e bianco, ciascuna loggia è triangolare, e si apre per una valvola fornita di ciglia, avente alla base poche glandolette tonde a guisa di perline: sono verdognole-giallicce e glabre. Il polline è giallo. Il pistillo è lungo quanto gli stami più lunghi. L'ovario è glabro, gialliccio, quadrilobo, con i lobi quasi orbicolari, piani di fuori e convessi di sotto, inseriti e abbracciati in parte da un disco il quale si prolunga inferiormente a guisa di una sijuama ovale, ottusa e spesso con qualche piccolo dente, più lunga dell'ovario e posta dalla parte inferiore. Lo stilo é lungo e filiforme, bianchiccio, glabro. Lo stimma è bifido, con le lacinie lesiniformi, dirergenti e l'anteriore diretto un po' in giù. Gli esterni sono obovato-orbicolari, quasi lenticolari, nericci, glabri. (I'arl. ms., descr. della pianta di l'rumone in Lombardia).

Deacrizione della var. a fiore giallo. - Pianta annua, alta da 5 a più decimetri, verde, ispida per peli lunghi, rigidi, formati 
di più cellule unite l'una all' estremità dell'altra, bianchi. La radice è ramosa, flessuosa, bianchiccia. Il fusto è eretto, ramoso con i rami eretto-patenti, o ascendente, quadrangolare, gonfiato molto sotto ogni nodo vitale, ispido, rossiccio-scuro in basso, spesso con gli angoli dello stesso colore, verdognolo nel resto. Le foglie sono opposte, alquanto lontane, patenti, molli, ovali-acuminate, tondeggianti e come troncate all'apice, verdi di sopra ed ivi con un solco longitudinale e con molti solchi laterali che corrispondono ai nervi di sotto, di un verde chiaro nella pagina inferiore ed ivi con un nervo longitudinale sporgente e quasi bianchiccio, da cui partono molti nervi laterali, anche sporgenti, e di colore più chiaro della stessa pagina inferiore, i quali si dirigono obliquamente e un po'curvi verso il mar. gine e l'apice della foglia e ivi si diramano poco: sono crenato-seghettate nel margine, pelose in ambedue le pagine, e fornite di un picciòlo più corto di esse, con un solco larghetto e profondo di sopra, convesso di sotto, peloso verdognolo, o rossiccio di sopra: questo picciòlo è gradatamente piủ corto nelle foglie superiori, tantochè le superne sono sessili, e quasi ovato-acuminate. I fiori sono circa 2 o 3 all'ascella di ciascuna foglia superiore del fusto e dei rami, sessili, accompagnati da bratteole più corte ed avvicinate ad esso, lanceolate, lesiniformi in alto, pelosi con i peli lunghi aventi all'apice una glandoletta tonda e rossiccia: ha il tubo bianchiccio, con dieci nervi sporgenti e verdognoli, pelosi, e cinque denti disuguali un po'tra loro, della lunghezza quasi del tubo, eretto-patenti nel fiore, più aperti nel frutto, lanceolato-lesiniformi, verdognoli, con peli glandolosi come le bratteole: la gola del calice è larga, pelosa, con i peli bianchi, diretti in su. La corolla è ocrolenca, il tubo e il labbro superiore sono di un giallo chiaro, il labbro inferiore è bianco con una macchia larghetta di un giallo quasi dorato alla base del lobo di mezzo di questo: peró varia essendo in una varietà di un colore violetto molto chiaro con la solita macchia giallo-dorata qui descritta. Il tubo è il doppio più lungo del calice, appena curvo, gradatamente un po più largo in alto. Il labbro superiore è più corto dell'inferiore, diretto in su, ovale, un po' concavo e glabro in avanti, un po' convesso di dietro ed ivi con peli lunghi e bianchi, tondeggiante all'apice e ivi con denti disuguali e profondi: il labbro inferiore è direito in avanti, trilobo, con i lobi laterali la metà circa più corti di a dello di mezzo, tutti quasi quadrati e leggermente e irreœolarr.ıente dentati nell'a apice, quello di mezzo è talvolta appena smar: ginato con un piccolo dente nella smarginatura: alla sua base vicino 
ai lobi laterali è da ciascun lato un gobbo lungo, a guisa di dente, diretto un po'in fuori. Gli stami sono didinami, due poco sopra de. gli altri due: questi giungono alla metà del labbro superiore, da cui tutti sono coperti. I filamenti sono saldati con il tubo sino all' apice di questo, per cui sono assai corti nella parte libera, un po'curvati in avanti verso l'apice, quasi piani d'avanti in dietro, bianchi, glabri. Le antere sono biloculari, con le logge opposte, quasi tonde, che si aprono per una valva ovata, ottusetta, fornita di ciglia nel margine; sono gialle. Il polline è giallo, il pistillo è lungo quanto le antere più lunghe. L'ovario è diviso in quattro lobi, quasi orbicolari, appena convessi di fuori, molto convessi di dentro, giallicci, glabri. Lo stilo è lungo, filiforme, bianchiccio, glabro. Lo stimma è bifido, con le lacinie poco disuguali, quasi acute, l'una diretta in su e l'altra curvata in giù, bianchiccio o roseo. (Parl. ms., descr. della pianta del Casentino in Toscana).

Osservazioni. - Questa specie varia poco per le foglie, molto per i fiori, che sono rossi, bianchi e gialli, o variamente screziati, col tubo corollino ora uguale al calice o più corto, ora piủ lungo e sino al doppio e più.

\section{SAIUIA.}

Horminum, Sclarea, Salvia Tourn. inst. p. 178-180. t. 82, 83. Salvia Linn. gen. pl. ed. 1. p. 6. Mirb. in ann. mus. 15. t. 14. f. 16 18. t. 15. f. 1-12. Gien. plant. fl. germ. 6. t. 8. Benth. Hook. gen. pl. 2. p. 1194. Ces. Pass. Gib. comp. fl. ital. p. 305. $t$. 45. f. 2.

Calyx tubuloso-campanulatus vel campanulatus, validiuscule 1013-nervatus. Corolla manifeste bilahiata, labio superiore galeato. Stamina 2 (inferiora), conniventia, filamentis apice sæpe d:nticulo aut appendiculo auctis, antheris dimidiatis 1-locularibus, Irngitudinaliter dehiscentibus, loculo altero in extrenitate inferiore connectivi elongati cum filamento articulati atrophico vel nullo. S'ylus lobis plus minus inæqualibus. Cocci apice convexi leves.

Portamento. - I te nostre Salvie sono frutici, o piiu spesso erbe perenui, di rado annue, di statura generalmente alta, $\mathrm{Ja} \% / 2$ metro a 1 metro, con foylie piccinolate, larghe, spesso rugos:, dentate, e talora maggionmente divise: innalzandosi sul fusto, le f yglie tendono 
a farsi più piccole e sessili, e passano nelle brattee dell'infiorescenza. Questa è terminale, ed è una pannocchia o un lungo grappolo di glomeruli piủ o meno ravvicinati, per lo piủ triflori, accompagnati da brattee ora grandi, ora piủ piccole, larghe, acute, erbacee e allora persistenti, o pure membranose o colorate e allora caduche. I fiori possono essere fra i piủ grandi della famiglia, ma anche relativamente piccoli, e sono di colore violaceo, lilacino, roseo o bianco, rarissimamente giallo.

Osservazione. - Sui processi d'impollinazione in questo genere vedasi Hildebrand, Ueber die Befruchtung der Salbeiarten durch Insek. ten, 1864.

\section{Salvia officinalis.}

S. frutescens sempervirens, foliis oblongis, crenulatis, interdum basi auriculatis, rugosis, plus minus canescentibus, bracteis mediocribus, membranaceis, ovatis, acuminatis, glomerulis distinctis, calycis campanulati labio superiore $\overline{3}$-dentato, inferiore 2-fido, corolla calyce duplo longiore.

Salvia officinalis Linn. sp. pl. ed. 1. p. 23. Zer. fl. mel. thes. p. 68. Bert. f. ital. 1. p. 136. Colla herb. ped. 4. p. 390. De Not. rep. fl. lig. p. 315. Koch syn. fl. germ. ed. 2. p. 637. Gren. Godr. f. Fr. 2. p. 670. Hausm. fl. Tir. p. 673. Rota prosp. fl. Berg. p. 69. Mor. fl. sard. 3. p. 295. Pir. fl. for. syll. p. 109. Bicch. agg. fl. lucch. p. 16. Car. prodr. fl. tosc. p. 509. Ard. fl. Alpes-mar. p. 293. De Vis. Sacc. cat. piant. Ven. p. 133. Pasq. fl. ves. p. 79. Mars. cat. pl. Cors. p. 113. Ces. Pass. Gib. comp. fl. ital. p. 306. Ing. cat. sp. Mond. p. 63. Terr.! quarta rel. Terra di Lav. p. 112. Arc. comp. fl. ilal. p. 543.

Figure, - Lam. ill. t. 515. Reich. ic. fl. germ. 18. t. 44.

Stazione, Abitazione e Fioritura. - Qua e là in luoghi sassosi montuosi. Essendo poi coltivata ovunque nei giardini ad uso di condimento, insalvatichisce con sufficiente facilità e frequenza perchè riesca bene spesso arduo il decidere se sia realmente spontanea o no in certi dati luoghi. Allioni ( $f$. pedem. 1. p. 12) la dà delle parti più calde dei monti Valdensi e della valle d'Aosta, Ingegnati di Mondovi, Gaudin (fl. helv. 1. p. 52) dietro Schleicher e Koch, del Ticino; Rota l'indica subspontanea nel Bergamasco a Sarnico e Predore; nel Bresciano è stata raccolta a Edolo sui margini dell'Oglio 
(Ricca!); è quasi spontanea nei colli Veronesi secondo Pollini ( $f$. ver. 1. p. 28), come pure intorno a Bolzano nelle vigne secondo Hausmann, che osserva ch'è coltivata allo scoperto fino a 1260 metri sul monte Ritten presso Klobenstein; fa nelle rupi del Friuli e dell'Istria intorno a Duino (Pir.), a Contovello presso Trieste (Mo. retti!), a Pola (Poll.), a Fiume (Poll., Bert.); è indicata a Nizza, a Mentone, ma rara e subspontanea (Ard.); in Toscana fa presso Lucca, ma probabilmente avventizia (Bicchi), e nell'Elba (Bert.); è indicata ad Ostia (Seb. Maur. fl. rom. prodr. p. 10), e trovasi al lago di Castel Gandolfo (Rolli!), e al lago Fucino sul monte Salviano (Ten. syll. $f l$. neap.) dove dicesi che nasca in quantità (Bert.), in Campania a Pastena al monte Cappiello (Terr.), e ad Itri al monte Faito (Terr.!), nei Principati a Savignano (Ten.) e a Casalnuovo (Bert.), in Basilicata a Lagonegro (Ten.); trovasi in Corsica a Bonifacio (Bert.), in Sardegna nei monti d'Orgosolo dalla parte di Dorgali a circa 600 metri, certamente indigena secondo Moris. Fiorisce in maggio e giu. gno, e anche in luglio nei luoghi meno caldi.

Distribuzione geografica. - Nell'Europa meridionale, dalla Spagna alle sponde orientali dell' Adriatico.

Osservazione. - Il calice varia in lunghezza da 7 a 15 millimetri.

\section{Salvia triloba.}

S. frutescens sempervirens, foliis ovato-oblongis ovalibusve, sæpius basi auriculatis aut lobatis, crenulatis, rugosis, plus minusve canescentibus, bracteis minimis, membranaceis, ovatis, acuminatis, glomerulis distinctis, calycis urceolato-campanulati labio superiore 3-dentato, inferiore 2-fido, corolla calyce 2-3-plo longiore.

Salvia triloba Linn. fil. suppl. p. 88. Bert. fl. ilal. 1. p. 137. Parl.! R. pan. p. 18. Guss. R. sic. syn. 1. p. 24. Ces. Pass. Gib. comp. f. ital. p. 306. Terr.! quarta rel. Terra di Lav. p. 112. Arc. comp. fl. ilal. p. 513.

Figura.- $F l$. grace. $t .17$.

Stazione, Abitazione e Fioritura. - Nelle colline aride sassose di Sicilia: a Palermo a Mondello, e ai Colli (Todaro!), ma principalmente nella parte meridionale dell' isola a Pozzallo, Spacca. forno (Guss.), Noto (Inzenga!), Avola (Bianca!), Siracusa ad Epipoli (Iluet!), Floridia (Parl.!), Palazzolo (Guss.). Trołasi poi nella Penisola in Calabria a Ginosa, in Terra d'Otranto a Taranto, Lecce, 
Brindisi (Ten. syll.), in Terra di Lavoro presso Ausonia nei monti a Selvacava (Terr.!). Fiorisce in aprile e maggio.

Distribuzione geografica. - Nell' Italia meridionale, la Grecia, l'Asia piủ occidentale, l'Algeria.

Osservazione. - Abbenchè questa specie abbia l'aspetto della precedente, se ne distingue subito anche per il calice non mai piủ lungo di 7 millimetri, e molto più glandoloso.

\section{Salvia canariensis.}

S. frutescens sempervirens, foliis hastatis, crenulatis, rugosis, glabriusculis, bracteis mediocribus, membranaceis coloratis, ovat lanceolatis, acutis, glomerulis approximatis, calycis campanulati labio superiore emarginato, inferiore 2-partito, corolla calyce 2-plo longiore.

Salvia canariensis Linn. sp. pl.ed.1. p. 26. Presl fl. sic. (1826). Guss. fl. sic. prodr. 1. p. 15. Parl.! fl. pan. p. 23. Guss. fl. sic. syn. 1. p. 21. Ces. Pass. Gib. comp. N. ital. p. 306. Arc. comp. Al. ital. p. 544 .

Figure. - Mor. plant. hist. 3. s. 11. t. 13.f. 17. Webb phyt. can. t. 167. f. G.

Stazione, Abitazione e Fioritura, e Distribuzione geografica. Specie indigena esclusivamente delle Isole Canarie, che coltivata in Palermo per ornamento dei giardini e dei boschetti, vi si è naturalata nelle boscaglie sul mare a Mondello (Todaro!), ai Colli (Guss.), al bastione della Concezione (Parl.!). Fiorisce in aprile e maggio.

\section{Salvia glutinosa.}

S. perennis, foliis cordato-hastatis, grosse dentatis, lævibus, pilosulis, bracteis parvis, herbaceis, ovatis, acuminatis, glomerulis distinctis, calycis campanulati labio superiore integro, inferiore 2-fido, corolla calyce 3-plo longiore.

Salvia glutinosa Linn. sp. pl.ed. 1. p. 26. Bert. fl. ital. 1. p. 155. Mass. prodr. fl. valt. p. 160. Com fl. com. 1. p. 31. Coll. herb. ped. 4. p. 389. Trev. prosp. fl. eug. p. 25. De Not. prosp. fl. lig. p. 317. pucc. syn. pl. luc. p. 20. Rota pros». piant. Pav. p. 271. Simi! fl. alp. vers. p. 13. Hausm. fl. Tir. p. 673. Rola prosp. fl. Berg. p. 69. Pir. fl. for. syll. p. 109. Car. prodr. fl. tosc. p. 509. Tassi fl. prov. 
sen. p. 50. G. Bert. not. Porr. p. 48. Veg. monti Porr. p. 88. Ard. fl. Alpes-mar. p. 294. De Vis. Sacc. cat. piant. Ven. p. 133. Zersi prosp. piant. Bresc. p. 163. Pasq. fl. ves. p. 79. Terr. fl. Vult. syn. p. 134. Ces. el. piant. Maiella p. 24. Terr.! sec. rel. Terra di Lav. p. 94. Archb. fl. Alto-Serch. p. 60. Ces. Pass. Gib. comp. 1l. ital. p. 306. Cald. fl. fav. tent. p. 181. Arc. comp. fl. ital. p. 544. Gib. Pir. fl. Mod. $12 \%$.

Figura. - Reich. ic. fl. germ. 18. t. 45.f. 1.

Stazione, Abitazione e Fioritura. - Nei boschi delle parti basse e medie dei monti della Penisola, raramente in pianura. Ê comunis. sima nell'Alta Italia, in Piemonte (All. fl. ped. ecc.), nel Cantone Ticino, nel Comasco (Com. ecc.), in Valtellina (Mass.), nel Bergamasco e nel Bresciano (Rota, Zersi ecc.), dove sale sulla Corna Blacca a 1500-1700 metri (Parl.!), nel Tirolo, fino in Pusteria (Hausm.), nel Veneto (De Vis. Sacc. ecc.), a Mantova (Barbieri!), a Grumone sull'Oglio (Parl.!), a Pavia (Rota), a Novara (Bir. fl. acon.), nel Mo. denese (Gib. Pir. ecc.), a Bologna (Bert.) e nell' Appennino bolo. gnese (Gennari! ecc.), a Faenza (Cald.); trovasi in Liguria da Nizza (Ard.) fino a S. Martino Lantosca (Bourgeau!), a Rezzo sopra Porto Maurizio (Berti!), a Voltri (Bert.), sopra Chiavari (Delpino!), al golfo della Spezia (Bert.) ecc.; in Toscana trovasi a Fivizzano, nelle Alpi Apuane, nell'Appennino di Garfagnana!, nel Lucchese (Calandrini!), nell' Appennino pistoiese a Boscolungo a 1300 metri (Parl.!), nel Fiorentino, in Mugello!, in Casentino(Parl.!), nel monte Amiata (Savi! ecc.), a Soana (Santi viagg.); nelle Marehe è stata trovata al Sasso di Simone (Cherici!), al Catria (Piccinini!), a Montefortino (Marzialetti!), e nell’ Abruzzo a Caramanico, a Solmona (Ces.), e al Maiellone (Pedicino!); fa nei colli Albani (Seb. Maur. fl. rom. prodr.), a Montecassino (Terr.), a Roccamorfina verso Gaeta (Terr.!), infine a Napoli nella valle S. Rocco (Parl.!), e sul Vesuvio dov'è rara (Pasquale), e nel Vulture (Terr.). Fiorisce da luglio a settembre.

Distribuzione geografica. - Nell' Europa meridionale ed anche media dalla Spagna per la Svizzera, la Germania meridionale e l'Austria alla Transsilvania, la Crimea e il Ciaucaso, e nelle parti finitime dell' A sia .

Descrizione. - Pianta perenne, alta da 4 a 8 decimetri e tal. volta sino ad un metro, verde, pelosa, con peli orizzontali, bianchi e articolati, e in alto glutinosa per peli disuguali, orizzontali e glan. dolosi all'apice. Il rizoma è radicante, come talvolta il fusto nella parte sua inferiore negli internodii. II fusto è eretto o ascendente, 
quadrangolare, peloso per peli fitti e orizzontali, verdognolo, sem. plice o con un solo ramo fiorifero in alto e rami corti o cortissimi, opposti, nell'ascella delle fnglie. Le foglie sono erbacee, molli, opposte, un po'lontane tra loro, patentissime, cordato-astate, acuminate, con grossi denti quasi a sega nel margine, verdi di sopra ed ivi puberuli e con un solco longitudinale e con solchi laterali in rete che corrispondono ai nervi di sotto, di un verde bianchiccio nella pagina inferiore ed ivi puberule e con un nervo longitudinale grosso, da cui partono molti nervi laterali, i quali si dirigono un po'curvi verso il margine per unirsi tra loro ad arco e formare una rete rilevata nella pagina inferiore: sono fornite di un picciòlo, lungo quasi quanto la foglia, solcato di sopra, convesso di sotto, verdognolo, peloso, un poco piủ grosso verso la base. Le foglie superiori sono più piccole, con il picciòlo più corto. I fiori sono molti, per lo piủ 3 , talvolta 2 , all' ascella di ciascuna brattea, in modo che in ciascun verticillo vi sono per lo più 6 , talvolta 5 o 4 fiori, formando un lungo racemo spiciforme, con i verticilli un po'lontani. Le brattee sono poco piủ lunghe dei pedicelli, erbacee, quasi obovate, acuminate, dapprima patenti, poi rivolte in giu, quasi glabre di sopra, pubescenti-glandolose di sotto e cigliato-glandolose nel margine: vi sono poi due bratteole laterali, una per ciascun fiore laterale, assai fiủ piccole della brattea, più corte del pedicello corrispondente, lineariallungate, rivolte in giù e del resto simili alla brattea. I pedicelli sono cilindrici, o eretto-patenti, più corti del calice, verdognoli, pubescenti-glandolosi. Il calice è tubuloso-campanulato, verde, pubescente-glandoloso di fuori, con cortissimi peli e con glandolette quasi sessili di dentro, ha cinque nervi longitudinali rilevati e altri intermedii più corti, meno rilevati, ed è bilabiato, il labbro superiore è più corto dell'inferiore, quasi tondeggiante però con un corto acume, e con i margini rivolti in dentro verso la gola del calice stesso, il labbro inferiore è bidentato, con i denti triangolari, acuminati e con l'apice un po'curvato in giù a guisa di oncino. La corolla è più del doppio più lunga del calice, di color giallo-pallido con alcune righe e macchioline di color quasi amarante nella faccia interna 0 anteriore del labbro superiore e nel labbro inferiore. Il suo tubo è poco più lungo del calice, quasi cilindrico, soltanto slargato nella gola, puberulo-glandoloso di fuori, peloso per peli lunghi, diritti e non glandolosi di dentro. I labbri sono quasi uguali in lunghezza tra loro e poco più lunghi del tubo; il superiore è eretto, un po'curvo a guisa di falce, grandemente concavo per avere i margini quasi avvicinati 
l'uno all'altro, schiacciato lateralmente, smarginato-bilobo, con i lobi tondeggianti; Г'inferiore è trilobo, con i lobi laterali allungati, tondeggianti all' apice ch'è solo libero e piegato lateralmente in giủ in modo che sono concave di sotto longitudinalmente: il lobo medio è piủ grande e piủ lungo, diretto un po'in giù con l'apice ed il margine, smarginato, dentato-increspato e quasi lacero. Gli stami sono due, il filamento è inserito nella gola vicino al labbro inferiore, il ramo superiore del connettivo piủ lungo è un po'curvo, diretto in alto e racchiuso con l' antera nella concavità del labbro superiore, che esso, compresa l'antera, uguaglia: è bianchiccio, e glabro. Il ramo inferiore sterile è un po' curvo, diretto in avanti e sporge nella gola insieme al rudimento dell' antera ch'è quasi bilobo, un po'concavo di sopra e gialliccio. L'antera è eretta, allungata-lineare, ottusa, appena smarginata alla base, inserita sopra di questa nel dorso, con il connettivo che si continua grosso e rilevato sino all' apice di essa, biloculare, aprentesi longitudinalmente, di colore gialliccio con macchioline violette, glabra. Il polline è giallo. Il pistillo è piủ lungo dul labbro superiore, sporgendo per qualche tratto dalla smarginatura dello stesso. L'ovario è profondamente quadrilobo, con i lobi allun. gati, ottusi, verdognoli, glabri e inseriti sopra un disco circolare, gialliccio, più grosso della parte inferiore. Lo stilo è molto lungo, filiforme, curvato, bianchiccio, glabro. Lo stimma è bifido, con le lacinie molto disuguali, la più lunga eretta, la più corta rivolta in giù, quasi piane, acute e di color violetto chiaro. I frutti sono allungati, un po'schiacciati da avanti in dietro, ottusi, con tre righe longitudinali un po'rilevate. (Parl. ms., descr. della pianta di Grumone in Lombardia).

\section{Salvia Iorminum.}

S. annua, foliis ovalibus, crenulatis, rugosis, utrinque pilosulis vel subtus canescentibus, bracteis magnis herbaceis, rotundatis, acuminatis, supremis sterilibus amplioribus coloratis comantibus, glomerulis distinctis, calycis tubuloso-campanulati labio superiore minute 3 -dentato, inferiore 2 -secto, corolla calyce dimidio aut duplo longiore.

Salvia Horminum Linn. sp. pl. ed. 1. p. 2.t. Bert. A. ital. 1. p. 139. Coll. herl. ped. 4. p 392. Zan. prosp. A. ven. p. 26. De Vis. Sacc. cal. piant. Ven. p. 132. Ces. Pass. Gib.comp. R. ilal. p. 306. Arc. comp. R. ital. p. 544. 
Figure.-Fl. grcec. t. 20. Reich. ic. A. germ. 18. t. 45. f. 2.

$\beta$ viridis, bracteis sterilibus comantibus haud amplioribus, herbaceis vel parce coloratis.

Salvia viridis Linn. l. c. Bert. o. c. p. 138. Coll. o. c. p. 393. Parl.l fl. pan. p. 19. Guss. fl. sic. syn. 1. p. 22. De Not. rep. fl. lig. p. 319. Ard. fl. Alpes-mar. p. 293. Ces. Pass. Gib.l. c. Groves! contr. fl. Terr. d'Otr. p. 65. Arc. l. c.

Salvia Horminum Grov.! l. c.

Figure. - Desf. fl. atl. t. 1. Fl. graec.t. 19. Reich. ic. bot. t. 531. Ic. fl. germ. 18. t. 45. f. 3.

Stazione, Abitazione e Fioritura. - Qua e là sui colli aridi, nei pascoli asciutti, nei luoghi campestri incolti, rara: in Piemonte nella valle d'Oulx (All. fl. ped.); nella Riviera di ponente a Nizza a Carabacel (Ard.), a Portomaurizio (Gentili!), ad Oneglia (All.); nel Veneto in Vallesina (Nacc. $f$. ven., Bert.), e nel Polesine (De Vis. Sacc.); in Puglia a Manfredonia (Porta e Rigo!), a Conversano, in Terra d'Otranto a Ginosa (Ten. syll.), a Lecce (Porta e Rigo!), a Otranto (Groves 1); in Sicilia a Palermo nel monte Gallo presso Sferracavallo (Parl.!), a Castrogiovanni, Villarosa, Priolo, da Vittoria a Catania (Guss.), cosi a Vittoria, Comiso (Aiuti!), Avola (Bianca!), Siracusa (Heldreich!). Fiorisce da marzo ed aprile a maggio e gingno.

Distribuzione geografica. - In Spagna, in Italia, nella Penisola Balcanica, in Crimea, nell'Asia occidentale, nell'Affrica settentrionale.

Osservazione. - La Salvia Horminum e la S. viridis sono identiche in tutto, fuorchè nella chioma di brattee sterili; e siccome fra le grandi e colorate e le piccole e verdi sonovi parecchie gradazioni, non è possibile riguardare le due forme come specie distinte. La $S$. Horminum tipica è generalmente più grande, piủ snella, quale si vede soprattutto nei giardini; la $S$. viridis è più bassa, più tozza, e di essa sono tutti i saggi italiani da me veduti.

\section{f. Salvin Selarea.}

S. perennis, foliis ovatis, crenatis denticulatis, rugosis, nunc pilosulis nunc tomentoso-incanis, bracteis magnis, coloratis, rotundatis, acuminatis, glomerulis distinctis, calycis campanulati labio superiore 3-dentato, inferiore 2-partito, dentibus lobisque acuminato-spinescentibus, corolla calyce dimidio aut duplo longiore. 
Salvia Sclarea Linn. sp. pl. ed. 1. p. 27. Bert. fl. ilal. 1. p. 156. Mass. prodr. fl. valt. p. 160. Com. fl. com. 1. p. 32. Coll. herb. ped. 4. p. 393. Parl.! fl. pan. p. 22. Trev. prosp. R. eug. p 25. Guss. fl. sic. syn. 1. p. 21. De Not. rep. fl. lig. p. 317. Rota prosp. piant. Pav. p. 271. Koch syn. fl. germ. ed. 2. p.637. Pucc. add. ad syn. pl. luc. in giorn. bot. 1. p. 118. Hausm. f. Tir. p 674. Rota prosp. fl. Berg. p. 69. Guss. enum. pl. Inarim. p. 256 Mor. fl. sard. 3. p. 294. Pir. fl. for. syll. p. 110. Car. prodr. fl. tose. p. 510. Tassi fl. prov. sen. p. 50. Ard. cat. pl. Ment. p. 28. Tassi cenn. bot. Sien. p. 87. G. Bert. veg. Porr. p. 88. Not. Porr. p. 48. Ard. fl. Alpesmar. p. 293. De Vis. Sacc. cat. piant. Ven. p. 133. Zersi prosp. piant. Bresc. p. 163. Pasq. fl. ves. p. 79. Terr. sec. rel. Terra di Lav. p. 94. Ces. el. piunt. Waiell. p. 24. Archb. fl. Alto-Serch. p. 60. Ces. Pass. Gib.comp. fl. ital. p. 307. Ing. cat. sp. Mond. p. 63. Terr. quarta rel. Terra di Lav. p. 112. Arc. comp. fl. ital. p. 544. Gib. Pir. fl. Mod. p. 127.

Figure. - Fl. grue. t. 25. Reich. ic. fl. germ 18. t. 48.

Stazione, Abitazione e Fioritura. - Per tutta l'Italia, ma sparpagliatamente, nei luoghi erbosi asciutti di collina e di bassa montagna: in Piemonte in val d'Aosta presso Aosta (All. fl. ped.) e a Nus (Heldreich!), a Torino vicino ai Cappuccini, verso Pinerolo, nel Mlon. ferrato abbondante a Vinchio, Corticelle, Pareto ecc. (All.), nel Novese (De Not.), ad Alba (Bertero!), presso Mondovi nei monti di Roccaforte (lng.), presso Vinadio (Reuter!); in Lombardia presso Bergamo e Scanzo (Rota), e presso Gargnano sul lago di Garda (Zersi); in Tirolo, ma forse inselvatichita, a Bolzano, a Trento (Hausm.); n.! Veneto presso Verona a Tagliaferro (Bracht!) e Montorio, presso Vicenza nella valle di Trissino (Poll. $l$. verr.), negli Euganei presso Arquà (Montini!), nella Carnia secondo Suffren (Pir.); nell'Istria (Koch); nell' Emilia a Bologua presso Roncorio (Bert.), alla Porretta, rara (G. Bert.), a Montefiorino e Rocea di Montese nel Modanese (Gib. Pir.); in Liguria qua e là da Tenda a Mentone, Turbia e Nizza (Ard.), frequente da Nizza ad Albenga (De Not.), cosi a Nizza (Bar. la!), Dolcedo (Berti!), Oneglia, Diano, Cervo (Ricca!); in Toscana a Sarzana (Bert.), a Lucca (Pucc.), in val di Serchio intorno ai Bagni di Lucea, a Firenze a Montici!, a Volterra (Bundi!), Siena (Bart.), Rapolano (Sommier!), Giuncarico in Maremma, Pitigliano, Radicofani (Santi), nell' lilba a Rio (Marcucci!); nelle Marche al Catria (Piccinini!), alla Polverina presso Camerino (Rucci!); nell'Abruzzo presso Castelvecchio a 1000 metri (Groves!), e comunissima da Po. 
poli e Solmona fin sopra Caramanico (Levier!); nel Gargano a Monte S. Angelo e altrove (Ten. syll. fl. neap.); a Roma, dov'è comune (Seb. Naur. fl. rom. prodr.), e a Trevi sotto Filettino (Rolli!); in Terra di Lavoro a S. Biagio, a Traetto (Terr.); nell'isola d'Ischia, coltivata e inselvatichita (Guss.); nel Principato citeriore a Rocca di Cilento (Ten.); in Basilicata a Muro (Terr.!); in Calabria a Sinopoli vecchio, a Oppido (Ten.); in Sardegna a Cagliari e Pula (Mor.); in Sicilia a Palermo dov'è comune (Parl.!), Noara (Munafỏ!), Manda. nici (Seguenza!), Nicolosi (Arc.!), Avola (Bianca!) ecc. Fiorisce da maggio e giugno a luglio e agosto.

Distribuzione geografica. - Nell' Europa meridionale, dalla Spa. gna al Caucaso, nell' Asia occidentale.

Usi. - Questa pianta odora fortemente di uva moscadella, per cui se ne servono per dare quell'odore ai vini bianchi ordinari, nonchè ai gelati acidi che vogliono far credere sieno d'uva.

\section{\%. Salvia AEthiopis.}

S. perennis, foliis subovalibus, irregulariter grosse sinuato-dentatis crenulatis, rugosis, utrinque albo-lanatis, bracteis magnis, herbaceis basi membranaceis, rotundatis, acuminato-spinescentibus, glomerulis distinctis, calycis campanulati labio superiore 3 -fido, inferiore 2-secto, lobis acuminato-spinescentibus, corolla calyce duplo fere longiore.

Salvia Æthiopis Linn.sp.pl.ed. 1.p. 27. All. fl. ped.1.p. 15. Re fl. seg. Bert. fl. ital. 1. p. 159. Colla herb. ped. 4. p. 394. Ces. Pass. Gib. comp. fl. ital. p. 307. Arc. comp. fl. ital. p. 544.

Figura. - Reich. ic. fl. germ. 18.t. 47.

Stazione, Abitazione e Fioritura. - «Obviam mihi venit loco maxime sicco, et aprico supra oppidum Fenestrellarum. Prim in monte Geneure, Caccia in valle Ulciensi reperit; nascitur etiam circa Sospello in Comitatu Nicceensi. Ignatius Molineri in valle di Bardonache " (All. l. c.). "Inter Bossoleno et Foresto Re fl. seg. „ (Colla l. c.). Peraltro nessun botanico piủ moderno pare che abbia riveduta la pianta in quei luoghi del Piemonte. Dovrebbe essere fiorita di maggio e gingno.

Distribuzione geografica. - Nella Spagna e la Francia meridionale, nei paesi Danubiani, nella Russia meridionale, nella penisola Balcanica, nell'Asia Minore e fino alla Persia, nell'Affrica settentrionale. 


\section{Salvia argentea.}

S. perennis, foliis subovalibus, irregulariter grosse sinuato-dentatis crenulatis, rugosis, utrinque lanatis, bracteis magnis, herbaceis, rotundatis, acuminato-spinescentibus, glomerulis distinctis, calycis campanulati labio superiore 3 -dentato, inferiore 2-fido, dentibus lobisque breviter spinescentibus, corolla calyce duplo triplo longiore.

Salvia argentea Linn. sp. pl. ed. 2. p. 38. Bert. fl. ital. 1. p. 158. Guss. fl. sic. syn. 1. p. 22. Pasq. Lic. viagg. al Garg. p. 23. Ces. Pass. Gib. comp. fl. ilal. p. 307. Arc. comp. fl. ital. p. 545.

Salvia Gussonii Boiss.

Figure. - Fl. groc. 1. 27. Reich. ic. fl. germ. 18. t. 46.

Stazione, Abitazione e Fioritura. - In luoghi aridi solatii dei monti dell'Italia meridionale: nel Gargano al Passo di Ferro, a Montecalvo (Ten. syll.), presso Monte S. Angelo (Ten., Pasq. Lic.), a Manfredonia e altrove, a Martina, a Lecce, nel Pollino (Ten.), in Sicilia nel monte di Cammarata (Guss., Calcara!). Fiorisce in mag. gio e giugno.

Distribuzione geografica. - Nella penisola Iberica, l'Italia, la Dalmazia, la Serbia, la Turchia, la Grecia, l’Algeria.

\section{Salvia ceratophylloides.}

S. perennis, foliis ambitu ovato-lanceolatis, irregulariter incisopinnatifidis crenatis, rugosis, pilosulis, bracteis parvis, herbaceis, ovato-acuminatis, glomerulis remotis, calycis campanulati labio superiore minutissime 3 -dentato, inferiore 2 -partito lobis acuminatis, corolla calyce duplo longiore.

Salvia ceratophylloides Ard. anim. bot. spec. alt. p. 12 (176 ' ). Linn. mant. p. 26 (1767). Bert. fl. ital. 1. p. 159. Parl. 11. pan. p. 23. Guss. 1l. sic. syn. 1. p. 2.t. Ces. Pass. Gib. comp. f. ital. p. 307. Arc. comp. fl. ital. p. 545.

Figura. - Ard. o. c. t. 2.

Stazione, Abitazione e Fioritura. - L'Arduino racconta che questa specie gli nacque nell'Orto di Padova da semi di Sicilia, e difatti questa sarebhe patria della pianta giusta un sinonimo di Cupani panph. citato da Gussone e da Parlatore, e stando anche all'indica- 
zione di Presl fl. sic.: però nè Gussone nè altri dopo di lui ve l'hanno più trovata. Trovasi invece nei colli aridi a Reggio di Calabria (Ten.!). Fiorisce in giugno e luglio (Bert.).

Distribuzione geografica. - E stato scritto che fa anche in Egitto.

\section{Salvia pratensis.}

S. perennis, foliis ovatis ovato-oblongis oblongisve, duplicatocrenatis, nunc basi incisis, plus minusve rugosis, pilosulis, bracteis parvis, herbaceis, ovato-rotundatis, acuminatis, glomerulis distinctis, calycis campanulati labio superiore minutissime 3-dentato, inferiore 2-fido lobis acuminatis, corolla calyce 2-3-plo longiore.

a vulgaris, caule subeglanduloso, corolla cœrulea, raro alba aut rosea, galea pilosula.

Salvia pratensis Linn. sp. pl. ed. 1. p. 25. Bert. fl. ital. 1. p. 144; et auct. omn.

Salvia agrestis Cald. fl. fav. tent. p. 180.

Figura. - Reich. ic. fl. germ. 18. t. 51. f. 1-4.

$\beta$ homatodes, caule glanduloso, corolla cœruleo-purpurea ex Bert., cœrulea cum labio superiore albescente ex Ten., galea hirsuta.

Salvia hæmatodes Linn.? sp. pl. ed. 1. p. 24. Bert. o. c. p. 140. Ces. Pass. Gib. comp. fl. ital. p. 307. Arc. comp. A. ital. p. 545 .

Salvia Barrelieri Ten.! fl. nap. 1. p. 9. Terr.! f. Vult. syn. p. 135. Quarta rel. Tería di Lav. p. 112.

Figure. - Barr. ic. f. 185. Ten. o. c. t. 2.

Stazione, Abitazione e Fioritura. - La var. $\alpha$, ch'è il tipo della specie, è comunissima per i prati ed altri luoghi erbosi della Penisola settentrionale e centrale, dove va dalle pianure a parti assai alte dei monti, così in Piemonte, dov'è stata raccolta per esempio a Valdieri a 1140 metri (Parl. !) e al col di Tenda (Bourgeau!), in Valtellina a Bormio (Rampoldi!), nel Bresciano a Ponte di Legno a 1320 metri (Parl.!), in Tirolo sul Ritten fino a 1500 metri (Hausm. fl. Tir.), in Toscana a Boscolungo a 1300 metri (Parl.!), nei monti di Norcia al Castelluccio (Ricci!): il confine meridionale essendo a Rieti nel Romano (Rolli!), al Velino (Ten. syll.) e a Ciaramanico nell'Abruzzo (Ces. el. piant. Maiella). La var. $\beta$ subentra alla var. $\alpha$ 
nella parte meridionale della Penisola, e principalmente nei luoghi bassi, così a Rioma alle Tre fontane (Bert.) e nel monte Circello (Seb. Maur. fl. rom. prodr.), a Terracina (Rolli!), in Terra di Lavoro a Fondi, Itri (Ten.!), Mondragone (Terr.!), Pastena, Scavoli (Terr.), a Melfi (Terr.), in Calabria a Cosenza (Ten. syll. $f$. neap.), tra capo Spartivento e Brancaleone (Hut. Port. Rigo!), presso Stilo e Pecorara (Arc.!), in Terra d'Otranto a Ginosa, in Terra di Bari a Conversano (Ten.). Fiorisce secondo i luoghi in maggio e giugno, o giugno e luglio, o luglio ed agosto.

Distribuzione geografica. - In tutta Europa dalla Svezia meridionale in giù.

Descrizione. - Pianta perenne, alta da 2 decim. a 1 metro, verde, pelosa, fornita di un rizoma scuro che manda fibre radicali delicate e dall' apice delle foglie e dei fusti. Questi sono eretti o ascendenti, quadrangolari, con le facce leggermente scanalate e striate, verdi, pelosi, con i peli bianchi e orizzontali. Le foglie inferiori sono patenti o patentissime, ovali o ovali-lanceolate, un po'acute, scavate un poco in cuore alla base, inciso-crenate, verdi disopra ed ivi con un nervo longitudinale poco rilevato e bianchiccio e con dei nervetti laterali pubescenti, e con il parenchima formante tante gibbosità, di un verde un po' pallido di sotto ed ivi pubescente e con un nervo longitudinale rilevato e bianchiccio da cui partono dei nervetti laterali anche rilevati e bianchicci, i quali si dirigono un po'obliquamente e un po'curvi verso il margine diramandosi molto per formare una rete rilevata nella pagina inferiore delle foglie: il parenchima ha tante fossette che corrispondono alle gibbosità della pagina superiore della foglia; sono fornite di un picciòlo più corto della foglia, quasi piano di sopra, convesso di sotto, bianchiccio e pubescente. Le foglie del fusto sono poche, piccole, opposte, cordato-ovate, acuminate, scssili ed abbraccianti il fusto, del resto simili alle inferiori. I fiori sono da due a tre in ciascun lato all' ascella di ciascuna brat. tea, quasi sessili, orizzontali, grandi, in modo che in ciascun giro vi sono da 4 a 6 fiori. Le brattee sono erbacee, poco più corte o quasi uguali al calice, patenti, poi rivolte in giu, ovate, acuminate, pelose di fuori e nel margine, glabre di dentro. Il calice è verderossiceio, con molti nervi, peloso-glandoloso nei nervi e con glandole sessili giallicce nelle facce, con il labbro superiore avente due denti laterali acuti e un po'conniventi, nel seno formato dai quali si vede un dentino per la sporgenza del nervo corrispondente, con il labbro inferiore un po'piì lungo del superiore, avente due denti 
ovato-lanceolati, con una punta sporgente all'apice. La corolla è di color pavonazzo, da due a tre volte piủ lunga del calice. Il tubo '̀ piủ lungo del calice, glabro, bianchiccio, stretto e quasi strozzato di sotto sopra della base, gonfio in alto di sotto. Il labbro superiore è diretto in avanti ed in alto, curvato quasi ad arco nel margine superiore, con il margine inferiore appena concavo, schiacciato lateralmente, smarginato-bilobo all' apice, con i lobi che si toccano dalla parte interna, puberulo-glandoloso di fuori; il labbro inferiore è poco più corto, diretto in avanti, trilobo, con i lobi laterali ovali-lanceolati, diretti in avanti ed in su, e il lobo medio molto più grande, quasi orbicolare, concavo in avanti, appena smarginato. Gli stami sono due, inseriti all'apice del tubo, racchiusi nel labbro superiore, di cui sono appena piủ corti. I filamenti sono lesiniformi, curvati in avanti, di color violetto, glabri. Le antere sono lineari-bislunghe, ottuse, inserite nel dorso sopra della base, uniloculari, aprentisi per una fessura longitudinale scuretta, glabri: non vi è connettivo sterile. Il pistillo è poco piủ lungo degli stami. Lo stilo è lungo, curvo, filiforme, quasi glabro, di color violetto. Lo stimına è bifido con le lacinie disuguali, un po'divergenti, acute. (Parl. ms., descr. della pianta di Boscolungo nell' Appennino toscano).

Osservazioni. - Il calice varia per grandezza da $1 / 2$ centimetro a quasi 1 centimetro; dove col calice dei più piccoli si ha la corolla non più grande del doppio del calice, ne risultano fiori la metà più piccoli che d'ordinario, come già osservò Koch (Syn. fl. germ. ed. 2), ossia una forma micrantha, che alcuni autori ritengono essere la Salvia agrestis di Linneo. E forse da riferirsi a tale forma la $S$. scabrida Bert. (Fl. ital. 1. p. 855), S. Bertolonii De Vis. (Fl. dalm. 2. p. 189), indicata a Lecco da Bertoloni e in Istria da Nyman (Consp. $f$. eur. p. 570).

All'infuori dei caratteri indicati, non ne trovo altri per separare la $S$. hamatodes dalla $S$. pratensis tipica; e siccome queste forme sono collegate da qualche forma intermedia, ch' è difficile riferire all'una piuttostochè all'altra, non ho creduto doverle disgiungere specificamente. Nonostante la cosa va meglio studiata, sulla $S$. hoematodes viva.

\section{Salvia virgata.}

S. perennis, foliis ovatis vel ovato-oblongis, duplicato-crenatis subincisis, plus minusve rugosis, nunc pilosulis nunc subtus præser- 
tim hirtis, bracteis parvis, herbaceis, rotundatis, acuminatis, glomerulis distinctis, calycis campanulati labio superiore minutissime 3-dentato, inferiore 2-partito lobis acuminatis, corolla calyce 2-plo longiore.

Salvia virgata Jacq. hort. vind. sec. Ait. hort. kew. ed. 1. 1. p. 39. Bert. fl. ital 1. p. 143. Benth.! in Cand. prodr. 12. p. 290. Car. prodr. fl. tosc. p. 510. Terr. fl. Vult. syn. p. 135. Ces. Pass. Gih. comp. fl. ital. p. 307. Cald. fl. fav. tent. p. 180. Arc. comp. fl. ital. p. 545.

Salvia garganica Ten.! ind. sem. hort. neap. 1829. Bert. l. c. et p. 848. Terr.! fl. Vult. syn. p. 185.

Salvia tiberina Mauri! in Ten. succ. rel. viagg. in Abruzzo ecc. p. 41. Bert. o. c. p. 142, 848. Sang. cent. prodr. fl. rom. add.p.7.

Salvia scabrida Bert.? o. c. p. 855 (non Pohl). Com. fl. com. 1. p. 363.

Salvia Bertolonii De Vis.? fl. dalm. 2. p. 189.

Salvia pallidiflora Cald. o. c. p. 181.

Figura. - Fl. grcec. $t$. 22.

Stazione, Abitazione e Fioritura. - Nei luoghi erbosi della Penisola centrale e meridionale, rara: intorno a Faenza a Sarna, Errano, Rontana, Moronico ecc. (Cald.); altra volta a Firenze, sull'argine dell'Arno fuori porta alla Croce!, ma v'è stata distrutta dai lavori d'ingrandimento della città ; intorno a Roma, comunissima nella parte occidentale dell' agro, principalmente fuori porta Portese oltre il secondo miglio (Seb. Maur. fl. rom. prodr.), alla macchia de' Mattei, a Tivoli, al monte Gennaro (Bert.), sopra i monti di Pontefratta (Rolli!), a Frascati (Parl.!), a Pozzo Pantaleo, al monte délla Pica, nella via Ostiense (Sang.) ecc.; a Tagliacozzo, e nel monte Salviano sul lago Fucino (Bert.); a Melfi lungo la strada S. Venere (Terr.); a Polla nel Principato citeriore (Bert.); nel Gargano presso Apricena e S. Nicandro (Porta e Rigo !). Fiorisce da giugno ad agosto.

Distribuzione geografica. - Trovasi incltre nella Penisola Balcanica, nell'Asia Minore e nella Russia meridionale.

Descrizione. - Pianta perenne, alta da 5 a 8 decimetri, di un verde bianchiccio, pubescente-irsuta per peli lunghetti, orizzontali, quasi crespi, articolati e nelle parti superiori forniti di glandola all'apice. Il fusto è erbaceo, quadrangolare, diritto, ramoso in alto, con i rami opposti, quasi cretti, piủ corti o quasi uguali al fusto. Le foglie sono opposte, patenti o patentissime, le inferiori o radicali ovato o ovali-allungate, tondeggianti o scavate in cuore alla base, 
tondeggianti all'apice, rosicchiato-crenate, verdi di sopra ed ivi quasi glabre e con solchi che corrispondono ai nervi di sotto e rendono la foglia rugosa, di un verde pallido o bianchiccio di sotto ed ivi con un grosso nervo longitudinale da cui partono dei nervetti laterali molto obliqui e rilevati, che si diramano per fare una rete rilevata a maglie strette, in mezzo delle quali si vedono delle fossette formate dal parenchima della foglia, i nervi e le loro divisioni sono pelose con peli grossotti e bianchi: le foglie stesse poi sono fornite di un picciòlo il quale è più corto della foglia, piano di sopra, convesso di sotto, rossiccio o bianchiccio, peloso segnatamente verso la foglia e nei margini. Le foglie che sieguono sono lontane tra loro, con un picciòlo gradatamente più corto tanto che le superiori sono quasi sessili, cordato-ovate, ottuse o quasi acuminate, con le crenature un po'più profonde, del resto simili alle inferiori. Le foglie fiorali o brattee sono patenti o appena curvate in giù, le inferiori quasi uguali al calice e le superiori un po'più corte di questo, ovato-acuminate, intere, verdognole-scure o sfumate di rossiccio. I fiori sono per lo più sei in ciascun giro, tre per ciascuna ascella della foglia fiorale, eretto-patenti, distribuiti in racemi all' apice del fusto e dei rami, i quali racemi si allungano molto, essendo i giri numerosi e alquanto distanti. Ciascun fiore ha un pedicello, molto più corto del calice, eretto, delicato, rossiccio, peloso. Il calice è campanulato, verdognolo-rossiccio-scuro, striato, con le strie molto rilevate, peloso, con i peli parte piủ lunghi e numerosi, bianchi, senza glandola, parte più corti con una glandola all'apice, e con glandole grossette, giallognole, sessili nei solchi lasciati dalle strie, di dentro è glabro, verdognolo, con solchi longitudinali che corrispondono alle strie: è diviso in due labbri, il labbro inferiore è patente-ricurvo, avendo l'apice rivolto un po' in su e in dietro e ha tre piccoli denti acuti, il labbro inferiore è appena più lungo del superiore, quasi diritto, bifido, con le lacinie triangolari-lanceolate, acuminate. La corolla è più del doppio piủ lunga del calice, di color violetto con due macchioline bianchicce e divergenti alla base del lobo medio del suo labbro inferiore. Il tubo è quasi uguale o poco più lungo del calice, un po'più largo verso alto, quasi interamente glabro di fuori, puberulo di dentro verso alto. Il labbro superiore è un po'più corto dell'inferiore, eretto, quasi falcato, schiacciato lateralmente, appena smarginato all' apice, peloso di fuori e con glandole grossette, tonde, gialle e quasi sessili, sparse. Il labbro inferiore è patente, trilobo, con i lobi laterali piccoli, allungati, ottusi o quasi troncati e den. 
tellati all'apice, e il lobo medio molto maggiore, quasi orbicolarerombeo, un po' concavo e glabro di sopra, convesso e peloso di sotto. Gli stami sono inseriti nella parte superiore del tubo vicino alla gola, fuori della quale sporgono poco. I filamenti sono corti, cilindrici, bianchicci, quasi glabri. Il connettivo è curvato quasi ad arco, più grosso nel centro, assottigliato verso la loggia fertile, slargato e quasi petaloideo colorito verso la loggia sterile di cui c'è appena un rudimento. La loggia fertile è allungata gialla. Il pistillo è piủ lungo del labbro superiore della corolla. Il disco è più alto dell'ovario, quasi tondo, di un giallo-ranciato, glabro, come lobato all'apice, dove riceve l'ovario. Questo è diviso profondamente in quattro lobi, ovali-allungati, ottusi, striato-rugosi, glabri, verdognoli. Lo stilo è lungo, cilindrico, curvato in alto, bianchiccio e glabro in gran parte, puberulo e violetto verso alto. Lo stimma è bifido, con le lacinie disuguali, curvate in fuori, lesiniformi, scanalate dalla parte interna e violette. Le noci sono ovoidee, un po'angolate, nere, con tanti punti rilevati. (Parl. ms., descr. di pianta coltivata).

Osservazioni. - Questa specie malamente si distingue da alcune forme della Salvia pratensis, e soprattutto della sua var. $\beta$. Spesso coltivata negli orti, vi si palesa polimorfa al pari di quella, alla quale probabilmente si dovrà congiungere.

Non avendo io veduto saggi della $S$. scabrida Bert. , o S. Berto. lonii De Vis., trovata presso Lecco dal Comolli, non saprei che cosa fosse. Bertoloni la paragona alla $S$. campestris Bieb., che si considera una forma micrantha della $S$. virgata; De Visiani la dice intermedia fra la $S$. pratensis e la $S$. verbenaca.

\section{Salvia viseosa.}

S. perennis, foliis late ovatis vel ovato-ellipticis, duplicato-crenatis, vix rugosis, utrinque pilosulis, bracteis parvis, herbaceis, rotundatis, acuminatis, glomerulis distinctis, calycis campanulati labio supe. riore minutissime 3-dentato, inferiore 2-partito lobis acuminatis, corolla calyce triplo longiore.

Salvia viscosa Jacq. misc. austr. 2. p. 328. Trev. prosp. 1.eug. p. 25. De Vis. Sacc. cat. piant. Ven. p. 133. Ces. Pass. Gib. comp. 凡. ital. p. 307. Arc. comp. 1. ital. p. 545.

Stazione, Abitazione e Fioritura. - Nei colli Euganei (Trev., De Visiani sec. Ces. Pass. e Gib.) a Calaone e Serra presso Este 
(Sternberg sec. Reich. fl. germ. exc.); e nelle selve della valle di Valstagna presso Bassano (Montini!). Fiorisce da giugno ad agosto.

Distribuzione geografica. - Questa rarissima pianta trovasi oltracciỏ in qualche luogo dell'Asia occidentale.

\section{Salvia verbenaca.}

S. perennis, foliis oblongis ovato-oblongis ovatisve, duplicatocrenatis incisis inciso-pinnatifidis aut pinnatipartitis, plus minus rugosis, subglabris, bracteis parvis, herbaceis, rotundatis, acuminatis, glomerulis plus minus approximatis, calycis campanulati labio superiore minutissime 3-dentato, inferiore 2-partito lobis acuminatis, corolla calyce subduplo longiore.

Salvia verbenaca Linn. sp. pl. ed. 1. p. 25. Boiss. voy en Esp. 2. p. 484. Gris. spic. fl. rum. 2. p. 112. De Vis. fl. dalm. 2. p. 189. Mor. fl. sard. 3. p. 296. Willk. Lange prodr. 1. hisp. 1. p. 426. Ces. Pass. Gib. comp. fl. ital. p. 307. Arc. comp. fl. ital. p. 546.

a vulgaris, elatior, foliis minus divisis, corolla minore purpu. reo-violacea.

Salvia verbenaca Linn. o. c. ed. 2. p. 35. Bert. fl. ital. 1. p. 146; et auct. omn.

Salvia oblongata Ten.! Bert. o. c. p. 148.

Figure. - Triumf. obs. t. 66. Barr. ic. t. 208. Reich. ic. bot. t. $522,523$. lacina.

$\beta$ australis, humilior, foliis magis divisis, corolla maiore li-

Salvia clandestina Linn.? o. c. ed. 2. p. 36 ; auct. ital. omnium.

Salvia multifida Sibth. Sm. fl. grcec. prodr. 1. p. 16. Bert. 0. c. p. 149; et auct.

Salvia controversa Ten.! syll. fl. neap. p. 18.

Salvia horminoides Ard. cat. pl. Ment. p. 28.

Figure. - Barr. ic. t. 220. Fl. groec. t. 23. Hoffm. Link fl. port. t. 19. Reich. o. c. t. 524.

Stazione, Abitazione e Fioritura. - Nei luoghi erbosi, come prati, pascoli, argini, cigli dei campi ecc. Comparisce in qualche luogo dell'Alta Italia, e quasi sernpre la var. $\alpha$ : nelle colline Torinesi e intorno a Pinerolo secondo Allioni $(f l$. ped. $)$, ma non più secondo Colla (herb. ped.), a Mondovi dov'è comune (Ing. cat. sp. Mond.), in Agra sopra Lecco nel monte di San Martino, rarissima 
(Com. Al. com.), sui bastioni di Pavia (Rota!), nel Bergamasco a Sarnico e Somasca (Rota prosp. $l$. Berg.), nei colli Bresciani (Bracht!) presso Venzago, rarissima (Zersi prosp. piant. Bresc.), a Verona fuori porta S. Giorgio e in val Pantena a Costoli (Goiran app. bot.), presso Goito (Bert.) e Sabbioneta (D'Arco!), negli Euganei a Battaglia (Montini!), nel Polesine (De Vis. Sacc. cat. piant. Ven.), nel Lido di Venezia ovunque (Kellner!), in Istria a Pola e Dignano la var. $\beta$ (Bert.), quindi a Parma (Passerini!), a Modena comunissima la var. $\beta$ (Gib. Pir. l. Mod.), a Bologna tra Gaibolla e Paderno (Bert.), e al vicino Appennino alla Porretta (G. Bert. not. Porr.), e a Montese la var. $\beta$ (Cocconi terzo contr. $\{. B o l$.), in varie località intorno a Faenza (Cald. $f l$ fav. tent.), a Comacchio e a Ravenna (Bert.) la var. $\beta$. Quindi discendono lungo il lato orientale della Penisola le due varietà insieme, trovandosi a Rimini (Cald.!), Pesaro (Bert.), al Porto di Recanati (Narducci!), a Fermo (Moretti!), Ascoli (Parl.!), Pescara, al Gargano (Porta e Rigo!), a Foggia (Pasquale!), Taranto (Aiuti!), Otranto (Groves contr. fl. Terra d'Otr.). La var. $\beta$ è comune in tutta la Liguria, mentrechè la var. $\alpha$ assai piì rara non si trova che a Nizza (Bourgeau!) e S. Remo (Panizzi!). La specie è comunissima in tutta Toscana comprese le isole, la var. $\beta$ ristretta peró alla regione maremmana, nel mentre che la var. $\alpha$ ab. bonda di più in lontananza dal mare, e s'inoltra fino nei monti del Casentino a 800 metri (Parl.!). Seguitano piủ giủ, trovandosi a $\mathrm{F}_{0}$ ligno, Viterbo (Bert.), Roma dove ahbondano (Seb. Mauri $/ l$. rom. prodr.), Terracina (Bert.), Napoli dove sono comuni (Ten.! ccc.), la var. $\alpha$ sola salendo sul Vesuvio (Pasq. fl. ves.), e sola ad abitare Ischia (Guss. enum. pl. Inarim.), mentrechè ambedue abitano Capri (Pasq.). Più giủ ancora fa a Melfi la var. $\beta$ (Terr.!), a Potenza la Salvia oblongata (Ten.!), in Calabria la $S$. controversa (Ten.!). Trovasi la specie in Ciorsica a Bastia, S. Fiorenzo, Calvi, Aleria, Sartena (Mars. cat. pl. Corse), Bonifacio (Kralik!), in Sardegna, per esempio a Sorso!, in Sicilia, la var. $\beta$ ovunque nei luoghi bassi marittimi, e nelle isolette (Guss. $l$. sic. syn.), la var. $\alpha$ principalmente nei luo. ghi alti, come alla Pizzuta e altrove sopra Palermo, nelle Iladonie (Parl.!), a Caltanisetta, Caltagirone ecc. (Guss.), ma anche in basso come a Messina!; infine a Pantellaria (Guss.) e Lampedusa (Aiuti!). In Malta abbonda la var. $\beta$, l' $\alpha$ sembra meno frequente (Grech Del.). Fiorisce dall'autunno alla primavera nei lunghi più caldi, in primavera e per l'estate nei meno caldi.

Distribuzione geografica. - Nell' Luropa meridionale, dal Por- 
togallo alla Crimea, risalendo lungo l'Atlantico fino in Inghilterra, nell'Asia occidentale, nell' Affrica settentrionale.

Descrizione della var. $\alpha$. - Pianta perenne, di un odore meno forte e più soave di quello della Salvia officinalis $L$., alta da 2 decimetri e mezzo a 5 decimetri. La radice è grossa, fusiforme, verticale, ramosa, scura. Il fusto o i fusti sono eretti o ascendenti, duretti, quadrangolari, con gli angoli ottusi e le facce piane, verdognoli e in parte rossicci, pubescenti per peli piuttosto corti, quasi orizzontali 0 un po'diretti in giù, bianchi e forniti nella parte superiore di altri peli piủ lunghi, orizzontali, bianchi e aventi all'apice una glandola quasi tonda e trasparente: sono semplici o spesso con pochi rami in alto, i quali sono opposti, eretto-patenti e piủ corti del fusto. Le foglie sono alquanto consistenti; la maggior parte radicali, quasi in rosetta, giacenti quasi per terra, ovali-bislunghe o bislunghe, ottuse, appena scavate in cuore alla base, lobato-sinuate, con i lobi tondeggianti e crenulati, sono di un color verde scuro di sopra ed ivi rugose, glabre e con un nervo longitudinale bianchiccio 0 rossiccio, non sporgente, di un verde più chiaro di sotto con un nervo longitudinale assai sporgente da cui partono dei nervi laterali sporgenti, i quali si dirigono obliquamente verso il margine e l'apice e si diramano per formare una rete rilevata nella pagina inferiore della foglia, lasciando delle fossette poco profonde che corrispondono alle rugosità della pagina superiore: tutti questi nervi sono di colore più chiaro della pagina inferiore e quasi bianchicci e quello longitudinale anche verso la base rossiccio, e pubescenti anche nei loro rami; le foglie stesse sono inoltre fornite di un picciòlo, il quale è poco più corto o uguale alla foglia, piano di sopra, convesso di sotto, spesso rossiccio o verdognolo, pubescente. Le foglie cauline sono poche, opposte, lontane, patenti, le inferiori simili alle radicali, soltanto un poco piủ piccole, e con il picciòlo un po' più corto, le superiori che corrispondono alla base dei rami sono piủ piccole, sessili, ovali 0 ovate, acute 0 acuminate. Le foglie fiorali sono ovate, cuspidate, con un grosso nervo longitudinale di sotto, lunghe quasi o poco più corte del calice, patenti, poi un po'dirette in giù, verdi, pubescenti e con altri peli glandolosi. I fiori sono 3 o 2 all' ascella di ciascuna foglia fiorale in modo che vi sono da 6 a 4 fiori in ciascun giro: sono forniti di un pedicello assai corto, patenti. I giri sono lontani e fanno così un racemo interrotto in alto del fusto e dei rami. Il calice è campanulato, con molti nervi sporgenti, verde, pubescente-glando. loso, ha due labbri, il superiore poco più corto dell'inferiore, cur- 
vato un po'in su, con 3 piccoli denti, convergenti all'apice; il labbro inferiore è avvicinato al tubo della corolla, diviso in due lacinie lanceolate e fornite all' apice di una punta quasi lesiniforme, piuttosto lunghetta. La corolla è piccola, appena il doppio del calice, di color violetto scuro in tutte le parti eccetto nel tubo ch' è bianchiccio e racchiuso nel calice ch'esso uguaglia in lunghezza. Tale tubo è un po'più largo alla base, un po'piủ stretto verso il mezzo: è glabro; il lembo della corolla è bilabiato, il labbro superiore è lungo quanto il tubo della corolla medesima, un po'curvo, diretto in su, schiacciato lateralmente, smarginato-bilobo con i lobi tondeggianti, con qualche pelo nella carena e li vicino; l'inferiore è piủ corto del superiore, diretto in avanti ed in giù, trilobo, con i lobi laterali più stretti, ovali-bislunghi, tondeggianti all'apice e curvati in fuori e in giù e ripiegati all'apice, e con il lobo di mezzo più largo, quasi orbicolare, un po'concavo di sopra, non smarginato, con tre leggiere righe un po'piủ cariche verso la base. Gli stami fertili son due, poco piủ corti del labbro superiore dentro al quale sono racchiusi, inseriti nella parte superiore del tubo della corolla; i filamenti sono lesiniformi, un po'curvati in avanti verso alto, di color violetto chiaro, glabri. Le antere sono bislunghe-lineari, ottuse ad ambedue l'estremità, inserite nel dorso poco sopra della base, uniloculari, aprentisi per una fessura longitudinale in avanti, verdognolo-violette, glabre. Il polline è giallo. Le antere sterili sono piccole e portate su filamenti molto corti e filiformi. Il pistillo è appena più lungo della corolla, sporgendo con l'apice dello stimma dalla smarginatura del labbro superiore di questa. L'ovario è piccolo, diviso in quattro lobi ovali, verdognoli, glabri; il disco è circolare, gialliccio. Lo stilo è lungo, un po'curvo ad $S$, filiforme, glabro, bianchiccio in basso, violetto in alto. Lo stimma è bifido, con le lacinie avvicinate l'una all'altra, disuguali, la posteriore essendo quasi il doppio più lunga dell' inferiore, quasi acute, di color violetto. Il frutto è racchiuso dentro il calice appena gonfio e diretto orizzontalmente c un po'in giù. I carpelli sono ovali, neri, lisci. (Parl. ms., descr. di pianta di Prataglia nel Casentino in Toscana).

Descrizione della var. $\beta$. - Pianta di un verde piuttosto cupo, con peli glandolosi all' apice in alto del fusto, e mandante un odore piuttosto spiacevole. Rizoma verticale, ramoso, legnoso, scuro, che manda fibre radicali flessuose scurette. Fusti spesso ascendenti o eretti, quadrangolari, di un verde piuttosto chiaro, semplici o con due corti rametti in alto ed opposti. Foglie radicali molte, in ro. 
setta, giacenti per terra, bislunghe, quasi in cuore alla base, inciso-crenate o pennatifide, con le lacinie disugualmente rosicchiatodentate e i denti ottusi, di un verde cupo di sopra e ivi rugose, con una linea bianchiccia dove corrisponde di sotto il nervo longitudinale, di un verde pallido di sotto, ed ivi con il nervo longitudinale assai sporgente da cui partono dei nervi laterali meno sporgenti, uno per ciascuna lacinia e che si diramà in questa: vedute con una lente offrono tanti punti bianchicci in ambedue le pagine. Il picciòlo è piano di sopra, convesso di sotto, spesso di color violetto come la base dei fusti. Le foglie del fusto sono per lo piủ 4 , a due a due opposte, le prime verso la metà, le seconde quasi sotto il racemo, simili alle radicali ma eretto-patenti, più corte e più profondamente divise, con il picciòlo assai corto e quasi sessili. I fiori sono patenti, circa 6 in ciascun verticillo, con i verticilli alquanto avvicinati tra loro e formanti quasi un racemo corto e come troncato all' apice: sono accompagnati da brattee larghe ovato-tonde, un po'scavate in cuore alla base, acuminate, più corte del calice, patentissime, di un verde chiaro, pelose di fuori, con lunghi peli nel margine, per peli parte più lunghi senza glandole e parte piủ corti e glandolosi all'apice. I pedicelli sono cilindrici, verdognoli, pelosi, molto più corti del calice. Il calice è quasi campanulato, di un verde scuro, angolato, peloso-glandoloso massime negli angoli, con le glandole giallicce, con il labbro superiore avente tre denti piccoli, corti, quasi uguali e quasi acuti, e con il labbro inferiore diviso in due lacinie lanceolato-acuminate e mucronate all'apice, avvicinate al tubo della corolla: ambedue i labbri hanno delle lunghe ciglia non glandolose nei margini. La corolla è due a tre volte piủ lunga del calice, glabra. Il tubo è lungo quanto il calice, cilindrico e stretto in basso, slargato e quasi gobbo d'avanti in alto, bianchiccio. Il labbro superiore è di color violetto, diretto in su e un po' curvato in avanti, schiacciato lateralmente, smarginato all'apice. Il labbro inferiore più corto del superiore, patente, trilobo, con i lobi laterali più piccoli, diretti lateralmente in su, un po'patenti, ovali, quasi acuti, del colore stesso del labbro superiore e con il lobo medio molto piü largo, quasi tondo, molto concavo di sopra, di un bianco sfumato di violetto con qualche riga corta e violetta alla gola. Gli stami sono lunghi quasi quanto il labbro superiore della corolla, dentro al quale sono racchiusi, inseriti nella parte su. periore del tubo della corolla. Filamenti corti, cilindrici e di color violetto chiaro. Connettivo lungo, eretto, curvato in avanti, piano d'avanti, un po'convesso nel dorso, glabro come il filamento e quasi 
dello stesso colore, portante in alto una antera fertile, bislungo-lineare, di un verde scuro, glabra, uniloculare, aprentesi longitudinalmente in avanti, inserita nel dorso poco sopra della base. Il polline è giallo. L'altra antera o loggia sterile si vede alla base del connettivo, è quasi tonda, violetto-rosea, glabra. Il pistillo è più lungo del labbro superiore. L' ovario è piccolo, diviso in quattro lobi profondi, ovali-bislunghi, ottusi, verdognoli e posti sopra un disco grosso, carnoso, gialliccio. Lo stilo è lungo, filiforme, curvato in avanti, violetto in alto, bianchiccio in hasso, glabro. Lo stimma è profondamente bifido, con le lacinie disuguali, curvate in fuori, lineari-filiformi, acute. (Parl. ms., descr. di pianta di Pisa).

Osservazioni. - Le due varietà che ho adottate, seguendo Bois. sier (cambiando perỏ le sue appellazioni di serotina e vernalis, che non sono niente esatte), sono due forme estreme, fra le quali stanno molte altre intermedie, che spesso non si sa a quale varietà più propriamente riferire, massime trattandosi di saggi d'erbario.

Nella $1^{2}$ edizione delle Species Linneo riuni le due forme sotto la sua Salvia verbenaca, come si rileva dalle figure citate, e dall'avere egli antecedentemente nell' Hort. Cliff. p. 12 discorso chiaramente del dimorfismo della pianta. Nella $2^{\mathrm{a}}$ edizione egli distaccỏ un sinonimo di Barrelier, ch'è della forma $\beta$, e l'applicỏ alla nuova $S$. clandestina, di cui diè una descrizione che quadra anch'essa con quella forma: ma si dice che il saggio esistente nel suo erbario con quel nome sia di altra pianta, distintissima, quella figurata nella $\mathrm{Fl}$. grcec. t. 24 . 0 sia questa la vera $S$. clandestina Linn., o debba andare sotto l'altro nome di $S$. lanigera Poir., certo non può andare come hanno creduto Bentham ed altri sotto il nome di $S$. controversa Ten., ch' ̀̀ da riferirsi alla forma $\beta$.

L'epiteto di verbenaca, suggerito dal sinonimo di Triunfetti Horminum verbence laciniis, è un aggettivo e va scritto col $v$ minuscolo.

\section{Salvin silvestris.}

.S. perennis, foliis ovato vel oblongo-lanceolatis, subduplicatocrenatis, rngosis, sultus presertim minute pilosis, bracteis mediocribus, coloratis, rotundatis, acuminatis, glomerulis approximatis, calycis tubuloso-campanulati labio superiore 5-dentato, inferiore 2-partito lobis acuminatis, corolla calyce 2 -plo longiore. 
Verbascum Nigrum Salvifolium purpureo flore Lob.? stirp. add. nov. p. 241.

Salvia sylvestris Linn. sp. pl. ed. 1.p. 24. All. fl. ped.1.p. 13. De Not. rep. fl. lig. p. 315. Bicch. agg. fl. lucch. p. 16. Pir. fl. for. syll. p. 110. Car. prodr. A. tosc. p. 510. De Vis. Sacc. cat. piant. Ven. p. 133. Ces. Pass. Gib. comp. fl. ital. p. 307. Arc. comp. fl. ital. p. 545 .

Salvia elata Ten.? syll. fl. neap. p. 18.

Figure. - Reich. ic. bot. t. 526, 527.

Stazione, Abitazione e Fioritura. - "Pratis sabulosis familiaris hic Salvifolius, præsertim non procul Taurino: cosi scrisse Lobelio di questa pianta, se tanto è che vi si debba riferire il sinonimo di lui sopra citato; secus vineas in saxosis, et in apricis sylvis collium Taurinensium observavi, „ scrisse a sua volta Allioni; però Colla già nel 1835 (herb. ped. 4. p. 392) dichiarava non sapere che botanici più recenti l'avessero mai trovata ivi. Pirona l'ha indicata nel Friuli in genere "in sylvaticis, ad vias præcipue in collibus et montibus., De Notaris disse di averne raccolto un unico saggio nel letto della Sturla presso Genova, nel 1843. Bicchi l'ammise sulla fede di un saggio raccolto dall'abate Mezzetti in un campo alla base occidentale del monte di Quiesa presso Lucca. Tenore l'indicò al Gargano, se pure la sua Salvia elata sia un sinonimo come quella di Host. Nè Bertoloni nè io abbiamo veduti saggi italiani della specie. Dovrebbe fiorire in giugno e luglio.

Distribuzione geografica. - Nella metà orientale dell' Europa media e meridionale, nell'Asia Minore e la Persia, nella Siberia occidentale.

\section{Salvia hispaniea.}

S. annua, foliis ovatis, serratis, lævibus, pilosulis, bracteis parvis, herbaceis, ovato-lanceolatis, acuminatis, glomerulis valde approximatis, calycis tubulosi labio superiore integro, inferiore 2-partito lobis acuminatis, corolla calyce vix longiore.

Salvia hispanica Lirn. sp. pl. ed. 1. p. 25. All. A. ped. 1. p. 14. Reich. fl. germ. exc. 1. p. 332. Bert. fl. ital. 1. p. 153. Colla herb. ped. 4. p. 389. Ces. Pass. Gib. comp. fl. ital. p. 306. Arc. comp. f. ital. p. 544 .

Figura. - Bot. reg. t. 359 . 
Stazione, Abitazione e Fioritura. - Allioni, sullo scorcio del secolo passato, indicò questa specie come raccolta negli oliveti d' Oneglia nella Riviera di ponente, e Bertoloni scrisse di averla avuta dal medesimo luogo dal Molineri; anche Reichenbach scrisse sull'indicazione di Charpentier che nasce in Piemonte e in Liguria, principalmente intorno ad Oneglia. Peraltro De Notaris (rep. fl. lig. p. 490) la mette fra le specie di cui non aveva veduto saggio. Fiorisce in giugno e luglio secondo Bertoloni.

Distribuzione geografica. - Linneo diede per patria alla specie I'Italia, e la Spagna dov' era stata trovata nella Nuova Castiglia; ma ora non esiste più anche in quest'ultimo paese. II suo vero paese di origine è l'America intertropicale.

\section{Salvia verticillata.}

S. perennis, foliis late ovatis, grosse dentatis, lævibus, plus minus pilosis, inferioribus auctis in petiolo lobulis paucis parvis, bracteis parvis, herbaceis, late ovatis, acuminatis, glomerulis distinctis, calycis tubulosi labio superiore 3-dentato, inferiore 2-partito lobis acuminatis, corolla calyce paullo longiore.

Salvia verticillata Linn. sp. pl. ed. 1. p. 26. Bert. R. ital. 1. p. 153. Mass. prodr. fl. valt. p. 160. Com. fl. com. 1. p. 29. Coll. herb. ped. 4. p. 391 . Trev. prosp. fl. eng. p. 25. De Not. rep. fl. lig. p. 316. Rola prosp. piant. Pav. p. 271. Hausm. Al. Tir. p. 674. Rota prosp. fl. Berg. p. 69. Bicch. agg. fl. lucch. p. 16. Pir. fl. for. syll. p. 110. Car. prodr. fl. tosc. p. 511. Ard. R. Alpes-mar. p. 293. De Vis. Sacc. cat. piant. Ven. p. 133. Zersi prosp. piant. Bresc. p. 163. Archb. 1. Alto-Serch. p. 60. Ces. Pass. Gib. comp. fl. ital. p. 306. Cocc. contr. fl. Bol. p. 14. Arc. comp. fl. ital. p. 544.

Figure. - Barr. ic. t. 199. Reich. ic. fl. germ. 18. t. 54.

Stazione, Abitazione e Fioritura. - Qua c là nella Penisola settentrionale e centrale, specialmente per $\mathrm{i}$ monti: in Valtellina tra Bormio e i Bagni (Parl.!) e sul monte Valdone, vicino a Lecco (Com.), sulle rupi a Somasca nel Bergamasco (Rota), nel Bresciano sui colli di Capriano in luoghi umidi, rarissima (Zersi), in Tirolo ad Ampezzo, Bressanone, Cortina, Bolzano, Salorno, Someda, Sardagna, Roveredo (Hausm.), presso Verona in val Policella, nei margini dei campi, verso l'Ospedaletto e Domeiara, nel Vicentino in abbondanza alle radici del monte Sumano (Poll. A. ver.), nei monti Bassanesi, nei campi e nelle selve (Montini!), nel Friuli nelle col- 
line solatie di Gemona, S. Daniele, Chiusa, Ponteba, e per le vie d'Udine, di Fagagno ecc. (Pir.), nel Lido veneto (Kellner!), negli Euganei (Trev.), a Mantova al bosco Fontana (Barbieri!) e a S. Giorgio (Bert.), a Bologna sul ciglio delle fosse di circonvallazione fra S. Vitale e S. Mamolo, fuori di porta Galliera, e sulla scarpa settentrionale della Montagnola (Cocc.), comune nei pascoli montani delle Alpi Marittime e dell:Appennino Ligure, da dove scende al mare e alla pianura del Po (De Not.), cosi a Limone (Parl.!), Vinadio, Acqui alla Cà di Prà, Torino rei colli (All. fl. ped.) e verso Rivoli (Re fl. seg.), Bobbio a Pregola, a Cegni (Rota), nel monte Lesime (Moretti!), e cosi lungo la Roia tra Tenda e Fontano (Ard.), tra Giandola e Brera (Webb!), a Sospello (Parl.!), Men. tone dov'è rara, Nizza all’Ariana (Ard.), Rezzo (Berti!), Marcaruolo (Carrega!), Torriglia (Ardissone!), in Toscana fra Carrara e le cave di Miseia (Bert.), a Palodina in Garfagnana (Pucc.), a Quiesa presso Lucca (Mezzetti!), nel monte Pisano (Cocchi!), a Firenze sugli argini del Mugnone (Gemmi!), in Umbria a Spoleto (Bert.), infine nel Piceno a Montefortino a S. Giacomo (Marzialetti!). Secondo il Cupani (hort. cath.) questa pianta nascerebbe anche in Sicilia, ma gli erboratori moderni non ve l'hanno trovata (Guss. $f l$. sic. syn.). Fiorisce da giugno a settembre.

Distribuzione geografica. - Nell'Europa media e meridionale a levante della Francia, nell' Asia Minore e fino alla Persia.

Descrizione. - Pianta di color verdognolo-bianchiccio, irsuta per peli bianchi disuguali. Fusto quadrangolare, ramoso, rami eret. to-patenti, angoli ottusi, grossi, facce scanalate. Foglie carnosette, molli, di sopra verdognole-bianchiccie, nervo longitudinale bianchiccio, solchi corrispondenti ai nervi assai manifesti in rete, di sotto più chiare, nervo longitudinale e laterali a rete rilevata. Brattee ovatoacuminate, rivolte in giù, scuricce. Fiori circa $8-10$ in ogni semiverticillo, un po'secundi. Pedicelli eretti, piủ corti del calice, delicati, cilindrici, irsuti per peli subulati, un po'curvi in su, di color amarante. Calice tubuloso-campanulato, angolato, con molti nervi, cinque dei quali maggiori terminano all' apice dei denti, quasi bilabiato, con il labbro superiore tridentato, denti corti, un po' divergenti, triangolari, con una setola all'apice, l'altro inferiore con due denti più lunghi, lanceolati, acuminati, con una piccola setola all'apice, tutti con ciglia corte, il calice è amarante in parte e peloso nei nervi con nervi disuguali, patenti, amaranti, di dentro è verde, glabro. Corolla piccola, meno del doppio più lunga del calice, di color vio- 
letto, pelosa di fuori superiormente nel tubo e nel labbro superiore, glabra nella parte inferiore del tubo e nel labbro superiore di fuori. Tubo poco più corto del calice, fornito in alto di un anello obliquo di peli corti e fitti. Il labbro superiore è eretto, stretto, ovale, ottuso, smarginato, concavo; l'inferiore è patente, piủ corto, o cordato-bilobo con i lobi ottusi, di sotto carenato. Gli stami sono poco più corti del labbro superiore da cui sono ricoperti, inseriti alla parte superiore del tubo. I filamenti sono cilindrici, glabri, bianchicci. Il connettivo è a guisa di filamento, bianchiccio, glabro, e con la parte inferiore più corta, acuta, diretta in avanti, sterile, la parte superiore eretta porta una sola loggia. Questa è allungata, ottusa, inserita nel dorso sopra della base, nericcia, aprentesi per una fenditura longitudinale in avanti. Il polline è bianco. Il pistillo è più lungo del labbro inferiore al quale è avvicinato, uguale al superiore. L'ovario è diviso profondamente in 4 lobi, allungati, ottusi, verdognoli, glabri, inseriti sopra un disco più corto di esso. Lo stilo è filiforme, curvato ad arco nel terzo inferiore, poi diritto, di color violetto-bianchiccio, glabro. Lo stimma è bifido, con le lacinie strette, ottusette, di color violetto-chiaro. (Parl. ms., descr. della pianta di presso Bormio).

Osservazione. - Per certi particolari della struttura degli stami questa specie si allontana dalle congeneri, ed è stata posta la questione se non convenga farne un gencre a sè. Vedansi Bentham $L a b$. p. 310, Delpino Pensieri sulla biologia vegetale p. 81-82.

\section{SEZIONE SECONDA.}

Nepetee.

NePETEÆ Benth. lab.p. 462.

Stamina superiora longiora.

\section{NETPEA .}

Cataria et Calaminthe sp. Tourn. inst. p. 202, 194. t. 9).

Nepeta et Glechoma Linn. gen. pl. ed. 1. p. 170, 171. Gen plant. 1. germ. 6. t. $23,24$. 
Nepeta Benth. lab. p. 464. Benth. Hook. gen. pl. 2. p. 1199. Ces Pass. Gib. comp. fl. ital. p. 309. t. 45. f. 6.

Calyx tubulosus, valide 13-15-nervatus. Corolla manifeste bilabiata, labio superiore erecto, planiusculo. Stamina 4, conniventia, filamentis edentulis aut apice denticulo auctis, antheris 2-locularibus, loculis longitudinaliter dehiscentibus, confluentibus. Stylus lobis subæqualibus. Cocci apice convexi leves.

Portamento. - Erbe generalmente perenni, di aspetto vario. L'altezza n'è di 2 decimetri a 1 metro; il fusto è semplice 0 ramoso; le foglie sono di mezzana grandezza, ora larghe, ora strette, dentate. I fiori, piccoli, bianchi o cerulei, più o meno numerosi, ora sono in dicasi, ora in glomeruli, che accompagnati da brattee, poco appariscenti, oppure grandi e vistose, formano in cima al fusto e a'rami lunghe infiorescenze, rade o fitte. La sola Nepeta Glechoma ha fiori più grandi, ascellari a foglie fiorali, che seguitano a crescere dopo la fioritura, nel mentre che il fusto passando a fruttifero si sdraia per terra e vi mette radici, e si allunga, e l'anno dipoi si raddrizza di bel nuovo passando nuovamente a fiorifero; le quali cose sono state egregiamente descritte nella Morphologie végétale di A. de Saint-Hilaire a pag. 104, e poi in tante altre opere.

\section{Nepeta tuberosa.}

N. tomentosa, foliis ovato-oblongatis, basi cordatis, crenatis, inferioribus petiolatis, superioribus sessilibus, glomerulis multifloris, densissimis, in inflorescentias elongatas cylindricas approximatis, bracteis coloratis, ovalihus, corollæ tubo calyce vix longiore.

Nepeta tuberosa Linn. sp. pl. ed. 1. p. 571. Bert. fl. ital. 6. p. 72. Ces. Pass. Gib. comp. fl. ital. p. 309. Arc. comp. fl. ital. p. 546 .

Figura. - Reich. ic. bot. t. 439.

Stazione, Abitazione e Fioritura. - In Sicilia nei campi aridi arenosi presso Palermo a Mondello e a Pallavicini (Parl.!), a Priolo all'abbeveratoio di Buongiorno, fra Piazza e Pietrapersia (Guss. syn.). a Sponti (Bert.). Fiorisce in maggio e giugno.

Distribuzione geografica. - Nasce inoltre nella Penisola Iberica.

Descrizione. - Pianta di un verde bianchiccio, per una lanugine o lana bianca che la cuopre nel fusto e nelle foglie. Fusti qua- 
drangolari, con un solco in ciascuna faccia, in alto verdogoli bianchicci. Foglie opposte, quasi sessili, bislunghe, ottuse, scavate in cuore alla base, con leggieri smerli, fornite alla base di pochi denti più grandi degli smerli del resto del margine, molli, pubescenti di sopra ed ivi con leggieri solchi che corrispondono ai nervi di sotto, di un colore più bianchiccio per la detta lana di sotto ed ivi con un nervo longitudinale e dei nervi più delicati che partono pure ugualmente dalla base e altri lateralmente dal nervo longitudinale, i quali sono un po'curvi e vanno diramandosi e terminano all' apice degli smerli. I fiori sono distribuiti in piccole cime formanti all' apice del fusto e dei rami una spiga quasi cilindrica, interrotta un po'in basso. Le brattee sono lunghe quasi o poco più del calice, ovato-bislunghe, acuminate all' apice, un po' concave, di un verde sfumato di violetto, con molti nervi ramosi e in rete di colore più carico, pubescenti con peli aventi una glandoletta bianca all'apice, e fornite di ciglia nel margine. Le bratteole sono quasi uguali in lunghezza alle brattee, ma un po'più strette e bislunghe o bislunghe-lanceolate, crenate nel modo medesimo. Le cime hanno per lo più 4 o 5 fiori: questi hanno IIn pedicello cortissimo. Calice piuttosto stretto, tubuloso, con molti nervi, verdognoli e in parte violetti, pubescenti: lembo circa la metà più corto del tubo con cinque denti quasi uguali, lesiniformi, erettopatuli. Corolla quasi una metà più lunga del calice, di color violetto, pelosetta di fuori: tubo appena curvo, piủ lunga del calice, slargata in alto nella gola dove sono di dentro delle piccole macchioline tonde. Labbro superiore largo, smarginato-bilobo, con i lobi ottusissimi, un po'concavo d'avanti; labbro inferiore trilobo, con i lobi laterali piccoli, tondeggianti, con il lobo mediano appena più corto del labbro superiore, concavo, ottusissimo, smerlato, con una barba bianca alla base verso la gola. Stami $\dot{4}$, due più corti del labbro superiore e due piủ corti di essi, tutti e quattro presso la sommità di quel lab. bro. Filamenti inseriti nella parte superiore del tubo della corolla, curvati un po' ad arco in avanti verso l'apice, filiformi, glabri, violetti. Antere violettn-scure, glabre, con le logge divergenti, aprentisi per una fessura laterale. Polline bianco. Pistillo quasi uguale al lahbro superiore. Ovario piccolo, diviso in 4 lobi, ottusi, verdognoligiallicci, lisci, sopra un disco grossetto dello stesso colore. Stiln molte volte piủ lungo dell'ovarin, filiforme, glabro, violetto. Stimma bifido, lacinie acute, quasi uguali, dello stesso colore. (Parl. ms., descr. di pianta collivala nell Orlo bolanico di Palermo). 


\section{Nepetr Apuleii.}

N. minute hirta, foliis ovato-oblongatis ovatisve, basi subcordatis, urenatis, inferioribus petiolatis, superioribus sessilibus, glomerulis multifloris, densissimis, in inflorescentias elongatas cylindricas approximatis, bracteis subcoloratis, ovalibus, corollæ tubo calyce multo longiore.

Nepeta Apuleii Ucria pl. ad Linn. op. add., in Röm. Arch. 1. p. 69. Bert. fl. ital. 6. p. 71. Ces. Pass. Gib. comp. fl. ital. p. 310. Are. comp. fl. ital. p. 546.

Figura. - Boce. ic. et descr. rar. pl.t. 25. f. 2.

Stazione, Abitazione e Fioritura. - In Sicilia, nei colli solatii argilloso-calcarei : Sutera, Regalmuto, Favare, Girgenti, Amorosa al sud di Busambra, S. Catterina, Nicosia, Caltanissetta, Cuozzo di Miceli, Summatino; Castrogioranni (Guss. syn.), Lercara, S. Maria del Bosco (Todaro!). Fiorisce da aprile a giugno.

Distribuzione geografica. - Nasce oltracciò nella Penisola Iberica e nell' Affrica settentrionale.

\section{Nepeta italiea.}

N. minute canescens, foliis cordatis, crenatis, petiolatis, glomerulis multifloris, densissimis, in inflorescentiis elongatis distinctis, bracteis herbaceis membranaceo-marginatis, anguste lanceolatis, corollæ tubo calyce longiore.

Nepeta italica Linn. sp. pl. ed. 1. p. 571. De Not. rep. fl. lig. p. 323.

Figura. - Reich. ic. bot. t. 483.

Abitazione e Fioritura. - Linneo descrisse questa specie su di un saggio d'Italia avuto da certo Ratgeb. Dopo, non essendo più stata trovata nel nostro paese, ma in Oriente, i botanici hanno ritenuta erronea la patria italiana; ma ecco, da saggi esistenti nell'Erbario centrale italiano apparire che fin dal 1859 il Marzialetti aveva ritrovata la pianta nel Piceno presso Montefortino al Pretaro, e più recentemente il Paolucci l'ha trovata nel monte S. Vicino delle Marche; e prima di loro l'aveva ritrovata Viviani nei monti S. Spirito del Genovesato (De Not.). Fiorisce in giugno e Iuglio. 
Distribuzione geografica. - Nell'Asia piủ occidentale, fino al Kurdistan; in Italia, e in Spagna.

Osservazione. - I saggi italiani sono perfettamente compagni a quelli asiatici, segnatamente al n. 1729 delle collezioni di AucherEloy, citato per la specie da Bentham (in Cand. prodr.) e da Boissier (Fl, orient.).

\section{Nepeta Catsoria.}

N. pilusulu-canescens, foliis cordatis, crenatis, petiolatis, glomerulis multifloris, laxiusculis, in inflorescentias parce elongatas subapproximatis, l,racteis herbaceis, lanceolatis, corollæ tubo calyce iequali.

Nepeta Cataria Linn. sp. pl. ed. 1. p. 570. Bert. A. ital. 6. p. 65; el auct. omu.

Nepeta vulgaris Ing. cat. sp. Mond. p. 52.

Figure. - Fl. dan. t. 580. Reich. ic. fl. germ. 18. 1. 41.

Stazione, Abitazione e Fioritura. - Nelle siepi, le macerie ecc. É frequente nell'Alta Italia: in Piemonte a Susa (Webb!), Novalese (Bert.), Mondovi (Ing. cat.), nel Novese dov'è comune (De Not. prosp.); a Moncalieri (Delponte!), tra Torino e Collegno (Re fl. $(0 r$.), a Riva di Valsesia (Carestia!), nel Novarese (Bir. l. acon.), ad Arona (Ricca!); in Lombardia a Pavia (Nocc. Palb. fl. lic.), Mlonza (Rampoldi!), in Valtellina sopra Ponte, e tra Bormio e i Bagni a 1300 metri (Parl.!), nel Bergamasco dov'è comunissima (Rota prosp. fl. Berg.), nel Bresciano a Bovegno (Zersi prosp. pianl. Bresc.), Collio e Ponte di legno a 1320 metri, a Grumone (Parl.!), Nlantova al Bosco funtana, Solferino (Bert.); in Tirolo a Bolzano verso Leifers, verso Gries e altrove, tra Mezzolombardo e Zambana, nelle valli di Fiemme e di Fassa, tra Breguzzo e Bondo, a lioveredo, a Brentonico (Hausm. M. Tir.); nel Veneto a Verona in val d'Avesa (Rert), negli Euganei (Trev. prosp. /l. eng.), a Bassano ad Angarano (Bert.), nel Lido di Venezia (Kellner!), a Ldine, Faedi e altrove (Pir. M. for. syll.); in Istria a Lippizza (Bert.); in Komagna a Liavenua (Bert.); nell'Ernilia a Bologna al Sasso (Bert.), a Rocca di Montese, Fanano, Boscolungo (Cii). Pir. /l. Mod.), Parma a Felino (Passerini!). Nell' Italia inedia e soprattutto bassa è inolto meno freyuente: nella Riviera di punente a Liza, Ciandola, Saorgio (.Ird. /l. Alpes-mar.) e in valle d'Alloenga (Bert.), di levante a S. Stefano 
d'Aveto (De Not.); in Toscana nella Selva pisana (P. Savi!), a San Casciano de'Bagni (Arcangeli!), Siena (Bart. cat. piant. Sien.), Rapolano (Sommier!), Radda e Pian d'Albola (Beccari!), Tosi sotto Vallombrosa!, Frassineto in Casentino (Parl.!), nel monte Nero in val Tiberina (Amidei!), a Montelatrone presso il monte Amiata (Savi bot. etr.); nelle Marche al Catria presso le Scalette (Piccinini!), a Camerino a Pioraco, a Macerata ai Cappuccini vecchi (Bert.); nei monti del Lazio (Bert.); in Abruzzo a Pacentro (Ces. el. piant. Maiella); in Campania a Picinisco verso Canneta (Terracciano!), a Nola ai Camaldoli, e Pietraroia (Terr. quarta rel. Terr. di Lav.); in diversi siti sul Vulture (Terr. fl. vult. syn.); in Corsica a Vico, Bocognano, Bastelica, Aullene ecc. (Mars. cat. pl. Cors.); in Sicilia a Lercara (Sorrentino!), Roccapalumba (Todaro!), fra Cammarata e Sutera, e Campofranco ed Aragona (Guss. syn.). Fiorisce secondo i luoghi in luglio e agosto, o giugno e luglio, o maggio e giugno.

Distribuzione geografica. - Nell' Europa, specialmente media ed esclusa la sua parte piủ settentrionale, nell' Asia occidentale, fino all'India, introdotta nell'America settentrionale e al Capo.

Descrizione. - Pianta odorosa, perenne, alta da 3 a 8 decimetri, di un verde bianchiccio, pubescente e molle. Il fusto è eretto, quadrangolare, con gli angoli ottusi e non rilevati e con le facce leggermente scanalate, bianchiccio, pubescente, con peli curvati un po' in giủ, ramoso con i rami opposti, patenti o eretto-patenti. Le foglie sono molli, opposte, patentissime-reflesse, cordato-ovate, un po'prolungate verso l'apice, acute, con grandi crenature dirette un po'verso l'apice, ottuse e con un mucrone ottuso, di un verde pallido e pubescenti di sopra ed ivi con molti solchi piuttosto profondi che corrispondono ai nervi di sotto e alla rete da questi formata, di un verde bianchiccio e pubescente-tomentoso di sotto ed ivi con un nervo longitudinale rilevato, da cui partono sin dalla base dei nervi laterali molto ramosi e rilevati che formano una rete rilevata in tutta la pagina inferiore della foglia; sono fornite di un picciòlo più corto della foglia, solcato profondamente di sopra, convesso di sotto, pubescente, bianchiccio. I fiori sono molti, in piccoli corimbi fitti e forniti di un corto peduncolo, all' ascella delle foglie superiori o fiorali del fusto e dei rami, facendo cosi un racemo spiciforme, ottuso, interrotto alla base e diretto quasi da una sola parte. Le foglie fiorali o brattee sono più corte dei fiori, ovate, scavate in cuore 0 ristrette alla base nel picciòlo, con le crenature piccole, del resto simili alle foglie del fusto. Il peduncolo è corto, cilindrico, bianchiccio, peloso. I pedicelli 
sono pure corti, molto piủ corti del fiore e simili al peduncolo: ciascuno ha molte bratteole, eretto-patenti, piủ corte del calice, lineari, acuminate, pelose, verdognolo-bianchicce. Il calice è tubuloso, un po'curvato, verdognolo, con quindici nervi longitudinali rilevati e pelosi, divisi da stretti solchi bianchicci, ha cinque denti lanceolato-acuminati, un po'divergenti, un po'patuli e pelosi: gontia un po'dopo la lioritura e diventa ovoideo, allontanandosi i nervi e rimanendo visibile la parte intermedia dove erano prima i solchi, i denti sono allora eretto-patuli. La corolla è il doppio piủ lunga del calice, bianca con macchioline amarante chiare in ambedue i labbri, pubescente di fuori, glabra di dentro ma fornita di lunghi peli sul labbro inferiore vicino alla gola. Il suo tubo è poco più lungo del calice!, stretto, slargato soltanto in alto verso la gola. Il labbro superiore è diretto in su, molto più piccolo dell'inferiore, quasi orbicolare, bilobo, con i lobi rivolti un po'in dietro nella parte loro su. periore; il labbro inferiore è patente, trilobo, con i lobi laterali molto corti e piccoli, tondeggianti e rivolti in fuori e un po' in giù e con il lobo di mezzo grande, un po'più largo che lungo, concavo di sopra e crenulato con poche e rare crenature nel margine. Gli stami sono quattro, didinami, i due posteriori o interni poco più lunghi degli altri due, quasi uguali o appena più lunghi del labbro superiore della corolla, dal quale sono coperti dapprima. I filamenti sono eretti, paralleli, cilindrici, bianchicci, con qualche peletto verso basso : dopo la fioritura si curvano in fuori ed in basso. Le antere sono biloculari, con le logge ottuse, interamente divergenti e aprentisi per una fessura longitudinale comune alle due logge, di color violetto, glabre. Il polline é bianchiccio. Il pistillo è quasi uguale agli stami. L'ovario è piccolo, con quattro lobi ovali, verdognoli, inserito sopra un disco verdognolo. Lo stilo è filiforme, appena curvo verso alto, di color violetto-bianchiccio. Lo stimma è bifido, con le lacinie corte, un po'divergenti, quasi uguali e quasi acute. Le noci sono quasi ovali, ottuse, un po'schiacciate e come lenticolari, finissimamente punteggiate quando si guardano con una forte lente, quasi nericce. (Parl. ms., descr. della pianta di Grumone in Lombardia).

\section{Nepeta Vepetella.}

N. minutc canescens, foliis anguste: ovato-lanceolatis, basi subcordatis, grosse dentatis, petiolatis, glomerulis paucifloris, laxiuscu. lis, in inflorescentias breviusculas subapproximatis vel elongatas re- 
motis, bracteis herbaceis, lanceolato-subulatis, corollæ tubo calyce longiore.

Nepeta Nepetella Linn. sp. pl.ed. 2. p. 797. Benth. lab. p. 477. Bert. fl. ital.6. p. 69. De Not. rep. fl. lig. p. 323. Ard. fl. Alpesmar. p. 297. Ces. Pass. Gib. comp. fl. ital. p. 310. Ing. cat. sp. Mond. p. 52. Arc. comp. fl. ital. p. 547.

Figure. - All. fl. ped. t. 2. f. 1. Reich. ic. bot. t. $261,586$. Ic. fl. germ. 18. t. 42. f. 1.

Stazione, Abitazione e Fioritura. - Nei luoghi sassosi o arenosi, nel letto dei torrenti, delle parti inferiori delle Alpi occidentali: a Cogne in val d'Aosta (Carestia!), a Susa (Aiuti!), Novalese (Bertero!), Ferrero (Malinverni!), nel Cenisio a Bard a 1300 metri (Arc.!), presso Oulx (Aiuti!), a Bardonecchia (Beccari!), nel monte Genevre (Ball!), nella valle di Luserna (Rostan!), presso Valdieri (Bert.), a Mondovi nella ripa del Vitale (Ing.), al col di Tenda a 1900 metri (Parl.!), dove abbonda e scende verso il mare (Ard.), cosi trovasi a Ventimiglia (Bert.). Quindi saltando tutti i monti intermedi ricomparisce nell'Appennino piceno-abruzzese: nel monte Birro al Balzo rosso (Bert.), presso Montefortino alla Madonna dell'Ambro (Marzialetti!), a Foce (Gennari!), nel Vettore (Orsini!), alle radici del monte Costone (Bert.), al Corno (Ten. syll.). Fiorisce in luglio e agosto.

Distribuzione geografica. - Nell' Europa meridionale dalla Spagna all'Italia.

Descrizione. - Perenne, formante dei cespugli alti da 3 a 5 decimetri, di un odore forte, di un verde bianchiccio, in gran parte coperta di un velluto corto fitto di un bianco cinerino. Rizoma ramoso, con i rami che si continuano nei fusti. Fusti ascendenti, o eretti, ramosi in basso, con i rametti corti e ascendenti; sono duretti, quadrangolari, con gli angoli ottusi e con un solco longitudinale leggiero in ciascuna faccia : coperti di un velluto corto, fitto e con i peli curvati ad arco in giù. Foglie opposte, piccole, dirette in giù, lanceolate, ottuse 0 ottusette, leggermente in cuore alla base 0 ivi ristrette in un picciòlo che è in tutte le foglie, profondamente dentate nel margine con i denti disuguali ed ottusi, quasi in forma di sega: di un verde bianchiccio di sopra ed ivi con solchi in rete in mezzo ai quali sporge il parenchima, di un verde cinerino di sotto ed ivi con un nervo longitudinale, da cui partono dei nervetti laterali che si diramano per terminare all' apice dei denti: tutti i nervi sono rilevati e di colore più chiaro del resto della pagina inferiore. Il picciòlo è 
stretto, ha un solco longitudinale di sopra, convesso di sotto, ivi e nel margine con il velluto come quello della pagina inferiore della foglia. Fiori 4 a 6 in ciascun glomerulo: i glomeruli avvicinati in un racemo spiciforme, corto, ottuso, molto folto in alto, interrotto in basso. I fiori sono diretti quasi tutti da una parte, hanno un peduncolo corto e ciascuno ha un pedicello proprio, molto piu corto del calice. Il pedicello è accompagnato da una bratteola, la quale è più lunga di esso, lineare-subulata, assottigliata verso l'apice, un po'curva, di un verde bianchiccio, sfumata spesso in alto di rossiccio. Il calice ha il tubo un po'curvo, quasi cilindrico, un po'ingrossato e come ovoideo alla base, peloso-lanato per molti peli lun. ghi, bianchi: ha molte strie longitudinali rossicce; i denti sono un po'disuguali per la lunghezza, ma tutti più della metà più corti del tubo, larghetti, lanceclati, assottigliati in alto, patuli, con le strie come il tubo. La corolla è il doppio piủ lunga del calice, bianco-rosea, pelosa di fuori. Il tubo è eserto, molto curro, cilin. drico, verso alto si slarga gradatamente. Il labbro superiore è eretto, quasi piano, convesso longitudinalmente nel dorso, bifido o meglio bilobo, con i lobi tondeggianti all'apice. Il labbro inferiore è piủ lungo del superiore, diretto in avanti, trilobo e con il lobo medio molto più grande dei laterali, molto concavo di sopra, con denti disuguali ed ottusi nel margine, peloso-barbato di sopra e in parte di sotto, con i peli di sopra più lunghi e bianchi, i lobi laterali sono piccoli, diretti in giủ, quasi orbicolari, senza peli di sopra. Gli stami sono quattro, poco disuguali, i due posteriori un po' piủ lunghi, tutti po. sti avanti del labbro superiore. I filamenti sono inseriti in alto del tubo, lesiniformi, curvati ad arco in avanti, rosei, pelosi in avanti verso basso. Le antere sono piccole, con le due logge ovali e divergentissime, di color violetto scuro, glabre. Il pistillo è quasi uguale agli stami. L' ovario è piccolo, quadrilobo, glabro, verdognolo-gialliccio. Lo stilo è lungo, un po'curvo, filiforme, bianchiccio. Lo stimma è bifido con le lacinic corte, un po' divergenti, acute. Il frutto c̀ quasi ovale, con quattro lobi all'apice, verdognolo, glabro. (Parl. ms., descr. della pianta di Susa).

\section{Nepetu ngrestis.}

N. vix pilosula, foliis anguste ovato-lanceolatis, basi subcordatis, inciso-dentatis, petiolatis, glomerulis paucilloris, laxiusculis, in

Flona Italiana, - Vot, VI. 
inflorescentiis elongatis distinctis, bracteis herbaceis, lanceolato-subulatis, corollæ tubo calyce vix longiore.

Nepeta agrestis Lois. nouv. not. Bert. fl. ital. 6. p. 70. Gren. Godr. fl. Fr. 2. p. 675. Mars. cat. pl. Cors. p. 113. Ces. Pass. Gib. comp. fl. ital. p. 310. Arc. comp. fl. ital. p. 547.

Nepeta aragonensis Willk. Lange! prodr. fl. hisp. 2. p. 431 (non Lam.).

Abitazione e Fioritura. - Nei monti centrali di Corsica: a Ba. stelica (Requien!), lungo la Gravona e la Sellola, sopra Bocognano (Mars.), alla Vizzavona (Soleirol!, Mabille!) nel monte d'Oro, e verso i Pasciali (Bert.), a Ghisoni (erb. Jordan!), a Casamiccioli (Kralik!), Campotile, le case dei pastori d'Ascutzela nel Niolo (Gren. Godr.).

Distribuzione geografica. - Nasce inoltre in Spagna.

Osservazione. - Willkomm e Lange nel Prodr. fl. hisp. riferiscono la Nepeta agrestis Lois. alla $N$. aragonensis Lam., di pubblicazione anteriore, e difatti la pianta raccolta da Bourgeau a Benasque nell'Aragona, e citata da loro, è la nostra $N$. agrestis; perỏ la descrizione molto chiara di Lamarck nell'Enc. méth. bot. 1.p. 711 non si adatta punto a questa specie, e invece quadra bene con la $N$. Nepetella, alla quale Bentham in Cand. prodr. riferisce la $N$. arogonensis quale sinonimo.

\section{y. Nepeta foliosa.}

N. hirsuta, foliis ovato-oblongis ovatisve, basi subcordatis, inciso-dentatis, petiolatis, glomerulis plurifloris, densiusculis, in inflorescentias elongatas subapproximatis, bracteis foliaceis, corollæ tubo calyce paullo longiore.

Nepeta foliosa Mor.! stirp. said. el. fasc. 3. p. 10. Bert. ^. ital. 6. p. 73. Mor. fl. sard. 3. p. 300. Cies. Pass. Gib. comp. fl. ital. p. 310. Arc. comp. fl. ital. p. 547.

Figura. - Mor. fl. sard. t. 107.

Stazione, Abitazione e Fioritura. - Nei pascoli dei monti calcarei di Oliena (Ascherson!), principalmente al luogo detto il prato, tra 1000 e 1300 metri (Mor.). Fiorisce da maggio a luglio.

Distribuzione geografica. - Specie sarda. 


\section{Nepeta inuala.}

N. minutissime piloso-scabra, foliis ovato-oblongis, basi subcordatis, crenatis, subsessilibus, glomerulis pauci-multifloris, laxis, in inflorescentias elongatas subapproximatis, bracteis herbaceis, subulatis, tubo corollino calyce æquali.

Nepeta nuda Linn. sp. pl.ed.1. p. 570. Bert. fl.ital.6. p. 67. Hausm. fl. Tir. p. 683. Pir. fl. for. syll. p. 29. Ard. fl. Alpes-mar. p. 297. De Vis. Sacc. cat. piant. Ven. p. 136. Zersi prosp. piant. Bresc. p. 66. Ces. Pass. Gib. comp. fl. ital. p. 310. Anzi anct. fl. nov.-com. p. 196. Sacc. Bizz. agg. fl. trev. p. 29. Arc. comp. fl. ital. p. 547.

Figure. - Fl. grcec. t. 547. Reich. ic. fl. germ. 18. t. 42. f. 3.

Stazione, Abitazione e Fioritura. - Nell'Alta Italia, per i luoghi erbosi, o boschivi, specialmente di collina e di montagna: alla Certosa di Pesio sopra Cuneo (Thuret!), ai colli di Tenda e di Fenestre (Ard.), sopra Limone (Parl.!), a Valdieri (Delponte!), presso Mirabone sopra Bobbi (Rostan!), presso Susa (Webb!), nel Cenisio (Bert.), a Moncalieri (Rostan!), nel Novarese sul Po dov'è frequente (Bir. 7 . acon.), sụlla Sesia al di là di Mortara (Moretti!), a Craveggia in val Vigezzo (Bir.), presso Como sul monte Palanzo, rara (Anzi), sul lago di Garda nel Bresciano, frequente (Zersi), e nel Veronese, sul Baldo nelle valli Basiana, Ortigara, della Ferrara, fredda, nei Lessini (Poll. fl. ver.), verso Brentonico, nel Trentino (Hausm.), nel Vicentino (De Vis. Sacc.), a Bassano, Oliero, Carpanè (Bert.), nei monti Grappa e Collalti, rara (Sacc. Bizz.), nel Bellunese ad Agordo (Hausm.) e alla Pieve di Quero (Poll.), presso Purzuso e Clauzet nel Friuli (Pir.), in Istria a Lippizza (Bert.) e Fiume (A. M. Smith!). Quindi passando sopra al tratto intermedio ricomparisce nell'Appennino centrale, dove si trova in valle Canetra, nel monte Acuto al Fonte Vidone (Bert.), in capo del Tenna al prato di S. Antonio, al Pian grande del Castelluccio (Narzialetti!), al Corno (Orsini I); e termina nel Matese (Terraceiano!). Fiorisec in giugno e luglio.

Distribuzione geografica. - Nell' Europa dai Pirenei alla Rus. sia media e meridionale, nell'Asia occidentale e sino all' Altai.

Descrizione. - Pianta alta sino a I metro, verde e in parte violetta, odorosa. Pizoma obliquo, grossetto, fibre delicate. Fusti eretti, 
quadrangolari, facce con un solco piuttosto profondo, ivi verdognolo, con gli angoli ottusi e violetti, in alto tutto violetto, ramoso in alto, rami corti eretto-patenti. Foglie patenti, opposte, sessili, ovate o ovato-bislunghe, ottuse, scavate in cuore e abbraccianti il fusto con la base, verdi di sopra, ivi leggieri solchi corrispondenti ai nervi di sotto, verde pallido di sotto, nervo longitudinale sporgente di sotto e più chiaro da cui partono pochi nervi laterali curvi che si dirigono verso il margine dall'apice e si diramano verso il margine stesso per formare una rete alquanto sporgente nella pagina inferiore: questa ha tante piccole fosserelle tonde; le foglie sono pelosette-scabre nella pagina superiore e nei nervi della inferiore, le superiori sono gradatamente più piccole e anche le fiorali ma della stessa forma. Fiori piuttosto piccoli, in gruppetti per lo più di 3 o di 4 , e forniti di un peduncolo corto, formanti insieme dei racemi spiciformi nella parte superiore del fusto e dei rami, piuttosto delicati e radi o per dir meglio interrotti. Pedicelli eretti o eretto-patenti, corti, più corti del calice, e come i peduncoli e la rachide quadrangolari, violetti, irti. Brattee e bratteole piccole, lanceolate o lanceolato-lineari, acute, verdognole $o$ in parte violette, nervo longitudinale un po' irto massime nei margini. Calice quasi cilindrico, un po'ristretto alla base, verdognolo-violetto, con molte costole longitudinali, pubescenti-pelose per peli piuttosto lunghetti, con cinque denti, lunghi poco più di un terzo della lunghezza del tubo, uguali trạ loro, eretto-patuli, strettamente lanceolato-acuminati, pubescenti-pelosi come il tubo, verdi nel dorso, violetti nei margini. Nel frutto il calice è un po' gonfio, quasi ovale, e i denti più patenti. Corolla piccola, violetto-chiara con macchioline tonde disuguali più cariche nel labbro inferiore, pubescente di fuori. Tubo delicato, poco più lungo del calice, slargato in alto quasi ad un tratto e un po'pubescente di dentro. Labbri poco piủ corti del tubo: labbro superiore diretto in su, quasi orbicolare, bilobo, labbro inferiore appena più lungo ma più largo del superiore, concavo di sopra, fatto a guisa di una piccola conca, con una barba nella concavità verso la gola, formata di peli lunghi e bianchi, è dentato-smerlato irregolarmente nel margine ch'è diretto in su. Gli stami sono 4, 2 quasi uguali e 2 poco più corti del labbro superiore al quale sono adattati in avanti, inseriti in alto del tubo. Filamenti un po'curvi, filiformi, violetti-chiari, con qualche corto peletto in basso. Antere violetto-scure, con le logge in direzione opposta, uniloculari per la confluenza delle logge, glabre. Polline bianco. Pistillo poco piủ lungo degli stami più lunghi. Ovario piccolo, di- 
viso in 4 parti bislunghe, verdognole, un po'irsute all' apice, inse. rito sopra un disco grossetto e gialliccio. Stilo lungo, filiforme, un po'curvo in alto, glabro, violetto-chiaro, bifido all'apice, lacinie quasi uguali, appena divergenti. Frutti irsuti all'apice, racchiusi nel calice, molto più corti di questo. (Parl. ms., descr. della pianta di Limone nelle Alpi Marittime).

\section{Nepeta Glechoma.}

N. pubescens vel hirsuta, foliis rotundatis, basi cordatis, crenatis, longe petiolatis, glomerulis paucifloris, ad axillas foliorum, tubo corollino calycem superante.

Glechoma hederacea Linn. sp. pl. ed. 1. p.578. Bert. fl. ital. 6. p. 106; et auct.

Nepeta Glechoma Benth. lah. p. 485; et auct. recent.

Nepeta hederacea Trev. prosp. fl. eug. p. 26.

Figure, - Savi mat. med. veg. tosc. t. 20. Reich. ic. fl. germ. 18. t. 40.

Stazione, Abitazione, Fioritura e Fruttificazione. - Comunissima sotto alle siepi e nei luoghi boschivi ombrosi della Penisola superiore e media: cosi in tutta l'Alta Italia, trovandosi ancora in Valtellina presso Bormio a 1200 metri (Parl. !), e in Tirolo a Hafling presso Merano e a Welsberg (Hausm. $f l$. Tir.), in Liguria, dove si osserva a preferenza nei monti (Ard. fl. Alpes-mar.), in Toscana, dove scarseggia al mare, e s' inalza nei monti a circa 1000 metri!, nelle Marche, nell'Abruzzo (Ten. syll.), a Rona (Webb!), terminando a Napoli (Ten.), dov'è rara (Pasq. $l$. ves.). Manca nelle isole. Toscane, in Corsica è indicata soltanto a Calenzana (Bert.), in Sardegna nasce nei monti fino a circa 1500 metri (Mor. $f$. sard.), in Sicilia rarissima trovasi a Tortorici (Todaro!), a Castelbuono (Minà !), in Valdemone (Guss. $n$. sic. syn.), infine è indicata in Malta (Zer. $\mu$. mel. thes.). Fiorisce in marzo ed aprile, ed anche in maggio. Fruttifica in maggio o giugno.

Distribuzione geografioa. - In tulta Europa, scarseggiando nelle sue parti più nordiche e più meridionali, nell'Asia Minore, in Siberia e fino al Giapjone. 


\section{DRACOCEPIA HUM.}

Dracocephalum et Moldavica Tourn. inst. p. 181, 184. t. 83; 84 . Gen. pl. fl. germ. 6. t. 25, 26.

Dracocephalum Linn. gen. pl. ed. 1. p. 173. Benth. Hook. gen. pl. 2. p. 1199. Ces. Pass. Gib. comp. fl. ital. p. 310. t. 45. f. 5.

Calyx costatus. Corolla manifeste bilabiata, labio superiore porrecto, concavo. Stamina 4 , filamentis apice denticulo auctis, antheris 2-locularibus, loculis longitudinaliter dehiscentibus, confluentibus. Stylus lobis subæqualibus. Cocci apice truncati areola plana terminati.

Portamento. - Sono due erbe perenni, con fusti semplici, alti 2_3 decimetri, con foglie (o i loro lobi) strette lineari, e fiori grandi, turchini, riuniti all'apice dei fusti.

Osservazione. - Oltre alle due specie qui registrate, una terza è stata data come italiana, il Dracocephalum Moldavica Linn., indicata dal Re nel 1824 ( $a d f$. ped. app. 2) nei margini dei campi di Mantoula presso Fenestrelle in Piemonte (Colla herb. ped. 4. p. 459). Nessun altro botanico ha ritrovata questa specie, ch'è dell'Asia centrale, e soltanto avventizia in qualche luogo di Europa.

\section{Dracocephalum Ituysehians.}

D. foliis integerrimis lanceolato-linearibus, bracteis integerrimis. Dracocephalum Ruyschiana Linn. sp. pl. ed. 1. p. 595. Bert. 1. ital. 6. p. 233. Hausm. fl. Tir. p. 684. Ard. fl. Alpes-mar. p. 298. Ces. Pass. Gib. comp. fl. ital. p. 311. Arc. comp. fl. ital. p. 547.

Figure.-Fl. dan. t. 121. Reich. ic. fl. germ. 18. t. 39.f. 2.

Stazione, Abitazione e Fioritura. - Nelle Alpi, rarissima : sulla frontiera francese tra l'Enchastraye e il col della Maddalena nelle Alpi Marittime (Ard.), e alla Combe d'Ambin e al col della Roue verso Bardonecchia nelle Alpi Cozie, secondo Allioni ( $f l$. ped.), e al Cenisio (Bonjean!); quindi in luoghi sassosi del Tirolo meridionale (Sieber n. 184!), e piu precisamente presso Laas nella valle di Matsch e altrove (Hausm.). Fiorisce in luglio e agosto.

Distribuzione geografica. - Nei monti di Europa, in Scandina- 
via, Russia, Ungheria, Germania, Svizzera, Italia, e Francia fino ai Pirenei, nell'Asia centrale e settentrionale, al Giappone.

\section{Dracocephalum austriacum.}

D. foliis plus minus partitis lobis angustissimis, bracteis palmatifidis.

Dracocephalum austriacum Linn. sp. pl. ed. 1. p. 95. Bert. $f$. ital. 6. p. 232. Hausm. fl. Tir. p. 685. Ces. Pass. Gib. comp. fl. ital. p. 311. Arc. comp. fl. ital. p. 547.

Figura. - Reich. ic. fl. germ. 18. t. 39. f. 3.

Stazione, Abitazione e Fioritura. - Nelle praterie delle Alpi, rarissima: al Cenisio (Huguenin!); in Tirolo in val Venosta presso Tschengels (Bert.), fra Tschengels e Prad sotto la cappella (Hausm.). Fiorisce da giugno ad agosto.

Distribuzione geografica. - Nei Pirenei, nelle Alpi, nei Carpazi, nel Caucaso.

\section{SOTHOFAMICHAA SECONDA.}

\section{TEUCR INEE.}

TEuCrineæ.

Gemmularium breviter nec ultra medium 4-lobum. Gemmulæ a medio et ultra loculorum insertæ, hemianatropæ vel fere atropæ. Fructus cocci areola insertionis magna, laterali, a basi versus medium cocci extensa. Embryo cauliculo brevi, recto.

\section{xxviH. HOSHARENG.}

Piosmarinus Tourn. inst. p. 195. t. 92. Mirb. in ann. mus. 15. t. 2.

f. 10. Gen. pl. Al. germ. 6. t. 9. Benth. Hook. gen. pl. 2. p. 1197. Ces. Pass. Gib. comp. f. ital. p. 308. t. 45. f. 3.

Calyx campanulatus, obsolete nervatus. Corolla manifeste 2-labiata, labio superiore erecto demum patulo, concaviusculo, caduca. 
Stamina 2 (inferiora), conniventia, filamentis infra medium denticulo reflexo auctis, antheris 1-locularibus, loculo longitudinaliter dehiscente. Stylus lobis valde inæqualibus. Cocci apice convexi leves.

Portamento. - Il Ramerino è un frutice ramosissimo, con rami drizzati in su, alto un par di metri, sempreverde, gremito di foglie strettissime lineari, coi margini rivoltati, glabre e verdiscure di sopra, pelose biancheggianti di sotto. I fiori sono di mezzana gran. dezza, cerulei, in piccoli grappoli ascellari, nudi alla base o fogliati, e sono voltati da un medesimo lato, e accompagnati da piccole brattee.

Osservazione. - Il filamento degli stami porta verso basso un dentino rivolto in giù, al quale si è voluto dare il valore morfologico della seconda casella anterale delle Salvie, genere cui il Ramerino è stato ravvicinato per questo motivo, e perchè gli stami sono ridotti a 2; ma nelle Salvie il connettivo è articolato col filamento, adunque l'analogia non è evidente, e, salvo nuovi studi, è cosa piủ semplice ritenere il dentino del Ramerino simile a quello che si ha nel filamento di molte altre Lamiacee.

\section{IDosmarinus officinalis.}

Rosmarinus officinalis Linn. sp. pl. ed. 1. p. 23. Bert. fl. ital. 1. p. 133 ; et auct. omn.

Figure, - Fl. grcec. t. 14. Reich. ic. fl. germ. 18. t. 43.

Stazione, Abitazione e Fioritura. - Nei luoghi asciutti sul mare: da Nizza (Parl.!) a Mentone, comunissimo (Ard. fl. Alpesmar.), a Capo di Noli, a Sestri Ponente, nell'isole Palmaria (De Not. rep. fl. lig.) e Tino (Ad. Targioni!) del golfo della Spezia, qua e là nella marina Lucchese, e nella bassa valle del Serchio (Bicch. agg. fl. lucch.), comune nelle macchie della Maremma toscana da Castiglione della Pescaia al monte Argentaro!, e nelle isole di Gorgona (Lena!), di Capraia (Mor. e De Not. fl.capr.), dell'E!ba!, di Montecristo (Watson Taylor!) e di Giannutri!, comune nel littorale Romano (Seb. Maur. fl. rom. prodr.), per esempio ad Ostia (Bert.), intorno Gaeta a Montespaccato, Sperlonga, Traetto, sopra Caserta a Valvori nell' andare a S. Biagio Saracinesco (Terr. sec. e quart. rel. Terra di Lav.), a Napoli al Fusaro (Ten. syll.), nelle isole d'Ischia (Bolle!) sugli scogli in molti luoghi (Guss. enum. pl. Inar.), e di Capraia (Pasq. fl. ves.), comune nelle macchie d'Otranto (Groves 
contr. fl. Terr. d'Otr.), al Gargano verso Manfredonia, Matinata (Pasq. Lic. viagg. Garg.) e S. Angelo, nel littorale Abruzzese al Mattone di Pettorano (Ten.), e su in fondo all'Adriatico nel Lido veneto al Cavallino (Parl.!), presso Duino dov'è raro (Pir. fl. for. syll.), e presso Fiume (Poll. fl. ver.); in Corsica, comune nei terreni calcari, piủ raro nei silicei, cosi a Bastia, S. Fiorenzo (Mars. cat. pl. Cors.), Calvi (Bert.), Corte, Bonifacio ecc. (Mars.), in Sardegna verso Ovadda (Gennari!) e Laconi (Reinhardt!), a lersu, Perdos de fogu, S. Antioco, Porto Scuso, nelle isole S. Pietro, Tavolara (Mor. fl. sard.) e Maddalena (Genn. fl. Capr.), in Sicilia a Messina (Seguenza!), Castelbuono (Minà), Palermo (Parl.!), Marsala, Maretimo (Guss. fl. sic. syn.), Terranova, Vittoria (Aiuti!), a quanto sembra comune (Guss.), e in Pantellaria (Calcara!), Malta a Wied Babu (Duthie!) e altrove (Grech Del. $f$. mel.), e Larnpedusa (Calcara!). Lontano dal mare fa sulle rupi del lago di Garda (Porta!), abbondante presso Limone e Monte Castello (Poll.), quasi spontaneo sul lago d'Iseo presso Sarnico e Predore (Rota prosp. fl. Berg.), presso Susa a Mom. pantero (Aicti!). Fiorisce pressochè tutto l'anno.

Distribu zione geografica. - Nell' Europa mediterranea, nell'Asia Minore, nell'Affrica settentrionale.

\section{IXIX. TEUCMUUM.}

Chamædrys, Polium, Teucrium Tourn. inst.p.201-207. t.97, 98. Teucrium Linn. gen. pl. ed. 1. p. 167. Mirb. in ann. mus. 15. t. 15. f. 11-18. Benth. Hook. gen. plant. 2. p. 1221. Ces. Pass. Gib. comp. fl. ilal. p. 323. t. 48. f. 3.

Teucrium et Scorodonia Gen. pl. fl. germ. 6. t. $45,46$.

Calyx campanulatus vel tubuloso-campanulatus, obsolete nervatus. Corolla ut videtur 1-labiata, decidua. Stamina 4, conniventia, filamentis subedentulis, antheris 2-locularibus, loculis longitudinaliter dehiscentibus, confluentibus. Stylus lobis subrqualibus. Cocci apice convexi.

Portamento. - Erbe per lo piủ perenui, o suffrutici o frutici, assai ramosi e cespuglinti, per lo più variamente pelosi, molto amari, con foglic mezzane o piccole, intere o divise, con fiori mezzani o piccoli, rossi, turchini, gialli o bianchi, opposti o in falsi verticilli 
di 3 a 6 , ora disposti lungo $i$ fusti, ora in grappoli fogliati o bratteati, diradati 0 addensati.

Osservazione. - Il paragone della corolla in questo genere, chc l'ha apparentemente unilabiata con 5 lobi, con quella del vicino genere Aiuga, che l'ha evidentemente bilabiata, col labbro inferiore con 3 lobi, mostra quanto bene si fosse apposto Koch (Syn. fl. germ. ed. 2) quando chiari la corolla bilabiata anche nel genere Teucrium, col labbro superiore diviso fino alla base e perciò i suoi due lobi resi laterali da sembrare appartenere al labbro inferiore.

\section{Teucrium siculum.}

T. perenne, foliis ovato-ellipticis, crenatis, plus minus pilosis, floribus oppositis, in racemos elongatos paniculatos laxiusculos parvibracteatos (bracteis rotundatis subcordatis) dispositis, calyce bilabiato, late campanulato supra basin constricto, tubum corollinum subæquante, coccis lævibus glabris.

Scorodonia sicula Raf. specch.

Teucrium Scorodonia var. crenatifolium Guss. fl. sic. prodr. suppl. Fl. sic. syn. 2. p. 55. Ces. Pass. Gib. comp. fl. ital. p. 324. Arc.! comp. fl. ital. p. 558.

Teucrium Sçorodonia (ex locis) Seb. Maur. fl. rom. prodr. p. 189 (ex parte?). Ten. fl. nap. 2. p. 8. Syll. fl. neap. p. 276 (ex parte?). Bert. fl. ital. 6. p. 22 (ex parte). Terr. fl. Vult. syn. p. 140. Arc.! l. c. (var. $\beta$ ex parte).

Teucrium siculum Guss. fl. sic. syn. 2. p. 837. Enum. plant. Inarim. p. 266. Terr.! quarta rel. Terra di Lav. p. 114.

Teucrium Pseudoscorodonia Ces. Pass. Gib. l. c. (non Desf.!). Arc. l. $c$.

Teucrium Gasparrinii Nym. consp. fl. eur. p. 564 .

Stazione, Abitazione e Fioritura. - Nei luoghi boschivi dell'Italia meridionale. Comparisce nel Piceno, dov'è stato trovato a Montefortino (Marzialetti!), presso Trisungo, presso Umito (Parl.!), e senza dubbio nell'Abruzzo, e nasce da quel lato anche nel Gar. gano a S. Nicandro e Vico (Porta e Rigo!); dall' altro lato della Penisola comparisce nel Romano, dov'è stato trovato a Frascati (Webb!), e piủ giủ nasce in Terra di Lavoro a Spigno al Montone, e a Frasso Telesino sul monte S. Michele (Terr.), a Cusano presso il Matese (Avellino!), nelle vicinanze di Napoli (Ces. Pass. Gib.), in Ischia nei 
monti Montagnone (Bolle!) e Vezza, e dal Bagno a S. Nicola (Guss.), a Muro in Basilicata (Terr.!), in Calabria a Nicastro (Ricca!), presso) S. Eufemia d'Aspromonte e presso Cerasia sopra Palizzi (Arc.!); quindi in Sicilia a Messina al Campó (Nicotra!), a Capo di Orlando, presso Mistretta (Guss.), intorno a Castelbuono (Parl.!), nelle Madonie (Guss.), a Busambra, Ficuzza (Parl.!), Pizzuta, Monreale ecc. (Guss.). Fiorisce in maggio e giugno.

Distribuzione geografica. - Nasce oltracciỏ nei Pirenei (Franqueville!).

Osservazioni. - Questa specie sostituisce nel mezzogiorno il Teucrium Scorodonia, da cui non differisce che poco per le brattee, ma principalmente per il calice più grande (lungo $7-8$ millimetri, nel mentre che quello del $T$. Scorodonia non ha che $1 / 2$ centimetro), più decisamente spinoso, e di forma alquanto diversa: non la tengo separata che per difetto di saggi intermedi.

I fiori, d' ordinario giallorini, possono essere anche bianchi, o rossigni.

Rassomiglia molto al T. Pseudoscorodonia Desf., che differisce per essere un suffrutice, e per avere le foglie molto più pelose, e le brattec più strette.

\section{Tencrimu Sconodonis.}

- T. perenne, foliis ovato-ellipticis, crenatis, plus minus pilosis, floribus oppositis, in racemos elongatos paniculatos laxiusculos parvibracteatos (bracteis ellipticis acuminatis) dispositis, calyce bilabiato, campanulato medio constricto, tubo corollino plus minus breviore, coccis lævibus glabris.

Teucrium Scorodonia Linn. sp. pl.ed. 1. p. 564. Bert. /l. ital. 6.p. 22 (ex parte). Ces. Pass. Gib. comp. fl. ital.p. 324 (excl.var. $\gamma$ ). Arc. comp. 月. ital. p. 558 (excl. var. $\gamma$, et var. $\beta$ ex parle); et auct. omn. florarum dimidii superioris Italice.

Teucrium eugancum De Vis. in T'rev. prosp. /l. eug. p. 26.

Figure. - Fl. dan. 1. 485. Reich. ic. 1. germ. 18. 1. 36. 1. 2.

Stazione, Abitazione e Fioritura. - Per i luoghi boschivi, specialmente di collina e li montagna, della metà superiore della Penisola, distribuito molto disugualmente: cosi in Piemonte, dov'é frequente (All. $l$. ped.), per esempio ai bagni di Valdieri a 11.40 metri (Parl. 1), tra Chiusa e S. Michele (Re /.seg.), mei dintorni di Torino 
(Re fl. tor.), a Biella (Rampoldi!), a Riva di Valsesia (Carestia!), nel Novarese comunissimo (Bir. fl. acon.); in Lombardia presso Milano (Rampoldi! ecc.), presso Como, comune (Com. fl. com.), nel distretto di Chiavenna e nella Valtellina inferiore appena oltre $\mathbf{i}$ castagni (Anzi auct. fl. nov.-com.), per esempio a Monastero, Regoledo e Berbenno (Mass. prodr. fl. valt.), comunissimo nel Bergamasco fino a 1000 metri (Rota prosp. fl. Berg.), e nel Bresciano, per esempio a Sonico, e presso Collio tra 800 e 900 metri (Parl.!); in Tirolo a Roveredo, a Riccomassimo nelle Giudicarie (Hausm. fl. Tir.), in Valsugana (Kellner!); nel Vicentino presso Recoaro e Schio (Poll. fl. ver.), negli Euganei, comune la var. di fiore rosseggiante detta Teucrium euganeum (Rigo! ecc.); sul versante nord dell' Appennino verso la Porretta (Gennari!) dov'è comune (G. Bert. veg. Porr.), all'Acero, al lago Scaffaiolo, a Fiumalbo, al Ventasso, nella valle di Riarbero, nell'alpe di Succiso (Gib. Pir. fl. Mod.) ecc., nel Pavese (Rota prosp. piant. Pav.); in Liguria qua e là verso Nizza, e Mentone (Ard. fl. Alpes-mar.), a Dolcedo (Berti!), Oneglia (Ricca!), Ellera (Piccone!), nella valle di Polcevera (Carrega!), a Chiavari (Delpino!), intorno alla Spezia!; in Toscana comunissimo, per esempio in Lunigiana da Sarzana (Bert.) a Pontremoli, al Gotro e a Fivizzano!, in molti luoghi delle Alpi Apuane (Simi! ecc.), in val di Serchio da Lucca (Puccinelli! ecc.) all'alpe di Mommio, nel val d'Arno da Fucecchio a Firenze!, e nei suoi monti sino al Teso sopra Pistoia (Delpino!), a monte Senario in Mugello (Parl.!), Vallombrosa!, e l'Appennino casentinese (Parl.!), nel Senese!, a Monterotondo (Bert.) e al'Poggio di Montieri (Amidei!) nel Massetano. Delle isole le sole dove si trovi sono l'Elba, a Marciana!, e la Corsica, nella regione media (Mars. cat. pl. Cors.), per esempio a Bastia (erb. Webb!) e nella valle di Bastelica (Requien). Fiorisce in giugno e luglio, o in luglio e agosto secondo i luoghi.

Distribuzione geografica. - Nell' Europa occidentale, eccetto la piủ nordica.

Descrizione. - Pianta perenne, alta da 3 a 5 decimetri, verde, pubescente, fornita di un rizoma duro, legnosetto, che manda in giủ fibre radicali delicate, fibrillose e degli stoloni corti, bianchicci. I rami o fusti sono eretti o ascendenti, erbacei, semplici o ramosi, con pochi rami fioriferi in alto, quadrangolari, con gli angoli ottusetti, sfumati di rossiccio in basso, verdognoli in alto, pubescenti per peli lunghetti, fitti e bianchi per cui i fusti sono un po' biancheggianti. Le foglie sono opposte, alquanto lontane, patenti, molli, rugose, 
scavate in cuore ed ovate, ottuse, smerlate, con gli smerli alquanto disuguali, verdi di sopra ed opache ed ivi con molti solchi in rete a piccole maglie, in mezzo alle quali sporge il parenchima che fa rugose le foglie stesse, di un verde più chiaro di sotto ed ivi con un nervo longitudinale da cui parton dei nervetti laterali che si diramano per fare una rete fitta e rilevata nella pagina inferiore, tra le maglie della quale corrispondono tante piccole fossette: sono fornite di un picciòlo corto, scanalato di sopra, convesso di sotto, verdognolo, e pubescente con i peli lunghi e bianchi, massime di sopra. I fiori sono molti, piuttosto vicini, in racemi lunghi e unilaterali all' apice del fusto e dei rami che vengono dall' ascella delle foglie superiori. Le bratteole sono piccole, quasi uguali ai pedicelli, patenti, ovate, acuminate, verdi, pubescenti. I pedicelli sono corti, cilindrici, anch'essi verdi e pubescenti, patenti e poi un po' cernui. Il calice è quasi campanulato, verdognolo, pubescente, un po'curvo di sopra, con un gobbo di sotto verso la base, con cinque denti, dei quali quattro piủ corti, quasi uguali, triangolari-acuminati, un po' curvati in su, e il quinto ch'è superiore piủ largo, quasi orbicolare, con una piccola punta all' apice, 'curvato in su e in dietro, con nervi ramosi che fanno come una rete venosa in essa: ha in alto dei piccoli peli glandolosi all'apice; la gola ha pochi peli lunghi dalla parte che corrisponde sotto al dente superiore. La corolla è di un bianco-gialliccio, sfumato in alcune parti di verdognolo, pubescente di fuori. Il tubo è il doppio piủ lungo del calice, un po' curvo alla base, quasi diritto nel resto, appena più largo verso alto. Il labbro superiore è corto, bifido, con le lacinie portate in avanti e saldate con il labbro inferiore in modo che paiono due lobi di questo, concave, ottuse, il labbro inferiore è grande, poco più corto del tubo, pendentc, avvivicinato alquanto a questo, trilobo, con i lobi laterali diretti in avanti c un po' convergenti, ovati, acuti, con una leggiera carena ncl dorso, il lobo di mezzo molto più grande è concavo in avanti, ovale-orbicolare, quasi intero o con qualche piccolissimo smerlo. Gli stami sono didinami, piủ lunghi il doppio del labbro superiore, sporgono dalla divisione di questo e sono quasi eretti. I filamenti sono quasi lesiniformi, papillosi in bayo, pelosi, con peli lunghi e bianchi nol mezzo, e in quelli degli stami più lunghi anche verso alto, rossicci 0 in parte bianchicci. Le antere sono bislunghe, un po'schiacciate lateralmente, biloculari, con le logge aprentiși per una fessura comune, di un rosso-gialliccio. Il polline è roseo-rossiccio. Il pistillo é poco piủ lungo degli stami più lunghi. L'ovario è diviso in quattro lobi, 
quasi obovati, tondeggianti all' apice, verdognoli, papillosi ; il disco è di un verdognolo-bianchiccio, poco.alto. Lo stilo è lungo, filiforme, glabro, bianchiccio. Lo stimma è bifido, con le lacinie corte, quasi uguali, acute, divergenti, verdognole. (Parl. ms., descr. della pianta del Casentino in Toscana).

Osservazione. - La corolla varia per la lunghezza del tubo, ch'è talora uguale al calice, ma piủ spesso il doppio piủ lungo.

\section{Teucrium massiliense.}

T. perenne, foliis ovato-lanceolatis, crenatis, rugosissimis utrinque minute molliter cano-pilosis, floribus oppositis, in racemos elongatos paniculatos laxiusculos parvibracteatos (bracteis ellipticis acuminatis) dispositis, calyce bilabiato, campanulato medio constricto, tubum corollinum æquante, coccis lævibus, glabris.

Teucrium massiliense Linn. sp. pl. ed. 2. p. 789. Bert. fl. ital. 6. p. 25. Gren. Godr. fl. Fr. 2. p. 710. Mor.! fl. sard. 3. p. 338. Mars. cat. pl. Cors. p. 115. Ces. Pass. Gib. comp. fl. ilal. p. 324. Arc. comp. fl. ital. p. 558.

Figura. - Ger. fl. gallo-prov. $t .11$.

Stazione, Abitazione e Fioritura. - In Corsica e in Sardegna, lungo i ruscelli, sul margine dei boschi ecc. Linneo, e altri copian. dolo, l'han dato di Villafranca (presso Nizza), ma i moderni non ve l'hanno trovato. In Corsica a Calvi (Soleirol!), Sartene (Jordan!), Patriciale, Galeria (Bert.), Prunelli, Vico, Bonifacio (Gren. Godr.), nelle valli di Porto, di Sagone e del Liamone, ad Afa a piè delle rupi di Gozzo, ad Aiaccio sulla cresta dietro la cappella S. Giuseppe, al col S. Rocco sopra Vico, a Portovecchio, al capo Corso a Ersa e nella strada di Rogliano (Mars.). In Sardegna, abbastanza frequente (Mor.), cosi a Tempio (Bert.), sopra la miniera d'Ingurtosu in quel d'Iglesias (Ascherson!), a Malganai, nei monti di Arizzo (Gennari!), nelle isole La Maddalena e Asinara (Mor.). Fiorisce da maggio a luglio secondo i luoghi.

Distribuzione geografica. - Si trova inoltre nella Spagna e la Francia meridionali, e in Creta.

Osservazione. - Per quanto assai vicina per i caratteri alle due specie precedenti, questa qui ha un aspetto tutto proprio, dovuto principalmente alle foglie piccole e molto rugose. I fiori sono rossicci, e tutta"la pianta ha odore grato. 


\section{Temcrinun spinosum.}

T. annuum, foliis oblongis, superne præsertim inciso-dentatis aut integris, pubescentibus, floribus oppositis vel false verticillatis, in racemos elongatos ample paniculatos laxos parvifoliatos dispositis, calyce bilabiato, tubuloso-campanulato, tubum corollinum excedente, coccis lævibus, minute glandulosis.

Teucrium spinosum Linn. sp. pl.ed. 1. p. 566. Zer. fl. mel. thes. p. 73. Bert. fl. ital. 6. p. 41 . Grech Del. f. mel. p. 26. Mor.! fl. sard. 3. p. 337. Ces. Pass. Gib. comp. fl. ital. p. 324. Arc. comp. fl. ital. p. 558.

Figure. - Cav. ic. et descr. t. 31. Fl. grace. t. 539.

Stazione, Abitazione e Fioritura. - Nei prati asciutti della Penisola piủ meridionale a Conversano e a Lecce (Ten. syll.); nei colli aridi di Sardegna (Mor.); in Sicilia nei campi umidi argillosi di Villafrati, Trapani (Todaro!), Alcamo, Nicosia, Gangi, Lentini, Mineo nel piano, Pietrapersia, Canicatti, Caltanisetta, Delia, Cata. nia, Fondaco nuovo presso Termini, Piazza, Terranova, Alicata, Castrogiovanni nel piano, Alimena, Caltauturo (Guss. syn.); in Mlalta nei colli (Zer.) ad Aalia (Grech). Fiorisce da giugno ad agosto.

Distribuzione geografica. - Nelle isole Cianarie, in Barberia, nella penisola Iberica, nell' Italia meridionale, nell'Asia mediterranea.

Osservazione. - Specie subito riconoscibile nel genere per i suoi rami spinescenti.

\section{Teucrium Mavum.}

T. fruticosum, foliis ovatis, crenatis, velutinis, floribus false verticillatis, in racemos elongatos laxiusculos bracteatos (bracteis ova. to-lanceolatis) dispositis, calyce subregulari, campanulato, tubum corollinum aquante, coccis rugulosis, glabris.

Teucrium flavum Linn. sp. pl. ed. 1. p. 565. Zer. /l. mel. thes. p. 73. Pucc. syn. pl. luc. p. 298. De Not.! rep. 月. lig. p. 930. Bert. 1l. ilal.6. p. 31. Gren. Ciodr. /l. Fr. 2. p. 7/I l'ar. prodr. 1. tosc. p. 526. Giuss. enum. pl. Inarim. p. 265. Grech Lel.! П. mel. p. 27. Mor.! H. sard. 3. p. 334. Pir. Al. For. syll. p. 114. Tussi R. prov. sen 
p. 51. Ard. cat. pl. Ment. p. 29. Car. fl. Montecr. p. 28. Ard. fl. Alpes-mar. p. 305. De Vis. Sacc. cat. piant. Ven. p. 141. Mars. cat. pl. Cors. p. 116. Terr.! sec. rel. Terra di Lav. p. 96. Ces. el. piant. Maiell. p. 23. Archb. fl. Alto-Serch. p. 61. Ces. Pass. Gib. comp. fl. ital. p. 321. Groves contr. fl. Terra d'Otr. p. 66. Terr. quarta rel. Terra di Lav. p. 114. Loj. is. eol. p. 124. Cald. fl. fav. tent. p. 184. Arc.! comp. fl. ital. p. 558.

Figure. - Fl. grcec. t. 533. Reich. ic. fl. germ. 18.t. 35. f. 2.

Stazione, Abitazione e Fioritura. - Nei luoghi aridi sassosi sul mare, o non molto discosti, raramente in maggiore lontananza da esso: a Nizza (Webb! ecc.), Villafranca (Bert.), Mentone (Ard.), Capo di Noli (De Not.! ecc.), Portofino, Rapallo, Zoagli, al golfo della Spezia, e nelle sue isole Palmaria, Tino e Tinetto, a Massa (Bert.), nel monte Pisano fra Asciano ed Agnano (P. Sav.!), e a Gattaiola (Bicchi!) e Pozzuoli (Calandrini!), quindi su per val di Serchio a Sesto (Pucc.), a Vinchiana (Calandrini!) e tra Diecimo e Borgo a Mozzano (Giannini!), tra Livorno e Cecina (Parl.!), a Campiglia (Bert.), nel monte Argentaro (Santi viagg.), nel monte Amiata (Bert.), nelle isole dell' Elba a Bagnaia! e al Volterraio (Cald.!), di Montecristo (Bert.), di Giannutri! e nell' isolotto di Port'Ercole (Aivti!), a Terni (Groves!) e Nerina presso Visso (Ricci!) in Umbria, a Roma (Fiorini! ecc.), a Terracina (Bert.), Arpiro (Terr.!), Sca:oli, Traetto, Castelvolturno, Piedimonte (Terr.), al Matese e monte Vergine (Ten. syll.), nei monti di Castellamare (Avellino! ecc.), in Ischia (Bolle! ecc.) e Capri (Pasq. $\pi$. ves.), in Calabria a Pizzo (Ricca!), Palme, Stilo (Arc.!), a Otranto (Groves), al Gargano (Ten.), a Caramanico (Pedicino!), al monte di S. Marco sopra Ascoli!, nelle Marche al monte S. Vicino (Bucci!), ad Ancona (Paolucci!), Arcevia, e al Furlo (Bert.), presso Faenza al monte Mauro (Cald. ecc.), negli Euganei a Torreglia, a Monfalcone (Bert.), Duino, Gorizia (Pir. ecc.), Trieste, Grignano (Bert.), Fiume (Noé!); in Corsica a Bastia!, S. Fiorenzo (Mars.), Calvi (Soleirol!), Calenzana (Bert.), Aiaccio (erb. Webb!), Sartena, Bonifacio (Gren. Godr.), Pontenovo, Curzo ecc. (Mars.); in Sardegna, per esempio a Sassari (Marcucci!); nell'isolé Salina (Loj.) e Lipari (Guss. syn.) delle Eolie, in Sicilia a Messina!, Noara (Munafỏ!), Cefalù, nelle Madonie (Guss.), nei monti Palermitani (Parl.!), a Marsala (Todaro!), in val di Mazzara (Guss.), a Castrogiovanni (Heldreich!), in val di Noto (Guss.), ad Avola (Bianca!), Lentini (Guss.), Catania (Parl.!), Nicolosi ecc., in Ustica, nelle Egadi (Guss.), in Malta (Grech! ecc.), in Lampedusa (Calcara!). Fiorisce 
da aprile e maggio a giugno nelle parti piủ meridionali, e in giugno e luglio nelle piủ settentrionali.

Distribuzione geografica. - Nell' Europa meridionale, e nell'Affrica settentrionale.

\section{G. Teucrium Incidums.}

T. perenne, foliis ovatis, inciso-dentatis, glaberrimis, floribus false verticillatis secus ramos parvifoliatos, calyce regulari, campa. nulato, tubum corollinum æquante, coccis lævibus, glabris.

Teucriun lucidum Linn. sp. pl.ed.2.p.790. Bert.fl.ilal.6.p. 28. De Not. rep. fl. lig. p. 330. Ard.! cat. pl. Ment. p. 29. Fl. Alpes-mar. p. 305. Ces. Pass. Gib. comp. fl. ital. p. 325. Ing. cat. sp. Mond. p. 69. Arc. comp. fl. ital. p. 559.

Figure. - Magn. hort. reg. monsp. p. 52. Reich. ic. fl. germ. 18. t. 38. f. 5 .

Stazione, Abitazione e Fioritura. - Nei luoghi sassosi dei monti piủ occidentali della Penisola : in quei di Savona, e d'Albenga (I) Not.) nel monte S. Callocero (Bert.), nei boschi di Garessio, dov' ̀̇ comune (Ing.), alla Certosa di Pesio sopra Cuneo (Bert.), sopra Mentone a Castiglione a 700 metri (Ard.!), a Sospello, a Lucerame (All. fl. ped.), a Utelle, e in generale fino a Duranus, S. Salvatore (Ard.), S. Martino Lantosca (Burnat!), Tenda (Bourgeau! ecc.) e Limone (Parl.! ecc.); quindi al Gran S. Bernardo secondo Allioni ( $\mu$. ped.), e secondo Biroli ( $f$. acon.) in valle di Vegezzo, soprattutto alle Vallette, e alle radici dei monti di Crodo. Fiorisce in luglio e agosto.

Distribuzione geografica. - Trovasi inoltre nei monti contermini di Francia, in Spagna, e in Barberia.

Descrizione. - Glabro, verde chiaro, in parte violetto. Fusti quasi frutescenti alla base, ivi ramosi e come i rami ascendenti o eretti, quadrangolari, violetti o in parte verdognoli. Foglie patenti, verdi e un po'lucenti di sopra, leggieri solchi che corrispondono ai nervi di sotto, verde più chiaro di sotto, nervo longitudinale pọco sporgente, nervetti laterali idem, ovate, romboidali, intere in basso, marginatodentate con $\mathrm{i}$ denti disuguali, ottusi, cigliolato-scabri. Fiori due o tre all' ascella delle foglie superiori e delle fọglie fiorali, in verticilli percio di 4 a 6 fiori, in un lungo racemo, assoltigliato in alto, e fogliato. Foglic fiorali inferiori simili a quelle dei fusti, piủ lunghe dei 
fiori, poi gradatamente piủ piccole, meno dentate e infine intere, ovali o ovali-bislunghe, e più corte dei fiori. Pedicelli delicati, piủ corti o quasi uguali al calice, violetto-rossicci, puberuli, e con piccole glandolette sessili, tonde. Calice quasi campanulato, rossiccio, puberulo o quasi glabro e con tante piccole glandolette tonde e sessili e bianchicce, un po' glabro alla base in avanti, con dieci nervi concolori, e con cinque denti più corti del tubo, triangolari-lanceolati, acuti. Corolla più piccola e piủ stretta del Teucrium Chamcedrys, più del doppio più lunga del calice, pubescente di fuori, tuho più lungo del calice, roseo-bianchiccio, labbro superiore profondamente diviso in due lacinie le quali fanno parte del labbro inferiore in modo che questo comparisce con cinque lobi, due appartenenti al labbro superiore che sono obliquamente lanceolato-lineari, ottusi, di un roseo-chiaro, cigliati nei margini, e tre spettanti al labbro inferiore medesimo dei quali due laterali più piccoli e uno intermedio molto più grande, quasi orbicolare, concavo di sopra, roseo come i due lobi laterali, questo labbro ha dei peli quasi una barba rosea verso la base. Gli stami sono quattro didinami, eretti, lontani dal labbro inferiore, e più corti di questo, sporgenti in mezzo alla fessura del superiore. I filamenti sono grossetti, cilindrici, un po'curvati in avanti verso l'apice, violetti, glabri, inseriti in alto del tubo. Le antere piccole, schiacciate lateralmente, violetto-scure, glabre. Polline bianchiccio. Pistillo in mezzo ai quattro stami. Ovario piccolo, quasi tondo, oscuramente quadrilobo, verdognolo, glabro; disco piccolo, gialliccio. Stilo lungo, un po'curvo in avanti verso alto, cilindrico, violettoroseo in alto, glabro. Stimma bifido, lacinie poco disuguali, corte, divergenti, lesiniformi, del colore dello stilo. (Parl. ms., descr. della pianta di Limone).

\section{\%. Teucrium Chamredrys.}

T. perenne, foliis ovalibus, grosse dentatis, plus minusve pilosis, floribus false verticillatis secus ramos parvifoliatos, calyce regulari, campanulato, tubum corollinum æquante, coccis rugosulis, glabris.

Teucrium Chamædrys Linn.sp.pl.ed.1.p.565; et auct. omn.

Figure. - Sav. mat. med. veg. tosc. t. 8. Reich. ic. fl. germ. 18. t. 38. f. 4.

Stazione, Abitazione e Fioritura. - Per tutta Italia, comu- 
nissimo nei luoghi boschivi asciutti, nelle prode dei campi ecc., più specialmente in collina e in montagna. Nelle Alpi trovasi fin nella regione degli abeti sul monte Cramont (Parl.!), e nel monte Ritten in Tirolo fino a 1300 metri (Hausm. fl. Tir.), negli Appennini sale in Toscana fin'oltre i 1500 metri. Sembra meno comune nelle grandi isole, ed è escluso da molte delle minori, non essendo stato trovato che nell'Elba!, in Ischia (Guss. en. pl. inarim.), in Capri (Pasq. $f$. ves.), e in Malta (Zer., Grech). Fiorisce da maggio e giugno a luglio e agosto.

Distribuzione geografica. - Nell' Europa media e meridionale e nell'Asia occidentale fino al Caspio; nell'Affrica settentrionale (Boiss. 1. or.).

Descrizione. - Pianta perenne, alta da uno a due decimetri e mezzo, di un verde chiaro, pubescente, fornita di un rizoma molto lungo, orizzontale, piuttosto delicato, che manda molte fibrille radicali scure e quasi nericce e in alto molti fusti ossia rami, i quali sono dapprima sdraiati, poi ascendenti, un po' legnosi alla basc: fanno dei cespuglietti assai graziosi quando sono tutti fioriti; tali rami sono semplici o con pochi rami corti, eretto-patenti, anch'essi fioriferi, che nascono dall'ascella delle foglie della base del racemo, ottusamente quadrangolari, rossicci in basso, verdognolo-biancheggianti in alto, pubescenti quasi pelosi, con i peli lunghetti, orizzontali, bianchi. Le foglie sono opposte, alquanto lontane tra loro, patenti, piuttosto molli, cuneato-ovali, inciso-crenate, ristrette alla base quasi in un corto picciòlo, di un verde piuttosto chiaro di sopra, non lucenti! ed ivi con leggieri solchi che corrispondono ai nervi di sotto, di un verde chiaro di sotto ed ivi con un nervo longitudinale rilevato, di colore più chiaro, dal quale partono dei nervetti laterali che si diramano per mandare un rametto ad ogni smerlo della foglia: sono pubescenti in ambedue le pagine e più nella inferiore. Le foglie fiorali sono gradatamente più piccole, cuneato-ovali, meno incise e via via soltanto smerlate verso l'apice. I fiori sono a due a due o a tre a tre all' ascella delle foglie della parte superiore dei rami; tutti fanno un racemo bislungo, piuttosto fitto, fogliato, e sono rivolti da una parte sola in avanti, gl'inferiori sono più corti o quasi uguali alle foglie fiorali corrispondenti, i superiori piủ lunghi di queste: hanno un pedicello poco piu corto del calice, ascendente, spesso rossiccio e pu. bescente. Il calice è tubuloso-campanulato, un po'gobbo in avanti verso la base, con cinque angoli poco sporgenti, rossiccio, con molte piccole glandolette tonde e bianchicce; ha pochi peli nel tubo, e 
cinque denti poco disuguali, più della metà più corti del tubu, eretti, quasi triangolari-acuminati, con una leggiera carena nel dorso ch'è la continuazione dell' angolo corrispondente del tubo, forniti di ciglia lunghe e rade. La corolla è circa tre volte più lunga del calice, di color violetto-chiaro e quasi roseo, talvolta di un roseo bianchiccio; il tubo è più lungo del calice, grossotto, quasi cilindrico, un po'an. golato, un po'curvo, di color più chiaro del lembo della corolla, glabro di fuori con poche glandolette in alto come quelle del calice, peloso di dentro! verso il mezzo, con peli radi, piuttosto corti e bianchi. Il labbro superiore è corto, profondamente diviso in due lacinie, poste lateralmente, dirette in su, avvicinate tra loro in modo che si toccano, lanceolato-acuminate, peloso-cigliate; il labbro inferiore è grande, patente, trilobo, con i lobi laterali piccoli, diretti in su e un po'in fuori, ovali-acuti, e il lobo di mezzo grande, obovatocuneiforme, concavo di sopra quasi a guisa di un cucchiaio, irregolarmente dentellato-crenulato nell' apice: tale labbro è glabro, eccetto tra i lobi laterali e il lobo di mezzo dove ha dei peli lunghi e bianchi. Gli stami sono quattro, didinami, sporgenti in mezzo alla divisione del labbro superiore e piủ lunghi delle lacinie di questo, eretti, un po'curvi verso alto e in avanti. I filamenti sono quasi lesiniformi, un po'schiacciati in basso, ivi puberuli, con qualche pelo lunghetto verso la metà loro, di color violetto-rossiccio. Le antere sono bislunghe, violetto-rossicce, glabre, con qualche glandoletta piccolissima e bianca nel punto che corrisponde all' apice del filamento, con due logge poste l'una all'estremità dell' altra e aprentisi per unà fessura longitudinale comune. Il pistillo è poco più lungo de. gli stami più lunghi. L'ovario è quadrilobo, con i lobi ovali-tondi, verdognoli, glabri, forniti di piccole glandolette bianche a guisa di papille verso il margine. Il disco è piccolo, gialliccio. Lo stilo è lungo, filiforme, eretto, glabro, del colore dei filamenti. Lo stimma è bifido, con le lacinie poco disuguali, corte, divergenti. (Parl. ms., descr. della pianta del Casentino in Toscana).

Usi. - Questa pianta è un medicinale popolare, noto per le sue virtủ febbrifughe.

\section{8. 'Teucrium Scordiums.}

T. perenne, foliis ovato-ellipticis aut elliptico-oblongis, grosse dentatis, pubescentibus villosis aut lanuginosis, floribus subopposi- 
tis vel false verticillatis secus ramos foliatos, calyce regulari, campanulato, tuhum corollinum æquante, coccis rugulosis, glabris.

Teucrium Scordium Linn.sp.pl. ed. 1.p. 565. Re 1. at. prodr. p. 62. Zer. 1. mel. thes. p. 73. Trev. prosp. n. eug. p 26. Pucc. syn. pl. luc. p. 298. Bert. fl. ital.6.p. 26. Com. fl. com. 4. p. 281. Car. prodr. fl. tosc. p. 525. Zan. prosp. fl. ven. p. 26. Rota prosp. piant. Pav. p. 272. Hausm. 11. Tir. p. 701. Rota prosp. fl. Berg. p. 71. Mor. fl. sard. 3. p. 336. Pir. fl. for. syll. p. 114. Tassi fl. prov. sen. p. 51. Cenno bot. Sien. p. 89. Ard. Al. Alpes-mar. p. 30\%. G. Bert. veg. Porr. p. 90. Not. Porr. p. 49. Zersi prosp. piant. Bresc. p. 170. De Vis. Sacc. cat. piant. Ven. p. 141. Terr.! sec. rel. Terra di Lav. p. 96. Ces. Pass. Gib. comp. fl. ital. p. 325. Ing. cat. sp. Mond. p. 70. Sacc. Bizz. agg. fl. trev. p. 29. Arc. comp. fl. ital. p. 559. Gib. Pir. fl. Mod. p. 13 3ง.

Figure. - Fl. dan. t. 593. Reich. ic. fl. germ. 18. t. 38. f. 2. $\beta$ scordioides, pilosius, foliis latioribus basi præsertim.

Teucrium scordioides Schreb. pl. vert. unilab. De Not. rep. fl. lig. p. 330. Gren. Godr. Al. Fr. 2. p. 709. Grech Del. fl. mel. p. 26. Pir. l. c. Ard. cat. pl. Ment. p. 29. De Vis. Sacc. l. c. Mars. cat. pl. Cors. p. 115. Ces. Pass. Gib. l. c. Groves! contr. fl. Terr. d'Otr. p. 66. Cocc. terzo contr. fl. Bol. p. 29. Gib. Pir. l. c.

Figura. - Savi mat. med. tosc. $t .4$.

Stazione, Abitazione e Fioritura. - Nei luoghi umidi e palıdosi di pianura, ed anche di collina e di montagna non molto alta, la forma $\beta$ essendo piu abbondante nelle parti centrali e meridionali d'Italia, e l'altra forma nelle parti settentrionali. Cosi in Piemonte presso Mondovi a Vico (Ing.), lungo il Tanaro (Bert.), a Bruno (Delponte!), a Torino, ad Avigliana a S. Antonino (Colla herb. ped.), al lago di Candia (Malinverni!), sul Po nel Novarese (Bir. fl. acon.); in Lombardia nel Pavese (Rota), a Nilano (Rampoldi!), nel Comasco, ad Agno nel Cantone Ticino, a Morbegnno in Valtellina (Com.), nel Bergamasen (Pota), ad Iseo (Parl.!) ed altrove nel Presciano (Zersi), a Grumone (Parl.!); in Tirolo verso Bolzano, ed altrove (Hausm.); nel Veneto intorno a Mantova (Barbieri! ecc.), a Verona (Porta! ecc.), negli Euganei (Trev.), a Chinggia (Bert.), a Venezia (Kellner I), a Bassano (Bert.), a Tarzo (Sacc. Bizz.), in Friuli a Doberdo (Bert.), a Monfalcone (Pir.); in Istria a Zaule presso Trieste (Bert.) e a Pola (Poll. /1. ver.); nell'Emilia verso Cinastalla (Passerini! ecc.), nei colli di Castelvetro, Campigglio, Ventasso (Re), intorno a liologna (Hert. ecc.), e nel vicino Appennino alla Porretta 
(G. Bert.) e all'Acero (Cocc.); in Romagna a Riolo e a Ravenna (Bert); in Liguria a Nizza e a Mentone dov' è raro (Ard.), a S. Remo (Panizzi!), ad Albenga (Berti!), alla Spezia!; in Toscana comune nel littorale dalla Magra a Livorno!, e a Lucca (Beccari! ecc.), quindi a Firenze!, Siena (Bert.), Rapolano (Sommier!), Montepulciano, Chianciano, nel monte Amiata (Santi viagg. ecc.), nel monte Argentaro (Aiuti!); nelle Marche e giủ lungo l'Adriatico a Sinigaglia (Bert.), Cupra (Piccinini!), Montefortino (Marzialetti!), Pescara, Caramanico a 1000 metri (Porta e Rigo! ecc.), Pescocostanzo (Bert.), Barletta (Bruni!), Otranto (Groves); a Civitavecchia e Roına (Bert.); in Campania sopra Cassino (Terr.), a Caserta (Terr.!), intorno a Napoli (Parl.!, Ten. ecc.), in Calabria ad Anoia (Pasquale!); in Corsica a Biguglia (Mabille!), S. Fiorenzo, Rogliano (Mass.); in Sardegna (Mor.); in Sicilia dov'è comune (Guss. fl. sic. syn.), per esempio a Palermo (Parl.!), Mistretta (Seguenza!), Messina (Bert.), Catania (Parl.!), Avola (Bianca!); in Malta (Zer., Grech). Fiorisce da luglio a settembre, e sin dalla primavera nei luoghi piủ caldi.

Distribuzione geografica. - In tutta Europa, al di sotto della Svezia meridionale, nell'Asia occidentale fino in Siberia e al Cascemir, nell'Affrica settentrionale e fino in Abisșinia.

Descrizione. - Pianta perenne, alta da 2 a 6 e talvolta 8 decimetri, di un verde chiaro, pubescente e con peli anche glandolosi nelle parti superiori, fornita di un rizoma delicato, radicante, che manda degli stoloni corti e giacenti per terra, i quali portano delle foglie opposte, piuttosto piccole, obovato-ovali, un po' ristrette alla base, del resto simili alle foglie del fusto e dei rami. Il fusto è ascen. dente, radicante nei nodi inferiori, quadrangolare, di un verde assai chiaro, pubescente per lunghi peli bianchi orizzontali, e fornito in alto di altri peli corti spesso numerosi, aventi all'apice una glandoletta, egualmente bianco in tutte le sue parti, ramoso in alto, con i rami corti, eretti o ascendenti. Le foglie sono erbacee, opposte, alquanto lontane, patenti, sessili, bislunghe, ottuse, crenato-dentate, con i denti piuttosto profondi e rari, fin quasi alla base, e con corte ciglia curve nel margine, di un verde chiaro di sopra ed ivi con solchi che corrispondono ai nervi di sotto e con peli corti e rari, av. vicinati alla foglia, di un verde più chiaro di sotto ed ivi con un nervo longitudinale rilevato, da cui partono molti nervetti laterali un po'rilevati che si dirigono obliquamente verso il margine e l'apice e si diramano per unirsi tra loro e terminare all' apice dei denti: sono pubescenti in questi nervi e hanno delle papille tonde bianche nel 
parenchima di sotto, visibili con una forte lente. I fiori sono 504 , talvolta 2, all'ascella di ciascuna foglia superiore, molto piủ corti di questa, quasi rivolti tutti da un lato. I pedicelli sono delicati, eretti, verdognoli, pelosi, quasi uguali al calice. Questo è tubuloso, inserito un poco obliquamente sul pedicello, un po'curvo e come gobbo in avanti alla base, verdognolo, peloso, con leggieri nervi longitudinali e con cinque denti più corti del tubo, quasi uguali tra loro, lanceolati, acuti o quasi acuminati, larghetti, avvicinati alla corolla. La corolla è quasi una volta e mezzo piủ lunga del calice. Il suo tubo è lungo quasi quanto questo, un po' curvo in su, cilindrico, pelosetto di fuori, senza anello di peli di dentro; il lembo è di color violetto assai chiaro e quasi roseo, diviso in due labbri, il superiore è profondamente bifido con le lacinie lanceolate, acute, concave di dentro, carenate di fuori e talmente avvicinate al labbro inferiore che sem. brano far parte di questo, il quale perciò pare diviso in cinque lobi, mentre in realtà è trilobo con i lobi laterali eretto-patenti, lanceolati, simili alle lacinie del lobo superiore ma privi di carena: il lobo medio è molto maggiore, diretto un po'in giù, orbicolare-obovato, appena concavo di sopra, spesso con macchioline o poche righe longitudinali piủ cariclıe e con peli corti e ritti in due linee verso la gola. Gli stami sono quattro, didinami, sporgenti in mezzo alle lacinic del labbro superiore, più lunghi di questo ma molto piủ corti del labbro inferiore, inseriti nella parte superiore del tubo. I filamenti sono eretti, filiformi, con qualche pelo lunghetto in avanti verso la base, quasi rosei. Le antere sono verticali, quasi reniformi, aprentisi per una fenditura comune alle due logge, di un giallo quasi ranciato, glabre, con papille tonde, piccole e bianche verso l'inserzione del filamento. Il pistillo è quasi uguale agli stami in mezzo ai quali sta. L'ovario è quasi tondo, quadrilobo, con i lobi quasi tondi, verdognolo, glabro, circondato alla base da un disco piccolo, circo. lare e verdognolo. Lo stilo è lungo, eretto, filiforme, glabro, quasi verdognolo o bianchiccio. Lo stimma è bifido, con le lacinie acule, quasi uguali, appena divergenti. (Parl. ms., descr. della pianta di Grumone in Lombardia).

Descrizione della varieta. - La pianta ha un rizoma delicato e quasi orizzontale, il quale manda le fibre radicali e alcuni stoloni. II fusto o ramo è diritto, alto da 5 o 6 pollici sino a un piede c a un piede e mezzo, poco ramoso, con i rami piuttosto corti, qualrangolare, e coperto di peli che lo rendono quasi lanato. Le foglie sono opposte, allungato-ovate o ovate, un poco incarate in cuore.alla base, 
le superiori ovate, tutte con crenature un poco seghettate: sono di color verde pallido, più pallido nella pagina superiore, ovunque coperte della stessa pelurie quasi lanata come il fusto. I fiori sono da uno o due all' ascella di ciascuna foglia delle parti superiori del fusto e dei rami, onde formano piccoli verticilli. I peduncoli sono delicati, cilindrici, patenti, con lunghi peli bianchi, e sono quasi uguali al calice. Questo è tubuloso, un poco gonfio di sotto alla base, diviso in cinque lacinie o denti quasi uguali, acuti: è verde con qualche sfumatura rossiccia di sopra, coperto dei soliti peli. La corolla ha il tubo lungo quasi quanto il calice, un poco curvato, con qualche raro pelo verso alto: è privo di anello di dentro. Il labbro superiore è diviso profondamente in due lacinie, le quali sono saldate con il margine del labbro inferiore in modo che nel punto dove dovrebbe essere il labbro superiore vi è solo una profonda smarginatura e il labbro inferiore apparisce come formato di cinque lobi: queste lacinie sono dirette in avanti, in alto e con l'apice rivolto in fuori, essendo tra loro divergenti, sono lanceolate, acute, con una carena di fuori, concave di dentro, hanno piccolissime ciglia nei margini, quasi del colore stesso del labbro inferiore ma verdognole verso l'apice. Il labbro inferiore è diretto in avanti e in giù, diviso in tre lobi, uno grande quasi obovato, tondeggiante all' apice, un poco concavo nel mezzo, quasi piano nel margine ch'è leggermente crenulato-increspato, ristretto verso la base dove sono due prominenze longitudinali, fornite di lunghi peli bianchi, e divise da un solco: ivi e longitudinalmente nel mezzo è di colore lilla, che diviene più pallido nel resto del labbro; i due lobi laterali sono piccoli, poco più corti delle lacinie del labbro superiore, diretti in avanti e avvicinati al lobo intermedio, ovati, ottusetti, di color lilla chiaro, con una riga longitudinale più carica. Gli stami sono inseriti nel tubo della corolla e sortono di mezzo alle lacinie del labbro superiore corrispondendo all'incavo ch'è tra essi, sono diretti in alto e poco più corti del labbro inferiore. I filamenti sono filiformi, verdognoli con alcuni punti glandolosi rossicci verso la base e con lunghi peli diretti in giu e bianchi poco sotto della metà, glabri nel resto. Le antere sono allungate, un poco ristrette nel mezzo e quasi reniformi, di color rossiccio; le logge sono confluenti e si aprono per una fessura longitudinale; il polline è rossiccio; nel punto di unione del filamento con l'antera ho veduto con una forte lente alcune piccolissime glandoline a guisa di perline bianche. L'ovario è piccolo, quasi globoso, verde. Lo stilo è lungo quanto gli stami più lunghi, filiforme, bianchiccio, leg. 
germente verdognolo verso l'apice llove è bifido. Gli stimmi sono acuti. (Parl. ms., descr. della pianta della Marinella di Luni).

Osservazioni. - Generalmente si distingue come specie il Teu. crium scordioides, per la pelurie piủ abbondante, per gli stoloni con appendici fogliari squamose, e non fogliacee, per le foglie abbraccianti, cioè slargate e non ristrette alla base. Ma in ambedue le forme le appendici degli stoloni sono squammose o fogliacee secondochẻ gli stoloni stessi sono sotto, o sopra terra; la pelurie varia all'infi. nito; e le foglie presentano molti intermedi fra i due estremi delle bislunghe e ristrettc in basso, e delle larghissime abbraccianti alla base. Non che specie diverse, le due forme rappresentano appena varieta distinte. Giả Bertoloni e Moris furono dello stesso parere.

\section{Teucrimn Hotrys.}

T. annuum, foliis ovatis, laciniatis, villosulis, floribus false verticillatis secus ramos foliatos, calyce subregulari, tubuloso-campanulato, tubum corollinum excedente, coccis rugoso-foveolatis, glabris.

Tencrium Botrys Linn. sp. pl. ed. 1. p. 562. Trev. prosp. 1 . eug. p. 26. Pucc. syn. pl. luc. p. 298. De Not. rep. R. lig. p. 390. Bert. fl. ital. 6. p. 17. Com. fl. com. 4. p. 280. Car. prodr. fl. tosc. p. 525. Rota prosp. piant. Pav. p. 272. Hausm. 月. Tir. p. 701. Rola prosp. fl. Berg. p. 71. Pir. fl. for. syll. p. 114. Ard. cat. pl. Ment. p. 29. F. Bert. veg. Porr. p. 90. Not. Porr. p. 49. Ard. A. Alpesmar. p. 30\%. Zersi prosp piant. Bresc. p. 170. De Vis. Sacc. cat. piant. Ven. p. 141. Ces. el. piant. Maiella p. 93. Archb. fl. Alto-Serch. p. 61. Ces. Pass. Gib. comp. 1. ital. p. 395 . Ing. cat. sp. Mond. p. 69. Arc. comp. 1. ital. p. 559. Gib. Pir. fl. Mod. p. 132. Coce. 11. Bol. p. 389.

Figura. - Reich. ic. fl. germ. 18. t. 38. f. 1.

Stazione, Abitazione e Fioritura. - Nei campi e nei luoghi incolti montuosi della Penisola settentrionale e centrale: in Piemonte in val Grisanche (Carestia!), intorno a Susa ! Aiuti! ecc.), a Fenestrelle (Colla herb. ped.), presso Mondovi (Ing.), ad Alba (Colla); in Lombardia presso Lecco, in val Menaggio e specialmente in vicinanza di Porlezza, e nel distretto di Lugano del prossimo Cantone Ticino (Com.), a Bergamo dov'è enmune (linta), presso Lirescia a Bogliaco, Toscolano, Palazzolo (Zersi); in Tirolo sopra Eppan, presso 
Tione (Hausm.), a Trento (Perini!), in Valsugana, a Roveredo (Hausm.); nel Veneto sul Baldo (Barbieri!), sui Lessini, sopra Verona a Grezzana (Poll. $f l$. ver.) e Stallavena (Bert.), sopra Vicenza a Trissino (Poll.), negli Euganei (Trev.), a Bassano, alle Vette di Fel. tre in Vallazza (Bert.), nel Friuli ad Aviano (Bracht!) ecc.; a Trieste (Bert.); in Liguria a Tenda (Bourgeau!), Nizza, Mentone (Ard.), Oneglia (Berti!), Genova (Carrega!) ecc.; nel Pavese a Gariasco (Nocc. Balb. fl. tic.), a Carposasso (Bert.), nel Modenese a Ranocchio di Montese (Gib. Pir.), nel Bolognese alla Porretta (Beccari! ecc.) e monte Beni (Bert.); nelle Marche e gli Abruzzi nei monti Sitria e Catria (Piccinini!), a Serra S. Quirico (Bucci!), ad Arcevia, nei monti di Sernano (Bert.), a Montefortino (Marzialettil), nei monti Birro (Bert.), Bove (Sang. cent.), Vettore, dei Fiori!, Amaro (Groves!), a Caramanico (Pedicino!), alla Maiella (Ten. syll.); in Toscana nel Pontremolese (Parl.!), in Garfagnana (Ad. Targ.!), a Montefegatese nel Lucchese!, a Cutigliano (P.. Sav.!) e Gavinana (Parl.!) nel Pistoiese, in Casentino a Pratovechio (Parl.!) e Camaldoli (Marcucci!), in valle Tiberina (Amidei!); nell'Umbria nei monti di Rieti (Rolli!). Tenore (syll.) l'indica ancora a Gallipoli, molto fuori della regione della pianta. Fiorisce in giugno e luglio.

Distribuzione geografica. - Nell'Europa media e in parte nella meridionale, dalla Spagna a Mosca e ai paesi Danubiani. In Algeria.

\section{A. Teucribu campanulatum.}

T. perenne, foliis cuneatis, partitis, glabris, floribus oppositis, in racemum elongatum laxum foliatum dispositis, calyce regulari, late campanulato, tubum corollinum excedente, coccis superne reticulato-rugosis, glandulosis.

Tencrium campanulatum Linn. sp. pl. ed. 1. p. 562. Bert. 凡. ital. 6. p. 16. Ces. Pass. Gib. comp. fl. ital. p. 325. Arc. comp. f. ital. p. 559.

Stazione, Abitazione e Fioritura. - In Sicilia, in luoghi argillosi umidi: Palermo al fiume Oreto (Parl.!) sotto la Guadagna (Guss. syn.), Mazzara (Citarda!), Selinunte, Sciacca, Girgenti (Parl.!), Siracusa (Avola!), Bronte (Todaro!), Pandazzo (Guss.). Nella Penisola sull'Adriatico a Lecce, Conversano, Bari, e nel Tavoliere (Ten. syll.). Fiorisce da maggio a luglio.

Distribuzione geografica. - Oltre che in Italia, in Spagna. 


\section{Teucrium fruticans.}

T. fruticosum, foliis ovalibus, integerrimis, subtus canis, flo. ribus oppositis, in racemum aut paniculan laxam foliatan dispositis, calyce regulari, late campanulato, tubum corollinum excedente, coccis reticulato-rugosis, superne pilosis.

Teucrium fruticans Linn. sp. pl. ed. 1.p. 563. Cand. fl. fr. 3. p. 515. Zer. 月. mel. thes. p. 73. De Not. rep. fl. lig. p. 329. Bert. fl. ilal. 6. p. 18. Grech Del.! fl. mel. p. 27. Guss. enum. pl. Inarim. p. 266. Car. prodr. 凡. tose. p. 524. Tassi 月. prov. sen. p. 51. Car. fl. Montecr. p. 28. Ces. Pass. Gib. comp. fl. ital. p. 324. Arc. comp. fl. ital. p. 558 .

Teucrium latifolium Bert. o. c. p. 20. Ces. Pass. Cib. l. c.

Figure. - Dill. hort. elth. t. 384. f. 366, 367. Fl. graec. t. 527.

$\beta$ rufescens, foliis subtus rufescentibus.

Stazione, Abitazione e Fioritura. - Sui poggi aridi sassosi del littorale più caldo. Trovasi in Sicilia da per tutto, come pure in Ustica, nelle Egadi, in Pantellaria (Guss. ecc. ecc.), in Lampedusa (Aiuti!), in Nalta (Grech Del.! ecc.), mancando però nelle isole Eo. lie; manca pure in Sardegna, e in Corsica è indicato senza luogo speciale soltanto da Candolle. Sulla costa occidentale della Penisola trovasi nel Napoletano e nel Romano a Casalicchio alla Torre delli pioppi (Ten. syll.), ad Amalfi (Heldreich!), in Ischia al Fonolillo presso la punta di S. Angelo (Guss.), presso Fondi (Ten.), a Terracina (Webb!) dov'è comune (Bert.), sul Circello, intorno a Civitavecchia (Seb. Maur. fl. rom. prodr.); quindi nella maremma Toscana, dov'è comune dal monte Argentaro! a Talamone (Parl.!), e nelle isole vicine di Giannutri! , del Giglio (Parl.!), di Montecristo, di Pianosa (Bert.) e dell' Elba!. Nasce infine isolatamente da una parte al Capo delle Mele in Liguria (Berti!, Ricea!), dall'altra parte nel Cargano presso Viesti (Porta e Rigo!). La var. $\beta$ è stata raccolta a Nizza da Parlatore. Fiorisce da febbraio e marzo a maggio.

Distribuzione geografica. - Nel bacino occidentale del Mlediterraneo, cioè oltreché in Italia, nella Penisola iberica e la Barberia.

Osservazioni, - Le foglie sono più o meno larglıe, i Inbi cali. cini ottusi o acuti; sono piccole rariazioni graduate, che non possono 
servire a distinguere varietà come vogliono alcuni, e molto meno specie come altri opinano.

Dubito della spontaneità in Italia della var. $\beta$, ch'è una forma più speciale alla Spagna.

\section{Teucrinn creticum.}

T. fruticosum, foliis margine revolutis linearibus, integerrimis, subtus canis, floribus suboppositis, in racemum laxum elongatum parvifoliatum dispositis, calyce regulari, late campanulato, tubum corollinum excedente, coccis reticulato-rugosis, superne pilosis.

Teucrium creticum Linn. sp. pl. ed. 1. p. 563. Bert. fl. ital. 6. p. 20. Ces. Pass. Gib. comp. f. ital. p. 324. Arc. comp. fl. ital. p. 558.

Teucrium rosmarinifolium $\boldsymbol{L} a \mathrm{~m}$.

Figura. - Fl. grce. $t$. 529.

Stazione, Abitazione e Fioritura. - Nell' isola di Lampedusa (Pasquale!), sugli aridissimi colli calcarei (Guss. syn.). Fiorisce in aprile e maggio (Guss.).

Distribuzione geografica. - Nel bacino orientale del Mediterraneo.

\section{Teucrium Marum.}

T. fruticosum, foliis ovatis lanceolatisve, integerrimis margine revolutis, supra pilosulis subtus incanis, floribus oppositis aut false verticillatis, in racemos breviusculos densiusculos foliatos dispositis, calyce regulari, tubuloso-campanulato, tubum corollinum æquante, coccis rugosis, glandulosis.

Teucrium Marum Linn. sp. pl. ed. 1.p. 564. Bert. fl. ital. 6. p. 21. Gren. Godr. fl. Fr. 2. p. 712. Car. prodr. f. tose. p. 526. Mor.! fl. sard. 3. p. 333. Car. fl. Montecr. p. 28. Mars. cat. pl. Cors. p. 116. Genn. 1l. Capr. p. 111. Ces. Pass. Gib. comp. fl. ital. p. 324. Arc. comp. fl. ital. p. 558.

Figura. - Reich. ic. fl. germ. 18. t. 35. f. 3.

Stazione, Abitazione e Fioritura. - Nei luoghi aridi sassosi delle isole: di Gorgona (P. Sav.!), di Capraia (Mor. De Not. fl. Capr.) e di Montecristo (Tayl.) dov'è comune, di Corsica dov'è pure comune fino a 1000 metri di altezza, per esempio a Bastia!, Calvi (Soleirol!), 
Vico, Guagno (Gren. Godr.), Aiaccio, e fino al Niolo (Requien!), a Sartena, al monte di Cagno (Gren. Godr.), a Bonifacio (Requien! eç), a Corte (Gren. Godr.), di Sardegna, principalmente sul mare, nell'isole La Maddalena (Mor.) e S. Stefano (Genn.), a Sassari (Marcucci!), nell'isola S. Pietro (Mor.), a Cagliari dov'è comune (Ascherson! ecc.), nei monti di Dorgali, nell'isola Tavolara, ma anche lontano dal mare, a Arcidano (Hor.). Fiorisce in maggio e giugno.

Distribuzione geografica. - Trovasi inoltre nella Spagna e la Francia più meridionali. Bentham (Lab. p. 68I) l'indica ancora nelle isole del Lago Naggiore, secondo un saggio dell' erbario Candolleano: ma trattasi senza dubbio di pianta coltivata, e forse inselvatichita.

Osservazione. - Il Teucrium subspinosum Pourr.! in Willd. enum. hort. berol. p. 596, giusta un saggio autentico nell' erbario Webb, è uno stato particolare di questa pianta, da' fiori rimpiccioliti, fino ad essere atrofici. Il sig. Biondi l' ha trovato in Sardegna a San Filippo presso Iglesias.

\section{T'eucrium Polium.}

T. suffruticosum, foliis oblongis vel ob margines valde revolutos linearibus, superne præsertim crenatis, tomentoso-incanis, 1loribus false verticillatis, in glomerulos foliatos subcorymbosos dispositis, calyce regulari, tubuloso-campanulato, tubum corollinum æequante, coccis rugosissimis, glabris.

Teucrium Polium Linn. sp. pl. ed. 1.p. 566. Benth. lab. p. 685. Bert. Al. ital. 6. p. 36; et auct.

Teucrium capitatum Linn. l. c. Bert. o. c. p. 40. Gren. Godr. fl. Fr. 2. p. 71 1. Mor. 11. sard. 3. p. 331. Mars. cal. pl. Ciors. p. 116. Genn. 1l. Capr. p. 110.

T'eucrium aureum Bert. o. c. p. 39.

Teucrium Pscudoliyssopus Guss. enum. pl. Inarim. p. 267. Pasq. 1. ves.p. 81. Terr. /l. Vult. syn. p. 110.

Teucrium tomentosum Ing. cal. sp. Mond. p. 69.

Figure. - Cav. ic. 1. 117, 119. Fl. grac. 1. 535, 536. Reich. ic. 1. germ. 18. 1. 3\%. . 4-7.

Stazione, Abitazione e Fioritura. - Nei luoghi selratici asciutti d'ogni maniera, dalle arene del littorale fino ai luoghi sassosi dei monti, in tutta Italia fuorchi nella sua parte pii settentrionale. Cosi in tutta la Liguria, da Nizza (Barla! ecc.) alla Spezia (Bert.!), co- 
mune, però non esteso a quanto pare al di là degli uliveti (De Not. rep. fl. lig. ecc.); in tutta la Toscana, comunissimo, dalla Magra! al monte Argentaro (Cherici! ecc.), non inalzandosi però nelle Alpi Apuane, e in val di Serchio non esteso al di là di Gomitelli presso Lucca (Pucc. syn. pl. luc.), ma estesa altrove dal mare verso l'in. terno fino al Mugello, al Casentino (Bert.) e alla montagna di Cetona (Santi viagg.), assente peraltro da tutto l'arcipelago toscano; quindi più giù a Terni (Sang. cent.), Viterbo, Civitavecchia (Bert.), Roma dov'è comune (Webb! ecc.), Massa presso Avezzano (Cherici!), Terracina (Fiorini!), Cairo (Terr.), nel Matese (Bert.), a Na. poli al Fusaro (Bert.), a Granatello (Avellino!) dove ora è quasi di. strutto e a Torre del Greco (Pasq.), in Ischia, rarissimo (Guss.), a Salerno, Castellabate, Licosa (Ten. syll.), Reggio (Pasquale!) e Bova (Biondi!) in Calabria; dall'altra parte della Penisola a Taranto (Aiuti!), Otranto (Groves!), Lecce (Ten.), a Melfi (Terr. ecc.), nel Tavoliere, ad Agnone in Molise (Ten.), nelle isole Tremiti (Bert.), nei monti di Caramanico (Ces. el. piant. Maiell.), a Pescara (Kuntze!), Ascoli (Parl.!), Montefortino (Marzialetti!), nel monte Corona, a S. Elpidio, Sinigaglia, Fano, al Furlo (Bert.), nel monte Catria (Piccinini!), a Cesena, Ravenna, Comacchio, Chioggia (Bert.), Venezia (Kellner! ecc.), Caorle (Pir. fl. for. syll.), Aquileia (Sieber!), Monfalcone, Trieste (Bert.), Pola (Kerner!), Fiume (Noé!). Ricomparisce nell'alto Piemonte a Castelrochero (Delponte!), ad Alba (Bertero!), fra Murazzano e S. Benedetto (Ing.), e secondo Bellardi in val d'Aosta (Poll. fl. ver.). In Corsica fa a Bastia (Mabille! ecc.), Corte (Jordan! ecc.), Bonifacio, nel Niolo (Requien! ecc.) ecc., e su taluni monti a grandi altezze come il Pigno, monte Rotondo, il Nebbio, quando i fiori sono generalmente rosei (Mars.). In Sardegna fa nelle isole S. Stefano (Genn.), Maddalena, Tavolara, Tonara (Mor.), Cagliari (Gennari! ecc.), S. Antioco (Mor.), Sassari (Marcucci!), innalzandosi a circa 900 metri sul mare (Mor.). In Sicilia sembra che sia comune, e ricco di forme, avendolo il Gussone potuto dividere in 4 specie, e queste in varietà; più particolarmente trovasi lungo tutta la costa meridionale e orientale, da Trapani (Guss.) a Siracusa e a Catania (Parl.! ecc.), quindi nel Messinese a S. Stefano (Seguenza!) e a S. Filippo di Argirò (Guss.), sulla costa settentrionale a Mistretta (Guss.) e Cefalù, e nei monti interni a Gangi (Parl. !), Nicosia, Leonforte (Guss.), Castrogiovanni (Parl. ! ecc.). Manca nelle isole siciliane, e nel gruppo di Malta. Fiorisce da aprile a giugno nelle parti piủ meridionali, e da giugno ad agosto nelle più settentrionali. 
Distribuzione geografica. - Nell' Europa meridionale, dal Portogallo alla liussia, nell'Asia occidentale, sino alla Persia, nell'Affrica settentrionale.

Osservazioni. - Questa specie presso noi varia nıltissimo: per il colore della pelurie, ch'é generalmente cenerognola, ma puỏ essere più bianca, o gialliccia, o decisamente gialla, in particolare nelle parti superiori fiorifere della pianta, lo che ne costituisce la variazione più vistosa fra tutte; - per la grandezza e soprattutto la larghezza, vera 0 apparente, delle foglie; - per l'addensamento maggiore o minore dei glomeruli di fiori; - per il colore di questi, più spesso bianco, talora gialliccio, o roseo. Tali variazioni sono combinate fra loro in più modi, cosicchè riesce difficilissimo precisare varietà distinte, e non è maraviglia se gli autori che ne hanno proposto sieno tanto discordi fra loro, e se i medesimi sinonimi di credute specic sono riferiti ora all' una ora all'altra varietà. La forma più comune fra noi è quella cenerognola, di foglie strette, di fiori bianchi fittamente addensati.

\section{5. 'Teucrom montanum.}

T. suffruticosum, foliis ex oblongo lanceolatis linearibusve, sub. integris margine revoluto, supra pilosulis subtus tomentoso-incanis, lloribus false verticillatis in glomerulum foliatum confertis, calyce regulari, turbinato-campanulato, tubum corollinum iequante, coccis rugoso-foveoiatis, glabris.

Teucrium montınum Linn. sp. pl. ed. 1. p. 565. Berl. /l. ital. 6. p. :4; et auct. omn.

Teucrium supinum Linn. o. c. p. 566. Mass. prodr. $\uparrow$. valt. p. 181.

Teucrium angustifulium Pir. fl. for. syll. p. 1/4.

Figure. - Fl. griec. 1. 539. Reich. ic. 1l. germ. 18. 1. 397. 1. $1-3$.

Stazione, Abitazione e Fioritura. - Nei luoghi solatii aridi, arenosi o pietrosi, specialmente nei montuosi, di tutta l'Italia, ad eccezione della Penisola piu meridionale e della Corsica, cotnune in certe parti, meno frequente in altre. Nell'dlta ltalia trovasi sulla sinistra del Po a Susa, presso Oulk fra i larici (.Iiuli! ecc.), nel Cenisio a Ronche (Bucci!), lungo la Stura (Colla herb. ped.), a Courmayeur (Webb!), al Cramont fra gli abeti (Parl.!), a Cressoney-la- 
Trinité (Piccone!), a Riva di Sesia (Carestia!), nel Novarese dovunque (Bir. ll. acon.), ad Arona (Ricca!), al Sempione (Parl.!), a Locarno (Franzoni!), nel Comasco comune (Anzi auct.), cosi a Cernobbio (Rampoldi!) e Chiasso (Bert.), e presso Lecco al monte Barro (Parl.!) e verso Morterone (Aiuti!), in Valtellina a Sondrio (Bert.), Pendolasco, Trevisio (Mass.), Bormio a 1350 metri (Parl.!), nel Bergamasco comunissimo (Rota prosp.), per esempio sopra Clusone (Bert.), nel Bresciano a Ronchi, Guglielmo, Calcinatello (Zersi prosp.), Ponte di legno a 1320 metri, Corna Blacca a 1700 metri (Parl.!), e Solferino (Barbieri!), nel Veronese dove pare che abbondi (Poll. $\mu$. ver.), in Tirolo sul Garda, a Roveredo (Hausm. fl. Tir.), Trento (Perini!), Tione (Hausm.), in Valsugana (Ambrosi!), in Fiemme e Fassa, a Bolzano, Laaso ecc. (Hausm.), negli Euganei (Trev. prosp.), a Venezia, nel Bassanese (Bert.); nel Friuli, per esempio a Monfalcone (Pir.), a Trieste (Bert.), a Fiume (Smith!); e sulla destra del Po trovasi vicino a Stroppo (Delponte!), al col di Tenda a 1900 metri (Parl.!), ad Alba (Colla), nel Pavese (Rota prosp.), nel Parmense, per esempio a Borgotaro e al monte Prinzera (Parl. !), nel Modanese a Campogalliano, Rubiera, Sassuolo, Scandiano, Salto di Montese, Sparavara (Gib. Pir. fl. Mod.), nel Bolognese a Montecastello, San Luca, Paderno, al Sasso (Bert.), Iula, Monteveglio, Casola Canina ecc. (Cocc. fl. Bol.), Porretta (G. Bert. not. Porr.), a Faenza nel monte Mauro e lungo il Lamone (Cald. fl. fav.), nella pineta di Ravenna (Beccari!), a Ciesena (Bert.). Quindi nel lato orientale della Penisola al Furlo e a Rio sacro (Bert.), sui monti Catria (Piccinini!) e S. Vicino a 1300 (Bucci!) e 1500 metri (Paolucci!), nel Piceno sui monti Regnolo (Marzialetti!), e Vettore a S. Gemma!, in Abruzzo a piè del Velino (Cherici!) e sulla Maiella sopra Caramanico e nei monti S. Antonino (Ces. el.) e Coccia (Pedicino!). Nel lato occidentale della Penisola occupa tatta la Riviera di ponente, essendo comune da Nizza (Durando!) a Voltri (Figari!); quindi buona parte della Toscana, cioè la Lunigiana a Sarzana (Bert.) e Pontremoli!, le Alpi "Apuane dov'è frequente nelle loro parti medie e altissime!, il monte Pisano (P. Sav.! ecc.), l'Appennino lucchese a Campagnaia (Giannini!), e pistoiese a Mandromini (Vitm. sagg.), Firenze (dov'è raro) all' Impruneta (Bechi!) e a S. Felice a Ema (Bucci!), il Chianti sopra l'Incisa! e presso Radda (Beccari!), il Senese a Rapolano (Sommier!) e in val di Rosia (Santi viagg.), la Maremma a Castiglioncello (Pampana!), nel monte Calvi!, verso Paganico (Santi), a Cala di Forno (Savi bot. etr.), al monte Argentaro, sul monte Amiata 
a Seggiano (Santi) e ai Bagni di S. Filippo (Savi!); infine per la Serra S. Antonio nel Romano (Bert.), per Picinisco (Terr. sec. rel.), Itri (Terr.!), Spigno, Pastena e Frasso Telesino (Terr. quarl. rel.) in Campania, termina al monte S. Angelo di Castellamare (Avellino!). In Sardegna ritrovasi nei monti d'Oliẹna a circa 1500 metri (Mor. fl. sard.); e in Sicilia a Palermo a S. Martino e al monte Occhio (Parl.!), a Busambra, Cammarata, al monte Pirredda secondo Ucria (Guss. syn.), nelle Madonie a Quacedda (Parl.!). Fiorisce da maggio e giugno a luglio e agosto.

Distribuzione geografioa. - Nell' Europa media e meridionale, nell' Asia Minore, in Songaria (Boiss. fl. or.).

Descrizione. - Il fusto è assai ramoso, giacente per terra, i rami sono cilindrici, delicati, bianchicci, pubescenti-tomentosi. Le foglie sono opposte, piccole, lineari-lanceolate, integerrime, di un verde pallido di sopra e ivi pubescente e con un solco longitudinale, con i margini rovesciati, bianchicce di sotto per un tomento e ivi con un nervo longitudinale grossetto, da cui partono pochi nervetti obliqui e diretti verso il margine dove si diramano in piccole vene. I fiori sono circa $7 \circ 8$, ravvolti in piccoli corimbi all'apice dei rami. Le brattee sono simili alle foglie ma piú piccole. I peduncoli sono cortissimi, cilindrici, quasi tomentosi. Il calice è tubuloso, un poco gonfio di sotto nella base, diviso in cinque lacinie o denti quasi uguali, acuminati: è di colore bianco-giallognolo, un poco tomentoso, con cinque nervi longitudinali che si continuano nelle lacinie e nell'acume di queste. La corolla è di un bianco-gialliccio, o quasi gialliccia. Il suo tubo è poco piủ lungo del calice, piủ largo verso alto dove è quasi inginocchiato, e pubescente. Il labbro superiore $\dot{e}$ diviso profondamente in due lacinie allungate, ottuse o come troncate, diritte e avvicinate tra loro in modo che si toccano con la loro faccia interna, con una carena formata da un nervo verdognolo che si dirama, ed ha nei rami qualche sfumatura rossiccia : sono pubescenti di fuori e fornite nei margini di peli corti e piuttosto fitti. All' incavatura profonda che divide le due lacinic corrispondono gli stami e il pistillo che sortono cosi tra esse e le lacinie. Il labbro inferiore è diretto in avanti $e$ in giu, bifido, con le lacinie laterali, strette, allungato-lineari, scanalate in avanti, convesse e un poco pubescenti di fuori, e dirette in fuori quasi orizzontalmente, con l'apice ottuso un poco rivolto in dietro e con la base fornita di una piccola orecehiella formata dalla base del margine superiore; tra essa e la base del labbro inferio.e stesso sono due linee longitudinali sporgenti dove sono dei peli lun-

Forma Italiaka. - Vol. Vi. 
ghi e grossetti messi in linea. La lacinia media è quasi tonda, concava di sopra, con una carena di sotto dove è appena pubescente, leggermente crenulato nel margine verso la base. Gli stami sono inseriti sul tubo della corolla: i piủ alti sono poco più alti delle lacinie del labbro superiore, e i più bassi poco più corti di queste. I filamenti sono filiformi, bianchicci o giallicci, con peli lunghetti e bianchi che sono piủ rari verso alto. Le antere sono rossicce, allungate, ristrette un poco nel mezzo e con le logge confluenti. Il polline è rossiccio. Nel punto di unione delle antere con i filamenti vi sono alcuni piccoli punti tondi come perline e pochi peli. L'ovario è piccolo, quasi globoso, verde, con quattro lobi: Lo stilo è lungo quasi quanto gli stami più lunghi, filiforme, glabro, bianchiccio, bifido all'apice. (Parl. ms., descr. della pianta di Ozzano nel Parmense).

\section{AIUGA.}

Chamæpitys et Bugula Tourn. inst. p. 208. t.98.

Ajuga Linn. gen. pl. ed. 1. p. 167. Mirb. in ann. mus. 15. t. 15. f. 13, 19. Benth. Hook. gen. pl. 2. p. 1222. Ces. Pass. Gib. comp. fl. ital. p. 325. t. 48. f. 5.

Ajuga et Chamæitys Gen. plant. fl. germ. 2. t. 47, 48.

Calyx campanulatus, obsolete nervatus. Corolla manifeste 2-labiata, labio superiore minino, marcescenti-persistens. Stamina 4 , conniventia, filamentis edentulis, antheris 1-locularibus (ob confluentiam loculorum), longitudinaliter arcuatin dehiscentibus. Stylus lobis subæqualibus. Cocci apice convexi.

Portamento. - Sono piante erbacee, basse, che per il portamento si dividono in 2 gruppi ben distinti. Quelle del primo (sp. 1-5) sono perenni, con una rosetta di foglie slargate, e un fusto semplice, fornito in alto di falsi verticilli di fiori turchini con foglie fiorali piủ o meno differenti dalle altre. Le specie del secondo gruppo $(6,7)$ sono annue, ramose, con fiori rossi o gialli in minor numero all'ascella di foglie simili alle altre, strette in una specie, profondamente divise nell' altra.

\section{Aiuga orientalis.}

A. estolonosa, caule erecto, basi ramoso, ramisque ascendentibus, tetragonis, piloso-lanatis, foliis inferiorihus oblongis, obtusis, 
inæqualiter crenato-lobatis, rugoso-bullatis, longiuscule petiolatis, petiolis margine alatis, superioribus sessilibus, basi angustatis, floralibus sensim minoribus, minusque crenatis-lobatis crenatisque, superioribus subintegris vel grosse subtrilobis floribus subæqualibus brevioribusve, verticillastris inferioribus sub-5-floris, reliquis sensim paucifloris, floribus brevissime pedunculatis, calyce piloso-lanato, ovato-campanulato, profunde 5-fido, laciniis subæqualibus, lanceolato-acuminatis, corollæ tubo calyce duplo longiore, basi globoso-in. flato, glabro, superne stricto, subtereti, contorto, limbo calyce subdimidio breviore, subresupinato, labio superiore brevissimo, bifido, laciniis ovatis, acutis, inferiore trifido, lacinia media majore, subobcordata, in emarginatura denticulata, laciniis lateralibus longitudine subæqualibus, angustioribus, sublinearibus, apice submucronulatis, stamine 5 rudimentario, stylo filiformi, staminibus breviore, nuculis subrugosis, pilosulis. (Parl. ms.).

Ajuga orientalis Linn. sp. pl. ed. 1. p. 561. Bert. fl. ital. 6. p. 4. Ces. Pass. Gib. comp. fl. ital. p. 326. Loj. is. eol. p. 123. Arc.! comp. fl. ital. p. 560.

Figura. - Dill. hort. elth. t. 53.

Stazione, Abitazione e Fioritura. - In luoghi ombrosi, al margine dei boschi, lungo le siepi ecc. dell' Italia piủ meridionale: cioè in Calabria presso Monteleone (Arc.!), presso S. Alessio nella valle di Gallico a 6-700 metri (Huter Porta Rigo!), in Aspromonte, a Staiti, nel vallone di Pendola, a Monticello, a Scrisi (Ten. syll.); e in Sicilia sopra Palermo verso il Parco (Guss. syn., Bert.), alla Pizzuta (Todaro!), a Renda e alla Ficuzza (Parl.!), a Castellobuono (Guss.), a Messina (Prestandrea! ece.), Fiumedinisi, Bronte, Milo, Aidone (Guss.), Siracusa (Cassia!), Avola (Bianca!), Terranuova, Chiaromonte, Biseari, Ragusa, Cassaro, Ferla, S. Michele, Floresta, Capizzi, Mistretta (Guss.), e nell' isola Salina delle Eolie (Guss. Loj.). Fiorisce in marzo, aprile e maggio.

Distribuzione geografica. - In Portogallo e Spagna, nell' Italia meridionale, in Grecia, in Asia Minore, in Soria, in Crimea.

Descrizione. - Fusto ramoso alla base, eretto, alto circa 6-8 pollici, quadrangolare, peloso-lanato, di un verde chiaro, con i rami ascendenti, alti quasi quanto il fusto, e privo di stoloni. Le foglie inferiori sono allungate, ottuse, son fornite di un picciỏlo lungo, disugualmente crenato-lobate nel margine, rugoso-bollate in vicinanza del margine stesso, di color verde scuro di sopra e verde pallido di sotto ed ivi con un grosso nervo longitudinale bianchiecio, 
che manda nervi laterali i quali si dirigono verso il margine e l'apice diramandosi in nervetti che si uniscono con quelli degli altri nervi e formano una rete a larghe maglie presso il margine e l'apice dove di sopra sporge il parenchima a guisa di bolle. Il picciòlo è poco più corto della lamina, scanalato di sopra, convesso di sotto, alato nei margini perchè la lamina della foglia vi scorre in una stretta striscia. Le foglie del fusto sono opposte, sessili, ristrette alla base a guisa di cuneo, simili nel resto alle foglie inferiori, delle quali sono più piccole. Le foglie fiorali sono gradatamente più piccole, crenato-lobate 0 crenate nel margine e gradatamente meno crenate, in modo che le superiori sono quasi intere o talvolta solo come trilobe: queste superiori sono più vicine tra loro, e spessso di colore rossiccio o in totalità o nel margine, quasi uguali o poco più lunghe dei fiori, mentre le inferiori sono costantemente piủ lunghe dei fiori. Tutte le foglie e i peduncoli sono pelosi. I verticillastri inferiori si compon. gono per lo più di cinque fiori, quelli superiori gradatamente di meno in modo che nei verticilli superni spesso vi è un sol fiore. Ciascuno di questi ha un peduncolo cortissimo, di color violetto. Il calice è ovato-campanulato, diviso in cinque lacinie quasi uguali, lanceolate, acuminate; è verde con un nervo più carico in ciascuna lacinia, e pe. loso con peli lunghi e bianchi che lo rendono quasi lanato. La co. rolla è piủ del doppio e quasi tre volte piủ lunga del calice. Il suo tubo è il doppio più lungo di questo, gonfio, bianco e glabro alla base, poi ristretto e contorto in modo che il lembo è un poco resupinato, di color violetto come il lembo, al pari di questo peloso-lanato per peli bianchi e lunghi, e con punti bianchi tra i peli. Il labbro superiore, divenuto quasi inferiore per la contorsione del tubo, è corto, bifido, con le lacinie ovate, acute o come mucronulate all'apice; il labbro inferiore, divenuto quasi superiore per la ragione qui detta, è molto piủ largo e piủ lungo del superiore, trifido, con la lacinia maggiore quasi in forma di cuore a rovescio, con un piccolo dente o mucrone nella smarginatura alla base di dentro, essa ha due righe cerulee chiare: le lacinie laterali sono più strette ma quasi della stessa lun. ghezza, quasi lineari con una piccola punta all'apice. Il lernbo e la parte superiore del tubo della corolla sono glabri di dentro, ma la parte di questo ch'è sotto all'inserzione degli stami è pelosa, con peli lunghi, rivolti in alto. Gli stami sono inclusi. I due piủ lunghi corrispondono al labbro maggiore, gli altri due poco più corti al labbro minore. I filamenti sono lesiniformi, quasi bianchicci, saldati in gran parte con il tubo. Le antere sono scure, allun. 
gato-bilobe, con i lobi divergenti e le logge confluenti. Il polline è giallo. Il quinto stame abortivo si vede ridotto alla sola antera di color giallo pallido, sessile e posta alla parte interna dell' ovario che corrisponde dal lato del labbro minore della corolla. L'ovario è quasi tondo, diviso in quattro lobi allungati, ottusi, lisci e verdi. Lo stilo è filiforme, bianchiccio, più corto anche degli stami piủ corti, giungendo solo alla inserzione dei loro filamenti nel tubo, bifido all'apice, con le lacinie quasi uguali, filiformi, ottuse e divergenti. Le nucule, non perfettamente mature, sono scure, appena appena rugose e pelosette. (Parl. ms., descr. di pianta coltivata).

\section{Aiuga reptans.}

A. perennis, stolonibus epigæis donata, foliis sinuato-crenatis, glabriusculis, floralibus sensim minoribus, ovatis, subintegris, flores subæquantibus, floribus false verticillatis, tubo corollino arcuato, staminibus exsertis.

Ajuga reptans Linn. sp. pl. ed. 1. p. 561. Bert. fl. ital. 6. p. 9; et auct. omn.

Figure. - Savi mat. med. tosc. t. 10. Ten. fl. nap. t. 239. f. 2, 240. f. 2. Reich. ic. fl. germ. 18. t. 33. f. 3.

Stazione, Abitazione e Fioritura. - Comunissima in tutta la Penisola, eccettochẻ la più meridionalę, per ogni sorta di luoghi erbosi, prati, boschi ecc., dalle basse pianure a parti altissime dei monti. Cosi in tutta la valle del $P_{0}$, e nell'Istria, salendo nei pascoli delle Alpi; in Liguria; in Toscana dal littorale alle cime più alte dell'Appennino, assente però dalle isole; nella Romagna; nelle Marche e negli Abruzzi, anche ivi fino alle cime dei monti più alti; nell' Umbria, nel Lazio, nella Campania, compresa l'isola di Capri (Bert., Pasq. $l$. ves.), fino al Vulture (Terr. $\mu$. Vult.). Più giủ fa in Calabria, a Monteleone e presso Serra S. Bruno (Arcangeli!), e fa a Otranto (Groves!). In Sicilia fra altri luoghi a Monreale (Parl.!), Castelbuono (Minà!), Capizzi (Seguenza!); e trovasi pure in Malta (Grech $/$. mel.). Manca in Corsica e in Sardegna. Fiorisce da marzo a maggio nei luo. ghi bassi, e in giugno e luglio nei più alti.

Distribuzione geografica. - In Europa dalla Norvegia meridionale in giù, e nell' $\Lambda$ sia Minore.

Osservazione. - Nei pascoli alpini questa pianta difficilmente mette stoloni; nella qual forma è stata batlezzata da alcuni autori Aiuga alpina. 


\section{Aiuga genevensis.}

A. perennis, stolonibus epigæis destituta, foliis sinuato-crenatis aut grosse dentatis, nunc hispidulis nunc glabriusculis, floralibus sensim minoribus, ex cuneato ovatis, crenatis incisisve, flores subæquantibus, floribus false verticillatis, tubo corollino recto, staminibus exsertis.

Ajuga genevensis Linn. sp. pl. ed. 1. p. 561. Bir. fl. acon. p. 194. Mass. prodr. fl. valt. p. 7. Colla herb. ped. 4. p. 402. Trev. prosp. fl. eug. p. 27. De Not. rep. fl. lig. p. 331. Bert. fl. ital. 6. p. 6. Com. fl. com. 4. p. 274. Car. prodr. fl. tosc. p. 528. Zan. prosp. fl. ven. p. 26. Rota prosp. piant. Pav. p. 272. Hausm. fl. Tir. p. 700. Rota prosp. fl. Berg. p. 71. Pir. fl. for. syll. p. 114. Ard. fl. Alpes-mar. p. 304. Zersi prosp. piant. Bresc. p. 169. De Vis. Sacc. cat. piant. Ven. p. 140. Ces. Pass. Gib. comp. fl. ital. p. 326. Ing. cat. sp. Mond. p. 12. Anzi auct. fl. nov.-com. p. 196. Bizz. sec. agg. fl. ven. p. 6. Arc. comp. fl. ital. p. 560. Gib. Pir. fl. Mod. p. 132.

Ajuga alpina Bir.? l. c. Ten.? syll. fl. neap. p. 275. Mass.? l. c. Ing.? cat. sp. Mond. p. 12.

Ajuga pyramidalis Ten.? l. c. (ex loco).

Figura. - Reich. ic. fl. germ. 18. t. 33. f. 1.

Stazione, Abitazione e Fioritura. - Nei luoghi boschivi, o erbosi, come pure nei campi, specialmente di collina e di montagna, della Penisola. Piủ comune nell'Alta Italia, trovasi a Trieste e Lippizza (Bert.), nei monti Friulani, per esempio alle sorgenti del Tagliamento (Pir.), nel Lido veneto (Kellner!), negli Euganei (Trev.), nel monte Berico, presso Feltre (Bizz.), alle Vette di Feltre sulla Vallazza, a Bassano sul Grappa, a S. Michele d'Angarano, Rossan, e Castiglione (Bert.), nel Mantovano (Barbieri!), a Verona a Tagliaferro, e sulle cime del Baldo (Bert.), in Tirolo sul Garda, a Trento, in Fiemme verso Predazzo, in Fassa, presso Tione, a Castel Brughier in val di Non, intorno a Bolzano, comune a Merano, Bressanone, Welsberg (Hausm. ecc.), presso Modena, e lungo l'argine di Secchia a villa Freto (Gib. Pir.), nel Parmense (Pass.!), nel Pavese, dov'è frequente nella Lomellina, a Belgioioso ecc. (Nocc. Balb.), nel Bresciano, frequente, per esempio alla Bornada e a Nuvolento (Zersi), nel Bergamasco (Rota), nel Comasco, dove abbonda presso Como, a 
Garzola, Camnago, Solzago, Ponzate, nell'alta Brianza, sui Corni di Canzo, nel Cantone Ticino verso Lugano, Mendrisio e Locarno (Com.), in Valtellina a Ponchiera sopra Sondrio (Mass.) e alle radici dei monti Bormensi (Anzi), nel Novarese (Bir.), a Bard in val d'Aosta (Carestia!), al Cenisio (Aiuti!), a Bardonecchia (Beccari!), a Torino (Parl.!), nelle vallate Valdensi (Rostan!), ad Alba (Bert.), verso Vicoforte (Ing.), al col di Tenda (Bourgeau!), al vallon di Fe. nestre, a Malaussena (Ard.), nei monti d'Albenga (De Not.). Nell'Italia Media riducesi rara, non essendo stata trovata che presso Firenze all'Incontro!, piủ giủ in Toscana a Pienza (Sav.), a Viterbo, nel Piceno al monte De' Fiori, a Castel Manardo e nel monte Priore (Bert.), in Abruzzo, nel Gargano a Carbonara di monte S. Angiolo (Ten.). Manca nella Bassa Italia e nelle isole. Fiorisce da aprile a giugno nei luoghi bassi, e in luglio e agosto nei piủ alti.

Distribuzione geografica. - In Europa dalla Scandinavia meridionale alla Francia, alla Turchia e al Caucaso, nell' Asia Minore, in China.

Osservazioni. - Molti botanici dubitano che questa specie non sia sufficientemente diversa dalla seguente. Lo Zersi, il Bizzozero (It. cc.) ed altri hanno anche indicato forme intermedie.

Lagrèze-Fossat ha scoperto in questa pianta sottili stoloni ipogei (Bull. soc. bot.'de Fr. 2. p. 793).

\section{Ainga pyramidalis.}

A. perennis, stolonibus epigxis destituta, foliis sinuato-crenatis, hispidulis, floralibus subæqualibus, ovatis, subintegris, flores valde superantibus, floribus false verticillatis, tubo corollino recto, staminibus exsertis.

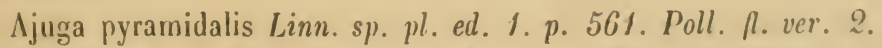
p. 264. Mass. prodr. 月. valt. p. 7. De Nol.! rep. /l. lig. p. 331. Bert. n. ital. 6. p. 7. Com. R. com. 1. p. 272. Hausm. R. Tir. p. 700. Rota prosp. 月. Berg. p. 71. Pir. 月. for. syll. p. 11\%. Ard. /1. Alpes-mar. p. 304. Zersi prosp. piant. Brese. p. 169. De Vis. Sacc. cat. piant. Ven. 1. 140. Ces. Pass. Gib. comp. R. ital. p. 326. Anzi auct. A. nov.-com. p. 196. Ing. cat. sp. Mond. p. 19. Arc. comp. R. ital. p. 560 .

Figure, - Fl. dan. t. 185. Reich. ic. R. germ. 1. 38. 1. 2.

Stazione, Abitazione e Fioritura. - Nei pascoli e nei prati delle 
Alpi, ad altezze molto diverse: nel Friuli, ovunque secondo Pirona, nel Bellunese e nel Vicentino, secondo De Visiani e Saccardo rara come nelle provincie venete in generale, più precisamente ad Aun e altrove nelle Vette di Feltre, a S. Michele di Marostica, e sopra Asiago, nel Baldo, soprattutto a Mon maor (Poll.), in Tirolo a Campiglio in Rendena, in val di Pelugo, nei monti Gazza e Scanucchia, presso Alba (Hausm.), nei monti di Torcegno, di Telve ecc. in val Sugana (Ambrosi!), intorno a Bolzano, per esempio sul Rittner da Pemmern fino in cima, in val di Laaso, e in Pusteria a Welsherg e altrove (Hausm.), nel Bresciano e Bergamasco a Ravenola sulle Colombine, nei monti Cigoletto e Rondenino (Zersi), nell'alpe Ruscara, sopra Pontedilegno (Ricca!), nel Comasco (Com., Anzi), in Valtellina a Togno e in valle Malenco (Mass.), al San Bernardino (De Not.!), al Sempione (Parl.!), a Riva di Valsesia (Carestia!), nel Novarese secondo Biroli ( $f$. acon.), nella catena del monte Bianco al Col du Géant, nel Cenisio a Ronche (Parl.!), presso Bardonecchia (Aiuti!), tra Cels e Exilles (All. $f l$. ped.), presso Torino alla Mandria secondo Re (Colla herb. ped.), nelle Alpi Marittime di S. Stefano, in Valdiblora, al col di Fenestre, al lago d'Entrecoulpes, al col di Salese (Ard.), al monte Formose presso il col di Tenda (Bourgeau!), in Vallasco (Bert.), a Ceva (Ing.), alla Scaggia (Bert.), nell'Appennino di Savona e di Genova (De Not.). Fiorisce da maggio e giugno a luglio e agosto.

Distribuzione geografica. - In Europa dalla Scandinavia al Portogallo, alla regione Danubiana e al Caucaso.

\section{Aiuga acaulis.}

A. perennis, stolonibus epigæis destituta, foliis sinuato-crenatis, pilosulis, floralibus subconformibus, elongatis, flores valde superantibus, floribus false verticillatis, tubo corollino recto, staminibus exsertis.

Ajuga acaulis Brocchi oss. Bert. fl. ital. 6. p. 8. Ces. Pass. Gib. comp. fl. ital. p. 326. Terr. quart. rel. Terra di Lav. p. 114. Arc.! comp. fl. ital. p. 560.

Figura. - Ten. fl. nap. t. 239. f. 3.

Stazione, Abitazione e Fioritura. - Nei pascoli dei monti dell'Italia Meridionale: in Abruzzo al Corno, al Velino (Ten. syll.) e al Morrone (Ces. Pass. Gib.), sul Cantro (Rolli!), in Terra di Lavoro a Setlefrati a Pietrorosiello (Terr.), in Calabria sul Pollino, a 
Mongiana, a Suveria (Telı.), sopra S. Cristina (Arc.!), a Soria (Bert.), in Aspromonte sopra S. Stefano a 1000-1100 metri (Huter Porta Rigo!), in Sicilia nel monte Soro (Guss. syn., Todaro!), a Cartolari in Valdemone (Tod.!), fra Caronia e S. Fratello (Guss.). Fiorisce da maggio a luglio.

Distribuzione geografica. - Specie italiana.

\section{Aiuga Iva.}

A. annua, foliis parce dentatis vel integris, hispidis, floralibus conformibus, linearibus, margine revolutis, flores valde superantibus, floribus oppositis aut false verticillatis, tubo corollino recto, staminibus exsertis.

Ajuga Iva Schreb. pl. vert. unilab. Bert. fl. ital. 6. p. 14. De Not. rep. fl. lig. p. 331. Gren. Godr. fl. Fr. 2. p. 707. Car. prodr. fl. tosc. p. 528. Grech Del. fl. mel. p. 27. Mor. fl. sard. 3. p. 329. Tass. fl. prov. sen. p. 51. Ard. cat. pl. Ment. p. 29. Fl. Alpes-mar. p. 304. Pasq. Lic. viagg. Garg. p. 22. Genn. fl. Gapr. p. 111. Mars. cat. pl. Cors. p. 115. Ces. Pass. Gib. comp. fl. ital.p. 326 . Arc. comp. fl. ital. p. 560.

Figure, - Cav. ic. t. 120. Fl. grac. t. 525. Reich. ic. fl. germ. 18. t. 34. f. 3, 4.

Stazione, Abitazione e Fioritura. - Nei luoghi asciutti, specialmente campestri, delle parti piủ calde d'Italia. In Sicilia trovasi ovunque (Guss. syn.), per esempio a Palermo (Parl.!), Militello (Seguenza!), Avola (Bianca!), Scoglitti, Girgenti (Aiuti!) ecc.; e nelle Eolie, le Egadi, in Pantellaria (Guss.), e in Malta (Todaro e Calcara! ecc.); in Sarilegna trovasi a Cagliari (Ascherson!), Laconi (Biondi!), Sassari (Marcucci!), nell' isola S. Stefano (Genn.); in Corsica a Bonifacio (Requien!), Ostriconi, Torre della Lossa (Bert.), Calvi, Isola Rossa, S. Fiorenzo, i Stretti (Mars.); nell'Elba a Campo (Caldesi!), in Pianosa (Bert.). E comune nella Liguria occidentale, da Nizza (Parl.!) a Finalmarina (Piccone!); ricomparisce in Toscana, assai rara peró, presso Campiglia (Savi!), e nel monte Argentaro verso Port' Ercole (Santi viagg.) e Porto S. Stefano (Aiuti!); quindi piủ giủ nel Romano a Civitavecchia (Bert.), Tivoli (Seb. Maur. $\pi$. rom. prodr.), e Castel di Guido (Rolli!), nel Napoletano sul Matese, sul monte Vergine, nei monti di Caserta (Ten. syll.), e a Capri (Bolle!), e infine in Calabria (Ten.). Dall'altro lato della Penisola si 
ritrova sul Gargano a Carpino (Pasq. Lic.) e verso Matirata (Porta e Rigo!), e nella Maiella secondo Tenore (syll.). Il Biroli ( $f$. acon.) la dả del Piemonte a Maggiora e Vergano sopra Novara. Fiorisce da marzo ed aprile all' autunno inoltrato.

Distribuzione geografica. - In tutto il bacino del Mediterraneo, nelle isole Canarie e a Madera.

Osservazioni. - Questa pianta sa generalmente di muschio. I fiori variano per essere ora rosei ora gialli. Ai fiori primaverili dicesi che manchi per lo più la corolla.

\section{\%. Aiuga Chamrepitys.}

A. annua, foliis tripartitis, hirtulis, floralibus conformibus, lohis linearibus, flores valde superantilus, floribus oppositis, tubo corollino recto, staminibus exsertis.

Ajuga Chamæpitys Schreb. pl. vert. unilab. Bert. fl. ital. 6. p. 11 ; et auct. omn.

Figure. - Savi mat. med. veg. tosc. t. 2. Reich. ic. fl. germ. 18. t. 34. f. 2.

$\beta$ grandiflora, floribus maioribus folia floralia subæquantibus.

Ajuga chia Schreb.? o. c. Guss. enum.pl. Inarim. p. 267. Grech Del. fl. mel. p. 27. De Vis. Sacc.cat. piant. Ven. p. 140. Groves! contr. fl. Terr. d'Otr. p. 66. Loj. is. eol. p. 123. Cald. fl. fav. tent. p. 184. Goir. app. bot. p. 25.

Ajuga Chamæpitys var. Ces. Pass. Gib. comp. fl. ital. p. 326. Arc. comp. fl. ital. p. 560.

Figure. - Fl. graec. t. 524. Reich. l. c. f. 1.

Stazione, Abitazione e Fioritura. - Nei campi asciutti e nei luoghi incolti, di quasi tutta l'Italia, dal piano fino ad una certa al. tezza nei monti. In Piemonte sembra rara, vi è stata trovata a Monpantero presso Susa (Aiuti!), Torino (Parl.! ecc.), Mondovì (Ing. cat.), Moncalvo (Malinverni!), Borgomanero, Borgo Ticino (Bir. $f l$. acon.). In Lombardia nasce nella Brianza, a Merate, Paderno, Montevecchio, in Valsolda e Valmadrera, in Valtellina sopra Berbenno (Com. $f l$. com.), nel Bergamasco (Rota prosp.), per esempio a Villa d'Adda (Bert.), nel Bresciano, frequente (Zersi prosp.), nel Pavese (Nocca Balb. h. tic.), a Grumone sull' Oglio (Parl.!), nel Mantovano (Barbieri!). In Tirolo nasce a Roveredo, Trento, Comano, Kaltern, Appiano (Hausm. fl. Tir.). Nel Veneto trovasi, sempre parcamente 
(De Vis. Sacc. cat.), a Verona (Bert.), e la var. ivi in valle di Pigozzo, presso Rivole, e in val Pantena alla Biondella, presso Alce. nago ecc. (Goir.), ad Angarano (Bert.), negli Euganei (Trev. prosp.), a Venezia (Zan. prosp.), nel Friuli a Faedis, Ignano, S. Odorico (Pir. syll.), Duino (Bert.); e nell'Istria a Trieste (Bert.), Pirano (Poll. fl. ver.), e Fiume (Rossi!). Nell' Emilia trovasi presso Ozzano e sul monte Prinzera nel Parmense (Parl.!), nel Modenese dov' ̇ comunissima, e sale sull'Appennino fino al Ventasso (Gib. Pir. $f$. Mod.), nel Bolognese dove parimente abbonda (Bert., Cocc. fl. Bol.), fino alla Porretta (G. Bert. not. Porr.). In Romagna a Faenza, comune, con la var. in Rivalta (Cald.), a Castrocaro (Cald.!), a Sassofeltrio (Bert.); nelle Marche a Sinigaglia (Bert.), Cupra (Piccinini!), Albaccina (Bucci!), S. Severino (Aiuti!), Macerata (Bert.), S. Angelo in Pontano (Marzialetti!), Grottamare (Bert.), Ascoli (Parl.!); in Abruzzo al monte de'Fiori!, la var. (Ten. syll.), a Pescara (Kuntze!), Caramanico (Ces. el. Haiell.), Assergio (Ten. syll.); sul Gargano (Pasq. Lic. viagg.), la var. (Ten.), ad Otranto, la var. (Groves!). E comune nella Liguria (De Not. rep.), da Nizza (Ard. fl.) a Genova (Carrega!). In Toscana pure comunissima, a Sarzana (Bert.), Lucca (Calandrini! ecc.), Pisa (P. Savi! ecc.), Firenze!, Vallombrosa (Parl.!), Siena (Campani! ecc.), Volterra (Amidei!), nella Maremma Grossetana (Parl.!); nell' Umbria a Fossato (Parl. !); nel Lazio a Canino, Sant' Oreste (Bert.), Roma', comune (Seb. Maur. fl. rom.), la var. (Rolli!), Terracina (Bert.); in Campania a Roccasecca (Terr.!), Cassino (Terr. sec. rel.), Caserta (Bert.), Napoli, la var. (Ten.), in Ischia, la var. (Guss.), in Capri (Bert.); in Basilicata a Melfi (Terr. $f l$. Vult.), e Castelgrande (Bert.); in Calabria a Cosenza (Aiuti!). In Sicilia, dove predomina la var., nasce a $\mathrm{Pa}$ lermo a S. Martino (Bivona!), a Mistretta, Militello (Guss. syn.), S. Marco (Citarda!), Noara (Munafó!), Fiumedinisi, Bronte, Catania (Guss.), frequente poi nella parte piủ meridionale dell isola, nella provincia di Noto ed anche nella vicina provincia di Caltanisetta; fa eziandio nelle Egadi (Guss.), e nella Panaria delle Lipari (Loj.). Fa infine in Malta, con la var. (Zer., Grech). Manca assolutamente in Corsica, nonchè nell' arcipelago toscano; è rarissima in Sardegna non vi è notata che dei monti di Oliena (Mor. $l l$. sard.). Fiorisce da aprile e maggio all'autunno inoltrato, e nelle parti più meridionali in primavera soltanto, da marzo a maggio.

Distribuzione geografica. - Nell'Europa media e meridionale, nell' Asia Minore e fino alla Persia, nell'Affrica settentrionale. 
Descrizione. - Pianta annua, alta da uno a un decimetro e mezzo, verde, pelosa. La radice è filiforme, lunga, flessuosa, bian. chiccia. Il fusto è erbaceo, ramoso alla base con i rami diffusi, ascendenti, semplici o biforcati, lunghi quasi quanto il fusto e come que. sto quadrangolari, con gli angoli un po' rilevati, con le facce piane, verdognolo 0 in parte sfumato di rossiccio, peloso nelle due facce opposte alternativamente negli internodii, con i peli lunghetti, bianchi, un po'curvati in giù. Le foglie inferiori sono bislunghe-obovate o bislunghe, intere 0 con pochi denti o dentato-incise, strette alla base in un picciòlo corto. Le superiori sono opposte, poco lontane, eretto-patenti, divise fin verso 0 per più della metà in tre lacinie lineari, ottuse, divergenti, hanno il margine rovesciato, sono verdi di sopra con una leggiera scanalatura dalla base fin verso il punto dove la foglia è divisa, con un leggiero solco longitudinale in ciascuna lacinia, di un verde chiaro e quasi bianchiccio di sotto ed ivi con un nervo longitudinale rilevato che manda un rametto in ciascuna lacinia un po'rilevato, sono glabre in ambedue le pagine con rari peli nel margine, i quali sono più abbondanti e più lunghi verso la base della foglia. I fiori sono solitarii, quasi sessili, all' ascella delle foglie di quasi tutta la pianta, due o tre volte più corti delle foglie medesime, quasi unilaterali. I peduncoli sono cortissimi, quadrangoJari, grossetti, verdognoli. Il calice è un po'gonfio nel mezzo, stretto in alto, angolato, verdognolo, peloso, ha cinque denti, dei quali uno superiore è quasi triangolare e molto più piccolo degli altri quattro i quali sono lanceolati e forniti di una carena ottusa, tutti sono eretti ed avvicinati alla corolla. Questa è più del doppio più lunga del calice, pelosa di fuori. Il tubo è lungo appena quanto il calice, gon. fio alla base ed ivi quasi tondo, poi un po'ristretto ed ivi di dentro con un anello di peli corti quasi orizzontale che corrisponde sotto l'inserzione degli stami, poi quasi cilindrico, appena piủ largo verso alto, e di un color bianchiccio-giallognolo. Il lembo è diviso in due labbri molto disuguali : il superiore è cortissimo con due piccoli denti triangolari, l'inferiore è grande patente, di un giallo chiaro, trifido, con le lacinie laterali piccole, bislunghe, quasi acute, con l'apice e in parte con i margini rovesciati, e con la lacinia di mezzo molte volte più grande, quasi in forma di cuore a rovescio: questa lacinia ha verso la gola diverse macchioline quasi tonde, disuguali, di colore amarante scuro o quasi nericce, e le lacinie laterali hanno ivi pure tre o due linee dello stesso colore: tra tali lacinie e quella di mezzo è una linea longitudinale sporgente, barbata per peli piuttosto corti. 
Gli stami sono quattro, due più lunghi quasi uguali alle lacinie laterali del labbro inferiore e due piủ corti, avvicinati al labbro superiore, del quale sono molto più lunghi. I filamenti sono cilindrici, bianchicci, pelosi in alto, con peli radi, lunghi e orizzontali. Le antere sono quasi tonde, aprentisi per una fessura comune alle due logge, con pochi peletti verso l'inserzione del filamento, nel resto glabre, gialle. Il pistillo è poco più lungo degli stami. piủ lunghi. L'ovario è diviso in quattro lobi, bislunghi, tondeggianti all'apice, verdognoli, glabri, lisci e ha alla base un piccolo disco circolare dello stesso colore. Lo stilo è cilindrico, bianchiccio, glabro, con pochi peli lunghi in alto. Lo stimma è bifido, con le lacinie quasi uguali, divergenti-recurve, acute, del colore dello stilo. Il frutto è quasi tondo, racchiuso dentro il calice persistente e poco più corto delle lacinie di questo, diviso in quattro carpidii bislunghi, lisci. (Parl. ms., descr. della pianta di Grumone in Lombardia).

Osservazioni. - L'Áiuga chia dei botanici italiani certamente non può essere considerata che come una varictà dell' $A$. Chamcepitys; anzi resta da indagarsi se non sia piuttosto una forma sessuale.

L'A. chia com' è intesa da Boissier (Fl. orient. 4. p. 802) è specie diversa, perenne.

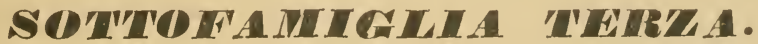

\section{SCUTELHARINER.}

Scutellarinee De Vis. fl. dalm. 2. p. 219.

Genmularium profunde fere ad basim 4-partitum. Gemmula medio loculorum insertæ, amphitropæ. Fructus cocci areola insertionis parva, laterali fere mediana. Embryo cauliculo longo, incurvo.

Osserrazione. - Altri generi della sottofamiglia sono Perilomia Humb. Bonpl. Kunth e Salazaria Torr. Sarebbe da studiarsi il genere Catopheria Benth. (in Benth. Ilook. gen.pl. 2. p. 1173), cui si dả un embrione dal fusticino ricurvo.

\section{SCUTEMAABIA.}

Cassila Tourn. inst. p. 181. 1. 8\%.

Scutellaria Linn. gen. pl.ed. 1.p. 167. Mirb. in ann, mus. 15. t. 21. 
f. 12-14. Gen. pl. fl. germ. 6.t. 22 A, B. Benth. Hook: gen. pl. 2. p. 1201. Ces. Pass. Gib. comp. fl. ital. p. 311. t. 46. f. 2 (male quoad gemmulam $h$ ).

Calyx campanulatus, obsolete nervatus, fructifer auctus compresso-clausus, demum lapsu dimidii superioris dehiscens. Corolla manifeste 2-labiata, labio superiore galeato. Stamina 4, conniventia, filamentis edentulis, antheris staminum lateralium 2-locularibus, loculis longitudinaliter dehiscentibus, a connectivo seiunctis, anteriorum dimidiatis 1-locularibus. Stylus lobis valde inæqualibus. Cocci apice convexi.

Portamento. - Erbe perenni, non molto grandi, con foglie brevemente picciolate, ovate 0 ovate-allungate, intere 0 smerlate, con fiori grandetti, turchini o porporini, spesso mischiati di bianco, solitari all'ascella di foglie fiorali o di brattee, ora distanti fra di loro, ora più o meno ravvicinati, dalla corolla lunga, dal calice cospicuo per un'appendice dorsale del suo labbro posteriore.

\section{Scutellaria mpina.}

S. procumbens, foliis ovatis, crenatis, pubescentibus, floribus in inflorescentiam brevem valde approximatis, undique versis, bracteis magnis, membranaceis, corolla $2-2 \%$ centim. longa, coccis griseo-canescentibus.

Scutellaria alpina Linn. sp. pl. ed. 1. p. 599. Mass. prodr. fl. valt. p..171. Bert. fl. ital. 6. p. 240. De Not. rep. fl. lig. p. 322. Coin. fl. com. 4. p. 383. Pir. fl. for. syll. p. 113. Ard. fl. Alpes-mar. p. 303. De Vis. Sacc. cat. piant. Ven. p. 139. Ces. Pass. Gib. comp. fl. ital. p. 312. Ing. cat. sp. Mond.p. 65. Arc. comp. fl. ital. p. 548.

Figura. - All. fl. ped. t. 26.f. 3.

Stazione, Abitazione e Fioritura. - Nei luoghi sassosi 0 arenosi delle Alpi : nelle Alpi Marittime al Frontero (Gentili!), al col di Tenda a 1900 metri (Parl.! ecc.), presso Mondovi nei territorii di Villanova e di Roccaforte dov'è comune sulle rupi calcari (Ing.), ad Entraunes (Bornet!), al col di Fenestre (Ard.), nelle Alpi Valdesi (Rostan!), a Bardonecchia (Aiuti!), nel Cenisio presso il lago (Parlatore! ecc.), nel monte Cramont a 1700 metri (Parl.!), in val d'Aosta a Courmayeur (Webb! ecc.), al San Bernardo (Heldreich!), al Col S. Teodulo a 2000 metri (Favrat!), a Champorcher sull' alpe Dondena e a Gressoney La Trinité, in Valsesia all'Ospizio di Valdobbia (Care- 
stia!), nella valle di Bugnanco nel Novarese (Bir. $f$. acon.), sul monte Codeno nel Comasco (Com.), nelle valli d'Ambria e del Livrio in Valtellina (Mass.), nel Vicentino (De Vis. Sacc.), in Friuli nel monte Cren (Bert.). Rarissima nell'Appennino vi si trova al Velino in Abruzzo (Rolli!), e al Pollino in Calabria (Ten. syll.). Fiorisce in luglio e agosto.

Distribuzione geografica. - Nella Spagna, la Francia meridionale, la Svizzera e l'Italia, la penisola Balcanica, la regione Danubiana, la Russia media e meridionale, la Siberia.

Descrizione. - Pianta alta da 1 a 2 decimetri, che fa dei graziosi cespuglietti, verde, in parte violetta, parte mollemente pelosa e pubescente, parte glabra. Rizoma lunghetto, duro, corto, ramoso; fibre radicali giallicce. Fusti molti, decumbenti e in parte radicanti, ramosi, con i rami anch'essi decumbenti, quadrangolari, in basso violetti, verdognoli in alto, pelosi, con peli lunghetti, molli e bianchi. Foylie opposte, patenti, ovate o quasi ovali, ottuse, spesso scavate un po'in cuore alla base, o ivi quasi tonde, disugualmente smerlate, verdi e con solchi, corrispoudenti ai nervi di sotto, nella pagina superiore, di un verde chiaro di sotto ed ivi con un nervo longitudinale delicato da cui partono pochi nervi laterali anche delicati, tutti sporgenti, i quali per lo piủ si biforcano presso l'apice per unirsi tra loro: sono pubescenti in ambedue le pagine e piu nei nervi della in. feriore, talvolta quasi glabre di sopra, hanno un picciỏlo corto, scanalato di sopra, convesso di sotto, pubescente peloso, di color piu chiaro: le superne sono più avvicinate tra loro, quasi sessili, spesso ovali-bislunghe. I fiori sono grandi, in spighe corte e bislunghe all'apice dei rami e del fusto, che si allungano e sono interrotte nel frutto, accompagnati da brattee molto più corte dei tiori medesimi, perỏ larghe, ascendenti, membranacee, quasi trasparenti, ovali!, acuminate! intere, un po concave alla base, rossiccio-pallido-verdognole, con molti nervi delicati e ramosi : son persistenti, piủ grandi, più con. cave di sopra nel frutto. Il calice è piccolo, rossiccio, peloso-glandoloso per peli aventi una piecola glandola all'apice, bilabiato, con i labbri corti, interi, quasi tondi, il superiore poco più grande e fornito di fuori di una appendice quasi orbicolare trasversale diretta in dietro, e un po'convessa di sopra e concava di sotto. La corolla è molto grande, di color violetto, con il labbro inferiore che diviene poi più chiaro e quasi bianco: è pubescente di fuori. Il tubo è molto lungo, un po'curvo e quasi inginocchiato alla base, poi quasi diritto e gradatanente piủ largo in alto. I labbri sono disuguali, il supe. 
riore diretto in su, trilobo, con il lobo intermedio più lungo e con. cavo quasi ad elmo, smarginato, e i due laterali corti e tondeggianti; l'inferiore è diretto in avanti ed in giù, è piủ largo, smarginato bilobo!. Stami 4, due più lunghi e due più corti, racchiusi dentro il lobo concavo del labbro superiore, inseriti nella parte superiore del tubo della corolla. Filamenti filiformi, curvati ad arco in avanti verso alto, violetto-chiari e quasi bianchicci, glabri. Antere schiacciate lateralmente, violetto-scure, uniloculari, pelose nei margini della fessura. Pistillo lungo quasi quanto gli stami più corti. Stilo lungo, curvo, glabro, filiforme, bianchiccio-violetto. Stimma lesiniforme, non diviso. Frutto racchiuso dentro al calice gonfiato e divenuto duro, con la appendice del labbro superiore molto più grande, verdognola. Achenii ovoidei, coperti di un tomento di peli stellati, corti e bianchicci. (Parl.ms., descr. della pianta del col di Tenda).

\section{Scutellaria Columnæe.}

S. erecta, foliis ovatis, crenatis, pubescentibus, floribus in inflorescentia elongata remotiusculis, secundis, bracteis parvis, herbaceis, corolla $2-2 \frac{1}{2}$ centim. longa, coccis atris, minutissime tuberculoso-hispidis.

Scutellaria peregrina Linn. sp. pl. ed. 1. p. 599 (pro maxima parte).

Scutellaria Columnæ All. fl. ped. 1. p. 40. Pucc. syn. pl. luc. p. 314. Bert.! fl. ital.6. p. 246. De Not. rep. fl. lig. p. 322. Rota prosp. piant. Pav. p. 272. Car. prodr. fl. tosc. p. 513. Tass. fl. prov. sen. p. 51. G. Bert. not. Porr. p. 48. Veg. Porr. p. 88. Terr.! fl. Vult. syn. p." 140. Sec. rel. Terra di Lav. p. 96. Ces. el. piant. Maiell. p. 24. Archb. fl. Alto-Serch. p. 60. Ces. Pass. Gib. comp. fl. ital. p. 312. Ing. cat. sp. Mond. p. 65. Terr. quarta rel. Terra di Lav. p. 114. Arc. comp. fl. ital. p. 548. Gib. Pir. fl. Mod. p. 131.

Figure. - All. o. c. t.84. f. 2. Reich. ic. fl. germ. 18.t. 56. f. 1.

$\beta$ Sibthorpii, bracteis maioribus.

Scutellaria Gussonii Ten. fl. nap. 5. p. 29.

Scutellaria Columnæ $\beta$ Sibthorpii Benth. in Cand. prodr. 12. p. 419.

Figura. - Fl. grace. t. 582.

Stazione, Abitazione e Fioritura. - Nei boschi ed altri luo- 
ghi ombrosi. Rara nell'Italia superiore, trovasi nella ripa del Vitale in Mondovi-Piazza (Ing.), a Rezzo nella Riviera di ponente (Berti!), lungo il torrente che scende a Grognardo in quel d'Acqui (All. fl. ped., Bert.), nella valletta di Erasta in val di Polcevera (Carrega !), nell'Appennino di Bobbio (De Not.), nel Modenese sui colli presso Maranello, Rotteglia, Grotta del Ventasso (Gib. Pir.), presso Bologna a Montecastello (Bert.), presso Porretta nel monte della Croce poco sopra le Donzelle (G. Bert.). Si fa molto piủ comume nell'Italia centrale, dove si trova nel lato orientale a Bagno in Romagna (Marcucci!), nel monte di S. Marino (Bert.), nel Catria (Piceinim!), nel monte S. Vicino a 1000 metri (Bucci!), tra Foligno e Colfiorito (Parlatore!), a Baregna nel Camerinese, a Fiastra nel Haceratese, a San Leonardo nel Piceno (Bert.), in Abruzzo a Pietracamela (Ber't. !), a Caramanico (Pedicino!) e nella valle Orfenta (Ces.), nel Gargano fra 500 e 1500 metri (Porta e Rigo !); e nel lato occidentale trovasi in Toscana tra Codolo e Noce nel Pontremolese (Parl.!), presso Sarzana nel monte Caprione (Bert.), alle sorgenti del Frigido nelle Alpi Apuane (P. Savi!), a Pisa nella Selva (Savi fl. pis.), e nel Monte a Rigoli! e a Pozzuolo (Calandrini!), in val di Serchio a Valdottavo (Giannini!), ai Bagni (Parl.!), a Tereglio (Giannini!) e Pratofiorito (Bert.), a Pescia (Chiostri!), intorno a Firenze in monte Senario (Grilli!) e monte Ceceri (Bechi!), alle Cascine (Bert.), e nelle valli dell'Ema! e della Pesa (Ricci!), ai Camaluoli di Casentino (Parl.!), a Montecatini di Val di Ćecina (Amidei!), a Siena (Bart. cat. piant. Sien.), nel Senese a Rapolano (Sommier!), Montisi, Campo Redaldi (Sant. viagg.), nel Massetano lungo la Milia (P. Savi!) e a Costa S. Rocco alla Sassetta (Bert.), nel monte Amiata!, a Cava presso il monte Lal)bro, a Monteti, Pitigliano (Sant. viagg.), e trovasi piủ giủ a Viterbo, Caninu (Bert.), Roma dov'è comune (Seb. Maur. R. rom. prodr.), Frascati (Parl.!), Albano (Webb!), Velletri (Rolli!), in Terra di Lavoro ad Arpino (Terr.!) e Pastena (Terr.), nel Matese, nel monte Vergine (Ten. syll.), a S. Angelo in Castellammare (Avellino!), nel Vulture ('Terr. !), in Basilieata presso Castelgrande, e infine si prulunga in Calabria a Corigliano (Guss. pl. rar.), alla Sile (Rert.), a Pizzo (Biondi !), S. Eufermia (Are.!), Anora (Ten.!), Gilloni (Guss.!), Stilo (Arc.!), e nell'Aspromonte (Bert.). Finalmente nasce in Sicilia sull'Etna (Bivona!) al Milo, a Bronte, sopra Ciaronia, e Floresta (Guss. syn.), a Castelbuono (Parl.! ece.), Ficuzza (Todaro! ece.), Buccheri, Militello di Val di Noto (Guss), Avola (lnzenga!). Fiorisce da maggio a lughlio. 
Distribuzione geografica. - Oltre l'Italia, questa specie abita la Grecia, i paesi Danubiani, e l'Affrica settentrionale (Boiss.).

Osservazione. - La var. $\beta$ è appena distinta dal tipo principale della specie ; la figura della Flora grœca rappresenta una forma estrema per la grandezza delle brattee, che spesso sono minori.

\section{Scutellaria Limnaeana.}

S. erecta vel adscendens, foliis ovatis, serrato-crenatis, glabriusculis, floribus in inflorescentia elongata remotiusculis, secundis, bracteis parvis, herbaceis, corolla $1 \frac{1}{y}$ centim. longa, coccis atris, minutissime tuberculoso-hispidis.

Scutellaria peregrina Linn. herb. (ex auct.). Bert. fl. ital. 6. p. 244. Ces. Pass. Gib. comp. fl. ital. p. 312. Arc. comp. fl. ital. p. 548 .

Figura. - Reich. ic. fl. germ. 18. t. 56. f. 3.

Stazione, Abitazione e Fioritura. - In Sicilia, nei luoghi sas. sosi, nei luoghi boschivi montuosi: Palermo a monte Cuccio (Guss. syn.), a Sagana, alla Pizzuta, a Piana de’ Greci, Castelbuono, Isnello (Parl.! ecc.), Alcamo (Sorrentino!), Caccaci, Busambra, monte de'Cani e di Cammarata, Madonie, Randazzo, Linguaglossa, Nicolosi, Spaccaforno, Modica, Siracusa, Palazzuolo (Guss.), Avola (Bianca!), Sponti, l'Etna (Bert.). Fiorisce in giugno e luglio.

Distribuzione geografica. - Trovasi inoltre in Grecia, nell'Asia più occidentale, in Abissinia.

Osservazione. - Da quanto ha scritto Linneo intorno alla sua Scutellaria peregrina, si rileva che egli ebbe principalmente in vista la pianta che poi Allioni chiamò S. Columnce; per cui si dovrebbe piuttosto applicare a questa l'altra denominazione. Ma cosi facendo si genererebbe confusione, per l'uso oramai da tutti accettato del nome di $S$. Columnce in un senso ben definito, e per la probabilità che Linneo intendesse per $S$. peregrina in parte anche quella che al presente si considera (quantunque a torto) come tale. Meglio è in questo stato di cose abbandonare un nome che potrà sempre essere controverso. Nella sinonimia della presente specie ve ne sono diversi, però tutti non pubblicati altrimenti che in schede da erbari, e poco adatti, ad eccezione di uno, S. rubicunda Horn. in Spreng. syst. veg. 2. p. 702, dove però la descrizione non corrisponde alla nostra pianta. Per cui mi è parso più conveniente proporne uno nuovo, S. Linnoana, che ricordi la parte che Linneo ha avuto nella storia della specie. 


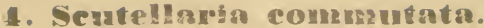

S. pallide viridis puherula, pilis brevibus, incurvis, nomnullis in racerno glandulosis, ratice librosa, ramosa, caule tetragono, ramoso, ramisque erectis, foliis oppositis, petiolatis, ovatis, obtusis, basi subcordatis, grosse crenatis, ciliatis, builatis, supra et subtus ad nervos puberulis, racemis 12-50-floris, secundis, folis florali. bus ovato-orbicularibus, apice acutis, breviter netiolatis, calyce fructifero subbrevioribus, pedicellis calyce paulo breviorıbus, subcompressis, superne crassioribus, calycis labio inferiore rotundato, integro, superiore majore, rotundato, integro, supra concavo, corolla calyce multo longiore, tubo extus puberulo, superne incrassato, labio superiore erecto, galeato, emarginato, labio inferiore patenti, trilobo, lobo intermedio majore emarginato, lateralibus, erectis cum labio superiore coalitis, apice libero, rotundato, stamimbus didynamis, exsertis, filamentis filıformibus, inferne villosis, antheris pendulis, oblongis, lateraliter compressis, subincurvis, unilocularibus, rima longe ciliatis, pistillo stamina breviora æquante, ovario gynophoro crasso basi disco inæquali cincto insidente, 4-lobo, lobis ovalibus, subrotundatis, divergentibus, styloque filitormi, glabro, stigmatis bilobi lobo superiore brevissimo, nuculis ovalibus, (fuscis), grosse obtuseque tuherculatis, tuberculis apice foveolatis et breviter pilosis. (Parl. ms.).

Scutellarıa altissima Linn.? sp. pl. ed. 1. p. 600. Ces. P'ass. Gib. comp. A. ital. p. 312. Arc. comp. A. ital. p. $5 / 8$.

Scutellaria commutata Guss. fl. sic. prodr. 2. p. 136 . Bert. fl. ital 6.p. 2.18.

Figure. - Waldst. Kit. pl. rar. Hung t. 195 Reich. ic. 1 . yerm. 18. 1.56. f. 2 (excl. 7, que certe nec Lamiacea cuiuscumque).

Abitazione e Fioritura. - Nell' Appennino centrale-meridiona. le, molto rara: nel moute Volubrio in Piceno (Narzialetti!), a Molise, nel monte Capraro (liert.), nel Monte di mezzo (Ten. /l. nap.), alla Serra S. Antunio sopra Filettino (Rollı!). Fiurisce da giugrio ad acosto.

Distribuzione geografica. - Questia sperie è molto pii dillusa nella Penisola Baleanica, nella regione Danubiana, nella Russia merillinnale fino al Caucaso.

Desorizione. - La radice è raunsa, en molte fihre llessunse, 
quasi orizzontali e giallicce. Il fusto è alto da 2 decimetri e $1 / 2$ a 3 decimetri e mezzo, compresa la infiorescenza, quadrangolare, con gli angoli acuti e un po'bianchicci, puberulo, con i peli corti, bianchi e curvati in alto, più abbondanti negli angoli che nelle facce, di un verde pallido e inferiornente sfumato di rossiccio, ramoso con i rami eretti e lunghi quanto o quasi quanto il fusto. Le foglie sono opposte, ovate, ottuse, scavate quasi in cuore alla base, con grosse crenature nel margine, cigliate segnatamente verso la base con le ciglia corte bianche e curvate verso l'apice, verdi, con prominenze come bolle e con peli rari e assai corti nella pagina superiore, di un verde molto pallido nella inferiore ed ivi glabre eccetto che nei nervi i quali son puberuli, e per lo più cinque che partendo dalla base della foglia si diramano per fare con le loro diramazioni e con le venette delicatissime una rete minuta nel parenchima: dalla sovrabbondanza di questo di fronte alle maglie e dalle diramazioni principali derivano le bolle della pagina superiore, alle quali nella inferiore corrispondono delle fossette. Tutte le foglie sono fornite di un picciòlo stretto, scanalato di sopra, convesso di sotto, puberulo per peli simili a quelli del fusto e delle foglie, lunghetto ma gradatamente più corto nelle foglie superiori. Le brattee sono opposte, patenti, ovatoorbicolari, acute, di un verde pallido e quasi glabre di sopra, di un verde più pallido e appena puberule di sotto, ed ivi con circa cinque nervetti, uno longitudinale e quattro laterali curvi, sono cigliate nel margine, hanno un corto picciòlo e sono quasi uguali o poco più corti del calice fruttifero. I fiori sono da 12 a 30 distribuiti in racemi eretti, e rivolti da una parte. La rachide è pelosetta, con i peli più lunghi e più abbondanti che nella parte inferiore del fusto e con alcuni di essi glandolosi all'apice. I pedicelli sono quasi eretti, un po'più larghi verso alto che in basso, alquanto schiacciati da avanti in dietro, poco piủ corti del calice ma molto piủ corti di questo nel frutto, verdognoli, pelosetti, con qualche pelo glandoloso. Il calice è pelosetto, con i peli parte glandolosi. Il labbro inferiore è poco meno della metà più corto del superiore, tondeggiante nel margine ed intero, di colore verdognolo; il superiore è diviso anteriormente in una parte inferiore poco grande e diretta verso il labbro inferiore, al quale si adatta chiudendosi dopo la fioritura, e in una parte superiore quasi tonda, convessa in avanti e concava di dietro, la quale s'ingrandisce dopo la fioritura: tale labbro superiore è più o meno rossiccio. La corolla è lunga circa 18 millimetri, di color violetto, piủ carico nel labbro superiore e nei lobi laterali del labbro inferiore, 
e quasi bianchiccio nel labbro inferiore e nella parte vicina anteriore del tubo: è puberula di fuori segnatamente nel tubo e nel labbro superiore, ha il tubo curvato in basso fuori del calice in modo che è ascendente, si allarga principalmente nella parte superiore. Il labbro superiore è eretto, smarginato, e il labbro inferiore è trilobo, con il lobo intermedio diretto in avanti, patente, più largo che lungo, tondeggiante in avanti ed ivi smarginato, bianchiccio e con i due lobi laterali eretti, tondeggianti all'apice e saldati in parte nel margine con il labbro superiore del quale hanno pure il colore violetto. Gli stami sono didinami, più lunghi del tubo, i più lunghi giungono sino alla smarginatura del labbro superiore, i più corti uguagliano i lobi laterali dei labbro inferiore. I filamenti sono filiformi, bianchi, pelosi inferiormente, tutti curvati all' apice in avanti e in giủ in modo che le antere sono pendenti. Queste sono avvicinate per paja, allungate, schiacciate alquanto lateralmente, un po'curve, bianchicce alla base, oppure nel resto, con lunghe e fitte ciglia bianche nella apertura dell'unica loggia che le formano. (Ho veduto tutte e quattro le antere adulte uniloculari e non come le descrive Bentham). Il pistillo è lungo quanto gli stami più corti. L'ovario è sostenuto sopra un ginoforo grosso, curvo, abbracciato alla hase da un disco piủ sporgente in avanti, tanto questo quanto il ginoforo sono glabri e giallicci: l'ovario è piccolo, diviso in quattro lobi profondi, quasi ovali, tondeggianti all'apice $e$ alla base, divergenti, glabri, giallicci. Lo stilo è lungo, filiforme, bianco, glabro. Lo stimma è bifido con le lacinie molto disuguali, essendo la superiore piccolissima e l'inferiore lunga e filiforme-lesiniforme. Le nocine sono ovali, poche, con grossi tubercoli ottusi e aventi una fossetta all'apice nella quale si vedono pochi e corti peli bianchi. (Parl. ms., descr. di pianta coltivala).

\section{ธ. Seutellaria Inastifolin.}

S. erecta vel adscendens, foliis ovato-lanceolatis, inferioribus præsertim hastatis, cxterum integris, subglabris, floribus remotiusculis, in inflorescentia elongata, inferne foliata superne bracteata (hracteis sensim minoribus, herbacris) secundis, corolla 2 centim. longa, coccis brunneis, minute tuherculatis.

Scutellaria hastifolia Linn. sp.pl. ed.1. p. 599. Pucc. syn. pl. luc. p. 814. Bert. 1. ital. 6. p. 2.42. Car. prodr. 1. tosc. p. 514. 
De Vis. Sacc. cat. piant. Ven. p. 139. Ges. Pass. Gib. comp. fl. ital. p. 313. Arc. comp. fl. ital. p. 548. Gib. Pir. Al. Mod. p. 132.

Figure. - Goll. herb. ped. t. 86. f. 2. Reich. ic. fl. germ. 18. t. $55 . f .1$.

Stazione, Abitazione e Fioritura. - Nei luoghi umidi dellæ Pe. nisola superiore e centrale, non comune : in Piemonte intorno a Villastellone (Balb.), nell'agro Milanese (Rampoldi!), nel Veneto a Monfalcone (Bert.), presso Parma a Frassinara (Passerini!), presso la Fiuma alla Rotta di Guastalla (Bert.), nelle valli di Rolo, nei dintorni di Modena, a Saliceta S. Giuliano (Gib. Pir.), nella valle di Sermide (Paglia!), presso Ferrara sopra il Buondeno, presso Bolo. gna a Monte Donato (Bert.), e al Battiferro (Gennari!) e lungo la Savena (Beccari !), presso Castelbolognese a Barrignano (Bert.), nella pineta di Ravenna presso il camposanto, presso Pisa a Castagnolo (Beccari!), presso Lucca a Porcari (Pucc.), a Colle di Compito (Bicchi !) e a Bientina (P. Savi!), presso Firenze a Poggio a Caiano!, presso Roma ad Acqua acetosa e a Ponte Molle (Seb. Maur. fl. rom. prodr.). Fiorisce da maggio ad agosto.

Distribuzione geografica. - Nell' Europa, esclusa la più meridionale e la più settentrionale, nell'Asia più occidentale.

\section{Seutellaria minor.}

S. erecta vel adscendens, foliis ovatis vel ovato-oblongis, inferioribus præsertim subhastatis, cæterum integris, pilosulis, floribus remotiusculis in inflorescentia elongata tota foliata (foliis sensim minoribus), secundis, corolla ${ }^{3} / 6$ centim. longa, coccis brunneis, minute tuberculatis.

Scutellaria minor Huds. fl. angl. p. 232 (1762). Linn. sp. pl. ed. 2. p. 835 (1763). Bert. fl. ital. 6. p. 244. Zers. prosp. piant. Bresc. p. 169. De Vis. Sacc. cat. piant. Ven. p. 139. Ces. Pass. Gib. comp. fl. ital. p. 313. Arc. comp. fl. ital. p. 548.

Figura. - Reich. ic. fl. germ. 18.t. 55. f. 3.

Stazione, Abitazione e Fioritura. - Questa rarissima specie non è stata trovata in Italia che in Piemonte lungo i fossi presso Zuhlena (All. $f l$. ped., Bert.), e alle rive del lago di Cusi verso Buscione (Bir. fl. acon.), presso Brescia verso S. Gervasio (Zersi), nel Mantorano (De Vis. Sacc.), e nel Veronese nei luoghi paludosi intorno alle risaie di Bovolone (Poll. fl. ver.). Fiorisce in luglio e agosto.

Distribuzione geografica.-Nell'Europa occidentale, in Siberia. 


\section{\%. Seutellaria galericulata.}

S. erecta vel adscendens, foliis ovato-lanceolatis basi subcorda. tis, obsolete crenatis, subglabris, floribus remotis inflorescentiam totam foliatam (foliis subconformibus) efformantibus, secundis, co. rolla $1 \frac{1}{2}$ centim. longa, coccis fulvis, minute tuberculatis.

Scutellaria galericulata Linn. sp. pl. ed. 1. p. 599. Mass. prodr. fl. valt. p. 171. Trev. prosp. fl. eug. p. 26. Pucc. syn. pl. luc. p. 314. De Not.1 rep. fl. lig. p 323. Bert. 凡. ital. 6. p. 241. Com. fl. com. 4. p. 384. Zan. prosp. fl. ven. p. 26. Rota prosp. piant. Pav. p. 272. Hausm. fl. Tir. p. 697. Rota prosp. fl. Berg. p. 70. Mor. 月. sard. 3. p. 321. Pir. fl. for. syll. p. 113. Car. prodr. fl. tosc. p. 514. Ard. fl. Alpes-mar. p. 303. Zersi prosp. piant. Bresc. p. 169. De Vis. Sacc. cat. piant. Ven. p. 139. Ces. Pass. Gib. comp. fl. ital. p. 312. Ing. cat. sp. Mond. p. 65. Anzi auct. fl. nov.-com. p. 196. Arc. comp. fl. ital. p. 548. Gib. Pir. fl. Mod. p. 131.

Figure. - Fl. dan. t. 637. Reich. ic. fl. germ. 18. t. 55. f. 2.

Stazione, Abitazione e Fioritura. - Nei luoghi paludosi, lungo le acque ferme o lentamente scorrenti. Nell'Alta Italia è comune: cosi in Piemonte lungo la Stura presso Cherasco (Ing.), al lago d’Avigliana, a Giavena e verso la Praglia di Pianezza (Re fl. tor.), presso il lago di Candia nel Canavese (Delponte!), al laghetto di S. Giuseppe presso Ivrea (Carestia!), a Oldenico nel Vercellese (Malinverni!), presso Novara lungo il Ticino e il Po, e alla Vesca (Bir. . acon.), ad Arona (Ricca!) e ad Orta sul lago Mlaggiore (De Not. !), in Lombardia intorno a Pavia a Roncaro, Linarolo, Parasacco ece. (Nocc. Balb. R. tic.), intorno a Milano (Bert.), nel Comasco al Bas. sone, nei piani di Colico e di Porlezza, nella Prabbia presso il lago di Varese (Com.) e ai laghetti d'Alserío (Parl.1) e di Mlergozzo (Anzi), in Valtellina sopra Carona (Nass.), e a Castello dell' acqua, Montagna, Traona, Berbenno, Rogolo, Ardenno (Anzi), a Bergamo (Rota), a Brescia (Zersi), a Olmeneta e Grumone nel Cremonese (Parl.!), lungo la Fiuma presso Guastalla, a Mantova (Bert.), in Tirolo a Brunecco, Bressanone, Bolzano, Gargazone, Salorno, Piné, Primolano (Hausm.), nel Veronese (Poll. fl. ver.), negli Euganei (Trev.), a Mestre (Bert.), nel Lido di Venezia (Kellner!), in Friuli a Udine, S. Daniele, Cordovado, Monfalcone (Pir.). Nel resto della Penisola è assai meno frequente: cosi in Liguria soltanto verso Tenda, a Nizza (Ard.) e ad 
Albenga (De Not.), in Toscana nella Selva pisana!, e presso Lucea ai Chiariti (Bicchi!), a Porcari (Beccari!) e al padule di Pientina (Mezzetti!), in Umhria al lago di Rieti, presso Roma a Fiumicino (Rolli!), in Abruzzo a Pescocostanzo e a Revisondoli (Ten. syll.), nel Matese alla Defanza (Terracciano!), in Calabria alla Sila (Bert.). Nasce inoltre in Sardegna presso Mamoiada, lungo la Flumendosa e alle radici del monte Corn'e boi (Mlor.). Fiorisce da giugno ad agosto.

Distribuzione geografica. - In tutta Europa, nell'Affrica settentrionale, in Asia Minore, nell'Asia settentrionale e centrale, nell'America settentrionale.

Descrizione - Pianta perenne, alta da 4 a 6 decimetri, quasi glabra, verde. Il rizoma è delicato, ramoso, bianchiccio, e manda fibre radicali egualmente delicate e bianchicce. Il fusto è procumbente o quasi eretto, ramosissimo, con i rami opposti e patenti e come questi quadrangolare, con gli angoli acuti e forniti di cortissimi peli rivolti in giù, e con le facce quasi piane o leggermente scanalate, glabro, verde. Le foglie sono membranose, molli, opposte, patentissime, alquanto avvicinate tra loro, lanceolate-allungate, scavate in cuore alla base, ottuse, leggermente crenato-seghettate, e un po'scabre nel margine con le crenature ottuse, di un verde chiaro di sopra ed ivi con solchi corrispondenti ai nervi della pagina inferiore, di un verde ancor più chiaro di sotto e ivi con un nervo longitudinale da cui partono pochi nervi laterali, curvi e rilevati, che si uniscono in rete tra loro: tali foglie hanno un picciỏlo corto, scanulato di sopra, un po'convesso di sotto, puberulo e verdognolo, assai chiaro. I fiori sono solitarii all' ascella di ciascuna foglia in modo che vi sono due fiori ad ogni nodo vitale, diretti da una parte, forniti di un peduncolo assai corto, quasi uguale o poco più corto del picciòlo, eretto o eretto-patente, cilindrico, verdognolo, il quale verso il terzo inferiore ha due bratteole, setacee, eretto-patenti, corte, verdi. Il calice è poco più lungo del peduncolo, diviso in due labbri uguali, troncati all' apice, interi, avvicinati tra loro nel margine nella parte inferiore e solo aperti all' apice per il passaggio della corolla, verdi, glabri: il superiore ha nel dorso al di sotto della metà una appendice orizzontale, larga, tondeggiante all'apice; nel frutto questa s'ingrossa, si dirige in alto, diventa lunga quanto i labbri e ha l'apice leggerissimamente crenulato, i labbri allora si chiudono interamente. La corolla è molte volte più lunga del calice, di color violetto, bianchiccia alla base e pelosa di fuori. Il tuho è curvato alla base, ascendente, gradatamente allargato verso alto. Il labbro superiore è poco 
piu corto dell'inferiore, concavo, trilobo, con il lobo intermedio smarginato e i laterali un po' più piccoli, ottusi all' apice : il labbro inferiore è patente, piủ lungo del superiore, appena smarginato, di color bianchiccio di sopra verso il centro dove ha pure dei punti violetti : la corolla di dentro è glabra. Gli stami sono quattro, poco più corti o quasi uguali al labbro superiore dal quale sono nascosti, didinami. I filamenti sono quasi filiformi, appena più grossi verso il mezzo, bianchi, saldati nella metà inferiore con il tubo della co. rolla, pelosi, segnatamente i due degli stami più corti, i quali sono meno curvi degli altri due, anżi quasi diritti per essere posteriori, mentre quelli vengono dalle parti laterali della corolla stessa: tutti sono curvati all'apice in modo che le antere sono quasi pendenti. Queste sono biloculari, con le logge un po'distanti, divise da un connettivo sul quale si vedono alcune glandolette bianche e grossette: le logge si aprono per una fenditura longitudinale ed hanno dei peli nel margine di questa e segnatamente all'apice e sono rossicce; una delle logge è rudimentare nelle antere degli stami più lunghi. Il pistillo è lungo quanto gli stami. L'ovario poggia sopra un disco grande, circolare, giallo, ottuso: è piccolo, diviso profondamente in quattro lobi tondi, come papillosi quando si guardano con una forte lente, e bianchi. Lo stilo esce dal mezzo dei lobi, è lungo, filiforme, ascendente, bianco, glabro. Lo stimma è acuto e irregolarmente bifido. (Parl. ms., descr. della pianta di Olmeneta in Lombardia).

\section{FAMIGLIA TERZA.}

\section{VIIE IE NACEE.}

Viticres Juss. gen. pl. p. 106.

Verbevace, Juss, in ann. mus. 5. p. $25 \%$.

Gemmularium 2-4-loculare, subintegrum, gemmulis binis collateralihus, vel solitariis, erectis anatropis, vel ascententihus hemia. natropis, cum raphe interiori. Fructus Irupa, aut hacca, ant polycoccus. Semen subexamygdalosum. Embryo radicula infera.

Desorizione. - Le pochissime Verhenacee nostrali sono erhe, 
annue o perenni, ad eccezione di una ch'è un frutice, anche un alberetto.

Le foglie sono composte digitate in quest'ultima, semplici nell'altre, dentate o maggiormente divise. Sono sempre opposte, e senza stipole.

I fiori sono piccoli, di color carnicino o turchiniccio, cortissimamente pedicellati, in spighe allungate $o$ contratte, $o$ in dicasi contratti disposti a spiga.

Il perianzio è 5-mero. Il calice è gamosepalo, regolare o irregolare, aperto nella preflorazione, persistente. La corolla è leggermente irregolare 2-labiata, il labbro posteriore essendo più piccólo e 2-lobo, l'anteriore più grande e 3-lobo. É embriciata nella preflorazione, caduca.

Gli stami sono 4, inseriti alla medesima altezza e allora i posteriori più lunghi, o questi inseriti più in alto e allora tutti di uguale lunghezza; rinchiusi, o sporgenti. Le antere sono attaccate per il dorso, sono ¿-loculari, deiscenti per lungo inentro. Il polline è pieghettato.

Il gemmulario è 2-loculare, o reso 4-loculare per la formazione di setti secondari, con 102 gemmule pér casella. Lo stilo è smarginato o brevemente 2-lobo.

Il frutto è drupaceo, o dirompente 2-4-cocco.

Il seme è pressochè senza mandorla, con embrione diritto, di radichetta infera.

Considerazioni geografiche. - Le Verbenacee, famiglia più pro. priamente tropicale, non sono rappresentate in Italia che da 4 specie di 3 generi, delle quali 1 largamente sparsa per i paesi temperati, 1 medesimamente per i paesi caldi, e 2 del bacino Mediterraneo.

\section{TRIBU் PRIMA.}

\section{Werbenee.}

Verbenea Dum. anal. des fam. p. 22. Schaner in Cand. prodr. 11. p. 525.

Gemmulæ e basi loculorum erectæ, anatropæ. 


\section{IIPIA A .}

Lippia Houst. ex Linn. gen. pl. ed. 1. p. 347. Benth. Hook. gen. pl. 2. p 1143. Ces. Pass. Cib. comp. fl. ital. p. 328. $t 49$.

Gemmularium biloculare, bigemmulatum. Fructus dicoccus.

Portamento. - Vedasi la descrizione dell'unica specie.

\section{Lippia nodiflorer.}

Lippia nodiflora Mich. fl. bor.-amer. 2.p. 15. Schauer in Cand. prodr. 11. p. 585. Car. prodr. fl. tosc. p. 499.

Verbena repens Pucc. syn. pl. luc. p. 326.

Zappania repens Bert. fl. ital. 6. p. 265. De Not. rep. fl. lig. р. 332.

Lippia repens Mars. cat. pl. Cors. p. 116. Ces. Pass. Gib. comp. fl. ital. p. 328. Are. comp. fl. ital. p. 561 .

Figura. - Fl. grcec. t. 553.

Stazione, Abitazione e Fioritura. - Nei prati arenosi umidi del littorale della Penisola, di Sicilia e di Corsica: presso Sarzana alla Marinella (Bert.), al Lago di Massaciuccoli (Bicchi!), e al vicino Confine (Pucc.), nella Selva pisana al Gombo!, presso S. Piero in Grado (Savi bot. etr.) e presso Coltano (Bert.), verso Livorno a Stagno (Savi); in Terra di Lavoro a Castelvolturno (Terracciano!), a Napoli a Bagnuoli, al Fusaro (Ten. syll.) al Pascone, al Licola (Pa. squale! ecc.), al Sebeto (Heldreich!); sull'Ionio a Cotrone, a Gallipoli (Ten.), presso Otranto ad Alimini; in Sicilia a Narsala (erb. Fior.!), a Palermo a S. Ciro (Todaro!), a Mondello e presso il fiume Oreto (Guss. syn.), presso Catania (Parl.! ecc.), a Lentini, nella marina di Noto, di Spaccaforno, di Scicli (Guss.); in Corsica al Capo Corso, di faccia all' isola Giraglia (Mars.). Fiorisce da giugno a set. tembre.

Distribuzione geografica. - Pianta latamente diffusa per i paesi raldi, nasce in Spagna, nell'Oriente, nell'Affrica settentrionale e tropicale, nell'Asia tropicale, in Cina, al Capo, nelle Americhe.

Descrizione. - Erba strisciante, in modo da far prato. Fusto pienn. Foglie opposte, dentate, carnosette, coperte come tutta la pianta da peli minutissimi, rigidl. Capolini lungamente peduncolati; 
peduncolo eretto, alto circa mezzo decimetro, nudo; brattee pao. nazze, rombee-cuneate, concave, submucronate. Calice membranoso, lungo $1 \frac{1}{2}$ millimetri, pelosetto, compresso, 2-carenato, 2-partito. Corolla : tubo cilindrico, poco più lungo del calice; lembo sporgente, bianco e poi rosseggiante, metà più corto del tubo, 2-labiato, dal labbro posteriore più piccolo 2-fido, dall' anteriore 3-partito coi lobi arrotonditi. Stami 2 posteriori inseriti alla metà del tubo, 2 anteriori presso alla sua bocca, dai filamenti cortissimi, dalle antere gialle, arrotondite, introrse, 2-loculari. Gemmulario pressochè globoso, glabro; stilo filiforme, corto, con stimma a capocchia e smarginato (Descr. della pianta di Pisa).

Osservazione. - Come bene notò lo Schauer o. c. p. 586 , la pianta europea non differisce neanco come varietà dalla estraeuropea, checchè abbia scritto in contrario il Bertoloni (Amoen. ital., p. 74).

\section{I1. VEIR IB ENA.}

Verbena Tourn.inst. p. 200. t. 94. Benth. Hook. gen. pl. 2. p. 1146. Gen. pl. fl. germ. 6. t. 50. Ces. Pass. Gib. comp. fl. ital. p. 327. t. 49.

Gemmularium 4-loculare, 4-gemmulatum. Fructus 4-coccus.

Portamento. - Erbe, l'ura perenne eretta, l'altra annua slraiata, con foglie lobate, e spighe lunghe sottili di fiori piccolini, di color gridellino.

\section{Verbenn offeinalis.}

V. perennis, suberecta, foliis subpinnatifidis, spicis paniculatis.

Verbena officinalis Linn. sp. pl. ed. 1. p. 20. Bert. fl. ital. 6 . p. 260 ; et auct. omn.

Figure. - Savi mat. med. tosc. t. 52. Reich. ic. fl. germ. 18. t. 91 .

Stazione, Abitazione e Fioritura. - Comunissima ovmnque nei luoghi erbosi, lungo le strade, ecc. In Tirolo è notata a 3200 piedi sotto il Ritten (Hausm. fl. Tir.); in Toscana sale fino alle parti medie dei monti. Delle piccole isole fa in quelle di Capraia (Mor. De Not. $f$. Capr.) e dell' Elba!, di Maddalena e di S. Stefano (Genn. $f$. 
Capr.), d'Ischia (Guss. enum.) e di Capri (Pasq. fl. ves.), di Ustica (Calcara!), di Lipari (Mandralisca!), di Favignana e di Pantellaria (Guss. syn.), infine di Malta (Grech! ecc.). Fiorisce dalla primavera all'autunno.

Distribuzione geografica. - In tutta Europa, meno la più nordica, nell'Affrica settentrionale, nell'Asia occidentale, nell' India settentrionale, in Giappone, nonchè in Australia, al Capo e nelle Americhe, ivi probabilnente naturalata.

Descrizione. - Pianta alta da 3 a 6 decimetri, di un verde sçuro. La radice è fusiforme, ramosa, flessuosa, bianchiccia. Il fusto $\dot{e}$ eretto, quadrangolare, con gli angoli rilevati nelle parti superiori, bianchi e scabri per rari e corti denti, con due delle facce opposte leggermente scanalate, verile scuro, ramoso, con i rami opposti, e distribuiti quasi in quattro linee longitudinali, eretto-patenti, più corti o quasi uguali al fusto. Le foglie sono opposte, patenti e curvate un po' ad arco in giù, di esse quelle del mezzo sono tripartite, con le lacinie disugnali, la intermedia piủ grande, quasi romboidale, tutte e tre incise e irregolarmente crenate, le superiori sono bislunghe o quasi lanceolate, crenate o inciso-crenate; tutte le foglie sono di un verde scuro di sopra ed ivi con profondi solchi in rete, strette alla base quasi in un picciỏlo, di un verde un po'chiaro di sotto, ed ivi con un nervo longitudinale rilevato, da cui partono pochi nervi laterali i quali si diramano per terminare all' apice dei denti delle lacinie e formano insieme una rète rilevata nella pagina inferiore della foglia. I fiori sono molti, piccoli, distribuiti in spighe lineari, strette, dapprima fitte, poi interrotte, nei rami e nei rametti di tutta la pianta: si aprono a pochi per volta, in modo che non vi sono aperti che uno, due, tre o al più quattro fiori in ciascuna spiga, mentre i fiori dell'apice di questa (ivi fitta) sono in boccia e la spiga si allunga in basso a seconda che passano i fiori e cadono le corolle di questi. La rachide è quadrangolare, con gli angoli spesso violetti, peloso-glandolosa. Ciascun fiore ha una brattea, piii corta del calice, avvicinata a questo, ovata, acuminata, un po' convessa nel dorso, verdognola, pelosetta e in parte grlandolosa, con ciglia non ylandolose nel margine. Il calice é quasi diritto, tubuloso, diviso, quasi quadraugolare, con gli angoli convessi, verdognolo, pelosetto, con alcuni peli glundolosi, con quattro denti eretti, quasi acuti. La corolla é più del doppio piủ lunga del calice, di color violetto assai chiaro, ha il tubo più lungo di questo, un po'curvo, quasi cilindrico in basso, un po'gontio nella parte che 
sporge dal calice, puberulo di fuori ed anche di dentro, e più con un anello di peli lunghi, e quasi barbato di dentro nella gola: il suo lembo è slargato quasi in imbuto, con cinque lobi disuguali, due superiori un po'più corti dei tre quasi inferiori, tutti un po'patenti, quasi orbicolari o come troncati all' apice. Gli stami sono quattro, due un poco più alti degli altri due, inseriti nella parte superiore del tubo della corolla. I filamenti sono filiformi, corti, glabri. Le antere sono quasi tonde, smarginate ad ambe le estremità, inserite appena so. pra della base, introrse, biloculari, verdognole, glabre. Il pistillo è poco più corto degli stami. L'ovario è ovato, ottuso, con quattro leggieri solchi longitudinali, verdognolo, glabro, liscio. Lo stilo è poco più lungo dell' ovario, eretto, un po'flessuoso, come angolato e un po' piủ grosso in alto, verdognolo, glabro. Lo stimma è quasi in capolino, come lobato, bianchiccio. Il frutto è eretto, nascosto dal calice persistente, meno l'apice che apparisce in mezzo all'apice dei denti del calice, appena più corto di questo : si divide in quattro carpelli i quali sono cilindrici, ottusi, con poche righe longitudinali nella parte esterna e con righe oblique nelle parti laterali ed interna, tutte rilevate ed ottuse, giallicci con l'apice rossiccio e contengono un solo seme. (Parl. ms., descr. di pianta di Lombardia).

\section{Verbena supina.}

V. annua, subdecumbens, foliis subbipinnatifidis, spicis subsolitariis.

Verbena supina Linn. sp. pl. ed. 1. p. 21. Bert. fl. ital. 6. p. 263. Mor. fl. sard. 3. p. 342. Ces. Pass. Gib. comp. fl. ital. p. 327. Arc. comp. fl. ital. p. 561.

Figure. - Fl. grcec. t. 554. Reich. ic. fl. germ. 18. t. 91.

Stazione, Abitazione e Fioritura. - In luoghi campestri, o in. colti, dell'Italia meridionale: in Calabria ultra, in Basilicata, a Lecce (Ten. syll.); in Sicilia a Dorillo presso Terranova, Spaccaforno (Guss. syn.), Avola (Bianca!), Agosta (Bert.), Lentini (Guss.), Catania (Guss.!), Palermo a monte Cuccio (Todaro!); in Sardegna presso Sedilo (Mor.). Fiorisce da maggio all'autunno.

Distribuzione geografica. - Pianta del bacino mediterraneo, si estende dalle Canarie al Caucaso. 


\section{TRIBU் SECONDA.}

Viticee.

Vitice※ Dum. anal. des fam. p. 22.

VITEÆ Schauer in Cand. prodr. 11.p. 620.

Gemmulæ ex angulo centrali loculorum adscendentes, hemianatropæ.

\section{VITEX.}

Vitex Tourn. inst. p. 603.t.373. Benth. Hook. gen. pl. 2. p. 1154. Ces. Pass. Gib. comp. R. ital. p. 327. t. 49.

Gemmularium 2-loculare, 4-gemmulatum. Fructus drupaceus.

Portamento. - Vedasi la deserizione della specie nostrale.

Osservazioni. - Ritengo che il gemmulario sia 2-loculare e non sembri 4-loculare (come generalmente si descrive) che per gli spermofori sporgenti tanto da toccare la parete delle caselle.

Non conosco altro che frutti sterili nel Vitex Agnus-castus. Sono globosi, larghi 3 millimetri, asciutti; l'interno è costituito da un ammasso di tessuto bianco, cuoioso, dentro al quale male si distinguono le caselle quasi scancellate, con rimasugli riseccati di gemmule.

\section{Vitex Aguus-Castus.}

Vitex Agnus-castus Linn. sp. pl. ed. 1. p. 698. Zer. R. mel. thes. p.78. Trev. prosp. A. eng. p 25. De Not. rep. Al. lig. p. 389. Bert. A ilal. 6. p. 455. Grech Del. 1. mel. p. 27. Guss. enum. pl. Inarim. p. 2j2. Pir. R. for. syll. p. 115. Mor.! 1. sard. 3. p. 34.3. Car. prodr. 1. lose. p. 499. Tassi fl prov. sen. p. 50 Ard. cat. pl Ment. p. 30. Car. 17. Montecr. p. 28. Ard. 11. Alpes-mar. p. soli De Vis. Sacc cat. piant. Ven. p. 1:1/. Pasq. /l. ves. p. 78. Terr. II Vult. syn. p. 134. Cenu. 1. Capr. p. 110. Mars. cat. pl. C'orse p. 116. Terr. sec. rel. Terra di Lav. p. 9.4. Cies. Pass. Gib. comp. R. ital. p. 327. Arc.! comp. fl. ital. p. 56 \%. 
Figure, - Lam. ill. t. 541. f. 1. Reich. ic. fl. germ. 18. t. 92. Moggr. contr. M. Ment. t. 14.

Stazione, Abitazione e Fioritura. - Nei luoghi umidi sul mare, nel letto dei torrenti, ecc., delle parti più calde d'Italia. Nella Penisola fa in Calabria al capo Spartivento (Arc.!) e a Reggio!, in Basilicata a Pomarico (Giordano!) e alla Rendina sotto Melfi (Terr.), nel Principato citeriore alle Marine di Agnone, a Pollica, a Casalicchio, presso Napoli a Bagnoli, Baia, Fusaro (Ten. syll.), nelle isole di Capri (Pasq.) e d'Ischia in più siti (Guss., Levier!), in Terra di Lavoro a Pontammare (Terr.), a Terracina (Fiorini!), a Ostia, a Civitavecchia (Seb. Maur. prodr.), in Toscana a Port'Ercole (Savi! ecc.), Castiglione della Pescaia (Santi viagg.), S. Vincenzo (Aiuti! ecc.), Pisa al Gombo dov'è rara!, e nelle isole dell' Elba in più siti!, di Montecristo (Watson Taylor) e di Capraia (Mor. De Not. $f l$.), nella Riviera di levante a Spezia (Caldesi!), di ponente ad Albissola (Piccone!), Pietra (Delponte!), Diano, Bordighera dove abbonda (Ricca! ecc.), Mentone e Nizza dov'è rara (Ard. ecc.); nell'altro lato orientale della Penisola fa a Barletta (Bruni!), a Giulianuova, Pedaso, Fermo (Bert.), Ancona (Piccinini !), Sinigaglia (Marzialetti!), quindi negli Euganei (Trev.) a Monselice (De Vis. Sacc.), ma forse non indigena, come neanco a Verona (Poll. $f l$.) dove pure si trova nelle siepi (Rigo !), nel Friuli presso Villaraspa ad Albaredo (Pir.), a Monfalcone e Duino (Poll.), in Istria a Trieste (Barbieri! ecc.), Parenzo (Poll.), Fiume (Rossi! ecc.). Nasce in Corsica al capo Corso (Mars.), a Bastia (Kralik! ecc.), a Calvi (Soleirol!), ad Aiaccio (Requien! ecc.) ed altrove (Mars.); in Sardegna a Caprera (Genn.), Cala d'0stia, Sulcis, S. Priamo, Carbonara, Sarrabus, Asinara (Mor.); in Sicilia, ovunque (Guss. syn.), così al Faro (Seguenza!), a Barcellona (Mallandrino!), Terranova (Todaro!), Cefalù, Termini (Parlatore! ecc.), Taormina (Nicotra!), Catania (Tornabene! ecc.), Siracusa (Guss.), Avola (Bianca!) ecc.; infine in Malta (Zer., Grech). Fiorisce da giugno e luglio a settembre.

Distribuzione geografica. - In tutto il Mediterraneo, e nell'Asia occidentale fino alla Persia.

Descrizione. - Frutice alto da 1 a 3 metri, forma dei cespugli o degli alberetti assai belli nel momento della fioritura per una gran quantità di fiori di color violetto o rosei, distribuiti in ispighe composte all'apice dei rami e dei rametti. Tronco legnoso, talvolta grossetto, ramoso, con i rami opposti, eretti o eretto-patenti, o ascendenti, gl'inferiori quasi cilindrici e i più giovani quasi quadrangolari, 

- Il prezzo di questa prima parte del Sesto Volume è di L. 10.50. Si trova in vendita presso il prof. Caruel al R. Istituto di Studi Superiori in Firenze; e presso i principali librai d'Italia.

Le altre parti dell'opera sinora comparse sono le seguenti:

VOLUNE I Lire 15. -

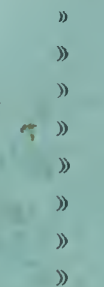

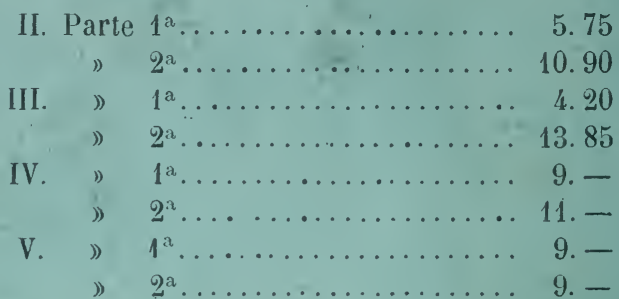

Lo sconto ai librai è del 20 per cento.

\section{NUOVO GIORNALE BOTANICO ITALIANO}

DiRetTo DA T. CARUEL.

Esce quattro volte all'anno in fascicoli che riuniti formano un volume di almeno 20 fogli di stampa, con tavole e figure nel testo. Il prezzo di associazione è di Lire 20 all'anno. Lo sconto ai librai è del 15 per cento. $\dot{\mathrm{E}}$ in pubblicazione il volume XVI.

\section{Altre Opere del prof. Caruel in vendita.}

Illustratio in hortum siccum Andreae Caesalpini. -Firenze, 1858. In-16 di pag. xi1-128......... Lire

Prodromo della flora toscana, ossia catalogo metodico delle piante che nascono selvatiche in Toscana, o che vi sono estesamente coltivate. - Firenze, 1860-64 In-8 di pag. xxir-767.............. 10. -

Guida del botaniço principiante, ossia compendio di consigli ed istruzioni per quelli che si vogliono iniziare nello studio della botanica. - Firenze, 1866.

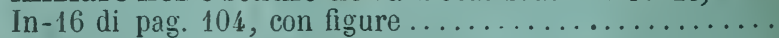

Statistica botanica della Toscana, ossia saggis di studi sulla distribuzione geografica delle piante toscane: - Firenze, 1871. In-8 di pag. 374 e una carta. .....

La morfologia vegetale. - Pisa, 1878. In-8 di pag. 433.

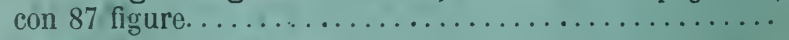

Pensieri sulla tassinomia botanica. - Roma, 1881. In-4

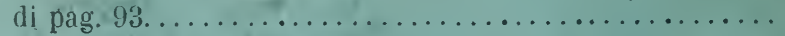

L'erborista italiano, chiave analitica per aiutare a trovare sollecitamente il nome delle piante che nascono selvatiche in Italia. - Pisa, 1883. In-16 di

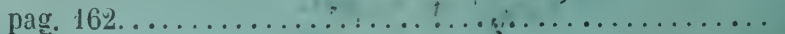




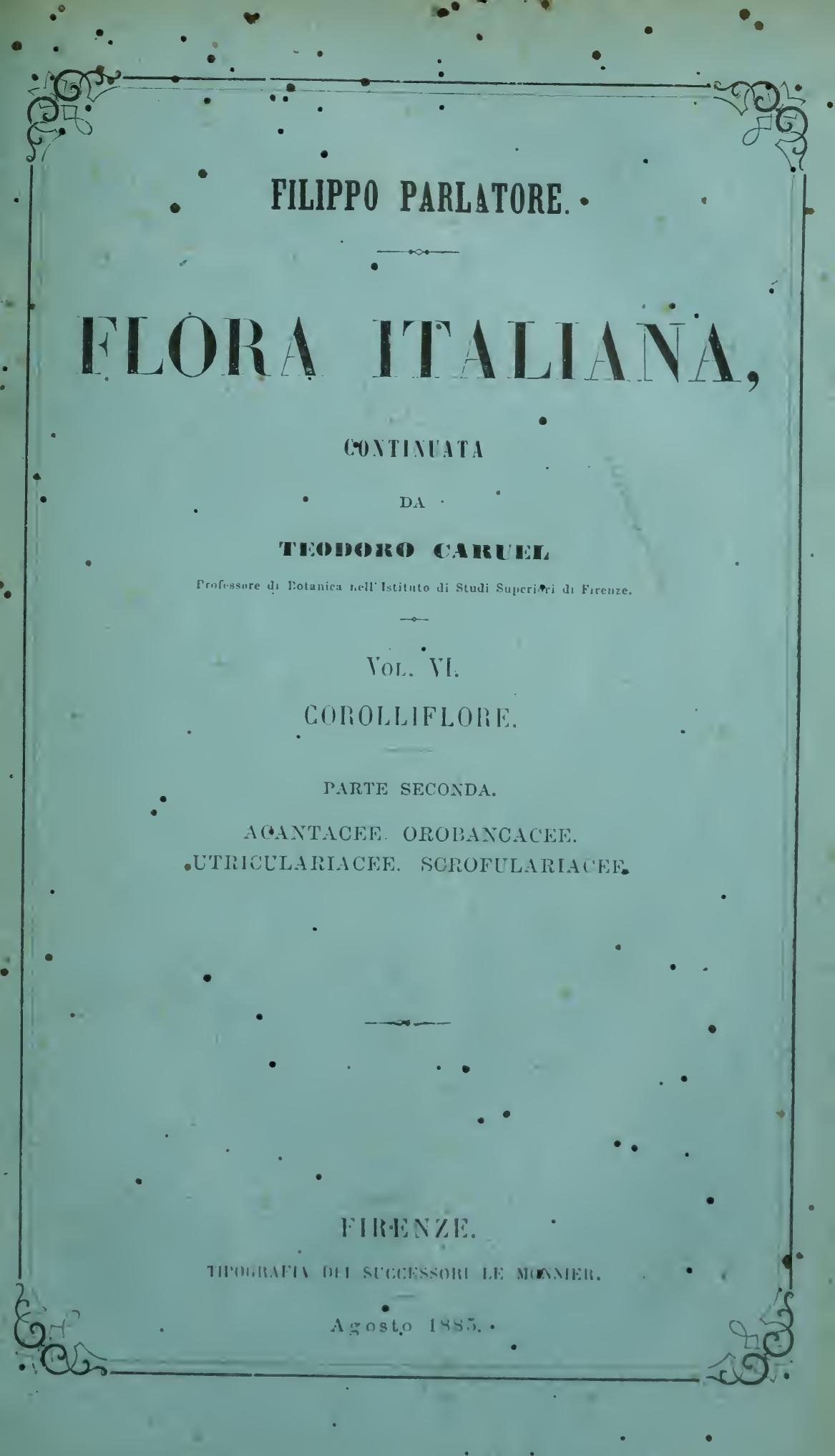



tomentosi per cortissimi peli, rossicci o verdognoli-cinerini. Foglie opposte, patenti, per lo più composte di 5 , talvolta di 5 o di 7 foglio. line, le quali sono lanceolate, acute, intere, di un verde scuro, glabre e con un solco longitudinale di sopra, e bianchicce di sotto ed ivi tomentose per peli cortissimi e fitti e con un nervo longitudinale sporgente e piú chiaro, da cui partono dei nervetti laterali i quali sono appena rilevati, si dirigono verso il margine e si diramano per unirsi in rete tra loro. Il picciòlo è piủ corto delle foglioline, con un leggerissimo solco longitudinale di sopra, tomentoso, di colore verdognolo-gialliccio. I fiori sono molti in piccoli racemi corti e laterali, formanti insieme delle spighe composte interrotte e verticillate, al. l'apice dei rami e dei rametti. I pedicelli sono corti, cilindrici, to. mentosi e hanno una bratteola lineare, stretta, scuretta. Il calice è piccolo, quasi campanulato, con cinque angoli, rerdognolo-bianchiccio o sfumato di rossiccio, tomentoso: ha cinque denti i quali sono corli, larghetti, triangolari! (non lineari Gr. e Godr), acuti ! La co. rolla è molto più lunga del calice, infundibuliforme, di color violetto o di un roseo chiaro e quasi bianchiccio, tomentoso di fuori. 11 tulso è corto, il lembo è quasi diviso in due labbri, il superiore un po'più corto, bilobo, l'inferiore trilobo, con i lobi tondeggianti. Gli stami sono 4, poco piủ lunghi del labbro superiore, didinami, inseriti nel tubo. I filamenti sono barbati nella loro inserzione, glabri nel resto, lesiniformi, curvati in alto ad areo in avanti, del colore della corolla. Antere fatte quasi a ferro di cavallo, con le logge lunghe, aprentisi separatamente, di color violetto chiaro, poi scure. Pistillo poco piủ lungo degli stami. Ovario piccolo, quasi tondo, verdognolo. Stilo lungo, eretto, un po'curvato in alto in avanti, violetto o roseo, glabro. Stimna bifido, lacinie poco disuguali, un po' divergenti, acute. (Parl. ms., descr. della pianta di monte Argentaro).

\section{FAMIGLia QUartí.}

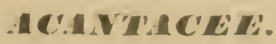

AcantiII Juss. gen. pl. p. 102.

Acanthacles Juss. 180\%.

fiemmularium.2-loculare, geınmulis septalibus, seriatis, adseen. dentibus, anatropis vel amphitropis, cum raphe subinteriori. Fructus 
capsula, rarissime drupa. Semen examygdalosum vel subexamygdalosum. Embryo radicula infera.

Descrizione. - Vedansi quelle delle specie dell'unico genere nostrale.

Considerazioni geografiche. - Questa gran famiglia è quasi esclusivamente tropicale, e non ha in Italia che 1 gencre e 4 specie a rappresentarla, tutte e quattro del bacino Mediterranco e più par. ticolarmente della sua parte orientale.

\section{ACANTITUS.}

Acanthus Tourn. inst. p. 176. Benth. Hook. gen.pl. 2. p. 1090. Ces. Pass. Gib. comp. fl. ital. p. 328. t. 48.

Portamento. - Le specie italiane del genere sono erbe perenni robuste, con un'ampia rosetta di grandissime foglie sinuate-lobate, inermi o spinose, che son quelle (dicesi) che figurano nel capitello delle colonne dell' ordine corintio. I fiori grandi, bianchicci, avvolti da grandi brattee spinose, sono addensati in grosse spighe all' estremità di scapi robusti, alti fino a 1 metro.

Osservazione. - Negli Ann. des sc. nat. $3^{\mathrm{a}}$ ser. 9 si troverà un lavoro di Planchon sulla gemmula e il seme degli Acanti; e rel Bull. de la soc. bot. de Fr. 13 un altro di Clos che spiega il meccanismo della deiscenza del frutto e della proiezione dei semi.

\section{Acanthus longifolius.}

A. foliis subinermibus, per totam longitudinem parlitis, costa late alata, lobis obtusiusculis.

Acanthus longifolius Host fl. austr. Koch syn. 7. germ. ed. 2. p. 664. Bert. fl. ital. 6. p. 460. Ces. Pass. Gib. comp. fl. ilal. p. 929. Arc. comp. fl. ital. p. 562.

Figura. - Reich. ic. fl. germ. 20.t. 193.

Abitazione e Fioritura. - In Istria presso Fiume a Pulucea (A. M. Smith!), a Volosca (Bert.), e nella baia di Pola nell' isolotio detto Scoglio degli ulivi (Koch). Fiorisce in giugno e luglio.

Distribuzione geografica. - Specie propria dell'Adriatico orientale e delle provincie Danubiane.

Descrizione. - Pianta peremne, alta circa 4 decimetri, di un 
verde chiaro, glabra o quasi glabra, quasi inerme, fornita di un rizoma il quale manda dall'apice il fusto e le foglie. Questo è eretto, grossotto, angolato, di un verde chiaro, glabro e semplice, e porta in basso una o due foglie e nel resto i fiori. Le foglie radicali sono in rosetta, esse e quelle del fusto, vicinissime alle prime, sono patenti, piủ piccole che nell'Acanthus mollis Linn., bislunghe, pennate divise, con le lacinie larghette, ovali-bislunghe 0 bislunghe, sinuato-pennatifide, o sinuato-dentate, con i denti aventi all'apice una piccola spina diretta verso l'apice della lacinia, con ciglia corte nei margini: tali lacinie sono disuguali, e la terminale è spesso piủ stretta e come lanceolata all'apice. Le foglie sono di color verde chiaro e lucenti di sopra ed ivi con solchi pubescenti che corrispondono ai nervi di sotto, di un verde piủ chiaro di sotto ed ivi in ciascuna lacinia con un nervo longitudinale spor. gente e bianchiccio dal quale partono pochi nervi laterali che vanno obliquamente verso l'apice e il margine della lacinia e terminano ai denti e fanno in parte una rete: i nervi di ciascuna lacinia partono da un nervo principale o costola della foglia ch'è molto più manifesta di sotto che di sopra, di colore bianchiccio, e che si continua in un picciỏlo corto, il quale è quasi piano di sopra, molto convesso di sotto, bianchiccio come la costola e con una stretta ala verde nel margine. I fiori sono molti, quasi opposti o opposti, un po'lontani, sessili, patenti, c distribuiti in quattro file longitudinali in una spiga bislunga, lunga circa tre decimetri. Ciascun fiore è ac. compagnato da tre brattee, uma inferiore piủ grande, poco più corta del labbro inferiore del calice, larga, ovata, acuta, convessả c un po' goblja di fuori e concava di dentro verso basso, con l'apice piano diretto in fuori, dentato-spinosa nel margine, con i denti per lo piủ tre o quattro per parte, lanceolati, stretti: è bianchiccio con nervi verdi che mandano venette in rete dello stesso colore e con $\mathrm{i}$ denti del margine verdi e con corte ciglia in tutto il margine. Le due brattee laterali sono erette, un po'ascendenti alla base, situate ai lati del calice, piii corte della lorattea inferiore, strette, lanceolato-lineari, assottigliate in basso, assottigliate di più all'apice dove terminano in una piccola spina: sono sinnili per il colore alla brattea esterra, sfumate spesso di russiccio in alto, hanno delle corte ciglia nel margine. Il calice ì profondamente diviso in due grandi labbri, uno superiore piì graude, che nguaglia o supera di poco la corolla, cli esso abbraccia in parte insieme agli stami, i convesso di sopra, concavo di daranti, ovale-bislungo, quasi troncato e con due o tre 
leggeri e larghi smerli all'apice, bianchiccio-verdognolo, sfunato di rossiccio in alto, con tre nervi longitudinali verdognolo-rossicci che giungono sino all'apice e mandano molti nervetti laterali obliqui; il labbro inferiore è poco più corto del labbro inferiore della corolla al qual è avvicinato, è bislungo-lineare, bilobo, con due lobi ottusi, ha due nervi longitudinali verdognoli-rossicci, i quali sono présso il margine, è rossiccio in alto nei lobi. La corolla ha il tubo cortissimo, barbato nella gola alla base dei filamenti, con la barba lunga e bianca; è bilabiata ma il labbro superiore manca, vi è l'inferiore il quale è più lungo del labbro inferiore e appena più corto del labbro superiore del calice, è eretto nella metà inferiore ed ivi stretto, gialliccio, con i margini larghi e di colore violetto assai chiaro, è largo patente e trilobo nella metà superiore, con i lobi quasi uguali, l'intermedio bianchiccio, smarginato all'apice, piegato in giù per la sua lunghezza, e i due lobi laterali quasi tondi all' apice, di color violetto chiaro, pendenti e avvicinati tra loro in modo da toccarsi con la faccia interna. Gli stami sono quattro, appena didinami, inseriti in basso della parte stretta del labbro inferiore della corolla e nella gola di questa; sono un po'più lunghi della parte stretta di quel labbro, un po'curvi. I filamenti sono grossi, schiacciati alquanto lateralmente; $i$ due superiori o posteriori appena più corti, curvati un po'ad arco in giủ, i due anteriori o inferiori curvati quasi ad S, tutti glabri e di un colore gialliccio sfumato di violetto chiaro. Le antere sono lineari-bislunghe, ottuse ad ambe le estremità, inserite nel dorso sopra della base presso il terzo inferiore, verdognole nel dorso, gialle e fornite di una lunga barba bianca in avanti, con una loggia che si apre longitudinalmente. Il pistillo è piủ lungo degli stami e più corto della corolla. L'ovario è conico; verdognolo, glabro, abbracciato alla base da un disco carnoso, a guisa di un anello gialliccio; è biloculare. Lo stilo è molto lıngo, curvato un po'ad arco in avanti ed in giù, un po'più largo in basso, gialliccio, glabro. Lo stimma è bifido, con le lacinie corte, un po'divergenti, ottusette, con un leggiero solco longitudinale dalla parte interna, del colore quasi dello stilo. (Parl. ms., descr. di pianta coltivata).

\section{Acanthus mollis.}

A. foliis subinermibus, inferne sectis superne fissis, lobis acutinsculis.

Acanthus mollis Linn. sp. pl. ed. 1.p.639. Zer. 1. mel. thes. 
p. 1. Bert. fl. ital. 6. p. 458. Grech Del.! fl. mel. p. 27. Guss. enum. pl. Inarim. p. 251. Mor. fl. sard. 3. p. 173. Car. prodr. fl. tosc. p. 498. Tassi fl. prov. sen. p. 50. Car. fl. Montecr. p. 27. Ard. fl. Alpes-mar. p. 306. De Vis. Sacc. cat. piant. Ven. p. 158. Pasq. fl. ves. p. 78. Terr. fl. Vult. syn. p. 133. Hars. cat. pl. Cors. p. 116. Ces. el. piant. Maiell. p. 23. Archb. 1. Alto-Serch. p. 59. Groves! contr. fl. Terra d'Otr. p. 64. Ces. Pass. Gib. comp. fl. ital. p. 329. Arc.! comp. fl. ital. p. 562. Nic. prodr. fl. mess. p. 334,

Acanthus spinulosus Host?. Ces. Pass. Gib. l. c.

Figura. - Reich. ic. fl. germ. 20.t. 190.

Stazione, Abitazione e Fioritura. - Nei luoghi sassosi ombrosi, sul margine dei campi ecc., per le parti piủ calde d'Italia, generalmente non comune e sparpagliato. Fa in Calabria a Stilo, Palizzi, Palmi (Arc.!), Pizzo (Ricca!), in Basilicata a Melfi (Terr.), presso Napoli sul Vesuvio, raro (Pasq.), ai Ponti rossi, a Baia ecc. (Ten. ${ }^{1}$ ), nonchè nelle isole di Capri (Bert., Pasq.) e d'Ischia (Guss.), in Terra di Lavoro a Sessa (Ten.), a Roma, comune (Seb. Maur.), in Toscana ad Ansidonia, nel monte Argentaro (Parl.! ecc.), a Siena!, a Firenze (Bert.), a Lucca (Calandrini! ecc.), a Viareggio (Beccari! ecc.), a Sarzana (Bert.), sempre raro, e nelle isole di Gorgona (P. Savi), di Montecristo (Taylor) e del Giglio (Bert.), a Nizza, raroe non veramente spontaneo (Ard.); e nell' altro lato orientale della Penisola fa ad Otranto (Groves!), sul Gargano presso Vico a 1-2000 piedi (Huter Porta Rigo!), in Abruzzo a Caramanico (Ces.), a Urbino (Serpieri!), a Verọna, probabilmente introdotto (Poll., Bert.), a Monfalcone (De Vis. Sacc.), in Istria a Fiume (Koch). Nasce ancora in Corsica a Bastia (Nabille!), Aleria (Soleirol!), Rogliano, Macinaggio (Mars.); in Sardegna a Sassari, Codrongianus, Oristano, Cagliari (Mor.); in Sicilia a Palermo (Parl.! ecc.), Messina (Nic.!), Mazzarino a $\mathbf{4 0 0}$ metri!, Avola dove abbonda (Bianca!), Vittoria (Aiuti!) ecc., e nelle Eolic (Guss.), in Ustica (Calcara!), nelle Egadi, in Pantellaria (Guss.); infine in Malta (Grech!). Fiorisce secondo i luoghi da marzo, aprile o maggio a giugno e luglio.

Distribuzione geografica. - Nel Mediterraneo occilentale.

Osservazioni. - Non so esattamente cosa sia l'Acanthus spinu. losus di Host, riferito in generale quale varietà all' A. mollis, e a

- Per maggiora brevili tralascierò di segnare d ora innanzi il litolo dell'oplera (non già indicata innanzi nella biblıgratia) dalla quale io abbla tralto (jualche località, bastando il nome dell'autore yer mettere sulla via per trovare l'opera stessa. 
quanto pare con ragione, se la tav. 1813 di Reichenbach o. c. ̇̀ rappresentazione fedele di quella forma.

L'A. longifolius è specie ben distinta; ma l'A. mollis e i due seguenti sembrano piuttosto forme di transizione di un medesimo tipo.

\section{Acanthus spinosus.}

A. foliis spinosis, per totam longitudinem partitis, lobis confluentibus acuminatis.

Acanthus spinosus Linn. sp. pl.ed. 1. p.639. Zer. fl. mel. thes. p. 1. Grech Del. fl. mel. p. 27. Ces. Pass. Gib. comp. /l. ilal. p. 329. Arc. comp. fl. ital. p. 562.

Figura. - Reich. ic. fl. germ. 20. t. 191 (A. spinulosus).

Abitazione e Fioritura. - $\dot{\mathrm{E}}$ indicato in Corsica dai Compendi, ma non si trova nel Catalogue di Marsilly; nasce alla Certosa di Firenze!, peraltro è da dubitarsi molto che vi sia spontaneo; nasce pure alla villa Giusti presso Verona (Rigo!), località assegnata dagli autori all'Acanthus mollis, ivi pure molto probabilmente introdotto; dove sembra veramente indigeno, si è in Malta, giusta le indicazioni di Zerapha e di Grech Delicata. Fiorisce da aprile a giugno.

Distribuzione geografica. - In Oriente.

Descrizione. - Pianta perenne, alta da 8 a 10 decimetri, verde, quasi glabra, spinosa, fornita di un rizoma grossetto, carnoso, ramoso, di un giallo scuro con cicatrici quasi romboidali un po'schiacciate da sopra in sotto per la cactuta delle foglie degli anni prece. denti, che manda delle fibre radicali grosse, carnose, del colore stesso del rizoma, e dall'apice dei rami, delle foglie ed il fusto. Questo è eretto, grossetto, quasi cilindrico, di un verde chiaro, glabro in basso, puberulo in alto nella rachide della spiga. Le foglie radicali sono da 5 a 5 , lunghe da 5 a 7 decimetri, erette o eretto-patenti, pinnatifide, con le lacinie dentato-pinnatifide, e le laciniette e i denti triangolari, acute e quasi acuminate, munite all'apice e nei piccoli denti laterali di una spina corta, delicata, bianchiccia e pungente; sono di un color verde scuro di sopra, con solchi che corrispondono ai nervi di sotto, di un verde pallido di sotto ed ivi con un nervo longitudinale grosso, rilevato, gialliccio-bianchiccio, da cui parte per ciascuna lacinia un nervo anche rilevato e dello stesso colore che si dirama nei denti o nelle laciniette per unirsi in rete larga 
e terminare nella spina; sono glabre, eccetto nei nervi di sotto dove sono puberule, e con qualche pelo anche nel nervo longitudinale di sopra, hanno un picciỏlo 3 o 4 volte più corto della lamina, grosso, quasi piano di sopra, convesso-tondo di sotto, puberulo. Le foglie del fusto sono 2 o $\overline{5}$, lontane, molto più piccole delle radicali, meno divise, con il picciòlo più corto, e le supreme sessili. I fiori sono molti, grandi, un po'lontani, sessili e patenti in una spiga lunga da 2 a 6 decimetri, ciascuno accompagnato da tre brattee, di queste una è inferiore grande, poco più corta del labbro inferiore del calice, ovata, patente con l'apice rivolto in giù, concava di dentro e convesso-gobba di fuori alla base, dentato-spinosa con i denti lunghi e lanceolati, verde, soltanto bianchiccia alla base, con nervi lon. gitudinali che si diramano lateralmente per unirsi tra loro formando una rete verde o verdognola: le due laterali sono quasi eretle, piu corte della inferiore e molto più strette di essa, quasi lineari-lanceolate, spinose all' apice e in qualche raro dente del margine anteriore verso l'apice, hanno un nervo longitudinale e nervetti laterali, il colore stesso della brattea inferiore e come questa sono puberule. Il calice è diriso in quattro lacinie assai disuguali, due laterali molto più piccole e due, una superiore e l'altra inferiore, grandi a guisa di due labbri. Il labbro superiore è poco più grande dell'inferiore, obovato-bislungo, ottusissimo o quasi troncato-dentato all'apice, convesso di sopra ed ivi puberulo, di color violetto nella parte superiore, verlognolo-bianchiccio verso la base, con cinque nervi longitudinali ramosi, dei quali i due intermedii si perdono sopra la metà, mentre gli altri tre giungono sino all'apice di tre dei denti dell'apice del labbro medesimo: è concavo di sotto e fa quasi una volta agli stami ed al petalo; il labbro inferiore è più corto e più stretto del superiore, quasi spatolato, quasi troncato-dentato all'apice e ivi bifido, slargato alla base quasi in due orecchiette bianchiccie e dentellate, nel resto simile per il colore, per i nervi e per la pubescenza al labbro superiore. Le lacinie laterali del calice sono nolte volte pii corte della superiore ed inferiore, nascoste dalle basi di queste, crette n avricinate al tubo della corolla, del quale sono quasi uğnali, ovatoorbicolari, dentellate nel margine, verdognolo-bianchicce, glabre. La corolla i grande, più lunga del labbro superiore del calice, unilabiata, con il tubo corto, largo, cilindrien, di un bianco gialliccio, shabra di fuori, con la gyola chinsa da un anello di lunghi e fitti peli diretti in dentro ed in su. Il labbro superiore manca interamente, l'interiore i largo, trilobo, con i lobi quasi tondeggianti, i laterali 
un po'piủ larghi del lobo intermedio; è stretto nella parte inferiore, bianco o in parte violetto-chiaro e quasi roseo, con molti nervi che a guisa di raggi vanno dai lati della parte stretta del labbro all'apice dei lobi di questo; è quasi glabro, eccetto nella parte stretta del labbro poco sopra dei filamenti degli stami dove è peloso-barbato per peli bianchi. Gli stami sono quattro, un po' didinami, inseriti in basso della parte stretta del labbro della corolla. I filamenti sono schiacciati lateralmente, di un bianco-gialliccio, glabri, disuguali, i due posteriori o superiori più corti, curvati appena ad arco in giù, e i due anteriori o inferiori quasi ad $\mathrm{S}$ perchè sono molto curvati in su verso l'apice. Le antere sono quasi uguali, lineari-bislunghe, ottuse ad ambedue le estremità, verdognole nel dorso, gialle verso il margine anteriore, ed ivi fornite di lunghe e folte ciglia bianche, inserite nel dorso, quelle degli stami posteriori poco più in sopra della base e quelle degli stami anteriori verso la metà, uniloculari, aprentisi per una fessura longitudinale. Il pistillo è più lungo degli stami e più corto della corolla. L'ovario è conico, verdognolo, glabro, con qualche pelo verso l'apice, abbracciato e saldato alla base da un disco, quasi un anello, stretto e gialliccio: è biloculare e contiene due(?) ovoli. Lo stilo è molto lungo, grosso, lesiniforme, un po'curvato in su, di un bianco gialliccio, glabro. Lo stimma è bifido, con le lacinie corte, lesiniformi, ottuse, un po'divergenti, del colore stesso dello stilo. (Parl.ms., descr. della pianta di Firenze).

\section{Acmuthus spinosissimus.}

A. foliis spinosissimis, per totam longitudinem partitis, lobis confluentibus acuminatis iterum partitis.

Acanthus spinosissimus Pers. syn. plant. 2. p. 179. Desf.! cat. hort. par. p. 85. Bert. fl. ital. 6. p. 461. Groves contr. fl. Terra d'Otr. p. 64. Cald. fl. fav. tent. p. 176. Ces. Pass. Gib. comp. fl. idal. p. 329. Arc. comp. fl. ital. p. 562.

A. spinosus Ten. sec. Bert.

Figura. - Reich. ic. fl. germ. 20.t. 194.

Stazione, Abitazione e Fioritura. - Nei campi della Penisola meridionale: in Calabria (Compendi), tra Lecce e Taranto, comune (Groves), a Conversano, Bari (Ten.) e Trani (Bert.), in Molise (Ten.). E stato altresi trovato dal Mlagnaguti nel Faentino al Castelluccio presso Samoggia (Cald.). Fiorisce di luglio sec. Bertoloni.

Distribuzione geografica. - Mediterraneo orientale. 


\section{FAMIGLIA QUINTA.}

OIROAHACACIEL.

Orobancioide Ee Vent. tabl. règne vég. 2. p. 292 (1799).

OrOBANCHACEA Lindl. nat. syst. bot. ed. 2. p. 287.

Gemmularium (superum) 1-loculare, gemmulis plurimis, vel raro diminutis, in spermophoris parietalibus subhorizontalibus, anatropis. Fructus capsula. Semen amygdalosum. Embryo in basi amygdali minimus.

Descrizione. - Le Orobancacee sono tutte piante paraşsite, di consistenza carnosa, di colore che varia dal bianchiccio al bruno, passando per varie tinte di violaceo e di rosso, sono glabre o pelose, i peli essendo spesso glandolosi. Esercitano il parassitismo innestando le radici a quelle di altri vegetali, sia erbacei sia legnosi. Il modo di attacco è nelle Clandestinee per succiatoi (cosi malamente chiamati) laterali delle diramazioni radicali, ossia per. radicelle ingrossate e perforanti; è nelle Orobanchee per la estremità della radicetta dell'embrione, che allungandosi nel germogliamento penetra nella radice invasa e vi si fissa, rigonfiandosi sopra il punto d'attacco e da quel rigonfiamento producendo ralici secondaric, dopo di che l'estremità opposta del germoglio si foggia a gemma, dà lateralmente appendici fogliari e crescendo in sı si trasforma in scapo fiorifero. II corpo vegetativo delle Clandestinee è un rizoma strisciante, ramoso, perenne, con polloni ascellari fioriferi, che vengono fuori di terra nella Lathrcea, e che nella Clandestina restano sotterra, non ne uscendo che i fiori; quello delle Orobanche e liopsia è uno stipite eretto, semplice, di durata necessariamente annua, almeno cosi generalmente si ritiene, abbenchè qualche osservatore ablia asserito essere talvolta perenne nella sụa base.

Le appendici fogliari sono squammose, le sotterranec carnose, anclie le epigee quando sono giovani, ma presto si riseccano. Sono sparse.

L'altezza dei fusti fioriferi, o polloni che siano, sta fra 1 decimetro e 7. Sono indefiniti, eccettochè .nella Clandestina, che lia fiori ascellari e terminali, mentrechè tutte l'altre Orobancacec li 
hanno solitari all' ascella di brattee squammose simili alle squamme fogliari.

Ogni fiore laa un pedicello, per lo piủ cortissimo, nudo o pure. con due bratteole laterali.

Il calice è tipicamente 5-mero, gamosepalo: spesso si atrofizza o abortisce il sepalo posteriore, per cui il calice risulta 4-lobato e non 5-lobato, e nelle Orobanche risulta spaccato in dietro fino alla base, ed essendo del pari spaccato in avanti viene ad essere diviso in due segmenti laterali, suddivisi in due lobi o puranche interi. $\grave{E}$ di preflorazione aperta, ed è persistente.

La corolla è gamopetala, più o meno ovviamente 2-labiata, di preflorazione embriciata, marcescente nelle Clandestinee, persistente riseccata nelle Orobanchee. È lunga da $1 \frac{1}{2}$ a $31 / 2$ centimetri.

Gli stami sono 4 didinami, essendo i 2 anteriori piu lunghi. Le antere sono biloculari, attaccate per il dorso, deiscenti inentro per fenditure longitudinali, le due caselle essendo distaccate inferiormente l'una dall'altra.

Il gineceo poggia sopra un disco carnoso, poco evidente eccettochè in avanti dov'è ingrossato. Costa di due pistilli intimamente congiunti, per modo che ne risulta un gemmulario 1-loculare, 1 stilo più o meno lungo, filiforme, curvato in avanti, e 1 stimma terminale a capocchia, intero, o smarginato-bilobo coi lobi posti lateralmente in corrispondenza sopra alle commettiture del gemmulario. In questo sonovi 2 spermofori lungo le commettiture, oppure 2 coppie di spermofori, uno da ciascun lato della commettitura. Sopra ogni spermoforo stanno moltissime minutissime gemmule; soltanto nella Clandestina sono poche.

Il frutto è una cassula bivalve, coronata dallo stilo persistente nelle Orobanche, dalla base dello stilo nella Clandestina e nella $L a$. throea, mentrechè nelle Kopsia lo stilo è caduco. Le valve si staccano per tutta la lunghezza nei tre ultimi generi, ma nelle Orobanche restano attaccate insieme in cima a motivo della persistenza dello stilo.

I semi sono piccolissimi, col guscio affossettato, di rado liscio; nel solo genere Clandestina sono grossi, e alla maturità sono lanciati fuori del frutto per l'arricciolamento in dentro delle sue valve. L'embrione è minutissimo, non misurando che una piccola frazione di millimetro in diametro. E sferico indiviso nelle Orobanchee, nelle Clandestinee ha una radicetta e due embriofilli corti ma distinti.

La struttura e la vita di queste piante sono state schiarite da 
molti lavori, fra i quali sono da rammentarsi: Guettard, Second mémoire sur les plantes parasites (Hist. de l'acad. des sc. 1746), Vaucher, Mémoire sur la germination des Orobanches (Mém. du mus. 10), Bowinan, On the parasilical connection of Lathroa Squamaria (Linn. trans. 16. 1829), Unger, Beitr. zur henntniss der parasit. Pflanz. (Ann. Wien. mus. 2. 1840), P. Savi, Sulla Clandestina rectiflora (Giorn. tosc. di sc. 1. 1813), Duchartre, Observ. sur la Clandestine d'Europe (Ann. sc.nat.20. 1843), Observ. anat. et organog. sur la Clandestine d'Europe (Mém. sav. étr. 10. 1847), Lory, Observ. sur la respiration et la structure des Orobanches (Ann. sc. nat. $3^{\mathrm{a}}$ ser. 8), Caspary, Ueber Samen, Keimung, Specien und Nührpflanzen der Orobanchen (Flora. 1854), Chatin, Anat. du Lathrcea Squamaria comparée à celle du Clandestina rectifora (Bull. soc. bot. Fr. 3), Anat. comparée des végétaux fasc. 3-5. 1856, Clos in Bull. soc. bot. Fr. 8, Rochebrune, Rech. sur le Clandestina rectiflora (Ibid. 9), Solms-Laubach, De Lathruece generis positione systematica. 1865, Ueber den Bau und die Entwicklung der Ernührungsorgane parasit. Phanerogamen (Pringsh. Jahrl. 6), Caird, Noles on the structure and the leaves of Lathraa Squamaria (Trans. bot. soc. 12), L. Koch, Ueber die Entwickluny des Samens der Orobanchen (Verh. des naturhisl. Ver. zu Heidelberg. 1876), Gilbert, On the structure and function of the scale-leaves of Lathrcea Squamaria (Journ. micr. soc. 1880).

Considerazioni geografiche. - Dei 6 generi della famiglia abitatori dell' Europa e di parti finitime, l'Italia ne possiede 4, mancando i generi Cistanche e Phelipcea.

Le specie si spartiscono presso a poco ugualmente nelle tre serie: di quelle che si trovano per tutta Italia; di quelle piu specialmente settentrionali, che nella Italia meridionale o mancano o abitano soltanto i monti; di quelle meridionali.

Sono parecchie le specie localizzate, e fra queste parecchie ritenute esclusivamente italiane. Péró, riguardo tanto alle une che alle altre nulla si potrà dire di preciso, fino a che non si sapri me. glio che cosa si debba statuire come tipo specifico nel genere dominante Orobanche. 


\section{TRIBU் PRIMA.}

Claterestinee.

Cilandestinea.

Corolla marcescens. Embryo diphyllus.

Osservazione. - Solms (De Lathraece generis positione systematica) toglie queste piante dalle Orobancacee per metterlo nelle Rhinanthacece, prese nel senso ristretto di alcuni autori come famiglia distinta dalle Scrofulariacee. Egli si fonda su differenze istologiche, criterio che io ritengo non potersi adoperare nel presente stato della tassinomia botanica ${ }^{1}$ sul diverso portamento e la diversa simmetria fogliare, caratteri invero di troppo poco momento; infine sull'embrione difillo e non indiviso, carattere che, adottato qui, dovrebbe nell' istesso ordine e contro tutte le consuetudini levare le Cuscute dalle Convolvulacee. Hooker (Fl. brit. Ind. 4. p. 246), credendo seguire Solms ma oltrepassandolo, mette il genere addirittura nelle Scrofulariacee.

\section{CHANDESTHNA.}

Clandestina Tourn. inst. p. 652. t. 424. Endl. gen. plant. p. 726. Lathræa Linn. gen. pl.ed. 1. p. 5.

Lathræa ex parte Benth. Hook. gen. pl. 2. p. 985. Ces. Pass. Gib. comp. fl. ital. p. 335.

Flores corymbosi, ebracteolati. Calyx 4-lobatus, lobis subæqualibus. Stylus basi persistens. Spermophora 2. Capsula valvis involutis. Semina perpauca.

Portamento. - Vedasi la descrizione dell'unica specie.

\section{Clandestina rectiflora.}

Lathræa Clandestina Linn.sp.pl.ed.1. p. 605. Bert. fl. ital. 6. p. 309. Ces. Pass. Gib. comp. R. ital. p. 336. Arc.! comp. fl. ital. .p. 532.

- 'Le ragioni ne sono date neì miei Pensieri sulla tassinomia. 
Clandestina rectiflora, Lam. fl. fr. 2. p. 328. Car. prodr. fl. tosc. p. 498.

Figure, - Lam. ill.t.551.f. 1. Reich. ic. fl.germ. 20. t. 144.

Stazione, Abitazione e Fioritura. - Assai rara, non è stata finora trovata che presso Lucca, parassita sulle radici dei salci e dei pioppi lungo il torrente Freddana in vari punti (Puccinelli! ece. ecc.), in Abruzzo alla Maiella, sopra Napoli nel monte Vergine all' Incoronata, in altra localitá del Napoletano detta Cerealto (Ten.), e in Calabria a Nardello (Huet!), in Aspromonte (Pasquale! ecc.), nei faggeti del monte Alto a 1600 metri, del monte Pecoraro, sopra Santa Círistina (Arc.!). Fiorisce da marzo a maggio nei luoghi bassi, fino a giugno nei più alti.

Distribuzione geografica. - Nell' Europa occidentale, dalla Spagna al Belgio, e all' Italia.

Descrizione. - La pianta $\dot{e}$ in gran parte sotterranea, restando solo fuori del terreno i fiori. Il fusto è carnoso, bianco, ramosissimo, con i rami avvicinati molto tra loro, e come questi coperto da squame opposte, embriciate, carnose, bianche, lacunose di dentro, quasi orbicolari, ottusissime, scavate alla base quasi in forma di cuore, concave e glabre. I fiori sono circa 12 in ciascun ramo, talvolta anche fino a 16 , alterni ma per la disuguale lunghezza dei peduncoli, i quali sono gradatamente più corti nei superiori, fanno un corimbo: tali peduncoli sono cilindrici, eretti, un po'flessuosi, grossetti, sfu. mati di bianco e violetto-rossiccio, glabri, gl'inferiori sono alquanto distanti tra loro e più lunghi dei fiori, i superiori più avvicinati e piủ corti di questi; ciascuno nasce all' ascella di una brattea che é simile alle squame dei rami, ma avente una lamina quasi in forma di fagiuolo, tondeggiante nelle inferiori, un poco acuta nelle superiori, bianchiccia o sfumata di violetto, ed un picciòlo che parte assai largo dal margine concavo della lamina e si restringe poi alla base, scanalato-concavo di sopra per abbracciare la base del pecluncolo: in tale cavità si raccoglie del nettare simile all' acqua. Il calice è quasi tubuloso, leggermente angolato, lungo 14 a 17 millimetri, glabro, bianco inferiormente, sfumato di violetto nel resto, diviso all'apice in quattro denti profondi, larghi, quasi acuti e diritti o un poco patuli, i quali sono valvati nell'infiorescenza. La corolla é poco meno della meta piủ lunga del calice, eretta, ringente, con il labbro superiore maggiore fatto a ğuisa di elmo, ottusissimo, smarginato-bilobo all'apice, un poco schiacciato in alto in modo che si vecle un margine ottuso convesso, di colore violetto-russiccio, e con il lab- 
bro inferiore molto piủ piccolo, lungo quasi quanto il calice, eretto, trilobo, con i lobi laterali quasi tondi e crenulati rel margine e con il lobo intermedio piủ stretto, piegato quasi in canale e con qualche piccolo dente nel margine: tutti e tre sono di color violetto-rossiccio scuro, e la parte interna del labbro è di color violetto-rossiccio chiaro. Gli stami sono quattro, quasi della stessa altezza; $\mathrm{i}$ due superiori sono inseriti in alto del tubo della corolla dalla parte del labbro superiore, e i due inferiori in basso di questo dalla parte che corrisponde al labbro inferiore. I filamenti nella loro origine hanno un ciuffetto di peli giallini quasi una piccola barba, nel resto sono filiformi schiacciati, più delicati e giallicci in basso, rossicci in alto. Le antere sono grandi, ellittico-allungate, ottuse e smarginate all'apice, bifide alla base, inseriti nel mezzo del dorso, introrse, biloculari, con le logge aventi un ciuffetto di peli in alto, forniti di pochi e lunghi peli in basso e terminate inferiormente in una lunga cresta : sono di color giallo pallido é si aprono longitudinalmente da sotto in sopra. Il polline è sferico, liscio, giallo. Alla base dell'ovario vi è un gran disco posto nella parte anteriore di esso ossia dalla parte che corrisponde al labbro inferiore della corolla, lungo quasi quanto l'ovario, di forma quasi quadrilatera, concavo posteriormente dove abbraccia la parte anteriore dell'ovario, carnoso, bianco, con il margine superiore assai grosso e ivi con cinque crenature e con un prolungamento circolare alla base che contorna la base dell'ovario. Questo è quasi lenticolare, di color bianchiccio, sfumato di violetto, glabro, uniloculare e ha una placenta longitudinale in ciascuna fáccia interna dei due carpelli che lo formano: tali placente sono rilevate a guisa di un cordone bianchiccio, rigonfiato verso la metà donde partono due oruli per ciascuna di esse, i quali sono quasi orizzontali, anatropi. Lo stilo è lungo in modo che il suo apice e lo stimma sporgono fuori della galea formata dal labbro superiore, è curvo come la galea, cilindrico, violetto-rossiccio, glabro. Lo stimma è quasi in capolino, bilobo, gialliccio, papilloso. La cassula è quasi la metả più corta del calice, persistente, obovata, smarginata quasi in cuore all'apice ed ivi fornita dello stilo persistente, un po'gonfia nelle facce, alquanto schiacciata nel margine e verso l' apice, gialliccia e sfumata di violetto scuro. Le valve sono molto elastiche e nel momento della maturità dei semi si accartocciano sopra loro stesse facendo un piccolo schioppettio e scacciano i semi a gran distanza; sono grosse, carrose e quando sono attorcigliate non si possono piủ estendere. I semi sono quattro, due per ciascuna placenta laterale, di raro 30 o 2 , 
occupano tutta la cavità della cassula, sono grossi, quasi tondi, bianchi, lisci, un po' pendenti. La testa è grossa, l'alluume è quasi corneo, bianco. L'embrione è piccolo, ovato, con la radichetta ottusa e i cotiledoni appena distinti. (Parl. ms., deser. della pianta Lucchese).

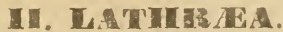

Anblatum ex parte Tourn. inst. cor. p. 48. 1.481. Squamaria ex parte Linn. gen. pl. ed. \% p. 183. Lathrea ex parte Linn. sp. pl. ed. 1. Gen. pl. A. germ. 5. t. 51. Hook. Benth. gen. pl. 2. p. 985. Ces. l'ass. Gib. comp. A. ilal. p. 335. t. 50.f. 4 .

Lathræa Endl. gen. plant. p. 727.

Flores racemosi, elbracteolati. Calyx 4-lobatus, lobis subsequa. liłus. Stylus basi persistens. Spermophora 2. Capsulá valvis extensis. Semina plurima.

Portamento. - Vedasi la descrizione della specie.

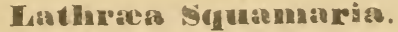

Lathraa Squamaria Linn. sp. pl. ed. 1. p. 606. Trev. prosp. 1 . eurg. p. 22. De Not. rep. R. lig. p. 305. Bert. /l. ital. 6. p. 310. Ciom. A. com. 5. p. 26. Rota mrosp. piant. Pav. p. 281. Ilausm. 1. Tir. p. 6ij2. Pir.! /l. for. syll. p. 107. Tassi fl. prov. sen. p. 50. Car. prodr. 1l. tose. p. 498. Tassi cenno bot. Sien. p. 84. De Vis. Succ. cat. piant. Ven. p. 160. Zersi prosp piant. Brese. p. 159. Ces. Pass. Gill. comp. 17. ilal. p. 396. Ing. cat. sp. Mond. p. 15. Anzi anct. R. nov.-com. p. 195. Sacc: Bizz. agg. Al. 1rev. p. 28. Gib. Pir. 1.. Hod. p. 121. Arc.! comp. Il ital. p. 532. Nic. prodr. /l. mess. p. 295.

Figure. - Fl. dan. t. 186. Gen. 11. 11. germ. 5. 1. 51. Reich. ic. 1l. yerm. 20. 1. 143 .

Stazione, Abitazione e Fioritura. - Nei boschiombrosi di lulillore a figlie caduche, parassita sulle radici del fagngio, del carfino, del nocciuolo, della queree, del noce, auche dell' olmo e dell'acero: in Pirmonte a Torino (Bertero!, Parl.!) non lungi dalla Vigna della liegina, alla Molinetla e a Mlitlefonti (Colla), presso Liastid-Mondori all' Ellero (ling.), ad Areglio (Colla), nel Novarese sul 
Po, sul Ticino e altrove (Bir.); nel Pavese, sul Ticino (Rota), presso Milano (Poll.), nel Comasco, intorno a Como all'Olmo, nella valle dei Molini (Anzi), al lago Alserio (Rampoldi!), tra Garzola e Camnago, nella val Duce sopra S. Croce, alle falde dei Corni di Canzo (Com.), nel Bresciano fra Clusane e Paratico, a Costalunga lungo il Garza e poco lungi dalla Chiesa (Zersi); in Tirolo presso Borgo a Civeron(Ambrosi!), in Fassa, presso Bolzano, a Welsberg (Hausm. ecc.); nel Baldo in valle dell' Artillon (Poll.), nel Mantovano (Barbieri!), negli Euganei (Trev.), presso Vicenza (Kellner!) verso il Lazzeretto (Poll.), a S. Michele d'Angarano nel Bassanese (Bert.), a Vittorio (Sacc. Bizz.), nel Bellunese (De Vis. Sacc.), in Friuli a Dignano (Pir.!), Fagagna, Quals (Pir.), Cimano, Suzano, Ragogna (Poll.); a Trieste, e Lippizza (Bert.); nel Modenese alle Lazze tra Fiumalbo e Pievepelago (Gib. Pir.); in Liguria all'Acqua fredda sopra Genova (De Not.), e al monte de' Sette pani (Bert.); in Toscana presso Siena al bosco della Monaca (Campani!), in Casentino a Pratorecchio e all'Alvernia!, in val Tiberina sopra Pieve S. Stefano (Cherici!); nell'Appennino umbro al Subasio (Gennari!), nel Piceno al monte Volubrio alle Svolte di S. Leonardo (Marzialetti !); presso Roma nel monte Albano (Bert.) all'Aricia (Seb. Maur.); a Roccamonfina in Terra di Lavoro (Terracciano!), a Napoli ai Ponti rossi(Pasquale!), presso S. Maria de'monti, nelle valli di S. Rocco, e di Orsolone, ai Camaldoli (Ten.); in Calabria al monte Alto a 1600-1900 metri(Arc.! ecc.); in Sicilia nei boschi di Valdemone (Guss., Nic.) e di Caronia (Bert.). Fiorisce in marzo ed aprile nei luoghi bassi, in maggio e giugno nei più alti.

Distribuzione geografica. - In tutta Europa fino alla Srezia e alla Russia media, nell'Asia occidentale fino all' Imalaia.

Descrizione. - Rizoma ramoso, come i rami cilindrico, carnoso, bianchiccio, coperto di molte squame carnose, grosse e bian. chicce, quasi cordato-orbicolari, convesse di fuori, quasi piane di dentro, che si cuoprono tra loro ad embrici. Fusto eretto, cilindrico, bianchiccio, con poche squame. Fiori molti, forniti di un pedicello corto o quasi sessili, diretti da una parte e pendenti, in una spiga fitta, pendente prima della fioritura, poi eretta, allungata, lunga da 5 a 15 centimetri, ottusa. Brattee grandi, quasi orbicolari, lunghe poco meno del fiore, bianchicce, sfumate di rossiccio, embriciate in due fila. Calice campanulato, diviso fin quasi alla metà in quattro lobi larghi, ovati, ottusi o ottusetti : è di colore quasi carneo, e peloso con i peli radi e aventi all'apice una piccola glandola. Corolla poco più 
lunga del calice, ha il tubo un po'gonfio in basso, il labbro superiore intero, convesso di fuori, concavo di sotto, di colore porporino scuro verso l'apice, il labbro inferiore è appena piủ corto del superiore, diretto in avanti, subtrilobo o trilobo, con i lobi superficiali, $\mathrm{i}$ due laterali un po'piủ larghi e tondeggianti, quello di mezzo un po' più corto e smarginato : è bianchiccio. Gli stami sono quattro, due appena piủ lunghi e due un poco più corti del labbro superiore. I filamenti sono inseriti sotto della metà del tubo della corolla, sono piủ grossi in alto, ristretti in basso, un po'curvi, bianchicci. Le antere sono scure, pelose. Il pistillo è poco più lungo del labbro superiore sotto del quale sporge. L'ovario è ovato, con un leggiero solco in avanti e in dietro, gialliccio-bianchiccio, glabro, uniloculare, ha due placente parientali da cui partono molti ovoli bislunghi, su un corto funicolo. Lo stilo è una volta e mezzo piủ lungo dell'ovario, lesiniforme, gialliccio, curvato in alto in avanti ed ivi di un color carneo porporino. Lo stimma è quasi capitato, ottusamente bilobo, porporino, papilloso. Il disco è posto in basso ed in avanti dell' ovario, principiando dalla metà della base di questo si prolunga quasi orizzontalmente in avanti e un po' in su a guisa di una linguetta carnosa, grossetta, ottusa, del colore stesso dell'ovario. (Parl. ms., descr. della pianta di val Tiberina).

\section{TRIBU் SECONDA.}

\section{Arobuneheo.}

Orobanchee (non alior.).

Corolla exsiccata persistens. Embryo aphyllus.

\section{KORSIA.}

Orobanche ex parte Tourn. inst. p. 175. 1. 81. Benth. Hook. gen. pl. 2. p. 984.

Kopsia Dum. comm. bot. p. 16 (1822), non Blume cat. Buitenz. (1823). Phelypxa C. A. .lley. in Led. fl. alt. 2. p. 459, non Tourn. o. c. cor. p. 17. t. 479 , nec Desf. in ann. mus. 10. p. 298. Gen. pl. 1. germ. 5. 1. 19. Reut. in Cand. prodr. 11. p. 4. Ces. Pass. Gib. comp. fl. ilal. p. 335. t. 49. 1. 5. 
Flores spicati, 2-bracteolati. Calyx 5-lobatus, lobis 4 subæqualibus, superiore minimo vel nullo. Stylus deciduus. Spermophora 4. Capsula valvis extensis. Semina plurima.

Portamento. - Lo stesso che nelle Orobanche, eccettochè talora lo scapo è ramoso.

Osservazioni. - Come bene hanno rilevato Bentham e Hooker (o. c. 2. p. 983), il genere Phelypcea, quale fu fondato da Tournefort, conservato da Linneo nella $1^{\text {a }}$ edizione dei suoi Genera, ripristinato da Desfontaines ( $l l . c c$.), poggia essenzialmente sulla $P$. coccinea Desf. (ascritto da autori più recenti agli Anoplanthus), e non corrisponde per conseguenza al genere Phelipcea di C. A. Meyer e dei moderni. Ritenuto questo come genere buono, e distintissimo dal vero Phelypcea, va riconosciuto col nome di Kopsia datogli da Dumortier fin dal 1822. É affine al Cistanche stabilito da Hoffmansegg e Link nella $\mathrm{Fl}$. port. 1. p. 318, ma questo differisce pei lobi del calice uguali, e per lo stilo persistente. Non v'è da tener conto della differenza di forma della corolla, perchè questa è variabile non poco nelle varie specie di Orobanche, ora evidentemente bilabiata e col labbro superiore a elmo, ora appena bilabiata coi lobi pressochè uguali e patuli.

\section{Kopsia coerulea.}

K. scapo simplici, spica laxa vel laxiuscula, lobis calycinis lanceolato-subulatis, corolla dimidio brevioribus, corolla lilacino-vio. lacea, $2-2 \frac{1}{2}$ centim. longa, tubuloso-infundibuliformi, sub medio constricta, antrorsum paullo curvata, labii inferioris lobis subacutis, antheris subglabris.

Orołsanche cœrulea Vill. hist. pl. Dauph. 2. p. 406. Ten. syll. fl. neap. p. 307. Mor De Not. fl. capr. p. 103. Bert. fl. ital. 6. p. 449. Com fl.com. 5. p. 89. Pucc. syn. pl. luc. p. 315. Rota prosp. piant. Pav. p. 270. Hausm. R. Tir. p. 652. De Vis. Sacc. cat. piant. Ven. p. 160. Zersi prosp. piant. Bresc. p. 159. Anzi auct. fl. nov.com. p. 195. Bizz. sec. agg. fl. ven. p. 7. Gib. Pir. fl. Mod. p. 123.

Kopsia cœrulea Dum. comm. bot. $p 17$.

Phelipæa cœrulea C. A. Mey. verz. pflanz. Cauc. p. 104. Trev. prosp. f. eug. $p$ 23. Guss, enum. pl. Inarim. p. 250. Car. prodr. fl. tosc. p. 494. Ard. fl. Alpes-mar. p. 288. Pasq. fl ves. p. 77. Terr. fl. Vult. syn. p. 133. Ces. Pass. Gib. comp. fl. ital. p. 335. Ing. cat. 
sp. Mond. p. 56. Loj. contr. fl. Sic. p. 16. Is. eol. p. 121. Crit. Orob. p. 30. Arc. comp. fl. ital. p. 531.

Figure, - Reich. ic. bot. t. 692, 784. Coss. Germ. atl. fl. Par. t. 19. f. K. Reich. ic. fl. germ. 20. t. 149.

Stazione, Abitazione o Fioritura - Parassita sulle Achillea e le Artemisia ed altre Asteracee: in Piemonte nei colli aridi solatii, cosi presso Torino a Moncalieri, a Pianezza, alla Veneria (Colla), a Mondovi, ovunque (Ing.), nelle valli S. Martino e d' Oulx (Rostan!), tra Foresto di Susa e S. Giuliano (Colla), a Riva di Sesia (Carestia!); a Pavia (Rota) nell'orto botanico (Nocc. Balb.), presso Como a Cam. nago (Com.), intorno a Bormio (Anzi, Rampoldi!), a Brescia nell'orto botanico (Zersi); nel Tirolo a S. Agata nel Trentino (Perini!), in Fiemme sotto Tesero presso Capriana (Bert.), a Bressanone, e in val Venosta (Hausm.); negli Enganei (Trev.), per esempio nel monte Ricco vicino a Monselice (una var. a fiori pallidi) (Bizz.); nel Modenese a Salto di Montese, e a Sparavara presso Castelnuovo ne' Monti (Gib. Pir.); nell'Urbinate al monte Cupiolo, e a Villa Grande (Ber. toloni); nel Nizzardo a S. Martino Lantosea (Ard.); in Toscana nella Selva pisana (Savi), presso Lucca alla Pieve S. Stefano (Pucc.), a Firenze lungo l'Arno alle Cascine!, e nell' isola di Capraia (Mor. De Not.); nel Piceno sul Vettore sopra S. Gemma!; presso Gaeta a Spigno, presso Napoli a Posilipo, a Fontanelle, ad Agnano (Ten.), sul Vesuvio a S. Anastasia (Pasq.), in Capri (Alioth!), in Ischia in varie località (Guss.); nel Vulture all'Incoronata (Terr.); nel Gargano, a Conversano sotto Bari (Ten.); in Calabria a Bagnara (Pasquale!); nelle Eolie a Salina sul monte Rivi (Loj.). Fiorisce in maggio, giugno o luglio secondo i luoghi.

Distribuzione geografioa. - Nell'Europa media e meridionale, nell'Asia occidentale fino alla Persia, in Siberia.

\section{Kopsia Borkhauseni.}

K. scapo simplici, spica laxiuscula vel densa, lobis calycinis lan. ceolato-subulatis, corolla dimidio brevioribus, corolla lilacino-violacea, 2-2 $1 / 2$ centim. longa, Lubuloso-infundibuliformi, sub medio constricta, recta vel antrorsum vix curvata, labii inferioris lobis obtusis, antheris subbarbatis.

Orobanche arenaria Borkh. in Raem. netues mag. bot. (1794), non Bieb. R. taur.-cauc. 2. p. 38 (1808), nec ideo Kiopsia arenaria Dum. 
comm. bot. p. 17. Rota prosp. piant. Pav. p. 270. Hausm. fl. Tir. p. 652 .

Orobanche Borkhauseni Bess. ser. Benth. in Cand. prodr. 11. p. 6 .

Phelipæa arenaria Walp. rep. bot. syst. 3. p. 459. Car. sec. suppl. prodr. fl. tosc. p. 22. Ard. fl. Alpes-mar. p. 287. Ces. Pass. Gib. comp. fl. ital. p. 335. Ing. cat. sp. Mond. p. 56. Arc. comp. fl. ital. p. 531 .

Figure. - Reich.ic. bot. t. 693, 694. Coss. Germ. at. fl. Par. t. 19. f. L. Reich. ic. fl. germ. 20. t. 145, 146.

Stazione, Abitazione e Fioritura. - Parassita sulle Artemisia, nella Penisola settentrionale e centrale : in Piemonte a Mondovi (Ing.), ad Alba (Bertero!), a Cambiano (Chevalier!); nel Pavese a Carbonara, Broni, Bobbio (Rota); in Tirolo a Gries presso Bolzano, ad Algund e S. Pietro presso Merano, a Castelbello e Laas in val Venosta, a Welsberg in Pusteria (Hausm.); nelle Alpi marittime presso Tenda, al col di Braus, tra Duranus e Figaret (Ard.). Fiorisce in maggio, giugno e luglio.

Distribuzione geografica. - Nell'Europa media e meridionale, nell'Asia Minore, nell' Affrica settentrionale.

Osservazione. - Specie appena distinta dalla precedente, checchè ne dicano le descrizioni degli autori, sempre propensi ad esagerare caratteri differenziali per rendere più ovvie le loro distinzioni specifiche.

\section{Kopsia stricta.}

K. scapo subsimplici, spica densa vel densiuscula, lobis calycinis longissime lanceolato-acuminatis, corollæ ${ }^{3} / 4$ partes æquantibus, corolla cœruleo-violacea, 2 centim. longa, tubulosa, sub medio vix constricta, antrorsum paullo curvata, labii inferioris lobis subacutis, antheris subbarbatis.

Orobanche stricta Mor.! herb. Bert. fl. ital. 6. p. 450.

Phelipæa stricta Mor.! herb. Reut. in Cand. prodr. 11. p. 7. Mor. fl. sard. 3. p. 240. Genn.! fl. Capr. p. 112. Ces. Pass. Gib. comp. fl. ital. p. 335. Loj. contr. fl. Sic. p. 16. Arc. comp. fl. ital. p. 532. Loj. crit. Orob. p. 28.

Figura. - Mor. o. c. t. 102.

Stazione, Abitazione e Fioritura. - Parassita sulla Ferula nodiflora, la Rubia peregrina ed altre piante, in Sardegna nelle isole 
Maddalena (Genn.!) e Caprera dove abbonda (Genn.), in quelle di Tavolara, Ogliastra ed altre intermedie (Mor.), nei monti di Capo. terra (Genn.!), ad Iglesias (Mor.), e in Sicilia presso Palermo a Chiarandà, rarissima, nelle Madonie a Timpe di Marfa a 1400 metri (Loj.), e a Feudo-nobile presso Terranuva (Mor.). Fiorisce da aprile a giugno.

Distribuzione geografica. - In Italia, in Algeria (Bové!, Duricu!), nell' India (Falconer!).

\section{Kopsia Iavandulacea.}

K. scapo simplici vel ramoso, spica densa vel densiuscula vel laxa, lobis calycinis lanceolato-acuminatis, corolla dimidio et plus brevioribus, corolla cœrulescente apice purpurea, $11 / 2-2$ centim. longa, tubuloso-infundibuliformi ac apice parum constricta, sub medio constricta, antrorsum curvata, labii inferioris lobis obtusissimis, antheris subbarbatis.

Orobanche lavandulacea Reich. ic. bot. 7. p. 48. Bert. fl. ital. 6. p. 451 . Grech Del. fl. mel. p. 29.

Phelipæa lavandulacea Reut. in Cand. prodr. 11. p. 7. Bicch.? agg. fl. lucch. p. 15. Car.? prodr. fl. tosc. p. 495. Ard. R. Alpes-mar. p. 288. Mars.? cat. pl. Corse p. 110. Ces. Pass. Gib. comp. fl. ilal. p. 335. Arc. comp. fl. ital. p. 531. Loj.! crit. Orob. p. 32. Nic. prodr. fl. mess. p. 338 .

Phelipæa Reuteri Mor.? fl. sard. 3. p. 343. Ces. Pass. Gib. l. c Arc. o. c. p. 532.

Figure. - Reich. o. c. t.697. Ic. fl. germ. 20. t. 147.

Stazione, Abitazione e Fioritura. - Parassita sulla Psoralea ed altre piante diversissime, nell' Italia centrale e meridionale: a Nizza a S. Rocco, Brancolar (Ard.), Vinaigrier (Sarato!), a Villafranca (Bourgeau!) al Bausrous (Ard.), a S. Remo a Mordebó (Panizzi!); presso Viareggio a Conca? (Bicch.); nel monte Argentaro (Beccari!); forse in Corsica a Bonifacio e a Portovecchio (Mars.); forse in Sardegna nei monti di Cuglieri e di Dorgali (Mor.); a Palermo al Caputo, a Buonriposo (Guss.), Sferracavallo (Loj.!) ecc., comunissima (Loj.), a Novara (Munafó!), a Messina, a Mandanici (Nic.), ad Avola (Bianca!); in Malta (Grech). Fiorisce da aprile a giugno. 
Distribuzione geografica. - In tutto il bacino del Mediterraneo, e fino alle Canarie da una parte e al Belucistan dall' altra parte.

Osservazione. - In questa specie le bratteole sono spesso ade. renti al calice e lo fanno comparire 5-6-lobato.

\section{Kopsia regyptiaca.}

K. scapo simplici vel ramoso, spica laxa vel laxiuscula, lobis calycinis lanceolato-acuminatis, corolla 2-.5-plo brevioribus, corolla cceruleo-lilacina, 2-2 $\frac{1}{2}$ centim. longa, tubuloso-infundibuliformi, sub medio constricta, antrorsum plus minus curvata, labii inferioris lobis obtusissimis interdum apiculatis, antheris subbarbatis.

Orobanche ægyptiaca Pers. syn. plant. 2. p. 181.

Phelipæa elongata Loj.! crit. Orob. p. 65.

Abitazione e Fioritura. - Trovata dal sig. Lojacono nel maggio 1882 nella pendice settentrionale del monte Gallo presso $\mathrm{Pa}$ lermo.

Distribuzione geografica. - Nell'Affrica settentrionale, nell'indicato luogo di Sicilia, nell'Asia occidentale, nell' India.

\section{Kopsia ramosa.}

K. scapo subramoso, spica laxa vel densiuscula vel densa, lobis calycinis lanceolato-acuminatis, corolla dimidio et plus brevioribus, corolla lilacina $1 \frac{1}{2}-1 \frac{2}{3}$ centim. longa, tubuloso-infundibuliformi, sub medio constricta, extrorsum plus minus curvata, labii inferioris lobis obtusis vel acutiusculis, antheris subglabris.

Orobanche ramosa Linn. sp. pl. ed. 1. p. 633. Zer. fl. mel. thes. p. 58. De Not. rep. fl. lig. p. 308. Bert. f. ital. 6. p. 452. Com. f. com. 5. p. 90. Rota prosp. piant. Pav. p. 270. Hausm. fl. Tir.p. 652. Rota prosp. fl. Berg. p. 67. Grech Del. fl. mel. p. 29. Pir. fl. for. syll. p. 107. De Vis. Sacc. cat. piant. Ven. p. 160. Zersi prosp. piant. Bresc. p. 158. Sacc. Bizz. agg. fl. trev. p. 28. Gib. Pir. fl. Mod. p. 124.

Kopsia ramosa Dum. comm. bot. p. 16.

Phelipæa ramosa C. A. Mey. verz. pflanz. in Cauc. p. 104. Trev. prosp. A. eug. p. 23. Zan. prosp. fl. ven. p. 25. Mor.l f. sard.3. p. 241. Tassi fl. prov. sen. p. 50. Car. prodr. fl. tosc. p. 495. Tassi cenno bot. Sien. p. 84. Ard. fl. Alpes-mar. p. 288. Pasq. fl. ves. p. 77. 
Genn. fl. Capr. p. 113. Ces. el. piant. Maiella p 23. Archb. fl. AltoSerch. p. 59 Ces. Pass. Gib. comp. fl. ital. p. 3.35. Ing. cat. sp. Mond. p. 56. Loj. is. eol. p. 122. Arc. comp. fl. ilal. p. 532. Loj. crit. Orob. p. 30. Cocc. fl. Bol. p. 369. Nic.! prodr. fl. mess. p. 337.

Phelipæa emarginata Loj. crit. Orob. p. 26. t. 8. f. 1.

Phelipæa olbiensis Loj. ibid. p. 31. t. 8. f. 2.

Figure. - Reich. ic. bot. t. 696. Ic. fl. germ. 20. t. 152.

$\beta$ Muteli, spicis abbreviatis densis.

Orobanche Muteli Schultz in Mut. fl. fr. Gib. Pir. l. c.

Orobanche cæsia Guss. fl. sic. syn. 2. p. 138. Ces. Pass. Gib. l.c. Arc. l. c.

Phelipæa Muteli Reut. in Cand. prodr. 11. p. 8. Guss. enum. pl. Inarim. p. 250. Mor o. c. p. 242. Ard. cat. pl. Ment. p. 28. Car. l. c. Fl. Hontecr. p. 27. Ard. fl. l. c. Genn.l l. c. Mar's. cat. pl. Cors. p. 110. Terr. sec. rel. Terra di Lav. p. 93. Ces. Pass. Gib. l. c. Loj.! is. eol. p. 121. Contr. Al. Sic. p. 16. Cald. A. fav. tent. p. 174. Arc. l. c. Loj. crit. Orob. p. 31.

Orobanche cæsia Grech Del.? fl. mel. p. 29.

Phelipæa ramosa $\beta$ et $\gamma$ Boiss. fl. orient. p. 499.

Phelipæa rufescens Loj. contr. Al. Sic. p. 17.

Phelipæa panormitana Loj.! crit. Orob. p. 25. 1. 6. f. 1.

Phelipæa Gussoneana Loj.! ibid. p. 27.

Figura. - Reich. ic. fl. germ. 20. t. 150.

Stazione, Abitazione e Fioritura. - Parassita sulla Canapa, sul Tabacco, su Faseolacee, insomma sopra una gran varietà di piante si coltivate che spontanee, piủ o meno comune per tutta quanta I'Italia. Nell'Alta Italia sale nei monti fino a 2000 ', nel monte Pastello (Bracht!). Fiorisce secondo $\mathrm{i}$ luoghi in aprile e maggio, o maggio e giugno, o giugno e luglio

Distribuzione geografica. - Nell' Europa media e meridionale, nell'Affrica settentrionale, nell'Asia occidentale, al Capo.

Osservazioni. - Come giustamente osserva Boissier l. c., le forıne di questa pianta polimorfa sono spesso difficili a spartirsi anche fra varietà. Le pieghettature pelose del labbro inferiore della co. rolla, sulle quali è stato insistito per distinguere specificamente la forma Muteli, è carattere comune a tutto il genere.

Il Bianca pare che abbia descritto nel gionale dell'Accallemia (Gioenia, nuova serie, vol. 5, una Pheliprea proboscisiyla, che da un saggio autentico risulterehbe una mostruosità di questa pianta, a fiori abortivi e chiusi. 


\section{DIROBANCIIE.}

Orobanche ex parte Tourn. inst. p. 175. t. 81. Benth. Hook. gen. pl. 2. p. 984 .

Orobanche G. A. Mey. in Led. fl. alt. 2. p. 450. Gen. pl. fl. germ.

5. t. 50. Reut. in Cand.prodr. 11. p. 15. Ces. Pass. Gib. comp. fl. ital. p. 330. t. 49. f. 4.

Flores spicati, ebracteolati. Calyx bisectus, segmentis (lateralibus) integris lobatisve. Stylus persistens. Spermophora 4. Capsula valvis ob stylum persistentem apice cohærentibus. Semina plurima.

Portamento. - E assai uniforme. Le radici essendo innestate sotterra a quelle della pianta attaccata dal parassita, da esse s'innalza uno scapo, o un fascio di scapi, eretto, alto da 1 a 5 decime. tri, semplice, spesso rigonfiato alla base ed ivi fittamente ricoperto di squamme lanciolate, le quali più diradate lo rivestono fino in cima. Nella porzione superiore dello scapo dette squamme portano all'ascella un fiore, per cui ne viene una spiga più o meno lunga, più 0 meno fitta. I fiori hanno la corolla lunga da $1 \frac{1}{2}$ a $2 \frac{1}{2}$ centimetri. Tutta la pianta è di consistenza molle quasi carnosa, ed è di un colore in tutte le sue parti, il quale varia dal giallo pallido o dal rossiccio al bruno rosso o violaceo; ed è più o meno abbondantemente provvista di peli glandolosi.

Osservazioni. - Di tutta la flora europea, il genere Orobanche sta peggio per la definizione delle specie. Qui non è, come per i generi Mentha ad esempio, o Rosa, l'abuso dell'analisi che esagerando ogni minima differenza la inalza a carattere specifico, e con la moltitudine di creazioni specifiche intangibili oscura il concetto delle specie più ovvie: è l'imperfetta cognizione dei limiti alle possibili variazioni di caratteri in una medesima pianta, che sparge la massima incertezza sul valore dei caratteri tutti quanti. Molti già si perdono coll'essiccazione, e cosi vien tolto di potere instituire completi esami comparativi sui saggi conservati in erbario; onde la necessità di fare pressochè tutti gli studi sul vivo, i quali da nessuno sono stati condotti con tanta estensione da dare risultati alquanto sicuri; anzi, senza farne colpa speciale ad alcuno, si può dire che ogni lavoro nuovo sul genere non ha fatto che accrescere la confusione. In questa condizione di cose, io non mi sono arrischiato a dare diagnosi 
specifiche, e mi sono ristretto a raccogliere le indicazioni meno fal. laci qua e là sparse per la delimitazione, la sinonimia e la distribuzione delle Orobanche italiane. In quanto ai saggi citati col segno esclamativo, tauto di questo genere quarito del precedente, io ho aruta la fortuna di poterli sottoporre alla revisione del dottor G. Beck di Vienna, autore di una monografia del gruppo, che sperasi sarà di prossima comparsa, e della quale grandemente duolmi per ragione di tempo di non potere fruire. A siffatta revisione, e a note altrettanto cortesemente comunicatemi per lettera dal dott. Beck, vado debitore della minore imperfezione di questo lavoro, rispetto a quello che altrimenti sarebbe riuscito.

\section{Orobanche gracilis.}

Orobanche gracilis Smith in linn. trans. 4. p. 172 (1798).

Orobanche cruenta Bert. rar. Ital. plant. dec. 3. p. 56 (1810). Trev. prosp. fl. eug. p. 22. De Not. rep. fl. lig. p. 306. Bert. fl. ital. 6. p. 430 (excl. syn. nonn.). Com. fl. com. 5. p. 82. Zan. prosp. fl.ven. p. 25. Pucc.! syn. pl. luc. p. 316. Sim.! fl. alp. vers. p. 138. Rota prosp. piant. Pav. p. 270. Hausm. fl. Tir. p. 647. Rota prosp. fl. Berg. p. 67. Pir. fl. for. syll. p. 106. Tassi fl. prov. sen. p. 50. Ard. cat. pl. Ment. p. 28. Car. prodr. fl. tosc. p. 495. G. Bert. veg. mont. Porr. p. 86. Ard. fl. Alpes-mar. p. 288. De Vis. Sacc. cat. piant. Ven. p. 159. Pasq. fl. ves. p. 77. Zersi prosp. piant. Bresc. p. 158. Mars.? cat. pl. Cors. p. 110. Archb. Al. Alto-Serch. p. 59. Ces. Pass. Gib. comp. 17. ital. p. 331. Ing. cat. sp. Mond. p. 54. Cald. fl. fav. tent. p. 174. Gib. Pir. f. Mod. p. 123. Arc. comp. fl. ital. p. 526. Loj. crit. Orob. p. 40. Cocc. fl. Bol. p. 370.

Orobanche grandiuscula Mor.? diagn. stirp. sard. Fl. sard. 3. p. 246.

Orobanche Levicri Loj.! contr. A. Sic. p. 14.

Figure. - Reich. ic. bot. t. 665, 667. Ic. fl. germ. 20. t. 159. $\beta$ concolor, corolla extus et intus sordide vel dilute flavescente. Orobanche procera Koch? in Röhl. deutschl. 1. 4. p. 13S. Ces. Pass. Gib. o. c. p. 33\%. Arc. o. c. p. 524.

Orobanche cruenta Coss. Germ. A. de Par. p. $309(\gamma)$ Bert.

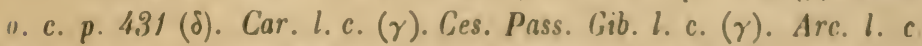

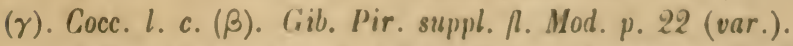

Btazione, Abitazione e Fioritura. - Parassita sulle Faseolacec 
selvatiche per i luoghi prativi e boschivi dei colli e dei monti della Penisola. Nell' Alta Italia è stata trovata nella sinistra valle del Po per i monti Valdensi (Rostan!), al Cenisio (Beccari!), a Riva di Sesia alla Balma (Carestia!), a Domodossola (Bert.), nel Cantone Ticino sul monte Generoso (Duthie!), nel Comasco a Tremezzo (Rampoldi!) e altrove (Com.), nel Bergamasco (Rota), nel Bresciano ad Urago (Lanfossi!), a Lavenone, sul lago di Garda (Zersi), nel Tirolo dov'è comune, cosi a Roveredo (Hausm.), in Valsugana (Ambrosi!), in Rendena, a Bolzano, sul Ritten fino a 5000', in val Venosta presso Laas, in Pusteria a Welsberg (Hausm.), poi nell' isola Pestrino (Bracht!) e altrove presso Verona (Poll.), negli Euganei (Trev.), nel Lido veneto (Kellner!), nel Bassanese a Oliero (Bert.), nel Friuli, ovunque (Pir.!), infine in Istria nell' isola S. Caterina (Bert.) e a Fiume (Noë! ecc.). Nella destra valle del Po è stata trovata a Mon. dovi, dov'è comunissima (Ing.), ad Alba (Bert.), nel Pavese (Rota), a Parma (Reich.), nel Modenese presso Modena, e nell' Appennino a Sestola e a S. Martino di Montese (Gib. Pir.), e altrove presso Bologna in molti siti (Bert., Cocc.), e nell'Appennino sino a Porretta (G. Bert.) e al monte Acuto (Cocc.), infine presso Faenza (Cald.!). In Liguria fa nel Nizzardo (Barla! ecc.), nel Genovesato (Bert., Baglietto! ecc.); in Toscana a Sarzana (Bert.), nella Versilia (Simi!), nell'Appennino di continuo dal monte Gotro al Teso, fino alle piủ alte cime!, presso Lucca (Calandrini! ecc.), nel monte Pisano (Beccari!), a Firenze!, in Mugello a monte Senario (Baroni! ecc.) e a Panna (Bert.), a Vallombrosa!, in Casentino a Bibbiena (Marcucci!), a Volterra (Amidei!); a Civitavecchia (Bert.); nel Napoletano a Spigno, nei monti di Frasso (Ten.), a Castellammare (Pasquale!), sul Vesuvio (Pasq.). Nell'altro lato orientale della Penisola fa nelle Marche al Catria (Piccinini!), al monte di Ancona (Narducci!), a Macerata (Bert.), ad Ascoli (Orsini!), nel Vettore!; in Abruzzo al Pizzo di Sivo (Parl.!), al Velino (Ten.); infine al Gargano (Ten., Reich.). Nasce forse in Corsica a Bastia (Mars.); in Sardegna ad Arizzo (Gennari!). Fiorisce da aprile e maggio a luglio e agosto secondo i luoghi.

Distribuzione geografioa. - Nell'Europa meridionale e media.

Descrizione. - Pianta parassita della Genista tinctoria, del Lotus cytisoides ecc., alta da 2 a 3 decimetri, pubescente-glandolosa, ros. siccia. Il fusto è semplice, eretto, un po' flessuoso, un po'angolato, ingrossato alla base ed ivi con scaglie largamente lanceolate, acuminate, che finiscono verso il terzo o la metà inferiore del fusto, pu. 
bescente glandoloso, segnatamente verso alto, per peli lunghetti, orizzontali, come articolati, bianchicci, aventi all'apice una glando. letta gialliccia. I fiori sono molti, distribuiti in una spiga un po' rada in basso, sessili, eretto-patenti. Le brattee sono poco piủ lunghe 0 quasi uguali alla corolla; ovato-lanceolate, lungamente acuminate, con lunghe ciglia bianche e glandolose nel margine verso basso, un po' convesse, con peli glandolosi e con molte righe nel dorso, concave e glabre dalla parte interna, rossicce, poi quasi scagliose. Il calice si compone di due sepali laterali, lunghi quasi quanto la corolla, ovato-lanceolati, bifidi con le lacinie quasi uguali lineari-acuminate, con molti nervi o righe longitudiuali, giallicci verso la base, rossicci in alto, peloso-glandolosi nel dorso, un po' nei margini e in alto della parte interna. La corolla è campanulato-tubulosa, appena piủ gonfia in avanti alla base, con una carena appena ad arco nel dorso, gialliccia e con pochi peli glandolosi di fuori, di color roseo-sanguigno alla gola e nella parte interna dei labbri; è divisa in due labbri uguali, frangiato-dentati e forniti di ciglia glandolose, il labbro superiore smarginato, l'inferiore trilobo, con i lobi patenti, quasi orbicolari, un po' concavi di sopra, dei quali i laterali sono quasi uguali a quello di mezzo. Gli stami sono lunghi quanto il tubo della corolla, due appena piủ alti degli altri due, inseriti alla base del tubo. I filamenti sono quasi cilindrici, un po' flessuosi e curvati in dentro all'apice, quasi glabri in basso, puberulo-glandolosi in alto, di colore quasi roseo. Le antere sono poste orizzontalmente, ottuse all'apice, bilobe alla base con una punta sporgente all' apice di ciascuna loggia, inserite nel dorso, biloculari, aprentesi longitudinalmente con una fenditura che per la situazione delle antere pare trasversale, giallicce, glabre. Il pistillo è piủ lungo degli stami e del tubo, tanto che lo stimma si vede sotto il labbro superiore. L'ovario è bislungo, quasi cilindrico, di un rosso bianchiccio, glabro, circondato alla base da un disco irregolare, come rosicchiato nel margine, e piủ sporgente in avanti. Lo stilo è una volta e mezzo piủ lungo dell' ovario, diritto, curvato in avanti all'apice, cilindrico, rossiccio, con pochi peli glandolosi. Lo stimma $\dot{e}$ grande, diretto in avanti, bilobo, con $\mathrm{i}$ lobi quasi tondi, divaricati, gialli in avanti, rossicci in dietro dove corrispondono allo stilo. (Parl. ms., descr. della pianta dell' alto Appen. nino pistoiese).

Osservazioni. - Lo stimma è ordinariamente descritto come giallo, nella descrizione di Parlatore qui sopra figura in parte giallo e in parte rossiccio, ed io l'ho veduto interamente rossoscuro. 
L'Orobanche grandiuscula di Moris parrebbe una forma meno glandolosa di questa specie; ma non ne ho veduto saggi autentici.

Riferisco alla 0 . procera dei nostri autori i saggi trovati dal dott. Levier nell'Abruzzo su varie Cinaree. Ė la forma con cui Loja. cono ha fatta la 0 . Levieri. Dicesi che la vera 0 . procera di Koch debbasi riferire alla 0 . pallidiflora Wimm. Grab.

\section{Drobanche CRapum-genistae.}

Orobanche Rapum-genistæ Thuill. fl. Par. ed. 2. p. 317. Trev. prosp. fl. eug. p. 23. De Not. rep. fl. lig. p. 305. Com. fl. com. 5. p. 83. Gren. Godr. fl. Fr. 2. p. 628. Req. in giorn. bot. 2. Rota prosp. piant. Pav. p. 270. Hausm. fl. Tir. p. 647. Rota prosp. fl. Berg. p. 67. Mor.? fl. sard. 3. p. 245. Tassi fl. prov. sen. p. 50. Car. prodr. fl. tosc. p. 496. Ard. fl. Alpes-mar. p. 288. De Vis. Sacc. cat. piant. Ven. p. 159. Mars. cat. pl. Cors. p. 110. Archb. fl. Alto-Serch. p. 59. Ces. Pass. Gib. comp. fl. ital. p. 330. Ing. cat. sp. Mond. p. 54. Gib. Pir. fl. Mod. p. 124. Arc.! comp. fl. ital. p. 524. Cocc. fl. Bol. p. 369.

Orobanche maior Bert. fl. ital. 6. p. 426. G. Bert. not. mont. porr. p. 46. Veg. Porr. p. 86. Pasq. fl. ves. p. 77. Terr. fl. Vult. syn. p. 132. Zersi prosp piant. Bresc. p. 158. Terr. sec. rel. Terra di Lav. p. 93.

Orobanche carnea Loj.! crit. Orob. p. 44. t. 6. f. 2.

Figure. - Reich. ic. bot. t. 668. Ic. fl. germ. 20. t. 157.

Stazione, Abitazione e Fioritura. - Parassitica sulla Saro. thamnus vulgaris ed altre Faseolacee frutescenti, per tutta Italia ad eccezione delle sue parti più meridionali. $\dot{\mathrm{E}}$ stata trovata in Piemonte a Mondovi dov'è comune (Ing.), a Riva di Sesia (Carestia!), ad Arona a S. Carlo (Ricca!), nel Cantone Ticino a Locarno alla Guta (Franzoni!), a Lugano e a Mendrisio (Com.), presso Como (Com.), nel Pavese a Bereguardo e S. Colombano, a Bergamo (Rota), presso Brescia sul monte Conche (Zersi), nel monte Baldo sull'Altis. simo (Hausm.), negli Euganei (Trev.), e generalmente per tutto il Veneto (De Vis. Sacc.), nel Bolognese e Modenese al Granaglione (G. Bert.), al Rio Maggiore, a Montese e Salto di Montese, al monte Acuto, a Sparavara (Cocc. , Gib. Pir.). Fa a Nizza (Ard.), a Montecarlo (Arc.!), a Genova (De Not.). In Toscana è piuttosto comune, essendo stata trovata in Garfagnana sopra Camporgiano!, presso 
Lucca nella valle della Freddana (Calandrini! ecc.), nell'Appennino pistoiese presso S. Marcello (P. Savi!), presso Firenze a Quarata!, nel Mugello a monte Senario (Arc.!) e Scarperia!, in Casentino a Romena !, Prataglia (Parl.!), Camaldoli (Arc.!), nel monte Amiata sopra Arcidosso!. Piủ giù trovasi in Terra di Lavoro presso Picinisco alla Pietra e al monte Meta (Terr.), sul Vesuvio (Pasq.), e sul Vulture in parecchi siti (Terr.). Nasce pure in Corsica (Bert.) a Portovecchio (Mars.), a Cervione (Gren. Godr.), e nella valle di Bastelica (Requien.); forse in Sardegna (Mor.); in Sicilia alla Ficuzza (Loj.!). Fiorisce da maggio a luglio secondo i luoghi.

Distribuzione geografioa. - Nell' Europa inedia-meridionale.

Osservazione. - Non so cosa sia l' Orobanche mudiflora Wallr., parassita su alberi nell' orto botanico di Napoli, che secondo il Compendio di Cesati, Passerini e Gibelli differirebbe dalla presente specie per la corolla affatto glabra. Wallroth (Sched. crit. 1. p. 310) cita per la sua specie la tav. 1219 della $F l$. danica, che dà piuttosto l'idea di una pianta del gruppo dell' 0 . minor, e anche le figure $(885,884$, 916) nell' Ic. bot. di Reichenbach danno l'istessa idea.

\section{Orobanche variegata.}

Orobanche variegata Wallr. Orob. Bert. A. ital.6. p. 4.43. Reut. in Cand. prodr. 11. p. 17. Rota prosp. piant. Pav. p. 270. Bicch. ngg. fl. lucch. p. 15. Car. prodr. fl. tosc. p. 496. Ard. fl. Alpes-mar. p. 289. Archb. fl. Alto-Serch. p. 59. Cies. Pass. Gib. comp. fl. ital. p. 331. Cald. fl. fav. tent. p. 174. Arc. comp. fl. ital. p. 526.

Orobanche superba De Not.? prosp. fl. lig. p. 53.

Orobanche lutea Loj. crit. Orob. p. 38 (non alior. sec. Beck).

Figure. - Reich. ic. bot. t.671, 672. Ic. /l. germ. 20. t. 160.

stazione, Abitazione e Fioritura. - Parassitica su Faseola. cee frutescenti, rara. $\dot{E}$ stata trovata nelle Alpi Apuane al Pisanino settentrionale (Bert.), nel Lucchese (Bicchi), a Chiavari sopra la Croce (Delpino!), nei colli sopra Genova (Vauch. sec. Reut.), e presso Genova sopra Rua? (De Not.), nel Nizzardo tra l'Escarcua c Sospello, tra Sospello e Giandola (Vauch.), tra Fontana e Tenda (Ard.), tra T'enda e Lupega (Reich.), presso Tenda (Reut.), e a S. Martino Lantosca (Ard.), di là dall'Appenuino nel Pavese a Broni (Rota), e a Faenza lungo il Lamone, a Castelraniero e a S. Lucia delle Spianate (Cald.), in Corsica (Mutel sec. Reut.), infine in Sici- 
lia presso Palermo a S. Martino (Parl.!) e alla Ficuzza (Loj.!), e nel Messinese a Noara (Munafö!). Fiorisce da maggio a luglio secondo i luoghi.

Distribuzione geografica. - Nella Francia meridionale, in Italia , in Algeria (Reut.).

Descrizione. - Pianta parasita, alta da 15 a 30 centimetri. Fusto quasi cilindrico, ingrossato quasi in forma di bulbo alla base, ed ivi coperto da molte squame embriciate, giallognolo-rossicce, delle quali le inferiori sono più lunghe e molto più corte delle altre che gradatamente diventano piủ lunghe, piủ strette e più rade in modo che si vedono poi un po'lontane o lontane nella parte media e superiore del fusto: questo è rossiccio-giallognolo, pubescente con peli glandolosi all'apice. I fiori sono sessili, distribuiti in una spica fitta, ma un po'rada in basso, accompagnati da una brattea scuretta, ovato-lanceolata, acuminata, poco più lunga o quasi uguale alla corolla, fornita di una carena, pubescente-glandolosa difuori, glabra di den tro, con ciglia lunghe e glandolose nel margine. Il calice si compone di due sepali distinti, poco più corti della corolla, alla quale sono avvicinati, ovato-lanceolati, divisi nella metà superiore in due lobi o lacinie quasi uguali, lineari-acnminate, ha molti nervi, è pubescente-glandoloso di fuori, glabro di dentro, di color rosso-violetto con il margine gialliccio; nei fiori superiori v'è un terzo sepalo in mezzo ai due sepali di sopra, il quale è distinto dai sepali laterali, lineare-acuminato, ora quasi lungo quanto questi, ora notabilmente più corto. La corolla è tubulosa, un po' gonfia di davanti alla base, curvata ad arco nel margine superiore 0 interno, pubescente-glandolosa di fuori verso alto, quasi glabra o glabra in basso, e glabra di dentro, di color gialliccio-sudicio, o di color violetto-rossiccio in vicinanza di ciascuno dei due lobi del labbro superiore massime della parte interna divisa in due labbri, con il labbro superiore smarginato-bilobo e i lobi uguali, larghi tondeggianti, dentati-increspati e cigliato-glandolosi, con il labbro inferiore trilobo, e i due lobi laterali la metà più piccoli del mediano il quale è lungo e grande quasi quanto ciascuno dei lobi del labbro superiore, tutti tondeggianti, un po' concavi di sopra, dentato-increspati e cigliato-glandolosi. Gli stami sono inseriti vicino alla base della corolla in avanti nella parte un po'gonfia di essa, due appena più alti degli altri due, lunghi quasi quanto il tubo della corolla. I filamenti sono un po'curvi in alto, più larghi in basso, solcati anteriormente, pelosetti con peli non glandolosi in basso e glandolosi in alto, di colore laterizio. Le antere 
sono giallicce, con le logge terminate da una punta quasi diritta. Il pistillo è poco piủ corto della corolla, in modo che il suo stimma si vede in mezzo alle labbra sotto al labbro superiore. Il nettario è a guisa di una squametta larga, poco carnosa, irregolarmente dentata nel margine, gialliccia e glabra. L'ovario è allungato, di color rossiccio-gialliccio, glabro. Lo stilo si continua quasi con l'ovario, è quasi lesiniforme, si allarga un po' all' apice, è un po'curvato in avanti, dello stesso colore e glabro come l'ovario in basso, di color violetto-rossiccio in alto ed ivi puberulo-glandoloso. Lo stimma è grande, bilobo, con i lobi quasi orbicolari, gialli, e diretti in avanti. (Parl. ms., descr. della pianta del Nizzardo).

\section{Orobanche Satyrus}

Orobanche Satyrus De Not. in Bert. fl. ilal.6. p. 446. Rep. fl. lig. p. 305. Reut. in Cand. prodr. 11. p. 717. De Not. prosp. fl. lig. p. 53. Ces. Pass. Gib. comp. fl ital. p. 330. Arc. comp. fl. ital. p. 525 Loj. crit. Orob. p. 39.

Stazione, Abitazione e Fioritura. - In luoghi boschivi mon. tuosi sopra Genova nella valle di Polcevera (De Not.). Parassita su Ginestre ed altre Faseolacee frutescenti nelle isole Eolie Salina, Vul. cano e Filicuri (Loj.). Fiorisce in aprile e maggio.

Distribuzione geografica. - Specie italiana.

Osservazione. - Est 0 . variegatce Wallr. forma spica bracteis flores superantibus comosa "(Beck in litt.).

\section{Grobanche epertii.}

Orobanche spartii Guss, fl. sic. prodr. 2. p. 182. Ten. fl. nap. г. p. 13. Syll. R. neap. p. 307. Guss. 1. sic. syn. 2. p. 135. Ces. Pass. lith. comp. 1l. ital. p. 331 . Loj. is. Eol. p. 122. Arc. comp. 1. ital. p 526. Loj. crit. Orob p. 33 Nic prodr. 11. mess. p. 336 .

Orobanche foetida Ten syll. ll. neap. l. c.

Orubunche cruenta ex parte Berl. fl. ilul. 6. p. 4,30.

Orobanche Todari $L$ oj crit. Orob. p. 36 .

Oroluanche sicula Loj ibid p. 37 .

Figura. - Reich. ic. 11. nerm 20. t. 218

stazione, Abitazione e Fioritura. - Parassitica su Faseolacee frutescentı, nell'Italia meridionale e media. Pare che sia comune in 
Sicilia, così intorno a Palermo (Parl!, Todaro! ecc.), a Castelbono (Minà!), sopra Messina a 3-400 metri (Huter Porta Rigo!), a Messina (Nic.), in Valdemone (Loj.), a Nicosia (Guss.), Comiso (Aiuti!), Terranova, nonchè in Salina, Basiluzzo (Guss.) e Lipari (Mandralisca!). Fa pure in Calabria a S. Gristina (Arc.!) e a Pizzo (Ten., Arc.!); a Napoli presso Posilipo a Gaiola, e alla Solfatara (Ten.); in Liguria sopra Genova, in quantità, e tra l'Escarena e Giandola, secondo Vaucher, nonchè a Diano (Ricca!). Fiorisce in aprile e maggio, e giugno.

Distribuzione geografica. - Nella Francia meridionale, in Ita. lia, in Algeria.

Osservazione. - Il dott. Beck riunisce anche questa specie, con la seguente, all' Orobanche variegata.

\section{Drobanche condensata.}

Orobanche condensata Mor. stirp. sard. el. 2. p. 8. Bert. fl. ital. 6. p. 433. Reut. in Cand. prodr. 11. p. 18. Mor. fl. sard. 3. p. 247. Ces. Pass. Gib. comp. fl. ital. p. 331. Arc. comp. fl. ital. p. 526. Loj. crit. Orob. p. 35.

Figure. - Reich. ic. bot. t. 666 (?). Ic. fl. germ. 20. t. 213.

Stazione, Abitazione e Fioritura. - Parassita su Faseolacee frutescenti: in Sardegna a S. Elia, a Cagliari, ad Iglesias, nelle isole S. Pietro (Mor.) e S. Antioco (Reut.), ad Arizzo (Mor.); in Sicilia secondo Lojacono; in Corsica secondo Mutel e Reuter; in Liguria a Cervo (Ricca!); in Istria a Fiume secondo Reichenbach. Fiorisce in aprile e maggio.

Distribuzione geografioa. - Nella Francia meridionale, in Italia, in Algeria.

Osservazioni. - Vedasi alla specie precedente. La figura della Icon. bot. di Reichenbach, abbenchè citata dal Moris stesso per la sua specie, non corrisponde affatto ai saggi autentici di lui; e neanco vi corrisponde bene l'altra figura delle Ic. $f$. germ.

\section{כ. Drobanche thyrsoidea.}

Orobanche rigens Lois. sec. Beck.

Orobanche thyrsoidea Mor.l in Bert. Al. ital. 6.p. 428, et in Cand. prodr. 11.p. 18. Fl. sard. 3. p. 244. Genn.! fl. Capr. p. 113. Ces. Pass. Gib. comp. fl. ital, p. 330. Arc. comp. fl. ital. p. 525. 
Orobanche Rapum Guss." enum.pl. Inarim.p. 244. Orobanche carnea o. c. p. 246.

Figura. - Mor. o. c. t. 103.

Stazione, Abitazione e Fioritura. - Parassita sul Cytisus tri. florus, sulla Genista corsica e sul Thymus Herba-barona in Sardegna (Mor.!), per esempio al capo S. Elia (Genn.!), presso Arizzo nel monte Tonneri d'Irgono (Ascherson!), all'isola La Maddalena (Mor., Genn.) ecc. Fiorisce in aprile e maggio.

Distribuzione geografioa. - Specie sarda.

\section{Orobanche sanguinea.}

Orobanche sanguinea Presl del.prag. p. 71 (1822). Ces. Pass. Gib. comp. fl. ital. p. 331. Arc. comp. fl. ital. p. 525. Loj.! crit. Orob. p. 62.

Orobanche crinita Viv. fl. cors. sp. nov. p. 11 (1824). Bert. . ital. 6. p. 445. Gren. Godr. fl. Fr. 2. p. 629. Guss. enum. pl. Inarim. p. 445. Mor. fl. sard. 3. p. 248. Pasq. fl. ves. p. 77. Mars. cat. pl. Cors. p. 110. Ces. Pass. Gib. l. c. p. 330. Arc. I. c. Loj. o. c. p. 61. Nic. prodr. fl. mess. p. 337.

Orobanche nebrodensis Tin. in Guss. f. sic. syn. 2. p. 845. Ces. Pass. Gib. o. c. p. 331.

Figura. - Mor. o. c. t. 103.

$\beta$ straminea, tota planta straminea.

Orobanche crinita var. straminea $L o j . ~ 0 . c . p .61$.

Stazione, Abitazione e Fioritura. - Parassita sul Lotus cylisoides ed anche su altri congeneri: in Corsica a Rogliano (Mlars.), Calvi (Gren. Godr.), Bonifacio (Viv., Requien!, Kralik। ecc.); in Sardegna a Cagliari, Lungon-sardo, scala di Ciocca (Mor.); in Sicilia presso Palermo alla Renella (Guss.), a Mondello (Iluet!, Loj.), Carini, S. Martino, monte Gallo al Malpasso (Loj.) e a Sferracavallo (Loj. !), nelle Madonie (Presl) sopra Isnello (Tin.), a Mlessina (Nic.), ad Arola (Bianca!), in Favignana e Levanzo (Griss.), e Lampedusa (Guss., Aiuti!); presso Napoli alla Gaiola (Ten., Rert), a Miseno, Coroglio (Ten.), Ottaiano, nel monte Somma, in Capri (Pasq.), in Ischia a Lacco e a Forio (Guss.). Fiorisce in aprile e maggio.

Distribuzione geografioa. - In Spagna, in Provenza, in Italia, in Dalmazia, if Algeria. 


\section{Drobanche densiflore.}

Orobanche densiflora Salzm.! in Bert. fl. ital. 6. p. 444, et in Cand. prodr. 11. p. 19. Mor. f. sard. 3. p. 249. Ces. Pass. Gib. comp. fl. ital. p. 330. Arc. comp. fl. ital. p. 525.

Figura. - Reich. ic. bot. f. 922.

Abitazione e Fioritura. - In Sardegna a Castelsardo (Viv. sec. Bert. e Mor.). Fiorisce in maggio.

Distribuzione geografica. - Nel Marocco, nella Spagna, in Sardegna.

Osservazione. - Secondo Beck, l' Orobanche densiflora di Moris non sarebbe che una forma dell' 0 . minor.

\section{Orobunche speciosa.}

Orobanche speciosa Cand. fl fr. 5. p. 393 (1815). Gren. Godr. fl. Fr. 2. p. 631. Bicchi agg. fl. lucch. p. 15. Ard. cat. pl. Ment. p. 28. Car. prodr. fl. tosc. p. 496. Ard. fl. Alpes-mar. p. 289. Mars. cat. pl. Cors. p. 110. Ces. Pass. Gib. comp. fl. ital. 331. Cocc. nuovo contr. fl. Bol. p. 23. Bizz. sec. agg. fl. ven. p. 8. Gib. Pir. fl. Mod. p. 124. Arc. comp. fl. ital. p. 527. Loj.! crit. Orob. p. 41. Cocc. Al. Bol. p. 370. Nic. prodr. fl. mess. p. 337.

Orobanche pruinosa Lap. hist. abr. pl. Pyr. suppl. p. 87 (1818). De Not. rep. fl. lig. p. 307. Grech Del. fl. mel. p. 29. Guss. enum. pl. Inarim. p. 246. Tassi f. prov. sen. p. 50. Car. l. c. Tassi cenno bot. Sien. p. 84. Pasq. fl. ves. p. 77. Terr. fl. Vult. syn. p. 133. Zersi prosp. piant. Bresc. p. 158. Groves! contr. fl. Terra d' Otr. p. 64. Loj. is. Eol. p. 122. Cald. fl. fav. tent.p. 175.

Orobanche alba Steph.? Guss. fl. sic. syn. 2. p. 134 sec. Loj. crit. Orob. Rota? prosp. fl. Berg. p. 67. Ces. Pass. Gib. l. c. Arc. l. $c$.

Orobanche pelargonii Cald. l. c.

Orobanche Tommasinii Loj.! crit. Orob. p. 41 (non Reich. fil.). Figure. - Vauch. mon. t. 5. Reich. ic. bot.f. 911.

Stazione, Abitazione e Fioritura. - Parassita su varie Faseolacee coltivate, e particolarmente sulle Fave, nonchẻ su altre spontanee e su piante di altre famiglie. Nell'Alta Italia è stata trovata a Fiume (Noë!), sul monte Calaone presso Este degli Euganei (Bizz.), 
nel Veronese a Monteforte, Soave, Montorio, S. Leonardo ecc. (Poll.), nel Bresciano presso il Goletto (Zersi), forse nel Bergamasco presso Adrara (Rota), a Faenza dov'è comunissima (Cald.), nel Bolognese agli Arienti, a Casalecchio, Montese, Porretta (Cocc.). In Liguria fa a Nizza, Monaco, Mentone (Ard.), Albenga (De Not.). In Toscana abbonda, cosi a Lucca (Beccari! ecc.), Pisa (Corinaldi! ecc.), Firenze!, Siena (Bartalini! ecc.); nel Lazio nasce a Viterbo (Bert.), e a Roma (Webb! ecc.), frequentissima (Seb. Maur.); nel Napoletano a Castelvolturno (Terr.!), a Portici, in Capri (Pasq.), in Ischia (Bolle!); in Basilicata a Melfi, comune (Terr.), e Ciastelgrande (Bert.); nelle Marche ad Arcevia, Macerata, S. Benedetto (Bert.), Montefortino (Marzialetti!); a Pescara (Kuntze!); in Puglia (Ten.) a Zittarella presso Taranto (Aiuti!), a Otranto (Groves !). In Corsica è comunissima a Bastia (Mabille! ecc.). Manca in Sardegna. Per contro ab. bonda in tutta la Sicilia (Guss.), cosi a Palermo (Loj.! ecc.), Messina (Nic.), Catania (Guss.), Avola (Bianca!), Girgenti (Aiuti!), Palazzo Adriano (Guss.), e comprese Lipari (Loj.), Panaria, Ustica e Pantellaria (Guss.); e fa ancora in Malta (Grech!). Fiorisce secondo i luoghi in aprile, maggio o giugno.

Distribuzione geografioa. - In tutto il bacino del Mediterra. neo, e sino ai paesi Transcaucasici e alle Canarie.

Osservazione. - Questa pianta detta in Toscana Succiamele, Fiamma, Hal d'occhio, pullula facilmente nei campi di Fave, a tal segno che la raccolta ne resta sovente scarsa. Di ciò scrisse il Micheli, nella sua Relazione dell'erba detla da'botanici Orobanche, 1723, la quale fu poi riprodotta in un Compendio pubblicato nel 1754.

\section{Orobanche caryophyllacea.}

Orobanche caryophyllacea Smith in trans. linn. soc. 4. p. 169 (179S). Bir. fl. acon. 1. p. 217. Nocc. Balb. fl. tic. 1.p. 305. Ruch. 1. lidi ven. p. 156. Poll. R. ver. 2. p. 308. Colla herb. ped. 4. p. 379. Giuss. 月. sic. syn. 2. p. 8.4. Bert. fl. ital. 6. p. 425. G. Bert. not. porr. p. 86. Veg. Porr. p. 46. Ard. R. Alpes-mar. p. 290 Zersi prosp. piant. Bresc. p. 158. Arc.! comp. fl. ital. p. 597. Loj. crit. Orob. p. 43.

Orobanche vulgaris Lam.? enc. mith. bot. 4. p. 621 (1797). Cand R. fr. 3. p. 489 (1805). Trex. prosp. A. eug. p. 23.

Orobanche galii Dub. bot. gall. ed. 2. 1. p. 3.49 (1898). Ciuss.? 
fl. sic. syn. 2. p. 135. Com. fl. com. 5. p. 86. Zan. prosp. fl. ven. p. 25. Rota prosp. piant. Pav. p. 270. Hausm. fl. Tir. p. 649. Rota! prosp. fl. Berg. p. 67. Grech Del. fl. mel. p. 29. Pir.! fl. for. syll. p. 106. Bicch. agg. fl. lucch. p. 15. Ard. cat. pl. Ment.p. 28. Car. prodr. fl. tosc. p. 496. De Vis. Sacc. cat. piant. Ven. p. 159. Terr. fl. Vult. syn. p. 133. Archb. fl. Alto-Serch. p. 59. Ces. Pass. Gib. comp. fl. ital. p. 332. Ing. cat. sp. Mond. p. 54, Loj. is. Eol.p. 122. Gib. Pir. fl. Mod. p. 124.

Figure. - Vauch. mon. t. 7. Reich. ic. bot.f.890-895. Ic. fl. germ. 20. t. 162 .

Stazione, Abitazione e Fioritura. - Parassita sui Galium, e secondo alcuni anche su Faseolacee ed Asteracee e Lamiacee, per tutta Italia. Nell Alta Italia sembra piuttosto comune: a Mondovi, ovunque nei pascoli (Ing.), presso Susa alle Blacce, presso Torino a Superga, alla Venaria ecc. (Colla), in val di Sesia (Bir.), nel Pavese al monte Cesarino, verso Trumello (Nocc. Balb.), e a Bobbio (Rota), nel Comasco al monte Generoso e nelle selve di Brunate, Camnago e Ponzate presso Como (Com.), nel Bergamasco a Carenno (Rota!), in valle S. Martino e Caleppio, e a Sopracornola (Rota), nel Bresciano a Ronchi, al Buren e a Tremosine (Zersi), e a Ponte di legno (Ricca!), in Tirolo a Trento ai Zoccolanti (Perini!), a Campitello di Fassa (Bert.), e a Laas e Tanaas in val Venosta (Hausm.), negli Euganei (Poll., Trev.), presso Venezia a Malghera (Ruch.), a Udine (Pir.!). e in generale per il Veneto (De Vis. Sacc.), in val di Reno alla Porretta (G. Bert.), nel Modenese a Castelvetro e Fiumalbo (Gib. Pir.). Nelle altre parti d'Italia è molto più rara: è stata trovata in Liguria a Nizza, Eza (Ard.), verso Turbia (Vauch., Bert.), a Roccabruna, Castiglione (Ard.), a Villafaraldi sopra Cervò (Ricca!); nel Lucchese (Bicchi); nel Fiorentino a Firenze! e verso monte Senario (Arc.!); nelle Marche al Catria (Piccinini!), e a Valcimara (Smith); in Basilicata nel Vulture (Terr.); in Calabria nel monte Dirupato di Morano (Huter Porta Rigo!), e presso Paline (Arc.!); in Sicilia, comunissima sec. Lojacono, cosi intorno a Palermo, nelle Madonie, a Palazzo Adriano (Guss.), ad Avola (Bianca!), nelle Eolie a Salina (Loj.) e Basiluzzo (Guss.); in Malta (Grech). Fiorisce da aprile a luglio secondo i luoghi.

Distribuzione geografica. - Nell' Europa media e meridionale, e in Siberia.

Osservazione. - Dicesi che i fiori sappiano fortemente di garofano. 


\section{Drobanche teucrii.}

Orobanche tencrii Hol. A. Mos. (1829). F. W. Schultz in flora 1835. p. 200. Ard. fl. Alpes-mar. p. 290. Ces. Pass. Gib. comp. fl. ital. p. 332. Arc. comp. fl. ilal. p. 527.

Figure.-Schultz l. c. Reich. ic. fl. germ. 20. t. 169.

Stazione, Abitazione e Fioritura. - Parassita sui Teucrium, rarissima : nelle Alpi Marittime a Tenda (Ard.); nelle Alpi Apuane in Versilia (Simi!). Fiorisce in luglio.

Distribuzione geografica. - In Germania, in Francia, in Sviz. zera, in Italia.

\section{Orobanche epithymum.}

Orobanche epithymum Cand. fl. fr. 3. p. 490. Trev. prosp. $f$. eug. p. 23. Pucc. syn. pl. luc. p. 316. Bert. l. ilal.6.p. 434 (excl. syn. ex parte). (om. fl.com. 5. p. 84. Rota prosp. piant. Pav. p. 270. Hausm. fl. Tir. p. 648. Rota prosp. fl. Berg. p. 67. Ard. cat. pl. Ment. p. 28. Car. prodr. fl. tose. p. 496. G. Bert. veg. Porr. p. 86. Ard. 1. Alpes-mar. p. 290. De Vis. Sacc. cat. piant. Ven. p. 159. Zersi prosp. piant. Bresc. p. 158. Terr. sec. rel. Terra di Lav. p. 93. Archb. fl. Alto-Serch. p. 59. Ces. Pass. Gib. comp. fl. ital. p. 332. Ing. cat. sp. Mond. p. 54. Terr. quarta rel. p. 111. Anzi auct. fl. nov.com. p. 195. Gib Pir. fl. Mod. p. 124. Arc. comp. fl. ital. p. 527. Loj. crit. Orob. p. 42. Cocc. 1. Bol. p. 370. Gib. Pir. suppl. fl. Mod. p. 22.

Orobanche Alexandri Tin.! in Guss. 17. sic. syn. 2. p. 845. Ces. Pass. Gib. o. c. p. 331 . Arc. l. c.

Orobanche amethystea Car. o.c. p. 497 (non Thuill.).

Figure. - Reich. ic. bot.f. 887-889. Ic. f1. germ. 20. t. 163.

Stazione, Abitazione e Fioritura. - Parassita sui Timi ed altre Lamiacee, per tutta Italia. In Piemonte al col di Tenda a 1900 metri (Parl.!), presso Valdieri (Bert.), a Mondovi, ovunque (lng.), ad Alba (Bertero!), a Riva di Sesia (Carestia!); nelle vicinanze di Lugano e di Mendrisio, in valle Intelvi (Com.), presso Como al monte S. Fermo, ai Corni di Canzo (Bert.), at Inverigo in Brianza (Ram. poldi!); nel Bergamasco (Rota); nel Bresciano alla Badia e sui Ronchi (Zersi); in Tirolo sul Doss li Trento, in Fassa, intorno a Bol. 
zano fino a $5400^{\prime}$, a Merano, Laas (Hausm.), a Bressanone (Bert.); nel Veneto a Lusiana, nel monte Grappa, a S. Michele d'Angarano (Bert.), negli Euganei (Trev.); nel Bolognese e Modenese a Casalecchio, Castel S. Pietro (Bert.), Pontecchio, Porretta (Cocc.), Pietramala (Bert.), Novellano, Villa Minozzo, Boscolungo (Gib. Pir.); nel Pavese a Bobbio (Rota). Nel Nizzardo intorno a Mentone e altrove (Ard.). In Toscana sull'Appennino al Gotro, all'Orsaio, all'alpe di Mommio!, al Teso (Beccari!), nelle Alpi Apuane al Gramolazzo e a Campagliana sopra Massa (Ad. Targioni!), nel Lucchese al monte di Quiesa (Beccari !), a S. Alessio (Pucc.), Gomitelli e Lucignana (Bert.), nel monte Pisano, in Maremma alla Badiola !. Nell' Umbria ai monti Catria (Piccinini!) e Leone (Bert.); nel Piceno al Vettore (Bert.); al Gargano (Ten. !); presso Roma a Tivoli e al Lucretile (Bert.); in Terra di Lavoro a Cassino al monte Cairo, a Valcopella presso Picinisco, sopra Forchia nella valle Caudina (Terr.); a Castellammare (Pasquale!) e nei monti di Sarno (Bert.). In Sicilia a Messina, nelle Madonie (Bert.), e presso Palermo a Chiarandà (Loj.!), sopra S. Maria a Gesù, nel monte Pellegrino (Guss.), a Catalfano (Tineo!). Fiorisce da aprile a luglio secondo i luoghi.

Distribuzione geografica. - Nell'Europa media e meridionale, nell'Asia caucasica, nell' Imalaia.

Osservazione. - Bertoloni (Fl. ital. 10. p. 506), Lojacono e Beck si trovano d'accordo per riferire l'Orobanche Alexandri Tin. all'0. epithymum.

\section{Drobnelne punctatu.}

Orobanche punctata F. W. Schulz? in flora 26. p. 131. Guss. enum. pl. Inarim. p. 247. Ces. Pass. Gib. comp. fl. ital. p. 330. Arc. comp. fl. ital. p. 525.

Stazione, Abitazione e Fioritura. - Parassita su Faseolacee in Ischia a Bagno (Guss.). Fiorisce in aprile e maggio.

Distribuzione geografica. - Nasce inoltre in Grecia.

Osservazione. - Reuter, Boissier, Lojacono ravvicinano la vera Orobanche punctata Schulz alla 0. epithymum, di cui il dottor Beck ne fa un sinonimo; ma se la pianta d'Ischia sia la stessa, è questione. Anche al dottor Beck è sconosciuta. 


\section{Orobanche platystigna.}

Orobanche platystigma Reich. ic. bot. 7. p. 31 (1829). Reich. fil. ic. fl. germ. 20. p. 95. Ard. R. Alpes-mar. p. 289. Ces. Pass. Gib. comp. Al. ital. p. 334. Arc. comp. fl. ital. p. 524.

Orobanche scabiosæ Koch Deutschl. fl. 4. p. 440 (1833). Hausm. fl. Tir. p. 648. Ces. Pass. Gib. o. c. p. 332. Arc. o. c. p. 524.

Figura. - Reich. o. c. t. 164.

Stazione, Abitazione e Fioritura. - Parassita su specie di Carduus e di Scabiosa: nel Tirolo in val Venosta presso Trafoi a $6000^{\prime}$ (Hausm.); nel Nizzardo a Levenzo e nelle gole di Saorgio (Ard.); in Abruzzo nella Maiella (Huet!). Fiorisce da maggio a luglio.

Distribuzione geografioa. - Nell' Europa centrale ed anche nella meridionale.

Osservazioni, - L'Orobanche Deucalion Reich. fil. (Ic. N. germ. 20. p. 92. t. 206. f. 1, 2. Arc. comp. fl. ital. p. 525), trovata dall'autore al col di Tenda fiorita d'agosto, a sentenza del dottor Beck parrebbe una forma anormale, immiserita dell' 0 . platystigma.

Il dottor Beck riferisce l'0. platystigma alla specie seguente come varietà.

\section{Orobanche pallidifora.}

Orobanche pallidiflora Wimm. Grab. fl. Sil. Hausm. fl. Tir. p. 648. Asch. fl. prov. Brand. p. 496. Ces. Pass. Gib. comp. fl. ital. p. 332. Arc. comp. n. ital. p. 527.

Orobanche cirsii Fries nov. fl. suec. mant. Ces. Pass. Gib. o. c. p. 331 . Arc. 0.c. p. 525.

Figura. - Reich. ic. fl. germ. 20. t. 211.

Stazione, Abitazione e Fioritura. - Parassita su Cinaree: nel Tirolo presso Grigno in Valsugana (Reich.); in Piemonte nelle valli Valdesi (Ces. Pass. Gib.); in Abruzzo a Rocca di Ciorno e nel monte Coccorello (Levier!). Fiorisce in luglio e agosto.

Distribuzione geografica. - Nell' Europa centrale ed anche nella ineridionale.

Osservazione. - Pare che sia la stessa specie della precedente. 


\section{1\%. Orobanclie Incorum.}

Orobanche loricata Reich. ic. bot. 7. p. 41 (ex parte). Rota prosp. piant. Pav. p. 270. Prosp. fl. Berg. p. 67. Ces. Pass. Gib. comp. fl. ital. p. 332. Arc. comp. fl. ital. p. 528.

Orobanche lucorum Al. Braun in Schultz ann. gew. regensb. bot. ges. Hausm. fl. Tir. p. 650. Anzi auct. fl. nov.-com. p. 195.

Figure. - Reich. ic. bot. f. 918. Ic. fl. germ. 20. t. 178.

Stazione, Abitazione e Fioritura. - Parassita sul Berberi e sui Rovi, anche sull'Artemisia campestris, rara : in Tirolo a Bressanone, nella media valle Venosta comune tra $2000^{\prime}$ e $4000^{\prime}$, sul Ritten presso Klobenstein e presso Lengmoos, a Sprechenstein presso Sterzing, a Folgaria (Hausm.); nel Bresciano a Ponte di legno a 1330 metri (Parl.!); nel Bergamasco in valle Caleppio (Rota, Ces. Pass. Gib.); in Valtellina presso Bormio (Anzi) a 1220 metri (Parl.!), e in val Malenco (Anzi); nel Pavese a Carbonara, Broni, Bobbio (Rota); in Toscana nell' Appennino pistoiese al Pian degli ontani (Beccari!); in Umbria al monte S. Vicino (Bucci!). Fiorisce da giugno ad agosto.

Distribuzione geografica. - In Germania, Svizzera, Italia.

Descrizione. - Pianta alta da 2 a 3 decimetri, di un giallo-rossiccio, pubescente-glandolosa per peli lunghetti, radi, bianchi, più abhondanti nel fiore e forniti all'apice di una glandoletta piccola, tonda e gialliccia. Fusto semplice, eretto, ramoso, angoloso, con scaglie ovato-lanceolate, acuminate, scurette, che seccan presto. Fiori in una spiga bislungo-cilindrica, ottusa, piuttosto radi, eretto-pa. tenti, un po' curvati in fuori. Ciascuno è accompagnato da una brattea simile alle scaglie del fusto, lunga quasi quanto i fiori. Calice formato di due sepali, ciascuno dei quali è composto della saldatura di due. I due sepali considerati insieme per ciascun lato, ossia ciascun sepalo principale, è laterale, poco più corto del tubo della corolla, largo ovato e concavo in basso, bifido in alto, con le lacinie lanceolato-acuminate, disuguali, spesso una molto più piccola dell'altra, ha due nervi longitudinali, uno per ciascuna lacinia, è gialliccio, pubescente-glandoloso. La corolla è di color giallo-rossiccio, ha il tubo grosso, curvato in avanti, ottusamente angolato, il lembo è diviso in due labbri, uno superiore più corto, bilobo, con i lobi quasi tondi, smerlato-dentellati, cigliato-glandolosi, il labbro inferiore è diretto in avanti ed un po' in giù, è bilobo, con i lobi più lunghi di 
quelli del superiore, quasi tondi ma un po'stretti in basso, egualmente dentellati e cigliato-glandolosi, scanalati di sopra verso la base. Gli stami sono quattro, poco più lunghi del tubo della corolla, due poco più corti degli altri due, inseriti in basso del tubo della corolla medesima. I filamenti sono grossi in basso, lesiniformi in alto, curvati ad arco in su in avanti, giallicci, pelosi, più abbondantemente nella metà inferiore che in alto, i peli di alto sono glandolosi. Il pistillo è lungo quasi quanto gli stami piủ lunghi. L'ovario è ovatobislungo, gialliccio, glabro, peloso verso l'apice in avanti con pochi peli, ha in avanti un disco giallo, saldato con esso. Lo stilo è più lungo dell' ovario, curvato in alto in avanti, lesiniforme, gialliccio, pelosetto con rari peli in avanti. Lo stimma è grosso, diretto in avanti, bilobo, con i lobi quasi tondi, di color quasi di zafferano. Le antere sono quasi ovali, smarginate all'apice ed ivi ottuse in ciascuna loggia, bifide alla base e ivi mucronate all'apice per una punta corta e bianchiccia, inserite nel dorso sopra della base, biloculari, aprentesi per una fessura longitudinale, sono scurette e hanno molti corti peli nella sutura che la rendono pubescente. (Parl. ms., descr. della pianta di Ponte di legno).

\section{Orobanche concolor.}

Orobanche concolor Dub. bot. gall, ed. 2. 1. p. 430. Ard. fl. Alpes-mar. p. 289. Ces. Pass. Gib. comp. fl. ital. p. \$31. Arc. comp. fl. ital. p. 526.

Figura, - Vauch. mon. t. 11.

Stazione, Abitazione e Fioritura. - Parassita su di una Scabiosa a Mentone (Ard.). Fiorisce in maggio e giugno.

Distribuzione geografica. - Nasce inoltre nella vicina Provenza.

Osservazioni. - "Planta mihi omnino dubia "(Beck in litt.). Pure in Provenza all' isola S. Margherita nasce l'affine Orobanche fuliginosa Reut. (Ard. o. c. p. 290), che gli autori dei Compendi per indebita estensione di territorio hanno indicata del Nizzardo.

\section{Drobanche hyalina.}

Orobanche hyalina Sprun. in Cand. prodr. 11. p. 24. Giren. Godr. 1l. Fr. 2. p. 639. Mars. cat. pl. Cors. p. 110. Ces. Pass. Gib. comp. f. ital. p. 331 . Arc. comp. A. ital. p. $52 \%$. 
Orobanche Salisii Loj.? crit. Orob. p. 60.

Figura. - Reich. ic. fl. germ. 20. t. 165. f. 1.

Stazione, Abitazione e Fioritura. - Parassita sul Chrysanthenum Myconis in Corsica a Bonifacio e ad Aiaccio (Gren. Godr., Reich.). Fiorisce in maggio.

Distribuzione geografica. - Nasce inoltre in Grecia.

Osservazione. - "Forma 0. minoris esse videtur. "(Beck in litt.).

\section{Orobanche rubens.}

Orobanche rubens Wallr. sched. crit. p. 307. Trev. prosp. $f$. eug. p. 23. Rota prosp. piant. Pav. p. 270 Hausm. fl. Tir. p. 649. Rola prosp. fl. Berg. p. 6\%. Pir. fl. for. syll. p. 106. Tassi fl. prov. sen. p. 50. De Vis. Sacc. cat. piant. Ven. p. 159. Ces. Pass. Gib.! comp. fl. ital. p. 333. Arc. comp. fl. ital. p. 528. Gib. Pir. fl. Mod. p. 124. Cocc. fl. Bol. p. 371.

Orobanche elatior Bert. fl. ital.6. p. 428. Zersi prosp. piant. Bresc. p. 158.

Figure. - Vauch. mon. t. 2. Reich, ic. bot.f.901, 902. Ic. fl. germ. 20.t. 171.

Stazione, Abitazione e Fioritura. - Parassita sulle Mediche nell'Italia superiore: in Tirolo presso Bolzano in diversi punti(Hausm.); nel Bergamasco in valle Caleppio (Rota), nel Bresciano a Sarezzo, sul Cornablacca a Peio (Zersi), a Verona (Massalongo!), e nel Veronese sul monte Pastello a 2500' (Bracht!, Bert.), negli Euganei (Trev.), in Friuli intorno a Udine e altrove (Pir.); in Istria intorno a Trieste in diversi punti (Reich. fil.), e in val Draga presso Fiume (A. M. Smith!); nel Bolognese e Modenese agli Arienti (Cocc.), a Castelfranco, a Rubiera (Gib. Pir.); nel Parmense alla Baganza (Passerini!, Ces. Pass. Gib.); nel Pavese sui colli d'Oltre Po (Rota); ad Alba (Bertero!). Il Tassi la dà del Senese. Fiorisce da maggio a luglio.

Distribuzione geografica. - Nell' Europa media e in parte nella meridionale, dalla Spagna al Caucaso.

Osservazione. - L'Orobanche congesta Reich. fil. (Ic. fl. germ. 20. p. 117. t. 218. De Vis. Sacc. cat. piant. Ven. p. 159. Sacc. Bizz. agg. fl.trev. p. 28. Arc. comp. fl. ital. p. 528), indicata dal- 
l'autore parassita sul Trifoglio nei prati del Trivigiano a Serravalle, giusta il dottor Beck è la presente specie "forma spica densiore forsan parum evoluta. "

\section{Drobanche frograntissima.}

Orobanche fragrantissima Berl. f. ital. 6. p. 436. Reut. in Cand. prodr. 11. p. 718. De Vis. Sacc. cat. piant. Ven. p. 159. Cies. Pass. Gib. comp. fl. ital. p. 332. Arc. comp. f. ital. p. 529. Cocc. fl. Bol. p. 371. Gib. Pir. suppl. fl. Mod. p. 22.

Orobanche fragrans Gib. Pir. fl. Mod. p. 124 (non Koch).

Stazione, Abitazione e Fioritura. - Parassita sulle Mediche e altre Faseolacee erbacee nell'Alta Italia : nei colli bolognesi diS. Michele in bosco, di Barbiano, Paderno, Sabbione, Casalecchio, abbondante (Bert.), di Mongardino, Sasso alle lagune, a Porretta sopra la Puzzola e a Granaglione (Cocc.), nel Modenese a Montegibbio (Gib. Pir.); nel Padovano, nel Veronese a Bussolengo (Bert.); a Trieste (Bert.); in Piemonte ad Alba (Bert.). Fiorisce in maggio.

Distribuzione geografioa. - Pianta di quei luoghi.

Osservazione. - Secondo il dottor Beck, che ha veduto un sag. gio originale della pianta di Bertoloni, questa non è che l'Orobanche epithymum.

\section{Drobanche laserpitio-sileris.}

Orobanche laserpitii-sileris Rap. in Cand. prodr. 11. p. 25. Ces. Pass. Gib. comp. fl. ital. p. 333. Arc. comp. fl. ital. p. 529.

Figura. - Reich. ic. 月. germ. 20. t. 172.

Stazione Abitazione. - Parassita sul Laserpitium Siler, " nelle Alpi, , secondo i Compendi, senz'altra più precisa indicazione.

Distribuzione geografioa. - In Svizzera e in Francia.

\section{Orobanche centumpina.}

Orobanche centaurina Bert. 1. ital. 6. p. 430. Car. prodr. A. tosc. p. 496. Cés. Pass. Cib. comp. Al ital. p. 334. Arc. comp. 11 . ilal. p. 530 .

stazione, Abitazione e Fioritura. - Parassita sulla Centaurea 
paniculata nel littorale di S. Giuseppe presso Massa in Toscana (Bert.). Fiorisce in maggio e giugno.

Distribuzione geografica. - Pianta di quel luogo.

Osservazione. - Al dottor Beck sembra che questa pianta possa spettare all'Orobanche maior Linn., o 0. elatior Sutt., 0. stigmato. des Wimm., nonostante il carattere dato da Bertoloni di «stigma purpureum, "tolto forse dal secco.

\section{Drobanche denudata.}

Orobanche denudata Mor. app. ad el. stirp. sard. Stirp. sard. el. fasc. 3. p. 10. Bert. fl. ital. 6. p. 439. Mor. fl. sard. 3. p. 250. Ces. Pass. Gib. comp. fl. ital. p. 333. Arc. comp. fl. ital. p. 529.

Orobanche decora Mor. in Cand. prodr. 11. p. 26.

Orobanche chironii Loj.! contr. fl. Sic. p. 12. Crit. Orob. p. 46.

Figura. - Mor. o. c. t. 105.

Stazione, Abitazione e Fioritura. - In Sardegna a Belvi, Fonni (Mor.), e Arizzo (Gennari!); in Sicilia parassita sull'Opopanax Chiro. nium sopra Palermo a Busambra, Ficuzza, Muracca di Mezzo Zotta Nespola (Lıj.!). Fiorisce in maggio, giugno, luglio.

Distribuzione geografica. - Specie di quelle due isole italiane.

\section{Drobanche pieridis.}

Orobanche picridis $F$. W. Schultz in ann. gew. kund. reg. ges. Bert. fl. ital. 6. p. 438. Rent. in Cand. prodr. 11. p. 26. Rota prosp. fl. Berg. p. 67. Ces. Pass. Gib. comp. n. ital. p. 333. Bizz. sec. agg. fl. ven. p. 9. Arc. comp. fl. ital. p. 530. Loj.crit. Orob. p. 58. Beck).

Orobanche carotæ Desm. in ann. sc. nat. $2^{\mathrm{a}}$ ser. 3. p. 78 (sec.

Orobanche ambigua Mor. diagn. stirp. Sard. (sec. Beck). Fl. sard. 3. p. 252. Ces. Pass. Gib. l. c. Arc. l.c.

Orobanche Tommasinii Reich. fil. ic. fl. germ. 20. p. 92. $t .209$ (sec. Beck).

Orobanche littoralis Loj.! o. c. p. 45.

Orobanche canescens Loj.! o. c. p. 47.

Orobanche denudata Loj.! o. c. p. 48 (non Mor.).

Figure. - Vauch. mon. t. 12. Reich. ic. A. germ. 20. t. 175. 
Stazione, Abitazione o Fioritura. - Parassita sulla Picrishie. racioides: in Istria a Capo d'Istria, Parenzo, Orsero (Reut.), Pinguente (Reich.); nel Lido veneto (Kellner!); presso Arquà negli Euganei (Bizz.); ne'monti Bresciani (Ces. Pass. Gib.), e di Valcamonica (Rota, Ces. Pass. Gib.); nelle colline del Parmigiano (Ces. Pass. Gib.); nell'Appennino genovese sopra Ronco (Vauch. , Bert.); a Roma fuori porta Pia (Webb!); nell'Elba a S. Martino (Beccari!); in Capri (Kesselmayer!) e Ischia (Bolle !); in Sardegna (Mor.); presso Palermo a S. Martino (Loj.) e a Sferracavallo (Loj.!), a Palma (Loj.), a Terranova (Loj.!). Fiorisce da maggio a luglio secondo i luoghi.

Distribuzione geografioa. - Nell' Europa media e in parte nella meridionale.

\section{Drobanche salvige.}

Orobanche salviæ $F . W$. Schultz in ann. gew. kund. reg. ges. Reut. in Cand. prodr. 11. p. 26. Ard. fl. Alpes-mar. p. 289. Ces. Pass. Gib. comp. fl. ital. p. 333. Arc. comp. 月. ital. p. 529.

Figura. - Reich. ic. fl. germ. 20.t. 179.

Stazione, Abitazione e Fioritura. - Parassita sulla Salvia glutinosa: in Piemonte nella valle di Luserna a Bobbio (Rostan!); nel Nizzardo a S. Martino Lantosca (Ard.) e alle mine di Tenda (Bert., Reich.); nel Parmigiano sul monte Dosso (Ces. Pass. Gib.); in Ischia (Ces. Pass. Gib.). Fiorisce in gingno e luglio.

Distribuzione geografica. - Dai Pirenei alla Stiria.

\section{Drobnnelne Iorienta.}

Orobanche Ioricata Reich. ic. bot.7. p. 41 (ex parte). Koch Deutsch. fl. 4. 451. Bert fl. itnl. 6. p. 4.4. Car. prodr. fl. Losc. p. 497.

Orobanche artemisix Mars. cat.pl. Corse p. 111. Ces. Pass Gib. comp. A. ital. p. 334. Arc. comp. R. ilal. p. 531.

Figure. - Reich. 0. c. 1. 917. Ic. /l. germ. 20. t. 176.

Stazione, Abitazione - Fioritura. - Parassita su Asteracee: in Piemonte nelle vallate Valdesi (Ces. Pass. Gib.); nel monte Pisano sopra i Bagni di S. Giuliano (Bert.); in Corsica a liogliano? (Mars.). Fiorisce in maggio.

Distribuziono geografica. - Nell' Europa centrale e meridionale. 


\section{Drobanche pubescens.}

Orobanche pubescens Urv. enum. plant. Arch. p. 76. Guss. enum. pl. Inarim. p. 248. Mars. cat. pl. Corse p. 110. Ces. Pass. Gib. comp. fl. ital. p. 332. Arc. comp. fl. ital. p. 528. Loj. crit. Orob. p. 57. $t$. 9. f. 1.

Figura. - Reich. ic. fl.germ. 20.t.180.

Stazione, Abitazione e Fioritura, - Parassita su Asteracee e Faseolacee: nell'isola d'Ischia (Guss.); nell' isola Saline delle Eolie (Ces. Pass. Gib.); presso Palermo al monte Gallo (Loj.); in Corsica a Rogliano? (Mars.). Fiorisce in aprile e maggio.

Distribuzione geografica, - Nella Francia meridionale, in Italia, in Oriente.

\section{Drobanche crithm i.}

Orobanche crithmi Bert.fl. ilal. 6. p. 425 (1844-47). De Vis. Sacc. cat. piant. Ven. p. 159. Genn. fl. Capr. p. 113. Ces. Pass. Gib. comp. fl. ital. p. 333. Arc. comp. fl. ital. p. 530.

Figura. - Reich. ic. fl. germ. 20. t. 184.

Stazione, Abitazione e Fioritura. - Parassita sul Crithmum e altre Apiacee: in Liguria tra Savona e Nizza (Vauch., Bert.); nell'isola Maddalena di Sardegna (Genn.); a Venezia (Reich.). Fiorisce in maggio e giugno.

Distribuzione geografica. - Inoltre nella Francia meridionale.

Osservazione. - Ė probabilmente una forma dell' Orobanche minor, a parere del dottor Beck.

\section{Drobanche hederre.}

Orobanche hederæ Dub. bot. gall. 1. p. 350. De Not. rep. fl. lig. p. 308. Com. fl. com. 5. p. 91. Guss. enum. pl. Inarim. p. 247. Ard. cat. pl. Ment. p. 28. Car. prodr. fl. tosc. p. 497. Ard. fl. Alpesmar. p. 289. Pasq. fl. ves. p. 77. Mars. cat. pl. Corse p. 110. Ces. Pass. Gib. comp. fl. ital. p. 333. Groves contr. fl. Terra d'Otr. p. 64. loj. contr. fl. Sic. p. 12. Cald. fl. fav. tent. p. 175. Cocc. quarto contr. fl. Bol. p. 15. Sacc. Bizz. agg. fl. trev. p. 28. Arc. comp. fl. ital. p. 529. Loj.! crit. Orob. p. 47. Cocc. fl. Bol. p. 370. 
Orobanche minor $\beta$ Bert. l. ital. 6. p. 421. Hausm. fl. Tir. p. 651. Zersi prosp. piant. Bresc. p. 158.

Orobanche barbata Rota? prosp. fl. Berg. p. 67.

Orobanche helicis Rota prosp. piant. Pav. p. 287. Prosp. fl. Berg. l.c.

Figura. - Reich. ic. R. germ, 20. t. 182.

Stazione, Abitazione e Fioritura. - Parassita sull'Ellera per tutta Italia. Nella settentrionale è stata trovata in Piemonte a Susa (Ces. Pass. Gib.), presso Ciomo a S. Croce e presso Lecco (Com.), in Tirolo intorno a Bolzano, dov'è comune (Hausm.), e sul Dosso di Trento (Perini!), in Bergamo (Rota), nel Bresciano alla Bornada (Zersi), a Verona (Massalongo!), nel Trivigiano a Costa presso Vittorio (Sacc. Bizz.), presso Faenza alla Serra (Ciald.), in Bologna (Cocc.). Nella Penisola centrale e meridionale nasce in Liguria a Nizza (Ard.), Villa. franca (Bourgeau!), Mentone (Ard.), Portofino (Baglietto!), Genova (Bert., De Not.), in Toscana a Sarzana (Bert.), Massa di Carrara (Beccari!), in Versilia (Ball!), a Pisa, Firenze!, Volterra (Amidei!), Berignone di val di Cecina (Parl.!), a Roma (Webb!, Rolli!), presso Napoli a Portici (Bert., Pasq.), a S. Angiolo di Castellammare (Pasquale!), in Ischia (Guss.) ecc., nel Piceno a Montefortino (Narzialetti!), infine a Otranto (Groves). Trovasi in Corsica a Bastia (Mars.), in Sardegna ad Arizzo (Gennari!) e Laconi (Ascherson!, Gennari!), in Sicilia in Palermo e nei dintorni (Loj.), fino alla Fi. cuzza (Loj.!), e in Lipari (Loj.). Fiorisce da aprile a giugno.

Distribuzione geografica. - Nell'Europa media e meridionale, in Asia Minore.

Osservazione. - Il dottor Beck opina che possa essere una forma misera di questa pianta l'Orobanche Pyrrha di Reich. fil. (IC. fl. germ. 20. p. 105. t. 206. Arc. comp. M. ital.p. 531), da lui trovata di luglio in val di Tenda.

\section{Orobanche Iamrina.}

Orobanche laurina Bonap. in Bert. fl. ital. 6. p. 424. Reut. in Cand. prodr. 11.p. 719. Ces. Pass. Gib. comp. 1. ital. p. 334. Arc. comp. \%. ital. p. 531.

Figura. - Reich. ic. 11. germ. 20. t. 181.

8tazione, Abitazione e Fioritura. - Parassita sull'Alloro in Roma (Bert.). Fiorisce in maggio.

Distribuzione geografica. - Inoltre nella Francia meridionale. 
Osservazione. - Il dottor Beck opina che sia pianta identica coll' Orobanche pumila Noë, ossia 0. minor var. pumila, altri però l'hanno riferita all' 0 . hederce, nonostante lo stimına descritto por: porino.

\section{Drobanche glaberrima.}

Orobanche glaberrima Guss. in Cand. prodr. 11. p. 719. Ces. Pass. Gib. comp. fl. ital. p. 333. Arc. comp. fl. ital. p. 530. Loj. crit. Orob. p. 52.

Orobanche Hederæ var. Pasq.? fl. ves. p. 97.

Orobanche stenantha Loj.? o. c. p. 53.

Stazione e Abitazione. - Parassita sul Dianthus plumarius presso Napoli al barco di Portici (Guss.), e a Torre del Greco (?) (Pasq.), e in Sicilia (Loj.) in Valdemone (?) (Loj.).

Distribuzione geografica. - Pianta di quei luoghi.

Osservazione. - Al dottor Beck sembra pianta identica con una forma dell'Orobanche pumila Noë, che talora possiede uno stimma gialliccio.

\section{Drobanche australis.}

Orobanche australis Mor.! in Bert. fl. ital. 6. p. 440, et in Cand. prodr. 11. p. 29. Fl. sard. 3. p. 252. Ces. Pass. Gib. comp. fl. ital. p. 333. Arc. comp. fl. ital. p. 529. Loj. crit. Orob. p. 49.

Orobanche thapsoides Loj.? o. c. p. 50.

Orobanche sabulicola $L 0 j . !$ o. c. p. 51.

Stazione, Abitazione e Fioritura. - In Sardegna a Lungonsardo, S. Maria da mare presso Orosei, Arizzo (Mor.); in Sicilia parassita sull'Anthemis maritima presso Balestrate nel Palermitano (Loj.!), a Girgenti, Porto Empedocle, Terranova alle case della Lupa (?) (Loj.). Fiorisce in aprile, maggio, giugno.

Distribuzione geografica. - Pianta di quelle due isole.

\section{Orobanche vitalba.}

Orobanche vitalbæ Bert. fl. ital. 6. p. 441. Car. prodr. fl. tosc. p. 497. Ces. Pass. Gib. comp. fl. ital. p. 334. Arc. comp. fl. ital. p. 530 . 
Stazione, Abitazione e Fioritura. - Parassita sulla Vitalba a Campiglia nella maremma toscana (Bert.). Fiorisce in giugno.

Distribuzione geografica. - Pianta di quel luogo.

Osservazione. - Dalla descrizione questa pianta combina quasi interamente coll' Orobanche carota Desm., ossia 0 . picridis var. carotee, e con l'O. pallidiflora Wimm. Grab.; ma le due specie hanno la corolla sparsa di peli glandolosi, e Bertoloni invece la descrive irsuta, carattere che quadra soltanto coll'0. pubescens nostrale. Cosi mi scrive il dottor Beck.

\section{Orobanche minor.}

Orobanche minor Sutt. in trans. linn. soc. 4. p. 179. Trev.prosp. fl. eug. p. 23. Guss. fl. sic. syn. 2. p. 133. De Not. rep. fl. lig. p. 308. P. Sav. /l. gorg. p. 273. Bert. R. ital. 6. p. 420 (excl. $\beta, x)$. Com. fl. com. 5. p. 87. Zan. prosp. fl. ven. p. 25. Sim.! fl. alp. vers. p. 139. Rota prosp. piant. Pav. p. 270. Hausm. fl. Tir. p. 651. Rota prosp. fl. Berg. p. 67. Grech Del. fl. mel. p. 29. Guss. enum. pl. Inarim. p. 248. Mor. fl. sard. 3. p. 253. Tassi fl. prov. sen. p. 50. Ard. cat. pl. Ment. p. 28. Gar. prodr. fl. tosc. p. 497. Fl. Montecr. p. 27. Ard. fl. Alpes-mar. p. 290. De Vis. Sacc. cat. piont. Ven. p. 159. Genn. fl. Capr.p. 113. Zersi prosp. piant. Bresc. p. 158 (excl. ß). Mars. cat. pl. Cors. p. 110. Terr. sec. rel. Terra di Lav. p. 93. Archb. fl. Alto-Serch. p. 59. Ces. Pass. Gih. comp. fl. ital. p.334. Ing. cat. sp. Mond. p. 54. Gald. fl. fav. tent. p. 175. Arc. comp. fl. ital. p. 530. Nic. prodr. fl. mess. p. 336. Cocc. fl. Bol. p. 370. Gib. Pir. suppl. fl. Hod. p. 22.

Orobanche leonuri Rota prosp. piant. Pav. p. 270.

Orobanche barbata Loj. is. eol. p. 122.

Orobanche pumila Loj.? crit. Orob. p. 59. Nic.? l. c.

Figure.-Vauch. mon. t. 4. Reich. ic. bot. f. 876, 877, s79. It. fl. germ. 20. t. 183.

$\beta$ canescens, bracteis præsertim lanatis.

Orobanche canescens Presl? del. pray. Bert. o.c. p. 4.12. Grech Del.! o.c. p. 2S. Mor. o. c. p. 255. Terr. R. Vult. syn. p. 133. Ces. Pass. Gib. o. c. p. 392. Loj. is. eol. p. 192. Arc. o. c. p. 59S. Nic. o. c. p. 335 .

Urolanche arcuata Loj.? crit. Orob. p. 56 .

Figura. - Reich. ic. /l. germ. 20. 1. 210.

Froza IтALIAKa. - Vol. Vi. 
Stazione, Abitazione e Fioritura. - Parassita su moltissime piante diverse, ma principalmente su Faseolacee, per tutta l'Italia. Nell'Alta Italia è stata trovata a Susa (Parl.!), a Mondovi, ovunque (Ing.), nel Pavese (Rota ecc.), a Milano (Rampoldi !), a Ciomo, Mendrisio, Lugano, Morbegno (Com.), a Bergamo (Rota) e Brescia, frequente (Zersi), in Tirolo dal confine italiano a Bolzano, e in val Ve. nosta, comune (Hausm.), nel Veronese al monte Pastello, a Bassano (Bert.), negli Euganei (Trev.), a Chioggia (Bert.), Venezia (Zan.), Treviso, a Trieste (Bert.), Pola (Reich.), Fiume (Kocls) ecc., a Faenza (Cald.), a Bologna (Bert.) e altrove nel Bolognese (Cocc.), nel Modenese all'alpe di Succiso (Gib. Pir.), nel Parmense a Collecchio ecc. (Passerini!). In Liguria trovasi nel Nizzardo, comune (Ard.), cosi a Mentone (Bourgeau!), e fino a Tenda (Reich.), quindi a Bordighera (Ricca!), ecc.; in Toscana è comune assai, cosi a Sarzana (Bert.), nella bassa Versilia (Simi!), a Lucca (Calandrini! ecc.), e nell'appennino lucchese a Lucignana (Bert.), nel monte Pisano, nel Fiorentino!, in Casentino (Parl. !), nel Senese (Tassi), a Volterra (Bert.), nelle isole di Gorgona (P. Savi), di Capraia (Mor. De Not.) e di Montecristo (Taylor) ecc.; a Viterbo (Bert.), a Roma (Webb!) dov'è comune (Seb. Maur.); nelle Marche al monte Cupiolo nell'Urbinate (Bert.), e a Montefortino nel Piceno (Marzialetti!). Nasce poi in Campania a Picinisco a Valcopella (Terr.), e nel monte Marsico (Terr.!), a Portici (Bert.), in Ischia (Bolle!, Guss.), in Capri (Kesselmeyer!); in Calabria a Pizzo (Ricea!); in Ciorsica ad Aiaccio (Requien!) e a Rogliano (Mars.); in Sardegua comunemente (Mor.), cosi a Caprera e Maddalena (Genn.), a Cagliari (Genn.!), presso Bonasia e Piscinas, ad Arizzo ecc. (Ascherson!); in Sicilia a Lipari, Saline, Panaria, Ustica (Guss.), a Palermo (Guss., Todaro!), Tortorici (?) (Loj.), Messina (Nic.), Scoglitti (Aiuti!); in Malta (Grech). La var. parassita sui Chrysanthemum ed altre Asteracee nell' Italia meridionale: in Corsica a Bonifacio (Bert.), in Sardegna (Mor., Bert.), in Sicilia nelle Eolie (Guss., Bert., Loj.), a Palermo (Parl.!), Mirto (Todaro!), Messina (Nic.) ece., in Malta (Grech), nel Napoletano (Ten.) al Vulture (Terr.). Fiorisce da marzo ed aprile a giugno e luglio secondo i luoghi.

Distribuzione geografica. - Nell'Europa media e meridionale, nell'Asia Minore, nell'Affrica settentrionale.

Osservazioni. - Probabilmente di questa specie sono forme parecchie delle precedenti, e qualcuna delle seguenti.

L' Orobanche yucce Bert. (Fl. ital. 6. p. 423. Reut. in Cand. 
prodr. 11. p. 719. Car. prodr. 月. tosc. p. 497. Sec. suppl. p. 23. Ces. Pass. Gib. comp. 凡. ital. p. 334. Arc. comp. fl. ital. p. 531), già trovata nella Selva Pisana presso il mare, e nell'orto botanico di Pisa, parassita su di una Túcca, da P. Savi che la mandò a Bertoloni senza nome, secondo il dottor Beck è l'Orobanche minor "forma spica laxa. " Non so se sia medesimamente dell' 0 . yuccee trovata di recente dal sig. Lojacono nell' orto botanico di Palermo, parassita sulla Sanicula (Crit. Oroh. p. 54).

\section{Drobanche amethystea.}

Orobanche amethystea Thuill. fl. Par. ed. 2. p. 317. Guss. fl. sic. syn. 2. p. 135. Gren. Godr. fl. Fr. 2. p. 641. Hausm. fl. Tir. p. 651. Mor. fl. sard. 3. p. 254. Ard. f. Alpes-mar. p. 290. Mars. cat. pl. Cors. p. 110. Archb. fl. Alto-Serch. p. 59. Ces. Pass. Gib. comp. fl. ital. p. 333. Arc. comp. fl. ital. p. 530. Nic. prodr. fl. mess. p 33\%. Loj. crit. Orob. p. 60.

Figure. - Vauch. mon. t. 10. Reich. ic. bot. f. 920, 921. Ic. f. germ. 20. t. 185

Stazione, Abitazione e Fioritura. - Parassitica sugli Eryngium, come pure (dicesi) su Asteracee e Faseolacee: in Tirolo (Hausm.), in Pienıonte presso Susa (Vanch.), a Nizza e alla Rocchetta (Ard.), e presso Tenda (Reut.), in Toscana a Cercina presso Firenze (Bucci!), a Messina (Nic.), presso Palermo a Sferracavallo nel monte Gallo (Guss.), in Sardegna, non rara (Mor.), in Corsica (Gren. Godr.). Fiorisce da aprile a luglio secondo i luoghi.

Distribuzione geografica. - Nell'Europa media e meridionale, in Asia Minore, in Algeria.

\section{Orobanche cenvarise.}

Orobanche cervarix Suard in Godr. A. Lorr. 2. p. 180. Ces. Pass. (jib. comp. A. ital. p. 333. Are. comp. 1. ital. p. 599. p. 38 .

Orobanche caudata De Not. rep. 凡. lig. p. 306. Prosp. ^. lig.

Figura. - Reich. ic. /l. germ. ?0. 1. 17\%.

Stazione e Abitazione. - Parassita sul Peucedanum Certaria in Liguria a Sestri di ponente (De Not.).

Distribuzione geografica. - Trovasi piii particolarmente nel. I'Europa media. 


\section{Drolbanche litorea.}

Orobanche litorea Guss. fl. sic. prodr. 2. p. 184. Bert. fl. ital. 6 p. 443. Ces. Pass. Gib. comp. fl. ital. p. 334. Arc. comp. fl. ital. p. 531. Nic. prodr. fl. mess. p. 336.

Stazione, Abitazione e Fioritura. - Parassita su Asteracee nelle sabbie marittime di Sicilia, comunissima nelle spiaggie meridionali, cosi a Palma (Loj.), ma trovasi anche altrove, come a Messina (Nic.). Fiorisce in aprile e maggio.

Distribuzione geografica. - Specie siciliana.

Osservazione. - A parere del dottor Beck, non è che una forma dell' Orobanche minor.

\section{Orobanche conulescens.}

Orobanche cœrulescens Steph. in Willd. sp. plant. 3. p. 349. Pir. fl. for. syll. p. 107. De Vis. Sacc. cat. piant. Ven. p. 160. Ces. Pass. Gib. comp. fl. ital. p. 334. Arc. comp. fl. ital. p. 528.

Figure - Reich. ic. bot. f. 925. Ic. fl. germ. 20. t. 188.

Stazione, Abitazione e Fioritura. - Nel Friuli, parassita sụll'Artemisia campestris, raccoltavi dal Brumati (Pir.). Fiorisce in maggio e giugno.

Distribuzione geografica. - Nell' Europa media, nell'Asia occi. dentale e centrale.

Osservazione. - Secondo il dottor Beck, questa specie non si trova in Italia.

\section{Drobanche cermua.}

Orobanche cernua Lœfl. it. hisp. p. 152. Mars. cat. pl. Corse p. 110. Loj. contr. fl. Sic. p. 12. Is. eol. p. 122. Crit. Orob. p. 65.

Orobanche cumana Guss. fl. sic. syn. 2. p. 137 (non Wallr.). Loj. crit. Orob. p. 64.

Orobanche bicolor Bert. fl. ital. 6. p. 448. Ces. Pass. Gib. comp. fl. ital. p. 333. Arc. comp. fl. ital. p. 529. Loj. crit. Orob. p. 54.

Figura. - Reich. ic. fl. germ. 20. t. 187.

Stazione, Abitazione e Fioritura. - Parassita su Asteracee e anche su Faseolacee in Sicilia: nelle isole Eolie in Panaria (Guss., 
Bert.), in Vulcano nella contrada Vallonaccio, e in Salina (Loj.), nelle Madonie alle Serre delle Croci a 1200 metri, al Comunello, e ad Isnello al bosco di Mlontaspro (Loj.). Nasce pure in Corsica a Bo nifacio, e a Rogliano (?) (Mars.). Fiorisce da aprile a giugno secondo i luoghi.

Distribuzione geografica. - Nel bacino del Mediterraneo, nell'Asia occidentale, nell' India, in Australia.

\section{FAMIGLIA SESTA.}

UTRICULARIACER.

Lentibulaires L. C. Rich. an. du fr. (1808), nomen tantum.

Utriculine Hoffin. et Link fl. port. 1. p. 337 (1809).

Pinguiculace el Utriculariacea Dum. an. des fam. $p .23$.

Utriculariace⿸.

Gemmularium 1-loculare, gemmulis plurimis in spermophoro centrali subhorizontalibus, anatropis. Fructus capsularis. Semen examygdalosum.

Descrizione. - I due generi italiani di questa piccola famiglia hanno portamento diversissimo, come si può vedere riferendosi alle descrizioni particolari di ognuno.

I fiori sono sempre laterali, anche dove (Pinguicula) sembrano terminali. Sono all'ascella di una brattea, che peró può essere atrofica o abortita. Non esistono bratteole.

Il calice essendo כ̆-mero, il sepalo dispari è posteriore. È bi. labiato, 3 sepali andando al labbro posteriore, 2 al labbro anteriore. Nella preflorazione il labbro anteriore abbraccia il posteriore nel gen Utricularia, mentrechè nel gen. Pinguicula la preflorazione ė aperta. II calice è persistente.

La corolla è vistosa, non per le sue dimensioni ma per il suo colorito vivace e per essere lontana da altre parti della pianta che la potessero nascondere. $\dot{F}$ grandemente irregolare, bilabiata, il labbro anteriore essendo spronato o saccato alla base, e prominente alla gola. La preflorazione n'è embriciata; nelle Utricularia il labbro anleriore abbraccia il posteriore. Es caduca la corolla. 
Più irregolare ancora è l'androceo, attesochè dei suoi 5 stami che dovrebbero essere, i 3 posteriori sono totalmente abortiti, e non ne restano che 2 , alternanti coi lobi del labbro anteriore della corolla. Sono inclusi; hanno il filamento inarcato, dimodochè le antere stanno ravvicinate fra.loro; queste sono inserite a capocchia, sono uniloculari, deiscenti per il traverso: veramente a quanto parrebbe 2-loculari in origine, con le caselle confluenti. Il polline è striato, almeno nelle Utricularia.

Il gineceo costa di 2 pistilli antero-posteriori, connati. Ne risulta un gemmulario 1-loculare, la di cui cavità è tutta occupata da un grosso spermoforo centrale, globoso, con un piede, tutto coperto di gemmule. V'ha uno stilo grosso e corto, 2-lobo all' apice, il lobo posteriore essendo piccolo, l'anteriore invece slargato, solo stimma. tifero.

Le gemmule sono descritte come anatrope, con l'integumento scempio.

Il frutto è cassulare. Si apre per una rottura irregolare della sua parete, o per la scissione di questa in due valve. Contiene semi numerosissimi, senza mandorla.

L'embrione è afillo, monofillo o difillo.

Considerazioni geografiche. - L'Italia possiede tutti e due i generi europei della famiglia, e la maggior parte delle specie.

Queste sono tutte dell' emisfero boreale, e più particolarmente delle sue parti fredde; per cui la piccola famiglia rappresenta nella flora italiana un elemento nordico.

\section{UTHE ICULARIA.}

Lentibularia Riv. ord.pl. A. irreg. monop. (fig.).

Utricularia Linn. gen. pl. ed. 1. p. 5. A. de Cand. in prodr. syst. nat. 8. p. 3. Benth. Hook. gen. pl. 2. p. 987. Ces. Pass. Gib. comp. fl. ital. p. 416. t. 66 .

Calyx bilabiatus, labiis subæqualibus, integris. Corolla hypogyna, personata, basi antice calcarata, tubo subnullo, labio superiore breviore trilobo, inferiore majore, palato prominente. Stamina 2, labio superiore basi inserta. Filamenta complanata subclavata, incurva, apice conniventia. Antheræ e confluentia loculorum uniloculares, oblongo-lineares, utrinque obtusæ, longitudinaliter dehiscentes. Pol- 
len discoideum costatum. Ovarium subglobosum, uniloculare, placenta basilari, globosa. Ovula plurima, subglobosa, anatropa. Stylus brevis, cylindraceus, rectus. Stigma bilabiatum, labio superiore obsoleto, inferiore lamelliforme, dilatato, margine subfimbriato (Parlatore ms.).

Portamento. - Piante sommerse, galleggianti nelle acque col fusto ch'è ramoso, e con le foglie che sono ripetıtamente divise in lobi sottilissimi, talora tutti cosi, talora invece alcuni rigonfiati in vesciche. Dalle acque sorgono scapi fioriferi alti $1-\overline{3}$ decimetri, semplici, terminati da un grappolo semplice indefinito rado di pochi fiori, con pedicelli piuttosto lunghi ascellati da una piccola brattea. I fiori sono inchinati, grandetti, gialli.

Osservazione. - Sulla morfologia e istologia di queste piante assai curiose si può consultare: Schacht Beitr. zur. Anat. u. Phys. der Gew., Irmisch in Flora 1858, Crouan in Bull. soc. bot. de Fr. 5, Buchenau in Bot. Zeit. 1865, Van Tieghem in Ann. sc. nat. $5^{a}$ ser. 10, Pringsheim Zur Morph. der Utricularien, Warming Contrib. à lu conn. des Lentibulariarece, Gilbert e Marchal in Comptes rend. de la soc. de bot. de Belgique 1882, Clos Des org. interm. entre la rac. et la feuille.

\section{A. Unoieularia vulgaris.}

U. foliis ambitu ovatis, ciliato-spinulosis, vesiculiferis, corollæ calcare descendente, conico lateribus plano vel depresso, corolla ipsa duplo breviore, labio superiore palatum labii inferioris suhæquante, antheris coniunctis.

Utricularia vulgaris Linn. sp. pl. el. 1. p. 18. Re fl. seg. p \&. Bert. A.ital. 1. p. 120. Mass. prodr. A. valt. p. 194. Com 1l. com. 1. p. 26. Colla herb. ped. 4. p. 512. Trev. mosp. 月. eug. p. 22. Guss fl. sic. syn. 1. p 19. De Not. rep. fl. lig. p. 333. Zan. prosp. Al. ven. p. 26. Puce syn. pl. luc. p. 19. Rota prosp. piant. Pav. p. 272. Hausm. 11. Tir. p. 705. Rota prosp. 1. Berg. p. 71. Pir. A. fornj syll. p. 115. Car prodr. 1l. Losc. p. 435. De Vis. Sace. cat. piant. Ven. p. 160. Zersi prosp. piant. Brest. 1. 171. Cocc. contr. A. Bol. p. 18. Ces. Pass. Gib. comp. A. Ital. p. 416. Terr.! quart. rel. Terra di Lav. p. 111. Anzi auct. 11. nov.-com. 1. 196. Sace. Bizz. agg. 1.. trev. p. 30. Arc. comp. R. Ital. p. 564. (jib. Pir. R. Mod. p. 134. Cocc. /l. Bol. p. 13..

Figure. - Fl. dan. 1. 138. Reich. ic. 1. germ. 20. t. 201, 202. 
Stazione, Abitazione e Fioritura. - Nelle palıdi, nelle risaie, nei fossi ed in altri luoghi acquosi. Nell' Alta Italia è molto sparsa per i luoghi bassi, dal Piemonte al Friuli (Pir.), e a Ravenna (Caldesi!), e nasce anche in montagna, cosi nei monti Generoso e Cauzo del Co. masco (Com.), in Valtellina a Paluaccio sopra Oga, Livigno e Vallaccia (Anzi), e nell'Appennino modenese al lago di Pratignano (Gib: Pir.). Nel restante della Penisola è molto rara, non essendo stata trovata che presso Sarzana!, nei dintorni di Lucca (Bicchi! ecc.) e di Pisa!, in Terra di Lavoro a Minturno presso Traetto (Terr.!), a Napoli al Sebeto (Ten.) e al Pascone (Pasquale!). Fa in Sicilia negli stagni montuosi di Gurgo lo Drago e Gurgo lungo presso il Cotrano (Guss.) e a Spaccaforno (Bert.). Manca in Corsica e in Sardegna. Fio. risce in giugno e luglio.

Distribuzione geografica. - In tutta Europa meno la piủ nordica, in Algeria, nell'Asia settentrionale, nell'A merica settentrionale.

Descrizione. - Pianta sommersa nell'acqua con le foglie, e con gli scapi in gran parte fuori dell'acqua. Le foglie sono espanse, di un bel color verde, divise in molte lacinie capillacee, alterne, patenti, qua e là aventi verso gli angoli alcune piccolissime lacinie a guisa di spinette; esse hanno alcune vescichette piccole, verdi, a guisa di sacchetti conici, con la base diretta in giù e con l'apice ing alto, dove è un orifizio, guarnito di un piccolo coperchio e per lo più di due appendici, talvolta di tre o più, a guisa di setole lunghe e bianche che dapprima curvate a guisa di arco, si estendono poi e diventan diritte. Tali foglie vengono da uno stelo orizzontale, delicato, cilindrico, liscio, di un verde chiaro, il quale si prolunga mandando a qualche distanza gli scapi. Questi nascono da un punto laterale dello stelo: talvolta ve ne è un solo. Lo scapo s'innalza verticalmente per cinque o sei pollici, è gracile, cilindrico, liscio, alquanto flessuoso, di un verde chiaro, eccetto in alto dove corrisponde ai fiori, e dove è rossiccio e più manifestamente flessuoso; dalla base manda uno o due rami quasi orizzontali, vicinissimi tra loro, i quali portano le foglie con le vescichette come lo stelo, e due rametti un poco più in alto $\mathrm{i}$ quali discendono obliquamente o quasi verticalmente in giù e parrebbero dover essere considerati come due fibre radicali: questi hanno dei rametti cortissimi laterali, quasi alterni, ciascun rametto è per lo più bifido e termina in una quantità di piccole punte larghette e aguzze, unite in fascetti; nel resto lo scapo è nudo, e solo ha piccolissime brattee quasi ovate e verdognole, l' una a molta distanza dall'altra. I fiori sono da 5 a 6 , distribuiti in un racemo corto 
e terminale, si aprono successivamente in modo che non vi sono mai più di due fiori aperti. Ciascun fiore è portato all'apice di un pedun. colo delicato, cilindrico, rossiccio, lungo quasi quanto il fiore, un poco curvato in giù nel fiore, diritto e patente nel frutto, accompa. gnato da una piccola brattea simile a quella del fusto e di colore quasi rossiccio. Il calice è diviso in due lacinie a guisa di labbra, una superiore appena più stretta dell'altra ch'è inferiore: queste lacinie sono ovate, ottuse, concave in avanti, convesse posteriormente, ver. dognole alla base, di colore rossiccio con molte vene longitudinali di colore verdognolo. La corolla è grande, di color giallo quasi do. rato, personata, con il tubo quasi nullo, divisa in due labbra, uno superiore piủ piccolo dell' altro inferiore. Il primo è diretto quasi ver. ticalmente in alto, è leggermente trilobo con il lobo medio piủ lungo ma piủ stretto dei lobi laterali, i quali hanno il margine diretto un poco in avanti onde il labbro è ivi un poco concavo. Il labbro inferiore ha il palato assai sporgente, e gonfio, è di colore dorato nel mezzo e con nervetti longitudinali, interrotte, di un rossiccio scuro, nel resto il labbro è espanso quasi orizzontalmente, ha un margine tondeggiante e leggermente crenulato: di sotto ha in avanti uno sprone conico, corto, verdognolo, nel quale di dentro anteriormente si vedono prolungate le linee del palato. Gli stami sono 2 , inseriti alla base del labbro superiore. I filamenti sono alquanto schiacciati, più grossi in alto, onde hanno la forma quasi di una clava, curvati ad arco e avvicinati tra loro superiormente in modo che le antere si toccano. Queste sono bianchicce, allungate-lineari, ottuse alla estre. mità, ristrette alquanto nel mezzo nel punto dove dorrebbe essere la divisione delle logge, per la mancanza della quale esse sono uniloculari; si aprono con una fenditura longitudinale. Il polline è bianchiccio, discoideo con leggiere costole rilevate. Il pistillo sta dietro degli stami ed é poco più lungo di essi. L'ovario è quasi globoso, verdognolo, con leggiere vene longitudinali più cariche e con alcuni punti scabri verdognoli visibili con una forte lente, ha una sola loggia, con, una placenta basilare globosa, sulla quale stanno inolti oroli, quasi tondi, bianchicci, e anatropi, aventi una sola inembrana. Lo stilo é corto, diritto, cilindrico, un poco schiacciato in alto dove si allarga in uno stimma bilabiato il di cui labbro superiore è cortissimo, verdognolo, leggermente dentellato e il labbro inferiore è largo, quasi tondo, bianchiccio con il margine quasi frangiato: questo labbro corrisponde dietro e un poco al di sopra delle antere. (Parl. ms., descr. della pianta di Sarzana). 


\section{Utrieularia dubia.}

U. foliis...., corollæ calcare adscenderite, conico lateribus con. cavo..., labio superiore...., antheris coniunctis.

Utricularia dubia Ros. in Ces. Pass. Gib. comp. fl. ital.p. 416.

Stazione, Abitazione e Fioritura. - Nei paduli fiancheggianti il Po presso Casale Monferrato. Settembre. (Ces. Pass. Gib. 1. c.).

Distribuzione geografica. - Pianta propria della suddetta lo. calità.

Osservazione. - Non conoscendo altrimenti questa pianta, nè potendo giudicare quanto veramente differisca dalla precedente, riferirò ciò che di essa scrive Cesati in una nota del Compendio I. c.

a U. fauce palato bilobo inflato clausa, labio inferiore oblongo, convexo subintegro, margine undulato, palato duplo longiore; labii superioris margine erosulo undulato, palatum amplectente; calcare conico compresso obtuso adscendente (ad palatum inflexo) latitudine et longitudine æqualibus. Antheræ adnatæ. " Rosellini in sched. Calcar in vivo eximie biconcavum in lateribus, nec simpliciter compressum; confirmante etiam egr. dom. Negri qui una cum beato Auctore pluries stirpem collegit. Prima vice anno $1814 \mathrm{~m}$. sept. Amicus dilectissimus mecum detexit."

\section{Utricularia neglectr.}

U. foliis ambitu ovatis, ciliato-spinulosis, vesiculiferis, corollæ calcare adscendente, conico subcompresso...., labio superiore palato labii inferioris subtriplo longiore, antheris disiunctis.

Utricularia neglecta Lelim. ind. schol. in hamb. gymn. p. 38. Koch syn. fl. germ. ed. 2. p. 665. Nym. syll. fl. eur. p. 134. Ard. 17. des Alpes-mar. p. 306. Ces. Pass. Gib. comp. fl. ital. p. 416. Arc. comp. fl. ital. p. 564

Figura. - Reich. ic. fl. germ. 20. t. 203.

Abitazione e Fioritura. - A Venezia (Nym.); a Nizza verso il Varo (Ard.). Fiorisce da giugno ad agosto (Ard.).

Distribuzione geografica. - Nella Svezia meridionale, in Da. nimarca, nella Germania settentrionale, nella Francia settentrionaleoccidentale, in Svizzera, in Ungheria, in Italia, ovunque in poche località. 


\section{Utricularia intermedia.}

U. foliis ambitu oblongis, aliis sine resiculis ciliato-spinulosis, aliis vesiculiferis tantum, corollæ calcare adscendente, conico acuto, corolla ipsa subbreviore, labio superiore palato labii inferioris du. plo longiore, antheris disiunctis.

Utricularia intermedia Hayne in Schrad. journ. 1800. fasc. 1. p. 18. Koch syn. fl. germ. ed. 2. p. 665. Hausm. R. Tir. p. 705. Rota prosp. fl. Berg. p. 71. De Vis. Sacc. cal. piant. Ven. p. 160. Ces. Pass. Gib. comp. fl. ital. p. 416. Arc. comp. fl. ital. p. 504

Figure. - Hayne l. c. t. 5. Reich. ic. fl. germ. 20. t. 203.

Stazione, Abitazione e Fioritura. - Nelle acque stagnanti presso S. Anna Morosina nel Vicentino (De Vis. Sacc.), e lungo l'Adda nel Bergamasco (Rota); in Tirolo nei fossi e nei luoghi pa. ludosi presso Bolzano e sul Ritten verso Kumaten a Pfaffstall (Hausm.). Fiorisce in luglio e agosto.

Distribuzione geografica. - Nell' Europa settentrionale e centrale, in Siberia, nella Nord-America.

\section{Utriculariat minor.}

U. foliis ambitu oblongis, glabris, minute vesiculiferis, corollæ calcare descendente, ovato obtuso, corolla ipsa multoties breviore, labio superiore palatum labii inferioris æquante, antheris disiunctis.

Utricularia minor Linn. sp. plant. ed. 1. p. 18. Bert. A. ital.6. p. 122. Mass. prodr. R. valt. p. 194. Com. fl. com. 5. p. 27. Collı herb. ped. 4. p. 512. Trev. prosp. 月. eug. p. 22. Zan. prosp. /l. ven. p. 26. Pucc.! syn. pl. luc. p. 19. Rola prosp. piant. Pav. p. 272. Hausm. R. Tir. p. 706. Pir. fl. for. syll. p. 115. Car. prodr. fl. Losc. p. 435. Ard. 1. Alpes-mar. p. 307. De Vis. Sacc. cat. piant. Ven. p. 161. Zersi prosp. piant. Bresc. p. 171. Ces. l'ass. Gib. comp. A ital. p. 416. Are comp. 1. ital. 1. 56.4. Gib. Pir. A. Mod. p. 131.

Figure. - Fl. dan. 1. 128. Reich. ic. A germ. 20. 1. 20\%.

Stazione, Abitazione e Fioritura. - Nei paluli, negli acquitrini e lnoghi simili della Penisola. Non è tanto rara nell' Alta ltalia: a Torino a Stupinigi (Colla), a Niblia e Proh nel Novarese (Bir.). nel Pavese verso Martignoni (Nocca Balb.), fra Milano e Treviglio (Rampoldi!), nel Comasco verso Alserio e Pusiano e alla Cá morta 
(Com.), in Valtellina (Mass.), nel Bresciano alle lame Bissa e Pranduna (Zersi), in Tirolo a Bolzano (Hausm.), a Bovolone nel Veronese (Poll.), nel Mantovano, nel Polesine (De Vis. Sacc.), negli Euganei (Trev.), a Venezia (Zan.), a Treviso (Poll.), in Friuli a Virco, Morsano (Pir.); ma è molto rara altrove, non trovandosi che a Nizza al Varo (Ard.), presso Pisa a Erpici (Tassi! ecc.) e intorno al padule di Bientina!, e a Napoli al Sebeto (Ten.). Fiorisce da maggio ad agosto.

Distribuzione geografica. - Nell' Europa seltentrionale e centrale, e in parte nella meridionale, nell' Asia centrale.

Descrizione. - Pianta perenne, di un verde cupo. Fusto delicato, eretto, alto 12 a 15 centimetri, cilindrico, glabro, liscio, rossiccio. Fiori da 2 a 5 , di raro fino a 7 , per lo più 3 o 4 , più piccoli di metà circa della $U$. vulgaris, in un racemo rado, un po'curvato in giủ all'apice prima che vi si aprano i fiori. Pedicelli delicati, cilindrici, eretti o eretto-patenti, appena curvati in giù nel fiore, quasi uguali a questo, più lunghi nel frutto, rossicci, glabri. Brattea piccola, molto più corta del pedicello, abbracciante la base di questo, largamente ovata, ottusa, con qualche dente nel margine, scavata in cuore alla base, rossiccia. Calice diviso profondamente in due labbri, alquanto patuli, uno superiore e uno inferiore, poco disuguali, ovato-orbicolari, ottusi, interi, convessi un po'di fuori, concavi un po'di dentro, rossicci, glabri. Corolla delicata, divisa anch'essa in due labbri, uno superiore più della metà più piccolo dell'altro, lungo quanto il palato, più del doppio più lungo del calice, diretto in avanti e un po' coi margini l' apice in su, largamente ovato, come troncatosmarginato all'apice. Il labbro inferiore è diretto in avanti, quasi orbicolare, un po' ondeggiante nel margine e questo un po' diretto in giù, appena smarginato all' apice. Il suo palato è poco sporgente e appena trilobo, e i due labbri sono di un giallo chiaro con righe o vene rossicce nel palato e nella parte vicina del labbro inferiore, le quali si dirigono verso l'apice e il margine del labbro medesimo ma si perdono presto. Il tubo è cortissimo e prolungato in basso in uno sprone, il quale è larghetto, quasi conico, corto, ottuso, diretto in giù e del colore della corolla. Stami 2, inseriti nel tubo, posti in una concavità del labbro superiore che corrisponde al labbro superiore del calice, ascendenti e rilevati in modo da coprire un po da. vanti ed ai lati l'ovario. Filamenti larghi, piani d'avanti in dietro, un po' piủ grossi in alto, bianchiccio-verdognoli, glabri. Antere piccole, quasi ellittiche, uniloculari, aprentesi longitudiraalmente, gialle. 
Pistillo quasi uguale agli stami, ovario piccolo quasi tondo, rossiccio. Stilo eretto, lungo quanto l'ovario, grossetto, come schiacciato da avanti in dietro, bianchiccio. Stimma grandemente piegato sopra se stesso, come smerlato e gialliccio. (Parl. ms., descr. della pianta del lago Sibolla presso Pisa). L'embrione è afillo (Dickson).

\section{PINGUICUIA.}

Pinguicula Tourn. inst. p. 167. t. 74. A. de Cand. in prodr. 8. p. 26. Benth. Hook. gen. pl. 2. p. 988. Ces. Pass. Gib. comp. fl. ital. p. 417. 1. 66 .

Calyx bilabiatus, labio superiore trifido, inferiore bifido. Corolla hypogyna, bilabiata, tubo brevi basi antice calcarato, labio su. periore bilobo vel emarginato, inferiore majore trilobo, lobo medio majore, lobis oinnibus integris, vel emarginatis, palato subconvexo. Stamina 2, imæ corollæ inserta. Filamenta cylindrico-campanulata, ascendentia, apice crassiora. Antheræ terminales, adnatæ, uniloculares, transverse bivalves. Ovarium uniloculare, placenta basilari glo. bosa multiovulatum. Ovula erecta, anatropa. Stylus crassus, brevis. Stigına petaloideum, supra papillosum, bilabiatum, labio superiore bifido, inferiore majore, antheras tegente. Capsula.... (Parl. ms.).

Portamento. - Un cortissimo rizoma porta di sotto un fascio di radici, di sopra una rosetta di foglie ovali o bislunghe, intere, coi margini rivoltati in dentro, e cosperse di cortissimi peli glandolosi vischiosi. Di nezzo alla rosetta s' innalzano da uno a porhi scapi di $1 / 2-2$ decimetri, gracili, uniflori.

Osservazione. - Sulla vegetazione e la genesi fiorale di queste piante si può consultare Buchenan in Bot. Zeit. 1865 e Dickson in Trans. roy. soc. of Edinb. 25.

\section{Pingaicula vulgaris.}

P. corolla calcare elongato subulato, fructu ovato.

Pinguicula vulgaris Linn. sp. pl. ed. 1. p. 17. Bert. A. ital. I. p. 115. Mass. prodr. 11. vall. p. 131. Com. 11. com. 1. p. 24. Colla herb. ped. 4. p. 513. Trev. prosp. A. eng. p. 92. He Not. rep. A. lig. p. 3.95?. Hausm. /. Tir. p. Y04. Rota! prosp. A. Berg. p. 71. Pir.! A. for. syll. p. 115. Car. prodr. A. 10sc. p. 435. Ard. fl. Alpes- 
mar. p. 307. De Vis. Sacc. cat. piant. Ven. p. 160. Zersi prosp. piant. Bresc. 11. 171. Ces. el. piant. Maiella p. 21. Archb. fl. AltoSerch. p. 54. Ing. cat. sp. Mond. p. 57. Cies. Pass. Gib. comp. fl. ital. p. 417. Anzi anct. fl. nov.-com. p. 196. Sacc. Bizz. agg. fl. trev. p. 29. Arc.! comp. fl. ital. p. 565. Gib. Pir. fl. Mod. p. 133. Cocc. fl. Bol. p. 371.

Pinguicula hirtiflora Ten.l fl. nap. 1. prodr. $p 6$.

Pinguicula grandiflora Bert. o. c. p. 117. Com. o. c. p. 25. Colla o.c. p. 514. Sang. cent. prodr. fl. rom. add. p. 7. De Not. l. c. Pucc.! syn. pl. luc. p. 18. Sim.l fl. alp. vers. p. 13. Rota l. c. Ard. l. c. Mars. cat. pl. Cors. p. 96.

Pinguicula corsica Bern. Gren. in Gren. Godr. fl. Fr. p. 443. Mars. l. c.

Figure. - Fl. dan. t. 93. Fl. nap. t. 210. f. 1, 2 (pess.). Reich. ic. bot. t. 82. f. 171. t. 84.f. 175. Ic. fl. germ. 20.t. 198. f. 1-3. t. 199. f. 2-4. Bot. mag. t. 6785.

Stazione, Abitazione e Fioritura. - Per i monti, nei luoghi erbosi umidi, sulle rupi bagnate, alle fonti ecc. $\dot{\mathrm{E}}$ comune per tutte le Alpi, dalle maggiori altezze scendendo nelle vallate: in Friuli nel inonte Mataiura, presso Rodda, Pontebba, Tolmezzo, Verzegnis (Pir.), nei monti Cavallo (Kellner!), Portale, Grappa e Sumano (Montini! ecc.), presso Bassano in val di S. Felicita di Romano (Montini!), negli Euganei (Trev.), nel Baldo nelle valli Fredda e Losana (Poll.) e a Navole (Manganotti!), in Tirolo presso Borgo, in Fiemme e Fassa, in val di Rendena e sullo Spinale, a Bolzano e Olten e sul Ritten, in val Venosta a Taufers, in Pusteria a Welsberg (Hausm.), nel Bresciano alle Colombine (Zersi), al Dos alto a 1700-1800 metri (Parl.!), nel Bergamasco in molti luoghi (Rota), per esempio a Ponte di legno, al monte Venerocolo a circa 7000' (Parl.!), nel Comasco altresi frequente, nonchè in Valtellina (Com., Mass., Anzi) fino allo Stelvio a 2000-2100 metri (Parl.!), al S. Bernardino (De Not.!), al Gottardo (Heldreich!), al Sempione (Parl.!), in Piemonte nel Novarese alle radici dei monti (Bir.), a Riva di Sesia (Carestia!), nel Cenisio al lago a 2000 metri (Arc.!) e a Ronche (Bucci!), a Caldemozzo in quel di Susa (Re), nelle vallate Valdesi, frequente (Ro. stan!), nel Monviso, presso Sampeyre (Colla), nei monti di Valdieri (Bert.), in quelli di Viosenne (Ricca!), a Mondovi, ovunque (Ing.). Seguita per le Alpi marittime, dove si trova generalmente, per esempio al col delle Cerese a 6-8000' (Ball!), a S. Martino (Bert.), e dal col di Tenda (Bourgeau! ecc.) a Saorgio (Bert.) e a S. Agnese sopra 
Mentone (Ard.), e seguita per l'Appennino ligustico, qua e là, per esempio fra Montalto e Triora (Panizzi!), nei monti d'Albenga (De Not.), nel monte Moro sopra Campomorone (Carrega!), dietro Pegli alla Scaggia (Bert.). Si ritrova in tutte le parti più alte delle Alpi apuane!; medesimamente nel vicino Appennino, dall'alpe di Succiso (Gib. Pir.) al Corno alle scale (Cocc.). Quindi ricomparisce nell'alto Appennino piceno-abruzzese nei monti Acuto, Pizzo di Sivo, Pelone, Corno (Orsini!), Naiella a 2400-2500 metri (Levier! ccc.) e altrove (Ten.). Nasce ancora nel monte S. Angelo di Castellam. mare all'Acquasanta (Ten.!) presso la Cava (Pasquale!), e altrove (Ten.). Infine nasce in Corsica nei monti Grosso, Rotondo (Bert.), Cinto, d’ Oro, Cagno (Gren. Godr.), Renoso, Coscione ecc. (Mars.). Fiorisce in giugno, luglio ed anche agosto, e sin da maggio ne'luoghi meno freddi.

Distribuzione geografica. - In tutta Europa, in Asia Minore, in Siberia, nella Nord-America.

Descrizione. - La radice è formata di fibre delicate e hianchicce. Le fuglie sono allungate, ottuse, lunglıe poco più di un pollice, di co. lor verde assai chiaro e quasi giallicce, carnosette, contenenti un succhio limpido ma alquanto vischioso, con un grosso nervo longitudi. nale nel mezzo della pagina inferiore, ed hanno la pagina superiore coperta di tante papille sporgenti ed ottuse, all'apice delle quali è un pelo bianco, diritto, formato di poche cellule poste l'una all'estre. mità dell'altra a guisa di coroncina e terminate all' apice da una glan. doletta bianca e tonda a guisa di un capolino di spillo. Lo scapo è solitario ovrero ve ne sono due, è gracile, cilindrico, insensibilmente assottigliato rerso l'apice, alto tre o quattro pollici, cilindrico, coperto di peli corti, orizzontali e glandolosi all' apice come i peli delle foglie, è verde inferiormente, di color violetto verso l'apice dove $\dot{i}$ alquanto curvato per sostencre il fiore. Questo è grandicello per la pianta, di color violetto, con una macchia bianca verso il centro del labbro inferiore. Il calice è di color verde scuro, sfumato di violetto, è diviso in duc labbra, il labbro superione è trifido, il labbro inferiore è bifido, le lacinie sono più profonde in quello che in questo, son tutte allungate, ottuse e coperte di fuori di peli corti e glandulosi. La corolla la di fuori alcuni peli glandolosi, corti, ed è livisa in due labbra, cou il tubo corto e prolungato alla base in avanti in uno sprone lunghetto, quasi lesiniforme, diritto, ottuso e quasi troncato all'apice, coperto anch' esso di qualehe pelo glandoloso, assai corto. Il labbro superiore è piu corto, bilobo, con i lobi quasial. 
lungati, ottusissimi quasi troncati e come un poco smarginati e rosicchiati nel margine, il labbro inferiore è quasi il doppio più lungo del superiore, trilobo, con il lobo intermedio un poco più grande dei due laterali, tutti quasi della stessa forma dei lobi del labbro superiore, però di sopra verso il mezzo e la base offrono molti peli lunghi e densi i quali sono formati inferiormente da due o tre cellule lunghe e verso l'apice da cinque, sei ed anche più cellule, quasi sferiche e cortissime in modo da formarvi una coroncina. Gli stami sono inseriti alla infima base della corolla dalla parte del labbro superiore. I filanenti sono curvati ad arco inferiormente, quasi diritti e convergenti tra loro in alto, cilindrico-schiacciati da avanti in dietro, ingrossati in alto e bianchi. Le antere sono portate all'apice dei filamenti con i quali sono saldati, sono uniloculari, bivalvi trasversalmente e nascoste sotto il labbro inferiore dello stimma che interamente le ricopre: esse sono assai vicine tra loro in modo che si toccano. L'ovario è quasi globoso, verde, coperto di peli glandolosi, si vede in parte in mezzo e dietro dei filamenti nella parte dove essi sono piủ lontani tra loro, è uniloculare e contiene molti ovuli diritti e anatropi portati sopra una placenta globosa e basilare. Lo stilo è corto, cilindrico e verdognolo. Lo stimma è largo, petaloideo, di color violetto, coperto di sopra di molte papille di color violetto carico, è diviso in due labbra, uno superiore bifido con le lacinie quasi lineari, uno inferiore più grande e largo, quasi tondo e come smarginato all'apice. (Parl. ms., descr. della pianta dell'Appennino toscano). L'embrione è monofillo (Saint-Hilaire, Buchenau, Dickson).

Osservazioni. - Pianta assai variabile: per l’ altezza dello scapo, da $1 / 2$ decimetro a più di 1 decimetro; per la grandezza delle foglie, lunghe da $1 \frac{1}{2}$ a 6 centimetri, e per la loro forma, ovata 0 bislun. ga; per la grandezza dei fiori, che misurano nella corolla (compreso lo sprone) da $1 \frac{1}{2}$ a $21 / 2$ centimetri di lunghezza; per il loro colore, ordinariamente violaceo, talora roseo 0 gialliccio; per la proporzione dello sprone con il restante della corolla, ora della metà 0 dei ${ }^{2} / \mathrm{s}$ e anche più, ora di $1 / 3$ (Pinguicula corsica); per i lobi del labbro inferiore della corolla, interi, smarginati, o quasi bilobi ( $P$. hirtiflora).

\section{Pinguicula alpina.}

P. corollæ calcare abbreviato conico, fructu oblongo.

Pinguicula alpina Linn. sp. pl. ed. 1. p. 17. Bert. fl. ital. 1. p. 118. Mass. prodr. fl. valt. p. 131. Com. fl. com. 1. p. 25. Colla 
herb. ped. 4. p. 514. Trev. prosp. fl. eng. p. 22. Huusm. fl. Tir. p. 704. Rota! prosp. 1l. Berg. p. 71. Pir.l fl. for. syll. p. 115. Ard. f. Alpes-mar. p. 307. De Vis. Sacc. cat. piant. Ven. p. 160. Zersi prosp. piant. Bresc. p. 170. Ces. Pass. Gib. comp. fl. ital. p. 417. Anzi auct. fl. nov.-com. p. 196. Arc. comp. fl. ital. p. 525.

Figure - Fl.dan, i. 453. Reich, ic. bot. t. 81. Ic. fl. germ. 20. t. 200. f. 3, 4 .

Stazione, Abitazione e Fioritura - La stazione è quella stessa della specie precedente, ma l'abitazione è ristretta alle Alpi, dove parimente dalle vette cala in basso nelle vallate: nel Friuli, ovunque (Pir.!), per esempio a S. Daniele, Amaro, Verzegnis (Suffren), nel monte Cavallo di Belluno (Kellner!), presso Bassano nelle selve di Vallerano, Oliero e Grappa (Montini!), nei Sette Comuni (Bert.), negli Euganei (Trev.), nel Baldo all'Aque negre e al Campion, in Tirolo a Col santo presso Roveredo, nei monti Alba, Zevola, Bondone, Povo, Buco di Vela, presso Borgo, in Fiemme e Fassa, in val di Genova, e di Non alla Rocchetta, a Denno, al Gazza, nei dintorni di Bolzano (Ilausm.), al Tonale (Ricca!), nel Bresciano dalle vette del Muffetto e del Dossalto fino a Caino (Zersi), nel Bergarnasco a Carenno ecc. (Rota!), nel Comasco sui monti Resegone (Aiuti!), Corni di Canzo (Bracht!), Generoso, Coderio, Pizzo di Gino, Aigua, Tabano, S. Primo, nelle Grosgalle tra Lezzeno e Bellagio (Com.), nella val Tellina al Valdone (Mass.), alla Maloia, nei monti Bormiensi, molto frequente (Anzi), allo Stelvio a 2000 metri (Parl.!), nel monte Clamoghe del Canton Ticino (Com.), prešso Lugano (Bert.), in val Travaglia al lago Maggiore (Gibelli!), in Piemonte ai monti Rosa, Moro (Bir.), Marguzzollo (Bert.), a Riva di Sesia (Carestia!), al Cenisio (Beccari! ecc.), presso Susa alle Gorgie (Re), presso Torino a Peceto (Delponte! ecc.), in valle S. Martino (Rostan!), in val di Pesio, a Ciarlino, al monte Bego, al col di Fremamorta (Ard.). Fiorisce in griugno e luglio, sin da aprile e maggio nei luoghi bassi.

Distribuzione geografica. - In Europa dalla Lapponia ai Pirenei e alle Alpi.

Descrizione. - Pianta perenne, verde chiara, glahra. Rizoma corto, con fibre giallicce. Foglie in rosetta, piccolissime, recurve in fuori, sessili, ellittiche-bislunghe, ottuse, verdi chiare di sopra, od anche di solto e spesso sfumate di rossiccio verso $i$ margini I qquali sono interi e piegati in su, con nervo grossetto longitudinale di sotto. Peduncolo eretto, cilindrico, un po' assottigliato in alto, rossiccio, gla. bro. Calice diviso profondamente in 2 labbri, avvicinati alla corolla, 
ovali, come troncati all'apice, il superiore un po' più largo, con tre denti corti ed ottusi, l'inferiore ha due denti egualmente corti ed ottusi : tutti e due verdognolo-rossicci. Corolla piccola, irregolare, di un bianchiccio-gialliccio, con la gola gialla e l'apice dello sprone di un giallo carico, con il tubo corto, largo, prolungato in uno sprone corto, quasi conico, ottuso e come troncato-smarginato, curvato ad arco in su e con il lembo diviso in due labbri disuguali, il superiore bilobo, circa la metà piủ corto dell'inferiore ch'è trilobo, tutti i lobi sono tondeggianti, quello di mezzo del lobo inferiore è appena smarginato e più largo. Gola larga, aperta, come ho detto gialla e con peli parte corti e gialli e parte piủ interni lunghi e bianchi. Stami 2, inseriti in basso della corolla, lunghi quasi quanto il tubo di questa. Filamenti grossi in basso, quasi cilindrici nel resto, ascendenti da un lato e dall'altro dell'ovario, bianchi, glabri. Antere quasi tonde, piuttosto piccole, uniloculari, si aprono traversalmente in alto, gialle. Pistillo poco più lungo degli stami. Ovario quasi tondo, verdognolo, coperto di corti peli aventi una glandoletta tonda e come essi bianca e trasparente. Stilo corto, che si continua nello stimma, il quale è grande, quasi bilabiato, con il labbro superiore eretto, stretto, quasi setaceo, bianchiccio e avente pochi peli a guisa di ciglia in alto, il labbro inferiore è molto grande, largo, membrana. ceo, diretto in avanti, con i margini rovesciati in giù in modo che forma una volta che cuopre e nasconde le antere, è gialliccio di sopra nel mezzo, più chiaro nella circonferenza, papilloso di sopra, cortamente frangiato-cigliato nei margini. (Parl. ms., descr. della pianta del Tonale).

Osservazione. - Questa specie varia come la precedente per la statura, e per la grandezza del fiore, da 1 a 2 centimetri : la forma grandiflora è stata trovata al monte Cavallo e altrove in Friuli,

\section{FAMIGLA SETTIMA.}

\section{SCROFULARIACEE.}

Pediculares (part.), Scrophularia (part.) et Solanea (part.) Juss. gen. pl. p. 99, 117, 124.

Scrophularine et Solanee (part.) R. Br. prodr. fl. Nov-Holl. p. $433,443$. 
SchorhUlakiNe Bartl. ord. nat. p. 169.

Scropiularineæ Benth. in bot. reg. 21.

Scropilulariace e Lindl. nat. syst. ed. 2. p. 288.

Gemmularium 2-loculare, gemmulis plurimis in spermophoris septalibus subhorizontalibus, rare paucis pendulis, anatropis. Fructus siccus dehiscens, rarissime carnosus. Sernen amygdalosum. Embryo axilis.

Osservazioni. - Per yuesta famiglia io ho seguito con alcune modificazioni la classazione proposta da Bentham nel tomo $10^{\circ}$ del Prodromo Candolleano, preferibile secondo me all' altra adottata dallo stesso nei Gen. plant. Cosi lascio nella famiglia quale terza sottofamiglia anche le esotiche Salpiglossidinece meiostemoni, anzichè trasportarle nelle Solanacee isostemoni a motivo dell' abito, dell'infiorescenza e della corolla contorta nella preflorazione, caratteri tutti variabili in quest'ultimo gruppo.

I generi sono fondati sulla figura del calice, e della corolla, sulla preflorazione di questa, sul numero degli stami, sulla struttura dell'antere, sulla forma dello stinma, sulla natura del frutto, sul numero e la conseguente disposizione de'semi. Non vale la configurazione de'semi, stata pure adoperata, perchè variabilissima in un genere ammesso da tutti, il genere Linaria.

Le Scrofulariacee, da tutti riconosciute vicinissime alle Solana. cee, si legano d'altra parte intimamente alle Utriculariacee mediante il gen. Limosella che ha uno spermoforo quasi tutto centrale libero.

Ecco il prospetto delle Scrofulariacee nostrali:

I. Pedicularines.

1. TOzzIEs Reich.

1. Tozzia Mich

2. Euthrasies Benth.

2. Melampyrum Tourn........... 5

3. Pedicularis Linn.............. 17

4. Alectorolophus IIall. ............. I

5. Probosciphora Neck ............. 1

6. Odontites IIall............... \& \&

7. Euphrasia Tourn............... 1

8. Bartsia Stev.................. 1 
9. Bellardia All................ 1

10. Parentucellia Viv.............. 2

3. Digitalea Benth.

11. Veronica Tourn............... 29

12. Bonarota Mich............... 2

13. Wulfenia $J a c q . . \ldots \ldots \ldots \ldots \ldots \ldots$ 1.

14. Erinus Linn. ................ 1

15. Digitalis Tourn............. 5

II. Scrofularineæ.

1. Scrofulariea.

16. Limosella Lindl................. 1

17. Lindernia $A l l \ldots \ldots \ldots \ldots \ldots \ldots \ldots \ldots$,

18. Gratiola Linn.................. 1

19. Scrofularia Tourn............. 11

20. Celsia Linn.................. 1

21. Verbascum Linn............. 15

2. Linariek Reich.

22. Anarrhinum Desf.............. 1

23. Linaria Tourn................ 50

24. Antirrhinum Tourn........... 5

Species..... 142

Descrizione. - Le nostre Scrofulariacee sono erbe, annue, bisannue o perenni, o pure suffrutici, di statura variabile da $1 / 2$ decimetro a un par di metri, di aspetto variabilissimo. Quelle della tribù delle Eufrasiee si ritengono in generale parassite, dopo l'avviso datone da Decaisne nella sua nota pubblicata nel 1847: Sur le parasitisme des Rhinanthacées (Ann. sc. nat. $3^{\mathrm{a}}$ ser. $_{\%}$ ); seccando anneriscono; l'istologia n'è esposta in Chatin, Anat. comp. des vég. $\mathrm{Pl}$. par. p. 137-243. t. 28-48, e in Solms, De Lathrcece gen.

Le foglie sono talora tutte opposte, talora tutte sparse, spessissimo sparse le superiori, e le inferiori opposte o verticillate. Altro di generale non c'è da dire sul loro conto, poichè, salvo che sono semplici e senza stipole, variano moltissimo per dimensioni, per figura, per il grado della divisione quando sono divise, per la quantità e la 
natura de'peli quando insieme con altre parti erbacee della pianta ne sono rivestite.

Varia altresi la infiorescenza. Bene spesso i fiori sono solitari all'ascella, sia di una foglia ordinaria (sp. di Veronica, di Linaria), sia di foglie fiorali o di brattee, talora colorate, che situate nel. l'estremità del fusto e dei rami danno così grappoli o spighe terminali 0 ascellari, sempre indefinite (Pedicularinee in generale, delle Scrofularinee). Due generi hanno infiorescenza piủ complicata: Scro. fularia e Verbascum. Ivi sono dicasi ascellari, irregolari, spesso rac. corciati (Verbascum), che col loro insieme formano un grappolo oppure mia pannocchia. Mancano d'ordinario bratteole; pertanto ve ne sono due laterali nella Gratiola.

I fiori sono pentameri in tutti i verticilli, fuorchè nel gineceale.

Nel calice il sepalo dispari è posteriore. Ha tendenza all' aborto, per cui si vede rimpiccolito in certe Veroniche per esempio, e scomparso del tutto nella piủ parte, come nella generalità delle Eufrasiee, e il calice $\dot{e}$ allora ridotto tetramero. La preflorazione de'sepali $\dot{e}$ embriciata, o aperta. Sono congiunti fra di loro in calice gamosepalo, ora appena, ora di più, ora fin quasi in cima; da ciò e dalla loro figura e proporzione risultano calici assai svariati: persistenti sempre, talora (Alectorolophus) accresciuti alla fruttificazione.

La corolla è tinta di rosso, giallo, turchino o bianen, e non di rado screziata con vari di quei colori. Le sue dimensioni vanno dalle minutissime della Limosella (2 millimetri) alle grandissime della $\mathrm{Di}$ gitalis purpurea o dell' Antirrhinum maius (4 centimetri). È irrego. lare sempre, ma in vario grado. Al minimo si presenta con lobi pressochè uguali (Verbascum, Limosella, Tozzia), al massimo è divisa in due labbri distintissimi, l'anteriore costituito da tre pezzi, il po. steriore da due, i quali sono talora siffattamente congiunti da sembrare uno solo (Veronica). Tali ed altre variazioni del lembo, unitamente ad altre del tubo, danno una gran varietả di forme di corolle. La preflorazione dei lobi è embriciata, in due modi : il lobo anteriore essendo sempre interiore, i lobi laterali sono talora esteriori ai lobi posteriori (Pedicularinee), e talora sono interiori (Scrofularince). La corolla nelle Pedicularis si torce a destra al tempo dello sbocciamento. É caduca, di rado marcescente (Parentucellia latifolia).

Nei soli Verbascum è completo l'androceo, per cui altra volta il genere era posto nelle Solanacee; ma anche ivi si manifesta in alcune specie una diversiti nei 5 stami, sia fra $\mathrm{i} 2$ anteriori paragonati agli altri, sia per l'atrolia dello stane posteriore. Atrofico e 
fatto staminodio è pure quello stame in specie dei generi Scrofularia, Linaria e Antirrhinum; nella più parte degli altri è abortito del tutto, e l'androceo risulta didinamo con gli stami anteriori piủ lunghi dei laterali, eccettochè nella Lindernia e nella Lìmosella dove compariscono uguali. Nel genere Gratiola i due stami anteriori sono ridotti staminodi; sono soppressi del tutto nei generi diandri Veronica, Bonarota e Wulfenia. Le antere sono generalmente dorsifisse, però sono basifisse nei generi Verbascum, Celsia, Scrofularia e Limosella. Sono biloculari, introrse, deiscenti per lungo, le caselle ora rimangono distinte (Eufrasiee ecc.), ora confluiscono più o meno in una (Digitalee ecc.), e talora (Erinus, Limosella, Anarrhinum, Verbascum, Celsia e Scrofularia) la confluenza avviene innanzi la maturità dell'antera e questa risulta uniloculare con linea trasversale di deiscenza.

Il gineceo poggia sopra di un disco, anellare uguale, o pure sviluppato maggiormente sull'innanzi. Esso è dimero, i due pistilli essendo antero-posteriori; e sono ugualmente sviluppati, o uno più dell'altro, l'anteriore (Antirrhinum) o il posteriore (specie di Linaria). Dall'osservazione genetica (Payer, Traité d'organ. p. 543.t. 111) è provato che la porzione gemmulariana del gineceo risulta, almeno in parte, da escavazione del talamo in corrispondenza alla base dei due pistilli, e che perciò è di natura stipitale il setto che separa le escavazioni ossia le caselle; e l'osservazione genetica è pienamente confermata da fatti teratologici facili ad avverarsi, nei Verbascum per esempio. Ciò spiega come alcune volte (Melampyrum, Pedicularis) il gemmulario sia biloculare in fondo nella porzione talamica, e uniloculare in alto nella porzione pistillare, e come nella Limosella il setto non sia tale che nella sua porzione basilare, e piủ su sporga in spermoforo centrale. Il gemmulario si prolunga in stilo, ch'è quasi sempre lungo, sottile; solamente nei generi Limosella e specialmente Lindernia ed Erinus è corto e grosso. La porzione stimmatica n'è sempre molto ristretta: o meramente terminale senza ingrossamento, o con un leggero ingrossamento a capocchia, quando facilmente. si divide in due lobi, i quali nella Gratiola e nelle Digitalis sono appiattiti a laminette.

La parte mediana del setto non ha rialzo quando le gemmule sono poche, è rialzatissima in due grossi spermofori quando sono molte. Il numero ne varia da due collaterali per casella nella Tozzia, a poche ancora e biseriate nella più parte delle Eufrasiee, a un numero grandissimo nel restante della famiglia, quando sono ammuc- 
chiate e pressochè orizzontali, mentrechè stanno pendenti quando sono poche. Nel genere Veronica il numero n'è ridotto, nonostante sono ammucchiate orizzontali. Sono anatrope, con integumento scempio.

Nel solo genere Tozzia il frutto è indeiscente, ed è una drupa. Altrove è secco, deiscente in uno dei tre modi seguenti: nelle Eufrasiee è una vera cassula, hivalva (talora univalva), con semi attaccati nel mezzo delle valve; nelle Digitalee e Scrofulariee è un settifragio, di cui si staccano le valve lasciando in posto i semi; nelle Linariee infine è un treto, con le caselle che si aprono in alto per un foro i di cui margini si fendono in valve piủ o meno profonde. Giova però avvertire che non sempre riesce ovvia la distinzione delle tre sorte di frutti.

I semi contengono un embrione diritto, e di varia lunghezza, nell' asse di una mandorla carnosa. Sono piccoli, anzi generalmente piccolissimi; del resto svariati per forma, e per gli accidenti della loro superficie, non mancandovi neppure le ali. Sono attaccati per la estremità, o per un punto laterale. Curiosi sono quelli delle Pe. dicularis, strofiolati all'ilo, e dei Melampyrum, carnosi nella loro base. Curiosissimi poi quelli di certe Veroniche, illustrate già da Planchon (Mém. sur le développ. et les car. des vrais el des faux arilles, p. 35-46.t. 3), da Schleiden (Grundz. der wissensch. bot. ed. 2. § 171 ), in cui la nocella, dopo la fioritura ingrossando molto dal lato opposto alla rafe, finisce coll'uscire fuori e foggiarsi a scudetto, nella cui concavità sono i rimasugli dell'altre parti della gemmula.

La Linaria Cymbalaria e le specie alfini, nońchè la Veronica Cymbalaria, sono celebri quali piante che da sẻ sotterrano i loro frutti co'semi. Vedasi Bodard, Dissertation sur les plantes hypocarpogées.

Considerazioni geografiche. - Sono relativamente poche (circa $\%$ della totalità) le Scrofulariacee sparse per tutta Italia: sono 2 Odontites, 1 P'arenturellia, 12 Veronica, 2 Scrofularia, ¿̀ Verbascum. 5) Linaria, 2 Antirrhinum. La categoria più numerosa, giacchè an. novera circa $1 / 2$ della specie, ¿̇ quella delle settentrionali, che occupano il nord della Penisola esclusivamente, o da quello scendono verso il merzogiono d'Italia, fermandosi in qualche punto; comprende perintern i generi Tozsia, Melamp!yrum, Alectorolophus, Euphrasia, Bartsia, Bonarota, Wulfenia, Erinus, Limosella, Lindernia, Gratiola, Anarrhinum, tutte le Pelicularis meno una, quasi tutte te Digitalis, una maggionanza di Veronica, di Serofulariu, eer. Tre volte meno numerosa $\dot{e}$ la categoria inversa, delle specie meri. 
dionali; non ha in proprio che i generi Probosciphora, Bellardia e Celsia, e una maggiore proporzione di Linaria e di Antirrhinum.

In corrispondenza a questo fatto stanno altri due. L'uno, che quasi una metà delle specie è esclusa dalle isole, nonchè 9 generi, cioè: Tozzia, Melampyrum, Pedicularis, Alectorolophus, Bartsia, Bonarota, Wulfenia, Limosella e Lindernia. L'altro fatto si è che più di $1 /$ delle specie, e vari fra i summentovati generi, sono piante di montagna, che abitano solo gli alti monti, o da quelli scendono nelle sottoposte vallate e pianure.

Sono da notarsi fra le specie più localizzate: nelle Alpi: parec. chie Pedicularis, Bartsia alpina, Veronica bellidioides; nella Penisola settentrionale occidentale: Pedicularis Oederi, $P$. atrorubens, $P$. adscendens, Odontites viscosa, 0 . lanceolata, Veronica Allionii, Linaria mons. pessulana, L. supina; in Lombardia: Linaria bipunctata; nella Penisola settentrionale orientale: Pedicularis acaulis, Veronica longifolia, Bonarota chamodrifolia e B. rotundifolia, Wulfenia carinthiaca, Digitalis lavigata, Serofularia laciniata; nella Penisola orientale: Melampyrum barbatum; nell'Appennino centrale: Pedicularis elegans, Verbascum longifolium, Linaria pallida; nella Penisola meridionale: Verbascum niveum, V. angustifolium, Linaria pilosa; inoltre in Sicilia: Probosciphora Columnce, Linaria reflexa, L. heterophylla; in Sicilia soltanto: Odonittes Bocconei, 0. rigidifolia, Linaria pubescens; in Corsica: Veronica Gouani, Linaria hepaticcefolia; in Sardegna: Verbascum conocarpum, Linaria alsinefolia, L. Mülleri, L. parviflora; in Corsica e Sardegna: Odontites corsica, Digitalis purpurea, Scrofularia ramosissima, Linaria flava; inoltre nell' Arcipelago toscano: Scrofularia trifoliata, Linaria aquitriloba; nelle sole isole toscane: Linaria capraria; ecc.

Su 29 generi di Scrofulariacee europee, l'Italia ne possiede 24; mancano i generi: Dodartia, Sibthorpia, Lafuentea, Castilleja e Cymbaria, ristrettissimi, che 1 artico, 2 orientali e 2 occidentali. Stando al Conspectus di Nyman, essa possiecie un po' più dei ${ }^{2} / \mathrm{s}$ delle specie.

- Ne ha in proprio 14: Pedicularis elegans e Linaria pallida dell'Appennino centrale, Verbascum niveum, V. angustifolium e Linaria pilosa della Penisola meridionale, Odontites Bocconei e Linaria pube. scens di Sicilia, Odontites corsica e Scrofularia trifoliata di Corsica e di Sardegna, l'ultima estesa anche alle isole toscane, Linaria hepa. ticcefolia di Corsica, Verbascum conocarpum, Linaria alsinefolia e $\boldsymbol{L}$. Mülleri di Sardegna, L. capraria dell'Arcipelago toscano. Alle quali si puỏ aggiungere la Pedicularis gyroflexa, l' Odontites lanceolata e la 
Veronica Allionii, nonchè la IVulfenia carinthiaca, i limiti della cui abitazione quasi toccano il confine italiano.

Una specie, la Veronica peregrina, è oriunda americana, e naturalizzata.

Com'era da aspettarsi dalle indicazioni già date sulla distribu. zione interna delle Scrofulariacee italiane, le loro relazioni esterne palesano subito una forte prevalenza, del doppio, dei tipi nordici su quelli del mezzogiorno, s'intenda dei tipi specifici che dall' Europa settentrionale partendosi da latitudini più o meno alte scendono verso basso, 0 che (in minor numero) vengono dall'Europa media; alle quali va aggiunta la serie non piccola (una $20^{\mathrm{a}}$ ) di quelli che occupano le catene montuose estese dai Pirenei ai Carpazi. L'altra cate. goria, metà minore, dei tipi mediterranei presenta le stesse particolarità giả riscontrate nelle Lamiacec (vedasi a pag. 51), vale a dire una prevalenza di specie estese a tutto il Mediterraneo, c fra quelle ristrette sia al Mediterraneo orientale sia all'occidentale, prevalenti le ultime. I tipi non mediterranei che si trovano esclusivamente a oriente 0 a occidente dell' Italia, sono equivalenti, in numero di circa 12.

Se si toglie la quasi cosmopolita Veronica serpillifolia; se si tolgono una dozzina e piủ di Veroniche, di Verbasci, di Linarie, ecc., naturalizzate agli Stati-Uniti, e qualcuna inoltre nell'America meridionale e al Capo, ecco quali Scrofulariacee hanno singolarmente estesa l'area di abitazione. Le seguenti: Pedicularis verticillata, $P$. Oederi, P. palustris, Alectorolophus Crista-galli, Euphrasia offici. nalis, Bartsia alpina, Veronica officinalis, V. scutellata, V. Anagallis-aquatica, V.alpina, V. fruticulosa, Limosella aquatica, Scrofularia nodosa var., si ritrovano nella Nord-America, e soprattutto nelle sue parti più settentrionali, essendo difatti quasi tutte della flora artica. Si estendono nell'Asia tino all' estremo oriente la Veronica Bercabunga e la V. Anagallis-aquatica, con la Lindernia e la Linaria vulgaris. Al sud, compariscono in Abissinia le due suddette Veroniche, la Linaria Elatine, I'Antirrhinum Orontium, la Limosella. Infine la Bellardia trovasi in Alissinia, nell'Africa meridionale e nelI'America meridionale. Le altre Scrofulariacee, vale a dire la gran massa della farniglia, sta raccolta entro ai limiti medesimi già indicati (a pag. 52) per le Lamiacee.

Siffatta concordanza riguardo all'area di ahitazione (salvo poche varianti facilmente spiegabili), fra due famiglie che nell'insieme della llora italiana rappresentano due tipi opposti, l'uno nordico, me 
diterraneo l'altro, porta alla conclusione che l'Europa, l'Affrica più settentrionale, e l'Asia occidentale, costituiscono un unico do. minio botanico: dove, in ogni paese e nelle sue regioni analoghe, vi ha diversità per le specie, maggiore o minore secondo la varia pro. porzione delle specie comuni e delle proprie, ma le famiglie sono graduate medesimamente per la importanza numerica, e sono rap. presentate dai medesimi generi dominanti.

Io ho già cercato altrove (Stat. bot. della Tose. p. 104) di precisare il termine di regione, distinguendola da abitazione, quale a una estensione di paese avente sostanzialmente la medesima flora, cioè le medesime specie in maggioranza distribuite presso a poco in uguale abbondanza d'individui. "

Il termine didominio, nel concetto espresso qui sopra, va preso in un senso molto diverso da quello di Grisebach nell'opera celebre: Die Vegetation der Erde nach ihrer klimatischen Anordnung, 1872. I domini di Grisebach sono climatici e non hotanici, e ciò avute in mira soltanto le parti basse di ogni paese, e a di più con una targhezza d'interpretazione tale che per esempio il Capo Nord, l'Irlanda e la valle superiore del Po fanno parte di un medesimo dominio. Egli ha cercato, è vero, di stabilire una relazione fra il clima e le forme vegetali; ma dessa non poteva toccare che la fisonomia o certi tratti biologici di alcune piante, prescelte quali caratteristiche; poichè se v'ha una verità dimostrata in geografia botanica, si è che il clima non spiega la flora: si pongano a confronto rerbigrazia l'Australia temperata, il bacino Mediterraneo e la California.

L'opera di Grisebach è un'applicazione larga e dotta di idee già manifestate da Humboldt. D'altra parte, è noto che A. P. de Candolle, Schouw, ed altri ancora, hanno successivamente proposto divisioni della superficie terrestre in aree, equivalenti a quelli che oggigiorno si chiamano domini, fondate sı caratteri botanici : rna che nella loro scelta sanno troppo di arbitrio per avere potuto incontrare accoglienza favorevole. I caratteri che propongo, e che sono in sostanza gli individui e le specie per le regioni, $\mathrm{i}$ generi e le famiglie per $\mathrm{i}$ domini, mi sembrano invece confacenti allo scopo; molto più che non li ho concepiti a priori, ma che vi sono stato condotto per mano dai risultati delle mie investigazioni sulla distribuzione, prima delle piante toscane (Stat. bot. della Tosc., 1871), ed ora delle piante italiane.

Nella sua classica opera della Géographie botanique raisonnée. 1855 , Alf. de Candolle si pronunzia contrario a qualunque spartizione 
in domini, a motivo della molta difficoltà che si prova bene spesso a limitarli, e delle frequenti transizioni da uno in un altro. L'obiezione è valida; ma vale ugualmente contro a qualunque classazione, contro a qualınque aggruppamẹnto giusta criteri di qualsiasi natura. Nelle loro molteplici relazioni, le piante mostrano dovunque transizioni e passaggi, che danno l'unità e la continuità del regno vegetale. Se si dovesse tenere conto soltanto di quelli, non vi sarebbe piủ luogo alla tassinomia nella serie dei nostri metodi botanici, nè classazioni possibili, le quali si fondano appunto su ciò che si tien conto di certe relazioni più strette, tralasciando altre meno strette: e classazioni cosi intese possono esservi per i rapporti geografici delle piante come per i rapporti morfologici.

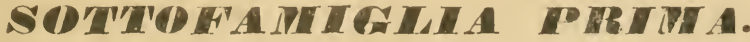

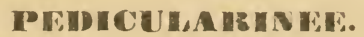

Rininantides Benth. in liand. prodr. 10.

Pedicularine.4.

Corollo lohi postici in præfloratione interiores.

\section{TRIBU் PRIMA.}

Tomiec.

Tozzifs: Reich. handl. nal. p/lanzen-sysl. p. 197.

Antherarum loculi distincti. Fructus drupa.

\section{1. 'TOZZTIA A.}

Tozicia Mich. nov. pl. gen. p. 19. Gen. pl. 1. germ. 5. 1. 48 Benth. Honk. gen. pl. 2. p. 9S0. Ces. Pass. Gib. comp. Al. ilal. p. 360. 1. 52. f. 6 (err. quond gemmulas).

Calyx campanulatus, 4-i-dentatus-fissus. Corolla limbo subxqualiter 5-Iobo. Stamina 4. Autherarum loculi basi mucronati. Stigma capitellatum. Gemunule in loculis hina rollaterales, pendula. Drupa abortı 1-sperma. 
Portamento. - Vedasi la descrizione dell' unica specie.

Osservazione. - Gaertner (De fruct. 3. p. 198), e dopo di lui gli autori in generale sulla sua scorta, descrivono i frutti come deiscenti in due valve, mentrechẻ sono drupe, dapprima erbacee e poi riseccate, ma che in niun modo si aprono.

\section{Tozzia alpina.}

Tozzia alpina Linn. sp. pl.ed. 1.p. 607. Mass. prodr. fl. valt. p. 187. Bert. fl. ital. 6. p. 313. Com. fl. com. 5. p. 29. Hausm. fl. Tir. p. 653. Rota prosp. fl. Berg. p. 67. Car. prodr. fl. tosc. p. 494. Ard. fl. Alpes-mar. p. 287. De Vis. Sacc. cat. piant. Ven. p. 155. Zersi prosp. piant. Bresc. p. 159. Ces. Pass. Gib. comp. fl. ital. p. 360. Arc. comp. fl. ital. p. 523.

Figure. - Mich. nov. pl. gen. . 16. Reich. ic. fl. germ. 20. t. 120.

Stazione, Abitazione e Fioritura. - In luoghi ombrosi freschi di montagna, nel terzo superiore della Penisola, rara. È stata trovata nel Friuli a Valdino sui monti di Sutrio (Morassi), e presso Zo. vello e Monaio (De Vis. Sacc.), nelle alpi di Cadore (Poll.), nel Tirolo sul Montalon (Bert.) e nell'alpe Sasso-rotto in val Sugana (Ambrosi!), nell'alpe di Lagorai in Fiemme (Bert.), in val Venosta presso Laas, nelle Giudicarie sull'alpe Gavardina (Hausm.) e in valle di Breguzzo (Bert.), nel Bresciano e Bergamasco al monte Paio presso il Cornablacca (Zersi), e al monte Epolo a Schilpario (Rota), nella val Tellina nelle alpi di Mara, Togno e Painale (Mass.), nel Piemonte ad Allagna nell'alpe Von Flua nel luogo detto Passii (Carestia I), nell'alpi di Prolugnan (All.), sopra Giaveno al collo della Rossa (Re), presso Vinadio nel vallon di S. Anna (All.), presso Valdieri in Vallasco (Ball!), e sull'altro versante delle Alpi Marittime al monte Bissa (Bourgeau!), al vallon di Nanduebis presso S. Martino Lanto. sca ('Thuret!), ai colli dell'Aabisso e di Fremamorta, presso S. Anna di Vinai (Ard.). Indi ricomparisce nell'Appennino toscano in Mugello (Savi!), in Casentino alla Falterona ai luoghi detti Fonte dei Conti (Baroni) e Fosso all'Abetio (Siemoni !), sopra Vallombrosa all'Elle. rema (Mich.). Fiorisce in giugno e luglio.

Distribuzione geografica. - In Europa nei Pirenei, e per la zona montuosa che si stende dal Delfinato e dalla Svizzera al Montenegro, alla Transsilvania e alla Silesia.

Descrizione. - Pianta perenne, alta 3 o 4 decimetri, di un verde chiaro, forse parassita. Rizoma corto, carnosetto, obliquo, co- 
perto di squame le quali sono molte, bislunghe, carnosette, bianchicce, distribuite in 4 fila, due per ciascun lato del rizoma, si toccano per la faccia piana in modo che si vedono soltanto ai margini che sono ottusi ; l'estremità del rizoma si continua in una radice duretta, corta, un po' ramosa e gialliccia. Fusto carnosetto, eretto, ramoso fin dalla base e con i rami eretto-patenti ed opposti, quadrangolare, con peli lunghetti e bianchi presso gli angoli alternativamente nelle due facce laterali e nelle facce antero-posteriori. Foglie piccole, opposte, patentissime, sessili e un po'abbraccianti il fusto ed i rami, ovate, ottuse, con pochi smerli a guisa di denti ottusi e diretti verso l'apice della foglia, di un verde chiaro, con leggieri solchi di sopra che corrispondono ai nervi di sotto, di un verde un po'più chiaro di sotto ed ivi con un nervo longitudinale e circa 3 altri nervi per parte che vengono dalla base, gradatamente piu corti da dentro in fuorie un po'curvi, i quali si dirigono verso il margine e l'apice della foglia. I tiori sono piccoli, solitarii all'ascella delle foglie fiorali, le quali sono simili alle foglie ma gradatamente piủ piccole e con gli smerli meno manifesti : sono opposti, peduncolati, piủ corti delle foglie corrispondenti, i superiori piủ lunghi di queste foglie. I peduncoli sono delicati, più corti lel fiore, sono pelosi per lunghi peli bianchi dalla parte interna. Il calice è quasi campanulato, di un verde chiaro, gla. bro: ha quattro denti corti, larghetti, quasi triangolari, acuti, eretti. La corolla è più del doppio più lunga del calice, con il tubo grada. tamente un po'pin largo in alto, pelosetto di fuori, e con il lembo piủ lungo del tubo diviso profondamente in due labbri quasi uguali, il superiore diviso in due lacinie dirette in su ed in dietro, anzi ro. vesciate in dietro, e l'inferiore in tre lacinie, dirette in giu ed in avanti, tutte le lacinie sono tondeggianti all'apice; è gialla, di un giallo più carico nelle lacinie del labbro inferiore nelle quali sono pure delle macchioline disuguali di colore rosso-scuro, verso la base; tutte le lacinie sono fornite di corti peli quasi cigli nel margine; la gola ¿ pelosetta. Gli stami sono quattro, didinani, piủ corti del labbro superiure vicino al quale essi stanno, inseriti nel tubo della corolla. Filamenti lesiniformi, delicati, giallicci, con qualche pelo in basso. Antere 2-loculari, ottuse all'apice, bifide in basso, e ivi con un'appendice corta a guisa di seta. Pistillo piủ corto degli stami. Ovario ovato, di un verde pallido, glabro, racchiuso nel tubo del calice. Stilo molto piì lungo dell'ovario, eretto, delicato, cilindrico, bianchiccio. Stimma in capolino, papilloso, hianchiccio. Frutto non maturo quasi tondo, poco più lungo del calice che vi è adattato con i suoi denti. 
(Parl. ms. descr. della pianta del Casentino). - Gemmulario 2-lo. culare, con 2 gemmule per casella, collaterali, pendenti, anatrope. Il frutto è una drupa erbacea, con endocarpio crostoso; è ridotto 1-loculare per atrofia di una delle due caselle, cosicchè il setto, mezzo-marcito, poggia su quel lato del frutto, e regge un solo seme, ch'è globoso, bianco, con guscio sottile membranoso, e l'embrione piccolissimo collocato superiormente (v. v.).

\section{TRIBU் SECONDA.}

Eufrasiee.

Euphrasiea Benth. in Cand. prodr. 10 (excl. Tozzia).

Antherarum loculi distincti. Fructus capsula.

\section{MELAMPYRUM.}

Melampyrum Tourn. inst. p. 173. t. 78. Gen. pl. fl. germ. 5. t. 47. Benth. Hook. gen. pl. 2. p. 979. Ces. Pass. Gib. comp. fl. ital. p. 359. t. 52. f. 4.

Calyx tubuloso-campanulatus, inæqualiter 4-fidus-partitus. Corolla limbo conspicue 2-labiato, labio postico galeato, antico 3-lobo. Stamina 4. Antherarum loculi basi mucronati. Stigma minusculum. Gemmulæ in loculis paucæ, subbiseriataæ, pendulæ. Capsula oligosper$\mathrm{ma}$, seminibus basi constrictis carnosis.

Portamento. - Piante annue, basse, erette, molto ramose, coi rami sottili. Foglie opposte, allungate lanciolate, intere. Fiori gialli, o misti di giallo e di rosso, quasi sessili, solitari all'ascella delle foglie superiori, le quali o sono simili alle altre, o pure di solito slargate e dentate inferiormente, talora anche colorate, e più 0 meno ravvicinate insieme in modo da costituire nell'estremità del fusto e dei rami una spiga bratteata.

\section{Melampyrum cristatum.}

M. spica densa, æquali, foliis floralibus imbricatis, inferne dilatatis rotundatis complicatis crebre serrato-pectinatis, calyce lobis valde inæqualibus, longioribus tubo suo subæqualibus, tubo corollino multo brevioribus, capsula 1-valvi, valva integra. 
Melampyrum cristatum Linn. sp. pl. ed. 1. p. 605. Bart. cat. piant. Sien. p. 20. Sant viagg. 3. p. 37, 125. Mass, prodr. 1 . valt. p. 111 Trev. prosp. /l. eng. p. 23. De Not. rep. /l. lig. p. 309. Bert. Al. ital.6. p. 300. Com. A. com. 5. p. 18. Hausm. fl. Tir. p. 654. Rota prosp. fl. Berg. p. 67. Pir. fl. for. syll. p. 107. Car. prodr. f. tosc. p. 493. Bert. not. porr. p. 46. Zersi prosp. piant. Bresc. p. 159. Ing. cat. sp. Mond. p. 50. Cies. Pass. Gib. comp. fl. ital. p. 360. Smilh. fl. Fiume p. 38. C'ald. R. fav. tent. p. 174. Gib. Pir. R. Mod. p. 124. Arc. comp. fl. ital. p. 523. Cocc. fl. Bol. p. 365.

Figure. - Fl. dan. t. 1104. Reich. ic. fl. germ. 20. t. 116.

Stazione, Abitazione e Fioritura. - Nei boschi montuosi e negli scopeti della Penisola superiore e media. Nell'Alta Italia nella sinistra valle del Po: a Susa (Parı.!), a Donnaz (Carestia!), nel No. varese alla Ciolma e nel monte Fenera dov'è comune (Bir.), sul S. Salvatore presso Lugano, presso Como (Com.), presso Lecco (Ball!), sul monte Barro a 7-800 metri, sui Corni di Canzo a 1000 metri (Parl.!), in val Tellina a Lanzada e Caspoggio (Mass.), a Bergamo (Rota), a Brescia, frequente (Zersi), al lago di Garda, presso Trento sul Bondone e altrove, sul Portale (Hausm. ece.), in val Arsa (Kerner!), nel Veronese quasi ovunque (Poll.), e nel Mantovano (Barbieri!), negli Euganei (Trev.), nel Bassanese (Bert.), in Friuli a Flambro, Fagagna, Iussago ecc. (Pir.), in Istria a Pirano (Poll.), e Fiume (Smith); nella destra valle del Po: a S. Dalmazzo (All.), Roccaforte (lng.), Alba (Colla), Superga dove abbonda (All.), presso Alessandria (Negri!), a Serravalle di Scrivia (De Not.), presso Pavia (Nocca Balb.), nel Parnense a Sala (Pass.!) e al monte Prinzera (A. Targioni!), a Modena, sul Ventasso (Gib. Pir.), presso Bologna in molti luoghi (Bert. , Cocc.), e sino alla Porretta (Beccari!), a Faenza (Cald.). Nasce poi in Toscana a Pracchiola in Lunigiana!, S. Marcello (P. Savi!), Fucecchio, intorno a Firenze dove non è raro!, in Mugello a Scarperia (Parl.!) e Borgo S. Lorenzo (Savi!), sui monti di Mliemo nel Volterrano (Amidei!), a Siena (Bart.), a S. Galgano e Monte Siepi uel Senese (Sant.), a S. Quirico di val d'Orcia (Parl.!), a Grosseto (Sant.); intine a Viterbo (Bert.). Dall'altro lato della Penisola è stato trovato al monte S. Vicino nell'Umbria (Bucci!); e giu nell' Abruzzo al Sirente a $3 \tilde{3} 000^{\prime}$ (Groves!). Fiorisce in giugno e luglio.

Distribuzione geografioa. - In Luropa, dalla Scandinavia me. dia alla Spagna e alla Russia meridionale, in Siberia fino all'Altai.

Desorizione. - Pianta annua, alta 2 a 5 decimetri, verde chiara, puberulo-irsuta, con i peli corti, più abbondanti in alto e curvati 
in giù. Radice ramosa, torta, bianchiccia. Fusto eretto, delicato, quadrangolare, rossiccio in basso, verde chiaro in alto, ramoso, rami patentissimi. Foglie opposte, patentissime e anche un po' dirette in giù, sessili, strette, lanceolato-lineari, acute, intere, con i margini un po'rovesciati e scabri, verdi chiare di sopra ed ivi con solco longitudinale, e altri verso la base leggieri che corrispondono ai nervi di sotto, di un verde ancora più chiaro di sotto ed ivi con un nervo longitudinale sporgente e più chiaro da cui in basso partono pochi riervetti a guisa di vene corte, che vanno obliquamente verso il margine e si diramano. Fiori piccoli, quasi sessili, formanti una spiga corta, molto fitta, quadrangolare, con gli angoli molto sporgenti e in cresta. Le brattee sono distribuite in quattro fila, molto fitte e coprentesi in parte tra loro, larghe alla base, piegate in due in modo da toccarsi nella faccia interna e a guisa di cuore, verdognolo-bianchicce, con i margini disugualmente pettinato-dentati, con i denti interi o bifidi, strette, lanceolato-acuminate, e cigliate per ciglia rade, corte e bianche: hanno all'apice una appendice fo. gliacea simile alle foglie superiori ma curvata in fuori ed in giù, e gradatamente più corta nelle brattee superiori, tantochè in queste è più corta della parte larga dentata o è quasi nulla. Il calice è tubuloso, appena curvo, bianchiccio, glabro, con due linee laterali longitudinali pubescenti, ha quattro lacinie, due superiori un po'più grandi delle inferiori, però piủ corte del tubo della corolla, tutte iineari-acuminate, cigliate per corte ciglia. Corolla bianchiccia con il labbro inferiore gialliccio; tubo bianchiccio, lunghetto, leggermente angolato, un po' curvo; lembo corto, molto più corto del tubo, bilabiato, labbro superiore in forma di elmo, schiacciato lateralmente e con i margini rovesciati e quasi diretti orizzontalmente, smarginato all' apice, bianchiccio, labbro inferiore lungo quasi quanto il superiore, diretto in avanti, trilobo, con il lobo medio piu piccolo dei due laterali, tutti ottusi di sopra, ha due gibbosità bislunghe, grosse, quasi parallele, di un giallo più carico. Stami 4, appena didinami, racchiusi dentro il labbro superiore. Filamenti filiformi, bianchicci, glabri. Antere bislunghe, ottusette e con una piccola punta ottusa, smarginate alla base, inserite nel dorso sopra di questa, verdognole nel dorso, rossicce verso il margine, pelose in questo ed in basso. Pistillo lungo quanto o poco più degli stami. Ovario ovatoacuminato, schiacciato, verdognolo-glabro. Stilo lungo, un po'curvato in alto in avanti, filiforme, bianchiccio, glabro. Stimma quasi in capolino, piccolo, bianco. (Parl.ms., descr. della pianta dei Corni 
di Canzo). - Gemmulario 2-loculare. Gemmule parecchie sovrapposte in ogni casella, ma le due superiori soltanto sono bene sviluppate, le altre sono atrofiche: sono pendenti, quasi conipletamente anatrope, con la rafe voltato inentro, pressochè conformi, cioè a dire senza quella gran diversità che secondo Tulasne (in Ann. sc. nat. $3^{a}$ ser. 12. p. 66) e Hofmeister (Neue Beitr. zur Kenntn. der Embr.p. 617) vedesi in altre specie, dove una gemmula è completamente anatropa e con corto funicolo, e l'altra appena curvata e con funicolo lungo. Cassula compressa, falcata in fuori, membranosa, 1-valve, ossia aprentesi sol. tanto lungo lo spigolo interno. Semi 4, grossi, ovali-cilindrici, falbi e lucidi, menochè alla loro base dove sono ristretti, carnosi e bianchi. Embrione cilindrico, parecchie volte piủ corto della mandorla ch'è carnosa, posto nell'estremità del seme opposta all'ilo, con la radicetta supera. (v. v.).

\section{Melampyrum arvense.}

M. spica laxiuscula, æquali, foliis floralibus ovato-lanceolatis, inferne præsertim pectinato-laciniatis, calyce lobis subæqualibus, tubo suo multo longioribus, tubo eorollino aqualibus, capsula 1-valvi valva 2 -loba.

Melampyrum arvense Linn. sp. pl. ed. 1. p. 605. Bart. cat. piant. Sien. p. 20. Sant. viagg. 2. p. 310. 3. p. 126. Mass. prodr. fl. valt. p. 111. Trev. prosp. A. eug. p. 23. De Not. rep. fl. lig. p. 309. Bert. f. ital. 6. p. 302. Com. fl. com. 5. p. 20. Pucc. syn. pl. luc. p. 302. Hausm. fl. Tir. p. 654. Rota prosp. fl. Berg. p. 67. Pir. f. for. syll. p. 107. Car. prodr. fl. tosc. p. 493. Ard. $l$. Al. pes-mar. p. 283. Zersi prosp. piant. Bresc. p. 159. Ces. el. Maiell. p. 22. Terr.! sec. rel. Terra di Lav. p. 93. Ing. cat. sp. Mond. p. 50. Ces. Pass. Gib. comp. fl. ital. p. 360. Terr. quarta rel. p. 111. Cald. f. fav. tent. p 174. Gib. Pir. H. Mod. p. 124. Arc. comp. M. ital. r 523. Cocc. fl. Bol. p. 365.

Figure. - Fl.dan. t. 911. Reich. ic. fl. germ. 20.t. 115. f. 1.

Stazione, Abitazione e Fioritura. - Nei campi fra le messi, principalmente di collina e di montagna, della Penisola. Nell Alta Italia nasce copioso in quel di Susa (Re), comune pure in quel di Pinerolo (All.), e trovasi fra Carlino e Viosenne (Ricca!), a Marsaglia (All.) e Dogliani (Ing.), è comunissimo nel Novarese (Bir.), e nel Pavese (Nocc. Balb.), fa nel Milanese (Rampoldi!), abbonda nella Brianza e presso Como (Com.), nasce in val Tellina a Bormio (Levier!), a Tirano enella valle Malenco (Mass.), nel Bergamasco (Ro- 
ta), frequentissimo nel Bresciano ('Lersi), si stende nella valle dell'Adige da Laas a Roveredo, quindi in val di Sol presso Peio, in Fiemme presso Predazzo, in val Sugana a Caldonazzo, nel Baldo sopra Brentonico (Hausm.), a Piovene sul monte Sumano (Bert.), nel Veronese è frequente (Poll.), fa ad Angarano nel Bassanese (Bert.), nel Friuli a Flambro, Virco e Fagagna (Pir.), nel Lido veneto (Kellner!), a Chioggia (Bert.), negli Euganei (Trev.), nel Mantovano (Barbieri!), nel Parmense ad Ozzano e altrove (Parl.!), fino alla Cisa (Bert.), nel Modenese da Reggiolo a Castelnuovo, a Fiumalbo e al Salto di Montese (Gib. Pir.), sopra Bologna fino alla Porretta e al Covigliaio ecc. (Bert. , Cocc.), presso Faenza (Cald.), a Castrocaro (Cald.!), a Ravenna (Bert.). Trovasi ad Entraunes, Tenda, Castiglione sopra Mentone (Ard.); e nella Riviera di levante a S. Stefano d'Aveto (Bert.). In Toscana è stato trovato nelle Alpi Apuane a Corfigliano (Pucc.), a Pisa (Savi), in Mugello a Scarperia, e nei monti Morello, Senario (Parl.!) e Giovi!, in Casentino all'Alvernia (Parl.!) e a Pratovecchio!, in Chianti fra Radda e la Castellina (Beccari!), nel Senese presso Monte Baroni (Campani!) e a Monte Guidi (Ant. Targioni!), Castelletto-Mascagni e Pienza (Santi), ai Bagni di S. Filippo nel monte Amiata (Ant. Targioni!), a Radicofani (Santi). Trovasi poi a Fossato in Umbria (Parl.!), nel Lazio a Tivoli ecc. dove abbonda (Seb. Maur.), in Campania a Cassino sul monte Cairo (Terr.!) e a Pietraroia (Terr.) e Cercemaggiore (Avellino!). Dall' altro lato della Penisola trovasi a Pergola nell'Urbinate (Bert.), nel monte della Sibilla (Marzialetti!), presso Caramanico (Groves!), e Campo di Giove, nel Morrone (Ces.). Fiorisce in giugno e luglio.

Distribuzione geografica. - Nell' Europa, dalla Svezia media alla Spagna, all' Italia e alla Russia meridionale, nell' Asia Minore.

Descrizione. - Pianta annua, alta, di un verde chiaro, coperta di peli corti ruvidi bianchi che la rendono un po'scabra. Radice profonda, torta, ramosa, scuretta. Fusto eretto, oscuramente quadrangolare, verdognolo, peli corti, duretti, curvati in giù, ramoso, con i rami opposti, corti, eretto-patenti. Foglie opposte, patenti, e curvate in giù, sessili, lanceolato-lineari, e nei rametti lineari, intere, verdi chiari, con solco longitudinale e quasi lisce di sopra, di un verde più chiare e con nervo longitudinale sporgente di sotto da cui partono dei nervi laterali molto ohliqui che vanno verso l'apice e il margine della foglia, scabre di sotto per i soliti peli. Fiori eretti, in una spiga quasi cilindrica, ottusa e piuttosto fitta. Brattee inferiori simili alle foglie del fusto, ma più larghe alla base per cui sono ovate- 
lanceolate, acuminate, e nelle parti larghe laciniate con le lacinie lanceolato-acuminate, verdi chiare, le superiori sono gradatamente più corte, quasi uguali o poco più lunghe dei fiori, larghe, ovate, laciniate, di colore violetto-rossiccio: hanno di sotto delle verruche tonde a guisa di punti neri distribuiti quasi in due linee 0 alquanto irregolarmente. Il calice e ristretto in tubo in basso, slargato in alto, verdognolo in parte o in gran parte di color violetto-rossiccio, pubescente-scabro di fuori, con lobi o lacinie che giungono fir quasi alla metà del calice e sono larghe, eretto-patenti, ovato-lanceolate, intere $o$ con qualche dente o lacinietta laterale e terminate all'apice in una punta setacea, lunga che uguaglia il tubo della corolla. Questa è circa la metà più lunga del calice. Il tubo è lungo, un po' curvo, gradatamente un po'più grosso in alto, puberulo, di colore violettorossiccio, giallo nella parte superiore. Il lembo è diviso in due labbri, quasi uguali, uno superiore fatto a gnisa di elmo, schiacciato lateralmente e con i margini rovesciati e barbati, è di color violettorossiccio eccetto alla base dove corrispende al tubo siccome è pure del labbro inferiore; il labbro inferiore supera appena il superiore, è quasi ovato, leggermente trilobo, con i lobi piccoli, quasi uguali, tondeggianti, di sopra ha due gibbosità longitudinali, bislunghe. Gli stami sono 4, didinami, inseriti due in alto e due in basso del tubo, racchiusi dentro il labbro superiore. I filarrenti più lunghi sono pelosi in basso, giallicci in alto come i filamenti più corti. Antere quasi sagittate, biloculari, aprentesi longitudinalmente, con le orecchiette prolungate in una punta, di colore rosso-scuro, pelose in basso. $\mathrm{Pi}$ stillo quasi uguale al labbro inferiore. Ovario avente alla base in avanti un disco verdognoló: è ovato, un po' schiacciato nelle facce, quasi acuto all'apice. Stilo lungo, un po' curvato in alto, filiforme, bianco, pelosetto. Stimma bilobo, bianco, papilloso in capolino. (Parl. ms., deser. della pianta del Casentino in Toscana).

\section{Velompyrum barlontum.}

II. spica laxiuscula, equali, foliis floralibus ovato-lanceolatis, inferne præsertim pectinato-laciniatis, calyce lobis subrequalibus, tubo suo longioribus, tubo corollino multo brevioribus, capsula 1-valvi valva 2-loba.

Melampyrum barhatum Waldst. Kit. in Willd. sp.pl. ஃ.p. 198. Trev. prosp. 月. eug. p. 99. Bert. /. ital. 6. p. 30\%. Pir. 月. for. syll. p. 107. De Vis. Sacc. cat. piant. Ven. p. 155. Ces. Pass. Cib. comp. 
fl. ital. p. 360. Terr. quart. rel. Terra di Lav. p. 111. Smith fl. Fiume p. 38. Arc. comp. fl. ital. p. 523.

Melampyrum arvense var. Godr. fl. Lorr. 3. p. 233.

Figure. - Waldst. Kit.pl. rar. Hung. t. 86. Reich. ic. fl.germ. 20. t. 115. f. 2.

Stazione, Abitazione e Fioritura. - Nei campi, ed anche in luo. ghi boschivi, della Penisola, quasi esclusivamente della sua parte orientale: a Fiume (Smith), a Trieste e Muggia (Bert.), nel Littorale Friulano (Pir.), a Treviso, presso Venezia alla Mira (Bert. ecc.), sugli Euganei verso Abano e Montortone dove abbonda (Poll.), nel Polesine (De Vis. Sacc.); quindi si ritrova nell'Appennino Umbro sotto Colfiorito!, nell'Aquilano (Ces. Pass. Gib.), nel Gargano presso Vico (Porta Rigo!), nel Beneventano a Pietraroia (Terr.). Fiorisce in giugno.

Distribuzione geografica. - Nell' Europa orientale, dalla Noravia all'Italia, alla Tessaglia, alla Transsilvania.

Osservazione. - Specie molto simigliante alla precedente, se ne distingue inoltre per le foglie fiorali ordinariamente verdi e non colorate, per i fiori ordinariamente gialli e non rossi, per il calice ispido, ecc.

\section{Melanpyrum nemorosum.}

M. spica laxa, secunda, foliis floralibus ovatis lanceolatisve acuminatis, basi grandidentatis, calyce lobis subæqualibus, tubo suo longioribus, tubo corollino plus minus brevioribus, capsula 1-valvi valva integra.

a latifolium.

Melampyrum nemorosum Linn. sp. pl. ed. 1. p. 605. De Not. rep. fl. lig. p. 309. Bert.! fl. ital. 6. p. 305. Zan. prosp. fl. ven. p. 25. Com. fl. com. 5. p. 22. Pucc.! syn. pl. luc. p. 320. Sim.! fl. alp. vers. p. 134. Rota prosp. piant. Pav. p. 271. Hausm. A. Tir. p. 654. Rota prosp. fl. Berg. p. 67. Pir. fl. for. syll. p. 107. Car. prodr. fl. tosc. p. 494. Ard. fl. Alpes-mar. p. 286. Zersi prosp. piant. Bresc. p. 159. Ces. el. piant. Maiell. p. 22. Archb. fl. Alto-Serch. p. 59. Ces. Pass. Gib. comp. fl. ital. p. 360. Smith fl. Fiume p. 39. Cald.! fl. fav. tent. p. 174. Gib. Pir. fl. Mod. p. 124. Arc. comp. fl. ital. p. 523. Cocc. fl. Bol. p. 365.

Figure. - Fl. dan.t. 305. Reich. ic. fl. germ, 20.t. 114. $\beta$ angustifolium Car. l. c. 
Melampyrum pratense Linn. l.c. De Not.l.c. Bert. o. c. p. 306. Com. o. c. p. 23. Hausm. l. c. Rota prosp. fl. Berg. l. c. Pir. l. c. Ard. o. c. p. 287. Zersi l. c. Ing. cat. sp. Mond. p. 50. Ces. Pass. Gib. l. c. Smith l. c. Arc.! l. c. Cocc. l. c.

Figura. - Reich. ic. fl. germ. 20. t. 112.

Stazione, Abitazione e Fioritura. - Nei boschi, particolarmente di collina e di montagna, della Penisola superiore e media. $\mathrm{Fa}$ in Piemonte al Cenisio (Parl.!), in quel di Susa (Re), in val 01. menco presso Dronero (Ball!), sotto Viosenne (Ricca!), presso Mondovi (Ing.), presso Alba (Bertero!), a Torino, comune (All.), a Locana (Carestia!), ad Arona (Ricca!), nel Novarese (Bir.); in Lombardia nel Pavese (Rota), in Brianza a Cavenago al laghetto d'Alserio (Parl.!), a Como, in valle Intelvi (Com.), nei monti Parro a 5-600 metri (Parl.!) e Resegone (Com.), nel Bergamasco (Rota), nel Bresciano, frequente (Zersi), cosi a Bovegno e Collio da 850-1000 metri, al lago d'Iseo (Parl.!), a Ponte di legno (Ricca!), in Tirolo a Welsberg, Brunecco, Bressanone, Bolzano, sul Ritten, in val Venosta presso Laas, a Salorno, in Fiemme (Hausm.), a Trento (Perini!), nelle Giudicarie presso Stelle (Hausm.), in val Sugana (Ambrosi!); nel Veneto sul Baldo in vari luoghi, nei Lessini, presso Verona (Poll.), nel Mantovano (Barbieri!), negli Euganei (Poll. ecc.), nel Lido veneto (Kellner!), sul monte Summano (Poll.), in parecchi siti del Bassanese (Bert.), e del Bellunese (Venzo!), in molti luoghi della valle del Tagliamento da Ampezzo a Muzzana (Suffren, Pir.); in Istria presso 'Trieste (Bert.) e Fiume (Smith); nell'Emilia a Parma (Jan!), al Ventasso, a Montese (Gib. Pir.), presso Bologna (Bert.), alla Porretta (Cocc.); in Romagna a Faenza (Cald.!). E abbastanza comune nelle Alpi Marittime, cosi a Bollena (Ard.), verso Tenda (Reuter!) e Briga (Sternberg!); e nasce pure nell'Appennino ligustico di Rezzo, Dolcedo, Albenga (De Not.), Voltri (Baglietto!), Pe. gli (Bert.), Genova (De Not.) e Novi (Ardissone!). In Toscana è pinttosto raro: sopra Pontremoli!, nelle Alpi Apuane al Pisanino (Ad. Targioni!), alle sorgenti del Frigido! e in Versilia (Simi!), nel Lucchese dal Rondinaio (P. Savi!) giì finn a Lucca (Pucc. ecc.), sopra Firenze a monte Senario (Ricasoli! ecc.), a Siena (Bart.), a Volterra (Amidei!, Biondi!). Nelle Marche è stato trovato al monte Cnceo (Piccinini!), e al viciho Catria a 1100 metri (Parl.!), nei monti di Sernano a S. Liberato (Bert.), nel monte Regnolo (Marzialetti!), sopra Uinito (Parl. I); nell'Abruzzo a Roccamorice (Ten.), a Caramanico a $\mathbf{3 0 0 0}$ piedi, alla Maiella (Groves!), al Maiellone (Ces.), in valle 
Vellavozzi presso Pettorano (Levier!). Infine è indicato nel Samnio da Tenore. Fiorisce in giugno, luglia e agosto.

Distribuzione geografica. - In tutta Europa, meno le sue parti più meridionali, nella Siberia occidentale.

Descrizione. - Pianta annua, di un verde chiaro, alta fino a 5 decimetri, puberula per peli cortissimi. Radice torta, fusiforme, ramosa, corta, bianchiccia. Fusto eretto, quadrangolare, verdognolo 0 in parte rossiccio, puberulo, ramoso, con i rami opposti, ascendenti. Foglie opposte, patenti, ovato-bislunghe, acuminate, verdi chiare di sopra e ivi con solchi che corrispondono ai nervi di sotto, verdi ap. pena piủ chiare di sotto ed ivi con nervo longitudinale sporgente, da cui partono rametti laterali curvi anche sporgenti che si diramano e uniscono tra loro verso il margine : intere, con picciỏlo corto, con un solco larghetto di sopra, convesso di sotto. Fiori solitarii all' ascella delle foglie superiori o fiorali, le quali sono gradatamente più piccole delle foglie cauline, le inferiori piủ lunghe dei fiori, le altre gradatamente più corte del fiore ma sempre più lunghe del calice, tutte con pochi denti, lunghetti o acuminati, alla base, le superne di color violetto carico, le altre del colore delle foglie cauline. Tali fiori sono forniti di un peduncolo molto corto, un po'cernuo, più grosso in alto, verdognolo e pubescente; i fiori sono unilaterali. Il calice è tubuloso, verdognolo, rossiccio in parte, con cinque leggieri angoli, diviso fino alla metà in cinque lacinie lanceolato-acuminate, avvicinate al tubo della corolla, con un nervo longitudinale sporgente con qualche ciglio nel margine, due delle quali sono superiori e tre inferiori. La corolla è quasi tre volte piủ lunga del calice, gialla, quasi dorata nelle labbra, e rossiccia all'apice dell' elmo del labbro superiore. Il tubo è gradatamente più largo verso alto, con una carena in alto che si continua a guisa di elmo nel mezzo del labbro superiore, pubescente di dentro. I labbri sono corti, molto più corti del tubo, il superiore è smarginato-bilobo, con i lobi e i margini rovesciati in su verso l'elmo e di sotto e per la rovesciatura di fuori irsuti per peli piuttosto corti, fitti e gialli. Il labbro inferiore è poco piủ lungo del superiore, diretto in avanti, trilobo, con i lobi laterali un poco più grandi di quello di mezzo, ottusi, ha tre vene longitudinali di sotto di colore violetto. Il palato è chiuso da due gibbosità bislunghe che si vedono in quasi tutta la pagina superiore del lobo inferiore, esse sono rossicce verso la base. Gli stami sono 4 , didinami, inseriti alla parte superiore del tubo, quasi filiformi, un po' curvati in alto in avanti, giallicci, puberuli. Le antere sono verdognole, ros- 
sicce nel margine, aderenti tra loro, pelose, cigliate nel margine esterno. Il pistillo è lungo più degli stami più lunghi. L'ovario è ovale, acuto, un po'convesso nelle facce e ivi con un solco longitudinale, verdognolo, glabro. Lo stilo è lungo, filiforme, bianco, curvato all'apice ad arco in avanti, glabro. Lo stimma è quasi in capolino, papilloso, bianco. (Parl. ms., descr. della pianla del Bresciano fra Pisogne e Tolina).

Osservazioni. - Generalmente si distinguono come specie le due varietà sopra ricordate, per: la diversa lunghezza e conseguente forma delle foglie; la maggiore o minore dentatura delle foglie fiorali; il loro colorimento; la pelosità o no del calice; la sua lunghezza rispetto alla corolla. Or bene: su di una vistosa raccolta di saggi italiani, io ho riscontrato talmente fallaci i suddetti caratteri differenziali, che le due varictá, piuttostochè tali, dovrebhero considerarsi (come sono rappresentate nelle figure citate del Reichenbach) due forme estreme, fra le quali stanno tutte le possibili intermedie.

\section{Melampyrum silvatieum.}

M. spica laxa, secunda, foliis floralibus lanceolatis acuminatis, subintegris, calyce lobis subæqualibus, tubo suo sublongioribus, tubo corollino æqualibus vel vix brevioribus, capsula 2-valvi.

Melampyrum silvaticum Linn. sp. pl.ed.1. p. 605. Mass. prodr. fl. valt. p. 111. Bert. f. ital. 6. p. 30\%. Com. fl. com. 5. p. 25. Hausm. 月. Tir. p. 655. Rota prosp. fl. Berg. p. 67. Pir.! /1. for. syll. p. 107. Ard. A. Alpes-mar. p. 287. Zersi prosp. piant. Bresc. p. 159. Ces. el. piant. Maiella p. 22. Ing. cat. sp. Mond. p. 50, Ces. Prss. Gib. comp. fl. ital. p. 360. Arc.l comp. fl. ital. p. 523.

Figure. - Fl. dan. t. 145. Reich. ic. fl. germ. 20. t. 113.

Stazione, Abitazione e Fioritura. - Nei boschi delle Alpi : nelle Marittime alla Fraca, al Boreon, al col di Salese, a S. Anna di Vinai, nei monti di S. Stefano (Ard.), verso T'enda (Ungern-Stern. berg!), ai Bagni di Valdieri (Bert.), dove abbonda, nei monti di Vi. nadio (Colla), in quel di Mundovi nella vallata del Carsaglia presso Frabosa, comune (lugo); in quel dı Susa presso i Cinati in Mocchie, e presso Cels in valle d' Exilles (Re), e su nel Cenisio al lago a 2000 metri (Arc.l ece.); nel Cramont (Parl. I) ; a Riva di Srsia (Carestia!); nelle valli di Vedra, e di Buggnanca (Bir.); al S. Rernardino (De Not !); in val Tellina, frequente (Mass.), per esempio sopra S. Caterina a 1800) metri (Parl.!), in val Intelvi (Ciom.); nel Bergamasen (Rota) al monte 
Epolo, a Pisgana sopra 2000 metri, a Ponte di legno a 1330 metri (Parl.!), nel Bresciano sul monte Conche e sopra Lavenone (Zersi); in Tirolo a Brunecco, presso Laas, sul Ritten, nel monte Pelenzana in Fiemme, a Predazzo, in Fassa, presso Trento nel monte Celva (Hausm.) e a Panevaggio (Perini !), a Roveredo (Hausm.); nei Lessini verso Chiesanova e Campofontana (Poll.), nei Sette Comuni a Marcesina (Bert.), in Friuli a Sappada (Pir. !), Forni di sotto, Pontebba (Pir.). Dicesi che nasca inoltre nell' Abruzzo (Ten.), e precisamente nei monti di Caramanico (Ces.), e nel Samnio (Ten.): ma non ho veduto saggi di quei luoghi. Fiorisce in luglio e agosto.

Distribuzione geografica. - In Europa, dalla Scandinavia ai Pirenei, alle Alpi, al Caucaso, in Siberia.

Descrizione. - Pianta annua, alta da 15 a 30 centimetri, di un verde chiaro, puberula o quasi glabra. Radice fusiforme, delicata, flessuosa. Fusto eretto, delicato, quadrangolare, con gli angoli ottusi, violetto-rossiccio o verdognolo, puberulo per peli corti nelle facce, ramoso con i rami patenti. Foglie opposte, le inferiori patentissime reflesse, le superiori patenti, lanceolate, ottusette 0 acute, ristrette in basso in un corto picciỏlo, intere nei margini ed ivi scabre, di un verde chiaro di sopra ed ivi con un solco longitudinale, di un verde piủ chiaro di sotto ed ivi con un nervo longitudinale più chiaro e delicato da cui partono dei nervetti delicati che si diramano per unirsi tra loro nel parenchima delle foglie. I fiori sono piccoli, solitarii all'ascella di ciascuna foglia superiore per cui a ciascun nodo vitale vi sono due fiori, unilaterali, molto più corti delle foglie fiorali corrispondenti le quali sono simili alle foglie ed intere. I peduncoli sono corti : piủ corti del calice, eretto-patenti, angolati e puberuli. Il calice è verdognolo, glabro, con il tubo angolato, diviso fin sopra la metà in quattro lobi quasi uguali, patenti, ovato-lanciolati, acuti, scabri nel margine e nella faccia interna o superiore. La corolla è piccola, poco più lunga del calice, di color giallo piuttosto chiaro con il labbro inferiore di sopra di un giallo più carico e quasi dorato. Il tubo è più lungo del tubo del calice, un po'curvato in fuori, quasi cilindrico e bianchiccio in basso, slargato in alto. Il lembo è diviso in due labbri, uno superiore, un po' più grande, quasi in forma di elmo, convesso di fuori, concavo e irsuto di dentro, smarginato all'apice con i denti della smarginatura ottusi, ha il margine piegato un po' in alto, il labbro inferiore è diretto in avanti e un po 'in giủ, quasi pianı, ovato-tondo, tridentato all' apice con i denti ottusi, quasi uguali, con due gibbosità appena sporgenti e puberule; la gola 
è aperta. Gli stami sono quattro, quasi uguali di altezza, con le antere poste nella concavità del labbro superiore, inseriti nel tubo della corolla. I filamenti sono filiformi, un po' curvi in alto in avanti, giallicci, con qualche corto peletto. Le antere sono quasi ovali, biloculari, pelosette, bilobe alla base con le logge terminate in una punta acuta, giallicce, pelosette o pelose. Il pistillo è più lungo degli stami ma non eserto per essere in alto molto curvato in avanti ed in giu dentro l'elmo. L'ovario è ovato, schiacciato, verde, glabro. Lo stilo è lungo, filiforme, curvato molto in alto come ho detto. Lo stimma è ottuso, papilloso. (Parl. ms., descr. della pianta di Ponte di legno).

\section{TII. PEITCULA IEIS.}

Pedicularis Linn. gen. plant. ed. 1. p. 184. Gen. pl. fl. germ. 5. t. 45 (mala quoad semina). Benth. Hook. gen. pl. 2. p. 978. Ces. Pass. Gib. comp. fl. ital. p. 357. t. 53. f. 6.

Calyx tubulosus vel subventricoso-campanulatus, inæqualiter 45-dentatus-fissus. Corolla limbo conspicue 2-labiato, labio postico galeato, antico 3-lobo. Stamina 4. Antherarum loculi basi mutici vel mucronati. Stigma minusculum. Gemmulæ in loculis paucæ, subbiseriatæ, pendulæ. Capsula oligosperma, seminibus ad hilum strophiolatis.

Portamento. -- Le Pedicularis sono erbe perenni; dotate di un rizoma dal quale si dipartono radici robuste, anzi. talora ingrossate affusate, e fusti eretti $o$ ascendenti, lunghi da 1 a 4 decimetri, semplici, fioriferi in cima. Le foglie sono grandi, lanciolate, profondamente divise a penna, e i lobi ancora divisi; per lo più ve n'è 'una rosetta basilare, sul fusto ve ne sono altre, o poche o molte, grandi o piccole. In cima al fusto stanno altre foglie, o brattee poco dissimili dalle rnedesime, con all' ascella fiori solitari, pressochè sessili, grandi, rossi o gialli, i quali formano cosi nel loro insieme una spiga, allungata o raccorciata, rada o fitta, di raro sono $i$ fiori pedicellati e formano piuttosto un corimbo.

- Corollæ galea erostris vel brevissime rostrata.

\section{Pedicularis menulis.}

P. foliis pinnatisectis Iobis ovatis partitis dentatis, omnibus hasilaribus, ralycis campanulati lobis foliaceis inciso-dentatis, corolla ex albo carnex galea er ostri obtusa ciliata, capsula.... 
Pedicularis acaulis Wulf. in Jacq. coll. 1.p. 207. Bert. fl. ital. 6. p. 317. Com. fl. com. 5. p. 42. Hausm. fl. Tir. p. 661. Rota! prosp.fl. Berg. p. 68. Pir. fl. for. syll. p. 108. De Vis. Sacc. cat. piant. Ven. p. 157. Zersi prosp. piant. Bresc. p. 160. Ces. Pass. Gib. comp. fl. ital. p. 358. Smith! fl. Fiume p. 39. Arc. comp. fl. ital. p. 522 .

Figure. - Wulf. o. c. t. 14. Reich. ic. fl. germ. 20. t. 140.

Stazione, Abitazione e Fioritura. - In luoghi erbosi o boschivi unidi dei monti della Penisola settentrionale orientale, generalmente rara: presso Fiume qua e là, a Castua (Smith!), sul monte Maggiore (Huguenin!), in val Recina, rara, cosi presso Bermani (Smith!); a Trieste (erb. centr!); sul monte Canino del Friuli (Pir.); nelle alpi Vicentine al monte Summano (De Vis. Sacc.), nelle Veronesi al monte Baldo sull'Altissimo (Barbieri!), sulla Colma di Malcesine, in valle Finestra (Poll.), al monte Maggiore, a Costabella e in valle delle Ossa (Hausm.); nelle Giudicarie sopra Crosette a piè dell'alpe Lenzada, e presso Tione (Hausm.), e in valle Vestino presso Moerna (Porta!); nel Bresciano nel monte S. Onofrio al Palosso (Bert.), alle coste di S. Eusebio, in val Bertone sopra Caino, frequente (Zersi); nel Bergamasco a Carenno (Rota!) in valle S. Martino (Bert.); nel Comasco al Resegone, assai rara (Com.); in val Tellina (Moretti!). Fiorisce secondo $\mathrm{i}$ luoghi in aprile e maggio, 0 in maggio e giugno.

Distribuzione geografica. - Nasce inoltre in Croazia e in Carnia.

\section{Pedicularis verticillata.}

P. foliis pinnatipartitis lobis ovalibus dentatis, plerisque basilaribus, caulinis subverticillatis, calycis inflati superne fissi dentibus brevissimis integris, corollæ rubræ galea erostri obtusa edentula, capsula 1-valvi.

Pedicularis verticillata Linn. sp. pl. ed. 1. p. 608. Vitm. ist. erb. p. 43. Mass. prodr. fl. valt. p. 127. Pucc.! syn. pl. luc. p. 320. Bert. fl. ital. 6. p. 320. Com.! fl. com. 5. p. 35. Hausm. fl. Tir. p. 661. Rota prosp. fl. Berg. p. 68. Pir.! fl. for. syll. p. 108. Car. prodr. fl. tosc. p. 492. Ard. fl. Alpes-mar. p. 285. Zersi prosp. piant. Bresc. p. 159. Ing. cat. sp. Mond. p. 55. Ces. Pass. Gib. comp. fl. ital. p. 359. Anzi auct. fl. nov.-com. p. 196. Paol. el. sib. p. 16. Sacc. Bizz. agg. fl. trev. p. 28. Arc.! comp. fl. ital. p. 522. 
Figura. - Reich. ic. fl. germ. 20. t. 141. f. 1.

Stazione, Abitazione e Fioritura. - Nei pascoli di tutta la catena delle Alpi: in Friuli nella valle della Resia, nei monti Plauris, Amariana, Cuch (Pir.), Iersadia (Pir.!); nel monte Cavallo (Kellner!), in Cansiglio (Sacc. Bizz.); nelle Vette di Feltre in Morsopian, in Collalti presso Bassano, a Marcesina presso Vicenza, nelle Vette dei Monzoni (Bert.), nel Baldo all'Altissimo, dove abbonda (Poll.); in Tirolo sul monte lombaa in val Vestino (Porta!), sul Bondone, nelle Giudicarie a Maria di Campiglio, sui monti Spinale e Lenzada (Hausm.), in valle di Campo (Caldesi!), in val di Non sul Proveis, alla Cima di Vallarga in val Fersina, in Fiemme (Hausm.), e Fassa (Bracht!) sul Davoi, nell'alpi Seiser e Schlern, comune, presso Weisenstein già a $4700^{\prime}$ (Hausm.), alla Cortina d'Ampezzo al passo delle tre croci (Smith!), intorno a Welsberg, e a Bressanone, in val Martello di val Venosta (Hausm.); in val Tellina sullo Stelvio tra 1700 e 2300 metri, a Plaghera (Parl.!), sul Braulio, nelle alpi Togno e Mara (Mass.), e Venina (Com.!), in val Furva (Rampoldi!); nel Bresciano a Ponte di legno (Ricca!), nel monte Venerocolo a 2000 metri, nel Tonale a 2000 metri, nei monti Ario a $1600-1700$ metri, Gerle e Dos Alto.a 1700-1900 metri (Parl.!), nelle vette del monte Guglielmo (Zersi); nel Bergamasco (Rota); nel Comasco sui monti Legnone, Darengo, Speluga, Camisolo in val Sassina (Com.); al S. Bernardino (De Not.!); nel Novarese, scendendo fino ai monti di Quarna e Margozzo (Bir.); in val Sesia nell'alpe Olen e alla Montà (Carestia!), in val d'Aosta alla Betta Furke (Piccone!), al Gran S. Bernardo a 6-7500' (Heldreich!), presso Courmayeur (Webb!), al lago Combal (Parl.!); nel Cenisio al lago a 2000 metri (Arc.! ecc.) e a Ronclıe presso le nevi eterne (Bucci!), e presso il Collet verso Fenestrelle (Re); nelle alpi Marittime al col della Maddalena e nell' altipiano di Giallorgues presso S. Dalmazzo (Ard.), nell'alpi di Valdieri (Bert.), e di Mondovi, comune (Ing.). Abita poi le più alte cime dell' $\Lambda$ ppennino toscano, cioè a dire l'alpi di Mommio al monte Prado e altrove, l'alpi di Corfino e di S. Pellegrino (Calandrini!), l’alpe di Controne (Vitm.), l'Alpi apuane (P'ucc.!), l'Appennino pistoiese e mugellano secondo Savi. Infine abita l'alto $\mathrm{Ap}$ pennino centrale, ossia i monti di Norcia (Sang.), il Pizzo di Sivo alla Macera della morte (Orsini!) e a Furca di Fonte Vidone!, il Corno sopra Campo Pericoli a $2400-2500$ metri (Levier!), il Ciostone (Bert.) e il Velino (Ten.!). Fiorisce in luglio ed agosto.

Distribuzioue geografica. - Nei monti dell' Luropa meridio- 
nale e centrale, dalla Sierra Nevada ai Carpazi e alla Macedonia, nell' Imalaia, e nell' Europa, Asia e America artiche.

Descrizione. - Pianta perenne, formante dei graziosi cespuglietti con i suoi fusti alti da 8 a 15 centimetri, fornita di un rizoma quasi verticale, duro, ramoso, dal quale partono delle fibre radicali durette, ramose, giallicce ed in alto i fusti e le foglie inferiori. I fusti sono diritti, semplici, verdognoli e inferiormente rossicci, quadrangolari, con gli angoli ottusi e con un solco longitudinale nelle facce, e forniti di peli lunghetti, e curvati in giù e distribuiti in quattro linee longitudinali. Le foglie inferiori sono in cespuglietti, erette $o$ eretto-patenti, piccole, di perimetro allungato-lanceolate, profondamente pinnatifide, con le lacinie ovali-allungate, ottuse, disugualmente dentate e con i denti acuti, verdi e glabre di sopra, di un verde piủ pallido di sotto ed ivi con un nervo longitudinale bianchiccio nel quale si vedono spesso alcuni peli lunghetti, con i margini un po'rovesciati in sotto. Il picciỏlo è quasi piano, leggermente solcato nella parte che corrisponde in mezzo alle foglioline, con i margini delicati e quasi acuti nella parte inferiore priva di foglioline, nella qual parte esso è per lo più rossiccio. Le foglie del fusto sono simili alle inferiori ma piủ piccole e quasi sessili, erettopatenti e acute, in un verticillo di quattro foglie. Le foglie fiorali in. feriori e le brattee sono pure a quattro a quattro, patenti, le inferiori simili alle foglie cauline, le altre gradatamente più piccole, meno divise, ovato-lanceolate e infine quasi intere: sempre però di consistenza e colore come le foglie. I fiori sono anch'essi in giri di quattro a quattro, quasi sessili all' ascella delle foglie fiorali e delle brattee, quasi uguali o poco più lunghi di queste, patenti e formanti per l'avvicinamento dei verticilli una spiga corta ed ottusa, talvolta interrotta per essere il verticillo inferiore dei fiori meno vicino agli altri. I pedicelli sono cortissimi, contorti sopra loro stessi. Il calice è quasi globoso, gonfio, delicatn, quasi trasparente, fesso irregolarmente in alto e con cinque denti tutti portati verso alto, i quali sono ottusi e dal mediano gradatamente più piccoli nei laterali da una parte e dall'altra: ha dieci nervi longitudinali rilevati i quali sono forniti di lunghi peli di color roseo-carico, il calice stesso è quasi roseo con i nervi di color più carico. La corolla è tre volte circa più lunga del calice, di color rosso-carico, bilabiata; il labbro superiore è quasi diritto, rivolto in su ed in avanti, ottuso, senza denti, schiacciato lateralmente e glabro; il labbro inferiore è lungo quasi quanto il superiore, ma patente, largo e trilobo, con il lobo intermedio un 
po' piủ lungo, e più stretto dei laterali, obovato-spatolato, e i lobi laterali quasi quadrilateri con gli angoli un po'tondeggianti. Gli stami sono 4, didinami: i due più lunghi uguagliano quasi il labbro superiore, $\mathrm{i}$ due piủ corti sono più corti, tutti sono racchiusi nel labbro medesimo e inseriti alla base del tubo della corolla. I filamenti sono filiformi, bianchicci, puberuli in basso, curvati in dentro verso l'apice e i due più lunghi ivi pelosetti. Le antere sono ovali, smarginate ed ottuse all'apice, bifide e con le logge acute alla base, inserite nel mezzo del dorso, biloculari, con le logge rossicce scure, glabre e aprentisi longitudinalmente e con il connettivo verdognolo, visibile solo di dietro. Il pistillo è lungo quanto il labbro superiore, anzi spesso lo supera appena in modo che lo stimma è eserto. L'ovario è ovato, un po'schiacciato dalle parti, verde, glabro, biloculare, con molti ovoli, inseriti nel setto carnoso. Lo stilo è filiforme, molto lungo, glabro, bianchiccio in basso, roseo-carico in alto. Lo stimma è quasi in capolino, oscuramente bilobo, papilloso, gialliccio. (Parl. ms., descr. della pianta delle Alpi Bresciane).

\section{Pedicularis AEderi.}

P. foliis pinnatisectis aut partitis lobis ovalibus dentatis, plerisque basilaribus, caulinis paucis sparsis, calycis tubulosi dentibus integris vel apice foliaceo parce dentatis, corollæ pallide luteæ apice rubro-maculatæ galea erostri obtusa edentula, capsula 1-valvi.

Pedicularis CEderi Vahl in Horn. plantel., sec. auct. rec. Ces. Pass. Gib. comp. fl. ilal. p. 358. Arc. comp. 月. ilal. p. 522.

Pedicularis versicolor Wahl. de veg. Helv. Bert. fl. ital.6. p. 318 . Hausm. fl. Tir. p. 660. Rota prosp. A. Berg. p. 68.

Figura. - Reich. ic. /l. germ. 20. t. 198. f. …

Stazione, Abitazione e Fioritura. - Nelle Alpi, rarissima. E stata trovata nel Cenisio (Thomas! ecc.), nell'alpe Savine in una rupe nevosa (All.), nel Cramont nei pascoli a 2510 metri (Parl.!), nel S. Bernardo (erb. Webb!); quindi al Brennero (Bert., Ilausm.), e nel monte Bianco di Sterzinga (Hausm.); infine secondo Rota nei pascoii sassosi delle prealpi Camoniche. Fiorisce in giugno, luglio, agosto.

Distribuzione geografica. - In tutte le terre artiche, in Scandinavia, nelle Alpi e nei Carpazi, nell'Asia centrale. 


\section{Pedicularis nosea.}

P. foliis pinnatisectis lobis anguste lanceolatis integris vel parce inciso-dentatis, omnibus fere basilaribus, caulinis perpaucis sparsis, calycis tubulosi dentibus integris, corollæ roseæ galea erostri obtusa edentula, capsula 2-valvi.

Pedicularis rosea Wulf. in Jacq. misc. austr. 2. p. 57. Bert. f. ital. 6. p. 319. Hausm. fl. Tir. p. 660. Rota prosp. fl. Berg. p. 68. De Vis. Sacc. cat. piant. Ven. p. 157. Zersi prosp. piant. Bresc. p. 160. Ces. Pass. Gib. comp. fl. ital. p. 358. Arc.! comp. fl. ital. p. 522. Gib. Pir. suppl. fl. Mod. p. 22.

Pedicularis Allionii Reich. fl. ic. Al. germ. 20, p. 77. Ard. fl. Alpes-mar. p. 285.

Figure, - All. rar. Ped. stirp. spec. prim. t. 12. f. 1, in $\mathrm{l}$. ped. t. 3. f. 1 repelita. Reich. o. c. t. 139. f. 1.

Stazione, Abitazione e Fioritura. - Nei pascoli e fra le rupi delle Alpi. Nasce nelle Marittime nell'alpe Ruscara (Ricca!), nelle Viosenne (Ard.), al Carlino (Reich.), al monte Formosa presso il col di Tenda (Bourgeau!), a Colla Lombarda (Ard.); quindi nel monte Viso (Colla), nell'alpi Valdensi alla Sea bianca a 5-7000' (Ball!), nel Cenisio a Ronche (Bonjean! ecc. ecc.), nell'alpi di Giaglione, e del Vallon (Re), a Roccamelone (Beccari!), alle valli di Viù, Groscavallo, Ponte, Ceresole (All.), nell'alpe Dondena (Carestia!) e a S. Marcello (Reich.) in val d'Aosta, nei monti Moro e del Turlo nel Novarese (Bir.). Ricomparisce nel Tirolo nell'alpi Seiser a piè del Plattkofel, Schlern e Ioch Latemar (Hausm.), allo Spinale, in Fiemme al Castellazzo (Perini!), e nell'alpe di Campitello (Sardagna!); e poi trovasi nelle Velte di Feltre (Ambrosi!) e nel Bellunese al monte Serva (Reich.) a levante, e a ponente nel Bresciano nel monte Catino, rarissima (Zersi), e nel Bergamasco nei monti Ferrante, Epolo, Presolana (Rota). Infine è stata recentemente scoperta dal signor Fiori nell' Appennino piủ settentrionale all'alpe di Mommio (Gib. Pir.). Fiorisce in luglio e agosto.

Distribuzione geografica. - Si stende dal Delfinato alla Transsilvania.

Descrizione. - Radice grossetta, bianchiccia. Foglie radicali erette; picciòlo di sopra leggermente scanalato, convesso di sotto, violetto, con i margini violetti e il canale verdognolo; lacinie strette 
lineari, acute, con qualche lacinietta, sono di un verde glaucescente. Foglie del fusto rade, poche, più piccole, meno divise. Fusto cavo, cilindrico, eretto, violetto, peloso, segnatamente in alto con peli bianchi, crespi. Fiori pochi in spiga corta, fitta; pedicello cortissimo. Brattea lanceolato-lineare, acuminata, lunga quasi quanto il calice, avvicinata a questo, pelosetta. Calice tubuloso, gonfio in alto, di un verde violetto, con cinque nervi verdognoli, diviso in 3 lacinie 0 denti uguali, lanceolato-acuminate, pelose e cigliate con lunghi peli bianchi. Corolla rosea, quasi il doppio pin lunga del calice. Tubo poco più lungo del calice. Labbro superiore eretto, appena curvato in giù, schiacciato lateralmente, tondeggiante all'apice e ivi smarginato, glabro, di colore un po' più carico dell' inferiore, questo diretto in avanti, trilobo. Stami didinami; filamenti filiformi, bianchicci, pelosetti; antere scure, mutiche. Pistillo lungo quanto il labbro superiore. Ovario ovato, quasi acuto, un po' convesso nelle facce, glabro, inserito sopra un disco verdognolo, disuguale. Lo stilo è lungo, filiforme, glabro, curvato ad arco in avanti ed in giù verso l'apice. Lo stimma è quasi tondo, piccolo, gialliccio. (Parl. ms., descr. della pianta del monte Cenisio).

Osservazione. - Reichenbach ha voluto distinguere la Pedicularis Allionii delle Alpi occidentali dalla $P$. rosea delle Alpi orientali per una leggiera differenza nella dentellatura delle foglie, ch' $\dot{e}$ vera. mente troppo poca per stabilire una specie.

\section{Pedieularis recutita.}

P. foliis pinnatipartitis lobis lanceolatis grosse serratis dentibus serrulatis, caulinis plurimis sparsis, calycis tubulosi dentibus integris, corollæ sordide purpurea galea erostri obtusa edentula, capsula demun 2-valvi.

Pedicularis recutita Linn. sp. pl. ed. 1. p. 608. Mass. prodr. A. valt. p. 128. Bert. fl. ital. 6. p. 321. Com. fl. com. 5. p. 31. Ilausm. 1l. Tir. p. 660 liota prosp. M. Berg. p. 68. De Vis. Sacc. cat. piant. Ven. p. 157. Zersi prosp. piant. Bresc. p. 160. Ces. Pass. (Gib. comp. fl. ital. p. 358. Anzi auct. 1l. nov.-com. p. 196. Arc. comp. 1l. ital. p. 521 .

Figura. - Reich. ic. R. germ. 20. t. 137.

Stazione, Abitazione e Fioritura. - Nei pascoli umidi delle Alpi. Nasce nel Gran S. Bernardo a 7600 piedi (Parl.! ecc.), in val d'Aosta nel monte Echallier fra Fraissen e Peyran (All.), nel monte 
Rusa (Anzi), in val Sesia nell'alpe Sella della Montata fra i larici (Carestia!), nel Sempione ai ghiacciai (Gaud.), nel Gottardo sopra il lago Lucendro (Levier!), al S. Bernardino, allo Spluga, al laghetto di Darengo nella valle di Livo sul lago di Como (Com.), in val Tellina nella valle di Tartano (Anzi), nelle alpi di Palù e Cavaia sopra Poschiavo (Mass.), e in Plaghera sopra S. Caterina a 2000 metri (Parlatore!), nelle prealpi di val di Scalve e Ciamonica (Rota), in Tírolo nella valle Suldner di val Venosta, sull' alpi Zil e Maiser presso Merano, in val di Sarn da Oberstücke verso Passeier, e sopra Reinswald di fronte all'alpe Villander, sullo Schlern sopra Ratzes, sull'alpe Seiser, a Lusia in Fiemme (Hausm.), in Fassa (Ambrosi !), nel Trentino a Paneveggio (Perini!), infine in Carnia nella valle d'Incaroio (Pir.!). Fiorisce in luglio e agosto.

Distribuzione geografica. - Specie delle Alpi di Europa.

Descrizione. - Pianta perenne, alta 5 o 6 decimetri, verde e in parte violetto-scura, glabra. Rizoma grossetto, obliquo, con lunghe fibre radicali, bianchicce. Fusto eretto, fistoloso, cilindrico, liscio, verdognolo e in parte violetto, semplice. Foglie radicali lunghe, erettopatenti, profondamente pennatifide, con le lacinie lanceolate, vicine tra loro, quasi opposte o opposte, pennatifido-dentate o dentate, con i denti aventi dei piccoli dentini, verdi di sopra e in parte violettoscure, con un solco longitudinale nella foglia e in ciascuna lacinia, verdi chiare di sotto ed ivi con un grosso nervo longitudinale più chiaro e un nervo longitudinale in ciascuna lacinia dal quale partono venette che formano una rete scarsa nella pagina inferiore, i margini sono rovesciati; picciòlo leggermente scanalato di sopra, convesso di sotto, verde chiaro. Foglie del fusto alterne, sino in alto, gradatamente più piccole, sinili alle inferiori ma con le lacinie largamente confluenti in basso e di color violetto scuro, e con il picciòlo più corto. Frutti in racemo stretto, più fitti in alto, interrotti in basso, eretto-patenti. Rachide angolata. Pedicelli eretto-patenti, più corti 0 quasi uguali al calice. Calice violetto-scuro, adattato al frutto, con denti disuguali, interi. Cassula (non matura) ovata, acuta, convessa nelle facce, di colore violetto-scuro. (Parl. ms.)

\section{Pedicularis foliosa.}

P. foliis pinnatisectis partitisque lobis lanceolatis iterum partitis lobulis serratis, caulinis plurimis sparsis, calycis campanulati denti- 
bus integris brevissimis, corollæ ochroleucæ galea erostri obtusa edentula, capsula 2 -valvi.

Pedicularis foliosa Lini. mant. p. 86. Bert. /. ital. 6. p. 322. De Not. rep. fl. lig. p. 310. Com.! /l. com 5. p. 46. Hausm. fl. Tir. p. 659. Rola prosp. fl. Berg. p. 68. Pir. A. for. syll. p. 108. Ard. 1. Alpes-mar. p. 285. Zersi prosp. piant. Bresc. p. 160. Ces. el. piant. Maiella p. 22. Ces. Pass. Gib. comp. 月. ital. p. 358. Anzi ancl. /. nov.-com. p. 196. Arc. comp. fl. ital. p. 521. Cocc. /. Bol. p. 366.

Pedicularis Hacquetii Graf in flora 1834. p. 40. Smith! /l. Fiume $p .39$.

Figura.-Reich.ic. fl.germ. 20. t. 135.

Stazione, Abitazione e Fioritura. - In luoghi erbosi delle Alpi e dell'Appennino, quasi sempre indicata come rara. È stata trovata in Istria nei monti Maggiore (Noë!, Smith! ecc.), Berlosnik (Smith) e Slavnik (Koch); in Friuli nel monte Lipgnac presso Lusevera (Pir.); nel monte Coppolo di Lamon di Feltre (Bert.); in Ti. rolo sul Brennero, in val Venosta nella valle di Roien, in Fassa nell'alpe di Busanto (Hausm.) e nel monte Bufaure, in val Sugana sopra Torcegno (Ambrosi!) e nell'alpe Sette Selle (Bert.); sulle Colombine verso Ravenola nel Bresciano (Zersi), in val di Scalve nel Bergamasco (Rota); in val Tellina nella valle di Fraele a 2000 metri (Anzi), al lago di Como nella Valmeria sopra Mandello (Bert., Com.) e nel monte Generoso (Ciom.! ccc.), e piủ oltre nell'alpi di Vegezzo sopra Salecchio (Bir.); nel S. Bernardo (All.), nel Cenisio al lago (Parl.!) e alla Ramasse (Bonjean!), nel piccolo Moncenisio, nel piccolo monte Colissè, a Bardonecchia (All., Re); nell' Alpi Marittime e nel contermine Appennino nel vallone di Nanduebis presso S. Martino Lantosea (Ard.), nei monti di Triora (Panizzi!), Frontero, Antola (De Not.), nel monte Figue (Carrega!). Quindi si presenta in un punto solo dell'Appennino bolognese al monte Acuto dell'alpe, statavi recentemente scoperta dal dottor Riva (Ciocc.), e poi nell'Appennino piceno-abruzzese, dov'è stata trovata nei monti Bove (Šang.), Birro (Marzialetti!), Pelone (Bert.), Acuto, al Pizzo di Sivo, al Corno (Orsini!), al Sirente a 5000' (Groves!), alla Maiella in valle d' Orfenta a J-4000' (Porta Rigo!); e fa un' ultima comparsa nei monti Stabiani (Ten.!) al monte S. Angrelo di Castellammare presso la cappella (Ten.). Fiorisce in giugrno e luglio.

Distribuzione geografioa. - In Europa dai Vosgi e dalla Galizia alla Catalogna, all'Italia, ai paesi Janubiani. 


\section{*. Pedicularis pulustris}

P. foliis semel vel bis pinnatisectis lobis oblongis partitis crenatis, omnibus caulinis sparsis, calycis tubulosi mox inflati 2-fidi dentibus foliaceis inciso-crenatis, corollæ roseæ galea suberostri acuta edentula, capsula 1-valvi.

Pedicularis palustris Linn. sp. pl. ed. 1. p. 607. Mass. prodr. fl. valt. p. 127. Trev. prosp. fl. eug. p. 23. Bert. fl. ital. 6. p. 315. Zan. prosp. A ven. p. 25. Com. Al. com. 5. p. 31. Rota prosp. piant. Pav. p. 271. Hausm. fl. Tir. p. 659. Pir.! fl. for. syll. p. 10\%. De Vis. Sacc. cat. piant. Ven.p. 156. Zersi prosp. piant. Bresc. p. 160. Ing. cat. sp. Mond. p. 55. Ces. Pass. Gib. comp. fl. ital. p. 359. Anzi auct. fl. nov.-com. p. 196. Arc. comp. fl. ital. p. 522.

Pedicularis insubrica Rota prosp. fl. Berg. p. 68.

Figura. - Fl. dan. $t .2055$.

Stazione, Abitazione e Fioritura. - Nei luoghi paludosi, principalmente montuosi, dell'Alta Italia : a Mondovi lungo l'Ellero (Ing.), al lago d'Avigliana (Re), al S. Bernardo (erb. Webb!), a Campolato nella valle del Ticino nel luogo detto Porta Lua, rara (Bir.), in Lomellina alla Menocca (Bert.), nel Pavese (Picaroli!) a Carbonara e Caselli Badia (Rota), nel Comasco a Brivio (Rota!), presso i la. ghetti d'Alserio, Pusiano, Sagrino, Ganna e sotto l'Aqua negra nelle vicinanze di Como, ad Agno nel Cantone Ticino, in val Tellina nel piano di Sondrio (Com.), nell'alpe di Entoa, nel maggese di Franscia (Mass.), a Colico, in val Malenco, in val Furva, a Livigno (Anzi), e sopra Montagna (Bert.), nel Bergamasco a Fopenico, Villasola (Rota), e Branzi (Bergamaschi!), in Tirolo al Brennero (erb. Cientrale!), a Welsberg, a Bolzano, sul Ritten comune intorno a Klobenstein, in Fiemme a Pinè, in Fassa a Mazzino (Hausm.), in val Sugana a Palù (Ambrosi) e sopra Telve (Hausm.), al sud del Benaco, frequente (Zersi), nel Mantovano (Barbieri!) a Padrella (Bert.), a Peschiera, Bovolon, Villimpenta, il Palù, Valeggio ecc., abbondante (Poll.), negli Eugarei (Trev.), a Venezia (Zan.), a Treviso (Kellner!), nel Friuli presso Amaro, e Fagagna alla Maranzona (Pir.), S. Daniele, Monfalcone ecc. (Poll.). Fiorisce da maggio e giugno ad ago. sto e settembre.

Distribuzione geografica. - Nell'Europa settentrionale e media, nell'Asia settentrionale, fino alla Dauria, nell'America più nordica. 


\section{Pedieularis silvatiea.}

P. foliis pinnatisectis lobis ovalibus inciso-dentatis, caulinis plurimis sparsis, calycis tubulosi $m \circ x$ inflati dentibus foliaceis dentatis, corollæ purpureæ galea erostri obtusa 2-dentata, capsula 2-valvi.

Pedicularis silvatica Linn. sp. pl. ed. 1. p. 60\%. Poll. fl. ver. 2. p. 339. Mass. prodr. fl. valt. p. 127. Bert. fl. ital.6.p.316. Com. fl. com. 5. p. 32. De Vis. Sacc. cat. piant. Ven. p. 156. Ing. cat. sp. Mond. p. 55. Ces. Pass. Gib. comp. R. ital. p. 359. Sacc. Bizz. agg. fl. trev. p. 29. Arc. comp. fl. ital. p. 522.

Figure. - Fl. dan. t. 225. Reich. ic. fl. germ. 20. t. 128. f. 1.

Stazione, Abitazione e Fioritura. - Nei luoghi umidi, sia pra. tivi sia hoschivi, delle Alpi, assai rara. $\grave{E}$ stata trovata in Piemonte presso Torre-Mondovi lungo il torrente Corsaglia (Ing.), nel Cenisio (Thomas! ecc.) e presso Susa (Ces. Pass. Gib.), in val Tellina nel piano di Sondrio, a Cavaia sopra Poschiavo (Mass.) e nell'alpe di Entoa, sul lago di Como nell'alpe di Darengo presso quel laghetto (Com.), nel Veronese presso Palù secondo Seguier, in Vallacia ${ }^{1}$ e all'Aun nelle Vette di Feltre secondo Tita e Zannichelli (Poll.), nel monte Grappa sopra Bassano (Sacc. Bizz.). Fiorisce in maggio, giugno, luglio.

Distribuzione geografica. - In Europa, dalla Svezia media al Portogallo, all'Italia e alla Volinia.

\section{Pediculneris commosa.}

P. foliis pinnatisectis lobis lanceolatis pinnatipartitis dentatis, caulinis plurimis sparsis, calycis tubulosi dentibus brevissimis integris, corollæ ochroleucae galea suberostri 2-dentata, capsula 2 -ralvi.

Pedicularis comosa Linn. sp. pl. ed 1. p. 609. Mass. prodr. fl. valt. p. 127. De Not. rep. /l. lig. p. 310. Com. 月. com. 5.p. 48. Rola prosp. piant. Pav. p. 271. Hausm. 凡. Tir. p. 659. Rota prosp. ^. Berg. p. 68. Pir.l 1. for. syll. p. 107. Ard /1. Alpes-mar. p. 2S5. Terr.! 凡. Vull. syn. p. 132. Ces. Pass. Gib. comp. 月. ilal. p. 359. Terr. quart. rel. Terra di Lav. p. 111. Sacc. Bizz. agg. 月. Irev. p. 29. Arc. comp. fl. ital. p. 522.

- Non so che cusa si debba intendere con questo nome, che non trovo in alcun dizionario geogralico. 
Figure. - All. fl. ped. t. 4. f. 1. Reich. ic. fl. germ. 20. t. 130.

Stazione, Abitazione e Fioritura. - Nei prati e nei pascoli delle Alpi e degli Appennini. Trovasi sul monte Mataiura del Friuli (Pir.!), sul monte Croce presso Vittorio (Sacc. Bizz.), nel Vicentino (Poll.), sul Baldo (Balbis! ecc.), principalmente verso la Corona e la Ferrara, al Prabazar, Basiana, Novesa in abbondanza (Poll.), e in val Fredda, nel Trentino (Hausm. ecc.), in val Sugana sul Montalone (Bert.), in val di Scalve del Bergamasco (Rota), nel Comasco nelle valli Cavargna e Sassina, di Dongo, Livo, sul Camogheo nel Cantone Ticino (Com.), in val Tellina nelle alpi di Mara, Zana, Arcoglio e monte Canale (Mass.), in val Vegezzo, frequente (Bir.), e in generale per il Piemonte (All.), cosi nel Cenisio al lago (Bucci! ecc.) e a Ronche (Huguenin!), presso Susa a Mompantero (Re), al collo della Croce (Rostan!); quindi per i colli di Tenda (Bourgeau! ecc.), di Raus, di Fenestre e S. Anna di Vinai (Ard.), si prolunga, comunissima, per gli appennini di Savona, di Genova e di Bobbio (De Not.), cosi alla Scaggia dietro Pegli, presso Campofreddo, al monte de'Buoi (Bert.), fino ai monti Pavesi detti Penice, Lesime, Boglelio e Ghiarolo (Nocca Balb.). Iri s' interrompe, difettando per tutto il tratto dell' Appennino toscano, per ricomparire nel monte Nerone dell'Urbinate (Bert.) e nel Catria a 900-1000 metri (Parlatore! ecc.), e proseguire per i monti di Fiastra nel Camerinese, di Norcia in valle Canetra (Bert.), della Sibilla alla Corona (Orsini!) e in altri siti (Bert.), e terminare da quel lato nell'Abruzzo al monte de’Fiori (Orsini!), al Corno (Bert.), alla Maiella, al Morrone e altrove (Ten.). Nell'altro lato occidentale della Penisola si presenta nell'appennino di Subiaco presso la Cammerata (Rolli!), quindi piủ giù nel Matese (Ten.), e presso Frasso al monte S. Michele e alla costa di Cepino (Ten., Terr.), nel monte S. Angelo di Castellammare (Bert.), nel Vulture al Pizzuto di S. Michele (Terr.! ecc.), nel Pollino (Ten.), per terminare finalmente alla Sila in Calabria (Ten.!). Fiorisce in giugno e luglio.

Distribuzione geografica. - Nell'Europa meridionale, dalla Spagna al Caucaso, nell'Asia Minore e fino alla Persia, e nella Siberia meridionale e media.

Osservazione. - Le radici sono ingrossate fusiformi. 


\section{Pedieularis petiolaris.}

P. foliis pinnatisectis lobis ovatis lanceolatisve semel vel bis pinnatipartitis, caulinis pluribus sparsis, calycis tubulosi dentibus lanceolatis integris, corollæ e flavo purpurascentis galea suberostri obtusa 2-dentata, capsula....

Pedicularis rosea Ten. fl. nap. 4. syll. p. 87 (non Wulf.).

Pedicularis petiolaris Ten. o. c. 5. syll. app. 4. p. 11. ^..p. 35 (1835).

Pedicularis Friderici-Augusti Tomm.! in linn. 13. p. 74 (1839). Bert. f. ital. 6. p. 326. Cies. Pass. Gib. comp. /. ital. p. 359. Arc. comp. fl. ilal. p. 522.

Figure. - Tomm. l. c. t. 2. Reich. ic. fl. germ. 20. t. 130.

Stazione, Abitazione e Fioritura. - In luoghi erbosi di monti, assai distanti gli uni dagli altri. Nasce in Istria sui monti Slavnik a 2400-3240', abbastanza comune (Tomm., ecc. ecc.), e Maggiore (Ces. Pass. Gib.); poi nell'Abruzzo-Piceno sui montí Corona (Ces. Pass. Gib.), Corno, Velino, Maiella (Ten.); poi sul monte S. Angelo di Castellammare all'acqua dei porci, rara (Ten.); poi in Basilicata sul Pollino (Ten. ecc.), al monte Dolcidormie a 2100 metri, e ab. bondantemente nei Piani di Pollino a 2000 metri (Huter Porta Rigo!). Fiorisce in maggio e giugno.

Distribuzione geografica. - Si stende inoltre nella penisola Balcanica.

Osservazione. - Veramente il Tenore descrisse come Pedicula. ris petiolaris ció che per l'innanzi egli aveva ritenuto essere una varietà della $P$. roseu, seguitando a tenere separata questa e dandola del Pollino; ma ivi non esiste che la $P$. petiolaris, mentrechè la $P$. rosea si ferma molto più a nord; e cosi per ragione di località è a ritenersi che tutta la $P$. rosea dalla Flora napolitana sia $P$. petiolaris.

\section{Pedienlaris atrorubens.}

P. foliis pinnatipartitis lobis lanceolatis grosse serratis imo pinnatifidis, caulinis pluribus sparsis, calycis campanulati dentibus lanceolatis integris, corollie purpurea galea brevissime rostrata rostro y-dentato, capsula demum 己-valvi.

Pedicularis atrorubens Schleich. exs. Cand. syn. pl. in R. gall. 
p. 212 (1806). Bert. fl. ital. 6. p. 326. Ces. Pass. Gib. comp. fl. ital. p. 358. Arc. comp. fl. ital. p. 521.

Pedicularis pennina Gaud.! in Mur. guid. bot. Val. p.83 (1810).

Figura. - Reich. ic. fl. germ. 20. t. 127.

Abitazione e Fioritura. - Nel Gran S. Bernardo al luogo detto la Baux (Tissières! ecc. ecc.); anche nel Cenisio secondo Bentham (in Cand. prodr. 10. p. 576). Fiorisce in luglio e agosto.

Distribuzione geografica. - Nasce inoltre in qualche luogo di Svizzera e del Tirolo.

Osservazioni. - La statura, il fogliame, l'infiorescenza, l'infruttescenza, insomma tutto l' aspetto è della Pedicularis recutita; ma i fiori sono differenti, e si accostano alquanto a quelli della $P$. incarnata: per cui è stato sospettato da taluni essere questa pianta un ibrido fra le due specie.

\section{Pedicularis gyroflexa.}

P. foliis pinnatisectis lobis lanceolatis pinnatipartitis lobulis dentatis, omnibus fere basilaribus, calycis campanulati dentibus foliaceis ovatis partitis, corollæ roseæ galea brevissime rostrata rostro denticulato, capsula 2-valvi.

Pedicularis gyroflexa Vill.hist.pl. Dauph.2. p. 426 (excl. var.). Gaud. fl. helv. 4. p. 131. Bert. fl. ital. 6. p. 327. Com.! fl. com. 5. p. 39.

Pedicularis fasciculata Bell.? in Willd. sp. plant. 3. p. 218. Poll. fl. ver. 2. p. 341. Mass. prodr. fl. valt. p. 127. De Not. rep. fl. lig. p. 309. Gren. Godr. fl. Fr. 2. p. 618. Hausm. fl. Tir. p. 657. Rota! prosp. fl. Berg. p. 68. Ard. fl. Alpes-mar. p. 285. De Vis. Sacc.? cat. piant. Ven. p. 156. Zersi prosp. piant. Bresc. p. 160. Ces. Pass. Gib. comp. fl. ital. p. 358 (excl. var.). Anzi auct. fl. nov.com. p. 196. Arc. comp. fl. ital. p. 521 (excl. var.).

Figure. - Hall. hist. stirp. ind. t. 11. Vill. o. c. t. 9. Reich. ic. fl. germ. 20.t. 123.

Stazione, Abitazione e Fioritura. - Nei pascoli umidi delle Alpi. Secondo De Visiani e Saccardo trovasi nel Friuli, Bellunese e Trivigiano, ma l'unico luogo di colà da dove io abbia veduto un saggio è il monte Serva di Belluno (Huter!). Fa nel Tirolo piủ meridionale, ma rara (Ambrosi!), cioè in Primiero sul Fedai, sopra il Broccon in Tesino, e in Fiemme presso Predazzo (Bert., Hausm.), 
nel monte Baldo (Barbicri!) in Malcesine, a piè dell'Artillon e in Costa bella (Poll.), nel Bresciano, sempre rara, cioè nei monti sopra Irma e presso Bovegno (Zersi), nel Bergamasco al Tonale a 2000 metri (Parl.!), in val di Scalve, in Selvino (Bergamaschi!), ne' monti Ocone, Resegone, Aralalta, Presolana (Rota), in Carenno (Rota!), sopra Selcico, e in val Taeggio (Bert.), nel Comasco, frequente (Com.), sul monte Barro a 800 metri (Parl.!), sul Resegone (Ainti!), in val Sassina sul monte Codeno (Daenen!), nei Corni di Canzo a $5000^{\prime}$ (Bracht! ecc.), nell'alpe Callivazzo sopra Mandello (Anzi), in val Cavargna (Com.) e nel monte Generoso (Com.!), quindi in val Tellina nelle alpi di Togno, Mara, Campiazzo e Venina (Mass.), nel Cantone Ticino sul Camogheo, nel Bolia (Com.!), sul Tamor e sul Calbege (Koch), al Sempione (Neneghini!), nel Novarese, non comune (Bir.), in val d'Aosta a Gressoney la Trinité nel luogo detto Sazic (Carestia!), e nei monti di Pianes (Manuel!), nel Cenisio (Bonjean! ecc.), nelle alpi di Foresto e di Giaveno (Re), nelle Valdensi, assai frequente, per esempio al col Lacroix (Rostan!) e nell'alpi di Fenestrelle (Colla), nel monte Genevre (Vill.), nel Monviso (Gren. Godr.), al col di Elva a 5-7000' (Ball!), nell'alpi di Vinadio presso S. Anna (Colla), c di Valdieri (Bert.), infine nelle Alpi Marittime al col di Tenda a Pierriaude (Bourgeau!), a Tanarello presso Briga (Ungern-Sternberg!), a Colmiane presso S. Martino Lantosca (Thuret!), a S. Anna di Vinai, nel vallone di Fenestre, e al lago di Rabuons (Ard.). Fiorisce da giugno ad agosto.

Distribuzione geografica. - Inoltre nelle contermini Alpi francesi.

Desorizione. - Pianta di un verde piutlosto scuro, pelosa, alta 5 e piủ centimetri. Rizoma obliquo, grossetto, con grosse fibre ra. dicali, le quali sono giallicce e quasi in fascio. Fusti ascendenti o quasi eretti, cilindrici, nudi in gran parte, verde-chiari, pelosi per peli piuttosto corti, bianchi, curvati in giu, più pelosi in alto per peli più lunghi. Foglie radicali molte, eretto-patenti, bipennati-divise, con le lacinie bislungo-lanceolate e le laciniette corte, ottuse, intere o più spesso con piccoli denti ottusi, ora superliciali ora pro. fondi, con i margini rovesciati e forniti di rade ciglia lunghette, di un verde pinttosto scuro di sopra ed ivi con un leggiero solco longitudinale in ciascuna lacinı o lacinietta, di un verde chiaro di sotto ed ivi con un nervo longitulinale grossetto e bianchiccio da cui parte un nervetto per ciascuna lacinietta: tutti i nervi sono pelosi per lunghi e radi peli bianchi. La rachide è stretta, con un solco profon- 
damente longitudinale di sopra, convesso di sotto si continua nel picciỏlo e come questo è peloso, spesso violetto-rossiccio in basso; vi è per lo più una piccola foglia in alto del fusto, di raro due, simili alle radicali ma meno divise e con il picciòlo corto, slargato a guisa di una guaina. I fiori sono molti in una spiga corta, non fitta, e ottusa. Le brattee sono simili alle foglie della parte superiore del fusto, quasi ugnali e le inferiori poco più lunghe del calice, di un verde scuro assai e quasi violetto all'apice delle lacinie. I pedicelli sono cortissimi. Il calice è tubuloso-campanulato, leggermente angolato, verdognolo e in parte con larghe righe violetto-scure, peloso-irsuto per peli lunghetti, curvati in giù e bianchi, ha cinque lacinie quasi uguali al tubo di esso, delle quali quattro sono simili alle laciniette delle brattee ma più piccole e la quinta è più piccola, è lanceolata, quasi intera o con qualche piccolo dente ottuso. La corolla è da due a tre volte più grande del calice, di color violetto-roseo, con il labbro superiore un po' piủ carico. Il tubo è stretto, un po' curvo, lungo quanto il calice, con due linee pelose longitudinali di sotto, peloso di dentro, si slarga in alto. Il labbro superiore è a guisa di elmo, curvato in giủ, ed in avanti assottigliato all'apice in un rostro corto, quasi troncato, smarginato e con denti disuguali. Il labbro inferiore è quasi uguale al superiore ma più largo e diretto in avanti e trilobo, con i lobi laterali molto piủ grandi dell' intermedio, e diretti lateralmente un po' in giù, tutti tondeggianti e con corti peli presso il margine a guisa di ciglia. Gli stami sono 4, due poco più corti degli altri due, nascosti nell'elmo, inserito nella parte inferiore del tubo della corolla. Filamenti delicati, filiformi, bianchicci, pelosi alla loro base e sotto delle antere. Antere ovate, bifide, scurette, glabre. Polline gialliccio. Pistillo lungo quasi quanto il labbro superiore. Ovario ovato-acuminato, un po'schiacciato, verdognolo, glabro, circondato alla base da un disco annulare, grossetto, verde. Stilo molto più lungo dell' ovario, filiforme, curvo, di color violetto, glabro. Stimma in capolino oscuramente bilobo, papilloso, gialliccio. (Parl. ms., descr. della pianta dei Corni di Canzo).

Osservazioni. - La figura di Villars, per quanto rozza, e quella da lui citata di Haller, chiariscono abbastanza essere la sua Pedicula. ris gyroflexa questa nostra pianta dal becco corollino corto; nè havvi ragione alcuna per credere ch'egli intendesse invece con quel nome la $\boldsymbol{P}$. cenisia dal becco lungo.

La $P$. fasciculata Bell. pare che si possa riferire a questa specie: è l'opinione anche di Bertoloni, che vide l'erbario di Bellardi. 


\section{Pedieularis elegans.}

P. foliis pinnatisectis lobis ovalibus pinnatipartitis lobulis incisodentatis, omnibus fere basilaribus, calycis tubulosi dentibus foliaceis oblongis partitis, corollæ purpureæ galea brevissime rostrata rostro denticulato, capsula 1-valvi.

Pedicularis rosea Ten. fl. nap. 4. syll. p. 87 (excl. var., non Wulf.).

Pedicularis fasciculata Ten. o. c. 4. syll. app. 3. p. 10 (non Bell.). Bert.l fl. ital. 6. p. 327. Ces. Pass. Gib. comp. fl ital. p. 358 (var.). Terr. quart. rel. Terr. di Lav. p. 111. Arc. comp. fl. ital. p. 521 (var.).

Pedicularis elegans Ten.! o. c. 5. p. 37.

Stazione, Abitazione e Fioritura. - Nei luoghii erbosi delle parti più alte dell'Appennino, dall' Umbria in giù: al Sasso Borghese (Bert.!), alla Sibilla sopra la Corona (Marzialetti!), al Vettore!, al monte de'Fiori (Parl. !), al monte Pelone, al Pizzo di Sivo, al Corno, al Velino (Orsini!), al Costone (Bert.), al Sirente a 6000' (Groves!), alla Maiella tra 2400 e 2600 metri in valle Canella, nel monte Amaro e altrove (Levier!, Grovès!), al monte Meta (Ten.), al monte Muria (Terr.), al monte S. Angelo di Castellammare dove però è rarissima, infine al Pollino (Ten.) Fiorisce in luglio e agosto.

Distribuzione geografioa. - Pianta propria di quei monti.

Osservazioni. - Specie distintissima dalla precedente.

E) difficile capire perchè Bertoloni abbia creduto che Willdenow descrivendo nelle Sp. plant. 3. p. 218 la Pedicularis fasciculata Bell., avesse in mira questa pianta e non quella delle Alpi piemontesi.

" Corollæ galea longe rostrata.

\section{1Pedicularis rostratso.}

P. foliis pinnatisectis Iobis ovalibus lancenlatisve pinnatipartitis aut inciso-dentatis, omnibus fere basilaribus, calycis tubuloso-campanulati dentibus foliaceis oblongis dentatis, corolla rosen-purpurascentis galea longe rostrata, capsula 1-valvi.

Pedicularis rostrata Linn. 8p. pl. ed. 1. p. G07. Vitm? ist. erb. p. 17, 33, 43. Savi? bot. etr. 2. p. I65 Mass. prodr. M. valt. p. 
127. Bert. fl. ital. 6. p. 330. Com. fl. com. 5. p. 37. Genn. cent. pl. rep. f. lig. add. p. 266. Hausm. fl. Tir. p. 656. Rota prosp. fl. Berg. p. 68. Pir.! fl. for. syll. p. 107. Car.? prodr. fl tosc p. 492. Ard. fl. Alpes-mar. p. 286. Zersi prosp. piant. Bresc. p. 160. Ces. Pass. Gib. comp. fl. ital. p. 358. Sacc. Bizz. agg. fl. trev. p. 28. Arc. comp. fl. ital. p. 521.

?Pedicularis cenisia Gaud. fl. helv. 4. p. 133

Pedicularis Jacquini Koch in Röhl. deutschl. fl. 4. p. 363. Hausm. o. c. p. 655. Rota 0. c. p. 67. Goir. pl. vasc. veron. p. 32. Anzi auct. fl. nov.-com. p. 195.

Pedicularis gyroflexa Ten.! fl. nap. 4. p. 37 (non Vill.). Ard. l. c. Terr.! sec. rel. Terra Lav. p. 91. Ces. Pass. Gib. l. c. Arc.! l. c.

? Pedicularis Bonjeani Colla! herb. ped. 4. p. 363. t. 83 (mala). Bert. o. c. p. 332.

Figure. - Reich. ic. fl. germ. 20. t. 121.f. 1, 2. t. 122.f. 3.

$\beta$ asplenifolia, humilis, foliorum lobis minus dentatis.

Pedicularis asplenifolia Floerke in Willd. sp. plant. 3. p. 208. Bert. o. c. p. 329. Hausm. l. c. Rota l. c. Zersi l. c.

Pedicularis Portenschlagii Saut.! in Reich. ic. bot. 5. p. 1. Hausm. o. c. p. 657. Ces. Pass. Gib. l. c. Arc. l. c.

Stazione, Abitazione e Fioritura. - Nei pascoli umidi di tutte le Alpi; la varietà nelle alte cime. Nasce in Friuli nei monti Amariana, Cren (Pir.!), Cuch, Plauris (Pir.), Cavallo (Kellner!), quindi nell'alpi d'Agordo (Ces. Pass. Gib.), nelle Vette di Feltre (Bert.), nel monte Grappa (Sacc. Bizz.), presso Recoaro al passo della Lora a 5-6000' (Smith!), nel Baldo, fra 5800' e 6200' (Bracht!) e nelle cime al Telegrafo (Manganotti!) e dell'Altissimo di Nago (Hausm.), vi è comune, nonchè nei monti Bondone, Scanuccia, Alba e Zevola (Poll.), in Tirolo sul Montalon (Kellner!), in Tesino da Tolva contro la Cima d'Asta sul granito (Hausm.), nei inonti di Papiria e di Paneveggio anche al di sopra dei mughi (Perini!), nella montagna di Denno (Hausm.), sul calcare e sul granito in Fiemme, e in Fassa ai Monzoni (Bert., Hausm.), comune sullo Schlern e sull'alpe di Seiss e di'Villanders, sul Peitler presso Bressanone, sul Brennero, sull'alpe Zil presso Merano, intorno a Welsberg (Hausm.), presso Laas (Tappeiner! ecc.), sull'Ortler, nel Falgamaierioch in valle d’ Olten, nell'alpe Colem tra Olten e val di Sol, nelle Giudicarie sul Frate in Breguzzo (Hausm.) e sullo Spinale (Sardagna!), nel Bergamasco e Bresciano sul Tonale (Rota), sulla Pisgana a 2600 metri, sul Venerocolo a $7000-7500^{\prime}$ (Parl.!), in val Brembana a Branzi (Rampoldi!), 
sulle Colombine tra 2000 e 2200 metri (Parl.!), sul monte Cigoletto, frequente, sul monte Gazza, rarissima (Zersi), in val Tellina in quasi tutte le alpi (Mass.), cosi sullo Stelvio tra 1700 e 2400 metri (Parlatore!), sul Braulio (Com.!), sul Dosso Reil (Levier!), sul Fraele, sul Gavia (Ball!), nel Comasco sul Biondino in val Sassina (Daenen!), e in generale abbondante nei subalpini delle valli Cavargna e Sassina, sul Legnore, sull'alpe di Darengo, nonchè nel Cantone Ticino (Com.) al S. Bernardino (De Notaris!) e al Gottardo (Siegfried!), nell'alpe Tolud (Koch), e in val Maggia sopra Campo a 2000 metri (Franzoni!), quindi al Sempione (Parl.!), al monte Rosa (Rosellini!), in val Formazza (Rampoldi!), in val Sesia al Corno bianco, a Valdobbia, e alle Selle della Montata (Carestia!), nei monti d'Allagna, e a Marguzzolo, rara (Bir.), al S. Bernardo (Heldreich !), all'Allée blanche (Webb!), in val S. Marcello presso Aosta (Müller!), e a Gressoney Saint Jean (Carestia!), al Cenisio in cima (Bonjean! ecc.), al piccolo monte Cenisio (Rostan!), a Rocca Melone presso Susa (Beccari!), nei monti di Giaveno, e di Lanzo (Colla), nel monte Genevre e nel monte Viso (Gren. Godr.), nelle alpi di Viù a Roccamorosa (Parl.!), Valdensi (Rostan!), di Valdieri (Bert.), nelle Marittime ai colli di Fenestre (Bourgeau!) e di Tenda (Reuter!), al monte Bissa (Parlatore!) ecc., e fino ai monti di Frontiero (Genn.), d'Ormea (Gentili!) e di Ceriale (Bert.), dove termina. Abbenchè indicata altra volta nell'Appennino toscano, io ho già chiarito nel mio Prodr. fl. tosc. l. $c$. e nel Sec. suppl. p. 22 come ciò sia avvenuto probabilmente per errore, e ad ogni modo la pianta non vi si trova più. Si riaffaccia nel. l'Appennino Piceno, dov'è stata scoperta di recente dal dottor Levier sulla cima del Vettore; era già indicata in Abruzzo al Velino, e nella Maiella alla cima della valle di Fara (Ten.), e nasce pure nel monte Meta (Terr.), e a Picinisco allo Zaffineto (Terr.!). Fiorisce in luglio e agosto.

Distribuzione geografica. - Nei Pirenei, nelle Alpi, nei Carpazi, nell' Imalaia.

Descrizione. - Pianta perenne, umile, alta da 6 a 12 centimetri, fornita di un rizoma corto, nericcio, obliquo da cui partono grosse fibre radicali, carnosette, semplici o fibrillose, quasi in fascetti $e$ in alto dei fusti o rami e le foglie. I fusti sono ascendenti, delicati, quasi uguali o poco piu corti delle foglie, quasi cilindrici, rossicci, glabri ma con due linee longitudinali di peli un po'rari e lunghetti, semplici, mudi o con una foglia verso la base. Le foglie radicali sono molte, patenti e quasi in rosetta, profondamente pinnatipartite, 
con le lacinie ovali-lanceolate, inciso-dentate, e le incisioni o denti aventi altri piccoli denti: tanto le lacinie quanto le incisioni sono acute e quasi mucronulate all' apice, le lacinie sono verdi, glabre e con un solco longitudinale di sopra, di un verde un po' più pallido di sotto ed ivi con un nervo longitudinale poco rilevato, nel quale e vicino al quale hanno dei peli diritti e orizzontali, nel resto della pagina inferiore sono glabre e un po'concave per essere le incisioni ed i denti rivolti un poco verso giù. Il picciòlo comune delle lacinie $\dot{e}$ stretto, profondamente scanalato di sopra, convesso di sotto, verdognolo 0 in parte sfumato di rossiccio, con qualche raro pelo. La foglia caulina quando esiste è uguale alle radicali ma un po' più piccola. Le brattee sono quasi opposte, patenti, corte, però lunghe sino ad uguagliare il calice, simili alle foglie ma più corte e quasi trian. golari con le lacinie inferiori più lunghe e più distanti dalle altre. I fiori sono per lo più due all'apice dei fusti, ciascuno ha un peduncolo che nasce dall'ascella della brattea: tali peduncoli sono diritti, piủ corti del calice, un po'angolati, rossicci, glabri, ma con una linea di peli quasi come nel fusto. Il calice è tubuloso, un po' gonfio, con cinque angoli a guisa di costole, rilevati, è rossiccio, glabro o quasi glabro nelle facce, peloso negli angoli; il suo labbro superiore è bifido con le lacinie pinnatifide, e l'inferiore è trifido con le lacinie laterali pinnatifide e l'intermedia intera un po'piủ corta e più piccola: le lacinie sono quasi tutte meno della metà più corte del tubo del calice, verdi e in parte rossicce; quelle pennatifide hanno le laciniette dentate, acute e rivolte in giù quasi ad uncino: tutte le lacinie sono fornite di ciglia lunghette. La corolla è più del doppio più lunga del calice. Il labbro superiore è di colore roseo-porporino, schiacciato lateralmente, prolungato all' apice in un rostro assottigliato in alto, troncato e smarginato all'apice; l'inferiore è patente, rivolto in giù e con i margini alquanto diretti in su, poco più lungo del labbro superiore, di color più chiaro, ovato, ottuso, trilobo, con i lobi laterali più grandi, ha due pieghe longitudinali rilevate alla base verso la gola: questa e la parte interna del tubo è pelosa. Gli stami sono quattro, più corti del petalo superiore dentro il quale sono racchiusi, didinami. I filamenti sono filiformi, i più corti son glabri eccetto verso la base nel punto dove sono saldati con il tubo della corolla! i piủ lunghi sono barbati dalla metà all'apice! Le antere sono grandi, ovali, bifide all'apice con le divisioni acute, ottuse e appena smarginate alla base (sono forse rovesciate?), inserite nel mezzo del dorso, biloculari, aprentisi longitudinalmente dalla parte interna o 
anteriore, gialle, glabre, con qualche pelo nel connettivo nel dorso. Il polline è giallo. Il pistillo è piủ lungo degli stami, uguale o appena più lungo del rostro. L'ovario è allungato, assottigliato un poco all'apice, un po'schiacciato lateralmente, verdognolo, glabro, biloculare, con molti ovoli inseriti nella placenta centrale. Lo stilo è molto lungo, filiforme, curvato in alto ed ivi roseo-carico, bianchiccio inferiormente. Lo stimma è quasi in capolino, gialliccio, papilloso. (Parl.ms., descr. della pianta delle Colombine).

Osservazioni. - I botanici sono oggigiorno generalmente d'accordo per considerare la Pedicularis Jacquini di Koch una semplice forma della $P$. rostrata, pressochè glabra nel calice.

La $P$. asplenifolia Floerke è certamente altra forma, stentata, coi lobi fogliari meno profondamente dentati: ma sono tante le gradazioni per rispetto alla divisione di essi lobi, da riscontrarsi perfino in una stessa pianta, che appena io so distaccare come varietà questa pretesa specie. In quanto alla $P$. Portenschlagii Saut., descritta e figurata da Reichenbach, è la stessissima $P$. asplenifolia, stando a tutti i saggi e d'Italia e di fuori che ne ho visti, compresi quelli distribuiti dal Reichenbach sotto il nnm. 1687, e che già Bunge (in Bot. zeit. 187\%.col. 89) e Hausmann (o. c.) avevano riconosciuto doversi riferire alla $P$. rostrata; la figura poi nella Icon. bot. ( $t$. 40l), riprodotta nelle $I c . f$. germ. (20. $t$. 122. f. J), è fantastica riguardo alla forma del labbro superiore della corolla (principale carattere distintivo della supposta specie), la quale non è di alcuna Pedicularis.

In quanto alla $P$. cenisia Gaud., o P. Bonjeani Colla, stata trovata nel Cenisio e in diversi altri siti delle Alpi occidentali, e ricondotta da Bentham (in Cand. prodr. 10. p. 578) alla P. rostrala, essa differisce dalla $P$. rostrata ordinaria per la statura piủ alta, i fusti piủ robusti, più eretti, i fiori piủ numerosi: tutto ciò le dả l'aspetto della $P$. luberosa. Osservo in una vistosa serie d'individui di questa forma che nessuna è fruttifera, e questa circostanza mi fa venire il sospetto che potrebbe trattarsi di un ibrido fra la $P$. Luberosa e la I'. rostrata pelosa, cume la $P$. erubescens lierner ! i ammessa come un ibrido tra la $P$. tuberosa e la $P$. rostrala glabra o $P$. Jacquini.

\subsection{Pealicularia tuberoma.}

P. foliis pinnatisectis lobis lanceolatis pinnatipartitis lobulis dentatis, caulinis plurilus sparsis parvis, calycis tubuloso-campanulati 
dentibus foliaceis oblongis dentatis, corollæ ochroleucæ galea longa rostrata, capsula 2-valvi.

Pedicularis tuberosa Linn. sp. pl. ed. 1. p. 610. Bir. fl. acon. p. 212. Mass. prodr. ft. valt. p. 128. Colla herb: ped. 4. p. 364. De Not. rep. fl. lig. p. 310. Bert.! fl. ital. 6. p. 333 (excl. $\beta$ ). Com. fl. com. 5. p. 44. Pucc. syn. pl. luc. p. 319. Sim.! fl.alp. vers. p. 134. Rota prosp. piant. Pav. p. 271. Hausm. Al. Tir. p. 658. Rota prosp. fl. Berg. p. 68. Pir.! fl. for. syll. p. 107. Bicch.! agg. fl. lucch. p. 15. Car. prodr. fl. tosc. p. 493. Ard. fl. Alpes-mar. p. 286. Zersi prosp. piant. Bresc. p. 160. Ces. Pass. Gih. comp. fl. ital. p. 358. Anzi auct. fl. nov.-com. p. 196. Paol. el. piant. sib. p. 16. Sacc. Bizz. agg. fl. trev. p. 28. Gib. Pir. fl. Mod. p. 125. Arc. comp. fl. ital. p. 521. Cocc. A. Bol. p. 365.

Figura. - Reich. ic. fl. germ. 20. t. 125.

Stazione, Abitazione e Fioritura. - Nei prati e nei pascoli delle Alpi e degli Appennini. Nasce nel Friuli presso Venzone (Pir.!), e nei monti Mediana, Tragonia, Crostis, Cavallo (Pir.), nel monte Cerva presso Belluno (Bert.), a Follina, presso Valdobbiadene, nei monti Grappa (Sacc. Bizz.), Summano (Poll.) e Portole (Bert.), nel Baldo (Manganotti!) all'Altissimo (Hausm.); è frequente per tutto il Tirolo, cosi intorno a Roveredo (Hausm.), e Trento (Perini! ecc.), in val Sugana (Ambrosi! ecc.) in Fiemme e Fassa (Bert. ecc.), intorno a Bolzano, per esempio sul Ritten dove comincia a $4600^{\prime}$ e va fino in cima, intorno a Bressanone, in Pusteria, presso Merano, in val Venosta, in val di Non, nelle Giudicarie (Hausm. ecc.); non è rara neppure in Lombardia, cosi nelle alpi di val Trompia, da 1300 a 2000 metri (Parl.!), in val Camonica al Tonale (Ricca!), e in generale nel Bergamasco (Rota), come pure tutt' attorno al lago di Como (Rampoldi!, Com.), e in val Tellina, fino al Braulio, e sullo Spluga (Mass., Com., Anzi); nel Cantone Ticino nasce sui monti Camogheo, Bolia e Cadro (Com.); in Piemonte fa nei monti sopra Canobbio (Ball!), in val Formazza (Gibelli!), al Sempione (Parl.!), al monte Rosa (Bert.), in val Sesia sopra Alagna (Piccone!) e in Valdobbia (Carestia!), in val Tournanche sopra Chatillon (Müller!), nel Cenisio (Colla); infine nelle Alpi Marittime a S. Anna di Vinai e al Garret sopra Entraunes (Ard.). Di li proseguendo per l'Appennino ligure orientale trovasi nel monte Antola sopra Torriglia (Ardissone!), nei monti di Bobbio (De Not.), nel monte Porcile, presso S. Stefano d'Aveto (Bert.), nell'alpe di Rigosa nel Parmense (Passerini!); indi occupa in buona quantità la regione alpestre e la montana per tutto 
il tratto dell' Appennino tra il monte Orsaio e il monte dell' Uccelliera in Toscana!, nonchè tutte le Alpi apuane (Bert.!, Simil ecc.); fa pure nel Mugello secondo Savi; quindi nelle Marche al monte Catria (Piccinini!), e piủ giủ nel monte Birro (Bert.), nel monte Regnolo (Marzialetti!), nel gruppo dei monti Sibillini, cosi al Vettore nella regione alpestre! ecc., e nell'Abruzzo contermine al monte de'Fiori (Orsini!), al Pizzo di Sivo!, al Corno a 2400-2500 metri (Levier!), al Velino (Bert. ecc.) ecc. Fiorisce in giugno, luglio e agosto.

Distribuzione geografica. - Nelle Alpi, nell'Appennino, nei Pirenei.

Descrizione. - Pianta perenne, alta da 2 a 3 decimetri, quasi glabra, verde, fornita di un rizoma ramoso, scuro, che manda fibre radicali e all'apice dei rami dei fusti e delle foglie. I fusti sono ascendenti, diritti, quasi quadrangolari, glabri nelle facce, con quattro fila di peli lunghetti e diretti un po' in giù negli angoli, semplici, verdognoli giallicci, nitidi. Le foglie sono delicate, piccole, patenti, le inferiori bipinnate-partite, con le lacinie patentissime, lanceolate, inciso-dentate e le laciniette o denti acuti, aventi i margini rivolti in giù, tali lacinie sono verdi 0 sfumate di rossiccio di sopra ed ivi con solchetti che corrispondono ai nervi di sotto, di un verde bianchic. cio di sotto ed ivi con un nervo longitudinale che manda un nervetto per ciascuna lacinietta, glabre in ambedue le pagine, fornite li un picciòlo più lungo di esse, solcato in mezzo alle lacinie, quasi piano di sopra inferiormente e convesso-angolato di sotto, un po'cigliato in basso, verdognolo o in parte rossiccio. Le foglie del fusto sono sparse, le inferiori piủ lontane, le superiori più vicine e talvolta quasi opposte, tutte gradatamente eretto-patenti, e piủ piccole delle foglie del rizoma, con il picciòlo anche più corto, slargato e cigliato, e le superiori sessili. I fiori sono solitarii e quasi sessili all'ascella delle brattee in un racemo corto, quasi in capolino, poi un poco allungato da circa 4 a 6 centimetri. Le brattee sono poco piủ lunghe o uguali al calice, avvicinate ad esso, trifide, con le lacinie inciso-dentate, con i denti acuti e i margini rivolti in giu come nelle foglie: la lacinia terminale é maggriore delle due laterali, sono fornite di ci. glia lunghe verso la base. I pedicelli sono corti o cortissini, cilindrici, eretti, glabri, verdognoli. Il calice è quasi campanulato, un po'stretto sopra la metà, verdognolo, glabro, diviso dalla metà in su in cinque lacinie quasi uguali, eretto-patule, fogliacee, incisodentate con i denti aculi e i margini un po'rivolti in fuori, ha dieci 
nervi, cinque dei quali vanno sino all'apice delle lacinie e cinque terminano nei seni intermedii. La corolla è quasi il doppio più lunga del calice, di un giallo assai pallido. Il labbro superiore è prolungato in un rostro, lungo quanto il labbro inferiore, ch'esso tocca con l'apice, stretto, diretto in giù e troncato, e smarginato all'apice, il labbro inferiore è patente, oscuramente trilobo, con i lobi laterali un po'piủ grandi del mediano, tondeggianti e irregolarmente crenulati, il lobo di mezzo è ottuso e appena marginato. Gli stami sono 4, didinaıni, più corti del labbro superiore dentro il quale sono racchiusi. I filamenti sono piani, un po'curvi, bianchi, quelli degli stami superiori peloso-barbati dalla metà in su, gli altri due glabri. Le antere sono ovali, ottuse all' apice, bilobe e con i lobi un po' acuti alla base, inserite nel dorso sopra di questa, con il connettivo larghetto, bilo. culari, giallicce e glabre. Il pistillo è più lungo degli stami ed uguale al labbro superiore. L'ovario è ovato, glabro, verde, biloculare. Lo stilo è lungo, curvo, bianchiccio, glabro. Lo stimma è quasi in capolino, verdognolo. (Parl. ms., descr. della pianta di val Trompia).

Osservazione. - Allo stato fruttifero questa specie si distingue bene da qualunque forma della precedente per il frutto rinchiuso nel calice e di colore chiaro, e bivalve, e non sporgente più o meno, nerastro e univalve. Allo stato fiorifero la pianta fresca si distingue per il colore dei fiori, ma seccata la distinzione riesce talora malagevole.

\section{Pediculenris adscendens.}

P. foliis pinnatisectis lobis lanceolatis pinnatipartitis lobulis dentatis, caulinis pluribus sparsis parvis, calycis tubuloso-campanulati dentibus lanceolatis integris, corollæ ochroleucæ galea longe rostrata, capsula 1 -valvi.

Pedicularis adscendens Gaud.fl. helv. 4. p. 145 (non Schleich.) (1829). Com. fl. com. 5. p. 45. Ces. Pass. Gib. comp. fl. ital. p. 538. Arc. comp. fl. ital. p. 520.

Pedicularis Barrelieri Reich. fl. germ. exc. p. 362 (1830). Rota prosp. fl. Berg. p. 68 .

Pedicularis tuberosa $\beta$ Bert. fl. ital. 6. p. 334. Zersi prosp. piant. Bresc. p. 160.

Figura, - Reich. ic. fl. germ. 20. t. 131.

Ssazione, Abitazione e Fioritura. - Nei pascoli delle Alpi, assai rara: allo Spluga, in val Tellina al Braulio, al Legnone (Bert.), 
al monte Cavallo sopra Valleve in val Brembana (Rota), sopra Lavenone in val Sabia (Zersi); sul Cenisio (Rostan!), nell'alpi di Tenda (Reuter!). Fiorisce in luglio e agosto.

Distribuzione geografica. - Nelle Alpi.

\section{Pedicularis incarnatr.}

P. foliis pinnatipartitis lobis lanceolatis pinnatifidis lobulis dentatis, plurimis caulinis sparsis, calycis tubuloso-campanulati dentibus lineari-lanceolatis integris, corollie roseæ galea longe rostrata, capsula $1-$ valvi.

Pedicularis incarnata Jacq. fl. austr. Bert. n. ital. $\dot{6}$. p. 331. De Not. rep. fl. lig. p. 310. Com.! fl. com. 5. p. 41. Gren. Godr. A. Fr. 2. p. 616. Hausm. fl. Tir. p. 658. Rota prosp. fl. Berg. p. 68. Ard. 1. Alpes-mar. 1. 286. Zersi prosp. piant. Pav. p. 160. Ing. cat. sp. Mond. p. 55. Cies. Pass. Gib. comp. fl. ital. p. 358. Arc. comp. ll. ital. p. 521.

Figure. - All. rar. Ped. stirp. spec. prim. t. 12. f. 2, in $\mu_{\text {. }}$ ped. t. 3. f. 2 repetita. Reich. ic. fl. germ. 20.t. 126.

Stazione, Abitazione e Fioritura. - Nei pascoli delle Alpi. Principia nelle Alpi Marittime: in quel di Mondovi, comune (Ing.), nell'alpe Ruscara (Ricca!), sull'alpi di Valdieri a Fuma morta (Bert.), sopra Limone (Boissier Reuter!), al col di Tenda (Bourgeau!), a Saorgio, all'Aution, al col di Raus, al lago d'Entrecoulpes, al col di Salese e nelle alpi d'Entrannes (Ard.); prosegue per il Monviso (Gren. Godr.), per l'alpi Valdensi (Rostan!), per il piccolo Cenisio (Beccari!) e il Cenisio a Ronche (Iluguenin!) e altrove, la valle di Moire (Manuel!), il col di Seigne e l'Allée blanche (Mercier!), fino al Gran S. Bernardo a 7700' (Parl.!); quindi per l'alpe Maccagno in val Sesia, e per Maccugnaga e monte Turlo nella valle dell' 0 ssola (Carestia!), abbondante in quelle parti secondo Biroli, passa alla val Tellina, dov'è stata trovata sulla cima del Reguzzo (Moretti!) nella valle di Arigna, e sul monte Coaglio (Com.), alle prealpi Bergamasche (Rota), e al Bresciano dov' i stata trovata sul monte Cigoletto alle Corti di Campo molle (Zersi). Finalmente dopo un lungo tratto intermedio termina al monte Cren nella valle dell'Isonzo a $6000-$ 6500 ' (Huter!). Fiorisce in luglio e agosto.

Distribuxione geografica. - Nelle Alpi.

Descrizione. - Rizoma obliquno, grossetto, scuro, con fibre radi. cali grossette, di un bianco sudicio. Fusto ascendente o quasi cretto,

Flona Italana. - Vul. Vi. 
appena angolato, verdognolo in basso, violetto in alto, ivi con linee pelose longitudinali che scendon dai lati di ciascuna foglia. Foglie. pennati-partite con le lacinie lanceolato-lineari pinnatifide, e con le laciniette piccole inciso-dentate, con i denti acuti e quasi in sega, di un verde piuttosto chiaro, con un solco longitudinale e dei solchi laterali nella pagina di sopra e con un nervo longitudinale sporgente in ciascuna lacinia di sotto, glabre in ambedue le pagine. I fiori sono distribuiti in una spiga bislunga e stretta, spesso interrotta in basso, sono sessili. Brattee trifide, le inferiori più grandi e glabre simili alle foglie superiori ma piủ piccole, le altre piủ corte del fiore e quasi uguali al calice, con le lacinie piủ strette e soltanto dentate, pelose, lanate come la rachide ch'è violetta. Calice violetto, peloso-lanato, tubuloso-campanulato, con cinque denti lunghi quasi quanto il tubo, eretti, lineari, ottusi, interi. Corolla quasi il doppio più lunga del calice, glabra, di un violetto-roseo, più carico nel labbro superiore. Tubo lungo quasi quanto il calice. Labbro superiore prolungato in un rostro lineare, troncato, smarginato e dentellato, curvato ad arco in giù, avvicinato alquanto lateralmente; labbro inferiore poco piủ corto del superiore, patente, trilobo, coi lobi laterali molto più grandi dell'intermedio e tondeggianti. Stami 4, didinami. Filamenti filiformi, gla. bri, bianchicci. Antere quasi ovali, bilobe in basso, inserite nel dorso sopra della base, scure. Pistillo più lungo del labbro superiore. Ova. rio ovato-lanceolato, con gli angoli quasi acuti, forse un po' convessi, verdognolo, glabro. Stilo lungo, curvato in giu ad arco in alto, violetto, glabro, filiforme. Stimma in capolino, oscuramente bilobo, papilloso, verdognolo. (Parl. ms., deser. della pianta del Cenisio).

Osservazione. - In qualche luogo delle Alpi Mlaritime, e segnatamente al Pizzo d'Ormea raccoltavi dal Gentili, esiste una pianta che ha l'aspetto, il fogliame e l'infiorescenza della Pedicularis incarnata, ma i fiori, a giudicarli sul secco, sembrano gialli anzichè rossi, e i denti calicini sono quelli della $P$. tuberosa: che sia forse un ibrido delle due specie, o una specie distinta?

\section{ALECTOROLOPIIUS.}

Rhinanthi sp. una Linn.

Alectorolophus Hall. enum stirp. Helv. (J742). Enum. plant. hort. et agri gott. p. 312 (1753). Hist. stirp. ind. Helv. 1. p. 137. Gen.pl. fl. germ. 5.t. 46. 
Rhinanthus Gileditsch (1749). Benth. Hook. gen. pl. 2. p. 979. Cies. Pass. Gib. comp. fl. ital. p. 356. t.50. f. 3.

Caly $x$ ventricoso-compressus, subæqualiter 4-dentatus, fructifer auctus. Corolla limbo conspicue 2-labiato, labio postico galeato, antico 3-lobo. Stanina 4. Antherarum loculi basi mutici. Stigma minusculum. Gemmulie in loculis pancæ, subbiseriatæ, pendulæ. Capsula oligosperma.

Portamento. - Vedasi la descrizione dell'unica specie.

Osservazione. - Il genere Rhinanthus venne foggiato da Linneo per comprendere le Crista galli degli antichi e le Elephas di Tournefort; ed egli v'incluse 5 specie. Haller pel primo ne distaccò il $K h$. Crista-galli per farne il genere Alectorolophus; poi Necker distaccò lo due prime specie per restituire il genere Tourneforziano Elephas sotto il none di Probosciphora; infine i moderni hanno riferito le dne ultime specie ai generi Geniosporum e Dasystoma: cosicchè del Rhinanthus di Linneo non resta più nulla.

\section{Alectorolophus Crosta-ginili.}

Rhinanthus Crista-galli Linn. sp. pl. ed. 1. p. 603. Bart.! cat. piunt. Sien. p. 19. Sant. viagg. 2. p. 17, 28. 3. p. 126. Sav. /l. pis. 2. p. 70. Balb. el. piant. Tor. p. 67. Re fl. seg. p. 51. Bir. fl. acon. p. 908. Nocc. Balb /l. tic. 1. p. 292. Poll. /l. ver. 2. p. 318. Nacc. 1l. ven. 2. p. 138. Pucc. syn. pl. luc. p. 317. Tassi fl. prov. sen. p. 50. Car prodr. 月. 1osc. p. 492. Ard. Al. Alpes-mar. p. 28j. Archb. l. Altn-Serch. p. 59 .

Nectorolophus Crista-galli Spreng. syst. 2. p. 792. Ten. syll. f. neap. p. 300. Colla herb. ped. 4. p. 360.

a maior, bracteis pallescentibus, calyce piloso aut glabrescente, corolla tubo calyce longriore.

Rhinanthus Alectorolophus Poll. hist. pl. in Palat. 2. p. 177. Bert. 17. ital. 6. p. 984. Com. f1. com. 5. p. 10. Coota prosp. piant l'av. p. 271. Prosp. 11. Berg. p. 68. Pir. fl. for. syll. p. 108. Bicch agg. 11. lucch. p. 15. De V'is. Sacc. cat. piant. Ven. p. 157. Zersi prosp. piant. Bresc. p 160. Terr. rel. Terra Lav. p. 1.99. Ters. rel. p. 77. Ces. Pass. Kiib. comp. 11. ital. p. 356. Terr.! yuart. rel. p. 111. Sacc. Bizz. agg-11. Irev. p. 29. Cib. Pir. R. Jod. p. 195 Are. lomp 11. ital. p. 520. Cocc. H. I3n' p. 366 .

Rhinanthus hirsutus Lam, fl. fr. ?. p. 353. Seb, Maur. fl rom 
prodr. p. 202. Mass. prodr. fl. valt. p. 153. Trev. prosp. fl. eug. p. 23. Ing. cat. sp. Mond. p. 61.

Rhinanthus maior Ehrh. beitr. De Not. rep. fl. lig. p. 311. Zan. prusp. fl. ven. p. 25. Rota ll. cc. Hausm. fl. Tir. p. 662. Pir. l. c. De Vis. Sacc. l. c. Ces. el. piant. Maiell. p. 22. Ces. Pass. Gib. l.c. Simith fl. Fiume p. 39. Gib. Pir. l. c. Are. l. c. Cocc. l. c.

Rhinanthus Crista-galli Ruch. fl. lidi ven. p. 152. Berl. A. alp. apuan. p. 380. Mass. l. c. Sim. fl. alp. vers. p. 132.

Alectorolophus maior Trev. l. c.

Figure. - Reich. ic. bot. t. 732, 733. Ic. fl. germ. 20. t. 118.

$\beta$ minor, bracteis herbaceis, calyce glabro, corollæ tubo calyce æquali.

Rhinanthus glaber Lam. fl. fr. 2. p. 352. Ing. l. c.

Rhinanthus minor Ehrh. o. c. Mass. l. c. Trev. l. c. De Not. l. c. Sim. l. c. Rota ll. cc. Hausm. l. c. Pir. l. c. Bicch. l. c. De Vis. Sace. l. c. Zersi l. c. Ces. Pass. Gib. l. c. Smith l. c. Cald. fl. fav. tent. p. 174. Cocc. quart. contr. fl. Bol. p. 15. Gib. Pir. l. c. Arc. l. c. Cocc. l. c.

Rhinanthus Crista-galli Bert. o. c. p. 282. ('. Bert. it. bon. p. 21. Com. o. c. p. 8. G. Bert. veg. porr. p. 85. Terr.! fl. Vult. syn. p. 132. Sec. rel. Terra di Lav. p. 93. Terz. rel. p. 77. Quart. rel. p. 111.

Rhinanthus alpinus Hausm. o. c. p. 663. Rota prosp. fl. Berg. l. $c$.

Rhinanthus angustifolius De Vis. Sacc. l. c.

Figure. - Reich. ic. bot. t. 731. Ic. fl. germ. 20. t. 117, 119.

Stazione, Abitazione e Fioritura. - Nei prati della Penisola, dalle pianure a parti altissime dei monti. Abbionda in tutta l'Alta Italia, dove s'inalza nelle Alpi, per esempio al lago del Cenisio (Parl.!), nel monte Campione presso Lecco a $4-6000^{\prime}$ (Ball!), in val Trompia alla Corna Blacca a 1800-1900 metri (Parl.!), in Tirolo sul Ritten $\sin 0$ a $50000^{\prime}$ (Hausm.). Abbonda eziandio nell' Italia centrale, specialmente nei monti, dove arriva alle più alte cime dell'Appennino; ma già in Liguria scarseggia sul mare (De Not. ecc.), in Toscana manca nella bassa Maremma, e nelle isole tutte quante, nel Romano non fa che nei monti (Seb. Maur.), e poi per quelli della Campania (Terr.!) s'inoltra fino at monte Vergine (Ten.) e al Vulture (Terr.); dall'altro lato della Penisola per i monti Catria (Piccinini!) e S. Vicino a 1100 metri (Bucci!) e per Tolentino (Ricci!) s'inoltra fino in Abruzzo ad Assergio (Ten.) e alla Maiella a 2400 metri 
(Levier!), e fino in Molise al monte di Mezzo (Bert.). Manca piủ al mezzodi, come manca in Sicilia, e in Sardegna, e, quel che più sorprende, in Corsica. Fiorisce secondo i luoghi da maggio ad agosto.

Distribuzione geografica. - In tutta Europa, eccetto le sue parti piủ meridionali, nell' Asia Minore e finı alla Persia, in Siberia, nell'America settentrionale.

Desorizione. - Pianta annua, alta da 2 a 5 decimetri, verde, glabra. La radice è fusiforme, ramosa, flessuosa, con i rami quasi orizzontali, bianchiccia. Il fusto è eretto, quadrangolare, con gli angoli un po'rilevati, verdognolo-rossiccio, ramoso, con i rami opposti, eretto-patenti o eretti o un po' ascendenti, quasi uguali al fusto. Le foglie sono opposte, patentissime o un po' dirette in giii, sessili, bislungo-lanceolate, acute, seghettate con i denti ottusetti, un po'scabri nei margini, con il margine un po'rovesciato, di un verde scuro di sopra ed ivi quasi lucenti e con un solco longitudinale e con solchi laterali che corrispondono ai nervi di sotto, di un verde pallido di sotto ed ivi con un nervo longitudinale e con dei nervi laterali ugualmente rilevati e quasi bianchicci che corrispondono in mezzo ai denti. Le brattee sono simili alle foglie, un po'più larghe e un po' concave alla base e perciò ovato-lanceolate, e con i denti ivi piủ lunghi e più acuminati; del resto son verdi eccetto alla base che nelle superiori è bianchiccia. I fiori sono quasi sessili, eretti, solitarii al. l'ascella delle brattee, in una spica fitta che poi si allunga e si fa rada. Il calice è più corto della brattea, gonfio-ventricoso, schiacciato un po'lateralmente, ovato-orbicolare, verdognolo-bianchiccio, con cinque denti divisi un po'più profondamente dove corrispondonn i due angoli del calice, eretti, e un po'divergenti in alto, triangolari-lanceolati, quasi acuti, scabri nei margini: diviene piủ grande e più gonfio nel frutto ed è allora di un verde bianchiccio, o rossiccio con i denti conniventi. La corolla $\grave{e}$ un po' più grande del doppio del calice, di un giallo chiarn con il tubo bianchiccio. Questo $\dot{e}$ ap. pena curvo, un po'schiacciato; il labbro superiore $\grave{e}$ in forma di elmo, poco più lıngo dell'inferiore, un po'schiacciato, puberulo di fuori verso l'apice e ivi peloso di dentro, come troncato all' apice e ivi di sotto fornito di due denti quasi quadrati, diretti in giì e arvicinati in modo da toccarsi per la loro faccia interna. Il labbro inferiore è trilobo, con il lobo medio diretto in avanti, quasi troncato e piegato in su in modo da toccarsi per la faccia interna e i lobi laterali diretti in avanti ed in giù, un po' concavi di sotto, obovato-orbicolari, lunghi quasi quanto il lobo medio. Gli stami sono quattro, 
didinami, racchiusi dentro il labbro superiore, inseriti nella parte superiore del tubo. I filamenti sono grossetti, un po' schiacciati da avanti in dietro, giallicci, papilloso-glandolosi. Le antere poste trasversalmente, inserite nel dorso, ottuse all' apice, bilobe alla base, bilocu. lari, giallicce, irsute nei margini delle logge, mutiche. Il pistillo è quasi uguale al labbro superiore o ai denti dello stesso. L'ovario è quasi ovale, un po' acuto, schiacciato dalle parti, verdognolo, con qualche macchiolina azzurrognola, glahro, biloculare, con molti ovoli in ciascuna loggia, è circondato alla base da un disco circolare, poco rilevato e verdognolo. Lo stilo è lungo, filiforme, un po' piủ grosso e curvo in alto, bianchiccio e pelosetto, azzurrognolo e glabro verso l'apice. Lo stinıma è quasi in capolino, papilloso, verdognolo. La cassula è racchiusa dentro il calice gonfio e persistente, quasi orbicolare, schiacciata, appena smarginata all'apice, verdognola, glabra, con due logge, contenenti molti semi. Questi sono pendenti, grandi, un po'schiacciati, ovali, troncati e un po' piủ grossi nell'ombelico, giallicci, glabri, con rughe fine concentriche, e cinti di un'ala bianchiccia ch' è più della metà piủ stretta della larghezza del seme. (Parl. ms., descr. della pianta di Boscolungo nell'Appennino toscano).

Osservazioni. - Questa specie varia: per la statura, da 1 decimetro (Rhin.alpinus) a ${ }^{1} / 2$ metro; - per le foglie, ora piủ larghe, bislunghe, ora strettissime lineari (Rh. angustifolius); - per i peli, assenti, o presenti e allora più o meno abbondanti, soprattutto sul calice (Rh. hirsutus); - per le brattee, erbacee verdi, o semimembranose e scolorite in parte; - per la corolla più o meno grande relativamente al calice; - per la larghezza dell' ala nei semi. Quando di questi caratteri differenziali parecchi trovansi riuniti in un individuo, e i caratteri opposti in un altro, confrontandoli si ha l'apparenza di due buone specie: ma non più cosi esaminando una serie di altri individui dove i medesimi caratteri vedonsi spartiti diversamente. Le due varietà proposte più sopra non rappresentano che due tipi estremi, fra i quali esistono molte forme intermedie. In questo caso, come in generale, è giuocoforza ritornare al concetto Linneano della specie, quello che, mandato ad atto, dà i resultati più sicuri.

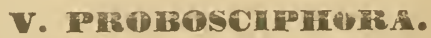

Elephas Tourn. inst. cor. p. 48. t. 482 (non zool.). Ces. Pass. Gib. comp. fl. ital. p. 357. t. 50 . f. 5.

Rhinanthi sp. Linn. 
Probosciphora Neck. elem. 1.p. 336 (1790).

Rhinanthus Bieb. fl. taur. 2. p. 68 (1808).

Rhynchocorys Gris. spic. fl. rum. 2. p. 12 (1844). Benth. Hook. gen. pl. 2. p. 978.

Elephantina Bert. fl. ital. 6. p. 279 (1844-47).

Calyx compressus 2-labiatus, labio postico subintegro, antico bipartito. Corolia limbo conspicue 2-labiato, labio postico galeato, antico 3-lobo. Stamina 4. Antherarum loculi basi mutici. Stigma capitellatum. Gemmulæ in loculis paucæ, subbiseriatæ, pendulæ. Capsula oligosperma.

Portamento. - L' unica specie nostrale è un'erba perenne, leggermente pelosa, con un fusto eretto 0 ascendente, alto da 2 a 5 decimetri, semplice 0 alquanto ramoso a pannocchia, con foglie opposte, quasi sessili, ovali, crenate, che hanno alla loro ascella i fiori, solitari, con corto pedicello, con calice grande, e corolla piuttosto piccola, gialla macchiata di rosso, dal labbro posteriore prolungato in un becco diritto. La cassula, globosa, è racchiusa nel calice, e contiene parecchi semi globosi, cenerognoli, con guscio rugoso, sugheroso.

\section{Probosciphorg Columnge.}

Elephas Columnæ Guss.! R. sic. prodr. 2. p. 155. Ces. Pass. Gib. l. c.

Rhynchocorys Elephas Gris. l. c. Arc. comp. fl. ital. p. 520. Nic prodr. 1. mess. p. 338.

Elephantina proboscidea Bert. o. c. p. 280.

Figure. - Col. ecphr. p. 188. Jaub. Spach ill. pl. orient. 1. 393.

Stazione, Abitazione e Fioritura. - Nei luoghi ombrosi, freschi ed umidi deiboschi montuosi dell'Italia meridionale. Colonna la scopri sul Matese nella valle di Campochiaro sopra un lnogo delto il vado di Scartocchia (o. c. p. 187); fa pure nel Molise, a Balvano in Basilicata (Ces. Pass. Gib.), in Calabria nell'Aspromonte a 1100 1200 metri (IItuter Porta IIigo!); abbonda nella Sicilia settentrionale orientale, nella cosidetta Valdemone: nei bosclii di Manılanici, loccella, Franeavilla, Floresta, Caronia, Capizzi, Mistretta (Guss.), Cannata (Huet!), al Gurgo di Scavioli a 1200 metri (Lojacono!). Fiori sce in maggio e gingno, o giugno e luglio. 
J) istribuzione geografica. - Nell'Italia meridionale, la Grecia e la Turchia, nell'Asia Minore fino al Caucaso e alla Persia.

\section{ODDNTITES.}

Odontites Hall. hist. stirp. Helv. p. 133. Gen. pl.fl. germ. 5. t. 41. Ces. Pass. Gib. comp. fl. ital. p. 354. t. 53. f. 1. Bartsia (ex parte) Benth. Hook. gen. pl. 2. p. 978.

Calyx tubulosus vel campanulatus, subæqualiter 4-dentatus. Corolla limbo conspicue 2-labiato, labio postico galeato, antico j-lobo. Stamina 4. Antherarum loculi hasi mucronati. Stigma capitellatum. Gemmulæ in loculis paucæ, subbiseriatæ, pendulæ. Capsula oligo. sperma.

Portamento. - L'Odontites Bocconei è un suffrutice, le altre specie sono erbe annue, erette, ramose, alte fino a $1 / 2$ metro, ad eccezione dell' 0 . corsica ch'è bassina e sdraiata. Il fusto e i rami sono sottili; le foglie sono opposte, lunghe, strette, intere o poco dentate; all'estremità del fusto e dei rami si fanno più piccole, e portano nella loro ascella un fiore, piccolino, brevemente pedicellato, rosso o giallo, e questi fiori formano per tal modo un grappolo interfogliato, unilaterale.

\section{Odontiter Bocconel.}

0. suffruticosa, glaberrima, caulibus erectis vel ascendentibus, basi ramosis, ramisque brevibus cæspitosis, snbtetragonis, foliis lanceolato-linearibus, apice breviter obtuse mucronatis, integerrimis, inferioribus laeviter subciliolato-scabris, bracteis angustioribus, flore longioribus, ciliolatis, floribus racemosis, breviter pedicellatis, pedun. culis puberulis, pedicellis glabris, erectis, calycibus tubulosis, subincurvis, puberulis, profunde quadridentatis, dentibus lanceolatis, acutis, carinatis, corollæ labio superiore concavo, emarginato-bilobo, in emarginatura ciliolato, inferiore breviore trilobo, lobis lateralibus brevioribus, suborbicularibus vel emarginatis, staminibus corolla et inter se æqualibus, antheris transversis, eglandulosis, loculis vix bar. hatis, submuticis, pistillo stamina superante, ovario oblongo, obtuso, apice subcompresso et pilosulo, stylo curvato, stigmate subcapitato. (Parl. ms.). 
Euphrasia Bocconi Guss. cat. hort. Boccad. p. 76. Bert. fl. ital. 6. p. 298 .

Odontites Bocconi Walp. rep. bot. syst. 3. p. 400. Ces. Pass. Gib. comp. fl. ital. p. 355. Arc comp. fl. ital. p. 519. Nic. prodr. fl. mess. p. 339.

Euphrasia Biancæ Guss. in Bianca fl. d'Av.

Figura. - Bocc. mus. piant. rare 1. 108.

Stazione, Abitazione e Fioritura. - Nelle rupi calcaree dei monti di Sicilia: presso Palermo a monte Pellegrino (Guss.) e monte Gallo (Todaro!), presso Gibilmessa (Huet!), alla Pizzuta (Parl. ecc.), al monte de' Cani, al monte Gebhia, e Quisquina, nelle Madonie ai luoghi detti monte Scalone e Acqua del canale (Guss.); nel Messinese a monte Scuderi (Guss.), in Mandanici all' Acqua d'Issula (Seguenza!), a Santissima (Nic.); ad Avola, però quasi scomparsa (Bianca!). Fiorisce da agosto a ottobre.

Distribuzione geografica. - Nasce oltracciò in Candia secondo Bentham (in Cand. prodr.), ma Boissier (Fl. orient. 4. p. 477) fa della pianta candiotta una specie distinta col nome di Odontites cretica.

Descrizione. - Pianta suffruticosa, quasi in cespuglio, alta da 4 a 6 o 7 decimetri, glaberrima. I fusti sono ascendenti 0 diritti, duri, quasi legnosi in basso ed ivi oscuramente quadrangolari, di co. lor rossiccio scuro, con le cicatrici per la caduta delle foglie, e mandanti corti rami sterili, quasi quadrangolari in alto ed ivi verdi $e$ vestiti di foglie. Queste sono lanceolato-lineari, con un corto ed ottusn mucrone all' apice, verdi e leggermente solcate di sopra, di un verde chiaro di sotto ed ivi con un nervo longitudinale da cui partono piccole venette laterali; intere nei margini e le inferiori di esse fornite di piccoli peli rari, grossetti alla base e giacenti nel margine, rivolti verso l'apice. I fiori sono distribuiti in racemi. Il peduncolo $i$ puberulo. I pedicelli sono cortissimi, glabri, eretti. Le brattee sono lanceolatn-lineari, acuminate, piủ lunglıe dei fiori, fornite di ciglia nel margine. Il calice è tubuloso, un po'curvo, verdognolo, puherulo, con pochi nervi longitudinali, diviso in quattro denti profondi, quasi uguali tra loro, lanceolati, acuti e carenati. La corolla è una metà piỉ lunga del calice, un po'curva, gialla. Il labbro superiore è concavo, smarginato, bilobo all'apice, con i lobi patenti e finamente cigliati verso la marginatura, il labbro inferiore è piu corto del superiore, trilobo, con il Jobo medio un po' più lungo e piủ smarginato dei laterali, i quali sono appena smarginati n quasi tondeggianti al. l'apice. Gli stami sono lunghi quanto il lahbro superiore della corolla, 
quasi tutti e quattro uguali. I filamenti sono giallicci, glabri, filiformi, inseriti in alto del tubo della corolla. Le antere sono trasversali, gialliccie, con le logge divaricate e quasi acuminate alla base, e all'apice ottuse e fornite di pochissimi peli bianchi che vi fanno un piccolo ciuffetto. Il polline è bianchiccio. Il pistillo è poco più lungo degli stami e del labbro superiore della corolla. L'ovario è verdognolo, allungato, ottuso, un po"schiacciato verso l'apice dove è pelosetto. Lo stilo è filiforme, gialliccio, glabro, un po'curvato in avanti. Lo stimma è quasi in capolino, papilloso, gialliccio-verdognolo. (Parl. ms., descr. della pianta di Palermo).

\section{2. Đdontites rigidifolin.}

0. minute pilosula, foliis anguste linearibus integerrimis, floralibus flore brevioribus, corolla rubro-lutea calyce dimidio longiore, staminibus corolla brevioribus, antheris apice barbulatis.

Euphrasia rigidifolia Biv. sic. plant. cent. 1. p. 36. Bert. fl. ital. 6. p. 292. Tar. Gerb. cat. pl. calat. p. 29.

Odontites rigidifolia Benth. in Cand. prodr. 10. p. 550. Ces. Pass. Gib. comp. fl. ital. p. 355. Arc. comp. fl. ital. p. 519.

Stazione, Abitazione e Fioritura. - In luoghi campestri aridi di Sicilia: Palermo a S. Martino (Parl.! ecc.), alla Grazia nel feudo del Salvatore (Biv.), alla Scala del Mesagno, al Chiarandà (Bert.), alla Pizzuta (Todaro!), e alla Ficuzza a Rocca d'Elce (Lojacono!), val di Mazzara, val di Noto, Nicosia, Troina, Caltanissetta, Riesi, Sommatino (Guss.), Caltagirone nel monte Cansaria (Tar. Gerb.). Fiorisce in settembre e ottobre.

Distribuzione geografica. - Oltre la Sicilia, questa specie abita anche l'Algeria.

Descrizione. - Radice annua, contorta, ramosa, nericcia. Fusti quadrangolari, con gli angoli ottusi, rossicci, pubescenti per peli assai corti e curvati quasi ad arco in giù e hianchicci. Rami opposti in croce, ascendenti, piủ corti assai del fusto, rossicci e puberuli come questo. Foglie opposte, piccole, carnosette, eretto-patenti, le superiori un po' curvate in fuori, lineari, ottuse, integerrime, di un verde pallido di sopra ed ivi coll un solco longitudinale quasi un canale, un po'convesse di sotto, di colore un po'più chiaro e con un leggerissimo solco longitudinale che non giunge sino all'apice; sono puberule di sotto e un po'anche di sopra per peli corti, adpressi, diretti verso l'apice della foglia e bianchicci. Le brattee sono deci- 
due, sono simili alle foglie superiori, ma gradatamente un po' più strette verso l'apice e quasi acuminate, avvicinate al calice, quasi uguali a questo. I fiori sono solitarii, eretto-patenti, un po'radi e quasi distichi in una spiga un po'racemosa all' apice del fusto e dei rami, ųuesta spiga è stretta, bislunga e quasi lineare, poi si allarga. Ciascun fiore ha un pedicello corto, molto più corto del calice, gros. setto, quasi roseo. Il calice è un po' curvo, tubuloso, 'con 8 angoli longitudinali, alcuni più alcuni meno sporgenti, diviso nel terzo superiore in 4 denti o lacinie lunghette, quasi lanceolate, acute, due superiori e due laterali, è verdognolo, spesso in parte rossiccio, con peli corti nella parte posteriore e in alcuni angoli che lo rendono un po'irtc. La corolla è poco piủ lunga del calice, di un giallo chiaro, sfumata di rossiccio, glabra, un po'curva. Il tubo è lungo quanto il calice, un po'curvo. Il labbro superiore è fornicato, come troncato all'apice, profondamente smarginato, diretto in su ed in avanti; il labbro inferiore è poco più della metà più corto del superiore, trilobo, con i lobi quasi tondi, l'intermedio un po'piủ largo e appena smar. ginato, tutti e tre diretti in su e avvicinati al superiore in modo che la corolla è chiusa. Gli stami sono 4, appena didinami, rinchiusi dentro il labbro superiore. I filamenti sono filiformi, gialli, glabri. Le antere gialle, biloculari, con le logge un po'divergenti in basso ed ivi quasi terminate in una piccola aresta, sono fornite di un ciuffo di peli bianchi all'apice. Il pistillo è più lungo del labbro superiore, per cui sporge in parte fuori. L'ovario è ovale-bislungo, ottuso, schiacciato lateralmente, gialliccio, puberulo all'apice, biloculare con molti ovoli inseriti in una placenta che fa da setto. Lo stilo è lungo, fatto quasi a $S$, curvato in alto assai in avanti, delicato, quasi cilin. drico appena ingrossato in alto, gialliccio, glabro. Lo stimma è quasi in rapolino, del colore dello stilo. (Parl. ms., deser. della pianla di S. Martino presso Palermo).

Osservazione. - La pianta di Caltagirone distinta da Todaro sotto il nome di Odontiles Cilarda, sembra una forma puủ robusta di questa specie, con le foglie meno strette.

\section{Mdontites corsien.}

0. minute pilosa, foliis anguste linearibus integerrimis, floralibus flore brevioribus, corolla purpurasente, nunc luteo aut alho (Nars.), calyec vix Iongiore, staminibus corolla breviorilus, autheris glabris. 
Euphrasia corsica Lois. fl. gall. p. 367. Bert. fl. ital.6. p. 293.

Odontites corsica G. Don gen. syst. 4. p. 611. Gren. Godr. fl. Fr. 2. p. 607. Mor.l fl. sard. 3. p. 237. Mars. cat. pl. Cors. p. 109. Ces. Pass. Gib. comp. fl. ital. p. 355. Arc. comp. fl. ital. p. 519.

Odontites albidula Mab.! rech. pl. cors.

Figura. - Lois. o. c. t. 10.

Stazione, Abitazione e Fioritura. - Nei luoghi rocciosi erbosi degli alti monti di Corsica e di Sardegna. In Sardegna nel monte Gennargentu a circa 1700 metri (Mor.). In Corsica alla Foce di Viz. zavona, a Morro Cinto, nei monti Rotondo e d'Oro, sopra i bagni di Guagno, d'onde scende al Fiume Grosso (Mars.), al lago di Nino, al Niolo, a Neori (Requien!), in cima al Pigno (Nabille!), a Pietramala di Bastelica, ad Aullena, da Portovecchio a Quenza (Mars.), alla Cagna (Gren. Godr.). Fiorisce in agosto e settembre.

Distribuzione geografica. - Specie corso-sarda.

\section{Odontites Intea.}

0 . minute pilosula, foliis anguste linearibus subintegris, flora. libus flore subbrevioribus, corolla aurea calyce duplo longiore, staminibus corolla longioribus, antheris glaberrimis.

Euphrasia lutea Linn.sp.pl. ed. 1.p. 604. Bart. cat. piant. Sien. p. 19. Sant. viugg. 1. p. 59. 2. p. 317. Mass. prodr. fl. valt. p. 68. Mor. De Not.? fl. Capr. p. 102. De Not. rep. fl. lig. p. 312. Bert. л. ital. 6. p. 294. Com. fl. com. 5. p. 16. Pucc.syn. pl. luc. p. 319. Sim.! fl. alp. vers. p. 133. Hausm. fl. Tir. p. 667. Rota prosp. fl. Berg. p. 68. Pir.! fl. for. syll. p. 196. Mor. fl. sard. 3. p. 237. Zersi prosp. piant. Bresc. p. 161. Smith! fl. Fiume p. 39. Anzi auct. fl. nov.-com. p. 196. Gib. Pir.! fl. Mod. p. 125.

Euphrasia linifolia Linn. l.c. Ed. 2. p. 842 (excl. $\beta$ ). Poll.? fl. ver. 2. p. 316. Mass.? o. c. p. 67. Colla herb. ped. 4. p. 354.

Odontites lutea Reich. fl. germ. exc. p. 359 (part.). G. Don gen. syst. 4. p.611. Trev. prosp. fl. eug. p. 23. Ard. cat.pl. Ment. p. 27. Car. prodr. fl. tosc. p. 490. Ard. fl. Alpes-mar. p. 284. G. Bert.veg. mont. Porr. p. 85. Genn. fl. Capr.p. 113. Terr. rel. Terra Lav. p. 149. Mars. cat. pl. Cors. p. 109. Terr. sec. rel. p. 93. Terz. rel. p. 77. Archb. fl. Alto-Serch. p. 58. Ing. cat. sp. Mond. p. 52. Groves contr. fl. Terr. d'Otr. p. 64. Ces. Pass. Gib. comp. fl. ital. p. 355. Cald.! f. fav. tent. p. 173. Arc. comp. fl. ital. p. 519. Cocc. fl. Bol. p. $36 \%$. 
Odontites linifolia G. Don l. c. Guss. enum. pl. Inarim. p. 244. Bartsia lutea Pasq. l. ves. p. 76.

Figure. - Coss. Germ. atl. fl. Par. t. 18. f. B. Reich. ic. fl. germ. 20. t. 108. f. 1.

Stazione, Abitazione e Fioritura. - Nei luoghi boschivi o cam. pestri aridi, specialmente di collina e di bassa montagna, per tutta l'Italia. In Piemonte è frequente (All.): fa a Susa (Aiuti!), Torino (Delponte!), Mondovi, ovunque (Ing.), Alba (Bertero!), Oldenico (Cesati!), verso Ivrea (Colla), alla Colma di Varallo (Bir.), ad Arona (Ricca!) e Cannero (Gib.!), al Sempione fra i larici e gli abeti(Par. latore!), ecc. ; nasce nel Cantone Ticino presso Locarno e Bellinzona (Com.); in Lombardia nel Pavese (Nocca Balb. ecc.), nel Milanese (Rampoldi! ecc.), nel Comasco (Com.), e sul monte Barro (Parl.!), su in val Tellina nel piano di Sondrio (Bert.) e nelle alpi di Togno e di Zana (Mass.), e a Montagna, Tresivio, Berbenno, Cosio (Anzi), nel Bergamasco, dal piano ai monti specialmente calcarei (Rota), nel Bresciano, frequentissima (Zersi), nel Mantovano (Barbieri!); in Ti. rolo a Merano, Bolzano (llausm.), Trento (Perini!), Levico, Piorgo, Brentonico ecc. (Hausm.); nel Veneto sul Baldo, nel Veronese, e Vicentino, negli Euganei (Poll.), a Venezia al Cavallino, rara (Nacc.), nel Friuli a Fagagna (Pir.!), S. Daniele ecc. (Pir.); in Istria a Trieste, sul monte Spaccato (Bert.), a Fiume (Smith!); in Emilia nei monti sopra Modena e Bologna, dalle loro radici al Salto di Montese c alla Porretta (Gib. Pir., Cocc. ecc.); in Romagna presso Faenza (Cald.!). È ugualmente comune nell'Italia media: così in tutta la Liguria (De Not.), da Tenda (Ungern-Sternberg!) e da Nizza (Durando! ecc.) a Chiavari (Delpino!); in Toscana nelle regioni maremmana, campestre e subinontana, da Sarzana (Bert.) al monte Argen. taro (Aiuti!), a Paterno sotto Vallombrosa (Borzi) e ai Bagni di San Filippo (Ant. Targioni!), nonchè nell' Elba! e forse in Capraia (Mor. De Not.); nelle Marche a Sasso Feltrio (Bert), a Cingoli (Narducci!), $\mathrm{e}$ in Abruzzo presso Filetto (Hluet!) e a lioccamorice (Ten.); in Un. bria (Figari!), intorno a Roma (Seb. Maur.). Nell'Italia meridionale trovasi ancora a Terracina (Bert.), Fondi ('Ten.), Sora, Cassino, Pi. cinisco (Terr.), Napoli verso Pozzuolo (Ileldreich! ecc.), in Ischia (Avellino! ecc.), in Capri (Pasq.), nel monte S. Angelo di Castellammare (Pasq. !), a Cava e Vietri (Ten.); e perfino a Otranto (Groves). In Corsica sembra comune, nelle regioni bassa e media (Mars.); cosi a Bastia (Pucci!), a Calvi (Soleirol!), nel monte Subigna (Cald.!). In Sardegna eziandio pare che sia comune (Mor.): per esempio nella 
Maddalena (Genn.). In Sicilia invece dev'essere rara, non essendo indicata che al Caputo secondo Ucria, a Nicolosi e a Adernò (Guss.). Fiorisce da agosto a ottobre.

Distribuzione geografica. - Nell'Europa media e meridionale dal Belgio e dalla Pommerania alla Spagna orientale e al Caucaso, nell' Asia Mlinore e sino al Libano.

Descrizione. - Radice molto tortuosa, ramosa, con i rami quasi orizzontali, scuri e glabri. Il fusto è eretto, alto da pochi pollici a un piede e mezzo, oscuramente quadrangolare perchè gli angoli sono oltusi, di color fosco-porporino, puberulo, ramoso, spesso quasi fin dalla base, con i rami opposti, gradatamente più lunghi verso alto, patenti o patentissimi. Le foglie del fusto sono opposte, patenti o curvate in giu, lanceolato-lineari, ottusette, con qualche raro e piccolo dente a guisa di sega nel margine, di un verde scuro, puberule, con un nervo longitudinale assai manifesto li sotto e piccoli nervetti laterali che vengono dalla base o presso la metà di questo e che diramandosi in venette fanno una vera rete nel parenchima. Le foglie dei rami sono numerose, vicine, patenti o patentissime, lineari, ottuse, con il margine ciliato-dentellato, puberule, con un nervo longitudinale e due venette laterali poco manifeste. I fiori sono solitarii all' ascella di ciascuna foglia fiorale ch'è simile alle foglie dei rami, ma quasi ovato-lanceolata e piủ corta del fiore, sono distribuiti in racemi all' apice del fusto e dei rametti o dei rami e sostenuti da un pedicello piủ corto della foglia fiorale, cilindrico, di un verdognolo assai chiaro e quasi glabro. Il calice è quasi campanulato, appena appena curvato, più largo in alto, di un verdognolo bianchiccio con quattro righe verdi e ha quattro denti corti, triangolari ed ottusi: è puberulo verso alto. La corolla è gialla. Il tubo è appena piủ lungo del calice: il labbro superiore è diretto in alto, smarginato-bilobo, con i lobi smarginati e forniti di ciglia, è concavo in avanti e puberulo di fuori; il labbro inferiore è poco più corto, diretto in avanti ed in su, bilobo, con i lobi quasi eguali, concavi di sopra, quasi obovati, smarginati o interi, forniti di ciglia nel margine e puberuli di sotto. Gli stami sono quasi uguali di altezza, i due posteriori avvicinati alquanto al labbro superiore, ciascuno degli altri due avvicinato al lobo laterale corrispondente del labbro inferiore: tutti sporgenti fuori in mezzo ai due labbri. I filamenti sono cilindrici, puberuli in avanti, dello stesso colore della corolla. Le antere sono di un giallo più carico, erette, glabre, biloculari, con le logge mucronate all'apice. Il polline è giallo. L'ovario è ovale, un po'schiac- 
ciato, verdognolo, glabro in basso, peloso in alto. Lo stilo è obliquamente inserito sull'ovario, passa in mezzo della base dei due stami anteriori ed è avvicinato al lobo medio del labbro inferiore del quale è piủ lungo, è filiforme, puberulo e griallo. Lo stimma è quasi in capolino, ma bilobo, verdognolo, con corte papille. La cassula è obovato-allungata, schiacciata, verilognola e glabra in basso, rossiccia e pelosa in alto. I semi non maturi sono allungati, rauciati, con costole longitudinali filiformi e righe trasversali cancellate. (I'arl. ms., descr. di pianta di Pisa).

\section{Dilontites viscosa.}

0. minute piloso-viscosa, foliis lineari-lanceolatis subintegris, floralibus flore subbrevioribus, corolla pallide flava calyce plus quam duplo longiore, staminibus corolla æqualibus, antheris subglabris.

Euphrasia viscosa Linn. mant.p. 86 Bert. fl. ital. 6. p. 297

Odontites viscosa Reich. fl. germ. exe. p. 960. Ard R. Alpesmar. p. 284. Ing. cat. sp. Mond. p. 52. Ces. L'ass Gib. comp. M.ital. p. 355. Arc. comp. 凡. ital. p. 519.

Figure. - Gar. hist. pl. Aix t. 80. Reich. ic. fl. germ. 20. t. 108. f. 2 .

Stazione, Abitazione e Fioritura. - Nei boschi delle Alpi occidentali: nella valle della Dora sopra Bussulino (III.), sopra Foresto e a Bardonecchia (Re); nella valle della Stura versu Berzesio (All.); nel Nizzardo a S. Anna di Vinai (Ard.); a Vicoforte in quel di Mondovi, comune (Ing.). Fiorisce in agosto e settembre.

Distribuzione geografica. - Nell'Europa meridionde-occidentale, dal P'ortogallo al Vallese in Svizzera.

\section{f. Dilontites Innceolatu.}

0. minute pilosa, foliis lanceolatis dentatis, lloralibus flore lougiuribus, corolla lutea calyce duplo Iongiore, staminibus curolla lon. gioribus, antheris glaberrimis.

Euphrasia lanceolala liaud. 12. helv. 4. p. $1 / 6$.

Odnntites lancenlata Reich. 1. germ. exc. p 862. Ard. fl. Alpesmar. p. 283. Ces. Pass. Ciib. comp. 1l. ilal, p. 955. Are. comp. 1 . ilal. p. 519

Bartsia lanrenlata Bert. 1l. ital. 6 p. ?73. 
Figura. - Reich. ic. fl. germ. 20. t. 107. f. 1.

Stazione, Abitazione e Fioritura. - Nei campi per le Alpi: nelle Marittime sopra Limone (Parl.!), al col di Tenda e a S. Stefano (Ard.), in val Maira tra Stroppo e Elva (Ball.!), nel Valdense a Massello (Rostan!), a Bardonecchia (Beccari!), sopra Balme nella valle di Lanzo (erb. Webb!), presso Val Grisanche (Carestia!), a Courmayeur (Ball!), a Riva di Sesia (Carestia!), infine presso Bormio a 1920 metri (Parl.!). Fiorisce da giugno ad agosto.

Distribuzione geografica. - Trovasi oltracciò nella Francia contermine.

\section{\%. Odontites serotina.}

0. minute pilosa, foliis lineari-lanceolatis dentatis, floralibus flore subbrevioribus, corolla purpurascente calyce duplo longiore, staminibus corolla vix longioribus, antheris apice barbulatis.

Euphrasia Odontites Linn. sp. pl. ed. 1. p. $604(\beta)$. Simi! fl. alp. vers. p. 133. Pir. fl. for. syll. p. 108 (excl. $\beta$ ).

Euphrasia serotina Lam. fl. fr. ed. 2. p. 350. De Not. rep. $/$. lig. p. 312. Koch syn. fl. germ. ed. 2. p. 629. Tar. Gerb. cat. pl. calat. p. 29. Ilausm. fl. Tir. p. 667. Rota prosp. /l. Berg. p. 68. De Vis. Sacc. cat. piant. Ven. p. 158. Zersi prosp. piant. Bresc. p. 161. Smith fl. Fiume p. 39. Gib. Pir. fl. Mod. p. 126. Suppl. p. 22.

Odontites serotina Reich. $l$. germ. exc. p. 359. Guss, enum. pl. Inarim. p. 244. Ard. fl. Alpes-mar. p. 283. G. Bert. veg. Porr. p. 85. Terr. A. Vult. syn. p. 131. Rel. Terra Lav. p. 148. Mar's. cat. pl. Cors. p. 109. Terr. sec. rel. Terra Lav. p. 93. Ces. Pass Gib. comp. fl. ital. p. 355. Cald. fl. fav. tent. p. 173. Arc. comp. fl. ital. p. 519. Coce: fl. Bol. p. $36 \%$.

Bartsia serotina Bert. fl. ital. 6. p. 274. Pucc. syn. pl. luc. p. $3 / 8$.

Odontites vulgaris Car. prodr. fl. tosc. p. $49 /$ ( $\beta)$. Nic.! prodr. R. mess. p. 339 .

Bartsia Odontites var. Pasq. fl. ves. p. 76 .

Figure, - Col. ecphr. p. 202. fig. sin.

$\beta$ sicula, corolla lutescente.

Euphrasia serotina b. sicula Guss. th. sic. syn. 2. p. 111.

Odontites vulgaris b. sicula Nic.! l. $c$.

Stazione, Abitazione e Fioritura. - Nei luoghi boschivi, o campestri, per tutta l'Italia. Nell'Alta Italia trovasi ad Alba (Berte- 
ro!), e probabilmente attorno a Torino (Balbis?, Colla?), a Novi (De Not.), in Brianza (Poll.), a Milano (Rampoldi I), a Pavia (Nocca Balb.), presso Cremona (Parl.!), nel Bergamasco (Rota), nel Bresciano, frequente (Zersi), nel Tirolo, dove non sembra comune, a Roveredo, Trento, Cavalese, Bolzano, Merano e Glorenza (Hausm.), a Peschiera (Rigo!), a Veroná, a Vicenza, negli Euganei (Poll.), a Chioggia (Bert.), a Venezia a Mestre (erb. Reboul!) e al Cavallino (Nocc.), a Treviso, a Bassano (Bert.), nel Friuli (Pir.), a Trieste (Bert.), a Fiume (Smith), a Parma (Passerini!), nel Modenese a Fiorano (Gibelli!) e alla Rocca di Fiumalbo (Gib. Pir.), presso Bólogna a Zola predosa (Bert.), e nel prossimo Appennino alla Porretta, a Rovinaglia e a Montese (Cocc.), a Castelbolognese (Bert.) e Facnza (Cald.), e nell'Appennino sovrastante a Valcoloreta (Bert.). Nell'Italia centrale trovasi nel Nizzardo a S. Dalmazzo di Tenda (Ungern-Sternberg!) e a Roubion (Ard.); quindi' in Toscana, dov'è comune assai nelle regioni basse, da Sarzana (Caldesi!) al monte Argentaro (Aiuti!), e fa anche nell'Elba!, e penetra poi nella regione montana, cosi nelle Alpi apuane (Simi!), a Vallombrosa!, in Casentino a Prataglia a 2200' (Parl.!); nasce ancora nelle Marche a Sinigaglia (Bert.), sul Catria (Piccinini!), e sul Vettore (Parl.!), nel Romano dov'è comune (Seb. Maur. ecc.). Nell' Italia meridionale è stata trovata a Cassino (Terr.), a Napoli! in parecchi punti, in Ischia (Parl.! ecc.) e Capri (Pasq.), a Sessa nel Principato citeriore (Ten.), nel Vulture (T'err.). Nasce in Corsica a Bastia (Pucci!), Vico, Bocognano, nella valle dell’Albitrone ecc. (Mars.). Manca in Sardegna. In Sicilia fa a Pizzuta (Todaro!), S. Maria del bosco, Ficuzza, Palizzi, Castellobuono (Parlatore! ecc.), Collerano, Aidone (Guss.), Mirto (Todaro!), Messina!, sull' Etna, a Catania (Parl.! ecc.), a Caltagirone (Tar. Gerb.): la var. sicula a quanto sembra più frequente del tipo. Fiorisce da agosto a ottobre.

Distribuzione geografioa. - In tutta Europa ad eccezione delle sue parti più nordiche, nell'Asia Minore, in Siberia.

Descrizione. - La radice è tortuosa, ramosa, con i rami quasi orizzontali, scura e quasi puberula. Il fusto ì eretto, alto da poco meno di un piede a un piede e mezzo e talvolta due piedi, qualran. golare, con gli angoli ottusi, scuro, puberulo, ramoso sin quasi dalla base con i rami opposti, patenti o patentissimi, anch' essi rossicci e puberuli. Le foglie sono opposte, patenti o curvate in hasso, sessili, lanceolate, acute, con pochi e larghi denti di sega nel margine, di color verde, talvolta sfumate di rossiccio, pubescenti, con un nervo 
longitudinale grossetto da cui partono dalla base e lateralmente più in sopra della metà della foglia dei nervetti, i quali si dirigono verso l'apice di questa e mandano diverse venette che fanno una rete nel parenchima. Tali foglie cadono presto nel fusto e alla base dei rami. I fiori sono distribuiti in racemi stretti, e accompagnati da brattee simili alle foglie dei rami, o un poco più larghette, alterne, alcune delle quali sono talvolta vicinissime da parere opposte: ciascun fiore è solitario, supera di poco la brattea ed ha un piccolo pedicello, lungo appena una linea, cilindrico, rossiccio, pubescente. Il calice è tubuloso, un poco curvo, con quattro denti grandi quasi uguali tra loro, quasi lanceolati, quasi acuti, è verdognolo rossiccio con quattro nervi longitudinali e altri intermedii meno manifesti, peloso. La corolla è di color violetto roseo o violetto bianchiccio con due righe più cariche nel punto di unione delle lacinie del labbro inferiore. Il tubo è poco più lungo del calice, quasi cilindrico, puberulo verso alto. Il labbro superiore è eretto, stretto, concavo di sotto, smarginato, pubescente, massime verso il margine e la smarginatura. Il labbro inferiore è piủ largo di sotto in avanti, diviso in tre lacinie, le quali sono scanalate concave di sopra, in modo che nel punto della loro unione si vedono due linee ottuse rilevate, che sono di colore violetto, hanno l'apice tondeggiante e in esso come nel margine alcuni cigli e come piccoli denti. Il lobo intermedio è poco piủ lungo e più largo dei laterali e smarginato. Gli stami sono didinami, tutti e quattro av. vicinati al labbro superiore della corolla, inseriti all'apice del tubo presso la gola. I filamenti sono un poco schiacciati, un poco assottigliati verso alto, del colore stesso della corolla e puberuli. Le antere superiori sono appena più lunghe del labbro superiore, le inferiori quasi uguali a queste, quasi trasversali, allungate, di color rosso scuro, con le logge distinte, fornite all' apice di un mucrone lunghetto e bianchiccio, glabre, nel punto d'inserzione con il filamento sono fornite di alcune lunghe glandole stipitate, che pajono tanti peli clavati, bianchicci o violetti chiari. I filamenti sono lesiniformi, verdognoli chiari, puberuli. L'ovario è ovato-allungato, verde, pubescente, biloculare e contiene in ciascuna loggia molti ovoli anatropi inseriti alle placente saldate con il setto. Lo stilo è posto tra gli stami più alti e il labbro superiore, del quale è piủ lungo, filiforme, piủ lungo degli stami superiori, bianchiccio e puberulo in basso, violetto e quasi glabro in alto. Lo stimma è globoso, in capolino, quasi bilobo, papilloso, di un verde giallognolo. Le cassule sono allungate-obovate, ottuse, un po' schiacciate verso alto, verdi, pube- 
scenti con i peli più lunghi verso alto. I semi (non maturi) sono longitudinalmente costati e come cancellati per altre strie trasversali più leggiere. (Parl. ms., descr. di piante di Pisa e di Firenze).

\section{Dolontites verna.}

0. minute pilosa, foliis lanceolatis dentatis, floralibus flore Iongioribus, corolla purpurascente, calyce dimidio longiore, staminibus corolla vix longioribus, antheris apice barbulatis.

Euphrasia Odontites Linn. sp.pl.ed. 1. p. 604 (excl. $\beta$ ). Nocca Balb. fl. tic. 1. p. $294(\beta)$. Seb. Maur. fl. rom prodr. p. 200. Poll. 1l. ver. 2. p. 317. Nacc. fl. ven. 3. p. 137. Ten. syll. fl. neap. p. 298. Zan. prosp. fl. ven. p. 25. Hausm. fl. Tir. p. 667. Rota prosp. Il. Berg p. 68. Pir. fl. for. syll. p. $108(\beta)$. De Vis. Sacc. cat. piant. Ven. p. 158. Zersi prosp. piant. Bresc. p. 161.

Euphrasia verna Bell.! oss. bot.p. 58. App. /l. ped. p. 33. Balb. el. piant. Tor. p. 37. De Not. rep. fl. lig. p. 312.

Odontites rubra Pers. syn. 2. p. $150(\beta)$. Ing. cal. sp. Mond. p. 53.

Odontites verna Reich. /l. germ. exc. p. 359 Trev. prosp. $l$. eug. p 23. Ard. fl. Alpes-mar. p. 283. Ces. Pass. Gib. comp. pl. ilal. p. 355. Cocc. nuov. contr. /1. Bol. p. 23. Arc. comp. fl. ilal. p. 519. Cocc. /l. Bol. p. 367 .

Bartsia Odontites Bert. /. ital. 6. p. 27.. Pucc. syn. pl. Iuc. p. $31 \%$.

Ddontites vulgaris Car. prodr. A. tosc. p. 4!)1.

Figure. - 17. desn. t. 695. Reich. ic. /l. germ. 20. ᄂ. 107. f. 2 .

Stazione, Abitazione e Fioritura. - Nei campi. listata trovata in I'iemonte presso Torino alla Dora nel luogo detto val d'Oeca inferiore, a Centello verso Cuneo (Balb.), a Mondovi, ovunque (Ing.), a Dego (Bert.), a Sassello (De Not.), ad Alba (Bert.), a Nontobbio e Torriglia (De Not.), a Riva di Sesia (Carestia!); in Lombardia a Pavia sul Po (Noce. Balb.), sopra Sesto Calenda, sul Balilone in val Tellina (Bert.), nel Bergamasco (Rota), nel Bresciano, frequentissima (Zersi); in Tirolo nel Trentino (Perini!), in Fienme e Fassa, a Bolzano, Laas, Bressanone, IVelsbery (Hausm.); nel Veneto a Verona, nou rara (l'oll.), negli Euganei (Trev.), a Padovia (liert.), presso Venezia a S. Amna e Loreo (Nace.), verso Bassano a Roman e Angarano (Bert.), in Friuli a S. Daniele, Cordovato, Udine (Poll.) 
e nel Littorale (Pir.); nel Bolognese, divenuta rara, nei monti a Sabbione, e alla Porretta (Cocc.). Nell' Italia media nasce molto più sparpagliatamente: in Liguria è stata trovata a S. Martino Lantosca, Nizza (Ard.), Viosenne, Borgomaro (Ricca!), Mendatica (Gentili!); in Toscana presso Sarzana alla Magra (Bert., Beccari!), e presso Lucca a S. Alessio (Beccari!); presso Roma in luoghi boschivi intorno a Castel Gandolfo, e nel monte Compatri (Seb. Maur.); finalmente in Abruzzo nel monte di Pizzoli (Ten.). Fiorisce da maggio ad agosto secondo i luoghi.

Distribuzione geografica. - Nell' Europa media occidentale, e in parte nella settentrionale e nella meridionale, introdotta nella NordAmerica.

Osservazioni. - Oltrechè per i caratteri indicati nella diagnosi, questa specie differisce dalla precedente, della quale molti la ritengono una varietà, per la diversa distribuzione geografica, e la diversa stagione di fioritura, per la diramazione a pannocchia e non sbracciata, e per i frutti la metà più corti.

VII. EUPIIRASIA.

Euphrasia Tourn. inst. p. 174. t. 78. Gen. pl. fl. germ. 5. t. 42. Benth. Hook. gen. pl. 2. p. 976. Ces. Pass. Gib. comp. fl. ital. p. 355. t. 53. f. 2.

Calyx tubulosus, inæqualiter 4-fidus. Corolla limbo conspicue ב-labiato, labio postico conicavo marginibus reflexis, antico 3-lobo lobis divisis, Stamina 4. Antherarum loculi basi mucronati, excepti 2 longe aristati. Stigma capitellatum. Gemmulæ in loculis paucæ, subbiseriatæ, pendulæ. Capsula oligosperma.

Portamento. - Vedansi le descrizioni appresso.

\section{Euphrasia offecinalis.}

Euphrasia officinalis Linn. sp. pl. ed. 1. p. 604. Bert. fl. ital. 6. p. 287 ; et auct.

Euphrasia tricuspidata Linn. l. c.: forma foliis angustissimis cum denticulis duobus apicem versus. Bert. o. c. p. 291. Hausm. fl. Tir. p. 666. Rola prosp. fl. Berg. p. 68. Pir.! fl. for. syll. p. 108 (et E. salisburgensis l. c.!). De Vis. Sacc. cat. piant. Ven. p. 158. Zersi 
prosp. piant. Bresc. p. 161. Ces. Pass. Gib. comp. fl. ital. p. 356. Arc. comp. fl. ital. p. 520.

Euphrasia alpina Lam. enc. méth. bot. 2. p. 400: forma humilis, foliis ovato-oblongis acuminato-dentatis, floribus maximis. Ard. $\mu$. Alpes-mar. p. 283.

Euphrasia salishurgensis Hoppe bot. tasch.: forma foliis anguste lanceolatis dentibus paucis acıminatis. Mass. prodr fl. valt. p. 68 . Com. fl. com. 5. p. 13. Rota prosp. piant. Pav. p. 271 . Hausm. $l$. c. Rota prosp. fl. Berg. l. c. Ard. l. c. Terr. rel. Terr. Lav. p. 148. Sec. rel. p. 93. Ces. Pass. Gib. l. c. Terr. quart. rel. p. 111. Goir. app. bot. p. 25. Arc. l. c.

Euphrasia minima Jacq. see. Cand. fl. fr. 3. p. 473: forma humilis, foliis latiusculis breve dentatis, floribus minimis luteis. Hausm. o. c. p. 665. Rota prosp. fl. Berg. l. c. Pir.! l. c. Ard. l. c. De Vis. Sacc. l. c. Ges. Pass. Gib. l. c. Anzi auct. fl. nov.-com. p. 196. Gib. Pir. fl. Mod. p. 125. Arc. l. c. Cocc. fl. Bol. p. 368. Bizz. contr. fl. ven. 3. p. 7.

Euphrasia pectinata Ten.! fl. nap. 1. prodr. p. 36: forma stricta glaucescens, foliis latis acuminato-dentatis. Terr. 1 rel. p. 148. Sec. rel.p. 92. Ces. el. piant. Maiella p. 22. Terr. terz. rel. p. 77.

Euphrasia micrantha Reich. fl. germ. exe. p. 358: forma gracilis, foliis latiusculis, floribus minimis. Terr.! sec. rel. p. 93.

Euphrasia nemorosa Soy.-Will. euphr. off. p. 7: forma subglabra eglandulosa, foliis acuminato-dentatis. Cocc. $l . c$.

Figure.-Bocc. mus. di piant. t. 60. Fl. dan. t. 1037. Lam. ill. t. 518. f. 1, 2. Reich. ic. fl. germ. 20. t. 109-111.

Stazione, Abitazione e Fioritura. - Comunissima, e per lo piú in società, nei prati, nei pascoli, nei luoghi erbosi dei boschi, per I'Italia settentrionale e media. Nell'Alta Italia si stende dalle pianure e dalle vallate a parti altissime dei monti (2000 metri e più). Nell'Italia Media è di collina e di montagna, ossia necupa i monti dalle loro cime più alte alle loro radici: ma già in Toscana non trovasi più nella regione maremmana, nè in alcuna isola, e scarseggia nella re. gione campestre, e nelle Marche l'ultimo luogo dove si trovi in prossimità al mare è Civitanova (Ricci!). l'iù in giủ si prolınga per l'Appennino, termina dal lato orientale della Penisola al Gargano (Bert.), e dall'altro lato per il monte Gennaro sopra Rioma (Seb. Maur.) e per i monti della Campania (Terr.! ecc.) arriva al monte Vergine (Ten.) e al Vulture (Terr.!), terminando alla Sila in Calabria. In Corsica, dove sembra assai rara, nasce ai monti Renoso (Kralik!), 
Rotondo (Soleirol!) e Grosso (Bert.). Manca interamente in Sardegna e in Sicilia; e soltanto per scambio con qualche altra pianta è indicata dallo Zerapha in Malta. Le forme nane sono proprie di luoghi altissimi, fra l'altre quella detta $E$. minima, ch è sparsa per le Alpi, e si ritrova nelle più alte cime dell'Appennino toscano, e nell Ábruzzo alla Maiella a 2400 metri (Levier!), e vi si riferiscono i saggi sopra indicati di Corsica. Le forme a foglie strette sono meno comuni : l' $E$. salisburgensis nel Friuli presso Erto a 2000' (Huter Porta!), nei monti Grappa e Sumano del Veneto (Bert.), nei Lessini e nel monte Baldo (Goir.), in molti luoghi del Tirolo (Hausm. ecc.), nelle Alpi lombarde (Parl.! ecc.) dove sembra comune, cosi giù in Brianza (Morelti!), e in val Tellina a Bormio a 2000 metri (Levier!) e al Braulio (Mass.), nel Cantone Ticino(Com.), in Piemonte nelle valli d'Ossola, e della Sesia (Bir.), nei colli a nord di Torino (Colla), nel Cenisio (Aiuti!), a Crissolo (Boissier Reuter!), nei monti di Vinadio (Colla), in val di Pesio (Burnat!), a S. Anna di Vinai, S. Martino Lantosca e S. Agnese sopra Mentone (Ard.), ad Alba (Bertero!), nel Pavese (Rota), presso Lucca alla fornace di Valdottavo (Gian. nini!), nell'Appennino di Spoleto (Ball!), nel piceno-abruzzese al Vettore (Parl.!), al Corno a 1800 metri (Levier!), a Pizzoli (Bert.), al Velino (Cherici!), al Sirente, presso Caramanico (Groves!) ecc., in Terra di Lavoro sul Matese (Ten.), a S. Biagio e Frasso Telesino (Terr.); mentrechè l'E. tricuspidata è ristretta alle Alpi orientali, trovandosi nel Friuli in valle Zellina (Ball!), in val di Resia, a Ospedaletto, Venzone (Pir.!), Cordovato (Bert.), presso Longarone nel Bellunese, in val Stagna presso Bassano (Ball!), nel monte Sumano, nel Baldo (Moretti! ecc.), nei monti di Maderno sul Benaco (Cesati!), in val Trompia (Bracht!) frequente, cosi nei monti Conche e Fronden (Zersi), in val Camonica sopra Breno (Rota), in Tirolo nella valle di Vestino tra 2000 e $5000^{\prime}$ (Porta!), a S. Giovanni presso Saone, in val d'Arsa, a Brentonico, Roveredo, Riva (Hausm.), nel Trentino (Perini!), in val Sugana (Hausm.), a Salorno (Müller!) ecc. Fiorisce secondo i luoghi in maggio e giugno, o giugno e luglio, o luglio e agosto.

Distribuzione geografica. - In tutta quanta l'Europa, meno alcune sue parti più meridionali, nell'Asia Minore, e fino all' Imalaia, in Siberia, nell'America settentrionale.

Descrizione. - Pianta annua, piccola, alta da 10 a 15 centimetri, di un verde rossiccio, pelosa. La radice è fusiforme, verticale, un po'flessuosa, duretta, di un giallo-scuro. Il fusto è eretto, 
quasi cilindrico, rossiccio-scuro, peloso con peli corti, curvati in giù e avvicinati al fusto (è la forma nemorosa) e bianchi, ramoso nella parte di mezzo, con i rami più corti del fusto, ascendenti o erettopatenti, semplici o ramosi anch'essi. Le foglie sono piccole, alterne, sessili, le inferiori più piccole, patenti, ovali o ovali-bislunghe, con cinque o tre denti, ottusi o ottusetti, di un verde rossiccio, con solchi di sopra che corrispondono ai nervi di sotto, con un nervo longitudinale di sotto da cui partono dei nervi laterali, tutti un po'rilevati, che vanno in mezzo ai denti delle foglie, pelosette in ambedue le facce: le superiori sono più grandi, eretto-patenti, inciso-dentate, con 7 o 9 denti acuti, e quasi arestati, nel resto simili alle inferiori. I fiori sono quasi sessili, solitarii all' ascella di tutte le foglie superiori. Il calice è tubuloso, verdognolo, pelosetto, con cinque nervi sporgenti, diviso in quattro lacinie erette, lanceolato-cuspidate, delle quali due sono più profonde, per essere il calice diviso quasi in due metà laterali. La corolla è piủ lunga del calice, ora un po' più grande ora un po' più piccola, pelosetta di fuori, di color bianchiccio, celestognolo, o violetto chiaro, con una macchia gialla di dentro alla base del labbro inferiore, e con due o tre righe violette scure alla base del labbro superiore e dei lobi dell'inferiore, con il tubo lungo quasi quanto questo o appena piủ corto, quasi cilindrico, e con i labbri un po' disuguali, il superiore quasi in forma di elmo, con l'apice smarginato-bilobo o con i lobi troncato-smarginati, diretti in su, l'inferiore patente, trilobo, con i lobi un po'disuguáli per essere il mediano un po'più lungo dei laterali, tutti profondamente smarginati all' apice: nella gola alla base del lobo medio del labbro inferiore vi sono dei peli lunghi, un po' piủ grossi verso l'apice: Gli stami sono quattro, didinarni, sotto il labbro superiore, del quale i due più lunghi sono poco più corti, inseriti presso la gola. I filamenti sono lesiniformi, un po' curvi, glabri, bianchicci o in parte gialli. Le antere sono poste sotto l'elmo del labbro superiore: sono rivolte in avanti ed in giü, di color violetto scuro, inserite sopra della base, biloculari, con le logge distinte in gran parte, quelle delle anterc superiori barbate all'apice con i peli bianchi, quelle delle antere degli stami più corti arestate all'apice e con una aresta più lunga di quella dell'altra loggia e quasi glabre. Il pistillo è più lungo degli stami e del labbro superiore, dal quale sporge stando appoggiato in mezzo alle due antere superiori. L'ovario è ovale, ottuso, un po' schiacciato, verde, peloso-irsuto in alto. Lo stilo è molto lungo, filiforme, eretto e glabro in basso, curvato in alto verso avanti e pelosetto, bianchic- 
cio. Lo stimma è in capolino, papilloso, bianchiccio. La cassula è quasi uguale al calice, bislunga-obovata, un po'schiacciata, appena smarginata e con una piccola punta all'apice, con un solco longitudinale in ciascuna faccia, pelosetta verso alto e gialliccia, si apre lungo il margine esterno in due logge. I semi sono molti, pendenti, bislunghi, giallicci-scuri, con costole longitudinali e bianche in mezzo alle quali con una forte lente si vedono molte righe trasversali fitte. (Parl. ms., descr. di pianta di Boscolungo nell' Appennino toscano).

Descrizione della forma detta Euphrasia minima. - Pianta piccola, annua, alta da 405 a 7 e 8 centimetri, verde alquanto scura, pubescente per peli corti, applicati e rivolti in giù nel fusto. Radice delicata, fusiforme, flessuosa, bianchiccia. Fusto eretto, cilindrico, semplice, o con due o pochi rametti opposti, corti e ascenderiti che vengono dal verso la metà o anche dal basso del fusto, è rossiccio e con i peli sopradescritti. Foglie poche, piccole, opposte, patenti, ovate, con cinque denti, due per parte e uno terminale, questo è il piủ grande di tutti che sono ottusi e quasi tondeggianti all'apice, le foglie superiori che accompagnano i fiori hanno i denti ottusetti e con una piccola punta, tutte le foglie sono verde-scure, con leggieri solchi di sopra, con leggieri nervi di sotto, pubescenti in ambedue le pagine. Fiori piccoli, solitarii all'ascella delle foglie superiori, quasi sessili e diretti da un sol lato. Calice campanulato, verdognolo-bianchiccio, con quattro strie rossicce, pubescenti, con quattro denti, eretto-pafuli, quasi uguali al tubo, lanceolato-subulati, con la stria che si prolunga dal tubo. Corolla meno del doppio piủ lunga del calice, gialla, con righe rosso-scure come ora dirò ; tubo poco piủ corto dei denti del calice, tubuloso, un po'curvo; labbro superiore quasi uguale al tubo, eretto, quasi in forma di elmo, bilobo all'apice, con i due lobetti corti, e un po' divergenti, come troncati e con qual. che piccolo dente all'apice; labbro inferiore quasi uguale al superiore, diretto in avanti, bifido, con le lacinie laterali appena piủ larghe ma quasi uguali in lunghezza alla lacinia media: tutte e tre sono smarginate all'apice, e hanno tre righe longitudinali di color rosso-scuro, e tali righe sono pure nei lobetti del labbro superiore; l'elmo è di colore più pallido del resto della corolla e pubescente per peli corti ed affusati. Gli stami sono quattro, didinami, racchiusi nell' elmo del labbro superiore ma si vedono di davanti; filamenti inferiori nel tubo; antere violette scure, pelose, le due degli stami più corti fornite in basso di un mucrone lunghetto. Pistillo quasi uguale agli stami più lunghi; stilo lungo, filiforme, curvato in alto in 
avanti, glabro, bianchiccio; stimma quasi in capolino. (Parl.ms., descr. di pianta di presso Rocciamelone in Piemonte).

Osservazioni. - L'Euphrasia varia oltremodo: per la statura, da $1 / 2$ decimetro a 3 decimetri; - per il fusto, semplice, o più 0 meno ramoso; - per la pelurie, sempre minuta, ma ora mista nelle parti superiori della pianta di peli glandolosi, ora no; - per le foglie, che dalla forma largamente ovata quasi rotonda e fortemente dentata passano per gradi nella strettissima quasi filiforme e quasi senza denti, i denti medesimi essendo ora ottusi, ora acuti, ora aguzzi; - per l'infiorescenza, quando corta di fiori ristretti insieme, quando allungata di fiori distanti fra loro; - per la grandezza dei fiori, tra $1 / 2$ centimetro e $1 \frac{1}{2}$ centimetri; - per il colore della corolla, per lo più gridellino con strie pavonazze e la gola gialla, ma che può essere più chiaro bianco, o con predominio sia del pavonazzo sia del giallo da far parere tutto il fiore di una di quelle tinte.

Reićhenbach figlio (Ic. fl. germ. 20. p. 58) opina che siffatte variazioni sieno in dipendenza diretta delle condizioni esterne, e mutabili di generazione in generazione. Ove cio fosse provato, ne resterebbe convalidata la sentenza di Babington (Man. brit. bot. p. 221) che sia quasi impossibile e neanco a desiderarsi la definizione di varietà in una specie talmente incostante. Cosi la pensa anche lo Zersi (l. c.), per osservazioni fatte sulle sole piante del Bresciano. Per contro l'ultimo minutissimo lavoro sulla specie, di Townsend (in Journ. of bot., 1884), considerandola sempre unica, la spartisce in 8 gruppi, e questi in sottospecie, varietà e sottovarietà : esempio imitabile di sottile analisi disgiunta da vane creazioni nominali.

Certo si è che l' Euphrasia è un esempio assai istruttivo di quei tipi polimorfi, che nel concetto Linneano della specie si possono intendere nel loro insieme, che altrimenti non si possono piủ afferrare ove si voglia scendere a un concetto specifico più ristretto. Coloro che l'hanno tentato per l'Euphrasia sono andati nelle maggiori discordanze. Linneo fece due specie, sulla forma delle foglic. Grenier e Godron ( Fl. de Fr. 2) ne ammettono pure due, ma diverse dalle Linneane, fondate sulla dentatura fogliare, sulla pelurie e su caratteri carpici poco tangibili. Soyer-Willemet (Euphr. o/f., Nancy J83.5) ne ha $\mathbf{3}$, dedotte dalle foglie fiorali. Kinch (Syn. fl. germ. ed. 2) ne ha 4 , ossia le due di Linneo, pin la forma nana parviflora detta E. minima e la forma a foglie strette detta L. salisburgensis. Per Janka. (Scroph. europ p. 33) sono 5, le medesime di lioch, con la sua E. officinalis divisa in due a motivo della dentatura delle foglie. E cosi 
si arriva alle specie molteplici della scuola Jordaniana, alle 9 di Bo. reau, alle 17 di Grenier o di Timbal-Lagrave. Ogni autore poi ha un modo suo di schierare le varietà nelle sue specie.

\section{HA RTSCHIA.}

Bartsiæ sp. Linn. sp. plant. ed. 1. p. 602. Benth. Hook. gen. pl. 2. p. 977. Ces. Pass. Gib. comp. fl. ital. p. 354. t. 53. f. 3. Bartsia Stev. in mem. soc. nat. Mosc. Gen. pl. fl germ. 5. $t$. 43.

Calyx campanulatus, subæqualiter 4-fidus. Corolla limbo conspicue 2-labiato, labio postico galeato, antico 3-lobo. Stamina 4. Antherarum loculi basi mucronati. Stigma capitellato-bilobum. Gemmulæ in loculis plurimæ coacervatæ, subhorizontales. Capsula polysperma.

Portamento. - Vedasi la descrizione della specie nostrale.

\section{Bantsahia alpina.}

Bartsia alpina Linn. sp. pl.ed.1. p. 602. Mass. prodr. fl. valt. p. 26. Bert. fl. ital. 6. p. 267. De Not. rep. fl. lig. p. 311. Com. Al. com. 5. p. 6. Hausm. fl. Tir. p. 664. Rota prosp. fl. Berg. p. 68. Pir.! fl. for. syll. p. 108. Ard. fl. Alpes-mar. p. 284. Zersi prosp. piant. Bresc. p. 161. Ces. Pass. Gib. comp. fl. ital. p. 354. Sacc. Bizz. agg. A. trev. p. 29. Arc. comp. fl. ital. p. 518.

Figure. - Fl. dan. t. 43. Reich. ic. fl. germ. 20. t. 104. f. 1 -3.

Stazione, Abitazione e Fioritura. - Nei prati e nei pascoli umidi delle Alpi, dalle Marittime alle Friulane, comune: nell'alpe Ruscara della Liguria (Ricca!), in quelle di Tenda ai monti Formose (Bourgeau!) e Orno (Ungern-Sternberg!), a Salsamorena, in val di Giallorgue (Ard.), nelle vallate Valdensi (Rostan!), sopra Giaveno e Monpantero (Re), al piccolo Cienisio (Beccari!), nel Cenisio al lago e a Ronche (Parl.! ecc.), al lago di Combal sotto il monte Bianco (Parl.!), in val Sesia nei monti Turlo e Tiglio (Carestia!), al Sempione (Parl.!), al S. Bernardino (De Not.!), in val Vegezzo al Pizzo d'Anzasco (Bert.), nel Cantone Ticino sul Camogheo (Com.), presso Chiavenna in valle di Lei (Rampoldi!) e sul Pizzo Stella (Gib.!), su quasi tutti i monti del Lario, nelle valli Sassina e Cavargna (Comolli ecc.), sullo Stelvio tra 1200 e 2000 metri (Parl.!), e gene- 
ralmente in val Tellina (Mass.), al Tonale a 2000 metri (Parl.!), nel Bergamasco (Rota), nei monti della val Trompia, per esempio sul Dossalto a 1800 metri (Parl.!), in Tirolo presso Welsberg, in val Venosta, intorno a Bolzano, per esempio sul Ritten da $4600^{\prime}$ in su, in Fiemme e Fassa (Hausm.), intorno a Trento (Perini!), nelle Giudicarie, sul Manasso presso Borgo, sulle Vette di Feltre, sul Portole, intorno a Roveredo ecc., sul Baldo all'Altissimo (Hausm.) e nella valle delle Ossa (Manganotti!), in generale nel Veronese e Vicentino (Poll.), sul monte Grappa, nel bosco Cansiglio (Sacc. Bizz.), sul monte Cavallo di Belluno (Kellner!), per la Carnia e il Friuli (Pir.). Fiorisce in luglio e agosto.

Distribuzione geografica. - Dall' Europa artica ai Pirenei, alle Alpi, e ai Carpazi, in Groenlandia e al Labrador.

Descrizione. - Pianta perenne, pelosa, con peli aventi una glandola violetto-rossiccia nella parte superiore del fusto, nei peduncoli, nel calice e nella corolla, verde, alta circa due decimetri, fornita di un rizoma delicato, che manda delle fibre radicali e all'apice il fusto il quale è diritto, duretto, ottusamente quadrangolare, semplice, peloso, maggiormente verso alto. Le foglie sono membranacee, molli, opposte, patenti, ovate, quasi abbraccianti il fusto con la base, ottusette o quasi acute, con crenature dentate nel margine, verdi e glabre di sopra ed ivi con solchi in rete corrispondenti ai nervi della pagina inferiore, di un verde un po'pallido di sotto ed ivi pelose e con nervi rilevati in rete: le foglie inferiori sono più piccole delle altre. I fiori sono solitarii all'ascella delle foglie superiori, quasi uguali o poco più lunghi di queste. I peduncoli sono eretto-patenti, corti, più corti del calice, grossetti, con peli lunghi e glandolosi. II calice è tubuloso-campanulato, diviso in quattro lacinie lineari-lan. ciolate, ottusette, eretto-patenti, appena più lunghe del tubo, ha otto nervi, quattro dei quali giungono sino all' apice delle lacinie e quattro terminano nei seni intermedii: è di color verdognolo-violetto, e peloso con i peli lunghi e glandolosi. La corolla è due o tre volte piủ lunga del calice, tubulosa, gradatamente piủ larga verso alto, di color violetto, con molte vene longitudinali, ramose, pelosa di fuori con peli lunghi e glandolosi; i labbri sono assai piccoli: il superiore in forma di elmo è tondeggriante, l'inferiore è trilobo, con i lobi laterali un po' più piccoli dell'inferiore, il quale è lungro quanto il labbro superiore: i tre lobi sono tondeggianti anch'essi all'apice. Gli stami sono quattro, due un poco più alti degli altri, alti quasi quanto la corolla, e inseriti verso la meta di questa. I filamenti sono piani, 
di color violetto, glabri, curvati in dentro verso alto. Le antere sono giallicce, biloculari, con le logge fornite di molti e lunghi peli bianchi che la rendono irsuta. Il pistillo è appena più lungo degli stami e della corolla. L'ovario è circondato alla base dalla parte inferiore di un disco glandoloso, gialliccio, liscio: è ovato, peloso verso alto, verdognolo. Lo stilo è lungó, filiforme, un po' curvo in alto, pelosetto, di color violetto. Lo stimma è quasi bilobo, ottuso, papilloso. (Parl. ms., descr. della pianta di val Trompia):

\section{BELISARIDA.}

Bartsiæ sp. Linn. sp. pl. ed. 1. p. 602. Benth. Hook. gen. pl. 2. p. 977. Ces. Pass. Gib. comp. ft. ital. p. 354.

Bellardia All. fl. ped. 1. p. 6 / (1785) (non Schreb. gen. 1791, nec Colla).

Trixago ex parte Stev. in mem. soc. nat. Mosc. (1823). Gen. pl. $n$. germ. 5. t. 44. f. 23-26.

Calyx campanulatus, antice et postice 2-fidus lobis $2-3$-dentatis. Corolla limbo conspicue 2-labiato, labio postico galeato, antico 3-lobo. Stamina 4. Antherarum loculi basi mucronati. Stigma capitato-bilobum. Gemmulæ in loculis plurimæ, coacervatæ, subhorizontales. Capsula myriosperma, lignosa.

Portamento. - Vedasi la descrizione dell' unica specie del ge. nere.

Osservazione. - Il nome generico di. Bellardia dev' essere preferito a Trixago non solo per ragione di anteriorità, ma perchè de. signa il genere come qui è accettato, nel mentrechè la Trixaga di Steven abbraccia inoltre l'altro genere che Grisebach più tardi ne separò col nome di Eufragia. D'altronde non sono state accettate le due Bellardia di Schreber nelle Rubiacee e di Colla nelle Asteracee.

\section{Bellardia Trixngo.}

Bartsia Trixago Linn. sp. pl. ed. 1. p. 602. Mor. De Not. fl. Capr. p. 101. De Not. rep. fl. lig. p 311. Bert. fl. itâl. 6. p. 270. Tar. Gerb. cat. pl. calat. p. 29. Mor. fl. sard. 3. p. 234. Ard. fl. Alpes-mar. p. 284. Pasq. fl. ves. p. 76. Terr. fl. Vult. syn. p. 131. Rel. Terr. Lav. p. 149. Duthie bot. malt. isl. p. 6. Ces. Pass. Gib. 
comp. fl. itul. p. 354. Terr. ! quart. rel. Terra di Lav. p. 111. Arc.! comp. fl. ital. p. 518. Cocc. fl. Bol. p. 367.

Bellardia Trixago All. $f$. ped. 1. p. 61.

Trixago apula Stev. in mem. soc. nat. ilosc. Grech Del.! f. mel. p. 28. Car. prodr. fl. tosc. p. 490. Genn.! fl. Capr. p. 113. Mars. cat. pl. Cors. p. 109. Groves contr. Terr. d' Olr. p. 6\%. Loj. is. eol. p. 121. Nic.! prodr. fl. mess. p. 339.

Figure, - Col. ecphr. p. 197. Cand. ic. pl. Gall. t. 10. Fl. groec. t. 585. Reich. ic. fl. germ. 20. t. 103.

Stazione, Abitazione e Fioritura. - Nei campi, nei prati ecc. dell' Italia meridionale e centrale. Fa nelle isole di Malta (Grech!) in parecchi luoghi (Grech), e Comino (Duthie); in Sicilia ovunque (Guss.): Girgenti (Aiuti!), Avola (Bianca!), Siracusa (Cassia!), Caltagirone (Tar. Gerb.), Catania (erb. Webb!), Messina (Nic.!), Castelbuono (Minà!), Palermo (Parl.!) ecc., e nelle Egadi e le Eolie (Guss. ecc.). In Sardegna è stata trovata a Clagliari (Ascherson!), a Macomer (Marcucci I), a Sassari!, e nelle isolette Caprera, Maddalena, S. Stefano (Genn.). In Corsica nasce nella costa a Bonifacio (Jordan!), Aiacció (Requien!), Calvi (Soleirol!), Bastia (Bert.). Nella Penisola principia dalla Calabria a Monteleone, Pizzo, S. Venere (Arc.!), la Sile (Ten.), e da Otranto (Porta Rigo!), e Zittarella presso Taranto (Aiuti!), e prosegue per la Basilicata a Castelgrande (Bert.) e IIelfi (Terr.), per il Principato citeriore ad Agropoli e Pesto (Ten.), per Capri (Pasq.), Capua (Terr.) e Traetto in Terra di Lavoro (Terr.!), Roma (Rolli! ecc.) e Tivoli (Seb.), Civitavecchia, Viterbo e Canino (Bert.), per terminare in Toscana al monte Amiati (Bert.), a Grosseto, Siena!, Monteguidi di val di Cecina (Bert), Volterra (Amidei!), Mortaiolo nel Pisano (P. Savil), Malmantile!, Firenze all'Impruneta (Ricci!) e in val di Mensola (lieboul!), e nelle isole del Giglio (Bert.), della Pianosa (Simonelli!), dell'Elba al monte Serrato (Caldesi!) e della Capraia (Mor. De Not.). Quindi saltando tutta la Liguria ricomparisce a Villafranca di Nizza (Durando!). Dall'altro lato della Penisola prosegue per la Puglia al Pantano salso presso Manfredonia (Ten.), per il monte Morrone in Abruzzo (Bert.), per Pescara (Kuntze!), Fermo (Marzialetti!), Civitanuova (Ricci!), Macerata (Bert.), e termina a Tossignano nel Faentino, e sotto il monte Sabbione di contro a Pontechio nel Bolognese (Bert.); ricomparendo poi in Istria (Nym.). Fiorisce secondo i luoghi in aprile e maggio, o in maggio e giugno.

Distribuzione geografioa. - Questa pianta largamente diffusa 
occupa tutto il bacino Mediterraneo, la Francia occidentale, l'Asia occidentale fino al Caspio, l'Abissinia, le Canarie, il Capo, il Brasile meridionale e l'Uruguai.

Descrizione. - Erba annua. Radici a ciuffo, sottili, scure. Fusto eretto, semplice, alto 3-6 decimetri, quadrangolare, rosseg. giante, coperto di peli minuti rivoltati in giù. Foglie opposte, o pressochè, patule, sessili, lineari-lanciolate, grossamente dentate, 1 -nervate, minutamente pelose in ambedue le pagine. Spiga terminale, folta, indefinita, di cui il graspo e le brattee con i calici fiorali dentro e fuori sono glandolosi-irsuti e vischiosi. Brattee inferiori simili alle foglie ma slargate alla base; le superiori ovate-aguzzate in. tere. Fiori ascellari, sessili. Calice campanulato, ampio, lungo un centimetro, 2-fido avanti e dietro, coi lobi eretti, ottusamente 23-dentati. Corolla gialla, inchinata, il doppio più lunga del calice, glabra dentro, e fuori glandolosa-pelosa, principalmente nell' elmo, dal tubo obliquo, della lunghezza del calice, dal lembo con i labbri sporgenti, il superiore concavo-carenato, intero, ottuso, l'inferiore un po'piủ lungo , 3-lobo (i lobi essendo arrotonditi, interi, spiegati, il me. diano molto più piccolo dei laterali), con nel mezzo di qua e di là una piega allungata e più alta in avanti. Stami inseriti nel mezzo del tubo, i 2 posteriori più corti un poco più in alto degli anteriori, racchiusi nell' elmo: talora avvene un $5^{\circ}$ più o meno sviluppato; filamenti gialli, glabri, compressi nelle faccie, lesiniformi, un po' ingrossati all'apice, assottigliati alla base, lunghi 1 centimetro; antere gialle, pressochè ovali, attaccate fortemente presso l'apice del loro dorso, smarginate all'apice, divise in basso oltre la loro metà, lunghe 3 millimetri, con le caselle mucronate alla base e lungamente barbate soprattutto in basso, deiscenti per lungo inentro e boccheggianti alla base: sono le antere collocate orizzontalmente sul fianco per maniera che le caselle stanno l'una sopra l'altra, che il loro apice sta voltato verso la parte posteriore del fiore, e che le antere degli stami posteriori sono poste sotto le altre, essendo tutte e quattro voltate faccia a faccia e riunite per mezzo dei loro lunghi peli intrecciati, i quali sono diretti in dietro, molli, bianchi, colorati e intrecciati alla fioritura, costituiti da una sola cellula tutta bernoccoluta; polline giallorino, ellittico, ottuso, lungo $0^{\mathrm{mm}}, 045$, pieghettato. Disco nullo. Gemmulario con le caselle uguali, ovato, irsuto (i peli diretti in su), lungo 3 millimetri, che passa nello stilo filiforme, pelosetto, più lungo degli stami e incurvato sopra di essi, terminato da uno stimma globoso a capocchia, alquanto 2-lobo, col lobo inferiore più grande; gemmule nu- 
merosissime, perpendicolari sullo spermoforo, anatrope, con integumento scempio (v. v., descr. della pianta di Malmantile presso Firenze).

Osservazione. - I fiori sono di più colori, ora interamente gialli, ora misti di giallo, di porporino e di bianco, ora di porporino e di bianco soltanto.

\section{PAR ENTUCELGA}

Trixago ex parte Stev. in mem. soc. nat. Mosc. Gen. pl. fl. germ. 5. t. 44. f. 1-22.

Parentucellia Viv. A. lib. spec. p. 31. t. 21.f. 2 (1824).

Trixago ex parte et Eufragia Gris. spic. fl. rum. 2. p. 13.

Eufragia Benth. in Cand. prodr. 10. p. 542.

Bartsia ex parte Benth. Hook. gen.pl. 2. p.977. Ces. Pass. Gib. comp. fl. ital. p. 354. t. 53. f. 3.

Calyx tubulosus, subæqualiter 4-dentatus aut fissus. Corolla limbo conspicue 2-labiato, labio postico galeato, antico 5-lobo. Stamina 4. Antherarum loculi basi mucronati. Stigma capitato-bilobum. Gemmulæ in loculis plurimæ, coacervatæ, subhorizontales. Capsula myriosperma, membranosa.

Portamento. - Sono due erbe annue, erette, alte 1-4 decimetri, semplici o ramose, coperte di peli glandolosi, con foglie piuttosto piccole, fortemente dentate. I fiori sono piccoli e porporini in una specie, grandetti e gialli nell'altra; in amendue sono solitari sessili all' ascella di foglie fiorali, e formano spighe fitte o rade nell'estremità del fusto e dei rami.

Osservazione. - Viviani fondò il genere Parentucellia sopra una pianta della Pentapoli, che chiamò $P$. foribunda, e che dopo di lui sembra non sia stata più veduta da alcun botanico. Per quanto malamente in più punti egli la descrivesse e la figurasse, nondimeno vi si riconosce facilmente una congenere dell' Euphrasia latifolia Linn., come ebbe subito a rilevare Zuccarini nella Flora pel 182.4 ( anzi una specie intermedia a quella, di cui ha il fogliame, e alla Bartsia viscosa Linn., di cui ha $\mathrm{i}$ fiori, senonchè sareblero porporini e non gialli. Si consultino in proposito De Notaris nell' Ind. seminum dell'orto di Genova, anno 18.40, a pag. 16, e Bertoloni, Fl. ital.6.p. 279 (questi elbe in esame saggi antentici della pianta), i quali 
videro che per quanto fosse tale pianta vicina all' Euphr. latifolia, pure non era da confondersi con lei, come hanno pensato Tenore (Syll. p. 300) e Webb (Phyt. can. 3. p. 152). Insomma il genere già antico Parentucellia Viv. corrisponde alla Eufragia dei moderni.

\section{Parentucellia Iatifolia}

P. foliis palmatinerviis, dentato-incisis, calyce dentato, corolla purpurascente, raro alba vel flavida, calyce vix longiore, antheris glabris.

Euphrasia latifolia Linn. sp.pl.ed.1.p.604. Bart.! cat. piant. sien. p. 19. Sant. viagg. 2. p. 311. Pucc. syn. pl. luc. p. 318. Pasq. fl. ves. p. 76. Pasq. Lic. viagg. Garg. p. 21.

Trixago purpurea Stev. (1823).

Bartsia latifolia Sibth. Sm. fl. groe. 6. p. 69. Mor. De Not. fl. Capr. p. 102. Trev. prosp. 1. eng. p. 23. Bert. fl. ital. 6. p. 276. Tar: Gerb. cat. pl. calat. p. 29. Ard. fl. Alpes-mar. p. 284. Terr. fl. Vult. syn. p. 132. Groves! contr. fl. Terr. d'Otr. p. 64. Terr. rel. Terr. Lav. p. 149. Ces. Pass. Gib. comp. fl. ital. p. 354. Arc.! comp. fl. ital. p. 518. Cocc. fl. Bol. p. 367.

Bartsia purpurea Dub. bot. gall. 1.p. 354. De Not.! rep. fl. lig. p. 312.

Trixago latifolia Reich. fl. germ. exc.p. 360. Koch syn. fl. germ. ed. 2. p. 627. Rota! prosp. piant. Pav. p. 271. Prosp. fl. Berg. p. 68. Mor.! fl. sard, 3.p. 232. De Vis. Sacc. cat. piant. Ven. p. 157. Zersi prosp. piant. Bresc. p. 161. Gib. Pir. fl. Mod. p. 125.

Eufragia latifolia Gris. spic. fl. rum. 2. p. 14. Guss. enum. pl. Inarim. p. 243. Car. prodr. fl. tosc. p. 489. Fl. Montecr. p. 27. Genn. fl. Capr. p. 113. Mars. cat. pl. Cor'se p. 109. Cald.! fl. fav. tent. p. 173. Nic. prodr. fl. mess. p. 340.

Eufragia atroviolacea Dur. in Blanco cat. pl. hisp. (1849).

Figure. - Barr. ic. t. 276. f. 3. Sibth. Sm. o. c. t.586. Reich. ic. fl. germ. 20. t. 104. f. 4.

Stazione, Abitazione e Fioritura. - Nei luoghi erbosi asciutti per tutta l'Italia, ma solo nelle regioni più basse e calde. Perciỏ scarseggia nell'Alta Italia, dove pertanto è stata trovata a Torino (Balbis! ecc.), Alba (Bertero!), Vercelli (Cesati!), Confienza (Bert.), Novara (Bir.), Pavia (Rota! ecc.), Bergamo (Rota), Brescia (Lanfossi!), Mantova (Magnaguti!), Valeggio e Custoza (Rigo!), Verona 
(Manganotti! ecc.), negli Euganei (Trev.), nel Polesine (De Vis. Sace.), in Istria tra Parenzo e Pola (Bert.), presso Parma (Passerini!), nel Modenese a S. Valentino (Pirotta!), Sassuolo e Pavullo (Gib. P'ir.), intorno a Bologna (Bert., Coce., Beccari!), a Faenza (Cald.!), ovurque in pochi siti. Seguita ed essere piuttosto rara in Liguria, vi nasce a Nizza, Berra e Fraca (Arú.), a Vado (Ardissone!), Savona (Carrega!), Sestri (De Not.!), Genova (Bert. ece.); abbonda più in Toseana, nella regione campestre e soprattutto nella maremma: a Sarzana (Bert.), a Lucca (Calandrini! ecc.), e su in val di Serchio fino ad Anchiano (Giannini!), a Pisa (P. Savi! ecc.), a Volterra (Amidei!), in val di Pesa (Baroni!), e sino intorno a Firenze risalendo il val d'Arno!, in Mlaremma a S. Vincenzo, nel Grossetano (Parl.!), e fino a Siena (Savi! ecc.) e a Pienza (Santi), nell' Orbetellano!, nelle isole di Capraia (Mor. De Tot.), dell' L'ba (WatsonTaylor!) e di Montecristo (Wats.-Tayl.); fa nelle Marche a Urbino, a Sinigaglia (Bert.), al Catria (Piccinini!), a Lorets (Bert.), Macerata (Narducci!), S. Severino (Aiuti!), Montefortino (Marzialetti!), Ascoli (Bert.), nell'abruzzo a Pescara (Kuntze!), nell' Umbria a Foligno (Ricei!), nel Lazio a Canino (Bert.), a Roma (Barbieri!) dove la si dice comunissima (Seb. Maur.), a Nettuno (Bert.). Nell'Italia meridionale è indicata al Saldo di Fondi (Ten.), a Caserta, nei monti Tifati (Terr.), a Napoli (Heldreich!) in piủ siti (Ten., Pasq.), in Ischia (Guss.) e Capri (Pasq.), a Melti (Terr.), ad Ariano (Bert.), nel Gargano (Pasq. Lic.), ad Otranto (Groves!), in Calabria a Pizzo (Arc.!) e Nicotera (Ricca!). É detta comunissima in Corsica, cosi a Bastia (erb. Webb!), Calvi (Soleirol!), Aiaccio (Bert.); frequente eziandio in Sardegua (Mor.), cosi a Cagliari, ed Iglesias (Gennari!). In Sicilia sembra abbondante del pari: a Palermo, Castelbono (Parl.!), Messina (Nic.), nel monte Scuderi (Seguenza!), a Catania, Militello (Heltreich!), Caltagiroue (Tar. Gerb.), Caltanisetta (HelAreich!), Avola (Bianca!) ecc. Fiorisce in aprile e maggio, o in inarzo ed aprile.

Distribuzione geografioa. - In tutto il bacino Mediterraneo, nell'Asia occidentale fino in Persia.

Desorizione, - Fiori sessili, solitarii all'aseclla di foglie fio. rali, le quali sono eretto-patenti, quasi crenate, palmato-lobate, le inferiori con cinque, le superiori con tre lobi, dei quali quello di mezzo ıи po piü grande, ottusi, verdornoli, peloso-glandolosi. Calice tubuloso, yıuasi un po'gonfio, verdogngolo-bianchiccio in basso con 4 nervi longitudinali verdognoli, con $\&$ denti o lacinie 
eretto-patule, lanceolate, ottusette, verdognolo-rossicce, pelosoglandoloso di fuori nei nervi e nelle lacinie. Corolla poco piủ lunga del calice, piccola, col tubo lungo quanto il calice, un po'curvo, un po' più grosso in basso e gradatamente un po'ristretto in alto, bianchiccio, glabro, pelosetto all'apice; labbri porporini e pelosi in basso di fuori, bianchi e glabri nel resto, l'inferiore con le due gibbositå del palato gialle; labbro superiore diretto un po' in su a guisa di elmo, cioè convesso assai di fuori ed ivi peloso verso l'apice, concavo di sotto con una fessura longitudinale piuttosto stretta; labbro inferiore diretto in avanti, patente, trilobo con i lobi quasi uguali, piccoli, ovali-tondi: gibbosità del palato lunghette, bislun. ghe-lineari, molto sporgenti, parallele, gialle e glabre. Stami 4 , racchiusi nel labbro superiore, due più corti e due più lunghi, inseriti nella parte superiore del tubo; filamenti ascendenti, lesiniformi, verdognoli in basso, bianchicci in alto, glabri; antere con due logge divergenti, bislunghe, ottuse, giallicce. Pistillo lungo quanto gli stami più lunghi. Ovario ovato-acuminato, un po'schiacciato la. teralmente, ivi con un solco longitudinale, altro più superficiale negli angoli, verdognolo, glabro; stilo più lungo dell'ovario, lesiniforme, un po' curvo, bianchiccio, glabro ; stimma grosso, in capolino, quasi bilobo, papillare, giallo. Fusto cilindrico, verdognolo, peloso, con peli corti, orizzontali, bianchi. Foglie un po' patenti, larghette, ovali-tonde, verdi, di sopra con solchi longitudinali profondi, di sotto più chiare con nervi o pieghe convesse longitudinali divise da solchi, coi margini un po'rovesciati. (Parl. ms., descr. della piantn. di S. Vincenzo in Toscana).

\section{Parentucellia viscosa.}

P. foliis penninerviis, dentatis, calyce fisso, corolla lutea, ca. lyce etiam duplo longiore, antheris pilosis.

Bartsia viscosa Linn. sp. plant. ed. 1. p. 602. Mor. De Not. fl. Capr. p. 101. De Nol. rep. fl. lig. p. 311. Pucc.! syn. pl. luc. p. 318. Bert. fl. ital. 6. p. 269. Ard. fl. Alpes-mar. p. 284. Tar. Gerb. cat. pl. calat, p. 29. Groves contr. fl. Terr. d'Otr. p. 64. Terr.! rel. Terr. Lav. p. 149. Ces. Pass. (Gib. comp. fl. ital. p. 354. Terr. quart. rel. p. 111. Arc.! comp. R. ital. p. 518.

Rhinanthụs viscosa Sant. viagg. 2. p. 125.

Trixago viscosa Reich. fl. germ. exc. p. 360. Mor.! fl. sard. 3. p. 233. 
Eufragia viscosa Benth. in Cand.prodr. 10. p. 543. Guss. enum. pl. Inarim. p. 243. Ard. cat. pl. Ment. p. 27. Car. prodr. fl. tosc. p. 490. Genn.! fl. Capr. p. 113. Hars. cat. pl. Cors. p. 109. Archb. n. Allo-Serch. p. 58. Nic. prodr. fl. mess. p. 340.

Figure. - Barr. ic. 655. Reich. ic. fl. germ. 20. t. 105.

Stazione, Abitazione e Fioritura. - Nei prati e nei campi umidi dell'Italia centrale e meridionale, e principalmente sul mare. Trovasi a Nizza al Varo, alla Rocchetta, a Mentone al capo Martino (Ard.), a Oneglia (De Not.), Diano (Ricca!), Albenga (Piccone!); quindi al golfo della Spezia (De Not.), a Sarzana (Bert.), Massa (Gemmi!), Lucca (Pucc.! ecc.), e sino in val di Lima (Archb.), a Pisa!, Volterra (Bert.), alla Badiola e a Port'Ercole nella llaremma toscana!; poi a Roma (Wcbb!) frequente, e 0stia (Scb. Maur.), in Terra di Lavoro a S. Giovanni Incarico, a Caserta (Terr.) e a Sora (Terr.!), a Napoli (Ten., Bert.), in Ischia (Guss.) e in Capri (Bert.), ad Eboli, a Omignano (Ten.), in Calabria a Pizzo e Rossano (Arc.!), a Otranto (Porta Rigo!), a Pietracatella in Molise (Avellino!). Trovasi ancora nell'isola di Capraia (Mor. De Not.); in Corsica a Bastia (Kesselmeyer!), Calvi (Bert.), Aiaccio (Mars.), Bo. nifacio (Kralik!) ecc.; in Sardegna (Mor.), e nelle isolette Caprera (Genn.!), Maddalena (Mor.) e S. Stefano (Genn.); in Sicilia a Palermo (Parl.!), Castelbuono (Minà!), Messina (Seguenza!), Siracusa (Cassia!), Avola (Bianca!), Scoglitti (Aiuti!), Caltagirone (Tar. Gerb.) Fiorisce in aprile e maggio, 0 in maggio e giugno.

Distribuzione geografica. - Nell'Europa sull'Atlantico, nelle isole Madera e Canarie, in tutto il Mediterraneo, nell'Asia occidentale fino in Persia.

Descrizione. - Pianta annua, alta da 1 a 4 decimetri, di un verde-chiaro, che diventa nera quando si secca, peloso-glandolosa, per peli bianchi, alcuni senza glandola e piủ corti e altri piủ lunghi aventi all'apice una glandoletta gialliccia, che rende la pianta un po'vischiosa. La radice ì corta, quasi orizzontale, ramosa, flessuosa, bianchiccia. Il fusto é eretto, semplice o talvolta ramoso, con i rami ascendenti o erelto-patenti, piü corti del fusto, cilindrico, verdognolo o in parte rossiccio. Le foglie sono sessili, patenti, le inferiori opposte, le supreme alterne, lontanette, bislunghe-lanceolate, e le inferiori piu corte, bislunghe-ovali o ovali, nttuse o ottusetle, crenato-seghettate per ilenti larghi, ottusi e un po'iliretti verso l'apice della foglia, di un verle-chioro di sopra ed ivi con un solco longiunlinale e con solchi laterali che corrispondono ai nervi di solto, di 
un verde un po'più pallido di sotto, e con un nervo longitudinale rilevato e dei nervi laterali quasi ugualmente rilevati che semplici 0 biforcati terminano ai denti del margine. I fiori sono quasi sessili, in una spiga ora più ora meno lunga, ottusa e rada, accompagnata da brattee che sono patenti, simili alle foglie superiori ma piủ strette, e con i denti del margine meno ottusi, piủ lunghe dei calici e talvolta uguali o quasi uguali ai fiori. Il calice è anch'esso peloso-glan. doloso, come le foglie, le brattee ed il fusto, è verdognolo, con nervi longitudinali, tuboloso, diviso fin poco più su della metà in quattro lacinie erette, lanceolato-lineari, ottusette. La corolla è poco più del doppio più lunga del calice, di un giallo chiaro, peloso-glandolosa di fuori. Il tubo è stretto, lungo, con un angolo di sopra; il labbro superiore ha una carena di sopra che si continua con l'angolo del tubo, è profondamente concavo di sotto, ovale-bislungo, ottuso all'apice ch'è diretto in avanti; il labbro inferiore è più lungo e più largo del superiore, trilobo, con i lobi quasi uguali, l'intermedio essendo un po' piủ stretto e un po'più lungo, tutti e tre bislunghi, tondeggianti all'apice, diretti in avanti: dal seno di ciascun lobo alla gola è di sopra una giblısità longitudinale, ottusa di sopra, concolore, cosi vi sono due gibbosità parallele. Gli stami sono quattro, didinami, nascosti nella concavità del labbro superiore, inseriti nella parte superiore del tubo. I filamenti sono quasi piani, un po' curvi in avanti verso l'apice, giallicci, con qualche corto peletto; le antere sono poste trasversalmente, biloculari, con le logge mucronate all'apice, aprentesi ciascuna per fessura distinta, giallicce, barbate. Il pistillo è più lungo degli stami e del labbro superiore, dal quale uscendo sporge curvato ivi in avanti ed in giù. L'ovario è bislungo, un po' schiacciato dalle parti, quasi acuto, verde, pelosetto in alto. Lo stilo è molto lungo, filiforme, pelosetto, gialliccio-bianchiccio, verdognolo verso l'apice dove è curvato in giù. Lo stimma è grosso, più largo trasversalmente, quasi in capolino, quasi bilobo, papilloso-peloso, verdognolo. (Parl. ms., descr. di pianta coltivata). 


\section{TRIBU் TERZA.}

\section{Digitalee.}

'Digitalee Benth. Hook. gen. pl. 2. p. 921.

Antherarum loculi vix listincti vel confluentes. Fructus septifragium.

\section{VERONICA.}

Veronica Tourn. inst. p. 143. t. 60. Duvau in ann. sc. nat. 8. p. 163. t. 26, 27. Gen. pl. 1. germ. 5. t. 38. Benth. Ilook. gen. pl. 2. p. 96 't (an excl. sect. 2 priores?). Ces. Pass. Gib. comp. fl. ital. p. 349. t. 53. f. 5.

Calyx patens, plus minus inæqualiter $4-5$-partitus. Corolla limbo rotato, inæqualiter 4-partito, lobo antico minore. Stamina 2. Antherarum loculi basi mutici. Stigma minusculum. Gemmula in loculis plures, subhorizontales, rare pendulæ. Septifragium poly oligospermum.

Portamento. - E assai svariato. Sonovi nel genere erlue, annue o perenni, di diverso modo di vegetare, suffrutici e frutici. Alcune specie alzano da terra pochi centimetri, altre vanno fino a 1 metro, alcune sono sdraiate radicanti, altre cespugliute. Le foglie sono opposte, talora verticillate, oppure (le fiorali) sparse, generalmente sessili o quasi sessili, dentate, di rado maggiormente divise o intere, di forma fra l'arrotondita e la lanciolata strettissima. I fiuri sono piü o meno piccoli, pur tuttavia i meno piceoli sono spesso vistosi per il bel colore turchino, altri sono smorti, altri bianchi; breve. mente o più lungamente pedicellati, stanno a distanza all'ascella di foglie fiorali sparse, oppure di brattee parimente sparse, e allora sono ravvicinati da formare un grappolo rado o filto, lungo o corto, ora terminale al fusto che da quello resta determinato, ora ascellare a foglie di un fusto indeterminato. Le infruttescenze conservano le faltezze delle inliorescenze.

- Caulis indefinitus, apice foliifer, ultra inflorescentiam protractus et eo modo perennans, inflorescentia e racemis axillaribus 


\section{Veronica apleylla.}

V. foliis ex obovato ovalibus, in petiolum brevem attenuatis, obtusis, crenato-subdentatis, racemis paucifloris, laxis, pedicellis fructiferis erectis, bracteis longioribus, lobis calycinis oblongis, subæqualibus, septifragio compresso, ovali vel oblongo leviter emarginato, vel obcordato-rotundato, calyce maiore, stylo septifragio breviore vel longiore.

Veronica aphylla Linn. sp. plant. ed. 1.p. 11. Vitm. ist. erb. p. 16, 46. Bert.! fl. ital. 2. p. 68. Mass. prodr. 1. valt. p. 199. Com. fl. com. 1. p. 11. Coll. herb. ped. 4. p. 336. Sang. cent. prodr. fl. rom. p. 5. De Not. rep. fl. lig. p. 302. Sim. fl. alp. vers. p. 12. Hausin. fl. Tir. p. 637. Rota prosp. fl. Berg. p. 66. Pir.l fl. for. syll. p. 105. Bicch. agg. fl. lucch. p. 15. Car. prodr. fl. 10sc. p. 487. Ard. fl. Alpes-mar. p. 275. Zersi prosp.piant. Bresc. p. 156. Terr.! sec. rel. Terr. Lav. p. 92. Ces. el. piant. Maiell. p. 22. Archb. fl. Alto-Serch. p. 58. C'es. Pass. Gib. comp. fl. ital. p. 351. Terr.! quart. rel. p. 110. Sacc. Bizz. agg. fl. trev. p. 27. Arc. comp. fl. ital. p. 514.

Veronica longistyla Ball! in ann. nat. hist. 1850. Car. suppl. prodr. fl. tosc. p. 42.

Veronica saxatilis Terr.l rel. Terr. Lav. p. 148 (non Linn. fil.).

Figure. - Bocc. mus, piant. rar. t. 1. Reich. ic. fl. germ. 20. t. $86 . f .2$.

Stazione, Abitazione e Fioritura. - Nei pascoli sassosi dei monti altissimi. Per tutte le Alpi : nella Carnia e nel Friuli (Pir.!), sul monte Cavallo (Kellner!), nel Cansiglio (Sacc. Bizz.), sulle Vette di Feltre, nei monti Portole e Grappa, e alle Laste presso Rubbio nel Vicentino (Montini!), sul Baldo a Costabella (Manganotti!) e nelle valli Fredda e Novesa (Poll.) e sull'Altissimo in Tirolo presso Roveredo, presso Folgaria, al Bec della Filadonna (Hausm.), in val Su. gana (Ambrosi!), in Fiemme e Fassa (Bert.! ecc.), sullo Schlern, in val Venosta sul Wormser, presso Trafoi e in val Martello (Hansm.), nel Trentino sul Bondone ecc. (Perini!), nelle Giudicarie sul calcare, rara, cosi in val Grande presso Bolbeno (Hausm.), nel Bresciano sui monti Dos alto a 1700-2000 metri (Parl.!) e Pesseda, frequente (Zersi), nel Bergamasco (Rota), in val Tellina generalmente (Mass.), cosi sullo Stelvio da 1700 a 2400 metri, sul monte Plaghera a 
2600 metri (Parl.!), in valle Malenco (Gib.!), nelle alpi di Zana, Forame e Saline, nel Comasco sui monti Legnone, Codeno, Camisolo, Pizzo dei tre Signori, Varrone nella val Sassina, Pizzo di Gino in Cavargna (Com.), nel S. Bernardino (De Not !), in val Sesia ad Alagna sull'alpe Olen, in val d'Aosta a St. Jacques in valle Challant (Carestia!) e a Gressoncy la Trinité sulla Betta Furke (Piccone!), al S. Bernardo (erb. Webb!), al Cenisio a Ronche e alla Ramasse (Parl.!), nei monti Valdensi (Rostan!), nelle alpi Marittime al col di Tenda, a Fontanalba, a Scianarè (Ungern-Sternberg!), nei monti Bego e Monnier (Ard.), sopra Viosenne (Ricca!), infune nei monti di Triora (Rosellini!) e d'Albenga (De Not.). In Toscana nelle alpi Apuane al Sagro, al Pizzo d'uccello, al Giovo (Bert.), alla Tambura! e alla Pania (Ball!), sull'Appennino presso Barga (Archb.), a Giovo (Bicch.), e a Mandromini (Vitm.). Nel Piceno-Abruzzo sui monti Priore, della Sibilla alla Corona (Marzialetti!), Vettore(Gemmi!), Costone (Bert.), le Moricane, la Stanca, Corno ad Arapietra (Orsini!) e al Corno grande a 2500-2700 metri, Coccorello (Levier!), Velino (Cherici!), della Naiella al monte Amaro (Pedicino!) e altrove (Ces.). Termina poco piu al sud nel monte Meta sopra Picinisca e Settefrati (Terr.!), e sopra il Cantro nei monti di Filettino (Rolli!). Fiorisce in luglio e agosto.

Distribuzione geografica. - In Spagna e nei Pirenei, nel Giura, nelle Alpi, nell'Appennino, nella penisola Balcanica e fino in Transsilvania e in Galizia.

Descrizione. - Pianta formante graziosi cespuglietti, alta appena 3 o 4 centimetri, fornita di peli bianchicci e lunghetti che sono glandolosi nella parte superiore del peduncolo, nelle brattec e nel calice. Ha un rizoma delicato e ramoso, che manda in giì fibre radicali, delicate e all'apice di ciascun ramo in su un cespuglietto di foglie, le quali sono avvicinate quasi in giro, sono verdi, membranacee, ovali o obovate, e le inferiori più piccole quasi orbicolari, un $0^{\circ}$ concave di sopra e un po'convesse di sotto dove hanno un nervo longitudinale poco rilevato, da cui partono dei nervetti laterali ramosi : sono dentate in margine con pochi denti oltusetti, orvero sono intere. Il peduncolo ì eretto, delicato, cilindrico, verdognolo, porta da 1 a 4 fiori e poco piu in sotto poche brattee eretto-patenti, simili per la consistenza ed il colore alle foglie ma di forma allungato-lanceolata, ottusette o ottuse e fornite di ciglia e di peli glandolosi. I pedicelli sono dapprima cernui, poi eretti nel frutto, delicati, rossicci, più lunghi del liore. Il calice è verdognolo- 
rossiccio, peloso-glandoloso, è diviso in quattro lacinie ovali-allungate, ottusette, eretto-patenti, poco più Junghe del tubo. La corolla è più del doppio più grande del calice, con il tubo cortissimo, bianchiccio, e con il lembo diviso in quattro lacinie molto aperte, delle quali la superiore è più larga, quasi orbicolari-rombee, ottusette, di colore violetto con vene ramosette più cariche: la gola è glabra, gialliccia con raggi corti ugualmente giallicci. Gli stami sono due, lunghi quasi quanto la corolla, inseriti in basso del tubo, posti tra la lacinia piủ larga e le due laterali. I filamenti sono filiformi e violetti. Le antere sono quasi ovate, smarginate alla base, inserite nel dorso sopra di questa, estrorse, biloculari, di colore violetto scuro. Il pistillo è lungo quasi quanto gli stami. L'ovario è ovale, schiacciato da avanti in dietro, verdognolo, con peli glandolosi. Lo stilo è molto più lungo dell' ovario, filiforme, violetto, glabro. Lo stimma è quasi in capolino, verdognolo-gialliccio. (Parl. ms., descr. della pianta di monte Gerle nelle alpi Bresciane).

Osservazione. - Questa pianta varia per la grandezza e la forma del frutto, e per la lunghezza dello stilo, ch'è di 2 a 6 millimetri. $\mathrm{Su}$ di ciò vedasi una mia Nota nel Nuovo giorn. bot. ital. 1. p. 292, e i Compendi.

\section{Veronica offeinalis.}

V. foliis breviter petiolatis, ovalibus, subacutis, serratis, racemis multifloris, laxiusculis, pedicellis fructiferis erectis, bracteis brevioribus, lobis calycinis 4 lanceolato-linearibus, subæqualibus, septifragio compresso, obverse triangulari, nunc late emarginato obcordato, nunc truncato, calycem conspicue superante, stylo longitudine septifragii.

Veronica officinalis Linn. sp. plant. ed. 1. p. 11. Bert. A. ital. 1. p. 65, 844; et auct. omn.

Figure. - Sav. mat. med. veg. tosc. t. 26. Targ. scelt. di piant. off. t. 10. Reich. ic. fl. germ. 20. t. 85. f. 1, 3.

Stazione, Abitazione e Fioritura. - Nei luoghi boschivi asciutti, principalmente nei montuosi, nonchè nei pascoli, di quasi tutta l'Italia. Nell'Alta Italia è molto comune, innalzandosi assai nelle Alpi, cosi in val Tellina a S. Caterina a 1700 metri (Parl.!), ecc. Seguita ad essere comune nell'Italia Media, dal mare a tutta la regione montana. Nella Bassa Italia si ristringe ai monti, estendendosi fino all' estrema Calabria a Giffone (Pasquale!), a Pecoraro (Arc.!). 
Abita poi le isole: di Corsica nelle parti alte e medie (Mars.), cosi a Cagna (Bert.); di Sardegna, dov'è rarissima, nei monti Limbara, verso Tempio (Moris!); dell' Elba a Campo (Marencci!); d'Ischia (Guss.), e di Capri (Bert.); di Sicilia, ristretta alla sua parte nordest, a Mistretta, Caronia; Capizzi, Bronte, Maletto, Francavilla, Mandanici (Guss.), e sull'Etna (Bert.). Fiorisce in maggio e giugno, e in luglio nei luoghi più alti.

Distribuzione geografica, - In tutta Europa, eccettochè alcune sue parti più meridionali, nell'Asia contermine, nel nord-est dell'America settentrionale.

Descrizione. - Pianta perenne, verde, pelosa, con peli glandolosi nelle parti superiori. Il fusto è giacente in basso per terra e manda ivi fibre radicali nei nodi vitali, è ascendente verso l'apice, cilindrico, ramoso, verdognolo o in parte rossiccio, peloso, con $\mathrm{i}$ peli bianchi, i quali nelle parti superiori hanno delle piccole glandole all' apice. Le foglie sono piuttosto molli, opposte, un po'lontane, patenti, ovali-bislunghe o obovato-ovali, seghettate, con i denti ottusetti all'apice, fornite di un corto picciòlo scanalato di sopra e convesso di sotto, sono verdi, con un solco longitudinale e leggieri solchi laterali un po'curvi di sopra, con un nervo longitudinale di sotto, da cui partono delicati nervetti laterali e curvi, che si diramano e si uniscono in rete, pelose o pelosette in ambedue le pagine. I fiori sono piccoli, distribuiti in un racerno fitto portato da un peduncolo piủ lungo delle foglie, eretto o eretto-patente, rigido, cilindrico, verdognolo o in parte rossiccio e peloso-glandoloso. I pedicelli sono piî corti del calice, eretti, cilindrici, rossicci, pelosoglandolosi, accompagnati da una brattea poco piu lunga di essi, lanceolato-lineare o lineare, ottusa, eretto-patente, verdognola, pelosoglandolosa. Il calice è arch'esso verde, peloso-glandoloso, quasi campanulato, diviso fin sopra la base in quattro lacinie lanccolatolineari, ottuse, erette con l'apice patulo; nel frutto sono quasi la metả più corte della cassula. La corolla è neno del doppio piủ lunga del calice, di color violetto con poche righe longitudinali piu scure che vanno sino all'apice delle lacinie di essa, e bianchiccia nel tubo e nella gola: il tubo è circa un terzo della metà della cotolla, peloso di dentro con i peli lunghi e orizzontali. Il lembo è patente cou le lacinie disuguali, la superiore essendo un poco piu larga delle laterali e la inferiore un po'pii stretta di queste: tutte ottuse all'apire e quuasi ovali. Gli stami sono due, quasi "guali alla corolla, crettnpatenti: i filamenti quasi filiformi, appena appena più grnssi verso 
il mezzo, violetti, glabri. Le antere sono ovato-orbicolari, ottusette, smarginato-bilobe alla base, inserite nel dorso, biloculari, violette, glabre. Il pistillo supera appena la corolla. L'ovario è quasi orbico. lare, un po' schiacciato, verdognolo, peloso-glandoloso. Lo stilo è più lungo dell'ovario, filiforme, violetto, glabro. Lo stimma è ottuso. La cassula è dura, quasi cartilaginea, triangolare-obcordata, con gli angoli della smarginatura tondeggianti, schiacciata, con un leggiero solco longitudinale nelle facce, peloso-glandolosa, gialliccia. I semi sono ovali, schiacciati, lisci, gialli. (Parl. ms., descr. di pianta di Boscolungo nell'Appennino toscano).

\section{Veronica Allionii.}

V. foliis breviter petiolatis, ovalibus, subacutis, subserratis, racemis multifloris, densissimis, pedicellis fructiferis erectis, bracteis brevioribus, lobis calycinis 4 lanceolato-linearibus, subæqualibus, septifragio compresso, obovato, emarginato, calycem paulo superante, stylo septifragio longiore.

Veronica Allionii Vill. fl. delph. in Gil. syst.pl. Eur. Hist.pl. Dauph. 2. p. 8. Bert. fl. ital. 1. p. 67. De Not. rep: fl. lig. p. 302. Gren. Godr. fl. Fr. 2. p. 591. Ard. fl. Alpes-mar. p. 275. Ces. Pass. Gih. comp. fl. ital. p. 351. Arc. comp. fl. ital. p. 515.

Figure. - All. rar. Ped. stirp. spec. prim. t. 4. f. 3. Reich. ic. bot. t. 782. Ic. A. germ. 20. t. 84. f. 1, 2.

Stazione, Abitazione e Fioritura. - Nei pascoli delle Alpi occidentali, non rara: al Cenisio (Bonjean! ecc.), al Piccolo Cenisio (Beccari!), presso Susa verso Rocca Melone, presso Oulx sul monte Praman (Aiuti!), al monte Genevre (Gr. Godr.), al Monviso (Jordan!), fra le valli Maira e Grana (Ball!), alla Madonna di Fenestre (Bourgeau!), presso S. Martino Lantosca (Bert.), al monte Bissa (Parl.!), verso Briga (Ungern-Sternberg!), sopra Viosenne (Ricca!), nei monti di Ceresole (Bert.). Altra volta era stata indicata allo Spinale in Tirolo, ma non vi è più stata trovata. Fiorisce in luglio e agosto.

Distribuzione geografica. - Nasce inoltre nelle Alpi francesi contermini.

Descrizione. - Fusti sdraiati per terra e radicanti con radici delicate, ramose. Rami giacenti per terra, cilindrici, verdognolorossicci, con piccolissimi peli avvicinati che si vedono con una forte lente. Foglie opposte, patenti, quasi coriacee, larghette, ovali o 
quasi obovate, ottusette, di un verde piuttosto chiaro di sopra, ivi con un leggiero solco longitudinale nel nervo e glabre, più chiare e quasi glancescenti di sotto ed ivi con un nervo longitudinale spor. gente, anche glabre, con leggieri denti a sega nel margine ch' ̀̀ anche cigliato con le ciglia rade, bianche, un po'curvate verso l'apice della foglia. Fiori in spiga fitta portata da peduncolo ascendente o eretto, duretto, cilindrico, pubescente, con i peli disuguali e curvati in su, verdognolo o piủ spesso rossiccio. I fiori sono molti, eretto-patenti. I pedicelli cilindrici, pubescenti, rossicci, più corti del calice. La brattea è poco piủ lunga del pedicello, erettopatente, lineare, oltusetta, verde, pubescente-glandolosa. Calice diviso fin poco sopra la base in quattro lacinie, eretto-patenti, lanceolato-lineari, quasi acute, piane, è verdognolo $o$ in parte rossiccio, pubescente-glandoloso. La corolla è violetto-carica, 3 a 4 volte più lunga del calice, con il tubo quasi tondo, assai chiaro e quasi bianchiccio; le 4 lacinie sono disuguali, eretto-patenti, lanceolate 0 bislunghe, ottuse o ottusette, glabre. Gli stami sono 2, poco più lunghi della corolla. I filamenti del colore di questa, filiformi, eretti, glabri. Le antere sono violette, glabre, ovate, bilobe alla base, ottusette all' apice, inserite nel dorso sopra la base. Il polline è giallo. Il pistillo è lungo quasi quanto la corolla. L'ovario è ovale-tondo, schiacciato da avanti in dietro, verdognolo, glabro. Lo stilo è lungo, filiforme, violetto, glabro, dapprima eretto, poi si curva ad arco in fuori ed in giù allungandosi nel giovine frutto. Lo stimma è oltuso, violetto. (Parl. ms., descr. della pianta del Cenisio).

\section{Veroniea Iatifolia.}

V. foliis sessilibus, cordato-ovatis ovalibus oblongis lanceolatis linearibusve, subobtusis, inciso-dentatis, racemis multifloris, densis, pedicellis fructiferis erectis, bracteis subæqualibus, lobis calycinis 5 lineari-lanceolatis, valde inæequalibus, septifragio compressu, obovato-rotundato, emarginato, calyce subequali, stylo septifragio paullo longiore.

Veronica latifolia Linn. sp. plant. ed. 1. p. 19. Bert /. ilal. 1. p. 7.9; el aucl. plur.

Vernnica Teucrium Linn. o. c. ed. 2. p. 16. Bert. o. c. p. 78; el allet.

Veronica prostrata Linn. o. c. ed. 9. p. 17. Berl. o. c. p. i6; el auct. 
Veronica Pseudochamædrys Mass. prodr. fl. valt. p. 198. De Vis. Sacc. cat. piant. Ven. p. 153.

Veronica Orsiniana Ten.! Ces. el. piant. Maiella p. 22.

Veronica polymorpha Ces. Pass. Gib. comp. fl. ital. p. 350 (excl. $\gamma)$.

Figure. - Pucc. syn. pl. luc. Reich. ic. fl. germ. 20.t. 87, 88 . f. 1-3.

Stazione, Abitazione e Fioritura. - Nei luoghi erbosi, principalmente di collina e di montagna, per la Penisola. $\dot{\mathrm{E}}$ molto frequente nell'Alta Italia, dove s'inalza a circa 1200 metri a Courmayeur (Webb!) e a Bormio (Parl.!), e più in su ancora, essendo indicata per esempio allo Spluga (Com.). È già meno frequente nell' Italia centrale, e nella meridionale sempre meno e vi si ristringe ai monti : in Liguria a Tenda (Ungern-Sternberg!), Malaussena, Nizza, Mentone (Ard.), da Porto Maurizio (Gentile!) a Capo del Mele (Bert.), a Capo di Noli (Piccone!), Genova, Sestri levante (Figari!); in Toscana si spinge fin oltre la regione montana, ma non trovasi più nella regione maremmana, cosi nelle Alpi Apuane al Pisanino (Bert.) e al Fatonero (Cocchi!), nell'Appennino a Montefegatese, Pratofiorito (Giannini!), Mandromini, la Piastra (Vitm.), nell'Agro fiorentino, comune!, in Mugello (Savi), nelle colline Pisane (Bert.), a Volterra, Montecerboli (Parl.!), Radda (Beccari!), Siena (Campani!), tra Siena e Grosseto!, presso Rocca d'Orcia nel monte Amiata (Parl.!); nelle Marche nasce a Urbino (Serpieri!), al Catria (Piccinini!), a S. Severino (Aiuti!), al monte Regnolo (Bert.), intorno ad Ascoli(Orsini!), al Vettore nella regione alpestre!; in Abruzzo al monte De'fiori (Orsini!), al Corno (Ten.), al Sirente (Groves!), alla Maiella tra 2000 e 2400 metri (Levier!); trovasi a Viterbo (Bert.), nei monti di Rieti (Rolli!); quindi in Terra di Lavoro presso Cassino, nelle dipendenze del monte Meta (Terr.!), nel Matese, poi nel monte S. Angelo di Castellammare, infine nel Pollino e nella Sile (Ten.). Fiorisce in aprile e maggio, e nei luoghi più alti in luglio.

Distribuzione geografica. - Nell'Europa centrale e meridionale, in Armenia, in Songaria.

Osservazione. - La forma di gran lunga più comune in Italia è quella che corrisponde alla Veronica prostrata degli antori, sdraiata ascendente e con le foglie di mezzana larghezza. 


\section{Veronica sustrinea}

V. foliis sessilibus, ambitu ovatis, bipinnatipartitis lobis oblongis linearibusve, racemis multifloris, densis, pedicellis fructiferis erectis, bracteis subæqualibus, lobis calycinis 5 lineari-lanceolatis, valde inæqualibus, septifragio compresso, rotundato, emarginato, calyce subæquali, stylo septifragio paullo longiore.

Veronica austriaca Linn. sp. pl. ed. 2. p. 17. Bert. fl. ital. 1. p. 74. Rota? prosp. fl. Berg. p. 66. Pir.! fl. for. syll. p. 105. De Vis. Sacc.? cat. piant. Ven. p. 152.

Veronica polymorpha $\gamma$ Ces. P'ass. Gib. comp. A. ilal. p. 350.

Veronica multifida Smith fl. Fiume p. 38.

Veronica Teucrium $\gamma$ Arc. comp. fl. ital. p. 513.

Figura. - Reich. ic. fl. germ. 20. t. 90.f. 1.

Stazione, Abitazione e Fioritura. - In luoghi erbosi sassosi dell'Istria presso Fiume (Noë!) a Braita (Loebisch!), Zakalj e Veprinac (Smith), e a-Trieste (Clementi!), e del Friuli presso Duino (Pir.), pressu Montenars (Pir.!), nel monte sopra Gemona (Barbieri!). E pure indicata nel Mantovano (De Vis. Sace.), nel beryamasco in valle Caleppio (Rota), in Pienonte a Roascia (All.). Fioririsce in maggio e giugno.

Distribuzione geografica. - Nell' Europa meridionale orientule, nell'Asia Minore.

\section{Veronica Clnamedrys.}

V. foliis subsessilibus, ovatis, subobtusis, inciso-dentatis, racemis multifloris, laxis, pedicellis fructiferis adscendentibus, bracteis plerumque longioribus, lobis calycinis 4 oblongo-lanceolatis, subaqualibus, septifragio compresso, obverse triangulari emarginato vel obcordato vel.truncato, calyce minore, stylo septifragio dimidio longiore.

Veronica Chamxdrys Linn. sp. pl.ed. 1. p. 13. Bert. R. ital. 1. p. 80; et auct. omn.

Figure. - 17. dan. t. Fis. Reich. ic. /. germ. 20. t. 83. f. $2-4$.

Stazione, Abitazione e Fioritura. - Alle siepi, nei luoghi boschivi freschi, per tutta la Penisola. Ahbonda in tutta l'Alta Italia, dove nasce ancora nei prati, e va dal piano alla regione montana, 
cosi sopra i Bagni di Valdieri a 1300 metri, presso Bormio a 1230 metri, a Ponte di legno a 1330 metri, sul Tonale a 2000 metri (Parlatore!). Nell' Italia centrale e meridionale si ristringe sempre più ai monti: in Liguria trovasi in parechi luoghi, da Tenda (Bourgeau!) a Chiavari (Delpino!); in Toscana, dove già quasi manca nella regione maremmana, a Sarzana, nelle Alpi Apuane (Bert. ecc.), a Lucca (Pucc.!), nel monte Pisano (P. Savi!), ma fa soprattutto nell'Appennino, nelle regioni montana e submontana, dall'alpi di Mom. mio! all'alpe della Luna (Cherici!), nonchè nelle sue dipendenze di monte Senario e di Vallombrosa!; nelle Marche è stata trovata al Catria (Piccinini!), a S. Severino, Tolentino (Ricci!), Montefortino (Marzialetti!), Ascoli (Orsini!); in Abruzzo al monte De'Fiori (Or. sini!) e nei monti di Caramanico (Pedicino!); è ancora comune presso Roma (Seb. Maur.), si ritrova nel monte Meta, a Itri (Terr.), nel Matese (Terr.!), a Benevento (Pasquale!), a Nola nel Taburno (Terr.), a Napoli nella valle di S. Rocco (Ten.), nel Vulture (Terr.!), e termina in Calabria nell'Aspromonte (Pasquale!) e a S. Cristina e Pimenovo (Arc.!). Fiorisce in maggio, giugno o luglio secondo i luoghi.

Distribuzione geografica. - Nell'Europa, scarseggiando però nelle sue parti piủ settentrionali e più meridionali, nell'Asia Minore, in Siberia, e (dicesi) nelle Isole Canarie.

Descrizione. - Pianta perenne, alta da 1 a 3 decimetri, di un verde chiaro, fornita di un rizoma lungo delicato, che manda fibre radicali delicate e bianchicce. Il fusto è ascendente, duretto, quadrangolare, ad angoli ottusi, rossiccio in basso, verdognolo in alto, glabro con due linee di peli curvati in su, che sono alterne con le. foglie, semplici o con pochi rami opposti, corti e eretto-patenti. Le foglie sono erbacee, opposte, poco lontane, patentissime, le inferiori un po' più piccole, ovali, ottuse, le altre ovate, acute, tutte scavate un po'in cuore alla base, inciso-crenate, seghettate, di un verde chiaro di sopra, con solchi corrispondenti ai nervi di sotto, di un verde più chiaro di sotto ed ivi con un nervo longitudinale da cui partono dei nervi laterali, tutti rilevati, i quali si diramano e si uniscono ad arco presso il margine; sono pelosette in ambedue le facce e nei margini, quasi sessili o con un picciòlo cortissimo, scanalato di sopra, convesso di sotto e rossiccio. I fiori sono molti, distribuiti in racemi laterali, solitarii, all'ascella di una o di ambedue le foglie che sono vicine alle supreme del tusto, eretti o eretto-patenti, più lunghi e nel frutto molto delle foglie corrispondenti. Il peduncolo 
e i pedicelli sono cilindrici, pubescenti, verdognoli o rossicci: questi sono diritti e lunghi quasi quanto i fiori. Il calice è piccolo, ver. dognolo, puhescente, diviso in quattro lacinie, circa due terzi della lunghezza del calice, patenti, lanceolate, ottusette. La corolla è molto piủ grande del calice, di color celestognolo-turchiniccio, con il tubo di dentro bianco, smerlato verso i lobi e peloso-barbato: questi sono patenti, $i$ tre superiori quasi ovali-tondeggianti, piủ grandi dell' inferiore che è obovato-ovale. I due stami sono quasi uguali alla corolla, patenti, inscriti nel suo tubo. I filamenti sono un po'piủ grossi nel mezzo, piủ assottigliati in basso, ivi bianchicci, nel resto celesto. gnoli. Le antere sono ovate, ottuse, smarginate alla base, tur. chine scure. Il pistillo è lungo quanto o poco più degli stami. L'ovario è piccolo, quasi orbicolare, schiacciato, verdognolo, puberulo. Lo stilo è molto più lungo dell'ovario, filiforme, di color turchiniccio, glabro. Lo stimma è quasi in capolino, papilloso, bianchiccio. (Parl. ms., descr. di pianta di Boscolungo nell'Appennino loscano).

\section{ๆ. Veronica montana.}

V. foliis longiuscule petiolatis, late ovatis, obtusis, profunde dentatis, racemis paucifloris, laxis, pedicellis fructiferis subpatulis, hracteis multo longioribus, lobis calycinis 4 obovatis, subæqualibus, septifragio complanato, transverse latiore, basi apiceque emarginato, Inargine denticulato-ciliato, calyce paullo maiore, stylo septifragio paullo breviore.

Veronica montana Linn. amoen. acad. 4. p. 263. Berl. f. ital. 1. p. 75. Com. fl. com. 1. p. 18. Pucc.! syn. pl. luc. p. 16. Trev. prosp. A. eug. p. 23. Guss. fl. sic. syn. 1. p. 17. De Not. rep. /.lig. p. 302. Sim. R.alp. vers. p. 12. Rota prosp. fl. Berg. p. 66. Pir. /1. for. syll. p. 105. Car. prodr. 凡. 10sc. p. 486. De Vis. Sacc. cat. piant. Ven. p. 152. Mars. cal. pl. Cors. p. 108. Terr. sec. rel. Terr. Lav. p. 92. Ing. cal. sp. Mond. p. 74. Ces. Pass. Gib. comp. A. ilal. p. 350. Terr. quart. rel. p. 1/1. Sacc. Bizz. agg. 11. trev. p. 27. Arc. comp. f. ital. p. 514. Nic. prodr. /l. mess. p. 341.

Figure. - Col. ecphr. p. 28S. Fl. dan. t 1201. Reich. ic. /l. germ. 20. 1. 84. f. 3, 4.

8tazione, Abitazione e Fioritura. - ( )ua e là nei boschi onnbrosi, sparsa per tutta l'ltalia: nella Carnia (Pir.), nel Bellunese al Cansiglio (Sacc. Bizz.), negli Euganei (Trev.), nel Bergamasco presso Schilpario (Rota), nel Comasco a Laino e Pellio in val In- 
telvi, sul Cornicciolo di Cianzo, nel Cantone Ticino a Cola (Com.), in quel di Mondovi a Monastero-Vasco (Ing.), in val di Pesio (Rostan!), nei monti d'Albenga, sopra Torriglia, nel monte di Portofino (De Not.), nel monte Gotro di Lunigiana!, nell'alpi di Mommio, a S. Pellegrino e nel contermine Modanese al Pian de' lagotti (Calandrini!), a Massa-Carrara (Bert.), in Versilia al monte Gab. beri (Simi), in molti luoghi tutt'intorno a Lucca (Giannini! ecc.), e nel vicino Appennino a Tereglio (Pucc.), nella Selva pisana!, a Boscolungo, e alla Madonna dell'Acero nell'Appennino bolognese, in Casentino al Sacr'eremo e in parecchi altri luoghi (Parl.!), in val Tiberina (Amidei!), al Catria (Piccinini!), nel Piceno al monte Acuto, in Abruzzo al Pizzo di Sivo, al Corno (Orsini !) e al Pelone (Bert.), presso Roma ad Albano (Seb. Maur.), nei monti Lepini (Rolli!), in Terra di Lavoro a Picinisco, a Pietraroia (Terr.), nel monte Vergine (Pasq.!), a Napoli in diversi luoghi, e a Sant'Angiolo di Castellammare (Ten.), in Calabria a Rosarno (Bert.), in Corsica a Bastia (Bert.), a Cardo, a Vivario, tra Vico e Aiaccio (Mars.), e a Bogomone (Bert.), in Sicilia nei monti di Caronia (Tineo!). Fiorisce in maggio, giugno o luglio secondo i luoghi.

Distribuzione geografica. - In quasi tutta Europa, ma principalmente nella centrale.

Descrizione. - Pianta perenne, di un verde chiaro, pelosa, con peli molli, lunghetti e bianchi, fornita di un rizoma delicato, e di fusti lunghi da 1 a 5 decimetri, giacenti per terra e un po' rilevati all'apice, cilindrici, radicanti ai nodi inferiori, pelosi con peli orizzontali, rossiccio-scuricci in basso, verdognoli in alto, sem. plici o ramosi. Le foglie sono molli, opposte, rivolte in su, patenti, ovali o ovali-orbicolari, ottuse, crenato-seghettate, con i denti grandi e il terminale piủ largo degli altri che decrescono verso la base, appena scavate in cuore alla base, di un verde chiaro di so. pra, ed ivi con solchi leggieri che corrispondono ai nervi di sotto, di un verde più pallido di sotto ed ivi con un nervo longitudinale rilevato da cui partono pochi nervetti laterali anche rilevati che si dirigono verso il margine per unirsi tra loro con i rami loro e mandare un rametto all'apice di ciascun dente: sono pelose con i peli molli, eretti e bianchi in ambedue le facce, e hanno un picciòlo il quale è circa la metà piủ corto di esse, con un solco longitudinale di sopra, convesso di sotto, verdognolo, peloso. I fiori sono pochi in un racemo rado portato da un peduncolo ascellare, il quale dapprima è quasi uguale, poi più lungo della foglia corrispondente. I pe- 
dicelli sono anch' essi filiformi, rossicci e pelosi come il peduncolo, più lunghi del calice, patenti e accompagnati da una bratteola lineare, ottusa, piủ corta del pedicello, verdognola e pelosa. Il calice è verdognolo, peloso, diviso fin sopra la base in quattro lacinie patonti, ovali, bislunghe, quasi acute, con una carena sul dorso, fornite di ciglia, pelosette anche di dentro; diventano più grandi nel frutto, quasi obovati, avvicinati alla cassula e poco piủ corti di questa. La corolla è quasi il doppio più grande del calice, piccola, con il tubo cortissimo, bianchiccio e il lembo patente, diviso in quattro lacinie, un po' disuguali, la superiore essendo la maggiore, quasi orbicolari, violetto-chiare con poche righe longitudinali più cariche nelle tre lacinie superiori. Gli stami sono due, appena piủ lunghi della corolla. I filamenti patenti, quasi filiformi, un po'piủ grossi verso il mezzo, di color violetto chiaro. Le antere piccole, quasi tonde, ottuse, smarginate alla base, biloculari, aprentisi longitudinalmente, di color violetto scuro. Il pistillo è più lungo della corolla. L'ovario è ovale, schiacciato, verdognolo, fornito di ciglia corte nel marginc. Lo stilo è quasi il doppio più lungo dell'ovario, filiforme, glabro, di color violetto chiaro. Lo stimma è quasi in capolino, gialliccio. La capsula è quasi orbicolare, piủ larga che lunga, smarginata al. l'apice, schiacciata dalle parti, con piccoli denti nel inargine, ciascuno dei quali ha un ciglio, e contiene quattro semi in ciascuna loggia, i quali sono orbicolari, schiacciati, che si cuoprono in parte tra loro. (Parl. ms., descr. della pianta di Boscolungo).

\section{Veroniea seutellato.}

V. foliis sessilibus, lineari-lanceolatis, acuminatis, subdenticulatis, racemis paucilloris, laxis, pedicellis fructiferis patentissimis, bracteis multo longioribus, lobis calycinis 1 ovalibus, subæqualibus, septifragio complanato, transverse latiore, emarginato-bilobo, margine integro, calyce multo maiore, stylo septifragio dimidio fere breviore.

Veronica scutellata Linn. sp. pl. ed. 1. p. 12. Bert. R. ilal. 1. p. 73. Com. R. com. 1. p. 15. Colla herb. ped. 4. p. 337. Sang. cent. prodr. A. rom. p. 5. Pucc! syn. pl. luc. p. 17. Tree. prosp. 1. cug. p. 23. Zan. prosp. M. ven. p. 25. Hausm. M. Tir. p. 695. Rota prosp. A. Berg. p. 66. Pir.! 1. for, syll. p. 104. Car. prodr. 1.. losc. p 186. Zersi prosp. piant. Bresc. p. 155. Ing. cat. sp. Mlond.

Flona Italana. - Vol. Vi. 
p. 74. Ces. Pass. Gib. comp. fl. ital. p. 351. Paol. el. piant. sib. p. 15. Sacc. Bizz. agg. fl. trev. p. 27. Arc. comp. fl. ital. p. 515.

Figure. - Fl. dan. t. 209. Reich. ic. fl. germ. 20. t. 82. f. 2, 3.

Stazione, Abitazione e Fioritura. - Nei fossi e nei paduli della Penisola, non tanto comune, e doventa pianta montanina nella sua porzione inferiore. Nasce presso Mondovi (Ing.), in più punti dell'agro Torinese (Colla ecc.), a Candia (Delponte!), Oldenico (Malinverni!), Obbiate (Bir.), a Pavia (Rampoldi! ecc.), presso Como in più punti (Parl.! ecc.), presso Treviglio (Rota), nel Bresciano a Torbole (Zersi), in Tirolo presso Pinè, presso Salorno, e sul Ritten (Hausm.), nel Veronese a Bovolone (Poll.), nel Mantovano in diversi siti (Paglia! ecc.), a Guastalla (Passerini!), a Ferrara (Bert.), negli Euganei (Trev.), a Venezia (Zan.), nel Trivigiano presso Roncade (Sacc. Bizz.), ad Aquileia (Pir.!) e Monfalcone (Pir.), nel Lucchese (Pucc.! ecc.), nel Pisano!, presso Montieri nella maremma toscana (Amidei!), in Umbria al Castelluccio, in Abruzzo a Pagliari (Orsini!), Pescocostanzo e Revisondoli (Bert.), infine a Potenza, e nella Sila (Ten.). Fiorisce da maggio a luglio.

Distribuzione geografica. - In tutta Europa, nell'Affrica settentrionale (dicesi), nell'Asia russa e nella Nord-America.

Descrizione. - Fusto giacente in basso e ivi con poche fibre radicali lunghe che partono dall' apice dei meritalli inferiori presso al nodo vitale: questi meritalli sono piủ delicati degli altri che seguono; è eretto 0 ascendente nel resto, cilindrico, rossiccio in basso, verdognolo in alto, glabro, ha di dentro un asse 0 cilindro vascolare grossetto e delle lacune piuttosto piccole, disuguali, in rete o retiformi. Foglie opposte, erette, sessili, spesso più corte o quasi uguali ai meritalli, lanceolato-lineari, acute, di un verde chiaro e le infeferiori un po'rossicce, con un solco longitudinale di sopra, con un nervo longitudinale sporgente e più chiaro di sotto, con i margini un po' rovesciati in dietro e forniti di rari denti poco sporgenti, ottusi, talvolta quasi a due a due insieme, e glandolosi. I fiori sono distribuiti in racemi alterni, ascellari, più lunghi delle foglie, erettopatenti e un po'curvati ad arco in su: tali fiori sono radi. I pedun. coli sono delicati, verdognoli. I pedicelli sono filiformi, piủ lunghi. dei fiori, eretti o eretto-patenti nel fiore, quasi orizzontali nel frutto. Le bratteole sono eretto-patenti, strette, quasi setacee, ottusette, più della metà più corte dei pedicelli, verdognole. Il calice è diviso fin poco sopra della base in 4 lacinie, poco disuguali, avvicinate alla corolla, ovali-bislunghe, ottusette, verdognole, sono più corte della 
cassula alla quale sono alquanto avvicinate. La corolla è quasi il doppio piủ grande del calice, divisa fin quasi alla base in quattro lacinie, patenti, poco*disuguali, obovato-sessili, è di un roseo-violetto con poche vene più cariche longitudinali. Il tubo è corto e gialliccio. Gli stami sono lunghi quanto la corolla, eretti. I filamenti sono inseriti alla gola di questa, fusiformi, cioc̀ un po'piủ grossi nel centro che nella estremità, dove, massime in basso, sono assottigliati, glabri, roseo-violetti e quasi bianchicci. Le antere sono quasi tonde, smarginate alla base. Il pistillo è lungo quasi quanto gli stami. L'ovario è quasi tondo, schiacciato, bilobo, verdognolo, glabro. Lo stilo è quasi tre volte più lungo dell'ovario, filiforme, bianchiccio; lo stimma quasi in capolino. La cassula è quasi tonda, schiacciata, smarginata all'apice ed ivi quasi biloba, intera nel margine, ivi quasi rosea. (Parl. $m s .$, descr. della pianta di Pisa).

\section{Veroniou Hecenbunga.}

V. foliis breviter petiolatis, ovalibus oblongisve, obtusis, absolete serratis, racemis multifloris, laxis, pedicellis fructiferis patentiusculis, bracteis subæqualibus, lobis calycinis 4 ovalibus, subæqualibus, septifragio turgido, subgloboso, vix emarginato, calyce maiore, stylo septifragio paullo breviore.

Veronica Beccabunga Limn. sp. pl. ed 1. p. 12. Bert. fl. ital.1. p. 69; et auct. omn.

Figure. - Fl. dan. 1.51\%. Targ. scelt. piant. off. 1. 22. Reich. ic. fl. gerin. 20. t. 80 .

Stazione, Abitazione e Fioritura. - Nei ruscelli e nei fossi di tutta l' Italia, dalle pianure a grandi altezze nei monti. Abbonda nell'Alta Italia, fino in luoghi alpini, come al S. Bernardino (De Not.!), in val Tellina a Tresero e Vallaccia (Anzi), al Tonale a 2000 metri (Parl.!), ecc. In Liguria scarseggia alqquanto, essendo però sparsa da Tenda (Ungern-Sternberg!) e da Nizza (Ard.) insino a Sarzana (Bert.); in 'Toscana è assai comune, fino alle cime dell'Appennino; nelle Marche è stata trovata ad Ancona (Calitesi!), ad Ascoli al Castelluccio (Orsini !) ecc., in Abruzzo nei monti Pelone (Bert.), Acuto (Orsinil), di Caramanico (Ces.); è ancora comune a Roma (Seb. Maur.); ritrovasi a Napoli ('asquale!), ad Avellino (Bruni!), sul Vulture (Terr.), in Calabria a Monteleone e sull' Aspromonte (Arc.!). Manca in Corsica e in tutte le isole minori del Tirreno; in Sardegna è notata di un luogo solo, montuoso, listerzili (Mor.); in 
Sicilia è ristretla alla parte nord-est dell'isola, nei monti : Madonie (Parl.!), Mistretta, Caronia, Montalbano, Milo, raramente in luoghi bassi come a Messina (Guss.); fa infine in Malta (Zer., Grech). Fiorisce in maggio e giugno, e in luglio e agosto nei luoghi più alti.

Distribuzione geografica. - In tutta Europa, in Siberia e al Giappone, nell'Asia occidentale sino all'Arabia e all'Imalaia, nell'Affrica settentrionale e in Abissinia.

Descrizione. - Pianta perenne, carnosetta, verde, glabra, fornita di un rizoma lungo , che manda fibre radicali delicate e bianchiccie e che si continua nell'altra estremità con il fusto. Questo è gros. setto, lungo da 2 a 4 decimetri, prostrato e radicante in basso, ascen. dente in alto, cilindrico, rossiccio o in parte verdognolo, semplice 0 con rami opposti, corti ed eretti. Le foglie sono opposte, un po'lontane, patenti, ovali o ovali-bislunghe, dentato-seghettate, con i denti superficiali, ottusetti e con un collo rossiccio all' apice, sono verdi, e un po'lucenti di sopra ed ivi con leggieri solchi che corrispondono ai nervi di sotto, di un verde pallido o rossicce di sotto ed ivi con un nervo longitudinale grossetto, da cui partono pochi nervi laterali i quali sono obliqui, curvi e poco rilevati e si dirigono verso l'apice e il margine: sono fornite di un picciòlo ch'è scanalato di sopra e convesso di sotto. I fiori sono pochi, distribuiti in racemi laterali, per lo più opposti, all' ascella delle foglie poco lontane dall' apice del fusto e dei rami, poco più lunghi delle foglie medesime, e patenti. I pedicelli sono delicati, patenti, poco piủ lunghi del fiore e della cassula, accompagnati da una bratteola, la quale è lineare, ot. tusa, lunga quasi quanto il pedicello, vérde. Il calice è diviso pro. fondamente in quattro lacinie patenti, lanceolate, ottusette, verdi. La corolla è poco più grande del calice; il tubo cortissimo, bianchiccio, puberulo di dentro; il lembo diviso in quattro lacinie un po'disuguali, le superiori maggiori, quasi orbicolari, l'inferiore più piccola, ovale, tutte di color turchino chiaro con tre righe più scure verso la base di ciascuna lacinia. Gli stami sono due, inseriti alla base dalla parte interna del tubo, più corti della corolla, patenti. I filamenti sono quasi fusiformi, cioè stretti in basso, più grossi verso alto e stretti di nuovo all'apice, di color bianchiccio, glabri. Le antere sono piccole, quasi tonde, smarginate alla base, inserite nel dorso sopra della base, aprentisi longitudinalmente, di color turchino scuro, glabre. Il pistillo è poco più lungo degli stami. L'ovario è quasi tondo, bilobo, verde, glabro. Lo stilo è due volte più lungo dell'ovario, diritto, filiforme, glabro, bianchiccio. Lo stimma è quasi in capo. 
lino, roseo-violetto, papilloso. La cassula è quasi orbicolare, smarginata, un po'gonfia, rossiccia, glabra, poco piủ lunga delle lacinie del calice: in ciascuna loggia sono molti semi piccoli, giallicci, glabri e lisci. (Parl. ms., descr. della pianta di Boscolungo nell'Appen. nino toscano).

\section{Veronica Anagallis-nquatica.}

V. foliis sessilibus, lanceolatis, subacutis, denticulatis, racemis multifloris, laxis, pedicellis fructiferis patentiusculis, bracteis lon. gioribus, lobis calycinis 4 ovalibus, subæqualibus, septifragio turgidulo, subrotundo, vix emarginato, calyce paullo minore, stylo septifragio paullo breviore.

Veronica Anagallis-aquatica Linn. sp. plant. ed. 1. p. 12. Bert.

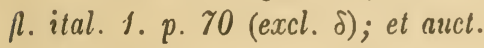

Veronica pusilla Benth? in Cand. prodr. 10.p. 468. Ces. Pass. Gib. comp. 月. ital. p. 350. Arc. comp. fl. ital. p. 514.

Figure. - Fl. dan. t. 903. Reich. ic. fl. germ. 20. t. 81. f. 1,2 .

Stazione, Abitazione e Fioritura. - Comunissima nei fossi e nei luoghi paludosi di tutta quanta l'Italia, comprese le isole minori dell'Elba (Marcucci!), del Giglio (Parl.!), d'Ischia (Guss.) e di Malta (Grech!). Sale nelle Alpi sino fra i larici, cosi in val Camonica a Ponte di legno a 1520 metri (Parl.!); nell'Appennino non sembra che oltrepasşi la regione submontana. Fiorisce in maggio e giugno, più presto e sin da marzo nei posti più caldi, piủ tardi nei meno caldi.

Distribuzione geografica. - In tutta Europa ad eccezione delle sue parti più nordiche, nell'Affrica settentrionale dalle isole Canarie all'Abissinia, nell'Asia occidentale e sino in India, nell'Asia settentrionale e sino in China e in Giappone, nell' America setten. trionale.

Osservazioni. - Variano e non poco le foglie, e talora essendo esse più larghe e più corte si accostano a quelle della V'eronica Beccabunga. Suppongo che a questa forma si debba riferire la $V$. intermedia descritta e figurata dal prof. Terracciano (Su di alcune piante della f. napol. p. 8. 1.1$)$, e da lui trovata nella valle del Vol. turno.

Nelle Species Linneo scrisse il nome triviale dalla pianta $A n a$. gall. $\Delta$, leggi : Anagallis aquatica, essendo quel $\Delta$ il segno da lui 
adoperato per significare la stazione acquaiola. Si sa che gli antichi designavano col nome di. Anagallis aquatica questa specie e le due precedenti.

Non so che cosa sia la Veronica pusilla Benth., indicata nelle risaie del Piemonte e in Sardegna: pertanto Boissier (Fl. orient. 4. p. 437) la riunisce alla presente specie.

\section{Veronica anagalloides.}

V. foliis sessilibus, lineari-lanceolatis, subacutis, subdenticulatis, racemis multifloris, laxis, pedicellis fructiferis patentiusculis, bracteis longioribus, lobis calycinis 4 lanceolatis, subæqualibus, septifragio turgidulo, ovali, 'vix emarginato, calyce paullo maiore, stylo septifragio dimidio fere breviore.

Veronica anagalloides Guss. pl. rar. p. 5. Parl.! fl. pan. 1. p. 10. Guss. fl. sic. syn. 1. p. 16. Gren. Godr. fl. Fr. 2. p. 589. Mars. cat. pl. Cors. p. 108. Terr.! rel. Terr. Lav. p. 147. Sec. rel. p. 92. Ces. Pass. Gib. comp. fl. ital. p. 350. Terr. quart. rel. p.110. Gib. Pir. A. Mod. p. 121. Arc.! comp. fl. ital. p. 514. Cocc. fl. Bol. p. 359.

Veronica Anagallis var. Bert. fl. ital. 1.p. $7 /(\delta$, excl.syn.Ten.). Mor. f. sard. 3. p. 224 ( $\beta$, excl. syn. Ten.). Car. prodr. f. tosc. p. $484(\beta)$. Bizz. sec. agg. A. ven. p. 7.

Figure. - Guss. pl. rar. t. 3. Reich. ic. fl. germ. 20. t. 81. f. 3.

Stazione, Abitazione e Fioritura. - Nei paduli, qua e là: in Sicilia presso Palermo a Mondello (Meli!), presso Partinico a Passarello (Sorrentino!), alla Piana dei Greci (Todaro!), al Gurgo di Ddingoli, presso Marineo al ponte di Mililli, ad Amorosa al sud di Busambra, a Lercara al Gurgo di Caccaci (Guss.), ad Altavilla, al monte de'Cani (Todaro!), a Caltavuturo, in val di Noto, presso Marsala (Guss.); in Calabria presso Pizzo al fume Angitola (Arc.!), e presso Catanzaro al fiume Magliacane (Guss.); in Terra di Lavoro a Dragoni, a Carditello (Terr.) e a Pontammare (Terr.); ad AscoliPiceno (Orsini!); presso Pisa ad Asciano (Reboul!), e presso Fi. renze verso Pratolino (Parl. !); presso Bologna a Gandazollo (Bert.), a Minerbio e altrove.(Cocc.); presso Modena ad Albareto e altrove (Gib. Pir.); presso Arquà negli Euganei (Bizz.); in Corsica a Campo di Loro (Mars.), e a Bonifacio (Gren. Godr.); in Sardegna (Mor.). Fiorisce in aprile, naggio, giugno. 
Distribuzione geografica. - In tutta l'Europa meridionale, e I'Asia occidentale, in Songaria, in Egitto, nell'Affrica australe.

Osservazioni. - Le infiorescenze sono più sottili in questa specie che nella precedente, i fiori più piccoli, e bianchicci: cosicchè nell'insieme riesce sufficientemente distinta.

\section{Veronica urticuefolia.}

V. foliis subsessilibus, ovatis, profunde serratis, superioribus acuminatis, racemis multifloris, laxis, pedicellis fructiferis patulis, bracteis multo longioribus, lobis calycinis 4 oblongis, inæqualibus, septifragio compresso, orbiculari, leviter emarginato, calyce multo maiore, stylo longitudine septifragii.

Veronica urticaefolia Jacq. R.austr., sec. Linn. fil. suppl. p. 83. Bert. fl. ital. 1. p. 81. Mass. prodr. fl. valt. p. 198. Com. 1. com. 1. p. 17. Coll. herb. ped. 4. p. 340. Sang. cent. prodr. A. rom. p.5. J. Beıt it. bon. p. 17. Pucc.! syn. pl. luc. p. 17. De Nol. rep. 凡. lig. p. 302. Rota! prosp. piant. Pav. p. 2i0. Hausm. R. Tir. p. 636. Rota prosp. fl. Berg. p. 66. Pir.! fl. for. syll. p. 105. Car. prodr. f. tosc. p. 485. G. Bert. not. porr. p. 46. Zersi prosp. piant Bresc. p. 156. Ces. Pass. Gib. comp. fl. ital. p. 350. Gib. Pir. 1 . Mod. p. 122. Arc. romp. fl. ital. p. 514. Cocc. fl. Bol. p. $35 \%$

Veronica latifolia Re fl. seg. p. 8. Ard. fl. Alpes-mar. p. 275. De Vis. Sacc. cat. piant. Ven. p. 152. Smith. fl. Fiume p. 38.

Figura. - Reich. ic. 月. germ. 20. t.82. f. 1.

Stazione, Abitazione Fioritura. - Nei boschi montuosi della Penisola settentrionale e centrale. Nasce a Fiume dov'è rara (Smith), presso Trieste (Bert.), nel Friuli (Pir.!), per esempin a Tolmezzo (Smith!), presso Passano (Montini!), tra Valdagno e Recoaro (Bert.), sul Baldo (Poll.), a Roveredo (Hausm.), intorno a Trento fra i faggi (Perini!); presso Borgo di val Sugana, in Fienume e Fassa, intorno a Bolzano in molti lnoghi, a Bressanone, a Merano, presso Laas in val Venosta, nelle Giudicarie presso Stelle e ai $\mathrm{ri}$ nali (Hausm.), nel Bresciano, frequente (Zersi), per esempio in fondo a val Trompia da $8: 30$ a 1000 metri, e sul lago d'Iseo, in val Camonica a Ponte di legno a 15.50 metri (Parl.!), nel Bergamaseo a Carenno (Rota!), e in val Bremhana a Branzi (Rampoldi!), nel Comasco, non rara, cosi nelle Grosgalle tra Lezzeno e Bellagio, sui Corni di Canzo, al Pertuso sopra Brunatr (Com.), in val Sassina 
sopra Bellano (Ramp.!) ecc., in val Tellina a Piatteda (Com.) e a Bormio (Ramp.!), a Riva di Sesia (Carestia!), a Courmayeur(Webb!), nel Cenisio (Parl.!), nei dintorni di Torino (Delponte! ecc.), sopra i Bagni di Valdieri a 1200-1300 metri (Parl.!), al col di Tenda (Bourgeau! ecc.), nelle Viosenne (Ricca!), a Fenestre, a Sant'Anna di Vinai (Ard.), nelle Riviere a Dolcedo (Berti!), Gandebella presso Oneglia (Bert.), Dego (Bertero!), sopra Voltri, a Genova (Figari!), sopra Chiavari (Delpino!) e a Graveglia (Berti!), nei monti del Pavese (Nocca Balb.), in tutto l'Appennino modenese (Gib. Pir.) e bolognese (Cocc.), nell'Appennino toscano nella regione montana dai monti Corneviglio (Bert.) e Gotro! al Mugello (Sav.) e al Cásentino (Bert.), scendendo nella regione submontana e persino nella campestre nelle valli della Magra!, e del Serchio sino ai dintorni di Lucca (Pucc.! ecc.), in tutte le alpi Apuane!, quindi, saltando il tratto intermedio, nell' Appennino piceno-abruzzese alla Sibilla (Bert.), al monte Acuto (Orsini !), al Cavaliere (Sang.), sopra Umito!, al Corno, a Chiarino (Ten.). Fiorisce da maggio a luglio secondo i luoghi.

Distribuzione geografica. - Nei monti dell'Europa meridionale-centrale dalla Spagna alla Rumenia.

Descrizione. - Pianta perenne, alta da 3 a 6 decimetri, di un verde chiaro, fornita di un rizoma duro, ramoso, che manda fibre radicali lunghette, delicate e bianchicce e dei fusti. Questi sono eretti 0 ascendenti, un po'obliqui, un po' flessuosi, cilindrici, pubescenti, con i peli curvati un po'in su, verdognoli. Le foglie sono molli, opposte, un po'lontane, patenti o patentissime, sessili, le inferiori più piccole, ovate, ottuse, le medie ovate o ovato-bislunghe, acute, e le superiori ovato-acuminate, tutte sessili, dentate, con i denti grossetti e ora più ora meno profondi e acuti: verdi di sopra con molti solchi che corrispondono ai nervi della pagina inferiore, di un verde chiaro di sotto ed ivi con un nervo longitudinale rilevato da cui partono molti nervi laterali, molto obliqui e rilevati, i quali si uniscono tra loro in rete verso il margine e terminano ai denti di questo: sono ruvidette in ambedue le pagine e massime nella inferiore per peli lunghi, rari, eretti e duretti, hanno qualche ciglio nel margine. I fiori sono distribuiti in racemi radi e ascendenti, quasi il doppio più lunghi delle foglie corrispondenti. Il peduncolo è delicato, cilindrico, verde, pubescente, ruvidetto. I pedicelli sono quasi capillari, patenti, pubescenti-glandolosi, verdognoli, poco piủ lunghi del fiore, più lunghi del frutto e accompagnati da una brattea bislungo-lineare, verde, 
fornita di ciglia glandolose e più corta del pedicello medesimo. Il calice è piccolo, verde, puberulo-glanduloso, con il tubo corto e il lembo diviso in quattro lacinie, due o tre volte piủ lunghe del tubo, patenti, bislunghe, ottuse, avvicinate poi al frutto. La corolla è molto più grande del calice. Il suo tubo è cortissimo, verdognolo e pelosetto di dentro, e il lembo apertissimo in modo che la corolla è quasi piana, di color roseo-violetto, con righe longitudinali più cariche, manifeste in basso e unite presso la gola quasi in un cerchio più carico che racchiude una parte verdognola che corrisponde alla parte interna del tubo della corolla; il lembo è diviso profondamente in quattro lacinie un po' disuguali, la superiore essendo la più larga, e quasi orbicolare, la inferiore la più stretta e quasi ovale, tutte pelosette di fuori con i peli patenti e glandolosi, glabre di dentro, con ciglia glandolose nel margine. Gli stami sono due, lunghi quanto la corolla, inseriti alla base del tubo di questa, patenti. I filamenti sono stretti alla base e all' apice, più grossi e quasi piani nel mezzo, del colore della corolla. Le antere sono bislunghe, ottuse, smarginate alla base, inserite nel dorso, biloculari, aprentisi longitudinalmente dalla parte interna e perciỏ introrse, violetto-rossicce, glabre. Il pistillo è piủ lungo degli stami. L'ovario è quasi orbicolare, schiacciato, appena smarginato, verdognolo, peloso. Lo stilo è molto piu lungo dell'ovario, filiforme, glabro, del colore della corolla. Lo stimma è ottuso. (Parl. ms., descr. di pianita di Boscolungo nell' Appennino toscano).

Osservazione. - Questa specie e le due precedenti segnano il passaggio all'altra sezione, inquantochè la gemma terminale del fusto o non si sviluppa o diventa fiorifera, e la pianta si fa perenne per gemme basilari.

" Ciaulis definitus, apice florifer, ope gemmarum basilarium perennans, aut planta annua.

+ Inflorescentia e racemo terminali, solitario aut pluribus axillarilus concomitato.

$\$$ Planta perennes.

\section{Veronica Gousani.}

V. foliis sulisessilibus, ovatis, oltusis vel (superioribus) acutinsculis, grosse serratis, racemis nultilloris, laxis, pedicellis fructife- 
ris erectis, bracteis multo longioribus, lobis calycinis 4 oblongis, inæqualibus, septifragio compresso, ovali-rotundato, emarginato, calyce multo maiore, stylo longitudine septifragii.

Veronica Ponæ Gouan ill. obs. bot.p. 1. Bert. fl. ital. 1.p. 83. Gren. Godr. fl. Fr. 2. p. 595. Mars. cat. pl. Cors. p. 108. Ces. Pass. Gib. comp. fl. ital. p. 35 I. Arc. comp. fl. ital. p. 515.

Veronica Gouani Mor. de quib. pl. Ital. dec. 2. p. 6.

Figura. - Gouan 0. c. t. 1.f. 1.

Abitazione e Fioritura. - Nei monti di Corsica, raccoltavi altra volta da Thomas (Bert., Gren. Godr.), ma, secondochè pare, non più riveduta. Fiorisce in giugno e luglio.

Distribuzione geografica. - E la Spagna con i Pirenei la patria sicura di questa specie.

Descrizione. - Pianta perenne, alta da 1 decimetro e mezzo a due decimetri e mezzo, pelosa, con i peli del racemo glandolosi all'apice. Il rizoma è delicato, cilindrico, e manda ai nodi delle fibre radicali. Il fusto che lo continua è eretto o ascendente, cilindrico, di color rossiccio-scuro, pubescente, con i peli delicati e bianchi, semplice o con qualche piccolo e corto rametto all' ascella delle foglie di mezzo e superiori. Le foglie sono opposte, alquanto distanti tra loro, patentissime, sessili, un po' consistenti, ruvidette perchè coperte di peli corti, rigidetti e bianchi, di color verde, più chiaro nella pagina inferiore, dove hanno un nervo longitudinale prominente dal quale partono dei nervi laterali molto obliqui, che vanno vęrso i denti dove si uniscono tra loro: sono dentato-seghettate con i denti distanti, quasi acuti e con una piccola glandola verde all'apice di ciascun dente, e fornite di ciglia nei margini; le inferiori sono piu piccole, quasi orbicolari o ovali, quelle di mezzo sono piủ larghe e piu lunghe, ovali o ovali-allungate, ottusette all'apice, le supreme sono più piccole e quasi ovate o ovali e un po'acute. Il racemo è solitario, terminale. La rachide e i pedicelli, il calice e la cassula sono pubescenti con i peli glandolosi all'apice. Le brattee sono allungate o quasi lineari, ottusette 0 acute, verdi, più corte dei pedicelli: questi sono poco più corti o quasi uguali al fiore, dapprima orizzontali, poi ascendenti $o$ quasi diritti, di color rossiccio quasi come la rachide. Il calice è quasi campanulato, quadrifido, con le lacinie lineari-allungate, ottuse, patenti, verdi, puberule nel dorso, cigliate nei margini con i peli glandolosi, e fornite di tre nervi longitudinali più chiari, uno nel mezzo e due nei margini. La corolla è molte volte più grande del calice; il tubo è cortissimo, verdognolo, il lembo è 
diviso in quattro lacinie apertissime, disuguali, essendo tre quasi uguali, ovate-orbicolari, e l'inferiore più stretta ovale-allungata, tutte ottuse, intere, di color lilla chiaro con poche righe longitudinali più cariche, più manifeste nella pagina superiore della fogliolina superiore e in parte delle due laterali. La gola è bianchiccia e pubescente, con i peli non glandolosi all'apice. Gli stami sono due, appena piủ corti della corolla, diretti un po'in giù verso la lacinia inferiore di questa, un po'divergenti e inseriti nella base dalla parte interna del tubo. I filamenti sono filiformi, un po' più grossi verso la metà dove sono di color lilla, assottigliati all' estremità ed ivi bianchicci. Le antere sono ovali, ottuse all'apice, profondamente bilobe alla base, inserite nel dorso verso la metà, biloculari, aprentisi longitudinalmente, violette, glabre come i filamenti. Il polline è giallo. Il pistillo è poco più lungo degli stami, un po'curvo e diretto verso giù. L'ovario è ovale, ottuso, un po'schiacciato, verde, appena puberulo, biloculare e contienẹ molti ovoli. Lo stilo è lungo, filiforme, glabro, di color lilla. Lo stimma è quasi in capolino, papilloso, bianchiccio. (Parl. ms., descr. di pianta coltivata).

Osservazione. - Come bene osservarono Moretti e Bertoloni, non può essere conservato a questa specie il nome di Veronica $P_{0}$ nce, poichè ribadisce lo sbaglio preso da Gouan nel riferirvi un sinonimo di Pona che spetta invece alla Bonarota rolundifolia.

\section{Veronica Iongifolia.}

V. foliis breviter petiolatis, anguste lanceolatis, acutis acuminatisve, anguste serratis, racemis subpaniculatis, multifloris, den. sissimis, pedicellis fructiferis erectis, bracteis brevioribus, lobis calycinis 4 lanceolatis, subæqualibus, septifragio turgido, subrotundo, emarginato, calycem breviter superante, stylo septifragio triplo longiore.

Veronica longifolia Linn. sp. pl. ed 1. p. 10. Hausm. A. Tir. p. 639. Pir. 1l. for. syll. p. 105. De Vis. Sacc. cat. piant. Ven. p. 153. Ces. Pass. Gib. comp. M. ital. p. 35\%. Arc. comp. /1. ital. p. 515 .

Veronica spuria Linn.? Ces. Pass. Gib.? I. c. Are.? I. c.

Veronica maritima Linn.?

Veronica ticinensis Poll. R. ver. 8. 1. 768. Bert. R. iial. 1. p. (i). Rota prosp. piant. Pav. p. 270).

Figura. - Reich. ic. 17. germ. 20. 1.93. 
Stazione, Abitazione e Fioritura. - Nei prati umidi in mezzo alle boscaglie fra il Ticino e il Gravellone a Pavia (Bert., Moretti!·ecc.); nel Friuli fra Cividale e Caporeto, e sul mare presso Grado, Aquileia e Monfalcone (Pir.); in Tirolo a Partschins sopra Merano, ma non sicuramente spontanea (Hausm.). Fiorisce in luglio e agosto.

Distribuzione geografica. - Nell' Europa orientale, dalla Scandinavia all'Italia e la Grecia e al Caucaso, in Siberia e Songaria.

\section{Veronica spicata.}

V. foliis subsessilibus vel (inferioribus) in petiolum attenuatis, ovalibus lanceolatisve, subacutis, crenato-serratis, apicem versus subintegris, racemo subsolitario, multifloro, densissimo, pedicellis fructiferis erectis, bracteis brevioribus, lobis calycinis 4 lanceolatis, inæqualibus, septifragio turgido, subrotundo, emarginato, calycem breviter superante, stylo septifragio 2-3-plo longiore.

Veronica spicata Linn. sp. plant. ed.1. p. 10. Bert. f. ital.1. p. 62; et auct.

Figure.-Barr. ic. 682. Fl. dan. t. 52. Reich. ic. fl. germ. 20. t. 92 .

Stazione, Abitazione e Fioritura. - Per i pascoli asciutti e gli scopeti della Penisola. É piủ o meno comune per tutta l'Alta Italia, in collina e in montagna ed anche in pianura; ivi sale nelle Alpi a grandi altezze: al Cenisio (Parl.!), ad Airolo a 1200 metri (Franzoni!), sopra Bormio a 1500 metri (Parl.!), sul Ritten in Tirolo a $4500^{\prime}$ (Hausm.), ecc. É molto meno comune nell'Italia centrale e molto meno ancora nella meridionale, e vi nasce soltanto in luoghi montuosi nelle Alpi marittime a Tenda (Ungern-Sternberg!), a S. Martino Lantosca (Ard.), a Ventimiglia (Bert.); in Toscana nel monte Bruciana presso Massa (Celi!), presso Livorno (P. Savi!), presso Casciana (Bastianini!), nel Volterrano (Amidei! ecc.), in più punti del Fiorentino!, in Mugello (Giannini!), nel Senese (Beccari! ecc.), a Pienza (Sav.), nel monte Calvi in Maremma (Aiuti !), nel monte Amiata (Sommier!); nel Piceno-Abruzzo al monte Priore (Marzia. letti!), presso Arquata (Orsini!), all'Incoronata, alla Maiella, al Piano di cinque miglia, a Capo d'acqua (Ten.); nel Romano ad Arcinazzo (Rolli!); in Terra di Lavoro sul Matese (Avellino!), a Frasso Telesino e Cardito, e ad Itri (Terr.); infine nel Principato ulteriore (Ten.). Fiorisce in giugno e luglio. 
Distribuxione geografica. - Nell'Europa media, e in parte uella settentrionale e la meridionale, nella Siberia occidentale.

Descrizione. - Perenne, alta circa un decimetro e mezzo a 2 decimetri, di un verde piuttosto bianchiccio, pubescente. Rizoma strisciante, radicante per fibre lunghette. Fusto ascendente, semplice, o di raro con qualche ramo alla base, eretto, cilindrico, verdognolo, pubescente, vestito di foglie sino in alto. Foglie opposte, patenti, le inferiori avvicinate tra loro quasi in rosetta, bislungoovali o bislungo-obovate, tondeggianti all' apice, ristrette alquanto alla base, ivi intere, smerlate nel resto, con gli smerli quasi a sega ma ottusi, di un verde un po'bianchiccio di sopra ed ivi con un solco longitudinale, di un verde un po'più bianchiccio di sotto ed ivi con un nervo longitudinale sporgente piủ chiaro, da cui partono dei nervetti laterali delicati che si diramano per unirsi tra loro nel tes. suto della foglia. Le altre foglie sono gradatamente piủ piccole e più strette, bislunghe o bislunghe-lanceolate, con minor numero di smerli e più superficiali. I fiori sono in una lunga spica all' apice del fusto, la quale è quasi cilindrica, assottigliata in alto perchè i fiori si aprano gradatamente da basso in alto e quelli della cima sono chiusi quando quelli di basso e del mezzo sono aperti: sono solitarii, eretto-patenti e sessili o quasi sessili all'ascella di una brattea 0 foglia fiorale, ch'è più lunga del calice, bislungo-lanceolata o quasi lineare, con pochi o punti smerli, del resto simile alle foglie. Il calice è quasi camıanulato, diviso fin sopra la base in 4 lacinie crettopatenti, ovali-bislunghe, ottuse, di un verde bianchiccio, puhescente. La corolla è piủ del doppio piủ lunga del calice, di colore violetto; il tubo è lungo quasi quanto il calice, un po'curvo; il lembo è diviso in quattro lacinie patenti, una delle quali superiore, e tre quasi inferiori in modo da formare quasi due labbri, la lacinia superiore è un po'piủ larga c piủ stretta della inferiore, quelle sono quasi lineari, ottusette. La gola è chiusa da una barba di peli bianchi. Gli stami sono 2, piủ lunghi della corolla, inseriti nel tubo di questa, eretto-patenti. Filamenti filiformi, glabri, del colore della corolla. Antere quasi ovali, ottuse all'apice, profondamente bilobc nella base, inserite nel dorso sopra di questa, bilobe, di color violetto scuro, glabre. Polline giallo. Pistillo piủ corto della corolla. Ovario quasi tondo, verdognolo, pubescente. Stilo cilindrico, diritto, violetto, glabro, poi si allunga. Stimma ottuso. (Parl. ms., descr. di pianta di Edolo nel Bresciano).

Osiervazioni. - Pianta molto variabile, ordinariamente prov. 
vista più o meno di peli cresputi o glandolosi, talora tutta glabra (Veronica nitens Host), col fusto semplice, ma talora ramoso e portante parecchi grappoli di fiori ( $V$. arguta Mor.), le foglie variabili per larghezza, talora le inferiori ovate o quasi cuoriformi ( $V$. hybrida Linn. ?).

\section{Veroniea bellidioides.}

V. foliis obovatis, basim versus attenuatis, obtusissimis, obsolete crenatis, racemis paucifloris, densis, pedicellis fructiferis erectis, bracteis nunc brevioribus nunc longioribus, lobis calycinis 4 .ovalioblongis, subæqualibus, septifragio compresso, ovali, vix emarginato, calyce multo maiore, stylo septifragio breviore.

Veronica bellidioides Linn. sp. plant. ed. 1. p. 11. Vitm. ist. erb. p. 17. Sav. bot. etr. 2. p. 2. Bert. fl. ital. 1. p. 84. Mass. prodr. fl. valt. p. 198. Com. Al. com. 1. p. 12. De Not. rep. fl.lig. p. 303. Hausm. fl. Tir. p. 640. Rota prosp. fl. Berg. p. 66. Pir.! f. for. syll. p. 105. Car. prodr. fl. tosc. p. 48\%. Ard. A. Alpes-mar. p. 276. Zersi prosp. piant. Bresc. p. 156. Ces. Pass. Gib. comp. fl. ital. p. 352. Anzi auct. fl. nov.-com. p. 195. Sacc. Bizz. agg. fl. trev. p. 27. Are. comp. fl. ital. p. 516.

Figura. - Reich. ic. fl. germ. 20. t. 95. f. 4, 5.

Stazione, Abitazione e Fioritura. - Nei pascoli più elevati di tutta la catena delle Alpi: in Friuli presso Sappada (Pir.), nel monte Novarza (Pir.!), sopra il Cansiglio (Sacc. Bizz.), nelle Vette di Fel. tre (Montini!), nel monte Grappa (Sacc. Bizz.), in Pusteria nella valle Rain in val di Taufers, e sull'Ellnerspitze presso Brunecco, intorno a Bressanone, nella valle di Laas in val Venosta, sull'Ifinger presso Merano, sul Falgamaierioch in val d'Olten, sull'alpe Rittner, dov'è comune, e dove principia sopra Pemmern a 5500'e va sino in cima, in Fassa e Fiemme, presso Borgo di val Sugana (Hausm.), presso Trento sul Bondone e sul Gazza (Perini!), nelle Giudicarie sul Frate in Breguzzo (Hausm.), nel Bresciano sul monte Mufetto (Zersi), a Bazzanale (Bert.) e sulle Colombine a 2200 metri, in val Camonica sul Tonale a 2030 metri (Parl.!) e sul monte Proalpe presso Incudine (Caldesi!), in val Tellina sullo Stelvio a $2520 \mathrm{~m}$., sul Braulio a 2810-2900 m., sul Plaghera in val Furva (Parl. !), sulle alpi di Zana, Motta, Acqua negra e in molte altre (Mass.), e in val Malenco (Com.), sul monte Barbelino (Bert.), in val Brem. bana (Rampoldi!), sul Legnone (Bert.) e nelle valli Cavargna e Sas- 
sina del Comasco, sul Camoghè nel Ticino (Com.), sul Sempione (Bert.), sul monte Rosa (Bert.), in val Sesia nell'alpe Rizzola presso Riva (Carestia!), e intorno ad Allagna (Bir.), sul Gran S. Bernardo a $6000^{\prime}$ (Heldreich!), al col du Géant a circa 5000 metri (Parl.!), ncll'Allée blanche (Webb!), sul Cramont a 2472 metri (Parl.!), nell'alpi Sevole (Bert.) e Nuvolet (Delponte!) di Ceresole, sul Ce. nisio a Ronche (Parl.!), sul piccolo Cenisio (Beccari!), nell'alpi di Bancet delle vallate Valdensi (Rostan!), nelle Alpi marittime ai monti Bego (Ard.) e Bissa (Bourgeau!), al col di Tenda (Ungern-Sternberg!), nelle Viosenne (Rosellini!), infine nei monti d'Albenga (Do Not.). E stata indicata nell'Appennino pistoiese in Toscana dal Savi, e prima di lui più precisamente a Mandromini dal Vitman, ma i recenti esploratori non ve l'hanno più trovata. Fiorisce da giugno ad agosto.

Distribuzione geografica. - Nei monti di Europa: Pirenei, Alpi, Sudeti, Carpazi, Balcani.

Descrizione. - Caulis teres, rubellus, hirsutus, pilis longiusculis, horizontalibus, albis, eglandulosis. Folia carnosula, pallide viridia, obtusa, subtus nervo longitudinali nervilisque paucis lateralibus obliquis margine patentibus prædita, supra et margine pilosa vel in pagina utraque glabrata. Pedicelli breves, flore brevio. res, tenues, rubelli, hirsuti. Bracteæ oblongæ, obtusæ, virides, vel partim rubellæ, plus minusve pilosæ, pedicellis longiores et calycem fere æquantes. Calyx 4-fidus, laciniis oblongo-linearibus, obtll. sis, subæqualibus, planis, tubo calycis 3-4-plo longioribus, rubentibus, dorso margineque hirsutis. Corolla calyce paulo longior, tubo albido, intus glabro, laciniis 4 , tubo paulo longioribus, subrotundis, subcrenulatis, concaviusculis, inæqualibus, violaceis sine venis saturatioribus. Stamina corolla breviora, filamentis albis, fauce insertis. Antheræ subrotundæ, fuscæ. Pollen luteolum. Pistillum stamina equans. Ovarium disco annulari albido basi cinctum, subrotundum, compressiusculum, viride, punctis minutis violaceis pilisque præditum, nervo pallidiore marginato. Stylus ovario equilongus, rectus, filiformis, albidus. Stigma subcapitato-hilobum, papillosum, violaccum. (Parl. ms., descr. di pianta dello Stelvio).

\section{1\%. Veronien alpina.}

V. foliis subsessilibus, ovalibus, obtusis vel acutinsculis, subintegris, racemo paucifloro, densissimo, pedicellis fructiferis erectis, 
bracteis brevioribus, lobis calycinis 4 lanceolatis, subæqualibus, septifragio turgidulo, ovali, leviter emarginato, calyce conspicue maiore, stylo septifragio pluries breviore.

Veronica alpina Linn. sp. pl. ed. 1. p. 11. Bert. fl. ital. 1.p. 88. Mass. prodr. fl. valt. p. 199. Com. fl. com. 1. p. 13. Colla herb. ped. 4. p. 335. Gren. Godr. fl. Fr. 2. p. 593. Hausm. fl. Tir. p. 641. Rota prosp. f. Berg. p. 66. Pir.! fl. for. syll. p. 105. Ard. f. Alpes-mar. p. 276. Zersi prosp. piant. Bresc. p. 157. Mars. cat. pl. Cors. p. 108. Terr. sec. rel. Terr. Lav. p. 92. Ces. Pass. Gib. comp. fl. ital. p. 352. Sacc. Bizz. agg. fl. trev. p. 27. Arc. comp. f. ital.p. 516.

Veronica apennina Tausch?. Cies. Pass. Gib. l. c. Paol. el. sib. p. 15.

Figure. - Linn. fl. lapp. t. 9. f. 4. Fl. dan. t. 16. All. rär. Ped. stirp. spec prim. t. 3. f. 3 in Fl. ped. І. 22. f. 5 repetila. Reich. ic. fl. germ. 20. t. 95. f. 1-3.

Stazione, Abitazione e Fioritura. - Nei pascoli ed in luoghi sassosi elevatissimi di tutta la catena delle Alpi: nel Friuli intorno a Pontebba (Pir.!), nel monte Cavallo di Belluno (Kellner!), nelle Vette di Feltre e nei monti Portole e Grappa (Montini!), in Tirolo dal Brennero al Pfitscherioch, e in valle di Pfitsch, intorno a Bressanone, sul Wormserioch, sull' alpe Zil e sull'lfinger presso Merano, sulle alpi Schlern, Seiser e Rittner, sul Penserioch, in Fassa e Fiemme, in Vallarga nella valle Fersina (Hausm.), nei monti Bondone e Gazza (Perini!), e sulla Cima d'Asta e presso Borgo in val Sugana, nelle Giudicarie nell'alpe Cengledino e in val di S. Valentino (Hausm.), nel Bresciano sulle Colombine a 2000_2200 metri (Parl.!) e in Pesseda (Zersi), nel Bergamasco (Rota), in val Tellina sul monte Cristallo a 2850 metri, sullo Stelvio a 2400-2800 metri, sopra S. Caterina, in Plaghera (Parl.!), nelle alpi di Entoa e Seneveda ecc., nel Comasco nei monti delle valli Cavargna e Sassina, del Legnone, di Arengo sopra Livo, nel Ticino nell'alpe di Maraggia (Com.), al S. Bernardino (De Not.!), al Gottardo, al Sempione (Parl.!), in val Sesia nell'alpe Fornaa (Carestia!), al Gran S. Bernardo a 7800', al col du Géant (Parl.!), presso la Tola e Usel verso Locana, nella valle d'Ala e di Monastero (Colla), al Cenisio (Beccari!), nell'alpe Albergian, nell'alpi di Limone (Colla), nelle alpi Marittime alla Madonna di Fenestre (Bourgeau!), al monte Bissa (Parl.!) ecc., infine nell'alpi di Viosenne (Ricca!). Ricomparisce nell' Appennino piceno-abruzzese al Vettore (?) (Paol.), alle Moricane, alla Stanca, al Pizzo di Sivo, alla 
Maiella (Orsini!) e al Costone (Bert.). Nasce ancora in Corsica al monte d'Oro (Gren. Grodr.). Fiorisce da giugno ad agosto.

Distribuzione gecgrafica. - Nell'Europa, dall'estremo nord ai monti dell'Italia e dell'Ungheria, nell'Asia settentrionale e media, nell'America piủ nordica e nei monti Rocciosi.

Descrizione. - Fibræ radicales albæ. Folia, caulis et calyx pallide virentes. Folia subcrenata vel integra, ovalia, vel fere obovata. obtusa, nervo longitudinali et nervulis 2 sublongitudinalibus subtus notata, supra hinc inde pilosa, versus basim et subtus nervo excepto glabrata. Scapus teres, pilosus, pilis albis subhorizontalibus, plerumque eglandulosus. Pedicelli erecti, flore longiores, etiam pilosi. Bractex oblongo-lanceolate, virides. Calyces pilosi, pilis sæpe minute glandulosis, profunde 4-fidi, laciniis erecto-patulis, oblongo-lanceolatis, obtusis, viridibus. Corolla calyce fere duplo longior, violacea cum venis plurinis longitudinalibus saturatioribus, tubo ex albido-luteolo, pilosulo, 4-fida, laciniis subæqualibus, ovato-rotun. datis, concaviusculis, tubo plus quam duplo longioribus. Stamina co. rolla breviora. Filamenta erecta, filiformia, violacea. Antheræ parvæ, atropurpureæ. Pollen albidun. Pistillum staminibus vix longius. 0varium disco annulari crasso, e viridi-luteolo, obtuso basi cinctum, ovale, compressum, viridulum, breviter piloso-glandulosum. Stylus rectus, ovario longior, filiformis, superne vix incrassatus, ex albo et violaceo. Stigma grandiusculum, subcapitato-bilobum, papillosum, album. Capsula oblongo-obovata, marginata, compressa, fusca, pilo. so-glandulosa. (Parl. ms., descr. della pianta dello Stelvio).

Osservazione. - La Veronica apennina di Tausch è ravvicinata dai nostri autori alla $V$. alpina; ma dal poco che ne posso rilevare sembrerelbbe più una qualche forma della $V$. serpillifolia, ed è forse la V. repens.

\section{Veronies fruticulosa.}

V. foliis superioribus ovalibus oblongisve, sessilibus, inferiori. bus hasim versus attenuatıs, obovatis, oinnibus obtusis, subintegris, racemo pancilloro, laxo, pedicellis fructiferis erectis, bracteis lon. gioribus, lobis calycinis 4 ovali-oblongis, subequalıbus, septifragio compresso, ovalı, subintegro, calice conspicue maiore, stylo longitudine septifragii.

Veronica fututiculosa L.mn. sp. plant. ed. 2. p. 15. Vitm. ist. erb. p. 16, 27. Re /l. seg. p. 7. Bir. 11. acon. p. 1. Berl. R. ital. I. 
p. 85. Mass. prodr. fl. valt. p. 199. Com. fl.com. 1. p. 12. De Nol. rep. fl. lig. p. 303. Hausm. fl. Tir. p. 640. Rola prosp. fl. Berg. p. 66. Pir.! fl. for. syll. p. 105. Car. prodr. fl. tosc. p. 487. Ard. fl. Alpes-mar. p. 276. De Vis. Sacc. cat. piant. Ven. p. 153. Zersi prosp. piant. Bresc. p. 157. Mars. cat. pl. Cors. p. 108. Ces. Pass. Gib. comp. fl. ital. p. 351. Anzi auct. fl. nov.-com. p. 195. Cocc. terzo contr. fl. Bol. p. 26. Sacc. Bizz. agg. fl. trev. p. 27. Gib. Pir. fl. Mod. p. 122. Arc.! comp. A. ital. p. 515. Cocc. fl. Bol. p. 359.

$\beta$, floribus maioribus, cœruleis nec roseis.

Veronica saxatilis Linn. fil. suppl. p. 83. Bert. o. c. p. 86. Mass. l. c. Com. o. c. p. 313. Coll. herb. ped. 4. p. 335. Pucc.! syn. pl. luc. p. 15. De Not. l. c. Hausm. o. c. p. 641. Rota l. c. Pir.! l.c. Ard. l. c. Anzi l. c. Sace. Bizz.l. c.

Figure. - Reich. ic. bot. t.903-905. Ic. fl. germ. 20. t. 96.

Stazione, Abitazione e Fioritura. - Sulle rupi e in luoghi sassosi delle parti elevatissime delle Alpi: in Friuli nella valle dell'Isonzo (De Vis. Sacc.), in Valcalda, nel monte Forca presso Ligosullo (Pir.!), nei monti Novazza, Parabba, Sierra, presso Sappada (Pir.), presso Forno di sotto, e Ampezzo (Poll.), nel monte Rite (Bizz.), nel monte Cavallo (Kellner!), al Cansiglio (Sacc. Bizz.), nel Marsupian (Bert.) e le Vette di Feltre, nel Bassanese verso Robbio (Poll.), e nei monti Grappa, Portale, Vallerana, Collalti (Montini!), nei Sette Cornuni a Piediscala (Bert.), nei Lessini (Poll., Bert.), nel Baldo (Manganotti! ecc.), in Tirolo sopra Terragnuolo presso Rove. redo (Hausm.), nel monte Scanuppia (Ambrosi!), in val Sugana a Sette Selle, Mendana e Torcegno, in Fiemme e Fassa (Hausm.), in Vallazza (Bert.), presso Bolzano in parecchi punti, cosi sul Ritten da $3800^{\prime}$ in su, nell'alpi Schlern e Seiser, in Ampezzo, sull' Ifinger presso Merano, sullo Spinale (Hausm.), sul monte Gazza (Perini!), sul Bondone, nelle Giudicarie in valle S. Valentino e a Campiglio in Rendena (Hausm.), ecc., in val Camonica al Tonale a $2000 \mathrm{~m}$., al Venerocolo a 7000-7500' (Parl.!), e nel monte Proalpe (Caldesi!), nel Bresciano sulle Colombine (Zersi), a Branzi in val Brembana (Rampoldi!), in val Tellina sullo Stelvio tra 1700 e $2400 \mathrm{~m}$., a S. Caterina a $1700 \mathrm{~m}$., a S. Colombano a 1000 m. (Parl.!), in val Malenco (Com.), presso Chiavenna in val di Lei (Rampoldi!), nel Comasco sui monti Codeno, Pizzo di Gino, Aigna, Camisolo, Legnone, delle valli Sassina e Cavargna, e nel Ticino sui monti Garzirola, Camoghè, Cola (Com. ecc.) e Serdena (Siegfried!), al S. Bernardino (De Not.!), al Gottardo (Parl.!), in val Intrasca alla Zeda (Bert.), in val For- 
mazza (Gib.!), al Sempione (Bir.), a Riva di Sesia (Carestia!), in val d'Aosta sopra Courmay"eur (Manuel!), al col du Géant, al Cramont a 1995 metri (Parl.!), nell' alpi di Monastero (Colla), nel Cenisio a Ronche (Parl.!), all'Assiette (Re), sopra i Bagni di Valdieri a 1140-1500 metri, al col di Tenda a 1900 metri (Parl. !), al col di Fenestre ecc. (Ard.), nell'alpi di Viosenne (Ricca!), di Triora (Rosellini!) e d'Albenga al monte Galle (Bert.). Ripiglia nell'Appennino nella Riviera di Levante (De Not.); quindi in Toscana al Succiso (Gib. Pir.), nell'alpi di Mommio! e di Corfino (Calandrini!), alla Tambura nelle Alpi apuane (Bert.), al Rondinaio!, le Tre Potenze (Beceari!), Boscolungo al Balzo (Parl.!), il Cimone (Gib. Pir.), il Corno (Parl.!), dove termina. Nasce ancora in Corsica nei monti Rotondo a 2200 metri (Soleirol!), d' Oro e Renoso (Mars.). Fiorisce da giugno ad agosto.

Distribuzione geografica. - In Europa dalle sue parti piủ nordiche alla Spagna, all'Italia e alla Transsilvania, in Groenlandia e al Labrador.

Descrizione. - La pianta è suffruticosa in basso e ivi ramosa, e manda nei rami inferiormente alcune fibre radicali bianchicce $e$ lisce. I rami sono ascendenti, lunghi da $20 \bar{j}$ a 4 o õ pollici, cilindrici, di colore rossiccio, e coperti di piccoli peli bianchi e curvati in alto $e$ in dentro a guisa quasi di piccoli uncini. Le foglie sono opposte, le inferiori piủ piccole, piủ vicine tra loro, aperte e quasi orizzontali, ovate a rovescio, ottusissime e quasi tondeggianti all'apice, le superiori sono dirette in alto e in fuori, piil lunghe, obovato-allungate, ottuse e ristrette inferiormente quasi in un picciỏlo, tutte poi sono verdi come carnosette, leggermente concave di sopra, con una carena ottusa di sotto e fornite nei margini e nella carena di pochi e piccoli peli simili a quelli dei rami. I fiori sono pochi, dei quali due stamo all'ascella delle due brattee che sono all' apice dei rami, ciascuno sostenuto da un gambetto delicato, rossiccio; in mezzo ad essi si trova un altro peduncolo poco più grosso e più lungo, il quale porta in alto due liori: tutti questi gambetti sono coperti di peli come quelli dei rami. Il calice è diviso fin quasi alla base in quattro lacinie quasi uguali, lanceolate, ottuse, di colore verdognolo sfumato di rosiccio, coperti di luori di peli come quelli dei rami. La corolla è di colore viuletto vivo, con un piecolo tubo, bianco tanto di fuori quanto di dentro, in modo che alla gola il bianco stacca benissimo sul color violetto delle lacinie: queste sono alı̣unto disuguali, due un poco piì grandi delle altre 
due, ovali, acute, il doppio più lunghe e molto più larghe delle lacinie del calice, con alcune righe ramosette di color violetto più carico, le quali vanno dalla base all' apice delle lacinie. Gli stami sono lunghi quanto le lacinie della corolla. I filamenti sono filiformi, glabri, di color violetto, eccetto alla base dove sono bianchicci: s' in. seriscono nella parte interna del tubo e sono ivi piu ristretti. Le antere sono ellittiche, bianchicce, ottuse all' apice, bilobe alla base, inserite nel mezzo del dorso. Il polline è gialliccio. (Parl. ms., descr. di pianta della catena del monte Bianco).

Descrizione della var. - Pianta di un verde chiaro, glabra in basso, puberula piủ sopra, pubescente-glandolosa nel fiore e frutto, formante dei graziosi cespuglietti. Fusti legnosi alla base, ivi radicanti, con fibre delicate e giallo-scure, ramosissimi e con i rami decumbenti, cilindrici, delicati, rossicci e glabri in basso, verdognoli in alto, ivi puberuli per peli corti, curvati ad arco e bianchi, forniti di foglie in alto. Foglie tutte opposte, patenti o eretto-patenti, un po'consistenti, bislunghe 0 obovato-bislunghe, ottuse e le inferiori più piccole, quasi spatolate, obovate, o ovali-tonde, intere 0 con pochi smerli superficiali, verdi chiare e lucide di sopra e ivi quasi scanalato-concave, di un verde un po'più chiaro di sotto ed ivi con un nervo lorgitudinale ottuso che le rende quasi carenate, glabre o con poche ciglia. Fiori pochi e solitarii all' ascella delle foglie supreme, formanti un capolino 0 un racemo corto. Pedicelli alterni, eretti, delicati, peloso-glandolosi, più lunghi della brattea corrispondente, la quale è pure alterna, bislungo-lanceolata o quasi lineare, ottusa, verdognola, pubescente di fuori. Calice diviso profondamente in 4 lacinie, patenti, bislunghe-lineari, ottuse, verdognole o rossicce, pubescenti-glandolose. Corolla grandicella, di un roseo-violetto, con righe longitudinali semplici o biforcate più cariche e con la gola gialliccio-verdognola; tubo corto, del colore della gola, con qualche pelo di dentro; lembo diviso profondamente in 4 lacinie ovali-tonde, poco disuguali, patentissime, glabre di sopra, puberule di sotto. Stami 2, eretto-patenti, poco più lunghi delle lacinie della corolla, inseriti in basso del tubo. Filamenti filiformi, glabri, di un violetto chiaro; antere bislunghe-ovali, profondamente bilobe alla base, inserite sopra di essa, introrse, biloculari, bianchicce-violette. Pistillo lungo quasi quanto gli stami. Ovario piccolo, ovale, un po' schiacciato, verdognolo, pubescente, glandoloso in alto, circondato alla base da un disco quasi un anello gialliccio. Stilo molto più lungo dell'ovario, più stretto in basso, violetto in alto, glabro. Stimma grossetto, in capo- 
lino, violetto, papilloso. Cassula eretta, piủ lunga del calice, ovale, schiacciata verso alto, sinarginata, pubescente-glandolosa, verdognola. Semi quasi ovali, schiacciati, giallicci, glabri, lisci. (Parl. ms., descr. di pianla del col di Tenda).

Osservazione. - Non può essere, per ragione di luogo, questa specie la Veronica saxatilis indicata da Terracciano (Rel. Terr. Lav. p. 148) al monte Mleta.

\section{Veronica senpillifollia.}

V. foliis subsessilibus, obovalis ovalibus ovatis oblongisve, obtusis vel acutiusculis, subintegris, racemo multifloro, laxo, pedicellis fructiferis erectis, bracteis nunc longioribus nunc brevioribus, lobis calycinis 4 ovalibus, subæqualibus, septifragio turgidulo, transverse latiori, profunde emarginato, calyce subæquali, stylo longitudine septifragii.

Veronica serpyllifolia Linn. sp. pl. ed. 1. p. 12. Bert.! 17. ital. 1. p. 89; et auct. omn.

Veronica repens Terr.! rel. Terra di Lav. p. 148. Quart. rel. p. 111.

Figure. - Fl. dan. t. 492. Reich. ic. fl. germ. 20. t. 97. f. $2-4$.

$\beta$ tenella, caule tenui prostrato, foliis parvis rotundatis, racemo paucifloro.

Veronica tenella All. f. ped. 1. p. 75.

Veronica repens Clar. in Cand. A. fr. 3. p. 727. Bert. o. c. p.98. Gren. Godr. fl. Fr. 2. p. 594. Mars. cat. pl. Cors. p. 108. Ces. Pass. Gib. comp. 1. ilal. p. 351. Arc. comp. 月. ital. p. 515.

Veronica serpyllifolia var. auct. nonn.

Figure. - 1ll. o. c. 1.22. f. 1. Lois. f. gall. t. 1. Reich. l. c. f. 1.

Stazione, Abitazione e Fioritura. - Ovunque nei luoghi erbosi umidi, nei campi ecc., dal littorale fino al termine della vegetazione nei monti. Peraltro nella Penisola meridionale, per quanto sia stata trovata in losso sino a Palmi in Calabria (Arc.!), si ristringe pii particolarmente ai monti, per esempio nel Vulture (Terr.). Delle isole minori nasce soltanto nell'litba (P. Savi!). Abita tulte e tre le maggiori, ma anche ivi fatta pianta esclusiramente montanina (Mars., Mor.), e in Sicilia nella sola parte settentrionale orientale, dalle Madonic (Parl.! ecr.) al Messinese (Guss. cce.). La var. i stata trovata 
qua e là nei monti, in Piemonte (All.), nel Vicentino, a Sarzana (Bert.), in Abruzzo (Ten.), e più particolarmente fa in tutte le cime di Corsica sopra i 2000 metri (Soleirol!, Requien! ecc.). Fiorisce secondo i luoghi in aprile e maggio, o maggio e giugno, o giugno e luglio.

Distribuzione geografica. - Pianta quasi cosmopolita : nell'Europa tutta quanta, in Algeria, nell'Asia Minore, in Siberia, nell' Imalaia, dall' America settentrionale per i monti sino all' estremità meridionale, al Capo, nei monti d'Australia, nelle terre antartiche.

Osservazione. - Pianta polimorfa, per la statura, la robustezza, la direzione, per la grandezza e forma delle foglie, per il numero dei fiori. La Veronica repens di Corsica è una forma estremə, alla quale si avvicina la $V$. tenella di alcuni luoghi della Penisola.

$\S \S$ Plantæ annuæ.

\section{Veronica peregrima.}

V. foliis ex ovali aut oblongo lanceolatis, obtusiusculis, in petiolum attenuatis, parce dentatis, supremis integerrimis, racemis multifloris, laxis, pedicellis fructiferis erectis, bracteis multoties brevioribus, lobis calycinis 4 oblongis, subæqualibus, septifragio compresso, obcordato, calyce æquali, stylo subnullo.

Veronica peregrina Linn. sp. pl. ed. 1. p. 14. Berl. R. ital. 1. p. 91. Trev. prosp. fl. eug. p. 23. De Vis. Sacc. cat. piant. Ven. p. 154. Ces. Pass. Gib. comp. 月. ital. p. 352. Sacc. Bizz. agg. fl. trev. p. 27. Arc. comp. fl. ital. p. 516.

Figure. - Reich. ic. bot. t. 36. f. 74-76. Ic. fl. germ. 20. t. 98. f. 1.

Stazione, Abitazione e Fioritura. - In luoghi coltivati della Penisola, assai rara: nell' orto botanico e negli altri orti di Padova, dove pare che abbondi (Saccardo! ecc.), negli Euganei (Trev.), presso Valdobbiadene nel Trivigiano (Sacc. Bizz.), presso Torino tra Nichelino e Stupinigi (Chevalier!), nelle vallate Valdensi fino a $800 \mathrm{me}$ tri (Rostan!), a Casale (Ces. Pass. Gib.), nell' Orto botanico di Firenze (Bucci!), presso Napoli al Fusaro (Ten.). Fiorisce in aprile, maggio, giugno.

Distribuzione geografica. - Oriunda dell'America, che abita da un estremo all'altro, ora inselvatichita in molti luoghi di Europa. 


\section{Veronica arvensis.}

V. foliis subsessilibus, ovatis, crenato-dentatis, obtusis, racemis multifloris, laxis, pedicellis fructiferis erectis, bracteis multoties brevioribus, lobis calycinis 4 lineari-lanceolatis, inæqualibus, septifragio compresso, obcordato, calyce breviore, stylo sinu septifragii breviore.

Veronica arvensis Linn. sp. pl. ed. 1. p. 13. Bert. fl. ital. 1. p. 92; et auct. omn.

Figure. - Col. phylob. t. 8. Reich. ic. fl. germ. 20. t. 99. f. 2.

Stazione, Abitazione e Fioritura - Abbonda per i campi e i luoghi incolti di tutta quanta l'Italia, comprese le isole minori. Sale nelle Alpi fino a 1200 metri a Bormio (Parl.!), e a 1800 metri sul monte Baldo (Goiran!), nell'Appennino fino a tutta la regione moritana!, in Sardegna fino a 1800 metri (Mor.). Fiorisce da marzo a luglio secondo i luoghi.

Distribuzione geografica. - In tutta l'Europa, nell' Affrica settentrionale, nell'Asia occidentale, in Siberia e Songaria, nell'America settentrionale, introdotta.

\section{Veronica verna.}

V. foliis subpetiolatis, inciso-dentatis pinnatipartitisque, lobo terminali maiore obtuso, racemis multifloris, laxis, pedicellis fructiferis erectis, bracteis multo brevioribus, lobis calycinis 4 linearilanceolatis, inæqualibus, septifragio compresso, transverse latiori, late emarginato, aut obcordato, calyce breviore, stylo sinum septi. fragii æquante.

Veronica rerna Linu. sp. plant. ed. 1. p. 14. Re fl. seg. p. 8. Bert. ח. ital. 1. p. 95. Mass. prodr. 1. valt. 1. p. 198. Com. R. com. 1. p. 22. Trev mosp. П. eug. p. 23. Rota mrosp A. Berg. p. 66. Hausm. 1. Tir. p. 619. De Vis. Sacc. cat. piant. Ven. p. 154. Zersi prosp. piant. Bresc. p. 15\%. Mar's. cat. pl. Cors. p. 108. Ing. cat. sp. Mond.p. 7.'. Cies. Pass. Gib. comp. A. ital. p. 352. Cib. Pir. /l. Mod. p. 12\%. Arc. comp A. ital. p. slo.

Figura. - Reich. ir. 1. germ. 20. t. 99. f. 1.

F longislylu Ces. Pass. Gib. l. c.

Veronica succulenta All. Al. ped. 1.p. 78. t. 99. f. 4 (mala). $\gamma$ brevistyla. 
Veronica triphyllos $\beta$ Bert. o. c. (excl. syn.).

Veronica brevistyla Mor. stirp. sard. ad calc. Mor. De Not. 1. Capr. Gren.. Godr. A. Fr. 2. p. 597. Mor. /. sard. 3. p. 226. Mars. cat. pl. Cors. p. 108. Ces. Pass. Gib. l. c. Arc. l.c.

Stazione, Abitazione e Fioritura. - Qua e là nei campi e in luoghi erbosi dell'Alta Italia: presso Torino alla Molinetta, a Stıpinigi, lungo la Stura (Re), lungo il Sangone (Chevalier!), a Susa (Re), nelle villate Valdensi (Rostan!), nei monti di Tenda (Reuter!), presso Mondovi (Ing.), ad Alba (Bertero!), ad Oldenico (Mlalinverni!), alla Bicocca (Bir.), a Riva di Sesia (Carestia!), nel Pavese (Piccaroli!), presso Milano a Bollate (Bert.), nel Comasco a Garzola e Geno (Gom.), in val Tellina (Nlass.), nel Bergamasco (Rota), a Ponte di legno (Ricca!), nel Bresciano a Pontoglio (Zersi), in Tirolo presso Bolzano e presso Welsberg (Hausm.), negli Euganei (Trev.), a Rovigo (Poll.), nel Modenese a Civago (Gib. Pir.) È pure indicata sui monti dell' Italia piủ inferiore al Velino e al Sile (Ten.), e di Corsica al monte Renoso ecc. (Mars.). La var. $\beta$ è propria delle vallate Valdensi del Piemonte, a Fenestrelle (All.), a S. Martino (Rostan!). La var. $\gamma$ è propria della Sardegna nei monti Linas e Genargentu a 1000-1500 metri (Nor.), cosi nella cima Paolino del Genargentu (Lovisato!), e della Corsica nei monti Pigno, Grosso (Bert.), alla foce di Vizzavona (Mars.), a Casarrosule (Gren. Godr.). Fiorisce da marzo a luglio secondo i luoghi.

Bistribuzione geografica. - In tutta Europa, meno le sue parti più scttentrionali e più meridionali, nell'Asia Minore, e fino all'Altai.

Osservazioni. - Si può consultare Bertoloni Fl. ital. e Moris Stirp. sard. sulla questione (iel resto di poco conto) se alla presente specie 0 alla precedente 0 ad una delle due seguenti appartengano la Veronica romana e la $V$. Bellardi descritte e figurate da Allioni nella $F l$. ped.

Io non saprei vedere altro nella $V$. brevistyla Mor. che una varietà della $V$. verna. I semi sono identici.

Re nell'Ad fl. pedem. app. alt. scrive a pag. 190 di aver trovata una volta sola l'affine $V$. digitata Vahl alla Venaria presso Torino, senz'alcuı dubbio avventizia.

\section{Veronien acinifolia.}

V: foliis subpetiolatis, ovatis, obtusiusculis, subdentatis, racemis multifloris, laxis, pedicellis fructiferis patentiusculis, bracteis 
subæqualibus, lobis calycinis 4 ovalibus, subæqualibus, septifragio compresso, transverse latiore, emarginato-bilobo lobis rotundatis, calyce paullo maiore, stylo sinum septifragii vix superante.

Veronica acinifolia Linn. sp. pl. ed. 2. p. 19. Balb. el. piant. Tor. p. 79. Re fl. seg. p. 8. Bert. fl. ital. 1. p. 93. Mass. prodr. fl. valt. p. 199. Pucc.! syn. pl. luc. p. 15. Trev. prosp. fl. eug. p. 23. De Not. rep. fl. lig. p. 303. Koch syn. 1 . germ. ed. 2. p. 608. Pir. f. for. syll. p. 106. Car. prodr. fl. tosc. p. 488. Ard Alpes-mar. p. 276. Ces. el. piant. Maiell. p. 22. Ces. Pass. Gib. comp. fl. ital. p. 352. Anzi auct. fl. nov.-com. p. 195. Cocc. nuov. contr. fl. Bol. p. 23. Arc. comp. fl. ital. p. 516. Cocc. fl. Bol. p. 359.

Veronica præcox Terr.! rel. Terr. Lav. p. 148 (non All.). Quart. rel.p. 111.

Figura. - Reich. ic. fl. germ. 20. t. 98. f. 2.

Stazione, Abitazione e Fioritura. - Qua e là nei campi umidi, ecc. della Penisola: in Piemonte presso Exilles (All.), presso Torino a Druent in abbondanza, e altrove (Re), verso Moncrivello e Borgomasino (All.), a Vercelli, rara (Ces.!), nel Monferrato (Negri!), presso Novara alla Bicocca (Bir.); in Lombardia presso Pavia a Linarolo, Belgioioso ecc. (Nocc. Balb.), nel Comasco al Bassone, ai laghetti di Alserio e di Pusiano, a Colico, a Porlezza ecc. ecc. (Com.), in valle di Liro, in val Telliua a Bormio (Anzi); nel Veneto ad Abano (Poll.), a Selva (Sacc.!), ad Angarano (Montini!), in Venezia (Moric.), nell'alto Friuli (''ir.); in Istria a Trieste (Poll.); nell'Emilia a Noceto nel Parmense (Pass.!), e nei monti Bolognesi al Granaglione e al monte Acuto (Cocc.); in Liguria a Nizza e alla Rocchetta (Ard.), e a Loano (De Not.); in Toscana a Sarzana al Corso, abbondantissima (Bert.), presso Massa (Celi!), a Lucca, pure comune (Calandrini! ecc.), nel monte Pisauo (P. Savi!), intorno a Firenze in parecchi punti!; in val Tiberina a Balcianclla (Amidei!); nel Piceno-Abruzzo a Montefortino (Marzialetti!), al monte de’ Fiori (Orsini!), al Corno (Bert.), all'Orfenta (Ces.), a Revisondoli, Roccarasa, il Piano di cinque miglia ('T'en.); a Roma alla villa Porghese (Barbieri!) e altrove; in Cimpania a Caserla (Terr.!), Carditello, Sandonato (Terr.), Napoli al lago di P'atria (Bert.) e al Fusaro (Ten.); in Calabria (Bert.). Finrisce in aprile $\mathrm{c}$ magrgin.

Distribuzione geografioa. - Nell Europa media e meridionale, in Madera, nell' Asia nccidentale. 


\section{Veronica troiplnyllos.}

V. foliis subsessilibus, digitato-partitis, imis ovatis subintegris, racemis multifloris, laxis, pedicellis fructiferis erectis adscendentibusve, bracteis subæqualibus, lobis calycinis 4 oblongis, subæqualibus, septifragio turgidulo, subrotundo, emarginato, calyce subæquali, stylo septifragio triplo breviore.

Veronica triphyllos Linn. sp. pl. ed. 1. p. 14. Balb. el. piant. Tor. p. 80. Re fl. seg. p. 8. Bert. fl ital. 1. p. 96 (excl. var.): Mass. prodr. fl. valt. p. 198. Com. Al. com. 1. p. 21. Trev. prosp. fl. eug. p. 23. De Not. rep. fl. lig. p. 304. Rota prosp. fl. Berg. p. 67. Hansm. fl. Tir. p. 643. De Vis. Sacc. cat. piant. Ven. p. 154. Zersi prosp. piant. Bresc. p. 15\%. Ces. Pass. Gib. comp. fl. ital. p. 352. Ing. cat. sp. Mond. p. 74. Arc. comp. fl. ital. p. 517.

Figure. - Fl. dan. t. 627. Reich. ic. fl. germ, 20. t. 100. f. $2-4$.

Stazione, Abitazione e Fioritura. - Nei luoghi coltivati dell'Alta Italia: a Torino, abbondante, a Susa, pure (Re), presso Mondovi alla Garzegna, ancora comune (Ing.), a Serravalle di Scrivia (De Not.), a Novara, comune (Bir.), a Magenta (Parl.!), a Pavia, non comune (Nocc. Balb.), a Milano (Rampoldi!), nel Comasco, comune (Com.), in val Tellina, pure (Mass.), nel Bergamasco (Rota) a Ponte di legno (Ricca!), nel Bresciano, frequente, cosi a Mombello (Zersi), nelle Giudicarie a Tione (Hausm.), a Trento (Perini!), a Borgo di val Sugana, intorno a Bolzano, comune, in val Venosta presso Goldrain, a Bressanone, a Welsherg (Hausm.), a Verona (Parl.!), comunissima (Poll.), a Montebello (Parl.!), negli Euganei (Trev.), a Bassano (Montini!), a Venezia al Lido ecc. (Moric.), a Mantova (Barbieri!), a Guastalla (Pass.!), a Parma (Jan!). Molto rara altrove, è stata trovata in Toscana a Fiesole (Gemmi!), e in Arezzo (Marcueci!), e in Corsica a Calvi (Bert.). Fiorisce da marzo a maggio.

Distribuzione geografica. - Nell' Europa media e meridionale, nell'Affrica settentrionale, nell' Asia occidentale.

Descrizione. - Annua, alta da 1 a 2 decimetri, delicata, verde piuttosto chiara, pelosa con peli delicati e nelle parti superiori aventi, all'apice una piccola glandola, per i quali è un po' vischiosa. Radice delicata, fusiforme, torta, fibrillosa, bianchiccia. Fusto ascendente 0 eretto, semplice o ramoso, con i rami patenti o eretto-patenti, 
cilindrici, verdognoli, peloso-glandoloso. Foglie inferiori piccole, ovali o ovali tonde, fornite di un corto picciòlo, tondeggianti all'apice, leggermente e irregolarmente sinerlate nel margine; quelle della parte media del fusto sono quasi sessili o sessili, palmatidivise, con 50 3 lacinie disuguali bislunghe o quasi spatolate, ottuse o tondeggianti all'apice; le superiori sono trifide o bifide, con le lacinie disuguali, la terminale essendo sempre maggiore delle laterali o della laterale; le foglie fiorali sono talvolta ridotte alla sola lacinia laterale: tutte le foglie sono verdi, di un verde piuttosto chiaro di sopra, ed ivi con un leggiero solco longitudinale al quale corrisponde di sotto un nervetto longitudinale, sono pelose in ambedue le pagine e le superiori peloso-glandolose. I fiori sono solitarii all' ascella delle foglie superiori, e formano quasi un racemo troncato che si allunga nel frutto. I pe. dicelli sono eretti o eretto-patenti, un po'ascendenti, più lunghi del fiore e della foglia fiorale corrispondente, delicati, cilindrici, verdognoli o sfumati di rossiccio, peloso-glandolosi. Il calice è diviso profondamente in 4 lacinie, le quali sono quasi uguali, patule in gran parte, bislunghe, ottuse, verdi, pelose di fuori. La corolla è appena più corta e quasi eguale al calice, ha il tubo corto, bianchiccio, fornito di dentro di pochi peli corti e bianchicci dove corrisponderebbe il mezzo di ciascuna lacinia di essa: con il lembo diviso in quattro lacinie, patenti, disuguali, quasi orbicolari, di color carnicino con poche righe larghette e raggianti dalla base di color piủ carico, e con pochi e corti peli di fuori verso l'apice della lacinia. Gli stami sono due, piǹ della metà più corti della corolla, inseriti nel tubo di questa. Filamenti filiformi, eretto-patenti, bianchicci, glabri. Antere piccole, quasi ovate, ottuse, smarginate alla base, inserite nel dorso sopra di questa, biloculari, di un violetto carico. Pistillo quasi uguale agli stami. Ovario piccolo, quasi tondo, un po'schiacciato, smarginato all'apice, verdognolo, pubescente-glandoloso. Stilo più lungo dell'ovario, diritto, cilindrico, di color violetto, glabro. Stimma in capolino, papilloso, bianchiccio. Cassula eretta, più corta del calice dal quale $\dot{e}$ in gran parte coperta, quasi tonda, con un solco longitudinale in ciascuna faccia che la rende quasi biloba, smarginato-biloha all'apice ed ivi con lo stilo persistente e lungo quasi il terzo di essa, convessa nelle facce in basso e schiacciata ivi in alto, verdognola e poi scuretta, con peli corti e glandolosi in alto e segnatamente nel margine: si apre longitudinalmente in due valve dall'apice in poi e conticne molti semi. I semi sono quasi ovati, con una faccia convessa e ivi con asperiti ottuse a guisa di tubercoli, Inolto concavi nel- 
l'altra faccia e ivi con il rafe e l'ombelico rossiccio, sono scuri e quasi nericci. (Parl. ms., descr. della pianta di Fiesole).

Osservazione. - I semi, che nel resto del genere hanno la faccia interna pianeggiante, sono singolarmente concavi a coppella in questa specie e nelle seguenti.

\section{Veronica prrecox.}

V. foliis subpetiolatis, ovatis, obtusis, inciso-dentatis, racemis pauci vel multifloris, laxis, pedicellis fructiferis erectis, bracteis brevioribus, lobis calycinis 4 oblongis, subæqualibus, septifragio turgido, ovali, emarginato, calyce æquali, stylo septifragio triplo breviore.

Veronica præcox All. auct. ad fl. ped. p. 5. Balb. el.piant. Tor. p 80. Re fl. seg. p. 8. Fl. tor. p. 31. Bert. fl. ital. 1. p. 99. Mass. prodr. fl. valt. p. 199. Com. 1l. com. 1. p. 23. Sang. cent. prodr. fl. rom. add. p. 6. Trev. prosp. A. eug. p. 23. Guss. fl. sic. syn. 1. p. 15. Rota prosp. piant. Pav. p. 270. De Vis. Sacc. cat. piant. Ven. p. 154. Ces. Pass. Gib. comp. ft. ital. p. 352. Ing. cat. sp. Mond. p. 74. Gib. Pir. fl. Mod. p. 123. Arc. comp. fl. ilal. p. 517.

Figure. - All. o. c. t. 1. f. 1. Reich. ic. fl. germ. 20. t. 100. f. 1.

Stazione, Abitazione e Fioritura. - Nei campi per tutta l'Italia, ma molto sparpagliata. Nasce in Piemonte presso Torino tra Grugliasco e Rivalta (Balb.), e a Lucengo ecc., nella valle d'Exilles, rarissima (Re), a Fenestrelle, comune (Rostan!), e presso MondoviMerlo (Ing.); in Lombardia nella Lomellina (Rota), a Como nel torrente Cosio (Com.), e in val Tellina (Mass.); nel Veneto nel Mantovano, nel Vicentino (De Vis. Sace.), e negli Euganei (Trev.); nel Modenese alla Rocca di Fiumalbo (Gib. Pir.); nel Piceno-Abruzzo al Vettore sopra Furca di Presto (Parl.!), al monte de'Fiori (Orsini!), e al Velino (Ten.); in Corsica (Bert.); in Sicilia nelle Madonie al Piano della Principessa (Gasparrini!), a Busambra, e al monte di Cammarata (Guss.). Fiorisce da aprile a giugno.

Distribuzione geografica. - Nell'Europa media e meridionale, nell'Affrica settentrionale, nell' Asia Minore.

* Inflorescentia e floribus in foliis floralibus axillaribus, solitariis. Plantæ annuæ. 


\section{Veronics rournefortib.}

V. foliis breviter petiolatis, ovato-rotundatis ovalibusve, grosse dentatis, obtusis, pedicellis fructiferis recurvatis, folia subsuperantibus, lobis calycinis 4 late lanceolatis, inæqualibus, septifragio compresso, transverse latiori, sinu latissimo emarginato-subbilobo lobis acute marginatis, calyce paullo minore, stylo septifragio paullo breviore.

Veronica Tournefortii Cmel. /l. bad. 1. p. 39 (1805). Ard. /l. Alpes-mar. p. 277. Ces. Pass. Gib. comp. /l. ilal. p. 352. Smith $/ l$. Fíume p. 38. Arc. comp. fl. ital. p. 517. Cocc. /l. Bol. p. 360.

Veronica persica Poir. in enc. méth. 8. p. 542 (1808). Com. $\mu$. com. 1. p. 20. Trev. prosp. 凡. eug. p. 23. Ard. cal. pl. Menl. p. 27. Car. prodr. 1t. losc. p. 488. Pasq. fl. ves. p. 76. Mars. cat.pl. Corse p. 108. Ces. el. piant. Maiell. p. 22. Nic. prodr. 11. mess. p. 3\%1.

Veronica Buxbaumi Ten.! fl. nap. 1.p. y (18/1). Bert. /l. ital. 1. p. 102; et auct. ital. plur.

Figure. - Ten. o. c. t. 1. Reich. ic. bot. t. 268. Ic. 凡. germ. 20. $t .78$.

Stazione, Abitazione e Fioritura. - Nei luoghi erbosi presso all'abitato, nei coltivati ecc., comunissima in alcuni siti, mancante in altri. Nasce a Torino (Delponte!), dove abbonda (Re), a Biella, comune (Zun.), a Novara (Bir.), a Pavia, in pochi punti (Nocc. Balb.), nel Ciremonese (Parl.!), a .lilano (Rampoldil), nel Comasco, abbondante (Com.), e sul monte Legnone (Ricasoli!), in val Tellina a Muntagna e altrove (Mass.), e a Belladore a 700 metri (Parl.!), nel Bergamasco (liota), e a Poute di legno a 1520 metri (Parl.!), nel Bresciano, Prequentissima (Zersi), in Tirolo presso Tione (Hausm.), a Trento (Perini!), in val Sugana (Amhrosi!), in Fiemme e Fassa, e a Bolzano (Hausm.), in tutto il Vencto, copiosamente (De Vis. Sacc.), e in modo più specificato a Verona (Poll.), negyli Euganei (Trev.), a Padora, Venezia (Parl !), Hassano (Montinil), Ijdine (Pir.!), Monfalcone ecc., a Trieste (Bert.), a Fiume (Smith), a Mo. dena, comune, e nell'Appennino a Fiumalbo e al Salto di Montese (Gib. Pir.), nel Bolognese, volgarissima (Coce.), e sino alla Porretla (G. Bert.), a Facnza, ovmnque (Calt.!), per tulta la Liguria (I) Not.), da Tenda (Ungern-Sternberg!) e Nizza (Ard.) a Cliaviri (Bert.), per la Toscana in generale, e più specialmente a Pontremoli 
(Parl.!), a Carrara (Bicchi!), nella bassa Versilia (Simi!), nel Lucchese (Bicchi! ece.), nel Pisano (Savi! e(c.), nel Fiorentino!, e in Casentino a Prataglia (Parl.!), nell'A ppennino umbro (Fiorini!), nelle Marche ad Avellana (Piccinini!), Macerata (Aiuti!) e Montefortino (Marzialetti!), a Pescara (Kuntze!), Chieti e S. Valentino (Ces.), a Roma (Sang.), a Caserta (Terr.), a Napoli (Ten.!), ovunque, in Capri (Pasq.), sul Vulture (Terr.), nel Principato citeriore (Ten.), sul Pollino presso Castrovillari a 1300 metri (Huter Porta Rigo!), in Sicilia nella punta nord-est a Messina (Seguenza! ecc.), Gesso, Milazzo, Fiumedinisi, Taormina (Guss., Nic.), in Corsica a Bastia, Aiaccio, Portovecchio (Mars.). Fiorisce nei luoghi più caldi da gennaio ad aprile, nei meno caldi da maggio ad agosto.

Distribuzione geografica. - Nell'Europa media e meridionale, nell'Asia occidentale e fino all'Imalaia, nell'America settentrionale, naturalata.

\section{2\%. Veromica agrestis.}

V. foliis breviter petiolatis, ovato-rotundatis ovalibusve, grosse dentatis, obtusis, pedicellis fructiferis recurvatis, folia subæquantibus, lobis calycinis 4 ovalibus, iræqualibus, septifragio turgido, transverse latiori, sinu angusto emarginato-bilobo lobis subglobosis subcarinatis, calyce paullo minore, stylo septifragio 2-3-plo breviore.

Veronica agrestis Linn. sp. plant. ed. 1. p. 13. Benth. in Cand. prodr. 10. p. 487.

$\alpha$, septifragio lobis evidentius marginato-carinatis, abortu oligospermis.

Veronica pulchella Bat. ess. fl. Maine et Loire p. 414; et auct.

Veronica agrestis Fries nov. fl. suec. p. 2. Bert. fl. ital. 1. p. 100; et auct. nonn.

Veronica opaca Fries o. c. p. 3; et auct.

Figure.-Reich. ic. bot. 1. 277, 278. Ic. fl. germ. 20. t. 79.

$\beta$, septifragio lobis obscure marginatis rotundatis, poly. spermis. et auct.

Vetonica didyma Ten.! fl. nap. prodr. p. 6. Bert. o. c. p. 101 ;

Veronica polita Fries o. c. p. I; et auct.

Figure.-Reich. ic. bot. t. 240. Ic. fl. germ. 20.t. 7\%. f. 1, 2. 
Stazione, Abitazione e Fioritura. - Ovunque nei luoghi coltivati o abitati. Io non ho alcuna indicazione precisa quanto possa salire nelle Alpi; nè che oltrepassi la regione submontana nell' Appenuino. $\dot{E}$ un fatto notevole che non sia stata trovata in Corsica che di recente, essendo ivi indicata soltanto dal Compendio di Cesati, Passerini e Gibelli; e che delle isole minori non abita che quelle d'Ischia (Guss.), di Capri (Pasq.), e di Malta (Zer., Grech). Fiorisce nei luoghi più caldi dall'autunno alla primavera, e nei meno caldi in primavera.

Distribuzione geografica. - In tutta Europa eccettoché la sua porzione artica, nell'Affrica settentrionale, nell'Asia occidentale fino all'India, nella Siberia fino all' Altai, nella Nord-America, introdotta.

Osservazioni. - Questa pianta varia nel fiore e nel frutto: per la larghezza dei sepali, che quando sono più larghi si ricuoprono, altrimenti no, per la loro forma, che dall'ovale tende ora all'ovato, ora allo spatolato, e per una differenza più o meno marcata nella loro grandezza; per la grandezza della corolla, e per il suo colore, turchino carico, o piủ pallido, o bianchiccio; per il frutto, che talora nasconde il suo spigolo in fondo a un solchetto dorsale quando ilati ne sono molto turgidi, e talora lo tiene alla pari dei lati, e talora lo mette in evidenza sopra questi quando sono poco turgidi : cose che vedonsi nei frutti di un medesimo individuo, e in un medesimo frutto secondo il punto della sua maturità; infine per il numero dei semi in relazione alla grandezza e gonfiezza del frutto. Peril cumulo delle mie osservazioni, fatte sul vivo e sul secco, non posso che consentire pienamente nella restituzione fatta da Bentham dell' antica Veronica agrestis Linneana, contro il parere dei moderni che lo spartiscono generalmente in tre specic.

\section{Veronies hedlerrefolia.}

V. foliis breviter ant longinsenle petiolatis, rotundatis, ohtusis, crenato-subynunquelobis, pedicellis fructiferis patulis refractisve, folia subequantibus, lubis calycius '́t cordatis, equalibus, septifra. gio turgido, subgloboso, leviter emarginato, i-lobo, calyce conspicue minore, stylo septifragio dinidio breviore

Veronica hederiefolia Linn. sp plant. ed. I. p. I.) (excl. \&) Bert. Al. ital. I. p. 101; el auct. omn. 
Figure, - Fl. dan. t. 428. Reich. ic. fl. germ. 20. t. 77. f. 3, 4 .

Stazione, Abitazione e Fioritura. - Nei luoghi coltivati di tutta l'Italia. É comunissima nell'Alta Italia, penetrando anche nelle vallate alpine e nelle appennine nella regione submontana (Cocc.); comune ancora in Liguria, da Tenda (Ungern-Stemberg!) e da Nizza (Ard.) a Genova (Carrega!) e a Chiavari (De Net.), e in Toscana, nelle regioni maremmana, campestre e submontana, comprese le isole di Gorgona (P. Sav.), di Capraia (Mor. De Not.) e dell'Elba; fa nelle Marche ad Urbino (Serpieri!), Avellana (Piccininil), Montefortino (Harzialetti!), e nel monte Acuto (Bert.), nell'Abruzzo al monte de'Fiori (Orsini!), a Pescara (Kuntze!), e più giù al Gargano (Pasq. Lic.) e sino ad Otranto (Groves); fa a Roma (Parl.!), sempre comune (Seb. Maur.), Caserta (Terr.), Napoli (Ten.), in Ischia (Guss.) e Capri (Pasq.), a Cusano (Pasq.!), nel Vulture (Terr.); nasce da per tutto in Sicilia (Guss. ecc.); si trova in Malta (Zer. ecc.), in Sardegna (Mor.), in Corsica ad Appieto, Aiaccio, Corte, Bastia ecc. (Mlars.). Fiorisce da gennaio e febbraio ad aprile, maggio, giugno.

Distribuzione geografica. - In tutta Europa, eccettuata la sua porzione artica, nell'Affrica settentrionale, nell'Asia occidentale, introdotta nell'America settentrionale.

\section{Verovacen Cyunbaliaroia.}

V. foliis breviter aut longiuscule petiolatis, rotundatis, obtusis, crenato-subquinquelobis, pedicellis fructiferis patulis refractisve, folia subsuperantibus, lobis calycinis 4 late ovalibus basi attenuatis, subæqualibus, septifragio turgido, subgloboso, leviter emarginato, 1-loho, calyce subæquali, stylo septifragio dimidio breviore.

Veronica Cymbalaria Bodard mém. sur la Vér. Cymb. (1798?). Bert. fi ital. 1. p. 105; et auct.

Veronica cymbalariæfolia Vahl enum. pl. 1. p. 81 (1805). Genn. fl. Capr. p. 114.

Weronica panormitana Tin.! in Guss. fl. sic. jrodr. suppl. 1. Bert. o. c. 2. p. 776. Parl.! fl. pan. p. 12. Guss. fl. sic. syn. 1. p. 17. Tar. Gerb. cat. pl. calat. p 5. Guss. enum. pl. Inarim. p. 241. 
Veronica cuneata Guss. enum. pl. Inarim. l. c. t. 8. f. 2. Loj. is. eol. p. 121.

Figure. - Viv. fl. ital. fragm. t. 16. f. 1. Pucc. syn. pl. luc. Reich. ic. fl. germ. 20.t. 77. f. 5.

Stazione, Abitazione e Fioritura, - Nei muri, nei rottami ecc. Rarissima nell'Alta Italia, vi è stata trovata a Verrone in Piernonte (Zum.), nel Bresciano (Huter!), nel Modenese a Montese (Gib. Pir.), nel Trivigiano a Mogliano (De Vis. Sacc.), in Venezia (Mor., Nacc.), a Trieste (Koch), a Fiume (Smith!). In Liguria abbonda(De Not.), da Nizza (Webb! ecc.) e Dolcedo (Berti!) a Genova (Carrega !), Chiavari (Delpino!) e Vernazza (Bert.); non è rara neppure in $\mathrm{T}_{0}$. scana, cosi a Sarzaua (Bert.), Lucca (Pucc.! ecc.), Tereglio nell’Appermino lucchese (Gianninil), Pisa (P. Savi!), Firenze!, Siena (Bartalini!), Volterra (Amidei!), Porto Santo Stefano!, e nelle isole di Gorgona (P. Sav.), di Capraia (Mor. De Not.), dell'Elba (Marcucci!), di Montecristo (Wats. Tayl.!) e del Giglio (Parl.!); nasce a Viterbo (Bert.), a Roma è comunissima (Rolli! ecc.), anche a Napoli (Pasquale! ecc.), e fa in Ischia (Guss.) e in Capri (Pasq.), e fa infine nel Vulture (Terr.), e a Otranto (Groves). In Corsica è stata trovata a Bastia, Calvi (Bert. ecc.), Aiaccio (Requien!); abita tutta la Sardegna (Mor.), comprese le isolette come Maddalena e S. Stefano (Genn.); abita tutta la Sicilia, cosi Palermo (Parl.! ecc.), Mlessina (Nicotra! ecc.), Taormina (Nic.), Siracusa (Cassia!), Avola (Bianca!), Vittoria (Aiuti!), Caltagirone (Tar. Gerb.), Girgenti (Webb!) ecc., e le isolette di Lipari (Guss.) e di Pantellaria (Cal. cara!); e nasce infine in Malta (Grech!). Fiorisce da gennaio e febbraio ad aprile.

Distribuzione geografioa. - In tutto il bacino del Mediterraneo.

Descrizione. - Pianta quasi glabra o pelosetta e carnosetta, segnatamente nel fusto e nei rami. La radice é delicata, ramosa, fibrillosa, di un bianco sudicio. Il fusto è giacente in parte per terra, ramoso fiu dalla base, con i rami allungati e come questi cilindrico, un po' gonfio nei nodi inferiori, verdognolo o in parte rossiccio, fornito di due linee laterali alterne di peli bianchi e molli, patenti o un po'rivolti in griù. Le foglie inferiori sono opposte, ovate, leggermente in cuore alla base, ottusette, con 7 o 9 crenature uel margine, delle quali è maggiore quella dell'apice, e con qualche ciglio, verdi e con qualche pelo eretto di sopra, più pallide di solto, ed ivi con un nervo longitudinale e due laterali poco manifesti che partono 
dalla base e si diramano presto, fornite di un picciòlo, quasi piủ lungo della lamina, solcato di sopra. Le foglie superiori o fiorali sono alterne, simili alle inferiori ma quasi cordato-orbicolari, per lo piủ con 7 crenature più profonde che si potrebbero dir quasi lobi, con le ciglia più manifeste nel margine, con i nervi un poco più manifesti e quasi cinque e con un picciòlo quasi uguale alla lamina o poco più corto di questa, il quale è piủ peloso. I peduncoli sono solitarii, ascellari, filiformi, eretti, gli inferiori più corti della foglia, i superiori quasi uguali a questa, glabri, rossicci, diventano poi orizzontali nel frutto o si curvano in basso, contorcendosi alla base, e $s$ ' ingrossano verso il loro apice. Il calice è diviso in quattro lacinie profonde, ovate, uguali, ottusette, fornite nel margine di lunghe. e grosse ciglia bianche e spesso verso la base da una parte di un dente o piccolo lobo, sono aperte e verdi, nel frutto ingrossano un poco e diventano maggiori ed apertissime, talvolta anche rossicce. La corolla è bianca, divisa in quattro lacinie uguali, ovate, ottusette, è glabra e appena più lunga del calice. Gli stami sono due, più corti della corolla, glabri, i filamenti filiformi, bianchi, le antere piccole e gialle. Il pistillo è glabro, poco più corto degli stami. L'ovario è quasi bilobo, verdognolo. Lo stilo è filiforme, bianco, diritto. Lo stimma è piccolo, in capolino, papilloso, bianco. Le capsule sono didime, con ciascuna metà quasi globosa e divisa da un solco longitudinale rossiccio, verdi nel resto e glabre. I semi sono quasi tondi, cimbiformi, glabri, lisci, eccetto alla base dove sono rugosetti. (Parl. ms., descr. di pianta coltivata, nata da semi di Palermo della Veronica panormitana).

Osservazioni. - Questa pianta varia secondo i saggi per il grado della pelosità, e in un medesimo saggio per la forma delle foglie nella loro base, ristretta o scavata, e per la loro dentatura. Su tali caratteri di niun mornento sono state fondate la Veronica panormitana e la $V$. cuneata. Si riscontrino pure le osservazioni di Moris in Fl. sard. 3. p. 231 , e di Pasquale in Fl. vesuv. p. 76.

\section{BONA IROTA.}

Bonarota Mich. nov. pl. gen. p. 19. t. 15. Adans. fam. pl. 2. p. 209. Scop. fl. carn. ed. 2. 1. p. 22.

Pæderota Linn. gen. pl. p. 4. Gen. pl. fl. germ. 5. t. 39. Benth. Hook. gen. pl. 2. p. 964. Ces. Pass. Gib. comp. fl. ital. p. 353. t. 50. f. 1 . 
Calyx erectus, plus minus inæqualiter 5-partitus. Corolla limbo 2-labiato, labio postico concavo, antico 3-partito. Stamina 2. Antherarum loculi basi mutici. Stigma capitellatım. Gemmulæ in loculis plurimæ, coacerratæ. Septifragium polyspermum.

Portamento. - E di certe Veroniche, come Veronica urticifo. lia, V. Gouani.

Osservazioni. - Stando alle piante di Europa, è sufficiente la distinzione fra questo genere e il precedente; peraltro non più se si prendono in considerazione certe specie esotiche di Veronica, come quelle di cui Nuttall fece il genere Septandra, e clse alcuni autori mettono nel presente genere, o pure come la Veronica speciosa o la $V$. salicifolia.

Linneo cambiò nome al genere per la ragione dichiarata a pa. gina 69 della sıa Critica botanica, ossia perchè gli parve immeritevole di essere cosi ricordato nella scienza il Senator Florentinus Filippo Buonarroti. Ebbe torto, giacchè non solo il Buonarroti, della faıniglia di colui che firmava le sue opere d'arte "Michelangelus Bonarotus, " fu uomo di molta dottrina, ma fu tra i fondatori della Società bota. nica fiorentina, e protettore di Micheli, e degno dell'onore di siffatta dedica quanto molti altri che l'hanno avuto incontrastato: vedasi in proposito la Vita del Micheli per Targioni-Tozzetti. Ad ogni modo la ragione non si mena più buona a' tempi nostri. Nè deve ostare alla restituzione del nome generico Bonarota l'essere stato coniato anteriormente a Linneo, come vorrebbe l'articolo 15 delle Leggi della nomenclatura botanica sancite dal Congresso internazionale botanico tenuto in Parigi nel 1867; poichè tale regola, giusta riguardo ai nomi specifici, di cui Linueo fu inventore, è ingiustissima riguardo ai generici, dopo che Tournefort, fondatore del genere, e dopo di lui un Plumier, un Vaillant, un Dillen, un Micheli, ebbero dato ai loro generi nomi conformi all'odierne regole di nomenclatura, salvo alcune ecegzioni da correggersi. II sommo Linneo fin riformatore felice in botanica, ma non fi creatore della scienza, e neanco della tassinomia vegetale.

\section{Bonsrotu chomatrifolin.}

B. foliis membranaceis, ovatis, infimis rotundatis superioribus lanceolatis, argute serratis, floribus llavis, corolla tubulosa calyce duplo longiore. 
Bonarota 3 Mich. nov. pl. gen. p. 19.

Pedærota lutea Scop. ann. II hist.-nat. p. 41 (1769).

Pæderota Ageria Linn. mant. pl. p. 22 (1771). Bert. fl. ital. 1. p. 110 (excl. syn. P. Zannichellii?). Koch syn. fl. germ. ed. 2. p.611. Hausm. fl. Tir. p. 645. Pir.! fl. for. syll. p. 106. De Vis. Sacc. cat. piant. Ven. p. 155 (excl. $\gamma$ ?). Ces. Pass. Gib. comp. fl. ital.p. 353. Arc. comp. fl. ital. p. 518.

Bonarota chamædrifolia Scop. fl. carn. ed. 2. 1. p. 22 (1772).

Pedærota urticæfolia Brign.! fase. rar. pl. for. p. 8.

Pedærota Zannichellii Brign.? o. c. p. 9. Pir. l. c.

Figura. - Reich.ic. fl. germ. 20. t. 76.f. 1.

Stazione, Abitazione e Fioritura. - Sulle rupi delle Alpi orientali, rara: presso Canziano nel Triestino (Kioch), in quel d'Idria, verso Gorizia, Vergenis, Canale e Caporetto lungo l'Isonzo (Poll.), nei monti Cren e Mariana (Brign.!), nel monte Matauer (Bert.), nei monti Campo presso Venzone (Pir.!), Sierra, Plauris ecc. (Pir), so. pra val di Raccolana (Ball!), nel monte Cavallo in Alpago sul calcare a 5-6000' (Huter Porta!), nel monte Summano (Poll.), sulla Cima d'Asta (Hausm.). Fiorisce in maggio, giugno o luglio secondo le altezze.

Distribuzione geografica. - Pianta propria delle Alpi orientali.

Osservazioni. - La Paederota Zannichellii, della Cima d'Asta (Bert.), dei monti Carallo (Brign.), Campo (Pir.), Cren (Brign.!), ha tutto l'aspetto della specie seguente, di cui Pollini ne ha fatta una varietà nonostante $\mathrm{i}$ fiori gialli. Accanto si può mettere la var. $\beta$ maior della $P$. Bonarofa trovata presso Raibl in Carinzia da Bentham (Cand. prodr. 10. p. 457), e altra pianta dello stesso posto distribuita da Huter come ibrido fra le due specie: ciò che dà forse la chiave di queste forme intermedie, se pure non sono tutte variazioni di un solo tipo specifico, sospetto questo avvalorato dalle molte discrepanze degli autori rispetto ai caratteri differenziali delle due forme.

\section{Bonarota rotundifolia.}

B. foliis carnosulis, subconformibus rotundatis vel ovatis, crenato-serratis, floribus cœeruleis rare carneis, corolla infundibuliformi calyce paullo longiore.

Bonarota 1, 2 Mich. nov. pl. gen. p. 19.

Veronica Bonarota Linn. sp. pl. ed. 1. p. 11. 
Pæderota Bonarota Linn. o. c. ed. 2. p. 20. Bert. fl. ital. 1.p. 108. Rota prosp. fl. Berg. p. 67. Hausm. fl. Tir. p. 645. Pir.! fl. for. syll. p. 106. De Vis. Sacc. cat. piant. Ven. p. 154. Zersi prosp. piant. Bresc. p. 15\%. Ces. Pass. Gib. comp. fl. ital. p. 353. Sacc. Bizz. agg. fl. trev. p. 28.

Pæderota cœrulea Scop. ann. II hist.-nat. p. 41.

Pæderota Buonarotta et P. chamædryfolia (non Scop.) Brign.! fasc. rar. pl. for. p. 6, \%.

Figure. - Mich. o. c. t. 15. f. 1, 2. Lam. ill. t. 13. f. 1. Reich. ic. fl. germ. 20. t. 76. f. 2.

Stazione, Abitazione e Fioritura. - Dove la specie precedente, ma piủ comune: in Friuli nel monte Strabut, intorno a Verzegnis (Brign.!), Tolmezzo, Pontebba (Brign.), presso Amaro (Pir.!), e Venzone, nei monti Amariana, Longarina, Culett (Pir.), nel Cadore (Brign.), sul monte Cavallo (Ball! ecc.), al Cansiglio, lungo la Piave a Covolo (Sacc. Bizz.), nelle Vette di Feltre, nei monti Grappa e Portole (Montini!), in val di Valstagna (Ball! ecc.), nel monte Sum. mano (Moretti! ecc.), nei Sette Comuni (Bert.), sul Baldo a $6000^{\prime}$ (Kellner! ecc.), al Col Santo presso Roveredo (Hausm.), nel Trentino al Bondone, al Castellazzo (Perini!), a Vel $\varsigma_{,}$, al Blemmone, allo Spinale, al monte Gazza, in val Sugana sullo Schiner tra Primiero e Fonzaso (Hausm.), e presso Borgo sul dolomitico (Ambrosi!), in Fiemme (Hausm.), e Fassa a Vaiolet sopra Vigo a $6-8000^{\prime}$ sul dolomitico (Ball!), sul calcare presso Salorno, fin giủ nella "alle, sul Joch Grimm e sullo Schlern presso Bolzano, nell'alpe Gavardina delle Giudicarie (Hausm.), nel Bresciano sui monti Ario a 1700 --1900 metri, Dos Alto a 1900-2000 metri, Ciorna Blacca a 1500-1900 metri (Parl.!), e Fronden (Zersi), nel Bergamasco in val Camonica (Ruta). Fiorisce in giugno, luglio, agosto.

Distribuzione geografica. - Pianta propria delle Alpi orientalicentrali.

Desorizione. - Planta perennis, e rupium fissuris dependens. Rhizoma duriusculum, gracile, filıras multas radicales, rigidiusculas, nigrescentes et apice ramos sive caules emıttens. Caules teretiusculi, sæpe purpurascentes vel virides, pilosi, pilis albis longiusculis. Folia carnosula, opposita, remotiuscula, subdisticha, ovata, obtusa, parce serrata, serraturis planiusculis, supra viridia et nitidula, subtus pallide viridia, sæpe rubentia, nervo longitudinali basim versus prominente ramisque lateralibus obliquis notata, nervo longitudinali pilosula, reliqua parte et pagina superiore glabra, margine ciliata; 
folia inferiora minora et sæpe ovato-oblonga, superiora ovata acuta vel acutiuscula. Flores in racemum brevern, densum, fere conicum dispositi, subcernui, subsecundi, inferiores bracteati, bractea viridi, sublineari, ciliata, florem subæquante. Pedicelli subnutantes, rubicundi, pilosi. Calyx 5-partitus, tubo brevissimo, laciniis linearibus, obtusiusculis, patentibus, apice patulis, viridibus, pilosis. Corolla laete vel intense violacea, calyce paullo longior, 4-fida, lacinia suprema paullo latiore ovali, obtusissima, subreflexa, reliquis ovatis, obtusiusculis, patulis. Ștamina 2, corolla subæqualia, tubo inserta. Filamenta filiformia, glabra. Antheræ parvæ, ovales, obtusæ, dorso insertæ. Orarium grande, oblongum, compressum, utrinque unisulcatum, gla. brum, viride, apicem versus purpurascens. Stylus elongatus, ovario plus quam duplo longior, filifor nis, demum recurvus. Stigma subcapitatum? Capsula oblonga, acuta, utrinque in medio longitudinaliter sulcata, purpurascens. (Parl.ms., descr. della pianta delle Alpi Bresciane).

Osservazione. - Vedansi quelle all'altra specie.

\section{WULFENIA.}

Wulfenia Jacq. misc. austr. 2. p. 62. t. 8. f. 2. Gen. pl. fl. germ. 5. t. 40. Benth. Hook. gen. pl. 2. p. 963. Ces. Pass. Gib. comp. fl. ital. p. 343. t. 50. f. 6 .

Calyx erectus, sulæqualiter 5-partitus. Corolla limbo campanulato inæqualiter 4-partito, lobo postico maiore subdiviso. Stamina 2. Antherarum loculi basi mutici. Stigma capitato-bilobum. Gemmulæ in loculis plurimæ, coacervatæ. Septifragium polyspermum.

Portamento. - Per quanto genericamente distinte appena dalle Bonarota, le Wulferia molto se ne allontanano per l'abito. La specie nostra è un' erba perenne, rizomatosa, con una rosetta di foglie basilari grandi, spatolate, crenate, dal mezzo della quale sorge uno 'scapo alto circa 3 decimetri, nudo in basso, guarnito in alto di piccole brattee sparse, e terminato da un grappolo fitto di fiori cerulei, tubulosi, inchinati. 


\section{Wulfenia carinthiaca}

Wulfenia carinthiaca Jacq. o. c. p. 60. Pir.! fl. for. syll. p. 106. De Vis. Sacc. cat. piant. Ven. p. 155. Ces. Pass. Gib. o. c. p. 344. Arc. comp. fl. ital. p. 518.

Figure.-Lam. ill. t. 13. 1. 2. Reich. ic. 1. germ. 20. t. 75.

Stazione, Abitazione e Fioritura. - Nell'estremo confine nordest delle alpi italiane presso Pontebba, e precisamente nel declive friulano del monte Nossfeld, in luoghi umidi (Pir.!), dove pare che la scoprisse Re Federigo Augusto di Sassonia (Pir.), e nel monte Auernick Kogel dal lato di mezzogiorno verso la valle Bambosch, dove la trovó il signor Ball!. Fiorisce in luglio.

Distribuzione geografica. - Questa pianta abita due altre località sole della contermine Carinzia, al Kühwegeralp (Lagger! ecc.) e al Watschiggeralp (Pittoni!) nella valle di Gail. I Compendi indicano ancora la Carniolia, non saprei con quale fondamento.

XIV. Ere INUS.

Ageratum Tourn. inst. p. 651. t. 422.

Erini sp. Linn.

Erinus Gutertn. de fruct. 1. p. 261. t. 55. Gen. pl. fl. germ. 5. t. 3.4.

Benth. Hook. gen. pl. 2. p. 961. Ces. Pass. Gib. comp. $1 l$. ital. p. 344. t. 51.f. 2.

Calyx erectus, subæqualiter 5-partitus. Corolla limbo patulo subxqualiter 5-partito, Iobis 2 posticis subminoribus. Stamina 4. Antheræ reniformes 1-loculares Stigma in stylo brevissimo capitatum, cornubus 2 comitatum. Gemmula in loculis plurimæ, coacervatæ. Septifragium polyspermum.

Portamento. - L'unica specie del genere è un'erholina perenne, avente l'apparenza di certe Verhene come quelle comunemente coltivate per ornamento, alta 4 decimetri, ramosa in basso, pelosa o quasi glatra, con foglie sparse, spatolate, crenate, e con grappoli terminali di fiori grandetti, porporini. 


\section{Erinus alpinus.}

Erinus alpinus Linn. sp. plant. ed. 2. p. 878. De Not. rep. fl. lig. p. 301. Bert.! fl. ital. 6. p. 412. Com. fl. com. 5. p. 79. Mor.! fl. sard. 3. p. 221. Ard. cat. pl. Ment. p. 27. Fl. Alpes-mar. p. 277. Ces. el. piant. Maiella p. 22. Ces. Pass. Gib. comp. fl. ital. p. 344. Arc. comp. fl. ital. p. 513.

Figure.-Lam. ill. t. 521. Bot. mag. t. 310. Reich. ic. fl. germ. 20.t. 74 .

Stazione, Abitazione e Fioritura. - In luoghi sassosi erbosi dei monti, sparso qua e là e sempre raro. Nel nord della Penisola è stato indicato nel Tirolo meridionale da Host, ma non più trovato; nasce invece nelle Alpi centrali-occidentali sul Legnone nel Comasco, oltre l'abitazione del mugo (Com.), in val Formazza sopra Crodo, in di Bugnanco sopra il monte d'Ossola (Bir.), nel monte Rosa (Kellner!), sul Cenisio (Bonjean!), presso i bagni di Vinadio e sopra il Pertus de Margon, tra Tenda e Saorgio (All.), tra Viosenne e Carlino (Ricca!), e da quelle alture scende sin presso Nizza a Sant'Andrea, e presso Mentone al ponte San Luigi, a Sant'Agnese a $660 \mathrm{~m}$., e nella montagna di Bress a 750-950 metri (Ard.). Ritrovasi poi nell'Appennino in Abruzzo nella Maiella in ralle di Santo Spirito (Bert.) e al Vado di Sant'Antonio (Ces.); e presso Roma a Frascati (Barbieri!). E poi ancora nasce in Sardegna in cima al monte Oliena sul calcare a 1300 metri (Mor.). Fiorisce da maggio e giugno a luglio e agosto.

Distribuzione geografica. - Nei monti di Spagna, della Francia meridionale, di Svizzera e d'Italia.

\section{DIGHTARS.}

Digitalis Tourn. inst. p. 165. t. 73. Lindl. Dig. monogr. 1821. Gen. pl. fl. germ. 5. t. 33. Benth. Hook. gen. pl. 2. p. 960. Ces. Pass. Gib. comp. fl. ital. p. 343. t. 51. f. 5.

Calyx patens, subæqualiter 5-partitus. Corolla limbo conspicue vel obscurius 2-labiato, labio postico brevi subdiviso, antico 3-lobo. Stamina 4. Antherarum loculi basi mutici. Stigma bilamellatum. Gemmulæ in loculis plurimæ, coacervatæ. Septifragium polyspermum. 
Portamento, - Erbe perenni o bisannue, grandi in tutte le loro parti. Il fusto n'è alto fino a un metro e più, semplice, le foglie sono lunghe, lanciolate, intere o dentellate, sparse, gradatamente descrescenti da basso in alto, $i$ fiori hanno la corolla lunga da $1 \frac{1}{2}$ a 4 centimetri, rossa o gialla, tubulosa-campanulata e rigonfia, sono inchinati, e formano un lungo grappolo terminale, unilaterale.

\section{A. Digitalis ferruginea.}

D. glaberrima, foliis oblongo-lanceolatis, subintegris, lobis calycinis obtusis, corollæ fulvæ tubo superne ventricuso subgloboso, lobo medio labii antici maximo tubum fere æquante, barbato.

Digitalis ferruginea Linn. sp. plant.ed. 1. p. 622. Sant. viagg. 1. p. 73, 155, 258. 2. p. 67, 86. Bert. fl. ital. 6. p. 410. Rola prosp. piant. Pav. p. 269. Pir. fl. for. syll. p. 104. Car. prodr. fl. tosc. p. 482. Terr.! fl. Vult. syn. p. 130. Rel. Terr. Lav. p. 147. Sec. rel. p. 92. Ces. el. piant. Haiell. p. 22. Cocc. contr. fl. Bol. p. 14. Ces. Pass. Gib. comp. fl. ilal. p. 343. Terr. quart. rel. p. 110. Paol. el. piant. sib. p. 14. Gib. Pir. fl. Mod. p. 120. Arc. comp. fl. ital. p. 513. Cocc. 1. Bol. p. 64.

Figure. - Lindl. mon. t. 12. Reich. ic. bot. t. 156. Ic. A. germ. 20. t. 73.

Stazione, Abitazione e Fioritura. - Qua e là in luoghi boschivi montuosi della Penisola, non comune. Nasce in Istria a Fiume, e presso Trieste tra Prosecco e Santa Croce (Poll.); in Friuli presso Monfalcone e Capriva, e presso Rigolato e Sappada (Pir.); nella destra valle del Po a Montese e Montespecchio nell'Appennino bolognese-modenese (Riva!, Cocc., Gib. Pir.), nel Parmense a Ravarano (Bert.), nel Pavese al monte Penice verso Pobbio (Nocc. Balb., Ces. Pass. Gib.), nell'Acquense a Rubbio e tra Gragnardo e Cavatore (All.); nel Genovesato a Pegli (Bert); in Toscana nel Fiorentino sui colli alla destra dell'Arno!, in Mugello (Bert.), in Casentino all'Alvernia (Ricasoli!), a S. Casciano (Ricci!), in Chianti (Ricasoli! ecc.), nel Senese a Torri!, nel monte Amiata (Sommier!), in val di Fiora (Sant.); nelle Marche al Catria a 1550 metri (Parl. !), ad Albaccina a 200 metri (Bucci!), nel monte S. Vicino, nel Camerinese, nel monte Regnolo (Bert.), in tutti i monti Sibillini (Paol.), in Abruzzo al monte de Fioril, al Corno (Bert.), al Velino (Cherici!), al Morrone (Huel!), alla Maiella (Groves! ecc.), in Puglia al Gargano 
a 2000' (Porta Rigo!); nel Lazio a Viterbo (Bert.), e presso Tivoli (Webb!), in Terra di Lavoro presso Fondi(Ten.), presso Picinisco (Terr.), nel Matese (Avellino!), presso Pietramelara (Terr.), e Ca. serta, nel Vulture (Terr.!), in Calabria (Gasparrini!) sul Pollino (Ten.). Fiorisce da giugno e luglio ad agosto e settembre.

Distribuzione geografica. - Nell Europa meridionale, dall'Italia alla Rumenia, nell'Asia Minore, al Libano.

Descrizione. - Laete viridis. Caulis firmus, erectus, subangulatus, viridis, hinc inde purpurascens. Folia radicalia erecto-patentia, hasi attenuata, caulina patula, basi vix cordata, omnia supra laete viridia, sulcis longitudinalibus profundis nervis paginæ inferioris re. spondentibus notata, subtus vix pallide viridia, nervo longitudinali crasso pallidiore nervisque paucis lateralibus fere longitudinaliter excurrentibus et prominentibus notata. Folia floralia sensim breviora, inferiora flores longe superantia, subreflexa, superiora linearia, flore breviora, reflexa. Flores in spicam sive racemum oblongo-cylindraceum, acutiusculum subconfertim dispositi et subpenduli. Pedicelli axillares breves, calyce breviores, cernui. Calyx fere ad basim 5-partitus, laciniis subinæqualibus, 2 inferioribus paulo maioribus, ovalibus, concavis, corollæ adpressis, 3 superioribus patentibus, concaviusculis, omnibus apice rotundato et denticulato reflexo, dorso et facie viridibus margine albido. Corolla calyce plus quam duplo longior, valde irregularis, luteo-fusca, intus venis crebre anastomosantibus rubellis picta, extus pilis brevibus glandulosis puberula, tubo inferne constricto et curvo cito abruptim inflato et subgloboso. Labium superius brevissimum, subbilobum, lobis rotundatis subreflexis, inferiore trilobo, lobis lateralibus patentibus, ovato-triangularibus, acutiusculis, brevibus, inferiore sive intermedio multo maiore et longiore, fere tubum longitudine et latitudine æquante, late ovali-oblongo, apice truncato, patenti-pendulo, supra piloso, pilis longis, articulatis, apice glandulosis. Stamina didynama sub labio superiore. Filamenta prope basim tubi corollæ inserta, ascen. dentia, inferne parum incrassata, subulata, basi luteo-rubra, reliqua parte pallide lutea et fere albida, pilis raris horizontalibus glandulosis præsertim extrorsum prædita. Antheræ transversales, apice vix emarginatæ, basi bifidæ, biloculares, rima dehiscentes, luteæ, margine piloso-glandulosæ ut filamenta. Pistillum stamina inferiora æquans et inde staminibus superioribus panlo brevius, erectum. Ovarium oblongo-canicum, subangulatum, viride, pilosoglandulosum, pilis paucis. Stylus rectus, ovario sublongior, cum 
ovario continuus, subulatus, e luteo-viridis, pilis raris glandulosis præditus. Stigna obtusum, subbilobum, e luteo-viride. (Parl. ms., descr. della pianta del monte Catria).

\section{Digitalis laevigata.}

D. glaberrima, foliis oblongo-lanceolatis, subintegris, lubis calycinis acuminatis, corollæ luteæ tubo superne ventricoso, lobo medio labii antici maximo tubo paullo breviore, puberulo.

Digitalis levigata Waldst. Kil. pl. rar. hung. Bert. f. ital. 6. p. 409. Ces. Pass. Gib. comp. fl. ital. p. 343. Smith! fl. Fiume p. 38. Arc. romp. f. ital. p. 512.

Figure. - Lindl. mon. t. 10. Reich. ic. bot. t. 155. Ic. fl. germ. 20. t.71.f. 1 .

Stazione, Abitazione e Fioritura. - In lıoghi salvatici rocciosi dell'Istria: intorno a Fiume (Noë!) in parecchi siti (Smith), nel monte Maggiore, verso Sestiana, e Orsei (Bert.), verso Risano (Pichler!), e alla grotta di S. Canziano (Bert.). Fiorisce in giugno e luglio.

Distribuzione geografica. - Nell' Europa meridionale, dall'Istria alla Transsilvania.

\section{Digitalis ambigua.}

D. minute parce glanduloso-pilosa, foliis ex oblongo vel ovali lanceolatis, serrulatis, lobis calycinis acutiusculis, corollæ ochroleucæ tubo superne ampliato campanulato, lobo medio labii antici brevi, lateralibus duplo latiore.

Digitalis ambigua Murr. des. stirp. gotting. p. 62 (1770). Balb. el. piant. Tor. p. 34. Re fl. tor. p. 327. Bir. A. acon. p. 216. Ard. 1. Alpes-mar p. 279. Ces. Pass. Gib. comp. fl. ital. p. 343. Smith n. Fiume p. 38. Arc. comp. fl. ital. p. 513.

Digitalis grandiflora Lam. l. fr. ed. 1. 2. p. 339 (1778). All. 月. ped. 1. p. 70 (1785). Mass. prodr. A. talt. p. 60. Trev. prosp. A. eng. p. 2. De Not.! rep. A. lig. p. 297. Bert. A. ital. 6. p. 40\%. Com. A. com. 5. p. 72. Zum. R. ped. 1. p. 207. Rola prosp. piant. Pav. p. 269. Prosp. 1. Berg. p. 66 Hausm. 1. Tir. p. 630. Pir.! /. for. syll. p. 104. Zersi prosp. piant. Bresc. p. 154. Ing. cat. sp. Mond. p. 32. 
Figure. - Bot. reg. t. 64. Lindl. mon. t. 7, 8. Reich. ic. bot. t. 159. Ic. fl. germ. 20. t. 69.

Stazione, Abitazione e Fioritura. - Nei luoghi prativi o boschivi asciutti montuosi dell' Italia superiore: in Istria presso Fiume, non comune (Smith), al monte Maggiore (Poll.), e presso Trieste al Farneto (Bert.), frequente nel Friuli (Pir.!), per esempio presso Pinzano, Suzano, Resinta (Poll.), Tarcento, e al monte Cavallo (Pir.), nelle Vette di Feltre (Poll.), nel Bassanese ad Angarano, Sollagna, Collalti, a Valstagna (Bert.), nel monte Summano, negli Euganei verso Montortone (Rigo!), nel monte Civillina, nel Baldo all' Artillon (Poll.), in Basiana (Goiran!), in val Fredda a $4500^{\prime}$, e nel monte Pastello a 3000' (Bracht! ecc.), intorno a Roveredo (Hausm.), nel Trentino nelle valli di Peio e di Passiria (Perini!), in val Sugana (Ambrosi!) alla Carota (Bert.), in Fiemme, in val di Rabbi, a Eppano, presso Bolzano sul Mendel, sul Torggele, rara, in Pusteria sull'Enneberg, in val di Taufers, e a Welsberg, a Merano, e in val di Passero, nelle Giudicarie sull'alpe Lenzada (Hausm.), nel Bresciano, frequentissima (Zersi), cosi a Collio, sopra S. Colombano ecc. a 850-1200 metri, e nel monte Gerle a 1000-1400 metri, nel Bergamasco a Ponte di legno a 1320 metri (Parl.!), e in val di Scalve, nel Comasco al Camoggia, sui monti d'Erba (Bert.), Corni di Canzo, Codeno, S, Primo, Resegone (Com.), in val Tellina al Legnone (Pass.!), nel bosco Valdone ecc. (Mass.), e in valle del Masino, presso Lugano (Bert.), e Locarno (Franzoni!), sul Gottardo (Heldreich!), ad Intragna in val Intrasca, rara (De Not. !), nel Pavese oltre il Gravelone in un luogo alto detto il Para. diso (Noce. Balb.), ad Albano nel Vercellese (Malinverni!), a Scopello in val Sesia (Carestia!), alla costa di Benna sopra l'Armondà, e sulla Bessa sopra Mongrando (Zum.), nelle colline di Torino (Bertero! ecc.), al Cimone (Parl.!), a Susa (Re), nelle vallate Valdensi (Rostan!), presso il santuario di S. Vico (Ing.), sopra Limone a 1600 metri (Parl.!), a Tenda (Bourgeau! ecc.), a S. Anna di Vinai, nelle valli di Libarè e di Giallorghe (Ard.), sopra Nizza a Mairis (Barla!), a Viosenne, abbondante (Ricca!), sul monte Ceppo in quel di S. Remo (Panizzi!), a Rezzo (Berti!), a Nava (Gentili!), e al monte Gallè (Bert.). Fiorisce in giugno, luglio, agosto.

Distribuzione geografica. - Nell' Europa media e in parte nella meridionale, dalla Spagna all'Ural, e in Asia Minore.

Descrizione. - Pianta perenne, alta da quattro decimetri a circa un metro, in parte pubescente per lunghi peli molli e bianchi, for- 
nita di un rizoma orizzontale, ramoso, grossetto, scuro, che manda in giủ fibre radicali fibrillose e di un bianco sudicio, e dall'apice i fusti. Questi sono diritti, quasi cilindrici, verdognoli, pubescenti. Le foglie sono un po'corsistenti, eretto-patenti o patenti, le inferiori lanceolate, acute, con piccoli denti acuti nel margine, segnatamente in alto, fornite di rare e corte ciglia, assottigliate alla base in un picciòlo corto, piano-scanalato di sopra, convesso di sotto, e alato nel margine: sono di un color verde-scuro e quasi glabre di sopra, ed ivi con un solco longitudinale e con solchi meno larghi molto obbliqui corrispondenti ai nervi di sotto, di un verde pallido di sotto ed ivi con un nervo longitudinale rilevato e verdognolo e con pochi nervi laterali, i quali sono anche rilevati, e nascendo quasi a paja dal nervo principale si dirigono molto obliquamente verso l'apice della foglia per unirsi tra loro ad arco poco lontano dal margine: in questi nervi, particolarmente in quello longitudinale, sono dei peli stellati. Le foglie di mezzo e superiori del fusto sono molte, alterne, vicine tra loro, o ovato-lanceolate o lanceolate, sessili, con i denti meno manifesti ma con ciglia assai piủ fitte e un po' più lunghe, anche più pelose di sotto, del resto simili alle inferiori. I fiori sono molti, solitarii all' ascella di una brattea fogliacea, distribuiti in un racemo unilaterale, dapprima corto, ma che si allunga nel frutto: sono un po'pendenti, piuttosto grandi: talvolta vi sono uno, due 0 pii rametti fioriferi alla base del racemo, i quali sono eretti e piuttosto corti. Le foglie fiorali o brattee sono patenti, più lunghe del peduncolo, ovato-lanceolate, acuminate, verdi, pubescenti sotto e cigliate nel margine, peloso-glandolose di sopra. I peduncoli sono piủ corti o quasi uguali al calice, dapprima eretto-patenti, poi patenti nel fiore, infine eretti nel frutto, cilindrici, pubescenti, con peli glandolosi all'apice. Il calice è quasi campaniforme, diviso profondamente in cinque lacinie lineari-lanceolate, ottusette, nell'apice rivolte in fuori ed in giủ, verdi e peloso-glandolose. La corolla è molto piii grande del calice, ristretta nel quarto inferiore, poi slargata e quasi campanulata, con la gola molto aperta, divisa all' apice quasi in due labbri, il labbro superiore piu corto, diretto un po' in su, bidentato-smarginato, talvolta con un dentino nella snsarginatura, e il labbro inferiore diviso in tre denti, quasi triangolari, acuti o ottusi, rivolti in giủ e in dietro: è puberula, con peli glandolosi all'apice di fuori, glabra di dentro eccetto verso i labbri dove ha lunghi e rari peli, i quali sono piì abbondanti segnatamente nei margini dei denti in molo che questi sono forniti di ciglia : è di un 
color giallo pallido con macchioline irregolari, in più punti unite ir. regolarmente insieme, di colore rossiccio-violetto nella parte inferiore soltarto di dentro. Gli stami sono 4, didinami, più corti della corolla, inseriti nella parte più stretta di essa. I filamenti sono cilindrici, un po'curvi, di un giallo-bianchiccio, glabri: quelli degli stami più lunghi sono piegati alla base a guisa quasi di un S. Le antere sono grandi, ovali-tonde, appena appena smarginate all'apice, profondaınente bi. lobe alla base, con i lobi un po'divergenti, inserite nel dorso sopra della base, biloculari, con le logge aprentisi longitudinalmente in avanti, giallicce, glabre, con pochi peli glandolosi verso l'apice di ciascun lobo cioè inferiormente. Il pistillo è poco più lungo degli stami più corti. L'ovario è allungato-acuminato, lungo quanto il calice, con quattro leggieri solchi Iongitudinali, verdognolo, peloso, con peli glandolosi all'apice. Lo stilo è più lungo dell'ovario, quasi diritto, verdognolo-bianchiccio, con qualche pelo glandoloso verso basso. Lo stimma è bifido, con le lacinie quasi piane, ottuse e avvicinate tra loro in modo da toccarsi con la faccia interna, dello stesso colore dello stilo. La cassula è cretta, ovoidea, acuminata all'apice, con quattro leggieri solchi longitudinali. (Parl. ms., descr. della pianta delle alpi Bresciane).

\section{Digitalis purpurea.}

D. velutino-canescens, folíis ex ovato vel ovali lanceolatis, rugosis, crenatis, lobis calycinis acutiusculis, corollæ purpureæ aut roseæ tubo superne ampliato campanulato, lobo medio labii antici lateralibus duplo maiore.

Digitalis purpurea Linn. sp. plant. ed. 1. p. 621. Bir. fl. acon. p. 215. Poll. A. ver. 2. p. 928. Mass. prodr. fl. valt. p. 61. Com. $\Omega$. com. 5. p. 71. Pir. fl. for. syll. p. 104. Mor.! fl. sard. 3. p. 218. Tass. cenn. bot. Siena p. 83. De Vis. Sacc. cat. piant. Ven. p. 150. Mars. cat. pl. Cors. p. 109. Ces. Pass. Gib. comp. fl. ital. p. 343. Arc. comp. f. ital. p. 513.

Digitalis Thapsi Bert. fl. ital. 6. p. 403 (non Linn.).

Figure.-Fi. dan. $t$. 74. Lindl. mon. t. 2. Reich. ic. fl. germ. 20. t. 67, 72. f. 1.

Stazione, Abitazione e Fioritura. - In Sardegna, in luoghi montuosi umidi, fino a 1400-1500 metri, frequente (Hor.); in Corsica, qua e là nei monti silicei, a quasi tutte le altezze (Mars.): 
ad Aiaccio (Bubani!), .a Sant'Antonio e al monte Lisa presso Aiaccio, nei monti della catena centrale, comune al Pigno, e al capo Corso (Mars.). Nella Penisola è indicata in qualche lıogo, ma quasi sempre dubbiamente: nel Veneto trovasi (dicono) qualche volta neiluoghi incolti e nelle ghiaie dei torrenti (De Vis. Sacc.), e cosi nel torrente But presso Paluzza in Carnia (Pir.); l'erbario del Huseo botanico di Firenze ne possiede un saggio del Baldo dato da Barbieri, ivi pure altra volta la trovó Calceolario nella valle di Caprino, se. condo Pollini, che pare non l'abbia più vista; è stata indicata nei monti-piủ alti del Lario, ma non vi cresce secondo Comolli; il $\mathrm{Bi}$. roli scrive di averla osservata una volta sola alla Cima Mullera in val d'Ossola; anticamente il Boccone (Mus. piant. rare $p .108$ ) la diede delle montagne di Toscana, ma certamente non vi nasce, peraltro secondo Caldesi (Fl. fav. tent. $)$. 17/) è stata trovata nel monte Coloreto presso Firenzuola nella Romagna toscana; tuttociỏ potendo essere spiegato da casuale comparsa d'individui avventizi di pianta molto frequentemente coltivata per ornamento o per uso me. dicinale, o pure da graduale scomparsa di pianta molto ricercata dagli erbojlai. Fiorisce in maggio, giugno, luglio.

Distribuzione geografica. - Nell' Europa occidentale, e princi. palmente nella sua parte media, ed esclusa la sua parte piu nórdica.

Osservazione. - La vera Digitalis Thapsi Linn., di Spagna, si distingue per la pelurie gialla, le fogglie più evidentemente scorrenti, il lobo medio del labbro anteriore della corolla più sviluppato, ece. Bentham (in Cand.prodr. 10. p. 4jof) sospetta nonostante che possa essere varietà della $D$. purpurea. Ad ogni modo la pianta italiana va riferita al tipo della specie, come già fece Bentham.

\section{Digitalis Iuten.}

D. glabra, foliis oblongo-lanceolatis, denticulatis, lobis calycinis acuminatis, corollæ flaræ tubo superne parum ventricoso, lobo medio labii antici lateralibus duplo maiore. •

Digitalis lutea Linn. sp. plant. ed. 1. p. 692. Bert. 1. ilal. 6. p. 406 ; et auct. omn.

Digitalis australis Ten.! Cald.! fl. fav. tent. p. 171 .

Digitalis micrantha Guss, enum. pl. Inar. p. 299. Pasq. 1. ves. p. 76 . 
Figure. - Bot. reg. t. 251. Lindl. mon. t. 23. Reich. ic. bot. t. 151, 153. Ic. fl. germ. 20. t. 70. f. 1.

Stazione, Abitazione e Fioritura. - Più o meno comune peri boschi di tutta la Penisola, in collina e in montagna, sul calcare e sul siliceo. Nelle Alpi sale fino a Courmayeur (1200 metri) (IVebb!), a 950 metri sopra Collio in val Trompia (Parl1), in Tirolo fino a $3800^{\prime}$ sulla pendice meridionale del Ritten (Hausm.), e in val di Ledro a $2-3000^{\prime}$ (Porta !), sul monte Pastello di Verona fino a $3000^{\prime}$ (Bracht!); nell'Appennino si spinge fino all'ultimo termine dei boschi, e scende ancora alle radici dei monti nell' Italia più meridionale, cosi a Otranto (Groves), o in Calabria a Murano a $4-500 \mathrm{~m}$. (Huter Porta Rigo !). Nasce eziandio nell' isola d'Ischia (Guss.), e in Corsica tra Nessa e Apricciani, e a Bocognano e Bastelica (Mars.). Fiorisce in giugno, luglio, agosto.

Distribuzione geografica.- Nell'Europa media e meridionale, e occidentale fino alla Transsilvania.

Descrizione. - Pianta perenne, alta da 5 a 8 decimetri, verde, glabra 0 in parte pelosa. La radice è ramosa, lunga, orizzontale, bianchiccia. Il fusto è eretto, duro, cilindrico, verdognolo, glabro, di raro peloso, con peli lunghi, orizzontali, duretti, semplice o raramente ramoso. Le foglie radicali sono lunghe circa un terzo del fusto, erette, bislıngo-lanceolate, ristrette in basso in un picciolo alato, acute, seghettate, con i denti radi e poco sporgenti, verdi e glabre di sopra, ed ivi con solchi che corrispondono ai nervi di sotto, di un verde pallido di sotto ed ivi glabre o di raro pelose nei nervi, e con un grosso nervo longitudinale bianchiccio da cui, segnatamente vicino alla base, partono altri nervi meno grossi che vanno quasi paralleli ad esso ma un po'obliqui e curvi verso il margine e l'apice per unirsi ivi tra loro: sono fornite di poche ciglia verso la base e talvolta fin quasi alla metà. Le foglie del fusto sono alterne, poco lontane, patenti o eretto-patenti, gradatamente più piccole, le inferiori strette alla base in un corto picciòlo alato, le superiori sessili, ovate alla base e quasi abbraccianti il fusto, del resto simili alle foglie radicali. I fiori sono molti, piccoli, fitti, quasi orizzontali e diretti da un lato in un racemo lungo e stretto. I peduncoli sono più corti del calice, cilindrici, verdognoli, con qualche pelo glandoloso, accompagnati da una brattea fogliacea, patente, più lunga di essi e quasi uguale al calice, lanceolato-lineare, acuminata, fornita di ciglia. Il calice è verdognolo, pubescente-glandoloso, quasi campanulato, diviso poco sopra della base in cinque lacinie 
DIGITALIS.

disuguali, patenti, lanceolate, acute. La corolla è piccola, circa 4 volte piủ lunga del calice, di un giallo pallido senza macchie di dentro, pubescente-glandolosa di fuori, puberulo-glandolosa di den. tro e pelosa per lunghi peli nel lobo medio del labbro inferiore, con il tubo stretto verso la base, poi gonfio di sotto, con il lembo bi. labiato, il labbro superiore piccolo, diretto un po' in su, bilobo, con i lobi quasi triangolari, ottusetti, appena divergenti, il labbro inferiore trilobo, con i lobi laterali più. piccoli, diretti un po' in avanti ed in fuori, quasi triangolari ed ottusetti, e il lobo medio quasi il doppio più largo e piủ lungo, patente-reflesso, ovato, ottusetto Gli stami sono quattro, didinami, più corti del labbro superiore, sotto del quale si vedono, inseriti verso la metà, o la parte inferiore del tubo della corolla. I filamenti sono un po' curvi, quasi piani, bianchi, glabri. Le antere sono quasi tonde, bilobe alla base con i lobi un po' divergenti, inserite nel dorso sopra della base, biloculari, e con le logge aprentisi longitudinalmente, giallicce, puberulo-glandolose verso la base. Il pistillo ė appena più corto degli stami più corti, diritto. L'ovario è ovoideo-conico, verdognolo, pubescente-glandoloso. Lo stilo è poco più lungo dell'ovario con il quale si continua in basso, cilindrico, bianchiccio, con qualche pelo glandoloso in basso. Lo stimma è ottuso, bilobo, con i lobi avvicinati nella parte interna piana. (Parl. ms., descr. di pianta di Boscolungo nell'Appennino toscano).

Osservazioni. - La corolla varia in grandezza da 1 a 2 centimetri. La-forma a fiori più grandi è prevalente nel nord della Penisola, quella a fiori più piccoli nel mezzogiorno.

Fra questa specie e la Digitalis ambigua pare che si formino ibridi, cui si riferisce la D. media di Roth (Reich. ic. bot. t. 152. Icon. fl. germ. 20. t. 70 f. 2). Ne suno stati trovati nelle Alpi marittime in val Sabbione sopra Entraque (Burnat!), e nel monte Resegone di Lecco (Rota prosp. fl. Berg.). Caldesi (Fl. fav. tenl.) vuole averne trovati in Toscana a Vallombrosa, ma ivi non possono essere per la mancanza della D. ambigua, e certamente per tali egli lıa preso individui grandiflori della (I). lutea. 


\section{SOTHOFA HITHIA SECONIDA.}

\section{SCROFULARINEE.}

Antirrhinide Eenth. in Cand. prodr. 10.

SCrOFUlaRiNEe (non alior.).

Corollæ lobi postici in præfloratione exteriores

\section{TRIBU் PRIMA.}

Serofulan i e e.

Scrophularue (non alior.).

Fructus septifragium.

\section{LIMOSELIA.}

Plantaginella Dill. app. ad cat. pl. circa Giss. p. 113. t. 6 (1719). Limosella Lind. Tourn. als. p. 156. t. 5 (1798). Linn. gen. $\mu l$. ed. 1.p. 187. Gen. pl. A. germ. 5. t. 37. Benth. Hook. gen. pl. 2.p. 958. Ces. Pass. Gib. comp. fl. ital. p. 353. t. 51. f. 4.

Calyx campanulatus, 5-fidus. Corolla limbo patulo subæqualiter 5̆-secto. Stamina 4, antheris I-locularibus. Stigma in stylo brevi capitatum. Gemmulæ in loculis (incompletis) plurimæ, coacervatæ. Septifragium polyspermum.

Portamento. - La Limosella è un' erbolina minuta, annua, stolonifera, con rosette di foglie lanciolate, intere, piccole ma do. tate di lunghissimo picciòlo, fra mezzo alle quali sonovi pedicelli piuttosto corti, con un fiore assai piccolo, bianchiccio.

Osservazione. - Per diritto d'anteriorità spetterebbe a questo genere il nome di Plantaginella, ma oramai ha contro di sè la prescrizione di un tempo troppo lungo. 


\section{Linuosella nquatica.}

Limosella aquatica Linn. sp. plant. ed. 1. p. 631. Trev. prosp. fl. eug. p. 23. Berl. fl. ital. 6. p. 419. Hausm. fl. Tir. p. 646. De Vis. Sacc. cat. plant. Ven. p. 155. Ces. Pass. Gib. comp. fl. ital. p. 354. Cocc. quart. contr. fl. Bol. p. 15. Gib. Pir. /. Mod. p. 123. Arc. comp. fl. ital. p. 518. Cocc. fl. Bol. p. 364.

Plantaginella aquatica Moench melh. pl. p. 427.

Figure. - Fl. dan. t. 69. Reich. ic. fl. germ. 20. t. 101.

Stazione, Abitazione e Fioritura. - Nei luoghi paludosi dell'Alta Italia, rara: nel Novarese al lago d'Orta, e intorno a Luzogno (Bir.), sul lago Maggiore a Intra (Gib.!), e a Locarno presso Rivapiana (Franzoni!), nel Milanese a Bolgiano (Zallinger!); in Tirolo sul Ritten al lago di Wolfgrub (Hausm.), presso Bolzano (Ambrosi!), in val Venosta presso Castelbel (Bert.); negli Euganei (Trev.), e nel Friuli presso S. Daniele (De Vis. Sacc.); nel Mode. nese a Guastalla (Jan!); nell'Appennino toscano-emiliano al lago Budellone sul Cimone (Gib. Pir.), e al lago di Pratignano (Cocc.). Fio. risce da luglio in là.

Distribuzione geografica. - In tutta Europa, meno le sue parti piủ meridionali, nell'Asia settentrionale fino all' Imalaia, in Egitto ed Abissinia, nell'America settentrionale, in Australia.

\section{INNDELENA.}

Pyxidaria Lind. Tourn. als. p. 156. t. 5 (1728).

Lindernia All. stirp. aliq. deser. in mél. de phil. el de math. de la soc. roy. de Turin (1762-65). Linn. mant. alt. p. 15 ' (1771). Gen. pl. fl. germ. 5. t. 36 Ces. Pass. Cib. comp. fl ilul. p. 315. เ. 52. f. 5 .

Vandellia Browne in Linn. mant. p. 19 (1967). lienth. Ilook. gen. pl. 2. p. 955 .

Calyx erectus, 5-partitus. Corolla limbo 2-labiato, labio postico planiusculo, antico 3-lobo. Stamina \& Antherarum loculi seiuncti basi ınutici. Stigma in stylo brevissimo bilamellatum. Gemmulæ in loculis plurime, coacervate. Septifragium polyspermum. 
Portamento. - L'unica specie europea è un' erbolina annua, ramosissima, con foglie opposte, ovali, intere, e fiori ascellari, lungamente pedicellati, piccolini, bianchicci.

Osservazioni. - Non ho restituito al genere il suo nome più antico di Pyxidaria, perchè diversi altri generi sono poi stati cosi chiamati, e perchè l'etimulogia da pyxis non concorderebbe con il significato odierno di quel termine.

Bentham e Hooker (l. c.) scrivono che Maximowicz è stato quello che ha additata l'identità, molto ovvia, della Lindernia Pyxidaria con la Vandellia erecta Benth., e costretto perciò alla riunione dei due generi. All'unico genere che ne risulta va dato il nome più antico dell'Allioni, e non, come essi propongono, il nome posteriore di Browne.

\section{Lindernia Pyxidaria.}

Lindernia Pyxidaria Linn. mant. alt. p. 252. Balb. el. piant. Tor. p. 50. Hass. prodr. /. valt. p. 104. Trev. prosp. fl. eug. p. 23. Bert. fl. ilal. 6. p. 417. Com. fl. com. 5. p. 77. Zum. 月. ped. 1. p. 207. Rola prosp. piant. Pav. p. 270. Prosp. fl. Berg. p. 67. Pir. fl. for. syll. p. 106. De Vis. Sacc. cat. piant. Ven. p. 155. Zersi prosp. piant. Bresc. p. 157. Terr.! sec. rel. Terr. Lav. p. 92. Ces. Pass. Gib. comp. fl. ital. p. 345. Arc. comp. fl. ital. p. 512.

Vandellia erecta Benth.

Figura. - Reich. ic. A. germ. 20. t. 102. f. 2.

Stazione, Abitazione e Fioritura. - Nei luoghi paludosi e specialmente nelle risaie dell'Alta Italia: a Torino lungo il Po (Balb. ecc.), ai laghi di Candia e di Vivrone, a Bollengo e Azeglio, tra Gaiani e Caudiel, al lago di Mlaion, presso Envie, e Bargia, verso Frossasco e la Marsaia (All.), a Massazza e Villanova (Zum.), a Vercelli (Ces.!) e Oldenico (Malinverni!), nel Novarese, comunissima (Bir.), a Confienza in Lomellina (Bert.), nel Pavese a Linarolo, Carbonara (Nocc. Balb.), Tromello, Valleggio (Poll.), nel Milanese (Rampoldi!), sull'Adda a Brivio, in val Tellina a Colico, Delebio (Com.), Spagna (Mass.), nel Bergamasco a Fopenico (Rota), nel Bresciano a Lama Scavola, rara (Zersi), a Guastalla (Pass.!), nel Mantovano (Barbieri!), a Migliaretto, Belforte, Luzara (Bert), nel Veronese a S. Giovanni in valle (Poll.), Vigasio ed Arcole (Goiran!), nel Padovano a Battaglia (Bert.), nel Vicentino (De Vis. Sacc.), in 
Friuli presso Moggio (Pir.). Ritrovasi poi in Campania presso Caianello al laghetto delle Correie (Ten.!, Terr.!). Fiorisce in agosto e seltembre.

Distribuzione geografica. - In Europa dalla Francia alla Rus. sia meridionale, nei paesi del Caucaso, in Persia, nell' India, nel paese dell'Amur.

\section{GIEATIOHA.}

Gratiola Linn. gen. plant. ed. 1. p. 333. Gen. pl. fl. germ. 5. t. 35. Benth. Hook. gen. pl. 2. p. 953. Ces. Pass. Gib. comp. fl. ital. p. 344.t. 53. f. 4.

Calyx erectus, 5̆-sectus. Corolla tubo æquali, leviter curvato, limbo 4-partito, lobo postico paullo latiore. Stamina 4, 2 antica antheris atrophicis. Antherarum loculi seiuncti, basi mutici. Stigna bilamellatum. Gemmulæ in loculis plurimæ, coacervatæ. Septifragium polyspermum.

Portamento. - Vedasi la descrizione dell' unica specie no. strale.

\section{Gratiola officinalis.}

Gratiola officinalis Linn. sp. plant. ed.1. p. 17. Bert. /. ilal. 1. p. 112; el auct. omn.

Figure. - Fl. dan. t. 363. Savi mal. med. veg. tosc. t. 5. Reich. ic. /l. germ. 20. t. 56 .

Stazione, Abitazione e Fioritura. - Nei prati umidi e nei lunghi paludosi della Penisola. É comune nell'Alta Italia: intorno a Torino (Re), ad Alba (Bertero!), presso Mondovi (Ing.), presso Susa (Re), a Ivrea (Carestıa!), a Oldenico (Malinverni!), nel Nora. rese (Bir.), ad Arona (Ricea!), a Locarno (Franzoni!) e Lugano (erb. Webb!), pressu Sondrio (Mass.), nel Comaseo (Parl!), a Milano (Bert.), nel Pavese (Rampoldi! ecc.), nel Cremonese (Par. latore! ecc.), nel Bergamascu (Rota), nel Bresciano (P'arl.! ecc.), nelle Gindicarie presso Tione e Prada (Hansm), nel Trentino (Perinil), in val Sugana (Ambrosil), in Pinc, a Salorno, Bolzano e Merano (Hausm.). nel Veronese (Goiran! ece.), a Mantora (Barbieri!), negh Euganei (Trev.), a Venezia (Kellner! ecc.), nel liassanese (Mon- 
tini!), nel Friuli (Pirona!), a Trieste (Bert.), Pola (Freyn), Fiume (Smith!), nel Modenese (Gib. Pir.), nel Bolognese (Gennari! ecc.). Trovasi in Liguria a Nizza (Ard.), Albenga, Arenzano, Sassello, Sestri Levante (De Not.), Chiavari (Caldesi!); in Toscana nel littorale da Sarzana a Pisa!, intorno a Lucca (Puccinelli! ecc.), ai Bagni di Lucca (Archb.), nell' Appennino pistoiese alle Panche (Vitm.), nel Fiorentino, fino al limite inferiore della regione montana!, a Scarperia (Parl.!), Figline (Ricasoli!), Siena (Bart.), Castelletto-Mascagni nel Senese (Sant.), Pieve S. Stefano in val Tiberina (Cherici!). Più giù nasce ad Ostia, ancora in copia (Seb. Maur.), a Terracina (Bert.), Fondi, S. Germano, e nell' altro lato della Penisola in Puglia (Ten.) e ad Otranto (Groves). Delle isole abita solamente la Sardegna nella valle di Bono (Mlor.!). Fiorisce da aprile a luglio.

Distribuzione geografica. - In tutta l'Europa media e in parte della meridionale, nell'Asia Minore, nella Siberia occidentale fino all' Altai, forse nell' America settentrionale.

Descrizione. - Pianta perenne, alta da 2 a 4 decimetri, di un verde piuttosto chiaro, glabra, fornita di un rizoma orizzontale, piuttosto delicato che manda delle fibre radicali delicate, lunghette e bianchicce. Il fusto è ascendente, diritto o decumbente in basso, semplice o ramoso con i rami eretti o ascendenti, quasi cilindrico in basso, quadrangolare (come i rami) in alto con gli angoli ottusi, fistoloso, articolato, sfumato di rossiccio in basso, verdognolo in alto. Le fo. glie sono un po' consistenti, opposte, formanti qualtro linee longitudinali, patentissinie, più lunghe dei meritalli, sessili, abbraccianti il fusto e i rami, lanceolate e le superiori lineari-lanceolate, ottusette, e le superiori acute, con piccoli denti acuti e quasi in forma di sega, rari nella metà o nei due terzi superiori del margine che è appena rovesciato; sono di un verde piuttosto chiaro e con un solco longitudinale di sopra, di un verde piủ chiaro di sotto ed ivi con un nervo longitudinale rilevato, e uno o due nervetti anche longitudinali ma delicati e quasi vene in ciascun lato, i quali non giungono sino all'apice. I fiori sono solitarii all'ascella delle foglie, un po'. pendenti all' apice di un peduncolo delicato, eretto-patente, quasi cilindrico, verdognolo o sfumato di rossiccio, più corto della foglia corrispon dente, quasi uguale al fiore. Questo è piuttosto grande, talvolta quasi la metà piủ piccolo. Il calice è diviso profondamente in cinque lacinie lanceolate, acute, avvicinate al tubo della corolla, con tre leg. gieri nervi longitudinali, verdi, ed è accompagnato da due brattee, un po'patule, poco più lunghe ma più strette delle lacinie del calice, 
lineari-lanceolate, verdognole. La corolla è molto piủ grande del calice, il suo tubo è un po' curvato in su, quasi quadrangolare, con le due facce laterali e la faccia inferiore aventi un profondo e largo solco longitudinale, è gialliccio con vene biforcate longitudinali piủ scure: il lembo è diviso in quattro lobi, di color violetto chiaro, e bianchicci verso la gola, quasi bilabiato, con il labbro superiore formato dal solo lobo superiore ch'è più largo e un po' piủ corto degli altri tre, quasi orbicolare, smarginato, diretto in su e in dietro e un po' barbato verso la gola, con i peli quasi in clava e giallicci, e con il labbro inferiore formato dal lobo inferiore e dai due lobi laterali, diretti anch'essi un po'in dietro, ovali-orbicolari, interi o l'inferiore appena appena smarginato. Gli stami sono quattro, due superiori fertili e due sessili o ridotti ai soli filamenti, tutti inclusi e inseriti sopra della metà del tubo. I filamenti sono eretti, cilindrici, bianchicci, glabri, quegli degli stami fertili piủ grossi di quelli de. gli stami sterili: il tubo della corolla è peloso intorno ad essi. Le antere sono tonde, bianchicce, uniloculari, glabre. Il pistillo è lungo quanto gli stami piủ lunghi. L'ovario è ovato, un po'schiacciato, con un solco longitudinale in ciascuna faccia, verdognolo-gialliccio, gla bro, biloculare. Lo stilo continua l'ovario, è circa tre volte più lungo di questo, un po'schiacciato in basso ed ivi con un leggerissimo solco che è la continuazione di quello di ciascuna fac. cia dell'ovario, quasi cilindrico, bianchiccio. Lo stimma è grande, laterale, a guisa di una linguetta ottusa all'apice, diretta orizzontalmente in avanti. La cassula è portata all' apice del peduncolo ch'è curvato in su verso l'apice, ed è abluracciata dalle lacinie del calice che sono quasi uguali o poco più lunghe di essa : è ovato-acuminata, un po'schiacciata nelle facce dov'è un legggiero solco longitudinale, ed è biloculare. I semi sono piccoli, ovoideo-bislunghi, angolati, delicati, con una rete piu scura assai delicata. (Parl. ms., descr. di pianta di Lombardia).

\section{SCHOFULARIA.}

Scrophularia Tuurn. insl.p. 166. t. 7\%. Wydler essai mon. 1898 (Scrofularia). Gen. pl. A. germ. 5. 1. 99. Benth. Hook. gen. pl. 2. p. 937. Ces. Pass. Gib. comp /l. ital. p. 3.41. I 5/. f. 3

Calyx cainpanulatus, 5-fidus vel ĩ-partitus. Corolla tubo urceolato, limbo inxugualiter 5-lobato, lobo antico reflexo. Stamina 4, sa- 
pius curn staminodio postico. Antheræ uniloculares. Stigma capitellatım. Gemmmulæ in loculis plurimæ, coacervatæ. Septifragium polyspermum.

Portamento. - Erbe annue o bienni o perenni, o suffrutici, di statura alta, da $1 / 2$ metro a 1 metro, spesso di odore ingrato. Fusto angoloso; foglie opposte, mezzane 0 grandi, più o meno divise; fiori piccoli, di color tetro, rosso-scuro o giallo verdognolo, numerosissimi, in pannocchie $o$ in grappoli di dicasi, generalmente radi, con brattee 0 con foglie fiorali frammiste.

\section{Serofularia vermalis.}

S. glanduloso-pilosa, caule acutangulo, foliis late ovatis, duplicato-inciso-dentatis, dichasiis pedunculatis inflorescentiam interfoliatain efformantibus, pedicello calyce subæquali, lobis calycinis ovalibus ovatisve immarginatis, staminibus exsertis, staminodio nullo.

Scrofularia vernalis Linn. sp. pl.ed. 1. p. 620: Sant. viagg. 1. p. 110. 2. p. 70. Bert. fl. iłal. 6. p. 388. De Not. rep. fl.lig. p. 296. Hausm. fl. Tir. p. 628. Rota! prosp. fl. Berg. p. 65. Car. prodr. fl. tosc. p. 480. Ard. fl. Alpes-mar. p. 277. Terr.! fl. Vult. syn. p. 129. De Vis. Sacc. cat. piant. Ven, p. 150. Zersi prosp. piant. Bresc. $p$. 154. Terr. sec. rel. Terr. Lav. p. 91. Ces. Pass. Gib. comp. fl. ilal. p. 351. Terr. quart. rel. p. 110. Arc. comp. fl. ital. p. 506.

Figure. - Fl. dan. t. 411. Reich. ic. fl. germ. 20. t. 55.f. 2.

Stazione, Abitazione e Fioritura. - Per i monti, nei boschi e in altri lnoghi ombrosi e freschi. Nasce per tutto il Veneto (De Vis. Sacc.), e cosi nella valle dell' Isonzo (Pirona !), a Chiapovano (Reich.), in Valdella delle Velte di Feltre (Poll.), nel Bassanese a Vallerana, Valstagna, e presso la Grappa, nel Portole (Bert.), nel Summano, nei Lessini (Poll.), nel Baldo in molti siti (Goiran!, Poll., Hausm.); in Tirolo fa nel Trentino (Poll., Hausm.), e in val Vestino presso Magasa (Porta!); è frequente nel Bresciano, cosi al Guglielmo, al Dragone (Zersi), allo Spino (Porta!), presso il Roccolo di Pezzeda a Sera (Parl.!); nel Bergamasco è stata trovata a Carenno (Rota!); quindi oltrepassando il tratto intermedio delle Alpi ritrovasi in Piemonte a Perrero di Pinerolo (Parl.!), nella valle di Lıserna (Ro. stan!), presso i Bagni di Vinadio (All.), ai Bagni di Valdieri (Bert.), verso Limone, abbondante in quel di Mondovi (All.), ed Ormea (Gentili!), e poi nell' alpi di Tenda in parecchi luoghi (Reuter! ecc.); ri- 
prende nell'Appennino al monte Antola, in quel di Bobbio (De Not.), nel Pavese a Boglielo e Ghiarolo (Nocca Balb.), e prosegue a salti all'Alvernia in Toscana!, nelle Marche al Catria (Piccinini!), alle Svolte di S. Leonarilo (Gennari!) e al Castelluccio (Bert.), in Abruzzo a Cafornio sul Velino (Cherici!), e dall'altro lato della Penisola in Toscana al monte Amiata a Ciastel del Piano, e a Pitigliano alla macchia del Lamone (Sant.), nel Lazio alla montagna di Viterbo (Bert.), e al monte Gennaro (Seb. Maur.), in Campania a Picinisco (Terr.), al monte Cairo (Terr. !), a Pastena (Terr.), Sessa (Ten.), Frasso Telesino, Pietraroia (Terr.), nel monte S. Angiolo di Castel. lammare (Ten.), nel Vulture (Terr.!), per terminare in Basilicata (Bert.) e al monte della Stella nel Principato citeriore (Ten.). Pas. sando infine nella Sicilia, vi nasce nelle Madonie sopra Castelbuono (Parl.!), a S. Guglielmo e a Isnello (Guss.). Fiorisce in aprile e maggio nelle parti meridionali, in maggio e giugno nelle settentrionali.

Distribuzione geografica. - Nell' Europa media, e in parte nella meridionale e la settentrionale.

\section{Scrofularia peregrina.}

S. glabra, caule acutangulo, foliis late ovatis, grosse duplicato-dentatis, dichasiis pedunculatis inflorescentiam inferne interfoliatam efformantibus, pedicello calyce multo longiore, lobis calycinis lanceolato-acutatis immarginatis, staminibus inclusis, staminodio subrotundo.

Scrofularia peregrina Linn. sp. pl. ed. 1. p. 621. Bert. fl. ital. 6. p. 389 ; et auct. omn.

Figure, - Fl. graec. t. 597. Reich. ic. R.germ. 20. t. 55. f. I.

Stazione, Abitazione e Fioritura. - Nei luoghi boschivi, lungo le siepi, nelle macerie ecc. delle regioni più calde. In Istria rara e sparsa, da Pirano a Pola (Bert., Freyn), e a Fiume (Smith). In Liguria abbastanza comune nel Nizzardo (Ard.), e poi in parecchi luoghi sino a Portofino (Baglietto!). In Toscana non rara nelle re. gioni maremmana e campestre, cosi da Sarzana (Lert.) al monte Argentaro, nel Fiorentino!, a Volterra (Amidei!), a Siena (Bert. ecc.), a Chiusi (Ball!), nel monte Amiata al Viro (Santi), e nelle isole di Gorgona (P. Sav.), di Capraia (Mor. De Not.) e dell' Eilba (Mar. cucci!). Nelle Marche principia a Sinigaglia e al Furlo, e poi ri. trovasi a Macerata (Bert.), e Sant'Angelo in Pontano (Marzialetti!), 
e in Abruzzo a Nereto (Bert.). Nel Lazio fa a Viterbo (Bert.), a Civitavecchia (Seb. Maur.), nei monti Albani (Rolli! ecc.), a Genzano (Bert); in Campania a S. Germano Cassino (Levier!), Mondragone, Caserta (Tur.), Napoli (Bruni! ecc.) dov'è frequente (Ten), nonchè in Ischia (Bolle!) e Capri (erb. Webb!); poi nel Vulture (Terr.); a Otranto (Groves); in Calabria a Cosenza (Aiuti!) e presso Pizzo (Arc!). In Corsica a Bastia!, Calenzana (Bert.), Vivario (Mars.), Aiaccio (Requien!), Bonifacio (Kralik!), Portovecchio (Bert.). In Sardegna, non rara (Mor.!), e nelle isole Ciaprera, S Stefano (Genn.), Maddalena, Tavolara (Mor.). In Sicilia ovunque (Guss.), cosi a Palermo (Parl.!), Messina (Borzi!), Avola (Pianca!), Comiso (Aiuti!) ecc., e nelle Eolie, in Ustica, nelle Egadi (Guss.), e poi in Pantellaria (Calcara!), Linosa, Lampedusa (Aiuti!); e in Malta (Grech! ecc.). Fiorisce in aprile e maggio, e sin da febbraio in Sicilia.

Distribuzione geografica. - In tutto il bacino del Mediterraneo.

Descrizione. - Pianta annua, alta da 2 a 5 e talvolta anche sei decimetri, quasi glabra 0 glabra, verde, puzzolente. La radice è duretta, fusiforme, tortuosa, ramosa, bianchiccia. Il fusto è fistoloso, quadrangolare, con gli angoli acuti, e un po'rilevati, con le faccie piane, puberulo in basso con i peli corti e glandolosi all' apice, glabro in alto, eretto, di color violetto scuro in gran parte o pure verdognolo sfumato di violetto, per lo più semplice, talvolta ramoso, con i rami che vengono anche dalla base, opposti, eretto-patenti o eretti, corti, dal resto simili al fusto ma più delicati di questo. Le foglie sono opposte, patenti, lontane, cordatoovate o cordato-ovali, ottusette o acute, doppiamente dentatoseghettate, con i denti disuguali forniti all'apice di un mucrone corto ed oblungo; sono di un verde chiaro, lucide di sopra ed ivi con molti solchi assai manifesti che corrispondono ai nervi di sotto, di un verde più chiaro e opache di sotto, ivi fornite di un nervo longitudinale e di nervi laterali rilevati, i quali si diramano per terminare con ciascun rametto all' apice di ciascun dente: i rametti sono poco o punto rilevati; le foglie inoltre sono glabre in ambe. due le pagine, eccetto nei nervi di sotto dove sono pochi peli corti simili a quelli della base del fusto e dei rami, tutte fornite di un picciỏlo poco più lungo o quasi uguale alla foglia, scanalato di sopra, angolato di sotto, gradatamente appena piủ largo verso la base, puberulo-glandoloso nei margini, di color violetto o verdo- 
gnolo. Le foglie fiorali sono alterne, gradatamente piủ piccole e più acute e quasi acuminate, piủ lunghe e gradatamente quasi uguali o poco più corte delle cime dei fiori, con un picciỏlo corto o cortissimo e poi quasi sessili. I fiori sono pochi, da 2 a 5 in cima all'ascella delle foglie superiori o fiorali. I peduncoli sono eretti 0 eretto-patenti; i pedicelli sono poco più corti o quasi uguali ai fiori, tanto gli uni che gli altri angolati, verdognoli e puberuliglandolosi. Le bratteole sono solitarie, piccole, setacee, verdognole, più corte dei pedicelli, glabre. Il calice è glabro, verdognolo, diviso fin poco sopra la base in cinque lobi o lacinie un po' patenti, ovato-lanceolate, ottusette, fornite di una carena. La corolla è quasi due volte piủ lunga del calice, di color porporino scuro, con il lobo medio del labbro inferiore di color più chiaro. Il tubo è un po'gonfio, quasi tondo. Il labbro superiore è più grande dell'inferiore, diretto in su, profondamente diviso in due lobi i quali si cuoprono in parte l'un l'altro con il margine interno; it labbro inferiore è trilobo con i lobi laterali eretti, il lobo inferiore patente, appena piủ lungo dei due laterali: tutti i lobi tanto del labbro superiore quanto dell'inferiore sono quasi tondi, con leggiere crenature nel margine e con vene ramose di colore più carico. L'appendice del labbro superiore corrisponde nella gola sotto della divisione dei due lobi di quello ed è piccola, diretta in su, quasi orbicolarecuneata e del colore stesso del labbro superiore. La gola è larga, aperta. Gli stami sono quattro, poco piủ lunghi della gola e più corti del labbro inferiore al lobo medio del quale sono avvicinati, due appena più alti degli altri, tutti inseriti nella parte anteriore ed interna della base del tubo. I filamenti sono grossetti, un po' piủ grossi verso l'apice, un po'schiacciati la avanti in dietro, bianchicci e in parte rossicci, quasi glabri. Le antere sono schiacciate da avanti in dietro, uniloculari, si aprono per una fessura trasversa ed in alto, e sono di color violetto scuro e glabre; il polline è giallo. Il pistillo è lungo quasi quanto gli stami. L'ovario è ovato-tondo, verdognolo, circondato alla base da un disco quasi un anello di color verde. Lo stilo ì appena curvo, filiforme, bianco, glabro, piủ lungo dell' ovario. Lo stimma è quasi in capolino, appena bilobo, papilloso, gialliccio. (Parl. ms., descr. di pianta di Fiesole). 


\section{Serofularia trifoliata.}

S. glabra, caule acutangulo, foliis inferioribus pinnatisectis lobis ovatis oblongisve, grosse duplicato-crenatis, dichasiis subsessilibus inflorescentiam interfoliatam efformantibus, pedicello calyce æquali vel longiore, lobis calycinis rotundatis albo-marginatis, staminibus inclusis, staminodio subrotundo.

Scrofularia trifoliata Linn. sp. plant. ed. 2. p. 865. P. Sav.! fl. gorg. p. 272. Bert. fl. ital. 6. p. 392. Mor. f. sard. 3. p. 189. Gren. Godr. fl. Fr. 2. p. 564. Car. prodr. fl. tosc. p. 480. Fl. Montecr. p. 27. Genn.! fl. Capr. p. 114. Mars. cat. pl. Cors. p. 106. Ces. Pass. Gib. comp. fl. ital. p. 342. Arc. comp. fl. ital. p. 506.

Scrofularia mellifera Bert. o. c. p. 391 (excl. pler. syn.).

Figure. - Pluk. phyt. t. 313. f. 6. Mor. o. c. t. 100.

Stazione, Abitazione e Fioritura. - In luoghi ombrosi umidi delle isole di Corsica e di Sardegna. In Corsica nelle regioni bassa e media: Capo Corso (Mars.), Bastia (Mabille!), Bagni di Guagno, Poggiolo (Requien!), Pozzo di Borgo (Soleirol!), Pastricciola (Bert.), Aiaccio (Bubani!), Olmeto (Mars.). In Sardegna specialmente in luoghi montuosi : Caprera (Genn.!), Maddalena, Tavolara, Asinara (Mor.), Tempio (Bert.), Milis, S. Lussurgiu, Paulli Gerei, Ercubatu, Arizzo, Belvi, Orosei, Tonneri d'Irgini (Mor.), Fonni (Lovisato!), Zinigas presso Siliqua (Biondi!), Capo S. Elia (Ascherson!). Nasce inoltre nelle isole di Gorgona (P. Savi!), e di Montecristo (Taylor!). Fiorisce in aprile, maggio, giugno.

Distribuzione geografica. - Specie propria di quelle isole.

Osservazioni. - Vedansi Bentham in Cand. prodr. 10. p. 307, e Moris $0 . c . p .191$ per i caratteri differenziali fra la presente specie e la Scrofularia sambucifolia Linn., di Spagna, Portogallo e Algeria, i quali sembrano invero molto próblematici.

L'affine $S$. Scorodonia Linn. fu già indicata da Allioni nel Niz. zardo, e più recentemente da Bertoloni; ma è pianta dell' Europa più occidentale, che nè De Notaris nè Ardoino riportano nelle loro flore, e di cui non conosco alcun saggio italiano.

\section{Serofularia Seopolii.}

S. plus minus pilosa, caule acutangulo, foliis ex ovato ovalibus oblongisve, crenato-serratis, dichasiis pedunculatis inflorescentiam 
aphyllam efformantibus, pedicello calyce æquali vel longiore, lobis calycinis rotundatis, albo-marginatis, staminibus inclusis, staminodio subrotundo.

Scrofularia Scopolii Hoppe exs. Pers. syn. pl. 2. p. 160. Bert. fl. ital. 6. p. 386. Car. prodr. fl. losc. p. 481. G. Bert. veg. Porr. p. 84. Terr.! rel. Terr. Lav. p. 147. Sec. rel. p. 92. Archb. fl. Alto-Serch. p. 58. Gib. Pir. fl. Mod. p. 120.

Scrofularia grandidentata Ten.! fl. nap. 1. prodr. suppl. 2. p. 69. Tar. Gerb. cat. pl. calat. p. 30. Ces. el. piant. Maiella p. 22. Ces. Pass. Gih. comp. fl. ital. p. 342. Terr.! quart. rel.p. 110. Arc. comp. fl. ital. p. 507. Cocc. fl. Bol. p. 362. Nic. prodr. fl. mess. p. 343.

Figure. - Scop. fl. carn. ed. 2. t. 32. Reich. ic. fl. germ. 20. t. 84. f. 3.

Stazione, Abitazione e Fioritura. - Nei boschi e nei pascoli delle parti più alte dell'Appennino, cioè nelle regioni montana e alpestre, d'onde scende qualche volta più in basso nella regione sub. montana. Principia in Toscana al monte Orsaio (Cocchi!), e si stende senza interruzione sino a Pieve S. Stefano in val Tiberina (Bert.), scendendo di là a Lustrola nel Bolognese (G. Bert.) e a Valcoloreta nel Faentino (Bert.), e di qua ai Bagni di Lucca (Archb.) e a Rassina in Casentino (Ricasoli!), e inoltrandosi a Vallombrosa (Borzi!), e fa eziandio in tutte le Alpi Apuane (Bert., Cocchi! ecc.); prosegue per le Marche alla Carpegna (Bert.), al Catria (Piccinini!), al monte Birro (Bert.), ai monti Sibillini (Gennari!, Parl.!), a S. Angelo in Pontano (Marzialetti!), e per l'Abruzzo al Monte de'Fiori (Bert.), al Pizzo di Sivo!, al Corno (Orsini!), e alla Maiella (Levier!, Groves!); dall'altro lato della Penisola nasce nel Lazio a Mentorella (Bert.), in Campania ad Arpino (Terr.), nel monte Meta, nel Matese (Terr.!), a Frasso, a Cerealto, nel monte Vergine (Ten.), e poi in Basilicata (Bert.), e termina in Calabria al Pullino (Ten.) a 2000 metri (Huter Porta Rigo!), e a Serra S. Bruno (Zwierlein!). Nasce altresi in Sicilia nel Valdemone (Todaro!), a Marabotto (Nic.), a Ca. ronia (Guss.), nelle Madonie (Parl.!), alla Ficuzza (Guss.), la Pizzuta (Todaro!), Renda (Sorrentino!), nel monte Cansaria di Cal. tagirone (Tar. Gerb.). Fiorisce da maggio ad agosto secondo $\mathrm{i}$ luoghi.

Distribuzione geografica. - Nell' Europa meridionale e media orientale, nell'Asia Minore, fino al Caucaso.

Desorizione. - Pianta perenne, alta 4 o 5 decimetri, pube. 
scente e in alto pubescente-glandolosa, fornita di un rizoma orizzontale o obliquo, grossetto, scuro che manda molte fibre radicali, lunghe e bianchicce e dall'apice un fusto o ramo. Questo è eretto, diritto, un po'flessuoso verso alto, quadrangolare, rossiccio-scuro e pubescente in basso, verdognolo e pubescente-glandoloso in alto per peli corti, bianchi, e forniti all'apice di una piccola glandola scura, semplice ma con rametti cortissimi portanti due foglioline patenti all'ascella delle foglie. Queste sono molli, opposte, un po'lontane, patenti o eretto-patenti, le inferiori ovate, le superiori ovatobislunghe, tutte scavate un po'in cuore alla base, ottuse, profondamente crenate o doppiainente crenato-dentate, con i denti ottusi, aventi all'apice una piccola punta: sono verdi di sopra, ed ivi puberule e con solchi in rete corrispondenti ai nervi della pagina inferiore, di un verde bianchiccio e pubescenti, massime nei nervi, di sotto ed ivi con un grosso nervo longitudinale da cui partono dei nervi laterali rilevati i quali si diramano, si uniscono ad arco e terminano alla punta dell'apice dei denti. Il picciỏlo è corto, scanalato di sopra, convesso di sotto, rossiccio, pubescente. I fiori sono distribuiti in una pannocchia, piuttosto corta, con i rami inferiori opposti, i superiori alterni, patenti, pubescenti-glandolosi con la glandola grossetta, rosso-scura, accompagnati da brattee o bratteole fogliacee, gradatamente più piccole, lanceolate, lanceolato-lineari o lineari, dentato-incise o quasi intere, verdi, pubescenti-glandolose di fuori. I pedicelli sono piủ lunghi o più corti dei fiori. Il calice è quasi campanulato-globoso, glabro, verde, diviso da sotto la metà in cinque lacinie quasi orbicolari, scagliose, scurette e come lacere nel margine. La corolla è appena il doppio piü lunga del calice, di colore rossiccio-scuro, eccetto nel lobo inferiore del labbro inferiore ch'è verdognolo-rossiccio, glabra; il suo tubo è quasi globoso, un po'gonfio, il lembo è poco più corto di questo, bilabiato, il labbro superiore bilobo, con i lobi eretti, quasi orbicolari, cuoprentisi un poco l' un l'altro con il margine interno, il lobo inferiore è un po'più corto del superiore, trilobo, con i lobi quasi orbicolari, i laterali eretti, e l'inferiore diretto in giù. Gli stami sono quattro fertili, quasi uguali, appena piủ lunghi del tubo della corolla. I filamenti sono cilindrici, slargati in alto sotto dell'antera, verdognoli, glandolosi, con le glandole rossicce. Lo stame sterile è posto sotto del labbro superiore ed è a guisa di una linguetta obovata, smarginata all'apice, verdognola, glandolosa di sopra. Il pistillo è poco più lungo degli stami. L'ovario è ovato-tondo, verde, glabro, biloculare, con gli 
ovoli piccoli, inseriti sopra una placenta grossa, posta dalla parte del setto; è circondato alla base da un disco circolare, ottuso, gialliccio. Lo stilo è molto più lungo dell' ovario, curvato in avanti, quasi lesiniforme, verdognolo-gialliccio, glabro. Lo stimma è quasi bilobo, giallo. La cassula è ovato-globosa, acuta. (Parl. ms., descr. di pianla di Boscoiungo in Toscana).

Osservazioni. - La forma coi denti fogliari più stretti e piủ lunghi (Serof. grandidentata Ten.) è prevalente al mezzogiorno, la tipica al settentrione; però in diversi luoghi trovansi frammiste. Vi sono anche forme di passaggio, e lo stesso Tenore non riteneva di. stinte le due specie.

\section{Serofularia anuatiea.}

S. glabra, caule angulis subalatis, foliis ex ovato ovalibus oblongisve, basi interdum pinnatisectis, serratis, dichasiis subpedun. culatis inflorescentiam aphyllam efformantibus, pedicello calyce longiore, lobis calycinis rotundatis, albo-marginatis, staminibus inclusis, staminodio retuso emarginato vel bilobo.

Scrofularia aquatica Linn. sp. plant. ed. 1. p. 620. Bert. fl. ital. 6. p. 382; et auct.

Scrofularia alata Gilib. $l$. lith. Ces. Pass. Gib.! comp. $l$. ital. p. 342. Bizz. piant. agg. /l. ven. p. 5. Arc. comp. fl. ilal. p. $50 \%$.

Scrofularia Balbisii Ilorn. hort. bot. hafn. p. 57\%. De Not. rep. f. lig. p. 296.

Scrofularia ublongifolia Lois, nouv. not. f. Fr. p. 26. Req. in giorn. bot. 2. 3. p. S. Nic. prodr. /1. mess. p. 3.13.

Scrofularia Ehrharti Stev. sec. Bab. man. brit. bot. p. 2/8. Pir. 11. flor. syll. p. 103.

Figure, - Fl. dan. t. 507. Reich. ic. /l. germ. 20. t. 51 , 52. $f$. 1 .

$\beta$ pubrscens.

Scrofularia auriculata Linn. sp. pl. ed. 1. p. 620. Mor. Al. sard. 9. p. 188. Ces. I'ass. liib. l. c. Arc. l. c.

Scrofularia subverticillata Mor. stirp. sart. el. 3. 1. 8. Bert. o. c. p. 39 ?.

Scrofularia aquatica var. 3 De Not. l. c. 
Stazione, Abitazione e Fioritura. - Per tutta l'Italia, nei fossi ed altri luoghi umidi. Abbonda nel Piemonte (All.), cosi a Torino, Susa (Re ecc.), Mondovi (Ing.), Alba (Bert.), Novara (Bir.), Valleggio (Com.); nasce nel cantone Ticino, presso Como (Com.), in val Tellina (Mass.), a Pavia (Nocc. Balb.), nel Bergamasco (Rota), nel Bresciano, poco frequente (Zersi), in Tirolo sul lago di Garda (Hausm.); trovasi in tutto il Veneto (De Vis. Sacc.), cosi nel Vero. nese in molti luoghi (Goiran!), negli Euganei (Trev.), a Chioggia (Bert.), Venezia (Nacc.), Bassano (Bert.), nel Friuli (Pir.); fa a Parma (Pass.!), nel Modenese a Fiumalbo (Gib. Pir.), a Bologna (Cucc.). Ė comunissima in Liguria (De Not.), cosi a Nizza (Durando!), Mentone (Ard.), Oneglia, Diano (Ricca!), Genova (Figari!) ; comune pure in Toscana, nelle regioni maremmana, campestre ed anche submontana: a Sarzana (Bert.), Lucca (Calandrini! ecc.), Bagni di Lucca (Archb.), Pisa, Livorno!, Firenze (Reboul!), Volterra (Amidei!), Siena (Bart.), Orbetello (Cherici!), Pitigliano (Sant.); nasce nelle Marche a Porlo Civitanuova (Ricci!), a Montefortino (Bertoloni); a Roma (Webb!), comune (Seb. Maur.); in Campania a Caserta (Bruni!), Picinisco (Levier!), Napoli (Ten.), Montecchio (Terr.). Manca nelle piccole isole, ma trovasi nelle grandi: in Corsica a Bastia (Mabille!), Calcataggio (Mars.), Vico, nel monte d'Oro (Requien!), a Bocognauo (Jordan!), Bonifacio (Bert.) ecc.; in Sardegna (Mor.), per esempio a Laconi (Ascherson!); in Sicilia a Palermo (Parl.! ecc.), nelle Madonie, a Fiumedinisi, Piazza, Siracusa, Noto, Spaccaforno (Guss.), Terranova (Aiuti!). La var., molto più rara, è stata trovata nella Riviera di ponente e nel Nizzardo (De Not.), in Toscana a Casciana (Baroni!), in Sardegna a Tempio (Gennari!), Ozieri (Bonjean!), Bono, Sassari, Scala di Ciocca, Limbara (Mor.). Fiorisce in maggio e giugno nei luoghi più caldi, e da giugno per l'estate nei mieno caldi.

Distribuzione geografica. - In tutta Europa, eccettuata la più nordica, nell'Asia occidentale fino al Cabul, nell'Asia media fino all'Altai, nell'Affrica settentrionale, nelle isole Canarie e Azore, nel Messico.

Descrizione. - La radice è grossa, come nodosa, bianchiccia e ramosa. Il fusto è alto da 4 a 5 o 6 piedi, quadrangolare, con gli angoli acuti o appena alati, glabro, rossiccio in basso, verdognolo in alto. I rami sono opposti, aperti o apertissimi. Le foglie sono opposte, le infime sono fornite di un picciòlo piủ lungo della lamina, mentre questa è piủ lunga del picciòlo in tutte le altre. Il picciòlo è 
largamente scanalato di sopra, con un angolo di sotto, rossiccio o in parte verdognolo, ed alato negli angoli laterali. La lamina è lunga circa 4 pollici e larga un pollice e mezzo, cordato-allungata, con la base obliquamente a cuore nelle foglie inferiori, con una o due appendici a guisa di orecchiette, obliquamente allungate o obliquamente quasi tonde, con i margini di essa e delle appendici crenate o duplicato-crenate, le crenature aventi un piccolo mucrone: nelle foglie superiori il margine è quasi seghettato con le seghe poco pro. fonde; la lamina infine è glabra, di color verde scuro di sopra, più pallido di sotto. Le foglie fiorali sono allungate, intere, ed ottuse o ottusissime, ed eccetto le due infime hanno il margine scaglioso e bianchiccio, nel resto sono verdi eccelto nel margine dove sono rossicce, alla loro ascella si vedono due piccole foglie quasi ellittiche, con il margine seghettato. La pannocchia è grande, lunga piủ di un piede, i suoi rami sono distanti, gl'inferiori opposti, i superiori alterni o sparsi, tutti apertissimi, duretti, quadrangolari e tricotomidicotomi, con una piccola brattea lineare a ciascun rametto. I pedicelli sono lunghi quasi quanto i fiori. Il calice ha le lacinie ovatotonde, con il margine scaglioso, bianchiccio, mentre è verde nel resto e sfumato solo di rosso verso il margine. Le due lacinie supe. riori del labbro superiore della corolla sono di color porporino scuro, le due laterali del labbro inferiore sono meno cariche, e verdognole in basso, tutte erette, la intermedia è piegata in giù, verdognola con il margine rossiccio. I filamenti sono piano-filiformi, di un bian乞̊o verdognolo, peloso-glandolosi, con le glandole porporine scure. Le antere sono gialle con il margine scuretto. Il polline è giallo. La la. minetta dell'antera sterile è porporino-scura, quasi nericcia, semiorbicolata e come smarginata e talvolta come biloba con un piccolissimo dente. Il disco è in forma di anello ottuso e verdognolo. L'ovario é ovato, verdognolo, papilloso. Lo stilo è filiforme, bianchiccio, glabro, curvato in avanti e in giù in modo che si adatta alla lacinia in. ternedia del labbro inferiore della corolla. Lo stimma è bilobo, con i lobi quasi tondi, verdognoli e papillosi. La cassula è ovata, acuta, quasi il doppio più lunga del calice. (Parl. ms., descr. di pianta Fio. rentina).

Osservazioni. - I moderni generalmente spezzano questa specie in due, o in tre, in base a vari caratteri, e soprattutto al fusto strettissimamente o largamente alato, e allo staminodio intero o diviso. Ora la larghezza delle ali varia al piủ possibile anche nei diversi tratti di un medesimo fusto; $e$ in quanto allo staminodio, gria diversi autori, 
quali Ascherson ( $F l$. Brand. p. 468), e Boissier (Fl. orient. 4. p. 400 ), ne hanno additata la variabilità di forma, confermata anche dalla descrizione di Parlatore qui sopra. Dicasi lo stesso di altri caratteri addotti, come le foglie scavate o no alla base, la forma dell'infiorescenza, ugualmente incerti.

La pianta di Parma, ossia Scrofularia alata dei Compendi, è una forma singolare, con le foglie aguzze, e l'infiorescenza assai ramosa a pannocchia.

Fuorchè la pelurie, non trovo nessun carattere che valga a separare la var. dal tipo specifico.

\section{G. Senofularia modosn.}

S. glabra, caule acutangulo, foliis ovato-acutatis, serratis, dichasiis pedunculatis inflorescentiam aphyllam efformantibus, pedicello calyce multo longiore, lobis calycinis ovato-rotundatis, vix albo-marginatis, staminibus inclusis, staminodio rotundato subemar. ginato.

Scrofularia nodosa Linn. sp. plant. ed. 1. p. 619. Bert. fl. ital. 5 . p. 380 ; el auct. omn.

Figure.-Fl. dan. t. 1167. Reich. ic. fl. germ. 20. t. 53.

Stazione, Abitazione e Fioritura. - Nei luoghi ombrosi umidi, alle siepi, nei boschi ecc. della Penisola, principalmente in collina e in montagna. In Piemonte nasce qua e là (All.): presso Roccaforte Mondovi (Ing.), in quel di Susa, a Torino (Re), Riva di Sesia (Carestia!), Oldenico (Malinverni!), nel Novarese (Bir.); trovasi nel Cantone Ticino (Com.), in val Tellina (Mass.), per esempio presso S. Caterina a 1700 metri (Parl!), abbonda nel Comasco (Com.), è pure frequente a Pavia (Nocc. Ball).), fa nel Cremonese, nel Bergamasco, eosi a Ponte di legno a 1520 nuetri (Parl.!), nel Bresciano, frequente (Zersi); nasce per tutto il Tirolo (Hausm. ecc.); per tutto il Veneto eziandio (De Vis. Sacc), cosi abbonda nel Veronese (Poll.), trovasi negli Eıganei (Trev.), a Venezia (Zan.), Bassano, Aquileıa (Bert.), Udiıe (Pir.) ecc.; non è rara nel Modenese, salenilo dai dintorni dı Modena alla regione montana nell'Appennino (Gib. Pir.), come pure nel Bolognese, da Bologna su nei monti (Cocc.), e ritrovasi a Faenza (Cald). Dicesi frequente nei monti Ligustici (De Not.): nelle Alpi marittime a S. Dalmazzo di Tenda (Un. gern-Sternberg!), S. Martino Lantosca, la Mairıs, Valdiblora (Ard.), 
e poi a Chiavari (Delpino!); in Toscana occupa le regioni campestre, submontana e montana, dalla Lunigiana! alla val Tiberina (Bert.) e al Pisano!, in val d'Elsa (Reboul!), nel Cliianti!, a Siena (Bart.), nel monte Amiata (Bert.); nelle Mlarche è stata trovata a S. Marino (Bert.), al Catria (Piccinini!), a Trisungo nell'Ascolano!. Ritrovasi presso Roma nei monti Albani (Webb! ecc.), e piủ giù in Campania a Treglia (Terr.!), Carditello (Terr.), Napoli (Parl.! ecc.), in Ischia (Guss.), e infine nel Vulture dove termina (Terr.). Fiorisce da maggio a luglio secondo i luoghi.

Distribuzione geografica. - Nell' Europa, eccettuata la sua parte arctica e le sue parti piủ meridionali, nei paesi del Caucaso, in Si. beria fino all'Altai, nell'America settentrionale (in una var. speciale).

Descrizione. - Pianta perenne, alta da 4 a 10 decimetri, verde, glabra, fornita di un rizoma orizzontale, nodoso-tubercoloso, bianco, che inanda fibre radicali delicate e bianche. Il fusto è eretto, appena flessuoso, quadrangolare, con gli angoli un po'rilevati e verdognoli, verde 0 in parte rossiccio, glabro, semplice o ramoso con i rami eretti. Le foglie sono erbacee, opposte, alquanto lontane, patenti o patentissime, ovali-bislunghe e le superiori ovali-lanceolate, acute, quasi scavate in cuore e come troncate alla base, doppiamente dentato-seghettate, con i denti della base più grandi, più acuti e un po' più patenti, verdi di sopra ed ivi con solchi che corrispondono ai nervi di sotto, di un verde pallido di sotto ed ivi con un nervo longitudinale rilevato e con altri nervi anche rilevati, parte che vengono dai lati della base del nervo longitudinale, e parte da questo verso la metà e l'apice, i quali obliquamente e curvi si dirigono verso il margine e l'apice, si diramano e formano una rete rilevata nella pagina inferiore della foglia: sono glabre e fornite di un picciólo il quale è corto, solcato di sopra, angolato di sotto, strettamente alato per la scorrenza della lamina della foglia. Le foglie superiori hanno il picciòlo cortissimo. I fiori sono molti, distribuiti in piccole cime laterali, formanti una pannocchia terminale, e lunga. I peduncoli sono patenti, quadrangolari, glandolosi per glandole tonde e scurette pedicellate: sono accompagnati da brattee poco più lunghe di essi, patenti, lanceolato-lincari olineari, acuminate, verili, con piccoli denti. I pedicelli sono più lunghi dei fiori e della cassula, quasi cilindrici, glanilolosi come i peduncoli nella metà inferiore, glabri nella meta superiore, verilognoli o rossicci, accompagnati alla base da bratteole lineari-setacee, verdi, glabre, più corte di essi. Il 
calice è verde, glabro, diviso fin poco sotto la metà in cinque lacinie ovali, ottuse, con una leggiera carena nel dorso, con un margine strettissimo membranaceo. La corolla è rosso-scura di sopra e nel labbro inferiore, verdognolo-gialliccia di sotto e nel labbro inferiore. Il suo tubo è quasi tondo; il labbro superiore è molto più lungo dell'inferiore, diretto in avanti, bilobo, con i lobi ovato-orbicolari, coprentisi in parte l'un l'altro; il labbro inferiore è trilobo, con i lobi quasi uguali, quasi orbicolari, perỏ un po'piủ larghi che lunghi, i due laterali diretti in su, l'intermedio patente-reflesso. 'Gli stami fertili sono quattro, inseriti alla base del tubo della corolla, didinami, due poco più alti degli altri due, quasi uguali o poco piủ corti del labbro inferiore, al lobo medio del quale sono avvicinati. I filamenti sono un pu'curvi, quasi piani, grossetti, bianchicci, puberulo-glandolosi. Le antere sono gialle, glabre. Lo stame sterile è in forma di una laminetta petaloidea, obovata, spesso smarginata e irregolarmente dentata nell' apice, è del colore del labbro superiore sotto del quale corrisponde, essendo diretto in avanti ed in su. Il pistillo è lungo quasi quanto gli stami piủ lunghi, avvicinato al lobo medio. L'ovario è ovato, verdognolo, glabro, circondato alla base da un disco circolare, ottuso e verdognolo. Lo stilo è poco piủ lungo dell'ovario, curvo, cilindrico, bianchiccio, glabro. Lo stimma è quasi in capolino, verdognolo, un po'papilloso. La cassula è ovata, acuta, con un soleo longitudinale in ciascuno dei lati. (Parl. ms., descr. di pianta di Boscolungo nell'Appenuino toscano).

\section{\%. Scrofuluria Inciniatu.}

S. glabra, caule obtusangulo, foliis inciso-dentatis pinnatifidis partitisque ımo basi pinnatisectis, dichasiis pedunculatis inflorescentiam aphyllam efformantibus, pedicello calyce subbreviore, lobis calycinis rotundatis, albo-marginatis, staminibus inclusis, staminodio subreniformi.

Scrofularia laciniata Waldst. Kit. pl. rar. Hung. Koch syn. fl. germ. ed. 2. p. 594. Simili fl. Fiume p. 38.

Figure.-Fl. grcec. t. 603. Reich. ic. fl. germ. 20. t.54.f. 1.

Stazione, Abitazione e Fioritura. - In Istria, sulle rupi e sui muri a fiume (Noë! ecc.), e sul monte Sissol (Reich.). Fıorisce da aprile a giugno.

Distribuzione geografica. - Oltracciò nella penisola Balcanica e nèi Paesi Damubiani. 


\section{Serofularia Incida.}

S. glabra, caule obtusangulo, foliis pinnatisectis segmentis lo. batis dentatis, dichasiis pedunculatis inflorescentiam aphyllam efformantibus, pedicello calyce subbreviore, lobis calycinis rotundatis, albo-marginatis, staminibus exsertis, staminodio subreniformi.

Scrofularia lucida Linn. sp. plant. ed. 2. p. 865. Ard.! cat. pl. Ment. p. 26. Fl. Alpes-mar. p. 278. Ces. Pass. Gib. comp. fl. ital. p. 342. Arc. comp. fl. ital. p. 507.

Scrofularia glauca Bert. A. ital. 6. p. 398.

Figure.-Guss. pl. rar. t. 43. Fl. griec. t. 599, 600.

Stazione, Abitazione e Fioritura. - Nei lıoghi sassosi e sui muri nella Penisola. Trovasi da una parte nel Nizzardo, dove pare che sia abbastanza comune, e fa da Nizza (Thuret!), Villafranca (Bourgeau!), Mentana (Ard.!) a Tenda (Ungern-Sternberg!); dall'al. tra parte nella costa meridionale orientale, a Bari, Polignano (Guss.), Monopoli, Brindisi, Lecce (Ten.), Otranto (Guss., Ten, Bert., Ces. Pass. Gib.), Gallipoli, Castro (Guss.), Ginosa, Martina (Ten.). Fiorisce in aprile e maggio nel mezzogiorno, da maggio ad agosto nel Nizzardo.

Distribuzione geografioa. - Oltracciò in Grecia e nell'Asia Minore.

\section{Serofularin canina.}

S. glabra, caule obtusangulo, foliis pinnatisectis segmentis inciso-dentatis partitisque, dichasiis pedunculatis inflorescentiam aphyllam efformantibus, pedicello calyce subbreviore, lobis calycinis rotundatis, albo-marginatis, staminibus exsertis, staminodio subulato vel nullo.

Scrofularia canina Linn. sp. pl. ed. 1. p. 621. Bert. A. ital. 6. p. 393; et auct.

Scrofularia lucida Seb. Maur.? fl. rom. prodr. p. 205 (non Linn.).

Scrofularia bicolor Sihth. Sin. fl. gripe. 7.p. 1. Cuss, enum. pl. Inarim. p. 239. Pasq. R. ves. p. 75. Terr. R. Vult. syn. p. 199. Ces. 
Pass. Gib. comp. fl. ital. p. 342. Loj. is. eol. p. 120. Gib. Pir. fl. Mod. p. 120. Nic.! prodr. fl. mess. p. 343.

Scrofularia Hoppii Terr.! rel. Terra Lav. p. 147 (non Koch).

Figure. - Guss. pl. rar. t. 44. Reich. ic. bot.t. 728. Fl. groec. t. 598, 602. Reich. ic. fl. germ. 20. t. 50. f. 2.

$\beta$ pinnatifida, foliis inciso-dentatis ad summum pinnatifidis.

Scrofularia pinnatifida Guss. fl. sic. prodr. suppl.

Stazione, Abitazione e Fioritura. - Nei luoghi aridi sassosi, nelle ghiaie, nei lnoghi arenosi ecc., per tutta l'Italia in pianura $\mathrm{e}$ in monte, e generalmente comune. In Piemonte ovunque (All.): Susa (Bucci! ecc.), Torino (Re), Mondovi (Ing.), Riva di Sesia (Ca. restia!), Novara (Bir.) ecc.; abbonda nel Cantone Ticino presso Mendrisio, Lugano, Locarno e Bellinzona, e nel Comasco (Com.), manca in val Tellina; nasce nel Milanese presso Cassano d'Adda (Rampoldi!), nel Pavese (Nocc. Balb.), nel Bergamasco (Rota), nel Bresciano, frequentissima (Zersi), e dove sale sino a 1500-1600 metri nei monti Gerle e Paio (Parl.!); in Tirolo fa nelle parti meridionali, presso Nago e Torbole (Hausm.), a Trento (Perini!), in val Sugana presso Borgo e Castel Tesino, in Fiemme e Fassa, in val di Non presso Denno e la Rocchetta, a Salorno, a piè del Seiser (Bert., Hausm.); trovasi in tutto il Veneto (De Vis. Sacc.) : Verona (Bracht!), Mantova (Barbieri!), Euganei (Trev.), Chioggia (Bert.), Venezia (Kellner!), Bassano (Bert.), Friuli, ovunque (Pir.!); in Istria è comune (Bert., Freyn, Smith); trovasi nell'Appennino parmense (Parl.!), è comune nel Modenese (Gib. Pir.), nel Bolognese pure (Cocc.) e fino alla Porretta (G. Bert.), e trovasi a Faenza (Cald.). In Liguria è stata trovata a Mentone (Ard.), Diano, Cervo (Ricca!), Genova (Bivona !), Chiavari (Delpino!); è piuttosto comune per tutta la Toscana, dalla regione maremmana alla montana, e fa nell' Elba!; nelle Marche è stata trovata a S. Marino, Senigallia, Sassoferrato (Bert.), nei monti Catria (Parl.!) e S. Vicino (Buccil), sotto Colfiorito!, a Fermo (Bert.), nell'Ascolano (Parl.!), nei monti Sibilla (Marzialetti!) e Vettore (Parl.!); in Abruzzo sul Corno (Orsini!), nei monti di Caramanico (Ces.) e sul Velino (Cherici!). Nasce nei monti a levante e scirocco di Roma (Rolli! ecc.); in Campania da Terracina (Bert.) al Matese (Terr.!) e a Napoli (Heldreich! ecc.), in Ischia (Guss.) e in Capri (Kesselmeyer! ecc.); a Melfi (Terr.); in Calabria a Cosenza (Aiuti!), Reggio! ecc. In Corsica è indicata a Calvi (Bert.), Corte, Vivario (Mars.); in Sardegna come non rara (Mor.); in Sicilia ovunque (Guss.), da Mlessina! a Palermo (Parl.! ecc.), sull'Etna (Bi- 
vona!), a Caltagirone (Tar. Gerb.), a Licata (Huet!). La var. è propria dell'isola di Pantellaria (Guss., Caleara!). Secondo i luoghii fiorisce da aprile, maggio o gingno alla fine dell'estate.

Distribuzione geografica. - Nell'Europa meridionale e media, nell'Affrica settentriunale, nell'Asia Minore.

Descrizione. - Pianta perenne, alta da 3 decimetri a un metro e più, verde, glabra, fornita di un rizoma grosso, che manda molti fusti i quali fanno un cespuglio. I fusti sono solidi, eretti, flessuosi, cilindrici o un po'angolati, verdi, glabri, semplici o con pochi rami verso il mezzo, i quali sono eretti o eretto-patenti. Le foglie sono appena carnosette, alterne, patenti, un po' lontane tra loro, pennatipartite, con le lacinie disuguali, inciso-dentate, con i denti rari e disuguali, verdi e quasi lucenti di sopra ed ivi con leggieri solchi che corrispondono ai nervi di sotto, di un verde pallido di sotto ed ivi con un nervo longitudinale rilevato in ciascuna lacinia da cui partono pochi nervetti laterali, poco rilevati che si diramano unendosi tra loro e terminando ai denti: sono fornite di un picciỏlo corto, scanalato di sopra, convesso di sotto, verdognolo, strettamente alato per la scorrenza della lamina della foglia. I fiori sono moltissimi, piccoli, in piccole cime patenti, formanti ura pannocchia lunga : $\mathrm{i}$ peduncoli sono divaricati ed angolati, i pedicelli quasi uguali o poco piủ corti del calice e cilindrici, tutti glandolosi, con piccole glandole portate all'apice di cortissimi peli: sono accompagnati di brattee e di brattèole, piccole, corte, lineari, ottusette, verdi. Il calice è verde, glabro, diviso fin sotto la metà in cinque lobi ovali-orbicolari, convessi nel dorso e largamente scagliosi e bianchicci lacero-frangiati nel margine e nell'apice. La corolla è piccola, di colore rosso-scuro con i margini dei lobi delle labbra di colore bianchiccio, glabra. Il tubo è quasi tondo, il lembo è bilabiato, piủ corto del tubo. Il labbro superiore è bilobo con i lobi un po' rivolti in su, quasi orbicolari, eretti nel margine, cuoprentisi in parte l'un l'altro: il labbro inferiore è poco più corto del superiore, trilobo, i lobi laterali sono un po' piủ larghi, eretti, quasi forniti di una orecchietta verso il labbro superiore, l'inferiore è quasi orbicolare, patente reflesso. Gli stami fertili son quattro, inseriti alla base del tubo della corolla, quasi uguali, tutti e quattro avricinati al lobo medio del labbro inferiore. I filamenti sono un po'curvi, cilindrici, peloso-glandolosi sino in alto, bianchi. Le antere sono quasi ovali, violetto-seurissime, uniloculari, aprentesi per una fessura trasversale, glabre. Il quinto stame è inse. rito nel mezzo del tubo sotto il labbro superiore, ed è ridotto a un 
filamento lesiniforme, diritto, puberulo-glandoloso verso il mezzo. Il pistillo è poco piủ lungo degli stami, avvicinato al lobo medio del labbro inferiore ed eserto come gli stami. L'ovario è piccolo, ovatotondo, verdognolo, glabro, circondato alla base da un disco circolare, più grosso in dietro a verdognolo. Lo stilo è un po'curvo, filiforme, un po' assottigliato in alto, bianchiccio e glabro. Lo stimma è ottuso e papilloso. (Parl. ms., descr. di pianta di Boscolungo nell’Appennino toscano).

\section{Scrofularia Mop'pei.}

S. glabra, caule obtusangulo, foliis pinnatisectis segmentis partitis, dichasiis pedunculatis inflorescentiam aphyllam efformantibus, pedicello calyce sublongiore, lobis calycinis rotundatis, albo-marginatis, staminibus exsertis, staminodio subulato vel nullo.

Scrofularia Hoppei Koch in Röhl. Deutschl. fl. 4. p. 410. Bert. fl. ital. 6. p. 397. Hausm. fl. Tir. p. 628. Rota prosp. fl. Berg. p. 65. Pir.! fl. for. syll. p. 103. Ard. fl. Alpes-mar. p. 278. De Vis. Sacc. cat. piant. Ven. p. 150. Zersi prosp. piant. Bresc. p. 154. Ces. el. piant. Maiell. p. 22. Goir.! pl. vasc. veron. p. 31. Cies. Pass. Gib. comp. fl. ital. p. 342. Terr. quart, rel. Terr. Lav. p. 110. Arc. comp. Al. ital. p. 507.

Scrofularia carina $\gamma, \delta$ Wydl. ess. mon. p. 44, 45.

Figure.-Guss. pl. rar. t. 45. Reich. ic. fl.germ. 20.t. 50.f. 3.

Stazione, Abitazione e Fioritura. - In luoghi sassosi dei monti della Penisola: Alpi marittime al col di Tenda e in val di Pesio (Ard.); Cenisio (Bert.); Tirolo in Pusteria presso Welsberg, nell'alpi Seiser, Schlern (Koch) e Rosszähne, e giủ presso Bolzano, in val di Non alla foce della Novella, nei monti Spinale (Hausm.) e Gazza (Perini!), nelle Giudicarie in val di Bolbeno (Hausm.); valli Brembana e Ca. monica (Rota), val Trompia sui monti Cornablacca, Paio, Ario (Zersi); Baldo all'Acque negre, sopra Brentonico (Hausm.), a Malga Canaletti (Kerner!), all' Artillonzin ecc. (Goir.!); Lessini, d’onde scende talvolta al piano (Goir.); Friuli nelle valli del Natisone (Pir.!) e della Rasia (Pir.); Istria presso Fiume (Koch); Appennino picenoabruzzese a Capo di Tenna, alla Sibilla, al Vettore, alla Corona, al monte de' Fiori (Bert.), al Corno (Orsini!), al Velino, al Morrone (Bert.), alla Maiella (Levier!); Campania al monte Meta (Terr., Levier!). Fiorisce in giugno, luglio, agosto. 
Distribuzione geografica. - Nell'Europa meridionale e in parte nella media.

Osservazioni. - Questa specie distinguesi inoltre dalla prece. dente per una maggiore quantità di peli glandolosi nell' infiorescenza, per i frutti generalmente più grossi, ma soprattutto per i fiori più grandi, con la corolla che arriva sino a 7 millimetri (mentre nella Scrofularia canina varia da 2 a 4 millinetri), coi lobi posteriori più grandi relativamente al tubo. Peraltro sono tutte differenze poco sicure, ed occorrono nuovi studi sulle piante vive per sincerarsi se la $S$. Hoppei sia altro che una varietà della $S$. canina.

\section{Serofulnria ramosissimn.}

S. glabra, caule obtusangulo, foliis dentatis, inferioribus partitis sectisque, dichasiis pedunculatis inflorescentiam aphyllam efforInantibus, pedicello calyce sublongiore, lobis calycinis rotundatis, albo-marginatis, staminibus exsertis, staminodio subulato vel nullo.

Scrofularia ramosissima Lois. fl. gall. p. 381. Bert. A. ital. 6 . p. 399. De Not. rep. fl. lig. p. 296. Gren. Godr. fl. Fr. 2. p. 568. Mor.! fl. sard. 3. p. 192. Ard. fl. Alpes-mar. p. 279. Genn.! fl. Capr. p. 114. Mars. cat.pl. Cors. p. 106. Ces. Pass. Cib. comp. fl. ital. p. 342. Arc. comp. fl. ital. p. 508.

Figura. - Reich. ic. fl. germ. 20. t. 54. f. 2.

Stazione, Abitazione e Fioritura. - Presso Nizza al ponte del Varo (Durando!), in luoghi incolti al ponte di Magnan (Canut!) e aila punta di Carras (Ard.) dove adesso è probabilmente distrutta. ${ }^{2}$ In Corsica nelle arene del littorale e in luoghi aridi dell'interno: Bastia (Mabille ]), capo Corso(Mars.), Galeria (Bert.), Aiaccio (Soleirol! ecc.), Bonifacio (Kralik!), Portovecchio (Mars.), Niolo (Reyuien!), Apricciani, la Soccia, Evisa, Cristinacce, Marignana (Mars.), Casamiccioli a piì di 2000$)^{\prime}$ (Gren. Godr.). In Sardegna nelle arene marittime: Maddalena (Genn.!), Tavolara, Orosei (Mor.), Piscinas (Asclierson!), Fluminimaior, Terralba (Mor.), Oristano (Bonjean! ecc.), Cabras (Mor.). Fiorisce in aprile e maggio.

Distribuzione geografica. - Oltraccio in Provenza, e in Tunisia (Kralik!).

\footnotetext{
'Dubitu che cun questi vari nomi s' indichi una localltà sola.
} 


\section{CELSIA.}

Celsia Linn. gen. plant. p. 186. Gen. pl. fl. germ. 5. t. 28. Benth. Hook. gen. pl. 2. p. 929. Ces. Pass. Gib. comp. fl. ital. p. 341. t. 52. f. 3 .

Calyx patulus, כ๊-partitus. Corolla rotata, limbo subæqualiter 5-partito. Stamina 4. Antheræ uniloculares. Stigma minusculum. Gemmulæ in loculis plurimæ, coacervatæ. Septifragium polyspermum.

Portamento. - È tale quale di un Verbascum.

\section{Celsia cretica.}

Celsia cretica Linn. syst. veg. ed. 13. p. 470. Linn. fil. suppl. plant. p. 281. Zer. fl. mel. thes. p. 11. Bert. fl. ital. 6. p. 401. Grech Del. fl. mel. p. 28. Mor.! fl. sard. 3. p. 184. Ces. Pass. Gib. comp. fl. ital. p. 341. Arc. comp. fl. ital. p. 506.

Figura. - Bot. mag. t. 964 .

Stazione, Abitazione e Fioritura. - In Sardegna, nei campi e nelle colline, principalmente al mare: Alghero, Sarrabus, S. Antioco, isola S. Pietro, Monastir (Mor.), capo S. Elia (Gennari!). In Sicilia, in luoghi erbosi montuosi: Palermo in parecchi siti (Parlatore! ecc.), da monte Pellegrino a Corleone e ad Amorosa, Cefalù, Cofani, Sciacca, Montallegro, Girgenti, Riesi, Caltanissetta (Guss.), Sponti (Bert.). Nei campi erbosi della Calabria orientale al. Bianco nuovo (Guss.). In Malta (Zer., Grech). Fiorisce in aprile e maggio.

Distribuzione geografica. - Nella Spagna meridionale, nell'Affrica settentrionale, nell' Italia meridionale, in Candia.

\section{VEIEBASCUM.}

Verbascum et Blattaria Tourn. inst. p. 146, 147. t. 61. Verbascum Linn. gen. plant. p. 153. Schrad. monogr. 1813, 1823. Gen. pl. fl. germ. 5. t. 27. Benth. Hook. gen. pl. 2. p. 928. Ces. Pass. Gib. comp. fl. ital. p. 338. 1. 51. f. 1.

Calyx erectus, 5-partitus. Corolla rotata, limbo subæqualiter 5--partito. Stamina 5 , sæpe dimorpha, nonnulla interdum atrophica. 
Antheræ 1-loculares. Stigma capitato-bilobum. Gemmulæ in loculis plurimæ, coacervatæ. Septifragium polyspermum.

Portamento. - Sono erbe, per lo più bisannue, e di alta statura, giacchè da $1 / 2$ metro alzano fino a 2 metri, pelose: di rado i peli sono scarsi, e semplici, sono quasi sempre stellati e in tal quantità da costituire alla pianta un rivestimento fitto e morbido, che poi col tempo facilmente si distacca a fiocchi. Le foglie sono di due sorte: le basilari grandi, ovali o bislunghe, sessili o picciuolate, crenate, di rado lobate, e le cauline gradatamente più piccole, sessili o scor. renti sul fusto. Questo finisce in una infiorescenza assai grande, a pannocchia, 0 a grappolo composto o semplice, fitta o rada, di fiori grandi, pedicellati o quasi sessili, gialli, di rado rossi, e soltanto casualmente bianchi, con un fiocco di stami barbuti, e con la corolla aperta e assai caduca.

Osservazioni.- Questo genere è imbrogliatissimo per la ricognizione delle specie negli autori. Ció si deve in parte ad un certo grado di polimorfismo, che però non è tale da impedire la costituzione di buone specie sopra caratteri stabili; ma in grandissima parte si deve alla frequenza degli ibridi, che spesso non riconosciuti per tali sono stati dati per specie o varietà. Da tutto ciò una grandissima confusione nella sinonimia, e la esistenza di nomi, che sono veri enimmi. Ad alcuni hanno accennato gli autori del primo Compendio della flora italiana; altri potranno forse essere chiariti da floristi locali, i più atti la mercè della loro minuta cognizione dei luoghi e dei documenti relativi, a sciogliere simili problemi.

Gl'ibridi si riconoscono alla loro sterilità. Koch ne lıa descritı buon numero nella sua Synopsis; Franchet ed altri pure in vari vo. lumi del Bullet. de la soc. bot. de France; e Wirtgen ne ha pubblicato saggi seccati.

" Stamina postica anthera reniformi, antica 2 anthera hinc in filamento decurrente.

\section{Verbascum Thapsus.}

V. dense tomentosum tomento lutescenti vel albido, caule subsimplici, foliis crenulatis, caulinis oblongis ovalibusve, longe atte. nuato-decurrentibus, racemo denso, floribus luteis raro albis, corolla limbo campanulato, staminibus posticis filamento albo-barbato, 2 anticis subglabro anthera brevissime decurrente, fructu ovato-globoso, 1 centun. longo. 
Verbascum Thapsus Linn. sp. plant. ed. 1.p. 177. Bert. fl. ital. 2. p. 570; et auct.

Verbascum Schraderi Trev. prosp. fl. eug. p. 24. Grech Del. fl. mel. p. 28. Hausm. fl. Tir. p 622.

Figure. - Fl. dan. t. 631. Sav. mat. med. veg. tosc. t. $5 \%$. Reich. ic. fl. germ. 20.t. 16.

Stazione, Abitazione e Fioritura. - Per tutta l'Italia nei terreni incolti, lungo le strade, nei campi ecc. $\grave{\mathrm{E}}$ comune in Piemonte (All.): Torino, dove al contrario è dichiarato raro, Susa (Re), Mondovi (Ing.), Alba (Bert.), Novara (Bir.); nasee nel Cantone Ticino specialmente a Bellinzona, è piuttosto raro nelle valli Intelvi, Sassina, di Mandello, intorno a Lecco (Com.), nasce anche in val Tellina (Mass., Com.), per esempio a S. Caterina a 1700 metri (Parl.!); altrove in Lombardia a Pavia (Nocc. Balb., Rota), Bergamo (Rota), Brescia, frequente (Zersi); in Tirolo: valle di Laas, Klobenstein, Gröden, Terlago (Hausm.); nel Veneto, parcamente (De Vis. Sacc.): Montalon, Sette Comuni, Portole, Grappa (Montini!), Verona (Bert.), monte Baldo, comunissino (Goiran!), Euganei (Trev.), Venezia (Bert. ecc.), Friuli superiore e Carnia (Pir.); in Romagna ed Emilia: Faenza (Cald.), Bologna (Cocc.) e Modena (Gib. Pir.), e Appennino sovrastante in molti luoghi delle regioni submontana e montana (G. Bert. ecc.), Parma (Pass.!). In Liguria è più comune in monte che in basso (De Not.): al col di Tenda (Ungern-Sternberg!), nel Nizzardo qua e là (Ard.), a Cervo (Ricca!); in Toscana è sparso dalla regione maremmana alla montana: presso Massa (Bert.), in Versilia (Sim.), nel Lucchese (Pucc.), nel Pisano dov'è comunissimo (Savi), a Firenze!, a Vallombrosa (Borzi!), in Casentino o Prataglia (Parl.!), nel Senese (Tass, ecc.), nel monte Amiata, in val di Fiora (Sant.), nelle isole di Gorgona (P. Savi) e di Capraia (Mor. De Not.); nelle Marche è stato trovato al monte S. Vicino (Bucci!), ad Ascoli!, in Abruzzo a Caramanico (Ces., Levier!). Più giù trovasi nei colli Albani (Bert.), a Caserta (Terr.), Napoli (Bert. ecc.), S. Angelo di Castellammare (Pasq.!), in Ischia (Guss.) e in Capri (Pasq.), infine nel Vulture (Terr.). Nasce in Corsica a Bastia e a Campo di Loro (Mars.); nella Sardegna centrale (Mor.!); in Sicilia a Palermo, nelle Madonie (Parl.!), a Messina, Spadafora, Catania, Milo (Guss.); in Malta (Zer., Grech). Fiorisce da maggio e giugno in là.

Distribuzione gecgrafica. - Nell' Europa media e meridionale, nell'Asia media, introdotto nell' America settentrionale.

Osservazioni. - Regna molta incertezza riguardo alle citazioni 
di autori e di localitá per questa specie, con la quale facilmente sono state scambiate le seguenti affini.

$\dot{\mathrm{E}}$ difficile sapere cosa ahbia inteso Tenore con le varietà del suo Verbascum Thapsus nella Syll. p. 105.

La pianta di Sicilia ha secondo Gussone, che la chiamerebbe più volentieri $V$. neglectum (Syn. 1. p. 262), i fiori assai più piccoli.

Bentham (in Cand.prodr. 10. p. 226) e i Compendi riferiscono a questa specie il V. plantagineum Mor. (Bert. o. c. p. 579. Mor. stirp. sard. el. 1. p. 33. Fl. sard. 3. p. 179) di Cála d'Ostia, Pula e Teulada in Sardegna, che ha pure i fiori più piccoli, e crocea la barba del filamento degli stami posteriori.

\section{Verbaseum montanum.}

V. dense tomentosum tomento lutescenti, caule subsimplici, foliis crenulatis, caulinis oblongis ovalibusve, breviter attenuato-decurrentibus, racemo denso, floribus luteis raro albis, corolla limbo carnpanulato, staminibus posticis filamento albo-barbato, 2 anticis subglabro anthera brevissime decurrente, fructu ovato-globoso, 1 centim. longo.

Verbascun montanum Schrad. hort. gott. Mass. prodr. fl. valt. p. 197. Berl. R. ital. 2. p. 578 (excl. syn. Mich.). De Not. rep. fl. lig. p. 243. Rota prosp. piant. Pav. p. 269. Prosp. fl. Berg. p. 65. Hausm. R. Tir. p. 623. Mor. A. sard. 3. p. 179. Car. prodr. A. tosc. p. 473 (excl. syn. Sim el .lich.). G. Bert. veg. porr. p. 83. Ard.! n. Alpes-mar. p. 273. Zer'si prosp. piant. Bresc. p. 153. Terr. sec. rel. Terr. Lav. p. 91. Archb. fl. Alı.-Serch. p. 57. Ces. Pass. Gib. comp. A. ital. p. 338. Anzi auct. 11. nov.-com. p. 195. Gib. Pir. 11. Moll p 119. Arc. comp 1t. ital. p. 503. Cocc. 1. Bol. p. 355. ljib. l'ir. suppl. fl. Hod. p. 22.

Verbascum phlornoides Mor. De Nol.? Il. capr.p. 97 (sec. Mor.).

Figura. - Retch. ic. fl. germ. 20.1.21.

Stazione, Abitazione e Fioritura. - In lunghi incolti, o boschivi ecc. dei monti della Penisola settentrionale: alpe Seiser in Tirolo, rarissimo, Bormio, nou raro (Hausm.), Carnate in val Tellina (Mass.), valle lutelvi presso Lainu, e Pellio (Conı.), val Sassina (Anzi), valli Caleppio e S. Martino nel Bergamasco (Roti), monte Dragone verso Serle nel Bresciano, raro (Zersi), Riva di Sesia (Ca. restia!), Cenisio, Bagni di Valdieri (Bert.), col di Tenda (Bourgean!), 
alpi di Garezzo, Triora e Frontero (De Not.), e S. Stefano d'Aveto (Bert.) in Liguria, Godiasco e Casale di Stafora nel Pavese (Rota), Appennino settentrionale a Torraqua presso Fiumalbo, sopra Barigazzo (Gib. Pir.), alla Porretta, all'Acero (G. Bert.), al Crocicchio, a Boscolungo (Parl.!), ai Bagni di Lucca (Archb.), alpi Apuane al monte Bruciana, tra Fornole e Rascetto, nella Tambura, e al Giovo sopra Vinca (Bert.). Quindi si ritrova in Campania nei monti di Pescosolido (Terr.), in Sardegna (Mor.), e nell' isola di Capraia (Mor. De Not.), quest'ultima località poco confacente invero alla pianta. Fiorisce in giugno, luglio ed agosto.

Distribuzione geografica. - Nell'Europa media-meridionale.

Osservazioni. - Specie ben poco distinguibile dalla precedente.

Gibelli e Pirotta $l$. $c$. indicano un Verbascum montano $\times$ Lychnitis raccolto alla Rocca di Fiumalbo.

\section{Verbascum thapsiforne.}

V. dense tomentosum tomento lutescenti vel albido, caule subsimplici, foliis crenulatis, caulinis ovatis, longe attenuato-decurrentibus, racemo denso vel laxiusculo, floribus luteis raro albis, corolla limbo explanato, staminibus posticis filamento albo-barbato, 2 anticis subglabro anthera longe decurrente, fructu ovato-globoso, 1 centim. longo.

Verbascum densiflorum Bert.? rar. Ital. plant. dec. 3. p. 52 (1810). Poll. fl. ver. 1. p. 243. Bert. fl. ital. 3. p. 574. Sang. cent. prodr. fl. rom. add. p. 35. Car. prodr. fl. tosc. p. 473. Freyn fl. Süd-Istr. p. 385.

Verbascum thapsiforme Schrad. mon. 1.p. 21 (1813). Mass. prodr. fl. valt. p. 197. Com fl. com.1. p. 295. Sang. l. c. Bert. fl. ital. 2. p. 573. Rota prosp. fl. Berg. p. 65. Hausm. fl. Tir. p. 622. Car. l. c. Ard. 1l. Alpes-mar. p. 272. De Vis. Sacc. cat. piant. Ven. p. 149. Zersi prosp. piant. Bresc. p. 153. Ces. el. piant. Maiell. p. 22. Ces. Pass. Gib. comp. fl. ital. p. 338. Ing. cat. sp. Morid. p. 73. Paol. el. piant. sib. p. 14. Gib. Pir. fl. Mod. p. 119. Arc. comp. Al. ital. p. 503. Cocc. fl. Bol. p. 355.

Verbascum thapsoides Com. prodr. fl. com. p. 40.

Verbascum macrurum Ten.? ad fl. neap. prodr. app. quinta $p .9$. Bert. fl. ital. 3. p. 573. Sang o. c. p. 34. Guss. fl. sic. syn. 1. p. 
262. Enum. pl. Inarim. p. 232. Terr. fl. Vult. syn. p. 127. Rel. Terr. Lav. p. 145. Ces. l. c. Terr. terz. rel. p. 76. Ces. Pass. Gib. o. c. p. 339. Terr. quart. rel. p. 109. Arc. l. c. Nic. prodr. fl. mess. p. 348.

Verbascum Thapsus Trev. prosp. fl. eug. p. 24.

Verbascum montanum Simi! l. alp. vers. p. 44.

Figure. - Schrad. mon. I. I. f. 1. Reich. ic. R. germ. 20. t. 17.

Stazione, Abitazione e Fioritura. - Nei luoghi incolti, o boschivi ecc. di quasi tutta l'Italia. Piemonte: Monpantero presso Susa (Parl.!), Mondovi, verso Vicoforte (Ing.), Alba (Bertero!), Acqui (Ces.), Riva di Sesia (Carestia I), Vercelli (Ces.), Varzi in val di Vedro, isola Madre del lago Maggiore (Bert.). Lombardia: Como, nel torrente Cosio, valle Intelvi presso S. Fedele, e Lanzo (Com.), val Sassina in valle di Varrone (Bert.), val Tellina a Montagna, a Sondrio (Mass.), monte Epolo vicino a Schilpario nel Bergamasco a 1500 metri (Parl.!), monte Conche (Zersi) e Collio a 850 metri (Parl.!) nel Bresciano. Tirolo: Roveredo (Poll.), Tione, val di Fassa, val di Non, Ritten, Bolzano, Eppan, Mals (Hausm.). Veneto: Ospedaletto in val Policella, Recoaro, Schio, raro (Poll.), Euganei ('Trev., De Vis. Sacc.). Istria: Pola, rarissimo (Freyn). Emilia : Pievepelago nel Modenese (Gib. Pir.), Lustrola nel Bolognese (Cocc.). Liguria : Tenda (Ard.). Toscana: llassa al moute Bruciana e verso Porti (Bert.), la Versilia tra Levigliani e la Corchia, nei monti Alto e Gabberi, e nell'alpe di Terrinca (Simi!), Tereglio nel Lucchese (Giannini!). Pi. ceno-Abruzzo: Caramanico (Ces., Pedicino!), lago di Fucino (Ten.). Lazio: Frascati (Bert.), Ariccia ecc., comune (Sang.). Campania: Piedimonte(Terr.), Agnano (Ten.), Ischia (Guss.). Basilicata: Nlelli, Rapolla (Terr.). Calabria: Dirupata di Mlorano, Campotenese (Ten.). Sicilia: Spadafora (Guss.), Messina (Seguenza!), Fiumedinisi ecc. (Nic.), Madonie (Parl. !). Fiorisce da maggio e gingno per l'estate.

Distribuzione geografioa. - Nell' Europa media e meridionale.

Descrizione. - Panta perenne, alta da 6 a 15 decimetri, coperta di un tomento bianchiccio, con peli stellati e alcuni molto lunghi, stellato-ramosi. Il fusto è diritto, semplice, angolato con gli angoli ottusi, alato per lo scorrere delle foglie negli angoli di esso. Le foglie sono molli, le inferiori patentissime o patenti, allungato-ellittiche, ottuse o ottusette, crenulate, ristrette alla base in un pieciolo corto, piano di sopra, conresso di sotto, e alate, suno di un verde hianchiccio di sopra con un nervo longitudinale piano, verdognolo- 
bianchiccio, di un verde ancora più bianchiccio di sotto ed ivi con un nervo longitudinale grosso e bianchiccio da cui partono molti nervi laterali obliqui, un po'curvi, che si dirigono verso il margine e l'apice e si uniscono tra loro ad arco vicino al margine mandando molti nervetti che fanno una rete tulta rilevata nella pagina inferiore della foglia. Le foglie di mezzo del fusto e le superiori sono patenti ed eretto-patenti, alterne, molto vicine tra loro, ovali-lanceolate, acuminate, crenulate, scorrenti alla base da foglia a foglia per tutto l'internodio: sono più tomentose delle inferiori. 1 fiori sono da 2 a 4 , in fascetti e i superiori solitarii all'ascella di una brattea ovata, ottusetta o ottusa, piủ corta dei fiori, glabra di dentro, con un fitto tomento di fuori, formando una spiga molto lunga e per lo piủ continua e fitta. I peduncoli sono corti o cortissimi, sempre molto piủ corti del calice, cilindrici, grossi e tomentosi. II calice è quasi cam. panulato, tomentoso, ha cinque lacinie quasi uguali tra loro e della lunghezza del tubo, patenti, triangolari-lanceolate, acute. La corolla è molto più grande del calice, con il tubo corto e larghetto in alto, è divisa in cinque lobi disuguali, patentissimi, dei quali gl'inferiori sono più grandi, ovali o ovali-tondi, larghi, è di un color giallo pallido e glabra di dentro, di un giallo ancora piủ pallido di fuori ed ivi con peli stellati. Gli stami sono disuguali e più del doppio più corti della corolla. I tre superiori sono un poco più corti, quasi diritti, cilindrici, giallicci e glabri in basso, da sotto la metà all'apice coperti di una lana lunga, gialliccia. Le loro antere sono lunghe, reniformi, inserite trasversalmente sull' apice delicato dei filamenti, uniloculari, aprentisi trasversalmente per il margine superiore oconvesso, gialle, glabre. I due stami inferiori un po' piủ lunghi, ascen. denti, cilindrici, glabri o con qualche raro pelo in basso, giallicci: le loro antere sono simili a quelle degli altri stami ma scorrenti sul filamento in modo che questo è nella parte libera circa una volta e mezzo a due volte più lungo dell' antera. Il pistillo è più lungo degli stami più lunghi e quasi uguale alla corolla. L'ovario è ovato, ottuso, tomentoso, biloculare, con molti ovoli inseriti in una placenta carnosa all'angolo interno di ciascuna loggia. Lo stilo è lungo, molte volte piủ lungo dell'ovario, curvato in giù, cilindrico e con pochi peli stellati in basso, un po'piano da avanti in dietro verso alto, ivi verdognolo e glabro. Lo stimma è scorrente da ambi i lati dello stilo in modo che ha la forma di un V rovesciato. (Parl. ms., descr. della pianta di Collio in Lombardia).

Osservazioni. - Nel caso che, giusta l'opinione di De Visiani 
(Fl. dalm. 2. p. 155) seguita dai Compendi, il Verbascum densiflorum di Bertoloni fosse veramente lo stesso del $V$. thapsiforme di Schrader, allora per ragione di data la specie dovrebbe portare il primo nome. Duolmi non avere potuto riscontrare in proposito i saggi dell'erbario Bertoloni, non accessibili se nou a chi si rechi a Bo. logna.

Tenore descrive il sıo $V$. macrurum con gli stami pressochè uguali, dai filamenti con barba gialliccia. Bertoloni, sopra un saggeio datogli da Tenore, descrive invece due stami molto piu lunghi, con le antere nolto allungate; cosi pure Gussone, che conferma la par. ticolarità della barba gialla I Compendi danno per caratteri differen. ziali dal V. thapsiforme il tomento bianco, e le ali del fusto piu larghe.

Un $V$. thapsiformi < Lychnilis è indicato nella Cosia presso Ciomo, e sul Matese nel Napoletano (Ces. Pass. Gib. o. c. p. 339).

\section{Verbageran plalomeides.}

V. dense tomentosum tomento lutescenti vel allido, caule sub. simplici, foliis crenulatis, caulinis ovatis, basi rotundata vix decur. rentibus, racemo denso rel laxo, floribus luleis raro albis, corolla limbo explanato, staminibus posticis filamento albo-barhato, 2 ans. ticis subglabro anthera longe decurrente, fructu ovato-globoso, 1 centim. fere longo.

Verbascum phlomoides Linn. sp. pl. ed. 2. p. 258 (excl. syn. Till.). Bert. fl. ital. 2. p. 575) (exil. syn nonn), el auct.

Verbascum comdensatum Mass. mrodr. H. vall. p. 19i. Bert. o. c. p. $5 i 7$.

Verbascum montanum Pucc.! syn. pl. luc. p. 116.

$\beta$ anstrale, ranescens.

Verbascum viminale Ginss.? plant. rar. p. 101. Bert. o. c. p. 5s?. C.es. Pass. (jib. comp. /1. ital. p. 939. Are. comp. /l. ial. p. 50 \%.

Verliascum argyrostachyou Ten, rel. riagy. in Abr. p. 59. Syll. p 107. Betl. o. c. p. Sisl. Sing. cent. prodr. 12. rom. add. p. 37. Terr. rel. Terr Lint. p. 1.5.5.

Verbascum australe Guss. /l. sic. syn. 1. p 96?. l'e's. el. piant. Ilaiell p. 92. Nir. prodr. 11. mess. p. 348.

Figure. - Silhrad mon 1. 1. f. ?, 1. ?. . IIofm. Link 1 .

liona itahana - Vin. II. 
port. t. 27. Fl. grec. t. 224. Reich. ic. fl. germ. 20. t. 18. f. 2, t. $19,20$.

Stazione, Abitazione e Fioritura. - Nei coltivati, nei luoghi incolti ecc. per tutta l'Italia. Piemonte: Cenisio (Bert.), Susa (Bucci!), Torino, comụnissimo (Re), monti d'Ormea, comunissimo (Ing.), cosi da Nava a Viosenne e Ciarlino (Ricca!), Arona (Ricca!), val Intrasca (Bert.). Ticino, abbondante (Com.). Lombardia: val Tellina (Mass.), Comasco, abbondante (Com.), Pavia (Bert. ecc.), Cremonese (Parl.!), Bergamasco (Rota), Bresciano, frequentissimo (Zersi), Mantova (Bert.). Tirolo: Giudicarie, Trentino, val Sugana (Hausm.). Veneto tútto, comunissimo: Verona (Bert.), Euganei.(Trev.), Chioggia (Bert.), Venezia (Kellner! ecc.), B-ssanese (Montini!), Fagagna, Ignano (Pir.), Monfalcone (Bert.). Istria: Trieste (Bert.), Pola (Freyn). Emilia: Nodenese (Grb. Pir.), Bolognese, fino all' Acero (P. Savi! ecc.) e alla Porretta (G. Bert.). Romagna: Faenza (Cald.). Liguria: monti Nizzardi, ahbastanza comune (Ard.), e Genovesi, cosi al monte Antola (De Not.). Toscana, nelle regioni montana e submontana, e forse più in basso: la Lunigiana a Sarzana (Bert.) e sopra Pontremoli!, alpi Apuane al Pisanino (Pucc.!) e a Levigliani (Beccari!), Appennino in molti luoghi dal monte Orsaio (Parl.!) al Teso!, monte Amiata (Santi), isole dell'Elba (?) (Marcucci!) e di Montecristo (?) (Taylor!). Lazio: Fumicino (Bert.), Ostia (Sang., Bert.), Rioma (Rolli! ecc.), Frascati (Parl.!), monte Gennaro (Bert.). Campania: Caserta (Terr.). Abruzzo (Bert.): Collebringione, Colli presso Carsoli, Giulianora, Pescara, Morrone (Ten.), monti di Caramanico, Maiellone (Ces.). Molise: Campobasso (Ten.); e più giù: Manfredonia (Guss.!), Castellaneta, Massafra, Taranto (Guss.). Basilicata (Bert.). Calabria: Stilo (Arc.!). Corsica nelle parti montuose calde (Mars.): Bastia (?) (Pucci!), Bonifacio (Bert.). Sardegna (Bert.). Sicilia: Madonie (Parl.!), Messina, Spadafora (Guss.). Fiorisce da maggio, giugno o luglio in poi.

Distribuzione geografica. - Nell'Europa meridionale e media.

Osservazioni. - La var. $\beta$ è una forma meridionale, alla quale si passa gradatamente andando dal nord al sud d'Italia, e che non so distinguere specificamente dal Verbascum plilomoides, nonostante l'aspetto diverso che forse cela buoni caratteri differenziali.

Due saggi autentici del V. viminale di Gussone posseduti dall' Erbario centrale corrispondono poco con la sua descrizione, e pochissimo poi con la figura che egli dà a tav. 21 delle Plant. rar. Tenore (Rel. $p$. 53) ebhe a fare la stessa osservazione. 


\section{Verbs semu longifolimm.}

V. dense tomentosum tomento luteo, caule subsimplici, foliis integris vel denticulatis, caulinis ovatis, sessilibus, racemo denso, vel inferne laxiusculo, floribus luteis, corolla limbo explanato, staminibus posticis filamento e purpurescenti albido-barbato, 2 anticis subglabro anthera longe decurrente, fructu ovato-globoso, 1 centiın. fere- longo.

Verbascum Iongifolium Ten. fl. nap. 1. prodr. p. 16. Bert. 1 . ital. 2. p. 595. Terr. 1. Vult. syn. p. 127. Rel. Terr. Lan. p. 145 Ces. el. piant. Maiella p. 22. Terr. sec. rel. p. 91. Ces. Pass. Gib. comp. fl. ital. p. 339. Terr. quart. rel. p. 103. Arc.! comp. fl. ital. p. 504 .

Figura, - Tell. o. c. t. 21.

Stazione, Abitazione e Fioritura. - Nelle praterie dell'Appennino centrale : nei monti Priore (G. Bert.!) e Vettore!, al Castelluccio, nel monte De'fiori (Parl!!), nei monti Acuto!, Corno (Orsini!), Vc. lino (Rolli!), Custone, S. Franco (Guss.), Maiellone, Amaro, al Prato della Corte (Ces.), nel Morrone a 1500 metri (Levier!), al Piano di cinque miglia (IIuet!), in valle Canctra (Bert.), a Pacentra (''edicino!), nel monte Meta e vicinanze in molti punti (Terr.), nei monti di Filettino (Rolli!). In luoghi distaccati da quel gruppo appennino è indicato al Vulture (Terr.), e al Gargano (Ces. Pass. Gib.). Fiorisce in luglio e agosto.

Distribuzione geografica. - Nasce inoltre in Serhia e in Grecia.

Osservazione. - Specie ben distinta.

\section{Verbaseum Battaria.}

V. subglabrum, caule simplici, rel paniculato-ramoso, foliis dentato-incisis, caulinis cordatis, sessilibus, racemis laxis, floribus luteis fundo purpureis, raro albis, corolla limbo explanato, staminibus posticis filamento purpureo-barbato, 2 snticis subbarbato anthera decurrente, fructu üloloso, $\%$ centiur. longo.

Verhascum Blattaria Linn. sp. plant.ed. 1. p. 178. Bert. Al. ital. 2. p. 586 ; el auct. omn.

Verbascum repandum Giss 17. sic. syn. 1. p. ?6i. Tar. Gerb. 
cat. pl. calat. p. 13. Guss. enum. pl. Inarim. p. 232. Pasq. fl. ves. p. 74. Terr. rel. Terr Lav. p. 146. Nic. prodr. A. mess. p. 349.

Figure. - Matth. ed. Valgr. 1565.p.1151. Reich. ic. fl. germ. 20.t. 32.

$\beta$ micropus, pedicello abbreviato calycem subæquante.

Verbascum virgatum With. bot. arr. Bert. o. c. p. 584 (excl. syn. Guss. el Ten.). Trev. prosp. fl. eug. p. 24. Zan. prosp. fl. ven. p. 25. De Vis. Sacc. cat. piant. Ven. p. 150. Ces. Pass. Gib. comp. fl. ital. p. 339. Cocc. terzo contr. fl. Bol. p. 26. Arc. comp. fl. ital. p. 504. Cocc. f. Bol. p. 355.

Figure. - Huffm. Link fl. porl. t. 28. Reich. o. c. t. 34.

Stazione, Abitazione e Fioritura. - Per i luoghi incolti, nei margini dei campi ecc. di tutta l'Italia, più o meno comune. Nell'Alta Italia abita il piano e i colli: Torino, il Segusino, ovunque (Re), Mondovi (Ing.), il Novarese (Bir.), Pavia (Nocc. Balb.), il Cremonese (Parl.!), il Comasco in diversi punti (Com.), il Bergamasco (Rota), il Bresciano, frequente (Zersi), il lago di Garda, le Giudicarie presso Corè (Hausm.), Trento (Perini!), Zambana, Salorno (Hausm.), Mautova (Bert.), Verona, Vicenza (Poll.), gli Euganei (Trev.), Padova (Poll.), Chioggia (Bert.), Venezia (Nacc. ecc.), il Bassanese (Montini!), il Friuli qua e là (Pir.), l’Istria, non comune (Freyn), cosi a Fiume (Smith), Faenza (Ciald.), il Bolognese, comunissimo (Cocc.), e il Modenese (Gib. Pir.), fino nei monti. In Liguria è assai frequente (De Not.): Nizza, Mentone (Ard.), S. Remo (Panizzi!), Diano (Ricca!), Genova (Carrega!) ecc. In Toscana non è raro, dalla regione maremmana alla montana: Pontremoli (Parl.!), Carrara (Ad. Targioni!), la Versilia bassa (Simi!), Lucca (Calandrini !), Bagni di Lucca (Parl.!), Pisa (Corinaldi!), Firenze!, monte Senario (Bucci!), Scarperia, Prataglia in Casentino (Parl.!), Siena (Tassi), Rapolano (Sommier!), Monte Pescali (Aiuti!), Castellazzara (Sauti), S. Sepolcro (Cherici!), Portoferraio (Marcucci!). A Roma è comunissimo (Seb. Maur.), cosi pure a Terracina (Fiorini!); nel Napoletano trovasi a Caserta (Terr.), sul Vesuvio, in Capri (Pasq.) ed Ischia (Guss.); in Basilicata a Melfi (Terr.). Fa nelle Marche al monte S. Vicino (Paolucci!), a Cupra (Piccinini!), ad Ascoli (Parl.!); in Abruzzo nei monti di Caramanico, e al Maiellone (Ces.); più giù a Barletta (Bruni!), a Otranto (Groves!). In Corsica sale fino a 1200 metri, ovunque, ma in generale poco abbondante: Bastia (Mars.), Calvi (Bert.) In Sardegna è abbastanza frequente (Mor.!). In Sicilia è stato trovato a Palermo, Piana de' greci (Parl.!), Castelbuono 
(Minà!), Messina (Seguenza!), Siracusa (Cassia!), Avola (Bianca!), Vittoria (Aiuti!), Caltagirone (Gerb. Tar.). La var., molto rara, è stata trovata in Piemonte, nel Parmigiano (Ces. Pass. Gib.), nel Bo. lognese sopra Pianoro all'Osteria nuova (Coce.), a Montegalda nel' Vicentino, una volta sóla (Poll., Bert.), a Venezia (Zaı.), a Cam. palto (Bert.), negli Enganei (Trev.), al Catria nelle Marche (Piccinini!), in Sicilia a Sagana (Parl.!). Fiorisce da maggio e giugno a luglio e agosto.

Distribuzione geografica. - Nell' Europa media e meridionale, nell'Asia fino al Cabul e all'Altai, nell' Affrica settentrionale, e poi al Capo, nell' India, nella Nord-America, nel Brasile, probabilmente introdotto.

Descrizione. - Pianta annua, con peli glandolosi nelle parti superiori, verde. La radice è fusiforme, verticıle, fibrosa, di un bianco sudicio. Il fusto è eretto, diritto, cilindrico ma con leggieri angoli derivati da due linee che in ciascuno internodio discendono dalla base delle foglie, verdognolo 0 in parte rossiccio, glabro, vestito di foglie nella metà circa inferiore, fiorifero nel resto, semplice o con pochi rami corti ed eretto-patenti. Le foglie sono sparse, le radicali sono in rosetta, bislunghe, ottuse, dentato-sinuate, ristrette alquanto alla base in un corto picciỏlo, le altre sparse, arvicinate tra loro, patenti, di esse le inferiori sono simili alle radicali, ma con un picciólo ancora piu corto, le superiori sono cordato-bislunghe, acute, abbrac. cianti il fusto, le une e le altre sono crenato-dentate nei marcrini con i denti radi e grandetti : tutte sono verdi di sopra ed ivi lucenti e con leggieri solchi bianchicci clie corrispondono ai nervi di sotto, di un verde pallido di sotto ed ivi con un nervo longitudinale bian. chiccio e rilevato da cui partono dei nervetti laterali rilevati e curvi che si diramano e si uniscono tra loro ad arco verso il margine. I fiori sono molti, solitarii, distribuiti in un lungo racemo terminale e rado: sono un po'cernui all'apice di pedicelli, i quali sono quasi uguali al fore, patenti, verdognoli o rossicci, forniti di peli glandolosi all'apice come la rachide, tali pedicelli sono meno aperti nel frutto, del quale sono più del doppio piủ lunghi Le brattee sono lanceolato-lnneari, acute, patenti, molto più corte dei pedicelli, verdi, peloso-glandolose. Il calice è verde, e con i peli glandolosi, diviso profondamente in cinque lacinie bislung-lineari, oltuse, patenti e reflesse nella metà superiore. La corolla è piu del doppio piủ lunga del calice, quasi piana, un po concava in avanti, il suo tubo è cortissimo, stretto, il lembo é diviso profondanente in cinque lobi di- 
suguali, dei quali i due superiori sono più piccoli, i due laterali un po'più grandi e l'inferiore anche più grande, tutti sono quasi orbicolari e gli inferiori un po' tondeggianti nel margine: è di colore giallo chiaro con una macchietta amarante alla base di ciascuno dei due lobi superiori verso la gola. Gli stami sono cinque, inseriti nel tubo della corolla, disuguali, però piủ corti della corolla. I filamenti sono fin verso alto barbati per lunghi peli clavati all' apice; questi come i filamenti sono di colore amarante. Le antere sono uniloculari, glabre, gialle. Il pistillo è quasi uguale agli stami piủ lunghi. L'ovario è ovato, di un verde bianchiccio, glandoloso. Lo stilo è lungo, appena curvo, un po'più grosso in alto, verdognolo alla base ed ivi con qualche pelo glandoloso, glabro e amarante nel resto. Lo stimma è quasi in capolino, verdognolo; papilloso. La cassula è quasi tonda, scura, duretta, più lunga delle lacinie del calice persistente, si apre in due valve nel setto. I semi sono molti, piccolissimi, neri, bislungo-quadrati, con grossi tubercoli. (Parl. ms., descr. di pianta coltivata).

Osservazioni. - Io ho lungamente esitato a congiungere il Verbascum virgatum al $V$. Blattario come varietà. Ni ci sono determinato per due considerazioni: $1^{\text {a }}$, che le due forme non differiscono per alcun altro carattere fuorchè la diversa lunghezza dei pedicelli, di $1-11 / 2$ centimetri nel $V$. Blattaria, di $3-9$ millimetri nel $V$. virgatum; 2a, che in un medesimo saggio di $V$. virgatum ne può variare la lunghezza dal semplice al doppio, e i saggi che li hanno più lunghi (7-9 millimetri) troppo si accostano ai saggi di $V$. Blattaria che li hanno più corti ( 1 centimetro) perchè la separazione riesca altro che artificiale. Osserverò poi che tutti i saggi di $V$. virgatum, tanto d'Italia che di fuori, da me esaminati, avevanovani i semi.

Freyn descrive nella $\mathrm{Fl}$. von Süd-Istrien p. 386 un suo V. geminatum di Pola, ibrido del V. Blattaria e del V. sinuatum.

** Stamina omnia anthera reniformi.

\section{ซ. Verbascum niveum.}

V. dense tomentosum tomento albido vel lutescenti, caule subsimplici, foliis crenatis, caulinis ovatis cordatisve, breviter decurrentibus, racemo denso, floribus luteis raro albis, corolla limbo explanato, staminibus filamento albo-barbato, fructu ovato-globoso, 1 centim. fere longo. 
Verbascum nireum Ten. fl. nap. 1. prodr. p. 16. Bert. fl. ital. 2. p.580. Sang. cent. prodr. fl. rom. add. p. 36. Ces. Pass. Gib. comp. A. ilal. p. 339. Arc. comp. fl. ilal. p. 50\%.

Figura. - Ten. o. c. t. 22.

Stazione, Abitazione e Fioritura. - Nei prati asciutti montuosi, nelle spiaggie arenose, della Penisola inferiore: Narche a Porto di Civitanuora (Narducci!, Cavanna!), Abruzzo al monte Salviano, al Pizzo della Carata (Bert.), a Pescara e Francavilla (Ten.), S. Polo nel Riomano (Sang.), monte di Spigno in Campania (Terr.!), Terra d'Otranto a Martina, a Ginosa (Ten.), a Otranto (Porta Rigo!, Groves!), Calabria a Cirò (Ten.), alla Dirupata di Norano (Huter, Porta Rigo!) e a Pizzo (Biondi!, Arc.!). Fiorisce in maggio, giugno, luglio.

Distribuzione geografica. - Specie italiana.

\section{Verbascum angustifolium.}

V. dense tomentosum tomento albido vel lutescente, caule subsimplici, foliis crenatis, caulinis ovatis, sessilibus, racemo denso, floribus luteis, corolla limbo explanato, staminibus filamento albobarbato, fructu ovato-globoso,....

Verbascum angustifolium Ten. fl. nap. 1. prodr. suppl. 2. p. 66 . Bert. 1.. ilal. 2. p. 601. Ces. Pass. Gib. comp. A. ital. p. 339. Arc. comp. A. tal.p. 504 .

Verbascum garganicum Ten. viagg. Abr. p. 53. Bert. o. c. p. 602. Ces. Pass. Gib. l. c. Arc. l. c. Groves contr. fl. Terr. d'Olr. p. 64 .

Figura. - Ten. fl. nap. 1. 122.

Stazione, Abitazione e Fioritura. - Nelle arene marittine, nei campi, della Penisola inferiore: Abruzzo a Gulianora (Ten., Orsini!), e all' Osteria di Salino, Gargano a Viesti (Ten.) e a S. Angelo (Ten., Bert.), Otranto (Groves), Calabria a Nicotera (Ten., Bert.), a Givia, a S. Ferdinando, a Corigliano (Bert.). Fırisce iı maggio e ginguo.

Distribuzione geografica. - Specie italiana

Osservazioni. - Erroneamente il Verlascum garganicum è stato riferito da Benthan (in Cand. prodr. 10. 1. . 292) al V. simplex d'Orienté, ch’̀ tutt’altra pianta.

Tenore (Fl. nap. 3. p. 2/ i) a ragione insiste sul caraltere delle 
foglie sessili e non semiscorrenti per distinguere il V. garganicum dal $V$. niveum, giacchè quello è difatti l'unico carattere differenziale.

\section{\$. Verbascum conocarpun.}

V. densiuscule tomentosum tomento lutescente, caule subsimplici, foliis crenatis, caulinis ovatis cordatisve, sessilibus, racemo laxo, floribus luteis fundo purpureis, corolla limbo explanato, staminibus filamento purpureo-barbato, fructu orato-acuminato, plus quam 1 centim. longo.

Verbascum conocarpum Mlor. stirp. sard. el. 2. p. 7. Bert. fl. ilal. 2. p. 603. Mor. 1. sard. 3. p. 180. Genn.! R. Capr. p. 114. Ces. Pass. Gib. comp. fl. ital. p. 340. Arc. comp. fl. ital. p. 505.

Stazione, Abitazione e Fioritura. - In Sardegna, abbastanza frequente, in luoghi rocciosi marittimi a Bosa, dell' isola S. Pietro (Mor.), e delle isole Maddalena e Caprera (Genn.!), e in luoghi montuosi di Oliena, Genargentu, Fonni e Tonara (Mor.). Fiorisce da maggio a luglio.

Distribuzione geografica. - Specie sarda.

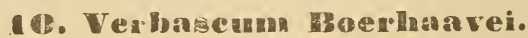

V. flocoso-tomentosum tomento albido, caule subsimplici, foliis crenatis, inferioribus interdum basi incisis, canlinis ovatis cordatisve, sessilibus, racemo laxo, floribus luteis, corolla limbo explanato, staminibus filamento purpureo-barbato, fructu ovato, 1 centim. longo.

Verbascum Boerhaavei Linn. mant. p. 45. Gren. Godr. fl. Fr. 2. p. 550. Ard.cat. pl. Ment.p. 26. Fl. Alpes-mar. p. 273. Mars.! cat. pl. Cors. p. 105. Ces. Pass. Gib. comp. 1. ital. p. 340. Arc. comp. fl. ital. p. 505.

Verbascum rotundifolium Ten. 1. nap. 1. prodr. suppl. 2. p. 66 . Bert. fl. ital. 2. p. 600. Guss.! fl. sic. syn. 1. p. 263. Pasq.! fl. ves. p. 74 .

Verbascum bicolor Bad. osserv. Bert. o. c. p. 599. De Not. rep. fl. lig. p. 294. Ces. Pass. Gib. l. c. Arc. l. c.

Figure. - Mill. abh. planz. t. 268. Ten. o. c. t. 23. Reich:ic. f. germ. 20. t. 33. 
Stazione, Abitazione e Ficritura. - Qua e là nell'Italia media e hassa: Nizza (Durando!) e Mentone, dov'è abbastanza comune ne' luoghi aridi sassosi (Ard.), Diano Cerro (Ricca!), Genova (Cald.!), Sestri di levante (Bert.), e in generale comune per la Liguria più meridionale (De Not.); il. Vesuvio nei monti Somma, e Vetrana, a S. Vito e Tironi di Torre del Greco (Pasq.), monti di Castellammare (Ten., Bert.) e di Cava, colline Amalfitane, Sorrento, Massa (Ten.) e Capri (Ten., Bert., Pasq.) nei campi (Ten.); monte Alpi in Basilicata a 1500-1700 metri, Pollino in Calabria (IIuter Porta Rigo!); pascoli montuosi di Sicilia a Busambra, le Madonie, il monte de'Cani sopra Altavilla (Guss.), il monte di Camarata (Bert.); Bonifacio, la Trinità (Mars.), S. Lucia (Mabille!), S. Fiorenzo (Soleirol!), Bastia dov'è coınunissimo, il liapo (Mars.). Fiorisce in maggio e giugno.

Distribuzione geografica. - Nella Spagna e la Francia meridionali, e in Italia.

Osservazione. - De Notaris fece già rilevare il polimorfismo di questa pianta per rispetto alle foglie inferiori, e alla lana più o meno facilmente asportabile, per cui non reggono le differenze fra il Verbascum bicolor e il V. Boerhaavei.

\section{Verbuscum sinuatum.}

V. dense tomentosum tomento lutescenti vel albido, caule paniculato-ramoso ramis brachiatis, foliis sinuato-lobatis, caulinis dentatis, ovatis, breviter decurrentibus, racemis laxissimis virgatis, flo. ribus luteis raro albis, corolla limbo explanato, staminibus filamento purpureo-barbato, fructu ovali, 4 millim. longo.

Verbascmm sinuatum Linn. sp. pl. ed. 1. p. 178. Bert. fl. ilal. 2. p. 583; et auct. omn.

Verbascum Gussonei Tineo! in Guss. 月. sic. syn. 2. p. i9i. Ces. l'ass. Gib. comp. fl. ital. p. 310. Arc. comp. A. ital. p. 505.

Verbascum pulverulentum var. Nic. prodr. fl. mess. p. 3 i9.

Figure. - Mallh. ed. Valgr. 1565, p. 11 18. Fl. grace. 1. 927. Reich. ic. /l. germ. ?0. t. 2i.

Stazione, Abitazione Fioritura. - Nei lnoghi incolti, per lc strade ece. commnissimo per tulta l'Ilalia, eecellochè nclla sua parte settentrionale. Nolla Penisola si stende da Regreio di Cialabria (.Ire.!) per il Vulture (Terr.), Napoli (Parl.! eer.), Capri (Pasq.) ed Ischia (Guss.), la Terra di Lavoro (Terr), Roma (Iebb! ece.), tutta la 
Toscana nelle regioni maremmana e campestre, comprese l'Elba! e la Pianosa (Simonelli!), fino in Liguria a Spezia (Bert.), e da Ge. nova (Ardissone!) a Nizza (Ard.); dall'altro lato della Penisola si stende da Otranto (Groves) per Chieti (Ces.) e Pescara (Kuntze!), Ascoli (Parl.!) e Cupra-marittima (Piccinini!), Porto Civitanuova (Cavanna!), Albacina (Bucci!), sino a Cesena (Bert.), Faenza (Cald.), Imola, Bologna (Cocc.) e Modena (Gib. Pir.) nella destra valle del Po, che risale perfino a Torino secondo Allioni, e nella valle sinistra trovasi a Prescia, ancora frequentissimo (Zersi), a Verona (Poll.), negli Euganei (Trev.), a Padova (Montini!) e nel Lido veneto (Kellner! ecc.), sino nell'Istria meridionale (Freyn) e a Fiume (Smith). In Corsica è pure comunissimo in tutta la regione bassa (Mars.): Bastia (Kralik!), Calvi (Soleirol!) ecc.; in Sardegna ancora comune (Mor.!), e nell'isola Haddalena (Genn.); in Sicilia a Palermo, ovunque (Parl.!), e a Patti (Profeta!), Messina (Seguenza! ecc.), Fiumedinisi (Tineo!), Catania (Tornabene!), Avola (Bianca!), Caltagirone (Tar. Gerb.) ecc., e in Lipari (Guss.), Ustica (Calcara!), le Egadi, Pantellaria e Lampedusa (Guss.); infine nasce in Malta (Tod. Calcara! ecc.). Fiorisce da maggio e giugno per l'estate.

Distribuzione geografica. - In tutta l'Europa meridionale, nell'Affrica settentrionale e le Canarie, nell'Asia occidentale sino in Persia.

Descrizione. - Perenne, alta 5 e più decimetri, verde bianchiccia, vellutata per peli stellati e corti. Fusto eretto, ramoso, con i rami ascendenti-crenati, un po' piủ lunghi del fusto medesimo, cilindrico in basso, un po'angolato in alto per la decorrenza delle foglie, duretto, sulido. Foglie inferiori o radicali grandi, in rosetta, giacenti per terra, lunghe sino a 5 decimetri e mezzo e larghe sino a 12 o 14 centimetri, bislunghe, un po'piu strette in basso, sinua. to-pennatifide, con i lobi o lacinie larghi, tondeggianti, piegati nel margine a onda, e con larghi smerli superficiali: sono di un verde bianchiccio di sopra ed ivi mostrano il nervo principale longitudinale largo, piano e bianchiccio da cui parte un nervo laterale bianchiccio che si dirama per formare tanti leggieri solchi a guisa di rete: sono di un verde più bianchiccio di sotto e ivi maggiormente vellntati per essere i peli stellati più lunghi e piủ fitti; il nerro longitudinale è grosso, molto sporgente, bianchiccio, i nervi laterali sono anch'essi sporgenti e bianchicci e si diramano per formare una rete sporgente nella pagina inferiore della foglia. Tali foglie hanno un corto picciòlo, che è in continuazione con il nervo longitudinale, leggermente sca- 
nalato di sopra, molto convesso di sotto, bianchiccio e vellutato come il nervo anzidetto. Le foglie del fusto sono alterne, eretto-patenti, lanceolate, quasi acute all'apice, smerlate e un po'ondeggianti nel margine, sessili e scorrenti per corto tratto con la base nel fusto. Le foglie dei rami sono patentissime o un po' dirette in giu, in forma di cuore, acute, abbraccianti ma non scorrenti nei rami: queste e quelle del fusto sono del resto simili per i nervi alle foglie inferiori. I fiori

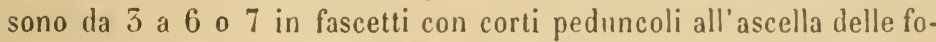
glie dei rami, le quali sono gradatamente piủ piccole da hasso in alto in modo che mentre le inferiori sono piì lunghe dei fiori, le superiori sono piủ corte di esse: ne resulta una grande pannocchia quasi piramidale. I peduncoli sono corti, piủ corti del calice, grossetti, cilindrici, vellutati per molti peli stellati. Calice quasi campanulato, diviso fin poco sotto della metà in cinque lacinic eretto-patenti, larghette, quasi triangolari, acute, verdognolo-bianchicce, come il tubo vellutate per molti peli stellati. Corolla grandicella, rotata, ipogina, che cade subito, con il tubo molto corto, con il lembo o lamina divisa fin sotto il terzo o quasi al quarto inferiore in cinque lacinie patenti, alquanto disuguali essendo le tre superiori un po' più piccole delle inferiori, bislunghe o ovali-obovate, di un giallo piuttosto chiaro. Boccia con le lacinie della corolla embriciate. Stami cinque, quasi uguali tra loro, molto più corti della corolla, inseriti nel tubo di questa. Filamenti eretti, quasi cilindrici, glabri, di color quasi di zafferano nella metà inferiore e all'apice, nel resto dalla parte esterna pelosi con i peli fitti e lunghi di colore amarante. Antere reniformi, inserite nel mezzo della base concava, schiacciate d'avanti in dietro, uniloculari, aprentisi longitudinalmente in tutto il margine. Pistillo quasi uguale aggli stami. Ovario ovato, bianchiccio, vellutato per molti peli fitti e stellati. Stilo molto più lungo dell' ovario, un po' curvo verso alto in su, un po' più grosso in basso ed ivi vellutato-stellato, sottile e quasi cilindrico nel resto, eretto verso l'apice dove è appena più grosso, glabro e verdognolo-bianchiccio. Stimma quasi in capolino, schiacciato da avanti in dietro, scorrente un po nei due lati dell'apice dello stilo, papilloso, giallo. Cassula piccola, quasi uguale al calice persistente le di cui lacinie sono avvicinate ad essa, oroideo-londa, con una piccola punta sporgente all' apice, vellutato-stellata, biloculare-bivalve. (Parl. ms., deser. di pianla di Lirorno).

Osservazioni. - Io riportato qui il Verbascum Gussonei di Tineo, perchè il saggio autentico dell'autore esistente nell' Erbario cen- 
trale è prettamente di $V$. sinuatum. Però giova avvertire che la descrizione da lui data nella Synopsis di Gussone è di altra pianta, piủ prossima al $V$. pulverulentum, al quale difatti Nicotra la riferisce come varietà. Che si tratti di un ibrido fre le due mentovate specie? Se ne danno, come quello descritto da Freyn (Fl. Süd-Istr. p. 385) sotto il nome di $V$. hybridum Brot., e da lui trovato nelle vicinanze di Pola.

\section{Verbascum pulverulentum.}

V. floccoso-tomentosum tomento albido, caule paniculato-ra. moso, foliis crenulatis, caulinis ovatis cordatisve, sessilibus, racemis laxis virgatis, floribus luteis, corolla limbo explanato, staminibus filamento albo-barbato, fructu globoso, 4 millim. longo.

Verbascum pulverulentum Vill. hist. pl. Dauph. 2. p. 490 (1787); et auct.

Verbascum floccosım Waldst. Kit. pl. rar. Hung. 1. p. 81 (1802). Bert. fl. ital. 2. p. 597; et auct. ital. plurim.

Figura. - Reich. ic. fl. germ. 20.t. 26.

Stazione, Abitazione e Fioritura. - Nei luoghi incolti asciutti, nelle ghiaie ecc., per tutta l'Italia. Alta Italia: Torino (Bert.), frequente (Re); val Tellina a Sondrio (Bert.) e Pendolasco (Mass.), Como (Com.!), valli Caleppio ed Imagna nel Bergamasco, e lungo il Brembo (Rota), frequente nel Bresciano (Zersi); il Tirolo tra Bressanone e Chiusa, a Bolzano (Hausm.), a Trento ecc. (Ambrosi!), monte Baldo (Jan!), frequentissimo a Verona, a Vicenza ecc. (Poll.), Mantova (Bert.), Euganei (Trev.), Padova, il Bassanese (Montini!), Venezia (Zan.), Udine (Pir.!), Monfalcone (Bert.), Fiume (Noë!), Istria meridionale (Freyn); e nella destra valle del Po a Mondovi (Ing.), a Godiasco, Varzi e Bubbio nel Pavese (Rota), a Montecchio nel Reggiano (Pass.!), a Porretta e presso Vergato in val di Reno (Cocc.). Manca in tutta la Liguria. Toscana, dalla regione marem. mana alla montana: Sarzana, Carrara (Bert.), Lucca (Pucc.! ecc.), Bagni di Lucca (Parl.!), Cardoso in Garfagnana (Calandrini!), Pisa!, S. Marcello (Parl.!), Firenze (Levier! ecc.), Vallombrosa (Reboul!), Prataglia in Casentino (Parl.!), Rapolano nel Senese, Borgo S. Sepolcro (Bert.), l'Elba a Marciana!. Umbria, Marche, Abruzzo: Gub. bio (Cherici!), Albacina, monte S. Vicino (Bucci!), Acquasanta (Parlatore!), monte Acuto (Bert.), Caramanico (Levier!), monte Coccia 
(Pedicino!), Terni (Bert.). Lazio: Ostia, Marino (Bert.), Castelgan. dolfo (Rolli!), Albano ecc., abbondante (Seb. Maur.). Campania: Cassino, Pietraroia, Caserta (Terr.), Napoli (Ten.). Gargano (Ten.). Melfi (Terr.!). Calabria (Pasq.!). Corsica, nelle regioni bassa e media (Mars.): Corte (Kesselmayer!), Aiaccio a Sartena ecc. (Mars.). Sardegna, non raro (Mor !). Sicilia settentrionale-orientale: Caronia, Floresta, Torrenova (Guss.), Mirto (Todaro!), S. Piero di Patti, monte Scuderi (Seguenza!), Acquedolci, Messina, Itala, Nicolosi, Milo (Guss.). Fiorisce da maggio per l'estate.

Distribuzione geografica. - Nell'Europa media e meridionale.

Descrizione. - Il calice è tubuloso, quinquelobo con i lobi stretti, lanceolato-lineari, alquanto acuti, è verde ma in parte con una pubescenza floccosa che lo fa biancheggiare come le foglie. La co. rolla $\dot{e}$ gialla, rotata, con un tubo cortissimo e quasi nullo, con il lenibo aperto, quinquefido; le lacinie sono obovate, ottusissime, un poco concave, pubescenti-floccose di sotto rerso il tubo, glabre nel resto, di sopra verso la gola offrono alcune righe più scure, cioè tre per ciascuna lacinia, la mediana delle quali è un poco piủ lunga delle taterali. Gli stami sono inseriti sulla gola, sono più corti della corolla e carenati. I filamenti da poco sopra la hase fin sopra la metả sono coperti di lunghi peli bianchi che li rendono barbati, in basso $\mathrm{e}$ in alto sono glabri, sono di color giallo carico come le antcre le quali sono reniformi, uniloculari, inserite per la concavita all' apice del filamento. L'ovario è ovato, tutto coperto di una pubescenza floccosa. Lo stilo è piì lıngo degli stami, cilindrico, grossetto, liscio, di colore verdognolo, un poco curvato in alto, s' ingrossa un poco verso l'apice dove è lo stimma che è grossetto, ottuso e di colore giallo carico. (Parl. ms., descr. di pianla di Murcia nell' Appennino umbro).

Osservazioni. - Fraquesta specie e il Verbascum nigrum havri un ibrido, stato descritto sotto il nome di $V$. Scholtianum, che il Rota (Prosp. 7 . Berg.) indica nel Bergamasco tra Lenna e Carona, Caldesi (Fl. fav. tent.) nel Faentino, e Genuari (Plant. lig. cent.3) nell' Appennino ligure sopra Voltaggio.

\section{Verbuseum Iychnitis.}

V. temuissime tomentosum tomento albido, caule simplici aut paniculato-ramoso, foliis crenatis, caulinis oratis lanceolatisse, suh. sessilibus, racemis laxiusculis, florilus luteis raro albis, corulla limbo 
explanato, staminibus filamento albo-barbato, fructu ovato, $1 / 2$ centim. et plus longo.

Verbascum Lychnitis Linn. sp. $\mu l . e d .1$. p. 177. Bert.! fl. ịtal. 2. p. 592; et auct.

Verbascum Weldenii el V. Mœnchii Mass. prodr. fl. valt. p. 197.

Figure. - Fl. dan. t. 586. Reich. ic. fl. germ. 20. t. 27.

Stazione, Abitazione e Fioritura. - Nei lnoghi aridi in montagna. Piemonte, comune (All.): Torino (Delponte! ecc.), Alba (Bertero!), Mondovi (Ing.), Viosenne (Ricca!), Ragni di Valdieri a 1200 metri, Susa (Parl !), Riva di Sesia (Carestia!), Novarese (Bir.). Ticino: Lugano (Bert.). Lombardia : val Tellina a S. Caterina (Parl.!), a Bormio (Rampoldi!), a Brusio (Mass.), intorno a Sondrio (Mass., Com.), il Comasco in molti luoghi (Com. ecc.), il Pavese, il Bergamasco (Rota), cosi a Pontagna a 1500 metri (Parl.!), il Bresciano, frequentissimo (Zersi). Tirolo: Pinera presso Tione (Hausm.), Trento (Perini!), Bolzano, Merano, Mals, Bressanone, Welsberg (Hausm.), Fassa (Bert.), Roveredo, Brentonico ecc. (Hausm.). Veneto: monte Baldo (Goiran! ecc.), monte Pastello a 2600' (Bracht!), val Pollicella in diversi punti (Poll.), Euganei (Trev.), Angarano presso Bassano (Montini!), friuli presso Amaro, e Tolmezzo ecc. (Pir.). Istria: Fiume (Smith). Emilia: Porretta, Montese (Cocc.), Fiumalbo, Pietra di Bismantova (Gib. Pir.) ecc. Liguria: Tenda (Ungern-Sternberg!), abbondante a Lantosca, a Robione e in parecchi altri siti vicini (Ard.), monti di Triora, di Frontero ecc. (De Not.), S. Ste. fano d'Aveto (Bert.). Toscana, nella regione montana: alpi Apuane al Pisanino (Pucc.), e nei monti Alto e Altissimo (Simi), S. Pellegrino (Calandrini!), Boscolungo a 1100 metri (Parl.!), Vallombrosa!, il Casentino all'Eremo (Arc.!), a Poggio Penna e altrove (Parl.!). Marche: Catria (Piccinini!), monte S. Vicino a 800 metri (Bucci!), S. Ginesio (Bert.), Castel Manardo (Marzialetti!), monti Birro (Bert.!) e Vettore!. Abruzzo: monte de'Fiori, monte Pelone (Bert.), Maiella (Levier!). Lazio: S. Polo, Lucretile (Sang.). Campania: Sora (Terr.), Vicalvi, S. Biagio Saracinesco (Terr.!), Frasso Telesino (Terr.), Napoli a Camaldoli (Bert.). Basilicata: Vulture a Monticchio (Terr.). Corsica: Bogomano (Bert.). Nanca in Sardegna, in Sicilia e nella Penisola più meridionale. Fiorisce in giugno, luglio, agosto.

Distribuzione geografica. - In tutta Europa meno le sue parti piủ settentrionali e più meridionali, nell'Asia caucasica, introdotto nella Nord-America.

Descrizione. - Pianta perenne, alta da 7 a 10 decimetri, di 
un verde cinericcio, in gran parte tomentoso-bianchiccia per peli corti, folti e stellati. Il fusto è diritto, per lo più ramoso in alto, e semplice, poco angolato in basso, angolato con gli angoli acuti in alto, puberuio o pubescente tomentoso. Le foglie inferiori nascono vicinissime che fanno quasi un giro alla base del fusto, e sono allun. gato-ellittiche o allungato-lanceolate, ottusette, crenate, ristrette alla base nel picciỏlo, verdi e puberule o quasi glabre di sopra, ed ivi con un solco longitudinale poco profondo e larghetto, rossiccio verso la base, e con leggieri solchi laterali che corrispondono ai nervi ed ai nervetti della pagina inferiore, tomentose e liancheggianti di sotto per peli corti, fitti e stellati ed ivi con un grosso nervo longi. tudinale bianchiccio, da cui partono molti nervi laterali curvi ed obliqui, i quali con i loro rami formano una rete rilerata nella pagina inferiore della foglia e si uniscono tra loro ad arco verso il margine; il picciỏlo è corto, quasi piano e rossiccio di sopra, convesso di sotto e tomentoso; le altre foglie del fusto sono molte, alterne, vicine tra loro, eretto-patenti, gradatamente più piccole, con un picciòlo più corto e le superiori sessili, ovato-acuminate, del resto simili alle inferiori. I fiori nascono da $\overline{3}$ a 7 in fascetti, i quali sono un poco lontani tra loro, in un racemo ramoso alla base e quasi pi. raınidale e sono accompagnati da una brattea piủ lunga o quasi uguale ai liori e ovato-acuminata o lanceolata nei fascetti inferiori, e più corta e lanceolata o lineare-acuminata nelle superiori. I peduncoli sono cilindrici, quasi uguali al calice, rerdognoli, tomentosi. Il calice è pure verdognolo e tomentoso, diviso in cinque lacinie piủ lun. ghe del tubn, eretto-patenti, lancenlate, ottusette o ottuse. La co. rolla é più del doppio più lunga del calice: il suo tulo è cortissimo e il lembo diviso in cinque lubi profondi $i$ quali sono disuguali, ovali, tondeggianti all' apice, essa è di color giallo-pallido, con qualche sfumatura rossiccia verso la gola, puberula di fuori, glabra di dentro. Gli stami sono disuguali, più corti della corolla. I lilamenti da poco sopra della base sino al terzo superiore sono forniti di peli lunghi, fitti, bianchi, un po'ingrossati all'apice: nella parte nuda sono quasi giallicci, all'apice sono ingrossati. Le antere sono uguali, sono reniformi, inserite sull'apice ingrossato del filamento, unilocu. lari, aprentisi trasversalmente nol margine superiore o conresso, sono gialle-ranciate e glabre. II pistillo é piu lungo drgli stami e piii corto della corolla. L'orario é poco piủ corto del calice, ovale, verdognolo-bianchiccio, peloso-tomentoso. Lo stilo é poeo meno del doppio più lungo dell'ovario, curvo, colindrico, ingrossato all' apice, 
verdognolo, peloso in hasso con peli stellati. Lo stimma è ottuso, quasi in capolino, verdognolo. (Parl. ms., descr. della pianta della valle di Seremondo nelle alpi lombarde).

Osservazione. - Nel nio Prodr. della 1 . 1osc. confusi con il vero Verbascum Ly:hnitis alcune forme spettanti al V. nigrum. Lo stesso è accaduto ad altri autori. II V. Lychnitis si distingue, anche sul secco, per il colore delle foglie, scurissime quasi nere di sopra, cenerognole di sotto.

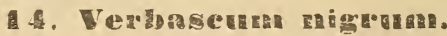

V. tomentosulum tomento albido, caule sinplici aut paniculato-ramoso, foliis crenatis, caulinis ovatis vel ovato-lanceolatis, subsessilibus, racemis laxiusculis, floribus luteis, corolla limbo explanalo, staminibus filamento purpureo-barbato, fructu ovato, $1 / 2$ centim. longo.

Verbascum nigrum Linn. sp. plant. el. 1. p. 178. Bert. fl. ital 2. p. 589 ; et auct.

Verbascum lanatum Schrad. mon. 2. p. 28. Hausm. fl. Tir. p. 625. Rota prosp. fl. Berg. p. 65. Smith fl. Fiume p. 38.

Figure. - Fl. dan. t. 1088. Schrad. o. c. t. 2. f. 1. Reich. ic. fl. germ. 20. t. $28,29$.

$\beta$ Chaixi, foliis basilaribus nec basi cordatis, sel truncatis rel attenuatis et quandoque inciso-lobulatis.

Verbascum Chaixi Vill. hist. pl. Dauph. 2. p. 491. De Not. rep. fl. lig. p. 94. Ard. cat. pl. Ment. p. 26. Fl. Alpes-mar. p. 273. Goir. 1l. vasc. veron. p. 32. Ces. Pass. Gib. comp. fl. ital. p. 340. Freyn fl. Süd-Istr. p. 386. Arc. comp. fl. ital. p. 506.

Verbascum nigrum $\beta$ Bert. o. c. p. 590.

Verbascum orientale Hausm. l. c. Goir? l. c.

Verbascum austriacum Smith fl. Fiume p. 37.

Figure. - Vill. o. c. t. 13. Reich. o. c. t. 36.

Stazione, Abitazione e Fioritura. - Nei lnoghi boschivi asciutti, nei pascoli ecc. di montagna, da dove scende ancora nelle sottoposte vallate e pianure della Penisola. Piemonte: Torino, raro (All., Re), Mondovi (Ing.), Sant'Anna di Valdieri (Bert), Susa (Parl.!), Courmayeur (Webb!), Scopello di ral Sesia (Carestia!), frequente nel Novarese (Bir), Arona (Ricca!), Renco in val Intrasca (De Not.!). Ticino: S. Gottardo (Parl.!). Lombardia: val Tellina, 
comunissimo (Mass.), cosi a Bormio (Rampoldi!), abbonda pure nel Comasco (Com.), Cassano d'Adda (Ramp.!), Pavia, sui colli oltrepadani (Nocc. Balb.), il Bergamasco, cosi nei monti Ocone, Resegone, Presolana (Rota), e a Ponte di legno a 1320 metri (Parl.!), frequente nel Bresciano (Zersi), per esempio sul lago d'Isco, alla Corna Blacca a 1800 metri, verso Collio a 900-1000 metri (Parl.!), Nantova (Bert). Tirolo: Welsberg, comune, Brunecco, Ritten, Bolzano, Merano, Laas, Goldrain e Mals in val Venosta, Pigenó, Ter. lago, Zambana, Roveredo (Hausm.), val Arsa (Kerner!), Folgaria, Tione (Hausm.). Veneto: inonte Baldo (Poll., Hausm.), monte Pastello, val Policella (Goir.), frequente nel Veronese e nel Vicentino (Poll.), Euganei (Kellner!), il Bassanese in molti siti (Mon(ini!), il Friuli a Fagagna, a Udine, a Femona (Pir.), Aquileia, Monfalcone (Bert.). Istria : Trieste (Bert), monte Maggiore (Smith), Dignano, Marzana, Pola (Freyn). Emilia: appennino Modenese e Bolognese nell'alpe di Succiso, sopra Civago a Fiumalbo, al lago Cerretano (Gib. Pir.), alle Pioppe di Salvaro (Cocc.). Liguria: alpi di Tenda (Reuter! ecc.), S. Martino Lantosca, val di Giallorgue, Saorgio, Fontana, abbondante, e fin sopra Mentone (Ard.), Dolcedo (Berti!), Castellerno, Genova (Bert.). Toscana, nelle regioni montana e submontana, ed anche nella reçione campestre: la Lunigiana al monte Corneviglio, ove abbonda (Bert.), e sopra Pontremoli!, alpi Apuane in parecchi siti della Versilia (Sini! ! ecc.), al Gioso, al Pisanino (Bert.), Appennino di Garfagnana (Calandrini! ecc.), lucchese e pistoiese in molti luoghi!, e giu fino a Lucca (Pucc.!), Firenze a monte .lorello (Reboul!), Pieve S. Stefano in val Tiberina (Berl.). Abruzzo: monte Velino Rert.). Puglia: Gargano, e altrove (Ten.). Lazio: Aíbano, monte Gentile (Sang.), campi di Aunibale (Gemmari!). Basilicata: Vulture (Terr.). Manca nella P'enisola piủ meritionale e nelle isole.

Distribuzione geografica. - In tulta Europa, meno la più seltentrionale, in Siberia.

Osservazioni. - Solto il nome di Verbascum Freynianum è stato indicato da Borbís un V. Chaixi Thapsus fra Veprinac e Vela Ucka presso fiume in Istria (Smith).

Descrizione. - Pianta perenne, alta da 6 a 12 decimetri, verde, putesecnte-tomentosa, fornita di un rizoma duretto, grossetto, scuro, che manda delle fibre radicali e il fusto. Questo è di. ritto, per lo piì semplice, angolato, segnatamente verso alto dove gli angoli sono acuti, verde, spesso rossiccio inferiormente, pube- 
scente-tomentoso con peli stellati, bianchi, piuttosto radi. Le foglie sono membranacee, molli, alterne, patenti, le inferiori allungatoovate, ottusette, scavate in cuore alla base, crenate, verdi di sopra ed ivi quasi glabre con un solco longitudinale rossiccio verso la base e con molti solchi in rete che corrispondono ai nervi della pagina inferiore, di un verde un po'pallido di sotto ed ivi con un grosso nervo longitudinale e con molti nervi laterali, i quali si dirigono obliqui e curvi verso il margine e l'apice per unirsi ad arco tra loro dopo avere mandato molti nervetti che formano una rete rilevata nella pagina inferiore della foglia: sono pubescenti-tomentose in questa pagina inferiore, segnatamente nei nervi, per peli stellati e bianchi forniti di un picciòlo più corto della lamina, scanalato e ros. siccio di sopra, convesso di sotto e pubescente-tomentoso, segnatamente nei margini che sono un poco alati; le foglie superiori sono ovato-allungate, acuminate, tondeggianti o leggermente scavate in cuore alla base, sessili o quasi sessili, del resto simili alle foglie inferiori, soltanto più piccole. I fiori nascono in fascetti di 5 a 10 fiori e formano un racemo a guisa di una spiga lunga, cilin. drica, per lo più semplice, continua o interrotta, tal volta ramosa, sono accompagnati da una brattea lanceolato-lineare, acuminata, e più lunga dei fiori nei fascetti inferiori, lineare-setacea e quasi uguale o più corta dei fiori nei fascetti superiori. I peduncoli sono disuguali, quasi il doppio più lunghi del calice, eretti o erettopatenti, quasi cilindrici, verdognoli, pubescenti-tomentosi per peli stellati. Il calice è diviso in cinque lacinie molto più lunghe del tubo, eretto-patenti, lineari, quasi acute, è verde e con $\mathrm{i}$ soliti peli. La corolla è piccola ma pur molto più grande del calice, con il tubo corto e il lembo diviso in cinque lobi profondi, patentissimi, un po'disuguali, ovali-tondi: è puberula di fuori in basso con peli stellati, glabra nel resto, gialla e appena violetta alla base dei lobi verso la gola, in una var. è bianca. Gli stami sono disuguali, piủ corti della corolla. I filamenti sono da poco sopra della base sin quasi al terzo superiore coperti di peli lunghi, di un violetto-porporino, diretti un po'in su e glandolosi all' apice: nel re. sto sono di color quasi croceo e nudi, sono ingrossati all' apice sotto dell'antera. Le antere sono uguali, quasi reniformi, inserite sull'apice slargato del filamento, uniloculari, aprentisi longitudinalmente nel margine superiore o convesso, gialle, glabre; il polline è giallo. Il pistillo è più lungo degli stami e quasi uguale alla corolla. L'ovario è quasi tondo, quasi la metà più corto delle lacinie del 
calice, peloso-irsuto, con peli stellati. Lo stilo è circa tre volte piủ lungo dell'ovario, un po'curvo, quasi cilmdrico, appena piủ grosso verso alto, ivi sfumato di violęto-porporino, bianchiccio in basso, glabro. Lo stimma è in capolino, papilloso, giallo, non scorrente nello stilo. (Parl. ms, ilescr. di pianla di sopra Ivino sul lago d'lseo).

\section{Verbascum phoeniceum.}

V. vix pilosulum, caule subsimplici, foliis subcrenatis, caulinis paucis parvis, racemis laxis, floribus rubris raro albis, corolla limbo explanato, staminibus filamento purpurco-barbato, fructu subgloboso, $1 / 2$ centim. longo.

Verbascum phœniceum Linn. sp. plant. ed. 1. p. 178. Bert. $\uparrow$. ilal. 2. p. 587. Sang. cent. prodr. fl. rom. add. p. 3S. Trev. prosp. R. eug. p. 24. Rota! rrosp. piant. Pav. p. 269. Hausm. fl. Tir. p. 626. Pir.! 11. for. syll. p. 103. Car. prodr. 17. tose. p. 474. Zersi prosp. piant. Bresc. p. 153. Pasq. Lic. viagg. Garg. p. 21. Ces. Pass. Gib. comp. 月. ital. p. 340. Freyn /l. Süd-Istr. p. 386. Smith! /1. Fiume p 38. Arce comp. Al. ital. p. 506.

Figure. - Bot.mag. t. 885 . Reich. ic. 11. germ. 20. t. 31.f. 1.

Stazione, Abitazione e Fioritura. - Qua e lá nei pascoli, nei luoghi boschivi ecc., della Penisola settentrionale e media: attorno a Torino in parecchi siti (Delponte! ecc.), a Borgone in quel di Susa, dove abbonda (Re), nell'Astigiano, nel Nonferrato, nel Canavese (All.), a Santhià (Malinverni!), al Ticino, ormuque (Bir.), a Turligo nel Milanese (Bert.), nel Pavese (Rota!), sul monte Prinzera nel Parmense (Y'ass.!), nel Bresciano da Jiago ai Campiani, frequente, altrove rarissinıo (Zersi), presso Roveredo in Vallunga (IIausm), a piè del Baldo, e in Covalsanto (Bert.), nel Veronese verso Villafranca in pui punti (Rigo!), e altrove, nei Rerici (Poll.), negli Emganei (Trev.), in Friuli, raro, a Nogaredo di Cormo (Pir !), pressu Silvella, intorno a Duino (Pir.), a Moufalcone, in Istria, sempre raro, a Trieste (Lert.), Rovigno (Smith!), Berbana (Bert.), nelI'isole Brioni, intorno a Pola (Freyn), presso Fiume (Emith!); quindi in Toscana nel inonte Pisano, rarissimo!, presso lientina (C; Targ.), e nel Fiorentino in ral di Marina (Rert.); poi in Ahruzzo al monte le' Fori (Orsni!); poi nel Romano a S. Gregorio (Sang.); infine nel Gargano a S. Nicanilro, rarissimo (Pasq. Lic.) Furisce in aprile, maggio, giugno, luglis. 
Distribuzione geografica. - Nell'Europa meridionale e media, dalla Germania e dall'Italia verso levante, nei paesi Caucasici e in Persia, in Siberia fino all'Altai.

Descrizione. - Pianta bienne, alta da 3 a 7 decimetri, con le foglie pelosette e con il fusto e i peduncoli pubescenti-glandolosi, verde e in parte sfumata di rossiccio. Il fusto è eretto, angolato, striato, rossiccio in basso, pubescente-glandoloso per peli corti, oriz. zontali, bianchi, aventi all' apice una glandola tonda, grossetta e trasparente come acqua, e fornito in basso di altri peli, piuttosto pochi, lunghi, bianchi e non glandolosi : è semplice o di raro con qualche rametto corto, un po' patente e fiorifero. Le foglie inferiori o radicali sono in rosetta, giacenti per terra, ovali o ovali-bislunghe, ottuse, crenate e un po'tondeggianti nel margine, di un verde cupo e pelosette di sopra per peli fini, bianchi e non glandolosi e ivi con un nervo longitudinale larghetto e rossiccio verso la base, non rilevato, anzi nel fondo di un solco longitudinale, con solchi in rete che corrispondono ai nervi della pagina inferiore e con il parenchima un po' sporgente in modo da render la foglia quasi con bolle, di un verde pallido di sotto ed ivi pubescenti per peli delicati, piủ abbondanti nei nervi, dei quali il longitudinale è grosso, sporgente e i laterali sono un po curvi, anch'essi rilevati, che si portano verso il margine e l'apice unendosi tra loro ad arco e con i loro rami, formando una rete rilevata nelle cui maglie sono quasi delle fossette lunghe e poco profonde che corrispondono alle bolle della pagina superiore. Le foglie inoltre sono fornite di un picciòlo corto, quasi piano, rossiccio e peloso di sopra, convesso, di un verde chiaro e pelosetto di sotto. Le foglie del fusto sono alterne, un po'lontane, molto più piccole, eretto-patenti, ovato-bislunghe, o ovate, ottuse o quasi acıte, sessili e le supreme abbraccianti un po'il fusto, con poche crenature e per lo più con peli glandolosi, del resto simili alle inferiori. I fiori sono belli, solitarii all'apice di lunghi pedicelli, in un racemo lungo e rado. Le brattee sono simili alle foglie supreme ma gradatamente più piccole, ovate o ovali, con poche e rare crenature, peloso-glandolose, molto più corte dei pedicelli. Questi sono patenti, un po'cernui all'apice, perciò il fiore è un po'cernuo, cilindrici, rossicci, pubescenti-glandolosi con le glandole spesso nericce; nel frutto sono diritti, sempre patenti. Il calice è diviso fin poco sopra la base in cinque lacinie quasi uguali, patenti, recurve, ovali, ottuse, con una leggiera carena, verdi, pubescenti-glandolose nel dorso e nei mar. gini con le glandule nericce. La corolla è patentissima, glabra, eccetto 
di fuori verso la gola dove ba dei peli lunghi, più abbondanti dalla parte che corrisponde ai lobi superiori; il tubo è cortissimo, verdognolo; il lembo è di un bel colore violetto-rossiccio, diviso profondamente in cinque lobi disuguali, $\mathrm{i}$ due superiori più piccoli degli altri tre, tutti quasi orbicolari, leggermente dentellati qua e là nel margine verso l'apice. Gli stami sono cinque, poco disuguali, piủ corti dei lobi superiori della corolla, diritti, inseriti all'apice del tubo. I filamenti sono appena più grossi alla base ed ivi giallicci e glabri, nel resto barbati con la barba che giunge sino all'apice del filamento nei tre stami superiori e poco sotto dell'apice nei dne stami inferiori : i peli sono del colore stesso del lembo della corolla in que. sti, e i superiori bianchi nei tre stami superiori, in tutti lunghi, patenti e gonfiati e come glandolosi all'apice quasi in forma di clava. Le antere sono schiacciate, quasi reniformi, inserite per la base, erette, uniloculari, aprentisi per una fessura trasversale nel margine superiore, di colore scuro quasi nericce, glabre. Il polline è giallo carico. Il pistillo è lungo quanto $\mathrm{i}$ due stami inferiori in mezzo ai quali è posto. L'ovario è piccolo, ovato, verdognolo, con pochi peli glandolosi verso alto, circondato all' infima base da un disco quasi un anello, del colore stesso dell' ovario: questo è poi biloculare, contiene molti piccoli ovoli, inseriti nella parte interna del setto. Lo stilo è un po'obliquo sull' ovario perchè diretto un po' in giủ, gradatamente un po' più grosso verso l'apice, glabro, di colore violettoscuro. Lo stimma è grossetto, papilloso, verdognolo. (Parl. ms., descr. di pianta coltivata).

\section{TRIBU் SECONDA.}

\section{Linn riee.}

LiNaRiese Reich.

Fructus tretum.

\section{ANA RIBHEUM.}

Anarrhinum Desf. R. atl. 2. p. 51. Chav. mon. Ant. p. 175. Gen. pl. A. germ. 5. t. 30. Benth. Hook. gen. pl. 2. p. 933. Ces. Pass. Cib. comp. 1. ital. p. 349. 1. 50. . . 2. 
Calyx erectus, 5-partitus. Corolla tubulosa, basi antice (in specie nostra) calcarata, limbo patulo bilabiato labiis subæqualiter partitis. Stamina 4. Antheræ 1-loculares. Stigma minusculum. Gem. mulæ in loculis plurimæ, coacervatæ. Tretum apice valvulatim foraminibus 2 dehiscens.

Portamento. - La specie nostrale è un' erba alta fino a $1 / 2$ metro, glabra, con una rosetta di foglie basilari mezzane, spatolate, dentate, e un fusto e spesso rami basilari sottili, semplici o ramosi a corimbo, ricoperti d'una quantità di foglie digitate dai lobi stret. tissimi, e terminati da lunghi grappoli di fiori piccolini, gridellini.

\section{Anarelhinum bellidifolium.}

Antirrhinum bellidifolium Linn. sp. plant. ed. 1. p. 617. Ing. cat. sp. Mond. p. 16 .

Anarrhinum bellidifolium Willd. sp. plant. 3. p. 260. Bert. f. ital. 6. p. 378. De Not. rep. A. lig. p. 301. Com.! fl. com. 5. p. 63. Rota prosp. piant. Pav. p. 270. Prosp. fl. Berg. p. 66. Zersi prosp. piant. Bresc. p. 155. Ces.Pass. Gib. l. c. Arc. comp. ft. ital. p. 512.

Anarrhinum corsicum Jord. sec. Mars. cat. pl. Cors. p. 106.

Figure. - Hoffm. Link fl. port. t. 32. Bot. mag. t. 2056. Chav. o. c. t. 10. Reich. ic. f. germ. 20. t. 57. f. 3.

Stazione, Abitazione e Fioritura. - Nell'Alta Italia: nel Trentino (Kellner!); nel Bresciano alle rive dell' Oglio poco sotto Monticelli, rarissimo (Zersi), nel Bergamasco negli ericeti tra Caleppio e Predore (Rota); nel Comasco, dove non è raro nelle selve e negli scopeti delle vicinanze di Como, cosi al monte Sant'Abbondio (Com.!), di Breccia, Cittiglio, nei territori di Lomazzo, Bregnano, Cermenate, ai piedi del monte Barro, nei siti aridi presso Lecco (Com.); tra Gallarate e Novara (Heldreich!), negli scopeti del Ticino, comune principalmente verso Cerano (Bir.), a Confienza, a Sannazzaro ed Alagna (Bert.), presso Pavia a Santa Sofia (Rota! ecc.), Torre d'Isola, lungo il Ticino e il Po (Nocc. Balb.); tra Borgoma. sino e Cossano in quel d' Irrea (All.); nei campi e nei pascoli asciutti del Novese, particolarmente intorno a Gavi (De Not.), sopra Grognardo nel Monferrato (All.); raramente sparso nei monti di Garessio nelle Alpi marittime (Ing.); nei colli intorno ad Oneglia (All.). 
Nasce poi in Corsica, sulle rupi e nei terreni magri della regione media, ai Bagni di Guagno, abbastanza raro, a Bocognano, salendo alla Foce, sopratutto sul versante del Pinso, sopra il Bronco, dov'è comune, a Bastelica, nelle pinete, ad Aullena (Mars.), ad Orezza (Bert.). Fiorisce in gingno, luglio, agosto.

Distribuzione geografica. - In Portogallo e Spagna, in Fran. cia, nella Germania occidentale e la Svizzera, in Italia.

Osservazione. - Chavannes nella sua monografia ( $p$. 177) aveva notato alcune differenze nella pianta corsa avuta da Orezza ; per cui Bentham (in Cand. prodr. 10. p. 289) sospetto trattarsi forse dell'Anarrhinum laxiflorum Boiss., specie vicinissima invero. Jordan ha fatto della pianta corsa un $A$. corsicum. Non avendola io veduta, non posso dire nulla in proposito.

\section{HNA HIA.}

Linaria Tourn. inst. p. 168. t. 76. Chav. mon. Ant. p. 91. t. 5. Gen. pl. fl. germ. 5. t. 31. Benth. Hook. gen. pl. 2. p. 932. Ces. Pass. Gib. comp. fl. ital. p. 346. t. 52. f. 1.

Calyx erectus, 5-partitus. Corolla basi antice calcarata, limbo bilabiato, labio postico porrecto 2-lobo, antico patulo 3-lobo palato prominente. Stamina 4, interdum cum staminodio filiformi. Antherarum loculi confluentes. Stigma minusculum. Genmula in loculis plurimæ, coacervatæ. Tretuın apice irregulariter valvatim foraminibus 2 dehiscens, interdum foramine 1 antico.

Portamento. - É assai svariato, ma si puỏ riferire a due tipi principali. Trattasi sempre di erbe o perenni o annue; ma le une sono deboli, suraiate, con foglie larghe e alquanto divise, con fiori ascellari sopra lunghi pedicelli; le altre piủ robuste sono erette, con foglie strettissime, e con grappoli terminali di fiori brevemente pedicellati. Certe specie poi sono intermedie fra i due tipi. I fiori sono mezzani o piccoli, gialli, o violacei, o bianchi, e spesso con quei colori frammisti.

Osservazioni. - Chavannes, e dopo di lui $\mathrm{i}$ botanici in gene. rale, hanno fatta una gran distinzione fra $i$ frutti delle Linaria, secondochè si aprono per un coperchietto, o per valve più o meno numerose, più o meno estese. In realıà non c' è tanta diversità di struttura. La parete del treto è reticolata-nervata, e seguendo in 
parte le linee di quelle nervature si apre, principiando verso l'alto, e in modo abbastanza irregolare, e variabile in ogni specie, e senza cosi ricise differenze.

Le specie di questo genere vanno soggette alla peloria, ossia. alla trasformazione dei fiori da irregolari in regolari. Su di ciò vedansi i trattati di teratologia vegetale, come l'opera Vegetable teratology di Masters.

*Folia sessilia aut petiolata, penninervata. Flores solitarii axillares, vel subracemosi, corollæ palato depresso.

Sect. Chænorrhinum C'and.

\section{Linaria minor.}

L. pubescenti-glandulosa, caule erecto a basi ramosissimo, ramis erecto-patentibus, patentibusve, foliis inferioribus oppositis, superioribus alternis, omnibus subsessilibus, lanceolato-linearibus, obtusis, uninerviis, pedunculis axillaribus, solitariis, capillaribus, erectis, vel erecto-patentibus, calyce sublongioribus, in fructu longioribus, laciniis calycinis sublinearibus, obtusis, corolla paulo brevioribus, corollæ labio superiore antice producto, bifido, laciniis apice rotundatis, divaricatis, labio inferiore trifido, laciniis oblongis, apice rotundatis, lateralibus antice productis, intermedia subreflexa, calcare conico, obtuso, dependente, corolla multo breviore, capsula ovoidea, obtusa, bilocủlari, seminibus oblongis, longitudinaliter sulcatis, lævibus. (Parl. ms.).

Antirrhinum minus Linn. sp. plant. ed. 1.p.617. Bert.fl. ital. 6. p. 348; et auct.

Linaria minor Desf. fl. atl. 2. p. 46; et auct.

Figure. - Fl. dan. t. 502. Reich. ic. fl. germ. 20. t. 61. f. $1,2$.

B litoralis, robustior, pedicellis brevioribus.

Linaria litoralis Willd. en. plant. hort. berol. p.641. Koch syn. fl. germ. ed. 2. p. 599. Ces. Pass. Gib. comp. 1. ital. p. 349. Freyn fl. Süd-Istr. p. 390. Smith fl. Fiume p. 38. Cald.! fl. fav. tent. p. 171. Arc. comp. fl. ital. p. 512.

Antirrhinum litorale Bert. fl. ital. 6. p. 350.

Figura. - Reich. l. c. f. 3.

Stazione, Abitazione e Fioritura. - Nei campi e nei luoghi 
incolti della Penisola, sino a notevoli altezze ne' monti, ed eccettuata la sua parte più meridionale. Piemonte, frequentissima, principalmente in luoghi montuosi solatii (All.): Torino (Re), Mondovi a Torre e Montaldo (Ing.), Susa (Ainti!), Cenisio (Bert.), Gressoney Saint Jean (Carestia!). Ticino: presso Agno (Rampoldi!). Lombardia: nel Pavese (Nocc. Palb.), nel Milanese (Bert.), nel Comasco ove abbonda (Com.), per esempio a Lecco (Bert.), e Ballabio inferiore (Parlatore!), in val Tellina a Tresivio (Mass.), a Bormio a 1225 metri (Parl.!) ecc., nel Bergamasco (Rota), per esempio a Ponte di legno a 1320 metri (Parl.!), nel Bresciano, frequente (Zersi), nel Man: tovano (Barbieri!). Tirolo: Tione, Roveredo, Trento, Fiemme e Fassa, Bolzano, sul Ritten ecc., Bressanone, Welsberg (Hausm.). Veneto tutto, frequentemente (De Vis. Sacc.): ovunque nel Veronese (Goiran!), negli Enganei (Trev.), a Chioggia (Bert.), Venezia (Kellner!), Possagno (Parl.!), Bassano, Feltre (Bert.), nel monte Serva del Bellunese (Venzo!), nel Friuli, comune (Pir.!). Istria: Trieste. (Bert.), Dignano, Pola ecc. (Freyn), Finme (Noë!). Emilia : nel Parmense al monte Prinzera (Parl.!), nel Modenese e Bolognese a Modena (Gib.!), Castelnuovo ne'monti, Salto di Montese, Fiumalbo (Gib. Pir.), Bologna (Bert.), la Porretla (G. Bert.), l'Acero (Cocc.). Pomagna: Faenza (Cald.!). Liguria, qua e là (De Not.): Nizza (Par. latore!), Mentone (Ard.), Tenda (Bourgeau!), Diano (Ricca!), Genova (Carrega!), Chiavari (Delpino!). Toscana, non rara, dalla regione maremmana alla montana: Alpi apuane al Sagro, a Massa (Bert.) e per la Versilia (Simi!), Lucca (Calandrini! ecc.), Bagni di lucca (Parl.!), Appennino Incchese a Tereglio (Giannini!), Appen. nino pistoiese a S. Marcello e al Teso (Parl.!), Firenze!, Vallom. brosa (Bert.), Prataglia in Casentino (Parl.!), Volterra (Amidei!), Siena, liosia!, Pienza (Santi), val Tiberina (Amidei!). Marche : Fano (Bert.), Pierosara (Bucci!), S. Ginesio (Bert.), Cupra (Piccinini!), Montefortino (Marzialetti!), Acquasanta (Parl.!), Castelluccio (Orsini!). Abruzzo: Caramanico, comune (Levier!), montagna di Rocca di Cambio (Cherici!). Umbria e lazio : Terni (Bert.), Roma, comune (Seb. Maur.) Campania: Villa Latina (Terr.!), S. Donato, Dragoni ('Terr.), Napoli, comune (Ten., P'asq.), Capri (Pasq.). Nasce pure in Corsica, dove pare che sia molto rara, essendo indiota soltanto a Calvi (Bert.). Manca in Sardegna e in Sicilia ; $\dot{e}$ peró data di Malta dallo \%erapha, ma non da Grech. Fiorisee dilla primavera all'autumno.

Distribuzione geografioa. - Nell'kurupa, principalmente me. dia, nell'Alf'rica settentrionale, nell'Asia Mnnore. 
Descrizione. - La pianta è pubescente-glandolosa, e vischiosetta nel fusto, nei rami, nelle foglie, nel peduncolo, nel calice e nella parte esterna della corolla. La radice è duretta, flessuosa, ramosa, bianchiccia, glabra. Il fusto è alto da pochi pollici a circa un piede, rossiccio, ramosissimo sin dalla base, con i rami patenti o erettopatenti e flessuosi, gl'inferiori opposti, gli altri alterni. Le foglie inferiori sono opposte, le superiori alterne, tutte aperte, quasi sessili, lanceolato-lineari, ottuse, intere nei margini, con un leggiero solco longitudinale di sopra più manifesto verso la base e con un nervo o venetta longitudinale di sotto. I fiori sono piccoli, solitarii all'ascella delle foglie. I peduncoli sono filiformi, poco più lunghi del calice nel fiore ma più lunghi di questo nel frutto, eretti e poi eretto-patenti. Il calice è verde, quinquefido; le sue lacinie sono quasi lineari, ottuse: le due inferiori un poco più strette. La corolla è un poco piủ lunga del calice, piccola, di un bianco-lilla con sfumature o righe di color lilla di sopra e di sotto del tubo. Le lacinie del labbro superiore sono dirette in avanti, tondeggianti e alquanto divaricate; le tre lacinie del labbro inferiore sono allungate, ottuse: le due laterali dirette in avanti, la intermedia diretta in giù e avvicinata al tubo. Il palato è poco sporgente e lascia aperta la gola; è dello stesso colore dei due labbri. Lo sprone è molte volte più corto della corolla, conico, ottuso, diretto in giù, di color lilla. I filamenti sono filiformi, bianchi, glabri eccetto verso alla base dove si vedono alcuni peli corti e grossetti e bianchi. Le antere sono violetto-scure. Il polline è bianchiccio. L'ovario è ovoideo, pubescente-glandoloso, di un verdognolo gialliccio. Lo stilo è filiforme, bianco, con pochi peli verso la base. Lo stimma di color lilla pallido, quasi bilamellato. La cassula è ovoidea, delicata, quasi uguale o poco più corta del calice, biloculare, con le logge che si aprono presso l'apice con un foro fatto dall'allontanamento di alcuni denti o valvette. I semi sono allungati, glabri, di colore quasi nericcio, solcati longitudinalmente con i solchi divisi da laminette delicate. (Parl. ms., descr. di pianta Fiorentina).

\section{Kinaria rubrifolia.}

L. erecta, glanduloso-pilosula, foliis sparsis oblongis, infimis oppositis subrosulatis obovatis, petiolatis, pedicellis elongatis, lobis calycinis angustissime oblongis, obtusis, corollæ purpureo- 
violaccæ $1 / 2$ centim. longæ calcare recto tubo breviore, treto calyce subbreviore, seminibus ovalibus, costato-muriculatis.

Antirrhinum origanifulium All. auct. all fl. ped. p. 5 (sec. Ard.).

Linaria rubrifolia Rob. Cast. in Cand. fl. fr. 5. p. 410. Mor.! fl. sard. 3. p. 206. Ard. fl. Alpes-mar. p. 281. Ces. Pass. Gib. comp. fl. ital. p. 349. Arc. comp. fl. ital. p. 511.

Linaria rupestris Guss.

Linaria origanifolia De Not.rep. fl.lig. p 299 (non Cand.).

Antirrhinum rubrifolium Bert. fl. iıal. 6. p. 3.47.

Figura. - Mor. o. c. t. 101.

Stazione, Abitazione e Fioritura. - Rarissima: nel Nizzardo al colle di Braus (De Not.), nelle rupi sopra Tenda e Scarena (All.), nel vallone della Volpe presso Scarena, a Levenzo (Ard.); presso Tivoli alle acque Albule sopra il travertino (Rolli!); in Abruzzo nell'anfiteatro di S. Vittorino (Bert.); in Sardegna nelle rupi calcaree di Tomneri d'Irgini (Mor.!), e de' monti d'Oliena, in luoghi arenosi verso la cima Scala di Dorgali, a 600-1000 metri sul mare (Mor.); in Sicilia in lnoghi rocciosi aridi, a Montallegro, Siculiana, Mineo (Guss.), Villafrati (Todaro!). Fiorisce da aprile a luglio secondo i luoghi.

Distribuzione geografica. - Nel bacino Nediterraneo, in Persia e in Belucistan.

Osservazione. - La Linaria origanifolia, indicata da Chavannes (Mon. p. 95) in Sicilia sulla fede di un saggio del Jan, è forse da riferirsi a questa specie.

\section{Minaria alsinefolia.}

L. diffusa?, villosa, foliis plerisque oppositis, petiolatis, ovatis ellipticisve, superioribus nunc oblongo-lanceolatis, pedicellis elongatis, lobis calycinis angustissime lineari-spathulatis, corolle pur. pureo-violacese? ..... raleare recto tubo breviore, treto calyce multo hreviore, seminilus angulato-uhoratis, sulcatis tuberculosis.

Antirrhinum alsincefolium Viv. fl. lib. spec. p. 67. Fl. cors. sp. nov. p. 10.

Linaria alsinafolia Spreng. syst. veg. 2. p. 792. Mor. A. sard. 3. p. 206 . Ces. Pass. Citb. comp./l. ilal. p. 347. Arr. comp. 16 . ilal. p. 509 .

Abitazione. - In litore boreali-orientali Sardiniar , in parvis insulis inter Corsicaın et Sardiniam. ( (Vir.). 
Distribuzione geografica. - Specie propria di quel luogo.

Osservazioni. - La pianta d'Oriente posta sotto il medesimo nome da Bentham (in Cand. prodr. 10. p. 269) è secondo Boissier tutt'altra cosa, la sua Linaria Acerbiana (Fl. orient. 4. p. 366).

Non si conosce altrimenti questa specie oscura, e non più rireduta. La diagnosi n'è stata fatta sulla descrizione di Moris, che vide $\mathrm{i}$ saggi del Viviani.

** Folia petiolata, penninervata. Flores solitarii, axillares, corollæ palato faucem arcte claudente.

Sect. Elatinoides Chav.

\section{Linaria spuria.}

L. diffusa, villosa viscidula, foliis sparsis vel imis oppositis, ovato-rotundatis, subintegris, pedicellis elongatis, lobis calycinis ovato-lanceolatis basi subcordatis, acutis, corollæ flavæ labio superiore violaceo $2 / 3$ centim. longæ calcare incurvato tubo duplo longiore, treto calyce breviore, seminibus ovali-globosis, reticulatorugosulis vix alveolatis.

Antirrhinum spurium Linn. sp. pl. ed. 1. p. 613. Bert. fl. ital. 6. $\mu .344$; et auct.

Linaria spuria Mill. gard. dict.; et auct.

Linaria Prestandreæ Terr.! fl. Vull. syn. p. 128 (non Tin.).

Figure. - Fl. dan. t. 913. Hoffm. Link fl. port. t. 34. Reich. ic. fl. germ. 20. t. 53. f. 2.

Stazione, Abitazione e Fioritura. - Nei campi, per quasi tutta l'Italia. Piemonte, specialmente in collina (All.): Torino, Susa (Re), Mondovì (Ing.), Monferrato (Delponte!), Novarese (Bir.). Lombardia, rara: colli transpadani del Pavese in parecchi luoghi (Nocca Balb.), considerata avventizia (Rota), Bergamasco, avventizia (Rota), Bresciano, poco frequente (Zersi), Mantovano a Volta e Sabbioneta (Paglia!). Veneto, non comune (De Vis. Sace.): Verona ad Avesa e Tagliaferro, Vicenza (Poll.). Emilia: Parma (Pass.!), Ozzano (Parl.!), Puianello presso Livizzano, valli di Sernide alla Guerrera (Gib. Pir.), Bologna, e per la valle del Reno fino a Porretta (G. Bert., Cocc.). Romagna: Faenza, Castrocaro (Cald.!). Liguria tutta (De Not.): Nizzardo, abbastanza comune (Ard.), S. Remo (Panizzi!) e Diano Cervo (Ricca!), comunissima, Genora (Bert.). Toscana, assai comune nelle regioni campestre e marem. 
mana: Lucca (Calandrini! ecc.), Pisa (P. Savi! ecc.), Livorno!, Casciana (Bastianini!), Volterra (Biondi!), Firenze!, Dudda nel Chianti (Ricasoli!), Siena (Bart.), S. Casciano de'Bagni (Arc.!), Elba (Car.). Marche: Fossato (Parl.!), Sinigallia (Ricci!), Porto Civitanova (Cavanna!), Cupra (Piccinini!), Montefortino (Marzialetti!). Abruzzo: Pescara (Porta Rigo!), monti di Carananico (Ces.). Lazio: Roma, comune (Seb. Maur.). Puglia: Barletta (Bruni!). Napoletano: Caserta (Terr.), Napoli al Vesuvio (Pasq.), Capri (Bert.). Basilicata: Melfi (Terr.!). Calabria: Anoia (Pasy.!). Sicilia, ovun. que (Guss.): Messina (Nic.), Catania (Tornabene!), Avola (Bianca!), Caltagirone (Tar. Gerb.), Palermo (Parl.! ecc.), Lipari (Mandralisca! ecc.). Malta (Grech!). Sardegna, abbastanza frequente (Mor.): Surso!. Fiorisce da giugno e luglio insino alla fine dell'autunno.

Distribuzione geografica. - Nell' Europa media e meridionale, e in parte nella settentrionale, in Madera e nelle Canarie, nell' Affrica settentrionale, nell'Asia Minore e fino alla Persia, introdotta al Capo e nell'America settentrionale.

Descrizione. - La radice è quasi verticale, tortuosa, bianchiccia, glabra e ha poche fibre o fibrille. Il fusto è ramosissimo alla base, con i rami apertissimi e quasi orizzontali, giacenti per terra, cilindrico, di un verde giallognolo, e coperto di peli lunghetti, bianchi, orizzontali e glandolosi all'apice e lateralmente, dei quali peli sono pure coperte le foglie, i peduncoli ed esternamente anche il calice: per tali peli le parti qui indicate sono un poco vischiose. Le foglie inferiori sono opposte e lornite di un corto picciòlo, le altre alterne e quasi sessili, tutte tondo-ovate, avvicinate alquanto ai rami ed al fusto, e come un poco scavate in cuore alla base, di co. lor verde pallido, con un nervo mediano dal quale partono due o tre nervi per parte, che si dirigono un poco curvi verso il margine e l'apice della foglia. I peduncoli sono dapprima crelli o eretto-patenti, ma poi divengono patenti e infine nel frutto quảsi orizzontali: sono solitarii, ascellari, capillari, e più lunghli delle foglie. I fiori sono resupinati, per il contorcimento dei peduncoli all'apice, in modo che il labbro superiore della corolla diventa inferiore e il labbro inferiore e lo sprone diventano superiuri. Il calice si compone di cinque foglioline erbacee, verdi, con il margine un poco rossiccio, tre superiori (inferiori) sono maggiori e due inferiori (superiori) un poco più piccole, tuitte sono ovato-lanceolate, acute. avvicinate alla corolla, coperte di fuori e segnatamente nei margini dai peli di sopra deseritti e glabre di dentro: hauno difuori un nervo 
a guisa di corona. La corolla, posta nella sua posizione naturale, ha il labbro superiore (inferiore) più piccolo dell' inferiore (superiore), bilobo, con i lobi tondeggianti all' apice, amaranti e glabri di sotto (sopra) e ivi con il inargine bianco, bianchicci di sopra (sotto) e ivi con qualche pelo simile a quelli del resto della pianta. Il labbro inferiore (superiore) è triloło, con i lobi quasi uguali e tondeggianti all' apice, rivolti in giù (in su), gialli e glabri, e con il palato chiuso da due grosse gibbosità anche gialle, nel resto questo labbro superiore è di colore gialliccio, ha qualche pelo simile a quelli della pianta e si continua in uno sprone dello stesso colore, lungo quanto la corolla, assottigliato all'apice e curvato in modo che è rivolto con l'apice in giù (in su). Gli stami sono quattro, due più alti e due più bassi. I filamenti sono cilindrici, bianchicci, glabri inferiormente, forniti in alto nella parte interna di lunghi peli bianchi e glandolosi. Le antere sono rivolte in dentro, e si trovano a paia, sono di colore rossiccio amaranto, con le logge confluenti. Il polline è bianchiccio. L'ovario è quasi tondo ma schiacciato lateralmente e verdognolo, è coperto di corti peli bianchi e glandolosi all'apice. Lo stilo è cilindrico, un po' ingrossato all' apice e un poco pubescente. Lo stimma è obliquo con lo stilo e bifido-lamellare. La cassula è più corta del calice, quasi tonda, verdognola, par glabra ma guardata con una lente si vede coperta di piccoli peli bianchi e glandolosi: si apre obliquamente con due coperchi laterali. I semi sono nericci, ovali o quasi tondo-ovali, con tante fossarelle divise da lamine delicate distribuite come in una rete. (Parl. ms., descr. di pianta Fiorentina).

Osservazione. - Secondo il signor Michelet (Bull. soc. bot. de France 7. p. 468), questa pianta produce alla base del fusto rami ipogei fioriferi e fruttiferi.

\section{Linaria Elatine.}

L. diffusa, pubescens aut villosa, foliis sparsis vel imis oppositis, ovatis et plerisque vel saltem nonnullis hastatis, cœterum subintegris, pedicellis elongatis, lobis calycinis ovato-lanceolatis lanceolatisve, acuminatis, corollæ flavæ labio superiore violace ${ }^{2} / 3$ centim. longæ calcare subincurvato tubo duplo longiore, treto calyce subbreviore, seminibus ovali-globosis, reticulato-rugosis alveolato-cristatis.

Antirrhinum Elatine Linn. sp. plant. ed. 1. p. 612. Bert. fl. ital. 6. p. 342 (ex parte); et auct. 
Linaria Elatine Mill. gard. dict.; et auct. p. 542.

Linaria Sieberi Reich. fl. germ. exc. p. 374. Nym. consp. fl. Eur.

Linaria Prestandreæ Tin.! in Guss. fl. sic. syn. 2.p. 842. Guss.! enum. pl. Inarim. p. 235. t. 9. f. I. Ilars. cat. pl. L'ors. p. 107. Terr.! rel. Terra di Lav. p. 146. Terz. rel. p. 76. Quart. rel. p. 109. Loj. is. eol. p. 121.

Linaria crinita Mab. rech. pl. Cors.

Linaria lasiopoda Freyn fl Süd-Istr. p. 389.

Linaria Biancæ Loj.! osserv. Lim. eur. p. 7, 13.

Figure. - Fl. dan. t. 426. Reich. ic. fl. germ. 20. t.59. f. 3.

Stazione, Abitazione e Fioritura. - Nei campi, e nei luoghi incolti, per tutta l'Italia. Piemonte, in abbondanza (All. ecc.): Torino, Susa (Re), Mondovi (Ing.), Alba (Bertero!), Novara (Bir.), Verrone (Zum.). Ticino: Coldrerio (Franzoni!), Mendrisio (Com.), Lombardia: il Comasco presso Como, nella valle Intelvi, nella Tremezzina, e a Varese (Com.), il Milanese in parecchi lıoghi (Rampoldil, Parl.!), il Pavese (Nocc. Balb.), il Cremonese (Parl.!), il Bergamasco (Rota), il Bresciano ad Iseo (Parl !), presso Brescia, e sul lago di Garda ecc. (Zersi). Veneto, non orunque (De Vis. Sacc.): Verona (Bracht!), Vilimpenta verso Ostiglia, Rovigo (Poll.), Euganei (Trev.), Vicenza (Poll.), Venezia (Kellner!), Manzano nel Friuli (Pir.!), Monfalcone (Pir.). Istria: Dignano, Brioni, Pola ecc. (Frènn), Fiume (Smith!). Emilia: Parma (Pass.!), valli di Sermide, Casinalbo, Sassuolo, Montespecchio (Gib. Pir.), Bologna, e valle del Reno lino a Porretta (Cocc.). Romagna: Faenza (Cald.!). Liguria, comunissima, particolarmente nella Riviera di levante (De Not.): il Nizzardo (Durando! ecc.), abbastanza comune, Mentone (Ard.), Chiavari (Del. pino!). Toscana, comune dalla regione maremmana alla submontana: Pontremoli (Parl.!), Lucca (Bicchi! ecc.), e val di Serchio fino a Tereglio (Giannini!), Pisa (Beccari! ecc.), Livorno (Bert.), Casciana (Bastianini!), Firenze!, Siena (Campani!), Arezzo (Gemmi!), Pratovecchio (Parl.!), Pieve S. Stefano (Amidei!), monte Argentario (Aiuti!), isole di Gorrona (P. Savi), di Capraia (Mor. De Not.) e dell' Ella (Marcucei!). Marche: Porto Civitanova (Cavanna!), Pedaso (Parl.!), Cupra (Pircinini!), Ascoli (Parl.!). lioma (Rolli l), comune (Seb. Maur.). Campania : Mola, Traetto, Roceasecca, 'Trifliseo (Terr.), Napoli, Capri (Pasy. !), Ischia (Guss.! (ecc.). Calalıria : Anoia (Pasq.!). Otranto (Groves 1). Corsica , comune (Mars.): Corte (Bert.), Rogliano, Bonifacio (Mars.). Sicilia, rara: Lipari (Loj.), Messina (Tineo! ecc.), 
S. Lucia (Nic.), Barcellona (Mallandrino!), Mandanici, Ali (Nic.), Avola (Bianca!). Malta (Zer.). Fiorisce da maggio, giugno o luglio insino nell' autunno.

Distribuzione geografica. - Nell' Europa meridionale e media e in parte nella seltentrionale, fino alle Azore, nell'Affrica settentrio. trionale, da Madera e le Canarie all'Abissinia, nell'Asia occidentale fino in Persia, introdotta nella Nord-America.

Descrizione. - Pianta annua, piccola, giacente per terra, coperta di lunghi peli molli che la fanno biancheggiare. La radice è fușiforne, flessuosa, semplice, bianchiccia. Il fusto è delicato, cilindrico, ramoso fin dalla base, con rami ancora più delicati, patentissimi e corti, gl'inferiori dei quali sono opposti e gli altri sparsi. Le foglie sono molli, le inferiori opposte, patenti, ovali, assottigliate un poco alla base, le altre alterne, eretto-patenti, ovato-astate, acute, con un nervo longitudinale e uno o due nervetti laterali verso la base, pelosette di sopra, pelose con lunghi peli molli di sotto come quasi tutte le parti della pianta. I fiori sono solitarii all'ascella delle foglie di quasi tutto il fusto e di quelle dei rami; sono forniti di un peduncolo filiforme, quasi glabro, patentissimo, più lungo delle foglie del fusto e molto più lungo di quelle dei rami, le quali sono assai piccole. Il calice è campanulato, diviso profondamente in cinque lacinie patenti, lanceolate, acuminate, con un nervo longitudinale in ciascuna di esse; è verdognolo, peloso con lunghi peli nel nervo e nei margini delle lacinie. La corolla è piuttosto piccola, superando appena il labbro superiore le lacinie del calice. Il labbro superiore è diretto in avanti, ed in su, carenato nel dorso ed ivi peloso con peli corti e diritti, smarginato-bilobo all'apice e con l'apice rivolto in su, il quale daranti è di color violetto e glabro. Il labbro inferiore è poco più lungo del superiore, più largo, trilobo, con i lobi quasi uguali in lunghezza, ma il lobo mediano ovale, ottuso e i due laterali estesi lateralmente, tondeggianti all'apice: in questo labbro superiore vicino alla gola sono due gibbosità assai rilevate e quasi tonde. La gola è chiusa e ivi sono molti peli corti e diritti. Lo sprone è delicato, lesiniforme, peloso, diritto o poco curvo, lungo quasi quanto la corolla, bianchiccio. Gli stami sono quattro, didinami, più corti del labbro superiore nella concavità del quale sono racchiusi: i filamenti sono cilindrici, un po'curvi in dentro verso l'apice, bianchi, quelli degli stami più lunghi pelosi quasi dalla base all' apice nella parte interna, quelli degli stami più corti pelosi soltanto verso alto dalla parte di davanti, tutti con peli lunghi e bianchi: laanuo inoltre 
un ciuffetto di peli corti sotto delle antere. Le antere sono violettonericce. Il pistillo è lungo quanto gli stami. L'ovario è piccolo, quasi tondo, verdognolo, peloso, biloculare. Lo stilo è piủ lungo dell'ovario, eretto, cilindrico e peloso in basso, un po'ingrossato verso l'apice e da ivi diretto un po' in avanti ed assottigliato e glabro, di color verdognolo gialliccio. Lo stimma è quasi in capolino, papilloso, bianchiccio. La cassula è tonda, racchiusa dentro il calice persistente. (Parl. ms., descr. di pianta di Lombardia).

Osservazioni. - Non ho potuto registrare la località data da Bertoloni, perchè egli ha confuso questa specie con la distintissima Linaria commutata.

La $L$. Prestandrexe dell' Italia inferiore è identica con la volgare L. Elatine d'Europa tutta, e non si comprende come alcuni autori persistano a considerarla forma autonoma. Non conosco altrimenti la L. lasiopoda Freyn, dell' Istria, già riferita da De Visiani ( $F l$. dalm. 2. p. 161) alla $L$. Elatine quale varietà, nè la $L$. crinita Mab., di Corsica, che Marsilly (Cat. pl. Cors. p. 107) non separa dalla L. Ela. tine: ma dalle descrizioni non risultano differenti che per una diversità di pelurie o di lunghezza di pedicelli o qualche altro carattere ugualmente insignificante perchè variabile. Cioloro che propongono o che accettano simili specie, pare che sempre dimentichino essere le piante organismi essenzialmente variabili, alle quali se non siconcede una certa latitudine per le loro variazioni più ovvie, è impossibile imporre delimitazioni specifiche, altrimenti si cade negli erramenti della scuola Jordaniana, che solto apparenza di maggiore oculatezza nell'osservazione praticamente sprofonda la scienza ncl caos.

\section{Linaria commutata.}

L. diffusa, pubescens aut glabrata, foliis sparsis vel imis oppositis, ovatis et plerisque hastatis, coterum subintegris, pedicellis elongatis, lobis calycinis angustissime lanceolatis, acuminatis, corollæ flavæ labio superiore violaceo $\% / 3$ centim. long $x$ calcare incurvato tubo duplo longiore, treto calyce subiequali, seminibus ovaliangulatis, tuberculatis.

Linaria commutata Bernh. in Reich. ^.germ. exc. p. 373 (18.30), et in Reich. ic. bot. 9. p. 6 (1831), lioch syn. R. germ. ed. 2. p. 599. De Not. rep. R. lig. p. 298. Mor. R. sard. 3. p. 203. Ces. Pass. Cib. comp. R. ilal. p. si7. Freyn! R. Sïd-Istr. p. 387. Arc. comp. A. ital. p. 509. Gib. Pir. $f^{\circ}$ suppl. fl. Mod. p. 22.

FLONA ITALAKA, - VOL. VI. 
Antirrhinum græcum Bory Chaub. exp. Mor. 3. 2. p. 175 (1832). Nouv. f. Pélop. p. 39 (1838).

Linaria graca Chav. monogr. p. 108 (1833). Ten. fl. nap. 5. p. 35. Guss. fl. sic. syn. 2. p. 118. Tar. Gerb. cat. pl. calat. p. 29. Gren. Godr. fl. Fr. 2. p. 575. Grech Del. fl. mel. p. 28. Guss, enum. pl. Inar. p. 236. Car. prodr. fl. tose. p. 476. De Vis. Sacc. cat. piant. Ven. p. 151. Terr. fl. Vult. syn. p. 128. Pasq.! fl.ves. p. 75. Mars. cat. pl. Cors. p. 107. Terr. rel. Terr. Lav. p. 146. Groves contr. fl. Terr. Otr. p. 64. Nic. prodr. fl. mess. p. 345.

Antirrhinum Elatine Bert. fl. ital. 6.p. 342 (ex parte).

Linaria Elatine Sim.! fl. alp. vers. p. 137. Terr.! sec. rel. p. 9 / (non Mill.).

Figure. - Reich. ic. bot. t. 815. Bory Chaub. exp. Mor: t. 21. Guss. enum. pl. Inar. t. 9. f. 2 (mala quoad sem.). Reich. ic. fl. germ. 20. t. 60.f. 2.

Stazione, Abitazione e Fioritura. - Nei luoghi coltivati ed in luoghi incolti di tutta l'Italia, eccettuata la massima parte della Penisola superiore. Fa in Istria nell' isola Brioni (Reich., Tommasini!), e nel distretto di Pola in molti punti (Freyn!); attorno Mantova (De Vis. Sacc.), e nel Modenese nelle valli di Sermide alla Guerrera (Gib. Pir.), e nei colli di Sassuolo (Gib.!); nella pineta di Ravenna (Beccari!); nei monti della Serra S. Quirico nelle Marche (Bucci!). De Notaris la dice molto frequente in Liguria, però non l'ho veduta che di Diano (Ricca!) e di Albenga (Gentili!). In Toscana è comune, nelle regioni maremmana e campestre, e forse più oltre: Montignoso!, la Versilia (Simi!), Viareggio!, Lucca (Calandriı:.") Bagni di Lucca (Parl.!), Pisa (Beccari!), Livorno!, Volterra (Amidei!), Pistoia (Parl.!), Firenze (Calandrini!), Rapolano (Sommier!), S. Vincenzo (Parl.!), Monte Pescali (Aiuti!), isole dell' Elba (Car.) e di Capraia (Mor.). Ritrovasi in Campania a Valvori (Terr.!), e Ciaserta (Terr.), a Portici (Pasq.!), in Ischia (Pedicino! ecc.) e Capri (Pasq.). Nasce al Gargano; in Basilicata a Castelgrande (Ten.) e a Melfi (Terr.); a Otranto (Groves); in Calabria a Pizzo (Ricca!), Sant'Eufemia, Palizzi (Arc.!) e Bova (Biondi!). In Corsica occupa le regioni bassa e media (Mars.): Bastia (Mabille!), Corte, Aiaccio, Bonifacio (Requien!). $\dot{\mathrm{E}}$ frequente in Sardegna (Mor.): Flumini Maggiore (Ascherson!). In Sicilia ovunque : Palermo (Parl.!), Godrano, Roccapalumba (Loj. !), Castellobuono a 5-600 metri (Huet!), Sampiero di Patti (Profeta!), Messina (Parl.!), Cal. tagirone (Tar. Gerb.), Scoglitti (Aiuti!), isole Eolie, Ustica, Egadi, 
Pantellaria (Guss.). Infine nasce in Malta (Grech). Fiorisce da giugno e luglio a settembre.

Distribuzione geografica. - Nel bacino del Mediterraneo e nelle Canarie.

Descrizione. - Pianta annua, giacente per terra, pelosetta, di un verde bianchiccio. La radice è fibrosa, ramosa, flessuosa, bianchiccia. Il fusto è ramosissimo sin dalla base e come i rami giacente per terra, radicante in basso, cilindrico, delicato, verdognolo, pelosetto, segnatamente in alto, con peli molli orizzontali, alcuni lunghi, altri più corti e aventi all'apice una piccola glandola. Le foglie sono erbacee, quasi distiche e un po' rivolte in su, le in. feriori più piccole, opposte o quasi opposte, tutte ovato-orbicolari, scavate in cuore, a guisa d'asta alla base o con pochi denti vicino a questa, quasi acute all'apice, un po'concave di sopra ed ivi con pochi leggieri solchi che corrispondono ai nervi di sotto, con pochi nervi palmati e lateralmente ramosi, un po' rilevati di sotto, pelo. sette in ambedue le facce e nel margine, con peli glandolosi al. l'apice, fornite di un picciòlo corto, con un solchetto longitudinale di sopra e convesso di sotto, di colore un po'più chiaro della foglia. I fiori sono solitarii all'ascella delle foglie, rovesciati, portati al. l'apice di peduncoli patenti e con l'apice un po diretti in su, delicati, ingrossati verso l'apice ed ivi pelosetti, quasi glabri nel resto, verdognoli: questi sono quasi uguali o poco più corti delle foglie in modo che i fiori uguagliano o superano appena le foglie. Il calice è verdognolo, peloso, con peli grossetti, diritti e bianchi, diviso in cinque lacinie profonde, patenti, quasi uguali, lanceolato-lineari, acute, circa la metà più corte della corolla. Questa è di un biancogiallognolo con il labbro superiore violetto-chiaro. Questo labbro per il rovesciamento del fiore pare inferiore, è poco piủ lungo dell'inferiore, diretto in avanti $e$ un po' in giü, bilobo, con i lobi tondeggianti. Il labbro inferiore (superiore per il rovesciamento del fiore) è trilobo, con i lobi rivolti in su e un po'indietro, tondeggianti all'apice; i due laterali sono appena più grandi di qquello di mezzo. Il palato ha due sporgenze, le quali sono quasi orali, molto rilevate, con macchioline tonde porporine e hanno dei peli nella parte inferiore che guarda e tocca il labbro superiore. Lo sprone per il rovesciamento del fiore é superiore, poco piủ lungo della corolla con la quale si confonde con la sua base molto larga, poi si restrin. ge, è lesiniforme, acuto, curvato o quasi ad arco in su e un po' in avanti. Gli stami sono didinami, lue piủ alti degli altri due: quelli 
giungono fin quasi alla smarginatura dei lobi del labbro superiore. I filamenti sono un po' curvati in dentro all' apice, verdognoli, pelosi in avanti verso il margine interno con peli lunghi. Le antere sono dirette in dentro ed in avanti, di color violetto scurissimo, con un ciuffo di peli bianchi, piuttosto corti e grossetti, in avanti. Il pistillo è più corto degli stami piủ lunghi. L'ovario è quasi tondo, un po'schiacciato dalle parti, verdognolo, peloso, inserito sopra un disco circolare, alto e verde. Lo stilo è più di due volte più lungo dell'ovario, quasi cilindrico, gialliccio, puberulo. Lo stimma è obliquamente diretto in avanti, acuto. La cassula è quasi tonda, gialliccia, quasi uguale o poco più corta delle lacinie del calice persistente e avvicinato ad esse. I semi sono pochi, quasi tondi, però angolati, scuri e con tanti grossi tubercoli. (Parl.ms., descr. della pianta di Pisa).

Osservazione. - Questa pianta si distingue bene dalla precedente, della quale ha tutto l'aspetto, anche per i frutti più piccoli e più duri.

Basta dare un' occhiata alla tavola del Reichenbach, per persuadersi che la Linaria commutata e la $L$. grceca son la stessa cosa. Vedansi le osservazioni di Boissier (Fl. orient. 4. p. 368) e di Freyn (l. c.).

\section{\%. Linuria cirnosa.}

L. diffusa, pilosula, foliis sparsis, lanceolatis hastatis, cœterum subintegris, pedicellis elongatis, lobis calycinis lanceolatis, acutissimis, corollæ lilacinæ $1 / 4$ centim. longæ calcare rectiusculo tubo sublongiore, treto calyce paullo longiore, seminibus subovalibus, tuberculatis.

Antirrhinum cirrhosum Linn. mant. alt: p. 249. Bert. fl. ital. 6. p. 346 .

Linaria cirrhosa Dum.-Cours. bot.cult. 2.p. 92 (1802). Willd. enum. hort. berol. 2. p. 639 (1809). Guss.! enum. pl. Inar. p. 235. Mor.! fl. sard. 3. p. 204. Car. prodr. fl. tosc. p. 476. Mars. cat. pl. Cors. p. 107. Ces. Pass. Gib. comp. fl. ital. p. 347. Arc. comp. fl. ital. p. 509.

Figure. - Till. hort. pis. t. 38.f. 2. Reich. ic. fl. germ. 20. t. 60 . f. 3 .

Stazione, Abitazione e Fioritura. - Qua e là per le parti più calde d'Italia. In Corsica : a Calvi, in luoghi erbosi umidi (So- 
leirol!), medesimamente dalla Parata ad Aiaccio (Mars.), ad Aiaccio stesso (Requien! ecc.), comunissima nei terreni granitici a Bonifacio e Portovecchio (Mars.). In Sardegna: nell' isola di Caprera (Ascherson!), nei monti di Domestica (Genn.!), nei campi tra Cagliari e Nonastir alla Cantoniera (Mor.). Nell' Istria (Ces. Pass. Gib.). In Toscana: intorno ad Altopascio (Chiostri!). Nel Lazio : comune nelle arene marittime a Fiumicino e Castelfusano (Sang.). Nell' isola d' Ischia, in luoghi sassosi aridissimi, e alle fumarole umide (Guss.). Nell'isole di Pantellaria (Guss.!) e di Linosa, sui colli aridi vulcanici (Guss.). Fiorisce in aprile, maggio, giugno.

Distribuzione geografioa. - Nel Mediterraneo occidentale, fino in Grecia.

Descrizione. - Pianta annua, delicata, piccola, di un verde chiaro, pelosa. La radice è delicata, fibrosa, ramosa, gialliccia. Il fusto è oscuramente quadrangolare, con gli angoli ottusi, ramoso o ramosissimo sin dalla base con i rami lunghi quanto esso e come questo eretto-patenti e diffusi, verdognolo e in parte rossiccio, peloso in basso, con i peli lunghi o lunghetti, articolati, bianchi, orizzontali, pelosetto in alto con peli molto disuguali, anche bianchi. Le foglie sono molli, le infime opposte, patentissime, allungate, ottuse, con pochi denti verso la base o intere, fornite di un picciòlo poco piủ corto della foglia, solcato di sopra, convesso di sotto, le altre sono alterne, patenti, astato-lanceolate, acute, intere o con qualche dente verso la base, fornite di qualche raro e corto ciglio, di un verde chiaro in ambedue le pagine, glabre o con pochi o rari peli verso la base nella pagina superiore, quasi glabre o con corti peli nel nervo principale e nei nervetti laterali della pagina inferiore, i quali sono un po' rilevati e bianchicci, fornite di un picciolo molto piủ corto della foglia, solcato di sopra, convesso di solto, peloso o pelosetto: le foglie superiori sono gradatamente piü piccole. I fiori sono piccolissimi, solitarii all' ascella di tutte le foglie, portati da peduncoli patenti o patentissimi, capillari, due o tre volte piu lungli delle foglie, glabri o quasi glabri, di colore violetto chiaro o bianchiccio, spesso flessuosi e talvolta avrolti quasi in forma di viticci. Il calice è profondamente quinquefido, con le lacinie patenti con l'apice un po'curvato in giù, lanceolate, acute, quasi uguali, con il dorso un po' rilevato, di un violetto rossiccio e con i margini larghi e bianchicci, coperte nel dorso di punte rilerate ottuse quasi come aculei bianchicci. La corolla è resupinata, quasi il doppio piu lunga del calice, posta quasi trasversalmente di fronte all' apice del 
peduncolo, è di color violetto chiaro, con punti più carichi nelle due sporgenze del palato, è pelosetta di fuori, con peli diritti. Il labbro superiore (infero per il rovesciamento della corolla), è poco più corto dell'inferiore, bilobo in alto con i lobi piccoli e quasi tondi; l'inferiore (supero per il detto rovesciamento) è trilobo con i lobi un po' piủ grandi di quelli del labbro superiore, il lobo di mezzo è anche un po' più grande dei due laterali e quasi smarginato. Il palato è chiuso da due sporgenze allungate. Lo sprone è lesiniforme, quasi diritto e un po' più lungo della corolla. Gli stami sono quattro, didinami; i filamenti filiformi, di color violetto chiaro, forniti alla base di peli acuti, lunghetti, a guisa di aculei di color violetto scuro. Le antere sono piccole, quasi tonde, nericce, glabre. Il polline è bianchiccio. Il pistillo è quasi uguale agli stami. L'ovario è tondo, pelosetto, gialliccio. Lo stilo è più lungo dell'ovario, filiforme, flessuoso, gialliccio. Lo stimma è quasi bidentato. La cassula è piccola, quasi il doppio piủ lunga del calice persistente e avvicinato ad essa, quasi tonda, e leggermente biloba, appena smarginata all'apice, quasi liscia, di color pallido gialliccio. Si apre in due fori larghi per la caduta di due coperchi o parti di valve laterali, riunendo il setto con una parte delle valve. I semi sono pochi, scuri, con tanti grossi tubercoli ottusi. (Parl. ms., descr. di pianta collivata).

*** Folia petiolata, palminervata. Flores solitarii, axillares, corollæ palato faucem arcte claudente.

Sect. Cymbalaria Chav.

\section{Linaria hepaticafolia.}

L. diffusa, glaberrima, foliis sæpius oppositis, rotundatis, lobatis lobis obtusissimis apiculatis, pedicellis elongatis, lobis calycinis angustissime lineari-lanceolatis, lævibus, corollæ lilacinæ 1 centim. ad summum (sine calcare) longæ calcare rectiusculo tubo breviore, treto calyce subbreviore, seminibus globosis, argute rugoso-costatis.

Antirrhinum hepaticæfolium Poir. enc. méth. suppl. 4. p. 19. Bert. fl. ital. 6. p. 339.

Linaria hepaticæfolia Spreng. syst. veg. 2. p. 790. Gren. Godr. fl. Fr. 2. p. 573. Mars. cat. pl. Cors. p. 106. Ces. Pass. Gib. comp. fl. ital.p.346. Arc. comp. fl. ital. p. 508.

Stazione, Abitazione e Fioritura. - Nei monti di Corsica, in 
luoghi pietrosi bagnati, comune tra 1000 e 2000 metri (Mars.): capo Corso (Gren. Godr.), monte Grosso, Vizzarona (Soleirol!), monte Renoso, Niolo (Requien!), monte Cagnnone (Kralik!), monte Ro. tondo, monte Cagno, Guagno (Gren. Godr.) ccc. Fiorisce da gingno ad agosto.

Distribuzione geografica. - Specie corsa.

\section{D) I Hanaria pallida.}

L. diffusa, pubescens, foliis sæpius oppositis, rotundatis, lobatis lobis obtusissimis apiculatis, pedicellis clongatis, lobis calycinis an. guste lineari-lanceolatis, lævibus, corollæ lilacinæ palato luteo $1-1]_{2}$ cention. longæ calcare recto tulıo sublongiore, treto calyce subbreviore, seminibus subglobosis, profunde argute rugosocostatis.

Antirrhinum pallidum Ten. fl. nap. 1. p. 920. Bert. fl. ital.6. p. 340 .

Linaria pallida Ten.! sec. Guss, pl. rar. p. 246. Terr.! rel. Terr. Lav. p. 146. Sec. rel. p. 91. Ces. el. piant. Maiell. p. 22. Cies. Pass. Gib. comp. fl. iial.p. 347. Arc. comp. fl. ilal. p. 509.

Figura. - Ten. o. c. t. 159. f. 2.

Stazione, Abitazione e Fioritura.- In luoghighiaiosi dei monti Alıruzzesi, ed altri finitimi, tra 2 -6000' (Porta Rigo!): nella Naiella in valle d'Orfenta, al monte Mucchia (Levier!), al monte Coccia, in valle Cupa (Pedicino!), al monte Amaro (Ces.), in valle di S. Spirito, alla Naielletta (Guss.), a Scrimacavallo (Ten.), nel Morrone in valle di Malacupa (Levier!), nel monte Heta (Terr.!), a Picinisco ai Treconfini ed allo Zaffineto (Terr.), sopra il Cantro presso lilettino (Rolli!). Fiorisce in luglio e agosto.

Distribuzione geografica. - Specie propria di quei monti.

Osservazione. - Tenore (o. c. 4. syll. $p$ 8r) cita come sinonimo di questa specic una Linaria apennina li Tausch descritta a pag. 217 dell'annata 1827 dal giornale l\%lora. Ora a quella pag̣ina non vi è $L$. apennina, in tutta l'annata nou vi è lavoro di Tausch, e nemmeno nell'altre annate ho trovata menzione alcuna di una L. apenninu, e di più ne li né altrove (giusta il Cat. of scient. papers i. p. 9/7) esiste lavoro suo dal titolo citato da Tenore. Beutham (in Cand. prodr.) e Bertoloni (l. c.) hanno ripetuta l'errata indicazione Tenoreana, senza verificare niente, secondo un uso molto 
comune fra' botanici, e molto comodo per darsi aria di ricerche erudite con poca fatica.

\section{Linaria pubescens.}

L. diffusa, pubescens, foliis oppositis sparsisque, rotundatis, lobatis lobis obtusis apiculatis, raro acutatis, pedicellis elongatis, lobis calycinis angustissime lineari-lanceolatis, nervatis, corollæ lilacinæ palato luteo $1 / 2-\left.{ }^{3}\right|_{4}$ centim. longæ calcare rectiusculo tubo subbreviore, treto calyce subbreviore, seminibus (atris) subglobosis, subtiliter argute rugoso-costatis.

Linaria pubescens Presl del. prag. p. 74. Fl. sic. 1. enum. $p$; 35. Guss. fl. sic. prodr. 2. p. 158. Fl. sic. syn. 2. p. 117, 842.

Antirrhinum pilosum Bert. fl. ital. 6. p. 340 (quoad pl. sic.).

Linaria pilosa (part.) Ces. Pass. Gib. comp. fl. ital. p. 340. Arc. comp. fl. ital. p. 509.

Figura. - Linaria clematites, saxatilis, rotundo Hederæfolio, hirsuta, purpurea. Cymbalaria dicta Bon. t. 24.

Stazione, Abitazione e Fioritura. - Sulle rupi calcaree umide ombrose dei monti di Sicilia: nelle Madonie (Meli!), a Polizzi, a Castellobuono (Guss.), tutt'intorno a Palermo in molti siti, da Busambra (Lojacono!), dalla Pizzuta a 1200-1500 metri (Huet!) e dal Caputo (Parl.!) al monte Catalfano (Guss.), a Tordiepi (Parl.!) e a Capaci (Aiuti!), nei monti di Castellammare e di Trapani (Guss.), nei colli d'Avola (Bianca!). Fiorisce da aprile a luglio.

Distribuzione geografica. - Specie siciliana.

\section{Tinaria pilosa.}

L. diffusa, pubescens vel villosa, foliis oppositis sparsisque, rotundatis, lobatis lobis obtusis apiculatis, pedicellis elongatis, lobis calycinis anguste lineari-lanceolatis, lævibus, corollæ lilacinæ palato luteo $1 / 2-3 / 4$ centim. longæ calcare rectiusculo tubo subbreviore, treto calyce longiore, seminibus subglobosis, profunde rugoso-tuberculatis.

Antirrhinum pilosum Jacq. obs. bot. 2. p. 28 (1767). Linn. mant. alt. p. 249 (1771). Bert. fl. ital. 6. p. 340 (excl. syn. pl. sic.). Linaria pilosa Cand. fl.fr. 3. p. 584. Terr.! rel. Terr. Lav. 
p. 146. Sec. rel. p. 91. Ces. Pass. Gil. comp. fl. ital. p. 347 (part.).

Terr. quart. rel. p. 109. Arc. comp. fl. ital. p. 509.

Linaria Cymbalaria var. De Vis. fl. dalm. 2. p. 160.

Figura. - Jacq. o. c. t. 48.

Stazione, Abitazione e Fioritura. - Sulle rupi umide e sui vecchi muri della Penisola inferiore: nella Sabina (Bert.), in Abruzzo verso Avezzano ad Albe, Coccorello (Cherici!), Grotta S. Benedetto (Levier!), e al Morrone (Ten.), nel Lazio sui monti Gennaro, Circello (Scb. Maur.) e Albano (Webb!), e a Terracina (Fiorini!), in Terra di Lavoro a Fondi (Orsini!), Itri (Ten., Bert.), Sperlonga (Terr.), Gaeta (Bert.), Mola (Ten.), Ausonia, Cassino (Terr.), Piedimonte (Terr. 1), nel Principato citeriore a Ciava (Pasq.!) ed Eboli, nel Gargano (Ten.!). Fiorisce dalla primavera all'autunno.

Distribuzione geografica. - Specie italiana.

Descrizione. - Pianta perenne, verde, pubescente per peli molli e bianchi. Fusto lungo, strisciante e in parte pendente dalle fessure delle rupi, pubescente e quasi peloso per peli lunghetti, delicati, cilindrico, ramosissimo, con fibre radicali delicate nei nodi inferiori, verdognolo-rossiccio. Foglie alterne, un po'lontane, carnosette, cordato-reniformi, con 5 o 7 lobi dei quali il mediano è il maggiore, gli altri poco piủ piccoli, e quando sono 7 con i due lobi più esterni molto piủ piccoli degli altri, tutti però tondeggianti ma con una piccola punta a guisa di un corto mucrone all' apice: sono un po'concave nella pagina superiore ed ivi verdi e con leggieri solchi che corrispondono ai nervi di sotto, un po' convesse e di un verde chiaro di sotto ed ivi con pochi nervi palmati i laterali dei quali si biforcano per terminare all'apice di cíascun lobo della foglia: sono pubescenti in ambedue le pagine, e fornite di un picciỏlo il quale è più lungo di esse, ha un solco longitudinale di sopra, è angolato con l'angolo ottuso quasi in forma di carena di sotto, verdognolo-rossiccio, pubescente. Le foglie superiori sono piu piccole. I fiori sono piuttosto piccoli, eretti, laterali all'ascella delle foglie, portati all' apice di un peduncolo il quale è diritto nel fiore, molto piủ lungo di questo, cilindrico, delicato, rossiccio-verdognolo, pubescente: nel frutto si allunga molto, si curva in giù, e si piega diversamente diventando flessuoso per portare il frutto sotterra ossia nelle fessure delle rupi. II calice è diviso profondamente in 5 lacinie, le quali sono quasi uguali, patule, lanceolato-lineari, quasi acute, hanno un nervo longitudiuale nel dorso quasi in forma di carena e sono verdognolo-rossicce e pubescenti. La corolla é di color violelto chiaro, con poche righe lon- 
gitudinali più cariche tanto nel tubo quanto nei lobi dei due labbri del lembo, e con le due gibhosità del palato di colore più chiaro e quasi bianchiccio, però all'apice di un giallo quasi dorato. Il tubo è poco più corto del labbro superiore, larghetto, con qualche solco di sopra verso il labbro superiore, alla base si prolunga in avanti cioè dalla parte del labbro inferiore in uno sprone ch'è poco più lungo di esso, diritto, parallelo al peduncolo, quasi cilindrico ma un po'assottigliato verso l'apice, ottuso. Il labbro inferiore è diretto in su o in avanti, bilobo, con i due lobi tondeggianti all'apice, larghetti, un po' convessi dalla parte anteriore e concavi dalla posteriore, formando insieme ivi quasi un canale. Il lobo inferiore è diretto in giù e un po' in avanti, è più grande e perciò più lungo e piủ largo del superiore, trilobo, con i lobi laterali un po' piủ grandi del lobo medio, tutti e tre però quasi tondi. Il palato è molto sporgente, e formato da due gobbe tondeggianti all' apice e divise a arco tra loro da un solco poco profondo: è pubescente dalla parte interna per peli corti e grossetti. Gli stami sono 4 , situati nella cavità che il tubo della corolla fa sotto dei lobi del labbro superiore, disuguali, due più alti degli altri due, tutti inseriti nel tubo della corolla, verso alto. I fila. menti sono un po'ascendenti, quasi lesiniformi, bianchicci, i due degli stami piủ lunghi puberuli alla base. Le antere sono piccole, ovali, bianchicce, glabre. Il pistillo è poco più lungo degli stami più corti e poco più corto dei piủ lunghi in guisa, che lo stimma corrisponde in mezzo ai quattro stami. L'ovario è piccolo, quasi tondo, ma schiacciato alquanto lateralmente, e ivi con un solco leggiero longitudinale nel mezzo, verdognolo-rossiccio, glabro. Stilo circa $\bar{j}$ volte piủ lungo dell'ovario, appena curvato ad $\mathrm{S}$, quasi cilindrico, bianchiccio, glabro. Stimma un po'grossetto, tondeggiante, un po'papilloso, bianchiccio. Cassula piủ lunga del calice, meno della metà, quasi tonda, con una punta corta e grossetta all'apice formata dalla base persistente dello stilo, con un solco longitudinale in ciascuna faccia, è del resto convessa e mostra la sporgenza per i serni che contiene, è gialliccia, biloculare e contiene in ciascuna loggia piủ semi, si apre dall' apice fin quasi sopra della base in sei valve. I semi sono tondo-ovali, giallo-scuri, con rughe o pieghe longitudinali un po' on. deggianti. (Parl. ms., descr. di pianta comunicala dal dottor Levier).

Osservazioni. - Nel mentre che in generale i semi di questa pianta sono come quelli della Linaria Cymbalaria, in alcuni saggi mentiscono quasi quelli della $L$. pubescens, ed in altri si accostano a quelli della $L$. cequitriloba. Si direbbe quasi una $L$. Cymbalaria fatta 
pelosa che accenna a passare nelle altre due specie. Havvi là un subbietto per più estese ricerche.

\section{Linaria Cymbalaria.}

L. diffusa, glaberrima, foliis sæpius sparsis, rotundatis, lobatis lobis obtusis apiculatis vel acutatis, pedicellis elongatis, lobis calycinis anguste lineari-lanceolatis, lævibus, corollæ lilacinæ palato luteo $1 / 2-3 / 5$ centim. longæ calcare rectiusculo tubo subbreviore, treto calyce longiore, seminibus (fuscis) subglobosis, fulvis, profunde rugoso-tuberculatis.

Antirrhinum Cymbalaria Linn. sp. plant. ed. 1. p. 612. Bert. f. ital. 6. p. 337; et auct. prcecipue veter.

Linaria Cymbalaria Mill. gard. dict.; et auct. rec.

Linaria acutangula Ten.! A. nap. 4. syll. p. 87. Guss. enum. pl. Inar. p. 234. Terr.! rel. Terr. Lav. p. 146. Sec. rel. p. 91. Ces. Pass. Gib. comp. R. ital. p. 347. Arc. comp. /l. ital. p. 509.

Figure. - Fl. dan. t. 1220. Curt. fl. lond. ed. Gr. 1. Reich. ic. fl. germ. 20. t. 59.f. 1. Guss. o. c. t. 8. f. 1.

Stazione, Abitazione e Fioritura. - Sui vecchi muri ombreggiati, sulle rupi umide, per tutta l'Italia, eccettuate la Corsica e la Sardegna. Piemonte, comune (All.): Torino, Susa, orunque (Re), Mondovi, comunissima (Ing.), Novara (Bir.). Cantone Ticino, co. mune (Com.). Lombardia: la val Tellina e il Comasco, comune (Comı). Pavia (Nocc. Balb.), il Bergamasco (Rota), fino a Edolo (Ricca!), il Bresciano, ovunque (Zersi!). Tirolo più meridienale: lago di Garda a 2-300' (Porta!), Arco (Hausm.), Roveredo (Kierner!), Trento, dietro il Dors Trent, a S. Martino (Hausm.) e alle Laste (Bert.), val Sugana (Ambrosi!), in tutta la valle (Hausm.). Veneto tutto quanto (De Vis. Sacc.): Verona, e monte Baldo a Campedello a 900 metri (Goiran!), Euganei (Trev.), Padova, Chioggia (Bert.), Venezia, volgarissima (Moric.), Bassano (Bert.), il Friuli, ovunque (Pir.). Istria meridionale, non comune (Freyn) : P'ola (Bert.), Fiume (Smith). Emilia: Modena, Fiumalbo, ece. (Gib. Pir.), il Bolognese, comunissima (Cocc.), fino a Porretta (G. Bert.). Roma. gna: Faenza (Cald.1). Liguria, volgarissima secondo De Notaris, peró nıanca nel Nizzardo, e nou l'ho veduta che dalla valle di Polcevera (Carrega!). Toseana, dalla regione maremmana alla submontana, comune: Sarzana (Bert.), la Versilia (Simi!), Lucca (Calan- 
drini!), Bagni di Lucca (Parl.!), Pisa (Savi), Firenze, Pratovecchio, Siena, monte Labbro! ecc.; manca nelle isole. Marche: monte di S. Marino (Bert.), Ascoli (Orsini!). Abruzzo, Boccaraso, Caramanico (Bert.), Maiella in valle d'Orfenta (Pedicino!), Morrone in valle di Malacupa a 1600 metri (Levier!). Puglia: Gargano (Ten.), Otranto (Groves). Umbria: Terni (Cherici!). Lazio: Roma, ovunque (Seb. Maur.), Tivoli (Bert.). Campania: Arpino (Terr.!), Caserta, comune (Terr.), Natese, Napoli, frequente (Ten.), monte S. An. gelo di Castellammare (Ten.!), Capri (Pasq.), Ischia (Guss.). Basilicata: Melfi e vicinanze (Terr.). Sicilia, dove non sembra comune: Messina (Guss.), Galati di Patti (Nic.), Palermo (Parl.!), Girgenti (Todaro!), Caltagirone (Tar. Gerb.), Cassaro e Ferla (Guss.). Malta (Zer., Grech). Fiorisce dalla primavera all'autunno.

Distribuzione geografica. - Nell'Europa meridionale, e nella media, dove si è diffusa in tempi moderni (Cand. géogr. bot.).

Descrizione. - Pianta perenne, pendente spesso lungamente dai muri e dai tetti, a guisa di una chioma, glabra, di un verde ora più ora meno chiaro, con i fusti delicati, cilindrici, ramosissimi, spesso lunghi e intrecciati tra loro, rossicci o verdognoli. Le foglie sono quasi carnosette, alterne, un po'lontane, dirette tutte in su dalla parte opposta al muro od al tetto, reniformi-cordate con cinque lobi, i quali sono tondeggianti e forniti di una piccolissima punta nelle foglie inferiori, più lunghi e quasi acuti o acuti nelle foglie superiori che sono generalmente più piccole e talvolta con tre lobi: le foglie sono di un verde chiaro di sopra ed ivi con leggerissimi solchi che corrispondono ai nervi di sotto, di un verde più chiaro e talvolta rossicce di sotto ed ivi con cinque leggieri nervi che partendo dalla base vanno come raggi un po' curvi alla punta dell'apice di ciascun lobo, e mandano delicati nervetti o vene late. tali : sono fornite di un picciòlo piủ lungo della lamina della foglia, delicato, quasi cilindrico, con uno stretto solco longitudinale di sopra. I fiori sono piccoli, solitarii all' apice di un peduncolo eretto, ascellare, delicato, cilindrico, rossiccio o verdognolo, poco più corto o poco più lungo della foglia: dopo la fioritura il peduncolo si allunga, si fa un po' flessuoso e si rivolge in giủ per mettere il frutto nelle fessure del muro. Il calice è piccolo, rossiccio o verdognolo, glabro, diviso fin poco sopra della base in cinque lacinie erettopatenti, avvicinate alla corolla, quasi uguali, bislungo-lineari, ottusette, con una leggiera carena nel dorso. La corolla è piccola, di un color violetto nel tubo, con i labbri di color violetto assai chiaro 
e quasi bianchi e con il palato giallo. Il labbro superiore è bilobo con i lobi lanceolato-lineari, ottusetti, rivolti in su, con tre o quat. tro leggiere linee violette, talvolta poco manifeste; il labbro inferiore è più grande assai del superiore, trilobo con i lobi quasi uguali, l'intermedio essendo appena più piccolo dei laterali, ovali-orbicolari, diretti in avanti e un po' in giu, il palato è sporgente con due gib. bosità quasi parallele, e chiude totalmente la gola, la quale è barbata per peli quasi in forma di clava, bianchicci o in parte gialli. Lo sprone è piccolo, da 2 a 3 volte più corto della corolla, quasi di. ritto, lesiniforme, ottusetto, del colore della corolla. Gli stami sono quattro, didinami, racchiusi nel tubo ed avvicinati alla parte di questo che corrisponde al labbro superiore. I filamenti sono pelosopapillosi alla base per peli grossi, ottusi, bianchi e quasi orizzontali, glabri nel resto, filiformi, un po'curvati in avanti all'apice, bianchicci. Le antere sono giallicce-bianchicce. Il pistillo è lungo quanto gli stami più corti. L'ovario è quasi tondo, glabro, rossiccio in alto, circondato da un disco verdognolo. Lo stilo è da 3 a 4 volte più lungo dell'ovario, filiforme, appena curvato ad S, bianchiccio. Lo stimma è quasi in capolino, bianchiccio, diretto in avanti. La cassula è quasi tonda, un po' gobba qua e là, poco piủ lunga delle lacinie del calice che sono persistenti ed avvicinate ad essa: è rossiccia dapprima, dipoi nera, ha due logge divise da un setto verti. cale e si apre in ciascuna loggia per due fori che si formano ciascuno per l'allontanamento di tre valve, poi i due fori si confondono in. sieme e ciascuna loggia sembra essersi aperta tutta con il contorno irregolarmente crestato. I scmi sono molti, quasi tondi, dapprima ranciati, poi neri, con rughe grosse e dense, molto rilevate. (!)arl. ms., descr. di pianta Fiorentina).

Osservazione. - La Linaria acutangula Ten. non è tampoco una varietà: su di una medesima pianta si possono trovare le due forme di foglie ritenute distintive, quelle a lobi più ottusi, e quelle a lobi più profondi e alquanto appuntati; e sono identici i semi, che Gussone vorrebbe diversi.

\section{3. Momsria Miilleri.}

L. diffusa, tomentoso-pubescens, foliis oppositis sparsisque. rotundatis, indivisis lobatisve lobis obtusis muticis, pedicellis elongatis, lobis calycinis lanceolatis, ..... corolla purpurea palato lute- 
scente 1 centim. et plus longæ calcare tubo multo breviore, treto calyce longiore, seminibus subglobosis, tuberculosis subrugosis.

Linaria Mulleri Mor. diagn. stirp. sard. nov. p. 1. Fl. sard. 3. p. 199. Ces. Pass. Gib. comp. fl. ital. p. 347. Arc. comp. fl. ital. p. 509.

Linaria æquitriloba Hook. in bot. mag. 56 (non Spreng.).

Figura. - Bot. mag. t. 2941.

Stazione e Abitazione. - In Sardegna sulle rupi dei monti presso Laconi (Müller).

Distribuzione geografica. - Specie propria di quel luogo.

Osservazione. - Non conosco questa pianta che per le descrizioni date da Hooker e da Moris e per la figura citata. Sembrerebbe specie molto distinta.

\section{Limaria aequitriloba.}

L. diffusa, pubescens, foliis sæpius oppositis, rotundatis, lobatis lobis obtusis apiculatis, pedicellis elongatis, lobis calycinis angustissime lineari-lanceolatis, lævibus, corollæ lilacinæ palato luteo $1 / 2$ centim. longæ calcare rectiusculo tubo multo breviore, treto calyce sublongiore, seminibus subglobosis, profunde rugoso-alveolatis.

Antirrhinum æquitrilobum Viv. fl. cors. spec. nov. p. 10. Bert. fl. ital. 6. p. 341 .

Linaria æquitriloba Spreng. syst. veg. 2. p. 790. Gren. Godr. 1. Fr. 2. p. 573. Car. prodr. fl. tosc. p. 476 . Fl. Montecr. p. 21. Mars. cat. pl. Cors. p. 106. Ces. Pass. Gib. comp. 1. ital. p. 347. Arc. comp. fl. ital. p. 509.

Linaria pilosa Mor.! fl. sard. 3. p. 199 (non Cand., et excl. syn. plur.). Genn.! fl. Capr. p. 114.

Stazione, Abitazione e Fioritura. - Sulle rupi bagnate, in luoghi ombrosi freschi dell'Arcipelago toscano, di Corsica e di Sardegna. Arcipelago: Gorgona (P. Savi!), Capraia (Mor. De Not.), Montecristo (Watson-Taylor!). Corsica: Capo, comune (Mars.), Pigno sopra Cardo (Mabille!), Calvi (Soleirol!), Aiaccio (Bubanil), Bagni di Guagno, Vico, valle di Porto, Bocognano, Bastelica (Mars.), Bonifacio (Jordan!) nel monte della Trinità (Bert.), Lavezzi (Requien!). Sardegna, frequente nei monti, fino a 1500 metri (Mor.): Caprera 
(Genn.), Arizzo, Malganai (Genn.!), Giovannibono presso S. Vito (Sommier!). Fiorisce da maggio a luglio.

Distribuzione geografica. - Dicesi che nasca inoltre nelle Baleari.

Descrizione. - I fusti sono delicatissimi, pendenti, ramosi, cilindrici, rossicci, glabri. Le foglie alterne sono fornite di un picciòlo lungo, delicato, cilindrico, glabro o con qualche raro e lungo pelo di sopra. La lamina è reniforme, scavata profondamente a guisa di cuore alla base, divisa in tre e più spesso in cintue lobi, larghi, tondeggianti, e aventi nel mezzo del margine un mucrone cortissimo, appena manifesto; è verde con pochi peli bianchi, eretti, qua e là sulla pagina superiore, di colore più pallido e glabra di sotto e con venette palmate e ramose, appena appena visibili. I peduncoli sono ascellari, lontani, capillari, rossicci, glabri o con qualche raro pelo. Il calice è glabro e quasi un terzo della lunghezza della corolla non compreso lo sprone: le sue lacinie sono lineari-lanceolate, acute e verdi sfumate di rossiccio. Il labbro superiore della corolla è bilobo, con i lobi allungato-lineari, ottusissimi e quasi tondi, diretti in alto e di color violetto chiaro con cinque vene più cariche verso la base $\mathrm{e}$ in basso dei lobi, delle quali le due laterali sono più manifeste e la mediana delicatissima è come biforcata all' apice. Il labbro infe. riore é trilobo, con i lobi ovati, tondeggianti, ottusissimi e come troncati all' apice, dello stesso colore del labbro superiore con qual. che sfumatura più carica verso le due próminenze che chindono il palato e che sono giallicce: il palato è coperto di peli fatti a guisa di mazza, bianchi e giallicci. Lo sprone è grossetto, lungo circa una metà del resto del fiore, ottuso, diretto in giù e in dietro e di colore più pallido della corolla. L filamenti sono liliformi, bianchicci, glabri, eccettochè in basso dove vi sono pochi peli corti e orizzontali. Le antere sono gialle. Anche il polline è giallo. L'ovario $\dot{c}$ quasi torido, rossiccio, glabro. Lo stilo é filiforme, bianco e glabro, un poco ingrossato nello stimma ch' ̀ quasi in capolino e come bilobo. (Parl. ms., descr. di pianta collivata).

-... Folia sessilia, peminervata. Flores racemosi, corollie palato faucem arcte claudente.

Sect. Linariastrum Chav.

$\doteqdot$ Semina exalata. 


\section{Hignria meflexa.}

L. diffusa, glabra, foliis inferioribus verticillatis superioribus sparsis, ex ovali vel oblongo lanceolatis, pedicellis elongatis, lobis calycinis lanceolatis acutatis, corollæ albidæ palato luteo $1-1^{1} / 2$ centim. longæ calcare recto tubo 2 -triplo longiore, treto calyce æquali, seminibus ovalibus, profunde rugoso-cristatis.

Antirrhinum reflexum Linn. sp. plant. ed. 2. p. 857. Bert. fl. ital. 6. p. 352.

Linaria reflexa Desf. fl. atl. 2.p. 42. Tar. Gerb. cat.pl. calat. p. 30. Grech Del.! fl. mel. p. 28. Mor.! fl. sard. 3. p. 208. Ces. Pass. Gib. comp. fl. ital. p. 348. Loj. is. eol. p. 121. Arc. comp. fl. ital. p. 511 . Nic.! prodr. fl. mess. p. 346.

Figure. - Fl. nap. t. 58. Fl. graec. t. 593.

Stazione, Abitazione e Fioritura. - Per i campi e i luoghi incolti dell' Italia piủ meridionale. È comunissima in Sardegna (Mor.!): Cagliari (Gennari! ecc.), Decimo (Thomas!). In Sicilia ovunque (Guss.): Palermo (Todaro! ecc.), Dula (Minà!), Messina (Nic.! ecc.), Catania (Parl.! ece.), Caltagirone (Tar. Gerb.), Siracusa (Webb!), Avola (Bianca!), Comiso, Vittoria, Girgenti (Aiuti!), Lipari (Loj.). Nasce nelle isole di Malta (Grech! ecc.) e di Lampedusa (Aiuti!). Nella Penirsola trovasi a Reggio (Magnaguti! ecc.), Taranto, Zittarella (Aiuti!), Ginosa(Ten.), Brindisi (Ball!), Bari, Manfredonia (Ten.). Fiorisce da gennaio ad aprile.

Distribuzione geografica. - Nasce inoltre in Barberia. È stata indicata in Grecia e in Soria, nonchè sull'autorità d'Allioni in Corsica, dove però non è piủ stata riveduta.

Descrizione. - Pianta annua, alta da $1 \frac{1}{2}$ a 3 decimetri, glabra, glaucescente. La radice è fusiforme, ramosa, fibrosa, flessuosa, bianchiccia. I fusti sono molti che partono dalla radice, dapprima giacenti per terra poi ascendenti, cilindrici, semplici o con qualche rametto verso alto, tutti fioriferi o alcuni piccoli, corti e sterili. Di questi le foglie come della parte inferiore dei fusti o rami fioriferi sono piccole, obovato-orbicolari o ovali-acute, a tre a tre in giro, le superiori dei rami o fusti fioriferi sono alterne, bislunghe o obovato-ovali, acute, tutte patenti, intere, piane, con tre leggieri nervi appena manifesti di sotto nella metà inferiore della foglia. I fiori sono tutti portati all' apice di lunghi peduncoli all' ascella 
delle foglie superiori e formano quasi un corimbo all'apice dei fusti o dei rami. I peduncoli sono quasi il doppio piủ lunghi delle foglie, eretti nel fiore, curvati ad arco in giủ nel frutto, cilindrici, verdognolo-rossicci. Il calice è diviso profondamente in cinque lacinie quasi uguali, patenti, delle quali due laterali e tre dirette un po' in avanti essendovi una distanza maggiore tra le due laterali in dietro nel posto dove corrisponde lo sprone: tali lacinie sono lanceolate, acute e quasi acuminate, di color verde-glaucescente. La corolla è piuttosto grande, bianca o di un bianco sfumato di giallicciı, con l'apice delle due gibbosità, che chiudono la gola, di color giallo-ranciato. Il labbro superiore è eretto, lungo, bifido, con le lacinie quasi lineari, ottuse, rivolte in dietro in mado che quasi si toccano con la faccia loro posteriore; il labbro inferiore è molto più corto, trilobo, con i lobi laterali quasi orbicolari, ap. pena smarginati e con il lobo medio più piccolo, piegato quasi in forma di tegolo, smarginato all' apice: le due gibbosità di questo labbro sono distinte soltanto all'apice, essendo confuse insieme e formando cosi una parte gonfia quasi tonda alla base del labbro in. feriore. La gola è pelosa dalla parte di questo, con i peli gialli quasi in due linee, nel resto i peli sono bianchicci. Lo sprone è piủ lungo della corolla, lesiniforme, diretto in giủ, bianco. Gli stami sono racchiusi nella gola, due più corti degli altri, inseriti alla base del tubo. I filamenti sono piủ grossi alla base ed ivi con peli grossi, corti e quasi conici, cilindrici nel resto, un po' curvi, bianchi e glabri. Le antere sono scure, con le logge verticali, aprentisi per una fessura comune. Il polline è giallo. Il pistillo $\dot{e}$ poco più corto degli stami più lunghi e poco più lungo legli stami piủ corti. L'ovario è quasi tondo, un po'schiacciato dalle parti, ver. dognolo, glabro, circondato alla base da un disco piu sporgente in avanti, come lobato e di color verde cupo. Lo stilo è da tre a quattro volte più lungo dell' ovario, cilindrico, un po'curvo in alto, ivi sfumato di violetto, bianchiccio in basso, glabro. Lo stimma è quasi in capolino, rivolto in avanti, bianco. (Parl. ms., descr. di pianta coltivata).

16. Linnrin triphylla.

L. erecta, glaberrima, foliis ternatis oppositisve, late ovalibus, integris, pedicellis brevissimis, lobis calycinis ovali-lanceolatis, obtu- 
sis vel acutiusculis, corollæ e cæruleo flavescentis $1 \frac{1}{2}$ centim. longæ calcare incurvato tubo duplo longiore, treto calyce æquali, seminibus ovali-triquetris, reticulato-rugosis.

Antirhirum triphyllum Linn. sp. plant. ed. 1. p. 613. Zer. fl. mel. thes. p. 5. Bert. fl. ital. 6. p. 350.

Linaria triphylla Mill. gard. dict. Tar. Gerb. cat. pl. calat. p. 30. Koch syn. fl. germ. ed. 2. p. 600. Gren. Godr. fl. Fr. 2. p. 579. Grech. Del.! fl. mel. p. 28. Mor.! fl. sard. 3. p. 210. Ard. fl. Alpes-mar. p. 282. Mars. cat. pl. Cors. p. 107. Ces. Pass. Gib. comp. fl. ital. p. 348. Arc. comp. fl. ital. p. 510. Nic. prodr. fl. mess. p. 347 .

Figure. - Bocc. ic. descr. rar. pl. t. 22. Bot. mag. t. 324. Reich. ic. fl. germ. 20. t. 63. f. 1.

Stazione, Abitazione e Fioritura. - Nei campi delle parti più calde d' Italia: in Malta (Grech! ecc.); in Sicilia a Trapani (Tod.!), Mazzara, Girgenti, Alicata, Terranova, Scicli (Guss.), Modica (Inzenga!), Ragusa, Spaccaforno, Noto (Guss.), Avola (Bianca!), Si. racusa (Cassia!), Mililli, Lentini (Guss.), Caltagirone (Tar. Gerb.), Catania (Guss.), Taormina (Nic.), e a Palermo (Bert.); in Sardegna è comune (llor.), cosi a Cagliari (Genn.! ecc.), a Tertenia (Sommier!); in Corsica a Bonifacio (Requien! ecc.), comune, a Rogliano, comunissima, a S. Fiorenzo, comune (Mars.); a Bastia (Soleirol!), rara al Capo (Mars.); nell' Elba a Monte di Reale (F. Picciỏli!); in Liguria a Nizza (Ard.), Bordighiera (Panizzi!), e Genova (Bert.); nella Calabria orientale (Guss.); in Puglia a Ginosa, Conversano, Bari, nel Tavoliere (Ten.), a Manfredonia (Porta Rigo!); in Istria (Koch, Reich.). Fiorisce in aprile e maggio.

Distribuzione geografica. - Nell'Europa meridionale, nell' Affrica settentrionale.

\section{1\%. Limaria fava.}

L. diffusa, glabra, foliis sparsis et infimis verticillatis, oblongolanceolatis, pedicellis brevissimis, lobis calycinis linearibus obtusis, corollae luteæ subpurpurascertis vix 1 centim. longæ calcare recto tubo duplo longiore, treto calyce longiore, seminibus ovali-angulatis, foveolatis.

Antirrhinum flavum Poir. voy. Barb. 2.p. 191. Bert. fl. ital. 6. p. 353. 
Linaria flava Desf. fl. atl. 2. p. 42. Gren. Godr. /l.Fr. 2. p. 582. Mor.! fl. sard. 3. p. 209. Mars. cat. pl. Cors. p. 10\%. Ces. Pass. Gib. comp. fl. ital. p. 348. Arc. comp. f. ital. p. 511.

Figura. - Desf. o. c. t. 136.

Stazione, Abitazione e Fioritura. - Nelle arene marittime di Corsica e di Sardegna. In Corsica a Galeria (Soleirol!), nel golfo d'Aiaccio dalla Pepiniera alla cappella S. Giuseppe, piuttosto rara, e tra le bocche della Gravona e del Prunelli, piuttosto comune (Mars.), ad Aiaccio (Requien!), a Campo di Loro (Bourgeau!). In Sardegna a Flumini, Portoscuso (Gennari!) e Bari (Mor.). Fiorisce in marzo, aprile, maggio.

Distribuzione geografica. - Inoltre nella Spagna meridionale e in Algeria.

\section{Linaria monspeswulana.}

L, erecta vel adscendens, glaberrima, foliis sparsis et infinis sub. verticillatis, angustissimis fere subulatis, pedicellis brevibus, lobis calycinis lineari-lanceolatis acutis, corollae albidæ e lilacino-luteolo variegatæ $2 / 3$ centim. longæ calcare rectiusculo tubo breviore, treto calyce duplo longiore, seminibus ovali-subtriquetris, reticulato-rugo. sissimis.

Antirrhinum repens Linn. sp. plant, ed. 1. p. 61:4. Bert. $\cap$. ilal. 6. p. 36 I.

Antirrhinum monspessulanum Linn. o. c. p. 616.

Linaria monspessulana Mill. gard. dict. ed. 8 (sec. ed. gall. i788. 4. p. 399).

Linaria decumbens Moench meth. pl. p. 523 (179. 1).

Linaria striata el L. monspessulana Dum.-Cours. bot. cult. ¿. p. 92, 195 (1802).

Linaria striata Cand. /l. fr. 3. p. 586 (1805). De Not. rep. A. lig. p. 300. Ard. cat. pl. Ment. p. 27. Car. prodr. 1. tosc. p. i7s. Fl. Monlecr. p. 27. Ard. Al. Alpes-mar. p. 281. Ces. Pass. Gib. comp. 1l. ital. p. 318. Arc. comp. fl. ital. p. 511.

Linaria repens Willd. en. pl. horl. berol. ?. p. 6 \%) (1809, non Mill.). Zum. /. ped. 1. p. 2)11.

Figure. - Reich. ic. bot. 1. 199. 1c. 12. germ. 20.1.69. 1.2.

Stazione, Abitazione e Fioritura. - In luoghi aridi sassosi de'monti del Piemonte e della Liguria: in val l' Ossola, e lungo il 
fiume Atison (Bir.!), alla Dora presso Torino (Re), intorno a Susa (Parl.! ecc.), nel Cenisio (Delponte! ecc.), a Oulx (Parl.!), Bardonecchia, Rochemolle (Beccari!), ad Andona, e Valdieri, a Limone, al col di Tenda a $1900 \mathrm{~m}$. (Parl.!), a Tenda (Ungern-Sternberg!), comune nel Nizzardo (Ard.), cosi a Nizza (Barla! ecc.), e a Mentone (Ard.), ai Piani presso Porto Maurizio (Ricca!), nelle Viosenne (Bert.), in quel d'Oneglia e d'Albenga, frequentissima (De Not.). Nasce inoltre nell' isola di Montecristo (Watson-Taylor!). Il Nyman (Consp. fl. eur. p. 539) l' indica pure di Lombardia. Fiorisce da giugno e luglio all' autunno.

Distribuzione geografica. - Nell' Europa occidentale, dạll' Irlanda alla Spagna, e per la Francia, la Svizzera e l'Italia sino in Dalmazia.

Descrizione. - Radice fusiforme, torta, fibrillosa. Fusti ramo. si, eretti, duretti, cilindrici, rossicci in basso, rami eretto-patenti. Foglie molli, conferte, eretto-patenti, strette, lineari, ottusette, glauche, leggermente solcate di sopra, di sotto quasi piane in basso $\mathrm{e}$ in alto convesso-carenate: foglie superiori più strette e più corte. Fiori in racemi stretti e piuttosto fitti in alto dei rami e del fusto. Pedicelli eretto-patenti, poco più corti del calice nel fiore e quasi uguali nel frutto, cilindrici, accompagnati da una brattea la quale è quasi uguale ad essi nel fiore e poco più corta nel frutto, stretta, lineare, acuta, glauca. Calice diviso profondamente in cinque lacinie strette, lineari-lanceolate, quasi acute, fornite nel dorso di una carena, glauche, eretto-patenti. Corolla piccola, poco piủ del doppio piủ lunga del calice, di un bianco-sudicio con vene violette più manifeste verso la base ossia nel tubo e con le sporgenze o gibbosità del palato gialle. Tubo lunghetto, lungo quanto il calice; sprone corto, lungo appena quanto il tubo, diritto, ottuso, grossetto. Labbro superiore diretto in alto ed in avanti, bilobo, con i lobi tondeggianti, concavi di sotto, dove si vedono bene le vene violette. Labbro inferiore poco più lungo del superiore, trilobo, con i lobi tondeggianti, l' intermedio poco meno largo, tutti un po' curvati in su, con le gibbosità del palato di un giallo dorato: peloso, barbato di sopra, peli alcuni gialli, altri bianchi. Stami 4 , rinchiusi, due più alti, due più bassi, inseriti in basso del tubo. Filamenti ingrossati un po' in basso, ivi verdognoli e papillosi per papille lunghette, quasi coniche e bianche, quasi cilindrici nel resto, e di color violetto chiaro e glabri, però appena più grossi verso alto: sono un po' curvąti in basso e all' apice. Antere bislunghe, uniloculari per la confluenza 


\section{LINARIA.}

delle logge, gialliccie e pallide, glabre, con una riga violacea e quasi amarante lateralmente alla fessura in modo che vi sono due righe violette longitudinali in ciascuna loggia. Polline gialliccio. Pistillo che giunge in mezzo agli stami, tra $i$ superiori e gl' inferiori. Ovario piccolo, quasi tondo, inserito sopra un disco grosso, verdognolo, angolato con gli angoli grossi ed ottusi: è glahro, verdognolo. Stilo eretto, un po'curvo, appena più grosso in alto, di color quasi violetto, glabro. Stimma quasi a capolino, papilloso, grossetto, gialliccio-verdognolo. Cassula quasi tonda, schiacciata lateralmente e in sII, con un solco longitudinale in ciascuna faccia, smarginata all' apice, gialliccia: si apre per sei denti corti, larghi e quasi troncati. Semi quasi triangolari, nericci, rugulosi. (Parl.ms., descr. della pianta di Valdieri).

Osservazione. - Non saprei cosa sia la Linaria slriala di Tenore, di Potenza, Ginosa, Martina (Syll. p. 303).

\section{Hinaria capraria.}

L. glaberrima, glaucescens, caulibus teretibus, basi ramosis, ramisque brevibus, decumbentibus vel ascendentibus, foliis crassiusculis, linearibus vel lineari-lanceolatis, acutiusculis, subtus obsolete uninerviis, inferioribus $3-4$ raro $5-6$ verticillatis, superioribus sparsis, ramorurn baseos minoribus, floribus laxe racemosis, racemis brevibus demum subelongatis, bracteis lanceolato-linearibus, acutiusculis, pedicello subæqualibus, pedicellis erecto-patentibus calyce subæqualibus, calycis subcampanulati laciniis linearibus vel lineari-lanceolatis, acutis, apice patulis, corolla calyce multo longiore, labio superiore erecto bilobo, lobis rotundatis dorso apice adpressis, labio inferiore patenti-dellexo, trilobo, lobis brevibus, rotundatis, intermedio lateralibus paulo minore, staminibus didynamis, filamentis basi pilis brevibus adspersis, antheris subhorizontalibus, glabris, pistillo stamina breviora superante, ovario subgloboso, disco annulari insidente, stylo apice incurvo, stigmate subcapitato, papilloso, capsula subglobosa, emarginata, utrinque unisulcata, calice subæquali aut paulo breviore, seminibus (nigricantibus) subtriquetris, angulis acutis, submarginatis, faciebus rugosis, lievibus. (Parl. ms.)

Linaria capraria Mor. De Not. n. Capr. p. 9S. Car. prodr. A. tosc. p. 478. Ces. Pass. (iib. comp. 11. ilal. p. 319. Arc. comp. П. ital. p. 511 . 
Antirrhinum caprarium Bert. fl. ilal. 6. p. 362.

Figura. - Mor. De Not. o. c. t. 3. f. 2.

Stazione, Abitazione e Fioritura. - Sulle rupi e sui muri delle isole dell'Arcipelago toscano: nella Gorgona (P. Savi!), nella Capraia alla città (Requien!), nell'Elba a Portoferraio (Bert.), al Volterraio, al Frasso (Durando!), alla Torre della Regina (Beccari!), alla Madonna di Monserrato (Caldesi!) ecc., nella Pianosa al Marchese e alla Cala dei Turchi (Simonelli!). Fiorisce da aprile e mag. gio a settembre.

Distribuzione geografica. - Pianta propria di quelle isole.

Descrizione. - Pianta glaberrima e glaucescente. La radice è grossotta, tortuosa, di un bianco sudicio, fibrilloso-ramosa e manda dal collo dei rami delicati, semplici o ramosi alla base, corti. I fusti sono decumbenti o ascendenti, alti da 2 a 4 decimetri, rugosi in basso, lisci nel resto, cilindrici, duretti, vestiti di foglie come i rami della base. Le foglie del fusto sono carnosette, ${ }^{7}$ lineari o lineari-lanceolate, quasi acute, intere, con un leggiero nervo longitudinale nella pagina inferiore; le inferiori di esse sono per lo più a quattro a quattro o a tre a tre, di raro a cinque o sei, le superiori sono opposte o quasi opposte o sparse; quelle dei rami della base sono piủ piccole: tanto queste quanto quelle sono spesso rivolte in su ossia quasi seconde. I fiori sono da 7 a 12 , distribuiti in un racemo piuttosto rado, che si allunga alquanto dopo la fioritura, accompagnati da una brattea carnosetta, lanceolato-lineare, acuta, uninerva e lunga quanto il pedicello: questo è cilindrico, eretto-patente, quasi uguale al calice o poco più corto di esso. Il calice è quasi campanulato, profondamente quinquefido con le lacinie quasi uguali, lineari o lineari-lanceolate, alquanto acute, fornite nei margini e nella pagina interna di qualche raro pelo, glandolose all'apice, patule all' apice, quasi nguali alla cassula o poco più lunghe di questa. La corolla è molto più lunga del calice, di color violetto, più carico nel labbro superiore, meno nei lobi dell' inferiore e quasi bianchiccio nella prominenza di questo e nello sprone. Il labbro superiore è eretto, bilobo, con i lobi tondeggianti e toccan. tisi con la pagina loro posteriore; il labbro inferiore è più largo, patente e rivolto un po'in giù con i lobi tondeggianti, l'intermedio dei quali è poco più piccolo dei laterali: talvolta questi lobi sono appena smarginati; il palato è molto prominente, e nella parte superiore ha dei peli grossetti e lunghetti di colore violetto. Lo sprone è stretto, lesiniforme, un po'curvato, acuto, poco più lungo della 
parte superiore della corolla. Gli stami sono didinami. I filamenti sono di color violetto chiaro, cilindrici, quasi diritti ma un po' curvati all'apice ed hanno verso la base, segnatamente i due piủ lunghi, alcuni corti peli quasi orizzontali. Le antere sono piccole, gialle o sfumate in parte di violetto, quasi orizzontali, ottuse all' apice, bilobe alla base, uniloculari per la confluenza delle due logge. Il polline è giallo. Il pistillo giunge fino a superare gli stami più corti in modo che lo stimma si vede in mezzo alle quattro antere. L'ovario è quasi tondo, verdognolo e in parte violetto, con qualche raro pelo glandoloso all'apice verso alto, e siede sopra un disco annulare, grossetto, verdognolo e glabro. Lo stilo è cilindrico, vio. letto, curvato all' apice in avanti, diritto nel resto, con qualclıe raro pelo glandoloso. Lo stimma è piccolo, quasi in capolino, bianco, papilloso, portato in aranti dell'apice curvato dello stilo. La cassula è quasi tonda, smarginata all' apice e con un solco longitudinale in ciascuna faccia, glabra, verdognola: si apre per due fori? presso l'apice e contiene molti semi nericci, quasi triangolari, con gli angoli acuti e quasi marginati e con le facce rugose e glabre. (Parl. ms., descr. di pianta coltivata).

Osservazione. - Alcuni sospettano che questa pianta sia una varietà della Linaria purpurea, alla quale è molto vicina.

\section{Linsria purpurea.}

L. erecta, glaberrima, foliis superioribus sparsis et inferioribus verticillatis, anguste lanceolato-acuminatis vel angustissimis linearibus imo filiformibus, pedicellis brevibus, lobis calycinis linearilanceolatis acutis, corollm purpurex $2 / 3$ centim. longæ calcare incurvato tubo duplo longiore, treto calyce duplo longiore, seminibus ovali-subtriquetris, rugosn-muriculatis.

Antirrhinum purpureum Linn. sp. plant. el. 1.p.6 6.9. Berl. A. ilal. 6. p. 359.

Linaria purpurea Mill. gard. dict. De Not. rep. M. lig. p. 999. Car. prodr. /l. losc. p. 477. Terr. /1. Vull. syn. p. 199. Lasil. /1. ves. p. 75. Terr. rel. Terr. Lat. p. I.6. Ces. el. piant. Maicll. p. 22. Terr.! lerza rel. p. zG. Groves! conlr. A. Terr. doir. p. Gi. Ces. Pass. Gib. comp. R. ital. p. Sis. Terr.! quarl rel. p. 109. Arc.! comp. 月. ilal. p. 510. Cib. Pir. suppl. R. Mod. p. 121. Nic. prodr. /l. mess. p. 3i\%. 
a robustior, foliis latioribus.

Linaria purpurea montana Car. l. c. Terr. fl. Vult. syn. l. c:

Figura. - Bot. mag. t. 99.

$\beta$ gracilior, foliis angustioribus.

Linaria purpurea littoralis $\mathrm{Car} . l$. $c$.

Figura. - Fl. grcec. $t .589$.

Stazione, Abitazione e Fioritura - In Sicilia e nella Penisola inferiore e media. In Sicilia ovunque nei luoghi boschivi montuosi (Guss.): Palermo a S. Martino, a Gibilmessa (Parl.!) e alla Pizzuta (Sorrentino!), Termini a S. Calogero (Parl.!), le Madonie sopra Isnello (Huet!), Castelbuono (Loj.!), Monticelli (Minà!), Mirto (Todaro!), Messina (Seguenza!), comune (Nic.), Mandanici (Aiuti!), Nicolosi (Tornabene!), Catania, sulle lave (Parl.!), Palazzolo (Bianca!). Nelia Penisola : in Calabria in luoghi erbosi a Palizzi, presso Scilla (Arc.!), S. Cristina e altrove (Biondi!); nelle messi presso Otranto (Groves!); sul Vulture, ovunque (Terr.), a Napoli, negli incolti!, in Capri (Kesselmayer! ecc.), in Terra di Lavoro a Caserta (Terr.), S. Donato, Collesammagno (Terr.!), Atina (Terr.); nel Lazio a Terracina (Fiorini!), intorno a Roma nei campi e per le vie, in copia (Seb. Maur.), cosi a Frascati (Parl.!), a Tivoli (Webb!) ecc., presso Viterbo sul monte Cimino (Parl.!) e a Canino (Bert.); in Umbria a Terni (Bert.); in Toscana al monte Argentario in più punti!, a Talamonaccio (Sant.), Castiglione della Pescaia!, Monte Pescali (Aiuti!), Torre S. Vincenzo, nel monte Calvi!, alla Cornata di Gerfalco (Bert.), a Rosia nel Senese!, quindi con notevole interruzione nelle alpi Apuane all'Altissimo, alla Corchia ecc. (Simi!), al Pizzo d' Uccello (Ad. Targioni!) e al Pisanino (Pucc.!), e nell'Appennino nelle regioni montana ed alpestre in parecchi luoghi dal Teso e da Boscolungo (Parl.!) a S. Pellegrino e all'alpi di Mommio (Calandrini!), dove termina; nell' altro lato orientale della Penisola occupa l'Abruzzo, dove abbonda ne'luoghi sassosi a 2-4000' alle radici della Maiella (Porta Rigo!) e del Morrone (Levier!), e trovasi ancora al Pizzo di Sivo e al monte de' Fiori (Parl.!), e si prolunga poi per le Marche ai monti Priore (Marzialetti!), e Regnolo (Bert.), in val Nerina presso Visso (Ricci!), al monte Birro (Bert.) e al monte S. Vicino (Narducci!) a 1100 metri (Bucci!), dove termina da quel lato. De Notaris l' indica di Nizza, manca però nella Flora di Ardoino. Fiorisce secondo $\mathrm{i}$ luoghi in aprile e maggio, $o$ in maggio e giugno, o in giugno e luglio, 0 in luglio e agosto, e nei luoghi piủ caldi da capo in autunno. 
Distribuzione geografica. - Specie italianà. Ė stata indicata inoltre in Grecia e in Tunisia.

Descrizione. - Glaberrima, glaucescens. Fibræ crassæ, fusiformes, flexuosæ, ex albido-luteolæ. Caulis teres, inferne purpureus. Rami steriles breves, pauci. Folia conferta, erecto-patentia, erecta vel adpressa, sessilia, lincari-lanceolata, acuminata, supra leviter sulcato-canaliculata, subtus nervo longitudinali concolori notata, marginibus integra et revoluta, levia. Flores parvi, racemosi, racemi in anthesi breves, in fructu elongati. Bracteolæ pedicello subæquales, lanceolato-lineares. Pedicelli in flore erecto-paten. tes, subrecurvi, glabri, flore 3-4plo breviores, in fructu crecti adpressive. Calyx parvus, rubellus, glaber, supra basin 5-partitus, laciniis subxqualibus, ovali-lanceolatis, acutis, dorso vix carinatis, patulis. Corolla parva, violacea, palato sæpe dilutiore; tubus latiusculus, rectiusculus; calcar subulatum, acutum, dependens, in. curvum, corollam subæquans. Labium superius erectum, bilobum, lobis ovalibus, obtusis, inferius majus, trilobum, lobis subæqualibus, ovali-orbicularibus, patentibus. Palatum prominens, bigibbosum, gibbis obtusis, supra ad fancem pilosum, pilis brevibus, subclavatis, violaceis. Stamina 4, didynama, sub labio superiore. Filamenta subulata, alba, glabra, crenata. Antheræ lutex, violaceo variegat $æ$, glabræ. Pollen luteum. Pistillum staminibus longioribus subbrevius. Ovarium ovato-orbiculare, apice emarginatum, viride, glabrun. Stylus longus, teres, glaber, albidus. Stigma obtusum, papillosum, e viridi-luteolum. Capsula calyce plus quam duplo longior, subglo. bosa, subdidyma, emarginata, viridis, glabra. Semina parva, subglo. boso-triquetra, transverse rugosa, fusca. (Parl. ms., descr. della pianta del monle S. Vicino).

Osservazione. - La diversita di aspetto, e la diversa abitazione in Toscana, mi avcvano altra volta fatto sospettare cho le due varictà di che sopra potessero essere specie distinte; ma un esame minuto di tutte le loro parti mi ha convinto non essere lifatti che varietà.

\section{Linurin chatepensis.}

L. erecta, glaherrima, foliis sparsis, infimis et ramorum sterilium verticillatis, anguste lineari-lanceolatis, pedicellis brevibus, Inbis calycinis angustissine lineari-acuminatis, corolle albidx ${ }^{2}{ }_{1}$ centim. 
longæ calcare incurvato tubo triplo longiore, treto calyce etiam dimidio breviore, seminibus ovali-angulatis, rugoso-scrobiculatis.

Antirrhinum chalepense Linn. sp. plant. ed. 1. p. 617. Bert. fl. ital. 6. p. 366 ; et auct.

Linaria chalepensis Mill. gard. dict.; et auct.

Figure. - Cyr. pl. rar. fasc. 2. t. 9. Reich. ic. fl. germ. 20. t. 66. f. 2, 3 (malce quoad semen).

Stazione, Abitazione e Fioritura. - Qua e là per i campi di molta parte d'Italia. Nell'Alta Italia fa nel Bresciano a Toscolano, rarissima (Zersi), nel Veronese nei colli di S. Leonardo, in val Policella verso l' Ospedaletto e Masurega (Poll.), a Guastalla (Goiran!), verso Garda e Torri a 240-800' (Rigo!); negli Euganei (Trev.); nel Friuli presso Duino (Pir.) e Monfalcone (Pir.!); in Istria presso Trieste a Contovello (Bert.), a Fiume (Koch), Dignano, Fasana, Stignano, Pola e in Brioni (Freyn). In Liguria è stata trovata a Nizza nel monte Calvo, a S. Agnese e Mentone, rara (Ard.), verso Briga (Colla), presso Genova nella valle di Borzoli (Carrega!), e sul monte Gazzo (Beccari!), a Chiavari (Bert.). È comune attorno a Firenze, e altrove in Toscana è stata trovata a Monsummano!, Volterra (Bert.), Radda, nonchè nelle isole dell' Elba a Rio (Beccari!) e a Portoferraio (Watson-Taylor!), e di Capraia (Mor. De Not.). Nelle Marche nasce a Macerata (Bert.) e a S. Lucia presso Tolentino (Narducci!). Fa presse Roma a Piofreddo (Seb. Maur.) e a Tivoli (Rolli!); in Terra di Lavoro a Cassino, Collesammagno e Sangiovanni Incarico (Terr.); in Ischia (Guss.); in Basilicata a Melfi (Terr.) e Maratea (Ten.); in Puglia a Bari, Conversano, Brindisi, Lecce (Ten.), Otranto (Groves!), Ginosa (Ten.), Zittarella (Aiuti!); in Calabria (Bert.). Fa in Corsica a Bastia, rara (Mars.), a S. Fiorenzo (Mabille!), comune, e a Rogliano, comune (Mars.). In Sardegna, rara (Mor.): Nurri, Laconi, Arizzo (Mor.), Pula (Bert.). In Sicilia: Palermo a Marineo (Todaro!), Castellobuono, Petralia, Gauci, le Madonie, da Trapani a Noto, e da Noto a Catania (Guss.), a Palazzo Adriano(Bert.), Delia (Guss.), Terranova (Parl.!), Caltagirone (Tar. Gerb.), Avola (Bianca!), Siracusa (Cassia!), Lentini (Huet!), Taormina (erb. Webb !), Salina, Panaria (Loj.), Ustica (Calcara!). Trovasi infine in Malta (Grech!). Fiorisce secondo i luoghi da marzo a giugno.

Distribuzione geografica. - Nell' Europa meridionale, nell' Asia occidentale fino in Persia.

Descrizione. - Pianta annua, alta da 5 centimetri a 5 decimetri, glaucescente, glabra. La radice è fusiforme, duretta, tortuosa, 
ramosa, bianchiccia. Il fusto è eretto, un po' flessuoso o flessuoso verso alto, quasi cilindrico, semplice o poco ramoso verso alto, con i rami eretto-patenti, corti; alla sua infima base nel punto dove principia la radice manda dei rami sterili, corti, ascendenti. Le foglie di questi sono quaterne o terne e le superiori spesso opposte, ovvero tutte opposte, patenti o patentissime, reflesse, carnosette, bislunghe-lanceolate, ottusette, intere nei margini, piane o appena solcato-concave di sopra, con un nervo longitudinale poco rilevato di sotto, di un verde glaucescente più chiaro delle foglie del fusto: queste sono anch' esse carnosette, le inferiori per lo piủ quaterne 0 terne, le altre quasi opposte e per lo piu alterne, tutte cretto-patenti o erette, lineari-lanccolate, ottusette, scanalato-concave di sopra, con un nervo longitudinale un po' rilevato di sotto che le rende quasi carenate, quasi glauche. I fiori sono distribuiti in un racemo all' apice del fusto e dei rami: un po' lontani tra loro. I pedicelli sono eretto-patenti o patenti, un po'nutanti, più corti del calice, verdognoli, ed hanno alla basc una bratteola lineare-setacea, verdognola, poco più lunga del pedicello. Il calice è verde, quasi glabro o con pochi peli glandolosi all' apice, i quali talvolta si vedono nelle bratteole e nei pedicelli, profondamente diviso in cinque lacinie fornite di una leggiera carena nel dorso, quattro delle quali sono lineari-lanceolate, acute, lunghe quasi quanto la corolla e patenti, la quinta o superiore piu piccola, lineare, acuta, quasi eretta. La corolla è bianca con il tubo di color violetto assai chiaro: questo è un po' curvo, larghetto, un po' schiacciato lateralmente. Il labbro superiore è diviso profondamente in due lacinie lunghe, lineari, ottuse, un po'divergenti 0 diritte in alto, il labbro inferiore $\dot{e}$ poco piủ lungo del superiore, trifido, con le lacinie uguali, dirette un po' in giù, lineari, larghette, quasi troncate all' apice e smarginate: verso la gola ha due gibbosita che la chiudono: in questa sono molti peli lunghi, grossi, bianchi, quasi una barba dalla parte del labbro inferiore; lo sprone è lungo, lesiniforme, un po' curvo, diretto in giủ, bianco. Gli stami sono quattro, due poco più corti degli altri due, tutti inclusi, poco più corti del tubo, inseriti verso la base di questo. I filamenti sono quasi diritti, lesiniformi, glabri, bianchicci. Le antere sono didime, aprentisi per una fessura comune in una sola loggia, gialle, glabre. Il pistillo è lungo quasi quanto gli stami piủ lunghi. L'ovario è tondo, verdognolo, gla. hro, circondato alla base da un disco a guisa di un anello verdo. gnolo. Lo stilo ¿̇ poco più lungo dell'ovario, quasi diritto, fili- 
forme, di color violetto chiaro, glabro. Lo stimma è papilloso, gialliccio, ottuso. (Parl. ms., descr. della pianta Fiorentina).

\section{Tinaria heterophylla.}

L. erecta, glabra racemo piloso, foliis sparsis, angustissimis subfiliformibus, ramorum sterilium autem brevioribus latioribus verticillatis, pedicellis brevibus, lobis calycinis anguste lanceolatis, acutiusculis, corollæ luteæ 1 centim. longæ calcare rectiusculo tubo 2-triplo longiore, treto calyce subæquali, seminibus ovali-angulatis, anfractuoso-rugosissimis.

Linaria heterophylla Desf.! fl. atl. 2.p. 48 (1800), non Spreng. syst. veg. 2. p. 790 (1825).

Antirrhinum aparinoides Willd. sp. plant. 3. p. 247 (ubi syn. Desf. citatur) (1800). Bert. fl. ilal. 6. p. 355.

Linaria stricta Guss.! pl. rar. p. 250 (1826). Tar. Gerb. cat. pl. calat. p. 30. Ces. Pass. Gib. comp. fl. ital. p. 348. Arc.! comp. fl. ital. p. 510. Nic. prodr. fl. mess. p. 347.

Linaria aparinoides Chav. mon. p. 138 (1833).

Figura. - Desf. o. c.t. 140.

$\beta$ humilis.

Linaria stricta var. humilis Guss. fl. sic. prodr. 2. p. 166.

Linaria humilis Guss. fl. sic. syn. 2. p. 123.

Linaria supina Bert. o. c. p. 354 (quoad pl. siculam).

Stazione, Abitazione e Fioritura. - Nei campi, e in luoghi incolti asciutti dell' Italia più meridionale. In Sicilia nasce ovunque (Guss.), per esempio a Palermo (Todaro! ecc.), alla Ficuzza (Lojacono!), nelle Madonie (Calcara!), a Messina!, Giardini, Acireale, Catania, sull' Etna (Parl.!), a Caltagirone (Tar. Gerb.), a Vittoria (Aiuti!), Licata, Sponti (Bert.). Nasce poi in Calabria a Villa S. Giovanni, Reggio!, Capo dell' Armi (Guss.). La var. nella Sicilia più meridionale a Comiso, Dorillo, Vittoria (Guss.!). Fiorisce tutto l'anno.

Distribuzione geografica. - Inoltre in Algeria.

Descrizione. - Pianta di un verde glaucescente, alta sino a 6 o 8 decimetri. Radice grossa, fusiforme, tortuosa, ramosa, fibrillosa, bianchiccia. Fusti molti, eretti o ascendenti, semplici, quasi cilin. drici, verde-glaucescenti. Rami sterili corti, decumbenti. Foglie dei rami sterili molte, avvicinate tra loro, quelle dei fusti in basso 
avvicinate tra loro e quasi in giro, le superiori meno vicine, solitarie e gradatamente più corte: tutte, anche nei rami sterili, erette, strettamente lineari, le inferiori piủ larghette e nei rami sterili quasi lineari-lanceolate, scanalate di sopra, convesso-carenate di sotto, ottuse, quasi carnosette, le superiori sono piủ strette e quasi filiformi. I fiori sono molti, piuttosto grandicelli, in un racemo piuttosto fitto, quasi conico dapprima perchè si svolgono prima i fiori inferiori. La raclide è pubescente con i peli orizzontali bianchi, glandolosi all' apice. I pedicelli sono quasi uguali o poco piủ corti del calice, eretto-patenti, cilindrici, verdognoli, pubescentiglandolosi come la rachide; alla base degl'inferiori è una brattea; poco piủ lunga del pedicello o quasi uguale a questo, bislunga-lanceolata, oltusa, convesso-carenata nel dorso ed ivi con una linea verdognola, biancliccia nel resto, cigliato-glandolosa con i peli radi nel margine. Il calice è diviso fin quasi alla base in cinque lacinie, quasi eguali, erette e quasi avvicinate al tubo della corolla, bislın. ghe-lanceolate, oltuse, convesso-carenate verdognole e pubescentiglandolose nel dorso, bianchicce nei margini ed ivi con ciglia piu abbondanti che nelle brattee. La corolla è di un giallo chiaro, quasi bianchiccio nel tubo e nello sprone, giallo-dorata nella gibbosità del labbro inferiore. II tubo è corto, un po' gonfio in basso, schiacciato lateralmente in alto, glabro, di sotto ha uno sprone diritto, lungo, lesiniforme, diretto in giủ, glabro. Il labbro superiore è poco piu lungo dell'inferiore, diretto in su, bilido, con le lacinie ovato-bislun. ghe, ottuse, convesse nel dorso e ivi con pochi peli lungli, bianchi e glandolosi, concave davanti. Il labbro inferiore è trilobo, con i lobi laterali un po' più grandi di quello di mezzo, tutti tondeggianti, di. retti in avanti ed in su, la gilbbosita o palato è grande, assai sporgente, ottusa retusa per un solco longitudinale verso alto, spesso con righe porporine, la gola i chiusa dal palato, pelosa nei margini grossi e ottusi e con due righe grosse, ottuse e longitudinali gialle e pubescenti-barbate. Gli stami sono 4, due piu alti, due poco piu bassi, racchiusi nella concavità del labbro superiore che corrisponde alla gola: $\mathrm{i}$ due più lunghi sono inseriti in basso del tubo della co. rolla, e i due piủ corti poco pii in alto. I filamenti dei primi sono grossi in basso e pubescenti, quelli dei secondi meno grossi in basso ed ivi glabri, tulti un po' ascendenti in basso, un po piu larghi verso l'apice, bianchi. Le antere sono ovali!, oltuse e appena smar. ginate all' apice, bilobe in basso, inserite nel dorso sotto dell'apice, introrse, con le logge aprentisi longitudinalmente ma con le fessure 
unite insieme in alto, gialle, glabre. Il pistillo è poco più corto degli stami piủ lunghi e poco più lungo dei due più corti. L' ovario è ovale-bislungo, un po' schiacciato da avanti in dietro, gialliccio, pubescente-glanduloso, con i peli corti diretti in su, è circondato in basso da un disco circolare, grossetto, verde, glabro. Lo stilo è il doppio piủ lungo dell' ovario, eretto, un po' curvato in avanti verso alto, cilindrico in basso e ivi con qualche raro pelo glandoloso, un po' più largo in alto, bianchiccio. Lo stimma è bifido, con le lacinie quasi uguali, un po' divergenti, corte, quasi acute, dirette in avanti, bianchiccie. La capsula è eretta, ovale, smarginata e quasi biloba 'all' apice, quasi glabra, scuretta. I semi sono molti, piccoli, neri, quasi uniformi, con sporgenze tonde disuguali poco rilevate e aventi piccolissimi tubercoli quando si guardano con una forte lente. (Parl. ms., descr. della pianta Messinese).

Osservazioni. - La pianta italiana è identica con quella dell'Algeria.

Boissier (Fl. orient. 4. p. 378) riferisce l'Antirrhinum strictum della Fl. grceca alla Linaria Sibthorpiana Boiss. Heldr.!, specie ben distinta dalla nostra $L$. heterophylla.

La var. ha un po' l' aspetto della $L$. supina, con la quale la confuse il Bertoloni; ma i suoi caratteri sono tutti della specie, di cui è piuttosto una forma nana che una varietà.

\section{Linaria genistifolia.}

L. erecta, glaberrima, foliis sparsis, lanceolato-acuminatis, in. tegris, pedicellis brevibus, lobis calycinis lanceolatis, acutis, corollæ luteæ $1-1 \frac{1}{2}$ centim. longæ calcare rectiusculo tubo æquali vel dimidio longiore, treto calyce subæquali, seminibus ovali-triquetris, rugulosis.

Antirrhinum genistifolium Linn. sp. plant. ed. 1.p. 616.

Antirrhinum linifolium Linn. o. c. ed. 2. p. 858.

Linaria genistifolia Mill. gard. dict. Ces. Pass. Gib. comp. $\{$. ital. p. 348. Boiss. fl. or. 4. p. 37\%. Arc. comp. fl. ital. p. 510.

Linaria linifolia Reich. ic. bot. 5. p. 14. Ces. Pass. Gib. l. c. Arc. l. c.

Figure,-Bol. mag. t. 2183. Reich. o. c. t. 422, 435. Ic. fl. germ. 20. t. 65. f. 1 .

Stazione, Abitazione e Fioritura. - Rarissima. Trovasi in 
Piemonte e Lombardia, secondo Arcangeli, a Novara, secondo Reichenbach (Fl. germ. exc. p. 374). Nasce nella Piviera di ponente (Ricasoli!); e in Calabria a Corregliano nei colli arenosi granitici a 3-400 metri (Huter Porta Rigo!). Fiorisce in giugno.

Distribuzione geografica. - Nell' Europa orientale media e me. ridionale, nell'Asia Ninore, in Songaria.

$\uparrow \dagger$ Semina applanata margine alato.

\section{Linaria Pellicierana.}

L. erecta, glaberrima, foliis sparsis, angustissime linearibus, ramorum sterilium verticillatis brevioribus oblongis, pedicellis brevibus, lobis calycinis lanceolato-acuminatis, corollæ violace 1 centim. longæ calcare rectiusculo tubo duplo longiore, treto calyce parum breviore, seminibus rotundatis applanatis, laciniato-ciliatis.

Antirrhinum Pellisserianum Linn. sp. pl. ed. 1. p. 615. Bert. f. ital. 6. p. 364 .

Linaria Pelisseriana Mill. gard. dict. Dum-Cours. bot. cult. 2. pag. 93. Cand. R. fr. 3. p. 589 (1805). De Not. rep. A. lig. p. 299. P. Sav. R. gorg. p. 273. Koch syn. fl. germ. ed. 2. p. 600. Rota! prosp. piant. Pav. p. 269. Guss. enum. pl. Inar. p. 238. Mor.! fl. sard. 3. p. 212. Ard.cal. pl. Ment. p. 26. Tassi f. prov. sen. p. 50. Car. prodr. 11. tosc. p. 478. Fl. Montecr. p. 27. Ard. R. Alpesmar. p. 281. Pasq. R. ves. p. 75. Terr. R. Vult. syn. p. 129. Genn. 1l. Capr. p. 114. Mar's cat. pl. Cors. p. 107. Terr. rel. Terr. Lav. p. 146. Terz. rel. p. 76. Groves! contr. A. Terr. d'0tr. p. 6.4. Ces. Pass. Cib. comp. 1I. ital. p. 318. Freyn A. Sud-Istr. p. 390. Loj. is. eol. p. 121. Arc.! comp. R. ilal. p. 510. Nic. prodr. 1l. mess. p. 317. Mil. suppl. fl. alp. vers. p. 26.

Figure. - Barr. ic. 1162 . Fl. greec. t. 59\%. Reich. ic. R. germ. 20. t. 62. f. 1.

Stazione, Abitazione e Fioritura. - Nei campi asciutti, in luoghi sterili ece., di quasi lutta l’Italia. Rarissima nell' Alta Italia, vi nasce solamente presso Pavia a S. Cristina, Campo Rinaldo, Motta Visconti (Noce. Balb.), Carbonara (liota!), e in Istria a Trieste (Bert.), e molto sparsa poi sotto llignano (Fregn). In Liguria seguita ad essere rara: Nizza (Barla! ecc.), hocchetta, Mentone (Ard.), Tenda (Bourgeau!), Albenga (l)e Not.), Albissola marina (Picione!). In Toscana si fa assai piú frequente, dalla regione maremmana alla 
montana: Massa (Bert.), la Versilia in parecchi punti (Simi!, Mil.), la valle del Serchio da Massarosa e dai contorni di Lucca (Calandrini! ecc.) ai Bagni (Parl.!), a Tereglio (Giannini!) e a Barga (Ball!), monte Pisano (Savi! ecc.), Fucecchio, il Fiorentino in molti punti, Vallombrosa!, Arezzo, val Tiberina (Bert.), Siena (Tassi), S. Vincenzo (Parl.!), monte Calvi (Bert.), monte Argentario (Parl.!), isole di Gorgona (P. Sav.), di Capraia (Mor. De Not.), dell' Elba (Bert.) e di Montecristo (Watson-Taylor!). È comune nel Romano (Seb. Maur., ecc.), e si ritrova a Porto d'Anzio (Rolli!), e piủ giù a Sora, Roccasecca, Mondragone (Terr.), e piủ giù ancora a Napoli (Ten. ecc.) e in Capri (Pasq., Bert.) ed Ischia (Bolle! ecc.). Dall' altro lato della Penisola è stata trovata in Puglia a Vico (Porta Rigo!), Tressanti (Bert.), Brindisi (Webb!), Otranto (Groves! ecc.), e in Basilicata a Melfi (Terr.). Fa poi in Calabria a Rossano, Cosenza (Aiuti!), Pizzo (Ricca!), Monteleone (Arc.!), Serra S. Bruno (Zwierlein!), Palmi (Arc.!). Occupa infine le grandi isole di Corsica, nella regione bassa e nelle parti calde della regione media (Mars.): Ostriconi (Soleirol!), Calvi (Bert.), Corte, Bonifacio (Requien!), isola Cavallo (Kralik!); di Sardegna, dov'è comune (Mor. !): Caprera, S. Stefano (Genn.), Pula (Bert.); e di Sicilia: intorno a Palermo in più punti (Parl.!, Guss. ecc.), a Cefalủ (Parl.!), Mistretta, Milazzo (Guss.), Messina (Seguenza!), sull'Etna (Guss.), a Catania (Webb!), Siracusa, Noto, Comiso, Vittoria, Terranova ecc. (Guss.), nelle isole Eolie, comunissima (Loj.), per esempio a Lipari (Mandra. lisca!). Fiorisce in maggio e giugno, 0 in aprile e maggio.

Distribuzione geografica. - Nell'Europa meridionale e in parte nella media, nell'Asia occidentale fino al Transcaucaso.

Descrizione. - Pianta annua, alta 3 decimetri e più, di un verde chiaro; glabra. La radice è delicata, flessuosa, bianchiccia. Il fusto è eretto, appena flessuoso, duretto, cilindrico, rossiccio un po' alla base, di un verde chiaro nel resto, semplice, 0 avente alla base uno o due rametti sterili, corti, lunghi da 3 a 8 centime. metri, giacenti per terra. Le foglie di questi rami sterili sono in giro a tre a tre, o a quattro a quattro, con i giri un po' lontani, sono carnosette, eretto-patenti o patenti, ovali-lanceolate, un po'strette alla base come in un picciòlo assai corto, quasi acute all' apice con un leggiero solco longitudinale di sopra verso la base, piane di sotto; quelle del fusto sono solitarie, un po'lontane, erette, lineari, ottusette, solcato-scanalate di sopra, convesso-carinate di sotto. I fiori sono pochi, in un racemo terminale, dapprima corto, 
poi lungo e con i fiori alquanto lontani. I pedicelli sono cilindrici, eretto-patenti nel fiore, eretti nel frutto e allora un po' piủ lunghi di questo, quasi uguali o poco più lunghi del calice, rossicci, accompagnati da una brattea la quale è lunga quanto il pedicello, avvicinata a questo, lineare-lanceolata, acuta, rerdognola con il margine un po' rossiccio. Il calice è profondamente diviso in cinque lacinie quasi uguali, eretto-patenti, lanceolato-lineari, acuminate, convesse e verdi nel dorso, trasparenti e rosee o rossicce nel margine verso la base ch'è ivi larghetta. La corolla è piủ del doppio piủ lunga del calice, di color violetto-chiaro, più chiaro e con linee in rete piu scure nelle gibbosità del labbro inferiore che chiudono la gola. II labbro superiore è eretto, bislungo, bilobo, con i lobi quasi lineari, ottusi, ha rivolti in dietro i margini esterni, come pure l'apice dei suoi lobi. Il labbro inferiore è molto piủ corlo, patente, trilobo, con i lobi quasi quadrati, i due laterali un po' più grandi del medio ch' è un po'smarginato; il labbro medesimo ha due grandi gibbositả rivolte in su che chiudono la gola: questa è pelosa dalla parte del labbro inferiore in mezzo alle due gibbosità. Lo sprone è lungo quanto la corolla, lesiniforme, diretto in giù. Gli stami sono quattro, due un poco più corti degli altri, racchiusi nella gola e inseriti alla base del tubo della corolla. I filamenti sono larghi e quasi conici alla base ed ivi bianchicci e con peli grossi, conici e corti, sono quasi cilindrici nel resto, un po' curvi in dentro verso su, di un bianca roseo e glabri. Le antere sono scurette, verticali, aprentisi con una fessura longitudinale comune. Il polline è giallo. Il pistillo è lungo quanto gli stani piủ corti. L'ovario è quasi tondo, un po' schiacciato dalle parti, smarginato all' apice, glabro, con piccolissime glandole in alto, verdognolo, circondato alla base da un disco quasi anuulare, verdognolo. Lo stilo è più lungo dell' ovario, quasi cilindrico, quasi diritto, curvato all' apice in avanti, di color violetto chiaro, glabro. Lo stimma è ottuso, papilloso, bianchiccio. La cassula i eretta, tondo-didima, glabra, più corta delle lacinie del calice che sono persistenti ed erette, ha due logge, ciascuna delle quali si apre con sei valve disuguali. I semi sono orati n quasi ovali, schiacciati, punteggiato-spinosi e neri nelle facce, frangiatocigliati nei margini con le ciglia cinericce. (Parl. ms., descr. della pianta Fiorentina).

Osservazioni. - Il nome della pianta ricorla un vescovo Pellicier che prino la notó, e va perciò scritto come qui s'é fatto. Védi Martins, Jard. des $p l$. de .lontpellier. 
Gussone e Nicotra hanno una var. micrantha, dalla corolla uguale al calice, che sarà probabilmente una forma anormale, atrofica.

\section{Finaria arvensia.}

L. erecta, glabra cum inflorescentia glanduloso-pilosula, foliis sparsis et inferioribus verticillatis, angustissime linearibus, pedicellis brevissimis, lobis calycinis oblongo-linearibus, corollæ cœruleæ $1 / 2$ centim. longæ calcare subcurvato tubo æquali, treto calyce longiore, seminibus rotundatis applanatis, late alatis subauriculatis.

Antirrhinum arvense Linn. sp. plant. ed. 1. p. 614. Balb. el. piant. Tor. p. 14. Re fl. seg. p. 52. Bir. fl. acon. p. 213. Zer. fl. mel. thes. p. 42.

Linaria repens Mill. gard. dict. (non Willd.).

Linaria arvensis Desf. fl. all. 2. p. 45. Zan. prosp. fl. ven. p. 25. Mor. fl. sard. 3. p. 214. Ard. fl. Alpes-mar. p. 282. Mars. cat. pl. Cors. p. 107. Ces. Pass. Gib. comp. 1. ital. p. 348. Arc. comp. fl. ital. p. 510.

Figura. - Reich. ic. 1. germ. 20.t. 62. f. 3.

$\beta$ flaviflora, corolla lutea.

Antirrhinum arrense var. $\beta, \gamma$ Linn. l. $c$.

Antirrhinum simplex Willd. $s p$. plant. 3. $\mu$. 243. Bert. $\uparrow$. ital. 6. p. 358 .

Linaria simplex Cand. f. fr. 3. p. 588. De Not. rep. fl. lig. p. 299. Rota prosp. piant. Pav. p. 269. Mor. l. c. Ard. cat. pl. Menl. p. 27. Car. prodr. fl. tosc. p. 478. Ard. fl. Alpes-mar. l. c. Mars. l.c. Ces. Pas. Gib l.c. Arc.! l. c. Nic.! prodr. 凡. mess. p. 346.

Linaria arvensis var. simplex Chav. mon.

Linaria arvensis var. flaviflora Boiss. fl. or. 4. p. 375.

Figure. - Fl. graec. t. 590. Reich. l. c. f. 2.

Stazione, Abitazione e Fioritura. - In luoghi asciutti, qua e là per tutta l'Italia: Susa a Mlonpantero (Parl.!) e a Foresto (Re), Torino a Superga (Balb.) e a Cambiano (Chevalier!), il Novarese (Bir.), Pavia al Rio (Rota), Venezia (Zan.), pineta di Ravenna (Bert.), Tenda (Bourgeau!), Nizza (De Not.), Mentone ecc. (Ard.), Diano (Ricca!), Spotorno, Serravalle (De Not.). Firenze a monte Ceceri (Baroni!) e all' Impruneta (Bert.), Assergio (Orsini!) e Massa d'Albe (Levier!) in Abruzzo, Tavoliere di Puglia (Ten.!), la Basilicata (Ten.), la Calabria a Scilla (Arc.!) e a Reggio (Bert.), la Corsica a 
Bastia, a Calvi (Soleirol!), fra S. Colombano e Olmi-Cappella, ad Aiaccio, a Bonifacio, a Portovécchio (Nlars.), la Sardegna, comune (Mor.), la Sicilia a Palermo al monte Occhio, alla Pizzuta, a Busambra, a Cammarata (Guss.), nelle Madonie alla Colma grande (Parl.!) e al Pizzo dell'Antenna (Mlinà!), a monte Scuderi (Guss.), a Messina a Curcurace, ai Cappuccini e al Paradiso (Nic.!), inline Malta (Zer.). Fiorisce in marzo ed aprile nei luoghi più caldi, in maggio e giugno nei meno caldi.

Distribuzione geografica. - Nell' Europa meridionale e in parte nella media, nell' Affrica settentrionale, nell'Asia occidentale fino in Persia.

\section{Linaria parviflora.}

L. erecta, glabra cum inflorescentia glanduloso-pilosula, foliis sparsis et inferioribus verticillatis, lanceolatis, pedicellis brevissimis, lobis calycinis oblongo-linearibus, corollæ cœrulescentis $\%$ centim. longæ calcare brevissimo, treto calyce longiore, seminibus rotundatis applanatis, late alatis muriculatis.

Antirrhinum micranthum Cav. ic. descr. 1. p. 51.

Linaria parviflora Desf. A. all. 2. p. 4.4 (1800).

Antirrhinum parviflorum Willd. sp. plant. 3. p. 2.45. Bert. 1 . ilal. 6. p. 357 . .

Linaria micrantha Spreng. syst. veg. 2. p. 79.' (1825). Mor.! fl. sard. 3. p. 213.

Figure.-Cav. 0. c. 1. 69. f. 3. Desf. o. c. t. 137. Fl. grace. t. 597 .

Staxione, Abitazione e Fioritura. - In Sardegna, nei coltirati, nei pascoli (Mor.): Cagliari (Bert.), isola Tavolara (Mor.). A Foggra (Bert.). Fiorisee in marzo ed aprile.

Distribuzione geografica. - Nell' Europa merilionale, in Algeria, nell'Asia occidentale fino in Persia.

Osservazione. - Ho seguito Moris e livissier (Fl. orient.) nel tenere questa specie separata dalla Linaria arvensis; perỏ Dufour (in Bull. soc. bot. de Fr. 7. p. 49S) e Willhomm e Lange (Prodr. A. hisp. 2. p. 570) ve la riuniscono, e darvero che le rassomiglia mol. tissimo. 


\section{2\%. Ginaria bigunctatue.}

L. erecta, glaberrima, foliis sparsis et verticillatis, angustissime linearibus crassiusculis, pedicellis brevissimis, lobis calycinis linea'ribus obtusis, corollæ e luteo purpurascentis ${ }^{2} / 3$ centim. longæ calcare recto tubo duplo longiore, treto calyce æquali, seminibus rotundatis applanatis, anguste alatis levibus.

Antirrhinum bipunctatum Linn. sp. plant. ed. 1.p. 614. Ed.2. p. 853.

Antirrhinun glaucum Linn. amæen. acad. 4. p. 277. Sp. plant. ed. 2. p. 856 .

Linaria bipunctata Dum.-Cours. bot. cult. 2. p. 93 (1802). Spreng. syst. veg. 2. p. 795 (1825).

Linaria glauca Ait. hort. kw. 4. p. 15 (1812). Spreng. o. c. p. 796. Ces. sagg. geogr. bot. Lomb. p. 55. Ces. Pass. Gib. comp. fl. ital. p. 348. Arc. comp. A. ital. p. 511.

Figura. - Cav. ic. 1. t. 33. f. 2.

Abitazione. - Lombardia (Ces.).

Distribuzione geografica. - In Portogallo e Spagna.

Osservazioni. - Questa specie è ammessa nella flora italiana sulla sola autorità di C.esati. Reichenbach ( $F l$. germ. exc. p. 373) la segna di Nizza, senza dubbio a causa dell' Antirrhinum bipuncta. tum dato dall' Allioni ( $F l$. ped. 1. p. 66) di colà: ma la pianta di Allioni, che manca nel suo erbario (Mor. fl. sard. 3. p. 215), è dubbia per alcuni (De Not. rep. l. lig. p. 490), ed altri (Bert. fl. ital. 6. p. 358) la riportano alla Linaria arvensis.

$\dot{\mathrm{E}}$ questa una pianta primaverile, annua, bassina, gracile, glauca.

\section{Hinaria alpina.}

L. diffusa adscendens, glabra, foliis plerisque verticillatis, oblongo-linearibus brevibus, pedicellis brevibus, lobis calycinis li. nearibus, corollæ violaceæ palato croceo $1-1 \frac{1}{2}$ centim. longæ calcare rectiusculo tubo duplo longiore, treto calyce æquali, seminibus rotundatis applanatis, late alatis levibus.

Antirrhinum alpinum Linn. sp. plant. ed. 1. p. 615. Com. prodr. fl. com. p. 121. Bert. fl. ital. 6. p. 363. 
Linaria alpina Mill. gard. dict. Re f. tnr. 1. p. 323. Mass. prodr. fl. valt. p. 1'. De Not rep. A. lig. p. 299. Com. fl. com 5. p. 58. Ilausm. fl. Tir. p. 632. Rola prosp. 1l. Berg. p. 66. Pir.! fl. for. syll. p 10\%. Ard. fl. Alpes-mar. p. 28\%. Zersi prosp. piant. Bresc. p. 155. Ces. Pass. Gib. comp. 凡. ilal. p. 3.48. Paol. el. piant. mont. sib. p. 15. Arc.! comp. il. ital. p. 511 .

Figure. - Bot. mag. t. 205. Reich. ic. fl. germ. 20. t. 60. f. 4 .

Stazione, Abitazione e Fioritura. - Nei lunghi sassosi delle alte Alpi, da dove scende con le ghiaie de'torrenti: nel Friuli (Pir.!), per esempio al monte Canin (Bert.) e intorno ad Amaro, Tolmezzo, Forno (Poll.) ed Ampezzo (Hansm.), nel monte Cavallo (Kellner!), nelle Vette di Feltre (Venzo!), nei monti Grappa e Portole (Rert.), nel Baldo alle Acque negre e in val Losanı ecc. (Goiran!, Poll., Bert.); nel Tirolo in Taufers, nell' alpe Kerschbaum, a Prax, nel Wormserjnch, in valle di Laas, nell' alpe Zil presso Merano, nel Falgamaierjoch, ad Ifinger, nell'alpi Mendel, Schlern, Seiser e Rittner, in giủ presso Bolzano ma rara (Hausm.), in Fassa (Bracht!) e Fiemme, a Cavalese (Hausm.), in ral Sugana al Montalon (Ambro. si!), nel monte Toccio, nel Trentino in val Fersina (IIausm.), sulla Scanuccia e al col Santo (Poll.), e nell'alpi Gazza, Spinale e Naunia 'Perini!), nelle valli di Breguzzo, di Genora (Hausm.) e di Daone (Porta!), ecc.; nelle prealpi Bresciane, frequente, cosi nel. l'alpi di Vaia e nel monte Rondenino (Zersi), nelle prealpi Bergamasche (Rota), in val Camonica a Pisgana sopra 2000 metri e a Ponte di Legno (Parl.!), a Clusone (Bert.), a Branzi in val Brem. bana (Rampoldi!), per il Comasco, per esempio al Legnone (Com.), in quasi tutte le alpi della val Tellina (Mass.), cosi da Bormio a 1550 metri alla cima del Braulio a 29100 metri (Parl.!); sul Bernardino (De Not. !; nel Ticino in valle Ravona, nell' alpi di Robbiei, e di Naret, in valle Maggio, sul siliceo a 2800 metri (Franzoni!), sul Gottardo (Bert.); sul Sempione (Parl.!), sul monte Rosa al Grauenliaupt a $11000^{\prime}$ (Ball!), all' Ospizio di Valdobbia (Carestia!), e alla Betta Furke(Piccone!), sul S. Bermardo (Mercier!), a Courmayeur (Webb!), sul Cenisio al lago a 2000 metri (Arc.!), c presso le nevi eterne (Bucci!), sopra Susa, sopra Oulx (Aiuti!), e trovata una volta lungo la Stura presso Torino Re), nelle alpi Valıesi (Rostan!); ai colli di Giallorghe (Ard.) e del Clapier (Reccari!), e di Tenda al monte Formose (Bourgeau!), infine al monte Gioia (Gentili!). Ricomparisce nell' alto Appennino piceno-abruzzese, dov' è stata trovata nei mon. 
ti: della Sibilla (Sang.), Vettore, Corno (Orsini!), Costone, Intermesole, Amaro, Maiella, Focaleto (Ten.). Fiorisce in luglio e agosto.

Distribuzione geografica. - Dalla Spagna alla Transilvania.

Descrizione. - Pianta glauca, glabra, giacente, poi ascendente. Radice fibrosa, bianchiccia. Fusti ramosi sin dalla base, con i rami prolungati a guisa di fusti e come questi cilindrici, violettorossicci. Foglie carnosette, patenti, diritte o un po' curve, un po'scanalate di sopra, un po' convesse di sotto, con margini ed apice ottusi, ristrette alla base, per lo più a 4 a 4 in verticilli piani o quasi unilaterali. Fiori solitari all'ascella di una brattea lineare, patente 0 erettopatente, in un racemo corto, poi allungato nel frutto. Peduncoli eretti, cilindrici, glabri, rossicci, poco più lunghi o quasi due volte più lunghi del calice, più corti della brattea. Calice diviso profondamente in cinque lacinie un po' patule, lineari o lineari-allungate, ottuse, un po' convesse di fuori, glabre, verdognole e in parte violette verso la base. Corolla di color violetto vivace con le due gibbosità del palato di color ranciato, molto più grande del calice. Labbro superiore più-lungo dell' inferiore, diretto in alto, diviso fin quasi alla metà in due lobi quasi lineari, larghetti e tondeggianti all' apice. Labbro inferiore patente, trilobo, co' lobi quasi uguali, orbicolari, appena smarginati. Gola chiusa da due gibbosità ottuse, quasi lineari e verticalmente quasi parallele, barbate di dentro per folti peli di color ranciato e alcuni bianchi. Sprone discendente, ottusetto, lungo quanto il labbro superiore o poco più. Stami 4, didinami, nascosti in una concavità che il labbro superiore fa nella gola del fiore, inseriti nella parte superiore del tubo; filamenti cilindrici e grossi in basso, un po' schiacciati e un po' curvi in alto, di un violetto chiaro, glabri, i due degli stami più lunghi papillosi alla base. Antere bilo. culari, con le logge divergenti sin dalla base in alto e in basso, aprentisi per una fenditura longitudinale comune, gialle, glabre. Polline giallo. Pistillo poco più corto dei due starni più lunghi, in modo che lo stimma sta in mezzo alle 4 antere. Ovario quasi tondo, un po' schiacciato dalle parti, verdognolo, glabro, 2-loculare; ovoli affissi alle placente del setto? Stilo diritto, un po' curvo in alto, cilindrico, di color violetto-chiaro, glabro. Stimma quasi in capolino, papilloso, bianco, diretto in avanti. Cassola quasi tonda, glabra, verdognola, appena più lunga delle lacinie del calice persistenti e un po' patule, aprentesi con tre denti all' apice di ciascuna loggia, cioè in tutto con sei denti acuti. Semi vari, orbicolari, schiacciati, da una parte un po'convessi e dall'altra un po' concavi, con un 
margine larghetto un po' rilevato, finissimamente punteggiati quando si guardano con una forte lente. (Parl. ms, deser. della pianta del Braulio).

\section{æ๖. Munria supina.}

L. diffusa adscendens, glabra cum inflorescentia glandulosopilosa, foliis sparsis et rerticillatis, angustissime linearibus, pedicellis brevibus, lobis calycinis oblongo-linearibus obtusis, corollæ lutea 1-1 $1 / 2$ centim. longx calcare rectiusculo tubo duplo etiam lon. giore, treto calyce paullo longiore, seminibus rotundatis applanatis poculiformibus, late alatis levibus.

Antirrhinum supinum Linn. sp. plant. ed. 1.p. 615. Bert. A. ital. 6 p. 354 (excl. syn. Gussonei et pl. sic.).

Linaria supina Desf. fl. all. 2.p. 44. De Not. rep. f. lig p. 200. Rota prosp. piant. Pav. p. 269. Ard. 1l. Alpes-mar. p. 252. Ces. Pass. Gib. comp. fl.ital. p. 349 (excl.pl. sic.) Arc. comp. 月. ilal. p. 511. Figure. - Cand. ic. pl Gall. 1. II. Reich. ic. A. germ. 20. t. 60.f. 5 .

Stazione, Abitazione e Fioritura. - In luoghi arenosi o ghiaiosi dell' Italia superiore occidentale: nel Cenisio, in valle di Rochemolle sotto il col d'Ambin (Beccari!), comune nella Dora verso Exille e Susa (All.), presso Torino lungo la Stura a Veneria (Re) e nel Po a S. Mauro (Balb.), nei monti Valdesi (Rostan!), tra Casteldellino e Sampeire (Boiss. Rieut.!), in valle di Maira al laghetto delle Monache (Manuel!), al col di Tenda a 1900 metri (Parl.!), e in generale in quella regione montana, da dove scende fino a Scarena, Torretta ecc. (Ard.), sopra Viosenne (Ricca!), ad Alba (Bertero!), nel Novarese al Po e al Ticino, ed anche in val d'Ossola (Bir.), nel Pavese lungo il Po alla Bastida de' Dossi (Nocc. Balb.), ne' monti di Chiavari (De Not.), nel Parmense al monte Prinzera (Parl !). Finrisce in giugno e lugrlio, e sin da maggio ne' luoghi bassi.

Distribuzione geografica. - Nell' Europa occidentale, tanto meridionale che media, in Algeria.

Descrizione. - La radice $\dot{e}$ duretta, poco ramosa, bianca, glabra. Il fusto é ramosissimo dalla base e furma con i suoi rami dei graziosi cespuglietti alti da tre a quattro pollici: $\dot{e}$ cilindrico, glabro, glaueo, con qualclic sfumatura rosicicia, o interamente rossiccio verso il basso. Le foglie sono opposte, o nate a tre o quattro in giro, e nelle parti superiori dei rami anche sparse, i giri sono 
vicini tra loro perchè gl'internodii sono assai corti. Tali foglie sono patenti, lineari, acute, carnosette, glabre, un poco convesse diso. pra e ivi di un verde pallido e glauche, con un solco longitudinale bianchiccio di sotto. I rami si diramano anch'essi superiormente, e questi rametti sono pelosetti con i peli orizzontali e glandolosi al. l'apice e portano i fiori, i quali sono da 2 a 6 distribuiti in un racemo poco denso. Ciascun fiore ha un peduncolo cilindrico, glauco come il resto della pianta e pelosetto, ed è accompagnato da una brattea simile alle foglie ma più piccola di queste, perỏ poco più lunga del peduncolo. Il calice è diviso in cinque lacinie disuguali, essendo la superiore di esse la piủ lunga e le altre gradatamente più corte da una parte e dall' altra: queste lacinie sono lineari-allungate, ottusette, verdognole, pelosette di fuori con i peli glandolosi all'apice. La corolla è gialla con il palato quasi dorato, e con lo sprone più pallido avente alcune righe longitudinali sottili amarante, delle quali le intermedie giungono fino all' apice dello sprone e le laterali descrivono una curva per tornare alla base dello sprone dalla parte superiore di questa. Il tubo della corolla è corto. Il labbro superiore è lungo il doppio del calice, bilobo, con i lobi quasi tondi all'apice, ha di sotto una linea sporgente longitudinale che divide la pagina inferiore di esso in due piccole cavità allungate, è glabro eccetto nella pagina superiore dove ha qualche raro pelo. Il palato è pro. minente, chiude la gola, la quale ha lateralmente e in avanti molti peli lunghetti, fitti, bianchi, diretti in avanti. Il lobo inferiore è leggermente trilobo, con i lobi laterali patenti e un poco diretti in giủ in avanti e con il lobo intermedio poco più piccolo, smarginato . e diretto un poco in giù. I filamenti sono filiformi, bianchi, pelosopapillosi alla base. Le antere sono gialle, allungate, biloculari. Il polline è giallo, piccolissimo, ellittico. L'ovario è quasi tondo, di color verde pallido, con qualche pelo glandoloso verso alto, biloculare. Gli ovoli sono molti e piccoli, inseriti sulle placente unite al setto. Lo stilo è lungo, filiforme, glabro, bianco. Lo stimma è sem. plice, ottusetto, gialliccio. La capsula è quasi tonda, appena schiac. ciata, membranacea, verdognola, poi bianchiccia, avente in alto il resio dello stilo persistente e circondata dalle lacinie del calice. Si apre in due logge quasi uguali, ciascuna delle due valve divisa in tre valvette minori che giungono fin quasi alla base: rimane allora il setto distinto e verticale. I semi sono pochi in ciascuna loggia, bianchicci, discoidei, concavi, con il margine trasparente. (Parl. ms., descr. della pianta del Parmense). 
Osservazione. - La pianta di Sicilia scambiata da Bertoloni per Linaria supina è invece una forma della $L$. heterophylla.

\section{4). Linaria vulgaris.}

L. glabra, rachi pedicellisque glanduloso-pubescentibus, caule a basi ramoso, ramisque erectis ascendentibusve, foliis sparsis, confertis, suhsessilibus, lineari-lanceolatis, acutis, integris, subtrinerviis, nervo medio crasso, racemo terminali, pedicellis flore brevioribus, erectis vel erecto-patentibus, laciniis calycinis ovato-lanceolatis, acutis, corolla multoties brevioribus, corollæ labio superiore erecto, laciniis apice rotundatis, laciniis lateralibus labii inferioris majorihus, medio minore subrotundo emarginato, calcare saccato-subulato, acuto, dependente, corolla subxquali, capsula erecta, oblonga vel ellipsoidea, ohtusa, ejus loculis apice dentibus sub-4 valvulæformihus ample dehiscentibus, seminibus compressis, tuberculosis, margine ala membranacea subareolata cinctis. (Parl. ms.).

Antirrhinum Linaria Linn. sp. plant. ed. 1. p. G16. Bert. 11. ital. 6. p. 36s; et anct.

Liuarid vulgaris Mill. gard. dict, ; et anct.

Linaria speciosa Ten.! ind. sem. hort. neap. 1827. Syll. 1. neap. p. 30\%. Terr.! 凡. Vult. syn.p. 12я. Rel. Terr. Lav. p. Íli.

Antirrhinum speciosum Bert. o. c. p. 370. Guss. enum. pl. Inar. p. 237.

Figure. - Fl.dan. 1. 9S2 (mala quoad fruclus). Fl. nap. 1. 159. f. 1 (mala). Reich. ic. /l. germ. 20. t. 64. f. 2.

B ilalica, floribus minoribus, ad summum 1 centim. longis.

Linaria italica Trev. in act. acad. car.-leop. Com. $\Omega$ com. is. p. 61. Rota prosp. A. Berg. p. 66. Hausm. /1. Tir. p. 6.93. De Vis. Sacr. cat. piant. Ven. p. 151. Ard. fl. Alpes-mar. p. 283.

Antirrhinum Bauhini Gaud. p. helv. 4. p. 154. Berl.o. c. p. sio.

Linaria angustifolia Rota prosp. piant. Pav. p. ?'Y0.

Limaria genistifolia Rota!' prosp. piant. Pav. p. 970. I'ir. fl. for. syll. p. 10\%.

Linaria Bauhini Zum. fl. ped. 1. p. 29?.

Linaria vulyaris var. italica Ces. Pass. (iil. comp. R. ital. p. "it. Arce comp. /l. ilal. p. 5lo.

Figure. - Reich. icon. lot. 1. 4?!. Icon. 11. germ. 90. 1. 6i. f. \% 
Stazione, Abitazione e Fioritura. - Nei coltivati, in luoghi incolti ecc. di tutta la Penisola. Piemonte, comune (All.): Torino (Re), Murazzano, comunissima (Ing.), Susa a Croale (Re), il No. varese, ovunque (Bir.). Lombardia: Buffalora (Bert.), il Pavese, comune (Nocc. Balb.), il Cremonese (Parl.!), il Comasco, comunissima (Com.), la val Tellina, ugualmente (Mass.), cosi a Bormio a 1220 metri (Parl.!), il Bergamasco (Rota), per esempio a Ponte di legno a 1320 metri (Parl. !), il Bresciano, frequentissima (Zersi), cosi a Bovegno a 750 metri, e sopra Collio a 1100 metri (Parl.!). Tirolo: Tione (Hausm.), Trento (Perini!), Bolzano, comune, e sale sul Ritten fino a 4000', Merano (Hausm.), la val Venosta presso Laas, valli di Fiemme e Fassa, Borgo, Roveredo ecc. (Hausm.). Veneto, frequente (De Vis. Sacc.): Belluno (Venzo!), Bassano (Bert.), gli Euganei(Trev.), Chioggia(Bert.), Venezia(Kellner!), il Friuli, ovunque (Pir. !). Istria: Trieste (Bert.), Istria meridionale (Freyn) e Fiume (Smith), comune. Emilia: il Modenese, comune, da Modena a luoghi alti dell'Appennino (Gib. Pir.), il Bolognese, volgare fino ai monti (Cocc.), cosi alla Porretta (G. Bert.), Faenza, ovunque (Cald.). Liguria, tutta (De Not.): il Nizzardo, rara (Ard.), Diano (Ricca!), Genova (Bert.), Chiavari (Delpino!). Toscana, comunissima dalla regione maremmana alla montana: la Lunigiana a Calice (Bert.) e Pontremoli (Parl.!), la Versilia (Simi!), il val di Serchio da Lucca (Pucc.) a Tereglio (Giannini!), il val d'Arno da Livorno (Bert.) e Pisa (Corinaldi!) a Boscolungo a 1300 metri e al Casentino (Parl.!), Siena!, Monte Pescali in Maremma (Aiuti!), la Capraia (Requien). Marche e Abruzzo: monte Catria (Piccinini!), Porto Civitanova (Cavanna!), Ascoli (Parl.!), Foce (Bert.), monti di Caramanico (Ces.). Pinglia: Gargano (Ten.!), Barletta (Bruni!), Otranto (Groves). Lazio: Corneto, Roma, abbondante (Rolli!), Terracina (Bert.). Campania : Roccamonfina (Terr.!), Picinisco, Dragoni, Pietravairano (Terr.), Napoli (Ten.), isola d'Ischia (Pasq.! ecc.). Basilicata : Castelgrande (Bert.), monte Vulture (T'err.!). Nasce inoltre in Corsica al nord dell'isola (Mars.), cosi a Bastia, da per tutto (Pucci!). La var. è ristretta all'Alta Italia: nel littorale Friulano, nel Mantovano (De Vis. Sacc.), in Tirolo nel paese dell'Adige (Bert.), a Bolzano, comune sulle pendici meridionali, in val Venosta sopra Schlanders (Hausm.) e presso Laas a piè della cima Gadria (Tappeiner!), nel Bergamasco (Rota), sopra Como a Garzola (Com.), presso Monza in più punti (Parl.!), nel Pavese a Carbonara (Rota!) e altrove, in Lomellina (Rota), in Piemonte in val Formazza, in val di Vedra 
(Bir.), a Mollia di val Sesia , a Val Savaranche (Carestia!), presso Aosta (Webh!), e verso il S. Bernardo (Gaud.), presso Susa (Parl.! ecc.) dove abbonda (Re), intorno a Torino! in diversi punti (Re), ad Alba (Bert.), da Borgo S. Dalmazzo al col di Tenda (Parl.!), a Viosenne (Ricca!), e in generale abbondante nelle Alpi marittime (Ard.). Fiorisce da gingno o luglio alla fine dell' autunno.

Distribuzione geografica. - In Europa, eccettuate le sue parti piủ settentrionali e più meridionali, in Songaria, in China, introdotta nella Nord-America.

Descrizione. - La radice è fusiforme, semplice o poco ramosa, flessuosa, bianchiccia, glabra. Il fusto è ramoso alla base, e tanto esso quanto i rami sono alti da uno a due piedi, diritti o ascendenti, cilindrici, verdi o rossicci in basso, glabri, eccetto in alto nella rachide la quale la spesso cortissimi peli glandolosi all'apice. Le foglie sono quasi sessili, molte, sparse, avvicinate tra loro, patenti, patentissime $n$ rivolte in giu, lineari-lanceolate, acute, intere, scanalate di sopra, con i margini alquanto rovesciati e con tre nervi, uno mediano molto grosso e due laterali, vicinissimi ai margini, poco manifesti. Il racemo è terminale, lungo da 2 a 4 o 5 pollici. I fori sono grandicelli, vicini in modo che si cuoprono in parte tra loro. Le brattee sono lanccolato-lineari, ottusette, verdi, quasi uguali ai pedicelli o poco piủ corte, alquanto rivolte in giui. I pedicelli sono lunghi due o tre linee, eretti, quasi cilindrici, verdognoli, eon qualche pelo glandoloso all'apice. Le lacinie del calice sono ovato-lanceolate, acute, verdi, glabre, con un nervo manifesto nella carena. La corolla è molte volte più lunga del calice: di color giallo pallido con la proninenza del palato giallo-dorata. Le lacinie del labbro superiore sono dirette in alto od in fwori, allungate, con l'apice tondeggiante ma non intere, concave di sotto; le lacinie del labbro inferiore sono disnguali, le due laterali sono maggiori, dirette in giii e in avanti, quasi quadre ma con l'angolo anteriore inferiore tondeggiante: la lacinia intermedia è piì piccola e piü corta, quasi tonda e come smarginata, diretta in avanti. Il palato $\dot{e}$ peloso, i peli non sono glandolosi, sono gialli o giallicci e si prolumgano in due linee nella parte interna del tulo. Lo sprone è lungo quasi yuanto la corolla, largo alla hase, assottigliato nel resto, acuto, pendente, un poco più carico del colore della corolla. I filamenti sono filiformi, bianchi, ghli anteriori o più lunghi un po'ingrossati alla hase dowe hanno pochi peli corti e bianchi: i posteriori sono glabri. Le antere sono gialle, biloculari, con le logge allungate, divarirate, conflucuti. 
Il polline è giallo. Il pistillo è poco più corto degli stami più lunghi. L'ovario è ellittico, schiacciato, glabro. Lo stilo è glabro, filiforme, un po' curvato in alto, dove è verdognolo, nel resto è bianco. Lo stimma è grossetto, quasi bilobo, verdognolo. La cassula è piủ del doppio più lunga del calice, allungata e quasi ellittica, ottusa, verdognola, biloculare. Ciascuna loggia si apre largamente all' apice per l'allontanamento di $\bar{\jmath}$ a 5 valve ottuse. I semi sono quasi schiacciati, nericci, con punti o tubercoli più scuri e cinti da un' ala membra. nacea scura e come areolata. (Parl. ms., descr. di pianta Fio. rentina).

Descrizione della var. - Pianta perenne?, alta da $1 \frac{1}{2}$ a 3 decimetri, glauca, glabra. La radice è fusiforme, con poche fibre delicate, bianchiccie. I fusti sono ascendenti o diffusi, cilindrici, rossicci in basso, glauchi nel resto, ramosi, con i rami sparsi, ascendenti, lunghi quasi quanto il fusto. Le foglie sono sparse, eretto-patenti, o patenti, piuttosto piccole, lineari, o lineari-lanceolate, acute, piane o appena concavo-solcate di sopra, con un nervo longitudinale poco rilevato di sotto, con i margini interi e ottusi, glauche in ambedue le facce. I fiori sono più piccoli di quelli della Linaria vulgaris, pochi in racemi corti (che si allungano appena nel frutto), all'apice del fusto e dei rami. 11 peduncolo e i pedicelli sono glabri, interamente privi di peli glandolosi, questi sono eretto-patenti, sempre più lunghi del calice, cilindrici, giallicci, accompagnati da una brattea poco più corta del pedicello, diretta in giù, lanceolato-lineare, simile alle foglie superiori ma più piccola. Il calice è diviso fin sopra la base in cinque lacinie patenti, lanceolate, acute, o quasi acute, appena convesse nel dorso e ivi verdognole, con uno stretto margine bianchiccio, glabre. La corolla è di un color giallo chiaro con le gibbosità che chiudono il palato di color appena più carico. Il tubo è corto, larghetto. Il labbro superiore è diviso fin quasi alla metà in due lobi ottusi, eretti, ha una carena oltusa in avanti dal seno dei lobi alla base con le parti laterali e i lobi diretti in dietro; il labbro inferiore è poco più corto del superiore, trilobo, con il lobo medio più piccolo, diretto in avanti, un 'po'convesso di sopra, quasi ovale-quadrato, appena smarginato all' apice: i lobi laterali sono due 0 tre volte maggiori, tondeggianti in avanti, diretti in avanti ed in giù, si cuoprono l'un l'altro con il margine interno e sono ivi coperti dal lobo di mezzo, e il palato è gonfio con due piccole gibbosità ottuse all'apice, dove corrisponde alla gola è tutto coperto di lunghi peli grossetti e giallicci diretti un po'in avanti. Lo sprone è 
lungo quanto il labbro superiore, lesiniforme, diretto in giủ, curvato alquanto in avanti, del colore della corolla. Gli stami sono quattro, didinami, inclusi, inseriti alla base del tubo della corolla. I filamenti sono più grossi in basso, cilindrici in alto, un po' curvi, glabri, bianchicci. Le antere hanno due logge bislunghe, ottuse, giallicce, glabre, che si aprono per una fessura comune. Il pistillo è lungo quasi quanto gli stami piì lunghi. L'ovario è quasi tondo, schiacciato lateralmente, verdognolo, glabro, ha alla base un disco circolare, grossetto, verde. Lo stilo è lungo, cilindrico, eretto, un po'curvo, bianchiccio, glabro. Lo stimma è quasi in capolino, papilloso, bianchiccio, abbracciato in gran parte dall' apice dello stilo, ivi concavo. La cassula è quasi tonda, appena piủ stretta alla base, con un piccolo ombelico all'apice, si apre per 8 o 4 denti disuguali all' apice in ciascuna valva. I semi sorio piccolicon un largo margine in modo che con questo pajono quasi tondi, schiacciati, nericci, con pic. coli tubercoli nelle facce, eccetto il margine. (Parl. ms., descr. di pianta di Bellusco in Lombardia).

Osservazioni. - Quesla pianta varia molto per la larghezza delle foglie, e per la grandezza dei fiori, anche in un medesimo saggio: vedasi quanto su di ciò e sulla pelurie lia scritto Bentham in Cand. prodr. 10. p. 273. La figura di Tenore rappresenta una forma estrema per sviluppo di parti, e forse esagerata, alla quale non corrispondono i saggi napoletani della Linaria speciosa, che sono tutta la ordinaria L. vulgaris. La $L$. ilalica invece è varietà ben distinta, che facilinente si potrebbe ritenere specie a sé, se possedesse qualche altro carattere differenziale, e se quelli che possiede nella grandezza c telle proporzioni del fiore e del frutto non fossero affetti da variabiliti.

\section{ANTHERHNUM.}

Antirhinum Tourn. inst. p. 167. 1. is. Chav. mon. Ant. p. 79. 1. 3. Gen. pl. 1. germ. 5. 1. 32. Benth. Hook. gen. pl. 2. p. 93i. Ces. P'ass. Cib. comp. 月. ital. p. 3\%15. 1. 5\%. 1. ?.

Calys obliquus, quinquepartitus, laciniis subagualibus. Corolla personata, hypogsua, tubo amplo, compressiusculo, basi saccato, intus antice biscratim piloso, labio superiore bilido, lacinis crects, inferiore patente trilobo, lobo medio minori, antice concaviusculo, 
palato amplo faucem claudente. Stamina corollæ tubo inserta, inclusa, 4 fertilia, didynama, quintum sterile brevissimum vel nullum. Filamenta basi ascendentia et subincrassata, reliqua parte subfiliformia, erecta. Antheræ biloculares, loculis oblongis, inferne divergentibus, confluentibus. Pollen minutissimum, subsphæricum, læve. Ovarium oblique ovatum vel pyriforme, biloculare, placentis septo adnatis, multiovulatis. Ovula minuta, anatropa. Stylus longus, subteres, apice curvatus, subsigmoideus. Stigma subbilobum. Capsula chartacea, oblique ovata vel pyriformis, bilocularis, loculo postico apice poro unico, antico poris geminis collateralibus prope apicem, omnibus multivalvatis, dehiscente. Semina minuta vel minutissima, plurima, oblique truncata, squamuloso-foveolata. (Parl. ms.).

Portamento. - Erbe erette, una annua con fiori ascellari pic. coli o mezzani, l'altre perenni con fiori grandi, gialli o rossi, vistosi, brevemente pedicellati, raccolti in grappoli terminali. Le foglie sono sparse, lanciolate o lineari.

\section{Antirehinum Drontium.}

A. annuum, glabrum superne glanduloso-pilosum, foliis anguste lineari-lanceolatis, floribus remotis, corolla (rosea) 1 centim. circiter longa, lobis calycinis linearibus, corollam subsuperantibus. Antirrhinum Orontium Linn. sp. plant. ed. 1. p. 617. Bert. fl. ital. 6. p. 376; et auct. omn.

Figure. - Barr. ic. 651, 652. Reich. ic. fl.germ. 20.t.57.f.1. $\beta$ grandiflorum, floribus duplo maioribus.

Antirrhinum Orontium var. grandiflorum Chav. mon. p. 90. Mor. fl. sard. 3. p. 195. Ces. Pass. Gib. comp. fl. ital. p. 346.

Antirrhinum Orontium var. calycinum Arc. comp. fl. ital. p. 508.

Figure. - Hoffm. Link fl. port. t. 52. Chav. o. c. t. 4. Reich. l. c. f. 2.

Stazione, Abitazione e Fioritura. - Per tutta l'Italia, nei luoghi coltivati, ora comunissimo, ora meno comune o anche raro. Piemonte: Torino, piuttosto raro, Condove, abbondante (Re), Susa (Parl.! ecc.), Mondovi (Ing.), Alba (Colla), Novara (Bir.). Ticino, rarissimo: Lugano (Com.). Lombardia: val Intelvi (Com.), val Tellina in valle Malenco e sopra Ponte (Ilass.), Impari in Brianza, e Bellusco (Parl.!), Pavia, comune (Nocc. Balb.), Grumone nel Gremonese (Parl.!), il Bergamasco (Rota), il Bresciano, frequente 
(Zersi). Tirolo: lago di Garda, Roveredo, Trento, Doss Trent, Fiemme a Capriana, Eppan (Hausm. ece.). Veneto: Revere (Paglia!), il Veronese, comune (Poll.), gli Euganei (Trev.), Venezia (Zan.), Bassano (Bert.), Fonzaso (Ball!), Sagrado (Pir.), Monfalcone (Pir.!). Istria: Trieste (Bert.), Pola ecc., qua e là (Freyn), Fiume (Sınith). Emilia: Modena, Ventasso, Fiumalbo (Gib. Pir.), Porretta (G. Pert., Cocc.). Romagna: Faenza (Cald.!). Liguria, ovunque (De Not.): Nizza (Durando! ecc.), Villafranca (Bert.), llentone (Aril.), Tenda (Ungern-Sternberg!), Genova (Bert.), Chiarari (Delpino!). Toscana, comune assai, dalla resione maremmana alla submontana ed anche (scarseggiando) alla montana: Pontremoli (Parl.!), Sarzana, Massa (Kert.), la Versilia (Sini !) sino all'Altissimo (Ad. Targioni!), Lucca (Calandrini! ecc.), Bagni di Lucca (Parl.!), Pisa (Reboul! ecc.), Casciana (Bastianini!), Firenze!, Prataglia in Casentino a 2200' (Parl.!), Siena (Campani!), S. Vincenzo e Campiglia (Parl.!), Pitigliano (Santi), isole di Gorgona (P. Savi), di Capraia (Mor. De Not.), dell'Elba (Picciòli! ecc.), di Hontecristo (Watson-Taylor!) e del Giglio (Parl.!). Marche: Urbino (Serpieri!), Ancona (Bert.), Ascoli (Parlatore!). Roma (Gennari! cce.), comune (Seb. Maur.). Campania: Mondragone (Terr.!), Tractto, inonte Marsico ecc. ('Terr.), Napoli, ovunque (Ten., I'asq.), isola d'Ischia, orunque (Guss.). Gargano a Monte S. Angelo (Ten.). Basilicata: sul Vulture, ovunque (Terr.). Calabria : Palizzi (Arc.!), Reggio (Aiuti!). Corsica, comune (Mars.): Bastia, Calvi (Soleirol!). Sardegna, comune (Mor.!): Caprera, S. Stefano (Genn.), Pula (Bert.). Sicilia, ovunque (Guss.): Castelbuono (Minả !), Messina (Seguenza! ecc.), Catania (Parl.! ecc.), Siracusa (Cassia!), Avola (Biancal), Caltagirone (Tar. Gerlı.), le Eolic, comunissimo (Loj ), Ustica (Caleara!), Favignana, Pantellaria (Guss.), Linosa (Aiuti!). Malta (Grech! ecc.). La var. i rara, e speciale al mezzogiorno. Finrisce dall' a prile, maggio o giugno insino all'autunno.

Distribuzione geografica. - Nell' Europa merilionale e media e in parte nella settentrionale, nell' Affrica settentrionale fino alle Ca. narie e all' Abissinia, nell' Asia occidentale fino all' Arabia e all' Imalaia, ora introdotto neg̣li Stati-Uniti di America.

\section{Antrrhinum siculum.}

A. caule gracili, ramosissino, ramis disaricatis, superne subpu. bescenti-glandulosis, foliis lineari-lanceolatis, linearibusve, acutis, 
uninerviis, glabris, patentibus vel patentissimis, oppositis ternisve, superioribus sparsis, foliis floralibus linearibus, acutis, glabris vel glabriusculis, pedicello longioribus subæqualibusve, floribus paucis laxe racemosis, pedicellis erectis calyceque pubescenti-glandulosis, laciniis calycinis orato-lanceolatis, acutinsculis, apice patulis, labii corollæ inferioris lobo medio oblongo-lanceolato obtuso, capsulis oblique ovatis, poris tribus hiantibus parviusculis, seminibus ninutissimis squamulosis, foveolatis. (Parl. ms.).

Antirrhinum siculum Ucria pl. ad Linn. op. add. in Roem. arch. bot. 1. p. 69.Zer. fl. mel. thes. p. 43. Bert. fl. ital. 6.. p. 375. Tar. Gerb. cat. pl. calat. p. 30. Grech Del. fl. mel.p. 27. Ces. Pass. Gib. comp. fl. ilal. p. 346. Arc.! comp. fl. ital. p. 508. Nic.! prodr. 1. mess. p. 344.

Antirrhinum assurgens Bianca! in giorn. accad. gioen.

Figura. - Barr. ic. 637.

Stazione, Abitazione e $\mathbf{F}$ ioritura. - Sui mụri e nelle macerie dell' Italia più meridionale: in Calabria a Trucoli (Ten.), e Reggio (Pasquale! ecc.); in Sicilia a Messina, comune (Nic.! ecc.), Catania (Magnaguti !), Lentini (Huet!), Caltagirone, comune (Tar. Gerb.), Siracusa (Bert.), Avola (Bianca!), Ragusa (Guss.), Girgenti (Aiuti! ecc.), Sciacca, Trapani, Carini (Guss.), Palerno, ovunque (Par. latore! ecc.); in Malta (Todaro Calcara! ecc.). Fiorisce quasi tutto l'anno.

Distribuzione geografica. - Nasce ancora in Spagna, se è da riferirsi a questa specie l'Antirrhinum Barrelieri Bor.

Descrizione. - Il fusto è cilindrico, glabro, piuttosto delicato, alto da un piede a un piede e mezzo, rossiccio o in parte verde, diritto 0 ascendente, alquanto flessuoso ramoso, con i rani aperti dei quali quelli fioriferi giungono ad uguagliare il fusto, delicati, glabri o verso alto con pochi peli corti, bianchi e glandolosi all'apice. Le foglie sono lineari-lanceolate o quasi lineari, strette, acute, glabre, di un verde pallido, con un sol nervo longitudinale e con venette laterali appena visibili, aperte o apertissime, opposte o terne, le superiori però sono sparse. I fiori sono pochi, circa cinque, in racemi all'apice dei rami e del fusto. Ciascuno di essi è accompagnato da una foglia fiorale stretta, lineare, glabra o con qualche raro pelo, poco più lunga e quasi ugnale al pedicello. Questo è lungo circa due linee, eretto, cilindrico, pubescente, con $\mathrm{i}$ peli corti e glandolosi all'apice. Il calice è campaniforme, diriso fin quasi alla base in cinque lacinie ovato-lanceolate, alquanto acute, con l'apice un po'ri- 

11 prezzo di questa SECoxd a Parte del Sasto Volume ie di $L .10$. Si trova in vendita presso il prof. Caruel al R. Istituto di Studi Superiori in Firenze, e presso i principali librai d'Italia.

Le altre parti dell'opera sinora comparse sono le seguenti:

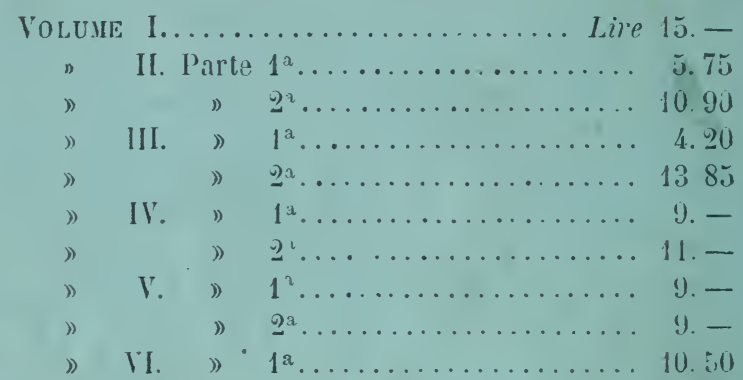

Lo sconto ai librai è del 20 jer cento.

\title{
NUOTO GIORNALE BOTANICO ITALIANO
}

\author{
DinetTo da T. CARLEL.
}

Esce quattro volte all' anno in fascicoli che riuniti formano un volume di almeno 20 fogli di stampa, con tavole e figure nel testo. Il prezzo di associazione è di Lire 20 all'anno. Lo sconto ai librai è del 15 per cento. $\dot{E}$ in pubblicizione il volume XVII.

\section{Altre Opere del prof. Caruel in vendita.}

Illustratio in hortum siccum Andreae Cassalpini. - Firenze, 1859. In-16 di pag. xII-123........... Lire 2.-

-Prodromo della flora toscana, ossia catalogo metodico delle piante che nascono selvatiehe in Toscana, o che vi sono estesamente coltivate. - Firull$z e, 1 s 60-6 \dot{t}$. In-s di pag. xxII-767................ 10. -

Guida del botanico principiante, ossia compendio di consigli ed istruzioni per quelli che si vogliono iniziare nello studio della botanica. - Fircnze, 1866. In-16 di pag. 10 ', con fi gure .....................

Statistica botanica della Toscana, ossia saggio di studi sulla distribuzione geografica delle piante toscane. - Fir nze, 1871. In-s di pas. 374 e una carta.....

La morfologia vegetale. - Pis 1,1373 . In- 8 di pag. 433 ,

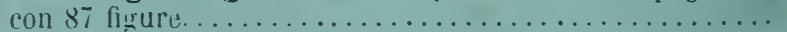

Pensieri sulla tassinomia botanica. - Ruma, 18si. In-1

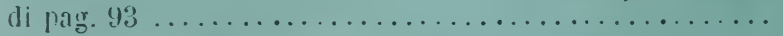

L'erborista italiano, chiave analitica per aiutare a trovare sollecitamente il nome delle piante che nascono selvatiche in Italia. - Pisa, 18ð3. In-16 di pag. $16 \%$. 






\section{New York Botanical Garden Library}

QK 332 .P33 v. 6

Parlatore, Filippo/Flora italiana; ossia || || || | || ||||||||||||| || ||| 


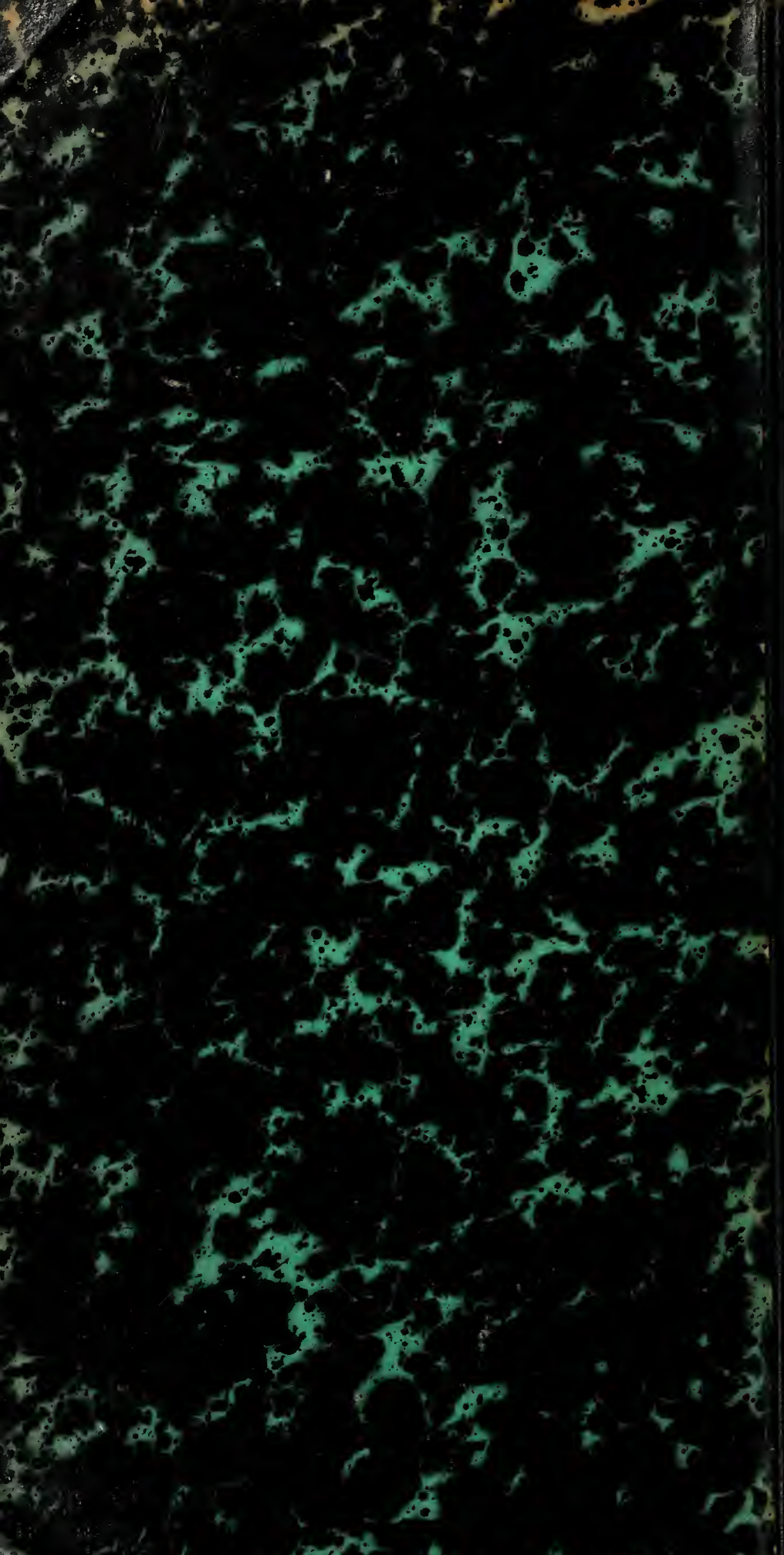

